\title{
C-H Activation by Iron(III), Manganese(II) and Rhoda(III)electro Catalysis
}

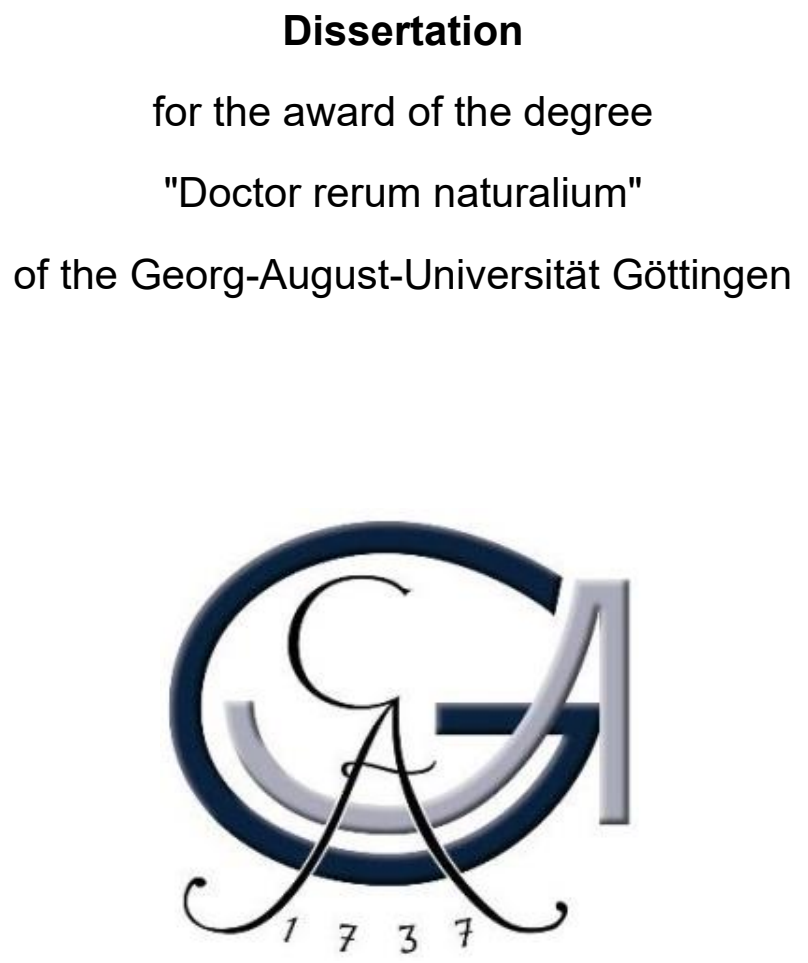

within the doctoral program of chemistry

of the Georg-August-University School of Science (GAUSS)

submitted by

Zhigao Shen

from Hunan, China

Göttingen, 2020 



\section{Thesis Committee}

Prof. Dr. Lutz Ackermann, Institute of Organic and Biomolecular Chemistry

Prof. Dr. Konrad Koszinowski, Institute of Organic and Biomolecular Chemistry

\section{Members of the Examination Board}

Reviewer: Prof. Dr. Lutz Ackermann, Institute of Organic and Biomolecular Chemistry

Second Reviewer: Prof. Dr. Konrad Koszinowski, Institute of Organic and Biomolecular Chemistry

\section{Further Members of the Examination Board}

Prof. Dr. Dr. h.c. Lutz-F. Tietze, Institute of Organic and Biomolecular Chemistry

Prof. Dr. Manuel Alcarazo, Institute of Organic and Biomolecular Chemistry

Dr. Michael John, Institute of Organic and Biomolecular Chemistry

Prof. Dr. Johannes C. L. Walker, Institute of Organic and Biomolecular Chemistry

Date of the Oral Examination: 02. 12. 2020 



\section{Contents}

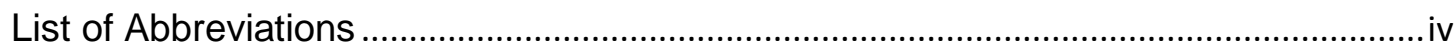

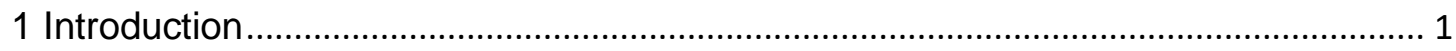

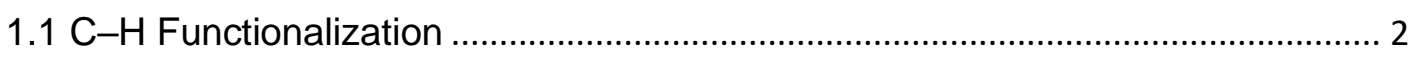

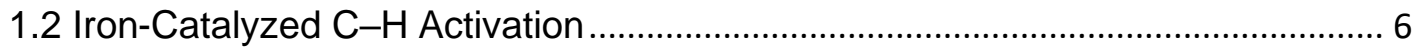

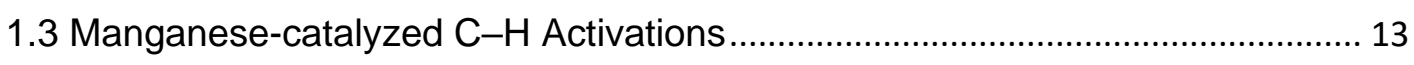

1.3.1 Manganese(I)-catalyzed C-H Functionalization......................................... 14

1.3.2 Low-valent Manganese(II)-catalyzed C-H Functionalization ...................... 22

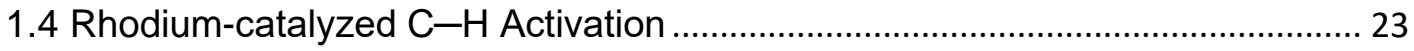

1.4.1 Rh-catalyzed Inner-sphere C-H Activation................................................ 24

1.4.2 Rhodaelectro-catalyzed C-H Activation......................................................... 37

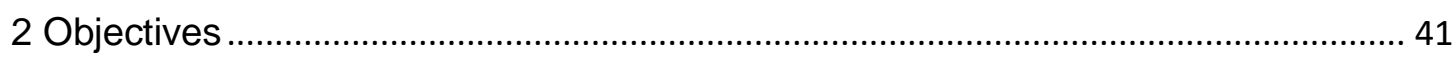

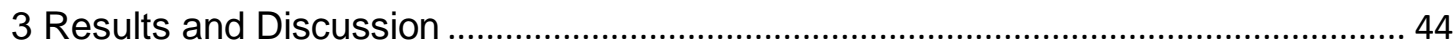

3.1 Triazole-Enabled C-H Activation of Benzylamines by Iron-Catalysis................ 44

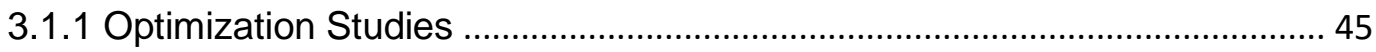

3.1.2 Scope of Triazole-Assisted C-H Mthylation ................................................ 46

3.1.3 KIE studies of Iron-catalyzed Methylation.................................................... 53

3.2 Manganese-catalyzed Pyridinyl C-H Alkylation.................................................. 55

3.3.1 Optimization of Pyridinyl Alkylation ............................................................ 55

3.2.2 Scope of Manganese Catalyzed Alkylation ................................................ 58

3.2.3 Mechanistic Studies of Manganese Catalyzed Alkylation ............................ 65

3.3 Rhodaelectro-Catalyzed Domino Alkyne Annulations to access Aza-Polycyclic

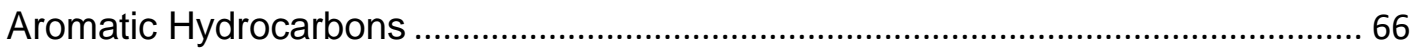

3.3.1 Optimization of Rhodaelectro-Catalyzed Domino Annulations ..................... 66

3.3.2 Scope of Rhodaelectro-catalyzed C-H Activation and Annulation Cascade 68

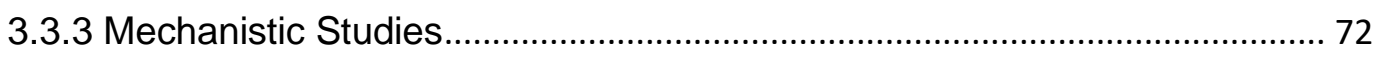

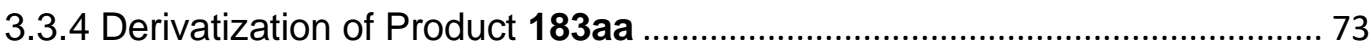


3.4.1 Optimization Studies for the Switchable Dienylation and Cyclopropylation74

3.4.2 Scope of the Switchable Dienylation and Cyclopropanylation..................... 80

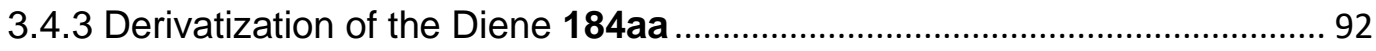

3.4.4 Mechanistic Investigation of Cyclopropylation and Dienylation ................... 93

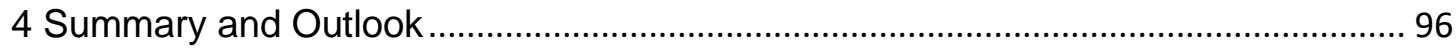

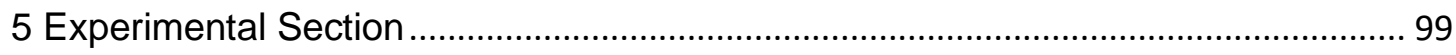

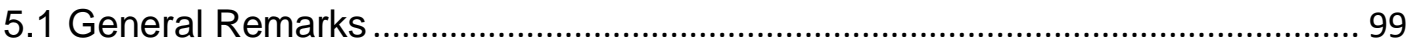

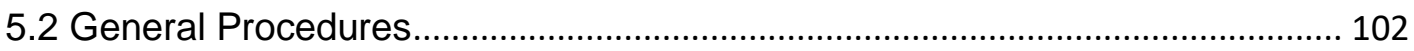

5.2.1 General Procedure A: Iron-catalyzed C-H Methylation.............................. 102

5.2.2 General Procedure B: Iron-catalyzed C-H Arylation.................................. 102

5.2.3 General Procedure C: Manganese-catalyzed Secondary Alkylation........ 103

5.2.4 General Procedure D: Manganese-catalyzed Primary Alkylation ............. 103

5.2.5 General Procedure E: Rhodaelectro-catalyzed Domino alkyne annulation

5.2.6 General Procedure F: Rhodaelectro-Catalyzed Dienylation ...................... 104

5.2.7 General Procedure G: Rhodaelectro-Catalyzed Cyclopropylation............ 104

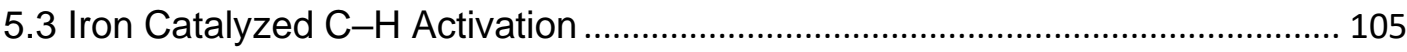

5.3.1 Experimental Procedures and Analytical Data ............................................ 105

5.3.2 Iron-catalyzed C-H methylation of chiral benzamide (S)-178a ................ 122

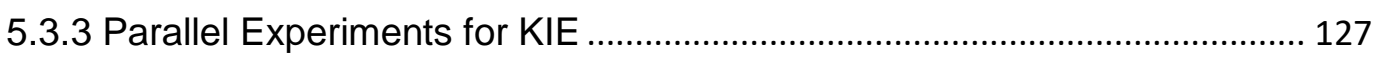

5.3.4 The Removal of Directing Group ............................................................. 128

5.4 Pyridinyl C-H Alkylation Catalyzed by Mn(II) ................................................. 132

5.4.1 Experimental Procedure and Analytical Data ........................................... 132

5.4.2 Racemization Free Alkylation of 88a ....................................................... 154

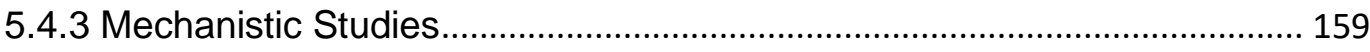

5.5 Rhodaelectro-Catalyzed Domino Alkyne Annulations to Access Aza-Polycyclic

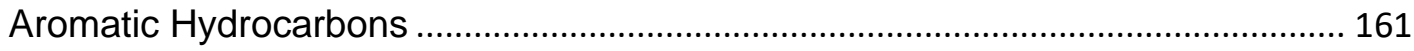

5.5.1 Experimental Procedure and Analytical Data........................................... 161

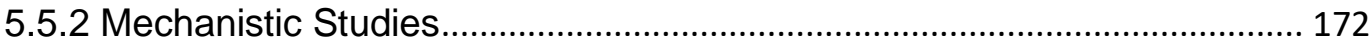

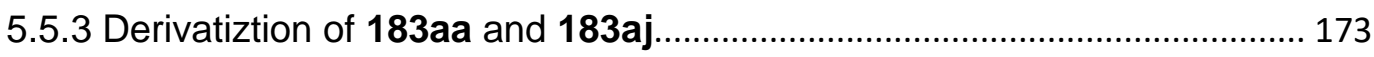


5.6 Rhodaelectro-catalyzed switchable Indole dienylation/ cyclopropylation 174

5.6.1 Experimental Procedure and Analytical Data............................................. 174

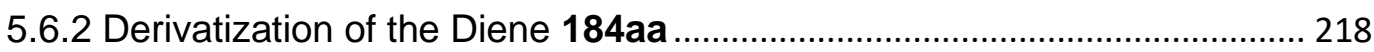

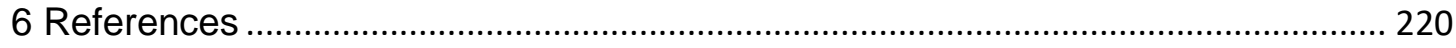

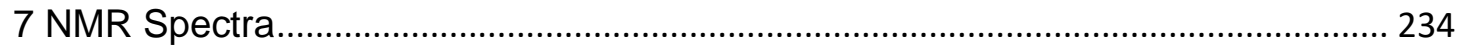




\section{List of Abbreviations}

\begin{tabular}{|c|c|}
\hline Ac & acetyl \\
\hline acac & acetylacetonate \\
\hline Alk & alkyl \\
\hline AMLA & ambiphillic metal-ligand activation \\
\hline aq & aqueous \\
\hline $\operatorname{Ar}$ & aryl \\
\hline atm & atmospheric pressure \\
\hline BIES & base-assisted internal electrophilic substitution \\
\hline $\mathrm{Bn}$ & benzyl \\
\hline Boc & tert-butyloxycarbonyl \\
\hline $\mathrm{Bu}$ & butyl \\
\hline Calc. & calculated \\
\hline cat. & catalytic \\
\hline CCE & constant current electrolysis \\
\hline CMD & concerted metalation deprotonation \\
\hline $\mathrm{Cp}$ & cyclopentadienyl \\
\hline$C p^{*}$ & pentamethylcyclopentadienyl \\
\hline CypCO ${ }_{2} \mathrm{H}$ & cyclopentanecarboxylic acid \\
\hline $\mathrm{CV}$ & cyclic voltammetry \\
\hline Cy & cyclohexyl \\
\hline$\delta$ & chemical shift \\
\hline$d$ & doublet \\
\hline DCE & 1,2-dichloroethane \\
\hline dd & doublet of doublets \\
\hline DG & directing group \\
\hline DMF & $N, N$-dimethylformamide \\
\hline DMSO & dimethylsulfoxide \\
\hline
\end{tabular}




\begin{tabular}{|c|c|}
\hline $\mathrm{dt}$ & doublet of triplets \\
\hline$e e$ & enantiomeric excess \\
\hline El & electron ionization \\
\hline equiv & equivalent \\
\hline ESI & electrospray ionization \\
\hline Et & ethyl \\
\hline g & gram \\
\hline GC & gas chromatography \\
\hline GF & graphite felt \\
\hline $\mathrm{h}$ & hour \\
\hline HFIP & 1,1,1,3,3,3-hexafluoro-2-propanol \\
\hline HRMS & high resolution mass spectromethy \\
\hline $\mathrm{Hz}$ & hertz \\
\hline$i$ & iso \\
\hline IMes & 1,3-bis(2,4,6-trimethylphenyl)imidazole-2-ylidene \\
\hline $\operatorname{IPr}$ & 1,3-bis(2,6-iso-propylphenyl)imidazole-2-ylidene \\
\hline IR & infrared spectroscopy \\
\hline$J$ & coupling constant \\
\hline KIE & kinetic isotope effect \\
\hline $\mathrm{L}$ & ligand \\
\hline$m$ & meta \\
\hline $\mathrm{m}$ & multiplet \\
\hline M & molar \\
\hline mol & mole \\
\hline$[\mathrm{M}]^{+}$ & molecular ion peak \\
\hline $\mathrm{mA}$ & Milliamper \\
\hline $\mathrm{Me}$ & methyl \\
\hline Mes & mesityl \\
\hline $\mathrm{mg}$ & milligram \\
\hline
\end{tabular}




\begin{tabular}{|c|c|}
\hline $\mathrm{MHz}$ & megahertz \\
\hline $\min$ & minute \\
\hline $\mathrm{mL}$ & milliliter \\
\hline $\mathrm{mmol}$ & millimolar \\
\hline M.p. & melting point \\
\hline MS & mass spectromethy \\
\hline$m / z$ & mass to charge ratio \\
\hline NMR & nuclear magnetic resonance \\
\hline 0 & ortho \\
\hline$p$ & para \\
\hline $\mathrm{Ph}$ & phenyl \\
\hline PMP & para-methoxyphenyl \\
\hline Piv & pivaloyl \\
\hline ppm & parts per million \\
\hline $\operatorname{Pr}$ & propyl \\
\hline Py & pyridyl \\
\hline pym & pyrimidyl \\
\hline$q$ & quartet \\
\hline$Q$ & 8-aminoquinoline \\
\hline rt. & room temperature \\
\hline s & singlet \\
\hline sat. & saturated \\
\hline SET & single electron transfer \\
\hline SPS & solvent purification system \\
\hline$t$ & tert \\
\hline $\mathrm{t}$ & triplet \\
\hline$T$ & temperature \\
\hline TFE & 2,2,2-trifluoroethanol \\
\hline THF & tetrahydrofuran \\
\hline
\end{tabular}




$\begin{array}{ll}\text { TLC } & \text { thin layer chromatography } \\ \text { TM } & \text { transition metal } \\ \text { TMS } & \text { trimethylsilyl } \\ \text { TS } & \text { transition state } \\ \text { V } & \text { Volt } \\ X & \text { (pseudo-)halide }\end{array}$




\section{Introduction}

Urea was first isolated in urine in $1773 .^{[1]}$ And almost half a century later, the German scientist Friedrich Wöhler from Göttingen realized the synthesis of urea by heating an aqueous solution of ammonium cyanate which is regarded as the first organic compound obtained from inorganic mixtures. ${ }^{[2]}$ Since then, organic chemistry has been viewed as an essential part of chemistry. Now, urea is widely used in agricultural industry improving the yields of crops, which helped to relieve the starvation all around the world significantly. Over the last century, different types of reactions have been developed. So, with all these chemical methods in hand, scientists synthesized different kinds of polymers, life-saving drugs, pesticides, dyes and finished the total synthesis of many natural compounds. Due to resource and energy consumption, waste generation, byproducts and the use of dangerous chemicals, chemistry continues to be regarded as one contributing factor to pollution. A new concept, sustainable development, was proposed by Brundtland that meets the present needs without compromising the ability of future generations to meet their own needs. ${ }^{[3]}$ It's urgent to remove or at least to minimize the disadvantages.

Thereafter, the development of more environment-friendly, resource-, ${ }^{[4]}$ atom- ${ }^{[5]}$ and stepeconomic $^{[6]}$ reactions are highly desirable. Many interests in chemical academia have been paid to Domino reactions ${ }^{[7]}$ which can construct several $\mathrm{C}-\mathrm{C}$ and $\mathrm{C}-\mathrm{Het}$ bonds in a single step and some reactions also have been modified to work in green solvents, such as biomass-derived glycerol. ${ }^{[8]}$ With the insightful understanding of chemical processes and the development of new ligands, stoichiometric reactions can work in catalytic version with high efficiency and the temperature of some reactions can be decreased dramatically. In addition, $\mathrm{CO}_{2}$ can also be used to synthesize value-added products, polymers and widely used chemical materials. ${ }^{[9]}$ Throughout the development of chemistry, chemists are guided by the principles 12 Principles of Green Chemistry proposed by Anastas and Warner. ${ }^{[10]}$ 


\subsection{C-H Functionalization}

Organic chemistry mainly studies the cleavage and formation of bonds in a practical and selective fashion, and the formation of carbon-carbon $(\mathrm{C}-\mathrm{C})^{[11]}$ and $\mathrm{C}-$ Heteroatom $(\mathrm{C}-$ Het $)^{[12]}$ bonds is the most important part, since it can serve as a valuable tool for scientists to design and synthesize different kinds of functional materials and bioactive molecules. Original works in coupling reactions were realized by Glase ${ }^{[13]}$ in the late of $19^{\text {th }}$ century and Ullmann ${ }^{[14]}$ in the early of $20^{\text {th }}$ century using stoichiometric or catalytic amounts of copper. Now highly efficient transition-metal catalyzed organic reactions have made a huge impact on organic transformations for $\mathrm{C}-\mathrm{C}$ and $\mathrm{C}-\mathrm{Het}$ bond formations. In the recent decades, significant progress have been further witnessed in this area by the invention of a variety of named cross-coupling reactions, such as the Kumada-Corriu-coupling, ${ }^{[15]}$ Mizoroki-Heck-coupling, ${ }^{[16]}$ Negishi-coupling, ${ }^{[17]}$ Stille-coupling, ${ }^{[18]}$ Hiyama-coupling, ${ }^{[19]}$ Suzuki-Miyaura-coupling, ${ }^{[20]}$ and Sonogashira-coupling ${ }^{[21]}$ reactions. Thus, transition metal-catalyzed cross-coupling reactions performed as a powerful tool in organic synthesis, with applications ranging from the construction of natural product and useful materials to the modifications of biologically active chemicals, ${ }^{[11,12]}$ and their significance was further reflected by Heck, Negishi and Suzuki being collectively awarded with the Nobel Prize in chemistry in 2010. ${ }^{[23]}$

Based on transition-metal catalysis, this newly acquired ability to forge carbon-carbon bonds between or within functionalized and often sensitive substrates provided new opportunities, particularly in total synthesis but also in medicinal chemistry as well as in chemical biology and nanotechnology. Prominent among these processes are the palladium-catalyzed $\mathrm{C}-\mathrm{C}$ bond-forming reactions. The historical, mechanistic, theoretical, and practical aspects of these processes have been amply discussed. Indeed, these protocols have revolutionized organic syntheses, albeit a few problems still exist. First, the use of pre-functionalized starting materials is needed, such as the organic (pseudo)halides. And even for the widely used organic nucleophiles, multiple synthetic steps, difficulty in storing and handling make them user unfriendly, e.g. $R M g X, R_{2} Z n$, and toxic $R_{3}{ }_{3} R S n$. 
Therefore, the selective $\mathrm{C}-\mathrm{H}$ functionalization can serve as an elegant tool to diminish these problems, ${ }^{[24]}$ combining the broad practicability of cross-couplings with the nature of green chemistry-atom economy and environmentally friendly methods (Scheme 1.1). Moreover, two-fold $\mathrm{C}-\mathrm{H}$ dehydrogenative activation also contribute to the formation of $\mathrm{C}-\mathrm{C}$ bonds while external oxidants are required in the dehydrative step. ${ }^{[25]}$

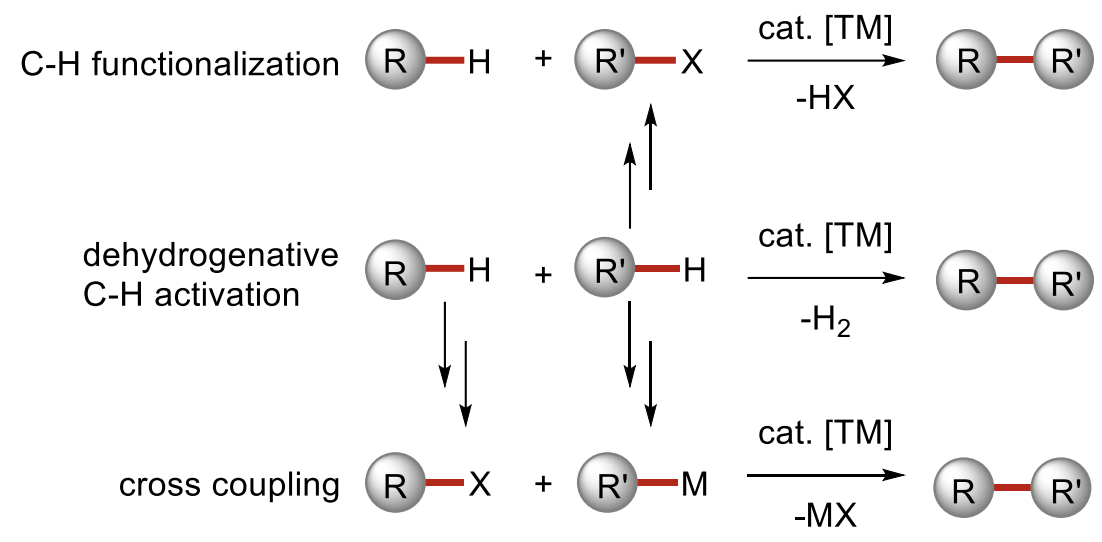

Scheme 1.1. Comparison of traditional cross-coupling vs. C-H activation.

The last thirty years have seen many examples of $\mathrm{C}-\mathrm{H}$ activation at different metal centers, usually with good regio- and chemoselectivity and under mild conditions. The selective transformation of ubiquitous but inert $\mathrm{C}-\mathrm{H}$ bonds to other functional groups has farreaching practical implications, ranging from more efficient strategies for fine chemical synthesis to the replacement of current petrochemical feedstocks by less expensive and more readily available alkanes. ${ }^{[26]}$ All the potential practical applications have inspired chemists to study how these organometallic reactions occur, and what their inherent advantages and limitations for practical alkane conversion and late-stage functionalization are. As the transition metal-facilitated cleavage of the $\mathrm{C}-\mathrm{H}$ bonds is the common key step in the above-mentioned $\mathrm{C}-\mathrm{H}$ functionalization strategies, it has been heavily examined. Excluding outer-sphere mechanisms, such as carbene/nitrene insertions ${ }^{[27]}$ or radical reactions $^{[28]}$, the bond dissociation proceeds generally via five different pathways, depending on the nature of the metal, the ligands and oxidation states. ${ }^{[29]}$ These methods (Scheme 1.2) are oxidative addition, electrophilic substitution, $\sigma$-bond metathesis, 1,2addition and base-assisted metalation. Electron-rich complexes of late transition metals 
are prone to cleave the inert $\mathrm{C}-\mathrm{H}$ bonds by oxidative addition, while this mode of action is unfavorable for early transition metals. ${ }^{[29 b]}$ Most late transition metals in higher oxidation states often act as a Lewis acid to cleave $\mathrm{C}-\mathrm{H}$ bond by an electrophilic substitution mode. The $\sigma$-bond metathesis is observed for early transition metals which cannot undergo oxidative addition. Metals containing an unsaturated $\mathrm{M}=\mathrm{X}$ bond tend to undergo $\mathrm{C}-\mathrm{H}$ activation via 1,2-addition. This fashion can be found in early transition metals.

a) oxidative addition

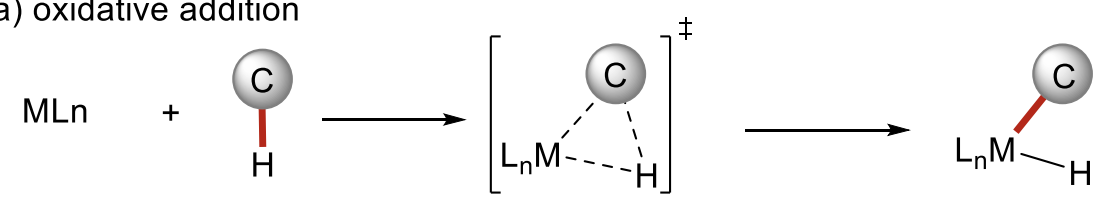

b) electrophilic substitution

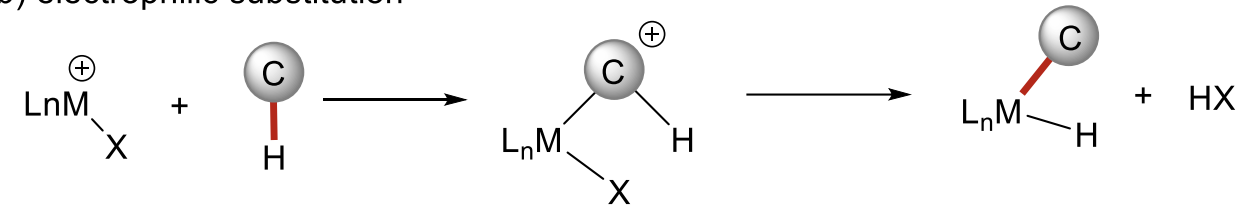

c) $\sigma$-bond metathesis

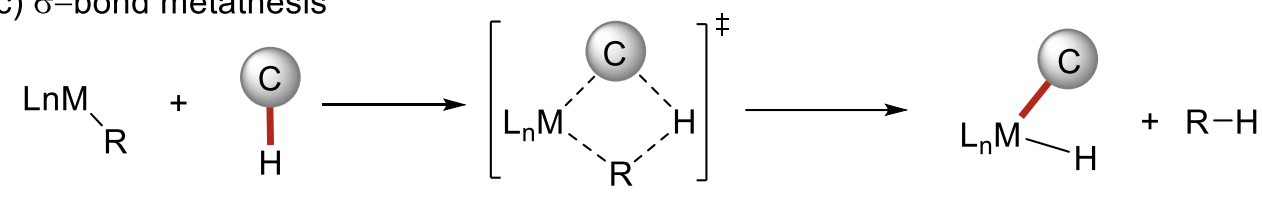

d) 1,2-addition

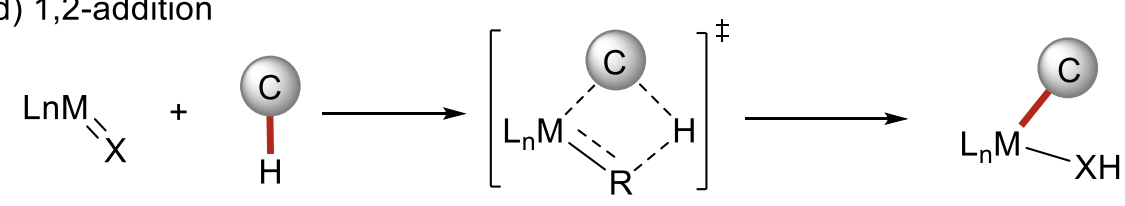

e) base-assisted metalation

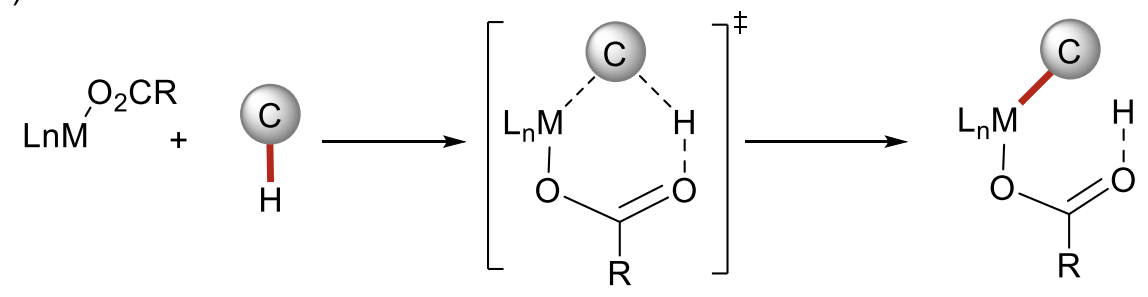

Scheme 1.2. Mechanistic pathways for the $\mathrm{C}-\mathrm{H}$ activation.

Besides the mechanistic scenarios, many examples proceed via the base-assisted $\mathrm{C}-\mathrm{H}$ metalation events (Scheme 1.3). Further research on this base-assisted $\mathrm{C}-\mathrm{H}$ activation led to the proposal of several transition states. The base-assisted deprotonation takes place via a six- or five-membered transition state respectively in the presence of 
carboxylate or a secondary phosphine oxide. ${ }^{[29 a, 30]}$ This $\mathrm{C}-\mathrm{H}$ cleavage mode was classified as Concerted Metalation-Deprotonation $(\mathrm{CMD})^{[30 \mathrm{~b}]}$ and ambiphillic metal-ligand activation (AMLA). ${ }^{[31]}$ An additional mechanism is the base assisted intramolecular electrophilic substitution (BIES), which is common for base assisted electrophilic transition metals. ${ }^{[32]}$

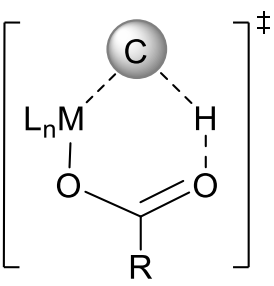

CMD<smiles>[M]OC1(C)CCCC=C1[R]</smiles>

AMLA

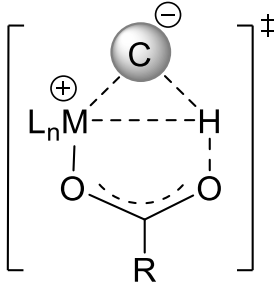

BIES

Scheme 1.3. Transition states for the $\mathrm{C}-\mathrm{H}$ cleavage in base-assisted $\mathrm{C}-\mathrm{H}$ metalation.

Although many modes have been proposed for the $\mathrm{C}-\mathrm{H}$ cleavage, another big challenge about $\mathrm{C}-\mathrm{H}$ activation is the regioselectivity due to the almost equal bond dissociation energies and acidities among several $\mathrm{C}-\mathrm{H}$ bonds. ${ }^{[33]}$ This issue can be tackled through steric hindrance, electronic bias, or the incorporation of directing groups. ${ }^{[34]}$ The lone pair of the directing groups can coordinate to the transition metal, thus bringing the catalyst in close proximity to the desired $\mathrm{C}-\mathrm{H}$ bond (Scheme 1.4). ${ }^{[35]}$ Remarkably, different templates $^{[36]}$ and strategies ${ }^{[37]}$ have been developed for the para- and meta- $\mathrm{C}-\mathrm{H}$ activation. Even free amine and hydroxyl groups could be used as directing groups to control the selectivity. ${ }^{[38]}$<smiles>c1cc2cc(c1)-c1ccccc1-c1ccccc1-c1ccccc1C2</smiles>

Scheme 1.4. Regioselective $\mathrm{C}-\mathrm{H}$ activation using directing groups(DGs). 
<smiles>Cc1ccccn1</smiles><smiles>Cn1cccn1</smiles><smiles>Cn1ccnn1</smiles><smiles>[R]n1cnnc1C</smiles><smiles>Cc1ncco1</smiles>

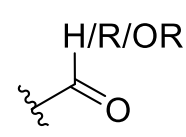<smiles>[R]C(=O)NCC</smiles><smiles>[R]C(=O)N([R])OC</smiles><smiles>[R]C(CC)=NO</smiles><smiles>[R]OC([Z9])=N</smiles>

Scheme 1.5. Common directing groups for proximity induced $\mathrm{C}-\mathrm{H}$ activation.

\subsection{Iron-Catalyzed C-H Activation}

Iron is the most abundant transition metal on the earth. The great abundance $(6.3 \%$ abundance of iron in the Earth's crust) and widespread distribution allow for the low cost as well as low biological toxicity in sharp contrast to the majority of other precious metals (e.g., $0.00000007 \%$ abundance of rhodium in the Earth's crust). ${ }^{[39]}$ So the use of iron as a substituent to precious metals is desirable in terms of both economy and sustainability.

Organoiron chemistry was arguably initiated by the synthesis of pentacarbonyliron in 1891 , independently by Mond ${ }^{[40]}$ and Berthelot. ${ }^{[41]}$ Another important breakthrough of ironchemistry was reported by the use of simple iron salt as precatalysts in reactions with Grignard reagent, including homocouplings, by Kharasch in 1941. ${ }^{[42]}$ A subsequent milestone was the preparation of ferrocene accomplished by Pauson and Keary in 1951, which was formulated as dicyclopentadienyl iron. ${ }^{[43]}$ Then a sandwich structure of ferrocene was proposed by Woodward and Wilkinson. ${ }^{[44]}$ Transformative application of ferrocene made a great contribution to organic chemistry, especially for asymmetric synthesis. ${ }^{[45]}$ A milestone in organoiron catalysis was the application of iron salts as catalysts in cross-couplings between Grignard reagents and vinyl bromides by Kochi in $1971,{ }^{[46]}$ which, remarkably, preceded before studies with palladium catalysts. Despite the loss of interest in iron-catalyzed cross-couplings due to the development of palladium catalysis, it has recently witnessed a renaissance. In fact, iron complexes are often too diverse in their reactivity compared with neighboring metals ${ }^{[47]}$ and noble metals, ${ }^{[48]}$ and 
hence it is difficult to design catalytic cycles of significance. Thus, the design of appropriate ligands for iron chemistry is in high demand.

Chemists have received many inspirations from enzymes to accelerate the design and exploitation of ligands compatible with iron, including heme proteins. ${ }^{[49]}$ In heme proteins, the iron is coordinated with four atoms that located in a macrocyclic porphyrin ring system. Thus, many types of porphyrins have been prepared to conduct different transformations catalyzed by iron (Scheme 1.6), which mostly limited to the carbene migration reactions and oxygenation reactions. ${ }^{[50]}$ Thus ligands with new scaffold are in high need for efficient $\mathrm{C}-\mathrm{H}$ activation.

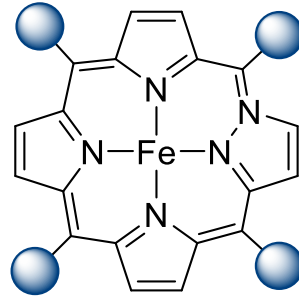

1

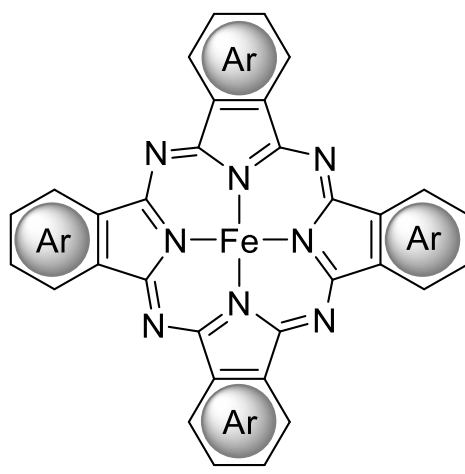

2

Scheme 1.6. Common structure of Fe-porphrin.

It should be highlighted that the first stoichiometric iron-mediated $\mathrm{C}-\mathrm{H}$ activation was described as early as in 1968 by Hata. ${ }^{[51]}$ Ultraviolet-light irradiation of $\mathrm{Fe}(\mathrm{dppe})_{2}\left(\mathrm{C}_{2} \mathrm{H}_{4}\right) 3$ resulted the loss of ethylene and shift of a hydrogen atom from a phenyl group of the ligand to the iron atom forming the new iron complex $\mathrm{HFe}\left(\mathrm{C}_{6} \mathrm{H}_{4} \mathrm{PPhCH}_{2} \mathrm{CH}_{2} \mathrm{PPh}_{3}\right)(\mathrm{dppe}) 4$ (Scheme 1.7).

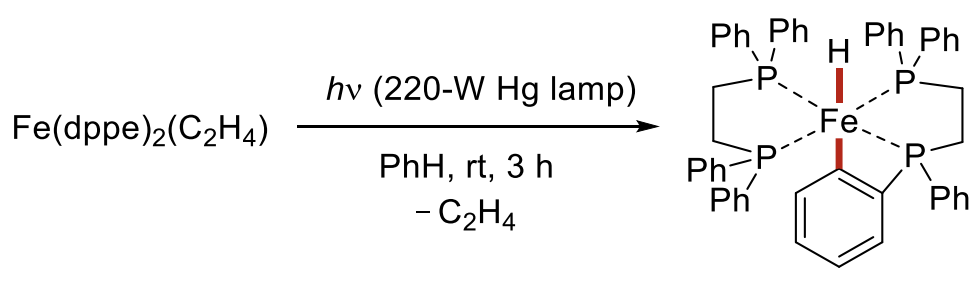

Scheme 1.7. Synthesis of cyclometalated complex 4. 
Among others, $\mathrm{Fe}\left(\mathrm{PMe}_{3}\right)_{4}$, first synthesized by Schmidabauer ${ }^{[52]}$ in 1975 , has shown significant efficiency in directing group-assisted $\mathrm{C}-\mathrm{H}$ cleavage, which indicating the feasibility of stoichiometric $\mathrm{C}-\mathrm{H}$ activation with low-valent iron complex (Scheme 1.8). ${ }^{[53]}$ Importantly, $\mathrm{Fe}\left(\mathrm{PMe}_{3}\right)_{4}$ tended to exist as an iron(II) species due to $\mathrm{C}-\mathrm{H}$ activation of the phosphine ligand. ${ }^{[54]} \mathrm{Fe}\left(\mathrm{PMe}_{3}\right)_{4}$ showed great potential for the application of catalytic $\mathrm{C}-\mathrm{H}$ activations.

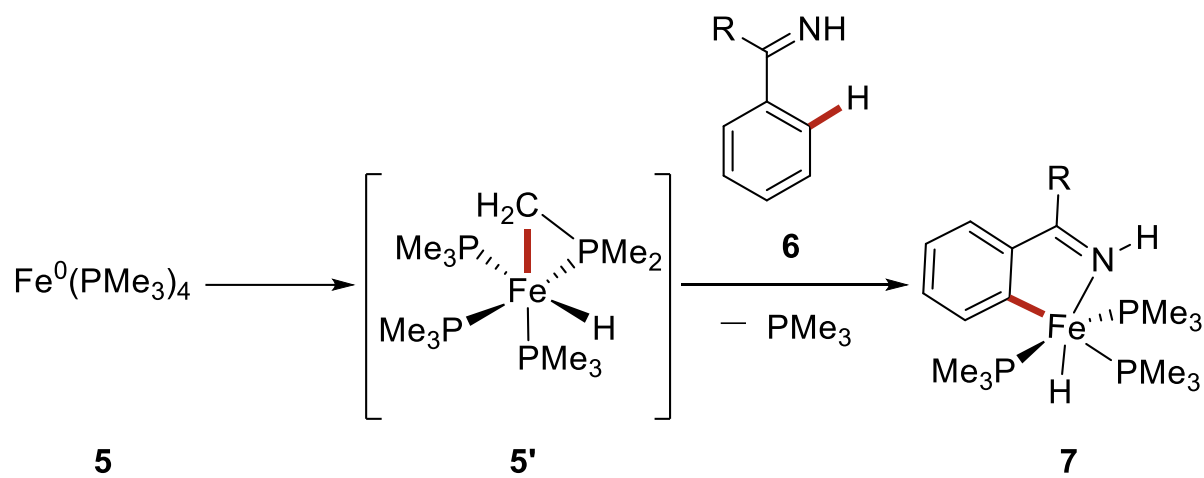

Scheme 1.8. Stoichiometric ortho-C-H metalation of imines with $\mathrm{Fe}\left(\mathrm{PMe}_{3}\right)_{4}$.

Indeed, the first contribution of a catalytic $\mathrm{C}-\mathrm{H}$ activation using an iron complex was made by Jones in $1987 .{ }^{[55]}$ Here, the combination between the catalyst derived from $\mathrm{Fe}\left(\mathrm{PMe}_{3}\right)_{4}$ and isocyanide ligands allowed for the formation of aldimines $\mathbf{1 0}$ from unactivated benzene under UV irradiation. Control experiment conducted in $\mathrm{C}_{6} \mathrm{D}_{6}$ proved that the solvent rather than the $\mathrm{PMe}_{3}$ ligand was the source of the aldimine's hydrogen. The authors suggested that light was indispensable for the coordination of an isocyanide ligand to generate a reactive intermediate 9 capable to insert into an inert $\mathrm{C}-\mathrm{H}$ bond (Scheme 1.9). Four decades after its original discovery, $\mathrm{Fe}\left(\mathrm{PMe}_{3}\right)_{4}$ was finally confirmed to be viable in ironcatalyzed $\mathrm{C}-\mathrm{H}$ activation free of additional ligands, as elegantly described by Kakiuchi and Ackermann for the carbonyl-assisted hydroarylation of alkenes and allenes respectively. ${ }^{[56]}$

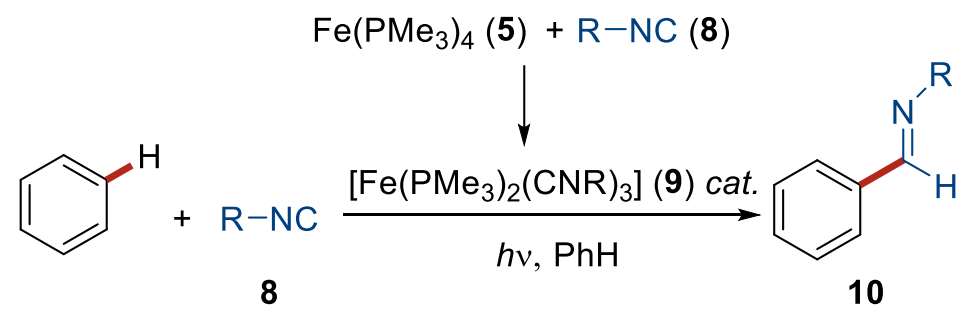


Scheme 1.9. Aldimine synthesis by iron-catalyzed $\mathrm{C}-\mathrm{H}$ activation.

In 2008, in the area of iron-catalyzed $\mathrm{C}-\mathrm{H}$ activation was disclosed by Nakamura and coworkers via an iron-catalyzed oxidative $\mathrm{C}-\mathrm{H}$ activation was disclosed. ${ }^{[57]}$ This discovery was originated from an intended iron-catalyzed cross-coupling reaction. ${ }^{[58]}$ Besides the desired product 12a, a small amount of the ortho-arylated phenylpyridine 13a was formed. Later, oxygen and 2,2'-bipyridine were identified as being pivotal to the $\mathrm{C}-\mathrm{H}$ arylation (Scheme 1.10).<smiles>Brc1ccccn1</smiles>

11

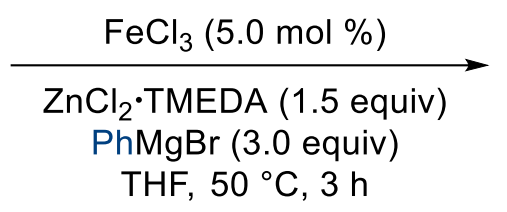

trace oxygen

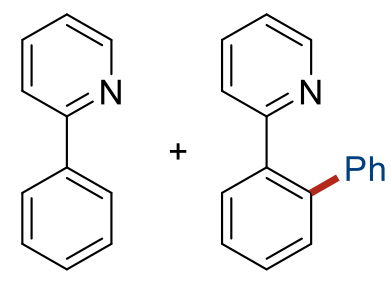

12a: $63 \%$

13a: $8 \%$

Scheme 1.10. Iron-catalyzed $\mathrm{C}-\mathrm{H}$ arylation as a byproduct of a cross-coupling reation.

Extensive optimization of this iron-catalyzed arylation proved that 1,2-dichloro-2methylpropane (DCIB) was the very essential oxidant and phenanthroline served as the best ligand.[57] Surprisingly, the zinc salt was essential for the reaction also. It was postulated that the zinc additive was responsible for the in situ generation of arylzinc species, while $\mathrm{Mg}$-free $\mathrm{Ph}_{2} \mathrm{Zn}$ and $\mathrm{PhZnBr}$ were unable to deliver the product, with or without TMEDA (Scheme 1.11).

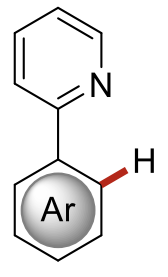

12

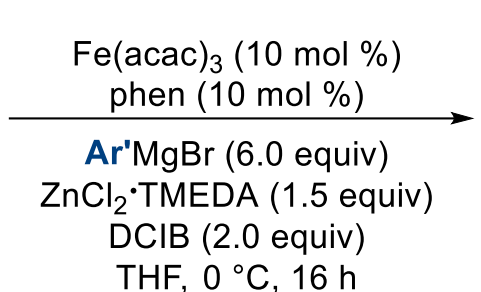

$\mathrm{THF}, 0{ }^{\circ} \mathrm{C}, 16 \mathrm{~h}$

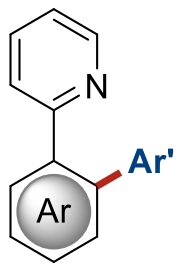

13

Scheme 1.11. First example of iron-catalyzed $\mathrm{C}-\mathrm{H}$ arylation.

Enormous efforts have been made on iron-catalyzed $\mathrm{C}\left(\mathrm{sp}^{2}\right)-\mathrm{H}$ arylation, including the application of more synthetically useful amides ${ }^{[59]}$ and imines ${ }^{[60]}$ as the directing groups, 
the use of environmentally benign oxygen as the oxidant, ${ }^{[61]}$ the $\mathrm{C}\left(\mathrm{sp}^{2}\right)-\mathrm{H}$ arylation of alkenes, ${ }^{[62]}$ the direct use of Grignard reagents without zinc additives, ${ }^{[63]}$ and the exploitation of metallic magnesium avoiding the use of dangerous and sensitive organometallic reagents. ${ }^{[64]}$

Despite the significant contributions, a well-established mechanism was not shown in their original reports, then Nakamura and coworkers suggested a catalytic cycle on the basis of KIE studies and stoichiometric reactions (Scheme 1.12). ${ }^{[63]}$ The catalytic cycle starts through the formation of an aryliron intermediate 14 by transmetalation from the aryl Grignard reagent to the iron center. Then, a reversible chelation of the iron center by the pyridine takes place followed by an irreversible $\mathrm{C}-\mathrm{H}$ metalation with synergistic elimination of an arene via $\sigma$-bond metathesis. Next, the cyclometalated species 16 undergoes a reductive elimination with the assistance of DCIB to produce the desired arylated product 13, isobutene and dichloroiron species. Finally, a transmetalation between dichloroiron species 17 and the Grignard reagent takes place to regenerate the active catalyst 14 .

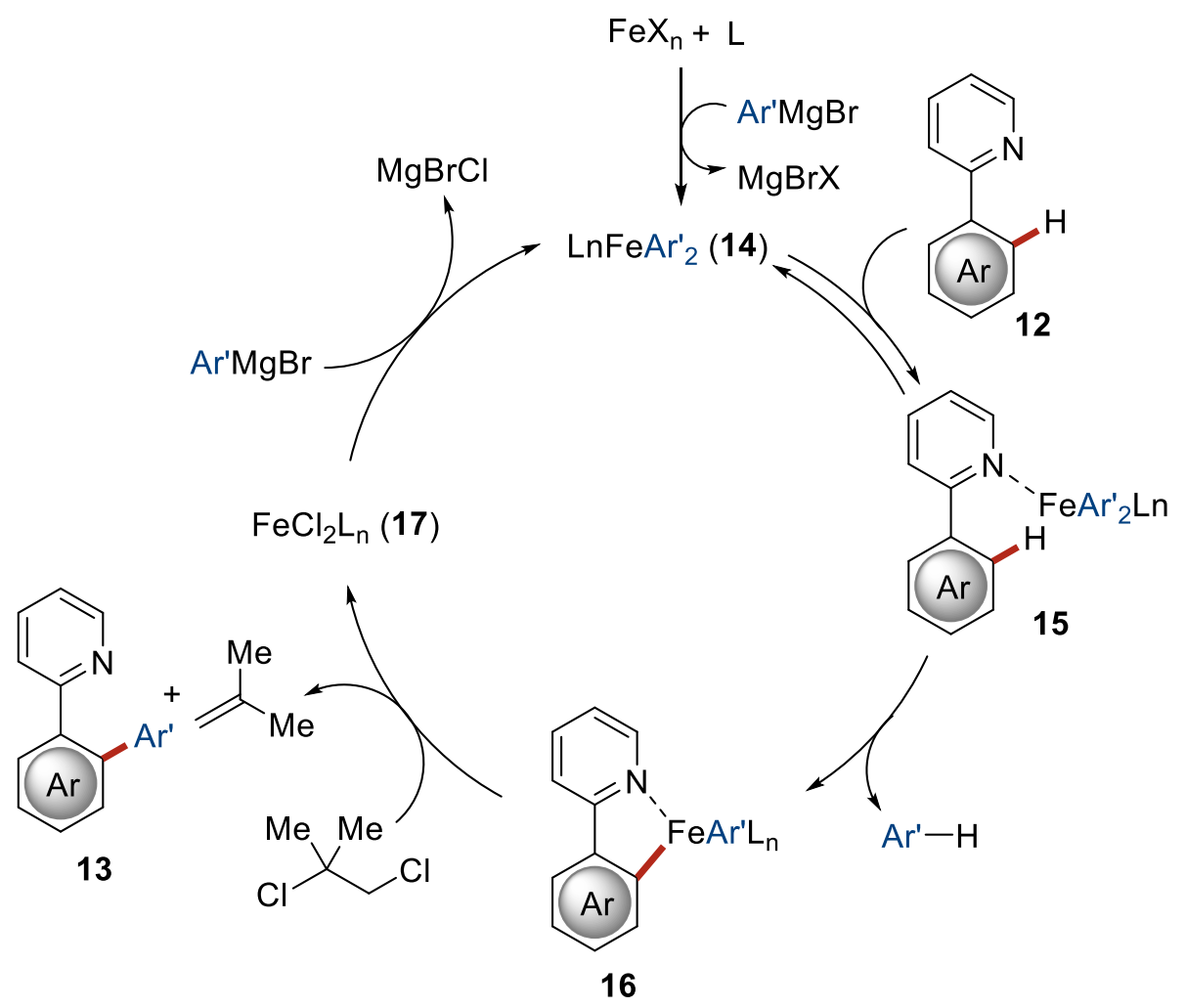

Scheme 1.12. Catalytic cycle of the oxidative iron-catalyzed $\mathrm{C}-\mathrm{H}$ arylation. 
Later the introduction of bidentate directing groups allowed for the challenging ironcatalyzed $\mathrm{C}\left(\mathrm{sp}^{3}\right)-\mathrm{H}$ activation expanding the scope beyond oxidative arylations with organometallic reagents (Scheme 1.13a). ${ }^{[65]}$ After optimization they discovered that 8aminoquinoline directing group was optimal which was initially introduced by Daugulis for palladium-catalyzed $\mathrm{C}-\mathrm{H}$ activations. ${ }^{[66]}$ The $\mathrm{KIE}$ study and priority for terminal methyl group over internal methylene groups suggested an inner-sphere $\mathrm{C}-\mathrm{H}$ activation process rather than a radical pathway. A biologically compatible triazole directing group was developed by Ackermann group which were effective for iron-catalyzed $\mathrm{C}\left(\mathrm{sp}^{3}\right)-\mathrm{H}$ and $\mathrm{C}\left(\mathrm{sp}^{2}\right)-\mathrm{H}$ arylations (Scheme 1.13b). ${ }^{[67]}$

a) E. Nakanura

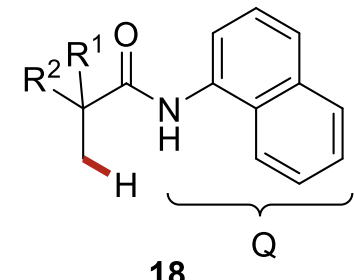

18

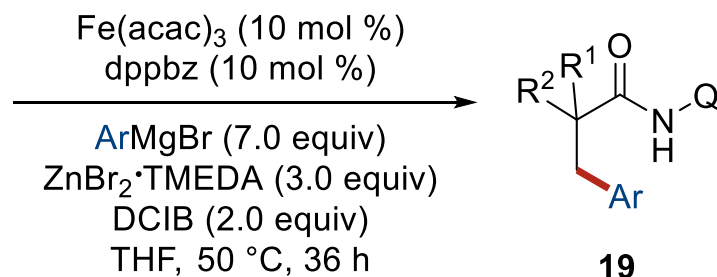

19

b) Ackermann
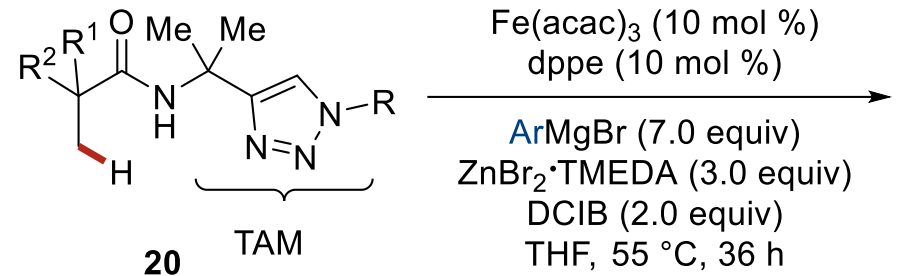<smiles>[Y10]NC(=O)C([R])([R])CBr</smiles>

$\mathrm{THF}, 55^{\circ} \mathrm{C}, 36 \mathrm{~h}$

21

Scheme 1.13. Bidentate directing group enabled Iron-catalyzed $\mathrm{C}\left(\mathrm{sp}^{3}\right)-\mathrm{H}$ arylation.

After those pioneering studies, under the assistance of bidentate directing groups further important progress were realized by the research groups of Nakamura, Ackermann and Cook, among others. Thus, C-H alkylations with alkyl halides, alkenes, alkyl tosylates or Grignard reagents became available. ${ }^{[68]}$ Alkynyl bromides were used for the preparation of the alkynylated product under iron-catalysis. ${ }^{[69]} \mathrm{N}$-chloroamines proved to be a good aminating reagents. ${ }^{[70]}$ Organoboron reagents proved viable for $\mathrm{C}-\mathrm{H}$ alkenylations and arylations. ${ }^{[71]}$ Various annulations with alkynes and allenes were reported to deliver 
heteroarenes in the presence of iron catalyst. ${ }^{[72]} \mathrm{C}\left(\mathrm{sp}^{3}\right)-\mathrm{H}$ and $\mathrm{C}\left(\mathrm{sp}^{2}\right)-\mathrm{H}$ methylations were also achieved. ${ }^{[73]}$

Yoshikai reported an iron-catalyzed $\mathrm{C}-\mathrm{H}$ activation in which a rare branched product 24 was formed enabled by a modification of the $\mathrm{N}$-heterocyclic carbene scaffold $\mathbf{2 5}$ (Scheme 1.14). Alkynes were also suitable coupling partner after a slight modification of the reaction conditions..$^{[74]}$

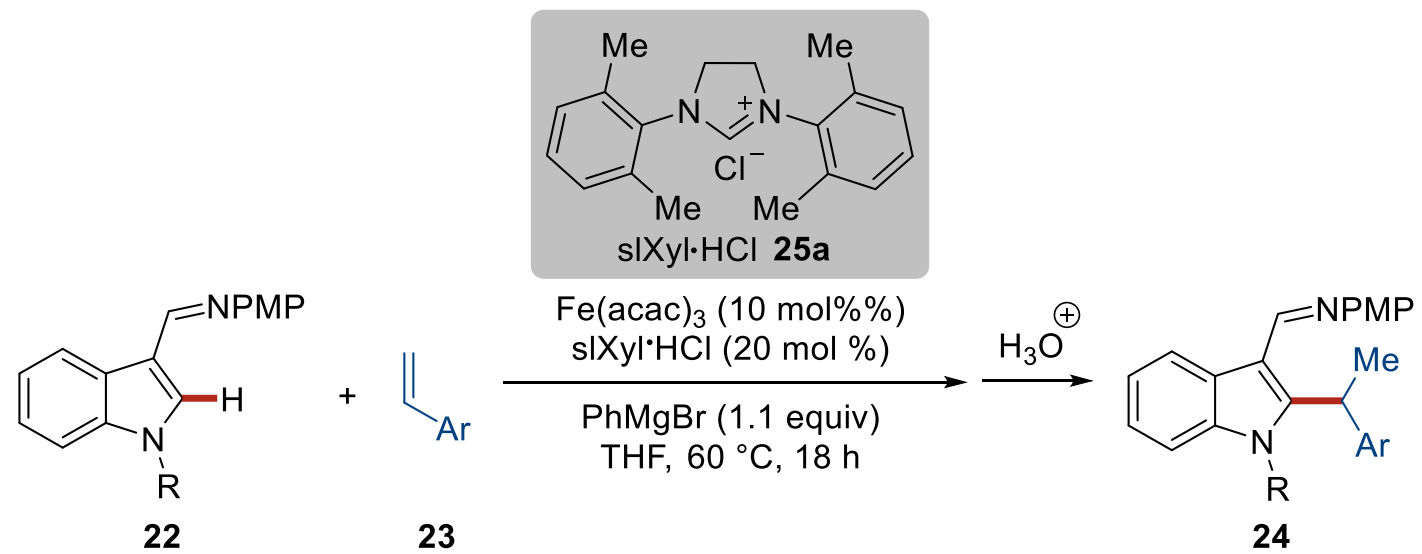

Scheme 1.14. Iron-catalyzed hydroarylation of styrenes.

The very first highly enantioselective iron-catalyzed $\mathrm{C}-\mathrm{H}$ activation was realized by Ackermann and coworkers (Scheme 1.15a). ${ }^{[75]}$ Shortly afterwards an asymmetric arylation of ferrocene was reported by Butenschoen, ${ }^{[76]}$ giving the planar-chiral product in moderate enantiomeric excess (Scheme 1.15b).

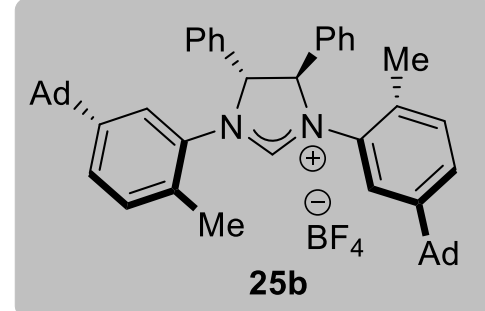<smiles>[Y16]/N=C\c1cn([R])c2ccccc12</smiles>

26
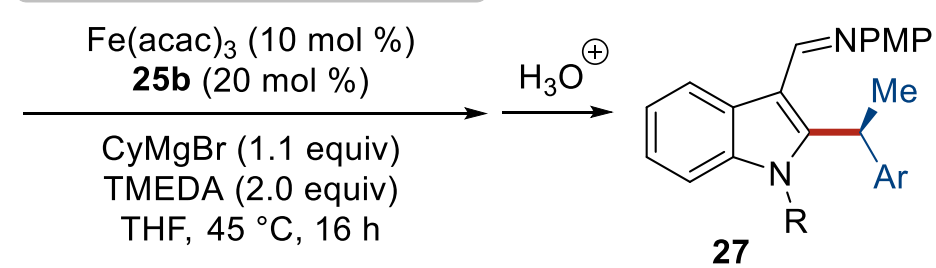

Scheme 1.15. Enantioselective iron-catalyzed C-H alkylation. 


\subsection{Manganese-catalyzed C-H Activations}

In the last few decades, significant attention has been paid to the development of sustainable and economic strategies for molecular synthesis. Despite significant advances, expensive transition metals, are predominantly needed to gain high efficiency, such as palladium, rhodium, ruthenium, and iridium complexes. ${ }^{[24 b, 24 c, 24 g]}$ Recent progress in this fast developing arena suggested the use of earth-abundant $3 \mathrm{~d}$ transition metal complexes as catalysts in $\mathrm{C}-\mathrm{H}$ activation. ${ }^{24 \mathrm{a}, 24 \mathrm{~d}]}$

Manganese is the third most abundant transition metal just after iron, titanium and the twelfth most abundant element on earth. ${ }^{[39]}$ In addition, it represents an essential trace element in the human body, with manganese cores served as an assistance factor. ${ }^{[77]}$ Therefore, it is promising to utilize it as a catalyst due to its low price, availability and low toxicity. ${ }^{[78]}$ The function of manganese in many enzymes inspired chemists to design ligands (Scheme 1.16), which can realize similar transformations accomplishing the $\mathrm{C}-\mathrm{H}$ functionalization by outer-sphere mechanisms for a variety of transformations. ${ }^{[79]}$

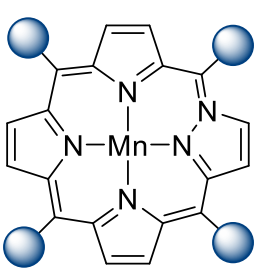

28

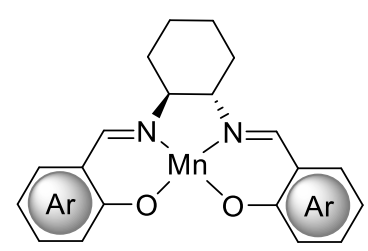

29

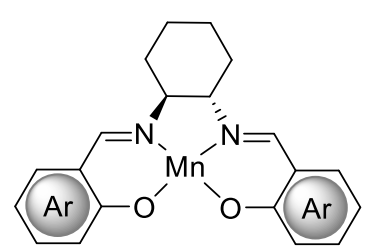

30

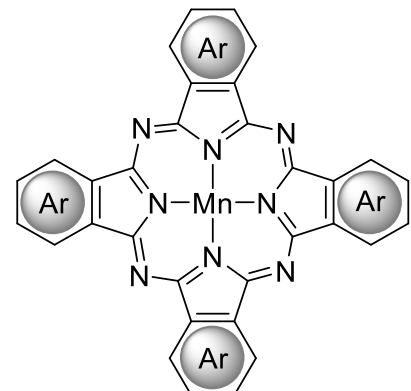

31

Scheme 1.16. Typical manganese complexes $28-31$ for $\mathrm{C}-\mathrm{H}$ functionalizations.

This chapter will focus on the manganese-catalyzed reactions under chelation assistance. There are two parts in this chapter: i) manganese(I)-catalyzed $\mathrm{C}-\mathrm{H}$ functionalization, ii) low-valent manganese(II)-catalyzed C-H functionalizations. 


\subsubsection{Manganese(I)-catalyzed C-H Functionalization}

The discovery of metal catalyzed $\mathrm{C}-\mathrm{H}$ bond cleavage is often inspired by the stoichiometric investigations with metal complexes. The first manganese-catalyzed $\mathrm{C}-\mathrm{H}$ activation traces back to the synthesis of the manganese complex 34 from $\left[\mathrm{MnMe}(\mathrm{CO})_{5}\right] 32$ through a $\mathrm{C}-\mathrm{H}$ scission event, reported by Stone and Bruce in 1970 (Scheme 1.17). ${ }^{[80]}$ The engagement of manganese $(\mathrm{I})$ in the $\mathrm{C}-\mathrm{H}$ activation was subsequently confirmed as various well-defined manganacycles were reported by different groups respectively, such as Woodgate ${ }^{[81]}$ and Liebeskind $^{[82]}$ among others.<smiles>C[C+](C)OC(C)(C)[O+]</smiles>
32

Scheme 1.17. Stoichiometric manganese-mediated C-H activation.

In 2007, the first catalytic manganese(I)-catalyzed $\mathrm{C}-\mathrm{H}$ activation was achieved by Kuninobu and Takai. ${ }^{[83]}$ They initiated the exploration by the stoichiometric $\mathrm{C}-\mathrm{H}$ bond activation and insertion into aldehyde $\mathbf{3 6}$ with the manganese complex $\left[\mathrm{MnBr}(\mathrm{CO})_{5}\right]$. After the reaction mixture was heated for 10 hours the desired alcohol 37 was obtained in $52 \%$ yield (Scheme 1.18a). Despite the fact that the stoichiometric $\mathrm{C}-\mathrm{H}$ bond scission and insertion of aldehyde proceeded smoothly with $\left[\mathrm{MnBr}(\mathrm{CO})_{5}\right]$, only trace amount of alcohol 37 was produced with catalytic amounts of the manganese complex 32. After optimization, triethylsilane 38 was found to be essential for regenerating the catalyst (Scheme 1.18b). A probable catalytic cycle was put forward based on mechanistic studies (Scheme 1.19). The catalytic cycle was initiated by the formation of a five-membered manganacycle $\mathbf{4 0}$ followed by the insertion into polar $\mathrm{C}=\mathrm{O}$ bond to form the seven-membered manganese complex 41. Finally, the silylethers 39 are formed via release of $\mathrm{H}_{2}$ and regeneration of the manganese(I) catalyst by the action of $\mathrm{Et}_{3} \mathrm{SiH} 38$. 
a) stoichiometric reaction

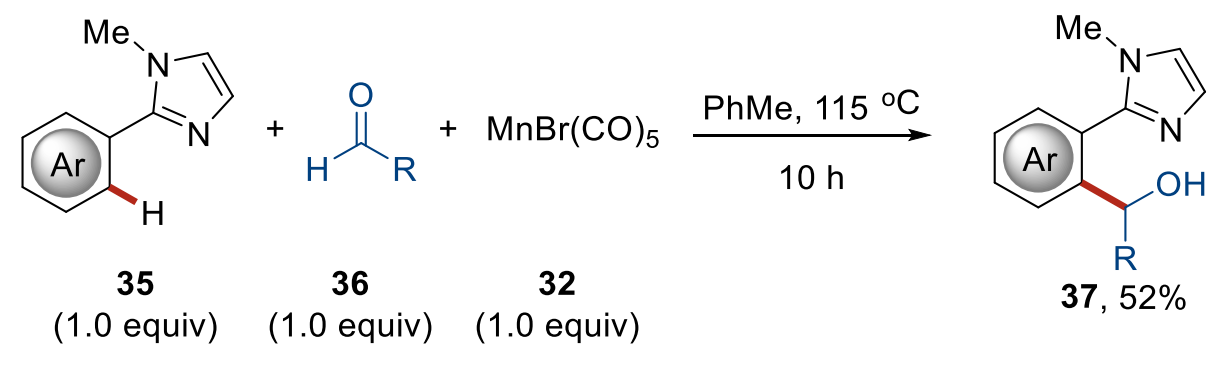

b) catalytic reaction

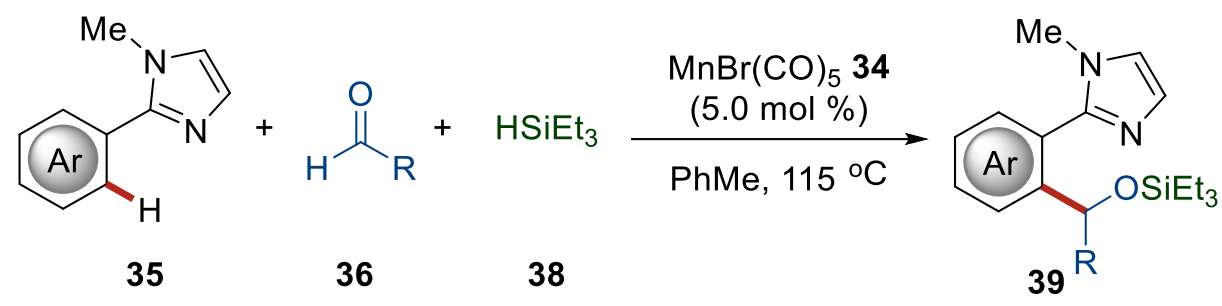

Scheme 1.18. Manganese(I)-catalyzed $\mathrm{C}-\mathrm{H}$ addition to aldehydes 36.

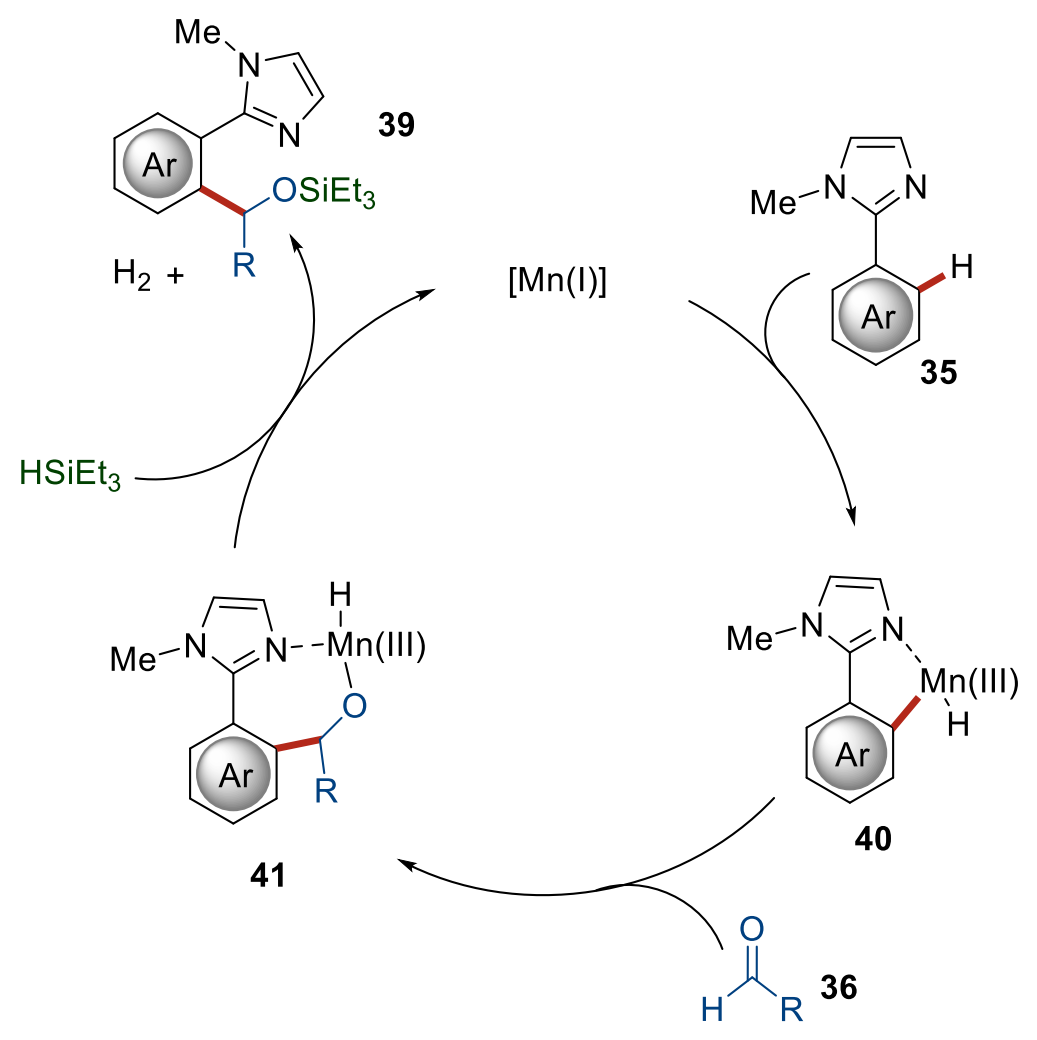

Scheme 1.19. Proposed catalytic cycle for manganese(I)-catalyzed $\mathrm{C}-\mathrm{H}$ addition to aldehydes 36 . 
In 2015, Wang developed a manganese-catalyzed Grignard-type nucleophilic addition of $\mathrm{C}\left(\mathrm{sp}^{2}\right)-\mathrm{H}$ bonds to aldehydes to access secondary alcohols (Scheme 1.20a), ${ }^{[84]}$ which circumvents the limitation of previous rhodium and palladium catalytic systems. During the mechanistic studies, the authors found that $\left[\mathrm{MnMe}(\mathrm{CO})_{5}\right]$ was an effective catalyst. Nitriles were also viable coupling partners to give ketones under this reaction. Later, Ackermann and Wang extended this kind nucleophilic transformation, as attack to $\mathrm{C}=\mathrm{Het}$ double bond led to functionalized amines (Scheme 1.20b). ${ }^{[85]}$

a) Wang's Grignard type addition

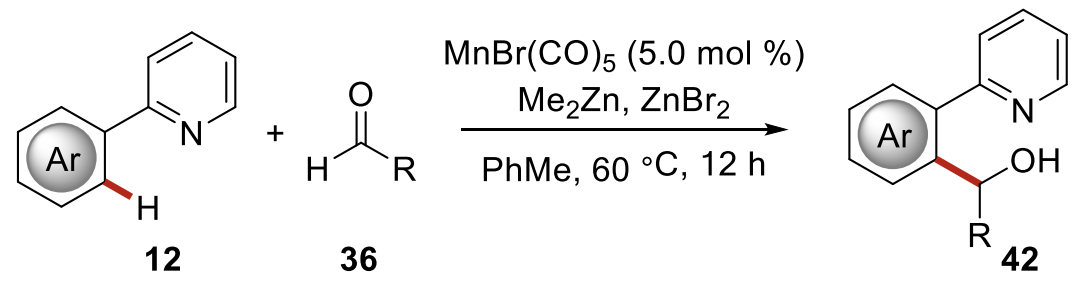

b) Ackermann's addition to imines

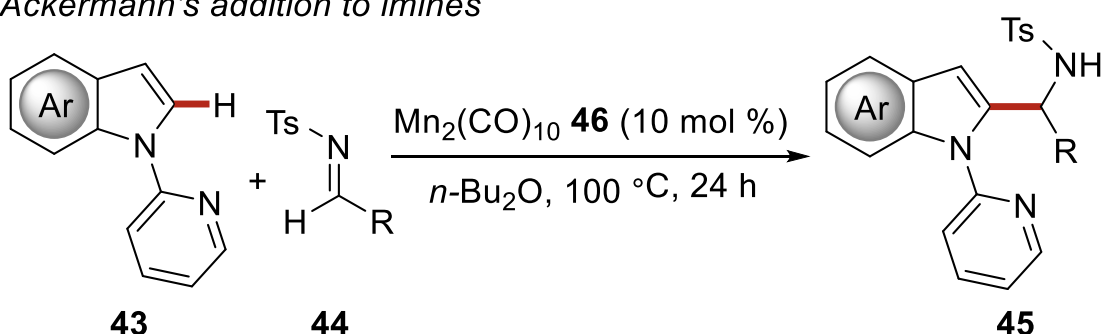

Scheme 1.20. Manganese-catalyzed nucleophilic addition.

The extension of these $\mathrm{C}-\mathrm{H}$ addition strategies from polar $\mathrm{C}=\mathrm{Het}$ multiple bonds to nonpolar $\mathrm{C}=\mathrm{C}$ double bonds was accomplished by Wang and co-workers. ${ }^{[86]}$ The addition reaction showed a broad substrate scope, good functional group tolerance, and complete mono-selectivity. Ketimines $\mathbf{4 7}$ are also good substrates to form five-membered manganacycle 48. Ackermann's group found an intramolecular nucleophile attack can occur to furnish useful $\beta$-amino acids $\mathbf{5 2}$ after the insertion of acrylate $\mathbf{4 9}$ to the complex 48 (Scheme 1.21). ${ }^{[87]}$ Using the same strategy, methylenecyclopropanes 53 (MCPs) containing electron-withdrawing groups were employed in the manganese-catalyzed $\mathrm{C}-\mathrm{H}$ activation. The intramolecular nucleophile addition followed by zinc-mediated hydroarylation of the alkene $\mathbf{5 4}$ provided the desired product 55 (Scheme 1.22). ${ }^{[88]}$ 


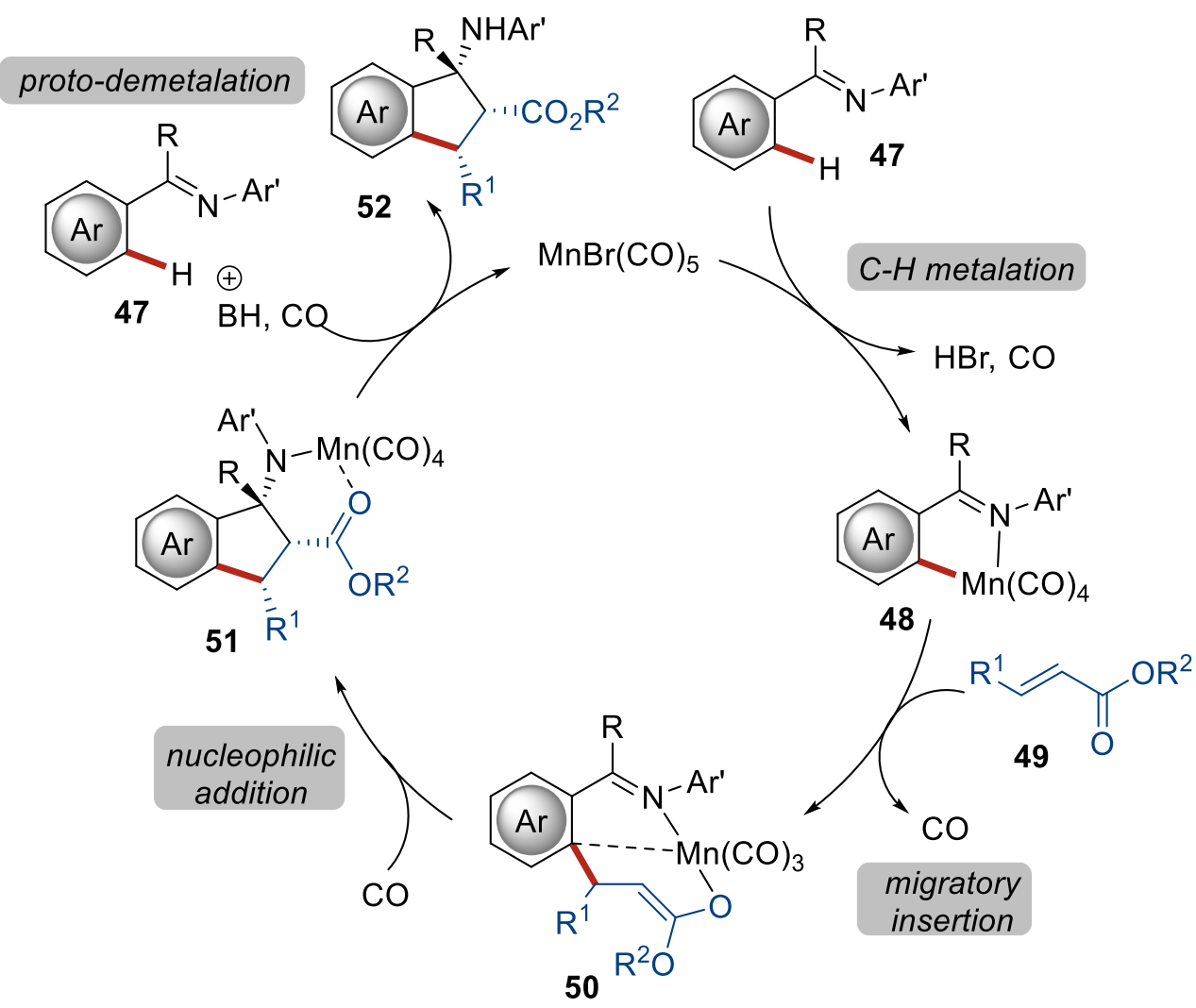

Scheme 1.21. Mechanism for the synthesis of $\beta$-amino acids.

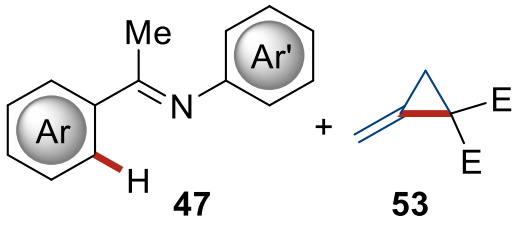

47

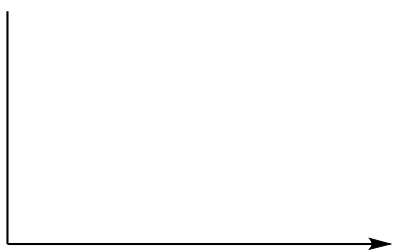

1) $\mathrm{MnBr}(\mathrm{CO})_{5}(5.0 \mathrm{~mol} \%)$ $\mathrm{NaOAc}(20 \mathrm{~mol} \%)$

1,4-dioxane, $80^{\circ} \mathrm{C}, 24 \mathrm{~h}$

2) $\mathrm{ZnCl}_{2}, 23{ }^{\circ} \mathrm{C}, 1 \mathrm{~h}$ $\mathrm{E}=\mathrm{CO}_{2} \mathrm{Et}$

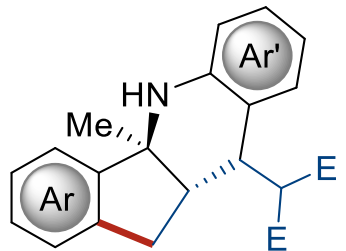

55
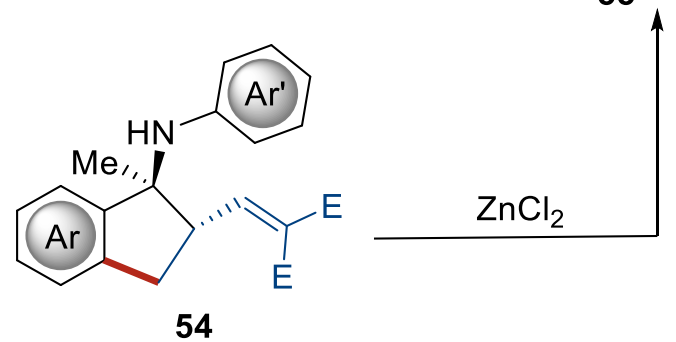

Scheme 1.22. C-H/C-C activation with MCP catalyzed by Manganese(I).

Alkenes with leaving groups are also good coupling partners in the manganese-catalyzed $\mathrm{C}-\mathrm{H}$ activations. Thus, alkenes with different leaving groups were designed by Ackermann for the unprecedented allylations on different functional molecules, ${ }^{[89]}$ remarkably this manifold was used for the diversification of peptides ${ }^{[00]}$ (Scheme 1.23). 


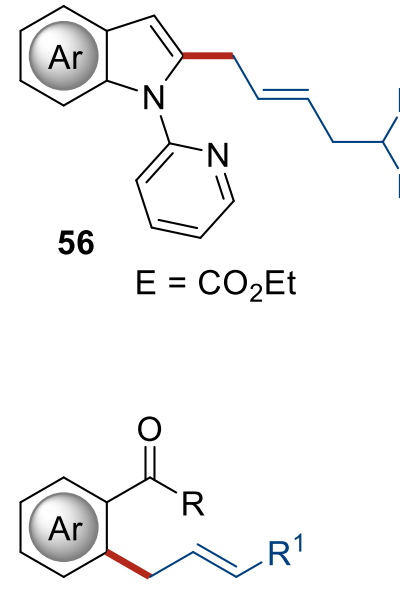

58<smiles>[R]C(=N[Al-])c1ccccc1CC=CC([R])O</smiles>

57

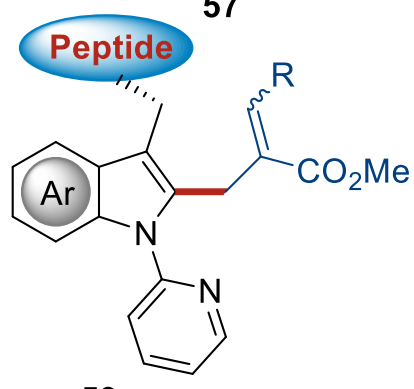

59

Scheme 1.23. Products derived by manganese(I)-catalyzed $\mathrm{C}-\mathrm{H}$ allylation.

Alkynes are very important synthons widely used in the synthesis of high-value products, showed high reactivity towards manganese-catalyzed $\mathrm{C}-\mathrm{H}$ activation. The first use of catalytic amounts of Brønsted acids in manganese-catalyzed $\mathrm{C}-\mathrm{H}$ activation enabling chemoselective hydroarylations was achieved by Ackermann, which is fully tolerant of $\beta-O$ leaving groups (Scheme 1.24) ${ }^{\left[{ }^{[1]}\right.}$ At the same time, indoles were successfully allenated by alkynes bearing a leaving group in the presence of $\left[\operatorname{MnBr}(C O)_{5}\right]$. Optically active multisubstituted allenes were prepared with high enantiomeric excess through a highly efficient chirality transfer. ${ }^{[92]}$ A general and scalable strategy was developed to regioselectively synthesize $\mathrm{N}$-heterocycles by using alkyne coupling partners with ketimine and a simple manganese-based catalyst. This procedure overcomes the previous limitations of $\mathrm{C}-\mathrm{H}$ activation with unsymmetrical alkyne coupling partners and was also demonstrated to be effective with unpolarized aliphatic alkynes, with the desired products obtained with complete regioselectivity. ${ }^{[93]}$ The manganese-catalyzed $\mathrm{C}-\mathrm{H}$ alkenylation with terminal alkynes was realized by Wang, highlighting not only a practical catalytic system comprised of easily available $\left[\mathrm{MnBr}(\mathrm{CO})_{5}\right]$ and $\mathrm{Cy}_{2} \mathrm{NH}$ but also high levels control in regio-, chemo- and stereoselectivity. ${ }^{[94]}$ 
Ackermann's hydroarylation<smiles></smiles>

43

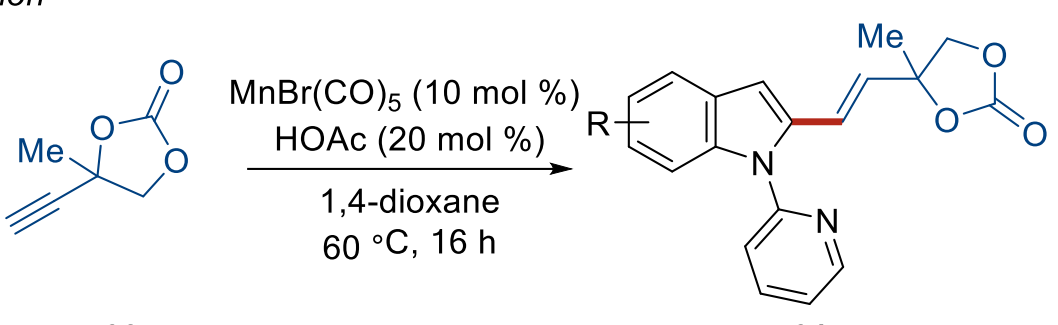

60

61

Scheme 1.24. $\mathrm{Mn}(\mathrm{I})$ catalyzed hydroarylation of alkynes.

Domino reactions via $\mathrm{C}-\mathrm{H}$ allenylation, Diels-Alder (DA) reaction, and retro Diels-Alder reaction pathway were realized by manganese catalysis in Ackermann and Li group respectively (Scheme 1.25). ${ }^{[95]}$ To shed light into this cascade process, a stable DA adduct 68 was isolated after the coupling of substrates 66 and 67 at a lower temperature, which proved to be an intermediate en route to the final product $\mathbf{7 0}$ via a retro-Diels-Alder event with extrusion of $\mathrm{HCN}$. 
a) Mn-Catalyzed DehydrocyanativeTransannulation of Heteroarenes and Propargyl Carbonates<smiles></smiles>

62<smiles>[R]C#CC(C)(C)OC(C)=O</smiles>

63

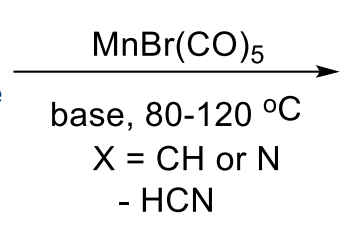

$-\mathrm{HCN}$<smiles></smiles>

64

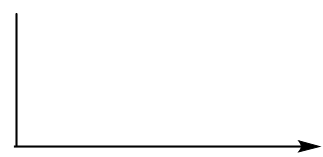

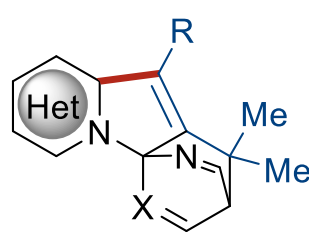

65

b) synthesis of key intermediate<smiles>CC(=O)C(C)(C#C[PH2+]c1ccccc1)C#Cc1ccc(-n2c([O-])cccc2=O)cn1</smiles>

67<smiles>CCC</smiles>

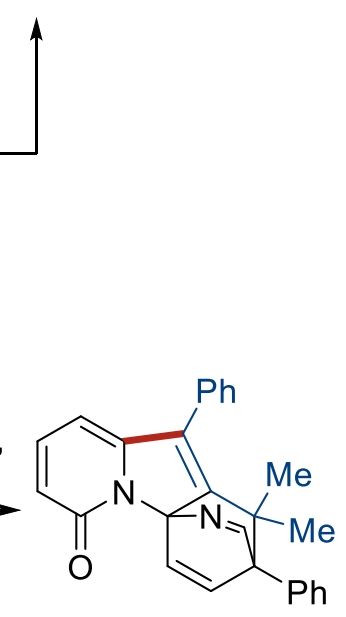

68

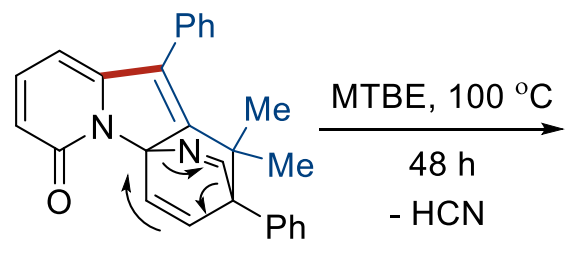

69<smiles>CC1(c2ccccc2)C(c2ccccc2)=CC=c2c1c(-c1ccccc1)n1c(=O)cccc21</smiles>

70

scheme 1.25. Manganese(I) catalyzed domino reactions.

With the great success of allenes in the construction of complex compounds, it represents an appealing partner in manganese-catalyzed $\mathrm{C}-\mathrm{H}$ functionalization. In 2017 , allylation of indole was achieved by Wang's group using 1,1-dimethylallene (Scheme 1.26a). ${ }^{\left[{ }^{[6]}\right.} \mathrm{A}$ manganese(I)-catalyzed region- and stereoselective 1,2-diheteroarylation of allenes was discovered by Wang and Rueping respectively, ${ }^{[97]}$ which features the combination of $\mathrm{C}-\mathrm{H}$ hydroarylation along with a Smiles rearrangement (Scheme $1.26 \mathrm{~b}$ ). In the same year, polycyclization enabled by manganese $(I) / s i l v e r(I)$ relay catalysis through one-pot manganese-catalyzed $\mathrm{C}-\mathrm{H}$ allylation and silver-catalyzed Pavarov reaction was established by Wang (Scheme 1.26c). ${ }^{[98]}$ 
a) Wang's allylation with allenes<smiles>c1cnc(-n2ccc3ccccc32)nc1</smiles>

71<smiles>[R]C(=C)C([R])=C=C</smiles>

72
$\mathrm{MnBr}(\mathrm{CO})_{5}(10 \mathrm{~mol} \%)$

$\underset{\mathrm{NaOAc}(40 \mathrm{~mol} \%)}{\text { 1,4-dioxane }}$

$100{ }^{\circ} \mathrm{C}, 24 \mathrm{~h}$

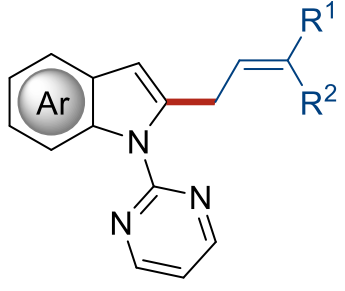

73

b) Wang and Rueping's cascade $\mathrm{C}-\mathrm{H}$ activation and cyclization

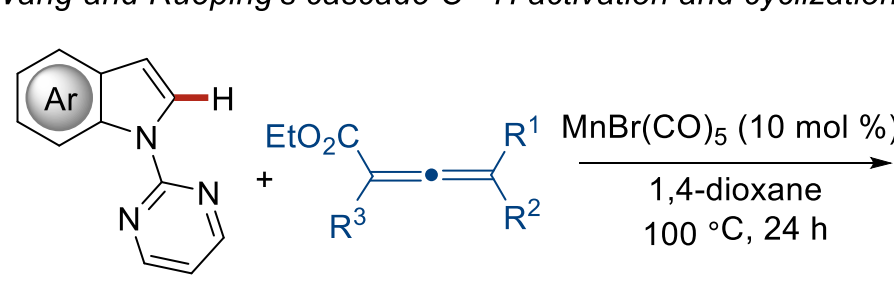

71

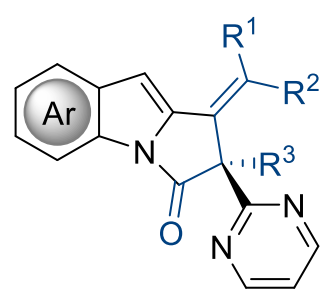

75

c) Wang's cascade $\mathrm{C}-\mathrm{H}$ activation and Povarov reaction<smiles>CC(=Nc1ccccc1)c1ccccc1</smiles>

47<smiles>[R]C(=C=C)C([R])C</smiles>

72
1) $\mathrm{MnBr}(\mathrm{CO})_{5}(5.0 \mathrm{~mol} \%)$ $\mathrm{NaOAc}(40 \mathrm{~mol} \%)$

AgOTf (20 mol \%)

1,4-dioxane/TFE (9:1) $100{ }^{\circ} \mathrm{C}, 24 \mathrm{~h}$

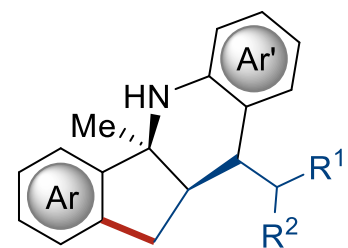

76

Scheme 1.26. Manganese(I) catalyzed transformations with allenes $\mathbf{7 2}$ and $\mathbf{7 4}$.<smiles>c1ccc(-n2ccc3ccccc32)nc1</smiles>

43<smiles>c1ccc(-n2ccc3ccccc32)nc1</smiles>

$43 a$<smiles>[R]C(Br)=C=C([R])C([R])Br</smiles>

77

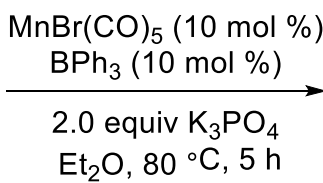

$\mathrm{Et}_{2} \mathrm{O}, 80^{\circ} \mathrm{C}, 5 \mathrm{~h}$ $\mathrm{MnBr}(\mathrm{CO})_{5}(10 \mathrm{~mol} \%)$

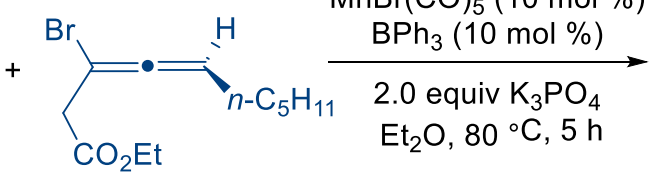

(S)-79 (97\% ee)

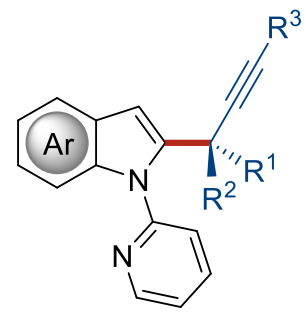

78<smiles>CCCCCC(C#CCC(=O)OC)c1cc2ccccc2n1-c1ccccn1</smiles>

80: $81 \%$ yield (91\% ee)

Scheme 1.27. Manganese $(\mathrm{I})$ catalyzed $\mathrm{C}-\mathrm{H}$ propargylation with bromoallenes 77 and $(S)-79$. 
Bromoallenes were efficiently employed in direct $\mathrm{C}-\mathrm{H}$ propargylations with high selectivity under the synergy of manganese $(\mathrm{I})$ and a Lewis acid. The axial chirality in the bromoallene (S)-79 could be successfully transferred to the central chirality at the propargylic position, a motif difficult to obtain by traditional methods (Scheme 1.27). ${ }^{[99]}$ Manganese-catalyzed $\mathrm{C}-\mathrm{H}$ annulation of ketimines $\mathbf{4 7}$ with allenes providing an approach to stereoselective synthesis of 1 -aminoindanes $\mathbf{8 1}$ was also described. ${ }^{[100]}$

With the rapidly growing interest in the application of photochemistry in organic synthesis, a photoredox Minisci reaction with unactivated iodoalkanes catalyzed by $\mathrm{Mn}_{2}(\mathrm{CO})_{10}$ was developed, which was successfully employed in the late-stage functionalization of complex drugs. ${ }^{[101]}$ Manganese-mediated photochemical generation of aryl radicals was likewise exploited for the direct $\mathrm{C}-\mathrm{H}$ arylations of (het)arenes (Scheme 1.28). ${ }^{[102]}$

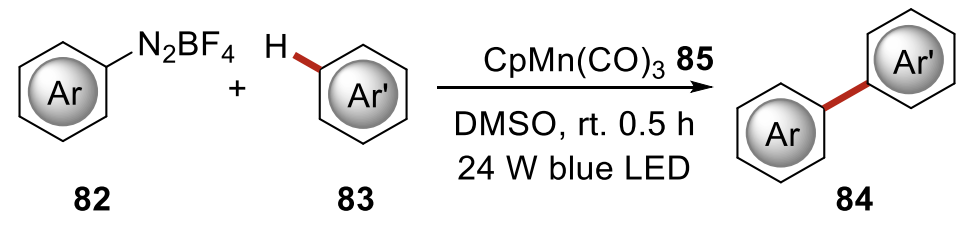

Scheme 1.28. Visible-light and manganese-catalyzed reactions.

Manganese $(\mathrm{I})$ catalysis displayed great power in meaningful cyanation and aminocarboxylation. ${ }^{103]}$ Lastly, challenging $\mathrm{C}-\mathrm{H} / \mathrm{C}-\mathrm{F}$ functionalization could be accomplished by manganese $(\mathrm{I})$. It is noteworthy that $\mathrm{C}-\mathrm{F}$ bonds are generally relatively inert due to the high bond energy and the reluctance of organofluorine compounds to coordinate to metal centers. ${ }^{[104]}$ Despite these challenges, $\mathrm{C}-\mathrm{H} / \mathrm{C}-\mathrm{F}$ activation has been successfully employed in the manganese-catalyzed perfluoroallylation and monofluoroalkenylation. ${ }^{[105]}$

\subsubsection{Low-valent Manganese(II)-catalyzed C-H Functionalization}

Despite a plethora of transformations enabled by manganese $(\mathrm{I})$-catalyzed $\mathrm{C}-\mathrm{H}$ functionalization, these methods are restricted to the addition of double bonds. The Ackermann group reported the first low-valent $\mathrm{MnCl}_{2}$-catalyzed $\mathrm{C}-\mathrm{H}$ alkylation with alkyl 
bromides 86 (Scheme 1.29a). The unprecedented manganese(II)-catalyzed C-H cleavage occurred without the use of expensive phosphine ligands in the absence of zinc, providing versatile access to alkylated benzamides through assistance of the removable TAM (triazolyl-methyl) group (Scheme 1.29a). ${ }^{[106]}$ Versatile manganese-catalyzed $\mathrm{C}-\mathrm{H}$ arylations on synthetically meaningful pyridines were accomplished with sustainable $\mathrm{MnCl}_{2}$ as the catalyst as disclosed one year later. ${ }^{[107]}$ The oxidative $\mathrm{C}-\mathrm{H}$ functionalization proved viable with a user-friendly and safe continuous flow setup.

a) Ackermann's alkylation

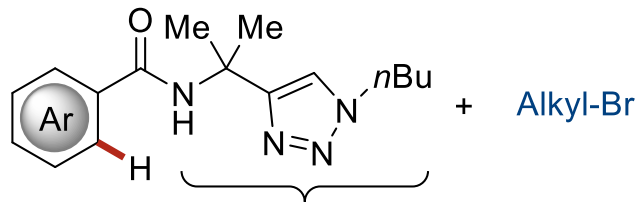

$85 \mathrm{TAM}^{\mathrm{Bu}}$

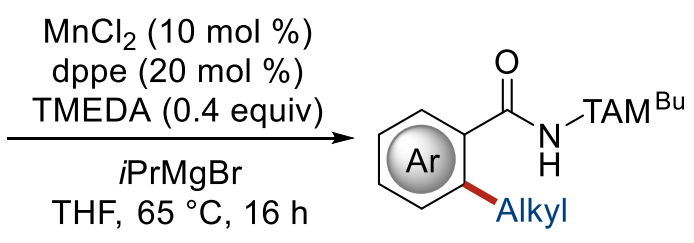

87

b) Ackermann's arylation<smiles>O=C(NF)c1ccccc1</smiles>

88

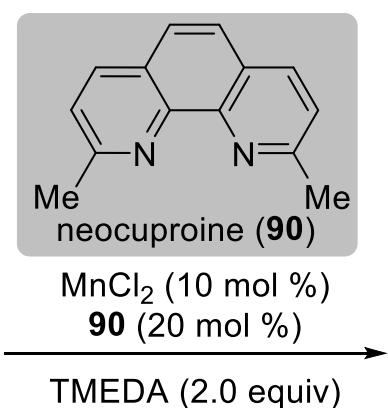

$\mathrm{THF}, 80^{\circ} \mathrm{C}$

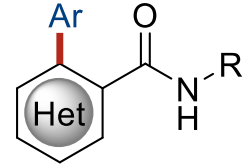

91

Scheme 1.29. Low-valent manganese(II)-catalyzed $\mathrm{C}-\mathrm{H}$ functionalization.

Later, Nakamura reported a manganese-catalyzed $\mathrm{C}-\mathrm{H}$ methylation, using $\mathrm{MeMgBr}$, a catalytic amount of $\mathrm{MnCl}_{2} \cdot 2 \mathrm{LiCl}$, and an organic dihalide as the oxidant (Scheme 1.29b). ${ }^{[108]}$

\subsection{Rhodium-catalyzed C-H Activation}

As the formation of $\mathrm{C}-\mathrm{C}$ bond is the most important objective within synthetic chemistry, different types of coupling reactions have been developed to achieve the formation of $\mathrm{C}-\mathrm{C}$ bonds. Most of these transformations proceed in the presence of palladium. Many new reactions catalyzed by rhodium complexes show great potential. ${ }^{[109]}$ In addition, rhodium often shuttles between rhodium(I) and rhodium(III) oxidation states in the catalytic cycle 
that does not require organometallic reagents. Cycloadditions, hydroacylation reactions, and allylic functionalization are representative examples for rhodium-catalyzed $\mathrm{C}-\mathrm{C}$ bonds formations. ${ }^{[110]}$ Rhodium-derived complexes also provide a powerful tool for the asymmetric hydrogenation of prochiral unsaturated compounds to give optically pure alcohols, amines and other hydrocarbons. ${ }^{[111]}$

The utility of $\mathrm{C}-\mathrm{H}$ activation in lieu of substrate prefunctionalization has featured a tantalizing alternative to classical cross-coupling reactions but the challenges ahead are the selectivity and reactivity associated with otherwise inert $\mathrm{C}-\mathrm{H}$ bonds. Literature reports on selectivity based on steric effects, acidity, and electronic and directing group effects are now numerous. The designs of directing groups and ligands allow the rhodium to conduct the cleavage of inert $\mathrm{C}-\mathrm{H}$ bond efficiently with good selectivity. For this part, rhodiumcatalyzed $\mathrm{C}-\mathrm{H}$ activation will be divided into two parts: (i) rhodium-catalyzed inner-sphere $\mathrm{C}-\mathrm{H}$ activation (ii) rhodaelectro-catalyzed $\mathrm{C}-\mathrm{H}$ activation.

\subsubsection{Rh-catalyzed Inner-sphere C-H Activation}

Due to the difficulty to control the selectivity, simple benzene and linear hydrocarbons were ideal model substrates to explore the process of $\mathrm{C}-\mathrm{H}$ cleavage in the early stages of $\mathrm{C}-\mathrm{H}$ activation. In 1984, Bergman and Jones found that photolysis of $\mathrm{Cp}^{*} \mathrm{Rh}\left(\mathrm{PMe}_{3}\right) \mathrm{H}_{2} 96$ in a hydrocarbon solvent led to the loss of $\mathrm{H}_{2}$ and the $\mathrm{C}-\mathrm{H}$ activation of solvent. ${ }^{[112]}$ Unfortunately, the 16-electron $\mathrm{Cp}^{*} \mathrm{Rh}\left(\mathrm{PMe}_{3}\right) 97$ viewed to be accountable for hydrocarbon oxidative addition was not detectable (Scheme 1.30).

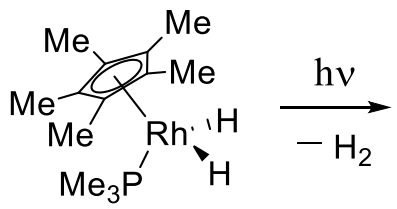

96

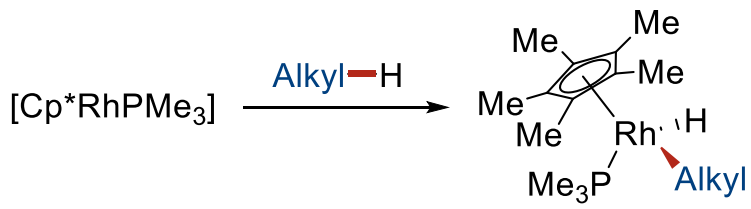

97
98

Scheme 1.30. Early study of $\mathrm{C}-\mathrm{H}$ activation by Bergman.

Inspired by the early rhodium-mediated $\mathrm{C}-\mathrm{H}$ activation, the inert methane successfully underwent carboxylation to release acetic acid catalyzed by $\mathrm{RhCl}_{3}$ under aqueous 
conditions with the use of oxygen as the oxidant (Scheme 1.31a). ${ }^{[113]}$ However, the yield of this process was not mentioned. Besides the alkane carboxylation, photochemical transformations of alkanes to aldehyde 38a catalyzed by $\mathrm{RhCl}\left(\mathrm{PMe}_{3}\right)_{3}(99)$ have also been observed (Scheme 1.31b). ${ }^{[114]}$ Mechanistic studies by Goldman provided evidence that the CO-dissociated species is not responsible for the $\mathrm{C}-\mathrm{H}$ activation step. Goldman proposed that the principal photo-process in this reaction involves direct $\mathrm{C}-\mathrm{H}$ oxidation addition to the four-coordinate rhodium(I) center to deliver a six-coordinate intermediate, which then undergoes $\mathrm{CO}$ insertion in the following step. ${ }^{[115]}$

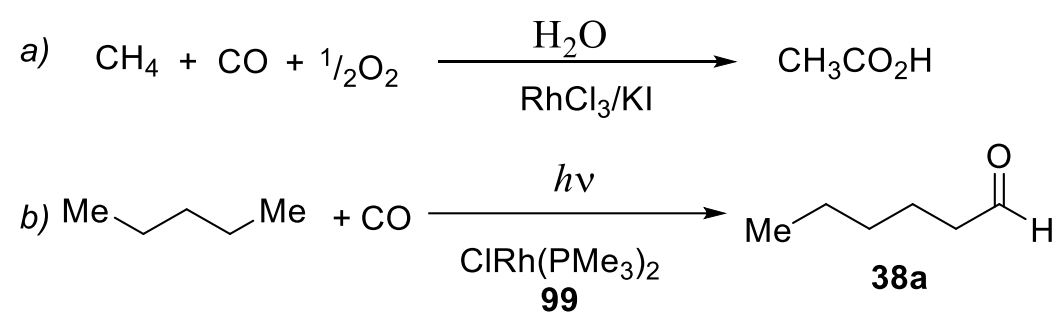

Scheme 1.31. $\mathrm{Rh}(\mathrm{I})$ catalyzed transformations of hydrocarbons.

Alkynes tend to experience dimerization or polymerization in the presence rhodium complexes especially at high temperatures. ${ }^{[116]}$ In the early stage of rhodium catalyzed $\mathrm{C}\left(\mathrm{sp}^{2}\right)-\mathrm{H}$ activation, alkenes are the ideal substrates for the position-selective $\mathrm{C}-\mathrm{H}$ activation. In one of the earliest reports of rhodium-catalyzed $\mathrm{C}-\mathrm{C}$ bond formation by $\mathrm{C}-\mathrm{H}$ activation, Lim and Kang showed that pyridine functioned as a directing group to achieve C-H alkylation, albeit without tolerance of any other functional groups. ${ }^{[117]}$

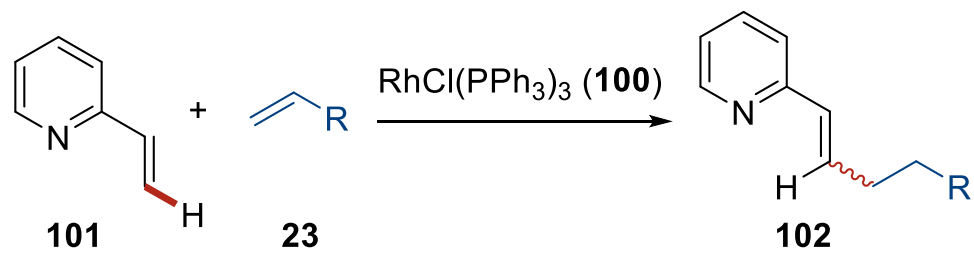

Scheme 1.32. Rhodium-catalyzed $\mathrm{C}-\mathrm{H}$ alkylation.

Subsequently, the imine functionality enjoyed widespread use and development in the realm of $\mathrm{C}-\mathrm{H}$ bond functionalization by Rhodium (I). Specifically, $\mathrm{RhCl}\left(\mathrm{PPh}_{3}\right)_{3}(100$ Wilkinson's catalyst) was determined to be the optimal catalyst for this transformation. ${ }^{[118]}$ Furthermore, the overalkylation that plagued aryl ketone alkylation could be avoided in this 
case. It was further discovered by Jun and co-workers that imines 103 were capable to undergo alkylation to generate the corresponding imines 104 (Scheme 1.33). ${ }^{[119]}$

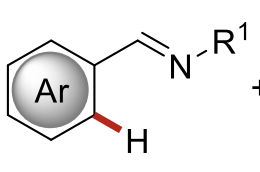

103<smiles>[R]C=CC</smiles>

23

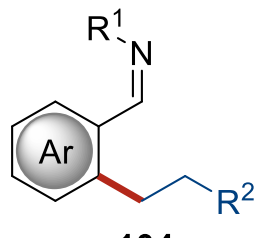

104

Scheme 1.33. Imine directed alkylation.

The widely accepted mechanism for chelation-assisted $\mathrm{C}-\mathrm{H}$ bond alkylation catalyzed by rhodium(I) demonstrated in Scheme 1.34. Initial coordination of rhodium by the chelating heteroatom of imine 105 followed by facile $\mathrm{C}-\mathrm{H}$ bond activation gives metallacyclic intermediate 107. Dissociation of a phosphine ligand, followed by olefin binding and hydride insertion, gives 108 . Reductive elimination of 109 produces the product 110 and closes the catalytic cycle. ${ }^{[120]}$ The reductive elimination step has been demonstrated to be rate limiting in $\mathrm{C}-\mathrm{H}$ alkylation reactions. 


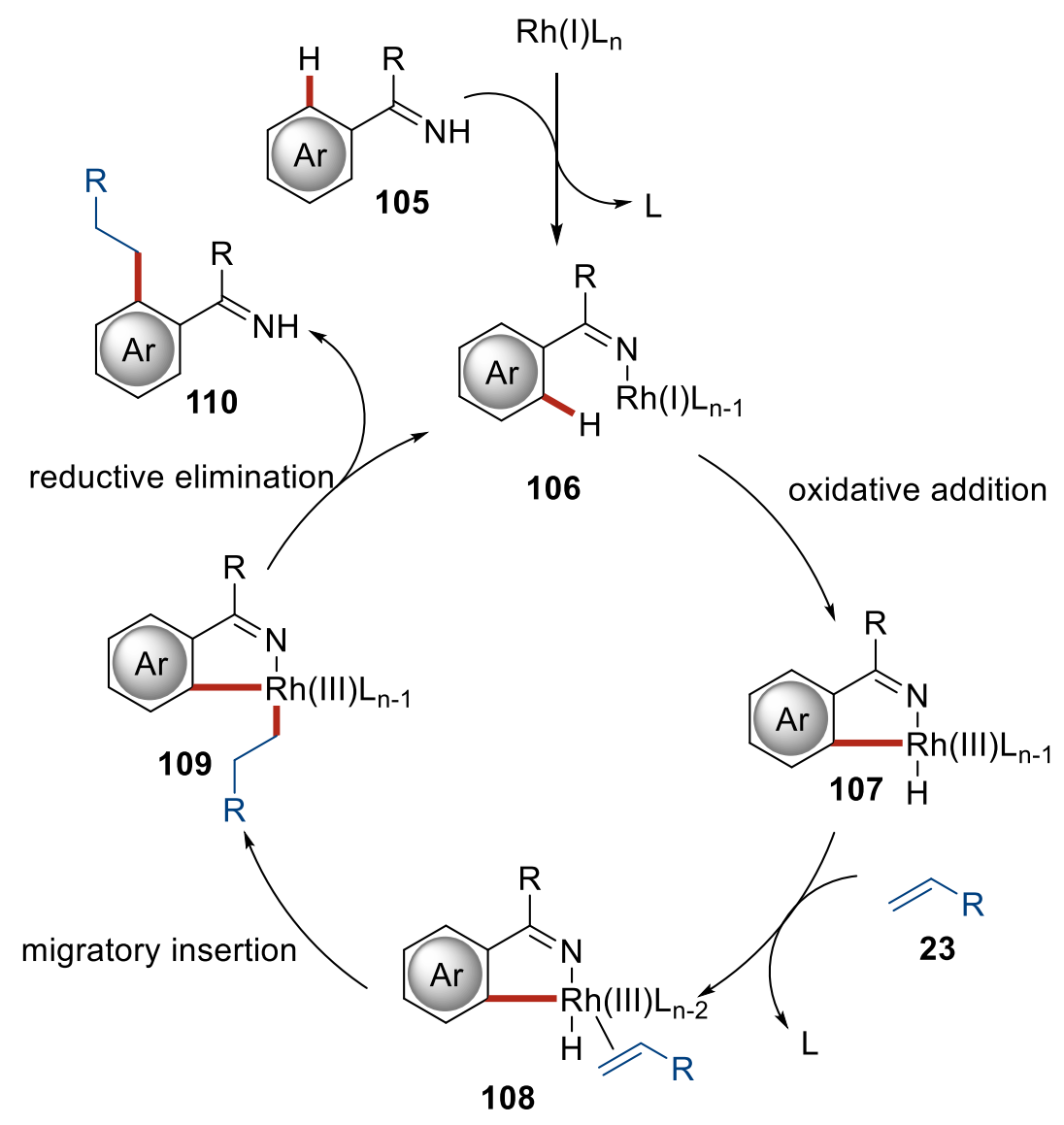

Scheme 1.34. Mechanism for rhodium(I) catalyzed alkylation.

While the hydroarylation of olefins has seen broad success using rhodium(I)-catalyzed chelation-assisted methods, the tendency of alkynes, in particular terminal alkynes, to undergo rhodium-catalyzed alkyne dimerization or trimerization has made their use problematic. ${ }^{[116]}$ Internal alkynes, much like internal olefins, are often unreactive in $\mathrm{C}-\mathrm{H}$ bond functionalization reactions. Under assistance of the reliable mechanism, several groups have successfully developed heteroatom-chelated vinylation methods. Lim and Kang reported the first example of chelation-assisted hydroarylation of an internal alkyne 113 realizing the ortho-alkenylation of 2-phenylpyridines by using Wilkinson's catalyst (Scheme 1.35a). ${ }^{[121]}$ The scope of alkyne was limited primarily to internal and symmetrical alkynes. Terminal alkynes generated mainly polymeric materials, ${ }^{[116]}$ and unsymmetrical alkynes, such as 2-hepyne, led to regioisomeric mixtures. ${ }^{[122]}$ Internal alkynes were smoothly used for the alkenylation with the assistance of imine using $\mathrm{RhCl}\left(\mathrm{PPh}_{3}\right)_{3}$ as the catalyst (Scheme 1.35b). It was also discovered by Jun that increased temperatures and 
prolonged reaction times ultimately produced isoquinoline products 116 in nearly quantitative amounts (Scheme 1.35c). ${ }^{[123]}$ When triisopropylsilylacetylene is used as the coupling partner, the substrate scope of the alkenylation in the presence of a $\mathrm{RhCl}\left(\mathrm{PPh}_{3}\right)_{3}$ catalyst was expanded to imidazole and benzimidazole directing groups (Scheme 1.36). ${ }^{[122]}$

a)<smiles>[C+]c1ccccc1-c1ccccn1</smiles>

112

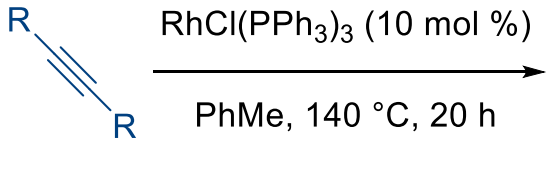

113<smiles>[R]C=C([R])c1ccccc1-c1ccccn1</smiles>

114

b)<smiles>[R]C(=N)c1ccccc1[18F]</smiles>
107
$\mathrm{R}$<smiles>[R]C#C</smiles>

113<smiles>[R]C([R])[PbH2]C=[13CH]</smiles>

PhMe, $120^{\circ} \mathrm{C}, 2 \mathrm{~h}$<smiles>[R]C=C([R])c1ccccc1C([R])=N</smiles>

115

c)<smiles>[R]C(=N)c1ccccc1[18F]</smiles>

107<smiles>c1ccc(-c2ccccc2)cc1</smiles>

$113 a$

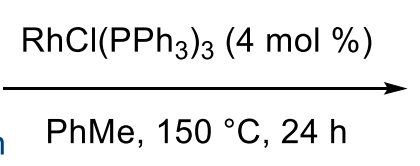<smiles>[R]c1nc(-c2ccccc2)c(-c2ccccc2)c2ccccc12</smiles>

116

Scheme 1.35. $\mathrm{Rh}(\mathrm{I})$ catalyzed $\mathrm{C}-\mathrm{H}$ alkenylation.<smiles>Cn1ccnc1-c1ccccc1/C=C/C=[Pt]</smiles>

TIPS<smiles></smiles>

$117 d, 87 \%$<smiles>CC(C)(C)[Pb]</smiles><smiles>C/C(=N\c1ccccc1)c1ccccc1/C=C/C(F)(F)F</smiles>

117 e, $80 \%$

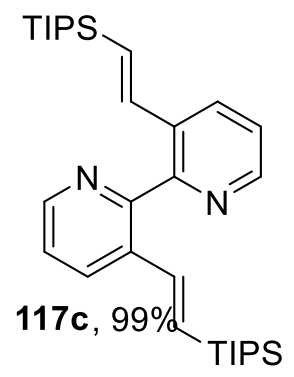<smiles>C/C(=N\O)c1ccccc1/C=C/C(F)(F)F</smiles>

117f, $66 \%$ 
Scheme 1.36. Alkenylation by using triisopropylsilylacetylene.

Carboxylate groups can also function as effective directing groups in oxidative coupling of benzoic acids and alkynes using a rhodium(III)-copper(II) co-catalytic system, where the copper(II) serves to oxidize the rhodium catalyst in order to reinitiate the catalytic cycle (Scheme 1.37). A series of isocoumarin derivatives 119 was thereby synthesized from benzoic acids and internal alkynes using $\left[\mathrm{Cp}^{*} \mathrm{RhCl}_{2}\right]_{2}$ as the catalyst. ${ }^{[124]}$ Both electrondeficient and electron-rich benzoic acids were suitable substrates, and alkyl or aryl substituted alkynes were used.

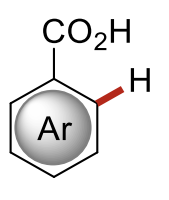

118<smiles>[R]C#C[R]</smiles>

113

$$
\begin{gathered}
\underset{\left[\mathrm{Cp}^{*} \mathrm{RhCl}_{2}\right]_{2}(1 \mathrm{~mol} \%)}{\mathrm{Cu}(\mathrm{OAc})_{2}(5 \mathrm{~mol} \%)} \\
\underset{\mathrm{DMF}, 120^{\circ} \mathrm{C}, 2 \mathrm{~h}}{\longrightarrow}
\end{gathered}
$$

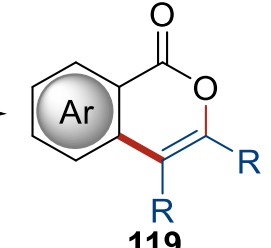

Scheme 1.37. Oxidative coupling of benzoic acids with alkynes.

A plausible mechanism for the reaction of benzoic acid 118 with alkyne 113 is shown in Scheme 1.38. Coordination of the carboxylate oxygen to $\mathrm{Cp}^{*} \mathrm{Rh}(\mathrm{III})(\mathrm{OAc})_{2} \mathbf{1 2 0}$ is followed by ortho rhodation to form a rhodacycle intermediate 121. Afterwards alkyne insertion and reductive elimination occurs to produce isocoumarin 119 . The resulting rhodium(I) species 123 is oxidized in the presence of the copper cocatalyst to regenerate $\mathrm{Rh}(\mathrm{III}) \mathrm{Cp}^{*}(\mathrm{OAc})_{2}$. 


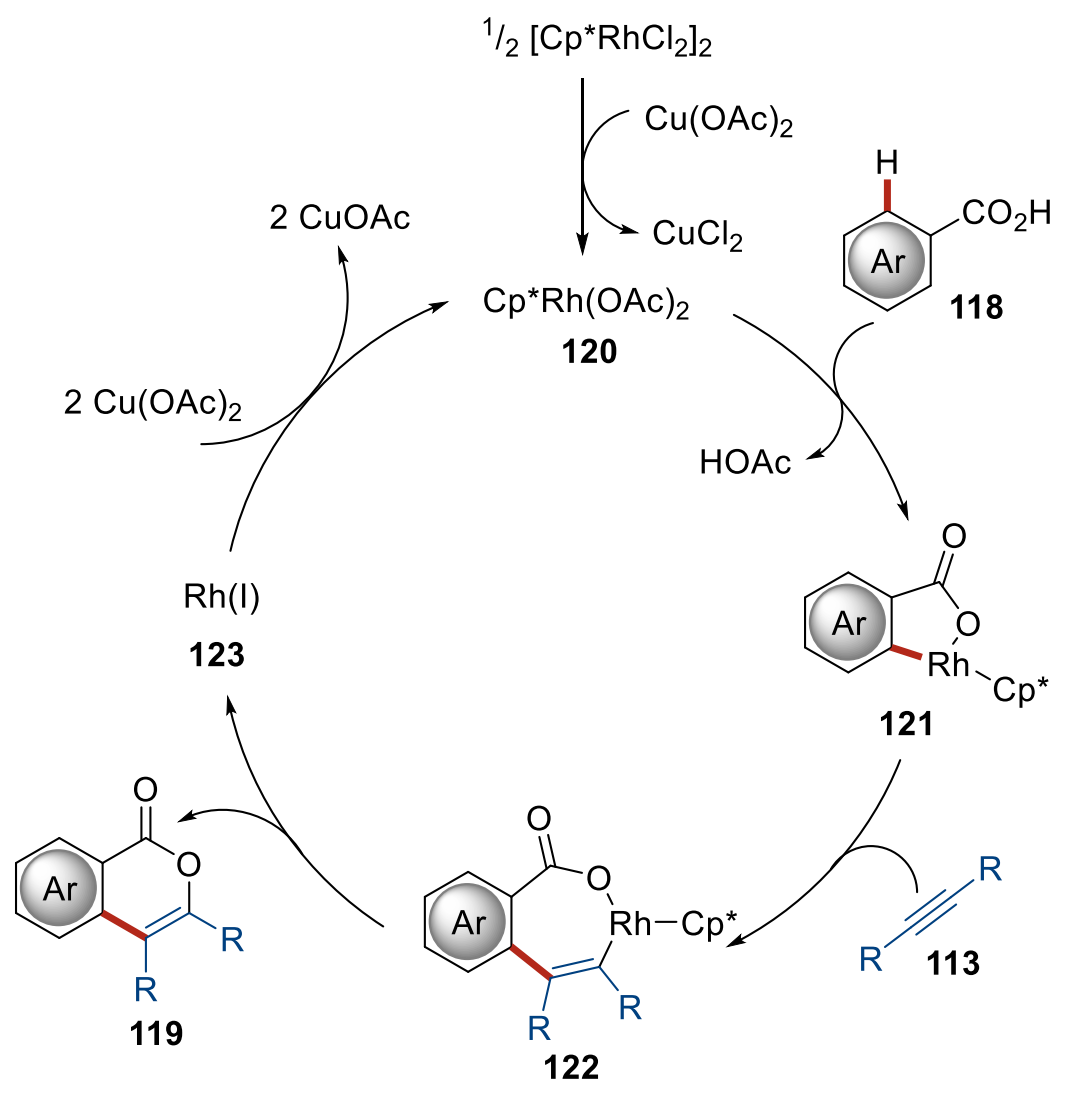

Scheme 1.38. Plausible mechanism for rhodium-catalyzed isocoumarin synthesis.

Encouraged by the significant breakthrough of the carboxylate-assisted $\mathrm{C}-\mathrm{H}$ activation reported by Miura, the reactions of 1-naphthols (124) (Scheme 1.39) and analogues including 4-hydroxycoumarin, quinolinone and 9-phenylxanthen-9-ol were discovered to undergo peri $\mathrm{C}-\mathrm{H}$ bond cleavage to produce fused pyran derivatives. ${ }^{[125]}$<smiles>Oc1cccc2ccccc12</smiles>

124

(2 equiv)

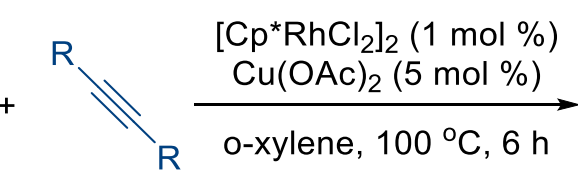

113

(1.0 equiv)<smiles>[R]C1=C([R])c2cccc3cccc(c23)O1</smiles>

125: $42-83 \%$

Scheme 1.39. The coupling of 1-naphthols with alkynes.

In contrast to the couplings described above (Scheme 1.39), treatment of equimolar amounts of 2-phenylphenol 126, diphenylacetylene, $\mathrm{Cu}(\mathrm{OAc})_{2} \cdot \mathrm{H}_{2} \mathrm{O}$, and $\mathrm{KI}$ in the presence 
of $\left[\mathrm{Cp}^{*} \mathrm{RhCl}_{2}\right]_{2}(1 \mathrm{~mol} \%)$ selectively furnished 5-(2-hydroxyphenyl)-1,2,3,4-tetraphenylnaphthalene 127 in $81 \%$ yield (Scheme 1.40). ${ }^{[125]}$

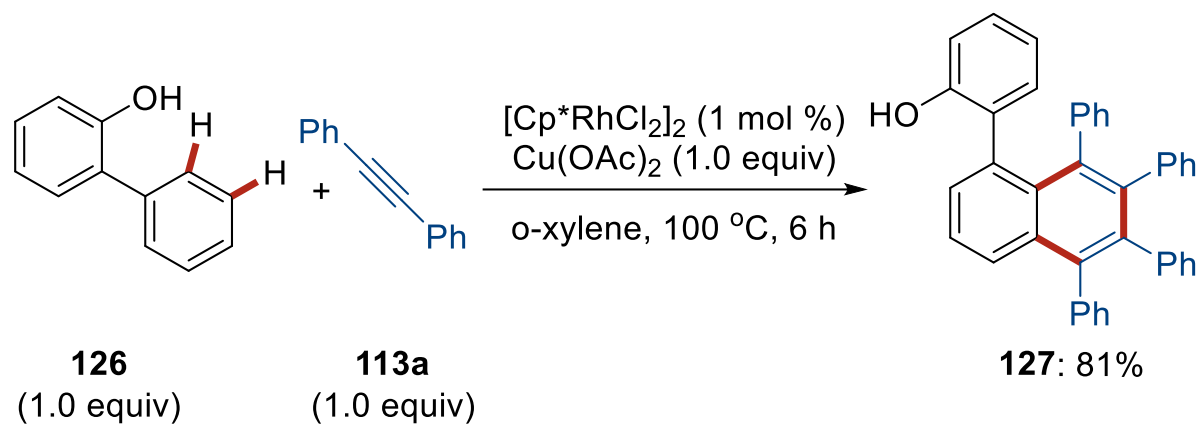

Scheme 1.40. The coupling of 2-phenylphenol with diphenylacetylene.

In 2008, Fagnou found that alkynes oxidatively coupled with acetanilides 128 by using rhodium(III) catalyst and $\mathrm{Cu}(\mathrm{OAc})_{2}$ as the oxidant through ortho $\mathrm{C}-\mathrm{H}$ bond cleavage to furnish $\mathrm{N}$-acetylindoles 129 (Scheme 1.41a). ${ }^{[126]}$ Meanwhile, Miura found that benzanilides 130, which possess two types of cleavable ortho $\mathrm{C}-\mathrm{H}$ bonds on aniline and benzoic acid moieties, undergo the oxidative coupling with alkynes involving the selective cleavage of the latter to produce isoquinolinone derivatives $131 .{ }^{[127]}$

a)

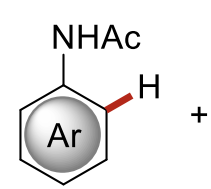

128

(1.0 equiv)

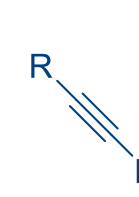

113

(1.1 equiv)

$$
\left[\mathrm{Cp}^{*} \mathrm{RhCl}_{2}\right]_{2}(2.5 \mathrm{~mol} \%)
$$

$$
\mathrm{AgSbF}_{6}(10 \mathrm{~mol} \%)
$$

$\mathrm{O}_{2} 1.0 \mathrm{~atm}$

$\mathrm{t}-\mathrm{AmOH}, 60^{\circ} \mathrm{C}, 16 \mathrm{~h}$

$$
\text { (1.1 equiv) }
$$

b)

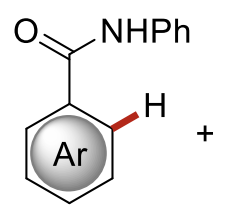

130<smiles>[R]C#C[R]</smiles>

113

(2.0 equiv) (1.0 equiv)

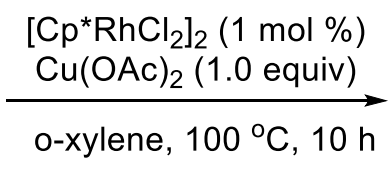

$\underset{\text { o-xylene, } 100^{\circ} \mathrm{C}, 10 \mathrm{~h}}{\mathrm{Cu}(\mathrm{OAc})_{2}(1.0 \text { equiv })}$
$\left.\mathrm{Cp} \mathrm{RhCl}_{2}\right]_{2}(1 \mathrm{~mol} \%)$

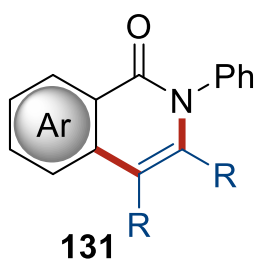

Scheme 1.41. The coupling of $\mathrm{N}$-acylanilines with alkynes.

Under similar conditions, $\mathrm{N}$-unsubstituted benzamides 132 undergo a cascade coupling accompanied by two $\mathrm{C}-\mathrm{H}$ and two $\mathrm{N}-\mathrm{H}$ bond cleavages to construct a tetracyclic dibenzoquinolizinone framework 133 (Scheme 1.42). 


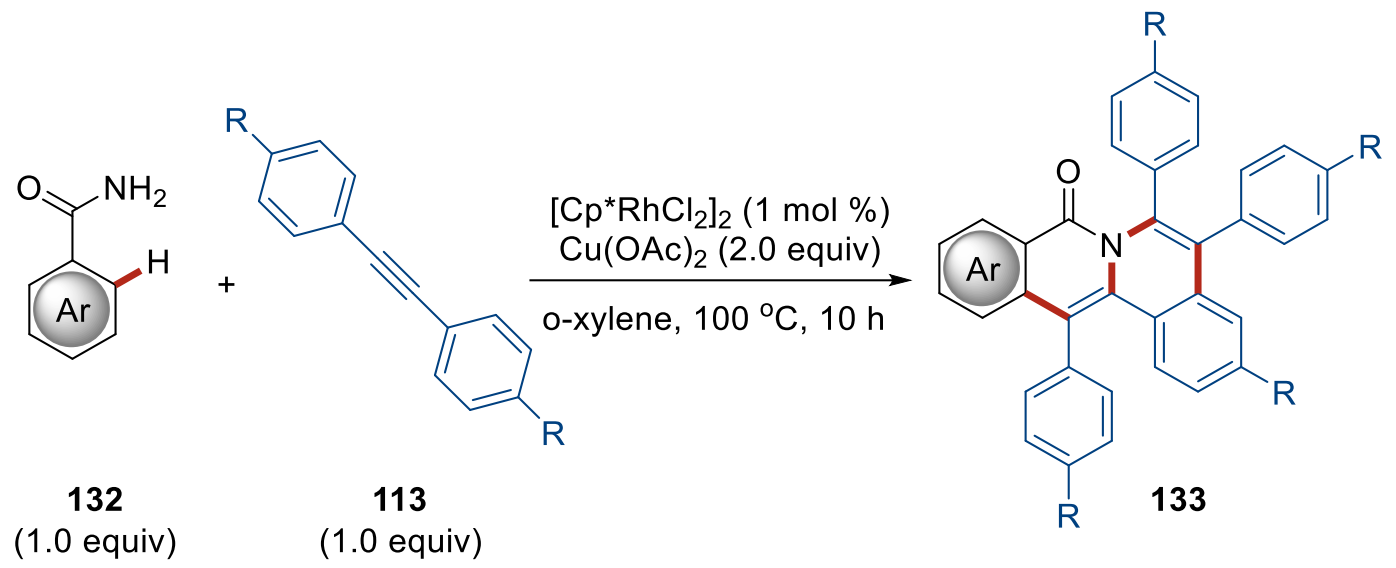

Scheme 1.42. The coupling of $N$-unsubstituted benzamides with diarylacetylenes.

In 2011, Glorius reported a rhodium-catalyzed oxidative olefination by chelation-assisted C-H activation of $\mathrm{N}$-methoxy benzamides 134 . In this mild process, the $\mathrm{N}-\mathrm{O}$ bond serves as an internal oxidant. In addition, a small modification of the substituent of the directing/oxidizing group results in the selective generation of valuable tetrahydroisoquinolinone products 135 (Scheme 1.43a). ${ }^{[128]}$ After changing the Cp ligand from $\mathrm{Cp}^{*}$ to much bulkier $\mathrm{Cp}^{t}$, synthesis of dihydroisoquinolones 137 from aliphatic alkenes and O-pivaloyl benzhydroxamic acids 136 mediated by a rhodium precatalyst was achieved with excellent regioselectivity (Scheme 1.43b). ${ }^{[29]}$

a)<smiles>CO[CH2+]c1ccccc1C(=O)NOC</smiles>
134<smiles>C=CCCC</smiles>

23
$\underline{\left[\mathrm{Cp}^{*} \mathrm{RhCl}_{2}\right]_{2}(2.5 \mathrm{~mol} \%)}$ $\mathrm{CsOAc}$ $\mathrm{MeOH}, \mathrm{rt}, 12 \mathrm{~h}$<smiles>[R]C=Cc1ccccc1C(N)=O</smiles>

135 b)<smiles>O=C(NOc1ccccc1)c1ccccc1[18F]</smiles>

136<smiles>[R]C=C</smiles>

23
$\underset{\left[\mathrm{Cp}^{t} \mathrm{RhCl}_{2}\right]_{2}(2.5 \mathrm{~mol} \%)}{\longrightarrow}$ $\mathrm{MeOH}, \mathrm{rt}, 12 \mathrm{~h}$<smiles>CC(C)(C)c1ccc(C(C)(C)C)cc1</smiles><smiles>[R]C1Cc2ccccc2C(=O)N1</smiles>

137

Scheme 1.43. Rhodium-catalyzed olefination and tetrahydroisoquinolinone synthesis. 
Inspired by this oxidizing group, an unprecedented rhodium-catalyzed $\mathrm{C}-\mathrm{H}$ activation of benzamides and methylenecyclopropanes 138 for the selective synthesis of spiro dihydroisoquinolinones 139 was reported by Cui (Scheme 1.44a). ${ }^{[130]}$ Interestingly, when the furan-derived amide 140 coupled with methylenecyclopropanes under higher temperature, a furan-fused azepinone 141 was formed in high yield (Scheme 1.44b).

a)

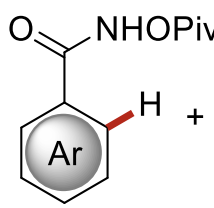

136

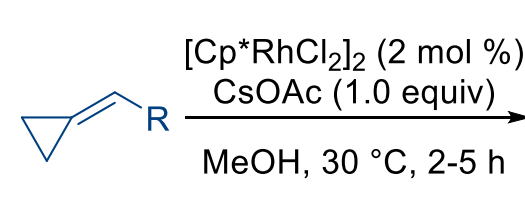

138<smiles>[R]C1NC(=O)c2ccccc2C12CC2</smiles>

139 b)

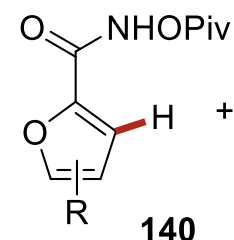

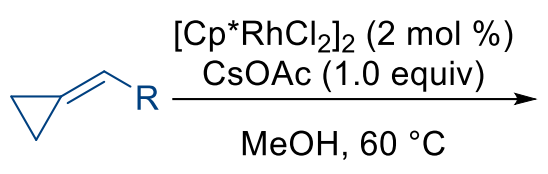
138<smiles>[R]C=C1CCNC(=O)c2oc[R]c21</smiles>

Scheme 1.44. Synthesis of spiro dihydroisoquinolinones 139 and furan-fused azepinone 141.

Another diastereoselective coupling of arylhydroxamates 136 and cyclopropenes 142 was successfully developed by rhodium catalysis (Scheme 1.45). Through ligand development, the diastereoselectivity of this reaction was improved using a heptamethylindenyl (Ind*) ligand. In addition, the nature of the O-substituted ester of benzhydroxamic acid proved important for achieving high diastereoselectivity. ${ }^{[131]}$

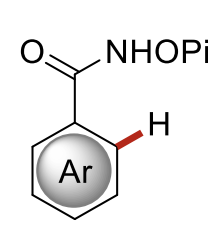

136

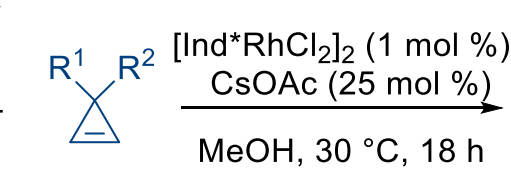

142

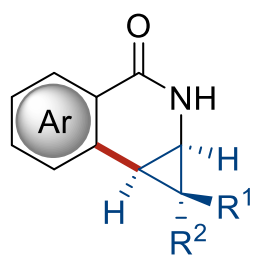

$143,>10: 1 \mathrm{dr}$<smiles>Cc1c(C)c(C)c2c(C)c(C)c(C)c(C)c2c1C</smiles>

Scheme 1.45. Benzamidation of cyclopropenes via rhodium(III) catalyzed C-H activation. 
In 2014, Wang group reported the use of cyclopropene as a three-carbon unit in rhodium(III)-catalyzed C-H bond activation. ${ }^{[132]}$ An efficient rhodium(III)-catalyzed synthesis of $2 \mathrm{H}$-chromene 145 from $\mathrm{N}$-phenoxyacetamides 144 and cyclopropenes through $\mathrm{C}-\mathrm{H}$ activation has been achieved (Scheme 1.46). The reaction proceeded at room temperature without using external oxidants.

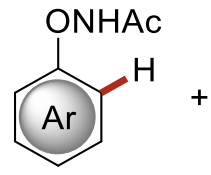

144

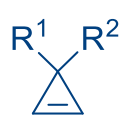

142

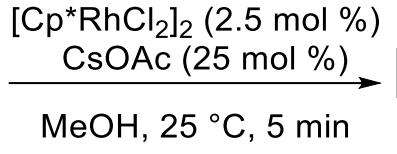

$\mathrm{MeOH}, 25^{\circ} \mathrm{C}, 5 \mathrm{~min}$

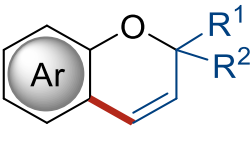

145

Scheme 1.46. Synthesis of $2 \mathrm{H}$-chromene 145 from $\mathrm{N}$-phenoxyacetamides 144 and cyclopropenes 142 .

A combination of $\mathrm{C}-\mathrm{H}$ activation with ring opening of cyclopropanols by rhodium(III)catalysis, offering an efficient route to access $\beta$-aryl ketones was reported by Li. ${ }^{[133]}$ The reaction proceeded under mild conditions with ample scope, high regioselectivity, and excellent functional group tolerance. Both oxime ethers and $N$-pyrimidylindoles proved to be viable substrates. In 2018, Li reported rhodium(III)-catalyzed C-H activation of nitrones 146 and azomethine imines 147, followed by a dipolar cycloaddition with alkylidenecyclopropanes 138 (ACPs) (Scheme 1.47). Taking advantage of the ring strain in ACPs, the reaction with aryl nitrones delivered bridged [3.2.1] bicyclic isoxazolidines 148, and reaction with azomethine imines afforded bridged tricyclic pyrazolones 149 under the same conditions. ${ }^{[134]}$

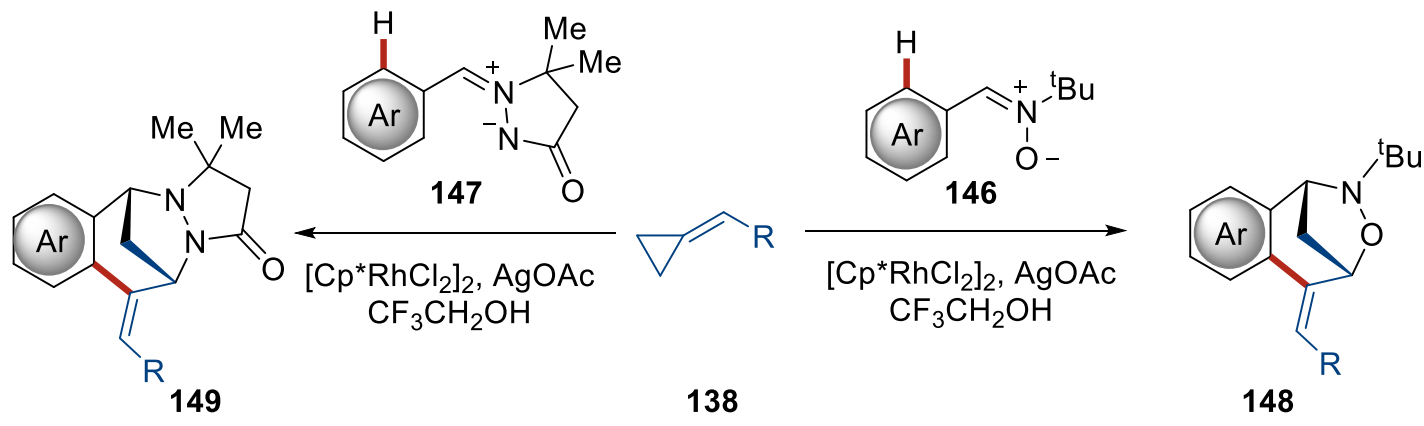

Scheme 1.47. Coupling of nitrones 146 and azomethine imines 147 with alkylidenecyclopropanes 138. 
The arylnitrones 150 were coupled with internal alkynes 113 to form indolines 151 in the presence of rhodium(III) catalyst free of external oxidants (Scheme 1.48). A dual role of the rhodium catalyst is proposed, initially enabling the $\mathrm{C}-\mathrm{H}$ cleavage and $\mathrm{O}$-atom transfer process. The cyclization worked well under mild conditions furnishing the desired products 151 with moderate to high diastereoselectivity. ${ }^{[135]}$<smiles>[O-][N+](=C[Br+])c1ccccc1</smiles>

150
$\mathrm{R}$<smiles>CC#CP</smiles>
113
$\left[\mathrm{Cp}^{*} \mathrm{RhCl}_{2}\right]_{2}(4 \mathrm{~mol} \%)$ $\mathrm{AgSbF}_{6}(16 \mathrm{~mol} \%)$

$\mathrm{PivOH}(0.5$ equiv) 1,4-dioxane, $50{ }^{\circ} \mathrm{C}$

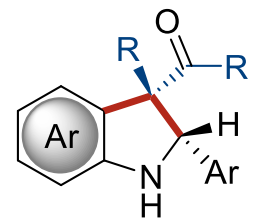

$151,40-80 \%$ yield, $4: 1$ to $9: 1 \mathrm{dr}$

Scheme 1.48. Cyclization of arylnitrones to indolines.

In 2012, Chang and coworkers reported the first rhodium(III)-catalyzed C-H amidation reaction using sulfonyl azides as the amino source, releasing $\mathrm{N}_{2}$ as the sole byproduct without the use of external oxidants (Scheme 1.49a). ${ }^{[136]} \mathrm{A}$ more challenging rhodium(III)catalyzed chelation-assisted activation of unreactive $\mathrm{C}\left(\mathrm{sp}^{3}\right)-\mathrm{H}$ bonds has been disclosed in You's lab (Scheme 1.49b), thus providing a practical and step-economic route to 2(pyridine-2-yl)ethanamine derivatives via an intermolecular amidation. In contrast to Chang's system, external oxidant $\mathrm{Phl}(\mathrm{OAc})_{2}$ was responsible for the generation of nitrene, which undergoes subsequent migratory insertion. ${ }^{[137]}$ Dioxazolone, $N$-methoxyamide and amidobenziodoxolones were also successfully developed as suitable nitrogenation sources to perform the rhodium(III)-catalyzed chelation-assisted $\mathrm{C}-\mathrm{H}$ activation (Scheme $1.49 \mathrm{c})^{[138]}$ 
a) Chang's $C\left(s p^{2}\right)-H$ amidation

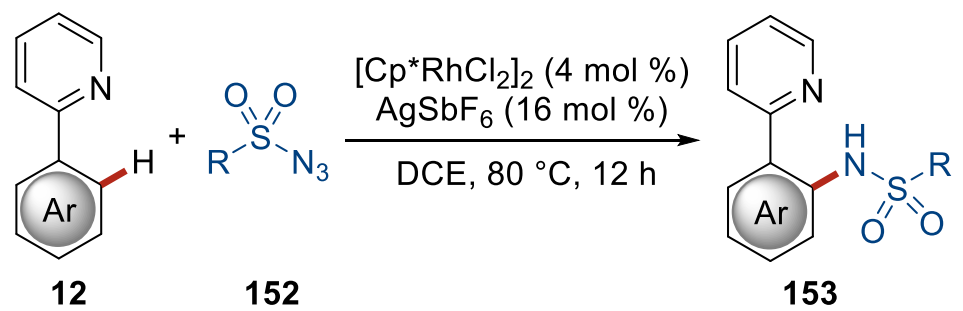

b) You's $C\left(s p^{3}\right)-H$ amidation

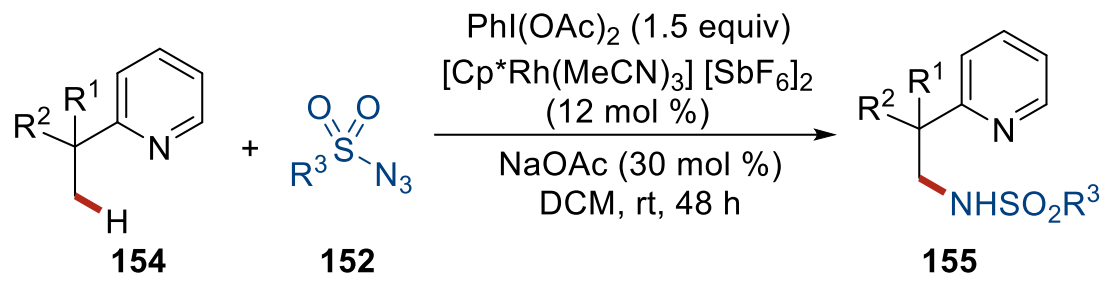

c) other amidation reagents<smiles>[R]c1noc(=O)o1</smiles>

156<smiles>O=C(NO)c1ccccc1</smiles>

157<smiles>[R]NI1OC(=O)c2ccccc21</smiles>

158

Scheme 1.49. Rhodium(III)-catalyzed C-H amidation.

Despite the plethora of $\mathrm{C} 2$ functionalization of indole, the selective $\mathrm{C} 7$ transformation of indole is scarce. Through elaborate modification of the directing groups, C7 olefination, arylation, methylation and alkylation were available in Shi's and Ma's lab (Scheme 1.50). ${ }^{[139]}$<smiles>[R]Oc1cccc2ccn(C(=O)C(C)(C)C)c12</smiles>

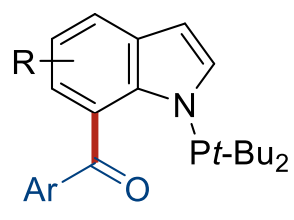

162<smiles>[R]c1ccc(-c2ccccc2)c2c1ccn2[PH](C)(C)C</smiles>

160<smiles>[R]c1cccc(/C=C/[Al])c1N([R16])CC</smiles>

161

Scheme 1.50. Products obtained via C7 functionalization of indole by rhodium(III). 
Further important functional groups have been directly introduced to useful molecules under the assistance of rhodium catalyst, such as $\mathrm{Br}, \mathrm{I}, \mathrm{CN}$ and $\mathrm{N}_{3} \cdot{ }^{[140]}$ Many structurally complex molecules become available via strategic chelation-assisted and rhodiumcatalyzed C-H functionalizations (Scheme 1.51). ${ }^{[141]}$

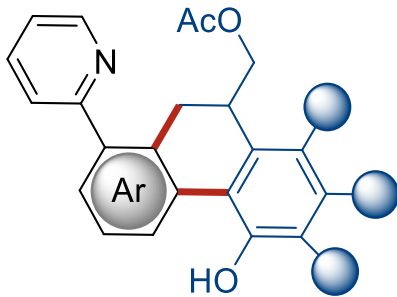

164

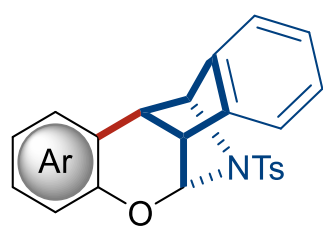

167

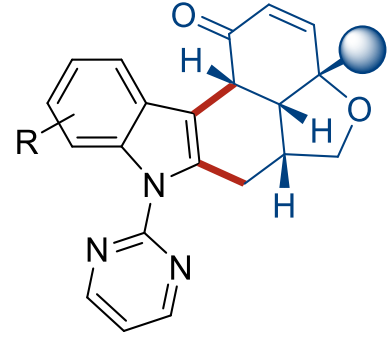

165

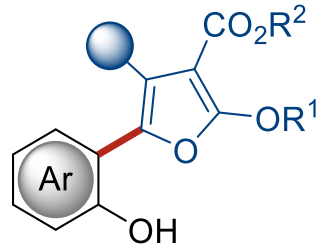

168

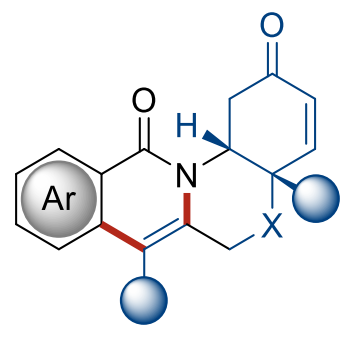

166

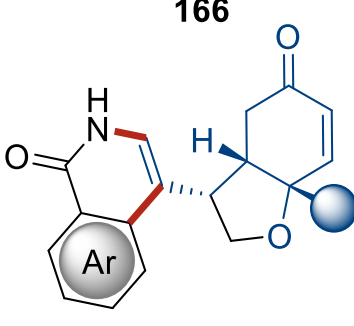

169

Scheme 1.51. Complex structures obtained by rhodium(III) catalyzed $\mathrm{C}-\mathrm{H}$ activation.

\subsubsection{Rhodaelectro-catalyzed C-H Activation}

In the last a few years, the use of electricity as a redox reagent to drive chemical reactions has emerged as an increasingly-viable platform. ${ }^{[142]}$ Significant achievements have been realized by the merger of metallaelectrocatalysis and $\mathrm{C}-\mathrm{H}$ activation, thus eliminating the use of toxic and expensive oxidants. ${ }^{[143]}$ The exploitation of efficient and more environmentally-friendly synthetic methods is still highly desirable. Many opportunities lie ahead to explore rhodaelectro-catalyzed $\mathrm{C}-\mathrm{H}$ activation. Thus, when replacing the chemical oxidants with electricity, new reaction pathway and new reaction manifold will be found

In 2018, Ackermann and coworkers reported the first rhodaelectro-catalyzed C-H activation (Scheme 1.52). ${ }^{[144]}$ Hence, cross-dehydrogenative $\mathrm{C}-\mathrm{H} / \mathrm{C}-\mathrm{H}$ alkenylations were realized via weakly-coordinating benzoic acids 118 and alkenes $\mathbf{2 3}$. This report serves as 
the proof of concept for the $4 \mathrm{~d}$-metal electrocatalyzed $\mathrm{C}-\mathrm{H}$ activation. The optimal reaction conditions featured a user-friendly undivided cell setup, KOAc as the additive and full water tolerance.

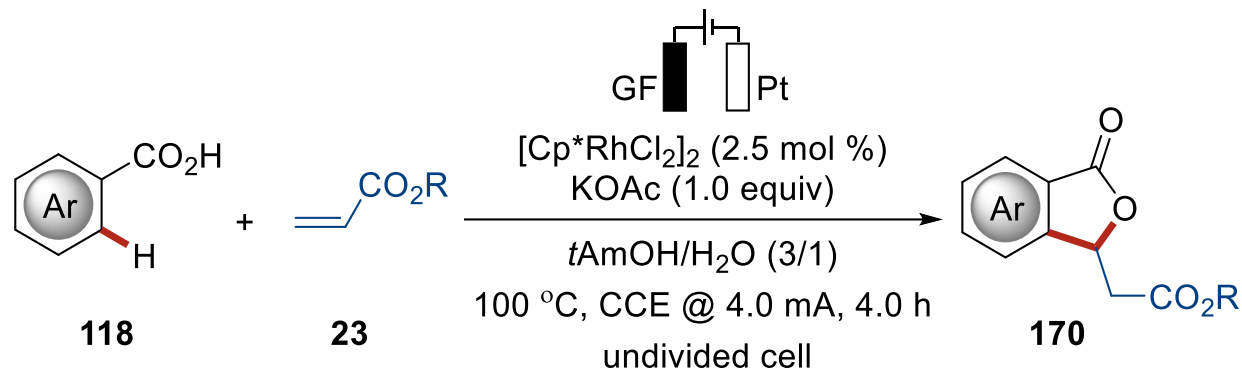

Scheme 1.52. First rhodaelectro-catalyzed $\mathrm{C}-\mathrm{H}$ activation.

In contrast to the alkenylation with $\alpha, \beta$-unsaturated carbonyl compounds under rhodaelectro-catalysis, Ackermann recently reported an intriguing alkenylation reaction using unactivated alkenes 23 with weakly coordinating benzamides 92 (Scheme 1.53). ${ }^{[145]}$ The rhodaelectro-catalyzed $\mathrm{C}-\mathrm{H}$ alkenylation was shown to proceed with ample substrate scope, including heterocycles and valuable electrophilic functional groups, such as chloride, bromo and nitrile. Likewise, a variety of alkenes proved to be amenable, especially oxidation-sensitive hydroxyl substituents. A gram-scale reaction without the decrease of yield highlighted the synthetic utility of the rhodaelectro-catalyzed $\mathrm{C}-\mathrm{H}$ activation.

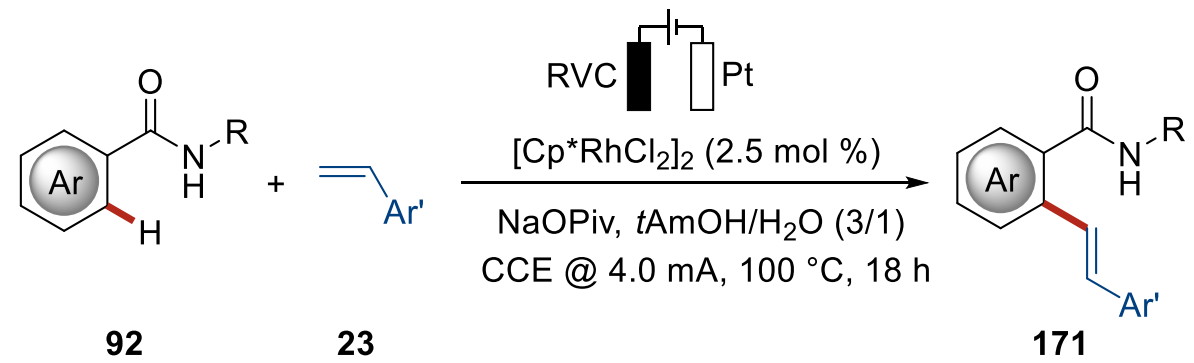

Scheme 1.53. Rhodaelectro-catalyzed C-H alkenylation.

Electrooxidative $\mathrm{C}-\mathrm{B} / \mathrm{C}-\mathrm{H}[2+2+2]$ cyclization was realized with a variety of boronic acids via versatile rhodium catalysis (Scheme 1.54). ${ }^{[146]}$ The $\mathrm{C}-\mathrm{B} / \mathrm{C}-\mathrm{H}$ annulation was efficiently established with ample scope and remarkable levels of functional group tolerance, such as chloro, ester and cyano substituents, in a user-friendly undivided cell setup. Notably, the 
chemoselectivity of the conversion of sensitive iodo-substituted boronic acids could be significantly improved as compared to transformations with the typical chemical oxidants, such as $\mathrm{AgOAc}$ and $\mathrm{Cu}(\mathrm{OAc})_{2}$.

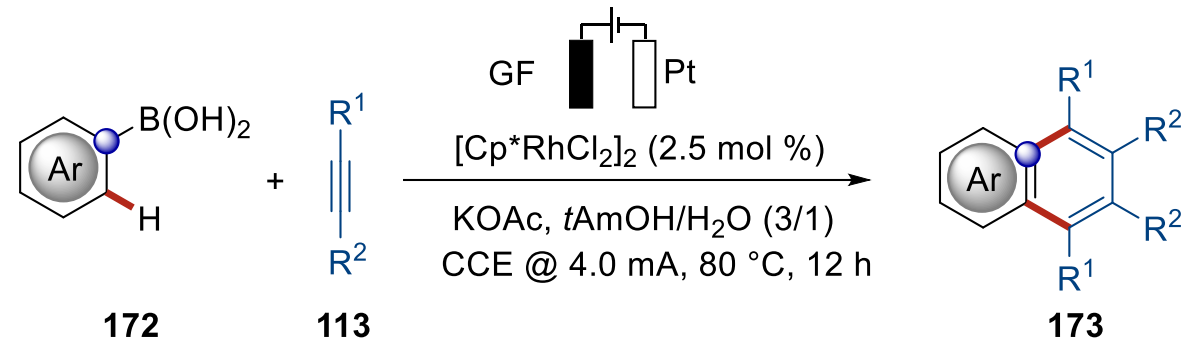

Scheme 1.54. Electrooxidative $\mathrm{C}-\mathrm{B} / \mathrm{C}-\mathrm{H}[2+2+2]$ cyclization.

Ackermann also found a robust flow-rhodaelectro-catalyzed alkynes cyclization utilizing aryl imidates 107 as the substrates (Scheme 1.55). ${ }^{[147]}$ It is worth noting that $\mathrm{C}-\mathrm{H} / \mathrm{N}-\mathrm{H}$ alkyne annulations were amenable to an electroflow setup. This strategy represents a userfriendly tool for the efficient upscaling of a reaction with significantly improved control of heat and mass transfer. This challenging flow-rhodaelectro-catalyzed alkyne annulations gave access to isoquinolines 174 as well as azo-tetracycles by an intramolecular reaction.

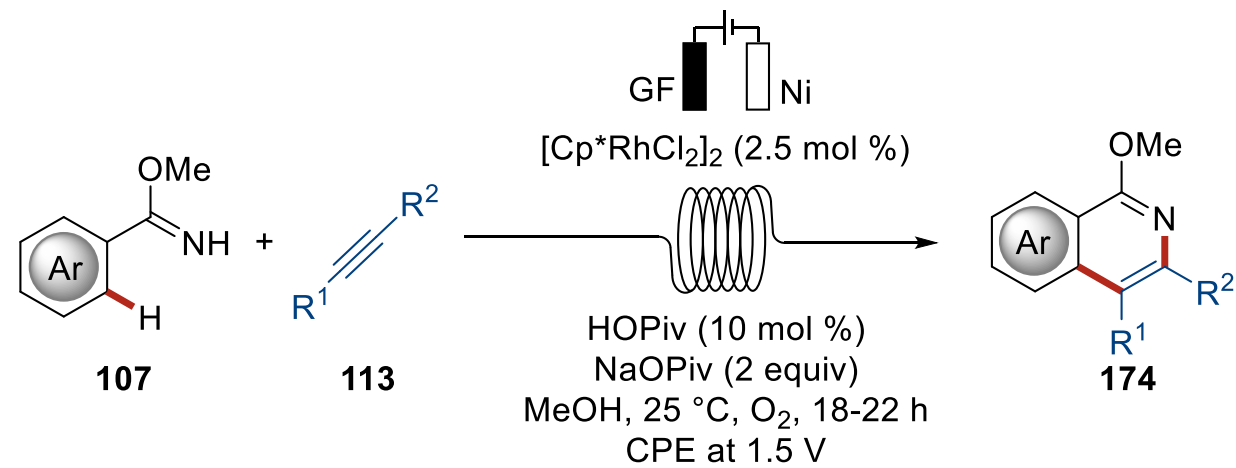

Scheme 1.55. Flow-rhodaelectro-catalyzed alkyne cyclization.

The Xu group then disclosed a phosphorylation using a $N$-coordinating directing groups (Scheme 1.56). ${ }^{[148]}$ The broadly applicable concept of rhodaelectro-catalysis was further utilized for the effective $\mathrm{C}-\mathrm{H}$ phosphorylation using diphenylphosphines 26 . To demonstrate the scalability, a decagram scale reaction was successfully performed, illustrating the potential for future industrial applications. 


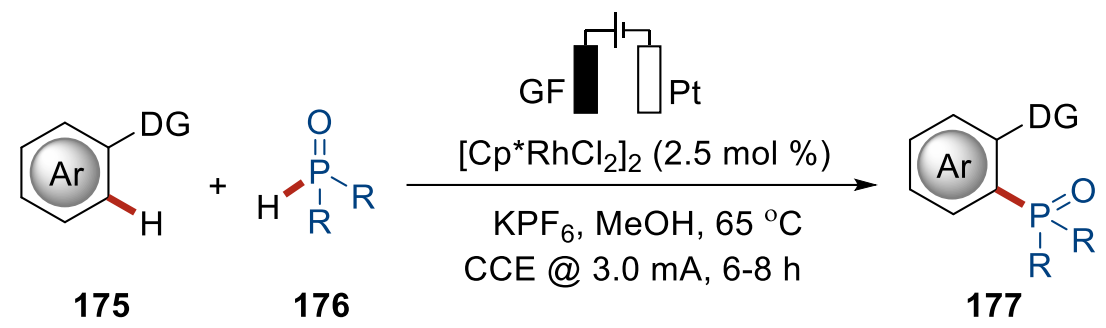

Scheme 1.56. Rhodaelectro-catalyzed C-H phosphorylation. 


\section{Objectives}

The methyl group is widely found among various bioactive compounds, and the introduction of a single methyl substituent can significantly impact the biological activities and physical properties of pharmacologically relevant drug molecules by favoring the bioactive conformation or changing drug metabolism and pharmacokinetic (DMPK) properties. $^{[149]}$ Ackermann ${ }^{[73 b]}$ and Nakamura ${ }^{[73 a]}$ introduced a widely applicable ironcatalyzed methylation protocol for $\mathrm{C}\left(\mathrm{sp}^{2}\right)-\mathrm{H}$ bonds. Benzylamines represent the key molecular motif of various natural products and bioactive molecules. ${ }^{[150]}$ With our continued interests in iron chemistry, the development of methylation of benzylamines catalyzed by iron continues to be in high demand (Scheme 2.1).

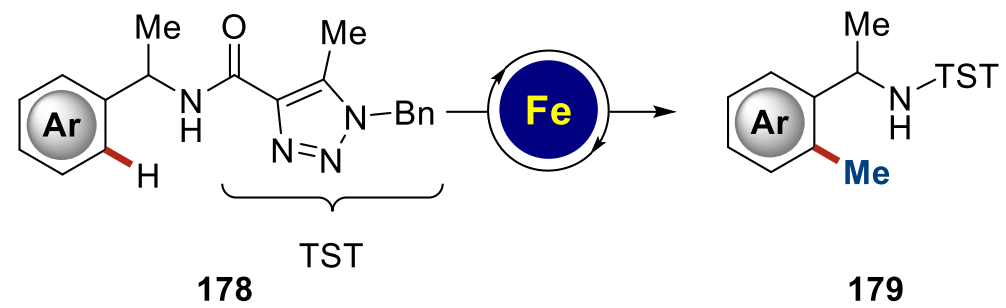

Scheme 2.1. Iron-catalyzed $\mathrm{C}-\mathrm{H}$ methylation.

Alkylation is a very important strategy to form $C\left(s p^{3}\right)-C\left(s p^{3}\right)$ and $C\left(s p^{2}\right)-C\left(s p^{3}\right)$ bonds. Noble metals played an important role in the $\mathrm{C}-\mathrm{H}$ alkyations. ${ }^{[151]}$ Recently inexpensive $3 \mathrm{~d}$ metal catalysts have gained considerable momentum for alkylations as a more environmentally-benign and economically-attractive alternative.[24a, $24 \mathrm{~d}]$ under the assistance of triazole directing groups, the first example of manganese(II)-catalyzed alkylation was reported by Ackermann's group. ${ }^{[106]}$ Pyridinylamides including picolinamide, nicotinamide, and isonicotinamide are key structural units of numerous biologically active molecules, with notable applications to the life science. ${ }^{[152]}$. Thus, it is of high importance to introduce alkyl groups into pyridinylamides in the presence of manganese(II) in order to achieve derivatization of pyridinylamides or gain mechanistic insight into manganese(II)catalyzed C-H activation (Scheme 2.2). 


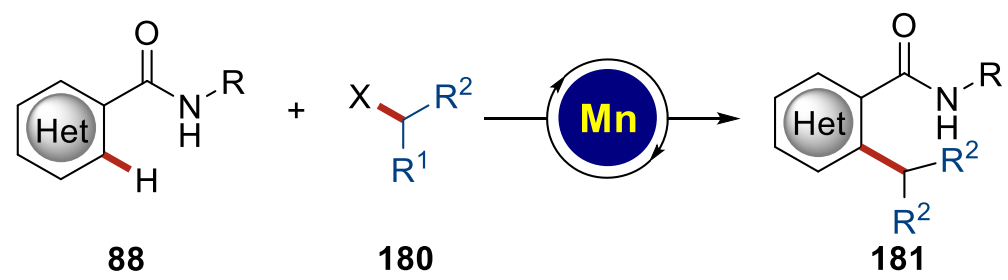

Scheme 2.2. Envisioned manganese(II)-catalyzed C-H alkylation.

Cross-dehydrogenative couplings present the most atom-economic transformation in $\mathrm{C}-\mathrm{H}$ activation. However, to achieve these transformations sacrificial oxidants are needed, which greatly reduces the atom economy of $\mathrm{C}-\mathrm{H}$ activation. Furthermore, most transformations rely on expensive silver salts as oxidants, which drive the cost of the overall reactions. ${ }^{[24,25]}$ The assembly of atomically precise aza-PAHs in an efficient and economic manner has received considerable attention. However, the synthesis of PAHs and aza-PAHs generally relies on stepwise elaborations, largely involving Diels-Alder cycloadditions, dehydrogenative cyclization, and transition-metal-catalyzed crosscouplings that require prefunctionalized substrates. ${ }^{[153]}$ Transition-metal-catalyzed oxidative $\mathrm{C}-\mathrm{H}$ activation/annulation has been proven to be a powerful tool for $\mathrm{PAH}$ syntheses. ${ }^{[154]}$ The electrooxidative alkyne annulation was recently likewise merged with a multiple $\mathrm{C}-\mathrm{H}$ domino strategy catalyzed by rhodium(III). ${ }^{[146]}$ With our ongoing interest in material syntheses by metalla-electrocatalysis, it is promising to develop a one-step approach to the assembly of aza-PAHs via rhodaelectro-catalyzed cascade $\mathrm{C}-\mathrm{H}$ annulations (Scheme 2.3).

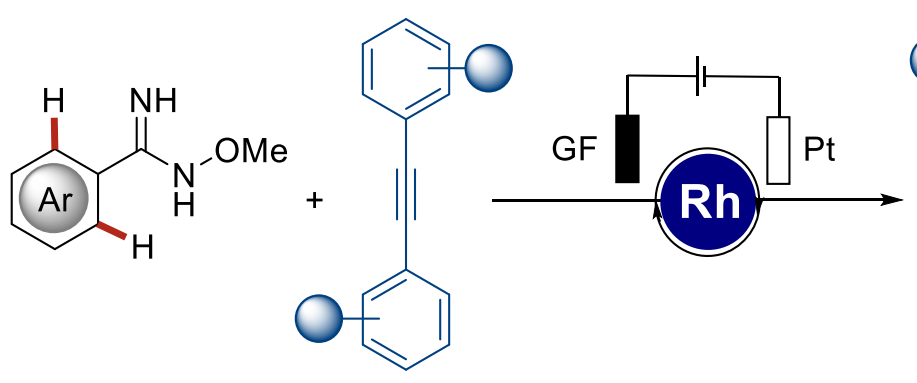

182<smiles>c1ccc(C2=C(c3ccccc3)n3c2cccc2c(-c4ccccc4)c(-c4ccccc4)c4c5ccccc5c(-c5ccccc5)c3n24)cc1</smiles>

183 
Scheme 2.3. Synthesis of aza-nanographene via rhodaelectro-catalyzed Domino $\mathrm{C}-\mathrm{H}$ annulations.

Among a variety of pharmaceuticals, biologically active molecules and natural products, 1,3-dienes ${ }^{[155]}$ and cyclopropanes ${ }^{[156]}$ represent a vital structure unit for their bioactivities. Ene-yne metathesis, Wittig-type olefinations, Mizoroki-Heck reactions and cross-coupling are among the traditional ways to synthesize 1,3 -dienes, ${ }^{[157]}$ while these procedures require the prefunctionalization of the substrates. Thus far, very few examples demonstrated the cyclopropylations by cleavage of $\mathrm{C}-\mathrm{H}$ bonds ${ }^{[158]}$ as cyclopropanes, the smallest rings, are prone to undergo ring-opening through metal-insertion or fragmentation. ${ }^{[159]}$ The exploitation of efficient and more environmentally-friendly synthetic methods is still highly desirable. With our continued interest in rhodaelectro-catalyzed C$\mathrm{H}$ activation, ${ }^{[144-147]}$ we wondered whether the $\mathrm{C}-\mathrm{H}$ dienylation and cyclopropylation could be realized through rhodaelectro-catalysis (Scheme 2.4).

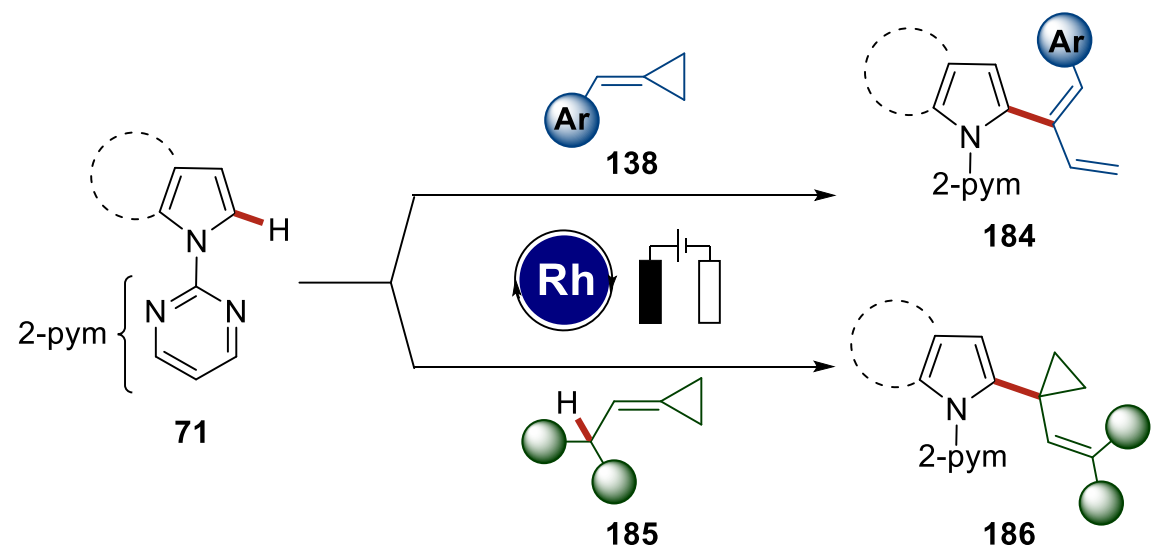

Scheme 2.4. Dienylation and cyclopropylation enabled by rhodaelectro-catalysis. 


\section{Results and Discussion}

\subsection{Triazole-Enabled C-H Activation of Benzylamines by Iron- Catalysis}

Since the methyl group plays a very important role for the biological activities and physical properties of pharmacologically relevant drug molecules, many methylation reagents have been developed for direct $\mathrm{C}-\mathrm{H}$ methylations, such as $\mathrm{MeMgBr}$ 187, $\mathrm{AlMe}_{3}$ 188, $\mathrm{Mel}$ 189, $\mathrm{Me}_{3} \mathrm{~B}_{3} \mathrm{O}_{3}$ 190, MeOTs 191 and $\mathrm{MeBF}_{3} \mathrm{~K} 192$ (Scheme 3.1). ${ }^{[160]}$ Bidentate directing groups were confirmed to be effective in iron-catalyzed $\mathrm{C}-\mathrm{H}$ methylation. ${ }^{[73]}$ In the previous reports, most of the methylation occurred on electron-deficient aromatic compounds.

$$
\mathrm{Me}-\mathrm{MgBr}
$$

187<smiles>CB1OB(C)OB(C)O1</smiles>

190<smiles>C[Al](C)C</smiles>

188

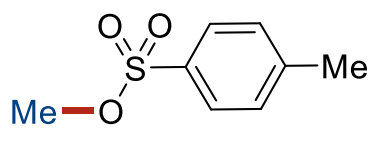

191
$\mathrm{Me}-\mathrm{I}$

189

$\mathrm{Me}-\mathrm{BF}_{3} \mathrm{~K}$

192

Scheme 3.1. Common methylation reagents.

Benzylamines are widely distributed among bioactive molecules (Scheme 3.2). ${ }^{[150]}$ In this context, we became interested in the development of an economical method for the methylation of electron-rich benzylamines. As shown in former studies, MeMgBr served as a suitable methylation reagent under the assistance of triazole directing group. ${ }^{[73 b]} \mathrm{We}$, hence, started to explore the feasibility of iron-mediated methylation of protected benzylamines $178 \mathrm{a}$ using $\mathrm{MeMgBr}$ as user-friendly and safe methylation reagent. 


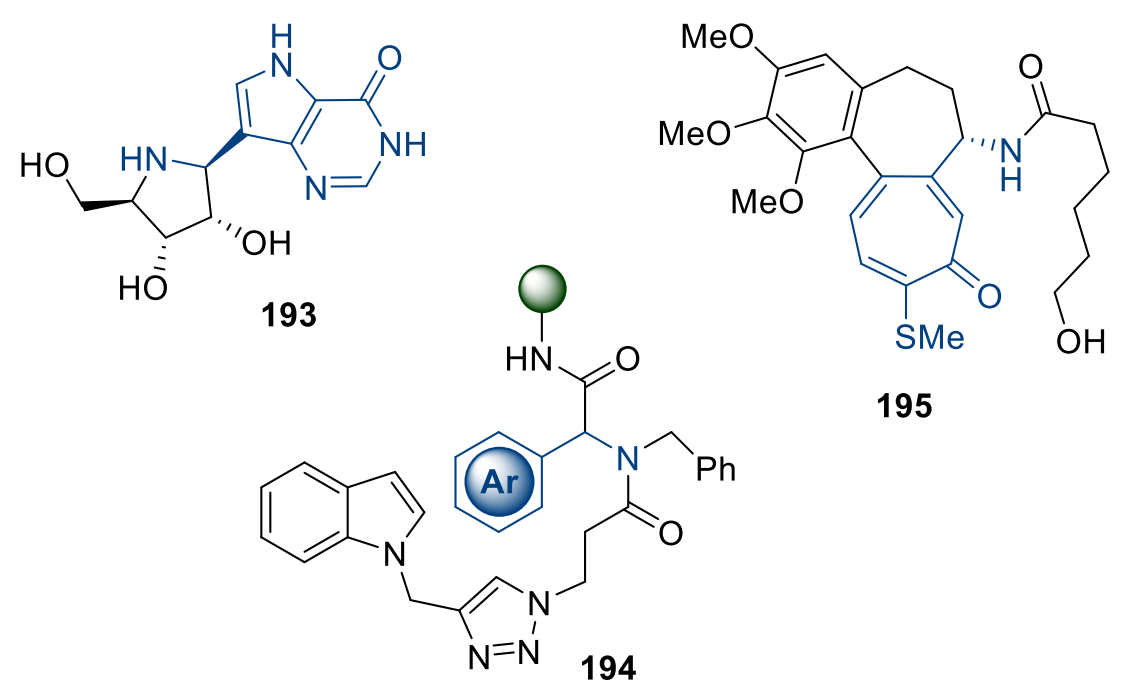

Scheme 3.2. Biological compounds 193-195 containing benzylamines.

\subsubsection{Optimization Studies}

At the outset of our studies, Dr. Cera designed the tri-substituted triazole TST, which enabled the envisioned iron-catalyzed $\mathrm{C}-\mathrm{H}$ methylation of benzylamide $178 \mathrm{a}$. Dr. Cera conducted the following optimization (Table 3.1). Preliminary results revealed that nitrogen or NHC ligands fell short in enabling the desired C-H methylation (Table 3.1, Entries 1-3). The bidentate dppe ligand gave only unsatisfactory result (Entry 4), which could be rationalized with the need for a more rigid coordination environment at iron. In good agreement with this hypothesis, dppz and dppen led to the formation of the desired compound 179a in synthetically useful yields (Entries 5 and 6), particularly, when using 2,3-dichlorobutane as the oxidizing agent. 
Table 3.1. Optimization of iron-catalyzed $\mathrm{C}-\mathrm{H}$ methylations of benzylamine $178 \mathrm{a} .{ }^{[a]}$

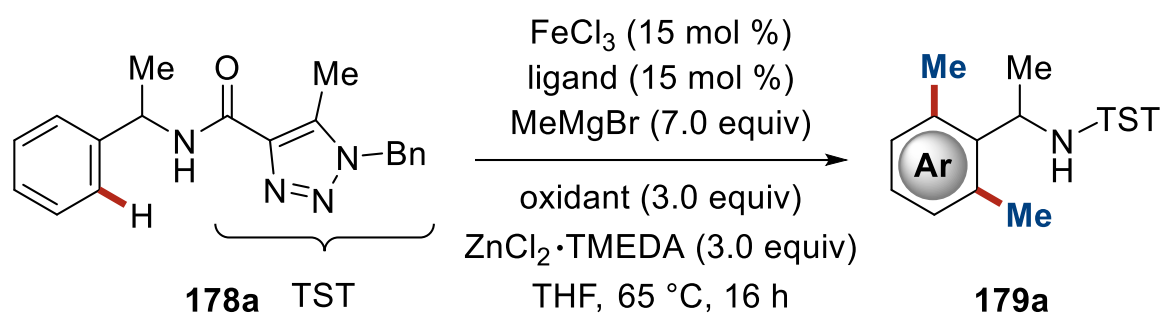

\begin{tabular}{cccc}
\hline Entry & Ligand & Oxidant & Yield [\%] \\
\hline 1 & -- & DCB & -- \\
2 & Dtbpy & DCB & -- \\
3 & IPr.HCl & DCB & -- \\
4 & dppe & DCB & 21 \\
5 & dppz & DCB & 45 \\
6 & dppen & DCIB & 53 \\
7 & dppen & DCB & 86 \\
\hline
\end{tabular}

[a] Reaction conditions: 178a (0.20 mmol), $\mathrm{FeCl}_{3}(15 \mathrm{~mol} \%)$, ligand (15 mol \%), $\mathrm{MeMgBr}(1.40 \mathrm{mmol})$, $\mathrm{ZnCl}_{2} \cdot \mathrm{TMEDA}(0.60 \mathrm{mmol})$, oxidant $(0.60 \mathrm{mmol})$, THF $(0.5 \mathrm{M}), 65^{\circ} \mathrm{C}, 16 \mathrm{~h}$, isolated yields. dppe $=1,2-$ bis(diphenylphosphino)ethane; $\quad \mathrm{dppbz}=1,2$-bis(diphenylphosphino)benzene; $\quad$ dppen $=1,2$ bis(diphenylphosphino)ethene. $\mathrm{DCB}=2,3$-dichlorobutane. $\mathrm{DCIB}=1$,2-dichloro-2-methylpropane.

\subsubsection{Scope of Triazole-Assisted C-H Methylation}

With the optimized reaction conditions in hand, we explored the influence exerted by the TST substitution pattern on the catalytic efficacy. Thus, various directing groups were tolerated in the $\mathrm{C}-\mathrm{H}$ methylation 178 (Table 3.2), with the sterically congested gemdisubstituted benzylamine $\mathbf{1 7 8 b}$ being efficiently converted. The iron-catalyzed $\mathrm{C}-\mathrm{H}$ activation proved amenable to differently $\mathrm{N}$-substituted triazoles, including alkyl- and aryldecorated derivatives $178 \mathrm{c}$ and $\mathbf{1 7 8 d}$. The amide $178 \mathrm{e}$ without free $\mathrm{NH}$-group failed to undergo $\mathrm{C}-\mathrm{H}$ methylation, probably due to an anionic bidentate coordination mode at iron. 
It is particularly noteworthy that the frequently used, picolinic acid-derived ${ }^{[161]}$ benzylamine $\mathbf{1 7 8 f}$ was less effective under otherwise identical reaction conditions.

Table 3.2. Influence of the TST substitution pattern.<smiles>[R]N(C(=O)c1nnn([R])c1C)C([R1])([R])c1ccccc1</smiles>

178

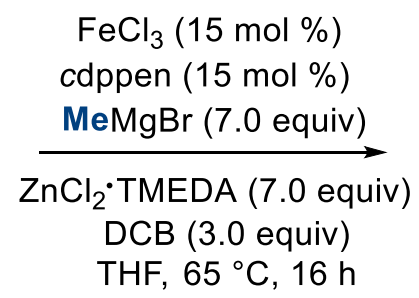<smiles>[R]N(C(=O)c1nnn([R])c1C)C([R14])([R14])c1c(C)cccc1C</smiles>

179

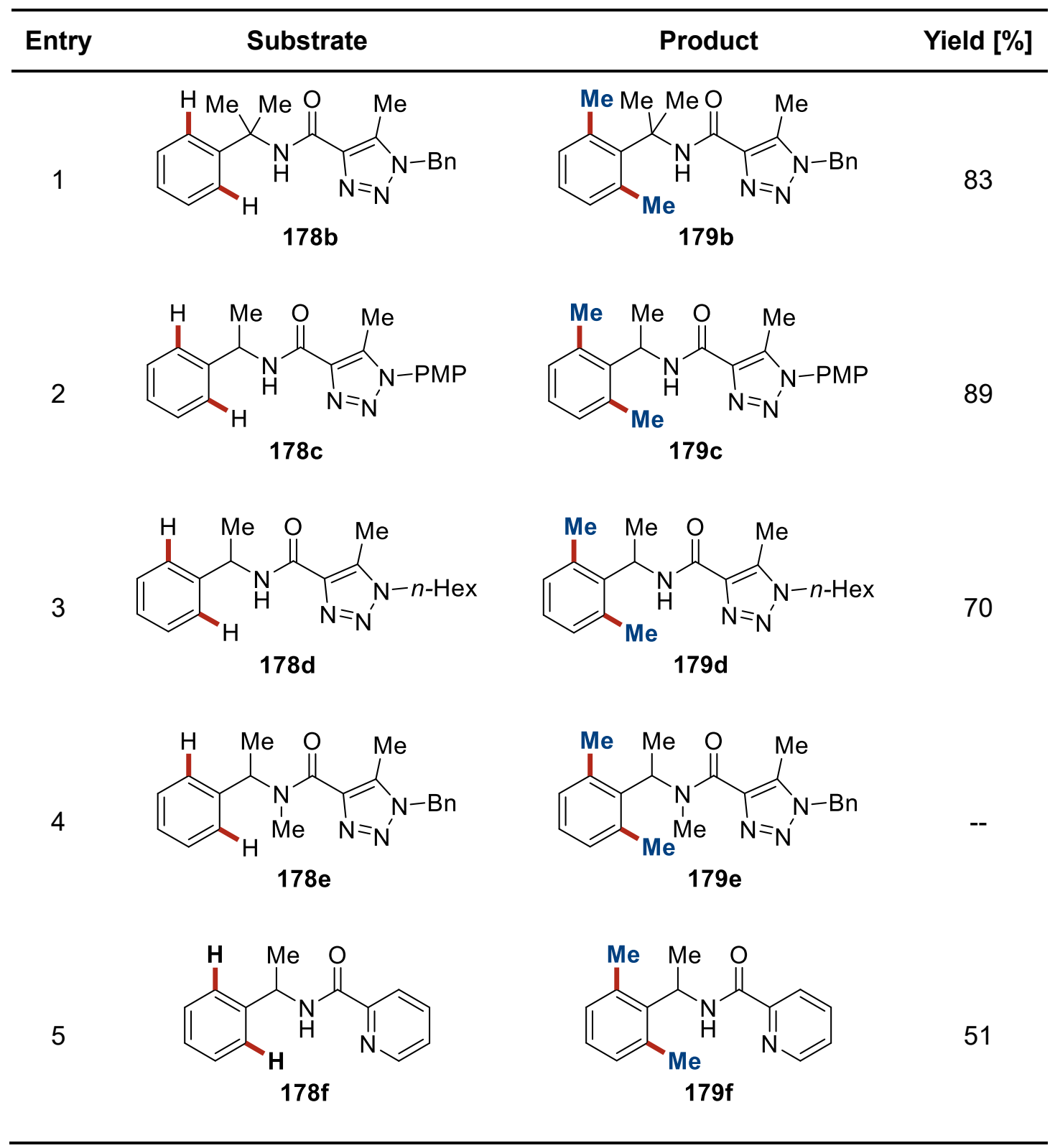

[a] Reaction conditions: 178 (0.20 mmol), $\mathrm{FeCl}_{3}(15 \mathrm{~mol} \%)$, dppen (15 mol \%), $\mathrm{MeMgBr}(1.40 \mathrm{mmol})$, $\mathrm{ZnCl}_{2} \cdot \operatorname{TMEDA}(0.60 \mathrm{mmol}), \mathrm{DCB}(0.60 \mathrm{mmol}), \mathrm{THF}(0.5 \mathrm{M}), 65^{\circ} \mathrm{C}, 16 \mathrm{~h}$, isolated yields. 
After the optimal TST group was confirmed, we probed the versatility of the iron-catalyzed $\mathrm{C}-\mathrm{H}$ activation with differently substituted benzylamines 178 (Table 3.3). To our delight, ortho-functionalized substrates furnished the products $\mathbf{1 7 8 g - 1 7 8 I}$ in good to excellent yields. The naphthalene derivative $178 \mathrm{~m}$ underwent an unusual peri-C-H methylation, thereby furnishing the product $179 \mathrm{~m}$ with excellent levels of positional selectivity. Paraand meta-substituted substrates were directly converted to the twofold methylated products $179 \mathrm{n}-179 \mathrm{u}$. Given the practical importance of nitrogen-containing heterocycles, it is noteworthy that pyridine $179 \mathrm{v}$ could be obtained in synthetically useful yields as well. Likewise, bicyclic substrate delivered the desired product $179 w$ featuring the sertraline motif - an important pharmacophore in medicinal chemistry. ${ }^{[162]}$

Table 3.3. TST-assisted iron-catalyzed $\mathrm{C}-\mathrm{H}$ methylation.

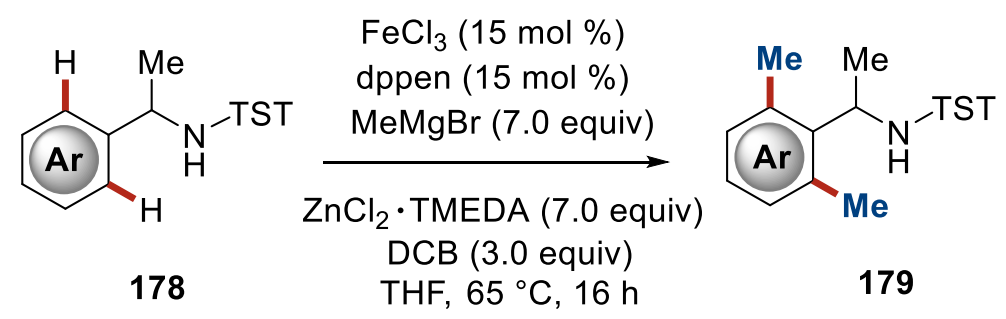

Entry Substrate


4<smiles>COc1ccccc1CN[Cl+][Si-]</smiles>

178j

5<smiles>CC(C)(C)[Mg][Te]</smiles>

6<smiles>FC(F)(F)c1cccc(I)c1C([Al-])N[Cl+]</smiles>

7<smiles>[Al-]Nc1cccc2ccccc12</smiles>

$178 \mathrm{~m}$

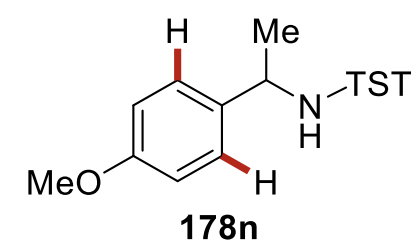

9

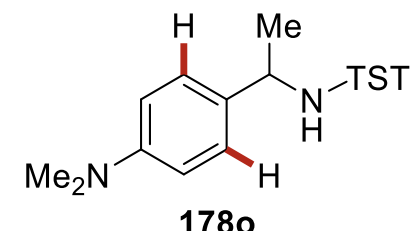

10<smiles>CC(N[Al-][Al-])c1ccc(Cl)cc1</smiles>

11<smiles>CC(C)Cc1cccc(C(C)N[Al-][SiH2])c1</smiles><smiles>COc1cccc(OC)c1C([Al-])NC[Al-]</smiles>

90

179j

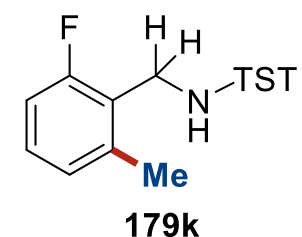

92

80

66 
12<smiles>CC(N[Al-][Al-])c1ccc(-c2ccccc2)cc1</smiles>

13<smiles>FC(F)(F)c1ccc([CH]N[Al-][Al-])cc1</smiles>

14

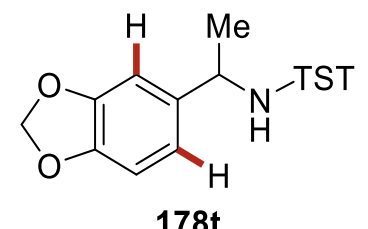

15<smiles>COc1ccccc1C(C)N[Al-][Al-]</smiles>

16<smiles>[CH2]C(N[Al-][Al-])c1ccncc1</smiles>

17<smiles>[Al-][Al-]NC1CCCc2cccc(P)c21</smiles>

18<smiles>CC(N[Cl+][Si-])c1ccc2ccccc2c1</smiles>

178x<smiles></smiles>

84<smiles>Cc1cc(C(F)(F)F)cc([N+](=O)[O-])c1C(C)N[Al-][SiH3]</smiles>

84 90 89<smiles>Cc1cc2ccccc2cc1C(C)N[Al-][SiH3]</smiles>

[a] Reaction conditions: $178(0.20 \mathrm{mmol}), \mathrm{FeCl}_{3}(15 \mathrm{~mol} \%)$, dppen (15 mol \%), $\mathrm{MeMgBr}(1.4 \mathrm{mmol})$, $\mathrm{ZnCl}_{2} \cdot \operatorname{TMEDA}(0.60 \mathrm{mmol}), \mathrm{DCB}(0.60 \mathrm{mmol}), \operatorname{THF}(0.5 \mathrm{M}), 65^{\circ} \mathrm{C}, 16 \mathrm{~h}$, isolated yields. 
The iron-catalyzed $\mathrm{C}-\mathrm{H}$ activation strategy was not limited to direct methylation reactions. Indeed, the versatility of our approach was reflected by a high-yielding $\mathrm{C}-\mathrm{H}$ ethylation of benzylamine $178 \mathrm{~h}$, with the best results being achieved by employing DCIB as the oxidant (Scheme 3.3).
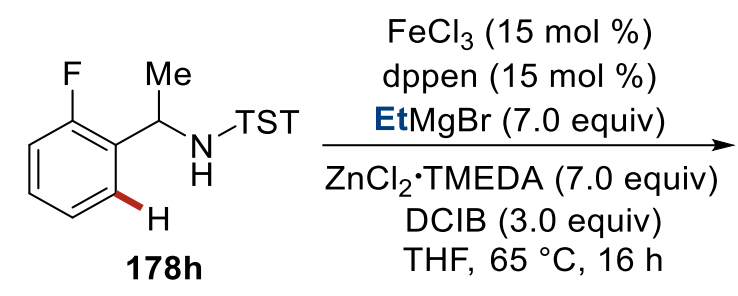<smiles>CCc1cccc(F)c1C(C)N[Cl+][Si]</smiles>

Scheme 3.3. TST-assisted iron-catalyzed C-H ethylation.

Then we did some optimization for the iron-catalyzed C-H arylation (Table 3.4). We found that DCIB was more effective than 2,3-DCB giving the desired product 197a in $87 \%$ yield (entry 3).

Table 3.4. Optimization of iron-catalyzed $\mathrm{C}-\mathrm{H}$ arylation. ${ }^{[\mathrm{a}]}$<smiles>[M]C(NC(=O)c1nnn(Cc2ccccc2)c1C)c1c(F)cc(C(C)(C)C)cc1-c1ccc(OC)cc1</smiles>

\begin{tabular}{cccc}
\hline entry & Oxidant & $\mathbf{X}$ & Yield [\%] \\
\hline 1 & $2,3-\mathrm{DCB}$ & $\mathrm{Br}$ & 17 \\
2 & $2,3-\mathrm{DCB}$ & $\mathrm{Cl}$ & -- \\
3 & $\mathrm{DCIB}$ & $\mathrm{Br}$ & 87 \\
\hline
\end{tabular}

[a] Reaction conditions: $178 \mathrm{~h}(0.20 \mathrm{mmol}), \mathrm{FeCl}_{3}(15 \mathrm{~mol} \%), \mathrm{ZnCl}_{2} \cdot \mathrm{TMEDA}(0.6 \mathrm{mmol})$, ligand (15 mol \%), PhMgX (1.4 mmol), Oxidant (0.6 mmol), isolated yield.

Next, we tested the versatility of the iron-catalyzed C-H arylation (Table 3.5). Both electrondeficient and electron-rich Grignard reagents furnished the products in excellent yields (197a-197c). Bicyclic amine 178w was also a reactive substrate in the $\mathrm{C}-\mathrm{H}$ arylation. 
Table 3.5. TST-assisted iron-catalyzed $\mathrm{C}-\mathrm{H}$ arylation. ${ }^{[a]}$

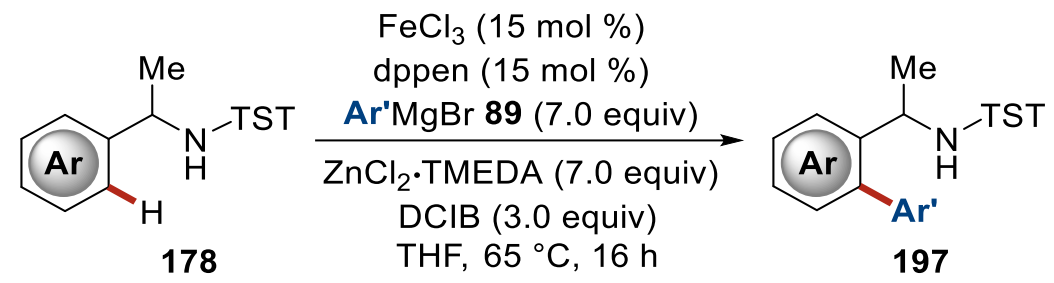

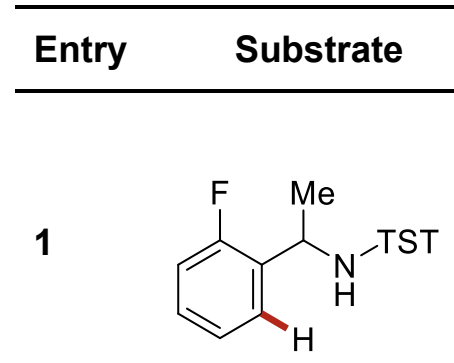

178h

2<smiles>[M]C(N[Al-])c1ccccc1F</smiles>

178h<smiles>CC(N[Al-][I-])c1ccccc1C(F)(F)F</smiles>

1781<smiles>[Si][N-]NC1CCCc2ccccc21</smiles>

178w<smiles>COc1ccc(N(Br)Br)cc1</smiles><smiles></smiles>

$89 \mathrm{~b}$<smiles></smiles>

$89 a$

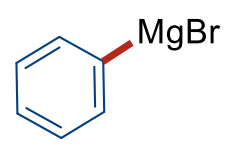

89c<smiles>[M]C(N[Al-][SiH2])c1c(F)cccc1-c1ccc(OC)cc1</smiles>

$197 a$<smiles>CC(N[Al-][Al-])c1c(F)cccc1-c1ccc(F)cc1</smiles>

197b<smiles>COc1ccc(-c2cccc(C(F)(F)F)c2C(C)N[Al-][SiH2])cc1</smiles>

197c<smiles>[Al-]NC1CCCc2cccc(-c3ccccc3)c21</smiles>

$197 d$

[a] Reaction conditions: 1a $(0.20 \mathrm{mmol}), \mathrm{FeCl}_{3}(15 \mathrm{~mol} \%), \mathrm{ZnCl}_{2} \cdot \operatorname{TMEDA}(0.60 \mathrm{mmol})$, dppen (15 $\mathrm{mol} \%), \operatorname{Ar}{ }^{\prime} \mathrm{MgBr}(1.4 \mathrm{mmol}), \mathrm{DCIB}(0.6 \mathrm{mmol})$, isolated yield. 
The synthetic utility of the iron-catalyzed $\mathrm{C}-\mathrm{H}$ activation strategy was among others mirrored by the racemization-free modification of the enantiomerically-enriched benzylamine (S)-178a (Scheme 3.4).

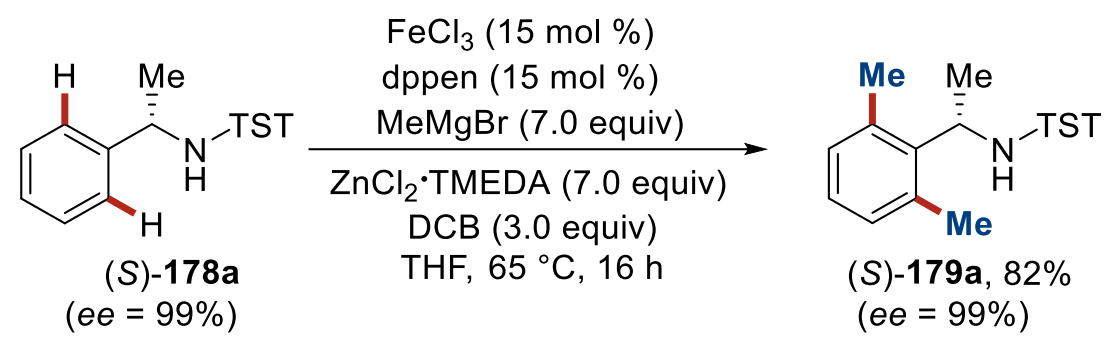

Scheme 3.4. Racemization-free $\mathrm{C}-\mathrm{H}$ methylation of benzylamine $(S)-\mathbf{1 7 8 a}$.

\subsubsection{KIE studies of Iron-catalyzed Methylation}

As to the catalyst mode of action, we conducted kinetic studies through independent experiments and the conversions were determined by ${ }^{19} \mathrm{~F}$ NMR. To this end, we unraveled a kinetic isotope effect $(\mathrm{KIE})$ of $k_{H} / k_{D} \approx 1.7$, being suggestive that the $\mathrm{C}-\mathrm{H}$ cleavage is before or in the rate-determining step (Scheme 3.5). The outstanding efficacy of the ironcatalyzed $\mathrm{C}-\mathrm{H}$ activation was further reflected by a $48 \%$ conversion of substrate $178 \mathrm{k}$ within only 25 minutes. 

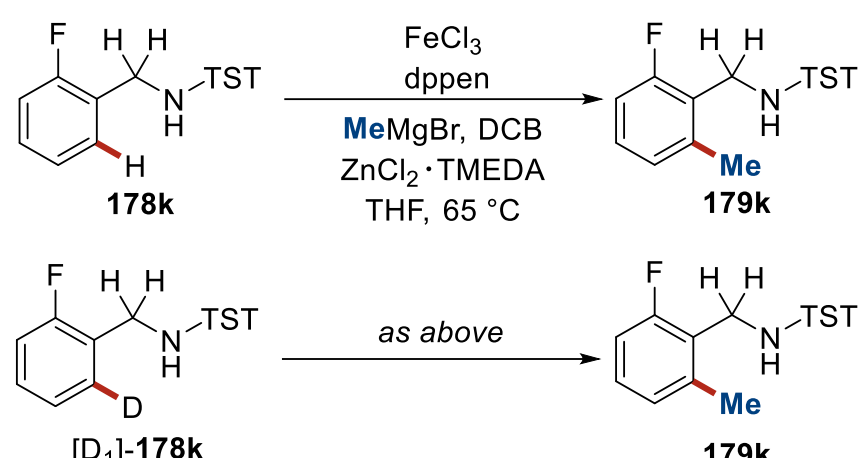

$\left[D_{1}\right]-178 k$

$179 k$

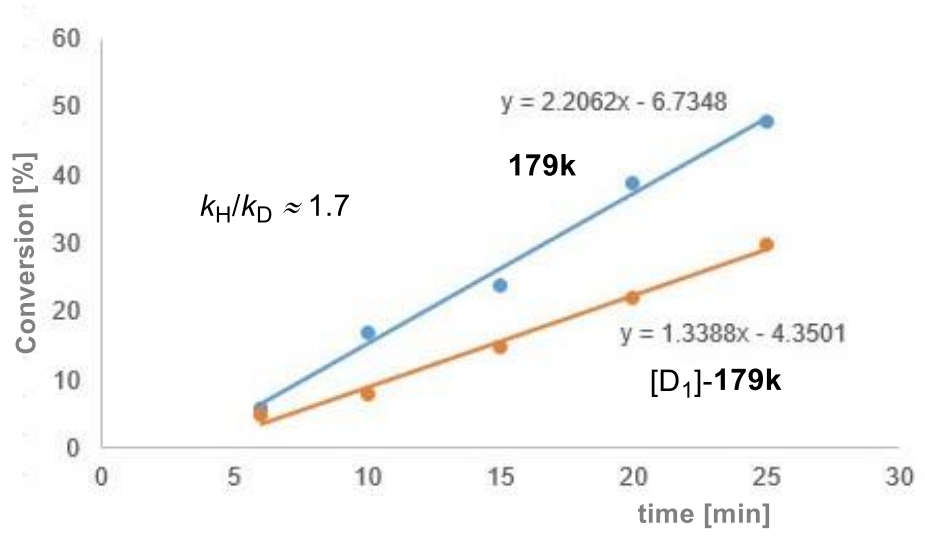

Scheme 3.5. KIE studies by independent experiments.

\subsubsection{Deprotection of 179}

Finally, the TST group could be removed in a traceless fashion, furnishing the free primary benzylamines $198 \mathrm{~m}$ and $198 \mathrm{~s}$, while the reusable TST acid 199a was recovered in high yield (Scheme 3.6). ${ }^{[163]}$
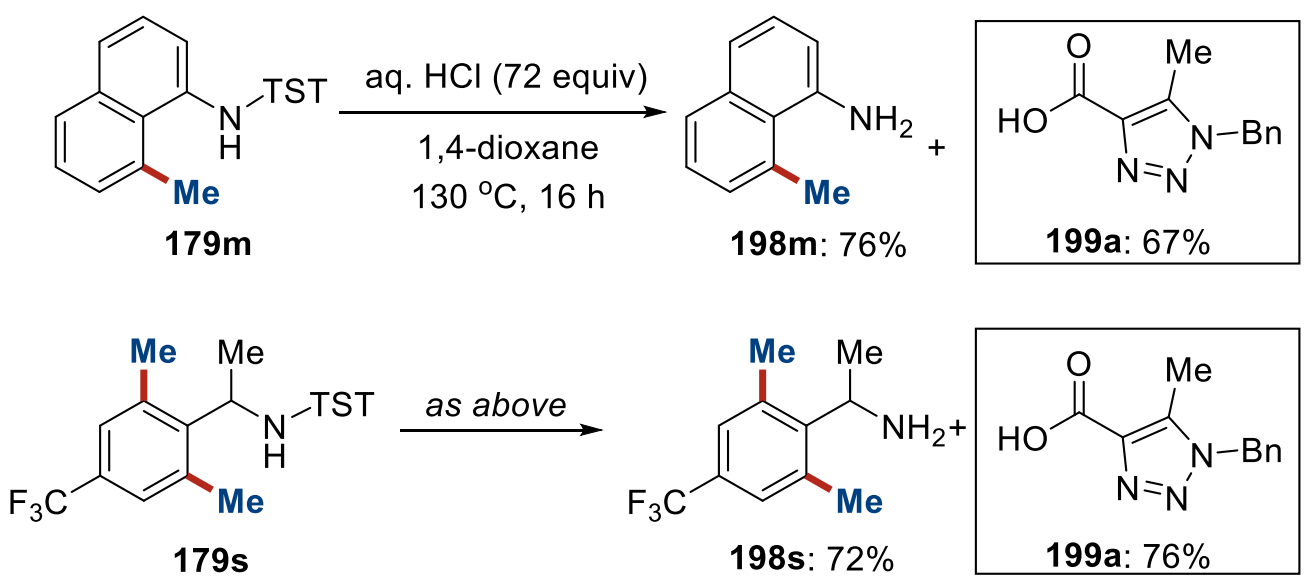

Scheme 3.6. Removal of TST group in a traceless fashion. 


\subsection{Manganese-catalyzed Pyridinyl C-H Alkylation}

In the past few years, direct $\mathrm{C}-\mathrm{H}$ alkylations of unreactive arenes with alkyl halides have been achieved in a site-selective manner by chelation assistance. ${ }^{[164]}$ However, those works were mostly realized by expensive and toxic rare $4 \mathrm{~d}$ and $5 \mathrm{~d}$ transition metals. ${ }^{[151]}$ Particularly, secondary $\mathrm{C}-\mathrm{H}$ alkylations continue to be scarce, since in many cases $\beta-\mathrm{H}$ elimination is more favorable in presence of those metals. Recently much attention has shifted to less expensive earth-abundant $3 \mathrm{~d}$ transition metals for $\mathrm{C}-\mathrm{H}$ activations such as nickel, ${ }^{[165]}$ iron $^{[68,73]}$ and manganese. ${ }^{[106]}$

\subsubsection{Optimization of Pyridinyl Alkylation}

Pyridinylamides represent a key unit in a variety of bioactive molecules. Therefore, it is urgent to develop some efficient approaches to perform alkylation of pyridinylamide. We initiated the study of pyridinyl $\mathrm{C}-\mathrm{H}$ alkylation by using cyclohexyl halide $\mathbf{1 8 0}$ as the alkylation reagents in the presence of $\mathrm{MnCl}_{2}$ (Table 3.6). Various bases including alkyl and phenyl Grignard reagents were tested, which showed that ethylmagnesium bromide was superior to others (entries 1-4). The chlorocyclohexane even featured higher reactivity for this pyridinyl $\mathrm{C}-\mathrm{H}$ alkylation reaction, while iodocyclohexane gave a modest yield (entries 5-6). It was found that no alkylated product was formed in the absence of the manganese catalyst or TMEDA additive (entries 7-8). Finally, the best result was obtained using $10 \mathrm{~mol} \%$ of $\mathrm{MnCl}_{2}$ combined with 1.0 equivalent of TMEDA (entries 9-12).

Table 3.6. Optimization of pyridinyl C-H alkylation with a secondary alkyl halide.[a]

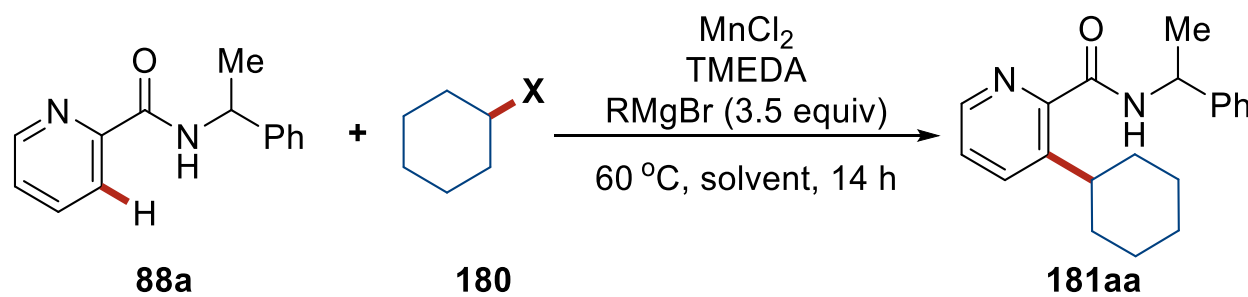

\begin{tabular}{llllll}
\hline Entry & {$[\mathrm{Mn}][\mathrm{mol} \%]$} & TMEDA & $X$ & $\mathrm{RMgBr}$ & Yield [\%] \\
& & & & \\
& & & & \\
\hline
\end{tabular}




\begin{tabular}{|c|c|c|c|c|c|}
\hline 1 & $\mathrm{MnCl}_{2}(20)$ & 2.0 & $\mathrm{Br}$ & $i-\mathrm{PrMgBr}(2.8 \mathrm{M}$ in THF) & 65 \\
\hline 2 & $\mathrm{MnCl}_{2}(20)$ & 2.0 & $\mathrm{Br}$ & EtMgBr (2.8 M in THF) & 69 \\
\hline 3 & $\mathrm{MnCl}_{2}(20)$ & 2.0 & $\mathrm{Br}$ & $t-\mathrm{BuCH}_{2} \mathrm{MgBr}(1.5 \mathrm{M}$ in THF $)$ & 56 \\
\hline 4 & $\mathrm{MnCl}_{2}(20)$ & 2.0 & $\mathrm{Br}$ & $\mathrm{PhMgBr}(2.3 \mathrm{M}$ in THF) & 45 \\
\hline 5 & $\mathrm{MnCl}_{2}(20)$ & 2.0 & $\mathrm{Cl}$ & EtMgBr (2.8 M in THF) & 78 \\
\hline 6 & $\mathrm{MnCl}_{2}(20)$ & 2.0 & I & EtMgBr (2.8 M in THF) & 48 \\
\hline 7 & -- & 2.0 & $\mathrm{Cl}$ & EtMgBr (2.8 M in THF) & 0 \\
\hline 8 & $\mathrm{MnCl}_{2}(20)$ & -- & $\mathrm{Cl}$ & EtMgBr (2.8 M in THF) & 0 \\
\hline 9 & $\mathrm{MnCl}_{2}(10)$ & 2.0 & $\mathrm{Cl}$ & $\mathrm{EtMgBr}(2.8 \mathrm{M}$ in THF) & 76 \\
\hline 10 & $\mathrm{MnCl}_{2}(5)$ & 2.0 & $\mathrm{Cl}$ & EtMgBr (2.8 M in THF) & 54 \\
\hline 11 & $\mathrm{MnCl}_{2}(10)$ & 1.0 & $\mathrm{Cl}$ & EtMgBr (2.8 M in THF) & 79 \\
\hline 12 & $\mathrm{MnCl}_{2}(10)$ & 0.5 & $\mathrm{Cl}$ & EtMgBr (2.8 M in THF) & 64 \\
\hline
\end{tabular}

[a] Reaction conditions: 88a (0.20 mmol), 180 (3.0 equiv), $\mathrm{MnCl}_{2}$ catalyst, TMEDA, $\mathrm{RMgBr}$ (3.5 equiv), THF $(0.6 \mathrm{~mL})$ under $\mathrm{N}_{2}$ at $60^{\circ} \mathrm{C}$ for $14 \mathrm{~h}$, isolated yield.

Dr. Huang finished the optimization of the primary alkylation by the reaction of $\mathrm{N}-(1-$ phenylethyl)picolinamide $\mathbf{8 8 a}$ and $n$-butyl bromide 86 a catalyzed by cost-efficient $\mathrm{MnCl}_{2}$ (Table 3.7). The assessment of different Grignard reagent showed that bulkier Grignard reagents are more efficient to form the butylated product. When $t-\mathrm{BuCH}_{2} \mathrm{MgBr}$ was used, only butylation was observed without formation of any alkylated product generated by Grignard reagent (entries 1-7). Dr. Huang also studied other manganese catalysts, such as $\mathrm{Mn}(\mathrm{OAc})_{2}, \mathrm{Mn}_{2}(\mathrm{CO})_{10}$ and $\mathrm{MnBr}(\mathrm{CO})_{5}$, without any alkylated product (entries 8-10). Control experiment without the manganese catalyst did not give the desired product, which confirmed that manganese is key for the catalytic reaction (entry 14). Decreased amounts of TMEDA gave less product as well (entry 15).

Table 3.7. Optimization of pyridinyl C-H primary alkylation. 


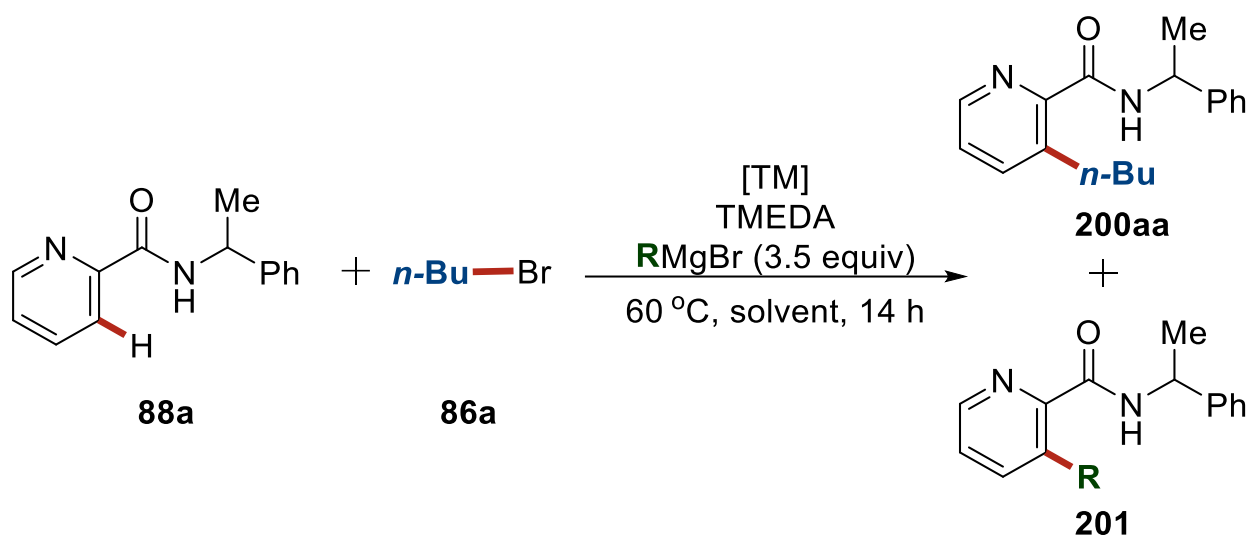

\begin{tabular}{|c|c|c|c|c|}
\hline Entry & [TM] $(\mathrm{mol} \%)$ & $\begin{array}{l}\text { TMEDA } \\
\text { [equiv] }\end{array}$ & $\mathrm{RMgBr}$ & $\begin{array}{c}\text { Yield [\%] } \\
\text { 200aa/201 }\end{array}$ \\
\hline 1 & $\mathrm{MnCl}_{2}(20)$ & 2.0 & $\mathrm{PhMgBr}(2.3 \mathrm{M}$ in $\mathrm{THF})$ & $45 / 35$ \\
\hline 2 & $\mathrm{MnCl}_{2}(20)$ & 2.0 & $\operatorname{MeMgBr}(3.0 \mathrm{M}$ in THF) & $55 / 29$ \\
\hline 3 & $\mathrm{MnCl}_{2}(20)$ & 2.0 & EtMgBr (3.4 M in THF) & $46 / 37$ \\
\hline 4 & $\mathrm{MnCl}_{2}(20)$ & 2.0 & $i-\operatorname{PrMgBr}(2.4 \mathrm{M}$ in THF) & $58 / 20$ \\
\hline 5 & $\mathrm{MnCl}_{2}(20)$ & 2.0 & i-PrMgBr (3.0 M in 2-MeTHF) & $59 / 22$ \\
\hline 6 & $\mathrm{MnCl}_{2}(20)$ & 2.0 & $i-\mathrm{PrMgCl}(2.0 \mathrm{M}$ in $\mathrm{THF})$ & $40 / 31$ \\
\hline 7 & $\mathrm{MnCl}_{2}(20)$ & 2.0 & $t-\mathrm{BuCH}_{2} \mathrm{MgBr}(1.5 \mathrm{M}$ in THF) & $69 / 0$ \\
\hline 8 & $\mathrm{Mn}(\mathrm{OAc})_{2}(20)$ & 2.0 & $t-\mathrm{BuCH}_{2} \mathrm{MgBr}(1.5 \mathrm{M}$ in THF $)$ & $0 / 0$ \\
\hline 9 & $\mathrm{Mn}_{2}(\mathrm{CO})_{10}(10)$ & 2.0 & $t-\mathrm{BuCH}_{2} \mathrm{MgBr}(1.5 \mathrm{M}$ in THF $)$ & $0 / 0$ \\
\hline 10 & $\operatorname{MnBr}(\mathrm{CO})_{5}(20)$ & 2.0 & $t-\mathrm{BuCH}_{2} \mathrm{MgBr}(1.5 \mathrm{M}$ in THF $)$ & $0 / 0$ \\
\hline 11 & $\mathrm{Fe}(\mathrm{acac})_{3}(10)$ & 2.0 & $t-\mathrm{BuCH}_{2} \mathrm{MgBr}(1.5 \mathrm{M}$ in THF) & $0 / 0$ \\
\hline 12 & $\mathrm{FeCl}_{3}(10)$ & 2.0 & $t-\mathrm{BuCH}_{2} \mathrm{MgBr}(1.5 \mathrm{M}$ in THF $)$ & $0 / 0$ \\
\hline 13 & $\mathrm{MnCl}_{2}(10)$ & 2.0 & $t-\mathrm{BuCH}_{2} \mathrm{MgBr}(1.5 \mathrm{M}$ in THF) & $42 / 0$ \\
\hline 14 & -- & 2.0 & $t-\mathrm{BuCH}_{2} \mathrm{MgBr}(1.5 \mathrm{M}$ in THF) & $0 / 0$ \\
\hline 15 & $\mathrm{MnCl}_{2}(20)$ & 1.0 & $t-\mathrm{BuCH}_{2} \mathrm{MgBr}(1.5 \mathrm{M}$ in THF) & $50 / 0$ \\
\hline
\end{tabular}

[a] Reaction conditions: 88a (0.20 mmol), $n$-BuBr (3.0 equiv), [Mn] catalyst, TMEDA, $\mathrm{RMgBr}$ (3.5 equiv), THF $(0.2 \mathrm{~mL})$ under $\mathrm{N}_{2}$ at $60^{\circ} \mathrm{C}$ for $14 \mathrm{~h}$, isolated yield. 


\subsubsection{Scope of Manganese Catalyzed Alkylation}

After the optimization of the pyridinyl $\mathrm{C}-\mathrm{H}$ alkylation, the scope and limitation of the secondary alkylation was evaluated (Table 3.8). A variety of 3-cyclohexylated picolinamides 181aa-181ja was synthesized with high catalytic efficiency. Quinoline was also viable to be cyclohexylated (181ka). Significantly, the importance of the reaction was underlined by furnishing the compound $\mathbf{1 8 1}$ fa on gram scale with a comparable catalytic efficiency.

Table 3.8. Scope of manganese-catalyzed alkylation with chlorocyclohexane.

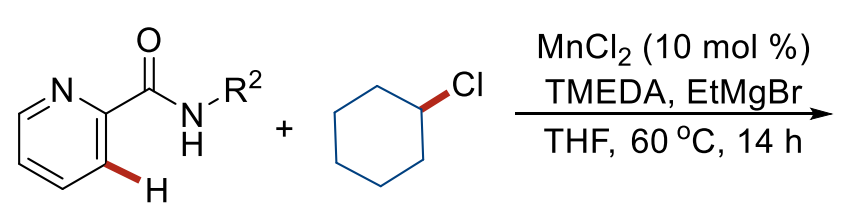

88

$180 a$<smiles>[R]NC(=O)c1ncccc1C1CCCCC1</smiles>

181<smiles>CC(NC(=O)c1ccccc1C(C)NC(=O)c1ncccc1P)c1ccccc1</smiles> 
4<smiles>O=C(NCc1ccc(F)cc1)c1ccccn1</smiles>

88d

5<smiles>CC(C)(C)OC(=O)c1ccccc1CNC(=O)c1ncccc1F</smiles>

6<smiles>COc1ccc(CNC(=O)c2ccccn2)cc1</smiles>

7<smiles>O=C(NC1CCCCC1)c1ccccn1</smiles>

$88 \mathrm{~g}$

8<smiles>O=C(NC1CCCc2ccccc21)c1ccccn1</smiles>

88h

9<smiles>CCCCNC(=O)c1ncccc1Br</smiles><smiles>O=C(NCc1ccc(F)cc1)c1ncccc1C1CCCCC1</smiles>

181da<smiles>O=C(NCc1ccccc1F)c1ncccc1C1CCCCC1</smiles>

181ea<smiles>COc1ccc(CNC(=O)c2ncccc2C2CCCCC2)cc1</smiles><smiles>O=C(NC1CCCCC1)c1ncccc1C1CCCCC1</smiles>

80

181ga<smiles>O=C(NC1CCCc2ccccc21)c1ncccc1C1CCCCC1</smiles>

181ha<smiles>CCCCNC(=O)c1ncccc1C1CCCCC1</smiles>

92

181ia 
10<smiles>O=C(NNC(=O)c1ncccc1Br)c1ccccc1</smiles>

11<smiles>CCCCNC(=O)c1ccc2ccccc2n1</smiles><smiles>N#[PH]NC(=O)c1ncccc1C1CCCCC1</smiles>

181ja<smiles>CCCCNC(=O)c1nc2ccccc2cc1C1CCCCC1</smiles>

$181 \mathrm{ka}$
46

26

[a] Reaction conditions: 88 (0.20 mmol), 180a (3.0 equiv), $\mathrm{MnCl}_{2}$ catalyst, TMEDA, EtMgBr (3.5 equiv), THF $(0.6 \mathrm{~mL})$ under $\mathrm{N}_{2}$ at $60^{\circ} \mathrm{C}$ for $14 \mathrm{~h}$, isolated yield.

Then, various secondary alkyl chlorides were tested (Table 3.9). The versatility of the optimized methodology was further documented through the cyclopentylation and isopropylation of picolinamides (181ib, 181ie). The cyclic secondary alkylchlorides with different ring size afforded the corresponding pyridinylamides 181if-181ik.

Table 3.9. Scope of manganese-catalyzed secondary alkylation with alkyl chlorides $\mathbf{1 8 0}$.<smiles>O=C(NPCBr)c1ccccn1</smiles>

$88 \mathbf{i}$<smiles>[R]C(Cl)Cl</smiles>

180

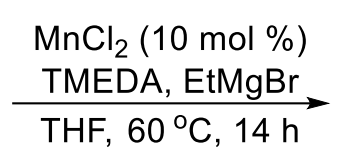<smiles>[R]C([R])c1cccnc1C(=O)NCCCC</smiles>

181

\begin{tabular}{llll}
\hline Entry & Substrate & Yield (\%) \\
\hline 1 & &
\end{tabular}


2<smiles>ClC1CCOCC1</smiles>

$180 \mathrm{c}$<smiles>C=CCN1CCC(Cl)CC1</smiles><smiles>CC(C)Cl</smiles>

5<smiles>CC(Cl)CBr</smiles>

6<smiles>CCCCC(C)Cl</smiles><smiles>CC(Cl)c1ccccc1</smiles><smiles>CC(Cl)CCc1ccccc1</smiles><smiles>CCCCNC(=O)c1ncccc1C1CCOCC1</smiles>

$181 \mathrm{ic}$<smiles>C=CCN1CCC(c2cccnc2C(=O)NPC(C)(C)C)CC1</smiles>

181id<smiles>CCCCNC(=O)c1ncccc1[Te]CCC</smiles>

181 ie

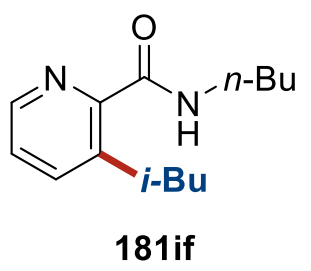<smiles>CC(C)CNC(=O)c1ncccc1C(C)CCc1ccccc1</smiles> 
9<smiles>CCC(Cl)c1ccccc1</smiles>

10<smiles>C=CCCC(C)Cl</smiles><smiles>CCCCNC(=O)c1ncccc1C(CC)c1ccccc1</smiles>

$181 \mathrm{ij}$<smiles>C=CCCC(C)c1cccnc1C(=O)NCCCC</smiles>

$181 \mathrm{ik}$
59

59

[a] Reaction conditions: $88 \mathrm{i}(0.20 \mathrm{mmol}), 180$ (3.0 equiv), $\mathrm{MnCl}_{2}$ (10 mol \%), TMEDA, EtMgBr (3.5 equiv), THF (0.6 mL) under $\mathrm{N}_{2}$ at $60^{\circ} \mathrm{C}$ for $14 \mathrm{~h}$, isolated yield.

Next, we turned our attention to the manganese-catalyzed $n$-butylation with differently substituted picolinamides 88 (Table 3.10 ). Variation of substituents on the amides 88 were tolerated in this reaction to achieve products 200aa-200ga.

Table 3.10. Scope of manganese catalyzed butylation.<smiles>[R]NC(=O)c1ccccc1</smiles>

88

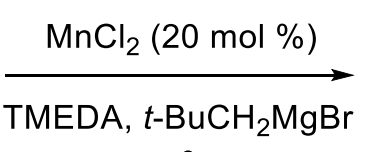
$\mathrm{THF}, 60^{\circ} \mathrm{C}, 14 \mathrm{~h}$<smiles>[R]NC(=O)c1ccccc1OCCC</smiles>

200

Entry Substrate


3<smiles>O=C(NCc1ccccc1F)c1ccccn1</smiles>

$88 \mathrm{c}$

4<smiles>COc1ccc(CNC(=O)c2ccccn2)cc1</smiles>

5<smiles>CC(C)CNC(=O)c1ncccc1PC(C)(C)C</smiles>

6<smiles>CCCCc1cccnc1C(=O)NCCc1ccccc1</smiles>

7<smiles>O=C(NC1CCCCC1)c1ccccn1</smiles>

$88 \mathrm{~g}$<smiles>CCCCc1cccnc1C(=O)NCc1ccccc1F</smiles>

200ca<smiles>CCCCc1cccnc1C(=O)NCc1ccc(OC)cc1</smiles>

56

$200 \mathrm{fa}$<smiles>CCCCc1cccnc1C(=O)NC1CCCCC1</smiles>

200ga

56<smiles>CCCCc1cccnc1C(=O)NCCc1ccccc1</smiles>

[a] Reaction conditions: 88 (0.20 mmol), $n-\mathrm{BuBr}$ (3.0 equiv), $\mathrm{MnCl}_{2}$ (10 mol \%), TMEDA (2.0 equiv), $t$ $\mathrm{BuCH}_{2} \mathrm{MgBr}$ (3.5 equiv), $\operatorname{THF}(0.2 \mathrm{~mL})$ under $\mathrm{N}_{2}$ at $60^{\circ} \mathrm{C}$ for $14 \mathrm{~h}$, isolated yield.

Different primary alkylbromides $\mathbf{8 6}$ were probed thereafter (Table 3.11). Interestingly, terminal alkene and alkyl chlorides were tolerated by this primary alkylation catalyst (200ee and 200ef).

Table 3.11. Scope of manganese catalyzed primary alkylation of pyridine.

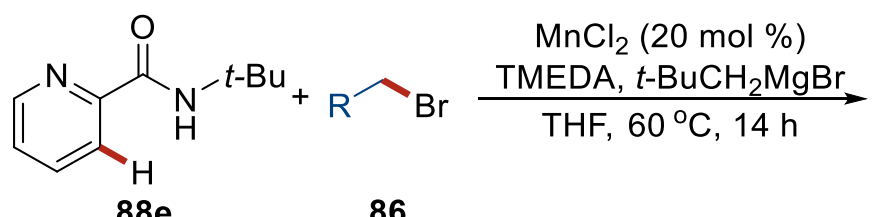

86<smiles>[R]Cc1cccnc1C(=O)NC(C)(C)C</smiles>

200 


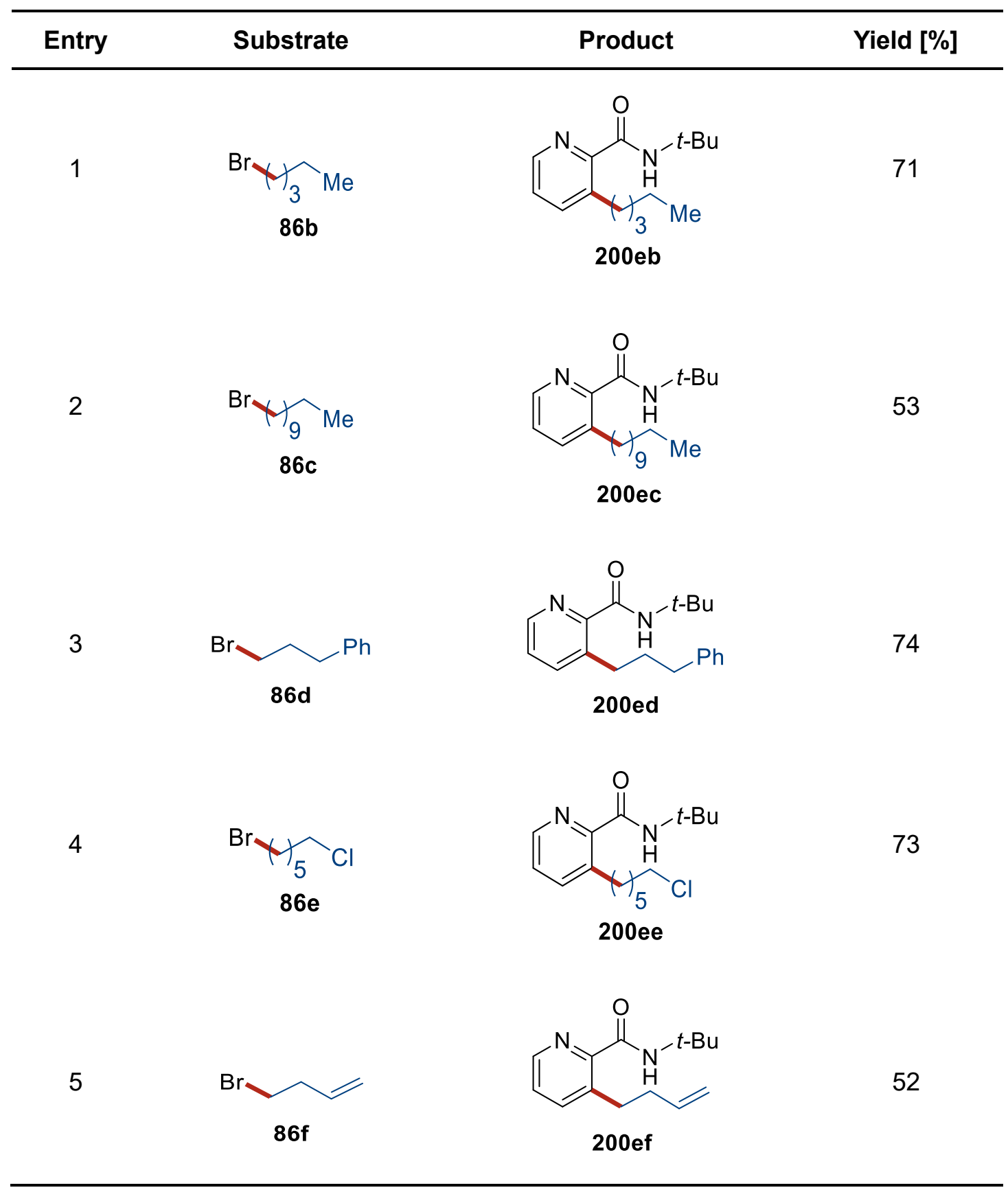

[a] Reaction conditions: $88 \mathrm{e}(0.20 \mathrm{mmol}), 86$ (3.0 equiv), $\mathrm{MnCl}_{2}(10 \mathrm{~mol} \%)$, TMEDA (2.0 equiv), $t$ - $\mathrm{BuCH} \mathrm{CHgBr}_{2} \mathrm{M}\left(3.5\right.$ equiv), THF $(0.2 \mathrm{~mL})$ under $\mathrm{N}_{2}$ at $60^{\circ} \mathrm{C}$ for $14 \mathrm{~h}$, isolated yield.

The synthetic utility of our manganese-catalyzed $\mathrm{C}-\mathrm{H}$ alkylation strategy was reflected by the derivatization of the optically enriched $N$-(1-phenylethyl)picolinamide 88a without racemization of the stereogenic center (Scheme 3.8). 


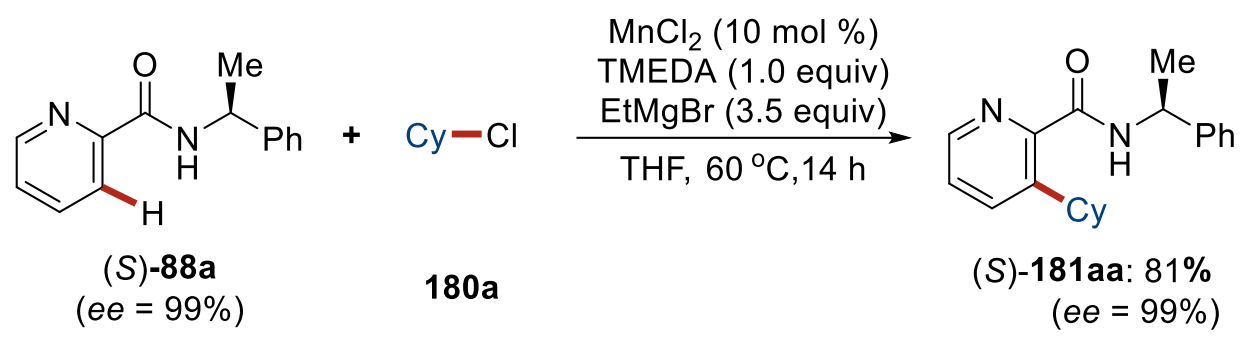

Scheme 3.8. Racemization-free C-H cyclohexylation of pyridine (S)-88a.

\subsubsection{Mechanistic Studies of Manganese Catalyzed Alkylation}

As to the elucidation of the reaction mechanism (Scheme 3.9), we subjected bromomethylcyclopropane $\mathbf{8 6 g}$ to the optimized conditions and obtained the ring-opening product $200 \mathrm{ef}$, which indicated a radical pathway in the activation of the $\mathrm{C}-\mathrm{Br}$ bond. However, the application of 6-bromohex-1-ene $\mathbf{8 6 h}$ only yielded the linear compound 200eh. Then we performed independent reactions observing a kinetic isotopic effect (KIE) of $k_{H} / k_{D} \approx 2.6$, thus indicating the $\mathrm{C}-\mathrm{H}$ cleavage as the rate-determining step.<smiles>CC(C)(C)NC(=O)c1ccccn1</smiles>

$88 e$

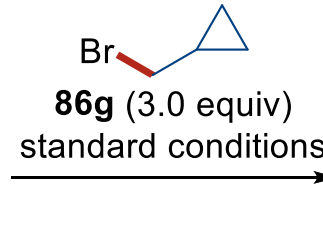<smiles>C=C[14CH2]Br</smiles><smiles>CC(C)(C)NC(=O)c1ccccn1</smiles>

$88 \mathrm{e}$<smiles>C=CCc1cccnc1C(=O)NC(C)(C)C</smiles>

200 ef +200 eg, $45 \%(>20: 1)$

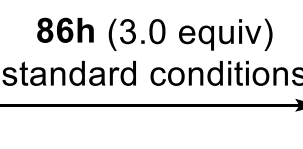<smiles>C=CCc1cccnc1C(=O)NC(C)(C)C</smiles>

$200 \mathrm{eh}+200 \mathrm{eh}, 68 \%(>20: 1)$<smiles>CCCCNC(=O)c1ncccc1P</smiles>

$88 \mathrm{i} /[\mathrm{D}]-88 \mathrm{i}$ $180 a$

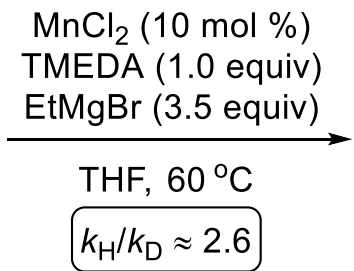

TMEDA (1.0 equiv) EtMgBr (3.5 equiv) $k_{\mathrm{H}} / k_{\mathrm{D}} \approx 2.6$<smiles>CCCCNC(=O)c1ncccc1Cl</smiles>

181ia

Scheme 3.9. Mechanistic studies of manganese-catalyzed $\mathrm{C}-\mathrm{H}$ alkylation. 


\subsection{Rhodaelectro-Catalyzed Domino Alkyne Annulations to access Aza-Polycyclic Aromatic Hydrocarbons}

To meet the rising demand for the development of sustainable and green synthetic methodology, electrosynthesis has emerged as a powerful strategy in which stoichiometric amounts of oxidizing and reducing reagents as well as electron transfer events can be replaced by electric current. Therefore, the generation of waste can be diminished and broader functional groups tolerance can be accomplished. The first rhodaelecto-catalyzed annulation to form polycyclic aromatic hydrocarbons was reported by Ackermann. ${ }^{[146]}$ Different transformations have been realized by rhodaelectro-catalysis, such as olefination, ${ }^{[145]}$ alkylation ${ }^{[144]}$ and annulation. ${ }^{[146,147]}$ Numerous examples of the formation of diverse heterocyclic compounds through the cleavage of $\mathrm{C}-\mathrm{H} / \mathrm{N}-\mathrm{H}$ or $\mathrm{C}-\mathrm{H} / \mathrm{O}-\mathrm{H}$ bonds followed by annulation reactions with alkynes catalyzed by Rhodium(III) have been reported. ${ }^{[127-134]}$ Thus far, only a few examples achieved the double $\mathrm{C}-\mathrm{H}$ activation and related $\mathrm{C}-\mathrm{C}$ or $\mathrm{C}-\mathrm{X}(\mathrm{X}=\mathrm{N}, \mathrm{O})$ bond formation. ${ }^{[127,166]} \mathrm{A}$ multiple $\mathrm{C}-\mathrm{H}$ activation and annulation strategy in the presence of rhodaelectro-catalysis to synthesize aza-polycyclic aromatic hydrocarbons is envisioned to be highly desirable.

\subsubsection{Optimization of Rhodaelectro-Catalyzed Domino Annulations}

On the basis of previous studies, ${ }^{[127,146,166]}$ Dr. Kong from the Ackermann team designed a new directing group to investigate the $\mathrm{C}-\mathrm{H}$ activation and alkyne annulation cascade via rhodaelectro-catalysis. The optimization was finished by Dr. Kong. Hence, I performed key control experiments. When cationic rhodium(III) catalyst $\left[\mathrm{Cp}^{*} \mathrm{Rh}\left(\mathrm{CH}_{3} \mathrm{CN}\right)_{3}\right]\left(\mathrm{SbF}_{6}\right)_{2}$ was employed as catalyst, the product was obtained in $90 \%$ yield (Table 3.12, entry 1). Higher loading of the catalyst did not show a decrease of the efficiency (entry 3 ). When the reaction was attempted without electricity, only trace amounts of the desired product 183aa was formed (entry 4). No product was observed in the absence of the rhodium catalyst 
(entry 5). A nitrogen atmosphere failed to improve the yield of this reaction (entry 6). The replacement of electricity by $\mathrm{Cu}(\mathrm{OAc})_{2}$ furnished the product in much lower yield (entry 7$)$. The use of other electrode materials led to a sharp decrease of the yield.

Table 3.12. Control experiments for the rhodaelectro-catalyzed cascade reactions. ${ }^{[a]}$

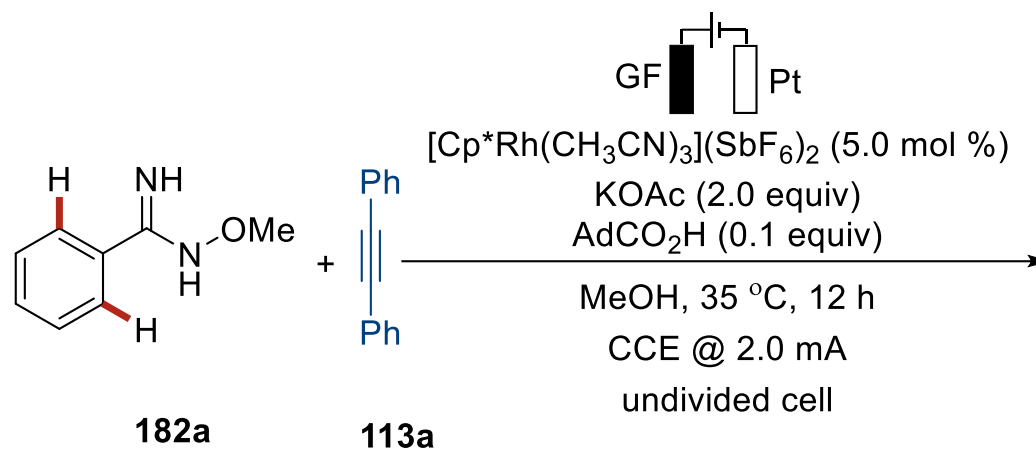<smiles>C1=CC2=C(c3ccccc3)c3c2c(c(-c2ccccc2)c2ccccc32)N2C(=C1)C(c1ccccc1)=C2c1ccccc1</smiles>

183aa

\begin{tabular}{ccc}
\hline Entry & Variation of standard condotion & Yield [\%] \\
\hline 1 & no & 90 \\
2 & {$\left[\mathrm{Cp}^{*} \mathrm{RhCl}_{2}\right]_{2}(2.5 \mathrm{~mol} \%)$} & 75 \\
3 & {$\left[\mathrm{Cp}^{*} \mathrm{Rh}\left(\mathrm{CH}_{3} \mathrm{CN}\right)_{3}\right]\left(\mathrm{SbF}_{6}\right)_{2}(2.5 \mathrm{~mol} \%)$} & 89 \\
4 & without electricity & trace \\
5 & Without rhodium catalyst & 0 \\
6 & under $\mathrm{N}_{2}$ & 60 \\
7 & $\mathrm{Cu}(\mathrm{OAc})_{2}(4$ equiv $)$ instead of electricity & 25 \\
8 & platinum plate as anode electrode & 67 \\
9 & nickel foam as cathode electrode & 65 \\
\hline
\end{tabular}

[a] Undivided cell, graphite felt anode (GF), platinum plate cathode $(\mathrm{Pt}), 182 \mathrm{a}(0.20 \mathrm{mmol}), 113 \mathrm{a}(0.70$ mmol), $\left[\mathrm{Cp}^{*} \mathrm{Rh}\left(\mathrm{CH}_{3} \mathrm{CN}\right)_{3}\right]\left(\mathrm{SbF}_{6}\right)_{2}(5.0 \mathrm{~mol} \%), \mathrm{KOAc}$ (2.0 equiv), $\mathrm{AdCO}_{2} \mathrm{H}$ (0.1 equiv), $\mathrm{MeOH}(4 \mathrm{~mL}), 35^{\circ} \mathrm{C}$ under air, isolated yield. 


\subsubsection{Scope of Rhodaelectro-catalyzed C-H Activation and Annulation Cascade}

With the optimal reaction conditions in hand, the scope of the electrocatalysis was examined with different imidamides 182 (Table 3.13). A broad range of aryl imidamides 182 bearing electron-donating (182b) and electron-withdrawing groups (182c) proved applicable to the electrocatalysis.

Table 3.13. Rhodaelectro-catalyzed C-H activation with imidamide 182.

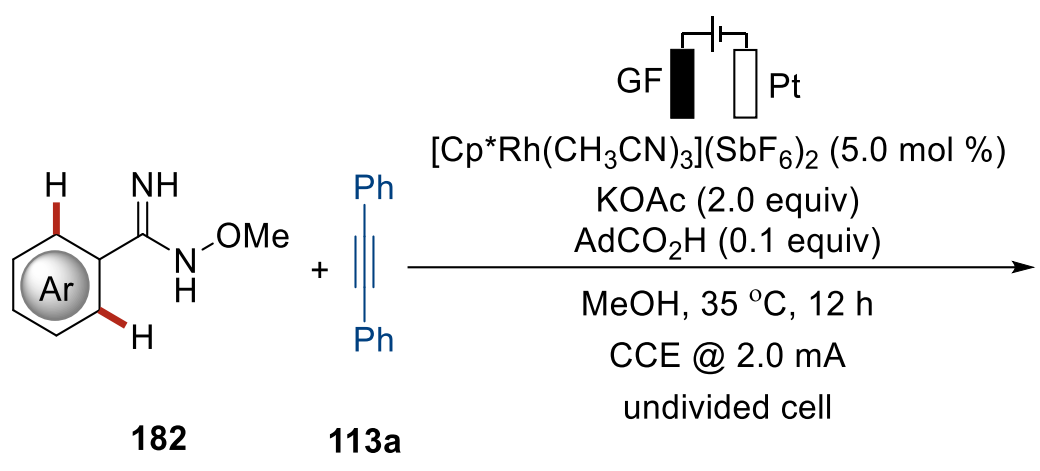<smiles></smiles>

183

Entry Substrate Yield [\%]


2<smiles>CONC(=N)c1ccc(OC)cc1</smiles>

3<smiles>CONC(=N)c1ccc(C(C)(C)C)cc1</smiles><smiles>CONC(=N)c1ccc(F)cc1</smiles><smiles></smiles>

$183 \mathrm{ba}$<smiles></smiles>

$183 \mathrm{ca}$<smiles></smiles>

183da
76

57

73

[a] Undivided cell, graphite felt anode (GF), platinum plate cathode (Pt), $182(0.20 \mathrm{mmol}), 113 a(0.70$ mmol), $\left[\mathrm{Cp}^{*} \mathrm{Rh}\left(\mathrm{CH}_{3} \mathrm{CN}\right)_{3}\right]\left(\mathrm{SbF}_{6}\right)_{2}(5.0 \mathrm{~mol} \%), \mathrm{KOAc}$ (2.0 equiv), $\mathrm{AdCO}_{2} \mathrm{H}$ (0.1 equiv), $\mathrm{MeOH}(4.0 \mathrm{~mL}), 35$ ${ }^{\circ} \mathrm{C}$ under air, isolated yield.

Subsequently, a variety of alkynes 113 was evaluated in the rhodaelectro-catalyzed cascade $\mathrm{C}-\mathrm{H}$ activations (Table 3.14). Alkynes 113 with electron-donating substituents on the arene motif delivered the desired products 183ab, 183ac, and 183ad. The trimethylsilyl 
group was well tolerated under the electrolysis conditions, serving as a handle for further transformations, such as Hiyama cross-couplings. ${ }^{[167]}$ The cascade annulative reaction proceeded equally well with meta-substituted alkyne $113 \mathrm{f}$, affording the desired product 183af in high yield and selectivity. Remarkably, challenging unsymmetrical alkyne $113 \mathrm{~g}$ delivered the corresponding product with only two regioisomers and good selectivity.

Table 3.14. Rhodaelectro-catalyzed C-H activation with alkyne 113.

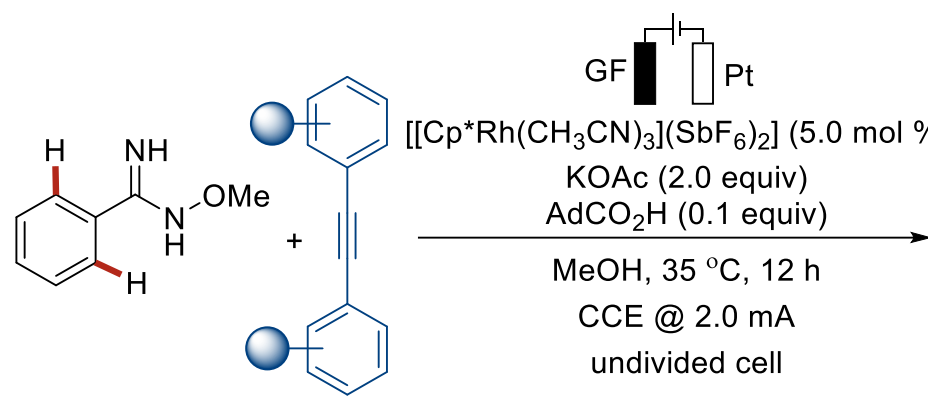

$182 a$

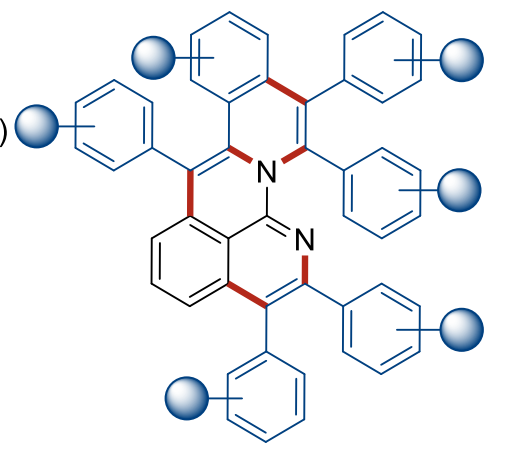

183

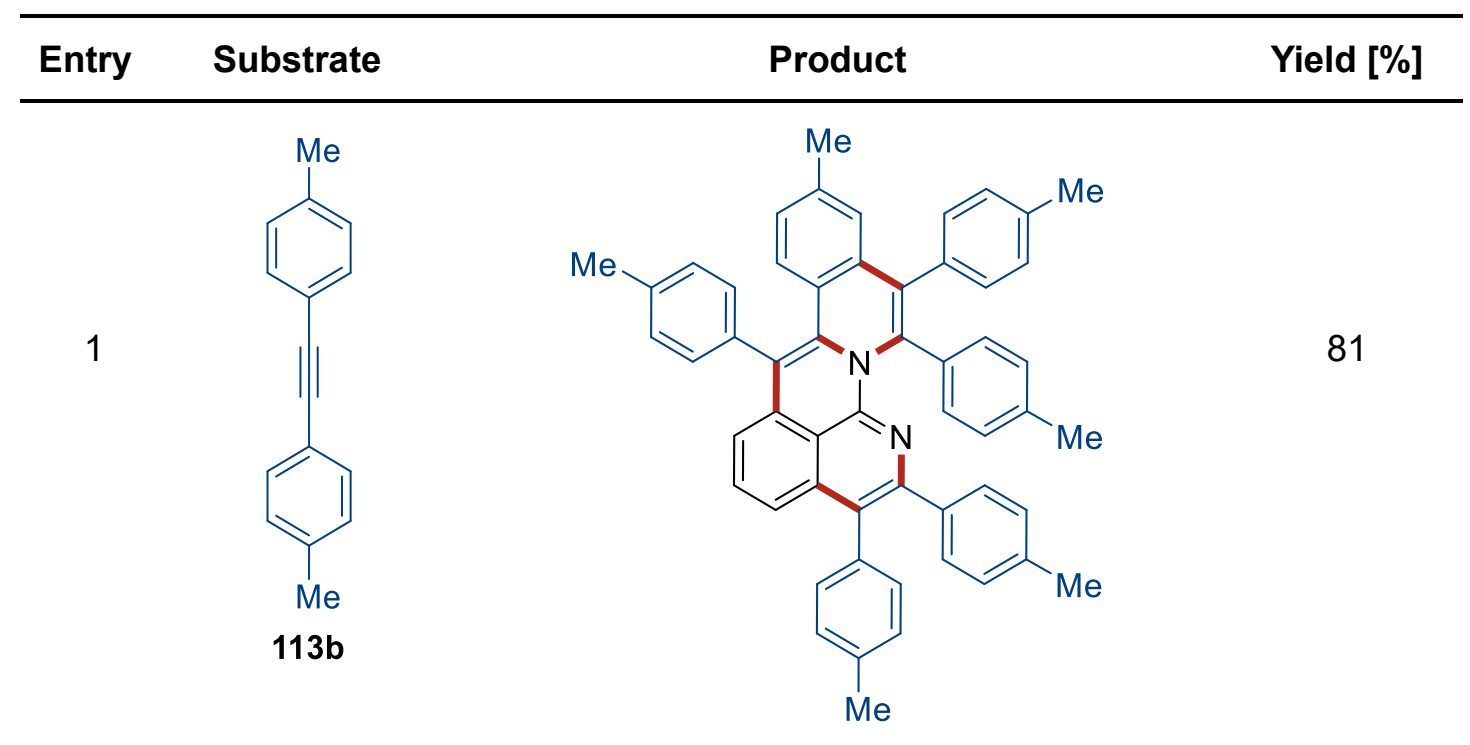

$183 a b$ 
2<smiles>COc1ccc(C#Cc2ccc(OC)cc2)cc1</smiles>

$113 c$

3<smiles>CC(C)(C)c1ccc(C#Cc2ccc(C(C)(C)C)cc2)cc1</smiles>

4<smiles>CS(=O)(=O)c1ccc(C#Cc2ccc([As])cc2)cc1</smiles>

$113 e$<smiles>COc1ccc(-c2c(-c3ccc(OC)cc3)n3c4c(-c5ccc(OC)cc5)c5ccc(OC)cc5c5c(-c6ccc(OC)cc6)c6cccc2c3c6c54)cc1</smiles> 
5<smiles>Cc1cccc(C#Cc2cccc(C)c2)c1</smiles>

$113 f$

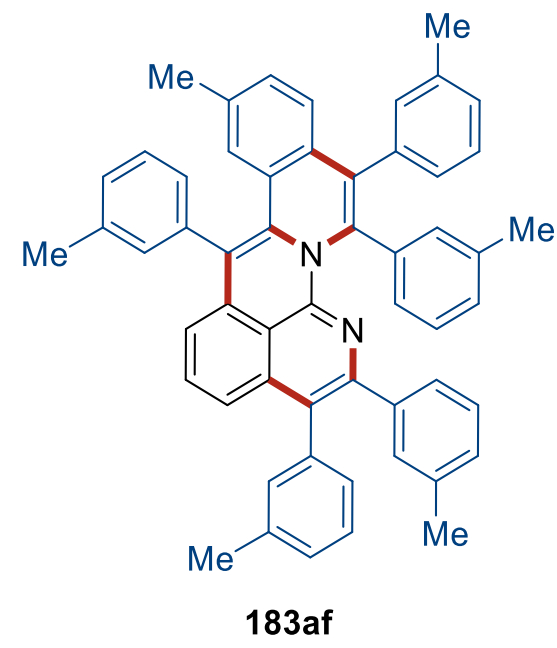

6<smiles>CCCC#Cc1ccccc1</smiles>

$113 g$<smiles></smiles>

183ag<smiles>CCCCC1=C(c2ccccc2)N2C(=C(C(C)C)c3cccc4c(-c5ccccc5)c(C(C)C)nc2c34)c2ccccc21</smiles>

183ag'
51 (183ga)

19 (183ga)

[a] Undivided cell, graphite felt anode (GF), platinum plate cathode (Pt), 182a $(0.20 \mathrm{mmol}), 113(0.70$ mmol), $\left[\mathrm{Cp}^{*} \mathrm{Rh}\left(\mathrm{CH}_{3} \mathrm{CN}\right)_{3}\right]\left(\mathrm{SbF}_{6}\right)_{2}(5.0 \mathrm{~mol} \%), \mathrm{KOAc}\left(0.40 \mathrm{mmol}, 2.0\right.$ equiv), $\mathrm{AdCO}_{2} \mathrm{H}$ (0.1 equiv), solvent (4.0 mL), $35^{\circ} \mathrm{C}$ under air, isolated yield.

\subsubsection{Mechanistic Studies}

The high efficacy of the rhodaelectro-catalyzed cascade $\mathrm{C}-\mathrm{H}$ activation for aza-PAHs synthesis motivated us to delineate its mode of action (Scheme 3.10). Both rhodacycles 202 and 203 showed catalytic reactivity for the electrocatalysis (Scheme 3.10a). This suggested that the three $\mathrm{C}-\mathrm{H}$ activation steps took place in an order of $1 \rightarrow 2 \rightarrow 3$ (Scheme 3.10b), which was further substantiated by the structure of PAH 183ja when unsymmetrical substrate $182 \mathrm{j}$ was employed. As $\mathrm{N}$-methoxylamide was widely used in rhodium- and ruthenium- catalyzed $\mathrm{C}-\mathrm{H}$ annulation, ${ }^{[168]}$ a similar pathway might proceed for our new $\mathrm{N}$ - 
methoxylamide directing group, as $\mathrm{N}-\mathrm{O}$ bond cleavage was observed on rhodacycle 203 and the products 183 .<smiles>CON1C(N)=C2c3ccccc3[Pb]([O+2])OC21</smiles>

202<smiles></smiles>

203 a) catalytical reactivities of 202 and 203<smiles>[R]ONC(=N)c1ccccc1[2H]</smiles>

113

b) the order of $\mathrm{C}-\mathrm{H}$ activation<smiles>CONC(=N)c1cccc(C)c1</smiles><smiles>[C+]1CCCCC1</smiles><smiles>[C]1C=CC=CC1</smiles>

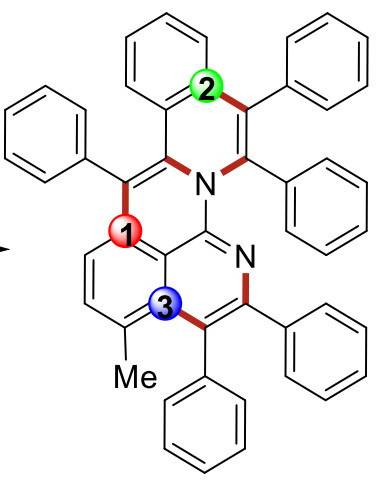

$183 \mathrm{ja}$

Scheme 3.10. mechanistic studies of Rhodaelectro-catalyzed C-H activation with alkyne 113.

\subsubsection{Derivatization of Product 183aa}

The obtained aza-PAHs 183 could be easily transformed to valuable functional molecular analogs. Treating aza-PAH 183aa with iodomethane thus afforded a cationic nitrogendoped nanographene 204 in $93 \%$ yield (Scheme 3.11). 


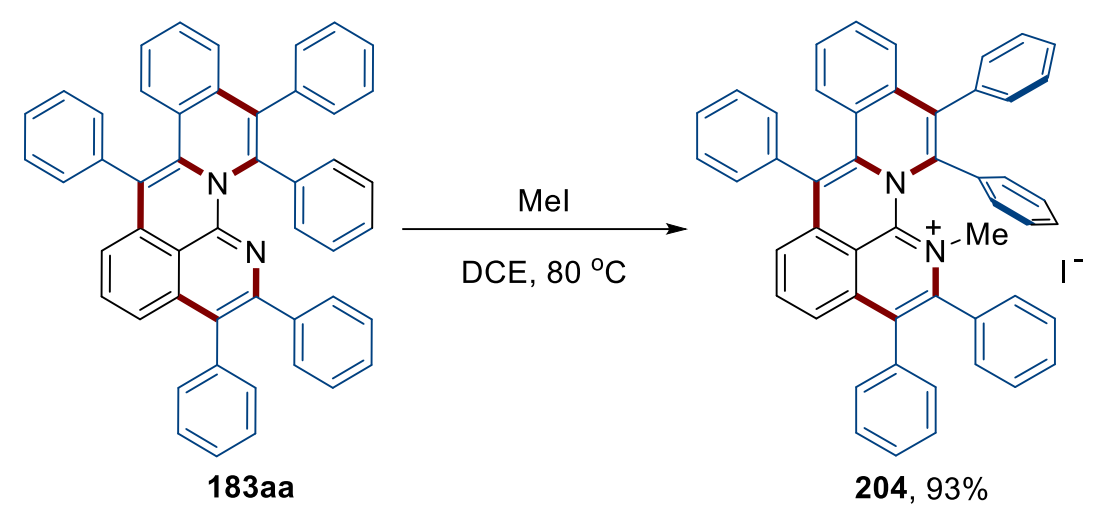

Scheme 3.11. Methylation of 183aa.

\subsection{Rhodaelectro-Catalyzed Switchable Indole Dienylation/ Cyclopropylation}

Conjugated dienes and cyclopropanes are key structural motifs in many natural products and drugs. ${ }^{[155,156]}$ These two structures can easily be transferred to other complex molecules. Rhodium(III) showed strong power in electricity-enabled $\mathrm{C}-\mathrm{H}$ activations.

\subsubsection{Optimization Studies for the Switchable Dienylation and Cyclopropylation}

Numerous examples of $\mathrm{C}-\mathrm{H}$ functionalization of indoles catalysed by rhodium(III) have been reported with the assistance of pyrimidine. ${ }^{[141 d]}$ Therefore, we chose 2indolepyrimidine $71 \mathrm{a}$ as the model substrate and a constant current of $3.0 \mathrm{~mA}$ was applied in an undivided cell setup. The optimization for the rhodaelectro-catalyzed dienylation was commenced by testing the effect of different carboxylate salts (Table 3.15). Initially, the commercially available NaOAc was used to give the dienylated product 184a in good yield with good $Z / E$ ratio. The sterically encumbered NaOPiv failed to improve the regioselectivity of this transformation. Aromatic carboxylate salts failed to increase the yield and selectivity of the reaction (entries 3-4). Fortunately, the desired product 184a was 
obtained in $85 \%$ yield and $4.5 / 1 \mathrm{Z} / \mathrm{E}$ ratio by $\mathrm{NaO}_{2} \mathrm{CAd}$ (entry 5 ). Then, weaker bases were also tested but not give better results (entries 6-7).

Table 3.15. Screening of the bases. ${ }^{[a]}$

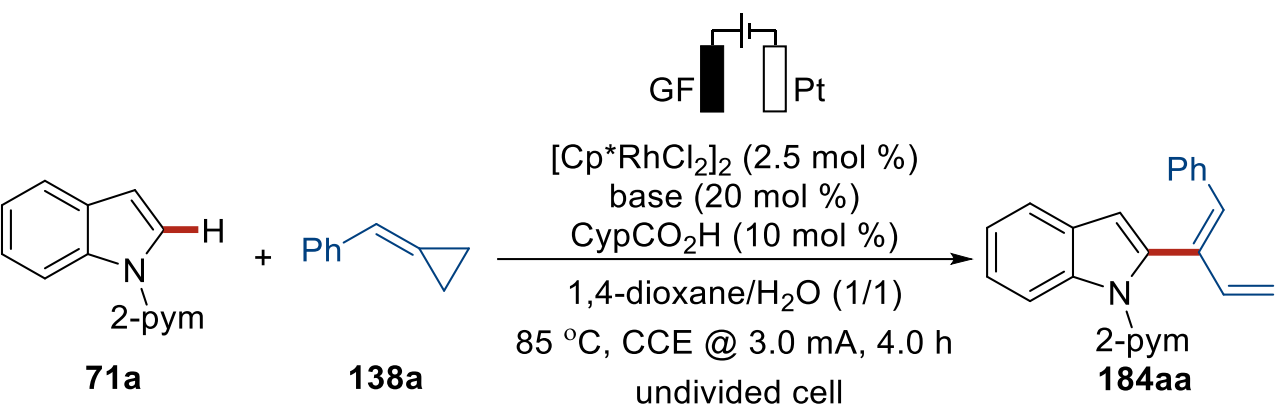

\begin{tabular}{cccc}
\hline Entry & Base & Yield [\%] & Z/E \\
\hline 1 & $\mathrm{NaOAc}$ & 72 & $3.9 / 1$ \\
2 & $\mathrm{NaOPiv}$ & 78 & $3.5 / 1$ \\
3 & $\mathrm{NaO}{ }_{2} \mathrm{CMes}$ & 60 & $4.0 / 1$ \\
4 & $\mathrm{NaO}_{2} \mathrm{CPh}$ & 82 & $3.6 / 1$ \\
5 & $\mathrm{NaO}_{2} \mathrm{CAd}$ & 85 & $4.5 / 1$ \\
6 & $\mathrm{NaO}_{2} \mathrm{CCF}_{3}$ & 72 & $3.5 / 1$ \\
7 & $\mathrm{NaO}_{3} \mathrm{SCF}_{3}$ & trace & - \\
\hline
\end{tabular}

[a] Undivided cell, graphite felt anode (GF), platinum plate cathode, 71a $(0.10 \mathrm{mmol}) 138 \mathrm{a}(0.16 \mathrm{mmol})$, $\left[\mathrm{Cp}^{*} \mathrm{RhCl}_{2}\right]_{2}(2.5 \mathrm{~mol} \%)$, base (20 mol \%), $\mathrm{CypCO}_{2} \mathrm{H}(10 \mathrm{~mol} \%)$, solvent $(4.0 \mathrm{~mL}), 85^{\circ} \mathrm{C}, \mathrm{CCE} @ 3.0 \mathrm{~mA}$, under air, yield of isolated product, $\mathrm{Z} / \mathrm{E}$ ratio determined by ${ }^{1} \mathrm{H} \mathrm{NMR}, \mathrm{CypCO}{ }_{2} \mathrm{H}=$ cyclopentanecarboxylic acid.

Next, we turned to the evaluation of the effect exerted by the acids (Table 3.16). The use of acetic acid showed lower efficiency than $\mathrm{CypCO}_{2} \mathrm{H}$ (entries 1-2). Other carboxylic acids with bulkier group also gave similar efficiency (entries 3-4). We also tried stronger carboxylic acids, but reaction efficiency was not improved (entries 5-6). 
Table 3.16. Screening of different acids. ${ }^{[a]}$

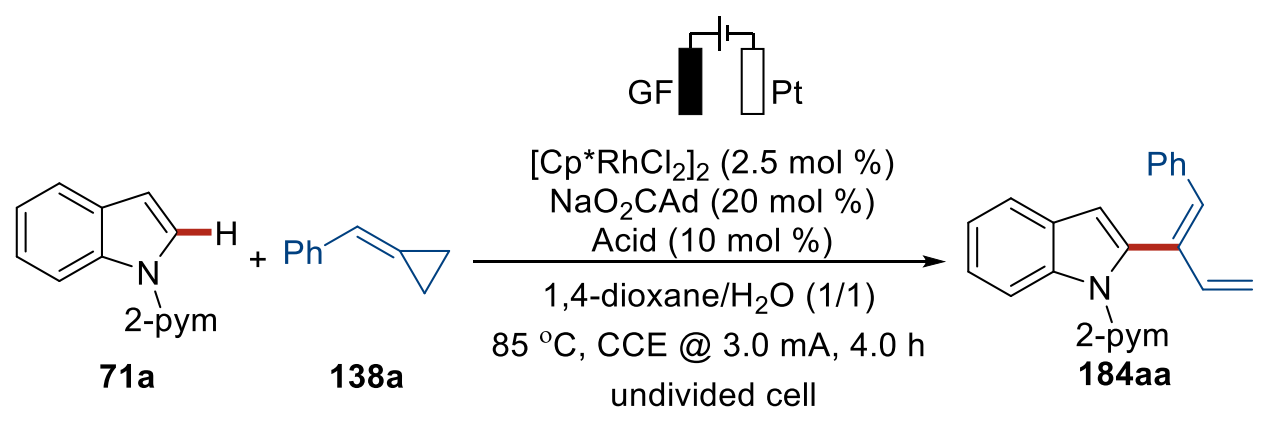

\begin{tabular}{|c|c|c|c|}
\hline Entry & Acid & Yield [\%] & Z/E \\
\hline 1 & СурCO ${ }_{2} \mathrm{H}$ & 85 & $4.5 / 1$ \\
\hline 2 & $\mathrm{AcOH}$ & 76 & $3.6 / 1$ \\
\hline 3 & $\mathrm{PivOH}$ & 82 & $3.3 / 1$ \\
\hline 4 & $\mathrm{AdCO}_{2} \mathrm{H}$ & 82 & $4.0 / 1$ \\
\hline 5 & $\mathrm{PhCO}_{2} \mathrm{H}$ & 79 & $3.7 / 1$ \\
\hline 6 & $\mathrm{MesCO}_{2} \mathrm{H}$ & 78 & $3.8 / 1$ \\
\hline
\end{tabular}

[a] Undivided cell, graphite felt anode (GF), platinum plate cathode $(\mathrm{Pt}), \mathbf{7 1 a}(0.10 \mathrm{mmol}) 138 \mathrm{a}(0.16 \mathrm{mmol})$, $\left[\mathrm{Cp}^{*} \mathrm{RhCl}_{2}\right]_{2}(2.5 \mathrm{~mol} \%), \mathrm{NaO}_{2} \mathrm{CAd}(20 \mathrm{~mol} \%)$, acid $(10 \mathrm{~mol} \%)$, solvent $(4.0 \mathrm{~mL}), 85^{\circ} \mathrm{C}, \mathrm{CCE} @ 3.0 \mathrm{~mA}$, under air, $4.0 \mathrm{~h}$, yield of isolated product, Z/E ratio determined by ${ }^{1} \mathrm{H}$ NMR.

Then, we resorted to the optimization of different solvents (Table 3.17). The variation of the ratio between 1,4-dioxane and water were conducted, yet not improve the efficiency of the reaction (entries 1-3). Another mixture of solvents $t-\mathrm{AmOH} / \mathrm{H}_{2} \mathrm{O}(3 / 1)$ (entry 4), often used for the electrochemistry, ${ }^{[144]}$ furnished the dienylated product 184aa in lower yield and Z/E ratio. The electrocatalysis was applicable to water-free system while with lower efficiency (entries 5-6). 
Table 3.17. Optimization of solvents.

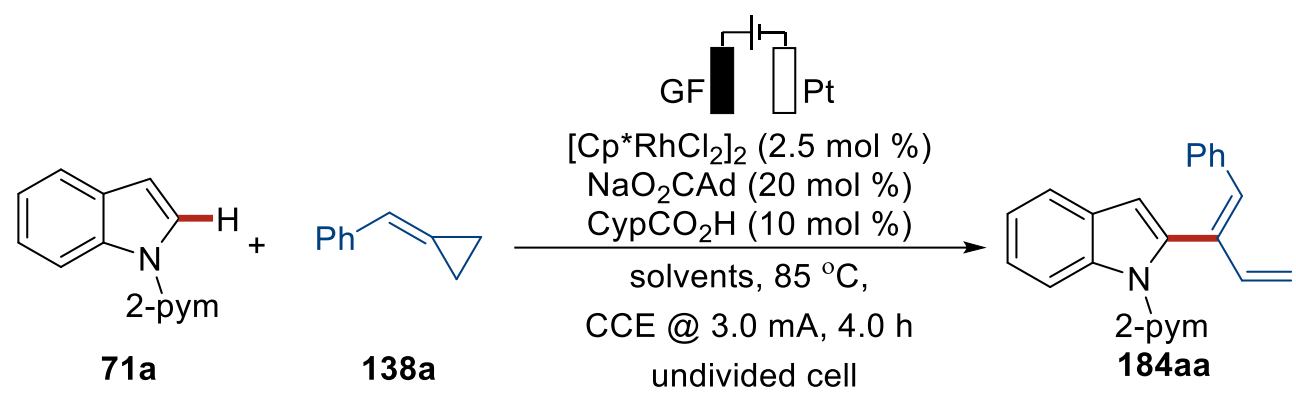

\begin{tabular}{cccc}
\hline Entry & Solvent & Yield (\%) & Z/E \\
\hline 1 & 1,4 -dioxane/ $\mathrm{H}_{2} \mathrm{O}(1 / 1)$ & 85 & $4.5 / 1$ \\
2 & $\mathrm{H}_{2} \mathrm{O}$ & trace & -- \\
3 & $1,4-$ dioxane $/ \mathrm{H}_{2} \mathrm{O}(2 / 1)$ & 72 & $3.4 / 1$ \\
4 & $t$-AmOH$/ \mathrm{H}_{2} \mathrm{O}(3 / 1)$ & 82 & $2.5 / 1$ \\
5 & $\mathrm{DMF}$ & trace & -- \\
6 & $\mathrm{EtOH}$ & 74 & $3.7 / 1$ \\
\hline
\end{tabular}

[a] Undivided cell, graphite felt anode (GF), platinum plate cathode $(\mathrm{Pt}), \mathbf{7 1 a}(0.10 \mathrm{mmol})$ 184a $(0.16$ mmol), $\left[\mathrm{Cp}^{*} \mathrm{RhCl}_{2}\right]_{2}(2.5 \mathrm{~mol} \%)$, base $(20 \mathrm{~mol} \%)$, acid $(10 \mathrm{~mol} \%)$, solvent $(4.0 \mathrm{~mL}), 85^{\circ} \mathrm{C}, \mathrm{CCE} @ 3.0$ $\mathrm{mA}$, under air, yield of isolated product, Z/E ratio determined by ${ }^{1} \mathrm{H} N M R$.

Subsequently, control experiments were conducted (Table 3.18). The absence of the catalyst $\left[\mathrm{Cp}^{*} \mathrm{RhCl}_{2}\right]_{2}$ resulted in no conversion of the starting material (Table 3.20, entry 2). Reactions in the absence of $\mathrm{NaO}_{2} \mathrm{CAd}$ or $\mathrm{CpCO}_{2} \mathrm{H}$ gave worse results. A low yield was obtained without electricity (entry 5). Other transition metal catalysts were tested, yet failed to assemble the desired product (entries 6-7). 
Table 3.18. Control experiments. ${ }^{[a]}$

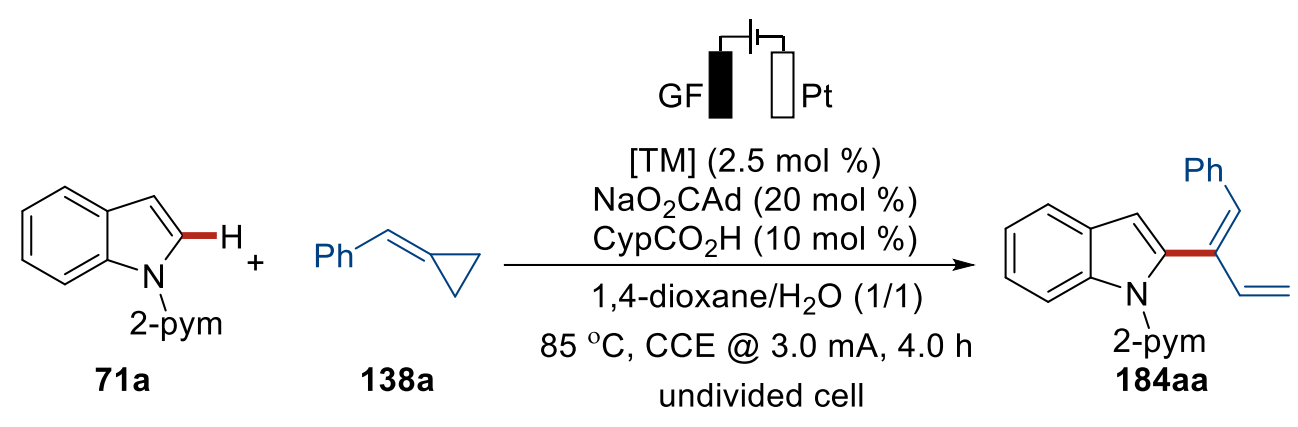

\begin{tabular}{cccccc}
\hline Entry & {$[\mathrm{TM}]$} & Base & Acid & Yield [\%] & Z/E \\
\hline 1 & {$\left[\mathrm{Cp}^{*} \mathrm{RhCl}_{2}\right]_{2}$} & $\mathrm{NaO}_{2} \mathrm{CAd}$ & $\mathrm{CypCO}{ }_{2} \mathrm{H}$ & 85 & $4.5 / 1$ \\
2 & -- & $\mathrm{NaO}_{2} \mathrm{CAd}$ & $\mathrm{CypCO}{ }_{2} \mathrm{H}$ & -- & -- \\
3 & {$\left[\mathrm{Cp}^{*} \mathrm{RhCl}_{2}\right]_{2}$} & -- & $\mathrm{CypCO}{ }_{2} \mathrm{H}$ & 32 & $3.2 / 1$ \\
4 & {$\left[\mathrm{Cp}^{*} \mathrm{RhCl}_{2}\right]_{2}$} & $\mathrm{NaO}_{2} \mathrm{CAd}$ & -- & 72 & $3.6 / 1$ \\
$5^{[\mathrm{b}]}$ & {$\left[\mathrm{Cp}^{*} \mathrm{RhCl}_{2}\right]_{2}$} & $\mathrm{NaO}_{2} \mathrm{CAd}$ & $\mathrm{CypCO}_{2} \mathrm{H}$ & 24 & $2.4 / 1$ \\
6 & {$\left[\mathrm{RuCl}_{2}\left(\mathrm{p}-\mathrm{Cymene}_{2}\right]_{2}\right.$} & $\mathrm{NaO}_{2} \mathrm{CAd}$ & $\mathrm{CypCO}_{2} \mathrm{H}$ & -- & -- \\
7 & $\mathrm{Pd}(\mathrm{OAc})_{2}$ & $\mathrm{NaO}_{2} \mathrm{CAd}$ & $\mathrm{CypCO}_{2} \mathrm{H}$ & -- & -- \\
\hline
\end{tabular}

[a] Undivided cell, graphite felt anode (GF), platinum plate cathode $(\mathrm{Pt}), 71 \mathrm{a}(0.10 \mathrm{mmol}) 138 \mathrm{a}(0.16$ mmol), [M] (2.5 mol \%), $\mathrm{NaO}_{2} \mathrm{CAd}(20 \mathrm{~mol} \%), \mathrm{CypCO}{ }_{2} \mathrm{H}(10 \mathrm{~mol} \%), 1,4-$ Dioxane/ $\mathrm{H}_{2} \mathrm{O}(1 / 1)(4.0 \mathrm{~mL}), 85$ ${ }^{\circ} \mathrm{C}, \mathrm{CCE} @ 3.0 \mathrm{~mA}$, under air, yield of isolated product, Z/E ratio determined by ${ }^{1} \mathrm{H} \mathrm{NMR}$. [b] Without electricity, $12 \mathrm{~h}$.

Small variation of the current did not give better results (Table 3.19, entries 2-3). An increased amount of $\mathrm{NaO}_{2} \mathrm{CAd}$ displayed better selectivity (entry 4). The $\mathrm{C}-\mathrm{H}$ dienylation proceeded well at larger scale and higher temperature with the desired product obtained in higher yield and selectivity. 
Table 3.19. Further optimization of rhodaelectro-C-H-dienylation. ${ }^{[a]}$

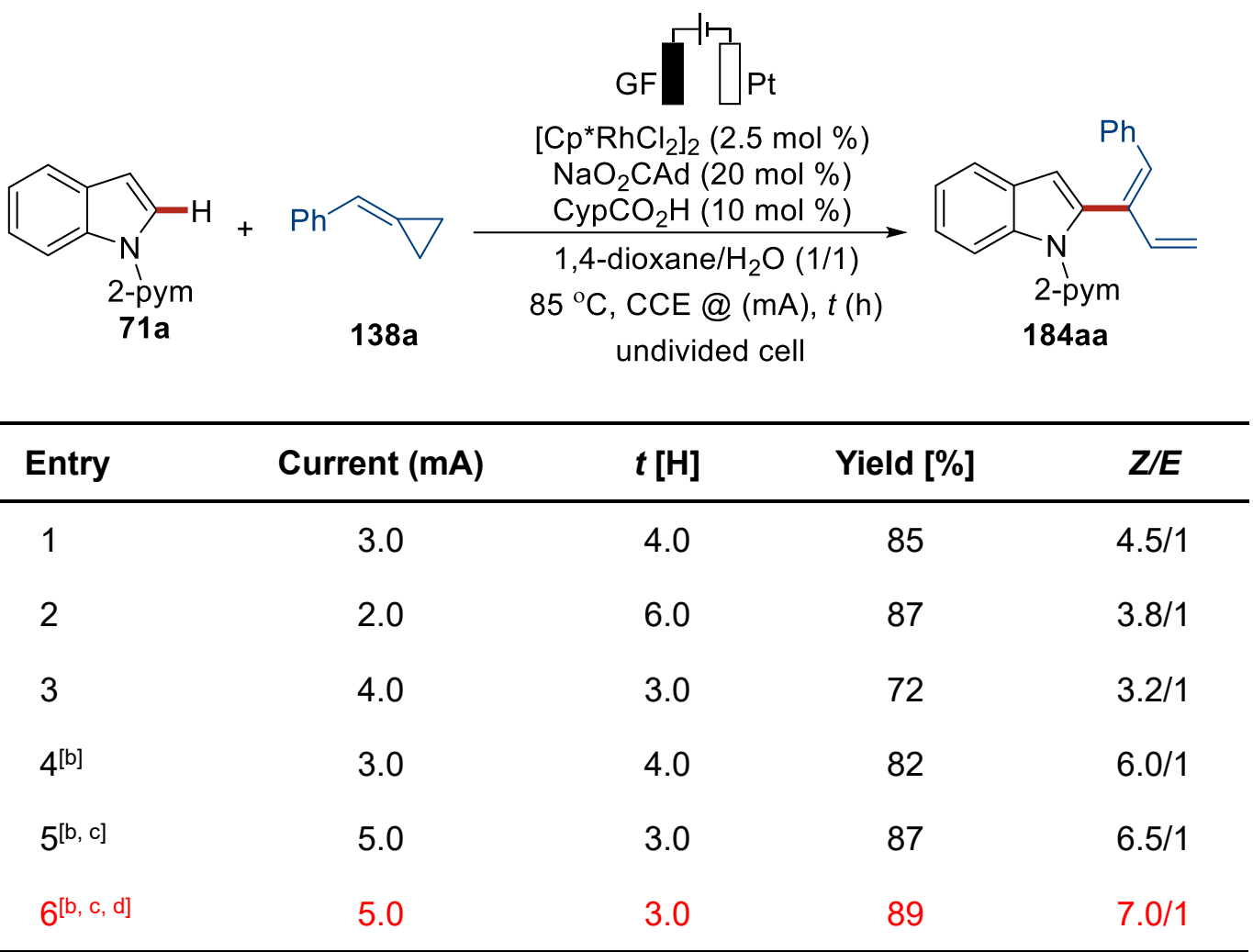

[a] Undivided cell, graphite felt anode (GF), platinum plate cathode (Pt), 71a $(0.10 \mathrm{mmol})$ 138a $(0.16$ $\mathrm{mmol}$ ), $\left[\mathrm{Cp}^{*} \mathrm{RhCl}_{2}\right]_{2}(2.5 \mathrm{~mol} \%), \mathrm{NaO}_{2} \mathrm{CAd}(20 \mathrm{~mol} \%), \mathrm{CypCO}_{2} \mathrm{H}(10 \mathrm{~mol} \%), 1,4-d i o x a n e / \mathrm{H}_{2} \mathrm{O}(1 / 1)$ $4.0 \mathrm{~mL}, 85^{\circ} \mathrm{C}$, CCE @ $3.0 \mathrm{~mA}$, under air, yield of isolated product, Z/E ratio determined by ${ }^{1} \mathrm{H}$ NMR. [b] $\mathrm{NaO}_{2} \mathrm{CAd}(40 \mathrm{~mol} \%)$. [c] reaction performed on $0.2 \mathrm{mmol}$ scale. [d] at $95^{\circ} \mathrm{C}$.

With the optimized reaction conditions for the novel $\mathrm{C}-\mathrm{H}$ dienylation reaction in hand, a benzyl substituted cyclopropane 185a was employed (Scheme 3.12), in which a unique direct $\mathrm{C}-\mathrm{H}$ cyclopropanylated indole 186aa was formed in high yield without the detection of (Z)-conformation.

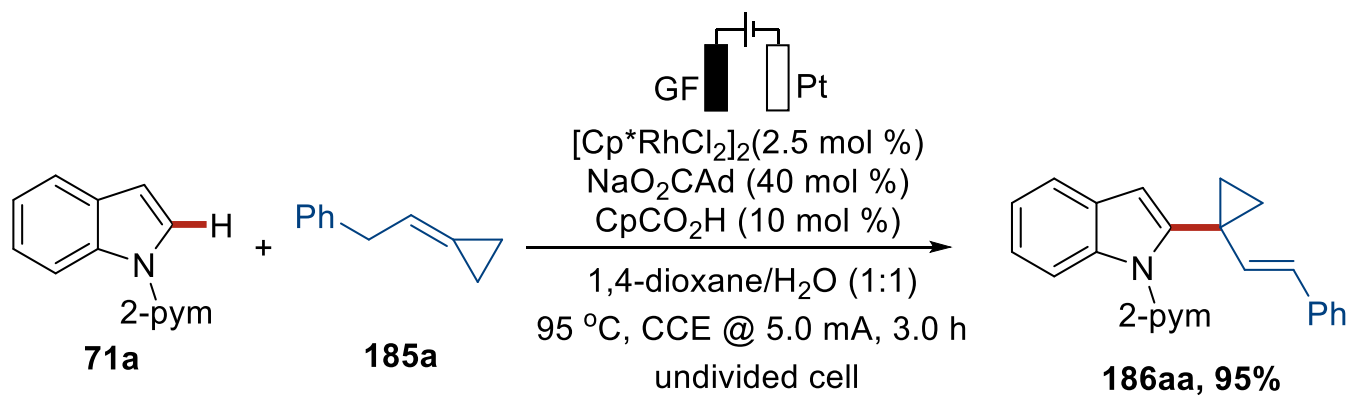

Scheme 3.12. Cyclopropanylation of indole. 


\subsubsection{Scope of the Switchable Dienylation and Cyclopropanylation}

With the optimized reaction conditions for electrochemical $\mathrm{C}-\mathrm{H}$ dienylations in hand, the versatility was explored with substituted indoles 71 (Table 3.20). Although 3- or 7-methyl indoles delivered the desired products in moderate yields, 3-methyl indole showed much a better selectivity (184ba, 1840a). Fluorine and methoxy-substituted indoles 71c and $\mathbf{7 1 f}$ were transformed efficiently, while 6-substituted indoles (184ka, 184ma) was converted less efficiently. Various functional group was tolerated, such as chloride, bromide and cyan. Interestingly, indoles $\mathbf{7 1 n}$ with ester functionality at the 6-position delivered 184na in high yield and good selectivity. This dienylation protocol was applicable to pyrrole $\mathbf{7 1 p}$.

Table 3.20. Electrochemical C-H dienylation of different indoles 71 with $185 \mathrm{a}$. ${ }^{[a]}$

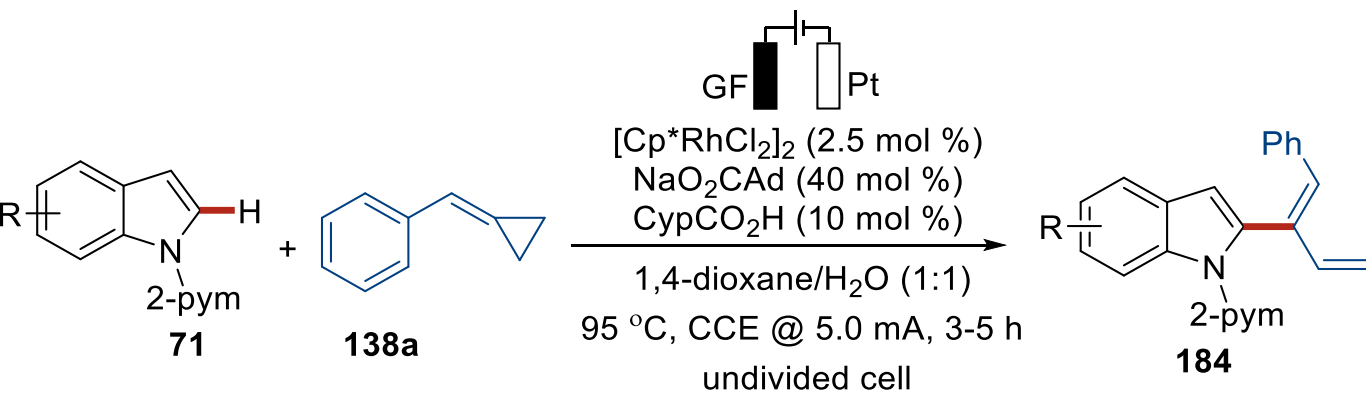

Entry Substrate


3<smiles>COc1cccc2c1ccn2[PbH]</smiles>

71c

4<smiles>N#Cc1cccc2c1cc([PH2+])n2P</smiles>

71d

5<smiles></smiles>

$71 \mathrm{e}$

6<smiles></smiles>

$71 f$

7<smiles>[PH3+]n1ccc2cc(Cl)ccc21</smiles>

$71 \mathrm{~g}$

8<smiles>[PH3+]n1ccc2cc(Br)ccc21</smiles>

$71 \mathrm{~h}$<smiles>COc1ccc2c(ccn2[Po+])c1</smiles>

$71 i$<smiles>C=C/C(=C/c1ccccc1)c1cc2c(OC)cccc2n1P(O)O</smiles>

184ca<smiles>C=C/C(=C/c1ccccc1)c1cc2c(C#N)cccc2n1[Po+]</smiles>

184da<smiles>C=C/C(=C/c1ccccc1)c1cc2cc(C)ccc2n1[Po+]</smiles>

184ea<smiles>C=C/C(=C\c1ccccc1)c1cc2cc(F)ccc2n1[Pb]</smiles>

$184 f a$<smiles>[R6]n1c(/C(C=C)=C\c2ccccc2)cc2cc(Cl)ccc21</smiles>

184fga<smiles>C=C/C(=C/c1ccccc1)c1cc2cc(Br)ccc2n1[PbH]</smiles>

184ha<smiles>C=C/C(=C\c1ccccc1)c1cc2cc(OC)ccc2n1[PbH]</smiles>

184ia 
10<smiles></smiles>

71j<smiles>Fc1ccc2ccc(CP)n2c1</smiles>

$71 \mathrm{k}$

12<smiles>Cc1cc2ccc(Cl)cc2n1P(O)O</smiles>

711<smiles></smiles>

$71 \mathrm{~m}$

14<smiles>CC(=O)c1ccc2cc(C)n([Pb])c2c1</smiles>

71n

15<smiles>Cc1cccc2cc(C)n(PO)c12</smiles>

16<smiles>O=C1CCCc2c1ccn2PO</smiles><smiles>C=C/C(=C\c1ccccc1)c1cc2cc(C#N)ccc2n1P(C)(=O)C(C)(C)C</smiles>

2-pym

184ka

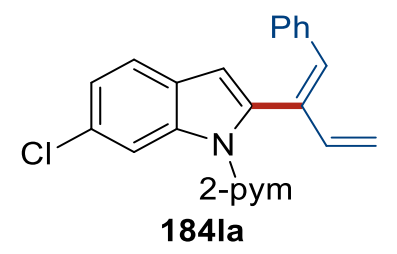<smiles>C=C/C(=C\c1ccccc1)c1cc2ccc(OC)cc2n1[Pb]</smiles>
$184 \mathrm{ma}$<smiles>C=C/C(=C/c1ccccc1)c1cc2ccc(C(C)(C)C)cc2n1[Pb]C(C)(C)C</smiles>

[a] Undivided cell, graphite felt anode (GF), platinum plate cathode (Pt), $71(0.20 \mathrm{mmol}) 138 \mathrm{a}(0.32 \mathrm{mmol}$, 1.6 equiv), $\left[\mathrm{Cp}^{*} \mathrm{RhCl}_{2}\right]_{2}(2.5 \mathrm{~mol} \%), \mathrm{NaO}_{2} \mathrm{CAd}(40 \mathrm{~mol} \%), \mathrm{CypCO}_{2} \mathrm{H}$ (10 mol \%), 1,4-dioxane/ $\mathrm{H}_{2} \mathrm{O}$ (1:1) $8.0 \mathrm{~mL}, 95^{\circ} \mathrm{C}, \mathrm{CCE} @ 5.0 \mathrm{~mA}, 3-5 \mathrm{~h}$, under air, yield of isolated product, Z/E ratio determined by ${ }^{1} \mathrm{H}$ NMR. 
Next, the robustness of the rhodaelectro-catalyzed dienylation was evaluated with a variety of functionalized cyclopropanes 138 (Table 3.21). We found that methy- and phenylgroups were tolerated by the electrocatalysis (184ab, 184ac). Substrates containing the bromo group delivered the products 184ae and 184am in good yields but with a lower Z/E ratio. Electron-deficient substrates $\mathbf{1 3 8 i}$ and $\mathbf{1 3 8}$ j showed a good reactivity in this method in contrast to previous studies ${ }^{[144-147]}$ and electron-rich compounds performed well in this transformation.

Table 3.21. Electrochemical C-H Dienylation with cyclopropane 138.[a]

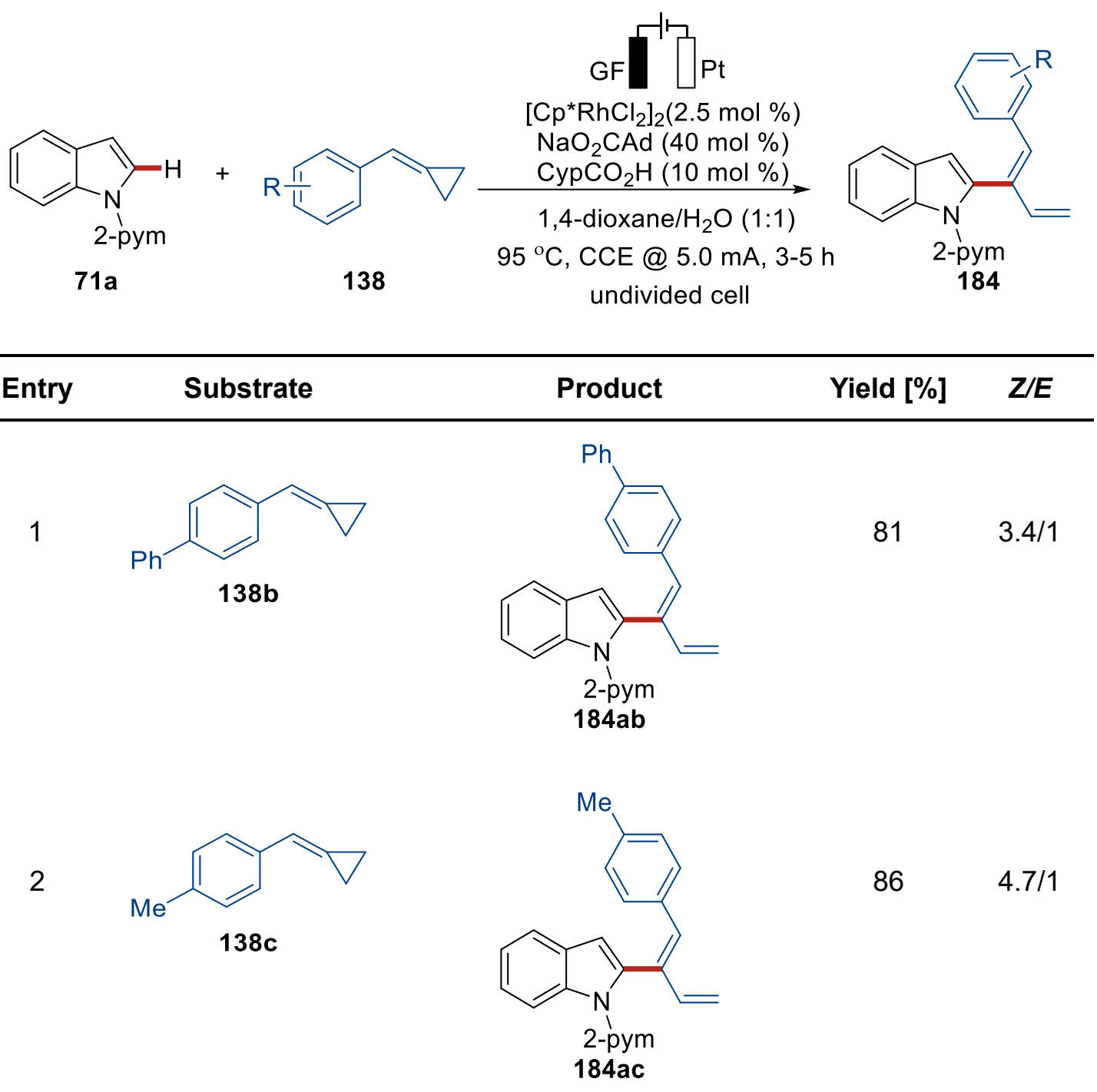


3<smiles>CC(C)(C)S(=O)(=O)c1ccc(F)cc1</smiles>

4<smiles>CS(=O)(=O)c1ccc(C=C2CC2)cc1</smiles>

5<smiles>FC(F)(F)c1ccc(C=C2CC2)cc1</smiles>

6<smiles>N#Cc1ccc(C=C2CC2)cc1</smiles><smiles>Fc1cccc(C=C2CC2)c1</smiles>

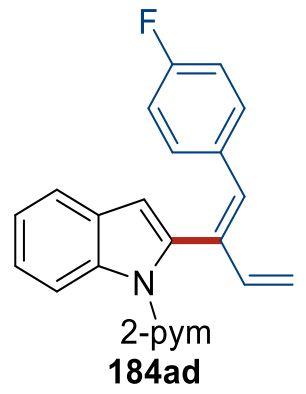
5.1/1<smiles>[R16][R16]([H])([H])n1c(/C(C=C)=C\c2ccc(Br)cc2)cc2ccccc21</smiles><smiles>[R16][R16]([H])([H])n1c(/C(C=C)=C\C)cc2ccccc21</smiles><smiles>C=C/C(=C/C)c1cc2ccccc2n1P(=O)(O)OC(C)(C)C</smiles>

184ag

7

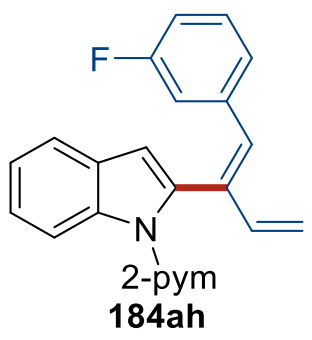

79

$4.4 / 1$ 
8<smiles>FC(F)(F)c1cccc(C=C2CC2)c1</smiles>

$138 \mathbf{i}$<smiles>COC(=O)c1cccc(C=C2CC2)c1</smiles>

138j<smiles>Oc1cccc(C=C2CC2)c1</smiles>

$138 \mathrm{k}$

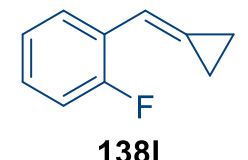<smiles>Brc1ccccc1C=C1CC1</smiles>

$138 \mathrm{~m}$<smiles>CCOc1ccccc1C=C1CC1</smiles>

13 $138 n$<smiles>[Z20]n1c(/C(C=C)=C\c2cccc(C(F)(F)F)c2)cc2ccccc21</smiles>

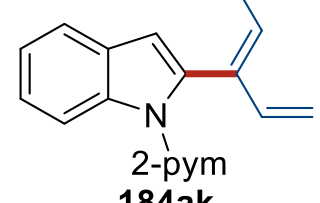

75
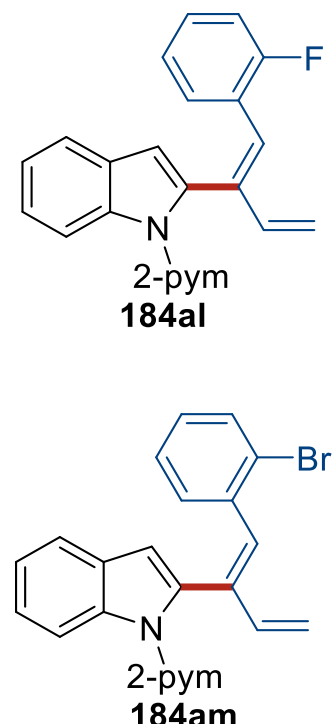

78 3.0/1<smiles>[Z20]n1c(/C(C=C)=C\c2ccccc2OCC)cc2ccccc21</smiles>

184an 
14<smiles>COc1cc(C=C2CC2)cc(OC)c1</smiles>

15<smiles>FC(F)(F)c1ccc(Br)c(C=C2CC2)c1</smiles>

$138 p$<smiles>C=C/C(=C\c1cc(OC)cc(OC)c1)c1cc2ccccc2n1[R16](=O)O</smiles>

CCDC: 2025011

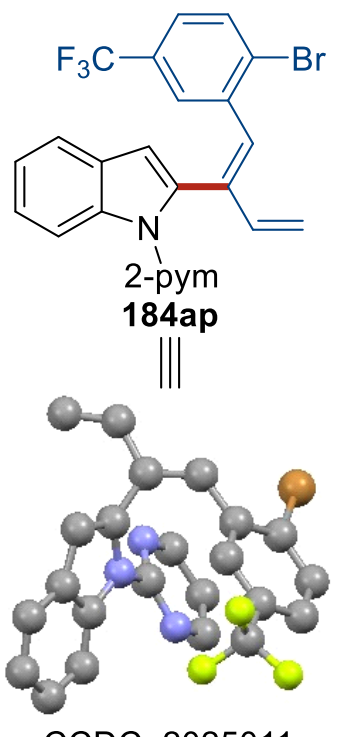

$10.5 / 1$

[a] Undivided cell, graphite felt anode (GF), platinum plate cathode (Pt), 71a (0.20 mmol) 184 (0.32 mmol),

$\left[\mathrm{Cp}^{*} \mathrm{RhCl}_{2}\right]_{2}(2.5 \mathrm{~mol} \%), \mathrm{NaO}_{2} \mathrm{CAd}(40 \mathrm{~mol} \%), \mathrm{CypCO}_{2} \mathrm{H}$ (10 mol \%), 1,4-dioxane/ $\mathrm{H}_{2} \mathrm{O}$ (1:1) $8.0 \mathrm{~mL}, 95$

${ }^{\circ} \mathrm{C}, \mathrm{CCE} @ 5.0 \mathrm{~mA}$, under air, yield of isolated product, Z/E ratio determined by ${ }^{1} \mathrm{H}$ NMR.

After the evaluation of the scope for the $\mathrm{C}-\mathrm{H}$ dienylation, we turned to probing the versatility of the unprecedented electrochemical cyclopropylation with functionalized indoles $\mathbf{7 1}$ (Table 3.22). We found that a reactive hydroxyl group is tolerated despite the steric hinderance 186qa. The halogen-containing indoles (186ga, 186ha and 186ra), even highly reactive iodo, were viable substrates. Indoles containing electron-withdrawing $71 \mathrm{~d}, 71 \mathrm{n}$ or electron-donating groups 71c, 71i were transfered efficiently. For 7-methyl indole, the cyclopropylation showed higher efficiency compared to the dienylation (1860a). To our delight, 2-phenyl pyridine could also be employed under the reaction condition though with moderate yield (186sa). Interestingly, a tryptamine derived substrate could form the challenging ring-opening product 186ta'. 
Table 3.22. Electrochemical C-H cyclopropylation of indole 71 with $185 a$.

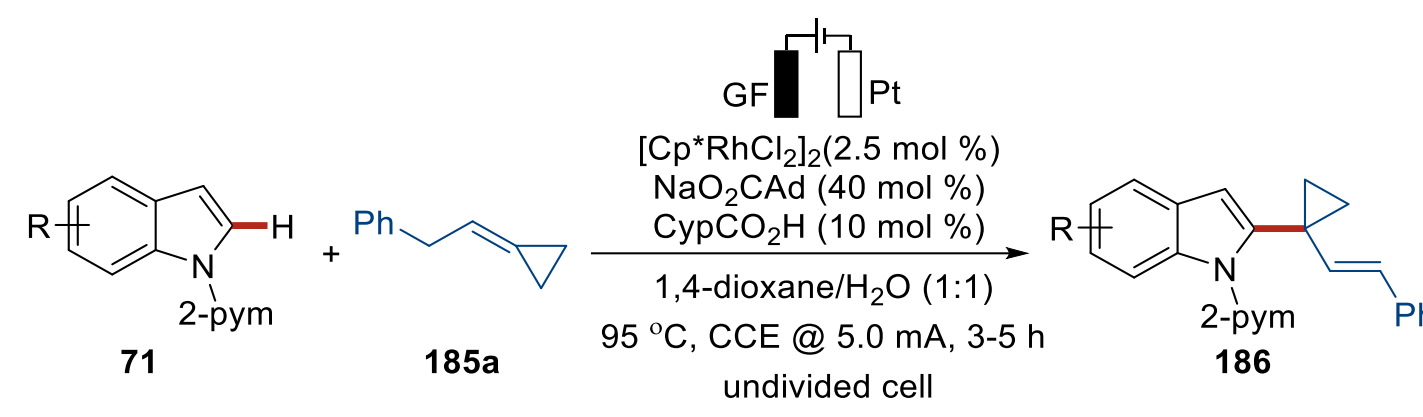

Entry [\%]


6<smiles></smiles>

$71 \mathrm{e}$

7<smiles>[PH3+]n1ccc2cc(Cl)ccc21</smiles>

$71 \mathrm{~g}$<smiles>Brc1ccc2c(ccn2[PbH2])c1</smiles>

$71 \mathrm{~h}$<smiles>[R6]n1ccc2cc(I)ccc21</smiles>

$71 \mathrm{r}$<smiles>COc1ccc2c(ccn2[Po+])c1</smiles>

71i<smiles></smiles>

71j<smiles>Fc1ccc2ccc(CP)n2c1</smiles>

71k

13<smiles>Clc1ccc2ccn([PbH2])c2c1</smiles>

71I<smiles>Cc1ccc2c(c1)cc(C1(/C=C/c3ccccc3)CC1)n2[12CH2]</smiles>

186ea<smiles>[R6]n1c(C2(/C=C/c3ccccc3)CC2)cc2cc(Cl)ccc21</smiles>

186ga<smiles>[R6]n1c(C2(/C=C/c3ccccc3)CC2)cc2cc(Br)ccc21</smiles>

186ha

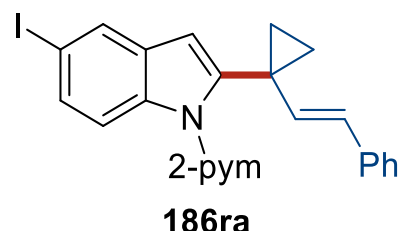

54<smiles></smiles>

186ia<smiles>N#Cc1ccc2c(c1)cc(C1(/C=C/c3ccccc3)CC1)n2[Po+]</smiles>

186ja<smiles>[Y6]n1c(C2(/C=C/c3ccccc3)CC2)cc2ccc(F)cc21</smiles>

186ka<smiles></smiles>

186la 
14<smiles></smiles>

$71 \mathrm{n}$

15<smiles>Cc1cccc2ccc(C)n12</smiles>

710

16<smiles></smiles>

$71 p$

17<smiles>COc1ccc(-c2ccccn2)c(C(C)(F)F)c1</smiles>

18

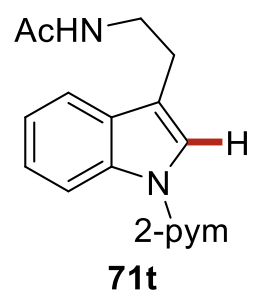<smiles></smiles>

186na

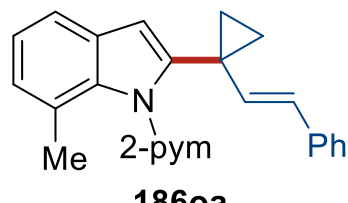

75

76

41<smiles>COc1ccc(-c2ccccn2)c(C2(/C=C/c3ccccc3)CC2)c1</smiles>

186sa

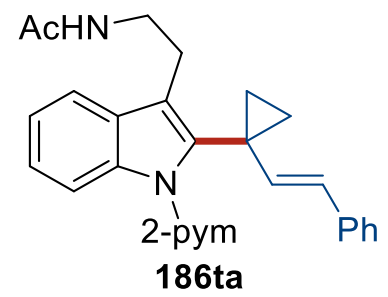

18

34

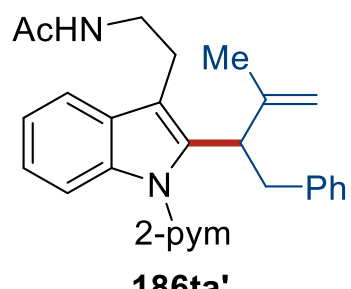

[a] Undivided cell, graphite felt anode (GF), platinum plate cathode (Pt), $71(0.20 \mathrm{mmol})$ 185a (0.32 mmol, 1.6 equiv), $\left[\mathrm{Cp}^{*} \mathrm{RhCl}_{2}\right]_{2}(2.5 \mathrm{~mol} \%), \mathrm{NaO}_{2} \mathrm{CAd}(40 \mathrm{~mol} \%), \mathrm{CypCO}{ }_{2} \mathrm{H}(10 \mathrm{~mol} \%), 1,4-$ dioxane $/ \mathrm{H}_{2} \mathrm{O}(1: 1) 8.0 \mathrm{~mL}, 95^{\circ} \mathrm{C}, \mathrm{CCE} @ 5.0 \mathrm{~mA}$, under air, yield of isolated product. 
Subsequently a series of cyclopropanes 185 were examined for the $\mathrm{C}-\mathrm{H}$ cyclopropylation reaction (Table 3.23). A substrate bearing the iodo-substituent gave the desired product 186ac in moderate yield along with a small amount of the deiodinated product (186aa/186ac 1:3). The reaction conditions were compatible with linear or branched-alkyl derived cyclopropanes (186ad-186af). The challenging cyclopropane bearing a terminal alkene was also found to be a viable substrate, affording product $186 \mathrm{ag}$ in $79 \%$ yield. The transformation was also tolerant for other changes to the backbone of the cyclic alkanes and generated the expected products in moderate yields (186ah, 186ai). Indeed, the structurally complex natural product Citronellol-derived starting material could be selectively converted to the desired product 186aj. 
Table 3.23. Electrochemical C-H cyclopropylation with methylenecyclopropanes 185 . $^{[a]}$

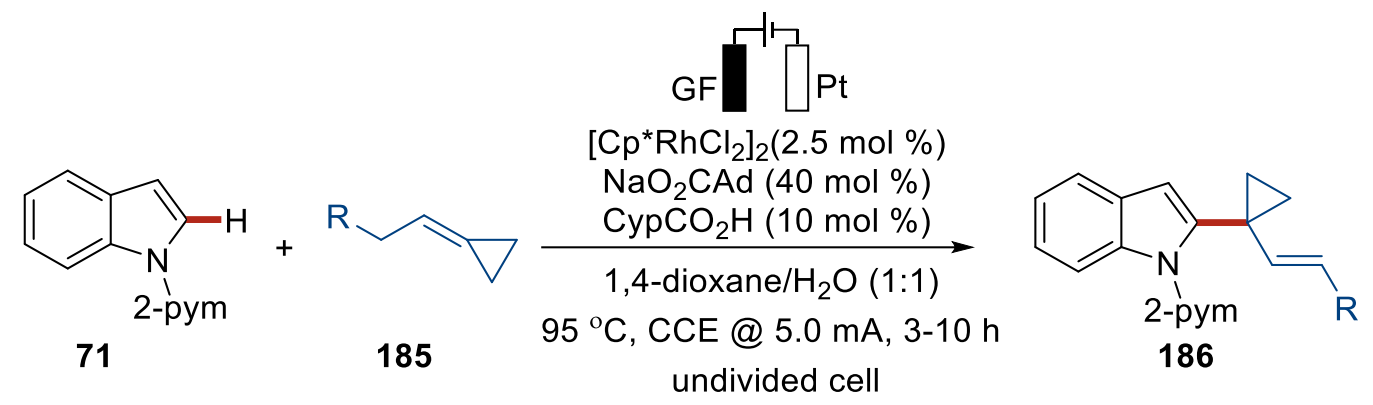

Entry Substrate

2
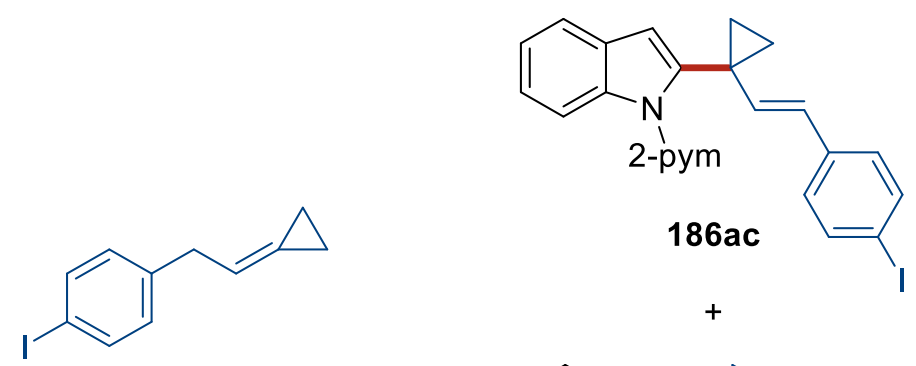

Yield [\%]

$185 c$

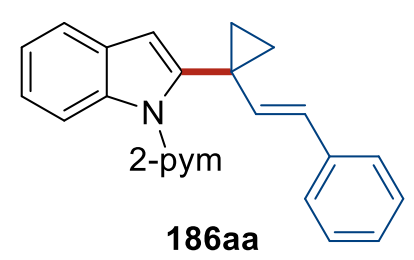

95

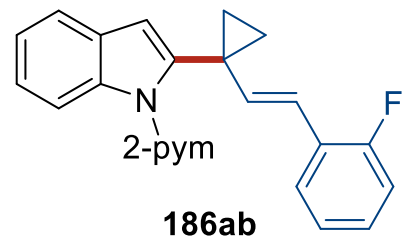

3<smiles>C(CCc1ccccc1)=C1CC1</smiles>

$185 d$

4<smiles>CC(C)(C)S(=O)(=O)C1=CC=C1</smiles><smiles>CC(C)(C)[P+]([18OH])n1c(C2(/C=C/Cc3ccccc3)CC2)cc2ccccc21</smiles>

74

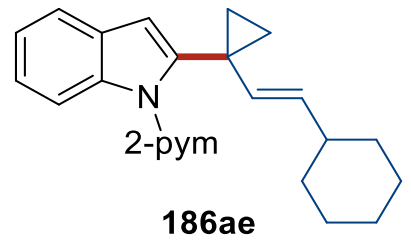


5<smiles>CCCCCCC=C1CC1</smiles>

6<smiles>C=CCCCCCC=C1CC1</smiles>

7

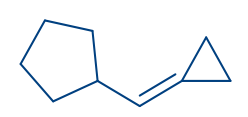

$185 \mathrm{~h}$

8

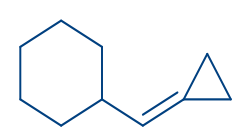

$185 i$<smiles>CC(C)C=CC1(c2cc3ccccc3n2P(O)c2ccccc2)CC1</smiles>

186af<smiles>[R6]n1c(C2(/C=C/N(C)C=C)CC2)cc2ccccc21</smiles>

186ag<smiles>[PH2+]n1c(C2(C=C3CCCC3)CC2)cc2ccccc21</smiles>

186ah<smiles>CC(=O)n1c(C2(C=C3CCCCC3)CC2)cc2ccccc21</smiles>

186ai
63

79

185j

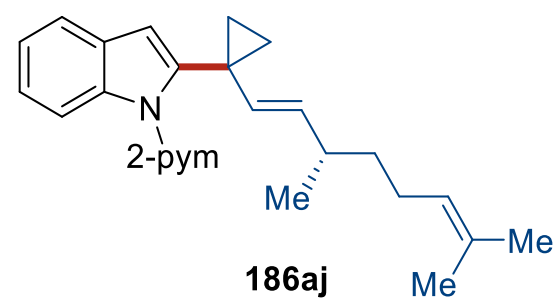

[a] Undivided cell, graphite felt anode (GF), platinum plate cathode (Pt), 71a $(0.20 \mathrm{mmol}) 185(0.32 \mathrm{mmol}$, 1.6 equiv), [ $\left.\mathrm{Cp}^{*} \mathrm{RhCl}_{2}\right]_{2}(2.5 \mathrm{~mol} \%), \mathrm{NaO}_{2} \mathrm{CAd}\left(40 \mathrm{~mol} \%\right.$ ), $\mathrm{CypCO}{ }_{2} \mathrm{H}(10 \mathrm{~mol} \%)$, solvent $(8.0 \mathrm{~mL}), 95{ }^{\circ} \mathrm{C}$, CCE @ $5.0 \mathrm{~mA}$, under air, yield of isolated product.

\subsubsection{Derivatization of the Diene 184aa}

Based on the scope evaluation of both $\mathrm{C}-\mathrm{H}$ dienylation and $\mathrm{C}-\mathrm{H}$ cyclopropylation, some transformations of product diene 184aa were conducted (Scheme 3.13). Thus, a DielsAlder reaction of dienylated product 184aa with alkyne 207 was tried for the construction of the 1,4-cyclohexadiene skeleton (Scheme 3.13a). Hydroquinone ${ }^{[169]}$ was confirmed to be a suitable catalyst to produce alkene $\mathbf{2 0 8}$ in good yield. With alkene $\mathbf{2 0 8}$ in hand, we extended the conjugation of the thus-formed product 208 through dehydrogenation of the 
diene moiety. Thus, the desired product 209 was obtained in good yield using catalytic amounts of $\mathrm{DDQ}$ as the redox mediator at room temperature with $\mathrm{CH}_{2} \mathrm{Cl}_{2}$ and $\mathrm{MeOH}$ as the solvent (Scheme 3.13b).

a) Die/s-Alder cyclization<smiles>[Z20]n1c(/C(C=C)=C/Pc2ccccc2)cc2ccccc21</smiles>

184aa

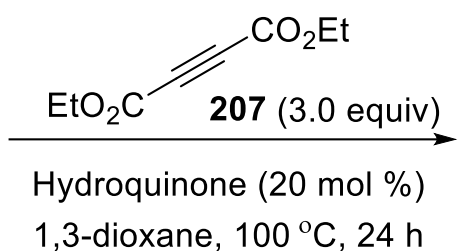

1,3-dioxane, $100{ }^{\circ} \mathrm{C}, 24 \mathrm{~h}$<smiles>[Z20]n1c(C2=CCC(C(=O)OCC)=C(C(=O)OCC)C2c2ccccc2)cc2ccccc21</smiles>

208, $72 \%$

b) dehydrogenative aromatization<smiles>[2H][PH]([O-])(c1ccccc1)n1c(C2=CCC(C(=O)OF)=C(C(=O)OCC)C2c2ccccc2)cc2ccccc21</smiles>

208

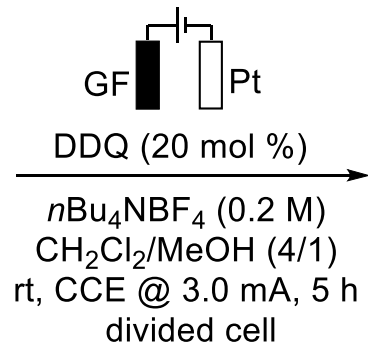

$209,65 \%$

Scheme 3.13. Derivatization of 1,3-diene 184aa.

\subsubsection{Mechanistic Investigation of Cyclopropylation and Dienylation}

Based on a previously reported mechanism, ${ }^{[170]}$ we propose a reasonable catalytic cycle for the $\mathrm{C}-\mathrm{H}$ cyclopropylation (Figure 3.1) and $\mathrm{C}-\mathrm{H}$ dienylation (Figure 3.2), respectively. The catalytic cycle is initiated by the formation of a catalytically competent mononuclear $\mathrm{Cp}{ }^{*} \mathrm{Rh}(\mathrm{III})$ species 210. As shown in Figure 3.1, coordination by the nitrogen of the pyrimidine indole $71 \mathrm{a}$ to $\mathrm{Cp}{ }^{*} \mathrm{Rh}(\mathrm{III})$ and the following $\mathrm{C}-\mathrm{H}$ activation at the 2-position affords rhodacycle $\mathbf{2 1 1}$. Then, insertion of alkene 185 a occurs to furnish intermediate $\mathbf{2 1 2}$, which undergoes $\beta$-H elimination to generate the cyclopropylated product 186aa along with a rhodium(I) complex. ${ }^{[125 a]}$ Finally the $\mathrm{Cp}^{*} \mathrm{Rh}(\mathrm{III})$ species 210 can be regenerated by reoxidation of rhodium $(\mathrm{I})$ at the anode, generating molecular hydrogen as the byproduct produced at the cathode. 


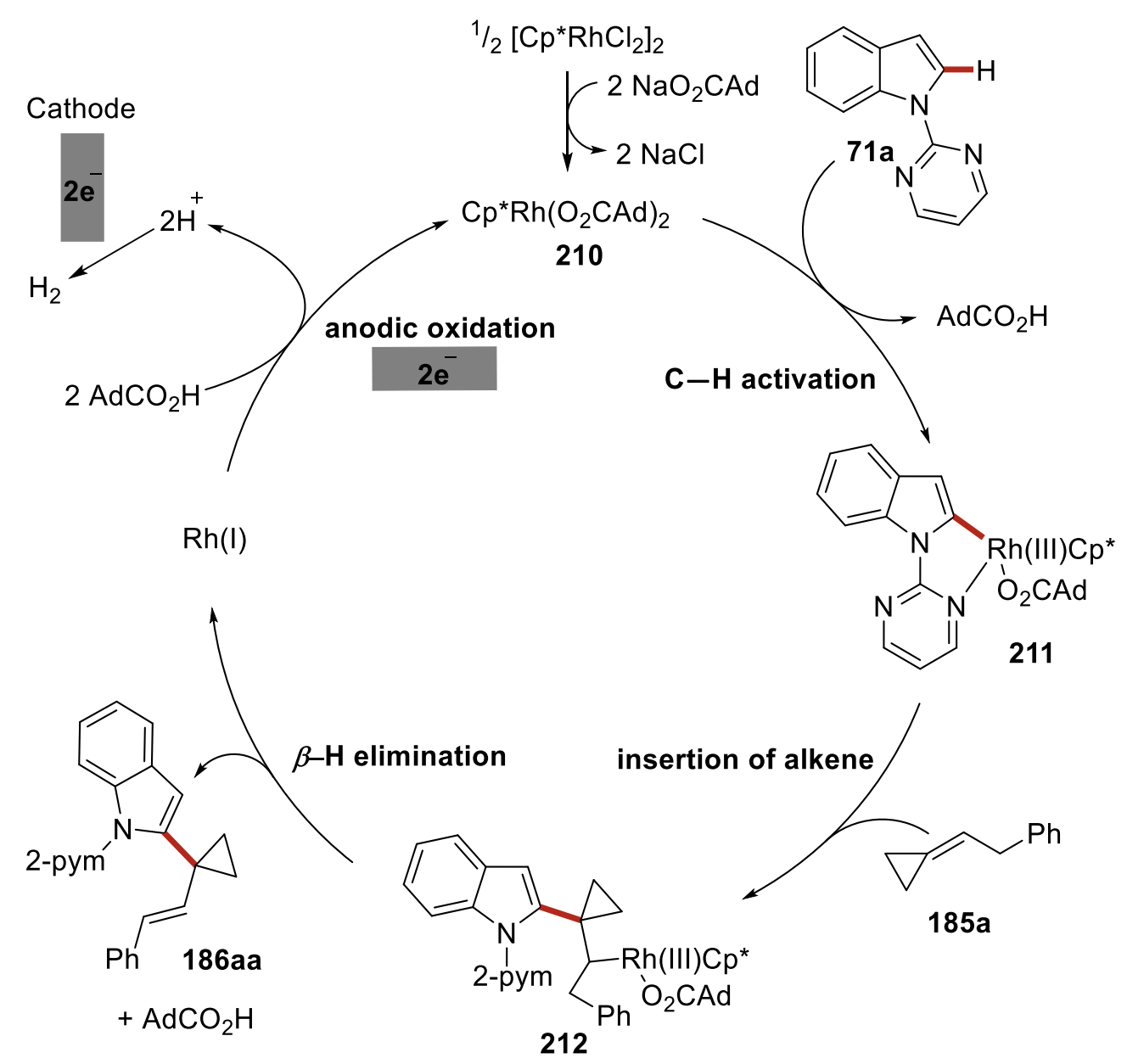

Figure 3.1. Proposed mechanism for rhodaelectro-C-H-cyclopropylation.

In terms of dienylation, the formed intermediate $\mathbf{2 1 3}$ after the insertion of alkene $138 \mathrm{a}$ to rhodacycle 211 can undergo $\beta$-C elimination to form intermediate 214 as there are no $\beta$-hydrogens in intermediate 213 (Figure 3.2). The resulted intermediate 214 undergoes $\beta$ $\mathrm{H}$ elimination and delivers the dienylated indole 184aa. 


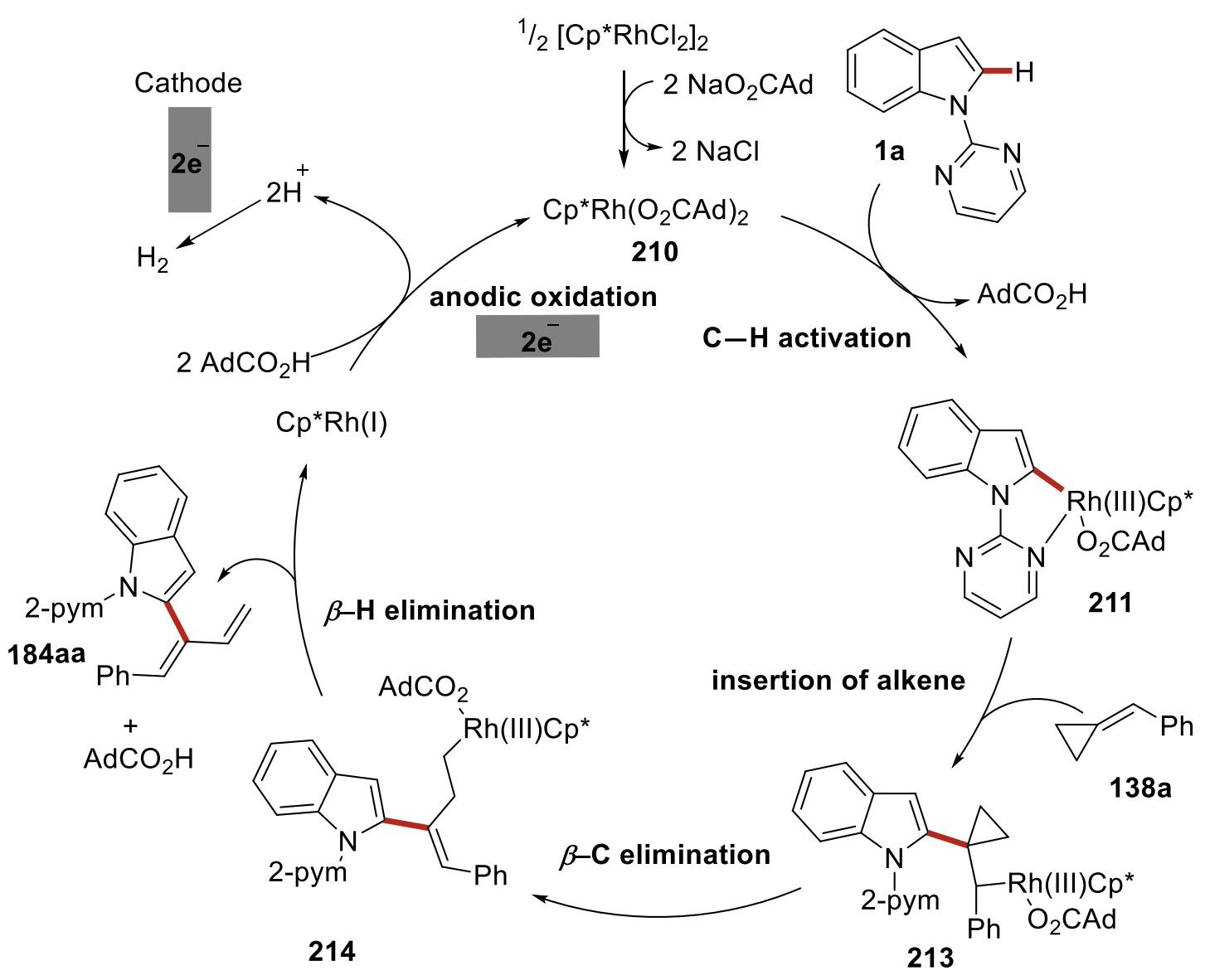

Figure 3.2. Proposed mechanism for rhodaelectro-C-H-dienylation. 


\section{Summary and Outlook}

The avoidance of time- and energy-consuming prefunctionalization which generate stoichiometric amounts of waste has made direct functionalization of $\mathrm{C}-\mathrm{H}$ bonds emerging as an environmentally benign alternative. While major progress was realized with noble transition metal catalysts, the employment of cost-effective and earth-abundant $3 \mathrm{~d}$ metals has gained significant momentum during the last decade. As stoichiometric amounts of oxidants are often needed for the $\mathrm{C}-\mathrm{H}$ activation, electricity has been identified as an inexpensive, economical, and environmental benign alternative for chemical redox equivalents. Thus, my projects have focused on both the earth-abundant $3 d$ metals and resource-economic electrochemical $\mathrm{C}-\mathrm{H}$ bond transformations.

In the first project, we achieved an expedient $\mathrm{C}-\mathrm{H}$ functionalization of electron-rich benzyl and aryl amines by sustainable iron-catalysis with the assistance of a new fully substituted triazole TST directing group enabling $\mathrm{C}-\mathrm{H}$ methylations, alkylations and arylations. This transformation featured racemization-free conditions and a newly developed reusable versatile TST group. Due to the magic contributions of methyl group to biological molecules, ${ }^{[149]}$ this method may help to accelerate the discovery of new drugs.

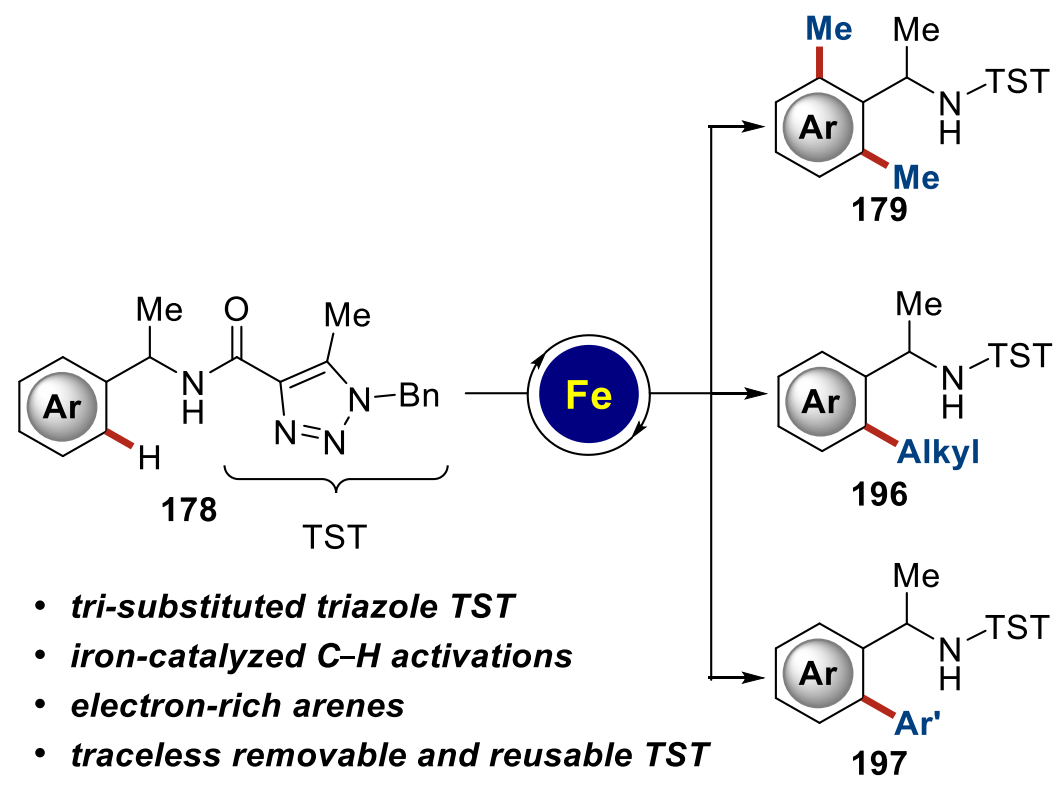

Scheme 4.1. Iron-catalyzed C-H functionalization by TST-assistance. 
With the significant progresses in $\mathrm{C}-\mathrm{H}$ activation, much attention has been paid to the use of weakly-coordinated directing groups and late-stage functionalization of biomolecules. In the second project, we developed a direct ortho-alkylation of pyridines by weak assistance of amides. A variety of alkylated pyridines were obtained via low-valent manganesecatalyzed alkylations. Mechanistic studies showed that SET-type C-X bond cleavage occurred during the $\mathrm{C}-\mathrm{H}$ functionalization.

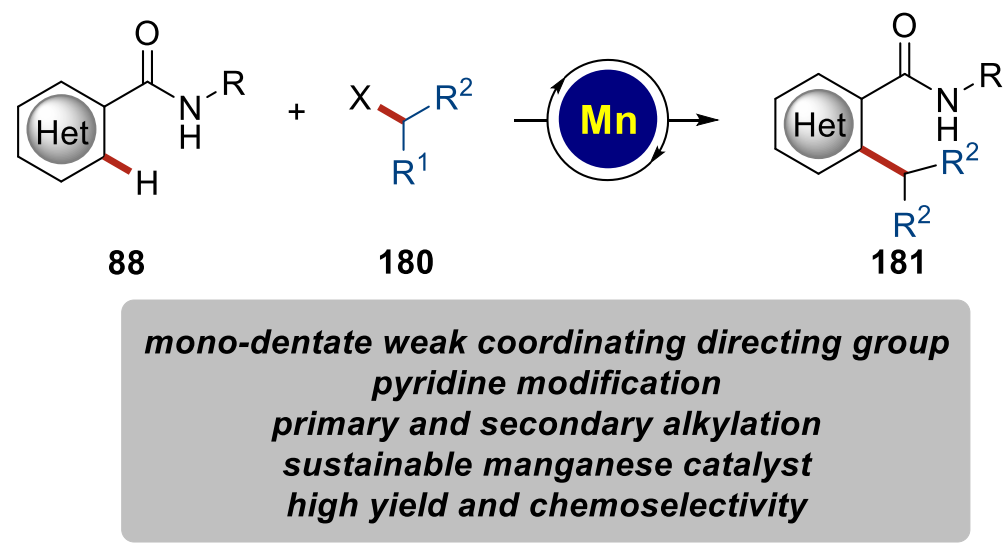

Scheme 4.2. Alkylation of pyridines catalyzed by manganese(II).

In the third project, a modular assembly of aza-PAHs enabled by rhodaelectro-catalyzed cascade $\mathrm{C}-\mathrm{H}$ functionalization was developed. A multifunctional and transformable $\mathrm{O}-$ methylamidoxime was designed to guarantee the reactivity and selectivity. The isolation of two $\mathrm{C}-\mathrm{H}$-activated rhodacycles offered the proof for sequence of the cascade $\mathrm{C}-\mathrm{H}$ activation. The electrosynthesis demonstrated broad substrate scope and excellent functional group tolerance, including iodo and azido groups. The practicality of this reaction was reflected by its mild conditions, most user-friendly setup, and easy scale-up. 


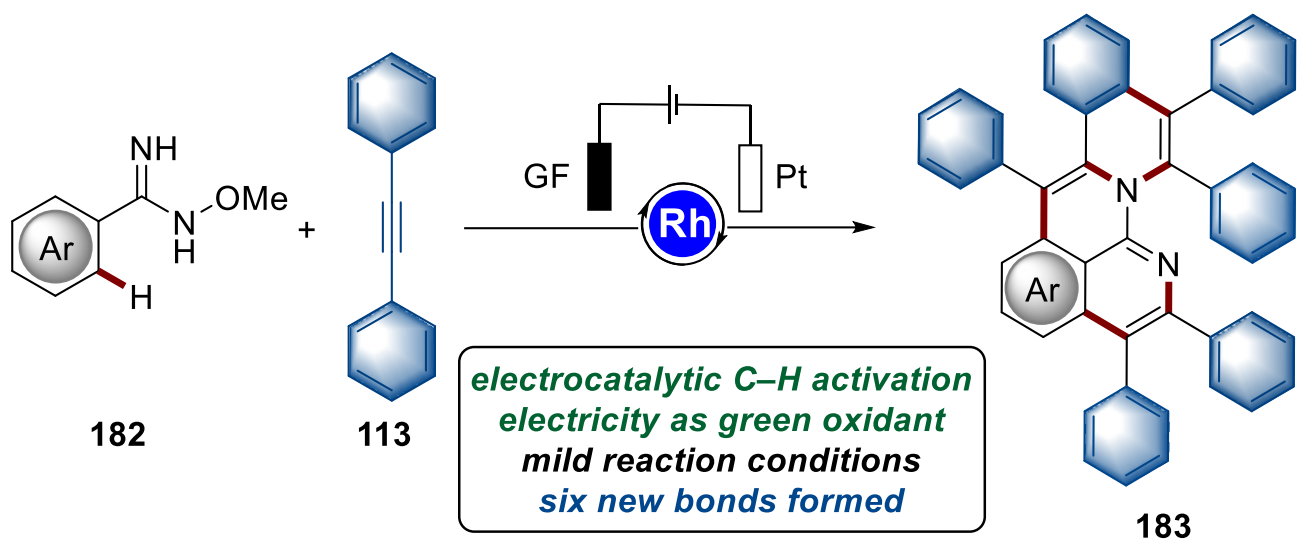

Scheme 4.3. Rhodaelectro-catalyzed Domino $\mathrm{C}-\mathrm{H}$ annulations for the assembly of azananographenes.

In the last project, we have developed a bifurcated $\mathrm{C}-\mathrm{H}$ cyclopropylation and dienylation of indoles by rhodaelectro-catalysis under aqueous conditions, avoiding the use of stoichiometric amounts of chemical oxidants. The reactive catalyst can be regenerated by anodic oxidation, producing hydrogen as the sole byproduct. Thereby, a wealth of indoles can be directly dienylated and cyclopropylated with good chemo-, position- and regioselectivities. Notably, electron-deficient compounds also performed well in this reaction. This method can serve as a new strategy for developing drugs containing cyclopropane motifs.

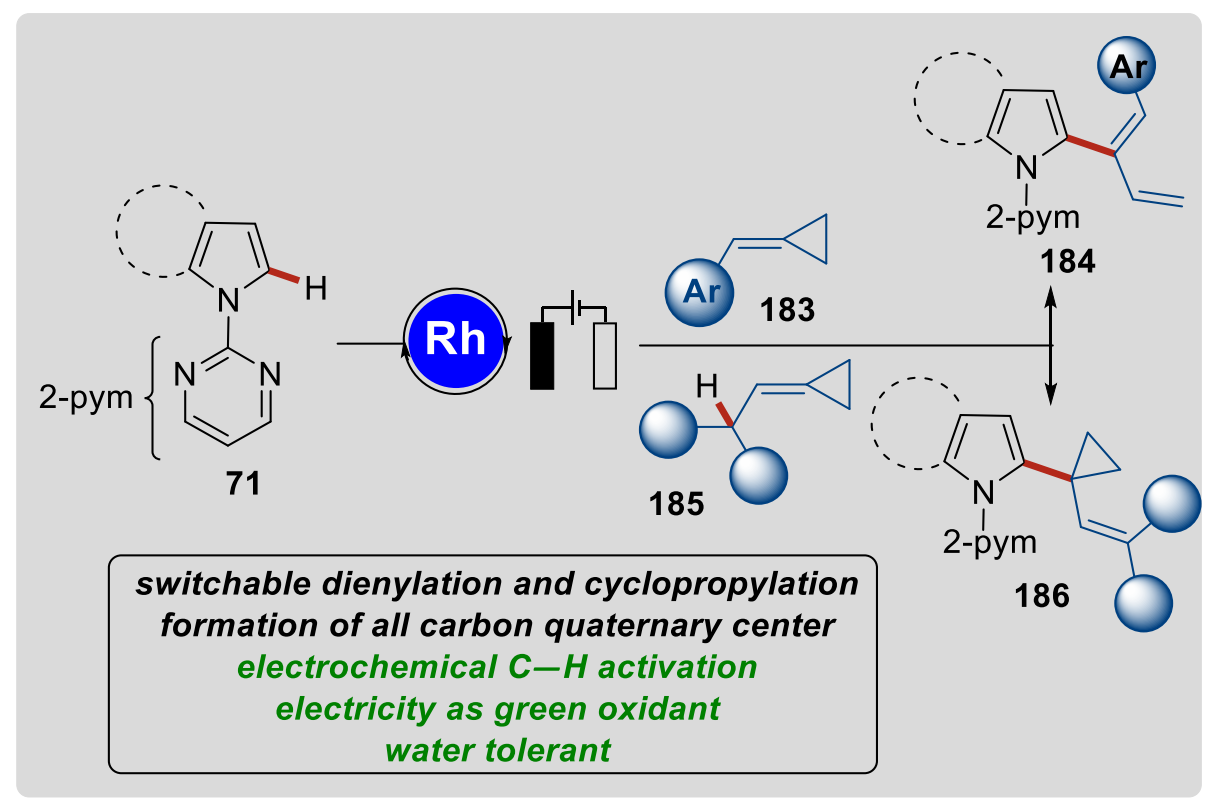

Scheme 4.4. Rhodaelectro-catalyzed bifurcated indole $\mathrm{C}-\mathrm{H}$ activation with MCPS. 


\section{Experimental Section}

\subsection{General Remarks}

All reactions involving reagents or products sensitive to moisture or air were performed under an atmosphere of nitrogen using standard Schlenk techniques and pre-dried glassware. If not mentioned otherwise, yields refer to isolated compounds, estimated to be $>95 \%$ pure as determined by ${ }^{1} \mathrm{H}$ NMR. In case NMR yields are reported, they were determined by using an internal standard.

\section{Vacuum}

The used Schlenk line was connected to a Vacuubrand® rotary vane pump. The following pressure was measured at the outlet of the used Schlenk line: 0.1 mbar. The values are not corrected.

\section{Melting points}

Melting points were measured by using a Stuart Melting Point Apparatus SMP3 from BarloworldScientific. The given values are not corrected.

\section{Chromatography}

Thin layer chromatography (TLC) was performed on Merck, silica gel 60 F254 aluminum TLC plates. Visualization of the substances was achieved by exposure to UV light at 254 $\mathrm{nm}$ or by treatment of the plates with a chemical staining agent. Purification by column chromatography refers to flash column chromatography using Merck silica gel, grade 60 (40-63 $\mu \mathrm{m}, 230-400$ mesh ASTM) and pre-distilled solvents.

\section{Gas Chromatography}

Sample analysis and reaction monitoring was performed via coupled gas chromatography mass spectrometry on a 7890B GC System from Agilent Technologies coupled with either a 5977A or a 5977B mass detector from Agilent Technologies. 


\section{Nuclear Magnetic Resonance Spectroscopy (NMR)}

NMR spectra were recorded on Varian VX 300, Bruker Avance 300, Bruker Avance 400 and 500 or Varian Inova 500 and 600 spectrometers in the solvent indicated. Chemical shifts are reported as $\delta$ values in ppm. ${ }^{1} \mathrm{H}$ - and ${ }^{13} \mathrm{C}-\mathrm{NMR}$ spectra were referenced using the residual solvent signal. The coupling constants $J$ are reported in $\mathrm{Hertz}(\mathrm{Hz})$.

\begin{tabular}{l|cc} 
NMR & ${ }^{1} \mathrm{H}$ NMR & ${ }^{13} \mathrm{C} \mathrm{NMR}$ \\
\hline $\mathrm{CDCl} 3$ & 7.26 & 77.16
\end{tabular}

Abbreviations for the characterization of the signals are: $s$ (singlet), $d$ (doublet), $t$ (triplet), $\mathrm{q}$ (quartet), dd (doublet of doublets), m (multiplet) and sbr (broad singlet) or analogous representations. For all spectra, a baseline and phase correction were performed. Mnova 10 from Mestrelab Research was used for the processing and analysis of the spectra.

\section{Mass Spectrometry}

Electrospray ionization (ESI) and high-resolution mass spectra (HR-MS) spectra were recorded on either a time-of-flight mass spectrometer micrOTOF (ESI-TOF-MS) or a quadrupole time-of-flight maXis (ESI-QTOF-MS) spectrometer, both from Bruker Daltonic.

\section{Infrared Spectroscopy}

Infrared (IR) spectra were recorded on a Bruker Alpha-P ATR spectrometer. Liquid samples were measured as a film and solid samples were measured neat. The spectra were recorded in the range from 4000 to $400 \mathrm{~cm}^{-1} 1$. The analysis was performed using Opus 6 from Bruker. Reported are the most significant peaks and their respective wave numbers $\left(\mathrm{cm}^{-1}\right)$.

\section{Electrochemistry}

Pt electrodes $\left(10 \mathrm{~mm} \times 15 \mathrm{~mm} \times 0.25 \mathrm{~mm}, 99.9 \%\right.$; obtained from ChemPur ${ }^{\circledR} \mathrm{Karlsruhe}$, Germany) and RVC electrodes (10 mm×15 mm×6 mm, SIGRACELL ${ }^{\circledR}$ GFA 6 EA, obtained from SGL Carbon, Wiesbaden, Germany) were connecteC using stainless steel adapters. Electrolysis was conducteC using an AXIOMET AX-3003P potentiostat in constant current 
mode. Divided cells separated by a P4-glassfrit were obtained from Glasgerätebau Ochs Laborfachhandel e. K. (Bovenden, Germany).

\section{Solvents}

Solvents used for column chromatography were purified by distillation under reduced pressure prior to use. Solvents purified by a solvent purification system (SPS-800) from M. Braun: dichloromethane, toluene, diethyl ether, tetrahydrofuran, and dimethylformamide. Solvents dried and distilled over sodium with benzophenone as an indicator: toluene, xylenes, mesitylene, 1,4-dioxane, di-n-butyl ether, and dimethoxyethane. Solvents dried and distilled over $\mathrm{CaH}_{2}$ : 1,2-dichloroethane, dimethylacetamide, dimethylformamide, dimethylsulfoxide. Solvents dried over molecular sieves and degassed by freeze-pumpthaw cycles: acetonitrile ( $3 \AA$ ). Water was degassed by sparging with $\mathrm{N}_{2}$ and simultaneous sonification for $2 \mathrm{~h}$.

\section{Reagents}

Chemicals obtained from commercial suppliers (with a purity $>95 \%$ ) were used without further purification. The following compounds have been previously reported and were synthesized according to previously described literature protocols:

TST amide $178,{ }^{[67]} N$-(1-phenylethyl)picolinamide $\mathbf{1 7 8 f},{ }^{[171]}$ heterocycles azine $\mathbf{8 8},{ }^{[172]}$ imidamides 182, ${ }^{[147]}$ alkynes $113,{ }^{[173]}\left[\mathrm{Cp}^{*} \mathrm{Rh}\left(\mathrm{CH}_{3} \mathrm{CN}\right)_{3}\right]\left(\mathrm{SbF}_{6}\right)_{2},{ }^{[174]} \mathrm{N}$-Pyrimidyl indoles $\mathbf{7 1 ,}{ }^{[175]}$ methylenecyclopropanes 138 and $185 . .^{[176]}$

The following chemicals were kindly supplied by the following coworkers:

Karsten Rauch: $\left[\mathrm{Cp}^{*} \mathrm{RhCl}_{2}\right]_{2}$.

Dr. Cuiju Zhu: amides 88. 


\subsection{General Procedures}

\subsubsection{General Procedure A: Iron-catalyzed C-H Methylation}

To a schlenk tube charged with amide $178(0.20 \mathrm{mmol}), \mathrm{TMEDA} \cdot \mathrm{ZnCl}_{2}(152 \mathrm{mg}, 0.60 \mathrm{mmol}$, 3.0 equiv) and $1.0 \mathrm{~mL}$ THF was added $\mathrm{MeMgBr}(457 \mu \mathrm{L}, 1.4 \mathrm{mmol}, 3.0 \mathrm{M}$ in ether, 7.0 equiv) in one portion at room temperature. Five minutes later, dppen (11.9 mg, $0.03 \mathrm{mmol}$, $15 \mathrm{mmol} \%)$ and $\mathrm{FeCl}_{3}(4.9 \mathrm{mg}, 0.03 \mathrm{mmol}, 15 \mathrm{mmol} \%)$ were added successively. Stirring at room temperature for another five minutes, 2,3-dichloridebutane was added and the reaction was moved to $65^{\circ} \mathrm{C}$ oil bath stirring overnight. Then quenched with sat. aqueous $\mathrm{NH}_{4} \mathrm{Cl}$, diluted with $10 \mathrm{~mL}$ water. The aqueous layer was extracted with EtOAc. The combined organic extracts were washed with brine and dried over $\mathrm{Na}_{2} \mathrm{SO}_{4}$. The filtrate was concentrated under reduced pressure and the crude product was purified by column chromatography on silica gel.

\subsubsection{General Procedure B: Iron-catalyzed C-H Arylation}

To a schlenk tube charged with amide $178(0.20 \mathrm{mmol}), \mathrm{TMEDA} \cdot \mathrm{ZnCl}_{2}(152 \mathrm{mg}, 0.60 \mathrm{mmol}$, 3.0 equiv) and $1.0 \mathrm{~mL}$ THF was added $\operatorname{ArMgBr}(457 \mu \mathrm{L}, 1.0 \mathrm{mmol}, 2.0 \mathrm{M}$ in ether, 5.0 equiv) in one portion at room temperature. Five minutes later, dppen (11.9 mg, $0.03 \mathrm{mmol}, 15$ mmol \%) and $\mathrm{FeCl}_{3}(4.9 \mathrm{mg}, 0.03 \mathrm{mmol}, 15 \mathrm{mmol} \%)$ were added successively. Stirring at room temperature for another five minutes, 2,3-dichloridebutane was added and the reaction was moved to $65^{\circ} \mathrm{C}$ oil bathe stirring overnight. Then quenched with sat. aqueous $\mathrm{NH}_{4} \mathrm{Cl}$, diluted with $10 \mathrm{~mL}$ water. The aqueous layer was extracted with EtOAc. The combined organic extracts were washed with brine and dried over $\mathrm{Na}_{2} \mathrm{SO}_{4}$. The filtrate was concentrated under reduced pressure and the crude product was purified by column chromatography on silica gel. 


\subsubsection{General Procedure C: Manganese-catalyzed Secondary Alkylation}

To a Schlenk tube charged with amide $88(0.20 \mathrm{mmol})$, TMEDA (30 $\mu \mathrm{L}, 0.20 \mathrm{mmol}) \mathrm{MnCl}_{2}$ (2.7 mg, $0.02 \mathrm{mmol})$ and THF (0.60 mL), EtMgBr (0.25 mL, $0.70 \mathrm{mmol}, 2.8 \mathrm{M}$ in THF) was added dropwise at ambient temperature under $\mathrm{N}_{2}$ atmosphere. After stirring for $5 \mathrm{~min}$, alkylchloride $2(0.60 \mathrm{mmol})$ was added and the reaction mixture stirred at $60^{\circ} \mathrm{C}$ for $14 \mathrm{~h}$. Then, saturated aqueous $\mathrm{NH}_{4} \mathrm{Cl}(5.0 \mathrm{~mL})$ and $\mathrm{H}_{2} \mathrm{O}(10 \mathrm{~mL})$ were subsequently added to the reaction mixture. The aqueous layer was extracted with EtOAc $(3 \times 15 \mathrm{~mL})$. The combined organic extracts were washed with brine and dried over $\mathrm{Na}_{2} \mathrm{SO}_{4}$. The filtrate was concentrated under reduced pressure and the crude product was purified by column chromatography on silica gel.

\subsubsection{General Procedure D: Manganese-catalyzed Primary Alkylation}

To a Schlenk tube charged with amide $88(0.20 \mathrm{mmol})$, TMEDA (60 $\mu \mathrm{L}, 0.40 \mathrm{mmol}) \mathrm{MnCl}_{2}$ (5.4 mg, $0.04 \mathrm{mmol})$ and THF (0.20 mL), t-BuCH $2 \mathrm{MgBr}(0.47 \mathrm{~mL}, 0.7 \mathrm{mmol}, 1.5 \mathrm{M}$ in THF) was added dropwise at ambient temperature under $\mathrm{N}_{2}$ atmosphere. After stirring for $5 \mathrm{~min}$, alkylchlorides $180(0.60 \mathrm{mmol})$ was added and the reaction mixture stirred at $60^{\circ} \mathrm{C}$ for 14 h. Then, saturated aqueous $\mathrm{NH}_{4} \mathrm{Cl}(5.0 \mathrm{~mL})$ and $\mathrm{H}_{2} \mathrm{O}(10 \mathrm{~mL})$ were subsequently added to the reaction mixture. The aqueous layer was extracted with EtOAc $(3 \times 15 \mathrm{~mL})$. The combined organic extracts were washed with brine and dried over $\mathrm{Na}_{2} \mathrm{SO}_{4}$. The filtrate was concentrated under reduced pressure and the crude product was purified by column chromatography on silica gel.

\subsubsection{General Procedure E: Rhodaelectro-catalyzed Domino alkyne annulation}

The electrocatalysis was carried out in an undivided cell with a graphite felt anode $(10 \mathrm{~mm}$ $\times 15 \mathrm{~mm} \times 6 \mathrm{~mm})$ and a Pt cathode $(10 \mathrm{~mm} \times 15 \mathrm{~mm} \times 0.25 \mathrm{~mm})$. The cell was charged with imidamide $182(0.20 \mathrm{mmol})$, alkyne $113(0.70 \mathrm{mmol}), \mathrm{KOAc}(74.4 \mathrm{mg}, 0.40 \mathrm{mmol}), 1-$ Adamantanecarboxylic acid $\left(\mathrm{AdCO}_{2} \mathrm{H}, 3.6 \mathrm{mg}, 10 \mathrm{~mol} \%\right),\left[\mathrm{Cp}^{*} \mathrm{Rh}\left(\mathrm{CH}_{3} \mathrm{CN}\right)_{3}\right]\left(\mathrm{SbF}_{6}\right)_{2}$ 
(8.4 mg, $5.0 \mathrm{~mol} \%)$ and $\mathrm{MeOH}(4.0 \mathrm{~mL})$. Electrocatalysis was performed at $35^{\circ} \mathrm{C}$ with a constant current of $2.0 \mathrm{~mA}$ maintained for $12 \mathrm{~h}$. The graphite felt anode was washed with $\mathrm{CH}_{2} \mathrm{Cl}_{2}(8 \times 5 \mathrm{~mL})$ in an ultrasonic bath. Evaporation of the solvents and subsequent column chromatography on silica gel afforded the corresponding product 183.

\subsubsection{General Procedure F: Rhodaelectro-Catalyzed Dienylation}

The electrolysis was carried out in an undivided cell with a GF $(10 \mathrm{~mm} \times 15 \mathrm{~mm} \times 6 \mathrm{~mm})$ and a Pt cathode $(10 \mathrm{~mm} \times 15 \mathrm{~mm} \times 0.25 \mathrm{~mm}) .\left[\mathrm{Cp}^{*} \mathrm{RhCl}_{2}\right]_{2}(3.0 \mathrm{mg}, 0.005 \mathrm{mmol}, 2.5$ mol \%), $\mathrm{NaO}_{2} \mathrm{CAd}(16.0 \mathrm{mg}, 0.80 \mathrm{mmol}, 40 \mathrm{~mol} \%$ ), cyclopentanecarboxylic acid (2.3 mg, $0.02 \mathrm{mmol}, 10 \mathrm{~mol} \%)$, cyclopropane 138 (0.32 mmol, 1.6 equiv) and indole 71 (0.20 mmol) were dissolved in 1,4-dioxane $(4.0 \mathrm{~mL})$ and then $\mathrm{H}_{2} \mathrm{O}(4.0 \mathrm{~mL})$ was added sequentially. At $95{ }^{\circ} \mathrm{C}$, electrolysis was conducted with a constant current of $5.0 \mathrm{~mA}$ for 3 to 10 hours. Then the mixture was transferred to a flask and the electrodes were rinsed with EtOAc $(3 \times 5.0$ $\mathrm{mL})$. The combined solvent was extracted with EtOAc $(3 \times 10 \mathrm{~mL})$ for three times, combined the organic layer, removed the solvent under reduced pressure and subsequent column chromatography on silica gel ( $n$-hexane/EtOAc) yielded the desired product 184 .

\subsubsection{General Procedure G: Rhodaelectro-Catalyzed Cyclopropylation}

The electrolysis was carried out in an undivided cell with a GF $(10 \mathrm{~mm} \times 15 \mathrm{~mm} \times 6 \mathrm{~mm})$ and a platinum cathode $(10 \mathrm{~mm} \times 15 \mathrm{~mm} \times 0.25 \mathrm{~mm}) .\left[\mathrm{Cp}^{*} \mathrm{RhCl}_{2}\right]_{2}(3.0 \mathrm{mg}, 0.005 \mathrm{mmol}$, $2.5 \mathrm{~mol} \%$ ), $\mathrm{NaO}_{2} \mathrm{CAd}(16.0 \mathrm{mg}, 0.80 \mathrm{mmol}, 40 \mathrm{~mol} \%$ ), cyclopentanecarboxylic acid (2.3 $\mathrm{mg}, 0.20 \mathrm{mmol}, 10 \mathrm{~mol} \%$ ), cyclopropane 185 (0.32 mmol, 1.6 equiv) and indole 71 (0.20 $\mathrm{mmol})$ were dissolved in 1,4-dioxane $(4.0 \mathrm{~mL})$ and then water $(4.0 \mathrm{~mL})$ was added sequentially. At $95{ }^{\circ} \mathrm{C}$, electrolysis was conducted with a constant current of $4.0 \mathrm{~mA}$ for 3 to 10 hours. Then the mixture was transferred to a flask and the electrodes were rinsed with EtOAc $(3 \times 5.0 \mathrm{~mL})$. The combined solvent was extracted with EtOAc $(3 \times 10 \mathrm{~mL})$ for three times, combined the organic layer, removed the solvent under reduced pressure and subsequent column chromatography on silica gel ( $n$-hexane/EtOAc) yielded the desired product 186. 


\subsection{Iron Catalyzed C-H Activation}

\subsubsection{Experimental Procedures and Analytical Data}<smiles>Cc1cccc(C)c1C(C)NC(=O)c1nnn(Cc2ccccc2)c1C</smiles>

1-Benzyl-N-[1-(2,6-dimethylphenyl)ethyl]-5-methyl-1H-1,2,3-triazole-4-carboxamide (179a)

Prepared following the general procedure $A$ by using $178 \mathrm{a}(64.0 \mathrm{mg}, 0.20 \mathrm{mmol})$. Purification by column chromatography ( $n$-hexane/EtOAc 2:1) yielded 179a (58.9 mg, 86\%) as a white solid. M.p. $=72-73^{\circ} \mathrm{C} .{ }^{1} \mathrm{H}$ NMR $\left(300 \mathrm{MHz}, \mathrm{CDCl}_{3}\right) \delta=7.76(\mathrm{~d}, J=7.7 \mathrm{~Hz}, 1 \mathrm{H})$, 7.37-7.30 (m, 3H), 7.15-7.12 (m, 2H), 7.06-6.97 (m, 3H), $5.70(\mathrm{q}, J=7.3 \mathrm{~Hz}, 1 \mathrm{H}), 5.49(\mathrm{~s}$, 2H), $2.54(\mathrm{~s}, 6 \mathrm{H}), 2.46(\mathrm{~s}, 3 \mathrm{H}), 1.61(\mathrm{~d}, J=7.3 \mathrm{~Hz}, 3 \mathrm{H}) .{ }^{13} \mathrm{C} \mathrm{NMR}\left(126 \mathrm{MHz}, \mathrm{CDCl}_{3}\right) \delta=$ $160.2\left(\mathrm{C}_{\mathrm{q}}\right), 139.0\left(\mathrm{C}_{\mathrm{q}}\right), 138.7\left(\mathrm{C}_{\mathrm{q}}\right), 135.9\left(\mathrm{C}_{\mathrm{q}}\right), 135.3\left(\mathrm{C}_{\mathrm{q}}\right), 134.0\left(\mathrm{C}_{\mathrm{q}}\right), 129.4(\mathrm{CH}), 129.0$ $(\mathrm{CH}), 128.4(\mathrm{CH}), 127.0(\mathrm{CH}), 126.7(\mathrm{CH}), 51.8\left(\mathrm{CH}_{2}\right), 45.2(\mathrm{CH}), 21.1\left(\mathrm{CH}_{3}\right), 19.8\left(\mathrm{CH}_{3}\right)$, $8.8\left(\mathrm{CH}_{3}\right)$. IR (ATR): 3359, 2963, 1650, 1505, 1073, 746, $402 \mathrm{~cm}^{-1} . \mathbf{M S}$ (ESI) $\mathrm{m} / z$ (relative intensity) 371 (100) [M+Na] $, 349(30)[\mathrm{M}+\mathrm{H}]^{+}, 217$ (10), 133 (5). HR-MS (ESI) m/z calcd for $\mathrm{C}_{21} \mathrm{H}_{25} \mathrm{~N}_{4} \mathrm{O}[\mathrm{M}+\mathrm{H}]^{+} 349.2028$ found 349.2021.<smiles>Cc1cccc(C)c1C(C)(C)NC(=O)c1nnn(Cc2ccccc2)c1C</smiles>

1-Benzyl-N-[2-(2,6-dimethylphenyl)propan-2-yl]-5-methyl-1H-1,2,3-triazole-4carboxamide (179b) 
Prepared following the general procedure A by using $178 \mathrm{~b}(66.4 \mathrm{mg}, 0.20 \mathrm{mmol})$. Purification by column chromatography ( $n$-hexane/EtOAc $2: 1$ ) yielded $179 \mathrm{~b}$ (65.3 mg, 89\%) as a colorless liquid. ${ }^{1} \mathrm{H}$ NMR $\left(300 \mathrm{MHz}, \mathrm{CDCl}_{3}\right) \delta=7.61$ (brs, $\left.1 \mathrm{H}\right), 7.38-7.31(\mathrm{~m}, 3 \mathrm{H})$, 7.17-7.14 (m, 2H), 6.99-6.97 (m, 3H), 5.49 (s, 2H), 2.52 (s, 6H), $2.43(\mathrm{~s}, 3 \mathrm{H}), 1.97$ (s, 6H). ${ }^{13} \mathrm{C}$ NMR $\left(75 \mathrm{MHz}, \mathrm{CDCl}_{3}\right) \delta=159.3\left(\mathrm{C}_{\mathrm{q}}\right), 143.0\left(\mathrm{C}_{\mathrm{q}}\right), 139.2\left(\mathrm{C}_{\mathrm{q}}\right), 136.0\left(\mathrm{C}_{\mathrm{q}}\right), 135.4\left(\mathrm{C}_{\mathrm{q}}\right)$, $134.1\left(\mathrm{C}_{\mathrm{q}}\right), 131.8(\mathrm{CH}), 129.1(\mathrm{CH}), 128.5(\mathrm{CH}), 127.2(\mathrm{CH}), 126.2(\mathrm{CH}), 58.7\left(\mathrm{C}_{\mathrm{q}}\right), 51.8$ $\left(\mathrm{CH}_{2}\right), 30.5\left(\mathrm{CH}_{3}\right), 25.6\left(\mathrm{CH}_{3}\right), 8.8\left(\mathrm{CH}_{3}\right)$. IR (ATR): 3342, 2983, 1657, 1503, 1257, 604, $573 \mathrm{~cm}^{-1}$. MS (ESI) m/z (relative intensity) $385(100)[\mathrm{M}+\mathrm{Na}]^{+}, 363(80)[\mathrm{M}+\mathrm{H}]^{+}, 257(50)$, 217 (55), 200 (25). HR-MS (ESI) m/z calcd for $\mathrm{C}_{22} \mathrm{H}_{27} \mathrm{~N}_{4} \mathrm{O}[\mathrm{M}+\mathrm{H}]^{+} 363.2185$ found 363.2180.<smiles></smiles>

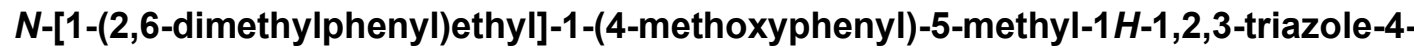
carboxamide (179c)

Prepared following the general procedure $A$ by using $178 \mathrm{c}(67.2 \mathrm{mg}, 0.20 \mathrm{mmol})$. Purification by column chromatography ( $n$-hexane/EtOAc 2:1) yielded 179c (65.3 mg, 89\%) as a yellow solid. M.p. $=104-106^{\circ} \mathrm{C} .{ }^{1} \mathrm{H}$ NMR $\left(300 \mathrm{MHz}, \mathrm{CDCl}_{3}\right) \delta=7.81(\mathrm{~d}, \mathrm{~J}=7.7 \mathrm{~Hz}$, 1H), 7.36-7.31 (m, 2H), 7.07-6.98 (m, 5H), 5.73 (q, J = 7.3 Hz, 1H), $3.88(\mathrm{~s}, 3 \mathrm{H}), 2.56$ (s, 6H), $2.55(\mathrm{~s}, 3 \mathrm{H}), 1.64(\mathrm{~d}, J=7.3 \mathrm{~Hz}, 3 \mathrm{H}) \cdot{ }^{13} \mathrm{C}$ NMR $\left(126 \mathrm{MHz}, \mathrm{CDCl}_{3}\right) \delta=160.45\left(\mathrm{C}_{\mathrm{q}}\right)$, $160.24\left(\mathrm{C}_{\mathrm{q}}\right), 139.00\left(\mathrm{C}_{\mathrm{q}}\right), 138.20\left(\mathrm{C}_{\mathrm{q}}\right), 136.57\left(\mathrm{C}_{\mathrm{q}}\right), 135.31\left(\mathrm{C}_{\mathrm{q}}\right), 129.4(\mathrm{CH}), 128.4\left(\mathrm{C}_{\mathrm{q}}\right)$, $126.7(\mathrm{CH}), 126.5(\mathrm{CH}), 114.6(\mathrm{CH}), 55.6\left(\mathrm{CH}_{3}\right), 45.3(\mathrm{CH}), 21.1\left(\mathrm{CH}_{3}\right), 19.8\left(\mathrm{CH}_{3}\right), 9.7$ $\left(\mathrm{CH}_{3}\right)$. IR (ATR): 3417, 2974, 1664, 1509, 1254, 766, $589 \mathrm{~cm}^{-1} . \mathbf{M S}$ (ESI) $\mathrm{m} / \mathrm{z}$ (relative intensity) 387 (65) [M+Na]+, 365 (100) [M+H], 233 (70), 188 (2), 133 (65). HR-MS (ESI) $m / z$ calcd for $\mathrm{C}_{21} \mathrm{H}_{25} \mathrm{~N}_{4} \mathrm{O}_{2}[\mathrm{M}+\mathrm{H}]^{+} 365.1978$ found 365.1972 . 


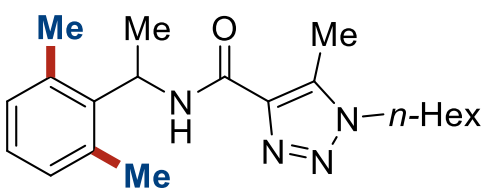

$N$-[1-(2,6-dimethylphenyl)ethyl]-1-hexyl-5-methyl-1H-1,2,3-triazole-4-carboxamide (179d)

Prepared following the general procedure A by using $178 \mathrm{~d}(62.9 \mathrm{mg}, 0.20 \mathrm{mmol})$. Purification by column chromatography ( $n$-hexane/EtOAc $2: 1$ ) yielded $179 \mathrm{~d}(48.1 \mathrm{mg}, 70 \%)$ as a yellow liquid. ${ }^{1} \mathrm{H}$ NMR $\left(300 \mathrm{MHz}, \mathrm{CDCl}_{3}\right) \delta=7.74(\mathrm{~d}, J=7.3 \mathrm{~Hz}, 1 \mathrm{H}), 7.05-6.96(\mathrm{~m}$, 3H), $5.70(\mathrm{p}, J=7.3 \mathrm{~Hz}, 1 \mathrm{H}), 4.23(\mathrm{t}, J=7.2 \mathrm{~Hz}, 2 \mathrm{H}), 2.56(\mathrm{~s}, 3 \mathrm{H}), 2.54(\mathrm{~s}, 6 \mathrm{H}), 1.91-1.78$ $(\mathrm{m}, 2 \mathrm{H}), 1.60(\mathrm{~d}, J=7.3 \mathrm{~Hz}, 3 \mathrm{H}), 1.34-1.26(\mathrm{~m}, 6 \mathrm{H}), 0.88(\mathrm{t}, J=6.9 \mathrm{~Hz}, 3 \mathrm{H}) .{ }^{13} \mathrm{C}$ NMR $(126$ $\left.\mathrm{MHz}, \mathrm{CDCl}_{3}\right) \delta=160.3\left(\mathrm{C}_{\mathrm{q}}\right), 139.0\left(\mathrm{C}_{\mathrm{q}}\right), 138.1\left(\mathrm{C}_{\mathrm{q}}\right), 135.3\left(\mathrm{C}_{\mathrm{q}}\right), 129.4(\mathrm{CH}), 126.7(\mathrm{CH})$, $47.8\left(\mathrm{CH}_{2}\right), 45.2(\mathrm{CH}), 31.2\left(\mathrm{CH}_{2}\right), 29.7\left(\mathrm{CH}_{2}\right), 26.1\left(\mathrm{CH}_{2}\right), 22.4\left(\mathrm{CH}_{2}\right), 21.0\left(\mathrm{CH}_{3}\right), 19.7$ $\left(\mathrm{CH}_{3}\right), 13.9\left(\mathrm{CH}_{3}\right), 8.7\left(\mathrm{CH}_{3}\right)$. IR (ATR): 3425, 2927, 1666, 1503, 1209, 772, $562 \mathrm{~cm}^{-1}$. MS (ESI) $\mathrm{m} / \mathrm{z}$ (relative intensity) $365(100)[\mathrm{M}+\mathrm{Na}]^{+}, 343(60)[\mathrm{M}+\mathrm{H}]^{+}, 211(30), 133$ (20). HRMS (ESI) $m / z$ calcd for $\mathrm{C}_{20} \mathrm{H}_{31} \mathrm{~N}_{4} \mathrm{O}[\mathrm{M}+\mathrm{H}]^{+} 343.2498$ found 343.2492.<smiles>Cc1cccc(C)c1C(C)NC(=O)c1ccccn1</smiles>

\section{N-(1-(2,6-Dimethylphenyl)ethyl)picolinamide (179f)}

Prepared following the general procedure A by using $178 \mathrm{f}(62.9 \mathrm{mg}, 0.20 \mathrm{mmol})$. Purification by column chromatography ( $n$-hexane/EtOAc 3:1) yielded $179 f(26.7 \mathrm{mg}, 51 \%$ ) as a colorless liquid. ${ }^{1} \mathrm{H}$ NMR $\left(300 \mathrm{MHz}, \mathrm{CDCl}_{3}\right) \delta=8.57(\mathrm{~d}, J=6.4 \mathrm{~Hz}, 1 \mathrm{H}), 8.48$ (ddd, $J$ $=4.8,1.7,0.9 \mathrm{~Hz}, 1 \mathrm{H}), 8.08(\mathrm{td}, J=7.7,0.9 \mathrm{~Hz}, 1 \mathrm{H}), 7.75(\mathrm{td}, J=7.7,1.7 \mathrm{~Hz}, 1 \mathrm{H}), 7.33$ (ddd, $J=7.6,4.8,1.3 \mathrm{~Hz}, 1 \mathrm{H}), 6.99-6.90(\mathrm{~m}, 3 \mathrm{H}), 5.72-5.62(\mathrm{~m}, 1 \mathrm{H}), 2.48(\mathrm{~s}, 6 \mathrm{H}), 1.57(\mathrm{~d}$, $J=7.3 \mathrm{~Hz}, 3 \mathrm{H}) \cdot{ }^{13} \mathrm{C}$ NMR $\left(75 \mathrm{MHz}, \mathrm{CDCl}_{3}\right) \delta=163.2\left(\mathrm{C}_{\mathrm{q}}\right), 150.0\left(\mathrm{C}_{\mathrm{q}}\right), 148.1(\mathrm{CH}), 139.5$ $\left(\mathrm{C}_{\mathrm{q}}\right), 137.3(\mathrm{CH}), 135.54\left(\mathrm{C}_{\mathrm{q}}\right), 129.5(\mathrm{CH}), 126.9(\mathrm{CH}), 126.0(\mathrm{CH}), 122.2(\mathrm{CH}), 45.6(\mathrm{CH})$, $21.0\left(\mathrm{CH}_{3}\right), 19.7\left(\mathrm{CH}_{3}\right)$. IR (ATR): 3402, 2997, 1679, 1497, 991, 740, $560 \mathrm{~cm}^{-1} . \mathbf{M S}$ (ESI) 
m/z (relative intensity) $277(100)[\mathrm{M}+\mathrm{Na}]^{+}, 255(40)[\mathrm{M}+\mathrm{H}]^{+}, 123(30)$. HR-MS (ESI) m/z calcd for $\mathrm{C}_{16} \mathrm{H}_{19} \mathrm{~N}_{2} \mathrm{O}[\mathrm{M}+\mathrm{H}]^{+} 255.1497$ found 255.1492 .<smiles>COc1cccc([N+](=O)[O-])c1C(C)NC(=O)c1nnn(Cc2ccccc2)c1C</smiles>

1-Benzyl-N-[1-(2-methoxy-6-methylphenyl)ethyl]-5-methyl-1H-1,2,3-triazole-4-car boxamide $(179 \mathrm{~g})$

Prepared following the general procedure A by using $178 \mathrm{~g}(70.2 \mathrm{mg}, 0.20 \mathrm{mmol})$. Purification by column chromatography ( $n$-hexane/EtOAc $2: 1$ ) yielded $179 \mathrm{~g}$ (64.3 $\mathrm{mg}, 88 \%$ ) as a colorless liquid. ${ }^{1} \mathrm{H}$ NMR $\left(400 \mathrm{MHz}, \mathrm{CDCl}_{3}\right) \delta=8.67(\mathrm{~d}, \mathrm{~J}=9.6 \mathrm{~Hz}, 1 \mathrm{H}), 7.34-7.27$ (m, 3H), 7.14-7.08 (m, 3H), 6.80-6.77 (m, 2H), $5.74(\mathrm{q}, J=7.0 \mathrm{~Hz}, 1 \mathrm{H}), 5.48(\mathrm{~s}, 2 \mathrm{H}), 3.96$ (s, 3H), 2.48 (s, 3H), 2.47 (s, 3H), 1.55 (d, J = 7.0 Hz, 3H). $\left.{ }^{13} \mathrm{C} \mathrm{NMR} \mathrm{(101} \mathrm{MHz,} \mathrm{CDCl}_{3}\right) \delta$ $=160.2\left(C_{q}\right), 158.1\left(C_{q}\right), 139.2\left(C_{q}\right), 136.3\left(C_{q}\right), 135.9\left(C_{q}\right), 134.2\left(C_{q}\right), 129.1\left(C_{q}\right), 129.0$ $(\mathrm{CH}), 128.4(\mathrm{CH}), 127.6(\mathrm{CH}), 127.1(\mathrm{CH}), 123.2(\mathrm{CH}), 109.3(\mathrm{CH}), 55.5\left(\mathrm{CH}_{3}\right), 51.7\left(\mathrm{CH}_{2}\right)$, $43.1(\mathrm{CH}), 20.2\left(\mathrm{CH}_{3}\right), 20.0\left(\mathrm{CH}_{3}\right), 8.7\left(\mathrm{CH}_{3}\right)$. IR (ATR): 3415, 2847, 1664, 1587, 1244, 7330, $581 \mathrm{~cm}^{-1}$. MS (ESI) m/z (relative intensity) $387(100)[\mathrm{M}+\mathrm{Na}]^{+}, 365(40)[\mathrm{M}+\mathrm{H}]^{+}, 217$ (15). HR-MS (ESI) $m / z$ calcd for $\mathrm{C}_{21} \mathrm{H}_{25} \mathrm{~N}_{4} \mathrm{O}_{2}[\mathrm{M}+\mathrm{H}]^{+} 365.1978$ found 365.1973.<smiles>Cc1cccc(F)c1C(C)NC(=O)c1nnn(Cc2ccccc2)c1C</smiles>

1-Benzyl-N-[1-(2-fluoro-6-methylphenyl)ethyl]-5-methyl-1H-1,2,3-triazole-4-carbox amide (179h)

Prepared following the general procedure $\mathbf{A}$ by using $178 \mathrm{~h}(67.7 \mathrm{mg}, 0.20 \mathrm{mmol})$. Purification by column chromatography ( $n$-hexane/EtOAc 2:1) yielded $179 \mathrm{~h}(60.8 \mathrm{mg}, 86 \%)$ as a white solid. M.p. $=92-94{ }^{\circ} \mathrm{C} .{ }^{1} \mathrm{H}$ NMR $\left(400 \mathrm{MHz}, \mathrm{CDCl}_{3}\right) \delta=7.97(\mathrm{~d}, J=9.0 \mathrm{~Hz}, 1 \mathrm{H})$, 
7.34-7.27 (m, 3H), 7.14-7.06 (m, 3H), 6.93-6.87 (m, 2H), 5.68-5.60 (m, 1H), $5.48(\mathrm{~s}, 2 \mathrm{H})$, $2.51(\mathrm{~s}, 3 \mathrm{H}), 2.46(\mathrm{~s}, 3 \mathrm{H}), 1.60(\mathrm{dd}, J=7.1,1.0 \mathrm{~Hz}, 3 \mathrm{H}) \cdot{ }^{13} \mathrm{C} \mathrm{NMR}\left(101 \mathrm{MHz}, \mathrm{CDCl}_{3}\right) \delta=$ $162.1\left(\mathrm{~d},{ }^{1} \mathrm{~J}_{\mathrm{C}-\mathrm{F}}=244.3 \mathrm{~Hz}, \mathrm{C}_{\mathrm{q}}\right), 160.4\left(\mathrm{C}_{\mathrm{q}}\right), 138.7\left(\mathrm{C}_{\mathrm{q}}\right), 137.4\left(\mathrm{~d},{ }^{4} \mathrm{~J}=5.0 \mathrm{~Hz}, \mathrm{C}_{\mathrm{q}}\right), 136.0\left(\mathrm{C}_{\mathrm{q}}\right)$, $134.1\left(\mathrm{C}_{\mathrm{q}}\right), 129.0(\mathrm{CH}), 128.7\left(\mathrm{~d},{ }^{3} \mathrm{~J}_{\mathrm{C}-\mathrm{F}}=11.4 \mathrm{~Hz}, \mathrm{C}_{\mathrm{q}}\right), 128.4(\mathrm{CH}), 128.0\left(\mathrm{~d},{ }^{4} \mathrm{~J}_{\mathrm{C}-\mathrm{F}}=9.8 \mathrm{~Hz}\right.$, $\mathrm{CH}), 127.1(\mathrm{CH}), 126.1\left(\mathrm{~d},{ }^{4} \mathrm{~J}_{\mathrm{C}-\mathrm{F}}=2.6 \mathrm{~Hz}, \mathrm{CH}\right), 113.9\left(\mathrm{~d},{ }^{2} \mathrm{~J}_{\mathrm{C}-\mathrm{F}}=22.6 \mathrm{~Hz}, \mathrm{CH}\right), 51.7\left(\mathrm{CH}_{2}\right)$, $42.6(\mathrm{CH}), 20.5\left(\mathrm{~d},{ }^{4} \mathrm{~J}_{\mathrm{C}-\mathrm{F}}=3.0 \mathrm{~Hz}, \mathrm{CH}_{3}\right), 19.6\left(\mathrm{~d},{ }^{4} \mathrm{~J}_{\mathrm{C}-\mathrm{F}}=2.5 \mathrm{~Hz}, \mathrm{CH}_{3}\right), 8.7\left(\mathrm{CH}_{3}\right) .{ }^{19} \mathrm{~F}$ NMR $\left(376 \mathrm{MHz}, \mathrm{CDCl}_{3}\right) \delta=-116.8$ (dd, $J=11.4,5.6 \mathrm{~Hz}$ ). IR (ATR): 3425, 2927, 1667, 1507, $1252,730,579 \mathrm{~cm}^{-1} . \mathbf{M S}(\mathrm{ESI}) \mathrm{m} / \mathrm{z}$ (relative intensity) $375(100)[\mathrm{M}+\mathrm{Na}]^{+}, 353(50)[\mathrm{M}+\mathrm{H}]^{+}$, 217 (40), 137 (10). HR-MS (ESI) $\mathrm{m} / z$ calcd for $\mathrm{C}_{20} \mathrm{H}_{22} \mathrm{FN}_{4} \mathrm{O}[\mathrm{M}+\mathrm{H}]^{+} 353.1778$ found 353.1772.<smiles>COc1cc(OC)c(C(C)NC(=O)c2nnn(Cc3ccccc3)c2C)c([N+](=O)[O-])c1</smiles>

1-Benzyl-N-[1-(3,6-dimethoxy-2-methylphenyl)ethyl]-5-methyl-1H-1,2,3-triazole-4carboxamide (179i)

Prepared following the general procedure $\mathbf{A}$ by using $178 \mathbf{i}(76.0 \mathrm{mg}, 0.20 \mathrm{mmol})$. Purification by column chromatography ( $n$-hexane/EtOAc $2: 1$ ) yielded $179 \mathrm{i}(63.2 \mathrm{mg}, 80 \%$ ) as a colorless liquid. ${ }^{1} \mathrm{H}$ NMR $\left(300 \mathrm{MHz}, \mathrm{CDCl}_{3}\right) \delta=8.58(\mathrm{~d}, J=9.6 \mathrm{~Hz}, 1 \mathrm{H}), 7.33-7.28$ (m, 3H), 7.13-7.10 (m, 2H), $6.34(\mathrm{dd}, J=20.2,2.4 \mathrm{~Hz}, 2 \mathrm{H}), 5.66(\mathrm{q}, J=7.0 \mathrm{~Hz}, 1 \mathrm{H}), 5.47$ (s, 2H), 3.93 (s, 3H), $3.76(\mathrm{~s}, 3 \mathrm{H}), 2.47(\mathrm{~s}, 3 \mathrm{H}), 2.44(\mathrm{~s}, 3 \mathrm{H}), 1.52(\mathrm{~d}, J=7.0 \mathrm{~Hz}, 3 \mathrm{H})$. ${ }^{13} \mathrm{C}$ NMR $\left(126 \mathrm{MHz}, \mathrm{CDCl}_{3}\right) \delta=156.0\left(\mathrm{C}_{\mathrm{q}}\right), 159.0\left(\mathrm{C}_{\mathrm{q}}\right), 158.9\left(\mathrm{C}_{\mathrm{q}}\right), 139.1\left(\mathrm{C}_{\mathrm{q}}\right), 136.9\left(\mathrm{C}_{\mathrm{q}}\right)$, $135.7\left(\mathrm{C}_{\mathrm{q}}\right), 134.1\left(\mathrm{C}_{\mathrm{q}}\right), 128.9(\mathrm{CH}), 128.3(\mathrm{CH}), 127.0(\mathrm{CH}), 121.8\left(\mathrm{C}_{\mathrm{q}}\right), 106.9(\mathrm{CH}), 97.1$ $(\mathrm{CH}), 55.5\left(\mathrm{CH}_{3}\right), 55.2\left(\mathrm{CH}_{3}\right), 51.7\left(\mathrm{CH}_{2}\right), 42.8(\mathrm{CH}), 20.6\left(\mathrm{CH}_{3}\right), 20.5\left(\mathrm{CH}_{3}\right), 8.8\left(\mathrm{CH}_{3}\right)$. IR (ATR): 3416, 2934, 1661, 1503, 1262, 1153, 731, $461 \mathrm{~cm}^{-1}$. MS (ESI) m/z (relative intensity) $417(100)[\mathrm{M}+\mathrm{Na}]^{+}, 395(70)[\mathrm{M}+\mathrm{H}]^{+}, 243$ (80), 217 (10), 179 (30). HR-MS (ESI) $m / z$ calcd for $\mathrm{C}_{22} \mathrm{H}_{27} \mathrm{~N}_{4} \mathrm{O}[\mathrm{M}+\mathrm{H}]^{+} 395.2083$ found 395.2078 . 


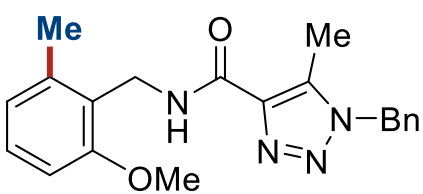

1-Benzyl-N-(2-methoxy-6-methylbenzyl)-5-methyl-1H-1,2,3-triazole-4-carboxamide $(179 j)$

Prepared following the general procedure $\mathbf{A}$ by using $178 \mathbf{j}(67.3 \mathrm{mg}, 0.20 \mathrm{mmol})$. Purification by column chromatography ( $n$-hexane/EtOAc $2: 1$ ) yielded 179j (63.2 mg, 90\%) as a white solid. M.p. $=83-85^{\circ} \mathrm{C} .{ }^{1} \mathrm{H}$ NMR $\left(300 \mathrm{MHz}, \mathrm{CDCl}_{3}\right) \delta=7.56(\mathrm{t}, J=5.6 \mathrm{~Hz}, 1 \mathrm{H})$, 7.35-7.28 (m, 3H), 7.18-7.10 (m, 3H), $6.79(\mathrm{~d}, J=7.6 \mathrm{~Hz}, 1 \mathrm{H}), 6.75(\mathrm{~d}, J=8.3 \mathrm{~Hz}, 1 \mathrm{H})$, $5.47(\mathrm{~s}, 2 \mathrm{H}), 4.67(\mathrm{~d}, \mathrm{~J}=5.6 \mathrm{~Hz}, 2 \mathrm{H}), 3.88(\mathrm{~s}, 3 \mathrm{H}), 2.50(\mathrm{~s}, 3 \mathrm{H}), 2.47(\mathrm{~s}, 3 \mathrm{H}) .{ }^{13} \mathrm{C}$ NMR $(126$ $\left.\mathrm{MHz}, \mathrm{CDCl}_{3}\right) \delta=160.7\left(\mathrm{C}_{\mathrm{q}}\right), 158.2\left(\mathrm{C}_{\mathrm{q}}\right), 138.9\left(\mathrm{C}_{\mathrm{q}}\right), 138.1\left(\mathrm{C}_{\mathrm{q}}\right), 135.8\left(\mathrm{C}_{\mathrm{q}}\right), 134.0\left(\mathrm{C}_{\mathrm{q}}\right)$, $128.9(\mathrm{CH}), 128.3(\mathrm{CH}), 128.2(\mathrm{CH}), 127.0(\mathrm{CH}), 124.4\left(\mathrm{C}_{\mathrm{q}}\right), 122.7(\mathrm{CH}), 108.0(\mathrm{CH}), 55.5$ $\left(\mathrm{CH}_{3}\right), 51.7\left(\mathrm{CH}_{2}\right), 34.6\left(\mathrm{CH}_{2}\right), 19.6\left(\mathrm{CH}_{3}\right), 8.8\left(\mathrm{CH}_{3}\right)$. IR (ATR): 3423, 2930, 1664, 1511, $1261,731,459 \mathrm{~cm}^{-1} . \mathbf{M S}(\mathrm{ESI}) \mathrm{m} / \mathrm{z}$ (relative intensity) $373(100)[\mathrm{M}+\mathrm{Na}]^{+}, 351(65)[\mathrm{M}+\mathrm{H}]^{+}$, 211 (10). HR-MS (ESI) $\mathrm{m} / z$ calcd for $\mathrm{C}_{20} \mathrm{H}_{23} \mathrm{~N}_{4} \mathrm{O}_{2}[\mathrm{M}+\mathrm{H}]^{+} 351.1821$ found 351.1816 .<smiles>Cc1cccc(F)c1CNC(=O)c1nnn(Cc2ccccc2)c1C</smiles>

\section{1-Benzyl-N-(2-fluoro-6-methylbenzyl)-5-methyl-1H-1,2,3-triazole-4-carboxamide} $(179 k)$

Prepared following the general procedure A by using $178 \mathrm{k}(65.3 \mathrm{mg}, 0.20 \mathrm{mmol})$. Purification by column chromatography ( $n$-hexane/EtOAc $2: 1$ ) yielded 179k $(62.3 \mathrm{mg}, 92 \%)$ as a white solid. M.p. $=107-110{ }^{\circ} \mathrm{C} .{ }^{1} \mathrm{H}$ NMR $\left(400 \mathrm{MHz}, \mathrm{CDCl}_{3}\right) \delta=7.38-7.29(\mathrm{~m}, 4 \mathrm{H})$, 7.17-7.11 (m, 3H), $6.96(\mathrm{~d}, J=7.6 \mathrm{~Hz}, 1 \mathrm{H}), 6.91(\mathrm{t}, J=9.0 \mathrm{~Hz}, 1 \mathrm{H}), 5.48(\mathrm{~s}, 2 \mathrm{H}), 4.67$ (dd, $J=5.7,1.7 \mathrm{~Hz}, 2 \mathrm{H}), 2.50(\mathrm{~s}, 3 \mathrm{H}), 2.45(\mathrm{~s}, 3 \mathrm{H}) .{ }^{13} \mathrm{C}$ NMR $\left(101 \mathrm{MHz}, \mathrm{CDCl}_{3}\right) \delta=161.9(\mathrm{~d}$, $\left.{ }^{1} J_{C-F}=245.7 \mathrm{~Hz}, C_{q}\right), 160.9\left(C_{q}\right), 139.5\left(d,{ }^{4} J_{C-F}=3.5 \mathrm{~Hz}, C_{q}\right), 138.6\left(C_{q}\right), 136.1\left(C_{q}\right)$, $134.0\left(\mathrm{C}_{\mathrm{q}}\right), 129.0(\mathrm{CH}), 128.9\left(\mathrm{~d},{ }^{4} \mathrm{~J}_{\mathrm{C}-\mathrm{F}}=9.3 \mathrm{~Hz}, \mathrm{CH}\right), 128.4(\mathrm{CH}), 127.1(\mathrm{CH}), 126.0(\mathrm{~d}$, 
$\left.{ }^{4} J_{\mathrm{C}-\mathrm{F}}=3.0 \mathrm{~Hz}, \mathrm{CH}\right), 123.1\left(\mathrm{~d},{ }^{3} \mathrm{~J}_{\mathrm{C}-\mathrm{F}}=14.3 \mathrm{~Hz}, \mathrm{C}_{\mathrm{q}}\right), 112.9\left(\mathrm{~d},{ }^{2} \mathrm{~J}_{\mathrm{C}-\mathrm{F}}=22.5 \mathrm{~Hz}, \mathrm{CH}\right), 51.7$ $\left(\mathrm{CH}_{2}\right), 33.6\left(\mathrm{~d},{ }^{4} \mathrm{~J}_{\mathrm{C}-\mathrm{F}}=5.3 \mathrm{~Hz}, \mathrm{CH}_{2}\right), 19.1\left(\mathrm{~d},{ }^{4} \mathrm{~J}_{\mathrm{C}-\mathrm{F}}=2.6 \mathrm{~Hz}, \mathrm{CH}_{3}\right), 8.7\left(\mathrm{CH}_{3}\right) .{ }^{19} \mathrm{~F} \mathrm{NMR}(376$ $\mathrm{MHz}, \mathrm{CDCl}_{3}$ ) $\delta=-118.0$ (ddt, $J=9.6,7.6,1.7 \mathrm{~Hz}$ ). IR (ATR): 3338, 2961, 1666, 1512, 1253, 730, $459 \mathrm{~cm}^{-1}$. MS (ESI) m/z (relative intensity) $361(100)[\mathrm{M}+\mathrm{Na}]^{+}, 339(60)[\mathrm{M}+\mathrm{H}]^{+}, 205$ (15), 149 (10). HR-MS (ESI) $\mathrm{m} / z$ calcd for $\mathrm{C}_{19} \mathrm{H}_{20} \mathrm{FN}_{4} \mathrm{O}[\mathrm{M}+\mathrm{H}]^{+} 339.1621$ found 339.1616.<smiles>Cc1cccc(C(F)(F)F)c1CNC(=O)c1nnn(Cc2ccccc2)c1C</smiles>

\section{1-Benzyl-5-methyl-N-[2-methyl-6-(trifluoromethyl)benzyl]-1H-1,2,3-triazole-4-} carboxamide (179l)

Prepared following the general procedure A by using $178 \mathrm{I}(75.0 \mathrm{mg}, 0.20 \mathrm{mmol})$. Purification by column chromatography ( $n$-hexane/EtOAc 2:1) yielded 179I (62.1 mg, 80\%) as a white solid. M.p. $=81-82^{\circ}{ }^{1}{ }^{1} \mathrm{H}$ NMR $\left(300 \mathrm{MHz}, \mathrm{CDCl}_{3}\right) \delta=7.54(\mathrm{~d}, J=7.7 \mathrm{~Hz}, 1 \mathrm{H})$, $7.40(\mathrm{~d}, J=7.2 \mathrm{~Hz}, 1 \mathrm{H}), 7.36-7.29(\mathrm{~m}, 4 \mathrm{H}), 7.17-7.12(\mathrm{~m}, 3 \mathrm{H}), 5.48(\mathrm{~s}, 2 \mathrm{H}), 4.78(\mathrm{~d}, J=$ $5.3 \mathrm{~Hz}, 2 \mathrm{H}), 2.52(\mathrm{~s}, 3 \mathrm{H}), 2.45(\mathrm{~s}, 3 \mathrm{H}) .{ }^{13} \mathrm{C} \mathrm{NMR}\left(101 \mathrm{MHz}, \mathrm{CDCl}_{3}\right) \delta=160.7\left(\mathrm{C}_{\mathrm{q}}\right), 140.2$ $\left(\mathrm{C}_{\mathrm{q}}\right), 138.4\left(\mathrm{C}_{\mathrm{q}}\right), 136.2\left(\mathrm{C}_{\mathrm{q}}\right), 134.5(\mathrm{CH}), 134.0\left(\mathrm{C}_{\mathrm{q}}\right), 133.4\left(\mathrm{~d},{ }^{3} \mathrm{~J}=1.3 \mathrm{~Hz}, \mathrm{C}_{\mathrm{q}}\right), 129.9\left(\mathrm{q},{ }^{2} \mathrm{~J}\right.$ $\left.=29.4 \mathrm{~Hz}, \mathrm{C}_{\mathrm{q}}\right), 129.0(\mathrm{CH}), 128.5(\mathrm{CH}), 128.0(\mathrm{CH}), 127.1(\mathrm{CH}), 124.5\left(\mathrm{q},{ }^{1} \mathrm{~J}_{\mathrm{C}-\mathrm{F}}=274.0\right.$ $\left.\mathrm{Hz}, \mathrm{C}_{\mathrm{q}}\right), 123.8\left(\mathrm{q},{ }^{3} \mathrm{~J}_{\mathrm{C}-\mathrm{F}}=5.8 \mathrm{~Hz}, \mathrm{CH}\right), 51.7\left(\mathrm{CH}_{2}\right), 36.7\left(\mathrm{~d},{ }^{3} \mathrm{~J}_{\mathrm{C}-\mathrm{F}}=2.0 \mathrm{~Hz}, \mathrm{CH}_{2}\right), 19.5\left(\mathrm{CH}_{3}\right)$, $8.7\left(\mathrm{CH}_{3}\right) .{ }^{19} \mathrm{~F}$ NMR $\left(376 \mathrm{MHz}, \mathrm{CDCl}_{3}\right) \delta=-58.1$. IR (ATR): 3328, 2974, 1664, 1509, 1254, 766, $589 \mathrm{~cm}^{-1}$. MS (ESI) m/z (relative intensity) $411(100)[\mathrm{M}+\mathrm{Na}]^{+}, 389(50)[\mathrm{M}+\mathrm{H}]^{+}, 292$ (10), 163 (10). HR-MS (ESI) $m / z$ calcd for $\mathrm{C}_{20} \mathrm{H}_{20} \mathrm{~F}_{3} \mathrm{~N}_{4} \mathrm{O}[\mathrm{M}+\mathrm{H}]^{+} 389.1589$ found 389.1584.<smiles>Cc1cccc2cccc(NC(=O)c3nnn(Cc4ccccc4)c3C)c12</smiles> 


\section{1-Benzyl-5-methyl-N-(8-methylnaphthalen-1-yl)-1H-1,2,3-triazole-4-carboxamide} $(179 m)$

Prepared following the general procedure A by using $178 \mathrm{~m}(317 \mathrm{mg}, 0.93 \mathrm{mmol})$. Purification by column chromatography ( $n$-hexane/EtOAc 2:1) yielded 179m (226 mg, 66\%) as a yellow solid. M.p. $=139-140{ }^{\circ} \mathrm{C} .{ }^{1} \mathbf{H}$ NMR $\left(300 \mathrm{MHz}, \mathrm{CDCl}_{3}\right) \delta=9.60(\mathrm{brs}, 1 \mathrm{H}), 7.91$ (dd, $J=7.5,0.8 \mathrm{~Hz}, 1 \mathrm{H}), 7.76-7.70(\mathrm{~m}, 2 \mathrm{H}), 7.47(\mathrm{t}, J=8.0 \mathrm{~Hz}, 1 \mathrm{H}), 7.41-7.32(\mathrm{~m}, 4 \mathrm{H})$, 7.30-7.27 (m, 1H), 7.24-7.20 (m, 2H), $5.53(\mathrm{~s}, 2 \mathrm{H}), 3.01(\mathrm{~s}, 3 \mathrm{H}), 2.58(\mathrm{~s}, 3 \mathrm{H}) .{ }^{13} \mathrm{C}$ NMR $\left(75 \mathrm{MHz}, \mathrm{CDCl}_{3}\right) \delta=159.8\left(\mathrm{C}_{\mathrm{q}}\right), 139.1\left(\mathrm{C}_{\mathrm{q}}\right), 136.9\left(\mathrm{C}_{\mathrm{q}}\right), 135.9\left(\mathrm{C}_{\mathrm{q}}\right), 134.0\left(\mathrm{C}_{\mathrm{q}}\right), 132.7\left(\mathrm{C}_{\mathrm{q}}\right)$, $132.7\left(\mathrm{C}_{\mathrm{q}}\right), 130.2(\mathrm{CH}), 129.2(\mathrm{CH}), 128.7(\mathrm{CH}), 128.4\left(\mathrm{C}_{\mathrm{q}}\right), 127.7(\mathrm{CH}), 127.7(\mathrm{CH}), 127.4$ $(\mathrm{CH}), 125.6(\mathrm{CH}), 125.2(\mathrm{CH}), 124.4(\mathrm{CH}), 52.0\left(\mathrm{CH}_{2}\right), 25.1\left(\mathrm{CH}_{3}\right), 8.9\left(\mathrm{CH}_{3}\right)$. IR (ATR): $3426,2974,1666,1487,813,726 \mathrm{~cm}^{-1}$. MS (ESI) $\mathrm{m} / \mathrm{z}$ (relative intensity) $379(100)[\mathrm{M}+\mathrm{Na}]^{+}$, 357 (55) $[\mathrm{M}+\mathrm{H}]^{+}, 217$ (10). HR-MS (ESI) $\mathrm{m} / \mathrm{z}$ calcd for $\mathrm{C}_{22} \mathrm{H}_{21} \mathrm{~N}_{4} \mathrm{O}[\mathrm{M}+\mathrm{H}]^{+} 357.1715$ found 357.1710.<smiles>COc1cc(C)c(C(C)NC(=O)c2nnn(Cc3ccccc3)c2C)c(C)c1</smiles>

1-Benzyl-N-[1-(4-methoxy-2,6-dimethylphenyl)ethyl]-5-methyl-1H-1,2,3-triazole-4carboxamide (179n)

Prepared following the general procedure A by using $178 \mathrm{n}(70.2 \mathrm{mg}, 0.20 \mathrm{mmol})$. Purification by column chromatography ( $n$-hexane/EtOAc $2: 1$ ) yielded $179 n$ ( $56.4 \mathrm{mg}, 75 \%$ ) as a colorless liquid. ${ }^{1} \mathrm{H}$ NMR $\left(300 \mathrm{MHz}, \mathrm{CDCl}_{3}\right) \delta=7.72(\mathrm{~d}, J=7.6 \mathrm{~Hz}, 1 \mathrm{H}), 7.36-7.29$ (m, 3H), 7.14-7.11 (m, 2H), $6.55(\mathrm{~s}, 2 \mathrm{H}), 5.62(\mathrm{q}, J=7.3 \mathrm{~Hz}, 1 \mathrm{H}), 5.48(\mathrm{~s}, 2 \mathrm{H}), 3.74(\mathrm{~s}$, 3H), $2.52(\mathrm{~s}, 6 \mathrm{H}), 2.46(\mathrm{~s}, 3 \mathrm{H}), 1.59(\mathrm{~d}, \mathrm{~J}=7.3 \mathrm{~Hz}, 3 \mathrm{H}) .{ }^{13} \mathrm{C} \mathrm{NMR}\left(126 \mathrm{MHz}, \mathrm{CDCl}_{3}\right) \delta=$ $160.2\left(C_{q}\right), 157.6\left(C_{q}\right), 138.7\left(C_{q}\right), 136.8\left(C_{q}\right), 135.8\left(C_{q}\right), 134.0\left(C_{q}\right), 131.5\left(C_{q}\right), 128.9$ $(\mathrm{CH}), 128.4(\mathrm{CH}), 127.0(\mathrm{CH}), 114.6(\mathrm{CH}), 55.0\left(\mathrm{CH}_{3}\right), 51.7\left(\mathrm{CH}_{2}\right), 44.8(\mathrm{CH}), 21.3\left(\mathrm{CH}_{3}\right)$, $20.1\left(\mathrm{CH}_{3}\right), 8.8\left(\mathrm{CH}_{3}\right)$. IR (ATR): 3423, 2934, 1663, 1497, 1151, 727, $574 \mathrm{~cm}^{-1}$. MS (ESI) 
m/z (relative intensity) $401(100)[\mathrm{M}+\mathrm{Na}]^{+}, 379(50)[\mathrm{M}+\mathrm{H}]^{+}, 163(10)$. HR-MS (ESI) m/z calcd for $\mathrm{C}_{22} \mathrm{H}_{27} \mathrm{~N}_{4} \mathrm{O}_{2}[\mathrm{M}+\mathrm{H}]^{+} 379.2134$ found 379.2128 .<smiles>Cc1cc(N(C)C)cc(C)c1C(C)NC(=O)c1nnn(Cc2ccccc2)c1C</smiles>

1-Benzyl-N-\{1-[4-(dimethylamino)-2,6-dimethylphenyl]ethyl\}-5-methyl-1H-1,2,3triazole-4-carboxamide (179o)

Prepared following the general procedure A by using $1780(72.7 \mathrm{mg}, 0.20 \mathrm{mmol})$. Purification by column chromatography ( $n$-hexane/EtOAc $2: 1)$ yielded 1790 (48.5 mg, $62 \%)$ as a yellow liquid. ${ }^{1} \mathrm{H}$ NMR $\left(300 \mathrm{MHz}, \mathrm{CDCl}_{3}\right) \delta=7.73(\mathrm{~d}, J=7.7 \mathrm{~Hz}, 1 \mathrm{H}), 7.37-7.29(\mathrm{~m}$, 3H), 7.14-7.11 (m, 2H), $6.42(\mathrm{~s}, 2 \mathrm{H}), 5.63(\mathrm{q}, J=7.2 \mathrm{~Hz}, 1 \mathrm{H}), 5.48(\mathrm{~s}, 2 \mathrm{H}), 2.90(\mathrm{~s}, 6 \mathrm{H})$, $2.52(\mathrm{~s}, 6 \mathrm{H}), 2.46(\mathrm{~s}, 3 \mathrm{H}), 1.62(\mathrm{~d}, J=7.2 \mathrm{~Hz}, 3 \mathrm{H}) .{ }^{13} \mathrm{C}$ NMR $\left(126 \mathrm{MHz}, \mathrm{CDCl}_{3}\right) \delta=160.1$ $\left(\mathrm{C}_{\mathrm{q}}\right), 148.9\left(\mathrm{C}_{\mathrm{q}}\right), 138.8\left(\mathrm{C}_{\mathrm{q}}\right), 136.2\left(\mathrm{C}_{\mathrm{q}}\right), 135.7\left(\mathrm{C}_{\mathrm{q}}\right), 134.0\left(\mathrm{C}_{\mathrm{q}}\right), 131.5\left(\mathrm{C}_{\mathrm{q}}\right), 128.9(\mathrm{CH})$, $128.3(\mathrm{CH}), 127.0(\mathrm{CH}), 113.7(\mathrm{CH}), 51.7\left(\mathrm{CH}_{2}\right), 44.7(\mathrm{CH}), 40.6\left(\mathrm{CH}_{3}\right), 21.6\left(\mathrm{CH}_{3}\right), 20.4$ $\left(\mathrm{CH}_{3}\right), 8.8\left(\mathrm{CH}_{3}\right)$. IR (ATR): 3423, 2928, 1662, 1593, 1497, 1356, 726, $551 \mathrm{~cm}^{-1} . \mathbf{M S}(\mathrm{ESI})$ $\mathrm{m} / \mathrm{z}$ (relative intensity) $414(100)[\mathrm{M}+\mathrm{Na}]^{+}, 392(50)[\mathrm{M}+\mathrm{H}]^{+}, 176(10)$. HR-MS (ESI) m/z calcd for $\mathrm{C}_{23} \mathrm{H}_{30} \mathrm{~N}_{5} \mathrm{O}[\mathrm{M}+\mathrm{H}]^{+} 392.2450$ found 392.2445 .<smiles>Cc1cc(C2CCCCC2)cc([N+](=O)[O-])c1C(C)NC(=O)c1nnn(Cc2ccccc2)c1C</smiles>

1-Benzyl-N-[1-(4-cyclohexyl-2,6-dimethylphenyl)ethyl]-5-methyl-1H-1,2,3-triazole4-carboxamie (179p)

Prepared following the general procedure A by using $178 \mathrm{p}(80.1 \mathrm{mg}, 0.20 \mathrm{mmol})$. Purification by column chromatography ( $n$-hexane/EtOAc $2: 1)$ yielded $179 p(69.4 \mathrm{mg}, 80 \%)$ 
as a yellow liquid. ${ }^{1} \mathrm{H}$ NMR $\left(300 \mathrm{MHz}, \mathrm{CDCl}_{3}\right) \delta=7.76(\mathrm{~d}, \mathrm{~J}=7.9 \mathrm{~Hz}, 1 \mathrm{H}), 7.37-7.30(\mathrm{~m}$, 3H), 7.14-7.11 (m, 2H), 6.83 (s, 2H), 5.68 (q, J = $7.2 \mathrm{~Hz}, 1 \mathrm{H}), 5.49(\mathrm{~s}, 2 \mathrm{H}), 2.52(\mathrm{~s}, 6 \mathrm{H})$, $2.46(\mathrm{~s}, 3 \mathrm{H}), 2.44-2.34(\mathrm{~m}, 1 \mathrm{H}), 1.84-1.71(\mathrm{~m}, 5 \mathrm{H}), 1.60(\mathrm{~d}, J=7.2 \mathrm{~Hz}, 3 \mathrm{H}), 1.41-1.24(\mathrm{~m}$, 5H). ${ }^{13} \mathrm{C} \mathrm{NMR}\left(126 \mathrm{MHz}, \mathrm{CDCl}_{3}\right) \delta=160.2\left(\mathrm{C}_{\mathrm{q}}\right), 146.2\left(\mathrm{C}_{\mathrm{q}}\right), 138.8\left(\mathrm{C}_{\mathrm{q}}\right), 136.3\left(\mathrm{C}_{\mathrm{q}}\right), 135.8$ $\left(\mathrm{C}_{\mathrm{q}}\right), 135.1\left(\mathrm{C}_{\mathrm{q}}\right), 134.0\left(\mathrm{C}_{\mathrm{q}}\right), 128.9(\mathrm{CH}), 128.4(\mathrm{CH}), 127.9(\mathrm{CH}), 127.0(\mathrm{CH}), 51.8\left(\mathrm{CH}_{2}\right)$, $45.0(\mathrm{CH}), 44.0(\mathrm{CH}), 34.4\left(\mathrm{CH}_{2}\right)$ and $34.3\left(\mathrm{CH}_{2}\right), 27.0\left(\mathrm{CH}_{2}\right), 26.2\left(\mathrm{CH}_{2}\right), 21.2\left(\mathrm{CH}_{3}\right), 20.0$ $\left(\mathrm{CH}_{3}\right), 8.8\left(\mathrm{CH}_{3}\right)$. IR (ATR): 3431, 2921, 1670, 1501, 1246, 726, $542 \mathrm{~cm}^{-1} . \mathbf{M S}(\mathrm{ESI}) \mathrm{m} / \mathrm{z}$ (relative intensity) $453(50)[\mathrm{M}+\mathrm{Na}]^{+}, 431(60)[\mathrm{M}+\mathrm{H}]^{+}, 243$ (100), $217(20), 200$ (20). HRMS (ESI) $m / z$ calcd for $\mathrm{C}_{27} \mathrm{H}_{35} \mathrm{~N}_{4} \mathrm{O}[\mathrm{M}+\mathrm{H}]^{+} 431.2811$ found 431.2805 .<smiles>Cc1cc(CC(C)C)cc(C)c1C(C)NC(=O)c1nnn(Cc2ccccc2)c1C</smiles>

\section{1-Benzyl-N-[1-(4-isobutyl-2,6-dimethylphenyl)ethyl]-5-methyl-1H-1,2,3-triazole-4-} carboxamide (179q)

Prepared following the general procedure $A$ by using $178 \mathrm{q}(75.0 \mathrm{mg}, 0.20 \mathrm{mmol})$. Purification by column chromatography ( $n$-hexane/EtOAc 2:1) yielded 179q (62.3 mg, 77\%) as a white solid. M.p. $=84-85^{\circ} \mathrm{C} .{ }^{1} \mathrm{H}$ NMR $\left(300 \mathrm{MHz}, \mathrm{CDCl}_{3}\right) \delta=7.77(\mathrm{~d}, J=7.9 \mathrm{~Hz}, 1 \mathrm{H})$, 7.37-7.28 (m, 3H), 7.15-7.12 (m, 2H), $6.78(\mathrm{~s}, 2 \mathrm{H}), 5.69(\mathrm{q}, J=7.3 \mathrm{~Hz}, 1 \mathrm{H}), 5.48(\mathrm{~s}, 2 \mathrm{H})$, $2.52(\mathrm{~s}, 6 \mathrm{H}), 2.46(\mathrm{~s}, 3 \mathrm{H}), 2.35(\mathrm{~d}, J=7.1 \mathrm{~Hz}, 2 \mathrm{H}), 1.89-1.76(\mathrm{~m}, 1 \mathrm{H}), 1.60(\mathrm{~d}, J=7.3 \mathrm{~Hz}$, 3H), $0.89(\mathrm{~d}, J=6.6 \mathrm{~Hz}, 6 \mathrm{H}) .{ }^{13} \mathrm{C}$ NMR $\left(126 \mathrm{MHz}, \mathrm{CDCl}_{3}\right) \delta=160.1\left(\mathrm{C}_{\mathrm{q}}\right), 139.9\left(\mathrm{C}_{\mathrm{q}}\right), 138.7$ $\left(\mathrm{C}_{\mathrm{q}}\right), 136.2\left(\mathrm{C}_{\mathrm{q}}\right), 135.8\left(\mathrm{C}_{\mathrm{q}}\right), 134.9\left(\mathrm{C}_{\mathrm{q}}\right), 134.0\left(\mathrm{C}_{\mathrm{q}}\right), 130.1(\mathrm{CH}), 128.9(\mathrm{CH}), 128.3(\mathrm{CH})$, $127.0(\mathrm{CH}), 51.7\left(\mathrm{CH}_{2}\right), 45.0(\mathrm{CH}), 44.8\left(\mathrm{CH}_{2}\right), 30.0(\mathrm{CH}), 22.5\left(\mathrm{CH}_{3}\right), 21.0\left(\mathrm{CH}_{3}\right), 20.0$ $\left(\mathrm{CH}_{3}\right), 8.8\left(\mathrm{CH}_{3}\right)$. IR (ATR): 3426, 2957, 1665, 1502, 1253, 729, $574 \mathrm{~cm}^{-1} . \mathbf{M S}(\mathrm{ESI}) \mathrm{m} / \mathrm{z}$ (relative intensity) $427(100)[\mathrm{M}+\mathrm{Na}]^{+}, 405(45)[\mathrm{M}+\mathrm{H}]^{+}, 243$ (80), 189 (6). HR-MS (ESI) $m / z$ calcd for $\mathrm{C}_{25} \mathrm{H}_{33} \mathrm{~N}_{4} \mathrm{O}[\mathrm{M}+\mathrm{H}]^{+} 405.2654$ found 405.2649 . 
<smiles>Cc1cc(-c2ccccc2)cc(C)c1C(C)NC(=O)c1nnn(Cc2ccccc2)c1C</smiles>

1-Benzyl-N-\{1-[3,5-dimethyl-(1,1'-biphenyl)-4-yl]ethyl\}-5-methyl-1H-1,2,3-triazole-4 carboxamide (179r)

Prepared following the general procedure A by using $178 \mathrm{r}(79.6 \mathrm{mg}, 0.20 \mathrm{mmol})$. Purification by column chromatography ( $n$-hexane/EtOAc $2: 1)$ yielded $179 \mathrm{r}(71.2 \mathrm{mg}, 84 \%)$ as a white solid. M.p. $=89-92{ }^{\circ} \mathrm{C} .{ }^{1} \mathrm{H}$ NMR $\left(300 \mathrm{MHz}, \mathrm{CDCl}_{3}\right) \delta=7.80(\mathrm{~d}, J=7.7 \mathrm{~Hz}, 1 \mathrm{H})$, 7.57-7.53 (m, 2H), 7.44-7.28 (m, 6H), $7.24(\mathrm{~s}, 2 \mathrm{H}), 7.16-7.12(\mathrm{~m}, 2 \mathrm{H}), 5.73(\mathrm{q}, \mathrm{J}=7.3 \mathrm{~Hz}$, 1H), $5.50(\mathrm{~s}, 2 \mathrm{H}), 2.62(\mathrm{~s}, 6 \mathrm{H}), 2.47(\mathrm{~s}, 3 \mathrm{H}), 1.66(\mathrm{~d}, J=7.3 \mathrm{~Hz}, 3 \mathrm{H}) \cdot{ }^{13} \mathrm{C}$ NMR $(126 \mathrm{MHz}$, $\left.\mathrm{CDCl}_{3}\right) \delta=160.3\left(\mathrm{C}_{\mathrm{q}}\right), 140.7\left(\mathrm{C}_{\mathrm{q}}\right), 139.4\left(\mathrm{C}_{\mathrm{q}}\right), 138.7\left(\mathrm{C}_{\mathrm{q}}\right), 138.1\left(\mathrm{C}_{\mathrm{q}}\right), 135.9\left(\mathrm{C}_{\mathrm{q}}\right), 135.8\left(\mathrm{C}_{\mathrm{q}}\right)$, $134.0\left(\mathrm{C}_{\mathrm{q}}\right), 129.0(\mathrm{CH}), 128.5(\mathrm{CH}), 128.4(\mathrm{CH}), 128.2(\mathrm{CH}), 127.0(\mathrm{CH}), 126.9(\mathrm{CH}), 126.9$ (CH), $51.8\left(\mathrm{CH}_{2}\right), 45.1(\mathrm{CH}), 21.2\left(\mathrm{CH}_{3}\right), 19.8\left(\mathrm{CH}_{3}\right), 8.8\left(\mathrm{CH}_{3}\right)$. IR (ATR): 3425, 2968, 2929, 1668, 1505, 1198, 731, $564 \mathrm{~cm}^{-1}$. MS (ESI) $\mathrm{m} / \mathrm{z}$ (relative intensity) 447 (100) [M+Na]+, 425 (50) $[\mathrm{M}+\mathrm{H}]^{+}, 243$ (90), 209 (10). HR-MS (ESI) $\mathrm{m} / z$ calcd for $\mathrm{C}_{27} \mathrm{H}_{29} \mathrm{~N}_{4} \mathrm{O}[\mathrm{M}+\mathrm{H}]^{+} 425.2341$ found 425.2336 .<smiles>Cc1cc(C(F)(F)F)cc(C)c1C(C)NC(=O)c1nnn(Cc2ccccc2)c1C</smiles>

1-Benzyl-N-\{1-[2,6-dimethyl-4-(trifluoromethyl)phenyl]ethyl\}-5-methyl-1H-1,2,3triazole-4-carboxamide (179s)

Prepared following the general procedure A by using $178 \mathrm{~s}(77.7 \mathrm{mg}, 0.20 \mathrm{mmol})$. Purification by column chromatography ( $n$-hexane/EtOAc $2: 1$ ) yielded 179 s $(70.3 \mathrm{mg}, 84 \%$ ) as a white solid. M.p. $=97-100{ }^{\circ} \mathrm{C} .{ }^{1} \mathrm{H}$ NMR $\left(300 \mathrm{MHz}, \mathrm{CDCl}_{3}\right) \delta=7.73(\mathrm{~d}, J=7.3 \mathrm{~Hz}, 1 \mathrm{H})$, 7.37-7.29 (m, 3H), $7.23(\mathrm{~s}, 2 \mathrm{H}), 7.15-7.12(\mathrm{~m}, 2 \mathrm{H}), 5.67(\mathrm{p}, \mathrm{J}=7.3 \mathrm{~Hz}, 1 \mathrm{H}), 5.49(\mathrm{~s}, 2 \mathrm{H})$, $2.58(\mathrm{~s}, 6 \mathrm{H}), 2.45(\mathrm{~s}, 3 \mathrm{H}), 1.61(\mathrm{~d}, J=7.3 \mathrm{~Hz}, 3 \mathrm{H}) .{ }^{13} \mathrm{C} \mathrm{NMR}\left(126 \mathrm{MHz}, \mathrm{CDCl}_{3}\right) \delta=160.3$ $\left(\mathrm{C}_{\mathrm{q}}\right), 142.9\left(\mathrm{C}_{\mathrm{q}}\right), 138.4\left(\mathrm{C}_{\mathrm{q}}\right), 136.2\left(\mathrm{C}_{\mathrm{q}}\right), 136.0\left(\mathrm{C}_{\mathrm{q}}\right), 133.9\left(\mathrm{C}_{\mathrm{q}}\right), 128.9(\mathrm{CH}), 128.4(\mathrm{CH})$, 
$127.7\left(\mathrm{~d},{ }^{2} \mathrm{~J}_{\mathrm{C}-\mathrm{F}}=112.9 \mathrm{~Hz}, \mathrm{C}_{\mathrm{q}}\right), 127.0(\mathrm{CH}), 126.0\left(\mathrm{q},{ }^{3} \mathrm{~J}_{\mathrm{C}-\mathrm{F}}=3.3 \mathrm{~Hz}, \mathrm{CH}\right), 124.0\left(\mathrm{q},{ }^{1} \mathrm{~J}_{\mathrm{C}-\mathrm{F}}\right.$ $\left.=271.6 \mathrm{~Hz}, \mathrm{C}_{\mathrm{q}}\right), 51.8\left(\mathrm{CH}_{2}\right), 45.2(\mathrm{CH}), 21.1\left(\mathrm{CH}_{3}\right), 19.2\left(\mathrm{CH}_{3}\right), 8.7\left(\mathrm{CH}_{3}\right) .{ }^{19} \mathrm{~F}$ NMR $(282$ $\left.\mathrm{MHz}, \mathrm{CDCl}_{3}\right) \delta=-62.6$. IR (ATR): 3373, 2983, 1647, 1581, 1513, 1323, 734, 611, 476 $\mathrm{cm}^{-1}$. MS (ESI) m/z (relative intensity) $439(100)[\mathrm{M}+\mathrm{Na}]^{+}, 417(70)[\mathrm{M}+\mathrm{H}]^{+}, 217$ (40). HRMS (ESI) $m / z$ calcd for $\mathrm{C}_{22} \mathrm{H}_{24} \mathrm{~F}_{3} \mathrm{~N}_{4} \mathrm{O}[\mathrm{M}+\mathrm{H}]^{+} 417.1902$ found 417.1897 .<smiles>[X]c1c2c(cc(C)c1C(C)NC(=O)c1nnn(Cc3ccccc3)c1C)OCO2</smiles>

\section{1-Benzyl-N-\{1-(4,6-dimethylbenzo[d][1,3]dioxol-5-yl)ethyl\}-5-methyl-1H-1,2,3-} triazole-4-carboxamide (179t)

Prepared following the general procedure A by using $178 \mathrm{t}(72.9 \mathrm{mg}, 0.20 \mathrm{mmol})$. Purification by column chromatography ( $n$-hexane/EtOAc 2:1) yielded $179 t$ (70.8 mg, 90\%) as a white solid. M.p. $=87-88^{\circ} \mathrm{C} .{ }^{1} \mathrm{H}$ NMR $\left(400 \mathrm{MHz}, \mathrm{CDCl}_{3}\right) \delta=7.70(\mathrm{~d}, \mathrm{~J}=7.4 \mathrm{~Hz}, 1 \mathrm{H})$, 7.35-7.30 (m, 3H), 7.14-7.12 (m, 2H), 6.49 (s, 1H), 5.86 (s, 2H), 5.56 (dq, J = 7.3, 7.4 Hz, 1H), $5.48(\mathrm{~s}, 2 \mathrm{H}), 2.45(\mathrm{~s}, 3 \mathrm{H}), 2.45(\mathrm{~s}, 3 \mathrm{H}), 2.39(\mathrm{~s}, 3 \mathrm{H}), 1.58(\mathrm{~d}, J=7.3 \mathrm{~Hz}, 3 \mathrm{H}) .{ }^{13} \mathrm{C}$ NMR $\left(101 \mathrm{MHz}, \mathrm{CDCl}_{3}\right) \delta=160.4\left(\mathrm{C}_{\mathrm{q}}\right), 145.1\left(\mathrm{C}_{\mathrm{q}}\right), 145.2\left(\mathrm{C}_{\mathrm{q}}\right), 138.7\left(\mathrm{C}_{\mathrm{q}}\right), 136.0\left(\mathrm{C}_{\mathrm{q}}\right), 134.0\left(\mathrm{C}_{\mathrm{q}}\right)$, $132.6\left(\mathrm{C}_{\mathrm{q}}\right), 129.0(\mathrm{CH}), 128.6\left(\mathrm{C}_{\mathrm{q}}\right), 128.5(\mathrm{CH}), 127.1(\mathrm{CH}), 117.1\left(\mathrm{C}_{\mathrm{q}}\right), 109.0(\mathrm{CH}), 100.4$ $\left(\mathrm{CH}_{2}\right), 51.7\left(\mathrm{CH}_{2}\right), 45.1(\mathrm{CH}), 20.94\left(\mathrm{CH}_{3}\right), 20.0\left(\mathrm{CH}_{3}\right), 12.7\left(\mathrm{CH}_{3}\right), 8.7\left(\mathrm{CH}_{3}\right)$. IR (ATR): $3311,2883,1647,1512,1247,743,462 \mathrm{~cm}^{-1}$. MS (ESI) $\mathrm{m} / \mathrm{z}$ (relative intensity) 415 (100) $[\mathrm{M}+\mathrm{Na}]^{+}, 393(60)[\mathrm{M}+\mathrm{H}]^{+}, 217(30)$. HR-MS (ESI) $\mathrm{m} / \mathrm{z}$ calcd for $\mathrm{C}_{22} \mathrm{H}_{25} \mathrm{~N}_{4} \mathrm{O}_{3}[\mathrm{M}+\mathrm{H}]^{+}$ 393.1927 found 393.1921 .<smiles>COc1ccc(C)c(C(C)NC(=O)c2nnn(Cc3ccccc3)c2C)c1C</smiles> 
1-Benzyl-N-[1-(3-methoxy-2,6-dimethylphenyl)ethyl]-5-methyl-1H-1,2,3-triazole-4carboxamide (179u)

Prepared following the general procedure A by using $178 \mathrm{u}(69.8 \mathrm{mg}, 0.20 \mathrm{mmol})$. Purification by column chromatography ( $n$-hexane/EtOAc 2:1) yielded $179 u$ (67.5 mg, 89\%) as a colorless liquid. ${ }^{1} \mathrm{H}$ NMR $\left(300 \mathrm{MHz}, \mathrm{CDCl}_{3}\right) \delta=7.79(\mathrm{~d}, J=7.7 \mathrm{~Hz}, 1 \mathrm{H}), 7.36-7.29$ (m, 3H), 7.14-7.11 (m, 2H), $6.96(\mathrm{~d}, J=8.3 \mathrm{~Hz}, 1 \mathrm{H}), 6.67(\mathrm{~d}, J=8.3 \mathrm{~Hz}, 1 \mathrm{H}), 5.70(\mathrm{q}, J=$ $7.3 \mathrm{~Hz}, 1 \mathrm{H}), 5.48(\mathrm{~s}, 2 \mathrm{H}), 3.78(\mathrm{~s}, 3 \mathrm{H}), 2.48(\mathrm{~s}, 3 \mathrm{H}), 2.45(\mathrm{~s}, 3 \mathrm{H}), 2.41(\mathrm{~s}, 3 \mathrm{H}), 1.62(\mathrm{~d}, J=$ $7.3 \mathrm{~Hz}, 3 \mathrm{H}) .{ }^{13} \mathrm{C}$ NMR (126 MHz, $\left.\mathrm{CDCl}_{3}\right) \delta=160.2\left(\mathrm{C}_{\mathrm{q}}\right), 156.30\left(\mathrm{C}_{\mathrm{q}}\right), 140.0\left(\mathrm{C}_{\mathrm{q}}\right), 138.7$ $\left(\mathrm{C}_{\mathrm{q}}\right), 135.8\left(\mathrm{C}_{\mathrm{q}}\right), 134.0\left(\mathrm{C}_{\mathrm{q}}\right), 128.9(\mathrm{CH}), 128.4(\mathrm{CH}), 127.2\left(\mathrm{C}_{\mathrm{q}}\right), 127.0(\mathrm{CH}), 124.1\left(\mathrm{C}_{\mathrm{q}}\right)$, $109.0(\mathrm{CH}), 55.6\left(\mathrm{CH}_{3}\right), 51.7\left(\mathrm{CH}_{2}\right), 45.5(\mathrm{CH}), 20.6\left(\mathrm{CH}_{3}\right), 19.9\left(\mathrm{CH}_{3}\right), 12.5\left(\mathrm{CH}_{3}\right), 8.8$ $\left(\mathrm{CH}_{3}\right)$. IR (ATR): 3351, 2935, 1644, 1580, 1450, 731, $472 \mathrm{~cm}^{-1}$. MS (ESI) m/z (relative intensity) $401(100)[\mathrm{M}+\mathrm{Na}]^{+}, 379(50)[\mathrm{M}+\mathrm{H}]^{+}, 243$ (10), 217 (20), 163 (25). HR-MS (ESI) $\mathrm{m} / \mathrm{z}$ calcd for $\mathrm{C}_{22} \mathrm{H}_{27} \mathrm{~N}_{4} \mathrm{O}_{2}[\mathrm{M}+\mathrm{H}]^{+} 379.2134$ found 379.2129 .<smiles>Cc1cncc(C)c1C(C)NC(=O)c1nnn(Cc2ccccc2)c1C</smiles>

\section{1-Benzyl-N-[1-(3,5-dimethylpyridin-4-yl)ethyl]-5-methyl-1H-1,2,3-triazole-4-carboxa} mide (179v)

Prepared following the general procedure A by using $178 \mathrm{v}(64.0 \mathrm{mg}, 0.20 \mathrm{mmol})$. Purification by column chromatography (EtOAc/n-hexane $1: 1$ to EtOAc) yielded $179 \mathrm{v}(36.3$ mg, $52 \%)$ as a yellow liquid. ${ }^{1} \mathrm{H}$ NMR $\left(500 \mathrm{MHz} \mathrm{CDCl}_{3}\right) \delta=8.21(\mathrm{~s}, 2 \mathrm{H}), 7.65(\mathrm{~d}, J=7.1$ $\mathrm{Hz}, 1 \mathrm{H}), 7.33-7.27(\mathrm{~m}, 3 \mathrm{H}), 7.13-7.11(\mathrm{~m}, 2 \mathrm{H}), 5.51$ (q, J = 7.4 Hz, 1H), 5.47 (s, 2H), 2.46 (s, 6H), $2.40(\mathrm{~s}, 3 \mathrm{H}), 1.56(\mathrm{~d}, J=7.4 \mathrm{~Hz}, 3 \mathrm{H}) .{ }^{13} \mathrm{C} \mathrm{NMR}\left(126 \mathrm{MHz}, \mathrm{CDCl}_{3}\right) \delta=160.5\left(\mathrm{C}_{\mathrm{q}}\right)$, $149.4(\mathrm{CH}), 148.9\left(\mathrm{C}_{\mathrm{q}}\right), 138.1\left(\mathrm{C}_{\mathrm{q}}\right), 136.2\left(\mathrm{C}_{\mathrm{q}}\right), 133.8\left(\mathrm{C}_{\mathrm{q}}\right), 130.6\left(\mathrm{C}_{\mathrm{q}}\right), 129.0(\mathrm{CH}), 128.5$ (CH), $127.1(\mathrm{CH}), 51.8\left(\mathrm{CH}_{2}\right), 45.0(\mathrm{CH}), 18.4\left(\mathrm{CH}_{3}\right), 17.3\left(\mathrm{CH}_{3}\right), 8.6\left(\mathrm{CH}_{3}\right)$. IR (ATR): 3419, $2931,1663,1503,1252,730,596 \mathrm{~cm}^{-1}$. MS (ESI) $\mathrm{m} / \mathrm{z}$ (relative intensity) $372(10)[\mathrm{M}+\mathrm{Na}]^{+}$, 
$350(100)[\mathrm{M}+\mathrm{H}]^{+}, 336(10), 117$ (8). HR-MS (ESI) $\mathrm{m} / z$ calcd for $\mathrm{C}_{20} \mathrm{H}_{24} \mathrm{~N}_{5} \mathrm{O}[\mathrm{M}+\mathrm{H}]^{+}$ 350.1981 found 350.1975 .<smiles>Cc1cccc2c1C(NC(=O)c1nnn(Cc3ccccc3)c1C)CCC2</smiles>

1-Benzyl-5-methyl-N-(8-methyl-1,2,3,4-tetrahydronaphthalen-1-yl)-1H-1,2,3-triazole4-carboxamide (179w)

Prepared following the general procedure A by using $178 \mathrm{w}(69.3 \mathrm{mg}, 0.20 \mathrm{mmol})$. Purification by column chromatography ( $n$-hexane/EtOAc 2:1) yielded $179 w$ (60.3 mg, 83\%) as a colorless liquid. ${ }^{1} \mathrm{H}$ NMR $\left(300 \mathrm{MHz}, \mathrm{CDCl}_{3}\right) \delta=7.37-7.29(\mathrm{~m}, 4 \mathrm{H}), 7.18-7.15(\mathrm{~m}, 2 \mathrm{H})$, $7.12(\mathrm{~d}, J=7.5 \mathrm{~Hz}, 1 \mathrm{H}), 7.01(\mathrm{t}, J=7.1 \mathrm{~Hz}, 2 \mathrm{H}), 5.50(\mathrm{~s}, 2 \mathrm{H}), 5.35-5.31(\mathrm{~m}, 1 \mathrm{H}), 2.92$ $2.72(\mathrm{~m}, 2 \mathrm{H}), 2.54(\mathrm{~s}, 3 \mathrm{H}), 2.28(\mathrm{~s}, 3 \mathrm{H}), 2.27-2.25(\mathrm{~m}, 1 \mathrm{H}), 1.91-1.79(\mathrm{~m}, 3 \mathrm{H}) .{ }^{13} \mathrm{C}$ NMR $\left(126 \mathrm{MHz}, \mathrm{CDCl}_{3}\right) \delta=159.9\left(\mathrm{C}_{\mathrm{q}}\right), 138.5\left(\mathrm{C}_{\mathrm{q}}\right), 137.9\left(\mathrm{C}_{\mathrm{q}}\right), 137.7\left(\mathrm{C}_{\mathrm{q}}\right), 136.0\left(\mathrm{C}_{\mathrm{q}}\right), 133.9\left(\mathrm{C}_{\mathrm{q}}\right)$, $133.3\left(\mathrm{C}_{\mathrm{q}}\right), 128.9(\mathrm{CH}), 128.4(\mathrm{CH}), 128.1(\mathrm{CH}), 127.4(\mathrm{CH}), 127.1(\mathrm{CH}), 51.8\left(\mathrm{CH}_{2}\right), 44.1$ (CH), $29.8\left(\mathrm{CH}_{2}\right), 29.6\left(\mathrm{CH}_{2}\right), 18.8\left(\mathrm{CH}_{3}\right), 18.0\left(\mathrm{CH}_{2}\right), 8.9\left(\mathrm{CH}_{3}\right)$. IR (ATR): 3412, 2930 , 1660, 1497, 1249, 774, $574 \mathrm{~cm}^{-1}$. MS (ESI) $\mathrm{m} / \mathrm{z}$ (relative intensity) $383(100)[\mathrm{M}+\mathrm{Na}]^{+}, 361$ (80) $[\mathrm{M}+\mathrm{H}]^{+}, 217$ (80), 145 (25). HR-MS (ESI) $\mathrm{m} / z$ calcd for $\mathrm{C}_{22} \mathrm{H}_{25} \mathrm{~N}_{4} \mathrm{O}[\mathrm{M}+\mathrm{H}]^{+} 361.2028$ found 361.2023 .<smiles>Cc1cc2ccccc2cc1C(C)NC(=O)c1nnn(Cc2ccccc2)c1C</smiles>

\section{1-Benzyl-5-methyl-N-[1-(3-methylnaphthalen-2-yl)ethyl]-1H-1,2,3-triazole-4-carbox amide (179x)}

Prepared following the general procedure $\mathbf{A}$ by using $178 \mathrm{x}(74.3 \mathrm{mg}, 0.20 \mathrm{mmol})$. Purification by column chromatography ( $n$-hexane/EtOAc $2: 1)$ yielded $179 x(71.2 \mathrm{mg}, 91 \%)$ 
as a white solid. M.p. $=65-71^{\circ} \mathrm{C} .{ }^{1} \mathrm{H}$ NMR $\left(300 \mathrm{MHz}, \mathrm{CDCl}_{3}\right) \delta=7.87(\mathrm{~s}, 1 \mathrm{H}), 7.81-7.72$ $(\mathrm{m}, 2 \mathrm{H}), 7.63(\mathrm{~s}, 1 \mathrm{H}), 7.59(\mathrm{~d}, J=8.0 \mathrm{~Hz}, 1 \mathrm{H}), 7.44-7.38(\mathrm{~m}, 2 \mathrm{H}), 7.36-7.30(\mathrm{~m}, 3 \mathrm{H}), 7.16-$ $7.12(\mathrm{~m}, 2 \mathrm{H}), 5.59(\mathrm{q}, J=6.8 \mathrm{~Hz}, 1 \mathrm{H}), 5.47(\mathrm{~s}, 2 \mathrm{H}), 2.6(\mathrm{~s}, 3 \mathrm{H}), 2.48(\mathrm{~s}, 3 \mathrm{H}), 1.68(\mathrm{~d}, J=$ $6.8 \mathrm{~Hz}, 3 \mathrm{H}) .{ }^{13} \mathrm{C}$ NMR $\left(126 \mathrm{MHz}, \mathrm{CDCl}_{3}\right) \delta=160.2\left(\mathrm{C}_{\mathrm{q}}\right), 140.2\left(\mathrm{C}_{\mathrm{q}}\right), 138.6\left(\mathrm{C}_{\mathrm{q}}\right), 136.1\left(\mathrm{C}_{\mathrm{q}}\right)$, $133.9\left(\mathrm{C}_{\mathrm{q}}\right), 133.7\left(\mathrm{C}_{\mathrm{q}}\right), 132.7\left(\mathrm{C}_{\mathrm{q}}\right), 132.1\left(\mathrm{C}_{\mathrm{q}}\right), 128.9(\mathrm{CH}), 128.6(\mathrm{CH}), 128.4(\mathrm{CH}), 127.4$ $(\mathrm{CH}), 127.0(\mathrm{CH}), 126.7(\mathrm{CH}), 125.7(\mathrm{CH}), 125.1(\mathrm{CH}), 123.7(\mathrm{CH}), 51.7\left(\mathrm{CH}_{2}\right), 45.2(\mathrm{CH})$, $21.7\left(\mathrm{CH}_{3}\right), 19.6\left(\mathrm{CH}_{3}\right), 8.8\left(\mathrm{CH}_{3}\right)$. IR (ATR): 3410, 2974, 1662, 1504, 1251, 732, 540 $\mathrm{cm}^{-1}$. MS (ESI) m/z (relative intensity) 407 (100) [M+Na] $]^{+}, 385(40)[\mathrm{M}+\mathrm{H}]^{+}, 258(10), 212$ (20), 169 (45). HR-MS (ESI) m/z calcd for $\mathrm{C}_{24} \mathrm{H}_{25} \mathrm{~N}_{4} \mathrm{O}[\mathrm{M}+\mathrm{H}]^{+} 385.2028$ found 385.2023.<smiles>CCc1cccc(F)c1C(C)NC(=O)c1nnn(Cc2ccccc2)c1C</smiles>

\section{1-Benzyl-N-[1-(2-ethyl-6-fluorophenyl)ethyl]-5-methyl-1H-1,2,3-triazole-4-carbox} amide (196h)

Prepared following the general procedure B by using $178 \mathrm{~h}(68.2 \mathrm{mg}, 0.20 \mathrm{mmol})$. Purification by column chromatography ( $n$-hexane/EtOAc $2: 1)$ yielded $196 \mathrm{~h}(63.7 \mathrm{mg}, 81 \%)$ as a colorless liquid. ${ }^{1} \mathrm{H}$ NMR $\left(400 \mathrm{MHz}, \mathrm{CDCl}_{3}\right) \delta=7.91(\mathrm{~d}, J=8.9 \mathrm{~Hz}, 1 \mathrm{H}), 7.26-7.20$ (m, 3H), 7.09-7.03 (m, 3H), 6.88-6.80 (m, 2H), 5.64-5.57 (m, 1H), 5.40 (d, J=2.6 Hz, 2H), $2.88(\mathrm{dq}, J=15.0,7.6 \mathrm{~Hz}, 1 \mathrm{H}), 2.72(\mathrm{dq}, J=15.0,7.6 \mathrm{~Hz}, 1 \mathrm{H}), 2.38(\mathrm{~s}, 3 \mathrm{H}), 1.55(\mathrm{~d}, J=$ $6.0 \mathrm{~Hz}, 3 \mathrm{H}), 1.20(\mathrm{t}, J=7.6 \mathrm{~Hz}, 3 \mathrm{H}) \cdot{ }^{13} \mathrm{C} \mathrm{NMR}\left(101 \mathrm{MHz}, \mathrm{CDCl}_{3}\right) \delta=162.3\left(\mathrm{~d},{ }^{1} \mathrm{~J}_{\mathrm{C}-\mathrm{F}}=244.3\right.$ $\left.\mathrm{Hz}, \mathrm{C}_{\mathrm{q}}\right), 160.3\left(\mathrm{C}_{\mathrm{q}}\right), 143.5\left(\mathrm{~d},{ }^{4} \mathrm{~J}_{\mathrm{C}-\mathrm{F}}=4.5 \mathrm{~Hz}, \mathrm{C}_{\mathrm{q}}\right), 138.8\left(\mathrm{C}_{\mathrm{q}}\right), 136.1\left(\mathrm{C}_{\mathrm{q}}\right), 134.1\left(\mathrm{C}_{\mathrm{q}}\right), 129.0$ $(\mathrm{CH}), 128.4(\mathrm{CH}), 128.3\left(\mathrm{~d},{ }^{3} \mathrm{~J}_{\mathrm{C}-\mathrm{F}}=9.8 \mathrm{~Hz}, \mathrm{CH}\right), 128.2\left(\mathrm{~d},{ }^{3} \mathrm{~J}_{\mathrm{C}-\mathrm{F}}=11.3 \mathrm{~Hz}, \mathrm{C}_{\mathrm{q}}\right), 127.08(\mathrm{CH})$, $124.6\left(\mathrm{~d},{ }^{4} \mathrm{~J}_{\mathrm{C}-\mathrm{F}}=2.6 \mathrm{~Hz}, \mathrm{CH}\right), 113.8\left(\mathrm{~d},{ }^{2} \mathrm{~J}_{\mathrm{C}-\mathrm{F}}=22.7 \mathrm{~Hz}, \mathrm{CH}\right), 51.7\left(\mathrm{CH}_{2}\right), 42.1(\mathrm{CH}), 29.6$ $\left(\mathrm{CH}_{2}\right), 26.4\left(\mathrm{~d},{ }^{4} \mathrm{~J}_{\mathrm{C}-\mathrm{F}}=2.2 \mathrm{~Hz}, \mathrm{CH}_{2}\right), 21.2\left(\mathrm{~d},{ }^{4} \mathrm{~J}_{\mathrm{C}-\mathrm{F}}=3.4 \mathrm{~Hz}, \mathrm{CH}_{3}\right), 15.7\left(\mathrm{CH}_{3}\right), 8.7\left(\mathrm{CH}_{3}\right)$. ${ }^{19} \mathrm{~F}$ NMR (376 MHz, CDCl 3 ) $\delta=-116.1$ (dd, $\left.J=11.5,5.6 \mathrm{~Hz}\right)$. IR (ATR): 3421, 2968, 1665, $1505,730,579 \mathrm{~cm}^{-1}$. MS (ESI) $\mathrm{m} / \mathrm{z}$ (relative intensity) $389(100)[\mathrm{M}+\mathrm{Na}]^{+}, 367(60)[\mathrm{M}+\mathrm{H}]^{+}$, 217 (40). HR-MS (ESI) $\mathrm{m} / \mathrm{z}$ calcd for $\mathrm{C}_{21} \mathrm{H}_{24} \mathrm{FN}_{4} \mathrm{O}[\mathrm{M}+\mathrm{H}]^{+} 367.1934$ found 367.1929. 
<smiles>COc1ccc(-c2cccc(F)c2CNC(=O)c2nnn(Cc3ccccc3)c2C)cc1</smiles>

1-Benzyl-N-\{1-(3-fluoro-4'-methoxy-[1,1'-biphenyl]-2-yl)ethyl\}-5-methyl-1 H-1,2,3-tria zole-4-carboxamide (197a)

Prepared following the general procedure B by using $178 \mathrm{~h}(67.9 \mathrm{mg}, 0.20 \mathrm{mmol})$. Purification by column chromatography ( $n$-hexane/EtOAc 2:1) yielded $197 \mathrm{a}(66.7 \mathrm{mg}, 75 \%)$ as a colorless liquid. ${ }^{1} \mathrm{H}$ NMR $\left(300 \mathrm{MHz}, \mathrm{CDCl}_{3}\right) \delta=7.83(\mathrm{~d}, \mathrm{~J}=8.9 \mathrm{~Hz}, 1 \mathrm{H}), 7.28-7.20$ (m, 5H), 7.16-7.10 (m, 1H), 7.06-6.88 (m, 6H), 5.45-5.33 (m, 3H), $3.76(\mathrm{~s}, 3 \mathrm{H}), 2.36(\mathrm{~s}$, $3 \mathrm{H}), 1.44(\mathrm{dd}, J=7.0,0.9 \mathrm{~Hz}, 3 \mathrm{H}) \cdot{ }^{13} \mathrm{C}$ NMR $\left(75 \mathrm{MHz}, \mathrm{CDCl}_{3}\right) \delta=162.1\left(\mathrm{~d},{ }^{1} \mathrm{~J}_{\mathrm{C}-\mathrm{F}}=245.2\right.$ $\left.\mathrm{Hz}, \mathrm{C}_{\mathrm{q}}\right), 160.1\left(\mathrm{C}_{\mathrm{q}}\right), 159.1\left(\mathrm{C}_{\mathrm{q}}\right), 142.9\left(\mathrm{~d},{ }^{3} \mathrm{~J}_{\mathrm{C}-\mathrm{F}}=5.2 \mathrm{~Hz}, \mathrm{C}_{\mathrm{q}}\right), 139.0\left(\mathrm{C}_{\mathrm{q}}\right), 136.0\left(\mathrm{C}_{\mathrm{q}}\right), 134.2$ $\left(\mathrm{C}_{\mathrm{q}}\right), 132.2\left(\mathrm{C}_{\mathrm{q}}\right), 130.4(\mathrm{CH}), 129.1(\mathrm{CH}), 128.5(\mathrm{CH}), 128.3\left(\mathrm{C}_{\mathrm{q}}\right), 128.0\left(\mathrm{~d},{ }^{3} \mathrm{~J}_{\mathrm{C}-\mathrm{F}}=9.9 \mathrm{~Hz}\right.$, $\mathrm{CH}), 127.1(\mathrm{CH}), 126.5\left(\mathrm{~d},{ }^{3} \mathrm{~J}_{\mathrm{C}-\mathrm{F}}=2.7 \mathrm{~Hz}, \mathrm{CH}\right), 115.0\left(\mathrm{~d},{ }^{2} \mathrm{~J}_{\mathrm{C}-\mathrm{F}}=22.7 \mathrm{~Hz}, \mathrm{CH}\right), 113.8(\mathrm{CH})$, $55.3\left(\mathrm{CH}_{3}\right), 51.8\left(\mathrm{CH}_{2}\right), 43.9(\mathrm{CH}), 21.6\left(\mathrm{~d},{ }^{3} \mathrm{~J}_{\mathrm{C}-\mathrm{F}}=2.6 \mathrm{~Hz}, \mathrm{CH}_{3}\right), 8.8\left(\mathrm{CH}_{3}\right) .{ }^{19} \mathrm{~F}$ NMR $(282$ $\left.\mathrm{MHz}, \mathrm{CDCl}_{3}\right) \delta=-115.9$. IR (ATR): $3422,2930,1668,1509,1248,730,569 \mathrm{~cm}^{-1} . \mathbf{M S}$ (ESI) m/z (relative intensity) $467(100)[\mathrm{M}+\mathrm{Na}]^{+}, 445(60)[\mathrm{M}+\mathrm{H}]^{+}, 288(10), 217$ (25), $177(20)$. HR-MS (ESI) $m / z$ calcd for $\mathrm{C}_{26} \mathrm{H}_{26} \mathrm{FN}_{4} \mathrm{O}_{2}[\mathrm{M}+\mathrm{H}]^{+} 445.2040$ found 445.2034 .<smiles>Cc1c(C(=O)NCc2c(F)cccc2-c2ccc(F)cc2)nnn1Cc1ccccc1</smiles>

1-Benzyl-N-\{1-[3,4'-difluoro-(1,1'-biphenyl)-2-yl]ethyl\}-5-methyl-1H-1,2,3-triazole-4carboxamide (197b)

Prepared following the general procedure B by using $178 \mathrm{~h}(67.9 \mathrm{mg}, 0.20 \mathrm{mmol})$. Purification by column chromatography ( $n$-hexane/EtOAc $2: 1)$ yielded $197 \mathrm{~b}(72.1 \mathrm{mg}, 83 \%)$ 
as a white solid. M.p. $=134-136{ }^{\circ} \mathrm{C} .{ }^{1} \mathrm{H}$ NMR $\left(300 \mathrm{MHz}, \mathrm{CDCl}_{3}\right) \delta=7.81(\mathrm{~d}, \mathrm{~J}=8.6 \mathrm{~Hz}$, 1H), 7.33-7.18 (m, 5H), 7.16-7.11 (m, 1H), 7.07-6.95 (m, 5H), 6.90-6.87 (m, 1H), $5.38(\mathrm{~d}$, $J=3.6 \mathrm{~Hz}, 2 \mathrm{H}), 5.3-5.25(\mathrm{~m}, 1 \mathrm{H}), 2.35(\mathrm{~s}, 3 \mathrm{H}), 1.43(\mathrm{dd}, J=7.0,0.9 \mathrm{~Hz}, 3 \mathrm{H}) .{ }^{13} \mathrm{C}$ NMR $\left(75 \mathrm{MHz}, \mathrm{CDCl}_{3}\right) \delta=163.9\left(\mathrm{~d},{ }^{1} \mathrm{~J}_{\mathrm{C}-\mathrm{F}}=25.7 \mathrm{~Hz}, \mathrm{C}_{\mathrm{q}}\right), 160.6\left(\mathrm{~d},{ }^{1} \mathrm{~J}_{\mathrm{C}-\mathrm{F}}=24.8 \mathrm{~Hz}, \mathrm{C}_{\mathrm{q}}\right), 160.2$ $\left(C_{q}\right), 142.1\left(d,{ }^{2} J_{C-F}=5.2 \mathrm{~Hz}, C_{q}\right), 138.9\left(C_{q}\right), 136.0\left(C_{q}\right), 135.8\left(t,{ }^{2} J_{C-F}=3.0 \mathrm{~Hz}, C_{q}\right)$, $134.2\left(\mathrm{C}_{\mathrm{q}}\right), 131.0\left(\mathrm{~d},{ }^{2} \mathrm{~J}=8.1 \mathrm{~Hz}, \mathrm{CH}\right), 129.1(\mathrm{CH}), 128.5(\mathrm{CH}), 128.4\left(\mathrm{C}_{\mathrm{q}}\right), 128.1\left(\mathrm{~d},{ }^{2} \mathrm{~J}=\right.$ $9.9 \mathrm{~Hz}, \mathrm{CH}), 127.2(\mathrm{CH}), 126.3\left(\mathrm{~d},{ }^{2} \mathrm{~J}_{\mathrm{C}-\mathrm{F}}=2.6 \mathrm{~Hz}, \mathrm{CH}\right), 115.5\left(\mathrm{~d},{ }^{1} \mathrm{~J}_{\mathrm{C}-\mathrm{F}}=22.7 \mathrm{~Hz}, \mathrm{CH}\right)$, $115.3\left(\mathrm{~d},{ }^{1} \mathrm{~J}_{\mathrm{C}-\mathrm{F}}=21.4 \mathrm{~Hz}, \mathrm{CH}\right), 51.8\left(\mathrm{CH}_{2}\right), 43.8(\mathrm{CH}), 21.5\left(\mathrm{~d},{ }^{2} \mathrm{~J}=2.6 \mathrm{~Hz}, \mathrm{CH}_{3}\right), 8.8\left(\mathrm{CH}_{3}\right)$. ${ }^{19} \mathrm{~F}$ NMR $\left(282 \mathrm{MHz}, \mathrm{CDCl}_{3}\right) \delta=-114.82(\mathrm{~s}),,-115.7(\mathrm{~s})$. IR (ATR): 3421, 2930, 1667, 1508, $1226,729,550 \mathrm{~cm}^{-1} . \mathbf{M S}(\mathrm{ESI}) \mathrm{m} / \mathrm{z}$ (relative intensity) $455(100)[\mathrm{M}+\mathrm{Na}]^{+}, 433(45)[\mathrm{M}+\mathrm{H}]^{+}$, 217 (30), 177 (20). HR-MS (ESI) $\mathrm{m} / \mathrm{z}$ calcd for $\mathrm{C}_{25} \mathrm{H}_{23} \mathrm{~F}_{2} \mathrm{~N}_{4} \mathrm{O}[\mathrm{M}+\mathrm{H}]^{+} 433.1840$ found 433.1834.<smiles>COc1ccc(-c2cccc(C(F)(F)F)c2CNC(=O)c2nnn(Cc3ccccc3)c2C)cc1</smiles>

1-Benzyl-N-\{[4'-methoxy-3-(trifluoromethyl)-(1,1'-biphenyl)-2-yl]methyl\}-5-methyl1H-1,2,3-triazole-4-carboxamide (197c)

Prepared following the general procedure B by using $178 \mathrm{I}(450 \mathrm{mg}, 1.2 \mathrm{mmol})$. Purification by column chromatography ( $n$-hexane/EtOAc $2: 1$ ) yielded 197c $(515 \mathrm{mg}, 89 \%)$ as a colorless liquid. ${ }^{1} \mathbf{H}$ NMR $\left(300 \mathrm{MHz}, \mathrm{CDCl}_{3}\right) \delta=7.67-7.61(\mathrm{~m}, 1 \mathrm{H}), 7.40-7.36(\mathrm{~m}, 2 \mathrm{H})$, 7.30-7.12 (m, 5H), 7.08-7.05 (m, 2H), $6.97(\mathrm{t}, \mathrm{J}=4.4 \mathrm{~Hz}, 1 \mathrm{H}), 6.82-6.77(\mathrm{~m}, 2 \mathrm{H}), 5.39(\mathrm{~s}$, $2 \mathrm{H}), 4.58(\mathrm{~d}, J=4.4 \mathrm{~Hz}, 2 \mathrm{H}), 3.70(\mathrm{~s}, 3 \mathrm{H}), 2.36(\mathrm{~s}, 3 \mathrm{H}) .{ }^{13} \mathrm{C} \mathrm{NMR}\left(126 \mathrm{MHz}, \mathrm{CDCl}_{3}\right) \delta=$ $159.9\left(\mathrm{C}_{\mathrm{q}}\right), 159.0\left(\mathrm{C}_{\mathrm{q}}\right), 145.5\left(\mathrm{C}_{\mathrm{q}}\right), 138.4\left(\mathrm{C}_{\mathrm{q}}\right), 135.9\left(\mathrm{C}_{\mathrm{q}}\right), 134.5(\mathrm{CH}), 134.0\left(\mathrm{C}_{\mathrm{q}}\right), 133.0$ $\left(\mathrm{C}_{\mathrm{q}}\right), 131.8\left(\mathrm{C}_{\mathrm{q}}\right), 130.1\left(\mathrm{q},{ }^{2} \mathrm{~J}_{\mathrm{C}-\mathrm{F}}=29.8 \mathrm{~Hz}, \mathrm{C}_{\mathrm{q}}\right), 129.9(\mathrm{CH}), 129.0(\mathrm{CH}), 128.4(\mathrm{CH}), 127.7$ $(\mathrm{CH}), 127.1(\mathrm{CH}), 125.4\left(\mathrm{q},{ }^{3} J_{\mathrm{C}-\mathrm{F}}=6.0 \mathrm{~Hz}, \mathrm{CH}\right), 124.3\left(\mathrm{q},{ }^{1} \mathrm{~J}_{\mathrm{C}-\mathrm{F}}=273.8 \mathrm{~Hz}, \mathrm{C}_{\mathrm{q}}\right), 113.8(\mathrm{CH})$, $55.2\left(\mathrm{CH}_{3}\right), 51.8\left(\mathrm{CH}_{2}\right), 37.7\left(\mathrm{CH}_{2}\right), 8.7\left(\mathrm{CH}_{3}\right) .{ }^{19} \mathrm{~F}$ NMR $\left(282 \mathrm{MHz}, \mathrm{CDCl}_{3}\right) \delta=-58.67$. IR (ATR): 3288, 2958, 1662, 1510, 1253, 693, $589 \mathrm{~cm}^{-1} . \mathbf{M S}$ (ESI) $\mathrm{m} / \mathrm{z}$ (relative intensity) 
$503(100)[\mathrm{M}+\mathrm{Na}]^{+}, 481(60)[\mathrm{M}+\mathrm{H}]^{+}, 217$ (30). HR-MS (ESI) $\mathrm{m} / z$ calcd for $\mathrm{C}_{26} \mathrm{H}_{24} \mathrm{~F}_{3} \mathrm{~N}_{4} \mathrm{O}_{2}$

$[\mathrm{M}+\mathrm{H}]^{+} 481.1851$ found 481.1846 .<smiles>Cc1c(C(=O)NC2CCCc3cccc(-c4ccccc4)c32)nnn1Cc1ccccc1</smiles>

1-Benzyl-5-methyl-N-(8-phenyl-1,2,3,4-tetrahydronaphthalen-1-yl)-1H-1,2,3-triazole 4-carboxamide (197e)

Prepared following the general procedure B by using $178 \mathrm{w}(69.3 \mathrm{mg}, 0.20 \mathrm{mmol})$. Purification by column chromatography ( $n$-hexane/EtOAc $2: 1$ ) yielded $197 \mathrm{~d}(63.9 \mathrm{mg}, 76 \%)$ as a colorless liquid. ${ }^{1} \mathrm{H}$ NMR $\left(300 \mathrm{MHz}, \mathrm{CDCl}_{3}\right) \delta=7.31-7.23(\mathrm{~m}, 3 \mathrm{H}), 7.17-6.99(\mathrm{~m}, 11 \mathrm{H})$, $5.37(\mathrm{~s}, 2 \mathrm{H}), 5.17-5.12(\mathrm{~m}, 1 \mathrm{H}), 2.89-2.71(\mathrm{~m}, 2 \mathrm{H}), 2.24(\mathrm{~s}, 3 \mathrm{H}), 2.08-2.00(\mathrm{~m}, 1 \mathrm{H}), 1.83-$ $1.72(\mathrm{~m}, 3 \mathrm{H}) .{ }^{13} \mathrm{C}$ NMR $\left(75 \mathrm{MHz}, \mathrm{CDCl}_{3}\right) \delta=159.2\left(\mathrm{C}_{\mathrm{q}}\right), 143.7\left(\mathrm{C}_{\mathrm{q}}\right), 141.0\left(\mathrm{C}_{\mathrm{q}}\right), 138.7\left(\mathrm{C}_{\mathrm{q}}\right)$, $138.3\left(\mathrm{C}_{\mathrm{q}}\right), 135.7\left(\mathrm{C}_{\mathrm{q}}\right), 134.3\left(\mathrm{C}_{\mathrm{q}}\right), 133.4\left(\mathrm{C}_{\mathrm{q}}\right), 129.1(\mathrm{CH}), 128.8(\mathrm{CH}), 128.7(\mathrm{CH}), 128.5$ $(\mathrm{CH}), 128.3(\mathrm{CH}), 127.7(\mathrm{CH}), 127.33(\mathrm{CH}), 127.1(\mathrm{CH}), 126.5(\mathrm{CH}), 51.7\left(\mathrm{CH}_{2}\right), 44.4(\mathrm{CH})$, $29.8\left(\mathrm{CH}_{2}\right), 29.7\left(\mathrm{CH}_{2}\right), 18.3\left(\mathrm{CH}_{2}\right), 8.6\left(\mathrm{CH}_{3}\right)$. IR (ATR): 3414, 2933, 1665, 1496, 1251, 730, $541 \mathrm{~cm}^{-1}$. MS (ESI) m/z (relative intensity) $445(100)[\mathrm{M}+\mathrm{Na}]^{+}, 423(60)[\mathrm{M}+\mathrm{H}]^{+}, 217$ (40), 158 (20). HR-MS (ESI) $m / z$ calcd for $\mathrm{C}_{27} \mathrm{H}_{27} \mathrm{~N}_{4} \mathrm{O}[\mathrm{M}+\mathrm{H}]^{+} 423.2185$ found 423.2179.

\subsubsection{Iron-catalyzed C-H methylation of chiral benzamide (S)-178a}<smiles>[I-][Al-]N[CH]c1ccccc1</smiles>

(S)-178a $(e e=99 \%)$

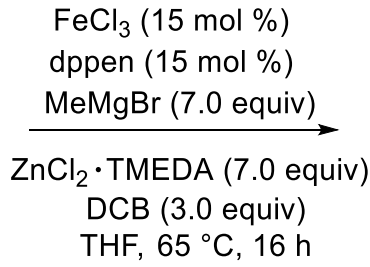

Prepared following the general procedure A by using $(S)-1 \mathrm{a}(64.0 \mathrm{mg}, 0.20 \mathrm{mmol})$. Purification by column chromatography ( $n$-hexane/EtOAc 2:1) yielded $(S)$-2a $(58.9 \mathrm{mg}$, 
$86 \%$ ) as a white solid. M.p. $=72-73{ }^{\circ} \mathrm{C}$. Chiral HPLC analysis (Chiralcel IC, $i-\mathrm{PrOH} / n$ -

hexane 30:70, flow rate $=1.0 \mathrm{~mL} / \mathrm{min}, \lambda=254 \mathrm{~nm}), \mathrm{t}_{\mathrm{r}}($ minor $)=8.7 \mathrm{~min}, \mathrm{t}_{\mathrm{r}}($ major $)=9.8 \mathrm{~min}$.

Data File E: \Chem32\1\Data\al1\def_LC_Purge 2017-05-03 10-10-28\OnlineEdited21.D

Sample Name: zsa16ra

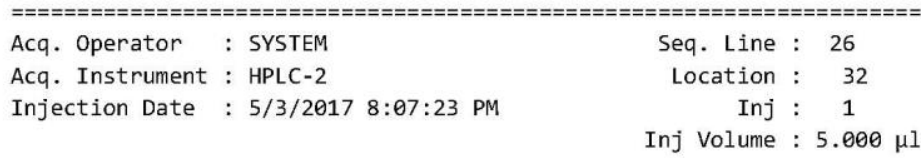

Different Inj Volume from Sample Entry! Actual Inj Volume : $2.000 \mu$

Acq. Method : E: \Chem32\1\Data \all\def_LC_Purge 2017-05-03 10-10-28\IC_hex70_iPrOH30.M

Last changed : 5/3/2017 1:45:14 PM by SYSTEM

Analysis Method : E: \Chem32\1\Data\all\def_LC_Purge 2017-05-03 10-10-28\IC_hex70_iPrOH30.M ( Sequence Method)

Last changed : 5/10/2017 3:49:49 PM by SYSTEM

Method Info : Test

Additional Info : Peak(s) manually integrated

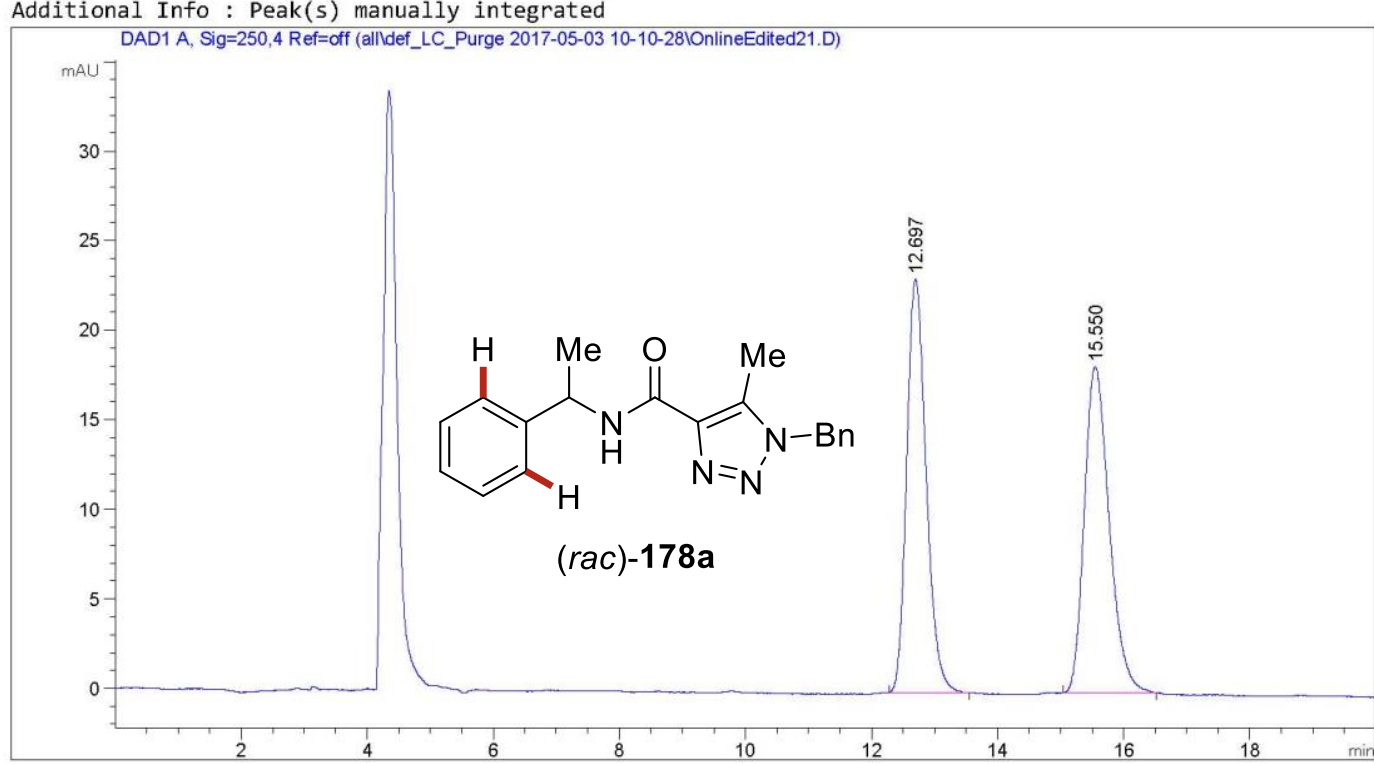

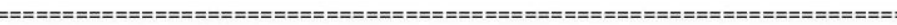

Area Percent Report

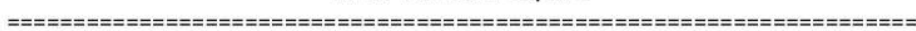

$\begin{array}{lll}\text { Sorted By } & : & \text { Signal } \\ \text { Multiplier } & : & 1.0000 \\ \text { Dilution } & : & 1.0000\end{array}$

Do not use Multiplier \& Dilution Factor with ISTDs

Signal 1: DAD1 A, Sig $=250,4$ Ref=off

\begin{tabular}{|c|c|c|c|c|c|c|}
\hline $\begin{array}{c}\text { Peak } \\
\#\end{array}$ & $\begin{array}{c}\text { RetTime } \\
\text { [min] }\end{array}$ & Type & $\begin{array}{l}\text { Width } \\
\text { [min] }\end{array}$ & $\begin{array}{c}\text { Area } \\
{[\mathrm{mAU} * \mathrm{~s}]}\end{array}$ & $\begin{array}{l}\text { Height } \\
\text { [mAU] }\end{array}$ & $\begin{array}{c}\text { Area } \\
\%\end{array}$ \\
\hline$\ldots$ & | & & - & - & - & - \\
\hline 1 & 12.697 & BB & 0.3259 & 494.62329 & 23.09455 & 49.7711 \\
\hline 2 & 15.550 & BB & 0.3999 & 499.17252 & 18.20427 & 50.2289 \\
\hline Toté & : & & & 993.79581 & 41.29882 & \\
\hline
\end{tabular}


Data File E: \Chem32\1\Data\all\def_LC_Purge 2017-05-03 10-10-28\OnlineEdited28.D Sample Name: zsa16asy

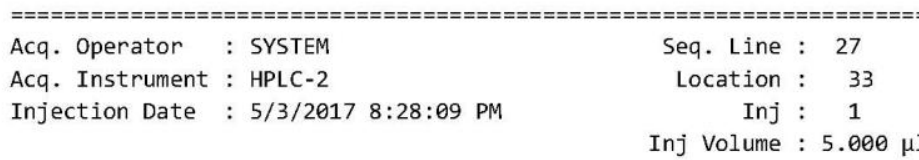

Different Inj Volume from Sample Entry! Actual Inj Volume : $2.000 \mu \mathrm{l}$

Acq. Method : E: \Chem32\1\Data\all\def_LC_Purge 2017-05-03 10-10-28\IC_hex70_iPrOH30.M

Last changed : 5/3/2017 1:45:14 PM by SYSTEM

Analysis Method : E: \Chem32\1\Data\all\def_LC_Purge 2017-05-03 10-10-28\IC_hex70_iPrOH30.M ( Sequence Method)

Last changed : 5/10/2017 3:49:49 PM by SYSTEM

Method Info : Test

Additional Info : Peak(s) manually integrated

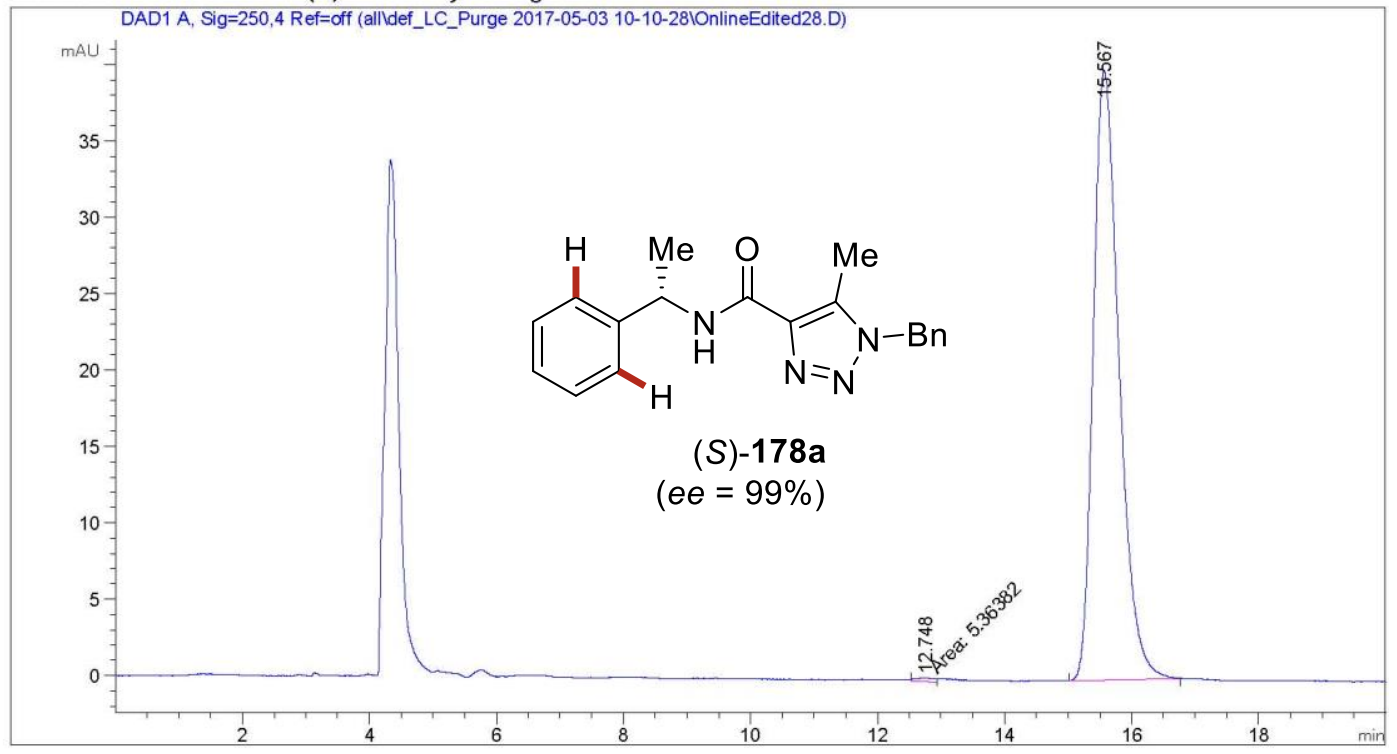

Area Percent Report

$\begin{array}{lll}\text { Sorted By } & : & \text { Signal } \\ \text { Multiplier } & : & 1.0000 \\ \text { Dilution } & : & 1.0000\end{array}$

Do not use Multiplier \& Dilution Factor with ISTDs

Signal 1: DAD1 A, Sig=250,4 Ref=off

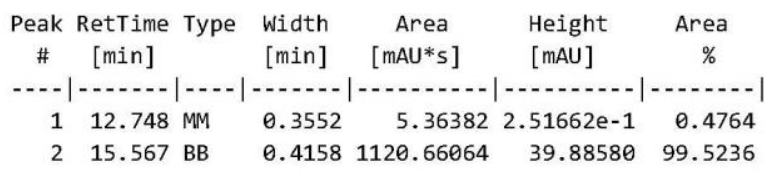

Totals : $\quad 1126.02446 \quad 40.13746$ 
Data File E: \Chem32\1\Data\all\def_LC_Purge 2017-05-05 23-02-06\031-0401.D Sample Name: zsa17ra1

\begin{tabular}{|c|c|c|c|}
\hline Acq. Operator & : SYSTEM & Seq. Line & 4 \\
\hline Acq. Instrument & : HPLC-2 & Location & 31 \\
\hline Injection Date & : 5/6/2017 12:04:42 AM & $\begin{array}{r}\text { Inj } \\
\text { Inj Volume }\end{array}$ & $\begin{array}{c}: \quad 1 \\
: 5.000 \mu 1\end{array}$ \\
\hline
\end{tabular}

Different Inj Volume from Sample Entry! Actual Inj Volume : $2.000 \mu 1$

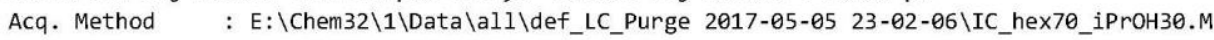

Last changed : 5/5/2017 11:02:07 PM by SYSTEM

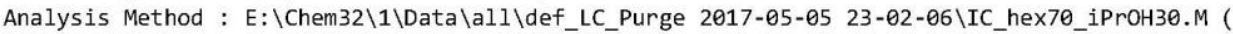
Sequence Method)

Last changed : 5/10/2017 7:31:46 PM by SYSTEM

(modified after loading)

Method Info : Test

Additional Info : Peak(s) manually integrated

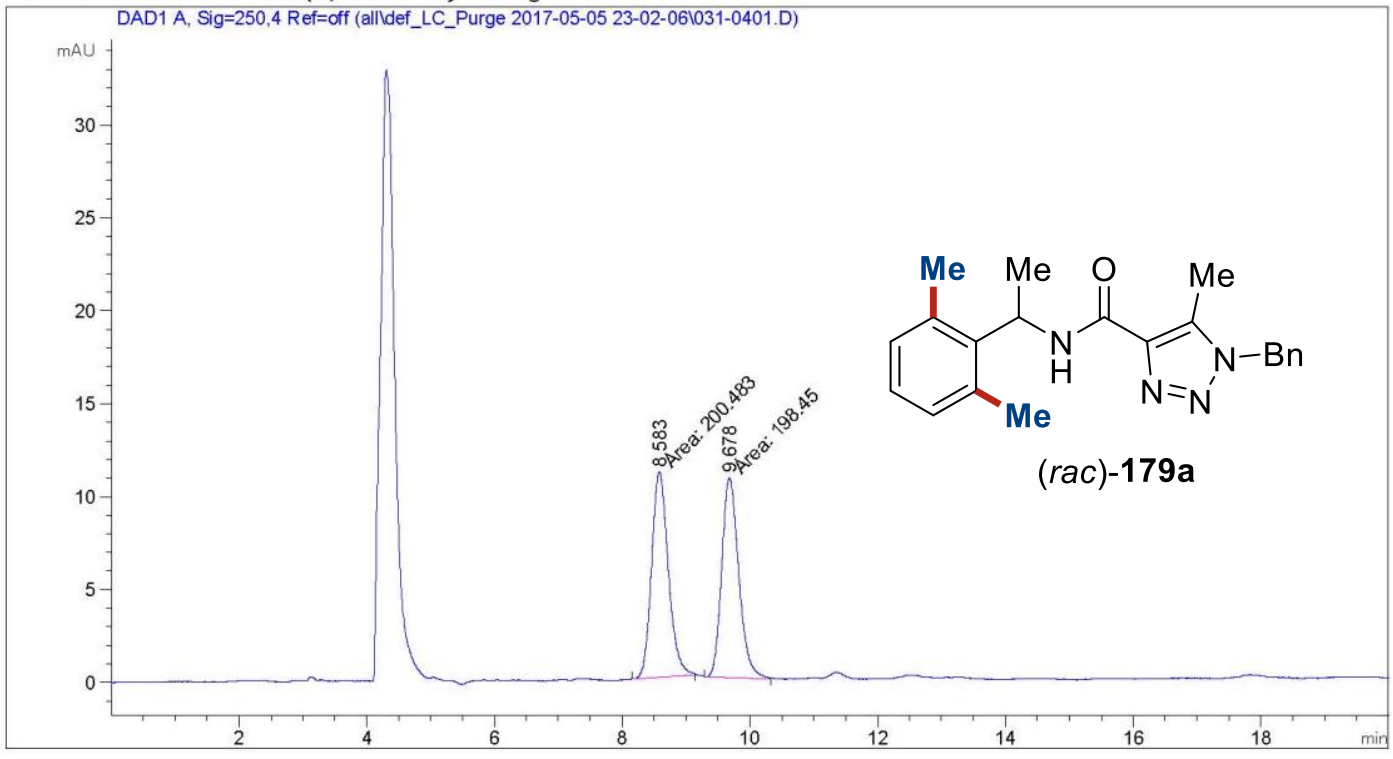

Area Percent Report

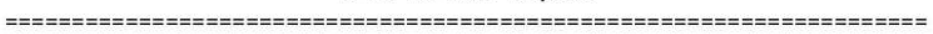

$\begin{array}{lll}\text { Sorted By } & : & \text { Signal } \\ \text { Multiplier } & : & 1.0000 \\ \text { Dilution } & : & 1.0000\end{array}$

Do not use Multiplier \& Dilution Factor with ISTDs

Signal 1: DAD1 A, Sig=250,4 Ref=off

\begin{tabular}{cccccc}
$\begin{array}{c}\text { Peak RetTime Type width } \\
\text { [min] }\end{array}$ [min] & $\begin{array}{c}\text { Area } \\
{[\text { mAU*s] }}\end{array}$ & $\begin{array}{c}\text { Height } \\
{[\mathrm{mAU}]}\end{array}$ & $\begin{array}{c}\text { Area } \\
\%\end{array}$ \\
\hline 1 & $8.583 \mathrm{MM}$ & 0.3023 & 200.48262 & 11.05368 & 50.2547 \\
2 & $9.678 \mathrm{MM}$ & 0.3082 & 198.45038 & 10.73153 & 49.7453
\end{tabular}


Data File E: \Chem32\1\Data\all\def_LC_Purge 2017-05-03 10-10-28\OnlineEdited28.D Sample Name: zsa16asy

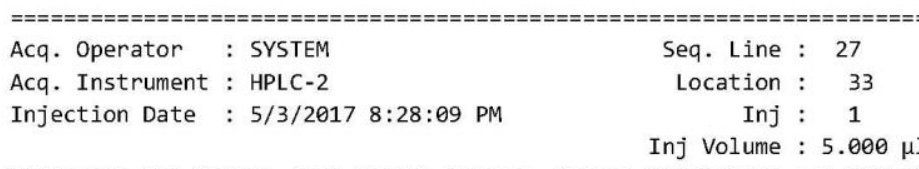

Different Inj Volume from Sample Entry! Actual Inj Volume : $2.000 \mu 1$

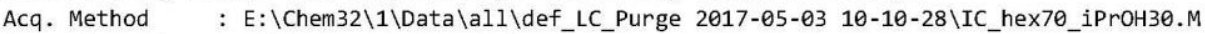

Last changed : 5/3/2017 1:45:14 PM by SYSTEM

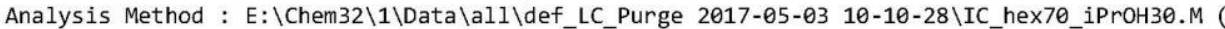
Sequence Method)

Last changed : 5/10/2017 3:49:49 PM by SYSTEM

Method Info : Test

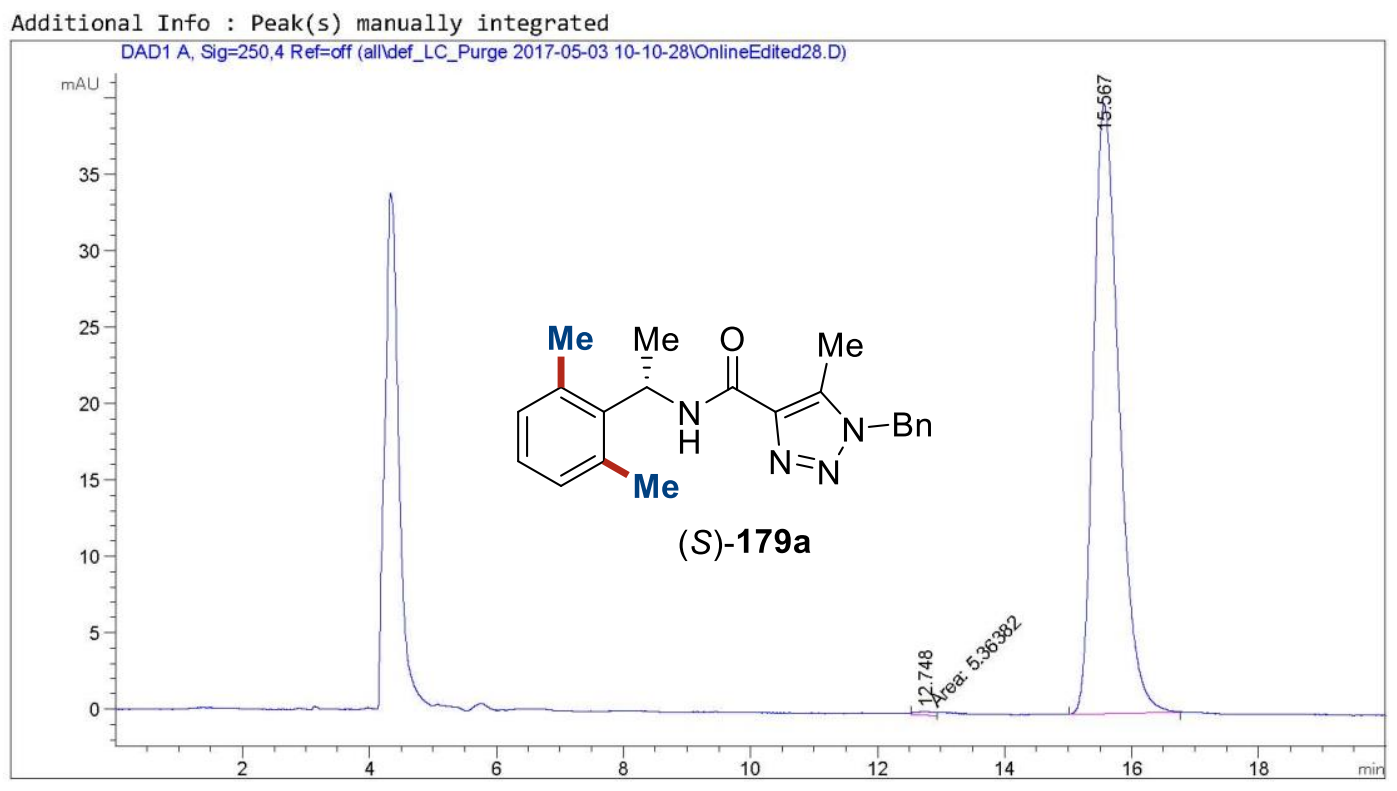

Area Percent Report

\begin{tabular}{|c|c|c|}
\hline Sorted By & : & Signal \\
\hline Multiplier & : & 1.0000 \\
\hline Dilution & : & 1.0000 \\
\hline
\end{tabular}

Do not use Multiplier \& Dilution Factor with ISTDs

Signal 1: DAD1 A, Sig=250,4 Ref=off

\begin{tabular}{|c|c|c|c|c|c|}
\hline $\begin{array}{c}\text { Peak } \\
\#\end{array}$ & $\begin{array}{l}\text { RetTime Type } \\
{[\mathrm{min}]}\end{array}$ & $\begin{array}{l}\text { Width } \\
\text { [min] }\end{array}$ & $\begin{array}{c}\text { Area } \\
{[\mathrm{mAU} * \mathrm{~s}]}\end{array}$ & $\begin{array}{l}\text { Height } \\
\text { [mAU] }\end{array}$ & $\begin{array}{c}\text { Area } \\
\%\end{array}$ \\
\hline - & 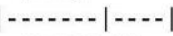 & 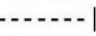 & | - & $\mid-\ldots-1$ & - \\
\hline 1 & $12.748 \mathrm{MM}$ & 0.3552 & 5.36382 & $2.51662 e-1$ & 0.4764 \\
\hline 2 & $15.567 \mathrm{BB}$ & 0.4158 & 1120.66064 & 39.88580 & 99.5236 \\
\hline Tota. & : & & 1126.02446 & 40.13746 & \\
\hline
\end{tabular}

HPLC-2 5/10/2017 7:47:18 PM SYSTEM 


\subsubsection{Parallel Experiments for KIE}<smiles>Fc1ccccc1CN[Te]</smiles>

$178 \mathrm{k}$<smiles>[2H]c1cccc(F)c1CN[Te]</smiles>

$\left[D_{1}\right]-178 k$
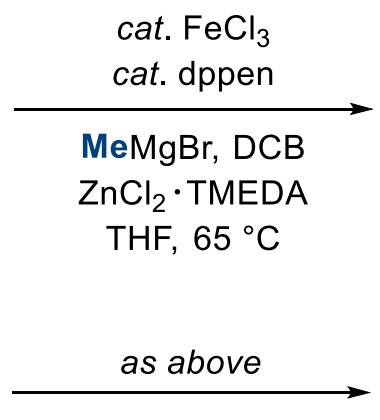<smiles>Cc1cccc(F)c1CN[Te]</smiles>

$179 k$

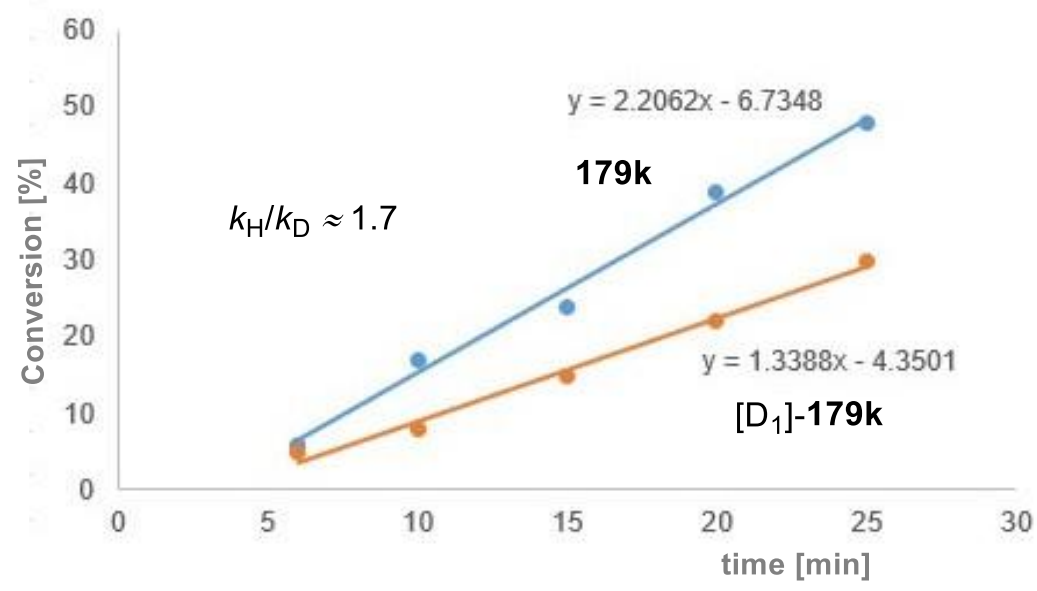

Ten independent reactions with $\mathbf{1 7 8} \mathbf{k}$ or deuterated substrate $\left[D_{1}\right]-\mathbf{1 7 8} \mathbf{k}$ under the standard conditions were performed. To a schlenk tube charged with amide $(0.20 \mathrm{mmol})$, TMEDA $\cdot \mathrm{ZnCl}_{2}$ (152 mg, $0.60 \mathrm{mmol}, 3.0$ equiv) and $1.0 \mathrm{~mL}$ THF was added $\mathrm{MeMgBr}(457$ ul, $1.4 \mathrm{mmol}, 3.0 \mathrm{M}$ in ether, 7.0 equiv) in one portion at room temperature under nitrogen gas. Five minutes later, dppen (11.9 mg, $0.030 \mathrm{mmol}, 15 \mathrm{~mol} \%$ ) and $\mathrm{FeCl}_{3}(4.9 \mathrm{mg}, 0.030$ $\mathrm{mmol}, 15 \mathrm{~mol} \%)$ were added successively. Stirring at room temperature for another one minute, 2,3-dichloridebutane was added and the reaction was moved to $65^{\circ} \mathrm{C}$ oil bath stirring for $5 \mathrm{~min}, 10 \mathrm{~min}, 15 \mathrm{~min}, 20 \mathrm{~min}, 25 \mathrm{~min}$ respectively. The yields of the products 179k were monitored by ${ }^{19} \mathrm{~F}$ NMR using benzotrifluoride as the internal standard. 


\begin{tabular}{c|ccccc}
$\mathrm{t}[\mathrm{min}]$ & 5 & 10 & 15 & 20 & 25 \\
\hline $179 \mathrm{k}[\%]$ & 6 & 17 & 24 & 39 & 48 \\
{$\left[\mathrm{D}_{1}\right]-179 k[\%]$} & 5 & 8 & 15 & 22 & 30
\end{tabular}

\subsubsection{The Removal of Directing Group}<smiles>Cc1cccc2cccc(N[Al-][SiH3])c12</smiles>

$179 m$

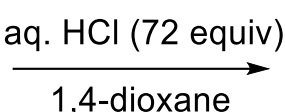
$130^{\circ} \mathrm{C}, 16 \mathrm{~h}$
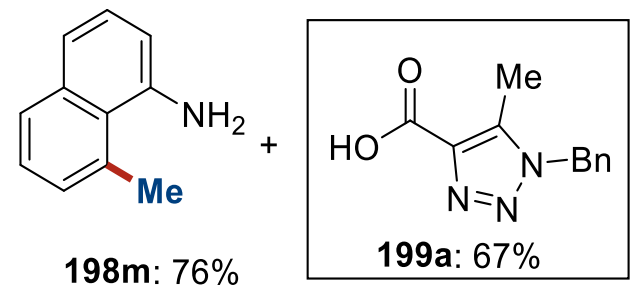

A solution of amide $179 \mathrm{~m} \mathrm{(178} \mathrm{mg,} 0.50 \mathrm{mmol})$ in $3.0 \mathrm{ml}$ concentrated hydrochloride and $1.5 \mathrm{ml}$ 1,4-dioxane under nitrogen was stirred at $130^{\circ} \mathrm{C}$ for 16 hours. Basified the mixture to $\mathrm{pH}=12$ by $3 \mathrm{M} \mathrm{KOH}$ aqueous solution and extracted with mixed solvent $(\mathrm{MeOH} / \mathrm{DCM}$ 1:8) for three times. Combined the organic layers, washed with brine and removed the solvent. The resulting crude material was purified by column chromatography ( $n$ hexane/EtOAc 1:4) yielding amine $198 \mathrm{~m}$ as a brown solid (59.4 mg, 76\%). M.p. $=68-69$ ${ }^{\circ} \mathrm{C} .{ }^{1} \mathrm{H}$ NMR $\left(300 \mathrm{MHz}, \mathrm{CDCl}_{3}\right) \delta=7.58(\mathrm{~d}, J=8.2 \mathrm{~Hz}, 1 \mathrm{H}), 7.26-7.16(\mathrm{~m}, 3 \mathrm{H}), 7.09(\mathrm{~d}, J=$ $7.0 \mathrm{~Hz}, 1 \mathrm{H}), 6.64$ (dd, J = 7.2, $1.5 \mathrm{~Hz}, 1 \mathrm{H}), 4.24$ (brs, 2H), 2.97 (s, 3H). ${ }^{13} \mathrm{C}$ NMR $(75 \mathrm{MHz}$, $\left.\mathrm{CDCl}_{3}\right) \delta=144.9\left(\mathrm{C}_{\mathrm{q}}\right), 136.5\left(\mathrm{C}_{\mathrm{q}}\right), 133.6\left(\mathrm{C}_{\mathrm{q}}\right), 128.2(\mathrm{CH}), 127.5(\mathrm{CH}), 126.0(\mathrm{CH}), 125.4$ (CH), $124.3\left(\mathrm{C}_{\mathrm{q}}\right), 120.0(\mathrm{CH}), 111.9(\mathrm{CH}), 25.2\left(\mathrm{CH}_{3}\right)$. IR (ATR): 3363, 2963, 1618, 1463, 814, 759, $671 \mathrm{~cm}^{-1}$. MS (ESI) $\mathrm{m} / \mathrm{z}$ (relative intensity) $180(100)[\mathrm{M}+\mathrm{Na}]^{+}, 158(40)[\mathrm{M}+\mathrm{H}]^{+}$, 141 (30). HR-MS (ESI) $m / z$ calcd for $\mathrm{C}_{11} \mathrm{H}_{12} \mathrm{~N}[\mathrm{M}+\mathrm{H}]^{+} 158.0970$ found 158.0964. The ${ }^{1} \mathrm{H}-$ ${ }^{1} \mathrm{H}$ COSY confirmed the structure of $198 \mathrm{~m}$ is 1 -Amino-8-methylnaphthalene rather than reported compound 1-Amino-2-methylnaphthalene. ${ }^{[177]}$

The aqueous layer was acidified to $\mathrm{pH}=3$ and extracted with mixed solvent (Methanol/DCM 1:8) for three times. Combined the organic layers, washed with brine and removed the solvent. The resulting residue was washed with very little amount of ether to afford the acid 199a as a yellow solid $(82.2 \mathrm{mg}, 76 \%)$. M.p. $=133-134{ }^{\circ} \mathrm{C} .{ }^{1} \mathrm{H}$ NMR $(300$ 
$\mathrm{MHz}, \mathrm{D}_{6}$-acetone) $\delta=7.45-7.31(\mathrm{~m}, 3 \mathrm{H}), 7.28-7.25(\mathrm{~m}, 2 \mathrm{H}), 5.67(\mathrm{~s}, 2 \mathrm{H}), 2.52(\mathrm{~s}, 3 \mathrm{H})$. ${ }^{13} \mathrm{C}$ NMR $\left(75 \mathrm{MHz}, \mathrm{D}_{6}\right.$-acetone) $\delta=167.2\left(\mathrm{C}_{\mathrm{q}}\right), 143.8\left(\mathrm{C}_{\mathrm{q}}\right), 141.7\left(\mathrm{C}_{\mathrm{q}}\right), 140.5\left(\mathrm{C}_{\mathrm{q}}\right), 134.1$ $(\mathrm{CH}), 133.4(\mathrm{CH}), 132.6(\mathrm{CH}), 56.4\left(\mathrm{CH}_{2}\right), 13.4\left(\mathrm{CH}_{3}\right)$. IR (ATR): 3443, 2923, 2854, 1716, 1570, 1183, 798, $747 \mathrm{~cm}^{-1}$. MS (ESI) m/z (relative intensity) $240(100)[\mathrm{M}+\mathrm{Na}]^{+}, 218(60)$ $[\mathrm{M}+\mathrm{H}]^{+}, 201$ (5), 91 (7). HR-MS (ESI) $\mathrm{m} / z$ calcd for $\mathrm{C}_{11} \mathrm{H}_{12} \mathrm{~N}_{3} \mathrm{O}_{2}[\mathrm{M}+\mathrm{H}]^{+} 218.0930$ found 218.0924 .

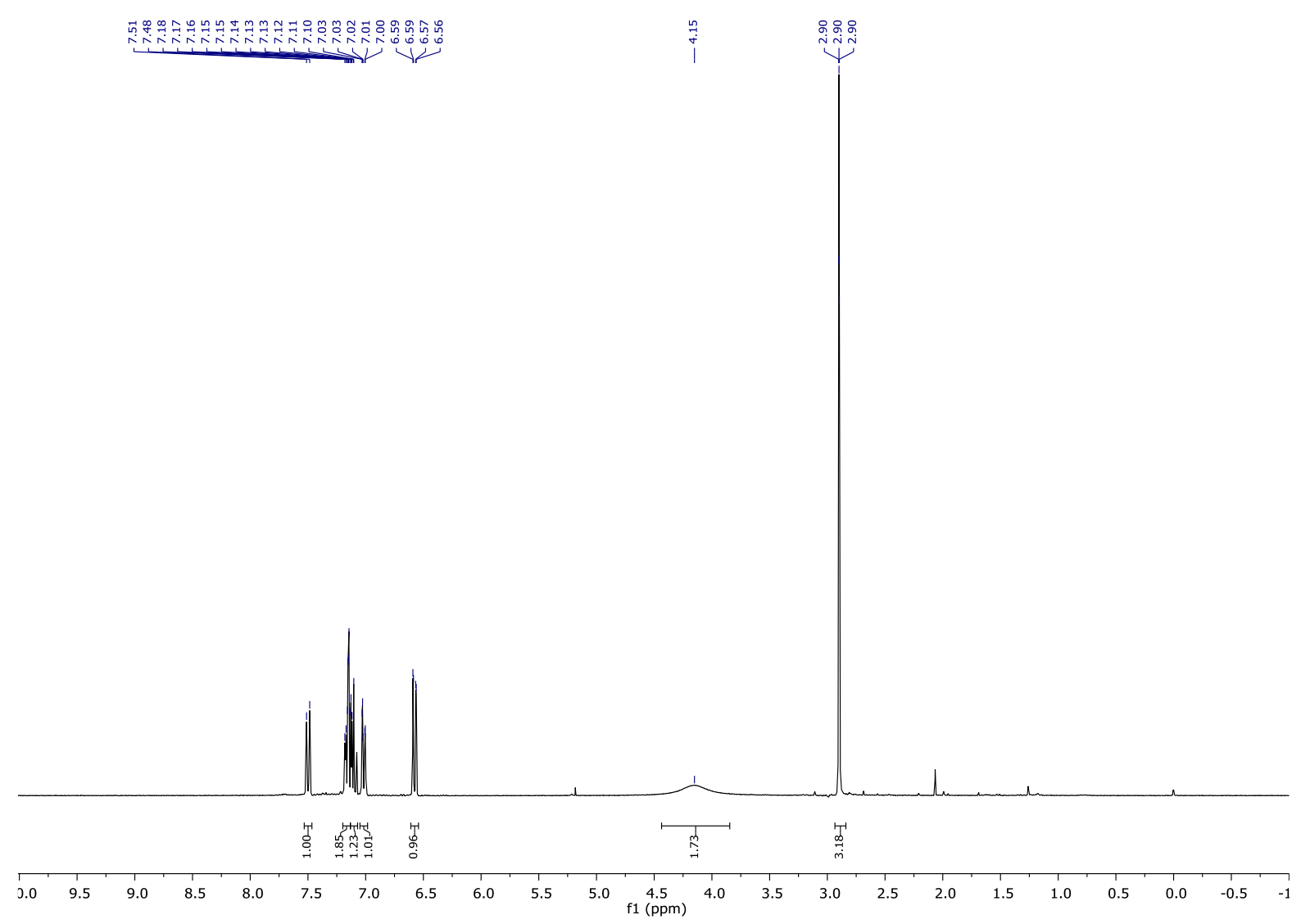



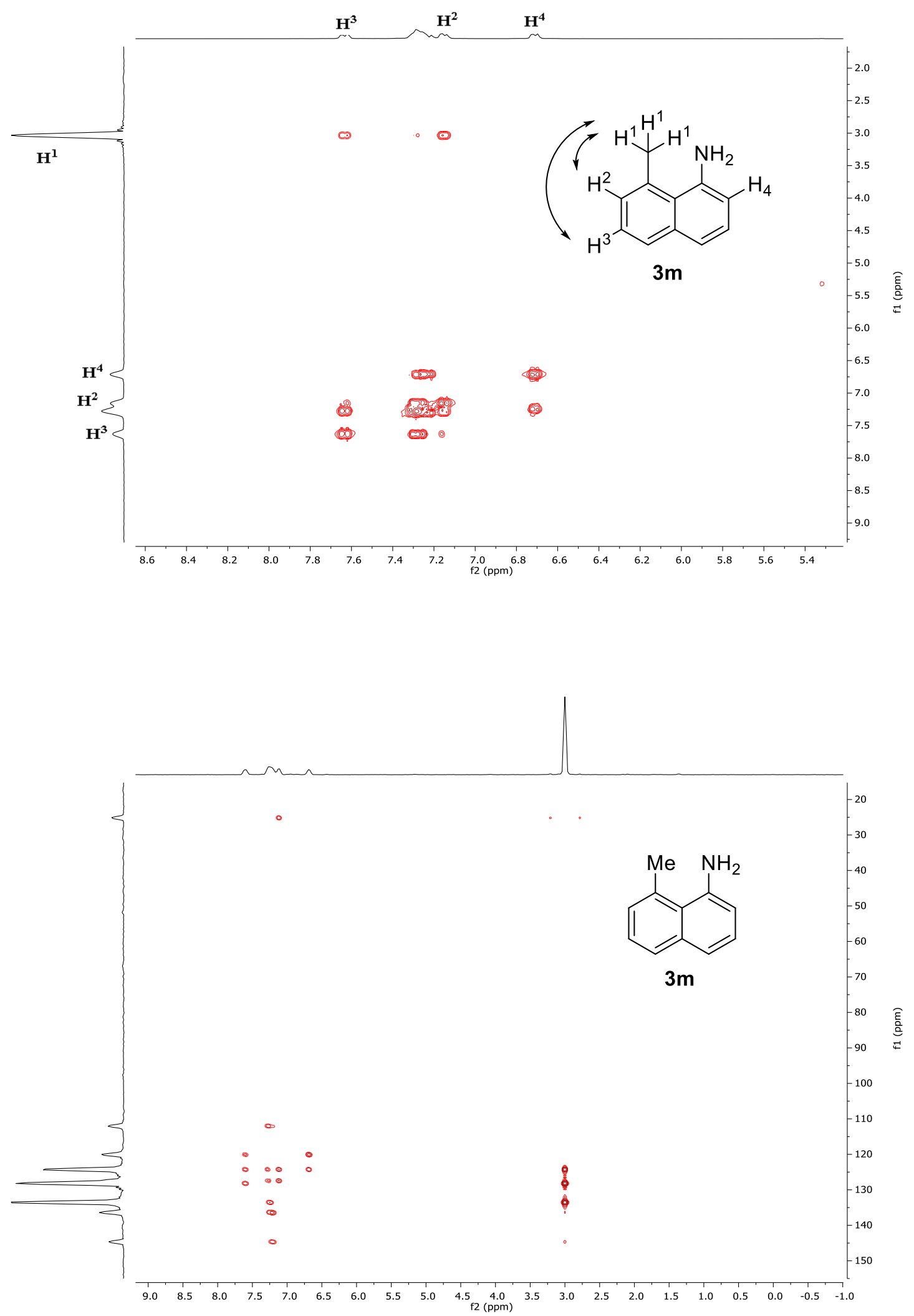


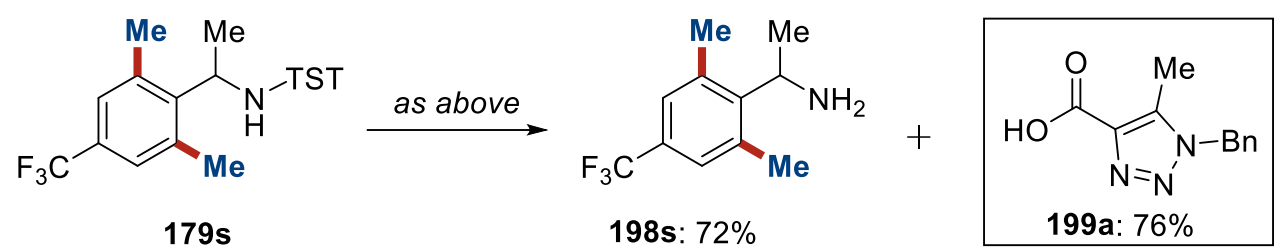

A solution of amide $179 \mathrm{~s}(215 \mathrm{mg}, 0.5 \mathrm{mmol})$ in $3.0 \mathrm{ml}$ concentrated hydrochloride and 1.5 $\mathrm{mL}$ dioxane under nitrogen was stirred at $130^{\circ} \mathrm{C}$ for 12 hours. Basified the mixture to $\mathrm{pH}$ = 12 by $3 \mathrm{M} \mathrm{KOH}$ aqueous solution and extracted with mixed solvent (Methanol/DCM 1:8) for three times. Combined the organic layers, washed with brine and removed the solvent. The resulting crude material was purified by column chromatography (gradient elution $n$-hexane/EtOAc $1: 1$ to EtOAc) yielding amine $198 \mathrm{~s}$ as a yellow liquid $(77.3 \mathrm{mg}, 72 \%)$. ${ }^{1} \mathrm{H}$ NMR $\left(300 \mathrm{MHz}, \mathrm{CDCl}_{3}\right) \delta=7.22(\mathrm{~s}, 2 \mathrm{H}), 4.67(\mathrm{q}, J=7.0 \mathrm{~Hz}, 1 \mathrm{H}), 2.51(\mathrm{~s}, 6 \mathrm{H}), 1.53$ (brs, 2H), $1.45(\mathrm{~d}, J=7.0 \mathrm{~Hz}, 3 \mathrm{H}) .{ }^{13} \mathrm{C}$ NMR $\left(101 \mathrm{MHz}, \mathrm{CDCl}_{3}\right) \delta=146.2\left(\mathrm{C}_{\mathrm{q}}\right), 136.6\left(\mathrm{C}_{\mathrm{q}}\right)$, $128.2\left(\mathrm{q},{ }^{2} \mathrm{~J}_{\mathrm{C}-\mathrm{F}}=31.9 \mathrm{~Hz}, \mathrm{C}_{\mathrm{q}}\right), 126.0\left(\mathrm{q},{ }^{3} \mathrm{~J}_{\mathrm{C}-\mathrm{F}}=2.9 \mathrm{~Hz}, \mathrm{CH}\right), 124.2\left(\mathrm{q},{ }^{1} \mathrm{~J}_{\mathrm{C}-\mathrm{F}}=271.8 \mathrm{~Hz}\right.$, $\left.\mathrm{C}_{\mathrm{q}}\right), 47.4(\mathrm{CH}), 21.8\left(\mathrm{CH}_{3}\right), 21.2\left(\mathrm{CH}_{3}\right) .{ }^{19} \mathrm{~F}$ NMR $\left(282 \mathrm{MHz}, \mathrm{CDCl}_{3}\right) \delta=-62.62$. IR (ATR): 3425, 2969, 2929, 1339, 1227, 1116, $881 \mathrm{~cm}^{-1}$. MS (ESI) m/z (relative intensity) 240 (100) $[\mathrm{M}+\mathrm{Na}]^{+}, 218(40)[\mathrm{M}+\mathrm{H}]^{+}, 201(80), 161(6)$. HR-MS (ESI) $\mathrm{m} / \mathrm{z}$ calcd for $\mathrm{C}_{11} \mathrm{H}_{15} \mathrm{~F}_{3} \mathrm{~N}[\mathrm{M}+\mathrm{H}]^{+}$ 218.1157 found 218.1152 .

The aqueous layer was acidified to $\mathrm{pH}=3$ and extracted with mixed solvent (Methanol/DCM 1:8) for three times. Combined the organic layers, washed with brine and removed the solvent. The resulting residue was washed with very little amount of ether to afford the acid $199 \mathrm{a}$ as yellow solid $(82.2 \mathrm{mg}, 76 \%)$. 


\subsection{Pyridinyl C-H Alkylation Catalyzed by Manganese(II)}

\subsubsection{Experimental Procedure and Analytical Data}<smiles>CC(NC(=O)c1ncccc1C1CCCCC1)c1ccccc1</smiles>

3-cyclohexyl-N-(1-phenylethyl)picolinamide (181aa)

Prepared following the general procedure $\mathbf{C}$ using $\mathrm{N}$-(1-phenylethyl)picolinamide $\mathbf{8 8 a}$ (45.2 mg, $0.20 \mathrm{mmol})$ and cyclohexylchloride 180a $(71 \mu \mathrm{L}, 0.60 \mathrm{mmol})$. Purification by column chromatography ( $n$-hexane/EtOAc $8: 1$ ) yielded 181aa (48.7 mg, 79\%) as a colorless oil. ${ }^{1} \mathrm{H}$ NMR $\left(300 \mathrm{MHz}, \mathrm{CDCl}_{3}\right) \delta=8.35$ (brs, $\left.1 \mathrm{H}\right), 8.33(\mathrm{dd}, J=4.5,1.5 \mathrm{~Hz}, 1 \mathrm{H})$, $7.74(\mathrm{dd}, J=8.1,1.5 \mathrm{~Hz}, 1 \mathrm{H}), 7.42-7.38(\mathrm{~m}, 2 \mathrm{H}), 7.35-7.29(\mathrm{~m}, 3 \mathrm{H}), 7.26-7.20(\mathrm{~m}, 1 \mathrm{H})$, $5.29(\mathrm{dq}, J=6.9,6.4 \mathrm{~Hz}, 1 \mathrm{H}), 4.07(\mathrm{tt}, J=11.7,3.0 \mathrm{~Hz}, 1 \mathrm{H}), 1.93-1.72(\mathrm{~m}, 5 \mathrm{H}), 1.59(\mathrm{~d}, J$ $=6.9 \mathrm{~Hz}, 3 \mathrm{H}), 1.54-1.16(\mathrm{~m}, 5 \mathrm{H}) .{ }^{13} \mathrm{C}$ NMR $\left(100 \mathrm{MHz}, \mathrm{CDCl}_{3}\right) \delta=165.3\left(\mathrm{C}_{\mathrm{q}}\right), 146.9\left(\mathrm{C}_{\mathrm{q}}\right)$, $144.9(\mathrm{CH}), 144.8\left(\mathrm{C}_{\mathrm{q}}\right), 143.6\left(\mathrm{C}_{\mathrm{q}}\right), 136.0(\mathrm{CH}), 128.6(\mathrm{CH}), 127.1(\mathrm{CH}), 126.1(\mathrm{CH}), 125.6$ $(\mathrm{CH}), 48.5(\mathrm{CH}), 37.7(\mathrm{CH}), 34.2\left(\mathrm{CH}_{2}\right), 34.0\left(\mathrm{CH}_{2}\right), 26.7\left(\mathrm{CH}_{2}\right), 26.7\left(\mathrm{CH}_{2}\right), 26.2\left(\mathrm{CH}_{2}\right)$, $22.3\left(\mathrm{CH}_{3}\right)$. IR (ATR): 3376, 2922, 2850, 1665, 1497, 806, 699, $625 \mathrm{~cm}^{-1}$. MS (ESI) m/z (relative intensity): $331(100)[\mathrm{M}+\mathrm{Na}]^{+}, 309(30)[\mathrm{M}+\mathrm{H}]^{+}, 188$ (30). HR-MS (ESI) m/z calcd for $\mathrm{C}_{20} \mathrm{H}_{25} \mathrm{~N}_{2} \mathrm{O}[\mathrm{M}+\mathrm{H}]^{+} 309.1961$ found 309.1958.<smiles>O=C(NCCc1ccccc1)c1ncccc1C1CCCCC1</smiles>

\section{3-Cyclohexyl-N-phenethylpicolinamide (181ba)}

Prepared following the general procedure C using $N$-phenethylpicolinamide $88 \mathrm{~b}$ (45.2 $\mathrm{mg}$, $0.20 \mathrm{mmol}$ ) and cyclohexylchloride 180a $(71 \mu \mathrm{L}, 0.60 \mathrm{mmol})$. Purification by column 
chromatography ( $n$-hexane/EtOAc $8: 1)$ yielded 181 ba $(58.0 \mathrm{mg}, 94 \%)$ as a colorless oil. ${ }^{1} \mathrm{H}$ NMR $\left(300 \mathrm{MHz}, \mathrm{CDCl}_{3}\right) \delta=8.22(\mathrm{dd}, J=4.5,1.5 \mathrm{~Hz}, 1 \mathrm{H}), 8.01(\mathrm{brs}, 1 \mathrm{H}), 7.67(\mathrm{dd}, J=$ 8.1, 1.5 Hz, 1H), 7.25-7.11 (m, 6H), 3.97 (tt, J = 11.8, 3.0 Hz, 1H), 3.69-3.54 (m, 2H), 2.86 $(\mathrm{t}, J=7.2 \mathrm{~Hz}, 2 \mathrm{H}), 1.78-1.67(\mathrm{~m}, 5 \mathrm{H}), 1.55-1.07(\mathrm{~m}, 5 \mathrm{H}) .{ }^{13} \mathrm{C} \mathrm{NMR}\left(100 \mathrm{MHz}, \mathrm{CDCl}_{3}\right) \delta=$ $166.2\left(\mathrm{C}_{\mathrm{q}}\right), 147.0\left(\mathrm{C}_{\mathrm{q}}\right), 144.9(\mathrm{CH}), 144.6\left(\mathrm{C}_{\mathrm{q}}\right), 139.1(\mathrm{CH}), 135.9(\mathrm{CH}), 128.7(\mathrm{CH}), 128.45$ $(\mathrm{CH}), 126.3(\mathrm{CH}), 125.6(\mathrm{CH}), 40.6\left(\mathrm{CH}_{2}\right), 37.8(\mathrm{CH}), 35.9\left(\mathrm{CH}_{2}\right), 34.1\left(\mathrm{CH}_{2}\right), 26.7\left(\mathrm{CH}_{2}\right)$, $26.2\left(\mathrm{CH}_{2}\right)$. IR (ATR): 3384, 2923, 2850, 1664, 1506, 1000, 806, 698, $497 \mathrm{~cm}^{-1}$. MS (ESI) m/z (relative intensity): $331(40)[\mathrm{M}+\mathrm{Na}]^{+}, 309(100)[\mathrm{M}+\mathrm{H}]^{+}, 284(10), 188$ (10). HR-MS (ESI) $\mathrm{m} / \mathrm{z}$ calcd for $\mathrm{C}_{20} \mathrm{H}_{25} \mathrm{~N}_{2} \mathrm{O}[\mathrm{M}+\mathrm{H}]^{+} 309.1965$ found 309.1961 .<smiles>O=C(NCc1ccccc1)c1ncccc1C1CCCCC1</smiles>

\section{N-Benzyl-3-cyclohexylpicolinamide (181ca)}

Prepared following the general procedure $\mathrm{C}$ using $\mathrm{N}$-phenethylpicolinamide $88 \mathrm{c}$ (42.4 mg, $0.20 \mathrm{mmol}$ ) and $180 \mathrm{a}$ cyclohexylchloride $(71 \mu \mathrm{L}, 0.60 \mathrm{mmol})$. Purification by column chromatography ( $n$-hexane/EtOAc $8: 1)$ yielded $181 \mathrm{ca}(45.2 \mathrm{mg}, 77 \%)$ as a colorless oil. ${ }^{1} \mathrm{H}$ NMR $\left(400 \mathrm{MHz}, \mathrm{CDCl}_{3}\right) \delta=8.36(\mathrm{brs}, 1 \mathrm{H}), 8.31(\mathrm{dd}, J=4.5,1.5 \mathrm{~Hz}, 1 \mathrm{H}), 7.76(\mathrm{dd}, J=$ 8.1, 1.5 Hz, 1H), 7.37-7.30 (m, 5H), 7.27-7.23 (m, 1H), $4.62(\mathrm{~d}, J=6.0 \mathrm{~Hz}, 2 \mathrm{H}), 4.10$ (tt, $J=11.8,3.3 \mathrm{~Hz}, 1 \mathrm{H}), 1.92-1.89(\mathrm{~m}, 2 \mathrm{H}), 1.84-1.75(\mathrm{~m}, 3 \mathrm{H}), 1.52(\mathrm{qt}, J=12.8,3.3 \mathrm{~Hz}, 2 \mathrm{H})$, $1.35(\mathrm{qd}, J=12.4,3.0 \mathrm{~Hz}, 2 \mathrm{H}), 1.24$ (qt, $J=13.0,3.6 \mathrm{~Hz}, 1 \mathrm{H}) .{ }^{13} \mathrm{C} \mathrm{NMR}\left(100 \mathrm{MHz}, \mathrm{CDCl}_{3}\right)$ $\delta=166.1\left(\mathrm{C}_{\mathrm{q}}\right), 146.8\left(\mathrm{C}_{\mathrm{q}}\right), 145.0(\mathrm{CH}), 144.9\left(\mathrm{C}_{\mathrm{q}}\right), 138.4\left(\mathrm{C}_{\mathrm{q}}\right), 136.0(\mathrm{CH}), 128.6(\mathrm{CH})$, $127.7(\mathrm{CH}), 127.3(\mathrm{CH}), 125.7(\mathrm{CH}), 43.3\left(\mathrm{CH}_{2}\right), 37.8(\mathrm{CH}), 34.1\left(\mathrm{CH}_{2}\right), 26.7\left(\mathrm{CH}_{2}\right), 26.2$ $\left(\mathrm{CH}_{2}\right)$. IR (ATR): 3381, 2922, 2849, 1665, 1505, 805, $698 \mathrm{~cm}^{-1} . \mathbf{M S}$ (ESI) $\mathrm{m} / \mathrm{z}$ (relative intensity): 317 (100) [M+Na] $]^{+}, 295(40)[\mathrm{M}+\mathrm{H}]^{+}, 188$ (20), 106 (10). HR-MS (ESI) m/z calcd for $\mathrm{C}_{19} \mathrm{H}_{23} \mathrm{~N}_{2} \mathrm{O}[\mathrm{M}+\mathrm{H}]^{+} 295.1800$ found 295.1805. 
<smiles>O=C(NCc1ccc(F)cc1)c1ncccc1C1CCCCC1</smiles>

\section{3-Cyclohexyl-N-(4-fluorobenzyl)picolinamide (181da)}

Prepared following the general procedure $\mathbf{C}$ using $\mathrm{N}$-(4-fluorobenzyl)picolinamide $\mathbf{8 8 d}$ (46.1 $\mathrm{mg}, 0.20 \mathrm{mmol})$ and cyclohexylchloride 180a $(71 \mu \mathrm{L}, 0.60 \mathrm{mmol})$. Purification by column chromatography ( $n$-hexane/EtOAc 8:1) yielded $181 \mathrm{da}(52.3 \mathrm{mg}, 84 \%)$ as a white solid. M.p. $=72-73{ }^{\circ} \mathrm{C} .{ }^{1} \mathrm{H}$ NMR $\left(300 \mathrm{MHz}, \mathrm{CDCl}_{3}\right) \delta=8.32$ (brs, $\left.1 \mathrm{H}\right), 8.25$ (dd, $J=4.5$, $1.5 \mathrm{~Hz}, 1 \mathrm{H}), 7.70(\mathrm{dd}, J=8.0,1.5 \mathrm{~Hz}, 1 \mathrm{H}), 7.28-7.23(\mathrm{~m}, 3 \mathrm{H}), 6.97-6.90(\mathrm{~m}, 2 \mathrm{H}), 4.51(\mathrm{~d}$, $J=6.0 \mathrm{~Hz}, 2 \mathrm{H}), 4.02(\mathrm{tt}, J=11.8,3.0 \mathrm{~Hz}, 1 \mathrm{H}), 1.85-1.67(\mathrm{~m}, 5 \mathrm{H}), 1.51-1.10(\mathrm{~m}, 5 \mathrm{H})$. ${ }^{13} \mathrm{C}$ NMR $\left(125 \mathrm{MHz}, \mathrm{CDCl}_{3}\right) \delta=166.0\left(\mathrm{C}_{\mathrm{q}}\right), 161.9\left(\mathrm{~d},{ }^{1} \mathrm{~J}_{\mathrm{C}-\mathrm{F}}=245.0 \mathrm{~Hz}, \mathrm{C}_{\mathrm{q}}\right), 146.6\left(\mathrm{C}_{\mathrm{q}}\right)$, $144.9(\mathrm{CH}), 144.8\left(\mathrm{C}_{\mathrm{q}}\right), 135.9(\mathrm{CH}), 134.2\left(\mathrm{~d},{ }^{4} \mathrm{~J}_{\mathrm{C}-\mathrm{F}}=3.1 \mathrm{~Hz}, \mathrm{C}_{\mathrm{q}}\right), 129.2\left(\mathrm{~d},{ }^{3} \mathrm{~J}_{\mathrm{C}-\mathrm{F}}=8.1 \mathrm{~Hz}\right.$, $\mathrm{CH}), 125.7(\mathrm{CH}), 115.3\left(\mathrm{~d},{ }^{2} \mathrm{~J}_{\mathrm{C}-\mathrm{F}}=21.4 \mathrm{~Hz}, \mathrm{CH}\right), 42.6\left(\mathrm{CH}_{2}\right), 37.9(\mathrm{CH}), 34.2\left(\mathrm{CH}_{2}\right), 26.8$ $\left(\mathrm{CH}_{2}\right), 26.3\left(\mathrm{CH}_{2}\right) \cdot{ }^{19} \mathrm{~F} \mathrm{NMR}\left(275 \mathrm{MHz}, \mathrm{CDCl}_{3}\right) \delta=-115.4-115.5$ (m). IR (ATR): 3187, 2925, 2851, 1655, 1505, 1221, 838, 728, $429 \mathrm{~cm}^{-1}$. MS (ESI) m/z (relative intensity): 335 (100) $[\mathrm{M}+\mathrm{Na}]^{+}, 313(15)[\mathrm{M}+\mathrm{H}]^{+}, 244$ (8). HR-MS (ESI) $\mathrm{m} / \mathrm{z}$ calcd for $\mathrm{C}_{19} \mathrm{H}_{22} \mathrm{FN}_{2} \mathrm{O}[\mathrm{M}+\mathrm{H}]^{+}$ 313.1708 found 313.1711.<smiles>O=C(NCc1ccccc1F)c1ncccc1C1CCCCC1</smiles>

\section{3-Cyclohexyl-N-(2-fluorobenzyl)picolinamide (181ea)}

Prepared following the general procedure C using $\mathrm{N}$-(2-fluorobenzyl)picolinamide $\mathbf{8 8 e}$ (46.0 $\mathrm{mg}, 0.20 \mathrm{mmol})$ and cyclohexylchloride 180a $(71 \mu \mathrm{L}, 0.60 \mathrm{mmol})$. Purification by column chromatography ( $n$-hexane/EtOAc $8: 1$ ) yielded 181ea $(51.7 \mathrm{mg}, 83 \%$ ) as a colorless oil. ${ }^{1} \mathrm{H}$ NMR $\left(400 \mathrm{MHz}, \mathrm{CDCl}_{3}\right) \delta=8.38(\mathrm{t}, J=6.4 \mathrm{~Hz}, 1 \mathrm{H}), 8.32(\mathrm{dd}, J=4.4,1.6$ 
$\mathrm{Hz}, 1 \mathrm{H}), 7.75(\mathrm{dd}, J=8.0,1.6 \mathrm{~Hz}, 1 \mathrm{H}), 7.40(\mathrm{td}, J=7.6,1.8 \mathrm{~Hz}, 1 \mathrm{H}), 7.31$ (ddd, $J=8.0$, 4.4, $0.4 \mathrm{~Hz}, 1 \mathrm{H}), 7.25-7.20(\mathrm{~m}, 1 \mathrm{H}), 7.08(\mathrm{td}, J=7.6,1.2 \mathrm{~Hz}, 1 \mathrm{H}), 7.06-7.00(\mathrm{~m}, 1 \mathrm{H}), 4.67$ $(\mathrm{d}, J=6.4 \mathrm{~Hz}, 2 \mathrm{H}), 4.07(\mathrm{tt}, J=11.6,3.0 \mathrm{~Hz}, 1 \mathrm{H}), 1.90-1.73(\mathrm{~m}, 5 \mathrm{H}), 1.56-1.45(\mathrm{~m}, 2 \mathrm{H})$, 1.39-1.20 (m, 3H). ${ }^{13} \mathrm{C}$ NMR $\left(100 \mathrm{MHz}, \mathrm{CDCl}_{3}\right) \delta=166.1\left(\mathrm{C}_{\mathrm{q}}\right), 161.0\left(\mathrm{~d},{ }^{1} \mathrm{~J}_{\mathrm{C}-\mathrm{F}}=246.0 \mathrm{~Hz}\right.$, $\left.\mathrm{C}_{\mathrm{q}}\right), 146.7\left(\mathrm{C}_{\mathrm{q}}\right), 145.0(\mathrm{CH}), 144.9\left(\mathrm{C}_{\mathrm{q}}\right), 136.0(\mathrm{CH}), 130.0\left(\mathrm{~d},{ }^{3} \mathrm{~J}_{\mathrm{C}-\mathrm{F}}=4.3 \mathrm{~Hz}, \mathrm{CH}\right), 129.0$ $\left(\mathrm{d},{ }^{3} \mathrm{~J}_{\mathrm{C}-\mathrm{F}}=8.1 \mathrm{~Hz}, \mathrm{CH}\right), 125.8(\mathrm{CH}), 125.5\left(\mathrm{~d},{ }^{2} \mathrm{~J}_{\mathrm{C}-\mathrm{F}}=14.9 \mathrm{~Hz}, \mathrm{C}_{\mathrm{q}}\right), 124.2\left(\mathrm{~d},{ }^{4} \mathrm{~J}_{\mathrm{C}-\mathrm{F}}=3.6 \mathrm{~Hz}\right.$, $\mathrm{CH}), 115.3\left(\mathrm{~d},{ }^{2} \mathrm{~J}_{\mathrm{C}-\mathrm{F}}=21.3 \mathrm{~Hz}, \mathrm{CH}\right), 37.8(\mathrm{CH}), 37.1\left(\mathrm{~d},{ }^{3} \mathrm{~J}_{\mathrm{C}-\mathrm{F}}=4.2 \mathrm{~Hz}, \mathrm{CH}_{2}\right), 34.1\left(\mathrm{CH}_{2}\right)$, 26.7 $\left(\mathrm{CH}_{2}\right), 26.2\left(\mathrm{CH}_{2}\right) .{ }^{19} \mathrm{~F}$ NMR $\left(275 \mathrm{MHz}, \mathrm{CDCl}_{3}\right) \delta=-118.7-118.8(\mathrm{~m})$. IR (ATR): 3382, 2922, 2850, 1668, 1501, 1227, 805, 757, $625 \mathrm{~cm}^{-1}$. MS (ESI) $\mathrm{m} / \mathrm{z}$ (relative intensity): 335 (100) $[\mathrm{M}+\mathrm{Na}]^{+}, 313(20)[\mathrm{M}+\mathrm{H}]^{+}, 244$ (15). HR-MS (ESI) $\mathrm{m} / \mathrm{z}$ calcd for $\mathrm{C}_{19} \mathrm{H}_{22} \mathrm{FN}_{2} \mathrm{O}[\mathrm{M}+\mathrm{H}]^{+}$ 313.1708 found 313.1709.<smiles>COc1ccc(CNC(=O)c2ncccc2C2CCCCC2)cc1</smiles>

\section{3-Cyclohexyl-N-(4-methoxybenzyl)picolinamide (181fa)}

Prepared following the general procedure $\mathbf{C}$ using $\mathrm{N}$-(4-methoxybenzyl)picolinamide $\mathbf{8 8 f}$ (48.4 mg, $0.20 \mathrm{mmol}$ ) and cyclohexylchloride $181 \mathrm{fa}(71 \mu \mathrm{L}, 0.60 \mathrm{mmol})$. Purification by column chromatography ( $n$-hexane/EtOAc $8: 1)$ yielded $181 \mathrm{fa}(57.1 \mathrm{mg}, 88 \%)$ as a yellow solid. M.p. $=107-108{ }^{\circ} \mathrm{C} .{ }^{1} \mathrm{H}$ NMR $\left(400 \mathrm{MHz}, \mathrm{CDCl}_{3}\right) \delta=8.29(\mathrm{dd}, J=4.4,1.6 \mathrm{~Hz}, 1 \mathrm{H})$, 8.27 (brs, 1H), 7.74 (dd, $J=8.0,1.6 \mathrm{~Hz}, 1 \mathrm{H}), 7.30$ (ddd, $J=8.0,4.4,0.4 \mathrm{~Hz}, 1 \mathrm{H}$ ), 7.27 (d, $J=8.8 \mathrm{~Hz}, 2 \mathrm{H}), 6.85(\mathrm{~d}, J=8.8 \mathrm{~Hz}, 2 \mathrm{H}), 4.54(\mathrm{~d}, J=6.0 \mathrm{~Hz}, 2 \mathrm{H}), 4.09(\mathrm{tt}, J=11.6,3.1 \mathrm{~Hz}$, $1 \mathrm{H}), 3.76(\mathrm{~s}, 3 \mathrm{H}), 1.93-1.72(\mathrm{~m}, 5 \mathrm{H}), 1.52(\mathrm{qt}, J=12.4,3.2 \mathrm{~Hz}, 2 \mathrm{H}), 1.34$ (qd, $J=12.4,3.2$ $\mathrm{Hz}, 2 \mathrm{H}), 1.23$ (qt, $J=12.8,3.6 \mathrm{~Hz}, 1 \mathrm{H}) .{ }^{13} \mathrm{C}$ NMR $\left(100 \mathrm{MHz}, \mathrm{CDCl}_{3}\right) \delta=166.0\left(\mathrm{C}_{\mathrm{q}}\right), 158.9$ $\left(\mathrm{C}_{\mathrm{q}}\right), 146.9\left(\mathrm{C}_{\mathrm{q}}\right), 144.9(\mathrm{CH}), 144.8\left(\mathrm{C}_{\mathrm{q}}\right), 136.0(\mathrm{CH}), 130.5\left(\mathrm{C}_{\mathrm{q}}\right), 129.1(\mathrm{CH}), 125.7(\mathrm{CH})$, $114.0(\mathrm{CH}), 55.2\left(\mathrm{CH}_{3}\right), 42.8\left(\mathrm{CH}_{2}\right), 37.8(\mathrm{CH}), 34.1\left(\mathrm{CH}_{2}\right), 26.7\left(\mathrm{CH}_{2}\right), 26.2\left(\mathrm{CH}_{2}\right)$. IR $(\mathrm{ATR})$ : 3306, 2925, 2848, 1652, 1513, 1242, 994, 807, $685 \mathrm{~cm}^{-1}$. MS (ESI) $\mathrm{m} / \mathrm{z}$ (relative intensity): 
$347(100)[\mathrm{M}+\mathrm{Na}]^{+}, 325(50)[\mathrm{M}+\mathrm{H}]^{+}, 188(15), 121$ (10). HR-MS (ESI) m/z calcd for $\mathrm{C}_{20} \mathrm{H}_{25} \mathrm{~N}_{2} \mathrm{O}_{2}[\mathrm{M}+\mathrm{H}]^{+} 325.1905$ found 325.1911 .<smiles>O=C(NC1CCCCC1)c1ncccc1C1CCCCC1</smiles>

\section{N,3-Dicyclohexylpicolinamide (181ga)}

Prepared following the general procedure $\mathbf{C}$ using $\mathrm{N}$-cyclohexylpicolinamide $\mathbf{8 8 g} \mathbf{g}(\mathbf{4 0 . 8} \mathbf{m g}$, $0.20 \mathrm{mmol}$ ) and cyclohexylchloride $180 \mathrm{a}(71 \mu \mathrm{L}, 0.60 \mathrm{mmol})$. Purification by column chromatography ( $n$-hexane/EtOAc $8: 1)$ yielded $181 \mathrm{ga}(45.9 \mathrm{mg}, 80 \%)$ as a colorless oil. ${ }^{1} \mathrm{H}$ NMR $\left(400 \mathrm{MHz}, \mathrm{CDCl}_{3}\right) \delta=8.30(\mathrm{dd}, J=4.4,1.6 \mathrm{~Hz}, 1 \mathrm{H}), 7.89(\mathrm{~d}, J=8.8 \mathrm{~Hz}, 1 \mathrm{H}), 7.72$ (dd, $J=8.0,1.6 \mathrm{~Hz}, 1 \mathrm{H}), 7.28(\mathrm{dd}, J=8.0,4.4 \mathrm{~Hz}, 1 \mathrm{H}), 4.04(\mathrm{tt}, J=11.6,3.2 \mathrm{~Hz}, 1 \mathrm{H}), 3.94-$ $3.85(\mathrm{~m}, 1 \mathrm{H}), 2.00-1.93(\mathrm{~m}, 9 \mathrm{H}), 1.63-1.16(\mathrm{~m}, 11 \mathrm{H}) .{ }^{13} \mathrm{C} \mathrm{NMR}\left(100 \mathrm{MHz}, \mathrm{CDCl}_{3}\right) \delta=$ $165.3\left(\mathrm{C}_{\mathrm{q}}\right), 147.2\left(\mathrm{C}_{\mathrm{q}}\right), 144.8(\mathrm{CH}), 144.7\left(\mathrm{C}_{\mathrm{q}}\right), 135.9(\mathrm{CH}), 125.5(\mathrm{CH}), 48.0(\mathrm{CH}), 37.9$ $(\mathrm{CH}), 34.1\left(\mathrm{CH}_{2}\right), 33.1\left(\mathrm{CH}_{2}\right), 26.7\left(\mathrm{CH}_{2}\right), 26.2\left(\mathrm{CH}_{2}\right), 25.6\left(\mathrm{CH}_{2}\right), 24.9\left(\mathrm{CH}_{2}\right)$. IR (ATR): 3378, 2922, 2850, 1663, 1502, 805, $625 \mathrm{~cm}^{-1}$. MS (ESI) m/z (relative intensity): 309 (30) $[\mathrm{M}+\mathrm{Na}]^{+}, 287(100)[\mathrm{M}+\mathrm{H}]^{+}, 202(20), 188(10) . \mathrm{HR}-\mathrm{MS}$ (ESI) $\mathrm{m} / \mathrm{z}$ calcd for $\mathrm{C}_{18} \mathrm{H}_{27} \mathrm{~N}_{2} \mathrm{O}$ $[\mathrm{M}+\mathrm{H}]^{+} 287.2119$ found 287.2118 .<smiles>O=C(NC1CCCc2ccccc21)c1ncccc1C1CCCCC1</smiles>

3-Cyclohexyl-N-(1,2,3,4-tetrahydronaphthalen-1-yl)picolinamide (181ha) 
Prepared following the general procedure C using $N$-(1,2,3,4-tetrahydronaphthalen-1yl)picolinamide $88 \mathrm{~h}$ (50.4 mg, $0.20 \mathrm{mmol}$ ) and cyclohexylchloride 180a (71 $\mu \mathrm{L}, 0.60 \mathrm{mmol})$. Purification by column chromatography ( $n$-hexane/EtOAc $8: 1$ ) yielded $181 \mathrm{ha}(55.1 \mathrm{mg}$, $82 \%)$ as a colorless oil. ${ }^{1} \mathrm{H}$ NMR $\left(400 \mathrm{MHz}, \mathrm{CDCl}_{3}\right) \delta=8.33(\mathrm{~d}, J=8.8 \mathrm{~Hz}, 1 \mathrm{H}), 8.28(\mathrm{dd}$, $J=4.4,1.6 \mathrm{~Hz}, 1 \mathrm{H}), 7.76(\mathrm{dd}, J=7.6,1.6 \mathrm{~Hz}, 1 \mathrm{H}), 7.36-7.33(\mathrm{~m}, 1 \mathrm{H}), 7.31$ (ddd, $J=8.0$, 4.4, $0.4 \mathrm{~Hz}, 1 \mathrm{H}), 7.17-7.09(\mathrm{~m}, 3 \mathrm{H}), 5.37-5.32(\mathrm{~m}, 1 \mathrm{H}), 4.15(\mathrm{tt}, J=12.0,3.2 \mathrm{~Hz}, 1 \mathrm{H})$, 2.94-2.71 (m, 2H), 2.22-2.09 (m, 1H), 1.96-1.77 (m, 8H), 1.63-1.46 (m, 2H), 1.43-1.22 $(\mathrm{m}, 3 \mathrm{H}) .{ }^{13} \mathrm{C}$ NMR $\left(100 \mathrm{MHz}, \mathrm{CDCl}_{3}\right) \delta=165.5\left(\mathrm{C}_{\mathrm{q}}\right), 146.9\left(\mathrm{C}_{\mathrm{q}}\right), 144.92(\mathrm{CH}), 144.8\left(\mathrm{C}_{\mathrm{q}}\right)$, $137.5\left(\mathrm{C}_{\mathrm{q}}\right), 137.0\left(\mathrm{C}_{\mathrm{q}}\right), 135.9(\mathrm{CH}), 129.0(\mathrm{CH}), 128.7(\mathrm{CH}), 127.0(\mathrm{CH}), 126.1(\mathrm{CH}), 125.6$ $(\mathrm{CH}), 47.3(\mathrm{CH}), 37.9(\mathrm{CH}), 34.2\left(\mathrm{CH}_{2}\right), 34.1\left(\mathrm{CH}_{2}\right), 30.3\left(\mathrm{CH}_{2}\right), 29.3\left(\mathrm{CH}_{2}\right), 26.8\left(\mathrm{CH}_{2}\right)$, $26.3\left(\mathrm{CH}_{2}\right), 20.2\left(\mathrm{CH}_{2}\right)$. IR (ATR): 3376, 2923, 2851, 1664, 1493, 1445, 805, 734, 624 $\mathrm{cm}^{-1}$. MS (ESI) m/z (relative intensity): $357(100)[\mathrm{M}+\mathrm{Na}]^{+}, 335(25)[\mathrm{M}+\mathrm{H}]^{+}, 188(30), 147$ (10). HR-MS (ESI) $\mathrm{m} / \mathrm{z}$ calcd for $\mathrm{C}_{19} \mathrm{H}_{22} \mathrm{FN}_{2} \mathrm{O}[\mathrm{M}+\mathrm{H}]^{+} 335.2123$ found 335.2120 .<smiles>CCCCNC(=O)c1ncccc1C1CCCCC1</smiles>

\section{N-Butyl-3-cyclohexylpicolinamide (181ia)}

Prepared following the general procedure $\mathbf{C}$ using $\mathrm{N}$-butylpicolinamide $\mathbf{8 8 \mathbf { i }}$ (35.6 $\mathrm{mg}, 0.20$ $\mathrm{mmol}$ ) and cyclohexylchloride 180a $(71 \mu \mathrm{L}, 0.60 \mathrm{mmol})$. Purification by column chromatography ( $n$-hexane/EtOAc $8: 1)$ yielded 181ia $(47.7 \mathrm{mg}, 92 \%)$ as a colorless oil. ${ }^{1} \mathrm{H}$ NMR $\left(400 \mathrm{MHz}, \mathrm{CDCl}_{3}\right) \delta=8.31(\mathrm{dd}, J=4.4,1.6 \mathrm{~Hz}, 1 \mathrm{H}), 7.97(\mathrm{brs}, 1 \mathrm{H}), 7.72(\mathrm{dd}, J=$ 8.0, 1.6 Hz, 1H), 7.29 (dd, $J=8.0,4.4 \mathrm{~Hz}, 1 \mathrm{H}), 4.04(\mathrm{tt}, J=12.0,3.2 \mathrm{~Hz}, 1 \mathrm{H}), 3.39(\mathrm{td}, J=$ 7.2, $6.0 \mathrm{~Hz}, 2 \mathrm{H}), 1.88-1.76(\mathrm{~m}, 5 \mathrm{H}), 1.62-1.18(\mathrm{~m}, 9 \mathrm{H}), 0.92(\mathrm{t}, J=7.2 \mathrm{~Hz}, 3 \mathrm{H}) .{ }^{13} \mathrm{C}$ NMR $\left(100 \mathrm{MHz}, \mathrm{CDCl}_{3}\right) \delta=166.2\left(\mathrm{C}_{\mathrm{q}}\right), 147.2\left(\mathrm{C}_{\mathrm{q}}\right), 144.9(\mathrm{CH}), 144.7\left(\mathrm{C}_{\mathrm{q}}\right), 136.0(\mathrm{CH}), 125.5$ (CH), $39.1\left(\mathrm{CH}_{2}\right), 37.8(\mathrm{CH}), 34.1\left(\mathrm{CH}_{2}\right), 31.7\left(\mathrm{CH}_{2}\right), 26.7\left(\mathrm{CH}_{2}\right), 26.2\left(\mathrm{CH}_{2}\right), 20.2\left(\mathrm{CH}_{2}\right)$, $13.8\left(\mathrm{CH}_{3}\right)$. IR (ATR): 3382, 2922, 2850, 1663, 1504, 694, $498 \mathrm{~cm}^{-1}$. MS (ESI) $\mathrm{m} / \mathrm{z}$ (relative 
intensity): 283 (40) [M+Na] $]^{+}, 261$ (100) [M+H] $]^{+}, 188$ (15), 160 (10). HR-MS (ESI) m/z calcd for $\mathrm{C}_{16} \mathrm{H}_{25} \mathrm{~N}_{2} \mathrm{O}[\mathrm{M}+\mathrm{H}]^{+} 261.1964$ found 261.1961.<smiles>O=C(NN=P)c1ncccc1C1CCCCC1</smiles>

\section{3-Cyclohexyl-N-(4-methoxyphenyl)picolinamide (181ja)}

Prepared following the general procedure $\mathbf{C}$ using $\mathrm{N}$-(4-methoxyphenyl)picolinamide $\mathbf{8 8 \mathbf { j }}$ (45.6 mg, $0.20 \mathrm{mmol}$ ) and cyclohexylchloride 180a $(71 \mu \mathrm{L}, 0.60 \mathrm{mmol})$. Purification by column chromatography ( $n$-hexane/EtOAc $8: 1$ ) yielded 181 ja $(28.7 \mathrm{mg}, 46 \%$ ) as a colorless oil. ${ }^{1} \mathrm{H}$ NMR $\left(300 \mathrm{MHz}, \mathrm{CDCl}_{3}\right) \delta=10.05$ (brs, $\left.1 \mathrm{H}\right), 8.41(\mathrm{dd}, J=4.5,1.5 \mathrm{~Hz}, 1 \mathrm{H})$, $7.85-7.80(\mathrm{dd}, J=8.1,1.5 \mathrm{~Hz}, 1 \mathrm{H}), 7.64(\mathrm{~d}, J=9.0 \mathrm{~Hz}, 2 \mathrm{H}), 7.39(\mathrm{dd}, J=8.1,4.5 \mathrm{~Hz}, 1 \mathrm{H})$, $6.91(\mathrm{~d}, J=9.0 \mathrm{~Hz}, 2 \mathrm{H}), 4.24-4.16(\mathrm{~m}, 1 \mathrm{H}), 3.81(\mathrm{~s}, 3 \mathrm{H}), 1.95-1.76(\mathrm{~m}, 5 \mathrm{H}), 1.57-1.24(\mathrm{~m}$, 5H). ${ }^{13} \mathrm{C}$ NMR $\left(125 \mathrm{MHz}, \mathrm{CDCl}_{3}\right) \delta=163.5\left(\mathrm{C}_{\mathrm{q}}\right), 156.1\left(\mathrm{C}_{\mathrm{q}}\right), 146.4\left(\mathrm{C}_{\mathrm{q}}\right), 145.4\left(\mathrm{C}_{\mathrm{q}}\right), 144.7$ $(\mathrm{CH}), 136.3(\mathrm{CH}), 131.3\left(\mathrm{C}_{\mathrm{q}}\right), 125.9(\mathrm{CH}), 121.5(\mathrm{CH}), 114.1(\mathrm{CH}), 55.5\left(\mathrm{CH}_{3}\right), 37.9(\mathrm{CH})$, $34.2\left(\mathrm{CH}_{2}\right), 26.8\left(\mathrm{CH}_{2}\right), 26.3\left(\mathrm{CH}_{2}\right)$. IR (ATR): 3319, 2922, 2847, 1673, 1513, 1240, 825, 689, $559 \mathrm{~cm}^{-1}$. MS (ESI) m/z (relative intensity): $333(35)[\mathrm{M}+\mathrm{Na}]^{+}, 211(100)[\mathrm{M}+\mathrm{H}]^{+}, 188$ (15), 160 (10). HR-MS (ESI) $m / z$ calcd for $\mathrm{C}_{19} \mathrm{H}_{23} \mathrm{~N}_{2} \mathrm{O}_{2}[\mathrm{M}+\mathrm{H}]^{+} 311.1756$ found 311.1754.<smiles>CCCCNC(=O)c1nc2ccccc2cc1C1CCCCC1</smiles>

\section{N-Butyl-3-cyclohexylquinoline-2-carboxamide (181ka)}

Prepared following the general procedure $\mathbf{C}$ using $\mathrm{N}$-phenethylpicolinamide $\mathbf{8 8 k}$ (48.4 mg, $0.20 \mathrm{mmol})$ and cyclohexylchloride $180 \mathrm{a}(71 \mu \mathrm{L}, 0.60 \mathrm{mmol})$. Purification by column 
chromatography ( $n$-hexane/EtOAc $8: 1)$ yielded 181ka $(16.7 \mathrm{mg}, 26 \%)$ as a white solid. M.p. $=92-93^{\circ} \mathrm{C} .{ }^{1} \mathrm{H}$ NMR $\left(400 \mathrm{MHz}, \mathrm{CDCl}_{3}\right) \delta=8.28(\mathrm{t}, J=5.6 \mathrm{~Hz}, 1 \mathrm{H}), 8.20$ (brs, $\left.1 \mathrm{H}\right)$, 8.12-8.07 (m, 2H), 7.70 (ddd, $J=8.4,6.8,1.6 \mathrm{~Hz}, 1 \mathrm{H}), 7.58(\mathrm{ddd}, J=8.4,6.8,1.6 \mathrm{~Hz}, 1 \mathrm{H})$, $3.51(\mathrm{td}, J=7.1,6.0 \mathrm{~Hz}, 2 \mathrm{H}), 3.33(\mathrm{tt}, J=11.6,3.0 \mathrm{~Hz}, 1 \mathrm{H}), 2.00-1.80(\mathrm{~m}, 4 \mathrm{H}), 1.85-1.80$ $(\mathrm{m}, 1 \mathrm{H}), 1.69-1.28(\mathrm{~m}, 9 \mathrm{H}), 0.96(\mathrm{t}, J=7.2 \mathrm{~Hz}, 3 \mathrm{H}) .{ }^{13} \mathrm{C} \mathbf{N M R}\left(100 \mathrm{MHz}, \mathrm{CDCl}_{3}\right) \delta=164.8$ $\left(\mathrm{C}_{\mathrm{q}}\right), 155.1\left(\mathrm{C}_{\mathrm{q}}\right), 149.8\left(\mathrm{C}_{\mathrm{q}}\right), 146.8\left(\mathrm{C}_{\mathrm{q}}\right), 130.6(\mathrm{CH}), 129.3(\mathrm{CH}), 127.8\left(\mathrm{C}_{\mathrm{q}}\right), 127.3(\mathrm{CH})$, $123.1(\mathrm{CH}), 115.2(\mathrm{CH}), 39.3(\mathrm{CH}), 33.4\left(\mathrm{CH}_{2}\right), 31.8\left(\mathrm{CH}_{2}\right), 26.9\left(\mathrm{CH}_{2}\right), 26.2\left(\mathrm{CH}_{2}\right), 20.2$ $\left(\mathrm{CH}_{2}\right), 13.8\left(\mathrm{CH}_{3}\right)$. IR (ATR): 3322, 2928, 2855, 1669, 1529, $762 \mathrm{~cm}^{-1}$. MS (ESI) $\mathrm{m} / \mathrm{z}$ (relative intensity): $333(30)[\mathrm{M}+\mathrm{Na}]^{+}, 311(100)[\mathrm{M}+\mathrm{H}]^{+}, 238$ (20). HR-MS (ESI) m/z calcd for $\mathrm{C}_{20} \mathrm{H}_{27} \mathrm{~N}_{2} \mathrm{O}[\mathrm{M}+\mathrm{H}]^{+} 311.2119$ found 311.2118 .<smiles>CCCCNC(=O)c1ncccc1C1CCCC1</smiles>

\section{N-Butyl-3-cyclopentylpicolinamide (181ib)}

Prepared following the general procedure $\mathbf{C}$ using $N$-butylpicolinamide $\mathbf{8 8 i}$ (35.6 mg, 0.20 $\mathrm{mmol})$ and cyclopentylchloride $180 \mathrm{~b}(63 \mu \mathrm{L}, 0.60 \mathrm{mmol})$ under stirring for $36 \mathrm{~h}$. Purification by column chromatography ( $n$-hexane/EtOAc 8:1) yielded $181 \mathrm{ib}(28.1 \mathrm{mg}, 57 \%)$ as a yellow oil. ${ }^{1} \mathrm{H}$ NMR $\left(300 \mathrm{MHz}, \mathrm{CDCl}_{3}\right) \delta=8.31$ (dd, $\left.J=4.5,1.5 \mathrm{~Hz}, 1 \mathrm{H}\right), 7.90$ (brs, $\left.1 \mathrm{H}\right)$, $7.74(\mathrm{dd}, J=8.1,1.5 \mathrm{~Hz}, 1 \mathrm{H}), 7.30(\mathrm{dd}, J=8.1,4.5 \mathrm{~Hz}, 1 \mathrm{H}), 4.39(\mathrm{tt}, J=9.6,7.5 \mathrm{~Hz}, 1 \mathrm{H}$ ), $3.40(\mathrm{td}, J=7.1,6.0 \mathrm{~Hz}, 2 \mathrm{H}), 2.17-2.07(\mathrm{~m}, 2 \mathrm{H}), 1.77-1.70(\mathrm{~m}, 4 \mathrm{H}), 1.62-1.36(\mathrm{~m}, 6 \mathrm{H})$, $0.93(\mathrm{t}, J=7.2 \mathrm{~Hz}, 3 \mathrm{H}) .{ }^{13} \mathrm{C}$ NMR $\left(125 \mathrm{MHz}, \mathrm{CDCl}_{3}\right) \delta=166.3\left(\mathrm{C}_{\mathrm{q}}\right), 147.9\left(\mathrm{C}_{\mathrm{q}}\right), 144.8(\mathrm{CH})$, $143.3\left(\mathrm{C}_{\mathrm{q}}\right), 136.0(\mathrm{CH}), 125.5(\mathrm{CH}), 39.7(\mathrm{CH}), 39.2\left(\mathrm{CH}_{2}\right), 34.7\left(\mathrm{CH}_{2}\right), 31.8\left(\mathrm{CH}_{2}\right), 25.8$ $\left(\mathrm{CH}_{2}\right), 20.3\left(\mathrm{CH}_{2}\right), 13.9\left(\mathrm{CH}_{3}\right)$. IR (ATR): 3385, 2947, 2859, 1660, 1508, 804, $654 \mathrm{~cm}^{-1}$. MS (ESI) m/z (relative intensity): $269(100)[\mathrm{M}+\mathrm{Na}]^{+}, 247(25)[\mathrm{M}+\mathrm{H}]^{+}, 174$ (15). HR-MS (ESI) $\mathrm{m} / \mathrm{z}$ calcd for $\mathrm{C}_{15} \mathrm{H}_{23} \mathrm{~N}_{2} \mathrm{O}[\mathrm{M}+\mathrm{H}]^{+} 247.1803$ found 247.1805 . 
<smiles>CCCCNC(=O)c1ncccc1C1CCOCC1</smiles>

N-Butyl-3-(tetrahydro-2H-pyran-4-yl)picolinamide (181ic)

Prepared following the general procedure C using $N$-butylpicolinamide $\mathbf{8 8 i}$ (35.6 mg, $0.20 \mathrm{mmol}$ ) and 4-chlorotetrahydro- $2 \mathrm{H}$-pyran $180 \mathrm{c}(65 \mu \mathrm{L}, 0.60 \mathrm{mmol})$. Purification by column chromatography ( $n$-hexane/EtOAc $8: 1$ ) yielded 181ic $(31.5 \mathrm{mg}, 60 \%$ ) as a colorless oil. ${ }^{1} \mathrm{H}$ NMR $\left(300 \mathrm{MHz}, \mathrm{CDCl}_{3}\right) \delta=8.36(\mathrm{dd}, J=4.5,1.5 \mathrm{~Hz}, 1 \mathrm{H}), 8.09(\mathrm{brs}, 1 \mathrm{H})$, $7.75(\mathrm{dd}, J=8.1,1.5 \mathrm{~Hz}, 1 \mathrm{H}), 7.36(\mathrm{dd}, J=8.1,4.5 \mathrm{~Hz}, 1 \mathrm{H}), 4.45-4.35(\mathrm{~m}, 1 \mathrm{H}), 4.06-4.01$ (m, 2H), 3.68-3,56 (m, 2H), $3.40(\mathrm{td}, J=7.2,6.0 \mathrm{~Hz}, 2 \mathrm{H}), 1.81-1.55(\mathrm{~m}, 4 \mathrm{H}), 1.65-1.54$ $(\mathrm{m}, 2 \mathrm{H}), 1.47-1.34(\mathrm{~m}, 2 \mathrm{H}), 0.94(\mathrm{t}, J=7.2 \mathrm{~Hz}, 3 \mathrm{H}) .{ }^{13} \mathrm{C} \mathrm{NMR}\left(125 \mathrm{MHz}, \mathrm{CDCl}_{3}\right) \delta=165.8$ $\left(\mathrm{C}_{\mathrm{q}}\right), 146.9\left(\mathrm{C}_{\mathrm{q}}\right), 145.3(\mathrm{CH}), 142.6\left(\mathrm{C}_{\mathrm{q}}\right), 136.0(\mathrm{CH}), 125.7(\mathrm{CH}), 68.4\left(\mathrm{CH}_{2}\right), 39.2\left(\mathrm{CH}_{2}\right)$, $35.4(\mathrm{CH}), 33.6\left(\mathrm{CH}_{2}\right), 31.7\left(\mathrm{CH}_{2}\right), 20.3\left(\mathrm{CH}_{2}\right), 13.9\left(\mathrm{CH}_{3}\right)$. IR (ATR): 3386, 2951, 2928, 2843, 1661, 1512, 1502, 1437, 1124, 630, $597 \mathrm{~cm}^{-1}$. MS (ESI) m/z (relative intensity): 285 (100) $[\mathrm{M}+\mathrm{Na}]^{+}, 263(10)[\mathrm{M}+\mathrm{H}]^{+}, 224$ (30), 190 (15). HR-MS (ESI) m/z calcd for $\mathrm{C}_{15} \mathrm{H}_{23} \mathrm{~N}_{2} \mathrm{O}_{2}$ $[\mathrm{M}+\mathrm{H}]^{+} 263.1754$ found 263.1754 .<smiles>C=CCN1CCC(c2cccnc2C(=O)NCCCC)CC1</smiles>

\section{3-(1-Allylpiperidin-4-yl)-N-butylpicolinamide (181id)}

Prepared following the general procedure $\mathbf{C}$ using $\mathrm{N}$-butylpicolinamide $\mathbf{8 8 i} \mathbf{i}$ (35.6 $\mathbf{m g}, 0.20$ mmol) and 1-allylpiperidine 180d (94 $\mu \mathrm{L}, 0.60 \mathrm{mmol})$. Purification by column chromatography ( $n$-hexane/EtOAc $8: 1)$ yielded 181id $(46.4 \mathrm{mg}, 77 \%)$ as a colorless oil. ${ }^{1} \mathrm{H}$ NMR $\left(400 \mathrm{MHz}, \mathrm{CDCl}_{3}\right) \delta=8.32(\mathrm{dd}, J=4.4,1.6 \mathrm{~Hz}, 1 \mathrm{H}), 8.04(\mathrm{brs}, 1 \mathrm{H}), 7.76(\mathrm{dd}, J=$ 8.0, 1.6 Hz, 1H), 7.30 (dd, $J=8.0,4.4 \mathrm{~Hz}, 1 \mathrm{H}), 5.88$ (ddt, $J=16.8,10.0,6.8 \mathrm{~Hz}, 1 \mathrm{H}$ ), 5.19- 
$5.11(\mathrm{~m}, 2 \mathrm{H}), 4.11(\mathrm{tt}, J=12.0,3.6 \mathrm{~Hz}, 1 \mathrm{H}), 3.37(\mathrm{td}, J=7.2,6.0 \mathrm{~Hz}, 2 \mathrm{H}), 3.03(\mathrm{dt}, J=6.8$, $1.2 \mathrm{~Hz}, 4 \mathrm{H}$ ), $2.18(\mathrm{td}, J=11.8,2.4 \mathrm{~Hz}, 2 \mathrm{H}), 1.93-1.80(\mathrm{~m}, 2 \mathrm{H}), 1.75$ (qd, $J=12.4,3.6 \mathrm{~Hz}$, 2H), 1.65-1.48 (m, 2H), 1.45-1.25 (m, 2H), $0.90(\mathrm{t}, J=7.2 \mathrm{~Hz}, 3 \mathrm{H}) .{ }^{13} \mathrm{C}$ NMR (100 MHz, $\left.\mathrm{CDCl}_{3}\right) \delta=166.0\left(\mathrm{C}_{\mathrm{q}}\right), 147.1\left(\mathrm{C}_{\mathrm{q}}\right), 145.2(\mathrm{CH}), 143.0\left(\mathrm{C}_{\mathrm{q}}\right), 136.0(\mathrm{CH}), 134.5(\mathrm{CH}), 125.7$ $(\mathrm{CH}), 118.4\left(\mathrm{CH}_{2}\right), 61.9\left(\mathrm{CH}_{2}\right), 53.9\left(\mathrm{CH}_{2}\right), 39.1\left(\mathrm{CH}_{2}\right), 35.9(\mathrm{CH}), 32.7\left(\mathrm{CH}_{2}\right), 31.6\left(\mathrm{CH}_{2}\right)$, $20.2\left(\mathrm{CH}_{2}\right), 13.7\left(\mathrm{CH}_{3}\right)$. IR (ATR): 3385, 2927, 2789, 1662, 1512, 1438, 994, 918, 626 $\mathrm{cm}^{-1}$. MS (ESI) m/z (relative intensity): $318(10)[\mathrm{M}+\mathrm{Na}]^{+}, 302(100)[\mathrm{M}+\mathrm{H}]^{+}, 229(30)$. HRMS (ESI) $\mathrm{m} / \mathrm{z}$ calcd for $\mathrm{C}_{18} \mathrm{H}_{28} \mathrm{~N}_{3} \mathrm{O}[\mathrm{M}+\mathrm{H}]^{+} 302.2227$ found 302.2224 .<smiles>CCCCNC(=O)c1ncccc1PCCC</smiles>

\section{$N$-Butyl-3-isopropylpicolinamide (181ie)}

Prepared following the general procedure $\mathbf{C}$ using $N$-butylpicolinamide $88 \mathbf{i}$ (35.6 mg, 0.20 mmol)and isopropylchloride $180 \mathrm{e}(53 \mu \mathrm{L}, 0.60 \mathrm{mmol})$ with stirring for $24 \mathrm{~h}$. Purification by column chromatography ( $n$-hexane/EtOAc $8: 1$ ) yielded 181ie $(27.3 \mathrm{mg}, 62 \%$ ) as a colorless oil. ${ }^{1} \mathrm{H}$ NMR $\left(400 \mathrm{MHz}, \mathrm{CDCl}_{3}\right) \delta=8.32(\mathrm{dd}, J=4.4,1.6 \mathrm{~Hz}, 1 \mathrm{H}), 7.92(\mathrm{brs}, 1 \mathrm{H})$, $7.75(\mathrm{dd}, J=8.0,1.6 \mathrm{~Hz}, 1 \mathrm{H}), 7.31(\mathrm{dd}, J=8.0,4.4 \mathrm{~Hz}, 1 \mathrm{H}), 4.40(\mathrm{p}, J=6.8 \mathrm{~Hz}, 1 \mathrm{H}), 3.39$ (td, $J=7.2,6.0 \mathrm{~Hz}, 2 \mathrm{H}), 1.62-1.55(\mathrm{~m}, 2 \mathrm{H}), 1.44-1.35(\mathrm{~m}, 2 \mathrm{H}), 1.23(\mathrm{~d}, J=6.8 \mathrm{~Hz}, 6 \mathrm{H})$, $0.92(\mathrm{t}, J=7.2 \mathrm{~Hz}, 3 \mathrm{H}) .{ }^{13} \mathrm{C} \operatorname{NMR}\left(100 \mathrm{MHz}, \mathrm{CDCl}_{3}\right) \delta=166.2\left(\mathrm{C}_{\mathrm{q}}\right), 147.2\left(\mathrm{C}_{\mathrm{q}}\right), 145.6\left(\mathrm{C}_{\mathrm{q}}\right)$, $145.0(\mathrm{CH}), 135.4(\mathrm{CH}), 125.6(\mathrm{CH}), 39.1\left(\mathrm{CH}_{2}\right), 31.7\left(\mathrm{CH}_{2}\right), 27.5(\mathrm{CH}), 23.6\left(\mathrm{CH}_{3}\right), 20.2$ $\left(\mathrm{CH}_{2}\right), 13.8\left(\mathrm{CH}_{3}\right)$. IR (ATR): 3386, 2958, 2866, 1662, 1511, 855, $655 \mathrm{~cm}^{-1}$. MS (ESI) $\mathrm{m} / \mathrm{z}$ (relative intensity): $243(100)[\mathrm{M}+\mathrm{Na}]^{+}, 221(20)[\mathrm{M}+\mathrm{H}]^{+}, 148$ (15). HR-MS (ESI) m/z calcd for $\mathrm{C}_{13} \mathrm{H}_{21} \mathrm{~N}_{2} \mathrm{O}[\mathrm{M}+\mathrm{H}]^{+} 221.1646$ found 2212.1648. 
$\overbrace{i-\mathrm{Bu}}^{\mathrm{N}_{\mathrm{H}}^{-n-\mathrm{Bu}}}$

\section{3-(S,ec-Butyl)-N-butylpicolinamide (181if)}

Prepared following the general procedure C using $N$-butylpicolinamide $\mathbf{8 8 i}$ (35.6 $\mathbf{m g}$, $0.20 \mathrm{mmol})$ and isobutylchloride $180 \mathrm{f}(64 \mu \mathrm{L}, 0.60 \mathrm{mmol})$. Purification by column chromatography ( $n$-hexane/EtOAc $8: 1)$ yielded 181 if $(35.7 \mathrm{mg}, 75 \%)$ as a colorless oil. ${ }^{1} \mathrm{H}$ NMR $\left(300 \mathrm{MHz}, \mathrm{CDCl}_{3}\right) \delta=8.28(\mathrm{dd}, J=4.5,1.5 \mathrm{~Hz}, 1 \mathrm{H}), 7.88(\mathrm{brs}, 1 \mathrm{H}), 7.66(\mathrm{dd}, J=$ 8.0, $1.5 \mathrm{~Hz}, 1 \mathrm{H}), 7.27(\mathrm{dd}, J=8.0,4.5 \mathrm{~Hz}, 1 \mathrm{H}), 4.23-4.11(\mathrm{~m}, 1 \mathrm{H}), 3.35(\mathrm{td}, J=7.2,6.0 \mathrm{~Hz}$, 2H), 1.60-1.48 (m, 4H), 1.41-1.29 (m, 2H), $1.16(\mathrm{~d}, J=6.9 \mathrm{~Hz}, 3 \mathrm{H}), 0.88(\mathrm{t}, J=7.2 \mathrm{~Hz}$, $3 \mathrm{H}), 0.78(\mathrm{t}, J=7.5 \mathrm{~Hz}, 3 \mathrm{H}) \cdot{ }^{13} \mathrm{C}$ NMR $\left(125 \mathrm{MHz}, \mathrm{CDCl}_{3}\right) \delta=166.1\left(\mathrm{C}_{\mathrm{q}}\right), 147.7\left(\mathrm{C}_{\mathrm{q}}\right), 144.9$ $(\mathrm{CH}), 144.5\left(\mathrm{C}_{\mathrm{q}}\right), 135.8(\mathrm{CH}), 125.4(\mathrm{CH}), 39.1\left(\mathrm{CH}_{2}\right), 34.0(\mathrm{CH}), 31.7\left(\mathrm{CH}_{2}\right), 30.8\left(\mathrm{CH}_{2}\right)$, $21.4\left(\mathrm{CH}_{3}\right), 20.3\left(\mathrm{CH}_{2}\right), 13.8\left(\mathrm{CH}_{3}\right), 12.1\left(\mathrm{CH}_{3}\right)$. IR (ATR): 3394, 2958, 2865, 1669, 1514, 146, 805, $398 \mathrm{~cm}^{-1}$. MS (ESI) m/z (relative intensity): $257(100)[\mathrm{M}+\mathrm{Na}]^{+}, 235(20)[\mathrm{M}+\mathrm{H}]^{+}$, 201 (60). HR-MS (ESI) $\mathrm{m} / \mathrm{z}$ calcd for $\mathrm{C}_{14} \mathrm{H}_{23} \mathrm{~N}_{2} \mathrm{O}[\mathrm{M}+\mathrm{H}]^{+} 235.1801$ found 235.1805.<smiles>CCCCNC(=O)c1ncccc1C(C)C</smiles>

\section{N-Butyl-3-(hexan-2-yl)picolinamide (181ig)}

Prepared following the general procedure C using $N$-butylpicolinamide $\mathbf{8 8 i}$ (35.6 $\mathbf{m g}$, $0.20 \mathrm{mmol}$ ) and 2-chlorohexane $181 \mathrm{~g}(83 \mu \mathrm{L}, 0.60 \mathrm{mmol})$. Purification by column chromatography ( $n$-hexane/EtOAc $8: 1)$ yielded $181 \mathrm{ig}(47.6 \mathrm{mg}, 82 \%)$ as a colorless oil. ${ }^{1} \mathrm{H}$ NMR $\left(400 \mathrm{MHz}, \mathrm{CDCl}_{3}\right) \delta=8.33(\mathrm{dd}, J=4.4,1.6 \mathrm{~Hz}, 1 \mathrm{H}), 7.88(\mathrm{brs}, 1 \mathrm{H}), 7.72(\mathrm{dd}, J=$ 8.0, 1.6 Hz, 1H), 7.32 (ddd, $J=8.0,4.4,0.4 \mathrm{~Hz}, 1 \mathrm{H}$ ), 4.30 (h, $J=7.2 \mathrm{~Hz}, 1 \mathrm{H}$ ), 3.43-3.39 $(\mathrm{m}, 2 \mathrm{H}), 1.63-1.48(\mathrm{~m}, 4 \mathrm{H}), 1.45-1.35(\mathrm{~m}, 2 \mathrm{H}), 1.30-1.19(\mathrm{~m}, 3 \mathrm{H}), 1.21(\mathrm{~d}, J=6.8 \mathrm{~Hz}, 3 \mathrm{H})$, 1.16-1.08 (m, 1H), $0.93(\mathrm{t}, J=7.2 \mathrm{~Hz}, 3 \mathrm{H}), 0.81(\mathrm{t}, J=7.2 \mathrm{~Hz}, 3 \mathrm{H}) .{ }^{13} \mathrm{C}$ NMR $(100 \mathrm{MHz}$, 
$\left.\mathrm{CDCl}_{3}\right) \delta=166.3\left(\mathrm{C}_{\mathrm{q}}\right), 147.7\left(\mathrm{C}_{\mathrm{q}}\right), 145.0(\mathrm{CH}), 144.9\left(\mathrm{C}_{\mathrm{q}}\right), 136.0(\mathrm{CH}), 125.6(\mathrm{CH}), 39.1$ $\left(\mathrm{CH}_{2}\right), 37.7\left(\mathrm{CH}_{2}\right), 32.3(\mathrm{CH}), 31.7\left(\mathrm{CH}_{2}\right), 29.8\left(\mathrm{CH}_{2}\right), 22.8\left(\mathrm{CH}_{2}\right), 22.0\left(\mathrm{CH}_{3}\right), 20.2\left(\mathrm{CH}_{2}\right)$, $14.0\left(\mathrm{CH}_{3}\right), 13.8\left(\mathrm{CH}_{3}\right)$. IR (ATR): 3387, 2955, 2925, 2863, 1665, 1511, 1457, 806, 708, $659 \mathrm{~cm}^{-1}$. MS (ESI) m/z (relative intensity): $285(30)[\mathrm{M}+\mathrm{Na}]^{+}, 263(100)[\mathrm{M}+\mathrm{H}]^{+}, 190(10)$, 162 (10). HR-MS (ESI) $\mathrm{m} / \mathrm{z}$ calcd for $\mathrm{C}_{17} \mathrm{H}_{29} \mathrm{~N}_{2} \mathrm{O}[\mathrm{M}+\mathrm{H}]^{+} 263.4050$ found 263.4046.<smiles>CCCCNC(=O)c1ncccc1C(C)c1ccccc1</smiles>

\section{N-Butyl-3-(1-phenylethyl)picolinamide (181ih)}

Prepared following the general procedure $\mathbf{C}$ using $\mathrm{N}$-butylpicolinamide $\mathbf{8 8 i i}$ (35.6 mg, 0.20 mmol) and (1-chloroethyl)benzene $180 \mathrm{~h}(80 \mu \mathrm{L}, 0.60 \mathrm{mmol})$. Purification by column chromatography ( $n$-hexane/EtOAc $8: 1)$ yielded 181ih $(20.9 \mathrm{mg}, 37 \%)$ as a colorless oil. ${ }^{1} \mathrm{H}$ NMR $\left(400 \mathrm{MHz}, \mathrm{CDCl}_{3}\right) \delta=8.38(\mathrm{dd}, J=5.2,0.8 \mathrm{~Hz}, 1 \mathrm{H}), 8.11-8.10(\mathrm{~m}, 1 \mathrm{H}), 8.03(\mathrm{brs}$, 1H), 7.30-7.24 (m, 2H), 7.21-7.16 (m, 4H), $4.17(\mathrm{q}, J=7.2 \mathrm{~Hz}, 1 \mathrm{H}), 3.44(\mathrm{td}, J=7.2,6.0$ $\mathrm{Hz}, 2 \mathrm{H}), 1.65(\mathrm{~d}, J=7.2 \mathrm{~Hz}, 3 \mathrm{H}), 1.61-1.55(\mathrm{~m}, 2 \mathrm{H}), 1.43-1.35(\mathrm{~m}, 2 \mathrm{H}), 0.93(\mathrm{t}, J=7.2$ $\mathrm{Hz}, 3 \mathrm{H}) .{ }^{13} \mathrm{C}$ NMR $\left(100 \mathrm{MHz}, \mathrm{CDCl}_{3}\right) \delta=164.3\left(\mathrm{C}_{\mathrm{q}}\right), 156.9\left(\mathrm{C}_{\mathrm{q}}\right), 150.1\left(\mathrm{C}_{\mathrm{q}}\right), 148.0(\mathrm{CH})$, $144.0\left(\mathrm{C}_{\mathrm{q}}\right), 128.7(\mathrm{CH}), 127.6(\mathrm{CH}), 126.7(\mathrm{CH}), 125.3(\mathrm{CH}), 121.3(\mathrm{CH}), 44.5(\mathrm{CH}), 39.1$ $\left(\mathrm{CH}_{2}\right), 31.7\left(\mathrm{CH}_{2}\right), 20.9\left(\mathrm{CH}_{3}\right), 20.1\left(\mathrm{CH}_{2}\right), 13.8\left(\mathrm{CH}_{3}\right)$. IR (ATR): 3387, 2960, 2868, 1668, 1523, 701, $585 \mathrm{~cm}^{-1}$. MS (ESI) m/z (relative intensity): $305(100)[\mathrm{M}+\mathrm{Na}]^{+}, 283(55)[\mathrm{M}+\mathrm{H}]^{+}$, 229 (35), 207 (20). HR-MS (ESI) m/z calcd for $\mathrm{C}_{18} \mathrm{H}_{23} \mathrm{~N}_{2} \mathrm{O}[\mathrm{M}+\mathrm{H}]^{+} 283.1805$ found 283.1805.<smiles>CCCNC(=O)c1ncccc1C(C)CCc1ccccc1</smiles>

N-Butyl-3-(4-phenylbutan-2-yl)picolinamide (181ii) 
Prepared following the general procedure C using $N$-butylpicolinamide $\mathbf{8 8 i}$ (35.6 $\mathbf{m g}$, $0.20 \mathrm{mmol}$ ) and (3-chlorobutyl)benzene $180 \mathrm{i}(101 \mu \mathrm{L}, 0.60 \mathrm{mmol})$. Purification by column chromatography ( $n$-hexane/EtOAc $8: 1)$ yielded $181 \mathrm{ii}(32.4 \mathrm{mg}, 52 \%)$ as a colorless oil. ${ }^{1} \mathrm{H}$ NMR $\left(300 \mathrm{MHz}, \mathrm{CDCl}_{3}\right) \delta=8.36(\mathrm{dd}, J=4.5,1.5 \mathrm{~Hz}, 1 \mathrm{H}), 7.93(\mathrm{~s}, 1 \mathrm{H}), 7.76(\mathrm{dd}, J=$ 8.1, 1.5 Hz, 1H), 7.34 (ddd, $J=8.1,4.5,0.6 \mathrm{~Hz}, 2 \mathrm{H}), 7.24-7.08(\mathrm{~m}, 4 \mathrm{H}), 4.45(\mathrm{~h}, J=7.2$ $\mathrm{Hz}, 1 \mathrm{H}), 3.42(\mathrm{td}, J=7.2,6.0 \mathrm{~Hz}, 2 \mathrm{H}), 2.70-2.57(\mathrm{~m}, 1 \mathrm{H}), 2.50-2.37(\mathrm{~m}, 1 \mathrm{H}), 1.99-1.82$ $(\mathrm{m}, 2 \mathrm{H}), 1.66-1.56(\mathrm{~m}, 2 \mathrm{H}), 1.49-1.36(\mathrm{~m}, 2 \mathrm{H}), 1.29(\mathrm{~d}, J=6.9 \mathrm{~Hz}, 3 \mathrm{H}), 0.95(\mathrm{t}, J=7.2 \mathrm{~Hz}$, 3H). ${ }^{13} \mathrm{C}$ NMR $\left(125 \mathrm{MHz}, \mathrm{CDCl}_{3}\right) \delta=166.1\left(\mathrm{C}_{\mathrm{q}}\right), 147.7\left(\mathrm{C}_{\mathrm{q}}\right), 145.10(\mathrm{CH}), 144.2\left(\mathrm{C}_{\mathrm{q}}\right), 142.5$ $\left(\mathrm{C}_{\mathrm{q}}\right), 135.8(\mathrm{CH}), 128.1(\mathrm{CH}), 128.1(\mathrm{CH}), 125.6(\mathrm{CH}), 125.5(\mathrm{CH}), 40.1\left(\mathrm{CH}_{2}\right), 39.2\left(\mathrm{CH}_{2}\right)$, $34.2\left(\mathrm{CH}_{2}\right), 32.8(\mathrm{CH}), 31.8\left(\mathrm{CH}_{2}\right), 22.18\left(\mathrm{CH}_{3}\right), 20.3\left(\mathrm{CH}_{2}\right), 13.9\left(\mathrm{CH}_{3}\right)$. IR (ATR): 3388, 2926, 2863, 1664, 1510, 1455, 807, $700 \mathrm{~cm}^{-1}$. MS (ESI) m/z (relative intensity): 333 (30) $[\mathrm{M}+\mathrm{Na}]^{+}, 311(100)[\mathrm{M}+\mathrm{H}]^{+}, 238(10)$. HR-MS (ESI) $\mathrm{m} / \mathrm{z}$ calcd for $\mathrm{C}_{20} \mathrm{H}_{27} \mathrm{~N}_{2} \mathrm{O}[\mathrm{M}+\mathrm{H}]^{+}$ 311.2120 found 311.2118 .<smiles>CCCCNC(=O)c1ncccc1C(CC)c1ccccc1</smiles>

\section{N-Butyl-3-(1-phenylbutyl)picolinamide (181ij)}

Prepared following the general procedure C using $N$-butylpicolinamide $\mathbf{8 8 i}$ (35.6 $\mathbf{m g}$, $0.20 \mathrm{mmol}$ ) and (1-chloropropyl)benzene $180 \mathrm{j}$ (100 $\mu \mathrm{L}, 0.60 \mathrm{mmol})$. Purification by column chromatography ( $n$-hexane/EtOAc $8: 1)$ yielded $181 \mathrm{ij}(36.7 \mathrm{mg}, 59 \%)$ as a colorless oil. ${ }^{1} \mathrm{H}$ NMR $\left(300 \mathrm{MHz}, \mathrm{CDCl}_{3}\right) \delta=8.37(\mathrm{dd}, J=5.1,0.9 \mathrm{~Hz}, 1 \mathrm{H}), 8.11(\mathrm{dt}, J=1.8,0.6 \mathrm{~Hz}, 1 \mathrm{H})$, 8.02 (brs, 1H), 7.29-7.17 (m, 6H), $3.82(\mathrm{t}, J=7.8 \mathrm{~Hz}, 1 \mathrm{H}), 3.43(\mathrm{td}, J=7.2,6.3 \mathrm{~Hz}, 2 \mathrm{H})$, 2.14-2.04 (m, 2H), 1.63-1.54 (m, 2H), 1.46-1.33 (m, 2H), $0.93(\mathrm{t}, J=7.2 \mathrm{~Hz}, 3 \mathrm{H}), 0.88(\mathrm{t}$, $J=7.5 \mathrm{~Hz}, 3 \mathrm{H}) \cdot{ }^{13} \mathrm{C}$ NMR $\left(125 \mathrm{MHz}, \mathrm{CDCl}_{3}\right) \delta=164.2\left(\mathrm{C}_{\mathrm{q}}\right), 155.7\left(\mathrm{C}_{\mathrm{q}}\right), 150.0\left(\mathrm{C}_{\mathrm{q}}\right), 147.9$ $(\mathrm{CH}), 142.7\left(\mathrm{C}_{\mathrm{q}}\right), 128.6(\mathrm{CH}), 127.8(\mathrm{CH}), 126.6(\mathrm{CH}), 125.5(\mathrm{CH}), 121.5(\mathrm{CH}), 52.9\left(\mathrm{CH}_{2}\right)$, $39.2\left(\mathrm{CH}_{2}\right), 31.8\left(\mathrm{CH}_{2}\right), 27.9\left(\mathrm{CH}_{2}\right), 20.2\left(\mathrm{CH}_{2}\right), 13.8\left(\mathrm{CH}_{3}\right), 12.6\left(\mathrm{CH}_{3}\right)$. IR (ATR): 3388, 2959, 2928, 2868, 1668, 1522, 700, $593 \mathrm{~cm}^{-1}$. MS (ESI) m/z (relative intensity): 319 (30) 
$[\mathrm{M}+\mathrm{Na}]^{+}, 297(100)[\mathrm{M}+\mathrm{H}]^{+}, 223(10)$. HR-MS (ESI) $\mathrm{m} / \mathrm{z}$ calcd for $\mathrm{C}_{19} \mathrm{H}_{25} \mathrm{~N}_{2} \mathrm{O}[\mathrm{M}+\mathrm{H}]^{+}$ 297.1966 found 297.1961.<smiles>C=CCCC(C)c1cccnc1C(=O)NCCCC</smiles>

\section{N-Butyl-3-(hex-5-en-2-yl)picolinamide (181k)}

Prepared following the general procedure C using $N$-butylpicolinamide $\mathbf{8 8 i}$ (35.6 $\mathbf{m g}$, $0.20 \mathrm{mmol}$ ) and 5-chlorohex-1-ene $180 \mathrm{k}(80 \mu \mathrm{L}, 0.60 \mathrm{mmol})$. Purification by column chromatography ( $n$-hexane/EtOAc $8: 1)$ yielded $181 \mathrm{k}(30.7 \mathrm{mg}, 59 \%)$ as a colorless oil. ${ }^{1} \mathrm{H}$ NMR $\left(300 \mathrm{MHz}, \mathrm{CDCl}_{3}\right) \delta=8.29(\mathrm{dd}, J=4.5,1.5 \mathrm{~Hz}, 1 \mathrm{H}), 7.85(\mathrm{brs}, 1 \mathrm{H}), 7.68(\mathrm{dd}, J=$ 8.1, $1.5 \mathrm{~Hz}, 1 \mathrm{H}$ ), 7.28 (ddd, $J=8.1,4.5,0.6 \mathrm{~Hz}, 1 \mathrm{H}$ ), 5.72 (ddt, $J=16.8,10.2,6.6 \mathrm{~Hz}, 1 \mathrm{H}$ ), 4.90-4.80 (m, 2H), $4.31(\mathrm{~h}, \mathrm{~J}=7.2 \mathrm{~Hz}, 1 \mathrm{H}), 3.39-3.32(\mathrm{~m}, 2 \mathrm{H}), 2.06-1.80(\mathrm{~m}, 2 \mathrm{H}), 1.66-$ $1.50(\mathrm{~m}, 4 \mathrm{H}), 1.44-1.27(\mathrm{~m}, 2 \mathrm{H}), 1.19(\mathrm{~d}, J=6.9 \mathrm{~Hz}, 3 \mathrm{H}), 0.89(\mathrm{t}, J=7.2 \mathrm{~Hz}, 3 \mathrm{H}) .{ }^{13} \mathrm{C}$ NMR $\left(125 \mathrm{MHz}, \mathrm{CDCl}_{3}\right) \delta=166.1\left(\mathrm{C}_{\mathrm{q}}\right), 147.7\left(\mathrm{C}_{\mathrm{q}}\right), 145.0(\mathrm{CH}), 144.3\left(\mathrm{C}_{\mathrm{q}}\right), 138.6(\mathrm{CH}), 135.9$ $(\mathrm{CH}), 125.5(\mathrm{CH}), 114.1\left(\mathrm{CH}_{2}\right), 39.2\left(\mathrm{CH}_{2}\right), 37.2\left(\mathrm{CH}_{2}\right), 32.2(\mathrm{CH}), 31.9\left(\mathrm{CH}_{2}\right), 31.8\left(\mathrm{CH}_{2}\right)$, $22.0\left(\mathrm{CH}_{3}\right), 20.3\left(\mathrm{CH}_{2}\right), 13.9\left(\mathrm{CH}_{3}\right)$. IR (ATR): 3385, 2924, 2864, 1664, 1512, 1373, 807, $654 \mathrm{~cm}^{-1}$. MS (ESI) m/z (relative intensity): $383(40)[\mathrm{M}+\mathrm{Na}]^{+}, 261(100)[\mathrm{M}+\mathrm{H}]^{+}, 188(10)$. HR-MS (ESI) $m / z$ calcd for $\mathrm{C}_{16} \mathrm{H}_{25} \mathrm{~N}_{2} \mathrm{O}[\mathrm{M}+\mathrm{H}]^{+} 261.1963$ found 261.1961 .<smiles>CCCCc1cccnc1C(=O)NC(C)c1ccccc1</smiles>

Butyl-N-(1-phenylethyl)picolinamide (200aa)

Prepared following the general procedure $\mathbf{D}$ using $N$-(1-phenylethyl)picolinamide $\mathbf{8 8 a}$ (45.2 $\mathrm{mg}, 0.20 \mathrm{mmol})$ and $n$-butylbromide $86 \mathrm{a}(64 \mu \mathrm{L}, 0.60 \mathrm{mmol})$. Purification by column 
chromatography ( $n$-hexane/EtOAc 8:1) yielded 200aa $(39.0 \mathrm{mg}, 69 \%)$ as a colorless oil. ${ }^{1} \mathrm{H}$ NMR $\left(400 \mathrm{MHz}, \mathrm{CDCl}_{3}\right) \delta=8.41(\mathrm{brs} 1 \mathrm{H}), 8.35(\mathrm{dd}, J=4.6,1.7 \mathrm{~Hz}, 1 \mathrm{H}), 7.56(\mathrm{dd}, J=$ 7.8, 1.7 Hz, 1H), 7.42-7.37 (m, 2H), 7.36-7.20 (m, 4H), 5.35-5.19 (m, 1H), 3.25-3.06 (m, 2H), 1.66-1.51 (m, 5H), 1.44-1.32 (m, 2H), $0.91(\mathrm{t}, J=7.3 \mathrm{~Hz}, 3 \mathrm{H}) .{ }^{13} \mathrm{C}$ NMR $(100 \mathrm{MHz}$, $\left.\mathrm{CDCl}_{3}\right) \delta=164.9\left(\mathrm{C}_{\mathrm{q}}\right), 147.1\left(\mathrm{C}_{\mathrm{q}}\right), 145.3(\mathrm{CH}), 143.7\left(\mathrm{C}_{\mathrm{q}}\right), 140.2\left(\mathrm{C}_{\mathrm{q}}\right), 139.9(\mathrm{CH}), 128.6$ $(\mathrm{CH}), 127.1(\mathrm{CH}), 126.1(\mathrm{CH}), 125.5(\mathrm{CH}), 48.5(\mathrm{CH}), 33.5\left(\mathrm{CH}_{2}\right), 32.7\left(\mathrm{CH}_{2}\right), 22.7\left(\mathrm{CH}_{2}\right)$, $22.3\left(\mathrm{CH}_{3}\right), 14.0\left(\mathrm{CH}_{3}\right)$. IR (ATR): 2958, 2864, 1669, 1496, 1208, $698 \mathrm{~cm}^{-1}$. MS (ESI) m/z (relative intensity) $305(100)[\mathrm{M}+\mathrm{Na}]^{+}, 283(50)[\mathrm{M}+\mathrm{H}]^{+}, 236$ (10). HR-MS (ESI) m/z calcd for $\mathrm{C}_{18} \mathrm{H}_{22} \mathrm{~N}_{2} \mathrm{O}[\mathrm{M}+\mathrm{H}]^{+}$283.1805, found 283.1797.<smiles>CCCCc1ccccc1CNC(=O)c1ccccn1</smiles>

\section{N-Benzyl-3-butylpicolinamide (200ba)}

Prepared following the general procedure D using $\mathrm{N}$-benzylpicolinamide $\mathbf{8 8 b}$ (42.4 $\mathrm{mg}$, $0.20 \mathrm{mmol})$ and $n$-butylbromide $86 \mathrm{a}(64 \mu \mathrm{L}, 0.60 \mathrm{mmol})$. Purification by column chromatography ( $n$-hexane/EtOAc $6: 1)$ yielded 200 ba $(34.8 \mathrm{mg}, 65 \%)$ as a colorless oil. ${ }^{1} \mathrm{H}$ NMR $\left(300 \mathrm{MHz}, \mathrm{CDCl}_{3}\right) \delta=8.44$ (brs $\left.1 \mathrm{H}\right), 8.34$ (brs, $\left.1 \mathrm{H}\right), 7.58(\mathrm{~d}, J=7.8 \mathrm{~Hz}, 1 \mathrm{H}), 7.46-$ $7.15(\mathrm{~m}, 6 \mathrm{H}), 4.62(\mathrm{~d}, J=4.8 \mathrm{~Hz}, 2 \mathrm{H}), 3.19(\mathrm{t}, J=7.5 \mathrm{~Hz}, 2 \mathrm{H}), 1.70-1.56(\mathrm{~m}, 2 \mathrm{H}), 1.51-$ $1.34(\mathrm{~m}, 2 \mathrm{H}), 0.93(\mathrm{t}, J=7.3 \mathrm{~Hz}, 3 \mathrm{H}) .{ }^{13} \mathrm{C}$ NMR $\left(125 \mathrm{MHz}, \mathrm{CDCl}_{3}\right) \delta=165.6\left(\mathrm{C}_{\mathrm{q}}\right), 147.0$ $\left(\mathrm{C}_{\mathrm{q}}\right), 145.3(\mathrm{CH}), 140.2\left(\mathrm{C}_{\mathrm{q}}\right), 139.9(\mathrm{CH}), 138.5\left(\mathrm{C}_{\mathrm{q}}\right), 128.5(\mathrm{CH}), 127.7(\mathrm{CH}), 127.2(\mathrm{CH})$, $125.5(\mathrm{CH}), 43.3\left(\mathrm{CH}_{2}\right), 33.7\left(\mathrm{CH}_{2}\right), 32.9\left(\mathrm{CH}_{2}\right), 22.9\left(\mathrm{CH}_{2}\right), 14.1\left(\mathrm{CH}_{3}\right)$. IR (ATR): 2926, 2862, 1668, 1505, 1433, 1127, 697, $602 \mathrm{~cm}^{-1}$. MS (ESI) m/z (relative intensity) 291 (100) $[\mathrm{M}+\mathrm{Na}]^{+}, 269$ (30) $[\mathrm{M}+\mathrm{H}]^{+}, 236(10)$. HR-MS (ESI) $\mathrm{m} / \mathrm{z}$ calcd for $\mathrm{C}_{17} \mathrm{H}_{20} \mathrm{~N}_{2} \mathrm{O}[\mathrm{M}+\mathrm{Na}]^{+}$ 291.1468, found 291.1463. 
<smiles>CCCCOc1cccnc1C(=O)NCc1ccccc1F</smiles>

\section{3-Butyl-N-(2-fluorobenzyl)picolinamide (200ca)}

Prepared following the general procedure $\mathbf{D}$ using $\mathrm{N}$-(2-fluorobenzyl)picolinamide $88 \mathrm{c}$ (46.0 mg, $0.20 \mathrm{mmol}$ ) and $n$-butylbromide $86 \mathrm{a}(64 \mu \mathrm{L}, 0.60 \mathrm{mmol})$. Purification by column chromatography ( $n$-hexane/EtOAc 6:1) yielded $200 \mathrm{ca}(32.0 \mathrm{mg}, 56 \%)$ as a white solid. M.p. $=55-57^{\circ} \mathrm{C} .{ }^{1} \mathrm{H}$ NMR $\left(300 \mathrm{MHz}, \mathrm{CDCl}_{3}\right) \delta=8.45(\mathrm{brs} 1 \mathrm{H}), 8.34(\mathrm{dd}, J=4.6,1.7 \mathrm{~Hz}, 1 \mathrm{H})$, $7.57(\mathrm{dd}, J=7.8,1.7 \mathrm{~Hz}, 1 \mathrm{H}), 7.39(\mathrm{td}, J=7.5,1.8 \mathrm{~Hz}, 1 \mathrm{H}), 7.29(\mathrm{dd}, J=7.8,4.6 \mathrm{~Hz}, 1 \mathrm{H})$, 7.26-7.16 (m, 1H), 7.13-6.96 (m, 2H), $4.67(\mathrm{~d}, J=6.3 \mathrm{~Hz}, 2 \mathrm{H}), 3.24-3.10(\mathrm{~m}, 2 \mathrm{H}), 1.68$ $1.55(\mathrm{~m}, 2 \mathrm{H}), 1.46-1.32(\mathrm{~m}, 2 \mathrm{H}), 0.92(\mathrm{t}, J=7.3 \mathrm{~Hz}, 3 \mathrm{H}) .{ }^{13} \mathrm{C} \mathrm{NMR}\left(125 \mathrm{MHz}, \mathrm{CDCl}_{3}\right) \delta=$ $165.6\left(\mathrm{C}_{\mathrm{q}}\right), 160.9\left(\mathrm{~d},{ }^{1} \mathrm{~J}_{\mathrm{C}-\mathrm{F}}=243.7 \mathrm{~Hz}, \mathrm{C}_{\mathrm{q}}\right), 146.9\left(\mathrm{C}_{\mathrm{q}}\right), 145.3(\mathrm{CH}), 140.2\left(\mathrm{C}_{\mathrm{q}}\right), 139.9(\mathrm{CH})$, $129.9\left(\mathrm{~d},{ }^{3} \mathrm{~J}_{\mathrm{C}-\mathrm{F}}=3.8 \mathrm{~Hz}, \mathrm{CH}\right), 128.9\left(\mathrm{~d},{ }^{3} \mathrm{~J}_{\mathrm{C}-\mathrm{F}}=7.5 \mathrm{~Hz}, \mathrm{CH}\right), 125.5(\mathrm{CH}), 125.4\left(\mathrm{C}_{\mathrm{q}}\right), 124.1$ $\left(\mathrm{d},{ }^{4} \mathrm{~J}_{\mathrm{C}-\mathrm{F}}=3.7 \mathrm{~Hz}, \mathrm{CH}\right), 115.2\left(\mathrm{~d},{ }^{2} \mathrm{~J}_{\mathrm{C}-\mathrm{F}}=21.3 \mathrm{~Hz}, \mathrm{CH}\right), 37.2\left(\mathrm{~d},{ }^{3} \mathrm{~J}_{\mathrm{C}-\mathrm{F}}=4.9 \mathrm{~Hz}, \mathrm{CH}\right), 33.6$ $\left(\mathrm{CH}_{2}\right), 32.8\left(\mathrm{CH}_{2}\right), 22.8\left(\mathrm{CH}_{2}\right), 14.1\left(\mathrm{CH}_{3}\right) .{ }^{19} \mathrm{~F}$ NMR $\left(275 \mathrm{MHz}, \mathrm{CDCl}_{3}\right) \delta=-118.0-117.9$ (m). IR (ATR): 2927, 2862, 1671, 1502, 1229, 754, $607 \mathrm{~cm}^{-1}$. MS (ESI) $\mathrm{m} / \mathrm{z}$ (relative

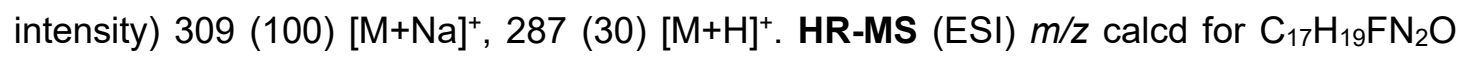
$[\mathrm{M}+\mathrm{H}]^{+}$287.1554, found 287.1550.<smiles>CCCCc1cccnc1C(=O)NCc1ccc(OC)cc1</smiles>

\section{3-Butyl-N-(4-methoxybenzyl)picolinamide (200da)}

Prepared following the general procedure $\mathbf{D}$ using $\mathrm{N}$-(4-methoxybenzyl)picolinamide $\mathbf{8 8 \mathbf { d }}$ (48.5 mg, $0.20 \mathrm{mmol}$ ) and $n$-butylbromide $86 \mathrm{a}(64 \mu \mathrm{L}, 0.60 \mathrm{mmol})$. Purification by column chromatography ( $n$-hexane/EtOAc $6: 1)$ yielded $200 \mathrm{da}(41.2 \mathrm{mg}, 69 \%)$ as a colorless oil. ${ }^{1} \mathrm{H}$ NMR $\left(400 \mathrm{MHz}, \mathrm{CDCl}_{3}\right) \delta=8.35(\mathrm{brs}, 1 \mathrm{H}), 8.31(\mathrm{dd}, J=4.6,1.7 \mathrm{~Hz}, 1 \mathrm{H}), 7.57(\mathrm{dd}, J=$ 7.8, 1.7 Hz, 1H), 7.30-7.25 (m, 3H), $6.85(\mathrm{~d}, J=8.8 \mathrm{~Hz}, 2 \mathrm{H}), 4.54(\mathrm{~d}, J=5.9 \mathrm{~Hz}, 2 \mathrm{H}), 3.77$ 
(s, 3H), 3.23-3.14 (m, 2H), 1.68-1.56 (m, 2H), 1.46-1.34 (m, 2H), $0.93(\mathrm{t}, J=7.3 \mathrm{~Hz}, 3 \mathrm{H})$.

${ }^{13} \mathrm{C}$ NMR $\left(125 \mathrm{MHz}, \mathrm{CDCl}_{3}\right) \delta=165.6\left(\mathrm{C}_{\mathrm{q}}\right), 158.8\left(\mathrm{C}_{\mathrm{q}}\right), 147.0\left(\mathrm{C}_{\mathrm{q}}\right), 145.3(\mathrm{CH}), 140.2\left(\mathrm{C}_{\mathrm{q}}\right)$, $140.1(\mathrm{CH}), 140.0\left(\mathrm{C}_{q}\right), 130.6(\mathrm{CH}), 129.1(\mathrm{CH}), 125.5(\mathrm{CH}), 114.0(\mathrm{CH}), 113.9(\mathrm{CH}), 55.2$ $\left(\mathrm{CH}_{3}\right), 42.7\left(\mathrm{CH}_{2}\right), 33.6\left(\mathrm{CH}_{2}\right), 32.8\left(\mathrm{CH}_{2}\right), 22.8\left(\mathrm{CH}_{2}\right), 14.0\left(\mathrm{CH}_{3}\right)$. IR (ATR): 2927, 2863, 1667, 1505, 1033, 813, $579 \mathrm{~cm}^{-1}$. MS (ESI) m/z (relative intensity) $321(100)[\mathrm{M}+\mathrm{Na}]^{+}, 299$ (30) $[\mathrm{M}+\mathrm{H}]^{+}$. HR-MS (ESI) $\mathrm{m} / \mathrm{z}$ calcd for $\mathrm{C}_{18} \mathrm{H}_{22} \mathrm{~N}_{2} \mathrm{O}_{2}[\mathrm{M}+\mathrm{Na}]^{+} 321.1573$, found 321.1569 .<smiles>CCCCNC(=O)c1ncccc1OCCCC</smiles>

\section{N,3-Dibutylpicolinamide (200ia)}

Prepared following the general procedure $\mathbf{D}$ using $N$-butylpicolinamide $88 \mathbf{i}$ (35.6 mg, 0.20 $\mathrm{mmol})$ and $n$-butylbromide $86 \mathrm{a}(64 \mu \mathrm{L}, 0.60 \mathrm{mmol})$. Purification by column chromatography ( $n$-hexane/EtOAc 8:1) yielded $200 \mathrm{ia}(34.5 \mathrm{mg}, 74 \%)$ as a colorless oil. ${ }^{1} \mathrm{H}$ NMR $(300 \mathrm{MHz}$, $\left.\mathrm{CDCl}_{3}\right) \delta=8.34(\mathrm{dd}, J=4.6,1.7 \mathrm{~Hz}, 1 \mathrm{H}), 8.05(\mathrm{brs} 1 \mathrm{H}), 7.56(\mathrm{dd}, J=7.8,1.7 \mathrm{~Hz}, 1 \mathrm{H}), 7.27$ (dd, $J=7.8,4.6 \mathrm{~Hz}, 1 \mathrm{H}$ ), 3.40 (td, $J=7.1,6.0 \mathrm{~Hz}, 2 \mathrm{H}), 3.14(\mathrm{t}, J=7.7 \mathrm{~Hz}, 2 \mathrm{H}), 1.64-1.54$ $(\mathrm{m}, 4 \mathrm{H}), 1.45-1.34(\mathrm{~m}, 4 \mathrm{H}), 0.92(\mathrm{td}, J=7.3,6.3 \mathrm{~Hz}, 6 \mathrm{H}) .{ }^{13} \mathrm{C} \mathrm{NMR}\left(125 \mathrm{MHz}, \mathrm{CDCl}_{3}\right) \delta=$ $165.7\left(\mathrm{C}_{\mathrm{q}}\right), 147.4\left(\mathrm{C}_{\mathrm{q}}\right), 145.2(\mathrm{CH}), 139.9\left(\mathrm{C}_{\mathrm{q}}\right), 139.8(\mathrm{CH}), 125.3(\mathrm{CH}), 39.1\left(\mathrm{CH}_{2}\right), 33.7$ $\left(\mathrm{CH}_{2}\right), 32.9\left(\mathrm{CH}_{2}\right), 31.8\left(\mathrm{CH}_{2}\right), 22.9\left(\mathrm{CH}_{2}\right), 20.3\left(\mathrm{CH}_{2}\right), 14.1\left(\mathrm{CH}_{3}\right), 13.9\left(\mathrm{CH}_{3}\right)$. IR (ATR): 2927, 2864, 1667, 1511, 1434, 807, $605 \mathrm{~cm}^{-1}$. MS (ESI) m/z (relative intensity) 257 (100) $[\mathrm{M}+\mathrm{Na}]^{+}, 235(40)[\mathrm{M}+\mathrm{H}]^{+}$. HR-MS (ESI) $\mathrm{m} / \mathrm{z}$ calcd for $\mathrm{C}_{14} \mathrm{H}_{22} \mathrm{~N}_{2} \mathrm{O}[\mathrm{M}+\mathrm{H}]^{+} 235.1805$, found 235.1801 .

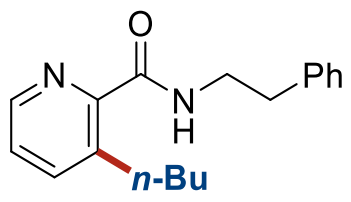

3-Butyl-N-phenethylpicolinamide (200fa) 
Prepared following the general procedure $\mathbf{D}$ using $N$-(2-phenylethyl)picolinamide $\mathbf{8 8 f}$ (45.2 $\mathrm{mg}, 0.20 \mathrm{mmol})$ and $n$-butylbromide $86 \mathrm{a}(64 \mu \mathrm{L}, 0.60 \mathrm{mmol})$. Purification by column chromatography ( $n$-hexane/EtOAc $8: 1)$ yielded $200 \mathrm{fa}(42.3 \mathrm{mg}, 75 \%)$ as a colorless oil. ${ }^{1} \mathrm{H}$ NMR $\left(400 \mathrm{MHz}, \mathrm{CDCl}_{3}\right) \delta=8.32(\mathrm{dd}, J=4.6,1.7 \mathrm{~Hz}, 1 \mathrm{H}), 8.16$ (brs $\left.1 \mathrm{H}\right), 7.57$ (dd, $J=$ 7.8, 1.7 Hz, 1H), 7.33-7.18 (m, 6H), 3.68 (td, $J=7.3,6.0 \mathrm{~Hz}, 2 \mathrm{H}), 3.16(\mathrm{t}, J=7.3 \mathrm{~Hz}, 2 \mathrm{H})$, $2.92(\mathrm{t}, J=7.3 \mathrm{~Hz}, 2 \mathrm{H}), 1.65-1.55(\mathrm{~m}, 2 \mathrm{H}), 1.46-1.34(\mathrm{~m}, 2 \mathrm{H}), 0.92(\mathrm{t}, J=7.3 \mathrm{~Hz}, 3 \mathrm{H})$. ${ }^{13} \mathrm{C}$ NMR $\left(100 \mathrm{MHz}, \mathrm{CDCl}_{3}\right) \delta=165.8\left(\mathrm{C}_{\mathrm{q}}\right), 147.2\left(\mathrm{C}_{\mathrm{q}}\right), 145.3(\mathrm{CH}), 140.1\left(\mathrm{C}_{\mathrm{q}}\right), 140.0(\mathrm{CH})$, $139.2\left(\mathrm{C}_{\mathrm{q}}\right), 128.8(\mathrm{CH}), 128.5(\mathrm{CH}), 126.3(\mathrm{CH}), 125.5(\mathrm{CH}), 40.6\left(\mathrm{CH}_{2}\right), 36.0\left(\mathrm{CH}_{2}\right), 33.6$ $\left(\mathrm{CH}_{2}\right), 32.8\left(\mathrm{CH}_{2}\right), 22.8\left(\mathrm{CH}_{2}\right), 14.0\left(\mathrm{CH}_{3}\right)$. IR (ATR): 2927, 2862, 1667, 1506, 1435, 745, $698 \mathrm{~cm}^{-1}$. MS (ESI) m/z (relative intensity) $305(100)[\mathrm{M}+\mathrm{Na}]^{+}, 283(30)[\mathrm{M}+\mathrm{H}]^{+}, 236(10)$. HR-MS (ESI) $m / z$ calcd for $\mathrm{C}_{18} \mathrm{H}_{22} \mathrm{~N}_{2} \mathrm{O}[\mathrm{M}+\mathrm{H}]^{+} 283.1805$, found 283.1798 .<smiles>CCCCc1cccnc1C(=O)NC1CCCCC1</smiles>

\section{3-Butyl-N-cyclohexylpicolinamide (200ga)}

Prepared following the general procedure $\mathbf{D}$ using $\mathrm{N}$-cyclohexylpicolinamide $\mathbf{8 8 g}(\mathbf{4 0 . 8} \mathrm{mg}$, $0.20 \mathrm{mmol})$ and $n$-butylbromide $86 \mathrm{a}(64 \mu \mathrm{L}, 0.60 \mathrm{mmol})$. Purification by column chromatography ( $n$-hexane/EtOAc $8: 1$ ) yielded $200 \mathrm{ga}(39.8 \mathrm{mg}, 77 \%$ ) as a colorless oil. ${ }^{1} \mathrm{H}$ NMR $\left(500 \mathrm{MHz}, \mathrm{CDCl}_{3}\right) \delta=8.33(\mathrm{dd}, J=4.6,1.7 \mathrm{~Hz}, 1 \mathrm{H}), 7.96$ (brs $\left.1 \mathrm{H}\right), 7.54$ (dd, $J=$ 7.8, 1.7 Hz, 1H), $7.26(\mathrm{dd}, J=7.8,4.6 \mathrm{~Hz}, 1 \mathrm{H}), 3.95-3.78(\mathrm{~m}, 1 \mathrm{H}), 3.13(\mathrm{t}, J=7.3 \mathrm{~Hz}, 2 \mathrm{H})$, 2.02-1.93 (m, 2H), 1.77-1.69 (m, 2H), 1.64-1.53 (m, 3H), 1.45-1.33 (m, 4H), 1.30-1.13 $(\mathrm{m}, 3 \mathrm{H}), 0.90(\mathrm{t}, J=7.4 \mathrm{~Hz}, 3 \mathrm{H}) \cdot{ }^{13} \mathrm{C}$ NMR $\left(125 \mathrm{MHz}, \mathrm{CDCl}_{3}\right) \delta=164.8\left(\mathrm{C}_{\mathrm{q}}\right), 147.5\left(\mathrm{C}_{\mathrm{q}}\right)$, $145.2(\mathrm{CH}), 140.1\left(\mathrm{C}_{\mathrm{q}}\right), 140.0(\mathrm{CH}), 125.3(\mathrm{CH}), 47.9(\mathrm{CH}), 33.7\left(\mathrm{CH}_{2}\right), 33.1\left(\mathrm{CH}_{2}\right), 32.9$ $\left(\mathrm{CH}_{2}\right), 25.6\left(\mathrm{CH}_{2}\right), 24.9\left(\mathrm{CH}_{2}\right), 22.8\left(\mathrm{CH}_{2}\right), 14.0\left(\mathrm{CH}_{3}\right)$. IR (ATR): 2927, 2854, 1667, 1503, 1440, 1122, 808, $590 \mathrm{~cm}^{-1}$. MS (ESI) $\mathrm{m} / \mathrm{z}$ (relative intensity) $283(100)[\mathrm{M}+\mathrm{Na}]^{+}, 261(50)$ $[\mathrm{M}+\mathrm{H}]^{+}, 133$ (5). HR-MS (ESI) $\mathrm{m} / \mathrm{z}$ calcd for $\mathrm{C}_{16} \mathrm{H}_{24} \mathrm{~N}_{2} \mathrm{O}[\mathrm{M}+\mathrm{H}]^{+}$261.1961, found 261.1960. 
<smiles>CCCCc1cccnc1C(=O)NC(C)(C)C</smiles>

\section{N-(tert-Butyl)-3-butylpicolinamide (200ea)}

Prepared following the general procedure D using $N$-(tert-butyl)picolinamide 88e (35.6 mg, $0.20 \mathrm{mmol})$ and $n$-butylbromide $86 \mathrm{a}(64 \mu \mathrm{L}, 0.60 \mathrm{mmol})$. Purification by column chromatography ( $n$-hexane/EtOAc $8: 1)$ yielded 200ea $(35.5 \mathrm{mg}, 75 \%)$ as a colorless oil. ${ }^{1} \mathrm{H}$ NMR $\left(400 \mathrm{MHz}, \mathrm{CDCl}_{3}\right) \delta=8.31(\mathrm{dd}, J=4.6,1.7 \mathrm{~Hz}, 1 \mathrm{H}), 7.83$ (brs $\left.1 \mathrm{H}\right), 7.54$ (dd, $J=$ 7.8, 1.7 Hz, 1H), $7.24(\mathrm{dd}, J=7.8,4.6 \mathrm{~Hz}, 1 \mathrm{H}), 3.13(\mathrm{t}, J=7.3 \mathrm{~Hz}, 2 \mathrm{H}), 1.65-1.52(\mathrm{~m}, 2 \mathrm{H})$, 1.45 (s, 9H), 1.32 (d, $J=7.3 \mathrm{~Hz}, 2 \mathrm{H}), 0.90$ (t, $J=7.3 \mathrm{~Hz}, 3 \mathrm{H}) .{ }^{13} \mathrm{C} \mathrm{NMR}\left(100 \mathrm{MHz}, \mathrm{CDCl}_{3}\right)$ $\delta=165.4\left(\mathrm{C}_{\mathrm{q}}\right), 148.6\left(\mathrm{C}_{\mathrm{q}}\right), 145.0(\mathrm{CH}), 139.8(\mathrm{CH}), 139.5\left(\mathrm{C}_{\mathrm{q}}\right), 125.1(\mathrm{CH}), 50.7\left(\mathrm{C}_{\mathrm{q}}\right), 33.4$ ( $\left(\mathrm{CH}_{2}\right)$, $32.6\left(\mathrm{CH}_{2}\right), 28.7\left(\mathrm{CH}_{3}\right), 22.6\left(\mathrm{CH}_{2}\right), 14.0\left(\mathrm{CH}_{3}\right)$. IR (ATR): 2960, 2865, 1674, 1508, 1450, 1227, 805, $603 \mathrm{~cm}^{-1}$. MS (ESI) m/z (relative intensity) $257(100)[\mathrm{M}+\mathrm{Na}]^{+}, 235(80)$ $[\mathrm{M}+\mathrm{H}]^{+}, 179$ (10), 149 (10). HR-MS (ESI) $\mathrm{m} / \mathrm{z}$ calcd for $\mathrm{C}_{14} \mathrm{H}_{22} \mathrm{~N}_{2} \mathrm{O}[\mathrm{M}+\mathrm{H}]^{+} 235.1805$, found 235.1805 .<smiles>CCCCNC(=O)c1ncccc1CC(C)(C)C</smiles>

\section{N-(tert-Butyl)-3-pentylpicolinamide (200eb)}

Prepared following the general procedure D using $N$-(tert-butyl)picolinamide 88e (35.6 mg, $0.20 \mathrm{mmol})$ and $n$-pentylbromide $86 \mathrm{~b}(74 \mu \mathrm{L}, 0.60 \mathrm{mmol})$. Purification by column chromatography ( $n$-hexane/EtOAc 10:1) yielded 200eb (35.3 mg, $71 \%)$ as a colorless oil. ${ }^{1} \mathrm{H}$ NMR $\left(400 \mathrm{MHz}, \mathrm{CDCl}_{3}\right) \delta=8.32(\mathrm{dd}, J=4.6,1.6 \mathrm{~Hz}, 1 \mathrm{H}), 7.83$ (brs $\left.1 \mathrm{H}\right), 7.54$ (dd, $J=$ 7.8, 1.6 Hz, 1H), $7.25(\mathrm{dd}, J=7.8,4.6 \mathrm{~Hz}, 1 \mathrm{H}), 3.13(\mathrm{t}, J=7.3 \mathrm{~Hz}, 2 \mathrm{H}), 1.66-1.54(\mathrm{~m}, 2 \mathrm{H})$, $1.45(\mathrm{~s}, 9 \mathrm{H}), 1.38-1.26(\mathrm{~m}, 4 \mathrm{H}), 0.89-0.81(\mathrm{~m}, 3 \mathrm{H}) .{ }^{13} \mathrm{C} \mathrm{NMR}\left(100 \mathrm{MHz}, \mathrm{CDCl}_{3}\right) \delta=165.4$ $\left(\mathrm{C}_{\mathrm{q}}\right), 148.6\left(\mathrm{C}_{\mathrm{q}}\right), 145.0(\mathrm{CH}), 140.0(\mathrm{CH}), 139.5\left(\mathrm{C}_{\mathrm{q}}\right), 125.1(\mathrm{CH}), 50.7\left(\mathrm{C}_{\mathrm{q}}\right), 32.8\left(\mathrm{CH}_{2}\right)$, 31.8 $\left(\mathrm{CH}_{2}\right), 30.9\left(\mathrm{CH}_{2}\right), 28.7\left(\mathrm{CH}_{3}\right), 22.6\left(\mathrm{CH}_{2}\right), 14.0\left(\mathrm{CH}_{3}\right)$. MS (ESI) $\mathrm{m} / \mathrm{z}$ (relative intensity) 
$271(100)[\mathrm{M}+\mathrm{Na}]^{+}, 249(70)[\mathrm{M}+\mathrm{H}]^{+}, 149$ (10). HR-MS (ESI) $\mathrm{m} / \mathrm{z}$ calcd for $\mathrm{C}_{15} \mathrm{H}_{24} \mathrm{~N}_{2} \mathrm{O}$ $[\mathrm{M}+\mathrm{H}]^{+}$249.1961, found 249.1960.<smiles>CCCCc1cccnc1C(=O)NC(C)(C)C</smiles>

\section{$N$-(tert-Butyl)-3-undecylpicolinamide (200ec)}

Prepared following the general procedure $\mathbf{D}$ using $N$-(tert-butyl)picolinamide $\mathbf{8 8 e}$ (35.6 $\mathbf{~ m g}$, $0.20 \mathrm{mmol}$ ) and 1-bromoundecane 86c (94 $\mathrm{L}, 0.60 \mathrm{mmol})$. Purification by column chromatography ( $n$-hexane/EtOAc $10: 1)$ yielded $200 \mathrm{ec}(35.3 \mathrm{mg}, 53 \%)$ as a colorless oil. ${ }^{1} \mathrm{H}$ NMR $\left(400 \mathrm{MHz}, \mathrm{CDCl}_{3}\right) \delta=8.32(\mathrm{dd}, J=4.6,1.6 \mathrm{~Hz}, 1 \mathrm{H}), 7.83$ (brs $\left.1 \mathrm{H}\right), 7.54$ (dd, $J=$ 7.8, 1.6 Hz, 1H), 7.25 (dd, $J=7.8,4.6 \mathrm{~Hz}, 1 \mathrm{H}), 3.13(\mathrm{t}, J=7.6 \mathrm{~Hz}, 2 \mathrm{H}), 1.63-1.54(\mathrm{~m}, 2 \mathrm{H})$, $1.45(\mathrm{~s}, 9 \mathrm{H}), 1.39-1.20(\mathrm{~m}, 16 \mathrm{H}), 0.85(\mathrm{t}, J=7.3 \mathrm{~Hz}, 3 \mathrm{H}) .{ }^{13} \mathrm{C} \mathrm{NMR}\left(100 \mathrm{MHz}, \mathrm{CDCl}_{3}\right) \delta=$ $165.4\left(\mathrm{C}_{\mathrm{q}}\right), 148.6\left(\mathrm{C}_{\mathrm{q}}\right), 145.0(\mathrm{CH}), 140.0(\mathrm{CH}), 139.6\left(\mathrm{C}_{\mathrm{q}}\right), 125.1(\mathrm{CH}), 50.7\left(\mathrm{C}_{\mathrm{q}}\right), 32.9$ $\left(\mathrm{CH}_{2}\right), 31.9\left(\mathrm{CH}_{2}\right), 31.2\left(\mathrm{CH}_{2}\right), 29.6\left(\mathrm{CH}_{2}\right), 29.6\left(\mathrm{CH}_{2}\right), 29.6\left(\mathrm{CH}_{2}\right), 29.6\left(\mathrm{CH}_{2}\right), 29.5\left(\mathrm{CH}_{2}\right)$, $29.3\left(\mathrm{CH}_{2}\right), 28.7\left(\mathrm{CH}_{3}\right), 22.7\left(\mathrm{CH}_{2}\right), 14.1\left(\mathrm{CH}_{3}\right)$. IR (ATR): 2922, 2853, 1676, 1509, 1455, 1228, 803, $604 \mathrm{~cm}^{-1}$. MS (ESI) m/z (relative intensity) $355(100)[\mathrm{M}+\mathrm{Na}]^{+}, 333(60)[\mathrm{M}+\mathrm{H}]^{+}$, 236 (10), 149 (10). HR-MS (ESI) $\mathrm{m} / z$ calcd for $\mathrm{C}_{21} \mathrm{H}_{36} \mathrm{~N}_{2} \mathrm{O}[\mathrm{M}+\mathrm{H}]^{+} 333.2900$, found 333.2901 .<smiles>CC(C)(C)NC(=O)c1ncccc1CCCc1ccccc1</smiles>

\section{$N$-(tert-Butyl)-3-(3-phenylpropyl)picolinamide (200ed)}

Prepared following the general procedure $\mathbf{D}$ using $\mathrm{N}$-(tert-butyl)picolinamide $88 \mathrm{e}$ ( $35.6 \mathrm{mg}$, $0.20 \mathrm{mmol}$ ) and (3-bromopropyl)benzene $\mathbf{8 6 \mathrm { d }}(119.4 \mathrm{mg}, 0.60 \mathrm{mmol})$. Purification by 
column chromatography ( $n$-hexane/EtOAc $7: 1)$ yielded $200 \mathrm{ed}(43.8 \mathrm{mg}, 74 \%)$ as a colorless oil. ${ }^{1} \mathrm{H}$ NMR $\left(400 \mathrm{MHz}, \mathrm{CDCl}_{3}\right) \delta=8.33(\mathrm{dd}, J=4.6,1.6 \mathrm{~Hz}, 1 \mathrm{H}), 7.90(\mathrm{brs} 1 \mathrm{H})$, $7.52(\mathrm{dd}, J=7.8,1.6 \mathrm{~Hz}, 1 \mathrm{H}), 7.29-7.21(\mathrm{~m}, 3 \mathrm{H}), 7.20-7.11(\mathrm{~m}, 3 \mathrm{H}), 3.22(\mathrm{t}, J=7.5 \mathrm{~Hz}$, 2H), $2.70(\mathrm{t}, J=7.3 \mathrm{~Hz}, 2 \mathrm{H}), 2.01-1.91(\mathrm{~m}, 2 \mathrm{H}), 1.47(\mathrm{~s}, 9 \mathrm{H}) .{ }^{13} \mathrm{C} \mathrm{NMR}\left(100 \mathrm{MHz}, \mathrm{CDCl}_{3}\right)$ $\delta=165.3\left(\mathrm{C}_{\mathrm{q}}\right), 148.5\left(\mathrm{C}_{\mathrm{q}}\right), 145.2(\mathrm{CH}), 142.4\left(\mathrm{C}_{\mathrm{q}}\right), 139.9(\mathrm{CH}), 139.0\left(\mathrm{C}_{\mathrm{q}}\right), 128.4(\mathrm{CH})$, $128.2(\mathrm{CH}), 125.6(\mathrm{CH}), 125.2(\mathrm{CH}), 50.7\left(\mathrm{C}_{\mathrm{q}}\right), 35.7\left(\mathrm{CH}_{2}\right), 32.8\left(\mathrm{CH}_{2}\right), 32.6\left(\mathrm{CH}_{2}\right), 28.7$ $\left(\mathrm{CH}_{3}\right)$. IR (ATR): 2963, 1672, 1508, 1448, 1227, 1096, 745, 700, $607 \mathrm{~cm}^{-1}$. MS (ESI) m/z (relative intensity) $319(100)[\mathrm{M}+\mathrm{Na}]^{+}, 297(70)[\mathrm{M}+\mathrm{H}]^{+}, 271$ (20), $236(15), 149$ (20). HRMS (ESI) $m / z$ calcd for $\mathrm{C}_{19} \mathrm{H}_{24} \mathrm{~N}_{2} \mathrm{O}[\mathrm{M}+\mathrm{H}]^{+}$297.1961, found 297.1960.<smiles>CC(C)(C)NC(=O)c1ncccc1C(=O)Cl</smiles>

\section{N-(tert-Butyl)-3-(6-chlorohexyl)picolinamide (200ee)}

Prepared following the general procedure D using $N$-(tert-butyl)picolinamide $88 \mathbf{e}(35.6 \mathrm{mg}$, $0.20 \mathrm{mmol}$ ) and 1-bromo-6-chlorohexane $86 \mathrm{e}(119.7 \mathrm{mg}, 0.60 \mathrm{mmol})$. Purification by column chromatography ( $n$-hexane/EtOAc $8: 1)$ yielded 200 ee $(43.5 \mathrm{mg}, 73 \%)$ as a colorless oil. ${ }^{1} \mathrm{H}$ NMR $\left(400 \mathrm{MHz}, \mathrm{CDCl}_{3}\right) \delta=8.33(\mathrm{dd}, J=4.6,1.7 \mathrm{~Hz}, 1 \mathrm{H}), 7.87(\mathrm{brs} 1 \mathrm{H})$, $7.54(\mathrm{dd}, J=7.8,1.7 \mathrm{~Hz}, 1 \mathrm{H}), 7.26(\mathrm{dd}, J=7.8,4.6 \mathrm{~Hz}, 1 \mathrm{H}), 3.50(\mathrm{t}, J=6.8 \mathrm{~Hz}, 2 \mathrm{H}), 3.14$ $(\mathrm{t}, J=7.5 \mathrm{~Hz}, 2 \mathrm{H}), 1.78-1.70(\mathrm{~m}, 2 \mathrm{H}), 1.66-1.57(\mathrm{~m}, 2 \mathrm{H}), 1.45(\mathrm{~s}, 9 \mathrm{H}), 1.49-1.32(\mathrm{~m}, 4 \mathrm{H})$. ${ }^{13} \mathrm{C}$ NMR $\left(100 \mathrm{MHz}, \mathrm{CDCl}_{3}\right) \delta=165.3\left(\mathrm{C}_{\mathrm{q}}\right), 148.5\left(\mathrm{C}_{\mathrm{q}}\right), 145.1(\mathrm{CH}), 139.9(\mathrm{CH}), 139.3\left(\mathrm{C}_{\mathrm{q}}\right)$, $125.2(\mathrm{CH}), 50.7\left(\mathrm{C}_{\mathrm{q}}\right), 45.1\left(\mathrm{CH}_{2}\right), 32.8\left(\mathrm{CH}_{2}\right), 32.5\left(\mathrm{CH}_{2}\right), 31.0\left(\mathrm{CH}_{2}\right), 28.8\left(\mathrm{CH}_{2}\right), 28.7$ $\left(\mathrm{CH}_{3}\right), 26.7\left(\mathrm{CH}_{2}\right)$. IR (ATR): 2928, 2858, 1672, 1509, 1447, 1361, 1227, 711, $610 \mathrm{~cm}^{-1}$. MS (ESI) $\mathrm{m} / \mathrm{z}$ (relative intensity) $319(100)\left({ }^{35} \mathrm{Cl}\right)[\mathrm{M}+\mathrm{Na}]^{+}, 297(80)\left({ }^{35} \mathrm{Cl}\right)[\mathrm{M}+\mathrm{H}]^{+}, 249(10)$, 149 (20). HR-MS (ESI) $\mathrm{m} / \mathrm{z}$ calcd for $\mathrm{C}_{16} \mathrm{H}_{25}{ }^{35} \mathrm{CIN}_{2} \mathrm{O}[\mathrm{M}+\mathrm{H}]^{+} 297.1728$, found 297.1729. 
<smiles>C=CCCc1cccnc1C(=O)NC(C)(C)C</smiles>

\section{3-(But-3-en-1-yl)-N-(tert-butyl)picolinamide (200ef)}

Prepared following the general procedure D using $N$-(tert-butyl)picolinamide $\mathbf{8 8 e}$ (35.6 mg, $0.20 \mathrm{mmol})$ and 4-bromobut-1-ene $86 \mathrm{f}(81.0 \mathrm{mg}, 0.60 \mathrm{mmol})$. Purification by column chromatography ( $n$-hexane/EtOAc $10: 1)$ yielded 200 ef $(24.1 \mathrm{mg}, 52 \%)$ as a colorless oil. ${ }^{1} \mathrm{H}$ NMR $\left(400 \mathrm{MHz}, \mathrm{CDCl}_{3}\right) \delta=8.33(\mathrm{dd}, J=4.6,1.7 \mathrm{~Hz}, 1 \mathrm{H}), 7.90$ (brs $\left.1 \mathrm{H}\right), 7.53(\mathrm{dd}, J=$ 7.8, 1.7 Hz, 1H), 7.26 (dd, $J=7.8,4.6 \mathrm{~Hz}, 1 \mathrm{H}), 5.84$ (ddt, $J=17.0,10.2,6.7 \mathrm{~Hz}, 1 \mathrm{H}), 5.03-$ $4.86(\mathrm{~m}, 2 \mathrm{H}), 3.25(\mathrm{t}, J=6.8, \mathrm{~Hz}, 2 \mathrm{H}), 2.44-2.33(\mathrm{~m}, 2 \mathrm{H}), 1.46(\mathrm{~s}, 9 \mathrm{H}) .{ }^{13} \mathrm{C}$ NMR $(100 \mathrm{MHz}$, $\left.\mathrm{CDCl}_{3}\right) \delta=165.2\left(\mathrm{C}_{\mathrm{q}}\right), 148.6\left(\mathrm{C}_{\mathrm{q}}\right), 145.3(\mathrm{CH}), 140.2(\mathrm{CH}), 138.5\left(\mathrm{C}_{\mathrm{q}}\right), 138.0(\mathrm{CH}), 125.1$ (CH), $115.1\left(\mathrm{CH}_{2}\right), 50.8\left(\mathrm{C}_{\mathrm{q}}\right), 35.1\left(\mathrm{CH}_{2}\right), 32.5\left(\mathrm{CH}_{2}\right), 28.8\left(\mathrm{CH}_{3}\right)$. IR (ATR): 2966, 1672, $1509,1441,1225,911,804,601 \mathrm{~cm}^{-1} . \mathbf{M S}$ (ESI) m/z (relative intensity) 255 (100) [M+Na] , $233(50)[\mathrm{M}+\mathrm{H}]^{+}, 177$ (10). HR-MS (ESI) $\mathrm{m} / \mathrm{z}$ calcd for $\mathrm{C}_{14} \mathrm{H}_{20} \mathrm{~N}_{2} \mathrm{O}[\mathrm{M}+\mathrm{H}]^{+} 233.1648$, found 233.1647.<smiles>C=CCc1cccnc1C(=O)NC(C)(C)C</smiles>

\section{N-(tert-Butyl)-3-(hex-5-en-1-yl)picolinamide (200eh)}

Prepared following the general procedure D using $N$-(tert-butyl)picolinamide $8 \mathbf{8 e}$ (35.6 mg, $0.20 \mathrm{mmol}$ ) and 6-bromohex-1-ene $86 \mathrm{~h}(97.2 \mathrm{mg}, 0.60 \mathrm{mmol})$. Purification by column chromatography ( $n$-hexane/EtOAc 10:1) yielded 200eh $(35.6 \mathrm{mg}, 68 \%)$ as a colorless oil. ${ }^{1} \mathrm{H}$ NMR $\left(400 \mathrm{MHz}, \mathrm{CDCl}_{3}\right) \delta=8.32(\mathrm{dd}, J=4.6,1.7 \mathrm{~Hz}, 1 \mathrm{H}), 7.85$ (brs $\left.1 \mathrm{H}\right), 7.54$ (dd, $J=$ 7.8, 1.7 Hz, 1H), 7.26 (dd, $J=7.8,4.6 \mathrm{~Hz}, 1 \mathrm{H}), 5.78$ (ddt, $J=16.9,10.2,6.7 \mathrm{~Hz}, 1 \mathrm{H}), 5.00$ $4.86(\mathrm{~m}, 2 \mathrm{H}), 3.15(\mathrm{t}, J=7.3 \mathrm{~Hz}, 2 \mathrm{H}), 2.14-1.99(\mathrm{~m}, 2 \mathrm{H}), 1.68-1.57(\mathrm{~m}, 2 \mathrm{H}), 1.51-1.40(\mathrm{~m}$, 11H). ${ }^{13} \mathrm{C}$ NMR $\left(100 \mathrm{MHz}, \mathrm{CDCl}_{3}\right) \delta=165.3\left(\mathrm{C}_{\mathrm{q}}\right), 148.6\left(\mathrm{C}_{\mathrm{q}}\right), 145.1(\mathrm{CH}), 139.9(\mathrm{CH}), 139.3$ $\left(\mathrm{C}_{\mathrm{q}}\right), 138.9(\mathrm{CH}), 125.2(\mathrm{CH}), 114.3\left(\mathrm{CH}_{2}\right), 50.8\left(\mathrm{C}_{\mathrm{q}}\right), 33.7\left(\mathrm{CH}_{2}\right), 32.7\left(\mathrm{CH}_{2}\right), 30.7\left(\mathrm{CH}_{2}\right)$, $28.8\left(\mathrm{CH}_{2}\right), 28.7\left(\mathrm{CH}_{3}\right)$. IR (ATR): 2924, 2858, 1673, 1509, 1445, 1227, 910, $607 \mathrm{~cm}^{-1}$. 
MS (ESI) m/z (relative intensity) $283(10)[\mathrm{M}+\mathrm{Na}]^{+}, 261(100)[\mathrm{M}+\mathrm{H}]^{+}, 205$ (10). HR-MS (ESI) $m / z$ calcd for $\mathrm{C}_{16} \mathrm{H}_{24} \mathrm{~N}_{2} \mathrm{O}[\mathrm{M}+\mathrm{H}]^{+} 261.1961$, found 261.1961 .

\subsubsection{Racemization Free Alkylation of 88a}<smiles></smiles>

(S)-88a $(e e=99 \%)$

$$
\begin{aligned}
& \mathrm{MnCl}_{2}(10 \mathrm{~mol} \%) \\
& \operatorname{TMEDA}(1.0 \text { equiv }) \\
& \text { EtMgBr }(3.5 \text { equiv }) \\
& \hline \text { THF, } 60^{\circ} \mathrm{C}, 14 \mathrm{~h}
\end{aligned}
$$

$180 \mathrm{a}$<smiles>C[C@H](NC(=O)c1ncccc1Cl)c1ccccc1</smiles>

(S)-181aa: $81 \%$ $(e e=99 \%)$

Prepared following the general procedure B using $N$-(1-phenylethyl)picolinamide $(S)$-88a (45.2 $\mathrm{mg}, 0.20 \mathrm{mmol})$ and cyclohexylchloride $180 \mathrm{a}(71 \mu \mathrm{L}, 0.60 \mathrm{mmol})$. Purification by column chromatography ( $n$-hexane/EtOAc $8: 1$ ) yielded $(S)-181$ aa $(49.8 \mathrm{mg}, 81 \%)$ as a colorless oil. Chiral HPLC analysis (Chiralcel IC, $i-\mathrm{PrOH} / n$-hexane 30:70, flow rate $=1.0$ $\mathrm{mL} / \mathrm{min}, \lambda=254 \mathrm{~nm}), \mathrm{t}_{\mathrm{r}}($ major $)=4.3 \mathrm{~min}, \mathrm{t}_{\mathrm{r}}(\operatorname{minor})=4.9 \mathrm{~min}$. 


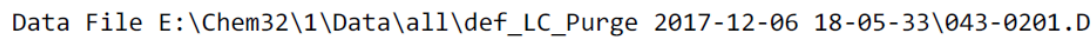
Sample Name: zsb132-2smrac1
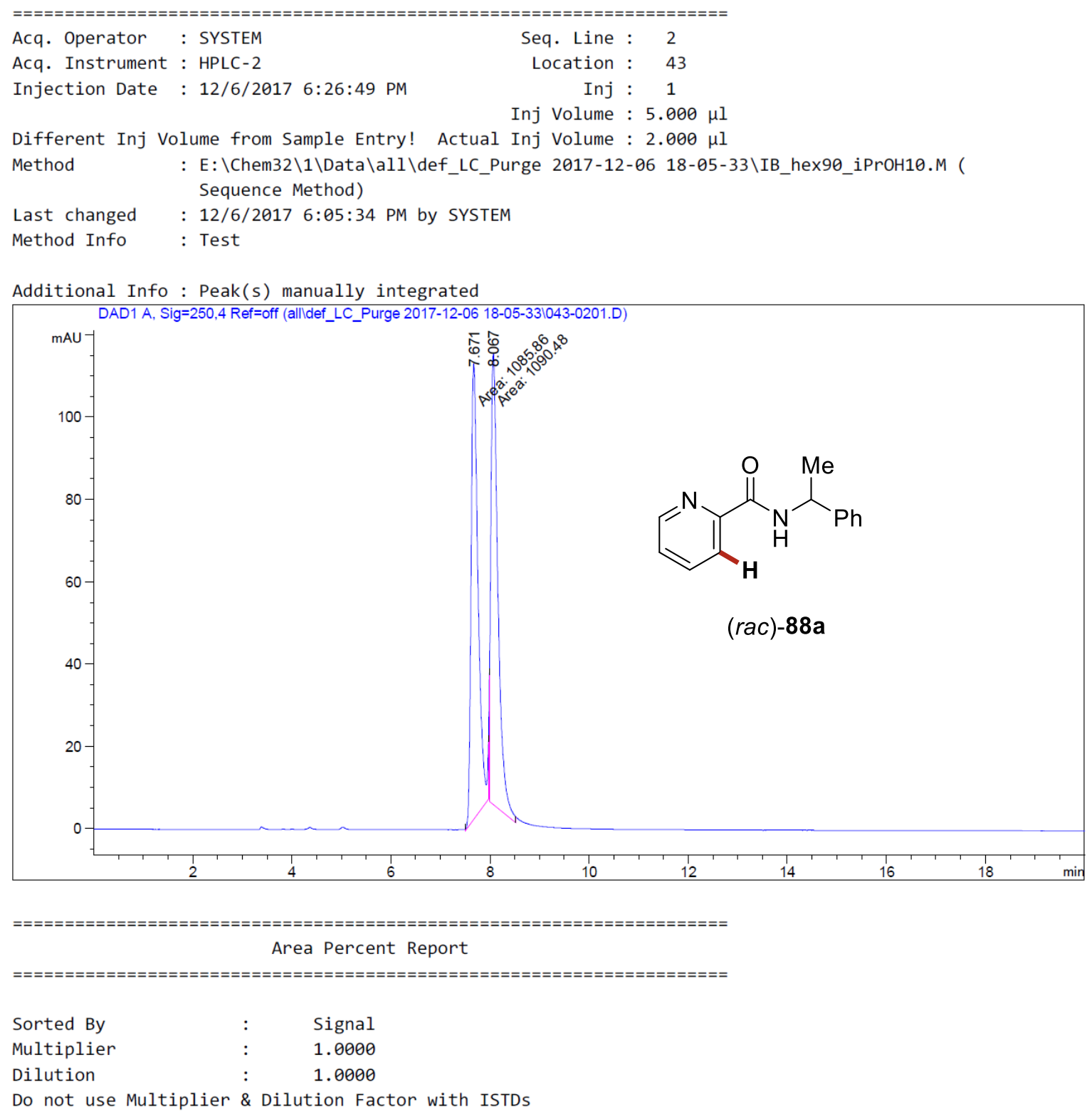

Signal 1: DAD1 A, Sig=250,4 Ref=off

\begin{tabular}{cccccc}
$\begin{array}{c}\text { Peak RetTime Type } \\
\text { Width }\end{array}$ & $\begin{array}{c}\text { Area } \\
{[\mathrm{min}]}\end{array}$ & $\begin{array}{c}\text { Height } \\
{[\mathrm{mAU} \text { [ }]}\end{array}$ & $\begin{array}{c}\text { Area } \\
{[\mathrm{mAU}]}\end{array}$ & $\%$ \\
\hline 1 & 7.671 MM & 0.1633 & 1085.86206 & 110.80559 & 49.8940 \\
2 & 8.067 MM & 0.1659 & 1090.47510 & 109.54958 & 50.1060
\end{tabular}

Totals : $\quad 2176.33716 \quad 220.35517$ 
Data File E: \Chem32\1\Data \al1\def_LC_Purge 2018-01-08 13-02-28\OnlineEdited14.D Sample Name: zsb130-2asytm
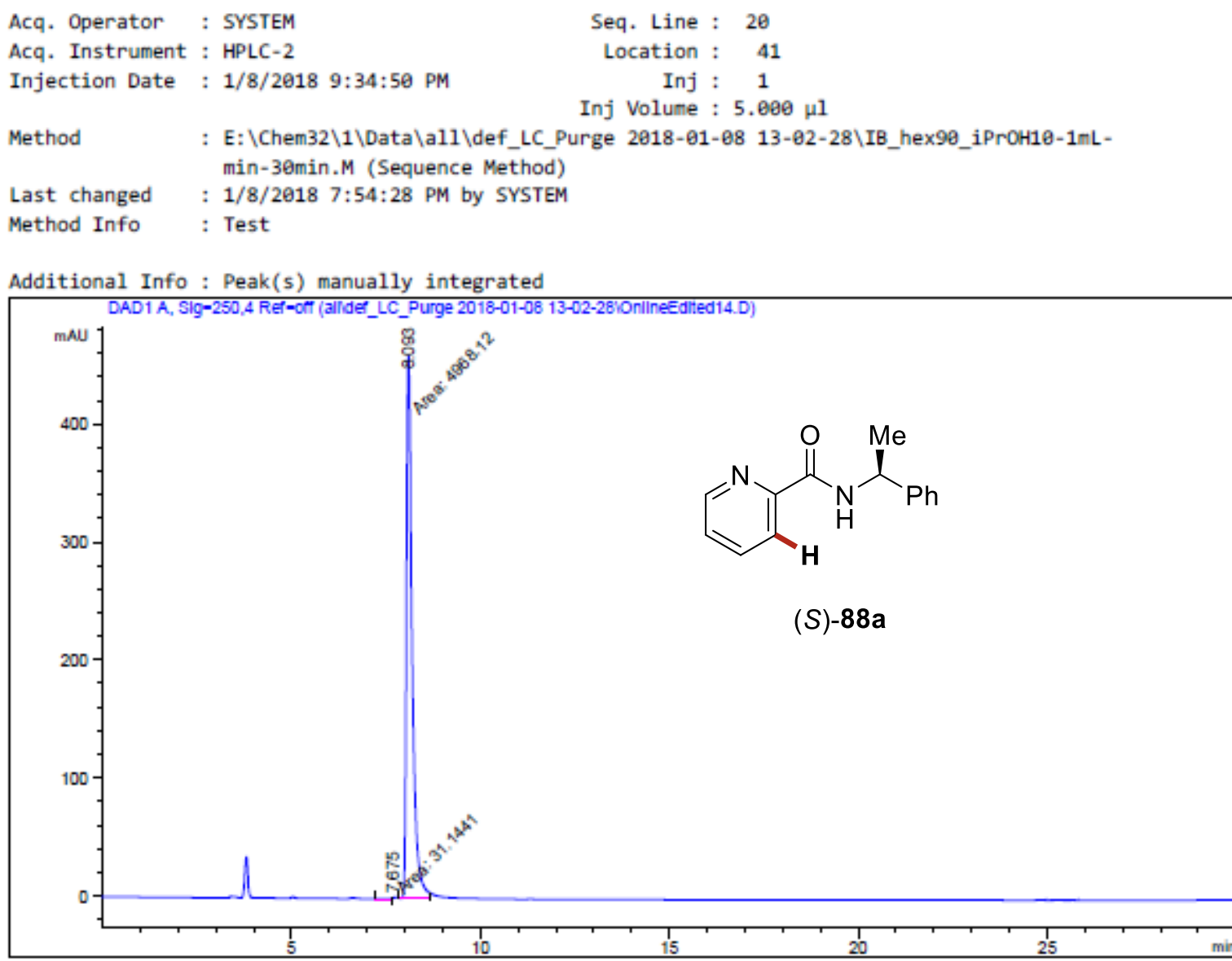

\section{Area Percent Report}

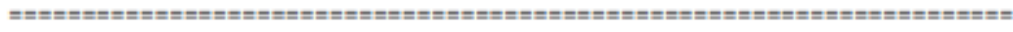

$\begin{array}{lll}\text { Sorted By } & : & \text { Signal } \\ \text { Multiplier } & : & 1.0000 \\ \text { Dilution } & : & 1.0090\end{array}$

Do not use Multiplier \& Dilution Factor with ISTDs

Signal 1: DAD1 A, Sig=250,4 Ref=off

\begin{tabular}{|c|c|c|c|c|c|}
\hline $\begin{array}{c}\text { Peak } \\
\quad \#\end{array}$ & $\begin{array}{l}\text { RetTime Type } \\
\text { [min] }\end{array}$ & $\begin{array}{l}\text { Width } \\
\text { [min] }\end{array}$ & $\begin{array}{c}\text { Area } \\
{\left[\mathrm{mAU} \mathbf{U}^{+\mathrm{s}]}\right.}\end{array}$ & $\begin{array}{l}\text { Height } \\
\text { [mAU] }\end{array}$ & $\begin{array}{c}\text { Area } \\
\%\end{array}$ \\
\hline$=-1$ & & & 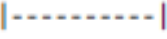 & | & | \\
\hline 1 & $7.675 \mathrm{MM}$ & 0.3221 & 31.14405 & 1.61171 & 0.6230 \\
\hline 2 & 8.093 MM & e.1801 & 4968.11621 & 459.88132 & 99.3770 \\
\hline al & ls & & 4999.26026 & 461.49303 & \\
\hline
\end{tabular}




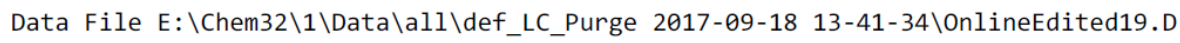
Sample Name: zsb130-2

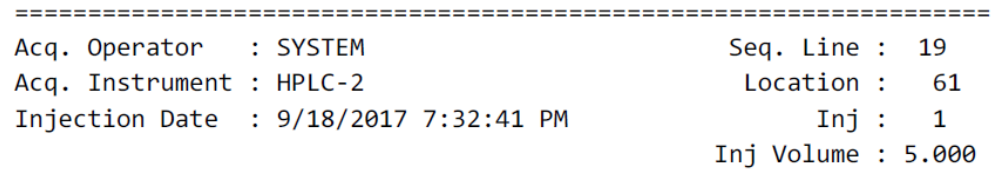

Different Inj Volume from Sample Entry! Actual Inj Volume : $2.000 \mu$

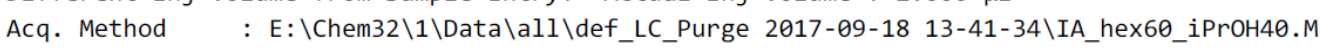

Last changed : 9/18/2017 5:53:09 PM by SYSTEM

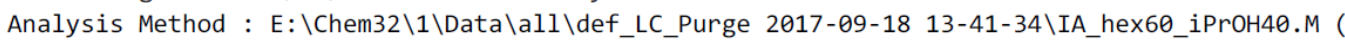
Sequence Method)

Last changed : 9/20/2017 1:57:07 PM by SYSTEM

Method Info : Test

Additional Info : Peak(s) manually integrated

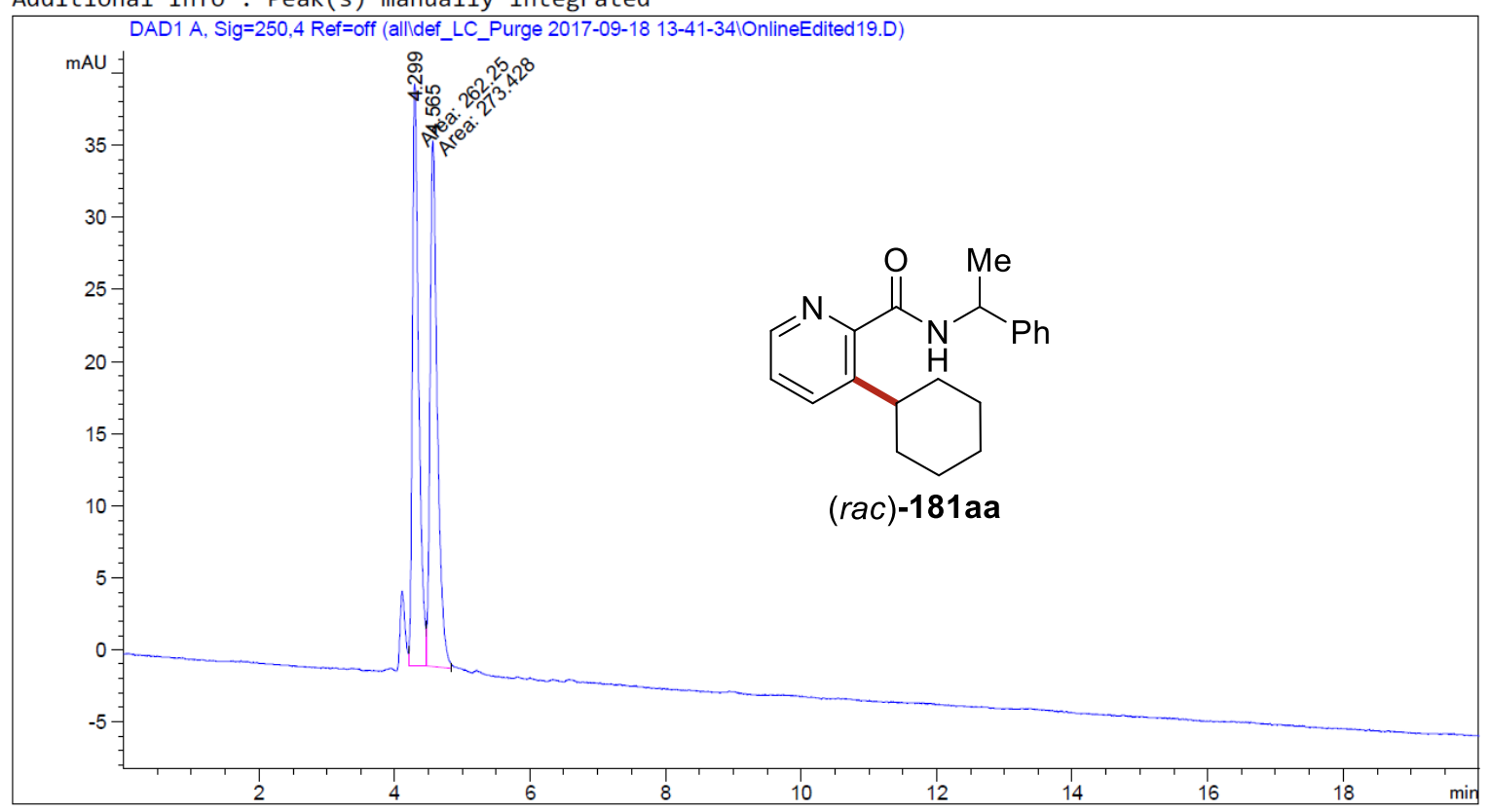

Area Percent Report

\begin{tabular}{|c|c|c|}
\hline Sorted By & : & Signal \\
\hline Multiplier & : & 1.0000 \\
\hline Dilution & : & 1.0000 \\
\hline
\end{tabular}

Do not use Multiplier \& Dilution Factor with ISTDs

Signal 1: DAD1 A, Sig=250,4 Ref=off

\begin{tabular}{llllll}
$\begin{array}{c}\text { Peak RetTime Type } \\
\text { Width }\end{array}$ & $\begin{array}{c}\text { Area } \\
{[\mathrm{min}]}\end{array}$ & $\begin{array}{c}\text { Height } \\
{[\mathrm{mAU} \text { ( }]}\end{array}$ & $\begin{array}{c}\text { Area } \\
{[\mathrm{mAU}]}\end{array}$ & $\%$ \\
\hline 1 & $4.299 \mathrm{MM}$ & 0.1082 & 262.24963 & 40.39529 & 48.9566 \\
2 & $4.565 \mathrm{MM}$ & 0.1248 & 273.42807 & 36.52723 & 51.0434 \\
& & & & & \\
Totals : & & 535.67770 & 76.92252 &
\end{tabular}

HPLC-2 3/14/2018 11:06:05 AM SYSTEM 


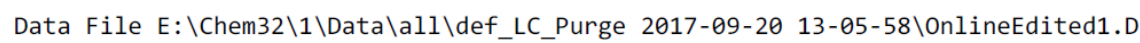
Sample Name: zsb148-2

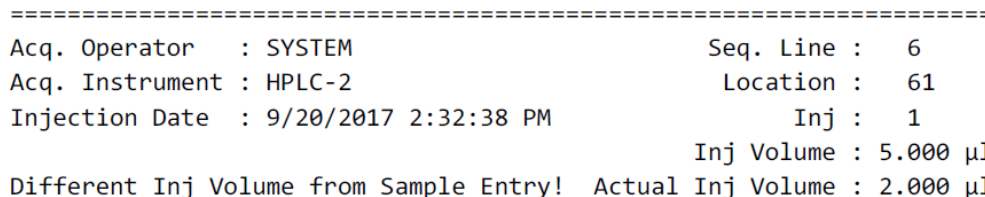
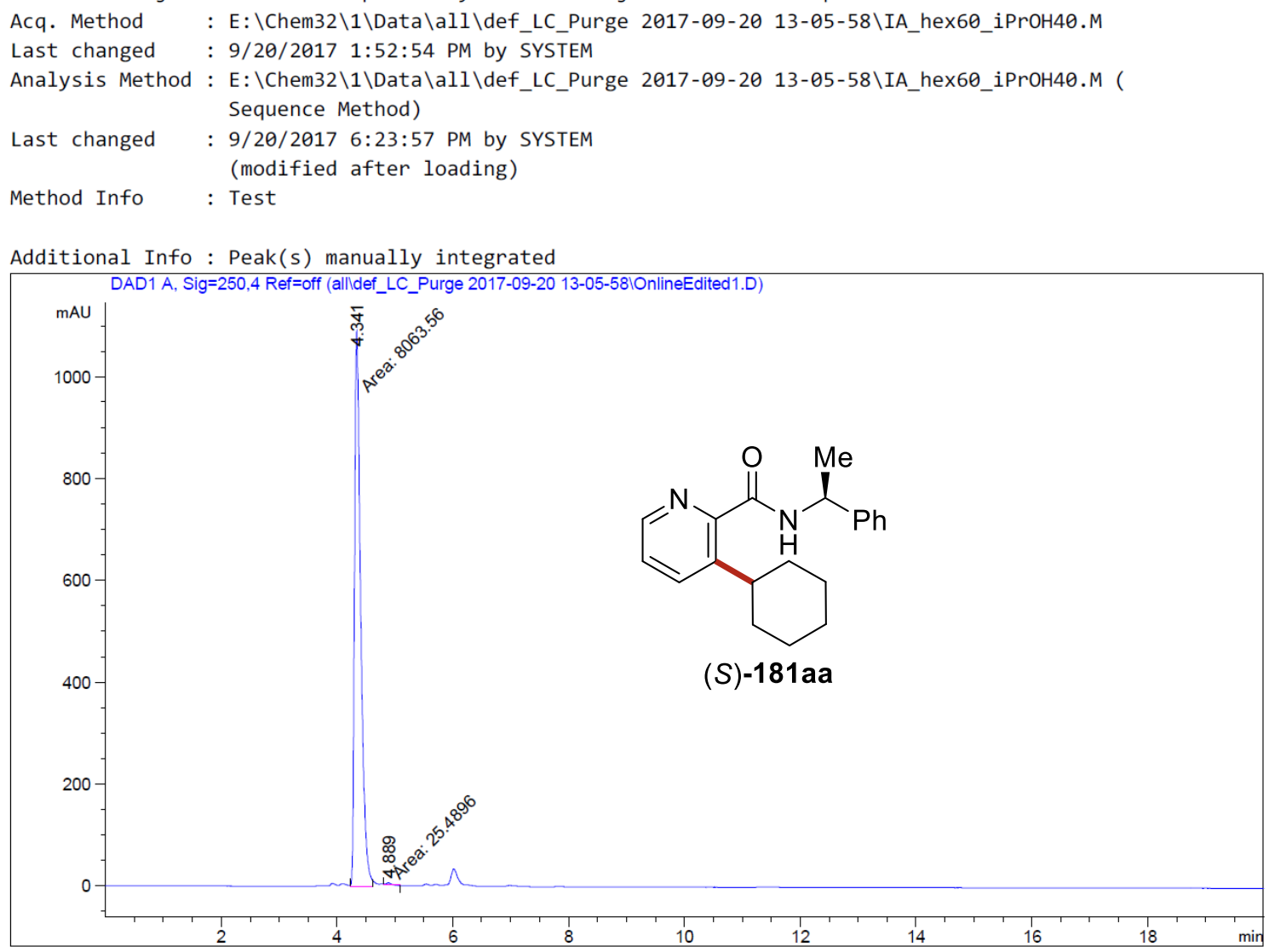

Area Percent Report

$\begin{array}{lll}================================ \\ \text { Sorted By } & : & \text { Signal } \\ \text { Multiplier } & : & 1.0000 \\ \text { Dilution } & : & 1.0000\end{array}$

Do not use Multiplier \& Dilution Factor with ISTDs

Signal 1: DAD1 A, Sig=250,4 Ref=off

\begin{tabular}{|c|c|c|c|c|c|c|}
\hline $\begin{array}{c}\text { eak } \\
\#\end{array}$ & $\begin{array}{c}\text { RetTime } \\
\text { [min] }\end{array}$ & Type & $\begin{array}{l}\text { Width } \\
\text { [min] }\end{array}$ & $\begin{array}{c}\text { Area } \\
{\left[\mathrm{mAU}^{*} \mathrm{~s}\right]}\end{array}$ & $\begin{array}{l}\text { Height } \\
{[\mathrm{mAU}]}\end{array}$ & $\begin{array}{c}\text { Area } \\
\%\end{array}$ \\
\hline & & & & & & \\
\hline 1 & & & & 8063. & 1091. & 349 \\
\hline & 9 & & 26 & 25.48958 & 4.14024 & .3151 \\
\hline
\end{tabular}




\subsubsection{Mechanistic Studies}

\section{Control experiments}

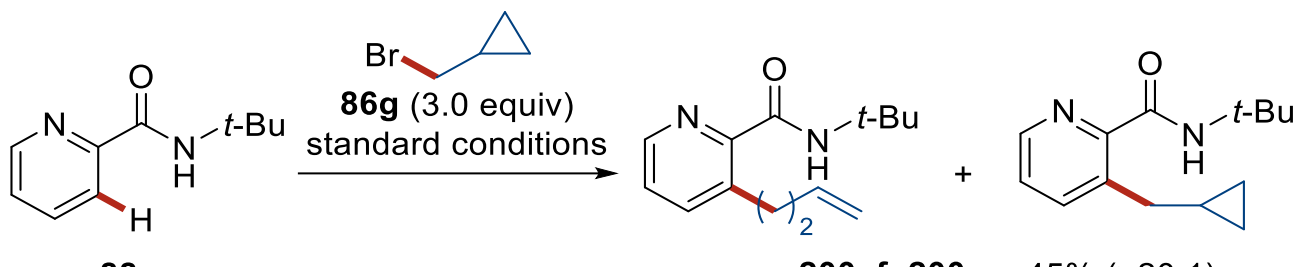

$88 e$

200ef+200eg, $45 \%(>20: 1)$

Prepared following the general procedure D using $N$-(tert-butyl)picolinamide 88e (35.6 mg, $0.20 \mathrm{mmol}$ ) and (bromomethyl)cyclopropane $86 \mathrm{~g}(58 \mu \mathrm{L}, 0.60 \mathrm{mmol})$. Purification by column chromatography ( $n$-hexane/EtOAc $10: 1)$ yielded $200 \mathrm{eg}(21.2 \mathrm{mg}, 45 \%)$ as a colorless oil, while no cyclopropane product (200eg') was detected.

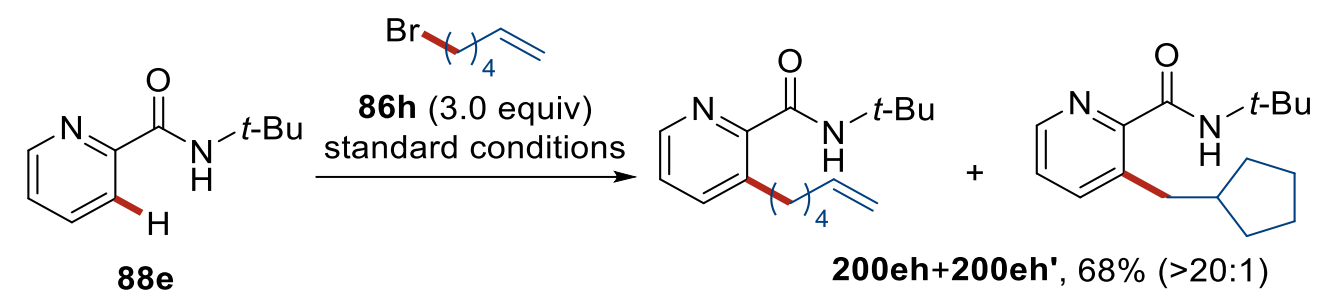

Prepared following the general procedure D using $N$-(tert-butyl)picolinamide $8 \mathbf{8 e}$ (35.6 mg, $0.20 \mathrm{mmol}$ ) and 6-bromohex-1-ene $86 \mathrm{~h}(97.2 \mathrm{mg}, 0.60 \mathrm{mmol})$. Purification by column chromatography ( $n$-hexane/EtOAc 10:1) yielded 200eh $(35.6 \mathrm{mg}, 68 \%)$ as a colorless oil, while no cyclopentane product (200eh') was detected.

\section{KIE Experiments}

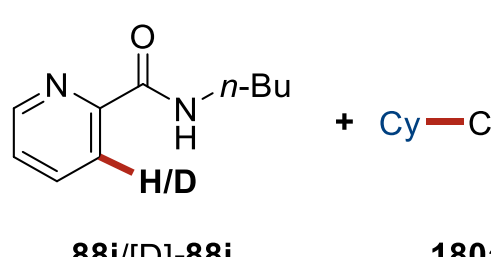

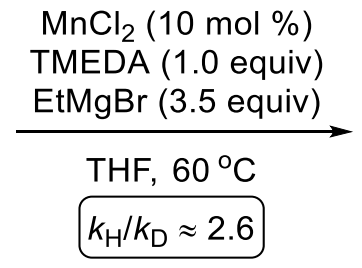

$180 a$<smiles>CCCCNC(=O)c1ncccc1Cl</smiles>

181ia

Twelve independent reactions with $\mathbf{8 8 \mathbf { i }}$ and deuterated substrate [D]-88i under the standard conditions were performed: To a schlenk tube charged with amide $\mathbf{8 8 i} \mathbf{i}(45.0 \mathrm{mg}$, $0.20 \mathrm{mmol})$, TMEDA (30 $\mu \mathrm{L}, 0.20 \mathrm{mmol}), \mathrm{MnCl}_{2}(2.7 \mathrm{mg}, 0.02 \mathrm{mmol})$ and THF $(0.6 \mathrm{~mL})$, 
EtMgBr $(250 \mu \mathrm{L}, 0.70 \mathrm{mmol}, 2.8 \mathrm{M}$ in THF) was added dropwise at ambient temperature under $\mathrm{N}_{2}$ atmosphere. After stirring for $5 \mathrm{~min}$, alkyl chloride $(0.60 \mathrm{mmol})$ was added and the reaction mixture stirred at $65^{\circ} \mathrm{C}$ for $16 \mathrm{~h}$. Then, to the reaction mixture sat. aqueous $\mathrm{NH}_{4} \mathrm{Cl}(5.0 \mathrm{~mL})$ and $\mathrm{H}_{2} \mathrm{O}(10 \mathrm{~mL})$ were subsequently added. The aqueous layer was extracted with EtOAc $(3 \times 15 \mathrm{~mL})$. The combined organic extracts were washed with brine and dried over $\mathrm{Na}_{2} \mathrm{SO}_{4}$, and the reaction mixture stirred at $60^{\circ} \mathrm{C}$ for $10 \mathrm{~min}, 20 \mathrm{~min}, 30 \mathrm{~min}$, $40 \mathrm{~min}, 50 \mathrm{~min}, 60 \mathrm{~min}$ respectively. The conversion of the product $181 \mathrm{ia}$ was monitored by ${ }^{1} \mathrm{H}$ NMR using $\mathrm{CH}_{2} \mathrm{Br}_{2}$ as the internal standard.

\begin{tabular}{c|cccccc}
$T(\min )$ & 10 & 20 & 30 & 40 & 50 & 60 \\
\hline Yield [H] (\%) & 6.1 & 14.0 & 19.0 & 25.5 & 29.7 & 36.9 \\
Yield [D] (\%) & 1.0 & 3.9 & 5.4 & 7.1 & 10.4 & 12.5
\end{tabular}

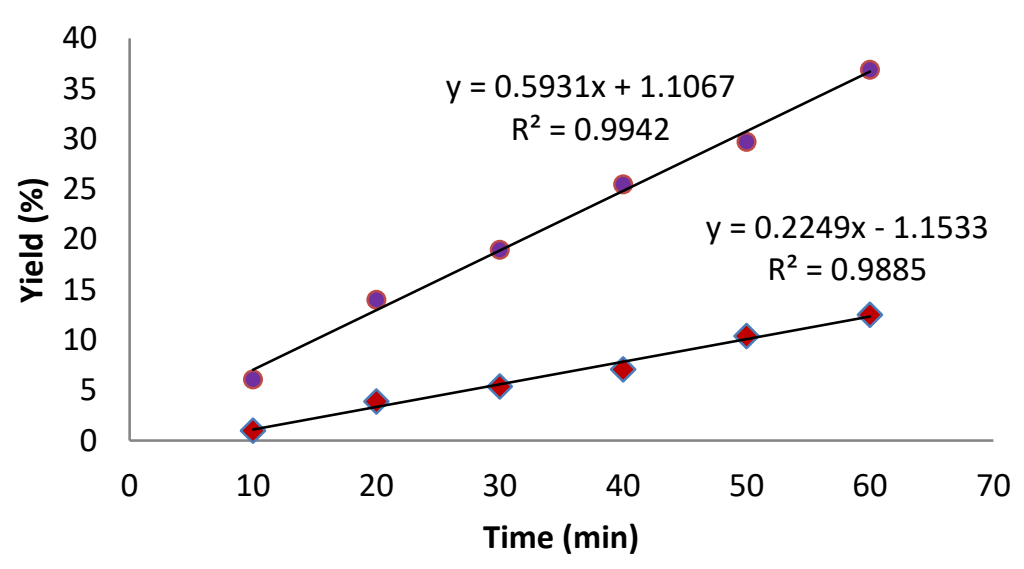




\subsection{Rhodaelectro-Catalyzed Domino Alkyne Annulations to Access Aza-Polycyclic Aromatic Hydrocarbons}

\subsubsection{Experimental Procedure and Analytical Data}<smiles></smiles>

\section{2,3,7,12,13-Pentaphenylbenzo[de]isoquinolino[2,1-a][1,8]naphthyridine (183aa)}

The general procedure $E$ was followed using $182 \mathrm{a}(30.0 \mathrm{mg}, 0.20 \mathrm{mmol})$ and $113 \mathrm{a}$ (124.6 mg, $0.70 \mathrm{mmol}$ ) at $35^{\circ} \mathrm{C}$ for $12 \mathrm{~h}$. Purification by column chromatography on silica gel ( $n$-hexane/EtOAc 50:1) yielded 183aa $(116.6 \mathrm{mg}, 90 \%)$ as a red solid. M.p. $=295-$ $296{ }^{\circ} \mathrm{C} .{ }^{1} \mathrm{H}$ NMR $\left(400 \mathrm{MHz}, \mathrm{CDCl}_{3}\right) \delta=7.62-7.42(\mathrm{~m}, 6 \mathrm{H}), 7.37-7.23(\mathrm{~m}, 9 \mathrm{H}), 7.21-7.02$ (m, 11H), 7.00-6.94 (m, 3H), $6.86(\mathrm{ddd}, J=8.3,7.1,1.5 \mathrm{~Hz}, 1 \mathrm{H}), 6.60-6.56(\mathrm{~m}, 2 \mathrm{H})$. ${ }^{13} \mathrm{C}$ NMR $\left(100 \mathrm{MHz}, \mathrm{CDCl}_{3}\right) \delta=150.1\left(\mathrm{C}_{\mathrm{q}}\right), 149.5\left(\mathrm{C}_{\mathrm{q}}\right), 140.7\left(\mathrm{C}_{\mathrm{q}}\right), 138.8\left(\mathrm{C}_{\mathrm{q}}\right), 137.9\left(\mathrm{C}_{\mathrm{q}}\right)$, $\left.137.8\left(\mathrm{C}_{\mathrm{q}}\right), 137.5 \mathrm{C}_{\mathrm{q}}\right), 137.1\left(\mathrm{C}_{\mathrm{q}}\right), 137.0\left(\mathrm{C}_{\mathrm{q}}\right), 135.8\left(\mathrm{C}_{\mathrm{q}}\right), 135.4\left(\mathrm{C}_{\mathrm{q}}\right), 134.7\left(\mathrm{C}_{\mathrm{q}}\right), 132.0(\mathrm{CH})$, $131.9(\mathrm{CH}), 131.4(\mathrm{CH}), 131.1(\mathrm{CH}), 130.4(\mathrm{CH}$, overlapped), $130.2(\mathrm{CH}), 129.9(\mathrm{CH})$, $128.6\left(\mathrm{CH}\right.$, overlapped), $128.4(\mathrm{CH}), 128.1(\mathrm{CH}), 128.0(\mathrm{CH}), 127.5\left(\mathrm{C}_{\mathrm{q}}\right), 127.1(\mathrm{CH}), 126.9$ (CH, overlapped), $126.8\left(\mathrm{CH}\right.$, overlapped), $126.7(\mathrm{CH}), 126.0(\mathrm{CH}), 125.7\left(\mathrm{C}_{\mathrm{q}}\right), 125.0(\mathrm{CH})$, $123.3\left(\mathrm{C}_{\mathrm{q}}\right), 122.0\left(\mathrm{C}_{\mathrm{q}}\right), 119.1(\mathrm{CH}), 117.7(\mathrm{CH}) . \mathrm{IR}(\mathrm{ATR})$ : 1738, 1610, 1536, 1342, 1234 , 757, $699 \mathrm{~cm}^{-1}$. HR-MS (ESI) $\mathrm{m} / z$ calcd for $\mathrm{C}_{49} \mathrm{H}_{33} \mathrm{~N}_{2}[\mathrm{M}+\mathrm{H}]^{+} 649.2638$, found 649.2635. 
<smiles></smiles>

5-Methoxy-2,3,7,12,13-pentaphenylbenzo[de]isoquinolino[2,1-a][1,8] naphthyridine (183ba)

The general procedure $\mathbf{E}$ was followed using $182 \mathbf{b}(36.0 \mathrm{mg}, 0.20 \mathrm{mmol})$ and $113 \mathbf{a}$ (124.6 mg, $0.7 \mathrm{mmol}$ ) at $35^{\circ} \mathrm{C}$ for $12 \mathrm{~h}$. Purification by column chromatography on silica gel ( $n$-hexane/EtOAc 50:1) yielded 183da (103.0 mg, 76\%) as a red solid. M.p. $>300{ }^{\circ} \mathrm{C}$. ${ }^{1} \mathrm{H}$ NMR $\left(400 \mathrm{MHz}, \mathrm{CDCl}_{3}\right) \delta=7.54-7.40(\mathrm{~m}, 5 \mathrm{H}), 7.29-7.16(\mathrm{~m}, 8 \mathrm{H}), 7.13-7.05(\mathrm{~m}, 6 \mathrm{H})$, 7.06-6.95 (m, 5H), 6.92-6.87 (m, 2H), $6.79(\mathrm{ddd}, J=8.4,6.6,1.9 \mathrm{~Hz}, 1 \mathrm{H}), 6.61(\mathrm{~d}, J=2.2$ $\mathrm{Hz}, 1 \mathrm{H}), 6.51-6.45(\mathrm{~m}, 3 \mathrm{H}), 3.64(\mathrm{~s}, 3 \mathrm{H}) .{ }^{13} \mathrm{C}$ NMR $\left(100 \mathrm{MHz}, \mathrm{CDCl}_{3}\right) \delta=162.0\left(\mathrm{C}_{\mathrm{q}}\right), 150.7$ $\left(\mathrm{C}_{\mathrm{q}}\right), 149.0\left(\mathrm{C}_{\mathrm{q}}\right), 140.7\left(\mathrm{C}_{\mathrm{q}}\right), 139.8\left(\mathrm{C}_{\mathrm{q}}\right), 138.6\left(\mathrm{C}_{\mathrm{q}}\right), 138.1\left(\mathrm{C}_{\mathrm{q}}\right), 137.4\left(\mathrm{C}_{\mathrm{q}}\right), 137.3\left(\mathrm{C}_{\mathrm{q}}\right), 137.0$ $\left(\mathrm{C}_{\mathrm{q}}\right), 136.4\left(\mathrm{C}_{\mathrm{q}}\right.$, overlapped, 2C $134.6\left(\mathrm{C}_{\mathrm{q}}\right), 131.9(\mathrm{CH}), 131.7(\mathrm{CH}), 131.2(\mathrm{CH}), 130.3(\mathrm{CH}$, overlapped, 2C), $130.1(\mathrm{CH}), 129.9(\mathrm{CH}), 128.6(\mathrm{CH}), 128.4(\mathrm{CH}), 128.4(\mathrm{CH}), 128.0(\mathrm{CH})$, $127.9(\mathrm{CH}), 127.3\left(\mathrm{C}_{\mathrm{q}}\right), 127.0(\mathrm{CH}), 126.7(\mathrm{CH}$, overlapped, 2C), $126.6(\mathrm{CH}), 126.5(\mathrm{CH})$, $125.9(\mathrm{CH}), 125.4\left(\mathrm{C}_{\mathrm{q}}\right), 124.9(\mathrm{CH}), 122.8\left(\mathrm{C}_{\mathrm{q}}\right), 118.5\left(\mathrm{C}_{\mathrm{q}}\right), 117.8(\mathrm{Cq}), 107.2(\mathrm{CH}), 100.2$ $(\mathrm{CH}), 55.1\left(\mathrm{CH}_{3}\right)$. IR (ATR): 1610, 1574, 1537, 1401, 1346, 1208, 759, $701 \mathrm{~cm}^{-1}$. HR-MS (ESI) $m / z$ calcd for $\mathrm{C}_{50} \mathrm{H}_{35} \mathrm{~N}_{2} \mathrm{O}[\mathrm{M}+\mathrm{H}]^{+} 679.2744$, found 679.2733 . 
<smiles>CC(=O)c1cc2c(-c3ccccc3)c(-c3ccccc3)c3c4c(cc(C(C)=O)c4c(c1)c1ccccc21)c1c2ccccc2c(-c2ccccc2)c(-c2ccccc2)n31</smiles>

Methyl 2,3,7,12,13-pentaphenylbenzo[de]isoquinolino[2,1-a][1,8]naphthyridine-5carboxylate (183ca)

The general procedure $\mathrm{E}$ was followed using $182 \mathrm{c}(41.6 \mathrm{mg}, 0.20 \mathrm{mmol})$ and $113 \mathrm{a}$ (124.6 mg, $0.7 \mathrm{mmol}$ ) at $35^{\circ} \mathrm{C}$ for $12 \mathrm{~h}$. Purification by column chromatography on silica gel ( $n$-hexane/EtOAc 50:1) yielded 183ca (81.0 mg, 57\%) as a red solid. M.p. $=193$ $194{ }^{\circ} \mathrm{C} .{ }^{1} \mathrm{H}$ NMR $\left(400 \mathrm{MHz}, \mathrm{CDCl}_{3}\right) \delta=7.97(\mathrm{~d}, J=1.2 \mathrm{~Hz}, 1 \mathrm{H}), 7.59-7.50(\mathrm{~m}, 6 \mathrm{H}), 7.33-$ $7.26(\mathrm{~m}, 8 \mathrm{H}), 7.17-6.99(\mathrm{~m}, 11 \mathrm{H}), 6.97-6.91(\mathrm{~m}, 2 \mathrm{H}), 6.86(\mathrm{ddd}, J=8.4,6.6,1.9 \mathrm{~Hz}, 1 \mathrm{H})$, $6.53(\mathrm{~d}, J=7.1 \mathrm{~Hz}, 2 \mathrm{H}), 3.84(\mathrm{~s}, 3 \mathrm{H}) .{ }^{13} \mathrm{C}$ NMR $\left(100 \mathrm{MHz}, \mathrm{CDCl}_{3}\right) \delta=167.4\left(\mathrm{C}_{\mathrm{q}}\right), 151.1$ $\left(\mathrm{C}_{\mathrm{q}}\right), 149.4\left(\mathrm{C}_{\mathrm{q}}\right), 140.4\left(\mathrm{C}_{\mathrm{q}}\right), 138.3\left(\mathrm{C}_{\mathrm{q}}\right), 137.6\left(\mathrm{C}_{\mathrm{q}}\right), 137.3\left(\mathrm{C}_{\mathrm{q}}\right), 137.3\left(\mathrm{C}_{\mathrm{q}}\right), 136.9\left(\mathrm{C}_{\mathrm{q}}\right), 136.8$ $\left(\mathrm{C}_{\mathrm{q}}\right), 136.8\left(\mathrm{C}_{\mathrm{q}}\right), 136.6\left(\mathrm{C}_{\mathrm{q}}\right), 135.9\left(\mathrm{C}_{\mathrm{q}}\right), 134.7\left(\mathrm{C}_{\mathrm{q}}\right), 132.6\left(\mathrm{C}_{\mathrm{q}}\right), 132.0(\mathrm{CH}), 131.8(\mathrm{CH})$, $131.4(\mathrm{CH}), 130.4(\mathrm{CH}), 130.3(\mathrm{CH}), 130.2(\mathrm{CH}), 128.8(\mathrm{CH}), 128.7(\mathrm{CH}), 128.7(\mathrm{CH})$, $128.5(\mathrm{CH}), 128.1(\mathrm{CH}), 128.1(\mathrm{CH}), 7.4\left(\mathrm{C}_{\mathrm{q}}\right), 127.3(\mathrm{CH}), 127.2(\mathrm{CH}), 127.0(\mathrm{CH}), 127.0$ $(\mathrm{CH}), 126.9(\mathrm{CH}), 126.2(\mathrm{CH}), 125.2(\mathrm{CH}), 124.2\left(\mathrm{C}_{\mathrm{q}}\right), 123.4\left(\mathrm{C}_{\mathrm{q}}\right), 120.8(\mathrm{CH}), 119.2\left(\mathrm{C}_{\mathrm{q}}\right)$, 117.5 (CH), 52.5 (CH). IR (ATR): 3058, 2919, 1725, 1609, 1541, 1339, 1229, 764, 669 $\mathrm{cm}^{-1}$. MS (ESI) m/z (relative intensity): $707(100)[\mathrm{M}+\mathrm{H}]^{+}, 739$ (15) $[\mathrm{M}+\mathrm{Na}]^{+}$. HR-MS (ESI) $\mathrm{m} / \mathrm{z}$ calcd for $\mathrm{C}_{51} \mathrm{H}_{34} \mathrm{~N}_{2} \mathrm{O}_{2}{ }^{+}[\mathrm{M}+\mathrm{H}]^{+}$707.2693, found 707.2695. 
<smiles>Fc1cc2c(-c3ccccc3)c3n4c(nc(-c5ccccc5)c(-c5ccccc5)c4c-2c1)=C(c1ccccc1)C(c1ccccc1)=c1ccccc1=3</smiles>

5-Fluoro-2,3,7,12,13- pentaphenylbenzo[de]isoquinolino[2,1-a][1,8]phthyridine (183da)

The general procedure $\mathbf{E}$ was followed using $182 \mathrm{~d}(33.6 \mathrm{mg}, 0.20 \mathrm{mmol})$ and $113 \mathbf{a}$ (124.6 mg, $0.7 \mathrm{mmol}$ ) at $35^{\circ} \mathrm{C}$ for $12 \mathrm{~h}$. Purification by column chromatography on silica gel ( $n$-hexane/EtOAc 50:1) yielded 183da $(98.0 \mathrm{mg}, 73 \%)$ as a red solid. M.p. $=278-$ $280{ }^{\circ} \mathrm{C} .{ }^{1} \mathrm{H}$ NMR $\left(300 \mathrm{MHz}, \mathrm{CDCl}_{3}\right) \delta=7.60-7.49(\mathrm{~s}, 5 \mathrm{H}), 7.34-7.22(\mathrm{~m}, 8 \mathrm{H}), 7.18-7.01$ (m, 11H), $6.95(\mathrm{t}, J=7.4 \mathrm{~Hz}, 2 \mathrm{H}), 6.89-6.82(\mathrm{~m}, 2 \mathrm{H}), 6.62(\mathrm{dd}, J=10.6,1.9 \mathrm{~Hz}, 1 \mathrm{H}), 6.53$ $(\mathrm{d}, J=7.3 \mathrm{~Hz}, 2 \mathrm{H}) .{ }^{13} \mathrm{C}$ NMR $\left(100 \mathrm{MHz}, \mathrm{CDCl}_{3}\right) \delta=165.1\left(\mathrm{C}_{\mathrm{q}},{ }^{1} \mathrm{~J}_{\mathrm{C}-\mathrm{F}}=247.1 \mathrm{~Hz}\right), 151.2$ $\left(C_{q}\right), 149.2\left(C_{q}\right), 140.4\left(C_{q}\right), 140.31\left(C_{q},{ }^{3} J_{C-F}=11.2 \mathrm{~Hz}\right), 138.7\left(C_{q},{ }^{3} J=11.0 \mathrm{~Hz}\right), 138.4$ $\left(\mathrm{C}_{\mathrm{q}}\right), 137.7\left(\mathrm{C}_{\mathrm{q}}\right), 137.4\left(\mathrm{C}_{\mathrm{q}}\right), 137.2\left(\mathrm{C}_{\mathrm{q}}\right), 137.1\left(\mathrm{C}_{\mathrm{q}}\right), 136.9\left(\mathrm{C}_{\mathrm{q}}\right), 134.7\left(\mathrm{C}_{\mathrm{q}}\right), 131.9(\mathrm{CH})$, $131.8(\mathrm{CH}), 131.8(\mathrm{CH}), 131.2(\mathrm{CH}), 130.4(\mathrm{CH}), 130.2(\mathrm{CH}), 130.2(\mathrm{CH}), 128.8(\mathrm{CH})$, $128.8(\mathrm{CH}) 128.7(\mathrm{CH}), 128.4(\mathrm{CH}), 128.1(\mathrm{CH}), 127.2(\mathrm{CH}), 127.2\left(\mathrm{C}_{\mathrm{q}}\right), 127.1(\mathrm{CH}), 127.0$ $(\mathrm{CH}), 127.0(\mathrm{CH}), 126.8(\mathrm{CH}), 126.2(\mathrm{CH}), 126.1\left(\mathrm{C}_{\mathrm{q}}\right), 125.3(\mathrm{CH}), 123.32\left(\mathrm{C}_{\mathrm{q}},{ }^{4} \mathrm{~J}_{\mathrm{C}-\mathrm{F}}=4.7\right.$ $\mathrm{Hz}), 119.2\left(\mathrm{C}_{\mathrm{q}}\right), 118.5\left(\mathrm{C}_{\mathrm{q}}\right), 105.9\left(\mathrm{CH},{ }^{2} \mathrm{~J}_{\mathrm{C}-\mathrm{F}}=27.3 \mathrm{~Hz}\right), 103.72\left(\mathrm{CH},{ }^{2} \mathrm{~J}_{\mathrm{C}-\mathrm{F}}=24.6 \mathrm{~Hz}\right)$. ${ }^{19} \mathrm{~F}$ NMR $\left(282 \mathrm{MHz}, \mathrm{CDCl}_{3}\right) \delta=-105.93$ (t, $\left.J=10.7 \mathrm{~Hz}\right)$. IR (ATR): 3051, 2961, 2161, 1609, 1572, 1342, 1021, $696 \mathrm{~cm}^{-1}$. MS (ESI) m/z (relative intensity): 649 (100), 667 (15) [M+H]. HR-MS (ESI) $m / z$ calcd for $\mathrm{C}_{49} \mathrm{H}_{32} \mathrm{FN}_{2}[\mathrm{M}+\mathrm{H}]^{+}$667.2544, found 667.2531. 
<smiles></smiles>

10-Methyl-2,3,7,12,13-penta-p-tolylbenzo[de]isoquinolino[2,1-a][1,8] naphthyridine (183ab)

The general procedure $\mathbf{E}$ was followed using $182 \mathrm{a}(30.0 \mathrm{mg}, 0.20 \mathrm{mmol})$ and $113 \mathrm{~b}$ (144.4 mg, $0.7 \mathrm{mmol}$ ) at $35^{\circ} \mathrm{C}$ for $12 \mathrm{~h}$. Purification by column chromatography on silica gel ( $n$-hexane/EtOAc 50:1) yielded 183ab (118.6 mg, 81\%) as a red solid. M.p. $=198-$ $199{ }^{\circ} \mathrm{C} .{ }^{1} \mathrm{H}$ NMR $\left(400 \mathrm{MHz}, \mathrm{CDCl}_{3}\right) \delta=7.48-7.33(\mathrm{~m}, 5 \mathrm{H}), 7.22-7.10(\mathrm{~m}, 7 \mathrm{H}), 7.04(\mathrm{~d}, J=$ $7.6 \mathrm{~Hz}, 4 \mathrm{H}), 7.00-6.90(\mathrm{~m}, 3 \mathrm{H}), 6.87(\mathrm{~d}, J=8.0 \mathrm{~Hz}, 2 \mathrm{H}), 6.80(\mathrm{~d}, J=7.9 \mathrm{~Hz}, 2 \mathrm{H}), 6.69(\mathrm{~d}$, $J=8.3 \mathrm{~Hz}, 1 \mathrm{H}$ ), 6.49 (d, $J=8.0 \mathrm{~Hz}, 2 \mathrm{H}), 2.51$ (s, 3H), 2.40 (s, 3H), 2.38 (s, 3H), 2.27 (s, 3H), $2.24(\mathrm{~s}, 3 \mathrm{H}), 2.20(\mathrm{~s}, 3 \mathrm{H}) .{ }^{13} \mathrm{C}$ NMR $\left(101 \mathrm{MHz}, \mathrm{CDCl}_{3}\right) \delta=145.0\left(\mathrm{C}_{\mathrm{q}}\right), 149.5\left(\mathrm{C}_{\mathrm{q}}\right)$, $138.10\left(\mathrm{C}_{\mathrm{q}}\right.$, overlapped 3C), $138.0\left(\mathrm{C}_{\mathrm{q}}\right), 137.6\left(\mathrm{C}_{\mathrm{q}}\right), 137.5\left(\mathrm{C}_{\mathrm{q}}\right), 136.1\left(\mathrm{C}_{\mathrm{q}}\right), 136.0\left(\mathrm{C}_{\mathrm{q}}\right)$, $136.0\left(\mathrm{C}_{\mathrm{q}}\right.$, overlapped, $\left.4 \mathrm{C}\right), 135.7\left(\mathrm{C}_{\mathrm{q}}\right), 135.2\left(\mathrm{C}_{\mathrm{q}}\right), 135.0\left(\mathrm{C}_{\mathrm{q}}\right), 134.34\left(\mathrm{C}_{\mathrm{q}}\right.$, overlapped, $\left.2 \mathrm{C}\right)$, $131.8(\mathrm{CH}), 131.7(\mathrm{CH}), 131.2(\mathrm{CH}), 130.8(\mathrm{CH}), 130.6(\mathrm{CH}), 130.3(\mathrm{CH}), 130.1(\mathrm{CH})$, $129.1(\mathrm{CH}), 128.6(\mathrm{CH}), 128.5(\mathrm{CH}), 127.7(\mathrm{CH}), 127.5(\mathrm{CH}) 127.0(\mathrm{CH}), 125.2\left(\mathrm{C}_{\mathrm{q}}\right), 125.2$ $(\mathrm{CH}), 122.8\left(\mathrm{C}_{\mathrm{q}}\right), 121.7\left(\mathrm{C}_{\mathrm{q}}\right), 118.7(\mathrm{CH}), 118.1\left(\mathrm{C}_{\mathrm{q}}\right), 117.1(\mathrm{CH}), 21.5\left(\mathrm{CH}_{3}\right.$, overlapped, 2C), $21.4\left(\mathrm{CH}_{3}\right), 21.3\left(\mathrm{CH}_{3}\right.$, overlapped, 2C) $21.2\left(\mathrm{CH}_{3}\right)$. IR (ATR): 2919, 1606, 1541, 1508, 1340, 907, 815, $730 \mathrm{~cm}^{-1}$. HR-MS (ESI) $\mathrm{m} / z$ calcd for $\mathrm{C}_{55} \mathrm{H}_{45} \mathrm{~N}_{2} \quad[\mathrm{M}+\mathrm{H}] 733.3577$, found 733.3568 . 
<smiles></smiles>

10-Methoxy-2,3,7,12,13-pentakis(4-methoxyphenyl)benzo[de]isoquinolino[2,1a][1,8]naphthyridine (183ac)

The general procedure $\mathbf{E}$ was followed using $182 \mathrm{a}(7.5 \mathrm{mg}, 0.05 \mathrm{mmol}), 113 \mathrm{c}(49.6 \mathrm{mg}$, $0.175 \mathrm{mmol})$ and $\left[\mathrm{Cp}^{*} \mathrm{Rh}\left(\mathrm{CH}_{3} \mathrm{CN}\right)_{3}\right]\left(\mathrm{SbF}_{6}\right)_{2}(4.2 \mathrm{mg}, 10 \mathrm{~mol} \%)$ at $35^{\circ} \mathrm{C}$ for $3 \mathrm{~h}$. Purification by column chromatography on silica gel ( $n$-hexane/EtOAc 50:1) yielded 183ac (38.2, 92\%) as a red solid. M.p. $=177-178^{\circ} \mathrm{C} .{ }^{1} \mathbf{H}$ NMR $\left(400 \mathrm{MHz}, \mathrm{CDCl}_{3}\right) \delta=7.45-7.39(\mathrm{~m}, 3 \mathrm{H}), 7.22-$ $7.14(\mathrm{~m}, 3 \mathrm{H}), 7.11-6.97(\mathrm{~m}, 7 \mathrm{H}), 6.91-6.82(\mathrm{~m}, 5 \mathrm{H}), 6.64(\mathrm{~d}, J=2.7 \mathrm{~Hz}, 1 \mathrm{H}), 6.60(\mathrm{dd}, J=$ 8.7, $5.3 \mathrm{~Hz}, 4 \mathrm{H}), 6.52(\mathrm{~d}, J=8.8 \mathrm{~Hz}, 2 \mathrm{H}), 6.48-6.42(\mathrm{~m}, 1 \mathrm{H}), 3.93(\mathrm{~s}, 3 \mathrm{H}), 3.84(\mathrm{~s}, 3 \mathrm{H})$, $3.82(\mathrm{~s}, 3 \mathrm{H}), 3.73(\mathrm{~s}, 3 \mathrm{H}), 3.71(\mathrm{~s}, 3 \mathrm{H}), 3.65(\mathrm{~s}, 3 \mathrm{H}) .{ }^{13} \mathrm{C} \mathrm{NMR}\left(100 \mathrm{MHz}, \mathrm{CDCl}_{3}\right) \delta=159.2$ ( $\mathrm{C}_{\mathrm{q}}$, overlapped, 2C), $158.3\left(\mathrm{C}_{\mathrm{q}}\right.$, overlapped, 2C), $158.2\left(\mathrm{C}_{\mathrm{q}}\right), 158.1\left(\mathrm{C}_{\mathrm{q}}\right), 149.6\left(\mathrm{C}_{\mathrm{q}}\right), 149.4$ $\left(\mathrm{C}_{\mathrm{q}}\right), 138.1\left(\mathrm{C}_{\mathrm{q}}\right), 137.9\left(\mathrm{C}_{\mathrm{q}}\right), 137.0\left(\mathrm{C}_{\mathrm{q}}\right), 135.9\left(\mathrm{C}_{\mathrm{q}}\right), 135.8\left(\mathrm{C}_{\mathrm{q}}\right), 133.6\left(\mathrm{C}_{\mathrm{q}}\right), 133.0(\mathrm{CH})$, $132.8(\mathrm{CH}), 132.4(\mathrm{CH}), 131.6\left(\mathrm{CH}\right.$, overlapped, 2C), $131.4(\mathrm{CH}), 131.0\left(\mathrm{C}_{\mathrm{q}}\right), 130.9(\mathrm{CH})$, $130.5\left(\mathrm{C}_{\mathrm{q}}\right), 130.1(\mathrm{CH}), 129.7\left(\mathrm{C}_{\mathrm{q}}\right), 129.4\left(\mathrm{C}_{\mathrm{q}}\right), 124.5\left(\mathrm{C}_{\mathrm{q}}\right), 122.1\left(\mathrm{C}_{\mathrm{q}}\right), 121.3\left(\mathrm{C}_{\mathrm{q}}\right), 121.0$ $\left(\mathrm{C}_{\mathrm{q}}\right), 118.4(\mathrm{CH}), 116.9\left(\mathrm{C}_{\mathrm{q}}\right), 116.8(\mathrm{CH}), 115.3(\mathrm{CH}), 113.9(\mathrm{CH}), 113.5(\mathrm{CH}), 112.5(\mathrm{CH})$, $112.3(\mathrm{CH}), 109.2(\mathrm{CH}), 55.3\left(\mathrm{CH}_{3}\right.$, overlapped, $\left.2 \mathrm{C}\right), 55.2\left(\mathrm{CH}_{3}\right.$, overlapped, $\left.2 \mathrm{C}\right), 55.1$ $\left(\mathrm{CH}_{3}\right), 55.0\left(\mathrm{CH}_{3}\right)$. IR (ATR): 2928, 1602, 1570, 1507, 1284, 1241, 1172, 1030, 826, 540 $\mathrm{cm}^{-1}$. HR-MS (ESI) $\mathrm{m} / z$ calcd for $\mathrm{C}_{55} \mathrm{H}_{45} \mathrm{~N}_{2} \mathrm{O}_{6}[\mathrm{M}+\mathrm{H}]^{+} 829.3272$, found 829.3268. 


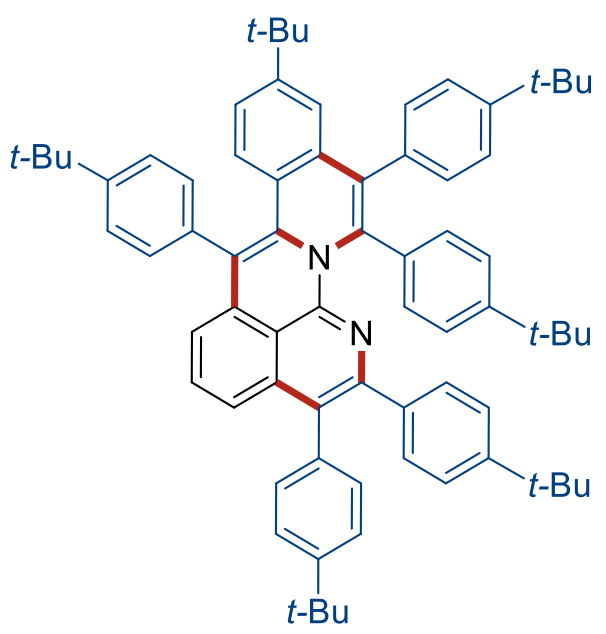

10-(tert-Butyl)-2,3,7,12,13-pentakis[4-(tert-butyl)phenyl]benzo[de]isoquinolino [2,1a][1,8]naphthyridine (183ad)

The general procedure $E$ was followed using $182 \mathrm{a}(30.0 \mathrm{mg}, 0.20 \mathrm{mmol})$ and $113 \mathrm{~d}$ (203.4 mg, $0.7 \mathrm{mmol}$ ) at $35^{\circ} \mathrm{C}$ for $12 \mathrm{~h}$. Purification by column chromatography on silica gel ( $n$-hexane/EtOAc 50:1) yielded 183ad (88.6 mg, 45\%) as a red solid. M.p. $=205-$ $206{ }^{\circ} \mathrm{C} .{ }^{1} \mathrm{H}$ NMR $\left(400 \mathrm{MHz}, \mathrm{CDCl}_{3}\right) \delta=7.58(\mathrm{~d}, J=8.3 \mathrm{~Hz}, 2 \mathrm{H}), 7.49(\mathrm{~d}, J=8.0 \mathrm{~Hz}, 2 \mathrm{H})$, $7.43(\mathrm{t}, J=7.9 \mathrm{~Hz}, 1 \mathrm{H}), 7.30$ (dd, $J=12.8,4.1 \mathrm{~Hz}, 6 \mathrm{H}), 7.21-7.11(\mathrm{~m}, 2 \mathrm{H}), 7.10-7.00(\mathrm{~m}$, $6 \mathrm{H}), 6.97-6.88(\mathrm{~m}, 4 \mathrm{H}), 6.85(\mathrm{dd}, J=8.7,2.0 \mathrm{~Hz}, 1 \mathrm{H}), 6.47-6.45(\mathrm{~m}, 2 \mathrm{H}), 1.48(\mathrm{~s}, 9 \mathrm{H})$, $1.37(\mathrm{~s}, 9 \mathrm{H}), 1.35(\mathrm{~s}, 9 \mathrm{H}), 1.26(\mathrm{~s}, 9 \mathrm{H}), 1.22(\mathrm{~s}, 9 \mathrm{H}), 1.18(\mathrm{~s}, 9 \mathrm{H}) .{ }^{13} \mathrm{C}$ NMR $(100 \mathrm{MHz}$, $\left.\mathrm{CDCl}_{3}\right) \delta=151.0\left(\mathrm{C}_{\mathrm{q}}\right), 150.8\left(\mathrm{C}_{\mathrm{q}}\right), 150.1\left(\mathrm{C}_{\mathrm{q}}\right), 149.5\left(\mathrm{C}_{\mathrm{q}}\right), 149.4\left(\mathrm{C}_{\mathrm{q}}\right), 149.3\left(\mathrm{C}_{\mathrm{q}}\right), 149.0\left(\mathrm{C}_{\mathrm{q}}\right)$, $148.9\left(\mathrm{C}_{\mathrm{q}}\right), 138.0\left(\mathrm{C}_{\mathrm{q}}\right), 137.9\left(\mathrm{C}_{\mathrm{q}}\right), 137.5\left(\mathrm{C}_{\mathrm{q}}\right), 136.0\left(\mathrm{C}_{\mathrm{q}}\right), 135.9\left(\mathrm{C}_{\mathrm{q}}\right), 135.8\left(\mathrm{C}_{\mathrm{q}}\right), 135.2\left(\mathrm{C}_{\mathrm{q}}\right)$, $134.5\left(\mathrm{C}_{\mathrm{q}}\right), 134.4\left(\mathrm{C}_{\mathrm{q}}\right), 134.3\left(\mathrm{C}_{\mathrm{q}}\right), 131.6(\mathrm{CH}), 131.4(\mathrm{CH}), 130.9(\mathrm{CH}), 130.7(\mathrm{CH}), 130.0$ $(\mathrm{CH}), 129.9\left(\mathrm{C}_{\mathrm{q}}\right), 128.2(\mathrm{CH}), 126.7(\mathrm{CH}), 125.6\left(\mathrm{C}_{\mathrm{q}}\right), 125.3\left(\mathrm{C}_{\mathrm{q}}\right), 125.1(\mathrm{CH}), 124.4(\mathrm{CH})$, $123.7(\mathrm{CH}), 123.5(\mathrm{CH}), 123.1(\mathrm{CH}), 122.6\left(\mathrm{C}_{\mathrm{q}}\right), 121.6(\mathrm{CH}), 121.5(\mathrm{CH}), 118.5(\mathrm{CH}), 117.8$ $\left(\mathrm{C}_{\mathrm{q}}\right), 117.0(\mathrm{CH}), 34.8\left(\mathrm{C}_{\mathrm{q}}\right), 34.6\left(\mathrm{C}_{\mathrm{q}}\right), 34.5\left(\mathrm{C}_{\mathrm{q}}\right.$, overlapped, $\left.2 \mathrm{C}\right), 34.4\left(\mathrm{C}_{\mathrm{q}}\right), 34.2\left(\mathrm{C}_{\mathrm{q}}\right), 31.5$ $\left(\mathrm{CH}_{3}\right), 31.4\left(\mathrm{CH}_{3}\right.$, overlapped, $\left.2 \mathrm{C}\right), 31.3\left(\mathrm{CH}_{3}\right.$, overlapped, $\left.2 \mathrm{C}\right), 31.0\left(\mathrm{CH}_{3}\right)$. IR $(\mathrm{ATR})$ : 2959, 2902, 1611, 1577, 1507, 1339, 1268, 1110, 1018, $823 \mathrm{~cm}^{-1}$. HR-MS (ESI) $\mathrm{m} / \mathrm{z}$ calcd for $\mathrm{C}_{73} \mathrm{H}_{81} \mathrm{~N}_{2}[\mathrm{M}+\mathrm{H}]^{+}$985.6394, found 985.6379. 


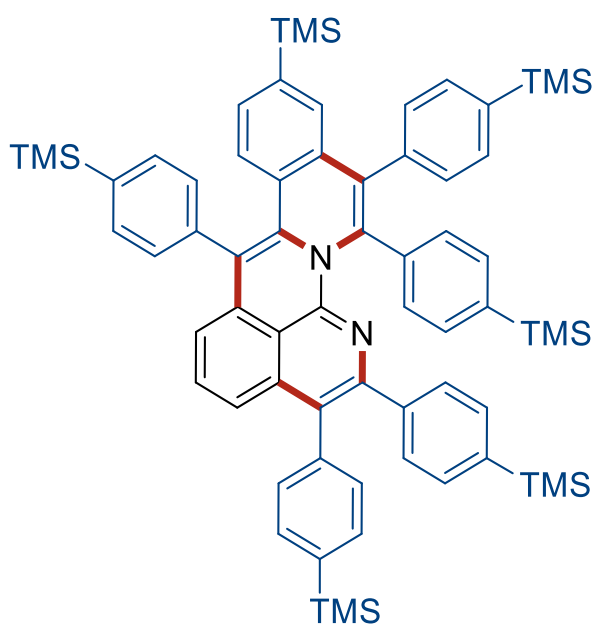

\section{0-(Trimethylsilyl)-2,3,7,12,13-pentakis[4-(trimethylsilyl)phenyl]benzo[de]isoqui} nolino[2,1-a][1,8]naphthyridine (183ae)

The general procedure $\mathrm{E}$ was followed using $182 \mathrm{a}(30.0 \mathrm{mg}, 0.20 \mathrm{mmol})$ and $113 \mathrm{e}$ (225.0 mg, $0.7 \mathrm{mmol}$ ) at $35^{\circ} \mathrm{C}$ for $12 \mathrm{~h}$. Purification by column chromatography on silica gel ( $n$-hexane/EtOAc 50:1) yielded 183ae $(145.0 \mathrm{mg}, 67 \%)$ as a red solid. M.p. $=219$ $220{ }^{\circ} \mathrm{C} .{ }^{1} \mathrm{H}$ NMR $\left(400 \mathrm{MHz}, \mathrm{CDCl}_{3}\right) \delta=7.68(\mathrm{~d}, J=7.8 \mathrm{~Hz}, 2 \mathrm{H}), 7.51(\mathrm{~d}, J=7.5 \mathrm{~Hz}, 2 \mathrm{H})$, 7.42-7.38 (m, 5H), $7.31(\mathrm{~s}, 1 \mathrm{H}), 7.23-7.15(\mathrm{~m}, 5 \mathrm{H}), 7.09-7.01(\mathrm{~m}, 6 \mathrm{H}), 6.94(\mathrm{~s}, 2 \mathrm{H}), 6.87$ (d, $J=7.6 \mathrm{~Hz}, 1 \mathrm{H}), 6.40$ (d, $J=8.1 \mathrm{~Hz}, 2 \mathrm{H}), 0.37$ (s, 9H), 0.28 (s, 9H), $0.26(\mathrm{~s}, 9 \mathrm{H}), 0.18$ (s, 9H), $0.14(\mathrm{~s}, 9 \mathrm{H}), 0.10(\mathrm{~s}, 9 \mathrm{H}) .{ }^{13} \mathrm{C}$ NMR $\left(100 \mathrm{MHz}, \mathrm{CDCl}_{3}\right) \delta=150.1\left(\mathrm{C}_{\mathrm{q}}\right), 149.6\left(\mathrm{C}_{\mathrm{q}}\right)$, $141.1\left(\mathrm{C}_{\mathrm{q}}\right), 140.9\left(\mathrm{C}_{\mathrm{q}}\right), 140.2\left(\mathrm{C}_{\mathrm{q}}\right), 139.5\left(\mathrm{C}_{\mathrm{q}}\right), 138.6\left(\mathrm{C}_{\mathrm{q}}\right), 138.5\left(\mathrm{C}_{\mathrm{q}}\right), 138.5\left(\mathrm{C}_{\mathrm{q}}\right), 138.4\left(\mathrm{C}_{\mathrm{q}}\right)$, $138.2\left(\mathrm{C}_{\mathrm{q}}\right), 137.8\left(\mathrm{C}_{\mathrm{q}}\right), 137.6\left(\mathrm{C}_{\mathrm{q}}\right), 137.6\left(\mathrm{C}_{\mathrm{q}}\right), 137.5\left(\mathrm{C}_{\mathrm{q}}\right), 135.9\left(\mathrm{C}_{\mathrm{q}}\right), 135.6\left(\mathrm{C}_{\mathrm{q}}\right), 134.9$ $(\mathrm{CH}), 133.5\left(\mathrm{C}_{\mathrm{q}}\right), 133.4(\mathrm{CH}), 132.6(\mathrm{CH}), 132.0(\mathrm{CH}), 131.9(\mathrm{CH}), 131.4(\mathrm{CH}), 131.1(\mathrm{CH})$, $131.0(\mathrm{CH}), 130.8(\mathrm{CH}), 130.7(\mathrm{CH}), 130.2(\mathrm{CH}), 129.6(\mathrm{CH}), 129.6(\mathrm{CH}), 128.0\left(\mathrm{C}_{q}\right), 127.6$ $(\mathrm{CH}), 125.9\left(\mathrm{C}_{\mathrm{q}}\right), 123.2\left(\mathrm{C}_{\mathrm{q}}\right), 122.0\left(\mathrm{C}_{\mathrm{q}}\right), 119.1(\mathrm{CH}), 119.0\left(\mathrm{C}_{\mathrm{q}}\right), 117.7(\mathrm{CH}),-0.8\left(\mathrm{CH}_{3}\right),-$ $0.9\left(\mathrm{CH}_{3}\right),-0.9\left(\mathrm{CH}_{3}\right),-1.0\left(\mathrm{CH}_{3}\right),-1.1\left(\mathrm{CH}_{3}\right),-1.3\left(\mathrm{CH}_{3}\right)$. IR (ATR): 2954, 2203, 1548, 1342, 1249, 1108, $840 \mathrm{~cm}^{-1}$. MS (ESI) m/z (relative intensity): 1081 (98) [M+H]+, $1082(100)$ $\left[\mathrm{M}\left({ }^{13} \mathrm{C},{ }^{29} \mathrm{Si}\right)+\mathrm{H}\right]^{+}, 1010$ (10). HR-MS (ESI) $\mathrm{m} / \mathrm{z}$ calcd for $\mathrm{C}_{67} \mathrm{H}_{80} \mathrm{~N}_{2} \mathrm{Si}_{6}[\mathrm{M}+\mathrm{H}]^{+}$1081.5010, found 1081.5017 . 


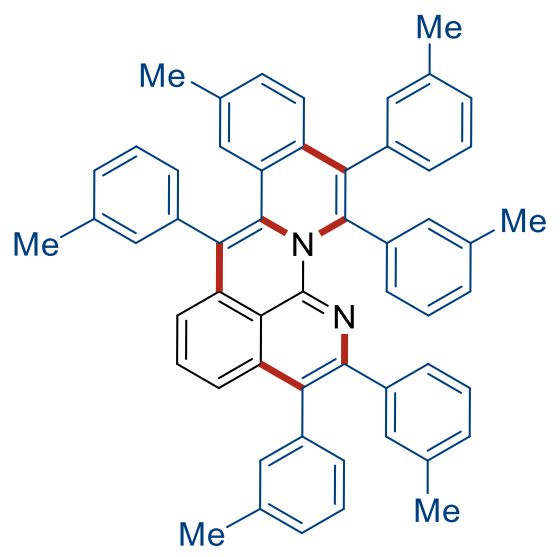

9-Methyl-2,3,7,12,13-penta-m-tolylbenzo[de]isoquinolino[2,1-a][1,8] naphthyridine (183af)

The general procedure $\mathrm{E}$ was followed using $182 \mathrm{a}(30.0 \mathrm{mg}, 0.20 \mathrm{mmol})$ and $113 \mathrm{f}$ (144.0 mg, $0.7 \mathrm{mmol}$ ) at $35^{\circ} \mathrm{C}$ for $12 \mathrm{~h}$. Purification by column chromatography on silica gel ( $n$-hexane/EtOAc 50:1) yielded 183af $(104.0 \mathrm{mg}, 71 \%)$ as a red solid. M.p. $=153-$ $154{ }^{\circ} \mathrm{C} .{ }^{1} \mathrm{H}$ NMR $\left(400 \mathrm{MHz}, \mathrm{CDCl}_{3}\right) \delta=7.51-7.44(\mathrm{~m}, 2 \mathrm{H}), 7.42-7.33(\mathrm{~m}, 3 \mathrm{H}), 7.29-7.19$ (m, 3H), 7.11-7.03 (m, 7H), 7.00-6.91 (m, 6H), 6.88-6.82 (m, 3H), $6.47(\mathrm{~s}, 2 \mathrm{H}), 2.47(\mathrm{~s}$, 3H), $2.35(\mathrm{~s}, 3 \mathrm{H}), 2.34(\mathrm{~s}, 3 \mathrm{H}), 2.18(\mathrm{~s}, 3 \mathrm{H}), 2.12(\mathrm{~s}, 3 \mathrm{H}), 2.00(\mathrm{~s}, 3 \mathrm{H}) .{ }^{13} \mathrm{C}$ NMR $(101 \mathrm{MHz}$, $\left.\mathrm{CDCl}_{3}\right) \delta=150.1\left(\mathrm{C}_{\mathrm{q}}\right), 149.6\left(\mathrm{C}_{\mathrm{q}}\right), 140.7\left(\mathrm{C}_{\mathrm{q}}\right), 139.5\left(\mathrm{C}_{\mathrm{q}}\right), 139.1\left(\mathrm{C}_{\mathrm{q}}\right), 138.1\left(\mathrm{C}_{\mathrm{q}}\right), 137.8\left(\mathrm{C}_{\mathrm{q}}\right)$, $137.7\left(\mathrm{C}_{\mathrm{q}}\right), 137.3\left(\mathrm{C}_{\mathrm{q}}\right), 137.3\left(\mathrm{C}_{\mathrm{q}}\right), 137.0\left(\mathrm{C}_{\mathrm{q}}\right)$ 136.7 $\left(\mathrm{C}_{\mathrm{q}}\right), 136.4\left(\mathrm{C}_{\mathrm{q}}\right.$, overlapped, 2C), 135.8 $\left(\mathrm{C}_{\mathrm{q}}\right), 135.5\left(\mathrm{C}_{\mathrm{q}}\right), 135.3\left(\mathrm{C}_{\mathrm{q}}\right), 132.6(\mathrm{CH}), 132.4\left(\mathrm{C}_{\mathrm{q}}\right.$, overlapped, $\left.2 \mathrm{C}\right), 132.2(\mathrm{CH}), 131.9$ $(\mathrm{CH}), 131.5(\mathrm{CH}), 130.9(\mathrm{CH}), 130.8(\mathrm{CH}), 129.7(\mathrm{CH}), 129.3(\mathrm{CH}), 129.3(\mathrm{CH}), 129.0(\mathrm{CH})$, $128.9(\mathrm{CH}), 128.6(\mathrm{CH}), 128.5(\mathrm{CH}), 128.3(\mathrm{CH}), 128.3(\mathrm{CH}), 127.7(\mathrm{CH}), 127.7(\mathrm{CH})$, $127.5(\mathrm{CH}), 127.4(\mathrm{CH}), 127.4(\mathrm{CH}), 127.2(\mathrm{CH}), 127.0(\mathrm{CH}), 126.7(\mathrm{CH}), 125.7\left(\mathrm{C}_{\mathrm{q}}\right), 125.0$ $(\mathrm{CH}), 123.3\left(\mathrm{C}_{\mathrm{q}}\right), 122.0\left(\mathrm{C}_{\mathrm{q}}\right.$, overlapped, $\left.2 \mathrm{C}\right), 118.9(\mathrm{CH}), 117.5(\mathrm{CH}), 21.6\left(\mathrm{CH}_{3}\right), 21.5$ $\left(\mathrm{CH}_{3}\right), 21.5\left(\mathrm{CH}_{3}\right), 21.4\left(\mathrm{CH}_{3}\right), 21.3\left(\mathrm{CH}_{3}\right), 21.3\left(\mathrm{CH}_{3}\right) .$. IR (ATR): 3023, 2914, 2091, 1997, 1606, 1538, 1337, 1289, $703 \mathrm{~cm}^{-1}$. MS (ESI) m/z (relative intensity): 733 (100) [M+H]+, 765 (10) $[\mathrm{M}+\mathrm{Na}]^{+}$. HR-MS (ESI) $\mathrm{m} / \mathrm{z}$ calcd for $\mathrm{C}_{55} \mathrm{H}_{45} \mathrm{~N}_{2} \mathrm{O}^{+}[\mathrm{M}+\mathrm{H}]^{+}$733.3577, found 733.3578. 
<smiles></smiles>

2,13-Diphenyl-3,7,12-tripropylbenzo[de]isoquinolino $[2,1-a][1,8]$ naphthyridine (183ag)

The general procedure was $\mathbf{G}$ followed using $182 \mathrm{a}(30.0 \mathrm{mg}, 0.20 \mathrm{mmol})$ and $\mathbf{1 1 3} \mathbf{g}$ (101.0 mg, $0.7 \mathrm{mmol}$ ) at $35^{\circ} \mathrm{C}$ for $12 \mathrm{~h}$. Purification by column chromatography on silica gel ( $n$-hexane/EtOAc $100: 1)$ yielded two regioisomers 183ag (55.5 mg ) and 183ag' (20.5 mg) $(76.0 \mathrm{mg}, 70 \%$, 183ag: 183ag' = $2.7: 1)$. Characterization data for 183ga: red solid. M.p. $=175-176{ }^{\circ} \mathrm{C} .{ }^{1} \mathrm{H}$ NMR $\left(400 \mathrm{MHz}, \mathrm{CDCl}_{3}\right) \delta=7.70(\mathrm{t}, J=8.0 \mathrm{~Hz}, 2 \mathrm{H}), 7.54(\mathrm{dd}, \mathrm{J}=$ 8.0, 1.1 Hz, 1H), 7.47-7.41 (m, 2H), 7.37-7.31 (m, 2H), 7.28-7.10 (m, 8H), 6.77-6.72 (m, 2H), 3.08-2.96 (m, 2H), 2.67-2.55 (m, 4H), 1.96-1.84 (m, 2H), 1.56-1.43 (m, 4H), $1.18(\mathrm{t}$, $J=7.3 \mathrm{~Hz}, 3 \mathrm{H}), 0.73(\mathrm{t}, J=7.4 \mathrm{~Hz}, 3 \mathrm{H}), 0.69(\mathrm{t}, J=7.3 \mathrm{~Hz}, 3 \mathrm{H}) .{ }^{13} \mathrm{C} \mathbf{N M R}\left(101 \mathrm{MHz}, \mathrm{CDCl}_{3}\right)$ $\delta=150.8\left(\mathrm{C}_{\mathrm{q}}\right), 147.7\left(\mathrm{C}_{\mathrm{q}}\right), 141.5\left(\mathrm{C}_{\mathrm{q}}\right), 137.9\left(\mathrm{C}_{\mathrm{q}}\right), 137.6\left(\mathrm{C}_{\mathrm{q}}\right), 137.4\left(\mathrm{C}_{\mathrm{q}}\right), 136.4\left(\mathrm{C}_{\mathrm{q}}\right), 134.5$ $\left(\mathrm{C}_{\mathrm{q}}\right), 134.2\left(\mathrm{C}_{\mathrm{q}}\right), 130.8(\mathrm{CH}), 129.6(\mathrm{CH}$, overlapped, $2 \mathrm{C}), 128.8\left(\mathrm{C}_{\mathrm{q}}\right), 128.7(\mathrm{CH}), 127.7$ (CH), $127.3(\mathrm{CH}$, overlapped, 2C), $127.1(\mathrm{CH}), 126.9(\mathrm{CH}), 126.1(\mathrm{CH}), 123.2(\mathrm{CH}), 122.5$ $\left(\mathrm{C}_{\mathrm{q}}\right), 121.0\left(\mathrm{C}_{\mathrm{q}}\right), 120.9\left(\mathrm{C}_{\mathrm{q}}\right), 117.5(\mathrm{CH}), 116.7\left(\mathrm{C}_{\mathrm{q}}\right), 115.3(\mathrm{CH}), 30.9\left(\mathrm{CH}_{2}\right), 30.4\left(\mathrm{CH}_{2}\right)$, $29.8\left(\mathrm{CH}_{2}\right), 22.8\left(\mathrm{CH}_{2}\right.$, overlapped, $\left.2 \mathrm{C}\right), 21.2\left(\mathrm{CH}_{2}\right), 14.5\left(\mathrm{CH}_{3}\right), 14.1\left(\mathrm{CH}_{3}\right), 13.9\left(\mathrm{CH}_{3}\right)$. IR (ATR): 3065, 2957, 2866, 1611, 1547, 1334, 1281, $757 \mathrm{~cm}^{-1}$. MS (ESI) m/z (relative intensity): $547(100)[\mathrm{M}+\mathrm{H}]^{+}, 579(40)[\mathrm{M}+\mathrm{Na}]^{+}$. HR-MS (ESI) $\mathrm{m} / z$ calcd for $\mathrm{C}_{40} \mathrm{H}_{39} \mathrm{~N}_{2}[\mathrm{M}+\mathrm{H}]^{+}$ 547.3108, found 547.3108. 


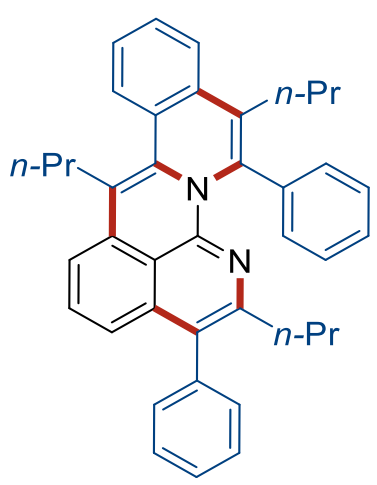

3,13-Diphenyl-2,7,12-tripropylbenzo[de]isoquinolino[2,1-a][1,8]naphthyridine (183ag')

Characterization data for 183ag': ${ }^{1} \mathrm{H}$ NMR $\left(400 \mathrm{MHz}, \mathrm{CDCl}_{3}\right) \delta=7.74(\mathrm{t}, J=7.9 \mathrm{~Hz}, 1 \mathrm{H})$, $7.65(\mathrm{~d}, J=7.5 \mathrm{~Hz}, 1 \mathrm{H}), 7.54(\mathrm{~d}, J=8.3 \mathrm{~Hz}, 1 \mathrm{H}), 7.49-7.37(\mathrm{~m}, 6 \mathrm{H}), 7.41-7.30(\mathrm{~m}, 5 \mathrm{H})$, 7.27-7.15 (m, 2H), 6.75 (dd, J = 7.6, 1.4 Hz, 1H), 3.19-3.01 (m, 2H), 3.03-2.98 (m, 2H), 2.85-2.79 (m, 2H), 1.94-1.81 (m, 2H), 1.65 (dq, $J=14.9,7.3 \mathrm{~Hz}, 2 \mathrm{H}), 1.17$ (t, $J=7.3 \mathrm{~Hz}$, $3 \mathrm{H}), 0.87$ (t, $J=7.3 \mathrm{~Hz}, 3 \mathrm{H}), 0.73(\mathrm{p}, J=7.4 \mathrm{~Hz}, 2 \mathrm{H}), 0.45$ (t, $J=7.3 \mathrm{~Hz}, 3 \mathrm{H}) .{ }^{13} \mathrm{C}$ NMR $\left(100 \mathrm{MHz}, \mathrm{CDCl}_{3}\right) \delta=151.0\left(\mathrm{C}_{\mathrm{q}}\right), 148.0\left(\mathrm{C}_{\mathrm{q}}\right), 142.1\left(\mathrm{C}_{\mathrm{q}}\right), 139.6\left(\mathrm{C}_{\mathrm{q}}\right), 138.8\left(\mathrm{C}_{\mathrm{q}}\right), 137.5\left(\mathrm{C}_{\mathrm{q}}\right)$, $136.5\left(\mathrm{C}_{\mathrm{q}}\right), 135.5\left(\mathrm{C}_{\mathrm{q}}\right), 133.9\left(\mathrm{C}_{\mathrm{q}}\right), 131.5(\mathrm{CH}), 131.1(\mathrm{CH}), 129.4(\mathrm{CH}), 128.6(\mathrm{CH}), 128.6$ $(\mathrm{CH}), 128.1(\mathrm{CH}), 127.5\left(\mathrm{C}_{\mathrm{q}}\right), 127.4(\mathrm{CH}), 127.2(\mathrm{CH}), 126.7(\mathrm{CH}), 125.5(\mathrm{CH}), 124.9(\mathrm{CH})$, $123.4\left(\mathrm{C}_{\mathrm{q}}\right), 122.1\left(\mathrm{C}_{\mathrm{q}}\right), 122.1\left(\mathrm{C}_{\mathrm{q}}\right), 117.6(\mathrm{CH}), 117.6\left(\mathrm{C}_{\mathrm{q}}\right), 115.7(\mathrm{CH}), 31.0\left(\mathrm{CH}_{2}\right), 30.7$ $\left(\mathrm{CH}_{2}\right), 30.7\left(\mathrm{CH}_{2}\right), 23.1\left(\mathrm{CH}_{2}\right), 21.3\left(\mathrm{CH}_{2}\right), 20.0\left(\mathrm{CH}_{2}\right), 14.5\left(\mathrm{CH}_{3}\right), 14.3\left(\mathrm{CH}_{3}\right), 13.5\left(\mathrm{CH}_{3}\right)$. IR (ATR): 3063, 2959, 2870, 1614, 1549, 1340, 1257, $767 \mathrm{~cm}^{-1}$. MS (ESI) m/z (relative intensity): 547 (100) $[\mathrm{M}+\mathrm{H}]^{+}, 579(20)[\mathrm{M}+\mathrm{Na}]^{+}$. HR-MS (ESI) $\mathrm{m} / \mathrm{z}$ calcd for $\mathrm{C}_{40} \mathrm{H}_{39} \mathrm{~N}_{2}[\mathrm{M}+\mathrm{H}]^{+}$ 547.3108, found 547.3110 . 


\subsubsection{Mechanistic Studies}

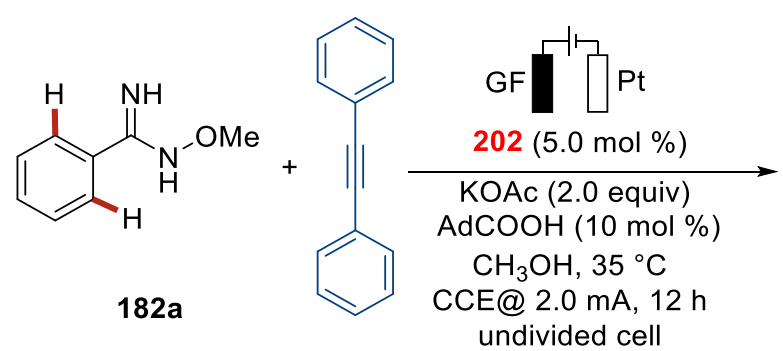

$113 a$<smiles></smiles>

183aa, $86 \%$

The electrocatalysis was carried out in an undivided cell with a graphite felt anode $(10 \mathrm{~mm}$ $\times 15 \mathrm{~mm} \times 6 \mathrm{~mm})$ and a Pt cathode $(10 \mathrm{~mm} \times 15 \mathrm{~mm} \times 0.25 \mathrm{~mm})$. The cell was charged with imidate 182a $(0.20 \mathrm{mmol})$, alkyne 113a $(0.7 \mathrm{mmol}), \mathrm{KOAc}(39.3 \mathrm{mg}, 0.4 \mathrm{mmol}), \mathrm{AdOH}$ (3.6 mg, $10 \mathrm{~mol} \%), 202(4.5 \mathrm{mg}, 5.0 \mathrm{~mol} \%)$ and $\mathrm{MeOH}(4.0 \mathrm{~mL})$. Electrocatalysis was performed at $35^{\circ} \mathrm{C}$ with a constant current of $2.0 \mathrm{~mA}$ maintained for $12 \mathrm{~h}$. The graphite felt anode was washed with $\mathrm{CH}_{2} \mathrm{Cl}_{2}(8 \times 5.0 \mathrm{~mL})$ in an ultrasonic bath. Evaporation of the solvents and subsequent column chromatography on silica gel ( $n$-hexane/EtOAc 50:1) afforded the corresponding product 183aa (112.0 mg, 86\%).

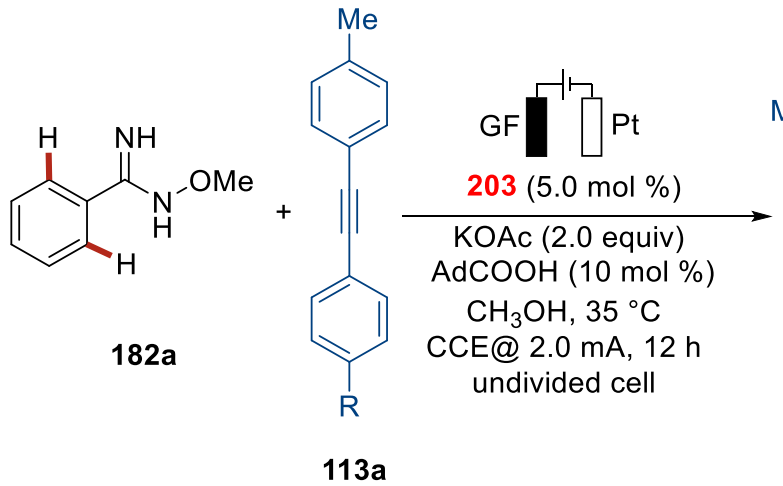

$113 a$<smiles></smiles>

$183 a b, 51 \%$

The electrocatalysis was carried out in an undivided cell with a graphite felt anode $(10 \mathrm{~mm}$ $\times 15 \mathrm{~mm} \times 6 \mathrm{~mm})$ and a Pt cathode $(10 \mathrm{~mm} \times 15 \mathrm{~mm} \times 0.25 \mathrm{~mm})$. The cell was charged with imidate 182a $(0.20 \mathrm{mmol})$, alkyne $113 \mathrm{a}(0.70 \mathrm{mmol}), \mathrm{KOAc}(39.3 \mathrm{mg}, 0.40 \mathrm{mmol})$, 
$\mathrm{AdCO}_{2} \mathrm{H}(3.6 \mathrm{mg}, 10 \mathrm{~mol} \%), 203(6.2 \mathrm{mg}, 5.0 \mathrm{~mol} \%)$ and $\mathrm{MeOH}(4.0 \mathrm{~mL})$. Electrocatalysis was performed at $35^{\circ} \mathrm{C}$ with a constant current of $2.0 \mathrm{~mA}$ maintained for $12 \mathrm{~h}$. The graphite felt anode was washed with $\mathrm{CH}_{2} \mathrm{Cl}_{2}(8 \times 5.0 \mathrm{~mL})$ in an ultrasonic bath. Evaporation of the solvents and subsequent column chromatography on silica gel ( $n$-hexane/EtOAc 50:1) afforded the corresponding product 183ab (75.2 mg, 51\%).

\subsubsection{Derivatiztion of 183aa and 183aj}

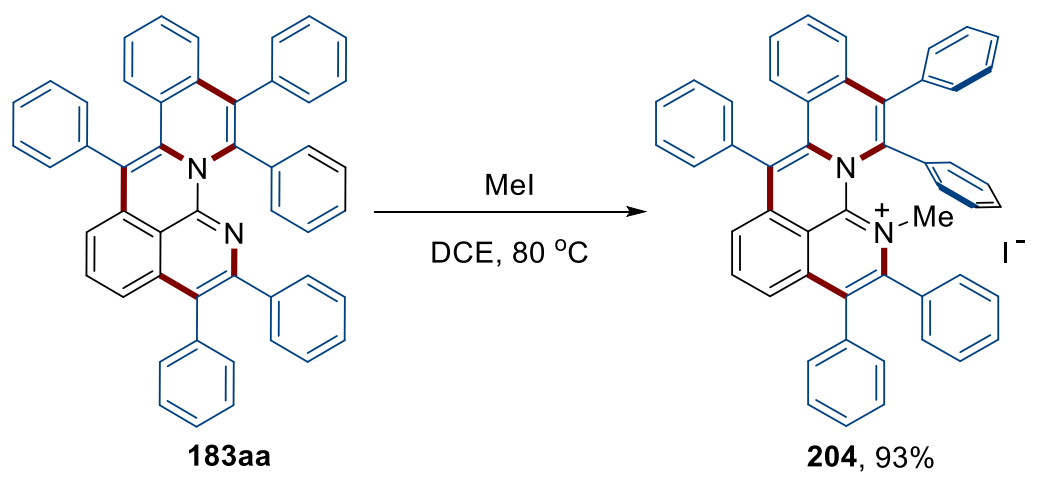

1-methyl-2,3,7,12,13-pentaphenylbenzo[de]isoquinolino[2,1-a][1,8]naphthyridin-1ium iodide (204)

To a $25 \mathrm{~mL}$ Schlenk tube was added 183aa $(65.2 \mathrm{mg}, 0.10 \mathrm{mmol})$, Mel $(85 \mathrm{mg}, 0.6 \mathrm{mmol}$, $20 \mu \mathrm{L}$ ) and $1.0 \mathrm{~mL}$ DCE. The reaction was stirred at $80{ }^{\circ} \mathrm{C}$ for 6 hours. Then the solvent was removed and purification of the residue by column chromatography (DCM/MeOH, 50:1) yielded the product $204(73.4 \mathrm{mg}, 93 \%)$ as a red solid. M.p. $=229-230^{\circ} \mathrm{C} .{ }^{1} \mathbf{H}$ NMR $(400$ $\left.\mathrm{MHz}, \mathrm{CDCl}_{3}\right) \delta=7.93(\mathrm{t}, J=8.0 \mathrm{~Hz}, 1 \mathrm{H}), 7.81(\mathrm{~d}, J=7.7 \mathrm{~Hz}, 1 \mathrm{H}), 7.74(\mathrm{t}, J=7.2 \mathrm{~Hz}, 1 \mathrm{H})$, $7.61(\mathrm{t}, J=7.4 \mathrm{~Hz}, 1 \mathrm{H}), 7.54-7.50(\mathrm{~m}, 3 \mathrm{H}), 7.45-7.35(\mathrm{~m}, 7 \mathrm{H}), 7.32-7.27(\mathrm{~m}, 3 \mathrm{H}), 7.26-$ $6.98(\mathrm{~m}, 13 \mathrm{H}), 6.55(\mathrm{~d}, J=7.6 \mathrm{~Hz}, 1 \mathrm{H}), 5.51(\mathrm{~d}, J=7.7 \mathrm{~Hz}, 1 \mathrm{H}), 3.63(\mathrm{~s}, 3 \mathrm{H}) .{ }^{13} \mathrm{C}$ NMR $\left(101 \mathrm{MHz}, \mathrm{CDCl}_{3}\right) \delta=151.9\left(\mathrm{C}_{\mathrm{q}}\right), 144.3\left(\mathrm{C}_{\mathrm{q}}\right), 136.0(\mathrm{CH}), 135.8\left(\mathrm{C}_{\mathrm{q}}\right), 135.5\left(\mathrm{C}_{\mathrm{q}}\right), 135.4\left(\mathrm{C}_{\mathrm{q}}\right.$ overlapped, $2 \mathrm{C}), 135.1\left(\mathrm{C}_{\mathrm{q}}\right), 135.0\left(\mathrm{C}_{\mathrm{q}}\right), 134.6\left(\mathrm{C}_{\mathrm{q}}\right), 134.1\left(\mathrm{C}_{\mathrm{q}}\right), 133.6\left(\mathrm{C}_{\mathrm{q}}\right), 132.5\left(\mathrm{C}_{\mathrm{q}}\right)$, $132.0(\mathrm{CH}), 131.7\left(\mathrm{C}_{\mathrm{q}}\right), 131.7\left(\mathrm{C}_{\mathrm{q}}\right), 131.6(\mathrm{CH}), 130.9(\mathrm{CH}), 130.7(\mathrm{CH}), 130.4(\mathrm{CH}), 130.3$ $(\mathrm{CH}), 130.0(\mathrm{CH}), 129.7(\mathrm{CH}), 129.5(\mathrm{CH}), 129.1(\mathrm{CH}), 129.0(\mathrm{CH}), 128.8(\mathrm{CH}), 128.7(\mathrm{CH})$, $128.4(\mathrm{CH}), 128.4(\mathrm{CH}), 128.3(\mathrm{CH}), 128.2(\mathrm{CH}), 128.1(\mathrm{CH}), 127.6\left(\mathrm{C}_{\mathrm{q}}\right), 126.8(\mathrm{CH}), 125.4$ $\left(\mathrm{C}_{\mathrm{q}}\right), 123.6(\mathrm{CH}), 122.6(\mathrm{CH}), 122.6\left(\mathrm{C}_{\mathrm{q}}\right), 46.9\left(\mathrm{CH}_{3}\right)$. IR (ATR): 3031, 3004, 1619, 1549, 
1439, 1328, 1246, 758, $697 \mathrm{~cm}^{-1}$. HR-MS (ESI) $\mathrm{m} / \mathrm{z}$ calcd for $\mathrm{C}_{50} \mathrm{H}_{34} \mathrm{~N}_{2}$ [M-I]+: 663.2795, found: 663.2799 .

\subsection{Rhodaelectro-catalyzed switchable Indole dienylation/ cyclopropylation}

\subsubsection{Experimental procedure and analytical data}<smiles>C=C/C(=C/c1cc2ccccc2n1[Po])c1ccccc1</smiles>

(Z)-2-(1-Phenylbuta-1,3-dien-2-yl)-1-(pyrimidin-2-yl)-1 H-indole (184aa)

The general procedure $\mathbf{F}$ was followed using 1-(pyrimidin-2-yl)-1 $H$-indole 71a (39.0 mg, $0.20 \mathrm{mmol}$ ) and (cyclopropylidenemethyl)benzene $138 \mathrm{a}(41.6 \mathrm{mg}, 0.32 \mathrm{mmol})$ for $3.0 \mathrm{~h}$. Isolation by column chromatography ( $n$-hexane/EtOAc 15:1) yielded 184aa (57.6 mg, 89\%, $Z / E=7.0 / 1$ determined by ${ }^{1} \mathrm{H}$ NMR $)$ as a yellow solid. M.p. $=137-139{ }^{\circ} \mathrm{C} .{ }^{1} \mathbf{H}$ NMR $(400$ $\left.\mathrm{MHz}, \mathrm{CDCl}_{3}\right): \delta=8.71(\mathrm{~d}, J=4.8 \mathrm{~Hz}, 0.29 \mathrm{H}, E$ isomer), $8.62(\mathrm{~d}, J=4.8 \mathrm{~Hz}, 2 \mathrm{H}, Z$ isomer), $8.33(\mathrm{~d}, J=8.3 \mathrm{~Hz}, 1 \mathrm{H}), 7.60(\mathrm{dd}, J=7.8,1.2 \mathrm{~Hz}, 1 \mathrm{H}), 7.32(\mathrm{td}, J=7.7,1.2 \mathrm{~Hz}, 1 \mathrm{H}), 7.24$ (t, $J=6.6 \mathrm{~Hz}, 1 \mathrm{H}), 7.08-7.06(\mathrm{~m}, 3 \mathrm{H}), 7.03-6.99(\mathrm{~m}, 3 \mathrm{H}), 6.69(\mathrm{dd}, J=17.4,10.6 \mathrm{~Hz}, 1 \mathrm{H})$, $6.68(\mathrm{~s}, 1 \mathrm{H}), 6.57(\mathrm{~s}, 1 \mathrm{H}), 5.06(\mathrm{~d}, J=10.6 \mathrm{~Hz}, 1 \mathrm{H}), 5.02(\mathrm{~d}, J=17.4 \mathrm{~Hz}, 1 \mathrm{H}) .{ }^{13} \mathrm{C}$ NMR $\left(101 \mathrm{MHz}, \mathrm{CDCl}_{3}\right) \delta=157.9(\mathrm{CH}), 157.5\left(\mathrm{C}_{\mathrm{q}}\right), 140.6(\mathrm{CH}), 136.7\left(\mathrm{C}_{\mathrm{q}}\right), 136.7\left(\mathrm{C}_{\mathrm{q}}\right), 135.4$ $\left(\mathrm{C}_{\mathrm{q}}\right), 134.0\left(\mathrm{C}_{\mathrm{q}}\right), 133.0(\mathrm{CH}), 129.5\left(\mathrm{C}_{\mathrm{q}}\right), 129.1(\mathrm{CH}), 128.1(\mathrm{CH}), 127.2(\mathrm{CH}), 123.4(\mathrm{CH})$, $122.0(\mathrm{CH}), 120.8(\mathrm{CH}), 117.0(\mathrm{CH}), 115.7(\mathrm{CH}), 114.2(\mathrm{CH}), 108.7\left(\mathrm{CH}_{2}\right)$. IR $(\mathrm{ATR}):$ 3000, $2201,1564,1437,1419,1360,1256,1075,812,769 \mathrm{~cm}^{-1}$. MS (ESI) $\mathrm{m} / \mathrm{z}$ (relative intensity): $346(80)[\mathrm{M}+\mathrm{Na}]^{+}, 324(100)[\mathrm{M}+\mathrm{H}]^{+}$. HR-MS (ESI) $\mathrm{m} / z$ calcd for $\mathrm{C}_{22} \mathrm{H}_{18} \mathrm{~N}_{3}[\mathrm{M}+\mathrm{H}]^{+} 324.1495$, found $324.1484 .^{+}$ 


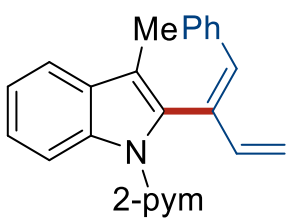

(Z)-3-Methyl-2-(1-phenylbuta-1,3-dien-2-yl)-1-(pyrimidin-2-yl)-1 H-indole (184ba)

The general procedure $\mathbf{F}$ was followed using 3-methyl-1-(pyrimidin-2-yl)-1H-indole $\mathbf{7 1} \mathbf{b}$ (41.9 mg, $0.20 \mathrm{mmol})$ and (cyclopropylidenemethyl)benzene 138a (41.6 mg, $0.32 \mathrm{mmol})$ for $3.5 \mathrm{~h}$. Isolation by column chromatography ( $n$-hexane/EtOAc 15:1) yielded 184ba (46.5 mg, $69 \%, Z / E=9.6 / 1$ determined by ${ }^{1} \mathrm{H}$ NMR) as a yellow solid. M.p. $=102-104{ }^{\circ} \mathrm{C}$. ${ }^{1} \mathrm{H}$ NMR $\left(300 \mathrm{MHz}, \mathrm{CDCl}_{3}\right) \delta=8.69(\mathrm{~d}, J=4.8 \mathrm{~Hz}, 0.18 \mathrm{H}, E$ isomer), $8.65(\mathrm{~d}, J=4.7 \mathrm{~Hz}$, 2H, $Z$ isomer), $8.44(\mathrm{~d}, J=8.1 \mathrm{~Hz}, 1 \mathrm{H}), 7.57(\mathrm{~d}, J=8.4 \mathrm{~Hz}, 1 \mathrm{H}), 7.39-7.24(\mathrm{~m}, 3 \mathrm{H}), 7.17-$ $7.10(\mathrm{~m}, 4 \mathrm{H}), 7.01(\mathrm{t}, J=4.8 \mathrm{~Hz}, 1 \mathrm{H}), 6.81(\mathrm{~s}, 1 \mathrm{H}), 6.65$ (ddd, $J=17.2,10.4,0.7 \mathrm{~Hz}, 1 \mathrm{H}$ ), $4.97(\mathrm{~d}, J=10.5 \mathrm{~Hz}, 1 \mathrm{H}), 4.81(\mathrm{~d}, J=17.2 \mathrm{~Hz}, 1 \mathrm{H}), 2.00(\mathrm{~s}, 3 \mathrm{H}) .{ }^{13} \mathrm{C}$ NMR $(101 \mathrm{MHz}$, $\left.\mathrm{CDCl}_{3}\right) \delta=157.9(\mathrm{CH}), 157.8\left(\mathrm{C}_{\mathrm{q}}\right), 140.3(\mathrm{CH}), 137.2\left(\mathrm{C}_{\mathrm{q}}\right), 136.5\left(\mathrm{C}_{\mathrm{q}}\right), 133.6\left(\mathrm{C}_{\mathrm{q}}\right), 133.5$ $(\mathrm{CH}), 132.0\left(\mathrm{C}_{\mathrm{q}}\right), 130.7\left(\mathrm{C}_{\mathrm{q}}\right), 128.8(\mathrm{CH}), 128.2(\mathrm{CH}), 127.2(\mathrm{CH}), 123.6(\mathrm{CH}), 121.6(\mathrm{CH})$, $119.0(\mathrm{CH}), 116.6(\mathrm{CH}), 115.2\left(\mathrm{CH}_{2}\right), 114.9\left(\mathrm{C}_{\mathrm{q}}\right), 114.4(\mathrm{CH}), 9.0\left(\mathrm{CH}_{3}\right)$. IR $(\mathrm{ATR}):$ 3048, 2920, 1565, 1425, 1354, 1223, 1017, 988, 801, 748, $697 \mathrm{~cm}^{-1}$. MS (ESI) $\mathrm{m} / z$ (relative intensity): $360(68)[\mathrm{M}+\mathrm{Na}]^{+}, 338(100)[\mathrm{M}+\mathrm{H}]^{+}$. HR-MS (ESI) $\mathrm{m} / z$ calcd for $\mathrm{C}_{23} \mathrm{H}_{20} \mathrm{~N}_{3}[\mathrm{M}+\mathrm{H}]^{+}$ 338.1657, found 338.1642.<smiles>C=C/C(=C/c1ccccc1)c1cc2c(OC)cccc2n1[Pb]</smiles>

(Z)-4-Methoxy-2-(1-phenylbuta-1,3-dien-2-yl)-1-(pyrimidin-2-yl)-1 H-indole (184ca)

The general procedure $\mathbf{F}$ was followed using 4-methoyl-1-(pyrimidin-2-yl)-1H-indole $71 \mathrm{c}$ (45.1 mg, $0.20 \mathrm{mmol})$ and (cyclopropylidenemethyl)benzene 138a (41.6 mg, $0.32 \mathrm{mmol}$ ) for $2.8 \mathrm{~h}$. Isolation by column chromatography ( $n$-hexane/EtOAc 15:1) yielded 184ca (67.1 
mg, $95 \%, Z / E=3.3 / 1$ determined by ${ }^{1} \mathrm{H}$ NMR) as a yellow solid. M.p. $=98-99{ }^{\circ} \mathrm{C} .{ }^{1} \mathbf{H}$ NMR $\left(400 \mathrm{MHz}, \mathrm{CDCl}_{3}\right) \delta=8.72(\mathrm{~d}, J=4.8 \mathrm{~Hz}, 0.6 \mathrm{H}, E$ isomer), 8.61 (d, $J=4.8 \mathrm{~Hz}, 2 \mathrm{H}, Z$ isomer), $7.90(\mathrm{~d}, J=8.5 \mathrm{~Hz}, 1 \mathrm{H}), 7.25(\mathrm{t}, J=8.1 \mathrm{~Hz}, 1 \mathrm{H}), 7.09-7.05(\mathrm{~m}, 3 \mathrm{H}), 7.02-6.98(\mathrm{~m}$, 3H), $6.73(\mathrm{~s}, 1 \mathrm{H}), 6.71$ (dd, $J=16.5,10.2 \mathrm{~Hz}, 1 \mathrm{H}), 6.70$ (d, $J=7.9 \mathrm{~Hz}, 1 \mathrm{H}), 6.66(\mathrm{~s}, 1 \mathrm{H})$, $5.09(\mathrm{~d}, J=16.5 \mathrm{~Hz}, 1 \mathrm{H}), 5.09(\mathrm{~d}, J=10.6 \mathrm{~Hz}, 1 \mathrm{H}), 3.98(\mathrm{~s}, 3 \mathrm{H}) .{ }^{13} \mathrm{C}$ NMR $(101 \mathrm{MHz}$, $\left.\mathrm{CDCl}_{3}\right) \delta=157.8(\mathrm{CH}), 157.5\left(\mathrm{C}_{\mathrm{q}}\right), 153.1\left(\mathrm{C}_{\mathrm{q}}\right), 140.6(\mathrm{CH}), 138.0\left(\mathrm{C}_{\mathrm{q}}\right), 136.7\left(\mathrm{C}_{\mathrm{q}}\right), 133.9$ $\left(\mathrm{C}_{\mathrm{q}}\right), 133.8\left(\mathrm{C}_{\mathrm{q}}\right), 133.0(\mathrm{CH}), 129.1(\mathrm{CH}), 128.0(\mathrm{CH}), 127.1(\mathrm{CH}), 124.2(\mathrm{CH}), 120.0\left(\mathrm{C}_{\mathrm{q}}\right)$, $117.1(\mathrm{CH}), 115.8\left(\mathrm{CH}_{2}\right), 107.4(\mathrm{CH}), 105.7(\mathrm{CH}), 102.1(\mathrm{CH}), 55.5\left(\mathrm{CH}_{3}\right)$. IR $(\mathrm{ATR}): 3026$, 2918, 1693, 1564, 1420, 1336, 1240, 1149, 910, 805, 740, $698 \mathrm{~cm}^{-1}$. MS (ESI) $\mathrm{m} / \mathrm{z}$ (relative intensity): $376(100)[\mathrm{M}+\mathrm{Na}]^{+}, 354(50)[\mathrm{M}+\mathrm{H}]^{+}$. HR-MS (ESI) $\mathrm{m} / \mathrm{z}$ calcd for $\mathrm{C}_{23} \mathrm{H}_{20} \mathrm{~N}_{3} \mathrm{O}$ $[\mathrm{M}+\mathrm{H}]^{+} 354.1606$, found 354.1601 .

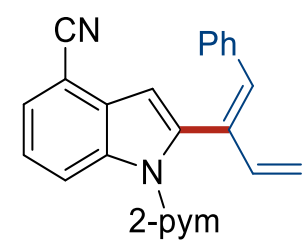

(Z)-2-(1-Phenylbuta-1,3-dien-2-yl)-1-(pyrimidin-2-yl)-1 H-indole-4-carbonitrile (184da)

The general procedure $\mathbf{F}$ was followed using 1-(pyrimidin-2-yl)-1H-indole-4-carbonitrile 71d (44.1 mg, $0.20 \mathrm{mmol})$ and (cyclopropylidenemethyl)benzene 138a (41.6 mg, 0.32 mmol) for $5.0 \mathrm{~h}$. Isolation by column chromatography ( $n$-hexane/EtOAc 15:1) yielded 184da (41.8 mg, 60\%, Z/E $=2.0 / 1$ determined by ${ }^{1} \mathrm{H}$ NMR) as a yellow oil. ${ }^{1} \mathbf{H}$ NMR (300 $\left.\mathrm{MHz}, \mathrm{CDCl}_{3}\right) \delta=8.76(\mathrm{~d}, J=4.8 \mathrm{~Hz}, 1 \mathrm{H}, E$ isomer), 8.65 (d, $J=4.8 \mathrm{~Hz}, 2 \mathrm{H}, Z$ isomer), $8.49(\mathrm{~d}, J=8.5 \mathrm{~Hz}, 1 \mathrm{H}), 7.59(\mathrm{~d}, J=7.5 \mathrm{~Hz}, 1 \mathrm{H}), 7.45-7.43(\mathrm{~m}, 1 \mathrm{H}), 7.35$ (dd, $J=8.5,7.4$ $\mathrm{Hz}, 1 \mathrm{H}), 7.11(\mathrm{t}, J=4.8 \mathrm{~Hz}, 1 \mathrm{H}), 7.09-7.04(\mathrm{~m}, 3 \mathrm{H}), 6.94-6.92(\mathrm{~m}, 1 \mathrm{H}), 6.82(\mathrm{~s}, 1 \mathrm{H}), 6.73$ (s, 1H), 6.71 (dd, $J=17.2,10.6 \mathrm{~Hz}, 1 \mathrm{H}), 5.13(\mathrm{~d}, J=10.6 \mathrm{~Hz}, 1 \mathrm{H}), 5.02(\mathrm{~d}, J=17.2 \mathrm{~Hz}$, 1H). ${ }^{13} \mathrm{C} \mathrm{NMR}\left(101 \mathrm{MHz}, \mathrm{CDCl}_{3}\right) \delta=158.0(\mathrm{CH}), 156.7\left(\mathrm{C}_{\mathrm{q}}\right), 139.8(\mathrm{CH}), 138.4\left(\mathrm{C}_{\mathrm{q}}\right), 136.7$ $\left(\mathrm{C}_{\mathrm{q}}\right), 136.1\left(\mathrm{C}_{\mathrm{q}}\right), 133.9(\mathrm{CH}), 132.7\left(\mathrm{C}_{\mathrm{q}}\right), 130.8\left(\mathrm{C}_{\mathrm{q}}\right), 128.8(\mathrm{CH}), 128.1(\mathrm{CH}), 127.5(\mathrm{CH})$, $126.8(\mathrm{CH}), 122.9(\mathrm{CH}), 118.7(\mathrm{CH}), 118.4\left(\mathrm{C}_{\mathrm{q}}\right), 117.9(\mathrm{CH}), 115.9\left(\mathrm{CH}_{2}\right), 106.6(\mathrm{CH})$, $103.1\left(\mathrm{C}_{\mathrm{q}}\right)$. IR (ATR): 3050, 2221, 1693, 1567, 1425, 1318, 1263, 1234, 1078, 913, 813, 
786, 739, $698 \mathrm{~cm}^{-1}$. MS (ESI) m/z (relative intensity): $371(40)[\mathrm{M}+\mathrm{Na}]^{+}, 349(100)[\mathrm{M}+\mathrm{H}]^{+}$. HR-MS (ESI) $m / z$ calcd for $\mathrm{C}_{23} \mathrm{H}_{17} \mathrm{~N}_{4}[\mathrm{M}+\mathrm{H}]^{+} 349.1448$, found 349.1449 .<smiles>C=C/C(=C/c1ccccc1)c1cc2cc(C)ccc2n1[Po+]</smiles>

(Z)-5-Methyl-2-(1-phenylbuta-1,3-dien-2-yl)-1-(pyrimidin-2-yl)-1H-indole (184ea)

The general procedure $\mathbf{F}$ was followed using 5-methyl-1-(pyrimidin-2-yl)-1H-indole 71e (45.1 mg, $0.20 \mathrm{mmol}$ ) and (cyclopropylidenemethyl)benzene 138a (41.9 mg, $0.32 \mathrm{mmol}$ ) for $3.5 \mathrm{~h}$. Isolation by column chromatography ( $n$-hexane/EtOAc 15:1) yielded 184ea (50.6 $\mathrm{mg}, 75 \%, Z / E=8.6 / 1$ determined by ${ }^{1} \mathrm{H}$ NMR $)$ as a yellow oil. ${ }^{1} \mathbf{H} \mathbf{N M R}\left(400 \mathrm{MHz}, \mathrm{CDCl}_{3}\right)$ $\delta=8.70(\mathrm{~d}, J=4.8 \mathrm{~Hz}, 0.23 \mathrm{H}, E$ isomer), $8.61(\mathrm{~d}, J=4.8 \mathrm{~Hz}, 1.97 \mathrm{H}, Z$ isomer $), 8.25(\mathrm{~d}, J$ $=8.6 \mathrm{~Hz}, 1 \mathrm{H}), 7.40(\mathrm{~s}, 1 \mathrm{H}), 7.15(\mathrm{~d}, J=8.2 \mathrm{~Hz}, 1 \mathrm{H}), 7.10-6.98(\mathrm{~m}, 6 \mathrm{H}), 6.72(\mathrm{~d}, J=17.6$, $10.6 \mathrm{~Hz}, 1 \mathrm{H}), 6.68(\mathrm{~s}, 1 \mathrm{H}), 6.50(\mathrm{~s}, 1 \mathrm{H}), 5.06(\mathrm{~d}, J=10.6 \mathrm{~Hz}, 1 \mathrm{H}), 5.01(\mathrm{~d}, J=17.6 \mathrm{~Hz}, 1 \mathrm{H})$. ${ }^{13} \mathrm{C}$ NMR $\left(101 \mathrm{MHz}, \mathrm{CDCl}_{3}\right) \delta=157.8(\mathrm{CH}), 157.5\left(\mathrm{C}_{\mathrm{q}}\right), 140.7(\mathrm{CH}), 136.8\left(\mathrm{C}_{\mathrm{q}}\right), 135.4\left(\mathrm{C}_{\mathrm{q}}\right)$, $135.0\left(\mathrm{C}_{\mathrm{q}}\right), 134.3\left(\mathrm{C}_{\mathrm{q}}\right), 132.8(\mathrm{CH}), 131.4(\mathrm{CH}), 129.8\left(\mathrm{C}_{\mathrm{q}}\right), 129.1(\mathrm{CH}), 128.1(\mathrm{CH}), 127.2$ $(\mathrm{CH}), 124.9(\mathrm{CH}), 120.6(\mathrm{CH}), 116.8(\mathrm{CH}), 115.6\left(\mathrm{CH}_{2}\right), 114.1(\mathrm{CH}), 108.5(\mathrm{CH}), 21.5\left(\mathrm{CH}_{3}\right)$. IR (ATR): 2919, 2860, 1565, 1423, 1217, 1080, 987, 806, 751, $699 \mathrm{~cm}^{-1}$. MS (ESI) m/z (relative intensity): $360(100)[\mathrm{M}+\mathrm{Na}]^{+}, 338(80)[\mathrm{M}+\mathrm{H}]^{+}$. HR-MS (ESI) $\mathrm{m} / \mathrm{z}$ calcd for $\mathrm{C}_{23} \mathrm{H}_{19} \mathrm{~N}_{3} \mathrm{Na}[\mathrm{M}+\mathrm{Na}]^{+} 360.1470$, found 360.1471 .<smiles>C=C/C(=C/c1ccccc1)c1cc2cc(F)ccc2n1[Po]</smiles>

(Z)-5-Fluoro-2-(1-phenylbuta-1,3-dien-2-yl)-1-(pyrimidin-2-yl)-1 H-indole (184fa)

The general procedure $\mathbf{F}$ was followed using 5-fluoro-1-(pyrimidin-2-yl)- $1 H$-indole $71 f(42.6$ $\mathrm{mg}, 0.20 \mathrm{mmol}$ ) and (cyclopropylidenemethyl)benzene $138 \mathrm{a}(41.9 \mathrm{mg}, 0.32 \mathrm{mmol})$ for 3.5 
h. Isolation by column chromatography ( $n$-hexane/EtOAc $15: 1$ ) yielded $\mathbf{1 8 4 f a}$ (48.5 mg, $71 \%, Z / E=5.0 / 1$ determined by ${ }^{1} \mathrm{H}$ NMR) as a yellow solid. M.p. $=104-106{ }^{\circ} \mathrm{C} .{ }^{1} \mathrm{H}$ NMR $\left(400 \mathrm{MHz}, \mathrm{CDCl}_{3}\right) \delta=8.67(\mathrm{~d}, J=4.8 \mathrm{~Hz}, 0.40 \mathrm{H}, E$ isomer $), 8.58(\mathrm{~d}, J=4.8 \mathrm{~Hz}, 2 \mathrm{H}, \mathrm{Z}$ isomer), 8.25 (dd, $J=9.1,4.7 \mathrm{~Hz}, 1 \mathrm{H}), 7.20(\mathrm{dd}, J=9.0,2.6 \mathrm{~Hz}, 1 \mathrm{H}), 7.07-7.02(\mathrm{~m}, 3 \mathrm{H})$, 7.01-6.94 (m, 4H), 6.67 (dd, J = 17.6, $10.5 \mathrm{~Hz}, 1 \mathrm{H}), 6.65(\mathrm{~s}, 1 \mathrm{H}), 6.48(\mathrm{~s}, 1 \mathrm{H}), 5.03(\mathrm{~d}, J=$ $10.5 \mathrm{~Hz}, 1 \mathrm{H}), 4.95(\mathrm{~d}, J=17.6 \mathrm{~Hz}, 1 \mathrm{H}) .{ }^{13} \mathrm{C}$ NMR $\left(101 \mathrm{MHz}, \mathrm{CDCl}_{3}\right) \delta=158.76(\mathrm{~d}, J=$ 297.2 Hz, $\left.\mathrm{C}_{\mathrm{q}}\right), 157.9(\mathrm{CH}), 157.89\left(\mathrm{~d}, \mathrm{~J}=2.7 \mathrm{~Hz}, \mathrm{C}_{\mathrm{q}}\right), 140.4(\mathrm{CH}), 137.1\left(\mathrm{C}_{\mathrm{q}}\right), 136.6\left(\mathrm{C}_{\mathrm{q}}\right)$, $133.9\left(\mathrm{C}_{\mathrm{q}}\right), 133.1(\mathrm{CH}), 132.9\left(\mathrm{C}_{\mathrm{q}}\right), 130.16\left(\mathrm{~d}, \mathrm{~J}=10.0 \mathrm{~Hz}, \mathrm{C}_{\mathrm{q}}\right), 129.1(\mathrm{CH}), 128.1(\mathrm{CH})$, $127.3(\mathrm{CH}), 117.6(\mathrm{~d}, J=23.3 \mathrm{~Hz}), 117.2(\mathrm{CH}), 115.7\left(\mathrm{CH}_{2}\right), 115.3(\mathrm{~d}, J=9.1 \mathrm{~Hz}, \mathrm{CH})$, $111.3(\mathrm{~d}, J=25.3 \mathrm{~Hz}, \mathrm{CH}), 108.4(\mathrm{~d}, J=4.1 \mathrm{~Hz}, \mathrm{CH}), 105.8(\mathrm{~d}, J=23.5 \mathrm{~Hz}, \mathrm{CH}) .{ }^{19} \mathrm{~F}$ NMR (377 MHz, $\left.\mathrm{CDCl}_{3}\right) \delta=-122.3$ (td, $J=9.1,4.6 \mathrm{~Hz}$ ). IR (ATR): 3053, 2130, 1961, 1710, 1568, 1427, 1419, 1196, 804, $698 \mathrm{~cm}^{-1}$. MS (ESI) m/z (relative intensity): 364 (95) [M+Na] ${ }^{+}, 342$ (100) $[\mathrm{M}+\mathrm{H}]^{+}$. HR-MS (ESI) $\mathrm{m} / z$ calcd for $\mathrm{C}_{22} \mathrm{H}_{17} \mathrm{~N}_{3} \mathrm{~F}[\mathrm{M}+\mathrm{H}]^{+} 342.1401$, found 342.1403<smiles>C=C/C(=C/c1ccccc1)c1cc2cc(Cl)ccc2n1[O+]=O</smiles>

\section{(Z)-5-chloro-2-(1-phenylbuta-1,3-dien-2-yl)-1-(pyrimidin-2-yl)-1 H-indole (184ga)}

The general procedure $\mathbf{F}$ was followed using 5-chloro-1-(pyrimidin-2-yl)-1H-indole $\mathbf{7 1 g}$ (45.9 mg, $0.20 \mathrm{mmol})$ and (cyclopropylidenemethyl)benzene 138a (41.9 mg, $0.32 \mathrm{mmol})$ for $3.5 \mathrm{~h}$. Isolation by column chromatography ( $n$-hexane/EtOAc 15:1) yielded 184ga (40.8 $\mathrm{mg}, 57 \%, Z / E=3.6 / 1$ determined by ${ }^{1} \mathrm{H}$ NMR $)$ as a yellow oil. ${ }^{1} \mathrm{H}$ NMR $\left(300 \mathrm{MHz}, \mathrm{CDCl}_{3}\right)$ $\delta=8.72(\mathrm{~d}, J=4.8 \mathrm{~Hz}, 0.55 \mathrm{H}, E$ isomer), $8.63(\mathrm{~d}, J=4.8 \mathrm{~Hz}, 2 \mathrm{H}, Z$ isomer), $8.28(\mathrm{~d}, J=$ $8.9 \mathrm{~Hz}, 1 \mathrm{H}), 7.58(\mathrm{~d}, J=2.1 \mathrm{~Hz}, 1 \mathrm{H}), 7.28(\mathrm{dd}, J=8.7,2.3 \mathrm{~Hz}, 1 \mathrm{H}), 7.15-7.04(\mathrm{~m}, 3 \mathrm{H})$, $7.05(\mathrm{t}, J=4.8 \mathrm{~Hz}, 1 \mathrm{H}), 7.00(\mathrm{dd}, J=6.7,3.0 \mathrm{~Hz}, 2 \mathrm{H}), 6.71(\mathrm{~s}, 1 \mathrm{H}), 6.70(\mathrm{dd}, J=17.2,10.2$ $\mathrm{Hz}, 1 \mathrm{H}), 6.52(\mathrm{~s}, 1 \mathrm{H}), 5.09(\mathrm{~d}, J=10.2 \mathrm{~Hz}, 1 \mathrm{H}), 5.00(\mathrm{~d}, J=17.2 \mathrm{~Hz}, 1 \mathrm{H}) .{ }^{13} \mathrm{C}$ NMR $(101$ $\left.\mathrm{MHz}, \mathrm{CDCl}_{3}\right) \delta=158.0(\mathrm{CH}), 157.2\left(\mathrm{C}_{\mathrm{q}}\right), 141.8\left(\mathrm{C}_{\mathrm{q}}\right), 140.3(\mathrm{CH}), 136.9\left(\mathrm{C}_{\mathrm{q}}\right), 136.5\left(\mathrm{C}_{\mathrm{q}}\right)$, $135.0\left(\mathrm{C}_{\mathrm{q}}\right), 133.7\left(\mathrm{C}_{\mathrm{q}}\right), 133.3(\mathrm{CH}), 130.6\left(\mathrm{C}_{\mathrm{q}}\right), 129.0(\mathrm{CH}), 128.1(\mathrm{CH}), 127.3(\mathrm{CH}), 123.5$ 
(CH), $120.1(\mathrm{CH}), 117.3(\mathrm{CH}), 115.7\left(\mathrm{CH}_{2}\right), 115.5(\mathrm{CH}), 108.0(\mathrm{CH}) . \mathbf{M S}(\mathrm{ESI}) \mathrm{m} / z$ (relative intensity): $380(40)\left({ }^{35} \mathrm{Cl}\right)[\mathrm{M}+\mathrm{Na}]^{+}, 358(100)\left({ }^{35} \mathrm{Cl}\right)[\mathrm{M}+\mathrm{H}]^{+}$. HR-MS (ESI) $\mathrm{m} / z$ calcd for $\mathrm{C}_{22} \mathrm{H}_{17} \mathrm{~N}_{3}{ }^{35} \mathrm{Cl}[\mathrm{M}+\mathrm{H}]^{+}$358.1106, found 358.1104.<smiles>C=C/C(=C/c1ccccc1)c1cc2cc(Br)ccc2n1[18OH]</smiles>

(Z)-5-Bromo-2-(1-phenylbuta-1,3-dien-2-yl)-1-(pyrimidin-2-yl)-1H-indole (184ha)

The general procedure $\mathbf{F}$ was followed using 5-bromo-1-(pyrimidin-2-yl)-1H-indole $\mathbf{7 1 h}$ (54.8 $\mathrm{mg}, 0.20 \mathrm{mmol})$ and (cyclopropylidenemethyl)benzene $138 \mathrm{a}(41.9 \mathrm{mg}, 0.32 \mathrm{mmol})$ for $3.5 \mathrm{~h}$. Isolation by column chromatography ( $n$-hexane/EtOAc 15:1) yielded $\mathbf{1 8 4 h a}(54.7$ $\mathrm{mg}, 68 \%, Z / E=6.9 / 1$ determined by ${ }^{1} \mathrm{H}$ NMR $)$ as a yellow oil. ${ }^{1} \mathrm{H}$ NMR $\left(400 \mathrm{MHz}, \mathrm{CDCl}_{3}\right)$ $\delta=8.72(\mathrm{~d}, J=4.8 \mathrm{~Hz}, 0.29 \mathrm{H}, E$ isomer $), 8.63(\mathrm{~d}, J=4.8 \mathrm{~Hz}, 2 \mathrm{H}, Z$ isomer $), 8.23(\mathrm{~d}, J=$ $8.9 \mathrm{~Hz}, 1 \mathrm{H}), 7.73(\mathrm{~d}, J=2.0 \mathrm{~Hz}, 1 \mathrm{H}), 7.40(\mathrm{dd}, J=8.9,2.0 \mathrm{~Hz}, 1 \mathrm{H}), 7.12-7.07(\mathrm{~m}, 3 \mathrm{H})$, $7.05(\mathrm{t}, J=4.8 \mathrm{~Hz}, 1 \mathrm{H}), 7.00-6.97(\mathrm{~m}, 2 \mathrm{H}), 6.71(\mathrm{dd}, J=17.3,10.5 \mathrm{~Hz}, 1 \mathrm{H}), 6.70(\mathrm{~s}, 1 \mathrm{H})$, $6.51(\mathrm{~s}, 1 \mathrm{H}), 5.08(\mathrm{~d}, J=10.5 \mathrm{~Hz}, 1 \mathrm{H}), 4.99(\mathrm{~d}, J=17.3 \mathrm{~Hz}, 1 \mathrm{H}) \cdot{ }^{13} \mathrm{C} \mathrm{NMR}\left(101 \mathrm{MHz}, \mathrm{CDCl}_{3}\right)$ $\delta=158.0,157.2\left(\mathrm{C}_{\mathrm{q}}\right), 140.3(\mathrm{CH}), 136.7\left(\mathrm{C}_{\mathrm{q}}\right), 136.5\left(\mathrm{C}_{\mathrm{q}}\right), 135.3\left(\mathrm{C}_{\mathrm{q}}\right), 133.6\left(\mathrm{C}_{\mathrm{q}}\right), 133.3$ $(\mathrm{CH}), 131.2\left(\mathrm{C}_{\mathrm{q}}\right), 129.0(\mathrm{CH}), 128.1(\mathrm{CH}), 127.4(\mathrm{CH}), 126.1(\mathrm{CH}), 123.2(\mathrm{CH}), 117.4(\mathrm{CH})$, $115.9(\mathrm{CH}), 115.7\left(\mathrm{CH}_{2}\right), 115.2\left(\mathrm{C}_{\mathrm{q}}\right), 107.8(\mathrm{CH}) . \mathbf{I R}(\mathrm{ATR}):$ 3049, 2964, 2927, 1692, 1561, $1414,1313,1264,1233,1171,1076,1035,989,912,808,736,696 \mathrm{~cm}^{-1}$. MS (ESI) m/z (relative intensity): $424(40)\left({ }^{79} \mathrm{Br}\right)[\mathrm{M}+\mathrm{Na}]^{+}, 402(100)\left({ }^{79} \mathrm{Br}\right)[\mathrm{M}+\mathrm{H}]^{+}$. HR-MS (ESI) m/z calcd for $\mathrm{C}_{22} \mathrm{H}_{17} \mathrm{~N}_{3}{ }^{79} \mathrm{Br}[\mathrm{M}+\mathrm{H}]^{+} 402.0600$, found 402.0594 . 
<smiles>C=C/C(=C/c1ccccc1)c1cc2cc(OC)ccc2n1[Po+]</smiles>

(Z)-5-Methoxy-2-(1-phenylbuta-1,3-dien-2-yl)-1-(pyrimidin-2-yl)-1H-indole (184ia)

The general procedure $\mathbf{F}$ was followed using 5-methoxyl-1-(pyrimidin-2-yl)-1H-indole 71i (45.1 mg, $0.20 \mathrm{mmol})$ and (cyclopropylidenemethyl)benzene 138a (41.9 mg, $0.32 \mathrm{mmol})$ for $2.8 \mathrm{~h}$. Isolation by column chromatography ( $n$-hexane/EtOAc 15:1) yielded 184ia (59.4 mg, $84 \%, Z / E=5.7 / 1$ determined by ${ }^{1} \mathrm{H}$ NMR $)$ as a yellow oil. ${ }^{1} \mathrm{H}$ NMR $\left(400 \mathrm{MHz}, \mathrm{CDCl}_{3}\right)$ $\delta=8.70(\mathrm{~d}, J=4.8 \mathrm{~Hz}, 0.36 \mathrm{H}, E$ isomer), $8.62(\mathrm{~d}, J=4.8 \mathrm{~Hz}, 2 \mathrm{H}, Z$ isomer), $8.32(\mathrm{~d}, J=$ $9.1 \mathrm{~Hz}, 1 \mathrm{H}), 7.12-7.03(\mathrm{~m}, 6 \mathrm{H}), 7.01-6.96(\mathrm{~m}, 2 \mathrm{H}), 6.73(\mathrm{~d}, J=17.4,10.5 \mathrm{~Hz}, 1 \mathrm{H}), 6.70$ (s, 1H), $6.51(\mathrm{~s}, 1 \mathrm{H}), 5.08(\mathrm{~d}, J=10.5 \mathrm{~Hz}, 1 \mathrm{H}), 5.03(\mathrm{~d}, J=17.4 \mathrm{~Hz}, 1 \mathrm{H}), 3.91(\mathrm{~s}, 3 \mathrm{H})$. ${ }^{13} \mathrm{C}$ NMR $\left(101 \mathrm{MHz}, \mathrm{CDCl}_{3}\right) \delta=157.8(\mathrm{CH}), 157.4\left(\mathrm{C}_{\mathrm{q}}\right), 155.5\left(\mathrm{C}_{\mathrm{q}}\right), 140.6(\mathrm{CH}), 136.8\left(\mathrm{C}_{\mathrm{q}}\right)$, $136.0\left(\mathrm{C}_{\mathrm{q}}\right), 134.3\left(\mathrm{C}_{\mathrm{q}}\right), 132.8(\mathrm{CH}), 131.6\left(\mathrm{C}_{\mathrm{q}}\right), 130.3\left(\mathrm{C}_{\mathrm{q}}\right), 129.1(\mathrm{CH}), 128.1(\mathrm{CH}), 127.2$ $(\mathrm{CH}), 116.8(\mathrm{CH}), 115.6\left(\mathrm{CH}_{2}\right), 115.4(\mathrm{CH}), 112.8(\mathrm{CH}), 108.6(\mathrm{CH}), 102.7(\mathrm{CH}), 55.8\left(\mathrm{CH}_{3}\right)$. IR (ATR): 3044, 2950, 2249, 1717, 1565, 1421, 1280, 1202, 1106, 908, 804, $728 \mathrm{~cm}^{-1}$. MS (ESI) m/z (relative intensity): $376(80)[\mathrm{M}+\mathrm{Na}]^{+}, 354(100)[\mathrm{M}+\mathrm{H}]^{+}$. HR-MS (ESI) m/z calcd for $\mathrm{C}_{23} \mathrm{H}_{20} \mathrm{~N}_{3} \mathrm{O}[\mathrm{M}+\mathrm{H}]^{+}$354.1601, found 354.1597.<smiles>C=C/C(=C/c1ccccc1)c1cc2cc(C#N)ccc2n1[Pb]</smiles>

(Z)-2-(1-Phenylbuta-1,3-dien-2-yl)-1-(pyrimidin-2-yl)-1H-indole-5-carbonitrile (184ja)

The general procedure $\mathbf{F}$ was followed using 1 -(pyrimidin-2-yl)-1 $H$-indole-5-carbonitrile $\mathbf{7 1} \mathbf{j}$ (44.0 mg, $0.20 \mathrm{mmol})$ and (cyclopropylidenemethyl)benzene 138a (41.9 mg, $0.32 \mathrm{mmol})$ for $5.0 \mathrm{~h}$. Isolation by column chromatography ( $n$-hexane/EtOAc $5: 1$ ) yielded 184ja (46.0 $\mathrm{mg}, 66 \%, \mathrm{Z} / \mathrm{E}=3.2 / 1$ determined by ${ }^{1} \mathrm{H}$ NMR $)$ as a yellow solid. ${ }^{1} \mathrm{H} \mathbf{N M R}\left(300 \mathrm{MHz}, \mathrm{CDCl}_{3}\right)$ $\delta=8.76(\mathrm{~d}, J=4.8 \mathrm{~Hz}, 0.63 \mathrm{H}, E$ isomer), $8.67(\mathrm{~d}, J=4.8 \mathrm{~Hz}, 2 \mathrm{H}, Z$ isomer), $8.34(\mathrm{~d}, J=$ 
8.7 Hz, 1H), $7.95(\mathrm{~s}, 1 \mathrm{H}), 7.55(\mathrm{dd}, J=8.7,1.7 \mathrm{~Hz}, 1 \mathrm{H}), 7.43(\mathrm{~s}, 1 \mathrm{H}), 7.13(\mathrm{t}, J=4.8 \mathrm{~Hz}$, $1 \mathrm{H}), 7.11-7.06(\mathrm{~m}, 2 \mathrm{H}), 6.96(\mathrm{dd}, J=7.6,2.2 \mathrm{~Hz}, 2 \mathrm{H}), 6.73(\mathrm{~s}, 1 \mathrm{H}), 6.69(\mathrm{dd}, J=16.9,10.0$ $\mathrm{Hz}, 1 \mathrm{H}), 6.63(\mathrm{~s}, 1 \mathrm{H}), 5.09(\mathrm{~d}, J=10.0 \mathrm{~Hz}, 1 \mathrm{H}), 4.97(\mathrm{~d}, J=16.9 \mathrm{~Hz}, 1 \mathrm{H}) .{ }^{13} \mathrm{C}$ NMR $(101$ $\left.\mathrm{MHz}, \mathrm{CDCl}_{3}\right) \delta=158.2(\mathrm{CH}), 156.8\left(\mathrm{C}_{\mathrm{q}}\right), 140.0(\mathrm{CH}), 138.2\left(\mathrm{C}_{\mathrm{q}}\right), 138.1\left(\mathrm{C}_{\mathrm{q}}\right), 136.3\left(\mathrm{C}_{\mathrm{q}}\right)$, $133.9(\mathrm{CH}), 132.9\left(\mathrm{C}_{\mathrm{q}}\right), 129.2\left(\mathrm{C}_{\mathrm{q}}\right), 129.0(\mathrm{CH}), 128.2(\mathrm{CH}), 127.6(\mathrm{CH}), 126.3(\mathrm{CH}), 125.9$ $(\mathrm{CH}), 120.4\left(\mathrm{C}_{\mathrm{q}}\right), 118.1(\mathrm{CH}), 115.9\left(\mathrm{CH}_{2}\right), 115.0(\mathrm{CH}), 108.3(\mathrm{CH}), 105.2\left(\mathrm{C}_{\mathrm{q}}\right)$. IR $(\mathrm{ATR})$ : $3050,2921,2221,1608,1565,1415,1316,1217,1030,986,886,809,735,698 \mathrm{~cm}^{-1}$. MS (ESI) m/z (relative intensity): $371(100)[\mathrm{M}+\mathrm{Na}]^{+}, 349(90)[\mathrm{M}+\mathrm{H}]^{+}$. HR-MS (ESI) $\mathrm{m} / \mathrm{z}$ calcd for $\mathrm{C}_{23} \mathrm{H}_{17} \mathrm{~N}_{4}[\mathrm{M}+\mathrm{H}]^{+} 349.1448$, found 339.1440 .

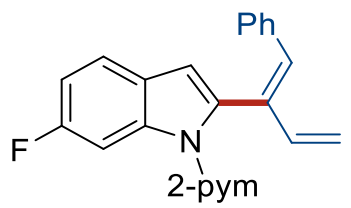

(Z)-6-Fluoro-2-(1-phenylbuta-1,3-dien-2-yl)-1-(pyrimidin-2-yl)-1 H-indole (184ka)

The general procedure $\mathbf{F}$ was followed using 6-fluoro-1-(pyrimidin-2-yl)-1H-indole $\mathbf{7 1} \mathbf{k}$ (42.6 mg, $0.20 \mathrm{mmol})$ and (cyclopropylidenemethyl)benzene138a (41.9 mg, $0.32 \mathrm{mmol}$ ) for $5.0 \mathrm{~h}$. Isolation by column chromatography ( $n$-hexane/EtOAc 15:1) yielded 184ka (42.3 $\mathrm{mg}, 62 \%, Z / E=2.7 / 1$ determined by ${ }^{1} \mathrm{H}$ NMR $)$ as a yellow oil. ${ }^{1} \mathrm{H}$ NMR $\left(300 \mathrm{MHz}, \mathrm{CDCl}_{3}\right)$ $\delta=8.73(\mathrm{~d}, J=4.8 \mathrm{~Hz}, 0.75 \mathrm{H}, E$ isomer), $8.64(\mathrm{~d}, J=4.8 \mathrm{~Hz}, 2 \mathrm{H}, Z$ isomer), 8.12 (dd, $J=$ 10.8, 2.4 Hz, 1H), $7.51(\mathrm{dd}, J=8.6,5.5 \mathrm{~Hz}, 1 \mathrm{H}), 7.44-7.37(\mathrm{~m}, 1 \mathrm{H}), 7.12-7.07(\mathrm{~m}, 3 \mathrm{H})$, 7.05-6.99 (m, 4H), $6.73(\mathrm{dd}, J=17.6,10.8 \mathrm{~Hz}, 1 \mathrm{H}), 6.69(\mathrm{~s}, 1 \mathrm{H}), 6.54(\mathrm{~s}, 1 \mathrm{H}), 5.08(\mathrm{~d}, J=$ $10.8 \mathrm{~Hz}, 1 \mathrm{H}), 5.02(\mathrm{~d}, J=17.6 \mathrm{~Hz}, 1 \mathrm{H}) .{ }^{13} \mathrm{C} \operatorname{NMR}\left(101 \mathrm{MHz}, \mathrm{CDCl}_{3}\right) \delta=160.6(\mathrm{~d}, J=238.2$ $\left.\mathrm{Hz}, \mathrm{C}_{\mathrm{q}}\right), 157.9(\mathrm{CH}), 157.3\left(\mathrm{C}_{\mathrm{q}}\right), 140.5(\mathrm{CH}), 137.2\left(\mathrm{C}_{\mathrm{q}}\right), 136.7\left(\mathrm{C}_{\mathrm{q}}\right), 135.8(\mathrm{~d}, \mathrm{~J}=4.2 \mathrm{~Hz}$, $\left.\mathrm{C}_{\mathrm{q}}\right), 133.9\left(\mathrm{C}_{\mathrm{q}}\right), 133.0(\mathrm{CH}), 129.1(\mathrm{CH}), 128.1(\mathrm{CH}), 127.2(\mathrm{CH}), 125.9\left(\mathrm{C}_{\mathrm{q}}\right), 121.3(\mathrm{~d}, \mathrm{~J}=$ 10.0 Hz, CH), $117.2(\mathrm{CH}), 115.7\left(\mathrm{CH}_{2}\right), 110.5(\mathrm{~d}, J=24.8 \mathrm{~Hz}, \mathrm{CH}), 108.5(\mathrm{CH}), 101.6(\mathrm{~d}$, $J=28.5 \mathrm{~Hz}, \mathrm{CH}) .{ }^{19} \mathrm{~F} \mathrm{NMR}\left(377 \mathrm{MHz}, \mathrm{CDCl}_{3}\right) \delta=-118.88--118.98$ (m). IR (ATR): 3050, 2925, 2854, 1724, 1566, 1418, 1423, 1261, 1149, 1077, 986, 852, 808, 738, $697 \mathrm{~cm}^{-1}$. 
MS (ESI) $m / z$ (relative intensity): $364(20)[\mathrm{M}+\mathrm{Na}]^{+}, 342(100)[\mathrm{M}+\mathrm{H}]^{+}$. HR-MS (ESI) $\mathrm{m} / \mathrm{z}$ calcd for $\mathrm{C}_{22} \mathrm{H}_{17} \mathrm{~N}_{3} \mathrm{~F}[\mathrm{M}+\mathrm{H}]^{+}$342.1401, found 342.1398.<smiles>C=C/C(=C/c1ccccc1)c1cc2ccc(Cl)cc2n1[18OH]</smiles>

(Z)-6-Chloro-2-(1-phenylbuta-1,3-dien-2-yl)-1-(pyrimidin-2-yl)-1H-indole (184la)

The general procedure $\mathbf{F}$ was followed using 6-chloro-1-(pyrimidin-2-yl)-1H-indole $71 \mathrm{l}$ (45.9 mg, $0.20 \mathrm{mmol}$ ) and (cyclopropylidenemethyl)benzene 138a (41.9 mg, $0.32 \mathrm{mmol}$ ) for $4.0 \mathrm{~h}$. Isolation by column chromatography ( $n$-hexane/EtOAc 15:1) yielded 184la (43.7 $\mathrm{mg}, 61 \%, Z / E=2.2 / 1$ determined by ${ }^{1} \mathrm{H}$ NMR $)$ as a yellow oil. ${ }^{1} \mathbf{H} \mathbf{N M R}\left(400 \mathrm{MHz}, \mathrm{CDCl}_{3}\right)$ $\delta=8.74(\mathrm{~d}, J=4.8 \mathrm{~Hz}, 0.91 \mathrm{H}, E$ isomer), $8.65(\mathrm{~d}, J=4.8 \mathrm{~Hz}, 2 \mathrm{H}, Z$ isomer $), 8.39(\mathrm{~s}, 1 \mathrm{H})$, $7.51(\mathrm{~d}, J=8.4 \mathrm{~Hz}, 1 \mathrm{H}), 7.40(\mathrm{t}, J=7.6 \mathrm{~Hz}, 1 \mathrm{H}), 7.23(\mathrm{dd}, J=8.3,1.9 \mathrm{~Hz}, 1 \mathrm{H}), 7.12-7.07$ (m, 3H), $7.06(\mathrm{~d}, J=4.8 \mathrm{~Hz}, 1 \mathrm{H}), 7.01-6.98(\mathrm{~m}, 1 \mathrm{H}), 6.69(\mathrm{~s}, 1 \mathrm{H}), 6.69(\mathrm{dd}, J=17.1,10.4$ $\mathrm{Hz}, 1 \mathrm{H}), 6.53(\mathrm{~s}, 1 \mathrm{H}), 5.07(\mathrm{~d}, J=10.4 \mathrm{~Hz}, 1 \mathrm{H}), 4.99(\mathrm{~d}, J=17.1 \mathrm{~Hz}, 1 \mathrm{H}) .{ }^{13} \mathrm{C}$ NMR $(101$ $\left.\mathrm{MHz}, \mathrm{CDCl}_{3}\right) \delta=158.0(\mathrm{CH}), 157.2\left(\mathrm{C}_{\mathrm{q}}\right), 140.4(\mathrm{CH}), 136.9\left(\mathrm{C}_{\mathrm{q}}\right), 136.6\left(\mathrm{C}_{\mathrm{q}}\right), 136.2\left(\mathrm{C}_{\mathrm{q}}\right)$, $133.7\left(\mathrm{C}_{\mathrm{q}}\right), 133.2(\mathrm{CH}), 129.3\left(\mathrm{C}_{\mathrm{q}}\right), 129.1(\mathrm{CH}), 128.1(\mathrm{CH}), 128.0\left(\mathrm{C}_{\mathrm{q}}\right), 127.3(\mathrm{CH}), 122.6$ (CH), $121.5(\mathrm{CH}), 117.4(\mathrm{CH}), 115.7\left(\mathrm{CH}_{2}\right), 114.5(\mathrm{CH}), 108.4(\mathrm{CH})$. IR (ATR): 3051, 1709, $1567,1426,1352,1315,1264,1218,908,811,748 \mathrm{~cm}^{-1}$. MS (ESI) $\mathrm{m} / z$ (relative intensity): $380(100) \quad\left({ }^{35} \mathrm{Cl}\right) \quad[\mathrm{M}+\mathrm{Na}]^{+}, \quad 358(80) \quad\left({ }^{35} \mathrm{Cl}\right) \quad[\mathrm{M}+\mathrm{H}]^{+}$. HR-MS (ESI) $\mathrm{m} / \mathrm{z}$ calcd for $\mathrm{C}_{22} \mathrm{H}_{16} \mathrm{~N}_{3}{ }^{35} \mathrm{CINa}[\mathrm{M}+\mathrm{Na}]^{+}$380.0925, found 380.0925 .<smiles>C=C/C(=C/c1ccccc1)c1cc2ccc(OC)cc2n1[Po+]</smiles>

(Z)-6-Methoxy-2-(1-phenylbuta-1,3-dien-2-yl)-1-(pyrimidin-2-yl)-1 H-indole (184ma) 
The general procedure $\mathbf{F}$ was followed using 6-methoxyl-1-(pyrimidin-2-yl)-1H-indole $71 \mathrm{~m}$ (45.1 mg, $0.20 \mathrm{mmol}$ ) and (cyclopropylidenemethyl)benzene 138a (41.9 mg, $0.32 \mathrm{mmol}$ ) for $3.0 \mathrm{~h}$. Isolation by column chromatography ( $n$-hexane/EtOAc 10:1) yielded 184ma (43.1 $\mathrm{mg}, 61 \%, Z / E=3.6 / 1$ determined by ${ }^{1} \mathrm{H}$ NMR $)$ as a yellow oil. ${ }^{1} \mathrm{H} \mathbf{N M R}\left(300 \mathrm{MHz}, \mathrm{CDCl}_{3}\right)$ $\delta=8.72(\mathrm{~d}, J=4.8 \mathrm{~Hz}, 0.56 \mathrm{H}, E$ isomer), $8.63(\mathrm{~d}, J=4.8 \mathrm{~Hz}, 2 \mathrm{H}, Z$ isomer $), 7.96(\mathrm{~d}, J=$ $2.3 \mathrm{~Hz}, 1 \mathrm{H}), 7.48(\mathrm{~d}, J=8.5 \mathrm{~Hz}, 1 \mathrm{H}), 7.12-7.06(\mathrm{~m}, 5 \mathrm{H}), 7.02(\mathrm{t}, J=4.8 \mathrm{~Hz}, 1 \mathrm{H}), 6.92(\mathrm{dd}$, $J=8.5,2.3 \mathrm{~Hz}, 1 \mathrm{H}), 6.71(\mathrm{~d}, J=10.5,17.3 \mathrm{~Hz}, 1 \mathrm{H}), 6.66(\mathrm{~s}, 1 \mathrm{H}), 6.49(\mathrm{~s}, 1 \mathrm{H}), 5.05(\mathrm{~d}, J=$ $10.5 \mathrm{~Hz}, 1 \mathrm{H}), 5.02(\mathrm{~d}, J=17.3 \mathrm{~Hz}, 1 \mathrm{H}), 3.92(\mathrm{~s}, 3 \mathrm{H}) .{ }^{13} \mathrm{C}$ NMR $\left(126 \mathrm{MHz}, \mathrm{CDCl}_{3}\right) \delta=157.9$ $(\mathrm{CH}), 157.6\left(\mathrm{C}_{\mathrm{q}}\right), 157.3\left(\mathrm{C}_{\mathrm{q}}\right), 140.8(\mathrm{CH}), 137.6\left(\mathrm{C}_{\mathrm{q}}\right), 136.9\left(\mathrm{C}_{\mathrm{q}}\right), 134.3\left(\mathrm{C}_{\mathrm{q}}\right), 134.2\left(\mathrm{C}_{\mathrm{q}}\right)$, $132.6(\mathrm{CH}), 129.2(\mathrm{CH}), 128.1(\mathrm{CH}), 127.1(\mathrm{CH}), 123.7\left(\mathrm{C}_{\mathrm{q}}\right), 121.2(\mathrm{CH}), 116.9(\mathrm{CH}), 115.6$ $\left(\mathrm{CH}_{2}\right), 111.3(\mathrm{CH}), 108.6(\mathrm{CH}), 98.7(\mathrm{CH}), 55.9\left(\mathrm{CH}_{3}\right)$. IR (ATR): 2924, 2855, 2245, 2200, 1948, 1612, 1565, 1484, 1418, 1265, 1159, 1030, 906, 808, $728 \mathrm{~cm}^{-1}$. MS (ESI) m/z (relative intensity): $376(80)[\mathrm{M}+\mathrm{Na}]^{+}, 354(100)[\mathrm{M}+\mathrm{H}]^{+}$. HR-MS (ESI) $\mathrm{m} / \mathrm{z}$ calcd for $\mathrm{C}_{23} \mathrm{H}_{20} \mathrm{~N}_{3} \mathrm{O}[\mathrm{M}+\mathrm{H}]^{+}$354.1606, found 354.1590.<smiles>C=C/C(=C/c1ccccc1)c1cc2ccc(C(C)=O)cc2n1[Po+]</smiles>

Methyl (Z)-2-(1-phenylbuta-1,3-dien-2-yl)-1-(pyrimidin-2-yl)-1H-indole-6-carboxy late (184na)

The general procedure $\mathbf{F}$ was followed using 1-(pyrimidin-2-yl)-1H-indole-6-carboxylate 71n (50.7 mg, $0.20 \mathrm{mmol}$ ) and (cyclopropylidenemethyl)benzene 138a (41.9 mg, 0.32 $\mathrm{mmol}$ ) for $3.0 \mathrm{~h}$. Isolation by column chromatography ( $n$-hexane/EtOAc $5: 1$ ) yielded 184na (59.5 mg, 78\%, Z/E = 3.8/1 determined by ${ }^{1} \mathrm{H}$ NMR) as a yellow oil. ${ }^{1} \mathbf{H}$ NMR (300 MHz, $\left.\mathrm{CDCl}_{3}\right) \delta=9.00(\mathrm{~s}, 1 \mathrm{H}), 8.77(\mathrm{~d}, J=4.8 \mathrm{~Hz}, 0.53 \mathrm{H}, E$ isomer), $8.68(\mathrm{~d}, J=4.8 \mathrm{~Hz}, 2 \mathrm{H}, Z$ isomer), 7.96 (d, $J=8.3 \mathrm{~Hz}, 1 \mathrm{H}), 7.63(\mathrm{~d}, J=8.3 \mathrm{~Hz}, 1 \mathrm{H}), 7.41$ (t, $J=7.6 \mathrm{~Hz}, 1 \mathrm{H}), 7.11-$ $7.06(\mathrm{~m}, 3 \mathrm{H}), 7.03-6.98(\mathrm{~m}, 2 \mathrm{H}), 6.72(\mathrm{~s}, 1 \mathrm{H}), 6.70(\mathrm{dd}, J=17.2,10.5 \mathrm{~Hz}, 1 \mathrm{H}), 6.61(\mathrm{~s}$, $1 \mathrm{H}), 5.08(\mathrm{~d}, J=10.5 \mathrm{~Hz}, 1 \mathrm{H}), 5.00(\mathrm{~d}, J=17.2 \mathrm{~Hz}, 1 \mathrm{H}), 3.97(\mathrm{~s}, 3 \mathrm{H}) .{ }^{13} \mathrm{C}$ NMR $(101 \mathrm{MHz}$, 
$\left.\mathrm{CDCl}_{3}\right) \delta=168.2\left(\mathrm{C}_{\mathrm{q}}\right), 158.1(\mathrm{CH}), 157.1\left(\mathrm{C}_{\mathrm{q}}\right), 140.1(\mathrm{CH}), 138.8\left(\mathrm{C}_{\mathrm{q}}\right), 136.4\left(\mathrm{C}_{\mathrm{q}}\right), 136.0$ $\left(\mathrm{C}_{\mathrm{q}}\right), 133.6(\mathrm{CH}), 133.4\left(\mathrm{C}_{\mathrm{q}}\right), 133.1\left(\mathrm{C}_{\mathrm{q}}\right), 129.0(\mathrm{CH}), 128.2(\mathrm{CH}), 127.4(\mathrm{CH}), 125.1\left(\mathrm{C}_{\mathrm{q}}\right)$, $123.1(\mathrm{CH}), 120.4(\mathrm{CH}), 117.6(\mathrm{CH}), 116.3(\mathrm{CH}), 115.8\left(\mathrm{CH}_{2}\right), 108.4(\mathrm{CH}), 52.1\left(\mathrm{CH}_{3}\right)$. IR (ATR): 3052, 2950, 2844, 1709, 1566, 1421, 1361, 1276, 1217, 1092, 990, 907, 804, 732, $696 \mathrm{~cm}^{-1}$. MS (ESI) m/z (relative intensity): $404(75)[\mathrm{M}+\mathrm{Na}]^{+}, 382(100)[\mathrm{M}+\mathrm{H}]^{+}$. HR-MS (ESI) $m / z$ calcd for $\mathrm{C}_{24} \mathrm{H}_{20} \mathrm{~N}_{3} \mathrm{O}_{2}[\mathrm{M}+\mathrm{H}]^{+} 382.1550$, found 382.1543.

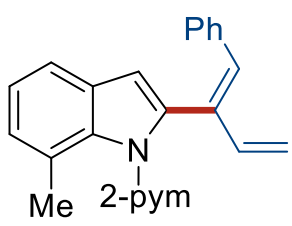

(Z)-7-Methyl-2-(1-phenylbuta-1,3-dien-2-yl)-1-(pyrimidin-2-yl)-1 H-indole (184oa)

The general procedure $\mathbf{F}$ was followed using 7-methyl-1-(pyrimidin-2-yl)-1H-indole 710 (41.6 mg, $0.20 \mathrm{mmol})$ and (cyclopropylidenemethyl)benzene 138a (41.9 mg, $0.32 \mathrm{mmol})$ for $3.0 \mathrm{~h}$. Isolation by column chromatography ( $n$-hexane/EtOAc 15:1) yielded 184na (41.2 $\mathrm{mg}, 61 \%, Z / E=3.6 / 1$ determined by ${ }^{1} \mathrm{H}$ NMR $)$ as a yellow oil. ${ }^{1} \mathrm{H}$ NMR $\left(400 \mathrm{MHz}, \mathrm{CDCl}_{3}\right)$ $\delta=8.78(\mathrm{~d}, J=4.8 \mathrm{~Hz}, 0.55 \mathrm{H}, E$ isomer), $8.64(\mathrm{~d}, J=4.8 \mathrm{~Hz}, 2 \mathrm{H}, Z$ isomer $), 7.55(\mathrm{~d}, J=$ $7.8 \mathrm{~Hz}, 1 \mathrm{H}), 7.39-7.35(\mathrm{~m}, 1 \mathrm{H}), 7.19(\mathrm{~d}, J=7.8 \mathrm{~Hz}, 1 \mathrm{H}), 7.15-7.11(\mathrm{~m}, 5 \mathrm{H}), 7.10(\mathrm{t}, J=$ $4.8 \mathrm{~Hz}, 1 \mathrm{H}), 6.69(\mathrm{~s}, 1 \mathrm{H}), 6.59(\mathrm{~d}, J=1.6 \mathrm{~Hz}, 1 \mathrm{H}), 6.58(\mathrm{dd}, J=17.0,10.6 \mathrm{~Hz}, 1 \mathrm{H}), 5.07$ $(\mathrm{d}, J=10.6 \mathrm{~Hz}, 1 \mathrm{H}), 5.00(\mathrm{dd}, J=17.0,1.5 \mathrm{~Hz}, 1 \mathrm{H}), 2.14(\mathrm{~s}, 3 \mathrm{H}) \cdot{ }^{13} \mathrm{C} \mathrm{NMR}\left(75 \mathrm{MHz}, \mathrm{CDCl}_{3}\right)$ $\delta=158.1\left(\mathrm{C}_{\mathrm{q}}\right), 157.6(\mathrm{CH}), 140.2(\mathrm{CH}), 136.4\left(\mathrm{C}_{\mathrm{q}}\right), 136.2\left(\mathrm{C}_{\mathrm{q}}\right), 136.2\left(\mathrm{C}_{\mathrm{q}}\right), 135.1(\mathrm{CH})$, $132.7\left(\mathrm{C}_{\mathrm{q}}\right), 129.9\left(\mathrm{C}_{\mathrm{q}}\right), 129.3(\mathrm{CH}), 128.1(\mathrm{CH}), 127.5(\mathrm{CH}), 125.9(\mathrm{CH}), 122.7\left(\mathrm{C}_{\mathrm{q}}\right), 121.5$ (CH), $118.8(\mathrm{CH}), 118.5(\mathrm{CH}), 116.5\left(\mathrm{CH}_{2}\right), 106.6(\mathrm{CH}), 20.7\left(\mathrm{CH}_{3}\right)$. IR (ATR): 3051, 1691, 1567, 1422, 1309, 1208, 1058, 913, 868, 802, 733, $697 \mathrm{~cm}^{-1}$. MS (ESI) $\mathrm{m} / \mathrm{z}$ (relative intensity): 360 (25) $[\mathrm{M}+\mathrm{Na}]^{+}, 338(80)[\mathrm{M}+\mathrm{H}]^{+}$. HR-MS (ESI) $\mathrm{m} / z$ calcd for $\mathrm{C}_{23} \mathrm{H}_{20} \mathrm{~N}_{3}[\mathrm{M}+\mathrm{H}]^{+}$ 338.1652, found 338.1647. 


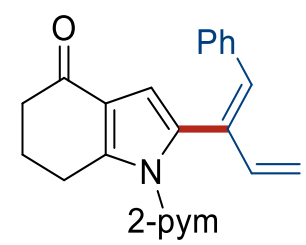

2-(1-Phenylbuta-1,3-dien-2-yl)-1-(pyrimidin-2-yl)-1,5,6,7-tetrahydro-4H-indol-4-one (184pa)

The general procedure $\mathbf{F}$ was followed using 1-(pyrimidin-2-yl)-1,5,6,7-tetrahydro-4Hindol-4-one $71 \mathrm{p}(42.4 \mathrm{mg}, 0.20 \mathrm{mmol}$ ) and (cyclopropylidenemethyl)benzene 138a (41.9 $\mathrm{mg}, 0.32 \mathrm{mmol}$ ) for $3.0 \mathrm{~h}$. Isolation by column chromatography ( -hexane/EtOAc $3: 1$ ) yielded (Z)-184pa (38.7 mg, 57\%) as a yellow solid and $(E)-184 \mathrm{pa}(19.2 \mathrm{mg}, 28 \%)$ as a yellow solid. (Z)-184pa: M.p. $=148-150{ }^{\circ} \mathrm{C} .{ }^{1} \mathrm{H}$ NMR $\left(400 \mathrm{MHz}, \mathrm{CDCl}_{3}\right) \delta=8.54(\mathrm{~d}, J=$ $4.8 \mathrm{~Hz}, 2 \mathrm{H}), 7.13-7.05(\mathrm{~m}, 4 \mathrm{H}), 6.88-6.82(\mathrm{~m}, 2 \mathrm{H}), 6.64(\mathrm{~s}, 1 \mathrm{H}), 6.60$ (dd, $J=17.2,10.5$ $\mathrm{Hz}, 1 \mathrm{H}), 6.49(\mathrm{~s}, 1 \mathrm{H}), 5.24(\mathrm{~d}, J=17.2 \mathrm{~Hz}, 1 \mathrm{H}), 5.14(\mathrm{~d}, J=10.5 \mathrm{~Hz}, 1 \mathrm{H}), 3.02(\mathrm{t}, J=6.0$ $\mathrm{Hz}, 2 \mathrm{H}), 2.60$ (t, $J=6.4 \mathrm{~Hz}, 2 \mathrm{H}), 2.18$ (tt, $J=6.0,6.4 \mathrm{~Hz}, 2 \mathrm{H}) .{ }^{13} \mathrm{C} \mathrm{NMR}\left(101 \mathrm{MHz}, \mathrm{CDCl}_{3}\right)$ $\delta=195.2\left(\mathrm{C}_{\mathrm{q}}\right), 157.9(\mathrm{CH}), 156.2\left(\mathrm{C}_{\mathrm{q}}\right), 145.6\left(\mathrm{C}_{\mathrm{q}}\right), 140.1(\mathrm{CH}), 136.4\left(\mathrm{C}_{\mathrm{q}}\right), 133.4(\mathrm{CH})$, $132.3\left(\mathrm{C}_{\mathrm{q}}\right), 130.1\left(\mathrm{C}_{\mathrm{q}}\right), 128.8(\mathrm{CH}), 128.1(\mathrm{CH}), 127.3(\mathrm{CH}), 122.3\left(\mathrm{C}_{\mathrm{q}}\right), 118.7(\mathrm{CH}), 116.1$ $\left(\mathrm{CH}_{2}\right), 109.4(\mathrm{CH}), 38.1\left(\mathrm{CH}_{2}\right), 24.3\left(\mathrm{CH}_{2}\right), 24.0\left(\mathrm{CH}_{2}\right)$. IR (ATR): 3051, 2945, 1657, 1562, 1413, 1261, 1179, 996, 903, 821, 730, $695 \mathrm{~cm}^{-1}$. MS (ESI) m/z (relative intensity): 1048 (10), 705 (15), 683(25), 364(20) [M+Na]+, $342(100)[\mathrm{M}+\mathrm{H}]^{+}$. HR-MS (ESI) $\mathrm{m} / z$ calcd for $\mathrm{C}_{22} \mathrm{H}_{20} \mathrm{~N}_{3} \mathrm{O}[\mathrm{M}+\mathrm{H}]^{+} 342.1601$, found 342.1601.

(E)-184pa: M.p. $=129-131^{\circ} \mathrm{C} .{ }^{1} \mathbf{H}$ NMR $\left(400 \mathrm{MHz}, \mathrm{CDCl}_{3}\right) \delta=8.73(\mathrm{~d}, \mathrm{~J}=4.8 \mathrm{~Hz}, 2 \mathrm{H})$, $7.35(\mathrm{~d}, J=4.4 \mathrm{~Hz}, 3 \mathrm{H}), 7.32-7.25(\mathrm{~m}, 2 \mathrm{H}), 7.22(\mathrm{t}, J=4.9 \mathrm{~Hz}, 1 \mathrm{H}), 6.79(\mathrm{~s}, 1 \mathrm{H}), 6.73(\mathrm{~s}$, 1H), $6.64(\mathrm{dd}, J=17.5,10.8 \mathrm{~Hz}, 1 \mathrm{H}), 5.05(\mathrm{~d}, J=17.5 \mathrm{~Hz}, 1 \mathrm{H}), 4.94(\mathrm{~d}, J=10.8 \mathrm{~Hz}, 1 \mathrm{H})$, $3.02(\mathrm{t}, J=6.1 \mathrm{~Hz}, 2 \mathrm{H}), 2.58(\mathrm{t}, J=6.4 \mathrm{~Hz}, 2 \mathrm{H}), 2.19$ (tt, $J=6.1,6.4 \mathrm{~Hz}, 2 \mathrm{H}) .{ }^{13} \mathrm{C}$ NMR $\left(101 \mathrm{MHz}, \mathrm{CDCl}_{3}\right) \delta=194.9\left(\mathrm{C}_{\mathrm{q}}\right), 158.5(\mathrm{CH}), 157.1\left(\mathrm{C}_{\mathrm{q}}\right), 145.8\left(\mathrm{C}_{\mathrm{q}}\right), 137.1\left(\mathrm{C}_{\mathrm{q}}\right), 135.8$ $\left(\mathrm{C}_{\mathrm{q}}\right), 134.2(\mathrm{CH}), 132.5\left(\mathrm{C}_{\mathrm{q}}\right), 131.4(\mathrm{CH}), 129.7(\mathrm{CH}), 128.3(\mathrm{CH}), 127.4(\mathrm{CH}), 121.9\left(\mathrm{C}_{\mathrm{q}}\right)$, $119.2(\mathrm{CH}), 118.0\left(\mathrm{C}_{q}\right), 109.3(\mathrm{CH}), 38.1\left(\mathrm{CH}_{2}\right), 24.1\left(\mathrm{CH}_{2}\right), 23.9\left(\mathrm{CH}_{2}\right)$. IR (ATR): 3051, 2926, 1727, 1658, 1564, 1418, 1265, 1182, 1000, 910, 733, $700 \mathrm{~cm}^{-1}$. MS (ESI) m/z 
(relative intensity): $364(70)[\mathrm{M}+\mathrm{Na}]^{+}, 342(100)[\mathrm{M}+\mathrm{H}]^{+}$. HR-MS (ESI) $\mathrm{m} / \mathrm{z}$ calcd for $\mathrm{C}_{22} \mathrm{H}_{20} \mathrm{~N}_{3} \mathrm{O}[\mathrm{M}+\mathrm{H}]^{+} 342.1601$, found 342.1606.

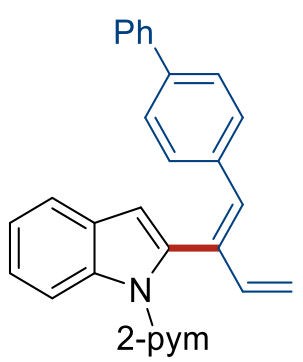

(Z)-2-\{[1-(1,1'-Biphenyl)-4-yl]buta-1,3-dien-2-yl\}-1-(pyrimidin-2-yl)-1H-indole (184ab)

The general procedure $\mathbf{F}$ was followed using 1-(pyrimidin-2-yl)-1H-indole $71 \mathrm{a}$ (39.0 mg, $0.20 \mathrm{mmol})$ and 4-(cyclopropylidenemethyl)-1,1'-biphenyl 138b $(66.0 \mathrm{mg}, 0.32 \mathrm{mmol})$ for 3.0 h. Isolation by column chromatography ( $n$-hexane/EtOAc 15:1) yielded 184ab (64.7 mg, $81 \%, Z / E=3.4 / 1$ determined by ${ }^{1} \mathrm{H}$ NMR) as a yellow solid. M.p. $=127-128{ }^{\circ} \mathrm{C}$. ${ }^{1} \mathrm{H}$ NMR $\left(400 \mathrm{MHz}, \mathrm{CDCl}_{3}\right) \delta=8.74(\mathrm{~d}, J=4.8 \mathrm{~Hz}, 0.59 \mathrm{H}, E$ isomer $), 8.65(\mathrm{~d}, J=4.8 \mathrm{~Hz}$, $2 \mathrm{H}, \mathrm{Z}$ isomer), $8.40(\mathrm{~d}, J=8.3 \mathrm{~Hz}, 1 \mathrm{H}), 7.64(\mathrm{~d}, J=5.7 \mathrm{~Hz}, 1 \mathrm{H}), 7.53(\mathrm{~d}, J=8.0 \mathrm{~Hz}, 2 \mathrm{H})$, 7.41-7.27 (m, 7H), $7.15(\mathrm{~d}, J=8.0 \mathrm{~Hz}, 2 \mathrm{H}), 7.04(\mathrm{t}, J=4.8 \mathrm{~Hz}, 1 \mathrm{H}), 6.75(\mathrm{~s}, 1 \mathrm{H}), 6.72(\mathrm{dd}$, $J=17.3,10.6 \mathrm{~Hz}, 1 \mathrm{H}), 6.61(\mathrm{~s}, 1 \mathrm{H}), 5.07(\mathrm{~d}, J=10.6 \mathrm{~Hz}, 1 \mathrm{H}), 5.00(\mathrm{~d}, J=17.3 \mathrm{~Hz}, 1 \mathrm{H})$. ${ }^{13} \mathrm{C}$ NMR $\left(126 \mathrm{MHz}, \mathrm{CDCl}_{3}\right) \delta=158.0(\mathrm{CH}), 157.6\left(\mathrm{C}_{\mathrm{q}}\right), 140.7(\mathrm{CH}), 140.6\left(\mathrm{C}_{\mathrm{q}}\right), 139.7\left(\mathrm{C}_{\mathrm{q}}\right)$, $136.7\left(\mathrm{C}_{\mathrm{q}}\right), 135.8\left(\mathrm{C}_{\mathrm{q}}\right), 135.5\left(\mathrm{C}_{\mathrm{q}}\right), 134.2\left(\mathrm{C}_{\mathrm{q}}\right), 132.7(\mathrm{CH}), 129.6(\mathrm{CH}), 129.6\left(\mathrm{C}_{\mathrm{q}}\right), 128.8$ $(\mathrm{CH}), 127.3(\mathrm{CH}), 126.9(\mathrm{CH}), 126.7(\mathrm{CH}), 123.4(\mathrm{CH}), 122.1(\mathrm{CH}), 120.8(\mathrm{CH}), 117.1(\mathrm{CH})$, $115.7\left(\mathrm{CH}_{2}\right), 114.4(\mathrm{CH}), 108.6(\mathrm{CH})$. IR (ATR): 3032, 2243, 1716, 1688, 1564, 1420, 1347, 1305, 1212, 904, 804, $727 \mathrm{~cm}^{-1}$. MS (ESI) m/z (relative intensity):, 422 (90) [M+Na] ${ }^{+}, 400$ (100) $[\mathrm{M}+\mathrm{H}]^{+}$. HR-MS (ESI) $\mathrm{m} / z$ calcd for $\mathrm{C}_{28} \mathrm{H}_{22} \mathrm{~N}_{3}[\mathrm{M}+\mathrm{H}]^{+} 400.1808$, found 400.1806 . 


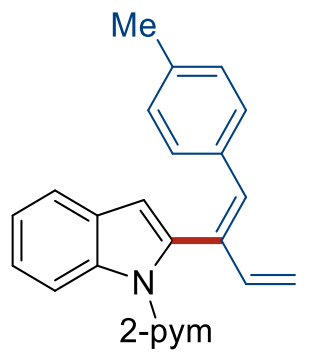

(Z)-1-(Pyrimidin-2-yl)-2-[1-(p-tolyl)buta-1,3-dien-2-yl]-1H-indole (184ac)

The general procedure $\mathbf{F}$ was followed using 1-(pyrimidin-2-yl)-1H-indole 71 a $(39.0 \mathrm{mg}$, $0.20 \mathrm{mmol}$ ) and 1-(cyclopropylidenemethyl)-4-methylbenzene 138c (46.1 mg, $0.32 \mathrm{mmol}$ ) for $3.0 \mathrm{~h}$. Isolation by column chromatography ( $n$-hexane/EtOAc 15:1) yielded 184ac (58.0 $\mathrm{mg}, 86 \%, Z / E=4.7 / 1$ determined by ${ }^{1} \mathrm{H}$ NMR $)$ as a yellow oil. ${ }^{1} \mathrm{H}$ NMR $\left(400 \mathrm{MHz}, \mathrm{CDCl}_{3}\right)$ $\delta=8.73(\mathrm{~d}, J=4.8 \mathrm{~Hz}, 0.43 \mathrm{H}, E$ isomer), $8.65(\mathrm{~d}, J=4.8 \mathrm{~Hz}, 2 \mathrm{H}, Z$ isomer), 8.39 (d, $J=$ $8.4 \mathrm{~Hz}, 1 \mathrm{H}), 7.63(\mathrm{~d}, J=7.8 \mathrm{~Hz}, 1 \mathrm{H}), 7.37-7.33(\mathrm{~m}, 1 \mathrm{H}), 7.31-7.23(\mathrm{~m}, 1 \mathrm{H}), 7.02(\mathrm{t}, J=$ $4.8 \mathrm{~Hz}, 1 \mathrm{H}$ ), $6.98(\mathrm{~d}, J=8.2 \mathrm{~Hz}, 2 \mathrm{H}), 6.92(\mathrm{~d}, J=8.2 \mathrm{~Hz}, 2 \mathrm{H}), 6.70(\mathrm{dd}, J=17.2,10.6 \mathrm{~Hz}$, 1H), $6.70(\mathrm{~s}, 1 \mathrm{H}), 6.59(\mathrm{~s}, 1 \mathrm{H}), 5.04(\mathrm{~d}, J=10.6 \mathrm{~Hz}, 1 \mathrm{H}), 4.98(\mathrm{~d}, J=17.2 \mathrm{~Hz}, 1 \mathrm{H}), 2.26(\mathrm{~s}$, 3H). ${ }^{13} \mathrm{C}$ NMR $\left(101 \mathrm{MHz}, \mathrm{CDCl}_{3}\right) \delta=157.9(\mathrm{CH}), 157.5\left(\mathrm{C}_{\mathrm{q}}\right), 140.8(\mathrm{CH}), 137.1\left(\mathrm{C}_{\mathrm{q}}\right), 136.6$ $\left(\mathrm{C}_{\mathrm{q}}\right), 135.7\left(\mathrm{C}_{\mathrm{q}}\right), 133.9\left(\mathrm{C}_{\mathrm{q}}\right), 133.2\left(\mathrm{C}_{\mathrm{q}}\right), 133.2(\mathrm{CH}), 129.6(\mathrm{CH}), 129.1\left(\mathrm{C}_{\mathrm{q}}\right), 128.9(\mathrm{CH})$, $123.3(\mathrm{CH}), 122.0(\mathrm{CH}), 120.8(\mathrm{CH}), 117.0(\mathrm{CH}), 115.1\left(\mathrm{CH}_{2}\right), 114.2(\mathrm{CH}), 108.4(\mathrm{CH})$, $21.3\left(\mathrm{CH}_{3}\right)$. IR (ATR): 3043, 2919, 1710, 1590, 1510, 1421, 1351, 1307, 1214, 1179, 985, 899, 804, $744 \mathrm{~cm}^{-1}$. MS (ESI) $\mathrm{m} / \mathrm{z}$ (relative intensity): $360(100)[\mathrm{M}+\mathrm{Na}]^{+}, 338(80)[\mathrm{M}+\mathrm{H}]^{+}$. HR-MS (ESI) $m / z$ calcd for $\mathrm{C}_{23} \mathrm{H}_{20} \mathrm{~N}_{3}[\mathrm{M}+\mathrm{H}]^{+} 338.1652$, found 338.1650 .

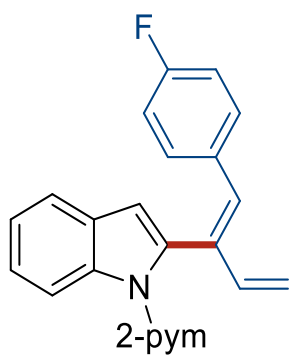

(Z)-2-[1-(4-fluorophenyl)buta-1,3-dien-2-yl]-1-(pyrimidin-2-yl)-1H-indole (184ad) 
The general procedure $\mathbf{F}$ was followed using 1-(pyrimidin-2-yl)-1H-indole 71a $(39.0 \mathrm{mg}$, $0.20 \mathrm{mmol}$ ) and 1-(cyclopropylidenemethyl)-4-fluorobenzene $138 \mathrm{c}(46.1 \mathrm{mg}, 0.32 \mathrm{mmol}$ ) for $3.5 \mathrm{~h}$. Isolation by column chromatography ( $n$-hexane/EtOAc 15:1) yielded 184ad (51.9 $\mathrm{mg}, 76 \%, Z / E=4.7 / 1$ determined by ${ }^{1} \mathrm{H}$ NMR $)$ as a yellow oil. ${ }^{1} \mathrm{H} \mathbf{N M R}\left(300 \mathrm{MHz}, \mathrm{CDCl}_{3}\right)$ $\delta=8.73(\mathrm{~d}, J=4.8 \mathrm{~Hz}, 0.39 \mathrm{H}, E$ isomer), $8.66(\mathrm{~d}, J=4.8 \mathrm{~Hz}, 2 \mathrm{H}, Z$ isomer $), 8.36(\mathrm{~d}, J=$ 8.2, $1 \mathrm{H}), 7.64(\mathrm{~d}, J=7.2,1 \mathrm{H}), 7.36(\mathrm{dd}, J=8.5,7.2 \mathrm{~Hz}, 1 \mathrm{H}), 7.28(\mathrm{dd}, J=8.5,6.8 \mathrm{~Hz}, 1 \mathrm{H})$, 7.06-6.99 (m, 3H), $6.79(\mathrm{t}, J=8.8 \mathrm{~Hz}, 2 \mathrm{H}), 6.69(\mathrm{dd}, J=17.0,10.6 \mathrm{~Hz}, 1 \mathrm{H}), 6.66(\mathrm{~s}, 1 \mathrm{H})$, $6.58(\mathrm{~s}, 1 \mathrm{H}), 5.08(\mathrm{~d}, J=10.6 \mathrm{~Hz}, 1 \mathrm{H}), 5.03(\mathrm{~s}, \mathrm{~d}, J=17.0 \mathrm{~Hz}, 1 \mathrm{H}) .{ }^{13} \mathrm{C}$ NMR $(101 \mathrm{MHz}$, $\left.\mathrm{CDCl}_{3}\right) \delta=161.9\left(\mathrm{~d},{ }^{1} \mathrm{~J}_{\mathrm{C}-\mathrm{F}}=247.7 \mathrm{~Hz}, \mathrm{C}_{\mathrm{q}}\right), 157.9(\mathrm{CH}), 157.4\left(\mathrm{C}_{\mathrm{q}}\right), 140.3(\mathrm{CH}), 136.7\left(\mathrm{C}_{\mathrm{q}}\right)$, $135.2\left(\mathrm{C}_{\mathrm{q}}\right), 133.85\left(\mathrm{~d},{ }^{4} \mathrm{~J}_{\mathrm{C}-\mathrm{F}}=2.0 \mathrm{~Hz}, \mathrm{C}_{\mathrm{q}}\right), 132.9\left(\mathrm{~d},{ }^{4} \mathrm{~J}_{\mathrm{C}-\mathrm{F}}=3.5 \mathrm{~Hz}, \mathrm{C}_{\mathrm{q}}\right), 131.7(\mathrm{CH}), 130.7$ $\left(\mathrm{d},{ }^{3} \mathrm{~J}_{\mathrm{C}-\mathrm{F}}=7.9 \mathrm{~Hz}, \mathrm{CH}\right), 129.4\left(\mathrm{C}_{\mathrm{q}}\right), 123.5(\mathrm{CH}), 122.1(\mathrm{CH}), 120.8(\mathrm{CH}), 117.1(\mathrm{CH}), 115.8$ $\left(\mathrm{CH}_{2}\right), 115.1\left(\mathrm{~d},{ }^{2} \mathrm{~J}_{\mathrm{C}-\mathrm{F}}=21.3 \mathrm{~Hz}, \mathrm{CH}\right), 114.2(\mathrm{CH}), 108.7(\mathrm{CH}) .{ }^{19} \mathrm{~F} \mathrm{NMR}\left(282 \mathrm{MHz}, \mathrm{CDCl}_{3}\right)$ $\delta=(-114.1)-(-114.2)(m)$. IR (ATR): 3044, 2245, 1717, 1690, 1565, 1503, 1420, 1347, 1307, 1220, 1153, 985,906, 805, $729 \mathrm{~cm}^{-1}$. MS (ESI) $\mathrm{m} / \mathrm{z}$ (relative intensity): 364 (70) $[\mathrm{M}+\mathrm{Na}]^{+}, 342(100)[\mathrm{M}+\mathrm{H}]^{+}$. HR-MS (ESI) $\mathrm{m} / z$ calcd for $\mathrm{C}_{22} \mathrm{H}_{17} \mathrm{~N}_{3} \mathrm{~F}[\mathrm{M}+\mathrm{H}]^{+} 342.1401$, found 342.1397.

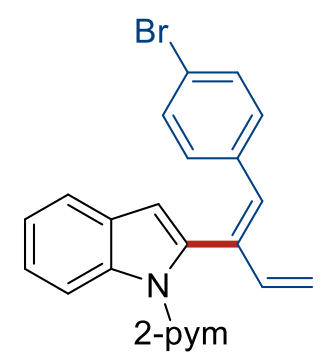

(Z)-2-[1-(4-Bromophenyl)buta-1,3-dien-2-yl]-1-(pyrimidin-2-yl)-1H-indole (184ae)

The general procedure $\mathbf{F}$ was followed using 1-(pyrimidin-2-yl)-1H-indole 71a $(39.0 \mathrm{mg}$, $0.20 \mathrm{mmol}$ ) and 1-(cyclopropylidenemethyl)-4-bromobenzene $138 \mathrm{e}(66.9 \mathrm{mg}, 0.32 \mathrm{mmol})$ for $3.0 \mathrm{~h}$. Isolation by column chromatography ( $n$-hexane/EtOAc 15:1) yielded 184ae (48.3 $\mathrm{mg}, 60 \%, Z / E=1.8 / 1$ determined by ${ }^{1} \mathrm{H}$ NMR) as a yellow solid. M.p. $=100-102{ }^{\circ} \mathrm{C}$. ${ }^{1} \mathbf{H}$ NMR (300 MHz, CDCl $) \delta=8.72(\mathrm{~d}, J=4.8 \mathrm{~Hz}, 1.09 \mathrm{H}, E$ isomer), 8.66 (d, $J=4.8 \mathrm{~Hz}$, 
2H, $Z$ isomer), $8.36(\mathrm{~d}, J=8.2 \mathrm{~Hz}, 1 \mathrm{H}), 7.62(\mathrm{~d}, J=7.7 \mathrm{~Hz}, 1 \mathrm{H}), 7.35-7.28(\mathrm{~m}, 2 \mathrm{H}), 7.21$ (d, $J=8.5 \mathrm{~Hz}, 2 \mathrm{H}), 7.05(\mathrm{t}, J=4.8 \mathrm{~Hz}, 1 \mathrm{H}), 6.93(\mathrm{~d}, J=8.5 \mathrm{~Hz}, 2 \mathrm{H}), 6.66(\mathrm{~d}, J=17.2,10.6$ $\mathrm{Hz}, 1 \mathrm{H}), 6.62(\mathrm{~s}, 1 \mathrm{H}), 6.54(\mathrm{~s}, 1 \mathrm{H}), 5.08(\mathrm{~d}, J=10.6 \mathrm{~Hz}, 1 \mathrm{H}), 5.01(\mathrm{~d}, J=17.2 \mathrm{~Hz}, 1 \mathrm{H})$. ${ }^{13} \mathrm{C}$ NMR $\left(126 \mathrm{MHz}, \mathrm{CDCl}_{3}\right) \delta=156.0(\mathrm{CH}), 157.5\left(\mathrm{C}_{\mathrm{q}}\right), 140.3(\mathrm{CH}), 140.2\left(\mathrm{C}_{\mathrm{q}}\right), 136.7\left(\mathrm{C}_{\mathrm{q}}\right)$, $135.7\left(\mathrm{C}_{\mathrm{q}}\right), 134.9\left(\mathrm{C}_{\mathrm{q}}\right), 131.6(\mathrm{CH}), 131.2(\mathrm{CH}), 130.6(\mathrm{CH}), 129.4\left(\mathrm{C}_{\mathrm{q}}\right), 123.6(\mathrm{CH}), 122.1$ $(\mathrm{CH}), 121.1(\mathrm{CH}), 120.9\left(\mathrm{C}_{\mathrm{q}}\right), 117.2(\mathrm{CH}), 116.3\left(\mathrm{CH}_{2}\right), 114.3(\mathrm{CH}), 108.7(\mathrm{CH}) . \mathbf{I R}(\mathrm{ATR})$ : $3044,1711,1561,1487,1451,1421,1346,1302,1070,1008,906,802,727 \mathrm{~cm}^{-1}$. MS (ESI) m/z (relative intensity): $364(75)\left({ }^{79} \mathrm{Br}\right)[\mathrm{M}+\mathrm{Na}]^{+}, 342(75)\left({ }^{79} \mathrm{Br}\right)[\mathrm{M}+\mathrm{H}]^{+}$. HR-MS (ESI) $m / z$ calcd for $\mathrm{C}_{22} \mathrm{H}_{14} \mathrm{~N}_{3}{ }^{79} \mathrm{Br}[\mathrm{M}+\mathrm{H}]^{+} 402.0606$, found 402.0604 .

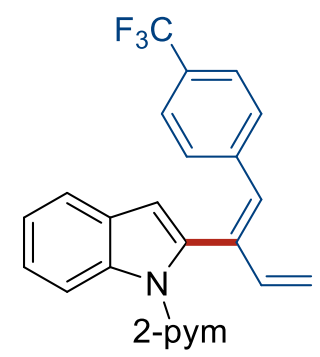

(Z)-1-(Pyrimidin-2-yl)-2-\{1-[4-(trifluoromethyl)phenyl]buta-1,3-dien-2-yl\}-1H-indole (184af)

The general procedure $\mathbf{F}$ was followed using 1-(pyrimidin-2-yl)-1 $H$-indole $71 \mathbf{a}$ (39.0 mg, $0.20 \mathrm{mmol}$ ) and 1-(cyclopropylidenemethyl)-4-trifluoromethylbenzene $138 \mathrm{f}(63.1 \mathrm{mg}, 0.32$ $\mathrm{mmol}$ ) for $3.0 \mathrm{~h}$. Isolation by column chromatography ( $n$-hexane/EtOAc 15:1) yielded 184af (40.7 mg, 52\%, Z/E = 3.6/1 determined by ${ }^{1} \mathrm{H}$ NMR) as a yellow oil. ${ }^{1} \mathbf{H}$ NMR $(300 \mathrm{MHz}$, $\left.\mathrm{CDCl}_{3}\right) \delta=8.74$ (d, $J=4.8 \mathrm{~Hz}, 0.56 \mathrm{H}, E$ isomer), 8.66 (d, $J=4.8 \mathrm{~Hz}, 2 \mathrm{H}, Z$ isomer), 8.42 $(\mathrm{d}, J=8.2 \mathrm{~Hz}, 1 \mathrm{H}), 7.64(\mathrm{~d}, J=8.3 \mathrm{~Hz}, 1 \mathrm{H}), 7.37(\mathrm{~d}, J=8.2 \mathrm{~Hz}, 2 \mathrm{H}), 7.35-7.27(\mathrm{~m}, 2 \mathrm{H})$, $7.20(\mathrm{~d}, J=8.2 \mathrm{~Hz}, 2 \mathrm{H}), 7.05(\mathrm{t}, J=4.8 \mathrm{~Hz}, 1 \mathrm{H}), 6.73(\mathrm{~s}, 1 \mathrm{H}), 6.72(\mathrm{dd}, J=17.3,10.7 \mathrm{~Hz}$, 1H) $, 6.58(\mathrm{~s}, 1 \mathrm{H}), 5.14(\mathrm{~d}, J=10.7 \mathrm{~Hz}, 1 \mathrm{H}), 5.07(\mathrm{~d}, J=17.3 \mathrm{~Hz}, 1 \mathrm{H}) .{ }^{13} \mathrm{C}$ NMR $(101 \mathrm{MHz}$, $\left.\mathrm{CDCl}_{3}\right) \delta=158.0(\mathrm{CH}), 157.5\left(\mathrm{C}_{\mathrm{q}}\right), 140.4\left(\mathrm{q}, J=1.4 \mathrm{~Hz}, \mathrm{C}_{\mathrm{q}}\right), 140.1(\mathrm{CH}), 136.7\left(\mathrm{C}_{\mathrm{q}}\right), 136.4$ $\left(\mathrm{C}_{\mathrm{q}}\right), 134.8\left(\mathrm{C}_{\mathrm{q}}\right), 131.1(\mathrm{CH}), 129.4\left(\mathrm{C}_{\mathrm{q}}\right), 129.2(\mathrm{CH}), 128.7\left(\mathrm{q}, J=32.3 \mathrm{~Hz}, \mathrm{C}_{\mathrm{q}}\right), 125.0(\mathrm{q}$, $J=3.9 \mathrm{~Hz}, \mathrm{CH}), 124.3\left(\mathrm{q}, J=271.9 \mathrm{~Hz}, \mathrm{C}_{\mathrm{q}}\right), 123.7(\mathrm{CH}), 122.2(\mathrm{CH}), 120.9(\mathrm{CH}), 117.2$ 
$(\mathrm{CH}), 117.1\left(\mathrm{CH}_{2}\right), 114.4(\mathrm{CH}), 109.0(\mathrm{CH}) .{ }^{19} \mathrm{~F} \mathrm{NMR}\left(377 \mathrm{MHz}, \mathrm{CDCl}_{3}\right) \delta=-62.48 . \mathbf{I R}$ (ATR): $3048,1573,1562,1453,1423,1352,1322,1124,1067,827,742 \mathrm{~cm}^{-1}$. MS (ESI) m/z (relative intensity): $414(100)[\mathrm{M}+\mathrm{Na}]^{+}, 392(90)[\mathrm{M}+\mathrm{H}]^{+}$. HR-MS (ESI) $\mathrm{m} / z$ calcd for $\mathrm{C}_{23} \mathrm{H}_{17} \mathrm{~N}_{3} \mathrm{~F}_{3}[\mathrm{M}+\mathrm{H}]^{+}$392.1368, found 392.1362.

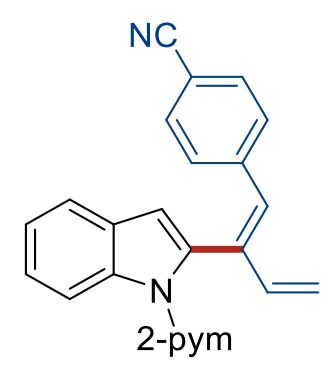

\section{(Z)-4-\{2-[1-(Pyrimidin-2-yl)-1 H-indol-2-yl]buta-1,3-dien-1-yl\}benzonitrile (184ag)}

The general procedure $\mathbf{F}$ was followed using 1-(pyrimidin-2-yl)-1 $H$-indole $71 \mathrm{a}$ (39.0 mg, $0.20 \mathrm{mmol}$ ) and 4-(cyclopropylidenemethyl)benzonitrile $138 \mathrm{~g}$ (49.7 mg, $0.32 \mathrm{mmol}$ ) for 3.0 h. Isolation by column chromatography ( $n$-hexane/EtOAc 15:1) yielded 184ag (62.7 mg, $90 \%, Z / E=1.4 / 1$ determined by ${ }^{1} \mathrm{H}$ NMR) as a yellow solid. M.p. $=140-141^{\circ} \mathrm{C} .{ }^{1} \mathbf{H}$ NMR $\left(400 \mathrm{MHz}, \mathrm{CDCl}_{3}\right) \delta=8.72(\mathrm{~d}, J=4.8 \mathrm{~Hz}, 1.46 \mathrm{H}, E$ isomer $), 8.65(\mathrm{~d}, J=4.8 \mathrm{~Hz}, 2 \mathrm{H}, Z$ isomer), $8.37(\mathrm{~d}, J=8.4 \mathrm{~Hz}, 1 \mathrm{H}), 7.61(\mathrm{~d}, J=7.6 \mathrm{~Hz}, 1 \mathrm{H}), 7.36(\mathrm{~d}, J=8.4 \mathrm{~Hz}, 2 \mathrm{H}), 7.28(\mathrm{t}$, $J=7.4 \mathrm{~Hz}, 2 \mathrm{H}), 7.13(\mathrm{~d}, J=8.4 \mathrm{~Hz}, 2 \mathrm{H}), 7.06(\mathrm{t}, J=4.8 \mathrm{~Hz}, 1 \mathrm{H}), 6.68(\mathrm{dd}, J=17.3,11.3$ Hz, 1H), $6.67(\mathrm{~s}, 1 \mathrm{H}), 6.54(\mathrm{~s}, 1 \mathrm{H}), 5.15(\mathrm{~d}, J=11.3 \mathrm{~Hz}, 1 \mathrm{H}), 5.08(\mathrm{~d}, J=17.3 \mathrm{~Hz}, 1 \mathrm{H})$. ${ }^{13} \mathrm{C}$ NMR $\left(101 \mathrm{MHz}, \mathrm{CDCl}_{3}\right) \delta=158.0(\mathrm{CH}), 157.4\left(\mathrm{C}_{\mathrm{q}}\right), 141.6\left(\mathrm{C}_{\mathrm{q}}\right), 139.8(\mathrm{CH}), 137.4\left(\mathrm{C}_{\mathrm{q}}\right)$, $136.7\left(\mathrm{C}_{\mathrm{q}}\right), 134.4\left(\mathrm{C}_{\mathrm{q}}\right), 131.8(\mathrm{CH}), 130.2(\mathrm{CH}), 129.5(\mathrm{CH}), 129.3\left(\mathrm{C}_{\mathrm{q}}\right), 123.9(\mathrm{CH}), 122.3$ $(\mathrm{CH}), 120.9(\mathrm{CH}), 119.2\left(\mathrm{C}_{\mathrm{q}}\right), 117.9\left(\mathrm{CH}_{2}\right), 117.2(\mathrm{CH}), 114.4(\mathrm{CH}), 110.1(\mathrm{CH}), 109.2\left(\mathrm{C}_{\mathrm{q}}\right)$. IR (ATR): 2221, 2135, 2023, 1701, 1563, 1420, 1346, 1307, 905, 800, $735 \mathrm{~cm}^{-1}$. MS (ESI)

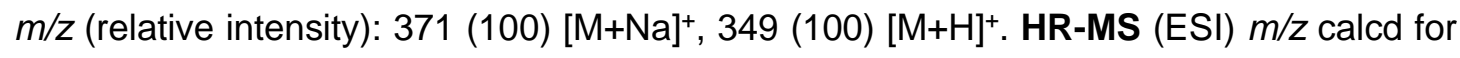
$\mathrm{C}_{23} \mathrm{H}_{17} \mathrm{~N}_{4}[\mathrm{M}+\mathrm{H}]^{+}$349.1448, found 349.1446. 


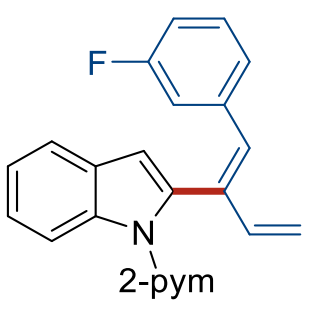

(Z)-2-[1-(3-Fluorophenyl)buta-1,3-dien-2-yl]-1-(pyrimidin-2-yl)-1 H-indole (184ah)

The general procedure $\mathbf{F}$ was followed using 1-(pyrimidin-2-yl)-1H-indole $71 \mathbf{a}$ (39.0 mg, $0.20 \mathrm{mmol}$ ) and 1-(cyclopropylidenemethyl)-3-fluorobenzene $138 \mathrm{~h}(47.4 \mathrm{mg}, 0.32 \mathrm{mmol})$ for $3.5 \mathrm{~h}$. Isolation by column chromatography ( $n$-hexane/EtOAc 15:1) yielded 184ah $(53.9$ mg, $79 \%, Z / E=4.4 / 1$ determined by ${ }^{1} \mathrm{H}$ NMR $)$ as a yellow oil. ${ }^{1} \mathrm{H}$ NMR $\left(300 \mathrm{MHz}, \mathrm{CDCl}_{3}\right)$ $\delta=8.73(\mathrm{~d}, J=4.8 \mathrm{~Hz}, 0.45 \mathrm{H}, E$ isomer), $8.66(\mathrm{~d}, J=4.8 \mathrm{~Hz}, 2 \mathrm{H}, Z$ isomer), 8.37 (dd, $J=$ 8.3, 1.0 Hz, 1H), $7.63(\mathrm{~d}, J=7.4 \mathrm{~Hz}, 1 \mathrm{H}), 7.36(\mathrm{t}, J=8.4 \mathrm{~Hz}, 1 \mathrm{H}), 7.33-7.21(\mathrm{~m}, 1 \mathrm{H}), 7.05$ $(\mathrm{d}, J=5.0 \mathrm{~Hz}, 1 \mathrm{H}), 7.02(\mathrm{t}, J=4.2 \mathrm{~Hz}, 1 \mathrm{H}), 6.84(\mathrm{~s}, 1 \mathrm{H}), 6.83-6.75(\mathrm{~m}, 2 \mathrm{H}), 6.69(\mathrm{dd}, J=$ 17.5, 11.2 Hz, 1H), $6.66(\mathrm{~s}, 1 \mathrm{H}), 6.59(\mathrm{~s}, 1 \mathrm{H}), 5.11(\mathrm{~d}, J=11.2 \mathrm{~Hz}, 1 \mathrm{H}), 5.06$ (d, J = 17.5 $\mathrm{Hz}, 1 \mathrm{H}) .{ }^{13} \mathrm{C}$ NMR $\left(101 \mathrm{MHz}, \mathrm{CDCl}_{3}\right) \delta=162.5\left(\mathrm{~d},{ }^{1} \mathrm{~J}_{\mathrm{C}-\mathrm{F}}=244.5 \mathrm{~Hz}, \mathrm{C}_{\mathrm{q}}\right), 157.9(\mathrm{CH}), 157.4$ $\left(\mathrm{C}_{\mathrm{q}}\right), 140.2(\mathrm{CH}), 139.1\left(\mathrm{~d},{ }^{3} \mathrm{~J}_{\mathrm{C}-\mathrm{F}}=8.0 \mathrm{~Hz}, \mathrm{C}_{\mathrm{q}}\right), 136.7\left(\mathrm{C}_{\mathrm{q}}\right), 135.4\left(\mathrm{C}_{\mathrm{q}}\right), 134.8\left(\mathrm{C}_{\mathrm{q}}\right), 131.4$ $\left(\mathrm{d},{ }^{4} \mathrm{~J}_{\mathrm{C}-\mathrm{F}}=2.6 \mathrm{~Hz}, \mathrm{CH}\right), 129.4,129.1\left(\mathrm{C}_{\mathrm{q}}\right), 124.8\left(\mathrm{~d},{ }^{4} \mathrm{~J}_{\mathrm{C}-\mathrm{F}}=2.8 \mathrm{~Hz}, \mathrm{CH}\right), 123.6(\mathrm{CH}), 122.1$ $(\mathrm{CH}), 120.8(\mathrm{CH}), 117.1(\mathrm{CH}), 116.6\left(\mathrm{CH}_{2}\right), 115.7\left(\mathrm{~d},{ }^{2} \mathrm{~J}_{\mathrm{C}-\mathrm{F}}=22.0 \mathrm{~Hz}\right), 114.3(\mathrm{CH}), 114.0$ $\left(\mathrm{d},{ }^{2} \mathrm{~J}_{\mathrm{C}-\mathrm{F}}=21.4 \mathrm{~Hz}, \mathrm{CH}\right), 109.0(\mathrm{CH}) .{ }^{19} \mathrm{~F} \mathrm{NMR}\left(282 \mathrm{MHz}, \mathrm{CDCl}_{3}\right) \delta=-113.79(\mathrm{td}, J=9.5$, 6.4 Hz). IR (ATR): 3045, 1998, 1718, 1566, 1420, 1347, 1307, 1244, 1143, 905, 803, 729 $\mathrm{cm}^{-1}$. MS (ESI) $\mathrm{m} / \mathrm{z}$ (relative intensity): 364( [M+Na] $]^{+}, 342(100)[\mathrm{M}+\mathrm{H}]^{+}$. HR-MS (ESI) $\mathrm{m} / \mathrm{z}$ calcd for $\mathrm{C}_{22} \mathrm{H}_{17} \mathrm{~N}_{3} \mathrm{~F}[\mathrm{M}+\mathrm{H}]^{+} 342.1712$, found 342.1400 . 


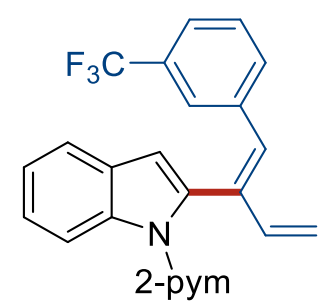

(Z)-1-(Pyrimidin-2-yl)-2-\{1-[3-(trifluoromethyl)phenyl]buta-1,3-dien-2-yl\}-1 H-indole (184ai)

The general procedure $\mathbf{F}$ was followed using 1-(pyrimidin-2-yl)-1H-indole $71 \mathrm{a}(39.0 \mathrm{mg}$, $0.20 \mathrm{mmol}$ ) and 1-(cyclopropylidenemethyl)-3-trifluoromethylbenzene $138 \mathrm{i}$ (63.1 mg, 0.32 mmol) for $3.5 \mathrm{~h}$. Isolation by column chromatography ( -hexane/EtOAc 15:1) yielded 184ai (64.2 $\mathrm{mg}, 82 \%, \mathrm{Z} / \mathrm{E}=5.7 / 1$ determined by ${ }^{1} \mathrm{H}$ NMR $)$ as a yellow oil. ${ }^{1} \mathrm{H}$ NMR $(300 \mathrm{MHz}$, $\left.\mathrm{CDCl}_{3}\right) \delta=8.74(\mathrm{~d}, J=4.8 \mathrm{~Hz}, 0.35 \mathrm{H}, E$ isomer), $8.63(\mathrm{~d}, J=4.8 \mathrm{~Hz}, 2 \mathrm{H}, \mathrm{Z}$ isomer), 8.32 (d, J = 8.2 Hz, 1H), $7.64(\mathrm{~d}, J=7.8 \mathrm{~Hz}, 1 \mathrm{H}), 7.37-7.32(\mathrm{~m}, 2 \mathrm{H}), 7.29$ (dd, J = 7.5, 1.2 Hz, 1H), 7.25-7.23 (m, 1H), 7.15-7.12 (m, 2H), $7.04(\mathrm{t}, J=4.8 \mathrm{~Hz}, 1 \mathrm{H}), 6.73(\mathrm{~d}, J=16.8,11.0$ $\mathrm{Hz}, 1 \mathrm{H}), 6.69(\mathrm{~s}, 1 \mathrm{H}), 6.61(\mathrm{~s}, 1 \mathrm{H}), 5.18(\mathrm{~d}, J=11.0 \mathrm{~Hz}, 1 \mathrm{H}), 5.17(\mathrm{~d}, J=16.8 \mathrm{~Hz}, 1 \mathrm{H})$. ${ }^{13} \mathrm{C}$ NMR $\left(101 \mathrm{MHz}, \mathrm{CDCl}_{3}\right) \delta=157.9(\mathrm{CH}), 157.3\left(\mathrm{C}_{\mathrm{q}}\right), 139.9(\mathrm{CH}), 137.6\left(\mathrm{C}_{\mathrm{q}}\right), 136.7\left(\mathrm{C}_{\mathrm{q}}\right)$, $135.9\left(\mathrm{C}_{\mathrm{q}}\right), 134.5\left(\mathrm{C}_{\mathrm{q}}\right), 131.7(\mathrm{CH}), 130.9(\mathrm{CH}), 130.3\left(\mathrm{q},{ }^{2} \mathrm{~J}_{\mathrm{C}-\mathrm{F}}=32.1 \mathrm{~Hz}, \mathrm{C}_{\mathrm{q}}\right), 128.5(\mathrm{CH})$, $125.9(\mathrm{CH}), 125.9\left(\mathrm{q},{ }^{3} \mathrm{~J}_{\mathrm{C}-\mathrm{F}}=3.8 \mathrm{~Hz}, \mathrm{CH}\right), 124.1\left(\mathrm{q},{ }^{1} \mathrm{~J}_{\mathrm{C}-\mathrm{F}}=273.7 \mathrm{~Hz}, \mathrm{CH}\right), 123.7(\mathrm{CH})$, $123.6\left(\mathrm{q},{ }^{3} \mathrm{~J}_{\mathrm{C}-\mathrm{F}}=3.8 \mathrm{~Hz}, \mathrm{CH}\right), 122.2(\mathrm{CH}), 120.8(\mathrm{CH}), 117.1(\mathrm{CH}), 117.1\left(\mathrm{CH}_{2}\right), 114.2(\mathrm{CH})$, 109.1 (CH). $\left.{ }^{19} \mathrm{~F} \mathrm{NMR} \mathrm{(282} \mathrm{MHz,} \mathrm{CDCl}_{3}\right) \delta=-62.9$. IR (ATR): 3045, 1720, 1594, 1420, 1347, 1224, 1090, 908, 800, $731 \mathrm{~cm}^{-1}$. MS (ESI) $\mathrm{m} / \mathrm{z}$ (relative intensity): $414(80)[\mathrm{M}+\mathrm{Na}]^{+}, 392$ (100) $[\mathrm{M}+\mathrm{H}]^{+}$. HR-MS (ESI) $\mathrm{m} / z$ calcd for $\mathrm{C}_{23} \mathrm{H}_{17} \mathrm{~N}_{3} \mathrm{~F}_{3}[\mathrm{M}+\mathrm{H}]^{+}$392.1369, found 392.1365.

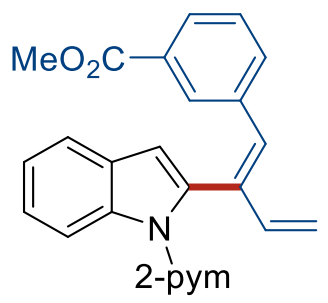

Methyl (Z)-3-\{2-[1-(pyrimidin-2-yl)-1H-indol-2-yl]buta-1,3-dien-1-yl\}benzoate (184aj) 
The general procedure $\mathbf{F}$ was followed using 1-(pyrimidin-2-yl)-1H-indole 71a $(39.0 \mathrm{mg}$, $0.20 \mathrm{mmol}$ ) and methyl-3-(cyclopropylidenemethyl)benzoate $138 \mathrm{j}(59.9 \mathrm{mg}, 0.32 \mathrm{mmol}$ ) for 3.0 h. Isolation by column chromatography ( $n$-hexane/EtOAc $8: 1)$ yielded 184aj $(58.7 \mathrm{mg}$, $77 \%, Z / E=4.5 / 1$ determined by ${ }^{1} \mathrm{H}$ NMR $)$ as a yellow oil. ${ }^{1} \mathrm{H}$ NMR $\left(400 \mathrm{MHz}, \mathrm{CDCl}_{3}\right) \delta=$ $8.73(\mathrm{~d}, J=4.8 \mathrm{~Hz}, 0.44 \mathrm{H}, E$ isomer), 8.62 (d, $J=4.8 \mathrm{~Hz}, 2 \mathrm{H}, Z$ isomer), 8.34 (d, $J=8.4$ $\mathrm{Hz}, 1 \mathrm{H}), 7.75(\mathrm{~d}, J=7.6 \mathrm{~Hz}, 1 \mathrm{H}), 7.65(\mathrm{~s}, 1 \mathrm{H}), 7.64-7.57(\mathrm{~m}, 1 \mathrm{H}), 7.34(\mathrm{t}, J=8.4 \mathrm{~Hz}, 1 \mathrm{H})$, $7.27(\mathrm{~d}, J=7.8 \mathrm{~Hz}, 1 \mathrm{H}), 7.21-7.12(\mathrm{~m}, 1 \mathrm{H}), 7.10$ (t, $J=7.6 \mathrm{~Hz}, 1 \mathrm{H}), 7.01$ (t, $J=4.8 \mathrm{~Hz}$, $1 \mathrm{H}), 6.73(\mathrm{dd}, J=17.2,10.4 \mathrm{~Hz}, 1 \mathrm{H}), 6.71(\mathrm{~s}, 1 \mathrm{H}), 6.59(\mathrm{~s}, 1 \mathrm{H}), 5.14(\mathrm{~d}, J=10.4 \mathrm{~Hz}, 1 \mathrm{H})$, $5.12(\mathrm{~d}, J=17.2 \mathrm{~Hz}, 1 \mathrm{H}), 3.75(\mathrm{~s}, 3 \mathrm{H}) .{ }^{13} \mathrm{C}$ NMR $\left(126 \mathrm{MHz}, \mathrm{CDCl}_{3}\right) \delta=167.0\left(\mathrm{C}_{\mathrm{q}}\right), 157.9$ $(\mathrm{CH}), 157.3\left(\mathrm{C}_{\mathrm{q}}\right), 140.2(\mathrm{CH}), 137.0\left(\mathrm{C}_{\mathrm{q}}\right), 136.7\left(\mathrm{C}_{\mathrm{q}}\right), 135.2\left(\mathrm{C}_{\mathrm{q}}\right), 134.9\left(\mathrm{C}_{\mathrm{q}}\right), 133.0(\mathrm{CH})$, $131.6(\mathrm{CH}), 130.5(\mathrm{CH}), 129.9\left(\mathrm{C}_{\mathrm{q}}\right), 129.4\left(\mathrm{C}_{\mathrm{q}}\right), 128.2(\mathrm{CH}), 128.1(\mathrm{CH}), 123.5(\mathrm{CH}), 122.0$ (CH), $120.8(\mathrm{CH}), 117.1(\mathrm{CH}), 116.6\left(\mathrm{CH}_{2}\right), 114.3(\mathrm{CH}), 108.9(\mathrm{CH})$. IR $($ ATR): 3045, 1720 , 1594, 1420, 1347, 1224, 1090, 908, 800, $731 \mathrm{~cm}^{-1}$. MS (ESI) $\mathrm{m} / \mathrm{z}$ (relative intensity): 414 (80) $[\mathrm{M}+\mathrm{Na}]^{+}, 392(100)[\mathrm{M}+\mathrm{H}]^{+}$. HR-MS (ESI) $\mathrm{m} / z$ calcd for $\mathrm{C}_{24} \mathrm{H}_{20} \mathrm{~N}_{3} \mathrm{O}_{2}[\mathrm{M}+\mathrm{H}]^{+}$382.1556, found 382.1559 .

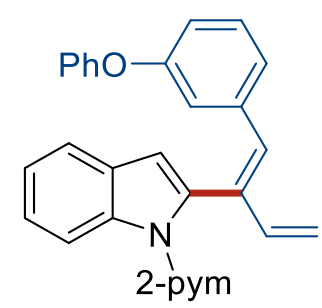

(Z)-2-[1-(3-Phenoxyphenyl)buta-1,3-dien-2-yl]-1-(pyrimidin-2-yl)-1 H-indole (184ak)

The general procedure $\mathbf{F}$ was followed using 1-(pyrimidin-2-yl)-1H-indole 71a $(39.0 \mathrm{mg}$, $0.20 \mathrm{mmol}$ ) and 1-(cyclopropylidenemethyl)-3-phenoxybenzene 138k (70.8 mg, $0.32 \mathrm{mmol})$ for $3.0 \mathrm{~h}$. Isolation by column chromatography ( $n$-hexane/EtOAc 15:1) yielded 184ak $(70.6$ mg, $85 \%, Z / E=7.0 / 1$ determined by ${ }^{1} \mathrm{H}$ NMR) as a yellow solid. M.p. $=86-88^{\circ} \mathrm{C} .{ }^{1} \mathbf{H}$ NMR $\left(300 \mathrm{MHz}, \mathrm{CDCl}_{3}\right) \delta=8.72(\mathrm{~d}, J=4.8 \mathrm{~Hz}, 0.28 \mathrm{H}, E$ isomer), $8.63(\mathrm{~d}, J=4.8 \mathrm{~Hz}, 2 \mathrm{H}, Z$ isomer), $8.34(\mathrm{~d}, J=8.2 \mathrm{~Hz}, 1 \mathrm{H}), 7.61(\mathrm{~d}, J=7.7 \mathrm{~Hz}, 1 \mathrm{H}), 7.37-7.25(\mathrm{~m}, 2 \mathrm{H}), 7.14-7.02$ (m, 2H), 7.03 (t, $J=5.1 \mathrm{~Hz}, 2 \mathrm{H}), 6.93(\mathrm{t}, J=7.3 \mathrm{~Hz}, 1 \mathrm{H}), 6.83-6.73(\mathrm{~m}, 5 \mathrm{H}), 6.66(\mathrm{~s}, 1 \mathrm{H})$, 
$6.65(\mathrm{~d}, J=17.3,10.8 \mathrm{~Hz}, 1 \mathrm{H}), 6.54(\mathrm{~s}, 1 \mathrm{H}), 5.06(\mathrm{~d}, J=10.8 \mathrm{~Hz}, 1 \mathrm{H}), 5.00(\mathrm{~d}, J=17.3 \mathrm{~Hz}$, 1H). ${ }^{13} \mathrm{C}$ NMR $\left(101 \mathrm{MHz}, \mathrm{CDCl}_{3}\right) \delta=157.8(\mathrm{CH}), 157.4\left(\mathrm{C}_{\mathrm{q}}\right), 157.2\left(\mathrm{C}_{\mathrm{q}}\right), 156.5\left(\mathrm{C}_{\mathrm{q}}\right), 140.2$ $(\mathrm{CH}), 138.6\left(\mathrm{C}_{\mathrm{q}}\right), 136.7\left(\mathrm{C}_{\mathrm{q}}\right), 135.1\left(\mathrm{C}_{\mathrm{q}}\right), 134.9\left(\mathrm{C}_{\mathrm{q}}\right), 132.1(\mathrm{CH}), 129.5(\mathrm{CH}), 129.4\left(\mathrm{C}_{\mathrm{q}}\right)$, $129.3(\mathrm{CH}), 123.6(\mathrm{CH}), 123.4(\mathrm{CH}), 123.2(\mathrm{CH}), 122.0(\mathrm{CH}), 120.8(\mathrm{CH}), 119.2(\mathrm{CH})$, $119.2(\mathrm{CH}), 118.8(\mathrm{CH}), 117.6(\mathrm{CH}), 117.0(\mathrm{CH}), 116.1\left(\mathrm{CH}_{2}\right), 114.3(\mathrm{CH}), 108.9(\mathrm{CH})$. IR (ATR): 3047, 1692, 1568, 1423, 1244, 1211, 1154, 1023, 1079, 900, 800, 732, $692 \mathrm{~cm}^{-1}$. MS (ESI) $m / z$ (relative intensity): $438(100)[\mathrm{M}+\mathrm{Na}]^{+}, 416(70)[\mathrm{M}+\mathrm{H}]^{+}$. HR-MS (ESI) m/z calcd for $\mathrm{C}_{28} \mathrm{H}_{22} \mathrm{~N}_{3} \mathrm{O}[\mathrm{M}+\mathrm{H}]^{+} 416.1757$, found 416.1744 .

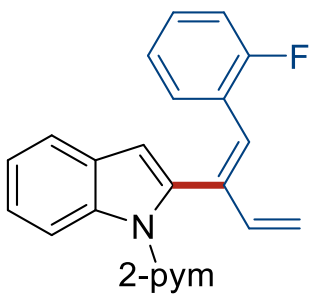

\section{(Z)-2-(1-(2-Fluorophenyl)buta-1,3-dien-2-yl)-1-(pyrimidin-2-yl)-1 H-indole (184al)}

The general procedure $\mathbf{F}$ was followed using 1-(pyrimidin-2-yl)-1H-indole $\mathbf{7 1 a}$ (39.0 mg, $0.20 \mathrm{mmol}$ ) and 1-(cyclopropylidenemethyl)-2-fluorobenzene $138 \mathrm{l}(47.1 \mathrm{mg}, 0.32 \mathrm{mmol}$ ) for $3.5 \mathrm{~h}$. Isolation by column chromatography ( $n$-hexane/EtOAc $15: 1)$ yielded 184 al $(51.2$ mg, $75 \%, Z / E=4.8 / 1$ determined by ${ }^{1} \mathrm{H}$ NMR $)$ as a yellow oil. ${ }^{1} \mathrm{H}$ NMR $\left(300 \mathrm{MHz}, \mathrm{CDCl}_{3}\right)$ $\delta=8.74(\mathrm{~d}, J=4.8 \mathrm{~Hz}, 0.42 \mathrm{H}, E$ isomer), $8.65(\mathrm{~d}, J=4.8 \mathrm{~Hz}, 2 \mathrm{H}, \mathrm{Z}$ isomer), 8.32 (dd, $J=$ 8.2, 1.2 Hz, 1H), $7.62(\mathrm{~d}, J=7.8 \mathrm{~Hz}, 1 \mathrm{H}), 7.34(\mathrm{td}, J=8.4,1.5 \mathrm{~Hz}, 1 \mathrm{H}), 7.26(\mathrm{td}, J=7.2$, $1.2 \mathrm{~Hz}, 1 \mathrm{H}), 7.12-7.06(\mathrm{~m}, 1 \mathrm{H}), 7.05(\mathrm{t}, J=4.8 \mathrm{~Hz}, 1 \mathrm{H}), 6.97(\mathrm{td}, J=7.8,1.8 \mathrm{~Hz}, 1 \mathrm{H}), 6.90$ (td, $J=8.4,1.2 \mathrm{~Hz}, 1 \mathrm{H}), 6.85(\mathrm{~s}, 1 \mathrm{H}), 6.75(\mathrm{t}, J=7.1 \mathrm{~Hz}, 1 \mathrm{H}), 6.74(\mathrm{dd}, J=18.0,9.6 \mathrm{~Hz}$, 1H), $6.61(\mathrm{~s}, 1 \mathrm{H}), 5.13(\mathrm{~d}, J=9.6 \mathrm{~Hz}, 1 \mathrm{H}), 5.12(\mathrm{~d}, J=18.0 \mathrm{~Hz}, 1 \mathrm{H}) .{ }^{13} \mathrm{C}$ NMR $(101 \mathrm{MHz}$, $\left.\mathrm{CDCl}_{3}\right) \delta=160.6\left(\mathrm{~d}, J=248.3 \mathrm{~Hz}, \mathrm{C}_{\mathrm{q}}\right), 157.9(\mathrm{CH}), 157.4(\mathrm{CH}), 140.2\left(\mathrm{C}_{\mathrm{q}}\right), 136.7\left(\mathrm{C}_{\mathrm{q}}\right)$, $135.5\left(\mathrm{~d}, J=1.6 \mathrm{~Hz}, \mathrm{C}_{\mathrm{q}}\right), 135.1\left(\mathrm{C}_{\mathrm{q}}\right), 130.1(\mathrm{~d}, J=2.8 \mathrm{~Hz}, \mathrm{CH}), 129.4\left(\mathrm{C}_{\mathrm{q}}\right), 128.7(\mathrm{~d}, J=$ 8.5 Hz, CH), $124.4\left(\mathrm{~d}, J=5.1 \mathrm{~Hz}, \mathrm{C}_{\mathrm{q}}\right), 123.8(\mathrm{CH}), 123.8(\mathrm{CH}), 123.6(\mathrm{CH}), 122.0(\mathrm{CH})$, $120.8(\mathrm{CH}), 117.1(\mathrm{CH}), 116.5\left(\mathrm{CH}_{2}\right), 114.8(\mathrm{~d}, J=21.9 \mathrm{~Hz}, \mathrm{CH}), 114.1(\mathrm{CH}), 109.0(\mathrm{CH})$. ${ }^{19} \mathrm{~F}$ NMR $\left(282 \mathrm{MHz}, \mathrm{CDCl}_{3}\right) \delta=-116.86-116.96$ (m). IR (ATR): 3044, 2244, 1565, 1437, 
1421, 1347, 1347, 1071, 1006, 906, 803, $727 \mathrm{~cm}^{-1}$. MS (ESI) $\mathrm{m} / \mathrm{z}$ (relative intensity): 414

(25) $[\mathrm{M}+\mathrm{Na}]^{+}, 392(100)[\mathrm{M}+\mathrm{H}]^{+}$. HR-MS (ESI) $\mathrm{m} / z$ calcd for $\mathrm{C}_{22} \mathrm{H}_{17} \mathrm{~N}_{3} \mathrm{~F}[\mathrm{M}+\mathrm{H}]^{+}$342.1407, found 342.1401 .

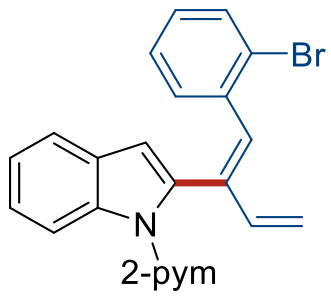

(Z)-2-(1-(2-Bromophenyl)buta-1,3-dien-2-yl)-1-(pyrimidin-2-yl)-1 H-indole (184am)

The general procedure $\mathbf{F}$ was followed using 1-(pyrimidin-2-yl)-1H-indole $71 \mathrm{a}$ (39.0 mg, $0.20 \mathrm{mmol}$ ) and 1-(cyclopropylidenemethyl)-2-bromobenzene $138 \mathrm{~m}$ (66.6 mg, $0.32 \mathrm{mmol}$ ) for $4.0 \mathrm{~h}$. Isolation by column chromatography ( $n$-hexane/EtOAc 15:1) yielded 184am (49.1 mg, $61 \%, Z / E=1.3 / 1$ determined by ${ }^{1} \mathrm{H}$ NMR) as a yellow solid. M.p. $=143-145^{\circ} \mathrm{C}$. ${ }^{1} \mathrm{H}$ NMR $\left(400 \mathrm{MHz}, \mathrm{CDCl}_{3}\right) \delta=8.75(\mathrm{~d}, J=4.8 \mathrm{~Hz}, 1.56 \mathrm{H}, E$ isomer), $8.65(\mathrm{~d}, J=4.8 \mathrm{~Hz}$, 2H, $Z$ isomer), $8.22(\mathrm{~d}, J=8.3 \mathrm{~Hz}, 1 \mathrm{H}), 7.59(\mathrm{~d}, J=7.8 \mathrm{~Hz}, 1 \mathrm{H}), 7.47(\mathrm{~d}, J=7.6 \mathrm{~Hz}, 1 \mathrm{H})$, 7.37-7.30 (m, 2H), $7.17(\mathrm{t}, J=8.0 \mathrm{~Hz}, 1 \mathrm{H}), 7.05(\mathrm{t}, J=4.8 \mathrm{~Hz}, 1 \mathrm{H}), 6.91(\mathrm{t}, J=7.4,1 \mathrm{H})$, $6.80(\mathrm{~s}, 1 \mathrm{H}), 6.80(\mathrm{~s}, 1 \mathrm{H}), 6.68(\mathrm{dd}, J=17.6,10.6 \mathrm{~Hz}, 1 \mathrm{H}), 6.59(\mathrm{~s}, 1 \mathrm{H}), 5.22(\mathrm{~d}, J=17.3$ $\mathrm{Hz}, 1 \mathrm{H}), 5.18(\mathrm{~d}, J=10.6 \mathrm{~Hz}, 2 \mathrm{H}) .{ }^{13} \mathrm{C}$ NMR $\left(101 \mathrm{MHz}, \mathrm{CDCl}_{3}\right) \delta=158.0(\mathrm{CH}), 157.2\left(\mathrm{C}_{\mathrm{q}}\right)$, $139.8(\mathrm{CH}), 139.4\left(\mathrm{C}_{\mathrm{q}}\right), 137.3\left(\mathrm{C}_{\mathrm{q}}\right), 136.8\left(\mathrm{C}_{\mathrm{q}}\right), 135.3\left(\mathrm{C}_{\mathrm{q}}\right), 132.0(\mathrm{CH}), 131.1(\mathrm{CH}), 130.8$ $(\mathrm{CH}), 129.2\left(\mathrm{C}_{\mathrm{q}}\right), 128.5(\mathrm{CH}), 127.1(\mathrm{CH}), 124.3\left(\mathrm{C}_{\mathrm{q}}\right), 123.6(\mathrm{CH}), 122.0(\mathrm{CH}), 120.8(\mathrm{CH})$, 117.2 (CH), $116.9\left(\mathrm{CH}_{2}\right), 113.9(\mathrm{CH}), 109.6(\mathrm{CH})$. IR (ATR): 3049, 2920, 1695, 1565, 1423, 1349, 1262, 1213, 1013, 987, 805, $741 \mathrm{~cm}^{-1}$. MS (ESI) $\mathrm{m} / \mathrm{z}$ (relative intensity): 424 (70)

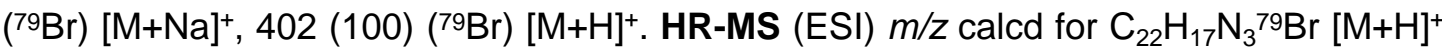
402.0606, found 402.0534. 


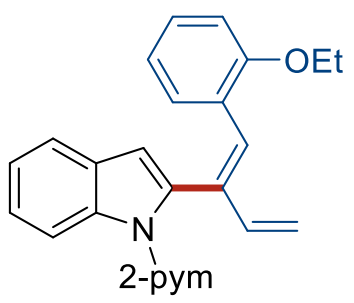

(Z)-2-(1-(2-Ethoxyphenyl)buta-1,3-dien-2-yl)-1-(pyrimidin-2-yl)-1 $H$-indole (184an)

The general procedure $\mathbf{F}$ was followed using 1-(pyrimidin-2-yl)-1H-indole 71a $(39.0 \mathrm{mg}$, $0.20 \mathrm{mmol}$ ) and 1-(cyclopropylidenemethyl)-2-ethoxybenzene 138n (66.6 mg, $0.32 \mathrm{mmol}$ ) for $3.5 \mathrm{~h}$. Isolation by column chromatography ( $n$-hexane/EtOAc 15:1) yielded 184an (57.3 mg, $78 \%, Z / E=3.0 / 1$ determined by ${ }^{1} \mathrm{H}$ NMR) as a yellow solid. M.p. $=86-89^{\circ} \mathrm{C} .{ }^{1} \mathbf{H}$ NMR $\left(300 \mathrm{MHz}, \mathrm{CDCl}_{3}\right) \delta=8.74(\mathrm{~d}, J=4.8 \mathrm{~Hz}, 0.66 \mathrm{H}, E$ isomer $), 8.64(\mathrm{~d}, J=4.8 \mathrm{~Hz}, 2 \mathrm{H}, Z$ isomer), 8.29 (d, $J=8.1 \mathrm{~Hz}, 1 \mathrm{H}), 7.61(\mathrm{~d}, J=7.6 \mathrm{~Hz}, 1 \mathrm{H}), 7.32$ (t, $J=7.2 \mathrm{~Hz}, 1 \mathrm{H}), 7.25$ (t, $J=7.6 \mathrm{~Hz}, 1 \mathrm{H}), 7.08-6.95(\mathrm{~m}, 5 \mathrm{H}), 6.78(\mathrm{dd}, J=16.4,11.2 \mathrm{~Hz}, 1 \mathrm{H}), 6.61(\mathrm{~s}, 1 \mathrm{H}), 6.58(\mathrm{t}$, $J=7.5 \mathrm{~Hz}, 1 \mathrm{H}), 5.08(\mathrm{~d}, J=16.4 \mathrm{~Hz}, 1 \mathrm{H}), 5.07(\mathrm{~d}, J=11.2 \mathrm{~Hz}, 1 \mathrm{H}), 3.99(\mathrm{q}, J=7.2 \mathrm{~Hz}$, 2H), $1.46(\mathrm{t}, J=7.2 \mathrm{~Hz}, 3 \mathrm{H}) .{ }^{13} \mathrm{C}$ NMR $\left(75 \mathrm{MHz}, \mathrm{CDCl}_{3}\right) \delta=157.8(\mathrm{CH}), 157.4\left(\mathrm{C}_{\mathrm{q}}\right), 156.7$ $\left(\mathrm{C}_{\mathrm{q}}\right), 140.8(\mathrm{CH}), 136.7\left(\mathrm{C}_{\mathrm{q}}\right), 135.9\left(\mathrm{C}_{\mathrm{q}}\right), 133.2\left(\mathrm{C}_{\mathrm{q}}\right), 129.9(\mathrm{CH}), 129.5\left(\mathrm{C}_{\mathrm{q}}\right), 128.4(\mathrm{CH})$, $128.1(\mathrm{CH}), 125.7\left(\mathrm{C}_{\mathrm{q}}\right), 123.2(\mathrm{CH}), 121.8(\mathrm{CH}), 120.7(\mathrm{CH}), 120.3(\mathrm{CH}), 116.8(\mathrm{CH}), 114.9$ $\left(\mathrm{CH}_{2}\right), 113.9(\mathrm{CH}), 111.0(\mathrm{CH}), 108.7(\mathrm{CH}), 63.7\left(\mathrm{CH}_{2}\right), 15.1\left(\mathrm{CH}_{3}\right)$. IR (ATR): 2978, 2508, 2162, 2088, 2011, 1957, 1563, 1422, 1239, 1039, $742 \mathrm{~cm}^{-1}$. MS (ESI) $\mathrm{m} / \mathrm{z}$ (relative intensity): 390 (60) $[\mathrm{M}+\mathrm{Na}]^{+}, 368(50)[\mathrm{M}+\mathrm{H}]^{+}$. HR-MS (ESI) $\mathrm{m} / z$ calcd for $\mathrm{C}_{24} \mathrm{H}_{21} \mathrm{~N}_{3} \mathrm{ONa}$ $[\mathrm{M}+\mathrm{H}]^{+} 390.1577$, found 390.1574 .

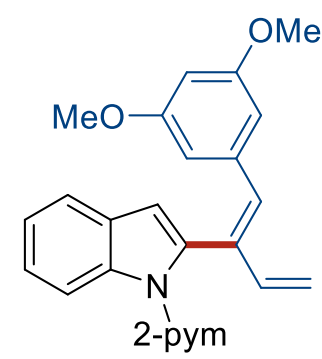

(Z)-2-[1-(3,5-Dimethoxyphenyl)buta-1,3-dien-2-yl]-1-(pyrimidin-2-yl)-1H-indole (184ao) 
The general procedure $\mathbf{F}$ was followed using 1-(pyrimidin-2-yl)-1H-indole $71 a$ (39.0 mg, $0.20 \mathrm{mmol}$ ) and 1-(cyclopropylidenemethyl)-3,5-dimethoxybenzene (1380) (60.6 mg, 0.32 mmol) for $3.5 \mathrm{~h}$. Isolation by column chromatography ( $n$-hexane/EtOAc 10:1) yielded 184ao (69.8 mg, 91\%, Z/E = 3.8/1 determined by ${ }^{1} \mathrm{H}$ NMR) as a yellow oil. ${ }^{1} \mathbf{H}$ NMR (300 $\left.\mathrm{MHz} \mathrm{CDCl}_{3}\right) \delta=8.73$ (d, $J=4.8 \mathrm{~Hz}, 0.52 \mathrm{H}, E$ isomer), 8.63 (dd, $J=4.8 \mathrm{~Hz}, 2 \mathrm{H}, Z$ isomer), $8.33(\mathrm{~d}, J=7.8 \mathrm{~Hz}, 1 \mathrm{H}), 7.64(\mathrm{dd}, J=7.8 \mathrm{~Hz}, 1 \mathrm{H}), 7.32(\mathrm{t}, J=7.2 \mathrm{~Hz}, 1 \mathrm{H}), 7.25(\mathrm{t}, J=7.2$ $\mathrm{Hz}, 1 \mathrm{H}), 7.01(\mathrm{t}, J=4.8 \mathrm{~Hz}, 0 \mathrm{H}), 6.78(\mathrm{dd}, J=17.2,10.5 \mathrm{~Hz}, 1 \mathrm{H}), 6.66(\mathrm{~s}, 1 \mathrm{H}), 6.59(\mathrm{~s}, 1 \mathrm{H})$, $6.22(\mathrm{t}, J=2.1 \mathrm{~Hz}, 1 \mathrm{H}), 6.07(\mathrm{~d}, J=2.1 \mathrm{~Hz}, 2 \mathrm{H}), 5.25(\mathrm{~d}, J=17.2 \mathrm{~Hz}, 1 \mathrm{H}), 5.21$ (d, $J=10.5$ $\mathrm{Hz}, 1 \mathrm{H}), 3.38(\mathrm{~s}, 6 \mathrm{H}) .{ }^{13} \mathrm{C}$ NMR $\left(75 \mathrm{MHz}, \mathrm{CDCl}_{3}\right) \delta=160.2\left(\mathrm{C}_{\mathrm{q}}\right), 157.8(\mathrm{CH}), 157.2\left(\mathrm{C}_{\mathrm{q}}\right)$, $140.4(\mathrm{CH}), 138.3\left(\mathrm{C}_{\mathrm{q}}\right), 136.6\left(\mathrm{C}_{\mathrm{q}}\right), 135.0\left(\mathrm{C}_{\mathrm{q}}\right), 134.5\left(\mathrm{C}_{\mathrm{q}}\right), 132.8(\mathrm{CH}), 129.4\left(\mathrm{C}_{\mathrm{q}}\right), 123.4$ $(\mathrm{CH}), 122.0(\mathrm{CH}), 120.6(\mathrm{CH}), 117.0(\mathrm{CH}), 116.3\left(\mathrm{CH}_{2}\right), 114.3(\mathrm{CH}), 108.7(\mathrm{CH}), 106.5$ (CH), $100.6(\mathrm{CH}), 55.0(\mathrm{CH})$. IR (ATR): 3047, 2937, 2835, 1730, 1590, 1423, 1344, 1303, 1263, 1201, 1152, 1061, 985, 904, 805, $735 \mathrm{~cm}^{-1}$. MS (ESI) m/z (relative intensity): 406 (100) $[\mathrm{M}+\mathrm{Na}]^{+}, 484(70)[\mathrm{M}+\mathrm{H}]^{+}$. HR-MS (ESI) $\mathrm{m} / z$ calcd for $\mathrm{C}_{24} \mathrm{H}_{22} \mathrm{~N}_{3} \mathrm{O}_{2}[\mathrm{M}+\mathrm{H}]^{+}$384.1707, found 384.1705 .

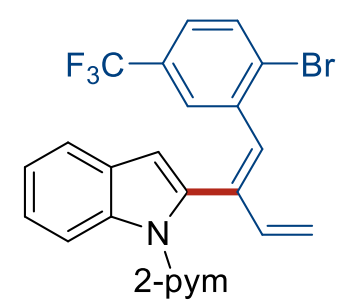

(Z)-2-\{1-[2-Bromo-5-(trifluoromethyl)phenyl]buta-1,3-dien-2-yl\}-1-(pyrimidin-2-yl)$1 H$-indole (184ap)

The general procedure $\mathbf{F}$ was followed using 1-(pyrimidin-2-yl)-1H-indole $71 \mathrm{a}$ (39.0 mg, $0.20 \mathrm{mmol}$ ) and 1-(cyclopropylidenemethyl)-2-bromo-5-trifluoromethylbenzene 138p (88.3 $\mathrm{mg}, 0.32 \mathrm{mmol}$ ) for $6.0 \mathrm{~h}$. Isolation by column chromatography ( $n$-hexane/EtOAc 15:1) yielded 184ap (48.0 mg, 78\%, Z/E $=10.5 / 1$ determined by ${ }^{1} \mathrm{H}$ NMR) as a yellow solid. M.p. $=71-72{ }^{\circ} \mathrm{C} .{ }^{1} \mathrm{H}$ NMR $\left(400 \mathrm{MHz}, \mathrm{CDCl}_{3}\right) \delta=8.70(\mathrm{~d}, J=4.8 \mathrm{~Hz}, 0.19 \mathrm{H}, E$ isomer), 8.60 (d, $J=4.8 \mathrm{~Hz}, 2 \mathrm{H}, Z$ isomer), $8.18(\mathrm{dq}, J=8.4,0.8 \mathrm{~Hz}, 1 \mathrm{H}), 7.54(\mathrm{dd}, J=7.7,1.4 \mathrm{~Hz}, 1 \mathrm{H})$, 
$7.45(\mathrm{~d}, J=8.4 \mathrm{~Hz}, 1 \mathrm{H}), 7.28-7.23(\mathrm{~m}, 1 \mathrm{H}), 7.19(\mathrm{dd}, J=7.7,2.4 \mathrm{~Hz}, 1 \mathrm{H}), 7.11$ (dd, 8.4, $2.3 \mathrm{~Hz}, 1 \mathrm{H}$ ), $7.02(\mathrm{t}, J=4.8 \mathrm{~Hz}, 1 \mathrm{H}), 6.73(\mathrm{ddd}, J=17.3,10.5,0.7 \mathrm{~Hz}, 1 \mathrm{H}), 6.72(\mathrm{dd}, J=$ 17.5, 10.7, $0.8 \mathrm{~Hz}, 1 \mathrm{H}$ ), $6.71(\mathrm{~s}, 1 \mathrm{H}), 6.56(\mathrm{~d}, J=0.8 \mathrm{~Hz}, 1 \mathrm{H}$ ), 5.27 (dt, $J=17.5,0.8 \mathrm{~Hz}$, $1 \mathrm{H}), 5.21(\mathrm{dt}, J=10.7,0.8 \mathrm{~Hz}, 1 \mathrm{H}) .{ }^{13} \mathrm{C}$ NMR $\left(101 \mathrm{MHz}, \mathrm{CDCl}_{3}\right) \delta=158.0(\mathrm{CH}), 157.2\left(\mathrm{C}_{\mathrm{q}}\right)$, $139.1(\mathrm{CH}), 137.9\left(\mathrm{C}_{\mathrm{q}}\right), 137.3\left(\mathrm{C}_{\mathrm{q}}\right), 136.9\left(\mathrm{C}_{\mathrm{q}}\right), 133.8\left(\mathrm{C}_{\mathrm{q}}\right), 132.4(\mathrm{CH}), 129.5(\mathrm{q}, J=33.5$ $\left.\mathrm{Hz}, \mathrm{C}_{\mathrm{q}}\right), 129.3(\mathrm{CH}), 129.0\left(\mathrm{C}_{\mathrm{q}}\right), 127.9(\mathrm{q}, J=3.9 \mathrm{~Hz}, \mathrm{CH}), 127.5\left(\mathrm{C}_{\mathrm{q}}\right), 124.6(\mathrm{q}, J=3.6 \mathrm{~Hz}$, $\mathrm{CH}), 123.9(\mathrm{CH}), 123.5\left(\mathrm{q}, J=272.5 \mathrm{~Hz}, \mathrm{C}_{\mathrm{q}}\right), 122.2(\mathrm{CH}), 120.8(\mathrm{CH}), 118.1\left(\mathrm{CH}_{2}\right), 117.2$ $(\mathrm{CH}), 113.9(\mathrm{CH}), 110.1(\mathrm{CH}) .{ }^{19} \mathrm{~F}$ NMR $\left(376 \mathrm{MHz}, \mathrm{CDCl}_{3}\right) \delta=-63.32$. IR (ATR): 3048, 2922, 1701, 1566, 1423, 1324, 1263, 1166, 1123, 1079, 1025, 912, 811, $741 \mathrm{~cm}^{-1}$. MS (ESI) $m / z$ (relative intensity): $492(100)\left({ }^{79} \mathrm{Br}\right)[\mathrm{M}+\mathrm{Na}]^{+}, 472(30)\left({ }^{79} \mathrm{Br}\right)[\mathrm{M}+\mathrm{H}]^{+}$. HR-MS (ESI) $\mathrm{m} / \mathrm{z}$ calcd for $\mathrm{C}_{23} \mathrm{H}_{16} \mathrm{~N}_{3}{ }^{79} \mathrm{BrF}_{3}[\mathrm{M}+\mathrm{H}]^{+} 470.0474$, found 470.0459 .

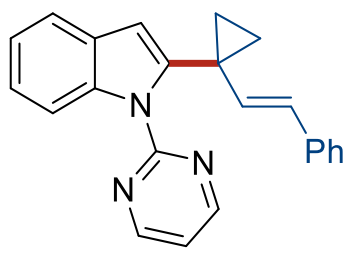

(E)-1-(Pyrimidin-2-yl)-2-(1-styrylcyclopropyl)-1 H-indole (186aa)

The general procedure $\mathbf{G}$ was followed using 1-(pyrimidin-2-yl)-1H-indole $71 \mathrm{a}$ (39.0 mg, $0.20 \mathrm{mmol}$ ) and (2-cyclopropylideneethyl)benzene $185 \mathrm{a}(46.1 \mathrm{mg}, 0.32 \mathrm{mmol})$ for $3.0 \mathrm{~h}$. Isolation by column chromatography ( $n$-hexane/EtOAc 15:1) yielded 186aa (64.1 mg, 95\%) as a yellow oil. ${ }^{1} \mathrm{H}$ NMR $\left(300 \mathrm{MHz}, \mathrm{CDCl}_{3}\right): \delta=8.77(\mathrm{~d}, J=4.7 \mathrm{~Hz}, 2 \mathrm{H}), 8.17(\mathrm{~d}, J=8.1$ $\mathrm{Hz}, 1 \mathrm{H}), 7.66(\mathrm{~d}, J=7.2 \mathrm{~Hz}, 1 \mathrm{H}), 7.37-7.30(\mathrm{~m}, 1 \mathrm{H}), 7.30-7.26(\mathrm{~m}, 1 \mathrm{H}), 7.25-7.14(\mathrm{~m}$, 5H), $7.09(\mathrm{t}, J=4.8 \mathrm{~Hz}, 1 \mathrm{H}), 6.69(\mathrm{~s}, 1 \mathrm{H}), 6.09(\mathrm{~d}, J=16.0 \mathrm{~Hz}, 1 \mathrm{H}), 6.00(\mathrm{~d}, J=16.0 \mathrm{~Hz}$, $1 \mathrm{H}), 1.50(\mathrm{dd}, J=4.6,4.6 \mathrm{~Hz}, 2 \mathrm{H}), 1.25(\mathrm{dd}, J=4.6,4.6 \mathrm{~Hz}, 2 \mathrm{H}) .{ }^{13} \mathrm{C}$ NMR $(101 \mathrm{MHz}$, $\left.\mathrm{CDCl}_{3}\right) \delta=158.3\left(\mathrm{C}_{\mathrm{q}}\right), 158.1(\mathrm{CH}), 142.7\left(\mathrm{C}_{\mathrm{q}}\right), 137.8\left(\mathrm{C}_{\mathrm{q}}\right), 137.2\left(\mathrm{C}_{\mathrm{q}}\right), 136.8(\mathrm{CH}), 128.8$ $\left(\mathrm{C}_{\mathrm{q}}\right), 128.4(\mathrm{CH}), 127.1(\mathrm{CH}), 126.6(\mathrm{CH}), 125.7(\mathrm{CH}), 123.1(\mathrm{CH}), 121.7(\mathrm{CH}), 120.3(\mathrm{CH})$, $117.6(\mathrm{CH}), 113.4(\mathrm{CH}), 107.4(\mathrm{CH}), 22.7\left(\mathrm{C}_{\mathrm{q}}\right), 18.4\left(\mathrm{CH}_{2}\right)$. IR (ATR): 3026, 1643, 1562, $1452,1417,1351,1298,1151,955,740 \mathrm{~cm}^{-1}$. MS (ESI) $\mathrm{m} / z$ (relative intensity): 360 (100) 
$[\mathrm{M}+\mathrm{Na}]^{+}, 338(85)[\mathrm{M}+\mathrm{H}]^{+}$. HR-MS (ESI) $\mathrm{m} / z$ calcd for $\mathrm{C}_{23} \mathrm{H}_{20} \mathrm{~N}_{3}[\mathrm{M}+\mathrm{H}]^{+} 338.1652$, found 338.1652 .<smiles>Cc1c(C2(/C=C/c3ccccc3)CC2)n(-c2ncccn2)c2ccccc12</smiles>

(E)-3-Methyl-1-(pyrimidin-2-yl)-2-(1-styrylcyclopropyl)-1 H-indole (186ba)

The general procedure $\mathbf{G}$ was followed using 3-methyl-1-(pyrimidin-2-yl)-1H-indole $71 \mathbf{b}$ (41.6 mg, $0.20 \mathrm{mmol}$ ) and (2-cyclopropylideneethyl)benzene 185a (46.1 mg, $0.32 \mathrm{mmol})$ for $3.5 \mathrm{~h}$. Isolation by column chromatography ( $n$-hexane/EtOAc 15:1) yielded $\mathbf{1 8 6 b a}(51.3$ $\mathrm{mg}, 73 \%)$ as a yellow oil. ${ }^{1} \mathrm{H}$ NMR $\left(400 \mathrm{MHz}, \mathrm{CDCl}_{3}\right) \delta=8.80(\mathrm{~d}, J=4.8 \mathrm{~Hz}, 2 \mathrm{H}), 8.23(\mathrm{~d}$, $J=7.8 \mathrm{~Hz}, 1 \mathrm{H}), 7.65(\mathrm{~d}, J=1.7 \mathrm{~Hz}, 1 \mathrm{H}), 7.40-7.30(\mathrm{~m}, 6 \mathrm{H}), 7.24-7.19(\mathrm{~m}, 1 \mathrm{H}), 7.13(\mathrm{td}$, $J=4.8,1.6 \mathrm{~Hz}, 1 \mathrm{H}), 6.37(\mathrm{~d}, J=15.8 \mathrm{~Hz}, 1 \mathrm{H}), 6.30(\mathrm{~d}, J=15.8 \mathrm{~Hz}, 1 \mathrm{H}), 2.44(\mathrm{~s}, 3 \mathrm{H}), 1.18$ $(\mathrm{s}, 2 \mathrm{H}), 1.04(\mathrm{~s}, 2 \mathrm{H}) .{ }^{13} \mathrm{C}$ NMR $\left(101 \mathrm{MHz}, \mathrm{CDCl}_{3}\right) \delta=158.1\left(\mathrm{C}_{\mathrm{q}}\right), 158.0(\mathrm{CH}), 138.2\left(\mathrm{C}_{\mathrm{q}}\right)$, $137.2\left(\mathrm{C}_{\mathrm{q}}\right), 136.5(\mathrm{CH}), 135.8\left(\mathrm{C}_{\mathrm{q}}\right), 130.1\left(\mathrm{C}_{\mathrm{q}}\right), 128.5(\mathrm{CH}), 127.2(\mathrm{CH}), 126.5(\mathrm{CH}), 125.9$ $(\mathrm{CH}), 123.3(\mathrm{CH}), 121.5(\mathrm{CH}), 118.3(\mathrm{CH}), 117.0(\mathrm{CH}), 116.4\left(\mathrm{C}_{\mathrm{q}}\right), 113.3(\mathrm{CH}), 21.3\left(\mathrm{C}_{\mathrm{q}}\right)$, $18.1\left(\mathrm{CH}_{2}\right), 9.5\left(\mathrm{CH}_{3}\right)$. IR (ATR): 3029, 2916, 1645, 1563, 1453, 1420, 1347, 1298, 1178, 956, $736 \mathrm{~cm}^{-1}$. MS (ESI) m/z (relative intensity): $374(80)[\mathrm{M}+\mathrm{Na}]^{+}, 352(100)[\mathrm{M}+\mathrm{H}]^{+}$. HR-MS (ESI) $m / z$ calcd for $\mathrm{C}_{24} \mathrm{H}_{22} \mathrm{~N}_{3}[\mathrm{M}+\mathrm{H}]^{+}, 352.1808$, found 352.1808 .<smiles>OCc1c(C2(/C=C/c3ccccc3)CC2)n(-c2ncccn2)c2ccccc12</smiles>

(E)-(1-(Pyrimidin-2-yl)-2-(1-styrylcyclopropyl)-1 H-indol-3-yl)methanol (186qa) 
The general procedure $\mathbf{G}$ was followed using (1-(pyrimidin-2-yl)-1H-indol-3-yl)methanol 71q (44.8 mg, $0.20 \mathrm{mmol}$ ) and (2-cyclopropylideneethyl)benzene 185a (46.1 mg, 0.32 $\mathrm{mmol}$ ) for $4.5 \mathrm{~h}$. Isolation by column chromatography ( $n$-hexane/EtOAc 5:1) yielded 186qa (33.1 mg, 45\%) as a colorless solid. M.p. $=145-146{ }^{\circ} \mathrm{C} .{ }^{1} \mathbf{H}$ NMR $\left(400 \mathrm{MHz}, \mathrm{CDCl}_{3}\right) \delta=$ $8.81(\mathrm{~d}, J=4.6 \mathrm{~Hz}, 2 \mathrm{H}), 8.11(\mathrm{~d}, J=8.0 \mathrm{~Hz}, 1 \mathrm{H}), 7.80(\mathrm{~d}, J=7.4 \mathrm{~Hz}, 1 \mathrm{H}), 7.36-7.26(\mathrm{~m}$, $6 \mathrm{H}), 7.21-7.17(\mathrm{~m}, 2 \mathrm{H}), 6.31(\mathrm{~s}, 2 \mathrm{H}), 5.06(\mathrm{~d}, J=5.0 \mathrm{~Hz}, 2 \mathrm{H}), 1.56(\mathrm{t}, J=5.0 \mathrm{~Hz}, 1 \mathrm{H})$, 1.23-1.15 (m, 2H), 1.15-1.06 (m, 2H). ${ }^{13} \mathrm{C}$ NMR (101 MHz, $\left.\mathrm{CDCl}_{3}\right) \delta=158.3(\mathrm{CH}), 158.0$ $\left(\mathrm{C}_{\mathrm{q}}\right), 139.4\left(\mathrm{C}_{\mathrm{q}}\right), 137.8\left(\mathrm{C}_{\mathrm{q}}\right), 137.0(\mathrm{CH}), 136.1\left(\mathrm{C}_{\mathrm{q}}\right), 128.6(\mathrm{CH}), 128.3\left(\mathrm{C}_{\mathrm{q}}\right), 127.7(\mathrm{CH})$, $126.8(\mathrm{CH}), 126.0(\mathrm{CH}), 123.8(\mathrm{CH}), 122.0(\mathrm{CH}), 119.2\left(\mathrm{C}_{q}\right), 118.8(\mathrm{CH}), 117.8(\mathrm{CH}), 113.3$ $(\mathrm{CH}), 56.4\left(\mathrm{CH}_{2}\right), 21.0\left(\mathrm{C}_{\mathrm{q}}\right), 17.8\left(\mathrm{CH}_{2}\right)$. IR (ATR): 3492, 3023, 2858, 1562, 1455, 1423, 1358, 1298, 1100, 953, $746 \mathrm{~cm}^{-1}$. MS (ESI) $\mathrm{m} / \mathrm{z}$ (relative intensity): 381 (100) [M+Na] ${ }^{+}, 368$ (50) $[\mathrm{M}+\mathrm{H}]^{+}$. HR-MS (ESI) $\mathrm{m} / \mathrm{z}$ calcd for $\mathrm{C}_{24} \mathrm{H}_{22} \mathrm{~N}_{3} \mathrm{O}[\mathrm{M}+\mathrm{H}]^{+}$368.1755, found 368.1757.

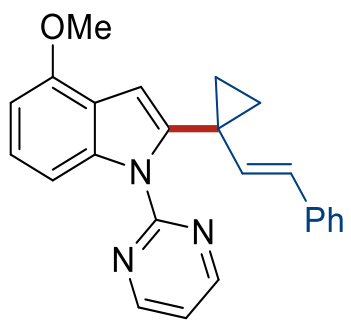

(E)-4-Methoxy-1-(pyrimidin-2-yl)-2-(1-styrylcyclopropyl)-1 H-indole (5da)

The general procedure $\mathbf{G}$ was followed using 4-methoxy-1-(pyrimidin-2-yl)-1H-indole 71c (44.8 mg, $0.20 \mathrm{mmol}$ ) and (2-cyclopropylideneethyl)benzene 185a (46.1 mg, $0.32 \mathrm{mmol})$ for $3.0 \mathrm{~h}$. Isolation by column chromatography ( $n$-hexane/EtOAc 15:1) yielded 186ca (55.1 mg, $75 \%)$ as a colorless solid. M.p. $=115-116{ }^{\circ} \mathrm{C} .{ }^{1} \mathbf{H}$ NMR $\left(300 \mathrm{MHz}, \mathrm{CDCl}_{3}\right) \delta=8.76(\mathrm{~d}$, $J=4.8 \mathrm{~Hz}, 2 \mathrm{H}), 7.75(\mathrm{~d}, J=8.4 \mathrm{~Hz}, 1 \mathrm{H}), 7.26-7.18(\mathrm{~m}, 3 \mathrm{H}), 7.17-7.12(\mathrm{~m}, 3 \mathrm{H}), 7.08(\mathrm{t}, J$ $=4.8 \mathrm{~Hz}, 1 \mathrm{H}), 6.82(\mathrm{~s}, 1 \mathrm{H}), 6.70(\mathrm{~d}, J=7.9 \mathrm{~Hz}, 1 \mathrm{H}), 6.08(\mathrm{~d}, J=16.0 \mathrm{~Hz}, 1 \mathrm{H}), 5.97(\mathrm{~d}, J=$ $16.0 \mathrm{~Hz}, 1 \mathrm{H}), 4.03(\mathrm{~s}, 3 \mathrm{H}), 1.53-1.48(\mathrm{~m}, 2 \mathrm{H}), 1.25-1.20(\mathrm{~m}, 2 \mathrm{H}) .{ }^{13} \mathrm{C} \mathrm{NMR}\left(75 \mathrm{MHz}, \mathrm{CDCl}_{3}\right)$ $\delta=158.4\left(\mathrm{C}_{\mathrm{q}}\right), 158.1(\mathrm{CH}), 152.8\left(\mathrm{C}_{\mathrm{q}}\right), 141.2\left(\mathrm{C}_{\mathrm{q}}\right), 138.5\left(\mathrm{C}_{\mathrm{q}}\right), 137.8\left(\mathrm{C}_{\mathrm{q}}\right), 136.8(\mathrm{CH})$, $128.3(\mathrm{CH}), 127.1(\mathrm{CH}), 126.5(\mathrm{CH}), 125.7(\mathrm{CH}), 123.9(\mathrm{CH}), 119.1\left(\mathrm{C}_{\mathrm{q}}\right), 117.7(\mathrm{CH}), 106.7$ 
(CH), $104.2(\mathrm{CH}), 101.8(\mathrm{CH}), 55.5\left(\mathrm{CH}_{3}\right), 22.6\left(\mathrm{C}_{\mathrm{q}}\right), 18.3\left(\mathrm{CH}_{2}\right)$. IR (ATR): 3000, 2201, 1564, 1437, 1419, 1360, 1256, 1075, 812, $769 \mathrm{~cm}^{-1}$. MS (ESI) $\mathrm{m} / \mathrm{z}$ (relative intensity): 390 (80) $[\mathrm{M}+\mathrm{Na}]^{+}, 368(100)[\mathrm{M}+\mathrm{H}]^{+}$. HR-MS (ESI) $\mathrm{m} / \mathrm{z}$ calcd for $\mathrm{C}_{24} \mathrm{H}_{22} \mathrm{~N}_{3} \mathrm{O}[\mathrm{M}+\mathrm{H}]^{+}$368.1755, found 368.1757 .<smiles>N#Cc1cccc2c1cc(C1(/C=C/c3ccccc3)CC1)n2-c1ncccn1</smiles>

(E)-1-(Pyrimidin-2-yl)-2-(1-styrylcyclopropyl)-1 H-indole-4-carbonitrile (186da)

The general procedure $\mathbf{G}$ was followed using 1-(pyrimidin-2-yl)-1H-indole-4-carbonitrile 71d (43.8 mg, $0.20 \mathrm{mmol})$ and (2-cyclopropylideneethyl)benzene 185a (46.1 mg, 0.32 $\mathrm{mmol}$ ) for $4.0 \mathrm{~h}$. Isolation by column chromatography ( $n$-hexane/EtOAc $4: 1$ ) yielded 186da (39.9 mg, 55\%) as a white solid. M.p. $=153-155^{\circ} \mathrm{C} .{ }^{1} \mathbf{H}$ NMR $\left(400 \mathrm{MHz}, \mathrm{CDCl}_{3}\right) \delta=8.80$ (d, $J=4.8 \mathrm{~Hz}, 2 \mathrm{H}), 8.28(\mathrm{~d}, J=8.2 \mathrm{~Hz}, 1 \mathrm{H}), 7.57(\mathrm{dd}, J=7.4,1.1 \mathrm{~Hz}, 1 \mathrm{H}), 7.31(\mathrm{td}, J=$ 8.1, 7.5, 1.4 Hz, 1H), 7.25-7.15 (m, 3H), 7.15-7.09 (m, 3H), $6.88(\mathrm{~s}, 1 \mathrm{H}), 6.01(\mathrm{~d}, J=16.0$ $\mathrm{Hz}, 1 \mathrm{H}), 5.95(\mathrm{~d}, J=16.0 \mathrm{~Hz}, 1 \mathrm{H}), 1.53-1.49(\mathrm{~m}, 2 \mathrm{H}), 1.29-1.25(\mathrm{~m}, 2 \mathrm{H}) .{ }^{13} \mathrm{C}$ NMR $(101$ $\left.\mathrm{MHz}, \mathrm{CDCl}_{3}\right) \delta=158.4(\mathrm{CH}), 157.7\left(\mathrm{C}_{\mathrm{q}}\right), 145.9\left(\mathrm{C}_{\mathrm{q}}\right), 137.4\left(\mathrm{C}_{\mathrm{q}}\right), 137.0\left(\mathrm{C}_{\mathrm{q}}\right), 135.6(\mathrm{CH})$, $130.5\left(\mathrm{C}_{\mathrm{q}}\right), 128.4(\mathrm{CH}), 127.7(\mathrm{CH}), 126.8(\mathrm{CH}), 126.5(\mathrm{CH}), 125.8(\mathrm{CH}), 122.8(\mathrm{CH}), 118.7$ $\left(\mathrm{C}_{\mathrm{q}}\right), 118.5(\mathrm{CH}), 118.0(\mathrm{CH}), 105.4(\mathrm{CH}), 102.7\left(\mathrm{C}_{\mathrm{q}}\right), 22.6\left(\mathrm{C}_{\mathrm{q}}\right), 18.4\left(\mathrm{CH}_{2}\right)$. IR $(\mathrm{ATR})$ : 3037, $2213,1646,1561,1411,1361,1302,1202,928,814,775,742 \mathrm{~cm}^{-1}$. MS (ESI) $\mathrm{m} / \mathrm{z}$ (relative intensity): $386(100)[\mathrm{M}+\mathrm{Na}]^{+}, 364(55)[\mathrm{M}+\mathrm{H}]^{+}$. HR-MS (ESI) $\mathrm{m} / z$ calcd for $\mathrm{C}_{24} \mathrm{H}_{19} \mathrm{~N}_{4}[\mathrm{M}+\mathrm{H}]^{+}$ 363.1604, found 363.1603 . 
<smiles>Cc1ccc2c(c1)cc(C1(/C=C/c3ccccc3)CC1)n2-c1ncccn1</smiles>

\section{(E)-5-Methyl-1-(pyrimidin-2-yl)-2-(1-styrylcyclopropyl)-1H-indole (186ea)}

The general procedure $\mathbf{G}$ was followed using 4-methoxy-1-(pyrimidin-2-yl)-1H-indole 71e (41.6 mg, $0.20 \mathrm{mmol}$ ) and (2-cyclopropylideneethyl)benzene 185a (46.1 mg, $0.32 \mathrm{mmol}$ ) for $3.0 \mathrm{~h}$. Isolation by column chromatography ( $n$-hexane/EtOAc 15:1) yielded 186ea (56.9 mg, $81 \%)$ as a yellow oil. ${ }^{1} \mathrm{H}$ NMR $\left(400 \mathrm{MHz}, \mathrm{CDCl}_{3}\right) \delta=8.72(\mathrm{~d}, J=4.8 \mathrm{~Hz}, 2 \mathrm{H}), 8.07(\mathrm{~d}$, $J=8.5 \mathrm{~Hz}, 1 \mathrm{H}), 7.41(\mathrm{~s}, 2 \mathrm{H}), 7.22-7.09(\mathrm{~m}, 6 \mathrm{H}), 7.03(\mathrm{t}, J=4.8 \mathrm{~Hz}, 1 \mathrm{H}), 6.59(\mathrm{~s}, 1 \mathrm{H}), 6.04$ $(\mathrm{d}, J=16.0 \mathrm{~Hz}, 1 \mathrm{H}), 5.98(\mathrm{~d}, J=16.0 \mathrm{~Hz}, 1 \mathrm{H}), 2.49(\mathrm{~s}, 3 \mathrm{H}), 1.48-1.36(\mathrm{~m}, 2 \mathrm{H}), 1.23-1.19$ $(\mathrm{m}, 2 \mathrm{H}) .{ }^{13} \mathrm{C}$ NMR $\left(101 \mathrm{MHz}, \mathrm{CDCl}_{3}\right) \delta=158.3\left(\mathrm{C}_{\mathrm{q}}\right), 158.0,142.7\left(\mathrm{C}_{\mathrm{q}}\right), 137.9\left(\mathrm{C}_{\mathrm{q}}\right), 137.0$ $(\mathrm{CH}), 135.5\left(\mathrm{C}_{\mathrm{q}}\right), 131.1\left(\mathrm{C}_{\mathrm{q}}\right), 129.1\left(\mathrm{C}_{\mathrm{q}}\right), 128.4(\mathrm{CH}), 127.0(\mathrm{CH}), 126.5(\mathrm{CH}), 125.7(\mathrm{CH})$, $124.6(\mathrm{CH}), 120.1(\mathrm{CH}), 117.3(\mathrm{CH}), 113.4(\mathrm{CH}), 107.3(\mathrm{CH}), 22.9\left(\mathrm{C}_{\mathrm{q}}\right), 21.5\left(\mathrm{CH}_{3}\right), 18.4$ $\left(\mathrm{CH}_{2}\right)$. IR (ATR): 3023, 1644, 1564, 1418, 1336, 1215, 1151, 952, 907, 802, 731, $688 \mathrm{~cm}^{-1}$. MS (ESI) m/z (relative intensity): 374 (95) [M+Na] ${ }^{+}, 352(100)[\mathrm{M}+\mathrm{H}]^{+}$. HR-MS (ESI) m/z calcd for $\mathrm{C}_{24} \mathrm{H}_{22} \mathrm{~N}_{3}[\mathrm{M}+\mathrm{H}]^{+} 352.1814$, found 352.1808.<smiles>Clc1ccc2c(c1)cc(C1(/C=C/c3ccccc3)CC1)n2-c1ncccn1</smiles>

\section{(E)-5-Chloro-1-(pyrimidin-2-yl)-2-(1-styrylcyclopropyl)-1H-indole (186ga)}

The general procedure $\mathbf{G}$ was followed using 5-chloro-1-(pyrimidin-2-yl)-1H-indole $\mathbf{7 1 g}$ (45.7 mg, $0.20 \mathrm{mmol}$ ) and (2-cyclopropylideneethyl)benzene 185a (46.1 mg, $0.32 \mathrm{mmol}$ ) for $3.0 \mathrm{~h}$. Isolation by column chromatography ( $n$-hexane/EtOAc 15:1) yielded $\mathbf{1 8 6 g a}(54.3$ mg, $73 \%)$ as a yellow oil. ${ }^{1} \mathrm{H}$ NMR $\left(400 \mathrm{MHz}, \mathrm{CDCl}_{3}\right) \delta=8.76(\mathrm{~d}, J=4.8 \mathrm{~Hz}, 2 \mathrm{H}), 8.07(\mathrm{~d}$, 
$J=8.9 \mathrm{~Hz}, 1 \mathrm{H}), 7.58(\mathrm{~d}, J=2.1 \mathrm{~Hz}, 1 \mathrm{H}), 7.25-7.18(\mathrm{~m}, 3 \mathrm{H}), 7.14-7.10(\mathrm{~m}, 4 \mathrm{H}), 6.60(\mathrm{~s}$, $1 \mathrm{H}), 6.02(\mathrm{~d}, J=16.0 \mathrm{~Hz}, 1 \mathrm{H}), 5.96(\mathrm{~d}, J=16.0 \mathrm{~Hz}, 1 \mathrm{H}), 1.47-1.44(\mathrm{~m}, 2 \mathrm{H}), 1.24-1.21(\mathrm{~m}$, 2H). ${ }^{13} \mathrm{C}$ NMR $\left(101 \mathrm{MHz}, \mathrm{CDCl}_{3}\right) \delta=158.2(\mathrm{CH}), 158.0\left(\mathrm{C}_{\mathrm{q}}\right), 144.1\left(\mathrm{C}_{\mathrm{q}}\right), 137.7\left(\mathrm{C}_{\mathrm{q}}\right), 136.4$ $(\mathrm{CH}), 135.6\left(\mathrm{C}_{\mathrm{q}}\right), 130.0\left(\mathrm{C}_{\mathrm{q}}\right), 130.0\left(\mathrm{C}_{\mathrm{q}}\right), 128.4(\mathrm{CH}), 127.2(\mathrm{CH}), 126.7(\mathrm{CH}), 125.8(\mathrm{CH})$, $123.2(\mathrm{CH}), 119.7(\mathrm{CH}), 117.9(\mathrm{CH}), 114.7(\mathrm{CH}), 106.8(\mathrm{CH}), 22.8\left(\mathrm{C}_{\mathrm{q}}\right), 18.4\left(\mathrm{CH}_{2}\right) . \mathbf{I R}$ (ATR): $3025,1644,1564,1416,1335,1186,1067,950,866,800,742,687 \mathrm{~cm}^{-1}$. MS (ESI) $\mathrm{m} / \mathrm{z}$ (relative intensity): $394(50)\left({ }^{35} \mathrm{Cl}\right)[\mathrm{M}+\mathrm{Na}]^{+}, 372(100)\left({ }^{35} \mathrm{Cl}\right)[\mathrm{M}+\mathrm{H}]^{+}$. HR-MS (ESI) $\mathrm{m} / \mathrm{z}$ calcd for $\mathrm{C}_{23} \mathrm{H}_{19} \mathrm{~N}_{3}{ }^{35} \mathrm{Cl}[\mathrm{M}+\mathrm{H}]+372.1268$, found 372.1262<smiles>Brc1ccc2c(c1)cc(C1(/C=C/c3ccccc3)CC1)n2-c1ncccn1</smiles>

(E)-5-Bromo-1-(pyrimidin-2-yl)-2-(1-styrylcyclopropyl)-1H-indole (186ha)

The general procedure $\mathbf{G}$ was followed using 5-bromo-1-(pyrimidin-2-yl)-1H-indole $\mathbf{7 1 h}$ (54.6 mg, $0.20 \mathrm{mmol}$ ) and (2-cyclopropylideneethyl)benzene 185a (46.1 mg, $0.32 \mathrm{mmol}$ ) for $3.0 \mathrm{~h}$. Isolation by column chromatography ( $n$-hexane/EtOAc $15: 1$ ) yielded $\mathbf{1 8 6 h a}(69.1$ mg, $83 \%)$ as a yellow solid. M.p. $=153-155^{\circ} \mathrm{C} .{ }^{1} \mathrm{H}$ NMR $\left(300 \mathrm{MHz}, \mathrm{CDCl}_{3}\right) \delta=8.76(\mathrm{~d}, \mathrm{~J}$ $=4.8 \mathrm{~Hz}, 2 \mathrm{H}), 8.03(\mathrm{~d}, J=8.8 \mathrm{~Hz}, 1 \mathrm{H}), 7.75(\mathrm{~d}, J=2.0 \mathrm{~Hz}, 1 \mathrm{H}), 7.38(\mathrm{dd}, J=8.9,2.0 \mathrm{~Hz}$, 1H), 7.25-7.18 (m, 2H), 7.16-7.09 (m, 4H), $6.60(\mathrm{~s}, 1 \mathrm{H}), 6.03(\mathrm{~d}, J=16.2 \mathrm{~Hz}, 1 \mathrm{H}), 5.97(\mathrm{~d}$, $J=16.2 \mathrm{~Hz}, 1 \mathrm{H}), 1.49-1.44(\mathrm{~m}, 2 \mathrm{H}), 1.26-1.21(\mathrm{~m}, 2 \mathrm{H}) .{ }^{13} \mathrm{C} \mathrm{NMR}\left(101 \mathrm{MHz}, \mathrm{CDCl}_{3}\right) \delta=$ $158.2\left(\mathrm{C}_{\mathrm{q}}\right), 158.0(\mathrm{CH}), 144.0\left(\mathrm{C}_{\mathrm{q}}\right), 137.6\left(\mathrm{C}_{\mathrm{q}}\right), 136.4(\mathrm{CH}), 135.9\left(\mathrm{C}_{\mathrm{q}}\right), 130.5\left(\mathrm{C}_{\mathrm{q}}\right), 128.4$ $(\mathrm{CH}), 127.2(\mathrm{CH}), 126.7(\mathrm{CH}), 125.8(\mathrm{CH}), 125.7(\mathrm{CH}), 122.8(\mathrm{CH}), 117.9(\mathrm{CH}), 115.1(\mathrm{CH})$, $114.9\left(\mathrm{C}_{\mathrm{q}}\right), 106.7(\mathrm{CH}), 22.7\left(\mathrm{C}_{\mathrm{q}}\right), 18.4\left(\mathrm{CH}_{2}\right)$. IR (ATR): 3022, 1644, 1563, 1415, 1334, 1185, 949, 907, 866, 799, 740, $688 \mathrm{~cm}^{-1}$. MS (ESI) $\mathrm{m} / \mathrm{z}$ (relative intensity): $438(60)\left({ }^{79} \mathrm{Br}\right.$ ) $[\mathrm{M}+\mathrm{Na}]^{+}, 416(100)\left({ }^{79} \mathrm{Br}\right)[\mathrm{M}+\mathrm{H}]^{+}$. HR-MS (ESI) $\mathrm{m} / \mathrm{z}$ calcd for $\mathrm{C}_{23} \mathrm{H}_{19}{ }^{79} \mathrm{BrN}{ }_{3}[\mathrm{M}+\mathrm{H}]^{+}$ 416.0762, found 416.0757 . 
<smiles>Ic1ccc2c(c1)cc(C1(/C=C/c3ccccc3)CC1)n2-c1ncccn1</smiles>

(E)-5-lodo-1-(pyrimidin-2-yl)-2-(1-styrylcyclopropyl)-1 $H$-indole (186ra)

The general procedure $\mathbf{G}$ was followed using 5-iodo-1-(pyrimidin-2-yl)-1H-indole 71r (64.0 $\mathrm{mg}, 0.20 \mathrm{mmol})$ and (2-cyclopropylideneethyl)benzene $185 \mathrm{a}(46.1 \mathrm{mg}, 0.32 \mathrm{mmol})$ for 4.0 h. Isolation by column chromatography ( $n$-hexane/EtOAc 15:1) yielded 186ra (50.0 mg, $54 \%)$ as a yellow oil. ${ }^{1} \mathrm{H}$ NMR $\left(400 \mathrm{MHz}, \mathrm{CDCl}_{3}\right) \delta=8.75(\mathrm{~d}, J=4.8 \mathrm{~Hz}, 2 \mathrm{H}), 7.95(\mathrm{~s}, 1 \mathrm{H})$, $7.91(\mathrm{~d}, J=8.8 \mathrm{~Hz}, 1 \mathrm{H}), 7.54(\mathrm{~d}, J=8.8 \mathrm{~Hz}, 1 \mathrm{H}), 7.24-7.17(\mathrm{~m}, 2 \mathrm{H}), 7.15-7.08(\mathrm{~m}, 4 \mathrm{H})$, $6.57(\mathrm{~s}, 1 \mathrm{H}), 6.00$ (d, J=16.2 Hz, 1H), 5.95 (d, $J=16.0 \mathrm{~Hz}, 1 \mathrm{H}), 1.49-1.42(\mathrm{~m}, 2 \mathrm{H}), 1.24-$ $1.19(\mathrm{~m}, 2 \mathrm{H}) .{ }^{13} \mathrm{C}$ NMR $\left(101 \mathrm{MHz}, \mathrm{CDCl}_{3}\right) \delta=158.2(\mathrm{CH}), 143.6\left(\mathrm{C}_{\mathrm{q}}\right), 137.6\left(\mathrm{C}_{\mathrm{q}}\right), 136.5$ $\left(\mathrm{C}_{\mathrm{q}}\right), 136.4\left(\mathrm{C}_{\mathrm{q}}\right), 131.4(\mathrm{CH}), 131.3\left(\mathrm{C}_{\mathrm{q}}\right), 129.1(\mathrm{CH}), 128.4(\mathrm{CH}), 127.2(\mathrm{CH}), 126.7(\mathrm{CH})$, $125.8(\mathrm{CH}), 117.9(\mathrm{CH}), 115.6(\mathrm{CH}), 106.4(\mathrm{CH}), 85.4\left(\mathrm{C}_{\mathrm{q}}\right), 22.6\left(\mathrm{C}_{\mathrm{q}}\right), 18.4\left(\mathrm{CH}_{2}\right)$. IR $(\mathrm{ATR})$ : $3025,2921,2849,1732,1643,1563,1415,1330,1183,1023,947,799,738,689 \mathrm{~cm}^{-1}$. MS (ESI) $m / z$ (relative intensity): $486(60)[\mathrm{M}+\mathrm{Na}]^{+}, 464(100)[\mathrm{M}+\mathrm{H}]^{+}$. HR-MS (ESI) m/z calcd for $\mathrm{C}_{23} \mathrm{H}_{19} \mathrm{~N}_{3} \mathrm{l}[\mathrm{M}+\mathrm{H}]^{+}$464.0624, found 464.0618.<smiles>COc1ccc2c(c1)cc(C1(/C=C/c3ccccc3)CC1)n2-c1ncccn1</smiles>

(E)-5-Methoxyl-1-(pyrimidin-2-yl)-2-(1-styrylcyclopropyl)-1H-indole (186ia)

The general procedure $\mathbf{G}$ was followed using 5-methoxyl-1-(pyrimidin-2-yl)-1H-indole 71i (44.8 mg, $0.20 \mathrm{mmol}$ ) and (2-cyclopropylideneethyl)benzene 185a (46.1 mg, $0.32 \mathrm{mmol}$ ) for $3.0 \mathrm{~h}$. Isolation by column chromatography ( $n$-hexane/EtOAc 15:1) yielded 186ia (61.7 
mg, $54 \%)$ as a yellow oil. ${ }^{1} \mathrm{H}$ NMR $\left(400 \mathrm{MHz}, \mathrm{CDCl}_{3}\right) \delta=8.74(\mathrm{~d}, J=4.8 \mathrm{~Hz}, 2 \mathrm{H}), 8.16(\mathrm{~d}$, $J=9.0 \mathrm{~Hz}, 1 \mathrm{H}), 7.25-7.20(\mathrm{~m}, 2 \mathrm{H}), 7.18-7.14(\mathrm{~m}, 3 \mathrm{H}), 7.12(\mathrm{~d}, J=2.5 \mathrm{~Hz}, 1 \mathrm{H}), 7.05(\mathrm{t}, J$ $=4.8 \mathrm{~Hz}, 1 \mathrm{H}), 6.98(\mathrm{dd}, J=9.0,2.6 \mathrm{~Hz}, 1 \mathrm{H}), 6.63(\mathrm{~s}, 1 \mathrm{H}), 6.09(\mathrm{~d}, J=16.0 \mathrm{~Hz}, 1 \mathrm{H}), 6.03$ $(\mathrm{d}, J=16.0 \mathrm{~Hz}, 1 \mathrm{H}), 3.93(\mathrm{~s}, 3 \mathrm{H}), 1.49-1.46(\mathrm{~m}, 2 \mathrm{H}), 1.26-1.23(\mathrm{~m}, 2 \mathrm{H}) .{ }^{13} \mathrm{C}$ NMR $(101$ $\left.\mathrm{MHz}, \mathrm{CDCl}_{3}\right) \delta=158.2\left(\mathrm{C}_{\mathrm{q}}\right), 158.0(\mathrm{CH}), 155.4\left(\mathrm{C}_{\mathrm{q}}\right), 143.2\left(\mathrm{C}_{\mathrm{q}}\right), 137.8\left(\mathrm{C}_{\mathrm{q}}\right), 137.0(\mathrm{CH})$, $132.1\left(\mathrm{C}_{q}\right), 129.5\left(\mathrm{C}_{q}\right), 128.4(\mathrm{CH}), 126.9(\mathrm{CH}), 126.5(\mathrm{CH}), 125.7(\mathrm{CH}), 117.2(\mathrm{CH}), 114.7$ $(\mathrm{CH}), 112.4(\mathrm{CH}), 107.6(\mathrm{CH}), 102.5(\mathrm{CH}), 55.9\left(\mathrm{CH}_{3}\right), 23.0\left(\mathrm{C}_{\mathrm{q}}\right), 18.4\left(\mathrm{CH}_{2}\right) .3002,1614$, 1564, 1418, 1341, 1214, 1154, 1030, 951, 801, 737, $688 \mathrm{~cm}^{-1}$. MS (ESI) $\mathrm{m} / z$ (relative intensity) $390(25)[\mathrm{M}+\mathrm{Na}]^{+}, 368(100)[\mathrm{M}+\mathrm{H}]^{+}$. HR-MS (ESI) $\mathrm{m} / \mathrm{z}$ calcd for $\mathrm{C}_{24} \mathrm{H}_{22} \mathrm{~N}_{3} \mathrm{O}$ $[\mathrm{M}+\mathrm{H}]^{+} 368.1757$ found 368.1759 .<smiles>N#Cc1ccc2c(c1)cc(C1(/C=C/c3ccccc3)CC1)n2-c1ncccn1</smiles>

\section{(E)-1-(Pyrimidin-2-yl)-2-(1-styrylcyclopropyl)-1H-indole-5-carbonitrile (186ja)}

The general procedure $\mathbf{G}$ was followed using 1-(pyrimidin-2-yl)-1H-indole-5-carbonitrile $71 \mathrm{j}(43.8 \mathrm{mg}, 0.20 \mathrm{mmol}$ ) and (2-cyclopropylideneethyl)benzene 185a (46.1 mg, $0.32 \mathrm{mmol}$ ) for $3.0 \mathrm{~h}$. Isolation by column chromatography ( $n$-hexane/EtOAc $5: 1$ ) yielded 186ja $(60.2$ mg, $83 \%)$ as a yellow oil. ${ }^{1} \mathrm{H}$ NMR $\left(400 \mathrm{MHz}, \mathrm{CDCl}_{3}\right) \delta=8.80(\mathrm{~d}, J=4.9 \mathrm{~Hz}, 2 \mathrm{H}), 8.09(\mathrm{~d}$, $J=8.7 \mathrm{~Hz}, 1 \mathrm{H}), 7.95(\mathrm{~s}, 1 \mathrm{H}), 7.51(\mathrm{dd}, J=8.6,1.5 \mathrm{~Hz}, 1 \mathrm{H}), 7.23-7.16(\mathrm{~m}, 3 \mathrm{H}), 7.18-7.07$ $(\mathrm{m}, 3 \mathrm{H}), 6.69(\mathrm{~s}, 1 \mathrm{H}), 5.99(\mathrm{~d}, J=16.0 \mathrm{~Hz}, 1 \mathrm{H}), 5.92(\mathrm{~d}, J=16.0 \mathrm{~Hz}, 1 \mathrm{H}), 1.49-1.46(\mathrm{~m}$, $2 \mathrm{H}), 1.26-1.23(\mathrm{~m}, 2 \mathrm{H}) .{ }^{13} \mathrm{C}$ NMR $\left(101 \mathrm{MHz}, \mathrm{CDCl}_{3}\right) \delta=158.4(\mathrm{CH}), 157.6\left(\mathrm{C}_{\mathrm{q}}\right), 145.3\left(\mathrm{C}_{\mathrm{q}}\right)$, $138.9\left(\mathrm{C}_{\mathrm{q}}\right), 137.3\left(\mathrm{C}_{\mathrm{q}}\right), 135.6(\mathrm{CH}), 128.5\left(\mathrm{C}_{\mathrm{q}}\right), 128.4(\mathrm{CH}), 127.6(\mathrm{CH}), 126.8(\mathrm{CH}), 126.0$ $(\mathrm{CH}), 125.7(\mathrm{CH}), 125.4(\mathrm{CH}), 120.6\left(\mathrm{C}_{\mathrm{q}}\right), 118.6(\mathrm{CH}), 114.0(\mathrm{CH}), 106.8(\mathrm{CH}), 104.7\left(\mathrm{C}_{\mathrm{q}}\right)$, $22.4\left(\mathrm{C}_{\mathrm{q}}\right), 18.3\left(\mathrm{CH}_{2}\right)$. IR (ATR): 3025, 2221, 1733, 1564, 1463, 1418, 1375, 1309, 1239, 1040, 952, 808, 743, $690 \mathrm{~cm}^{-1}$. MS (ESI) m/z (relative intensity): $385(100)[\mathrm{M}+\mathrm{Na}]^{+}, 363$ (65) $[\mathrm{M}+\mathrm{H}]^{+}$. HR-MS (ESI) $\mathrm{m} / z$ calcd for $\mathrm{C}_{24} \mathrm{H}_{19} \mathrm{~N}_{4}[\mathrm{M}+\mathrm{H}]^{+} 363.1604$, found 363.1603. 
<smiles>Fc1ccc2cc(C3(/C=C/c4ccccc4)CC3)n(-c3ncccn3)c2c1</smiles>

(E)-6-Fluoro-1-(pyrimidin-2-yl)-2-(1-styrylcyclopropyl)-1H-indole (186ka)

The general procedure $\mathbf{G}$ was followed using 6-fluoro-1-(pyrimidin-2-yl)-1H-indole $\mathbf{7 1 k}$ (42.4 mg, $0.20 \mathrm{mmol}$ ) and (2-cyclopropylideneethyl)benzene 185a (46.1 mg, $0.32 \mathrm{mmol})$ for 3.0 h. Isolation by column chromatography ( $n$-hexane/EtOAc 15:1) yielded 186ka (64.0 mg, $90 \%)$ as a yellow oil. ${ }^{1} \mathrm{H}$ NMR $\left(300 \mathrm{MHz}, \mathrm{CDCl}_{3}\right) \delta=8.76(\mathrm{~d}, \mathrm{~J}=4.8 \mathrm{~Hz}, 2 \mathrm{H}), 7.96(\mathrm{dd}$, $J=10.9,2.4 \mathrm{~Hz}, 1 \mathrm{H}), 7.54(\mathrm{dd}, J=8.6,5.5 \mathrm{~Hz}, 1 \mathrm{H}), 7.26-7.19(\mathrm{~m}, 2 \mathrm{H}), 7.15-7.13(\mathrm{~m}$, 3H), $7.03(\mathrm{td}, J=8.9,2.3 \mathrm{~Hz}, 1 \mathrm{H}), 6.65(\mathrm{~d}, J=0.8 \mathrm{~Hz}, 1 \mathrm{H}), 6.07(\mathrm{~d}, J=16.0 \mathrm{~Hz}, 1 \mathrm{H}), 6.00$ $(\mathrm{d}, J=16.1 \mathrm{~Hz}, 1 \mathrm{H}), 1.51-1.46(\mathrm{~m}, 1 \mathrm{H}), 2.28-1.22(\mathrm{~m}, 2 \mathrm{H}) .{ }^{13} \mathrm{C} \mathrm{NMR}\left(75 \mathrm{MHz}, \mathrm{CDCl}_{3}\right) \delta$ $=160.6\left(\mathrm{~d},{ }^{1} \mathrm{~J}_{\mathrm{C}-\mathrm{F}}=237.4 \mathrm{~Hz}, \mathrm{C}_{\mathrm{q}}\right), 143.2\left(\mathrm{~d},{ }^{4} \mathrm{~J}_{\mathrm{C}-\mathrm{F}}=3.9 \mathrm{~Hz}, \mathrm{C}_{\mathrm{q}}\right), 137.3\left(\mathrm{~d},{ }^{3} \mathrm{~J}_{\mathrm{C}-\mathrm{F}}=12.6 \mathrm{~Hz}\right.$, $\left.\mathrm{C}_{\mathrm{q}}\right), 120.8\left(\mathrm{~d},{ }^{3} \mathrm{~J}_{\mathrm{C}-\mathrm{F}}=10.0 \mathrm{~Hz}, \mathrm{CH}\right), 110.1\left(\mathrm{~d},{ }^{2} \mathrm{~J}_{\mathrm{C}-\mathrm{F}}=24.2 \mathrm{~Hz}, \mathrm{CH}\right), 100.8\left(\mathrm{~d},{ }^{2} J_{\mathrm{C}-\mathrm{F}}=28.3\right.$ $\mathrm{Hz}, \mathrm{CH}) .125 .1\left(\mathrm{~d},{ }^{5} \mathrm{~J}_{\mathrm{C}-\mathrm{F}}=1.4 \mathrm{~Hz}, \mathrm{C}_{\mathrm{q}}\right) 158.1(\mathrm{CH}), 158.1\left(\mathrm{C}_{\mathrm{q}}\right), 137.7\left(\mathrm{C}_{\mathrm{q}}\right), 136.7(\mathrm{CH}), 128.4$ $(\mathrm{CH}), 127.1(\mathrm{CH}), 126.6(\mathrm{CH}), 125.7(\mathrm{CH}), 117.7(\mathrm{CH}), 107.3(\mathrm{CH}), 22.8\left(\mathrm{C}_{\mathrm{q}}\right), 18.4\left(\mathrm{CH}_{2}\right)$. ${ }^{19} \mathrm{~F} \mathrm{NMR}\left(282 \mathrm{MHz}, \mathrm{CDCl}_{3}\right) \delta=-119.48$ (td, $J=10.1,5.5 \mathrm{~Hz}$ ). IR (ATR): 3025, 1734, 1565, 1417, 1357, 1261, 1192, 1150, 936, 813, 739, $689 \mathrm{~cm}^{-1} . \mathbf{M S}$ (ESI) $\mathrm{m} / \mathrm{z}$ (relative intensity): $378(90)[\mathrm{M}+\mathrm{Na}]^{+}, 356(100)[\mathrm{M}+\mathrm{H}]^{+}$. HR-MS (ESI) $\mathrm{m} / \mathrm{z}$ calcd for $\mathrm{C}_{23} \mathrm{H}_{19} \mathrm{~N}_{3} \mathrm{~F}[\mathrm{M}+\mathrm{H}]^{+}$ 356.1563, found 356.1558 .<smiles>Clc1ccc2cc(C3(/C=C/c4ccccc4)CC3)n(-c3ncccn3)c2c1</smiles>

(E)-6-Chloro-1-(pyrimidin-2-yl)-2-(1-styrylcyclopropyl)-1 H-indole (186la) 
The general procedure $\mathbf{G}$ was followed using 6-chloro-1-(pyrimidin-2-yl)-1H-indole $71 \mathrm{I}$ (45.7 mg, $0.20 \mathrm{mmol}$ ) and (2-cyclopropylideneethyl)benzene 185a (46.1 mg, $0.32 \mathrm{mmol}$ ) for $3.0 \mathrm{~h}$. Isolation by column chromatography ( $n$-hexane/EtOAc 15:1) yielded 186la (58.8 mg, $79 \%)$ as a yellow oil. ${ }^{1} \mathrm{H}$ NMR $\left(300 \mathrm{MHz}, \mathrm{CDCl}_{3}\right) \delta=8.77(\mathrm{~d}, J=4.8 \mathrm{~Hz}, 2 \mathrm{H}), 8.19$ (d, $J=1.9 \mathrm{~Hz}, 1 \mathrm{H}), 7.52(\mathrm{~d}, J=8.3 \mathrm{~Hz}, 1 \mathrm{H}), 7.25-7.18(\mathrm{~m}, 3 \mathrm{H}), 7.16-7.10(\mathrm{~m}, 4 \mathrm{H}), 6.63(\mathrm{~s}$, $2 \mathrm{H}), 6.04(\mathrm{~d}, J=16.0 \mathrm{~Hz}, 1 \mathrm{H}), 5.96(\mathrm{~d}, J=16.0 \mathrm{~Hz}, 1 \mathrm{H}), 1.50-1.45(\mathrm{~m}, 2 \mathrm{H}), 1.26-1.21(\mathrm{~m}$, 2H). ${ }^{13} \mathrm{C}$ NMR $\left(101 \mathrm{MHz}, \mathrm{CDCl}_{3}\right) \delta=158.2(\mathrm{CH}), 157.9\left(\mathrm{C}_{\mathrm{q}}\right), 143.5\left(\mathrm{C}_{\mathrm{q}}\right), 137.6\left(\mathrm{C}_{\mathrm{q}}\right), 137.5$ $\left(\mathrm{C}_{\mathrm{q}}\right), 136.5(\mathrm{CH}), 129.0\left(\mathrm{C}_{\mathrm{q}}\right), 128.4(\mathrm{CH}), 127.3\left(\mathrm{C}_{\mathrm{q}}\right), 127.2(\mathrm{CH}), 126.7(\mathrm{CH}), 125.7(\mathrm{CH})$, $122.3(\mathrm{CH}), 121.0(\mathrm{CH}), 117.9(\mathrm{CH}), 113.7(\mathrm{CH}), 107.2(\mathrm{CH}), 22.7\left(\mathrm{C}_{\mathrm{q}}\right), 18.4\left(\mathrm{CH}_{2}\right)$. IR (ATR): 3025, 2221, 1562, 1417, 1350, 1298, 958, 911, 811, 739, $688 \mathrm{~cm}^{-1}$. MS (ESI) m/z (relative intensity): $394(30)\left({ }^{35} \mathrm{Cl}\right)[\mathrm{M}+\mathrm{Na}]^{+}, 372(100)\left({ }^{35} \mathrm{Cl}\right)[\mathrm{M}+\mathrm{H}]^{+}$. HR-MS (ESI) m/z calcd for $\mathrm{C}_{23} \mathrm{H}_{19} \mathrm{~N}_{3}{ }^{35} \mathrm{Cl}[\mathrm{M}+\mathrm{H}]^{+} 372.1262$, found 372.1264.

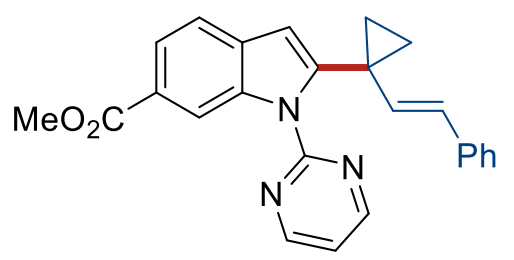

\section{Methyl (E)-1-(pyrimidin-2-yl)-2-(1-styrylcyclopropyl)-1 H-indole-6-carboxylate (186na)}

The general procedure $\mathbf{G}$ was followed using 1-(pyrimidin-2-yl)-1H-indole-6-carboxylate 71n (50.5 mg, $0.20 \mathrm{mmol}$ ) and (2-cyclopropylideneethyl)benzene 185a (46.1 mg, 0.32 mmol) for $3.0 \mathrm{~h}$. Isolation by column chromatography ( $n$-hexane/EtOAc $5: 1$ ) yielded 186 na $(62.5 \mathrm{mg}, 79 \%)$ as a yellow oil. ${ }^{1} \mathrm{H}$ NMR $\left(300 \mathrm{MHz}, \mathrm{CDCl}_{3}\right) \delta=8.80(\mathrm{~d}, J=4.8 \mathrm{~Hz}, 2 \mathrm{H})$, $8.78(\mathrm{~s}, 1 \mathrm{H}), 7.96(\mathrm{dd}, J=8.3,1.5 \mathrm{~Hz}, 1 \mathrm{H}), 7.65(\mathrm{dd}, J=8.3,0.6 \mathrm{~Hz}, 1 \mathrm{H}), 7.24-7.17(\mathrm{~m}$, 2H), 7.16-7.10 (m, 4H), $6.70(\mathrm{~s}, 1 \mathrm{H}), 6.04(\mathrm{~d}, J=16.2 \mathrm{~Hz}, 1 \mathrm{H}), 5.95(\mathrm{~d}, J=16.2 \mathrm{~Hz}, 1 \mathrm{H})$, $3.96(\mathrm{~s}, 3 \mathrm{H}), 1.52-1.46(\mathrm{~m}, 2 \mathrm{H}), 1.27-1.22(\mathrm{~m}, 2 \mathrm{H}) .{ }^{13} \mathrm{C} \mathrm{NMR}\left(101 \mathrm{MHz}, \mathrm{CDCl}_{3}\right) \delta=168.2$ $\left(\mathrm{C}_{\mathrm{q}}\right), 158.4(\mathrm{CH}), 157.8\left(\mathrm{C}_{\mathrm{q}}\right), 146.0\left(\mathrm{C}_{\mathrm{q}}\right), 137.5\left(\mathrm{C}_{\mathrm{q}}\right), 136.6\left(\mathrm{C}_{\mathrm{q}}\right), 135.9(\mathrm{CH}), 132.5\left(\mathrm{C}_{\mathrm{q}}\right)$, 128.4 (CH), $127.4(\mathrm{CH}), 126.7(\mathrm{CH}), 125.7(\mathrm{CH}), 124.7\left(\mathrm{C}_{\mathrm{q}}\right), 122.8(\mathrm{CH}), 119.9(\mathrm{CH}), 118.2$ (CH), $115.3(\mathrm{CH}), 107.0(\mathrm{CH}), 52.0\left(\mathrm{CH}_{3}\right), 22.6\left(\mathrm{C}_{\mathrm{q}}\right), 18.3\left(\mathrm{CH}_{2}\right)$. IR (ATR): 3023, 2949, $1708,1563,1418,1360,1279,1221,1127,1093,956,910,811,735,688 \mathrm{~cm}^{-1}$. MS (ESI) 
m/z (relative intensity): $418(100)[\mathrm{M}+\mathrm{Na}]^{+}, 396(100)[\mathrm{M}+\mathrm{H}]^{+}$. HR-MS (ESI) $\mathrm{m} / \mathrm{z}$ calcd for $\mathrm{C}_{25} \mathrm{H}_{22} \mathrm{~N}_{3} \mathrm{O}_{3}[\mathrm{M}+\mathrm{H}]^{+}$396.1712, found 396.1707.

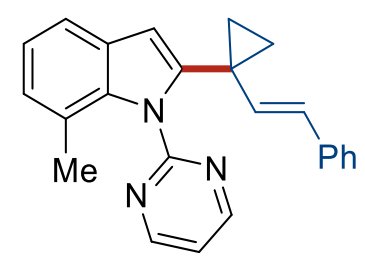

(E)-7-Methyl-1-(pyrimidin-2-yl)-2-(1-styrylcyclopropyl)-1H-indole (1860a)

The general procedure $\mathbf{G}$ was followed using 7-methyl-1-(pyrimidin-2-yl)-1H-indole 710 (41.6 mg, $0.20 \mathrm{mmol})$ and (2-cyclopropylideneethyl)benzene 185a (46.1 mg, $0.32 \mathrm{mmol})$ for $3.0 \mathrm{~h}$. Isolation by column chromatography ( $n$-hexane/EtOAc 15:1) yielded $\mathbf{1 8 6 0 a}(52.7$ mg, $75 \%)$ as a yellow oil. ${ }^{1} \mathrm{H}$ NMR $\left(300 \mathrm{MHz}, \mathrm{CDCl}_{3}\right) \delta=8.78(\mathrm{~d}, J=4.9 \mathrm{~Hz}, 2 \mathrm{H}), 7.54$ (d, $J=7.4 \mathrm{~Hz}, 1 \mathrm{H}), 7.28-7.20(\mathrm{~m}, 2 \mathrm{H}), 7.20-7.12(\mathrm{~m}, 5 \mathrm{H}), 7.04(\mathrm{~d}, J=7.2 \mathrm{~Hz}, 1 \mathrm{H}), 6.63$ (s, $1 \mathrm{H}), 6.01(\mathrm{~d}, J=16.0 \mathrm{~Hz}, 1 \mathrm{H}), 5.83(\mathrm{~d}, J=16.0 \mathrm{~Hz}, 1 \mathrm{H}), 2.00(\mathrm{~s}, 3 \mathrm{H}), 1.45-1.39(\mathrm{~m}, 2 \mathrm{H})$, 1.08-1.01 (m, 2H). ${ }^{13} \mathrm{C}$ NMR $\left(101 \mathrm{MHz}, \mathrm{CDCl}_{3}\right) \delta=159.5\left(\mathrm{C}_{\mathrm{q}}\right), 157.9(\mathrm{CH}), 143.2\left(\mathrm{C}_{\mathrm{q}}\right)$, $137.4\left(\mathrm{C}_{\mathrm{q}}\right), 136.9\left(\mathrm{C}_{\mathrm{q}}\right), 134.9(\mathrm{CH}), 128.9\left(\mathrm{C}_{\mathrm{q}}\right), 128.3(\mathrm{CH}), 127.7(\mathrm{CH}), 126.6(\mathrm{CH}), 125.8$ $(\mathrm{CH}), 125.4(\mathrm{CH}), 121.7\left(\mathrm{C}_{\mathrm{q}}\right), 121.2(\mathrm{CH}), 119.4(\mathrm{CH}), 118.4(\mathrm{CH}), 105.3(\mathrm{CH}), 21.5\left(\mathrm{C}_{\mathrm{q}}\right)$, $19.7\left(\mathrm{CH}_{3}\right), 16.8\left(\mathrm{CH}_{2}\right)$. IR (ATR): 3029, 1644, 1593, 1560, 1415, 1355, 1229, 955, 807, 739, $689 \mathrm{~cm}^{-1}$. MS (ESI) m/z (relative intensity): $374(95)[\mathrm{M}+\mathrm{Na}]^{+}, 352(100)[\mathrm{M}+\mathrm{H}]^{+}$. HRMS (ESI) $m / z$ calcd for $\mathrm{C}_{24} \mathrm{H}_{22} \mathrm{~N}_{3}[\mathrm{M}+\mathrm{H}]^{+} 352.1808$, found 352.1809 .<smiles>O=C1CCCc2c1cc(C1(/C=C/c3ccccc3)CC1)n2-c1ncccn1</smiles>

(E)-1-(Pyrimidin-2-yl)-2-(1-styrylcyclopropyl)-1,5,6,7-tetrahydro-4H-indol-4-one (186pa) 
The general procedure $\mathbf{G}$ was followed using 1-(Pyrimidin-2-yl)-1,5,6,7-tetrahydro-4Hindol-4-one $71 \mathrm{p}(42.5 \mathrm{mg}, 0.20 \mathrm{mmol})$ and (2-cyclopropylideneethyl)benzene 185a (46.1 $\mathrm{mg}, 0.32 \mathrm{mmol}$ ) for $3.0 \mathrm{~h}$. Isolation by column chromatography ( $n$-hexane/EtOAc 2:1) yielded 186pa (54.0 mg, 76\%) as a white solid. M.p. = 157-159 ${ }^{\circ} \mathrm{C} .{ }^{1} \mathbf{H}$ NMR $(400 \mathrm{MHz}$, $\left.\mathrm{CDCl}_{3}\right) \delta=8.75(\mathrm{~d}, J=4.8 \mathrm{~Hz}, 2 \mathrm{H}), 7.22-7.17(\mathrm{~m}, 3 \mathrm{H}), 7.14-7.08(\mathrm{~m}, 3 \mathrm{H}), 6.58(\mathrm{~s}, 1 \mathrm{H})$, $5.95(\mathrm{~d}, J=15.9 \mathrm{~Hz}, 1 \mathrm{H}), 5.75(\mathrm{~d}, J=15.9 \mathrm{~Hz}, 1 \mathrm{H}), 2.92(\mathrm{t}, J=6.2 \mathrm{~Hz}, 2 \mathrm{H}), 2.55$ (dd, $J=$ 7.2, 5.6 Hz, 2H), 2.19-2.13 (m, 2H), 1.32 (dd, $J=3.5,4.5 \mathrm{~Hz}, 2 \mathrm{H}$ ), 1.03 (dd, $J=3.5,4.5$

$\mathrm{Hz}, 2 \mathrm{H}) .{ }^{13} \mathrm{C}$ NMR $\left(101 \mathrm{MHz}, \mathrm{CDCl}_{3}\right) \delta=194.9\left(\mathrm{C}_{\mathrm{q}}\right), 158.4(\mathrm{CH}), 157.3\left(\mathrm{C}_{\mathrm{q}}\right), 145.5\left(\mathrm{C}_{\mathrm{q}}\right)$, $137.8\left(\mathrm{C}_{\mathrm{q}}\right), 137.5\left(\mathrm{C}_{\mathrm{q}}\right), 135.7(\mathrm{CH}), 128.4(\mathrm{CH}), 127.4(\mathrm{CH}), 126.7(\mathrm{CH}), 125.7(\mathrm{CH}), 121.0$ $\left(\mathrm{C}_{\mathrm{q}}\right), 119.5(\mathrm{CH}), 106.9(\mathrm{CH}), 38.0\left(\mathrm{CH}_{2}\right), 23.9\left(\mathrm{CH}_{2}\right), 23.8\left(\mathrm{CH}_{2}\right), 21.2\left(\mathrm{C}_{\mathrm{q}}\right), 17.5\left(\mathrm{CH}_{2}\right)$. IR (ATR): 2942, 1649, 1566, 1415, 1303, 1226, 974, 940, 805, 750, $692 \mathrm{~cm}^{-1}$. MS (ESI) m/z (relative intensity): 733 (100), $378(60)[\mathrm{M}+\mathrm{Na}]^{+}, 356(60)[\mathrm{M}+\mathrm{H}]^{+}$. HR-MS (ESI) m/z calcd for $\mathrm{C}_{23} \mathrm{H}_{22} \mathrm{~N}_{3} \mathrm{O}[\mathrm{M}+\mathrm{H}]^{+}$356.1763, found 356.1768.<smiles>COc1ccc(-c2ccccn2)c(C2(/C=C/c3ccccc3)CC2)c1</smiles>

(E)-2-(4-Methoxy-2-(1-styrylcyclopropyl)phenyl)pyridine (186sa)

The general procedure $\mathbf{G}$ was followed using 2-(4-methoxylphenyl)pyridine 71s (36.8 mg, $0.20 \mathrm{mmol}$ ) and (2-cyclopropylideneethyl)benzene $185 \mathrm{a}(46.1 \mathrm{mg}, 0.32 \mathrm{mmol})$ for $5.0 \mathrm{~h}$. Isolation by column chromatography ( $n$-hexane/EtOAc 10:1) yielded 186sa (26.8 mg, 41\%) as a colorless solid. M.p. $=103-106{ }^{\circ} \mathrm{C} .{ }^{1} \mathrm{H}$ NMR $\left(300 \mathrm{MHz}, \mathrm{CDCl}_{3}\right) \delta=8.71$ (dd, $J=5.0$, $1.9 \mathrm{~Hz}, 1 \mathrm{H}), 7.64(\mathrm{td}, J=7.7,1.9 \mathrm{~Hz}, 1 \mathrm{H}), 7.39(\mathrm{t}, J=8.0 \mathrm{~Hz}, 1 \mathrm{H}), 7.34(\mathrm{~d}, J=7.9 \mathrm{~Hz}, 1 \mathrm{H})$, 7.29-7.17 (m, 6H), $7.14(\mathrm{dd}, J=7.8,1.0 \mathrm{~Hz}, 1 \mathrm{H}), 6.96(\mathrm{dd}, J=8.3,1.0 \mathrm{~Hz}, 1 \mathrm{H}), 5.95(\mathrm{~d}, J$ $=15.9 \mathrm{~Hz}, 1 \mathrm{H}), 5.86(\mathrm{~d}, J=15.9 \mathrm{~Hz}, 1 \mathrm{H}), 3.77(\mathrm{~s}, 3 \mathrm{H}), 1.02-0.97(\mathrm{~m}, 2 \mathrm{H}), 0.76-0.70(\mathrm{~m}$, 2H). ${ }^{13} \mathrm{C}$ NMR (75 MHz, $\left.\mathrm{CDCl}_{3}\right) \delta=157.3\left(\mathrm{C}_{\mathrm{q}}\right), 156.8\left(\mathrm{C}_{\mathrm{q}}\right), 148.7(\mathrm{CH}), 142.0\left(\mathrm{C}_{\mathrm{q}}\right), 139.2$ $(\mathrm{CH}), 137.7\left(\mathrm{C}_{\mathrm{q}}\right), 135.5(\mathrm{CH}), 131.8\left(\mathrm{C}_{\mathrm{q}}\right), 129.1(\mathrm{CH}), 128.4(\mathrm{CH}), 127.5(\mathrm{CH}), 126.6(\mathrm{CH})$, $125.7(\mathrm{CH}), 125.6(\mathrm{CH}), 124.4(\mathrm{CH}), 121.7(\mathrm{CH}), 109.8(\mathrm{CH}), 55.9\left(\mathrm{CH}_{3}\right), 27.7\left(\mathrm{C}_{\mathrm{q}}\right), 16.3$ 
$\left(\mathrm{CH}_{2}\right)$. IR (ATR): 3004, 2937, 2837, 1640, 1574, 1459, 1426, 1352, 1254, 1056, 961, 784,

736, $694 \mathrm{~cm}^{-1}$. MS (ESI) m/z (relative intensity): $350(30)[\mathrm{M}+\mathrm{Na}]^{+}, 328(100)[\mathrm{M}+\mathrm{H}]^{+}$. HR-MS (ESI) $m / z$ calcd for $\mathrm{C}_{23} \mathrm{H}_{22} \mathrm{NO}[\mathrm{M}+\mathrm{H}]^{+} 328.1701$, found 328.1696.<smiles>CC(=O)NCCc1c(C2(/C=C/c3ccccc3)CC2)n(-c2ncccn2)c2ccccc12</smiles>

(E)-N-\{2-[1-(pyrimidin-2-yl)-2-(1-styrylcyclopropyl)-1 H-indol-3-yl]ethyl\}acetamide (186ta)

The general procedure $\mathbf{G}$ was followed using $\mathrm{N}$-(2-(1-(pyrimidin-2-yl)-1H-indol-3yl)ethyl)acetamide 71t (55.9 mg, $0.20 \mathrm{mmol}$ ) and (2-cyclopropylideneethyl)benzene 185a (46.1 mg, $0.32 \mathrm{mmol})$ for $5.0 \mathrm{~h}$. Isolation by column chromatography $\left(\mathrm{CH}_{2} \mathrm{Cl}_{2} / \mathrm{EtOAc} 20: 1\right)$ yielded 186ta (15.2 mg, 18\%) as a yellow oil and 186ta' (28.9 mg, 34\%) as a yellow solid. 186ta: ${ }^{1} \mathrm{H}$ NMR $\left(300 \mathrm{MHz}, \mathrm{CDCl}_{3}\right) \delta=8.82(\mathrm{~d}, J=4.8 \mathrm{~Hz}, 2 \mathrm{H}), 8.09(\mathrm{~d}, J=8.0 \mathrm{~Hz}, 1 \mathrm{H})$, $7.68(\mathrm{~d}, J=6.9 \mathrm{~Hz}, 1 \mathrm{H}), 7.35-7.25(\mathrm{~m}, 6 \mathrm{H}), 7.19(\mathrm{t}, J=4.9 \mathrm{~Hz}, 2 \mathrm{H}), 6.45(\mathrm{~d}, J=15.9 \mathrm{~Hz}$, 1H), $6.33(\mathrm{~d}, J=15.9 \mathrm{~Hz}, 1 \mathrm{H}), 5.62(\mathrm{t}, J=6.6 \mathrm{~Hz}, 1 \mathrm{H}), 3.68(\mathrm{dt}, J=6.6,6.9 \mathrm{~Hz}, 2 \mathrm{H}), 3.15$ (t, $J=6.9 \mathrm{~Hz}, 2 \mathrm{H}), 1.74(\mathrm{~s}, 3 \mathrm{H}), 1.22-1.09(\mathrm{~m}, 2 \mathrm{H}), 1.04-1.09(\mathrm{~m}, 2 \mathrm{H}) .{ }^{13} \mathrm{C}$ NMR $(101 \mathrm{MHz}$, $\left.\mathrm{CDCl}_{3}\right) \delta=170.3\left(\mathrm{C}_{\mathrm{q}}\right), 158.3(\mathrm{CH}), 158.0\left(\mathrm{C}_{\mathrm{q}}\right), 138.4\left(\mathrm{C}_{\mathrm{q}}\right), 137.7\left(\mathrm{C}_{\mathrm{q}}\right), 137.0(\mathrm{CH}), 136.1$ $\left(\mathrm{C}_{\mathrm{q}}\right), 129.1\left(\mathrm{C}_{\mathrm{q}}\right), 128.7(\mathrm{CH}), 127.7(\mathrm{CH}), 127.0(\mathrm{CH}), 126.0(\mathrm{CH}), 123.6(\mathrm{CH}), 121.8(\mathrm{CH})$, $118.9(\mathrm{CH}), 117.7(\mathrm{CH}), 117.0\left(\mathrm{C}_{\mathrm{q}}\right), 113.2(\mathrm{CH}), 39.5\left(\mathrm{CH}_{2}\right), 25.1\left(\mathrm{CH}_{2}\right), 23.3\left(\mathrm{CH}_{3}\right), 21.0$ $\left(\mathrm{C}_{\mathrm{q}}\right), 18.0\left(\mathrm{CH}_{2}\right)$. IR (ATR): 3293, 3051, 2923, 1648, 1562, 1455, 1424, 1356, 1296, 1204 , 963, 744, $696 \mathrm{~cm}^{-1}$. MS (ESI) $\mathrm{m} / \mathrm{z}$ (relative intensity): $445(50)[\mathrm{M}+\mathrm{Na}]^{+}, 423(100)[\mathrm{M}+\mathrm{H}]^{+}$. HR-MS (ESI) $m / z$ calcd for $\mathrm{C}_{27} \mathrm{H}_{27} \mathrm{~N}_{4} \mathrm{O}[\mathrm{M}+\mathrm{H}]^{+} 423.2185$, found 423.2179. 
<smiles>C=C(C)C(Cc1ccccc1)c1c(CCNC(C)C)c2ccccc2n1-c1ccccc1</smiles>

$N-\{2-[2-(3-M e t h y l-1-p h e n y l b u t-3-e n-2-y l)-1-(p y r i m i d i n-2-y l)-1 H$-indol-3-yl]ethyl\} acetamide (186ta')

The general procedure $\mathbf{G}$ was followed using $\mathrm{N}$-(2-(1-(pyrimidin-2-yl)-1H-indol-3yl)ethyl)acetamide 71t (55.9 mg, $0.20 \mathrm{mmol}$ ) and (2-cyclopropylideneethyl)benzene 185a (46.1 mg, $0.32 \mathrm{mmol})$ for $5.0 \mathrm{~h}$. Isolation by column chromatography $\left(\mathrm{CH}_{2} \mathrm{Cl}_{2} / \mathrm{EtOAc} 20: 1\right)$ yielded 186ta (15.2 mg, 18\%) as a yellow oil and 186ta' (28.9 mg, 34\%) as a yellow solid. 186ta': M.p. $=74-75^{\circ} \mathrm{C} .{ }^{1} \mathrm{H}$ NMR $\left(300 \mathrm{MHz}, \mathrm{CDCl}_{3}\right) \delta=8.77(\mathrm{~d}, J=4.8 \mathrm{~Hz}, 2 \mathrm{H}), 7.79$ $7.73(\mathrm{~m}, 1 \mathrm{H}), 7.61(\mathrm{dd}, J=6.3,2.8 \mathrm{~Hz}, 1 \mathrm{H}), 7.24-7.17(\mathrm{~m}, 3 \mathrm{H}), 7.12-7.05(\mathrm{~m}, 3 \mathrm{H}), 7.01-$ $6.96(\mathrm{~m}, 2 \mathrm{H}), 5.46$ (brs, 1H), 4.88 (d, $J=8.5 \mathrm{~Hz}, 2 \mathrm{H}), 4.57$ (t, $J=7.7 \mathrm{~Hz}, 1 \mathrm{H}), 3.53-3.27$ $(\mathrm{m}, 4 \mathrm{H}), 3.03-2.84(\mathrm{~m}, 2 \mathrm{H}), 1.93(\mathrm{~s}, 3 \mathrm{H}), 1.70(\mathrm{~s}, 3 \mathrm{H}) .{ }^{13} \mathrm{C}$ NMR $\left(101 \mathrm{MHz}, \mathrm{CDCl}_{3}\right) \delta=$ $169.9\left(\mathrm{C}_{\mathrm{q}}\right), 158.1(\mathrm{CH}), 158.0\left(\mathrm{C}_{\mathrm{q}}\right), 145.8\left(\mathrm{C}_{\mathrm{q}}\right), 140.5\left(\mathrm{C}_{\mathrm{q}}\right), 137.0\left(\mathrm{C}_{\mathrm{q}}\right), 136.5\left(\mathrm{C}_{\mathrm{q}}\right), 129.4$ $\left(\mathrm{C}_{\mathrm{q}}\right), 128.7(\mathrm{CH}), 128.0(\mathrm{CH}), 125.9(\mathrm{CH}), 123.0(\mathrm{CH}), 121.3(\mathrm{CH}), 118.4(\mathrm{CH}), 117.8(\mathrm{CH})$, $114.9\left(\mathrm{C}_{\mathrm{q}}\right), 112.5(\mathrm{CH}), 110.7\left(\mathrm{CH}_{2}\right), 44.6(\mathrm{CH}), 39.5\left(\mathrm{CH}_{2}\right), 38.7\left(\mathrm{CH}_{2}\right), 24.4\left(\mathrm{CH}_{2}\right), 23.4$ $\left(\mathrm{CH}_{3}\right), 23.1\left(\mathrm{CH}_{3}\right)$. IR (ATR): 3294, 3055, 2927, 1647, 1561, 1454, 1420, 1365, 1263, 1020, 803, 741, $698 \mathrm{~cm}-1$. MS (ESI) m/z (relative intensity): 447 (100) [M+Na] $]^{+}, 425(65)[\mathrm{M}+\mathrm{H}]^{+}$. HR-MS (ESI) $m / z$ calcd for $\mathrm{C}_{27} \mathrm{H}_{29} \mathrm{~N}_{4} \mathrm{O}[\mathrm{M}+\mathrm{H}]^{+} 425.2336$, found 425.2340 .

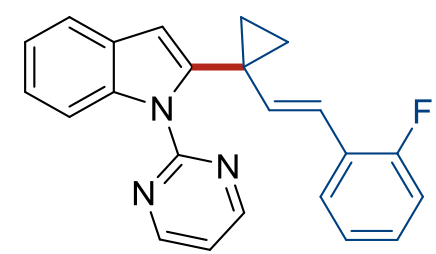

(E)-2-[1-(2-Fluorostyryl)cyclopropyl]-1-(pyrimidin-2-yl)-1 H-indole (186ab) 
The general procedure $\mathbf{G}$ was followed using 1-(pyrimidin-2-yl)-1 $H$-indole (71a) (41.6 mg, $0.20 \mathrm{mmol}$ ) and 1-(2-cyclopropylideneethyl)-2-fluorobenzene 185b (51.9 mg, $0.32 \mathrm{mmol})$ for $3.0 \mathrm{~h}$. Isolation by column chromatography ( $n$-hexane/EtOAc 15:1) yielded 186ab (57.6 $\mathrm{mg}, 81 \%)$ as a yellow oil. ${ }^{1} \mathrm{H}$ NMR $\left(300 \mathrm{MHz}, \mathrm{CDCl}_{3}\right) \delta=8.77(\mathrm{~d}, \mathrm{~J}=4.8 \mathrm{~Hz}, 2 \mathrm{H}), 8.15(\mathrm{dd}$, $J=8.3,1.0 \mathrm{~Hz}, 1 \mathrm{H}), 7.62(\mathrm{~d}, J=7.4 \mathrm{~Hz}, 1 \mathrm{H}), 7.33-7.15(\mathrm{~m}, 3 \mathrm{H}), 7.13-7.05(\mathrm{~m}, 2 \mathrm{H}), 7.01-$ $6.89(\mathrm{~m}, 2 \mathrm{H}), 6.67(\mathrm{~s}, 1 \mathrm{H}), 6.16(\mathrm{~s}, 2 \mathrm{H}), 1.49-1.43(\mathrm{~m}, 2 \mathrm{H}), 1.27-1.22(\mathrm{~m}, 2 \mathrm{H}) .{ }^{13} \mathrm{C}$ NMR $\left(101 \mathrm{MHz}, \mathrm{CDCl}_{3}\right) \delta=159.84\left(\mathrm{~d},{ }^{1} \mathrm{~J}_{\mathrm{C}-\mathrm{F}}=249 \mathrm{~Hz}, \mathrm{C}_{\mathrm{q}}\right), 158.3\left(\mathrm{C}_{\mathrm{q}}\right), 158.1(\mathrm{CH}), 142.6\left(\mathrm{C}_{\mathrm{q}}\right)$, $139.7\left(\mathrm{~d},{ }^{4} \mathrm{~J}_{\mathrm{C}-\mathrm{F}}=5.5 \mathrm{~Hz}, \mathrm{CH}\right), 137.2\left(\mathrm{C}_{\mathrm{q}}\right), 128.1\left(\mathrm{C}_{\mathrm{q}}\right), 127.7\left(\mathrm{~d},{ }^{4} \mathrm{~J}_{\mathrm{C}-\mathrm{F}}=8.0 \mathrm{~Hz}, \mathrm{CH}\right), 127.4$ $\left(\mathrm{d},{ }^{4} \mathrm{~J}_{\mathrm{C}-\mathrm{F}}=4.2 \mathrm{~Hz}, \mathrm{CH}\right), 125.6\left(\mathrm{~d},{ }^{3} \mathrm{~J}_{\mathrm{C}-\mathrm{F}}=12 \mathrm{~Hz}, \mathrm{C}_{\mathrm{q}}\right), 123.9\left(\mathrm{~d},{ }^{4} \mathrm{~J}_{\mathrm{C}-\mathrm{F}}=3.5 \mathrm{~Hz}, \mathrm{CH}\right), 123.2$ $(\mathrm{CH}), 121.8(\mathrm{CH}), 120.3(\mathrm{CH}), 119.8\left(\mathrm{~d},{ }^{4} \mathrm{~J}_{\mathrm{C}-\mathrm{F}}=2.8 \mathrm{~Hz}\right), 117.6(\mathrm{CH}), 115.6\left(\mathrm{~d},{ }^{2} \mathrm{~J}_{\mathrm{C}-\mathrm{F}}=22\right.$ $\mathrm{Hz}, \mathrm{CH}), 113.5(\mathrm{CH}), 107.6(\mathrm{CH}), 23.1\left(\mathrm{C}_{\mathrm{q}}\right), 18.5\left(\mathrm{CH}_{2}\right) .{ }^{19} \mathrm{~F}$ NMR $\left(377 \mathrm{MHz}, \mathrm{CDCl}_{3}\right) \delta=(-$ 117.8) - (-117.9) (m). IR (ATR): 3044, 1644, 1563, 1418, 1352, 1222, 1192, 958, 803, 743, 676, $629 \mathrm{~cm}^{-1}$. MS (ESI) $\mathrm{m} / \mathrm{z}$ (relative intensity): $378(90)[\mathrm{M}+\mathrm{Na}]^{+}, 356(100)[\mathrm{M}+\mathrm{H}]^{+}$. HR-MS (ESI) $m / z$ calcd for $\mathrm{C}_{23} \mathrm{H}_{19} \mathrm{~N}_{3} \mathrm{~F}[\mathrm{M}+\mathrm{H}]^{+} 356.1563$, found 356.1559.

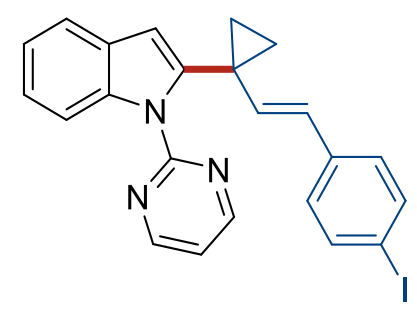

(E)-2-[1-(4-lodostyryl)cyclopropyl]-1-(pyrimidin-2-yl)-1 H-indole (186ac)

The general procedure $\mathbf{G}$ was followed using 1-(pyrimidin-2-yl)-1H-indole 71a (41.6 mg, $0.20 \mathrm{mmol}$ ) and 1-(2-cyclopropylideneethyl)-4-iodobenzene $185 \mathrm{c}(84.4 \mathrm{mg}, 0.32 \mathrm{mmol})$ for 4.0 h. Isolation by column chromatography ( $n$-hexane/EtOAc $15: 1$ ) yielded a mixture of 186ac and 186aa (51.8 mg, 45\% yield for 186ac, 15\% yield for $186 \mathrm{aa}$ ) as a yellow oil. ${ }^{1} \mathrm{H}$ NMR $\left(400 \mathrm{MHz}, \mathrm{CDCl}_{3}\right) \delta=8.75(\mathrm{~d}, J=4.7 \mathrm{~Hz}, 2 \mathrm{H}), 8.13(\mathrm{~d}, J=8.0 \mathrm{~Hz}, 1 \mathrm{H}), 7.62(\mathrm{dd}$, $J=8.0,1.1 \mathrm{~Hz}, 1 \mathrm{H}), 7.51(\mathrm{~d}, J=8.4 \mathrm{~Hz}, 2 \mathrm{H}), 7.24(\mathrm{td}, J=7.5,1.1 \mathrm{~Hz}, 1 \mathrm{H}), 7.10(\mathrm{t}, J=4.8$ $\mathrm{Hz}, 1 \mathrm{H}), 6.86$ (d, J = 8.5 Hz, 2H), 6.66 (s, 0.25H, 186aa), 6.65 (s, 0.75H, 186ac), 5.95 (s, $2 \mathrm{H}), 1.49-1.43(\mathrm{~m}, 2 \mathrm{H}), 1.27-1.22(\mathrm{~m}, 2 \mathrm{H}) .{ }^{13} \mathrm{C}$ NMR $\left(101 \mathrm{MHz}, \mathrm{CDCl}_{3}\right) \delta=158.3\left(\mathrm{C}_{\mathrm{q}}\right)$, 
$158.1(\mathrm{CH}), 142.3\left(\mathrm{C}_{\mathrm{q}}\right), 137.9(\mathrm{CH}), 137.41(\mathrm{CH}), 128.8\left(\mathrm{C}_{\mathrm{q}}\right), 128.4\left(\mathrm{C}_{\mathrm{q}}\right), 127.6(\mathrm{CH}), 126.1$ $\left(\mathrm{C}_{\mathrm{q}}\right), 125.8(\mathrm{CH}), 123.2(\mathrm{CH}), 121.8(\mathrm{CH}), 120.4(\mathrm{CH}), 117.6(\mathrm{CH}), 113.5(\mathrm{CH}), 107.6,91.4$ $\left(\mathrm{C}_{\mathrm{q}}\right), 22.8\left(\mathrm{C}_{\mathrm{q}}\right), 18.5\left(\mathrm{CH}_{2}\right)$. IR (ATR): 3044, 2924, 1710, 1564, 1453, 1424, 1354, 1301, 1218, 1004, 959, 804, 745, $697 \mathrm{~cm}^{-1}$. MS (ESI) m/z (relative intensity): 486 (45) [M+Na]+, 464 (60) [M+H] $]^{+}$. HR-MS (ESI) $\mathrm{m} / z$ calcd for $\mathrm{C}_{23} \mathrm{H}_{19} \mathrm{~N}_{3} \mathrm{I}[\mathrm{M}+\mathrm{H}]^{+} 464.0617$, found 464.0618.

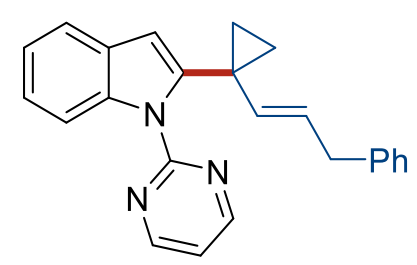

(E)-2-[1-(3-phenylprop-1-en-1-yl)cyclopropyl]-1-(pyrimidin-2-yl)-1 $H$-indole (186ad)

The general procedure $\mathbf{G}$ was followed using 1-(pyrimidin-2-yl)-1H-indole 71a (41.6 mg, $0.20 \mathrm{mmol}$ ) and (3-cyclopropylidenepropyl)benzene 185d (50.6 mg, $0.32 \mathrm{mmol}$ ) for $3.5 \mathrm{~h}$. Isolation by column chromatography ( $n$-hexane/EtOAc 15:1) yielded 186ad (52.0 mg, 74\%) as a colorless solid. M.p. $=81-82^{\circ} \mathrm{C} .{ }^{1} \mathbf{H}$ NMR $\left(300 \mathrm{MHz}, \mathrm{CDCl}_{3}\right) \delta=8.69(\mathrm{~d}, J=4.8 \mathrm{~Hz}$, 2H), $8.04(\mathrm{dd}, J=8.1,1.2 \mathrm{~Hz}, 1 \mathrm{H}), 7.57(\mathrm{~d}, J=6.6 \mathrm{~Hz}, 1 \mathrm{H}), 7.26-7.13(\mathrm{~m}, 5 \mathrm{H}), 7.05(\mathrm{t}, J$ $=4.8 \mathrm{~Hz}, 1 \mathrm{H}), 6.91(\mathrm{~d}, J=6.4 \mathrm{~Hz}, 2 \mathrm{H}), 6.58(\mathrm{~s}, 1 \mathrm{H}), 5.31(\mathrm{~d}, J=15.5 \mathrm{~Hz}, 1 \mathrm{H}), 5.22(\mathrm{dt}, J$ $=15.5,5.9 \mathrm{~Hz}, 1 \mathrm{H}), 3.11(\mathrm{~d}, J=5.9 \mathrm{~Hz}, 2 \mathrm{H}), 1.39-1.35(\mathrm{~m}, 2 \mathrm{H}), 1.10-1.06(\mathrm{~m}, 2 \mathrm{H})$. ${ }^{13} \mathrm{C}$ NMR $\left(101 \mathrm{MHz}, \mathrm{CDCl}_{3}\right) \delta=158.3\left(\mathrm{C}_{\mathrm{q}}\right), 157.9(\mathrm{CH}), 143.2\left(\mathrm{C}_{\mathrm{q}}\right), 140.9\left(\mathrm{C}_{\mathrm{q}}\right), 137.3\left(\mathrm{C}_{\mathrm{q}}\right)$, $137.2(\mathrm{CH}), 128.7\left(\mathrm{C}_{\mathrm{q}}\right), 128.5(\mathrm{CH}), 128.3(\mathrm{CH}), 126.5(\mathrm{CH}), 125.8(\mathrm{CH}), 123.0(\mathrm{CH}), 121.6$ $(\mathrm{CH}), 120.2(\mathrm{CH}), 117.5(\mathrm{CH}), 113.2(\mathrm{CH}), 106.8(\mathrm{CH}), 38.8\left(\mathrm{C}_{\mathrm{q}}\right), 22.0\left(\mathrm{CH}_{2}\right), 17.7\left(\mathrm{CH}_{2}\right)$. IR (ATR): 3026, 1728, 1562, 1418, 1352, 1302, 1198, 1024, 964, 802, 740, 693, $630 \mathrm{~cm}^{-1}$. MS (ESI) m/z (relative intensity): $374(100)[\mathrm{M}+\mathrm{Na}]^{+}, 352(75)[\mathrm{M}+\mathrm{H}]^{+}$. HR-MS (ESI) m/z calcd for $\mathrm{C}_{24} \mathrm{H}_{22} \mathrm{~N}_{3}[\mathrm{M}+\mathrm{H}]^{+}$352.1814, found 352.1817. 


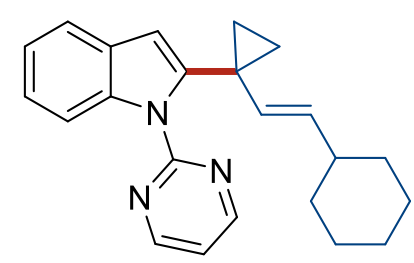

(E)-2-(1-(2-Cyclohexylvinyl)cyclopropyl)-1-(pyrimidin-2-yl)-1H-indole (186ae)

The general procedure $\mathbf{G}$ was followed using 1-(pyrimidin-2-yl)-1H-indole 71a (41.6 mg, $0.20 \mathrm{mmol}$ ) and (2-cyclopropylideneethyl)cyclohexane $185 \mathrm{e}(48.1 \mathrm{mg}, 0.32 \mathrm{mmol})$ for 3.0 h. Isolation by column chromatography ( $n$-hexane/EtOAc 15:1) yielded 186ae (52.9 mg, $77 \%)$ as a colorless oil. ${ }^{1} \mathrm{H}$ NMR $\left(400 \mathrm{MHz}, \mathrm{CDCl}_{3}\right) \delta=8.80(\mathrm{~d}, J=4.8 \mathrm{~Hz}, 2 \mathrm{H}), 8.04(\mathrm{~d}, J$ $=8.1 \mathrm{~Hz}, 1 \mathrm{H}), 7.60(\mathrm{~d}, J=7.7 \mathrm{~Hz}, 1 \mathrm{H}), 7.27$ (ddd, $J=8.4,7.1,1.5 \mathrm{~Hz}, 1 \mathrm{H}), 7.23-7.19(\mathrm{~m}$, 1H), $7.16(\mathrm{t}, J=4.8 \mathrm{~Hz}, 1 \mathrm{H}), 6.56(\mathrm{~s}, 1 \mathrm{H}), 5.07$ (d, $J=15.6 \mathrm{~Hz}, 1 \mathrm{H}), 4.95$ (dd, $J=15.6,7.0$ $\mathrm{Hz}, 1 \mathrm{H}), 1.67-1.54(\mathrm{~m}, 4 \mathrm{H}), 1.44-1.39(\mathrm{~m}, 2 \mathrm{H}), 1.35-1.29(\mathrm{~m}, 2 \mathrm{H}), 1.18-1.00(\mathrm{~m}, 5 \mathrm{H})$, $0.73(\mathrm{ddd}, J=12.2,12.2,3.3 \mathrm{~Hz}, 2 \mathrm{H}) .{ }^{13} \mathrm{C}$ NMR $\left(101 \mathrm{MHz}, \mathrm{CDCl}_{3}\right) \delta=158.4\left(\mathrm{C}_{\mathrm{q}}\right), 158.1$ $(\mathrm{CH}), 143.5\left(\mathrm{C}_{\mathrm{q}}\right), 137.4\left(\mathrm{C}_{\mathrm{q}}\right), 134.1(\mathrm{CH}), 133.0(\mathrm{CH}), 128.7\left(\mathrm{C}_{\mathrm{q}}\right), 122.9(\mathrm{CH}), 121.5(\mathrm{CH})$, $120.2(\mathrm{CH}), 117.5(\mathrm{CH}), 112.9(\mathrm{CH}), 106.2(\mathrm{CH}), 40.7(\mathrm{CH}), 33.2\left(\mathrm{CH}_{2}\right), 26.2\left(\mathrm{CH}_{2}\right), 26.1$ $\left(\mathrm{CH}_{2}\right), 21.7\left(\mathrm{C}_{\mathrm{q}}\right), 17.7\left(\mathrm{CH}_{2}\right)$. IR (ATR): 2925, 1561, 1421, 1351, 1324, 1206, 815, $747 \mathrm{~cm}^{-1}$. MS (ESI) $m / z$ (relative intensity): $366(100)[\mathrm{M}+\mathrm{Na}]^{+}, 344(40)[\mathrm{M}+\mathrm{H}]^{+}$. HR-MS (ESI) m/z calcd for $\mathrm{C}_{23} \mathrm{H}_{26} \mathrm{~N}_{3}[\mathrm{M}+\mathrm{H}]^{+}$344.2127, found 344.2129.

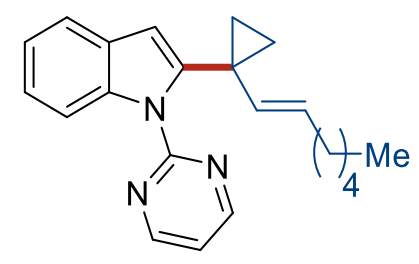

(E)-2-(1-(Hept-1-en-1-yl)cyclopropyl)-1-(pyrimidin-2-yl)-1 H-indole (186af)

The general procedure $\mathbf{G}$ was followed using 1-(pyrimidin-2-yl)-1H-indole 71a (41.6 mg, $0.20 \mathrm{mmol}$ ) and heptylidenecyclopropane $185 \mathrm{f}(44.2 \mathrm{mg}, 0.32 \mathrm{mmol})$ for $4.0 \mathrm{~h}$. Isolation by column chromatography ( $n$-hexane/DCM 8:1) yielded 186ag (41.8 mg, 63\%) as a colorless oil. ${ }^{1} \mathrm{H}$ NMR $\left(300 \mathrm{MHz}, \mathrm{CDCl}_{3}\right) \delta=8.80(\mathrm{~d}, J=4.8 \mathrm{~Hz}, 2 \mathrm{H}), 8.04(\mathrm{~d}, J=8.1 \mathrm{~Hz}, 1 \mathrm{H}), 7.58$ 
(d, $J=7.4 \mathrm{~Hz}, 1 \mathrm{H}), 7.26-7.14(\mathrm{~m}, 3 \mathrm{H}), 6.56(\mathrm{~s}, 1 \mathrm{H}), 5.14(\mathrm{~d}, J=15.5 \mathrm{~Hz}, 1 \mathrm{H}), 5.02(\mathrm{dt}, J=$ 15.5, 6.5 Hz, 1H), $1.72(\mathrm{dt}, J=6.5,6.5 \mathrm{~Hz}, 2 \mathrm{H}), 1.39-1.34(\mathrm{~m}, 2 \mathrm{H}), 1.23-1.16(\mathrm{~m}, 2 \mathrm{H})$, 1.10-0.95 (m, 6H), $0.83(\mathrm{t}, J=7.2 \mathrm{~Hz}, 3 \mathrm{H}) .{ }^{13} \mathrm{C}$ NMR $\left(101 \mathrm{MHz}, \mathrm{CDCl}_{3}\right) \delta=158.3\left(\mathrm{C}_{\mathrm{q}}\right)$, 158.0, $143.4\left(\mathrm{C}_{\mathrm{q}}\right), 137.2\left(\mathrm{C}_{\mathrm{q}}\right), 135.3,128.7\left(\mathrm{C}_{\mathrm{q}}\right), 128.2(\mathrm{CH}), 122.8(\mathrm{CH}), 121.5(\mathrm{CH}), 120.1$ $(\mathrm{CH}), 117.4(\mathrm{CH}), 112.9(\mathrm{CH}), 106.4(\mathrm{CH}), 32.3\left(\mathrm{CH}_{2}\right), 31.2\left(\mathrm{CH}_{2}\right), 29.3\left(\mathrm{CH}_{2}\right), 22.5\left(\mathrm{CH}_{2}\right)$, $21.7\left(\mathrm{C}_{\mathrm{q}}\right), 17.4\left(\mathrm{CH}_{2}\right), 14.0\left(\mathrm{CH}_{3}\right)$. IR (ATR): 2923, 2853, 1564, 1454, 1424, 1354, 1301, 1200, 967, 802, 743, $679 \mathrm{~cm}^{-1}$. MS (ESI) m/z (relative intensity): 354 (90) [M+Na] $]^{+}, 332$ (100) $[\mathrm{M}+\mathrm{H}]^{+}$. HR-MS (ESI) $\mathrm{m} / z$ calcd for $\mathrm{C}_{24} \mathrm{H}_{26} \mathrm{~N}_{3}[\mathrm{M}+\mathrm{H}]^{+}$332.2127, found 332.2133.

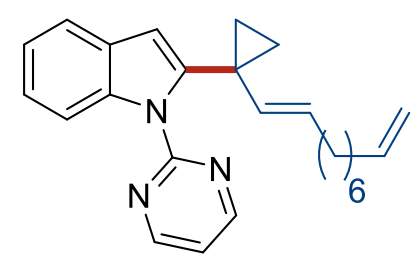

(E)-2-[1-(Deca-1,9-dien-1-yl)cyclopropyl]-1-(pyrimidin-2-yl)-1 H-indole (186ag)

The general procedure $\mathbf{G}$ was followed using 1-(pyrimidin-2-yl)-1H-indole (71a) (41.6 mg, $0.20 \mathrm{mmol})$ and dec-9-en-1-ylidenecyclopropane $185 \mathrm{~g}(57.1 \mathrm{mg}, 0.32 \mathrm{mmol})$ for $4.0 \mathrm{~h}$. Isolation by column chromatography ( $n$-hexane/DCM 8:1) yielded 186ag (58.7 mg, 79\%) as a colorless oil. ${ }^{1} \mathrm{H}$ NMR $\left(400 \mathrm{MHz}, \mathrm{CDCl}_{3}\right) \delta=8.80(\mathrm{~d}, J=4.7 \mathrm{~Hz}, 2 \mathrm{H}), 8.06(\mathrm{~d}, J=8.2$ $\mathrm{Hz}, 1 \mathrm{H}), 7.59$ (dd, $J=7.7,1.3 \mathrm{~Hz}, 1 \mathrm{H}), 7.30-7.25(\mathrm{~m}, 1 \mathrm{H}), 7.20$ (td, $J=7.4,1.1 \mathrm{~Hz}, 1 \mathrm{H}$ ), $7.15(\mathrm{t}, J=4.8 \mathrm{~Hz}, 1 \mathrm{H}), 6.57(\mathrm{~s}, 1 \mathrm{H}), 5.83$ (ddt, $J=17.0,10.3,6.7 \mathrm{~Hz}, 1 \mathrm{H}), 5.15$ (d, $J=$ $15.5 \mathrm{~Hz}, 1 \mathrm{H}), 5.09-4.94(\mathrm{~m}, 3 \mathrm{H}), 2.03(\mathrm{dt}, J=7.2,7.2 \mathrm{~Hz}, 1 \mathrm{H}), 1.74(\mathrm{dt}, J=7.2,7.2 \mathrm{~Hz}$, 1H), 1.39-1.29 (m, 4H), $1.20(\mathrm{tt}, J=9.9,6.6 \mathrm{~Hz}, 2 \mathrm{H}), 1.13-0.99(\mathrm{~m}, 6 \mathrm{H}) .{ }^{13} \mathrm{C}$ NMR $(101$ $\left.\mathrm{MHz}, \mathrm{CDCl}_{3}\right) \delta=158.4\left(\mathrm{C}_{\mathrm{q}}\right), 158.1(\mathrm{CH}), 143.5\left(\mathrm{C}_{\mathrm{q}}\right), 139.3(\mathrm{CH}), 137.3\left(\mathrm{C}_{\mathrm{q}}\right), 135.4(\mathrm{CH})$, $128.8\left(\mathrm{C}_{\mathrm{q}}\right), 128.2(\mathrm{CH}), 122.9(\mathrm{CH}), 121.6(\mathrm{CH}), 120.2(\mathrm{CH}), 117.5(\mathrm{CH}), 114.3\left(\mathrm{C}_{\mathrm{q}}\right), 113.1$ $(\mathrm{CH}), 106.5(\mathrm{CH}), 33.9\left(\mathrm{CH}_{2}\right), 32.4\left(\mathrm{CH}_{2}\right), 29.7\left(\mathrm{CH}_{2}\right), 29.0\left(\mathrm{CH}_{2}\right), 28.9\left(\mathrm{CH}_{2}\right), 28.9\left(\mathrm{CH}_{2}\right)$, $21.8\left(\mathrm{C}_{\mathrm{q}}\right), 17.6\left(\mathrm{CH}_{2}\right)$. IR (ATR): 2923, 2851, 1564, 1453, 1421, 1353, 1198, 965, 908, 802, 742, $679 \mathrm{~cm}^{-1}$. MS (ESI) m/z (relative intensity): $394(18)[\mathrm{M}+\mathrm{Na}]^{+}, 372(100)[\mathrm{M}+\mathrm{H}]^{+}$. HR-MS (ESI) $m / z$ calcd for $\mathrm{C}_{25} \mathrm{H}_{30} \mathrm{~N}_{3}[\mathrm{M}+\mathrm{H}]^{+} 372.2434$, found 372.2433. 


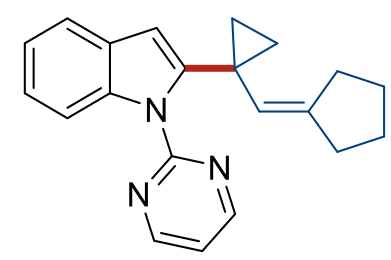

\section{2-[1-(Cyclopentylidenemethyl)cyclopropyl]-1-(pyrimidin-2-yl)-1 H-indole (186ah)}

The general procedure $\mathbf{G}$ was followed using 1-(pyrimidin-2-yl)-1H-indole 71a (41.6 mg, $0.20 \mathrm{mmol}$ ) and (cyclopropylidenemethyl)cyclopentane $185 \mathrm{~h}$ (39.1 $\mathrm{mg}, 0.32 \mathrm{mmol}$ ) for 5.0 h. Isolation by column chromatography ( $n$-hexane/DCM 8:1) yielded 186ah (29.0 mg, 46\%) as a white solid. M.p. $=113-115{ }^{\circ} \mathrm{C} .{ }^{1} \mathbf{H}$ NMR $\left(300 \mathrm{MHz}, \mathrm{CDCl}_{3}\right) \delta=8.84(\mathrm{~d}, \mathrm{~J}=4.8 \mathrm{~Hz}$, 2H), 7.99 (d, J = 7.2 Hz, 1H), $7.54(\mathrm{dd}, J=7.1,1.8 \mathrm{~Hz}, 1 \mathrm{H}), 7.25-7.14(\mathrm{~m}, 3 \mathrm{H}), 6.49$ (s, $1 \mathrm{H}), 5.18(\mathrm{~d}, J=2.5 \mathrm{~Hz}, 1 \mathrm{H}), 2.04-1.93(\mathrm{~m}, 4 \mathrm{H}), 1.55-1.45(\mathrm{~m}, 2 \mathrm{H}), 1.45-1.37(\mathrm{~m}, 2 \mathrm{H})$, 1.33-1.27 (m, 2H), 1.00-0.93 (m, 2H). ${ }^{13} \mathrm{C}$ NMR (101 MHz, $\left.\mathrm{CDCl}_{3}\right) \delta=158.5\left(\mathrm{C}_{\mathrm{q}}\right), 158.2$ $(\mathrm{CH}), 146.7\left(\mathrm{C}_{\mathrm{q}}\right), 145.4\left(\mathrm{C}_{\mathrm{q}}\right), 137.0\left(\mathrm{C}_{\mathrm{q}}\right), 128.9\left(\mathrm{C}_{\mathrm{q}}\right), 124.1(\mathrm{CH}), 122.6(\mathrm{CH}), 121.5(\mathrm{CH})$, $120.0(\mathrm{CH}), 117.5(\mathrm{CH}), 112.9(\mathrm{CH}), 105.0(\mathrm{CH}), 34.4\left(\mathrm{CH}_{2}\right), 28.6\left(\mathrm{CH}_{2}\right), 26.8\left(\mathrm{CH}_{2}\right), 26.0$ $\left(\mathrm{CH}_{2}\right), 19.9\left(\mathrm{C}_{q}\right), 17.9\left(\mathrm{CH}_{2}\right)$. IR (ATR): 2921, 2855, 1563, 1455, 1422, 1349, 1318, 1283, 1195, 1022, 803, 744, $680 \mathrm{~cm}^{-1}$. MS (ESI) m/z (relative intensity): 338 (30) $[\mathrm{M}+\mathrm{Na}]^{+}, 316$ (100) $[\mathrm{M}+\mathrm{H}]^{+}$. HR-MS (ESI) $\mathrm{m} / z$ calcd for $\mathrm{C}_{21} \mathrm{H}_{22} \mathrm{~N}_{3}[\mathrm{M}+\mathrm{H}]^{+} 316.1814$, found 316.1802.

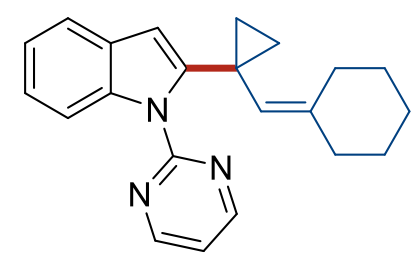

\section{2-(1-(Cyclohexylidenemethyl)cyclopropyl)-1-(pyrimidin-2-yl)-1H-indole (186ai)}

The general procedure $\mathbf{G}$ was followed using 1-(pyrimidin-2-yl)-1H-indole 71a (41.6 mg, $0.20 \mathrm{mmol}$ ) and (cyclopropylidenemethyl)cyclohexane $185 \mathrm{i}(43.6 \mathrm{mg}, 0.32 \mathrm{mmol})$ for 5.0 h. Isolation by column chromatography ( $n$-hexane $/ \mathrm{CH}_{2} \mathrm{Cl}_{2} 8: 1$ ) yielded 186ai (34.3 mg, $52 \%)$ as a colorless oil. ${ }^{1} \mathrm{H}$ NMR $\left(300 \mathrm{MHz}, \mathrm{CDCl}_{3}\right) \delta=8.85(\mathrm{~d}, J=4.8 \mathrm{~Hz}, 2 \mathrm{H}), 7.98(\mathrm{~d}, J$ 
$=7.6 \mathrm{~Hz}, 1 \mathrm{H}), 7.59-7.49(\mathrm{~m}, 1 \mathrm{H}), 7.25-7.13(\mathrm{~m}, 3 \mathrm{H}), 6.49(\mathrm{~d}, J=0.8 \mathrm{~Hz}, 1 \mathrm{H}), 5.08(\mathrm{~s}, 1 \mathrm{H})$, 2.23-2.10 (m, 2H), 1.83-1.72 (m, 2H), 1.37-1.25 (m, 8H), 1.02-0.92 (m, 2H). ${ }^{13} \mathrm{C}$ NMR $\left(101 \mathrm{MHz}, \mathrm{CDCl}_{3}\right) \delta=158.6\left(\mathrm{C}_{\mathrm{q}}\right), 158.2(\mathrm{CH}), 146.5\left(\mathrm{C}_{\mathrm{q}}\right), 144.1\left(\mathrm{C}_{\mathrm{q}}\right), 137.0\left(\mathrm{C}_{\mathrm{q}}\right), 129.0$ $\left(\mathrm{C}_{\mathrm{q}}\right), 125.5(\mathrm{CH}), 122.6(\mathrm{CH}), 121.5(\mathrm{CH}), 120.0(\mathrm{CH}), 117.5(\mathrm{CH}), 112.7(\mathrm{CH}), 104.0(\mathrm{CH})$, 36.8 $\left(\mathrm{CH}_{2}\right), 29.4\left(\mathrm{CH}_{2}\right), 28.6\left(\mathrm{CH}_{2}\right), 27.0\left(\mathrm{CH}_{2}\right), 26.6\left(\mathrm{C}_{\mathrm{q}}\right), 18.2\left(\mathrm{CH}_{2}\right)$. IR (ATR): 2921, 2849 $1559,1454,1420,1352,1262,1196,1024,967,801,737,681 \mathrm{~cm}^{-1}$. MS (ESI) $\mathrm{m} / \mathrm{z}$ (relative intensity): 354 (100) $[\mathrm{M}+\mathrm{Na}]^{+}, 330(25)[\mathrm{M}+\mathrm{H}]^{+}$. HR-MS (ESI) $\mathrm{m} / z$ calcd for $\mathrm{C}_{22} \mathrm{H}_{24} \mathrm{~N}_{3}[\mathrm{M}+\mathrm{H}]^{+}$ 330.1970 , found 330.1965 .

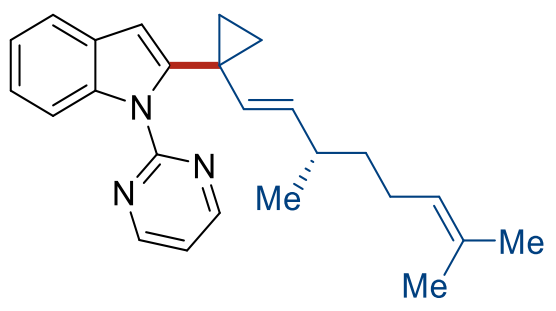

(S,E)-2-[1-(3,7-Dimethylocta-1,6-dien-1-yl)cyclopropyl]-1-(pyrimidin-2-yl)-1 $H$-indole (186aj)

The general procedure $\mathbf{G}$ was followed using 1-(pyrimidin-2-yl)-1H-indole 71a (41.6 mg, $0.20 \mathrm{mmol}$ ) and (S)-(3,7-dimethyloct-6-en-1-ylidene)cyclopropane 185j (57.1 mg, 0.32 mmol) for $7.0 \mathrm{~h}$. Isolation by column chromatography ( $n$-hexane/DCM 8:1) yielded 186aj (45.3 mg, $61 \%)$ as a colorless oil. ${ }^{1} \mathrm{H}$ NMR $\left(400 \mathrm{MHz}, \mathrm{CDCl}_{3}\right) \delta=8.78(\mathrm{~d}, J=4.8 \mathrm{~Hz}, 2 \mathrm{H})$, $8.05(\mathrm{~d}, J=8.2 \mathrm{~Hz}, 1 \mathrm{H}), 7.58(\mathrm{~d}, J=7.6 \mathrm{~Hz}, 1 \mathrm{H}), 7.27-7.13(\mathrm{~m}, 3 \mathrm{H}), 6.56(\mathrm{~s}, 1 \mathrm{H}), 5.08(\mathrm{~d}$, $J=15.5 \mathrm{~Hz}, 1 \mathrm{H}), 4.97(\mathrm{t}, J=7.1 \mathrm{~Hz}, 1 \mathrm{H}), 4.87(\mathrm{dd}, J=15.5,8.1 \mathrm{~Hz}, 1 \mathrm{H}), 1.87-1.78(\mathrm{~m}$, $2 \mathrm{H}), 1.72-1.67(\mathrm{~m}, 1 \mathrm{H}), 1.67(\mathrm{~s}, 3 \mathrm{H}), 1.54(\mathrm{~s}, 3 \mathrm{H}), 1.38-1.34(\mathrm{~m}, 2 \mathrm{H}), 1.08-0.94(\mathrm{~m}, 4 \mathrm{H})$, $0.66(\mathrm{~d}, J=6.7 \mathrm{~Hz}, 3 \mathrm{H}) .{ }^{13} \mathrm{C}$ NMR $\left(101 \mathrm{MHz}, \mathrm{CDCl}_{3}\right) \delta=158.4\left(\mathrm{C}_{\mathrm{q}}\right), 158.1(\mathrm{CH}), 143.6$ $\left(\mathrm{C}_{\mathrm{q}}\right), 137.4\left(\mathrm{C}_{\mathrm{q}}\right), 134.1(\mathrm{CH}), 133.8(\mathrm{CH}), 131.1\left(\mathrm{C}_{\mathrm{q}}\right), 128.8\left(\mathrm{C}_{\mathrm{q}}\right), 125.0(\mathrm{CH}), 122.9(\mathrm{CH})$, $121.6(\mathrm{CH}), 120.2(\mathrm{CH}), 117.5(\mathrm{CH}), 113.1(\mathrm{CH}), 106.5(\mathrm{CH}), 37.1\left(\mathrm{CH}_{2}\right), 36.2(\mathrm{CH}), 25.8$ $\left(\mathrm{CH}_{2}\right), 21.8\left(\mathrm{C}_{\mathrm{q}}\right), 21.0\left(\mathrm{CH}_{3}\right), 18.0\left(\mathrm{CH}_{3}\right), 17.8\left(\mathrm{CH}_{3}\right), 17.5\left(\mathrm{CH}_{2}\right)$. IR (ATR): 2915, 2855, $1564,1453,1423,1353,1199,965,803,743,679 \mathrm{~cm}^{-1}$. MS (ESI) m/z (relative intensity): 
$394(80)[\mathrm{M}+\mathrm{Na}]^{+}, 372(100)[\mathrm{M}+\mathrm{H}]^{+}$. HR-MS (ESI) $\mathrm{m} / z$ calcd for $\mathrm{C}_{25} \mathrm{H}_{30} \mathrm{~N}_{3}[\mathrm{M}+\mathrm{H}]^{+} 372.2440$, found 372.2434 .

\subsubsection{Derivatization of the Diene 184aa}

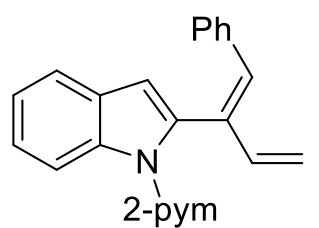

184aa

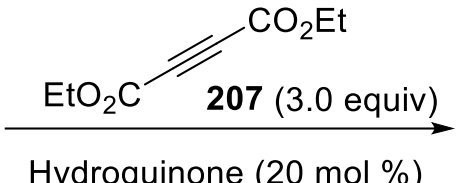

1,4-dioxane, $100^{\circ} \mathrm{C}, 24 \mathrm{~h}$

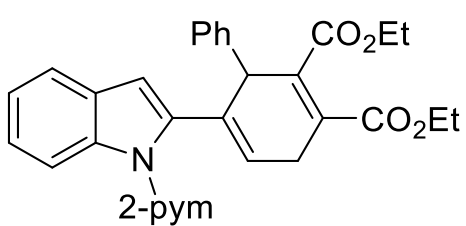

$208,72 \%$

Diethyl-6-[1-(pyrimidin-2-yl)-1H-indol-2-yl]-1,4-dihydro-[1,1'-biphenyl]-2,3dicarboxy-late (208)

To a $25 \mathrm{~mL}$ schlenk tube was added 184aa (135 mg, $0.40 \mathrm{mmol}$ ), diethyl acetylenedicarboxylate 207 (204 mg, 1.2 mmol, 3.0 equiv), hydroquinone (8.8 mg, 0.080 $\mathrm{mmol}, 20 \mathrm{~mol} \%$ ) and 1,4-dioxane $2.0 \mathrm{~mL}$. Then stirred at $100 \stackrel{\circ}{\circ}$ for $24 \mathrm{~h}$. Removed the solvent under vacuum and the residue was purified by column chromatography on silica gel ( $n$-hexane/EtOAc 5:1) affording 208 (140 mg, 72\%) as a yellow solid. M.p. $=149$ $150{ }^{\circ} \mathrm{C} .{ }^{1} \mathrm{H}$ NMR $\left(300 \mathrm{MHz}, \mathrm{CDCl}_{3}\right) \delta=8.83(\mathrm{~d}, \mathrm{~J}=4.8 \mathrm{~Hz}, 2 \mathrm{H}), 8.29(\mathrm{dd}, \mathrm{J}=8.3,1.0 \mathrm{~Hz}$, 1H), $7.44(\mathrm{~d}, \mathrm{~J}=7.7 \mathrm{~Hz}, 1 \mathrm{H}), 7.28-7.22(\mathrm{~m}, 1 \mathrm{H}), 7.21-7.12(\mathrm{~m}, 5 \mathrm{H}), 7.06(\mathrm{dd}, \mathrm{J}=7.8,1.8$ $\mathrm{Hz}, 2 \mathrm{H}), 6.20(\mathrm{~s}, 1 \mathrm{H}), 6.00(\mathrm{dd}, J=4.7,2.7 \mathrm{~Hz}, 1 \mathrm{H}), 4.61(\mathrm{t}, J=5.9 \mathrm{~Hz}, 1 \mathrm{H}), 4.27(\mathrm{q}, J=$ 7.1 Hz, 2H), 4.01 (dddd, $J=17.9,10.8,7.1,3.7 \mathrm{~Hz}, 2 \mathrm{H}$ ), 3.44 (ddd, $J=23.4,6.5,2.8 \mathrm{~Hz}$, $1 \mathrm{H}), 3.23(\mathrm{dt}, J=23.5,5.0 \mathrm{~Hz}, 1 \mathrm{H}), 1.34(\mathrm{t}, J=7.1 \mathrm{~Hz}, 3 \mathrm{H}), 1.02(\mathrm{t}, J=7.1 \mathrm{~Hz}, 3 \mathrm{H}) .{ }^{13} \mathrm{C}$ $\operatorname{NMR}\left(75 \mathrm{MHz}, \mathrm{CDCl}_{3}\right) \delta=167.8\left(\mathrm{C}_{\mathrm{q}}\right), 167.5\left(\mathrm{C}_{\mathrm{q}}\right), 158.4,158.1\left(\mathrm{C}_{\mathrm{q}}\right), 140.2\left(\mathrm{C}_{\mathrm{q}}\right), 139.1\left(\mathrm{C}_{\mathrm{q}}\right)$, $138.3\left(\mathrm{C}_{\mathrm{q}}\right), 137.1\left(\mathrm{C}_{\mathrm{q}}\right), 133.6\left(\mathrm{C}_{\mathrm{q}}\right), 129.8\left(\mathrm{C}_{\mathrm{q}}\right), 129.2\left(\mathrm{C}_{\mathrm{q}}\right), 128.8(\mathrm{CH}), 128.4(\mathrm{CH}), 127.2$ $(\mathrm{CH}), 123.5(\mathrm{CH}), 122.8(\mathrm{CH}), 122.1(\mathrm{CH}), 120.5(\mathrm{CH}), 117.3(\mathrm{CH}), 113.9(\mathrm{CH}), 109.3(\mathrm{CH})$, $61.4\left(\mathrm{CH}_{2}\right), 60.9\left(\mathrm{CH}_{2}\right), 48.2(\mathrm{CH}), 28.8\left(\mathrm{CH}_{2}\right), 14.2\left(\mathrm{CH}_{3}\right), 13.9\left(\mathrm{CH}_{3}\right)$. IR (ATR): 2983, 1715, 1567, 1423, 1344, 1248, 1147, 1068, 810, 744, $705 \mathrm{~cm}^{-1}$. MS (ESI) $\mathrm{m} / \mathrm{z}$ (relative intensity): $516(100)[\mathrm{M}+\mathrm{Na}]^{+}, 494(90)[\mathrm{M}+\mathrm{H}]^{+}$. HR-MS (ESI) $\mathrm{m} / \mathrm{z}$ calcd for $\mathrm{C}_{30} \mathrm{H}_{28} \mathrm{~N}_{3} \mathrm{O}_{4}$ $[\mathrm{M}+\mathrm{H}]^{+}$494.2074, found 494.2072. 


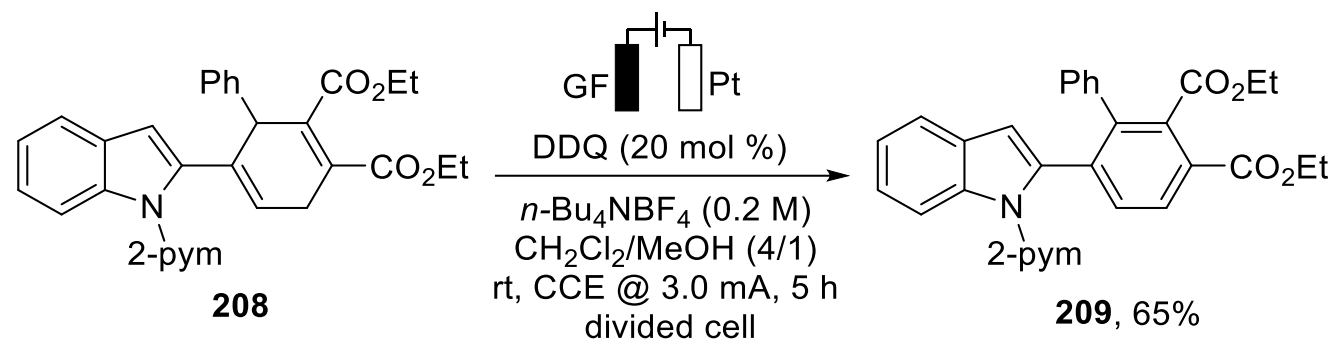

\section{Diethyl 6-[1-(pyrimidin-2-yl)-1 H-indol-2-yl]-[1,1'-biphenyl]-2,3-dicarboxylate (209)}

The electrochemical dehydrogenative annulation of 3 was carried out in a divided cell (P4 glass filter as separator) with a GF anode $(10 \mathrm{~mm} \times 15 \mathrm{~mm} \times 6 \mathrm{~mm})$ and a Pt cathode (10 $\mathrm{mm} \times 15 \mathrm{~mm} \times 0.25 \mathrm{~mm})$. The anodic cell was charged with $208(0.1 \mathrm{mmol}, 49.3 \mathrm{mg}), 2,3-$ dichloro-5,6-dicyano-1,4-benzoquinone (DDQ) $(0.02 \mathrm{mmol}, 20 \mathrm{~mol} \%, 5.5 \mathrm{mg}), n-\mathrm{Bu}_{4} \mathrm{NBF}_{4}$ (1.0 mmol, $32.9 \mathrm{mg}), \mathrm{CH}_{2} \mathrm{Cl}_{2}(4.0 \mathrm{~mL})$ and $\mathrm{MeOH}(1.0 \mathrm{~mL})$. The cathodic cell was charged with $n \mathrm{BuN}_{4} \mathrm{BF}_{4}(1.0 \mathrm{mmol}, 32.9 \mathrm{mg}), \mathrm{CH}_{2} \mathrm{Cl}_{2}(4.0 \mathrm{~mL})$ and $\mathrm{MeOH}(1.0 \mathrm{~mL})$. Electrolysis was performed at $25{ }^{\circ} \mathrm{C}$ with a constant current of $5.0 \mathrm{~mA}$ maintained for $5.0 \mathrm{~h}$. The anodic solution was transferred to a separating funnel charged with $\mathrm{H}_{2} \mathrm{O}(50 \mathrm{~mL})$ and $\mathrm{CH}_{2} \mathrm{Cl}_{2}(50$ $\mathrm{mL}$ ) and the GF anode was rinsed with $\mathrm{CH}_{2} \mathrm{Cl}_{2}(4 \times 10 \mathrm{~mL})$. The organic phase was collected and dried over anhydrous sodium sulfate. The evaporation of the solvent and subsequent column chromatography on silica gel ( $n$-hexane/EtOAc 5:1) afforded 209 (32.1 mg, $65 \%)$ as a yellow solid. ${ }^{1} \mathrm{H}$ NMR $\left(300 \mathrm{MHz}, \mathrm{CDCl}_{3}\right) \delta=8.50(\mathrm{~d}, J=4.8 \mathrm{~Hz}, 2 \mathrm{H}), 8.17$ $(\mathrm{d}, J=8.1 \mathrm{~Hz}, 1 \mathrm{H}), 8.06(\mathrm{~d}, J=8.1 \mathrm{~Hz}, 1 \mathrm{H}), 7.65(\mathrm{~d}, J=8.1 \mathrm{~Hz}, 1 \mathrm{H}), 7.58(\mathrm{~d}, J=7.5 \mathrm{~Hz}$, 1H), 7.26-7.18 (m, 2H), 7.10 (d, J = 7.5 Hz, 1H), 7.06-6.98 (m, 3H), 6.81 (d, J = 7.5 Hz, 2H), 6.60 (s, 1H), 4.40 (q, J = 7.2 Hz, 2H), 3.98 (q, J = 7.2 Hz, 2H), 1.40 (t, J = 7.2 Hz, 3H), $0.90(\mathrm{t}, J=7.2 \mathrm{~Hz}, 3 \mathrm{H}) .{ }^{13} \mathrm{C}$ NMR $\left(101 \mathrm{MHz}, \mathrm{CDCl}_{3}\right) \delta=168.7\left(\mathrm{C}_{\mathrm{q}}\right), 165.9\left(\mathrm{C}_{\mathrm{q}}\right), 157.9(\mathrm{CH})$, $157.2\left(\mathrm{C}_{\mathrm{q}}\right), 139.5\left(\mathrm{C}_{\mathrm{q}}\right), 138.5\left(\mathrm{C}_{\mathrm{q}}\right), 137.8\left(\mathrm{C}_{\mathrm{q}}\right), 137.2\left(\mathrm{C}_{\mathrm{q}}\right), 137.0\left(\mathrm{C}_{\mathrm{q}}\right), 136.3\left(\mathrm{C}_{\mathrm{q}}\right), 130.7$ $(\mathrm{CH}), 129.2(\mathrm{CH}), 129.1(\mathrm{CH}), 129.0\left(\mathrm{C}_{\mathrm{q}}\right), 127.5(\mathrm{CH}), 127.5(\mathrm{CH}), 127.3\left(\mathrm{C}_{\mathrm{q}}\right), 124.0(\mathrm{CH})$, $122.2(\mathrm{CH}), 120.7(\mathrm{CH}), 117.1(\mathrm{CH}), 114.0(\mathrm{CH}), 111.3(\mathrm{CH}), 61.7\left(\mathrm{CH}_{2}\right), 61.3\left(\mathrm{CH}_{2}\right), 14.3$ $\left(\mathrm{CH}_{3}\right), 13.7\left(\mathrm{CH}_{3}\right)$. IR (ATR): 2982, 2259, 1719, 1565, 1422, 1263, 1183, 1147, 1067, 1019, 911, 803, 733, $700 \mathrm{~cm}^{-1}$. MS (ESI) m/z (relative intensity): $514(100)[\mathrm{M}+\mathrm{Na}]^{+}, 492(35)$ $[\mathrm{M}+\mathrm{H}]^{+}, 446$ (35). HR-MS (ESI) $\mathrm{m} / \mathrm{z}$ calcd for $\mathrm{C}_{30} \mathrm{H}_{26} \mathrm{~N}_{3} \mathrm{O}_{4}[\mathrm{M}+\mathrm{H}]^{+}$492.1918, found 492.1912. 


\section{References}

[1] F. Kurzer, P. M. Sanderson, J. Chem. Educ. 1956, 33, 452.

[2] F. Wöhler, Ann. Phys. Chem. 1828, 87, 253.

[3] G. Brundtland, Our Common Future, Oxford University Press, Oxford, 1987

[4] P. Gandeepan, L. H. Finger, T. H. Meyer, L. Ackermann, Chem. Soc. Rev. 2020, 49, 4254-4272.

[5] a) B. M. Trost, Angew. Chem. Int. Ed. 1995, 34, 259-281; b) B. M. Trost, Science 1991, 254, 1471-1477.

[6] P. A. Wender, V. A. Verma, T. J. Paxton, T. H. Pillow, Acc. Chem. Res. 2008, 41, 4049.

[7] a) C. Grondal, M. Jeanty, D. Enders, Nat. Chem. Rev. 2010, 2, 167-178; b) D. Enders, C. Grondal, M. R. M. HHuttl, Angew. Chem. Int. Ed. 2007, 46, 1570-1581; c) L. F. Tietze, Chem. Rev. 1996, 96, 115-136.

[8] a) T. H. Meyer, G. A. Chesnokov, L. Ackermann, ChemSusChem. 2020, 13, 668671; b) C. J. Li, Chem. Rev. 2005, 105, 3095-3165.

[9] a) J. Zhong, X. Yang, Z. Wu, B. Liang, Y. Huang, T. Zhang, Chem. Soc. Rev. 2020, 49, 1385-1413; b) B. Grignard, S. Gennen, C. Jérôme, A. W, Kleij, C. Detrembleur, Chem. Soc. Rev., 2019, 48, 4466-4514; c) M. Aresta, A. Dibenedetto, A. Angelini. Chem. Rev. 2014, 114, 1709-1742.

[10] a) P. T. Anastas, M. M. Kirchhoff, Acc. Chem. Res. 2002, 35, 686-694; b) P. T. Anastas, J. C. Warner, Green chemistry: theory and practice, Oxford University Press, Oxford, 1998.

[11] a) J. Xie, H. Jin, A. S. K. Hashmi, Chem. Soc. Rev. 2017, 46, 5193-5203; b) S. W. M. Crossley, C. Obradors, R. M. Martinez, R. A. Shenvi, Chem. Rev. 2016, 116, 8912-9000; c) Z. Chen, B. Wang, J. Zhang, W. Yu, Z. Liu, Y. Zhang, Org. Chem. Front. 2015, 2, 1107-1295; d) T. Akiyama, K. Mori, Chem. Rev. 2015, 115, 92779306; e) C. C. C. Johansson Seechurn, M. O. Kitching, T. J. Colacot, V. Snieckus, Angew. Chem. Int. Ed. 2012, 51, 5062-5085.

[12] a) S. Bhunia, G. G. Pawar, S. V. Kumar, Y. Jiang, D. Ma, Angew. Chem. Int. Ed. 2017, 56, 16136-16179; b) Q. Wang, Y. Su, L. Li, H. Huang, Chem. Soc. Rev. 2016, 45, 1257-1272; c) M. N. Hopkinson, A. Tlahuext-Aca, F. Glorius, Acc. Chem. Res. 
2016, 49, 2261-2272; d) X.-H. Xu, K. Matsuzaki, N. Shibata, Chem. Rev. 2015, 115, 731-764; e) C. Shen, P. Zhang, Q. Sun, S. Bai, T. S. A. Hor, X. Liu, Chem. Soc. Rev. 2015, 44, 291-314; f) J. W. Beatty, C. R. J. Stephenson, Acc. Chem. Res. 2015, 48, 1474-1484.

[13] C. Glaser, Ber. Dtsch. Chem. Ges. 1869, 2, 422-424.

[14] a) P. E. Fanta, Synthesis. 1974, 9-21; b) F. Ullmann, J. Bielecki, Ber. Dtsch. Chem. Ges. 1901, 34, 2174-2185.

[15] a) R. J. P. Corriu, J. P. Masse, J. Chem. Soc, Chem. Commun. 1972, 144; b) K. Tamao, K. Sumitani, M. Kumada, J. Am. Chem. Soc. 1972, 94, 4374-4376.

[16] R. F. Heck, J. P. Nolley, J. Org. Chem. 1972, 37, 2320-2322.

[17] a) E. Negishi, S. Baba, J. Chem. Soc., Chem. Commun. 1976, 596-597; b) S. Baba, E. Negishi, J. Am. Chem. Soc. 1976, 98, 6729-6731.

[18] D. Milstein, J. K. Stille, J. Am. Chem. Soc. 1978, 100, 3636-3638.

[19] Y. Hatanaka, T. Hiyama, J. Org. Chem. 1988, 53, 918-920.

[20] N. Miyaura, A. Suzuki, Chem. Rev. 1995, 95, 2457-2483.

[21] K. Sonogashira, J. Organomet. Chem. 2002, 653, 46-49.

[22] a) R. D. J. Froese, C. Lombardi, M. Pompeo, R. P. Rucker, M. G. Organ, Acc. Chem. Res. 2017, 50, 2244-2253; b) K. Hirano, M. Miura, Chem. Commun. 2012, 48, 10704-10714; c) J. R. Cox, H. A. Kang, T. Igarashi, T. M. Swager, ACS Macro. Lett. 2012, 1, 334-337; d) J. Magano, J. R. Dunetz, Chem. Rev. 2011, 111, 2177-2250.

[23] The Nobel Prize in Chemistry 2010- Press Release: https://www.nobelprize.org/nobel_prizes/chemistry/laureates/2010/press.html (accessed on 19.09.2020).

[24] Selected reviews: a) P. Gandeepan, T. Mueller, D. Zell, G. Cera, S. Warratz, L. Ackermann, Chem. Rev. 2019, 119, 2192-2452; b) Y. Yang, J. Lan, J. You, Chem. Rev. 2017, 117, 8787-8863; c) J. Wencel-Delord, F. Glorius, Nat. Chem. 2013, 5, 369-375; d) L. Ackermann, Chem. Rev. 2011, 111, 1315-1345; e) D. Balcells, E. Clot, O. Eisenstein, Chem. Rev. 2010, 110, 749-823; f) X. Chen, K. M. Engle, D.H. Wang, J.-Q. Yu, Angew. Chem. Int. Ed. 2009, 48, 5094-5115; g) R. G. Bergman, Nature. 2007, 446, 391-393. h) L. Ackermann, Top. Organomet. Chem. 2007, 24, $35-60$.

[25] a) S. A. Girard, T. Knauber, C.-J. Li, Angew. Chem. Int. Ed. 2014, 53, 74-100; b) C. S. Yeung, V. M. Dong, Chem. Rev. 2011, 111, 1215-1292; c) C.-J. Li, Acc. 
Chem. Res. 2009, 42, 335-344; d) L. Ackermann, R. Vicente, A. R. Kapdi, Angew. Chem. Int. Ed. 2009, 48, 9792-9826.

[26] J. F. Hartwig, J. Am. Chem. Soc. 2016, 138, 2-24.

[27] a) Y. Xia, D. Qiu, J. Wang, Chem. Rev. 2017, 117, 13810-13889; b) F. Hu, Y. Xia, C. Ma, Y. Zhang, J. Wang, Chem. Commun. 2015, 51, 7986-7995; c) H. M. L. Davies, O. Loe, Synthesis 2004, 2595-2608; d) H. M. L. Davies, R. E. J. Beckwith, Chem. Rev. 2003, 103, 2861-2903.

[28] a) H. Yi, G. Zhang, H. Wang, Z. Huang, J. Wang, A. K. Singh, A. Lei, Chem. Rev. 2017, 117, 9016-9085; b) J.-T. Yu, C. Pan, Chem. Commun. 2016, 52, 2220-2236.

[29] a) L. Ackermann, Chem. Rev. 2011, 111, 1315-1345; b) D. Balcells, E. Clot, O. Eisenstein, Chem. Rev. 2010, 110, 749-823; c) Y. Boutadla, D. L. Davies, S. A. Macgregor, A. I. Poblador-Bahamonde, Dalton Trans. 2009, 5820-5831; d) J. Oxgaard, W. J. Tenn, R. J. Nielsen, R. A. Periana, W. A. Goddard, Organometallics 2007, 26, 1565-1567; e) J. A. Labinger, J. E. Bercaw, Nature 2002, 417, 507-514.

[30] a) D. Lapointe, K. Fagnou, Chem. Lett. 2010, 39, 1118-1126; b) S. I. Gorelsky, D. Lapointe, K. Fagnou, J. Am. Chem. Soc. 2008, 130, 10848-10849.

[31] a) Y. Boutadla, D. L. Davies, S. A. Macgregor, A. I. Poblador-Bahamonde, Dalton Trans. 2009, 5887-5893; b) D. L. Davies, S. M. Donald, S. A. Macgregor, J. Am. Chem. Soc. 2005, 127, 13754-13755.

[32] a) E. Tan, O. Quinonero, M. Elena de Orbe, A. M. Echavarren, ACS Catal. 2018, 8, 2166-2172; b) D. Zell, M. Bursch, V. Müller, S. Grimme, L. Ackermann, Angew. Chem. Int. Ed. 2017, 56, 10378-10382; c) D. Santrač, S. Cella, W. Wang, L. Ackermann, Eur. J. Org. Chem. 2016, 5429-5436; d) H. Wang, M. Moselage, M. J. González, L. Ackermann, ACS Catal. 2016, 6, 2705-2709; e) R. Mei, J. Loup, L. Ackermann, ACS Catal. 2016, 6, 793-797; f) C. Tirler, L. Ackermann, Tetrahedron 2015, 71, 4543-4551; g) W. Ma, R. Mei, G. Tenti, L. Ackermann, Chem. Eur. J. 2014, $20,15248-15251$.

[33] X. S. Xue, P. Ji, B. Zhou, J. P. Cheng, Chem. Rev. 2017, 117, 8622-8648.

[34] a) W. Ma, P. Gandeepan, J. Li, L. Ackermann, Org. Chem. Front. 2017, 4, 14351467; b) Z. K. Chen, B. J. Wang, J. T. Zhang, W. L. Yu, Z. X. Liu, Y. H. Zhang, Org. Chem. Front. 2015, 2, 1107-1295; c) L. Ackermann, in Directed Metallation (Ed.: N. Chatani), Springer-Verlag Berlin Heidelberg, Berlin, Heidelberg, 2007, pp. 35-60.

[35] M. Zhang, Y. F. Zhang, X. M. Jie, H. Q. Zhao, G. Li, W. P. Su, Org. Chem. Front. 2014, 1, 843-895. 
[36] a) A. Dey, S. K. Sinha, T. K. Achar, D. Maiti, Angew. Chem. Int. Ed. 2019, 58, 10820-10843; b) U. Dutta, S. Maiti, S. Pimparkar, S. Maiti, L. R. Gahan, E. H. Krenske, D. W. Lupton, D. Maiti, Chem. Sci. 2019, 10, 7426-7432; c) M. Ghosh, S. De Sarkar, Asian J. Org. Chem. 2018, 7, 1236-1255; d) D. Leow, G. Li, T.-S. Mei, J.-Q. Yu, Nature 2012, 486, 518-522.

[37] a) X. Ma, X. Zhao, R. Zhu, D. Zhang, J. Org. Chem. 2020, 85, 5995-6007; b) J. R. Montero Bastidas, T. J. Oleskey, S. L. Miller, M. R. Smith, R. E. Maleczka, J. Am. Chem. Soc. 2019, 141, 15483-15487; c) J. A. Leitch, C. L. McMullin, A. J. Paterson, M. F. Mahon, Y. Bhonoah, C. G. Frost, Angew. Chem. Int. Ed. 2017, 56, 1513115135; d) N. Hofmann, L. Ackermann, J. Am. Chem. Soc. 2013, 135, 5877-5884.

[38] a) M. Kapoor, P. Chand-Thakuri, M. C. Young, J. Am. Chem. Soc. 2019, 141, 79807989; b) Y.-Q. Chen, Z. Wang, Y. Wu, S. R. Wisniewski, J. X. Qiao, W. R. Ewing, M. D. Eastgate, J.-Q. Yu, J. Am. Chem. Soc. 2018, 140, 17884-17894; c) C. Vila, J. Rostoll-Berenguer, R. Sanchez-Garcia, G. Blay, I. Fernandez, M. C. Munoz, J. R. Pedro, J. Org. Chem. 2018, 83, 6397-6407. d) B. Haffemayer, M. Gulias, M. J. Gaunt, Chem. Sci. 2011, 2, 312-315. e) A. Lazareva, O. Daugulis, Org. Lett. 2006, $8,5211-5213$.

[39] https://periodictable.com/Properties/A/CrustAbundance.an.html (accessed on 19. 09. 2020).

[40] L. Mond, F. Quincke, J. Chem. Soc, Trans.1891, 59, 604-607.

[41] M. Berthelot, C. R. Hebd. Seances Acad. Sci. 1891, 112, 1343.

[42] M. Kharasch, E. Fields, J. Am. Chem. Soc. 1941, 63, 2316-2320.

[43] T. J. Kealy, P. L. Pauson, Nature 1951, 168, 1039-1040.

[44] G. Wilkinson, M. Rosenblum, M. Whiting, R. Woodward, J. Am. Chem. Soc. 1952, 74, 2125-2126.

[45] A. Togni, T. Hayashi, Organic Synthesis, Materials Science 1995, 2, 685-721.

[46] a) M. Tamura, J. Kochi, Synthesis. 1971, 1971, 303-305; b) M. Tamura, J. K. Kochi, J. Am. Chem. Soc. 1971, 93, 1487-1489.

[47] N. Yoshikai, E. Nakamura, Chem. Rev. 2012, 112, 2339-2372.

[48] S. T. Kochuveedu, Y. H. Jang, D. H. Kim, Chem. Soc. Rev. 2013, 42, 8467-8493

[49] P. Nordlund, Handbook on Metalloproteins, New York, 2001, 461-570.

[50] a) M. J. Weissenborn, R. M. Koenigs, ChemCatChem 2020, 12, 2171-2179; b) P. L. Holland, Nat. Chem. 2011, 3, 507-508. 
[51] G. Hata, H. Kondo, A. Miyake, J. Am. Chem. Soc. 1968, 90, 2278-2281.

[52] H. H. Karsch, H.-F. Klein, H. Schmidbaur, Angew. Chem. Int. Ed. 1975, 14, 637638.

[53] S. Camadanli, R. Beck, U. Flörke, H.-F. Klein, Organometallics 2009, 28, 2300 2310.

[54] J. Rathke, E. Muetterties, J. Am. Chem. Soc. 1975, 97, 3272-3273.

[55] W. D. Jones, G. P. Foster, J. M. Putinas, J. Am. Chem. Soc. 1987, 109, 5047-5048.

[56] a) N. Kimura, T. Kochi, F. Kakiuchi, J. Am. Chem. Soc. 2017, 139, 14849-14852;

b) A. M. Messinis, L. H. Finger, L. Hu, L. Ackermann, J. Am. Chem. Soc. 2020, 142, 13102-13111.

[57] J. Norinder, A. Matsumoto, N. Yoshikai, E. Nakamura, J. Am. Chem. Soc. 2008, 130, 5858-5859.

[58] E. Nakamura, Two stories of iron https://chemistrycommunity.nature.com/users/209773-eiichi-nakamura/posts/246018two-stories-of-iron (consulted on 22.09.2019)

[59] L. Ilies, E. Konno, Q. Chen, E. Nakamura, Asian. J. Org. Chem. 2012, 1, 142-145.

[60] a) J. J. Sirois, R. Davis, B. DeBoef, Org. Lett. 2014, 16, 868-871; b) N. Yoshikai, A. Matsumoto, J. Norinder, E. Nakamura, Angew. Chem. Int. Ed. 2009, 48, 29252928.

[61] N. Yoshikai, A. Matsumoto, J. Norinder, E. Nakamura, Synlett 2010, 2010, 313316.

[62] L. Ilies, S. Asako, E. Nakamura, J. Am. Chem. Soc. 2011, 133, 7672-7675.

[63] N. Yoshikai, S. Asako, T. Yamakawa, L. llies, E. Nakamura, Chem. - Asian. J. 2011, 6, 3059-3065.

[64] L. Ilies, M. Kobayashi, A. Matsumoto, N. Yoshikai, E. Nakamura, Adv. Synth. Catal. 2012, 354, 593-596.

[65] R. Shang, L. llies, A. Matsumoto, E. Nakamura, J. Am. Chem. Soc. 2013, 135, 6030-6032.

[66] V. G. Zaitsev, D. Shabashov, O. Daugulis, J. Am. Chem. Soc. 2005, 127, 1315413155.

[67] Q. Gu, H. H. Al Mamari, K. Graczyk, E. Diers, L. Ackermann, Angew. Chem. Int. Ed. 2014, 53, 3868-3871. 
[68] a) G. Cera, T. Haven, L. Ackermann, Angew. Chem. Int. Ed. 2016, 55, 1484-1488; b) L. Ilies, S. Ichikawa, S. Asako, T. Matsubara, E. Nakamura, Adv. Synth. Catal. 2015, 357, 2175-2179; c) B. M. Monks, E. R. Fruchey, S. P. Cook, Angew. Chem. Int. Ed. 2014, 126, 11245-11249; d) L. Ilies, T. Matsubara, S. Ichikawa, S. Asako, E. Nakamura, J. Am. Chem. Soc. 2014, 136, 13126-13129; e) E. R. Fruchey, B. M. Monks, S. P. Cook, J. Am. Chem. Soc. 2014, 136, 13130-13133.

[69] G. Cera, T. Haven, L. Ackermann, Chem. Eur. J. 2017, 23, 3577-3582.

[70] T. Matsubara, S. Asako, L. llies, E. Nakamura, J. Am. Chem. Soc. 2014, 136, 646649.

[71] R. Shang, L. Ilies, S. Asako, E. Nakamura, J. Am. Chem. Soc. 2014, 136, 1434914352.

[72] a) J. Mo, T. Müller, J. C. A. Oliveira, S. Demeshko, F. Meyer, L. Ackermann, Angew. Chem. Int. Ed. 2019, 58, 12874-12878; b) J. Mo, T. Mueller, J. C. A. Oliveira, L. Ackermann, Angew. Chem. Int. Ed. 2018, 57, 7719-7723; c) L. Ilies, Y. Arslanoglu, T. Matsubara, E. Nakamura, Asian. J. Org. Chem. 2018, 7, 1327-1329; d) G. Cera, T. Haven, L. Ackermann, Chem. Commun 2017, 53, 6460-6463; e) T. Matsubara, L. Ilies, E. Nakamura, Chem. - Eur. J. 2016, 11, 380-384.

[73] a) R. Shang, L. llies, E. Nakamura, J. Am. Chem. Soc. 2015, 137, 7660-7663; b) K. Graczyk, T. Haven, L. Ackermann, Chem. - Eur. J. 2015, 21, 8812-8815.

[74] M. Y. Wong, T. Yamakawa, N. Yoshikai, Org. Lett. 2015, 17, 442-445.

[75] J. Loup, D. Zell, J. C. A. Oliveira, H. Keil, D. Stalke, L. Ackermann, Angew. Chem. Int. Ed. 2017, 56, 14197-14201.

[76] D. Schmiel, H. Butenschön, Organometallics 2017, 36, 4979-4989.

[77] (a) J. Emsley, Nature's Building Blocks: An A-Z Guide to the Elements 'Manganese', Oxford University Press, Oxford, 2001; (b) N. A. Law, M. T. Caudle, V. L. Pecoraro, in Advances in Inorganic Chemistry; Ed.: A. G. Sykes, Academic Press, 1998, 46, pp. 305-440.

[78] A. Francis, C. Forsyth, Toxicity summary for manganese, 1995.

[79] (a) W. Liu, J. T. Groves, Acc. Chem. Res. 2015, 48, 1727-1735; b) S. M. Paradine, J. R. Griffin, J. Zhao, A. L. Petronico, S. M. Miller, M. C. White, Nat. Chem. 2015, 7, 987-994; (c) X. Huang, T. M. Bergsten, J. T. Groves, J. Am. Chem. Soc. 2015, 137, 5300-5303; d) X. Huang, W. Liu, H. Ren, R. Neelamegam, J. M. Hooker, J. T. Groves, J. Am. Chem. Soc. 2014, 136, 6842-6845; e) W. Liu, X. Huang, J. T. Groves, Nat. Protoc. 2013, 8, 2348-2354; (f) W. Liu, J. T. Groves, Angew. Chem. Int. Ed. 2013, 52, 6024-6027; (g) W. Liu, X. Huang, M.-J. Cheng, R. J. Nielsen, W. 
A. Goddard, J. T. Groves, Science 2012, 337, 1322-1325; (h) J.-L. Liang, J.-S. Huang, X.-Q. Yu, N. Zhu, C.-M. Che, Chem. - Eur. J. 2002, 8, 1563-1572; (i) X.Q. Yu, J.-S. Huang, X.-G. Zhou, C.-M. Che, Org. Lett. 2000, 2, 2233-2236.

[80] M. Bruce, M. Iqbal, F. Stone, J. Chem. Soc. 1970, 3204-3209.

[81] (a) R. C. Cambie, M. R. Metzler, P. S. Rutledge, P. D. Woodgate, J. Organomet. Chem. 1992, 429, 41-57; (b) R. C. Cambie, M. R. Metzler, P. S. Rutledge, P. D. Woodgate, J. Organomet. Chem. 1990, 398, 22-24; (c) R. C. Cambie, M. R. Metzler, P. S. Rutledge, P. D. Woodgate, J. Organomet. Chem. 1990, 381, 26-30.

[83] L. S. Liebeskind, J. R. Gasdaska, J. S. McCallum, S. J. Tremont, J. Org. Chem. 1989, 54, 669-677.

[83] Y. Kuninobu, Y. Nishina, T. Takeuchi, K. Takai, Angew. Chem. Int. Ed. 2007, 46, 6518-6520.

[84] B. Zhou, Y. Hu, C. Wang, Angew. Chem. Int. Ed. 2015, 54, 13659-13663.

[85] Y.-F. Liang, L. Massignan, L. Ackermann, ChemCatChem 2018, 10, 2768-2772. B. Zhou, Y. Hu, T. Liu, C. Wang, Nat. Commun. 2017, 8, 1169.

[86] B. Zhou, P. Ma, H. Chen, C. Wang, Chem. Commun. 2014, 50, 14558-14561.

[87] W. Liu, D. Zell, M. John, L. Ackermann, Angew. Chem. Int. Ed. 2015, 54, 40924096 .

[88] Y.-F. Liang, V. Müller, W. Liu, A. Münch, D. Stalke, L. Ackermann, Angew. Chem. Int. Ed. 2017, 56, 9415-9419.

[89] a) T. H. Meyer, W. Liu, M. Feldt, A. Wuttke, R. A. Mata, L. Ackermann, Chem. - Eur. Chem. 2017, 23, 5443-5447; b) H. Wang, M. M. Lorion, L. Ackermann, Angew. Chem. Int. Ed. 2017, 56, 6339-6342; c) Q. Lu, F. J. R. Klauck, F. Glorius, Chem. Sci. 2017, 8, 3379-3383.

[90] a) W. Wang, P. Subramanian, O. Martinazzoli, J. Wu, L. Ackermann, Chem. Eur. J. 2019, 25, 10585-10589; b) N. Kaplaneris, T. Rogge, R. Yin, H. Wang, G. Sirvinskaite, L. Ackermann, Angew. Chem. Int. Ed. 2019, 131, 3514-3518.

[91] H. Wang, F. Pesciaioli, J. C. A. Oliveira, S. Warratz, L. Ackermann, Angew. Chem. Int. Ed. 2017, 56, 15063-15067.

[92] Q. Lu, S. Greßies, F. J. R. Klauck, F. Glorius, Angew. Chem. Int. Ed. 2017, 56, 6660-6664.

[93] Q. Lu, S. Greßies, S. Cembellín, F. J. R. Klauck, C. G. Daniliuc, F. Glorius, Angew. Chem. Int. Ed. 2017, 56, 12778-12782. 
[94] B. Zhou, H. Chen, C. Wang, J. Am. Chem. Soc. 2013, 135, 1264-1267.

[95] a) C. Zhu, R. Kuniyil, L. Ackermann, Angew. Chem. Int. Ed. 2019, 58, 5338-5342;

b) G. Zheng, J. Sun, Y. Xu, S. Zhai, X. Li, Angew. Chem. Int. Ed. 2019, 58, 5090 5094 .

[96] S.-Y. Chen, Q. Li, H. Wang, J. Org. Chem. 2017, 82, 11173-11181.

[97] a) S.-Y. Chen, X.-L. Han, J.-Q. Wu, Q. Li, Y. Chen, H. Wang, Angew. Chem. Int. Ed. 2017, 56, 9939-9943; b) C. Wang, A. Wang, M. Rueping, Angew. Chem. Int. Ed. 2017, 56, 9935-9938.

[98] S.-Y. Chen, Q. Li, X.-G. Liu, J.-Q. Wu, S.-S. Zhang, H. Wang, ChemSusChem 2017, 10, 2360-2364.

[99] C. Zhu, J. L. Schwarz, S. Cembellín, S. Greßies, F. Glorius, Angew. Chem. Int. Ed. 2018, 57, 437-441.

[100] C. Lei, L. Peng, K. Ding, Adv. Synth. Catal. 2018, 360, 2952-2958.

[101] P. Nuhant, M. S. Oderinde, J. Genovino, A. Juneau, Y. Gagné, C. Allais, G. M. Chinigo, C. Choi, N. W. Sach, L. Bernier, Y. M. Fobian, M. W. Bundesmann, B. Khunte, M. Frenette, O. O. Fadeyi, Angew. Chem. Int. Ed. 2017, 56, 15309-15313.

[102] Y.-F. Liang, R. Steinbock, L. Yang, L. Ackermann, Angew. Chem. Int. Ed. 2018, 57, 10625-10629.

[103] a) W. Liu, S. C. Richter, R. Mei, M. Feldt, L. Ackermann, Chem. Eur. J. 2016, 22 , 17958-17961; b) W. Liu, J. Bang, Y. Zhang, L. Ackermann, Angew. Chem. Int. Ed. 2015, 54, 14137-14140.

[104] J. L. Kiplinger, T. G. Richmond, C. E. Osterberg, Chem. Rev. 1994, 94, 373-431.

[105] a) D. Zell, U. Dhawa, V. Müller, M. Bursch, S. Grimme, L. Ackermann, ACS Catal. 2017, 7, 4209-4213; b) S.-H. Cai, L. Ye, D.-X. Wang, Y.-Q. Wang, L.-J. Lai, C. Zhu, C. Feng, T.-P. Loh, Chem. Commun. 2017, 53, 8731-8734.

[106] W. Liu, G. Cera, J. C. A. Oliveira, Z. Shen, L. Ackermann, Chem. Eur. J. 2017, 23, 11524-11528.

[107] C. Zhu, J. C. A. Oliveira, Z. Shen, H. Huang, L. Ackermann, ACS Catal. 2018, 8, $4402-4407$.

[108] T. Sato, T. Yoshida, H. H. Al Mamari, L. Ilies, E. Nakamura, Org. Lett. 2017, 19, $5458-5461$.

[109] K. Fagnou, M. Lautens, Chem. Rev. 2003, 103, 169-196. 
[110] a) B.-M. Fan, J.-H. Xie, S. Li, L.-X. Wang, Q.-L. Zhou, Angew. Chem. Int. Ed. 2007, 46, 1275-1277; b) P. A. Wender, G. G. Gamber, R. D. Hubbard, L. Zhang, J. Am. Chem. Soc. 2002, 124, 2876-2877; c) K. Tanaka, G. C. Fu, J. Am. Chem. Soc. 2001, 123, 11492-11493; d) P. A. Evans, J. E. Robinson, J. Am. Chem. Soc. 2001, 123, 4609-4610.

[111] a) G.-H. Hou, J.-H. Xie, L.-X. Wang, Q.-L. Zhou, J. Am. Chem. Soc. 2006, 128, 11774-11775; b) A.-G. Hu, Y. Fu, J.-H. Xie, H. Zhou, L.-X. Wang, Q.-L. Zhou, Angew. Chem. Int. Ed. 2002, 41, 2348-2350.

[112] a) R. A. Periana, R. G. Bergman, Organometallics 1984, 3, 508-510; b) W. D. Jones, F. J. Feher, J. Am. Chem. Soc. 1984, 106, 1650-1663.

[113] M. Lin, A. Sen, Nature 1994, 368, 613-615.

[114] a) T. Sakakura, T. Sodeyama, K. Sasaki, K. Wada, M. Tanaka, J. Am. Chem. Soc. 1990, 112, 7221-7229; b) T. Sakakura, M. Tanaka, J. Chem. Soc, Chem. Comm. 1987, 758-759.

[115] G. P. Rosini, W. T. Boese, A. S. Goldman, J. Am. Chem. Soc. 1994, 116, 94989505.

[116] a) K. M. Oberg, E. E. Lee, T. Rovis, Tetrahedron 2009, 65, 5056-5061; b) M. Schäfer, J. Wolf, H. Werner, Dalton. Trans. 2005, 1468-1481; c) L. D. Field, A. J. Ward, P. Turner, Aust. J. Chem. 1999, 52, 1085-1092.

[117] Y.-G. Lim, J.-S. Han, J. Kang, Bull. Korean. Chem. Soc. 1999, 20, 1097-1100.

[118] C.-H. Jun, J.-B. Hong, Y.-H. Kim, K.-Y. Chung, Angew. Chem. Int. Ed. 2000, 39, $3440-3442$.

[119] C.-H. Jun, K.-Y. Chung, J.-B. Hong, Org. Lett. 2001, 3, 785-787.

[120] a) D. A. Colby, R. G. Bergman, J. A. Ellman, J. Am. Chem. Soc. 2008, 130, 36453651; b) C. P. Lenges, M. Brookhart, J. Am. Chem. Soc. 1999, 121, 6616-6623; c) T. Matsubara, N. Koga, D. G. Musaev, K. Morokuma, J. Am. Chem. Soc. 1998, 120, 12692-12693.

[121] Y.-G. Lim, K.-H. Lee, B. T. Koo, J.-B. Kang, Tetrahedr. Lett. 2001, 42, 7609-7612.

[122] T. Katagiri, T. Mukai, T. Satoh, K. Hirano, M. Miura, Chem. Let. 2009, 38, 118-119.

[123] S.-G. Lim, J. H. Lee, C. W. Moon, J.-B. Hong, C.-H. Jun, Org. Lett. 2003, 5, 27592761.

[124] a) K. Ueura, T. Satoh, M. Miura, Org. Lett. 2007, 9, 1407-1409; b) K. Ueura, T. Satoh, M. Miura, J. Org. Chem. 2007, 72, 5362-5367. 
[125] S. Mochida, M. Shimizu, K. Hirano, T. Satoh, M. Miura, Chem. Asian. J. 2010, 5, 847-851.

[126] D. R. Stuart, M. Bertrand-Laperle, K. M. N. Burgess, K. Fagnou, J. Am. Chem. Soc. 2008, 130, 16474-16475.

[127] S. Mochida, N. Umeda, K. Hirano, T. Satoh, M. Miura, Chem. Lett. 2010, 39, 744746.

[128] S. Rakshit, C. Grohmann, T. Besset, F. Glorius, J. Am. Chem. Soc. 2011, 133, 2350-2353.

[129] T. K. Hyster, D. M. Dalton, T. Rovis, Chem. Sci. 2015, 6, 254-258.

[130] S. Cui, Y. Zhang, Q. Wu, Chem. Sci. 2013, 4, 3421-3426.

[131] N. Semakul, K. E. Jackson, R. S. Paton, T. Rovis, Chem. Sci. 2017, 8, 1015-1020.

[132] H. Zhang, K. Wang, B. Wang, H. Yi, F. Hu, C. Li, Y. Zhang, J. Wang, Angew. Chem. Int. Ed. 2014, 53, 13234-13238.

[133] X. Zhou, S. Yu, L. Kong, X. Li, ACS Catal. 2016, 6, 647-651.

[134] D. Bai, T. Xu, C. Ma, X. Zheng, B. Liu, F. Xie, X. Li, ACS Catal. 2018, 8, 4194-4200.

[135] R. B. Dateer, S. Chang, J. Am. Chem. Soc. 2015, 137, 4908-4911.

[136] J. Y. Kim, S. H. Park, J. Ryu, S. H. Cho, S. H. Kim, S. Chang, J. Am. Chem. Soc. 2012, 134, 9110-9113.

[137] X. Huang, Y. Wang, J. Lan, J. You, Angew. Chem. Int. Ed. 2015, 54, 9404-9408.

[138] a) C. Zhou, J. Zhao, W. Guo, J. Jiang, J. Wang, Org. Lett. 2019, 21, 9315-9319; b) X.-H. Hu, X.-F. Yang, T.-P. Loh, ACS Catal. 2016, 6, 5930-5934; c) H. Wang, G. Tang, X. Li, Angew. Chem. Int. Ed. 2015, 54, 13049-13052.

[139] a) X. Quu, P. Wang, D. Wang, M. Wang, Y. Yuan, Z. Shi, Angew. Chem. Int. Ed. 2019, 58, 1504-1508; b) A. J. Borah, Z. Shi, J. Am. Chem. Soc. 2018, 140, 60626066; c) L. Xu, C. Zhang, Y. He, L. Tan, D. Ma, Angew. Chem. Int. Ed. 2016, 55, 321-325.

[140] a) J. Li, L. Shi, S.-P. Zhang, X.-Y. Wang, X. Zhu, X.-Q. Hao, M.-P. Song, J. Org. Chem. 2020, 85, 10835-10845; b) T.-J. Gong, B. Xiao, W.-M. Cheng, W. Su, J. Xu, Z.-J. Liu, L. Liu, Y. Fu, J. Am. Chem. Soc. 2013, 135, 10630-10633; c) M. Chaitanya, D. Yadagiri, P. Anbarasan, Org. Lett. 2013, 15, 4960-4963; d) N. Schröder, J. Wencel-Delord, F. Glorius, J. Am. Chem. Soc. 2012, 134, 8298-8301.

[141] a) D. Gao, X.-Y. Liu, H. Xu, Y.-X. Tan, Q. Liao, Q.-H. Li, X. Yang, G.-Q. Lin, P. Tian, Org. Lett. 2020, 22, 4300-4305; b) X. Wang, A. Lerchen, C. G. Daniliuc, F. 
Glorius, Angew. Chem. Int. Ed. 2018, 57, 1712-1716; c) D.-S. Kong, Y.-F. Wang, Y.-S. Zhao, Q.-H. Li, Y.-X. Chen, P. Tian, G.-Q. Lin, Org. Lett. 2018, 20, $1154-$ 1157; d)X. Zhou, Y. Pan, X. Li, Angew. Chem. Int. Ed. 2017, 56, 8163-8167; e) X. Wang, A. Lerchen, T. Gensch, T. Knecht, C. G. Daniliuc, F. Glorius, Angew. Chem. Int. Ed. 2017, 56, 1381-1384. f) Y. Fukui, P. Liu, Q. Liu, Z.-T. He, N.-Y. Wu, P. Tian, G.-Q. Lin, J. Am. Chem. Soc. 2014, 136, 15607-15614.; g) Z. Qi, X. Li, Angew. Chem. Int. Ed. 2013, 52, 8995-9000.

[142] a) T. H. Meyer, L. H. Finger, P. Gandeepan, L. Ackermann, Trends Chem. 2019, 1, 63-76; b) P. Xiong, H.-C. Xu, Acc. Chem. Soc. 2019, 52, 3339-3350; c) D. Wang, A. B. Weinstein, P. B. White, S. S. Stahl, Chem. Rev. 2018, 118, 2636-2679; d) M. Yan, Y. Kawamata, P. S. Baran, Chem. Rev. 2017, 117, 13230-13319.

[143] a) K.-J. Jiao, Y.-K. Xing, Q.-L. Yang, H. Qiu, T.-S. Mei, Acc. Chem. Soc. 2020, 53, 300-310; b) P. Gandeepan, L. H. Finger, T. H. Meyer, L. Ackermann, Chem. Soc. Rev. 2020, 49, 4254-4272; c) L. Ackermann, Acc. Chem. Res. 2020, 53, 84-104. d) N. Sauermann, T. H. Meyer, Y. Qiu, L. Ackermann, ACS Catal. 2018, 8, 70867103

[144] Y. Qiu, W.-J. Kong, J. Struwe, N. Sauermann, T. Rogge, A. Scheremetjew, L. Ackermann, Angew. Chem. Int. Ed. 2018, 57, 5828-5832.

[145] Y. Zhang, J. Struwe, L. Ackermann, Angew. Chem. Int. Ed. 2020, 59, 15076-15080.

[146] W.-J. Kong, L. H. Finger, J. C. A. Oliveira, L. Ackermann, Angew. Chem. Int. Ed. 2019, 58, 6342-6346.

[147] W.-J. Kong, L. H. Finger, A. M. Messinis, R. Kuniyil, J. C. A. Oliveira, L. Ackermann, J. Am. Chem. Soc. 2019, 141, 17198-17206.

[148] Z.-J. Wu, F. Su, W. Lin, J. Song, T.-B. Wen, H.-J. Zhang, H.-C. Xu, Angew. Chem. Int. Ed. 2019, 58, 16770-16774.

[149] H. Schönherr, T. Cernak, Angew. Chem. Int. Ed. 2013, 52, 12256-12267.

[150] (a) K. Hatzimouratidis,; D. G. Hatzi-Christou, Curr. Pharm. Des. 2009, 15, 34763485. (b) S. Maignan, J.-P. Guilloteau, S. Pouzieux, Y. M. Choi-Sledeski, M. R. Becker, S. I. Klein, W. R. Ewing, H. W. Pauls, A. P. Spada, V. Mikol, J. Med. Chem. 2000, 43, 3226-3232.

[151] a) K. Korvorapun, N. Kaplaneris, T. Rogge, S. Warratz, A. C. Stueckl, L. Ackermann, ACS Catal. 2018, 8, 886-892; b) S.-Y. Zhang, Q. Li, G. He, W. A. Nack, G. Chen, J. Am. Chem. Soc. 2015, 137, 531-539; c) J. Li, S. Warratz, D. Zell, S. De Sarkar, E. E. Ishikawa, L. Ackermann, J. Am. Chem. Soc. 2015, 137, 13894-13901; d) M. Graužinytė, J. Forth, K. A. Rumble, P. S. Clegg, Angew. Chem. Int. Ed. 2015, 127, 
1476-1480; e) R.-Y. Zhu, J. He, X.-C. Wang, J.-Q. Yu, J. Am. Chem. Soc. 2014, 136, 13194-13197; f) S.-Y. Zhang, G. He, W. A. Nack, Y. Zhao, Q. Li, G. Chen, J. Am. Chem. Soc. 2013, 135, 2124-2127; g) B. Xiao, Z.-J. Liu, L. Liu, Y. Fu, J. Am. Chem. Soc. 2013, 135, 616-619; h) N. Hofmann, L. Ackermann, J. Am. Chem. Soc. 2013, 135, 5877-5884; i) K. Gao; N. Yoshikai, J. Am. Chem. Soc. 2013, 135, 92799282; j) D. Shabashov, O. Daugulis, J. Am. Chem. Soc. 2010, 132, 3965-3972.

[152] a) K. A. Bollinger, A. S. Felts, C. J. Brassard, J. L. Engers, A. L. Rodriguez, R. L. Weiner, H. P. Cho, S. Chang, M. Bubser, C. K. Jones, ACS Med. Chem. Lett. 2017, 8, 919-924, b), G. Rosse ACS Med. Chem. Lett. 2016, 7, 1022-1023; c) L. Wang, B. Tan, H. Zhang, Z. Deng, Org. Process Res. Dev. 2013, 17, 1413-1418; d) H. Liu, S. Liu, Z. Miao, Z. Deng, B. Shen, X. Hong, Z. Cheng, J. Med. Chem. 2013, 56, 895-901.

[153] a) H. Ito, K. Ozaki, K. Itami, Angew. Chem. Int. Ed. 2017, 56, 11144-11164; b) A. Narita, X.-Y. Wang, X. Feng, K. Müllen, Chem. Soc. Rev. 2015, 44, 6616-6643; c) M. Ball, Y. Zhong, Y. Wu, C. Schenck, F. Ng, M. Steigerwald, S. Xiao, C. Nuckolls, Acc. Chem. Res. 2015, 48, 267-276.

[154] a) J. You, J. Yin, Angew. Chem. Int. Ed. 2019, 58, 302-306; b) H. Ito, Y. Segawa, K. Murakami, K. Itami, J. Am. Chem. Soc. 2019, 141, 3-10; c) C. Zhu, D. Wang, D. Wang, Y. Zhao, W.-Y. Sun, Z. Shi, Angew. Chem. Int. Ed. 2018, 57, 8848-8853; d) Z. She, Y. Wang, D. Wang, Y. Zhao, T. Wang, X. Zheng, Z. X. Yu, G. Gao, J. You, J. Am. Chem. Soc. 2018, 140, 12566-12573; e) Y. Koga, T. Kaneda, Y. Saito, K. Murakami, K. Itami, Science 2018, 359, 435-439; f) H. Kitano, W. Matsuoka, H. Ito, K. Itami, Chem. Sci. 2018, 9, 7556-7561; g) V. D. Kadam, B. Feng, X. Chen, W. Liang, F. Zhou, Y. Liu, G. Gao, J. You, Org. Lett. 2018, 20, 7071-7075; h) H. Huang, Z. Xu, X. Ji, B. Li, G. J. Deng, Org. Lett. 2018, 20, 4917-4920; i) K. Ozaki, W. Matsuoka, H. Ito, K. Itami, Org. Lett. 2017, 19, 1930-1933; j) W. Matsuoka, H. Ito, K. Itami, Angew. Chem. Int. Ed. 2017, 56, 12224-12228; k) W. C. Fu, Z. Wang, W. T. K. Chan, Z. Lin, F. Y. Kwong, Angew. Chem. Int. Ed. 2017, 56, 7166-7170; I) K. Ozaki, K. Kawasumi, M. Shibata, H. Ito, K. Itami, Nat. Commun. 2015, 6, 6251; m) T. Fujikawa, Y. Segawa, K. Itami, J. Am. Chem. Soc. 2015, 137, 7763-7768; n) J. Dong, Z. Long, F. Song, N. Wu, Q. Guo, J. Lan, J. You, Angew. Chem. Int. Ed. 2013, $52,580-584$.

[155] a) R. Feng, J. A. Smith, K. D. Moeller, Acc. Chem. Res. 2017, 50, 2346-2352; b) A. Misale, S. Niyomchon, N. Maulide, Acc. Chem. Res. 2016, 49, 2444-2458; c) S. Carosso, M. J. Miller, Org. Biomol. Chem. 2014, 12, 7445-7468; d) H. Irschik, P. Washausen, F. Sasse, J. Fohrer, V. Huch, R. Müller, E. V. Prusov, Angew. Chem. 
Int. Ed. 2013, 52, 5402-5405. e) W. Erb, J. Zhu, Nat. Prod. Rep. 2013, 30, $161-$ 174. f) S. D. Rychnovsky, Chem. Rev. 1995, 95, 2021-2040.

[156] a) L. A. Maslovskaya, A. I. Savchenko, C. J. Pierce, G. M. Boyle, V. A. Gordon, P. W. Reddell, P. G. Parsons, C. M. Williams, Chem. - Eur. J. 2019, 25, 1525-1534; b) D. Y. K. Chen, R. H. Pouwer, J.-A. Richard, Chem. Soc. Rev. 2012, 41, 4631-4642.

[157] a) A. M. Olivares, D. J. Weix, J. Am. Chem. Soc. 2018, 140, 2446-2449; b) J. J. Molloy, C. P. Seath, M. J. West, C. McLaughlin, N. J. Fazakerley, A. R. Kennedy, D. J. Nelson, A. J. B. Watson, J. Am. Chem. Soc. 2018, 140, 126-130; c) G.-P. Lu, K. R. Voigtritter, C. Cai, B. H. Lipshutz, Chem. Commun. 2012, 48, 8661-8663; d) G. Wang, S. Mohan, E. Negishi, Proc. Natl. Acad. Sci. USA 2011, 108, 11344-11349; e) S. T. Diver, A. J. Giessert, Chem. Rev. 2004, 104, 1317-1382; f) L. Zhu, R. M. Wehmeyer, R. D. Rieke, J. Org. Chem. 1991, 56, 1445-1453; g) J. K. Stille, B. L. Groh, J. Am. Chem. Soc. 1987, 109, 813-817.

[158] a) A. Clemenceau, P. Thesmar, M. Gicquel, A. Le Flohic, O. Baudoin, J. Am. Chem. Soc. 2020, 142, 15355-15361; b) N. Semakul, K. E. Jackson, R. S. Paton, T. Rovis, Chem. Sci. 2017, 8, 1015-1020; c) P. S. Thuy-Boun, G. Villa, D. Dang, P. Richardson, S. Su, J.-Q. Yu, J. Am. Chem. Soc. 2013, 135, 17508-17513; d) S. Cui, Y. Zhang, Q. Wu, Chem. Sci. 2013, 4, 3421-3426.

[159] A. de Meijere, Angew. Chem. Int. Ed. 1979, 18, 809-826.

[160] a) K. Feng, R. E. Quevedo, J. T. Kohrt, M. S. Oderinde, U. Reilly, M. C. White, Nature 2020, 580, 621-627; b) S. D. Friis, M. J. Johansson, L. Ackermann, Nat. Chem. 2020, 12, 511-519; c) Z.-T. He, H. Li, A. M. Haydl, G. T. Whiteker, J. F. Hartwig, J. Am. Chem. Soc. 2018, 140, 17197-17202; d) C. Le, Y. Liang, R. W. Evans, X. Li, D. W. C. MacMillan, Nature 2017, 547, 79-83.

[161] V. G. Zaitsev, D. Shabashov, O. Daugulis, J. Am. Chem. Soc. 2005, 127, 1315413155.

[162] Z.-J. Wang, Y. Gao, Y.-L. Hou, Z C. hang, S.-J. Yu, Q. Bian, Z.-M. Li, W.-G. Zhao, Eur. J. Med. Chem. 2014, 86, 87-94.

[163] a) W. Ma, P. Gandeepan, J. Li, L. Ackermann, Org. Chem. Front. 2017, 4, 14351467; b) M. R. Yadav, R. K. Rit, M. Shankar, A. K. Sahoo, J. Org. Chem. 2014, 79, 6123-6134; c) F. Zhang, D. R. Spring, Chem. Soc. Rev. 2014, 43, 6906-6919.

[164] Ackermann, L. Chem. Commun. 2010, 46, 4866.

[165] a) Z. Ruan, D. Ghorai, G. Zanoni, L. Ackermann, Chem. Commun. 2017, 53, 91139116; b) Z. Ruan, S. Lackner, L. Ackermann, Angew. Chem. Int. Ed. 2016, 55, 3153- 
3157; c) L. C. M. Castro, N. Chatani, Chem. Lett. 2015, 44, 410-421; d) O. Vechorkin, V. Proust, X. Hu, Angew. Chem. Int. Ed. 2010, 49, 3061-3064.

[166] J. Jayakumar, K. Parthasarathy, Y.-H. Chen, T.-H. Lee, S.-C. Chuang, C.-H. Cheng, Angew. Chem. Int. Ed. 2014, 53, 9889-9892.

[167] Y. Nakao, T. Hiyama, Chem. Soc. Rev. 2011, 40, 4893-4901.

[168] a) R.-Y. Zhu, M. E. Farmer, Y.-Q. Chen, J.-Q. Yu, Angew. Chem. Int. Ed. 2016, 55, 10578-10599; b) L. Xu, Q. Zhu, G. Huang, B. Cheng, Y. Xia, J. Org. Chem. 2012, 77, 3017-3024; c) L. Ackermann, S. Fenner, Org. Lett. 2011, 13, 6548-6551; d) N. Guimond, C. Gouliaras, K. Fagnou, J. Am. Chem. Soc. 2010, 132, 6908-6909. e) F. Yang, L. Ackermann, J. Org. Chem. 2014, 79, 12070-12082.

[169] T. Lomberget, I. Chataigner, D. Bouyssi, J. Maddaluno, G. Balme, Tetrahedr. Lett. 2004, 45, 3437-3441.

[170] Y. Q. Zhu, Y. X. Niu, L. W. Hui, J. L. He, K. Zhu, Adv. Synth. Catal. 2019, 361, 28972903.

[171] E. T. Nadres, G. I. F. Santos, D. Shabashov, O. Daugulis, J. Org. Chem. 2013, 78, 9689-9714.

[172] S. Cai, C. Chen, P. Shao, C. Xi, Org. Lett. 2014, 16, 3142-3145.

[173] N. Martínez-Yáñez, J. Suárez, A. Cajaraville, J. A. Varela, C. Saá, Org. Lett. 2019, 21, 1779-1783.

[174] P. Lu, C. Feng, T.-P. Loh, Org. Lett. 2015, 17, 3210-3213.

[175] a) R. Liu, J. Liu, Y. Wei, M. Shi, Org. Lett. 2019, 21, 4077-4081; b) K. Zhao, R. Du, B. Wang, J. Liu, C. Xia, L. Yang, ACS Catal. 2019, 9, 5545-5551.

[176] J. M. Medina, T. Kang, T. G. Erbay, H. Shao, G. M. Gallego, S. Yang, M. TranDubé, P. F. Richardson, J. Derosa, R. T. Helsel, R. L. Patman, F. Wang, C. P. Ashcroft, J. F. Braganza, I. McAlpine, P. Liu, K. M. Engle, ACS Catal. 2019, 9, 11130-11136.

[177] J. T. Markiewicz, O. Wiest, P. Helquist. J. Org. Chem. 2010, 75, 4887-4890. 


\section{NMR Spectra}
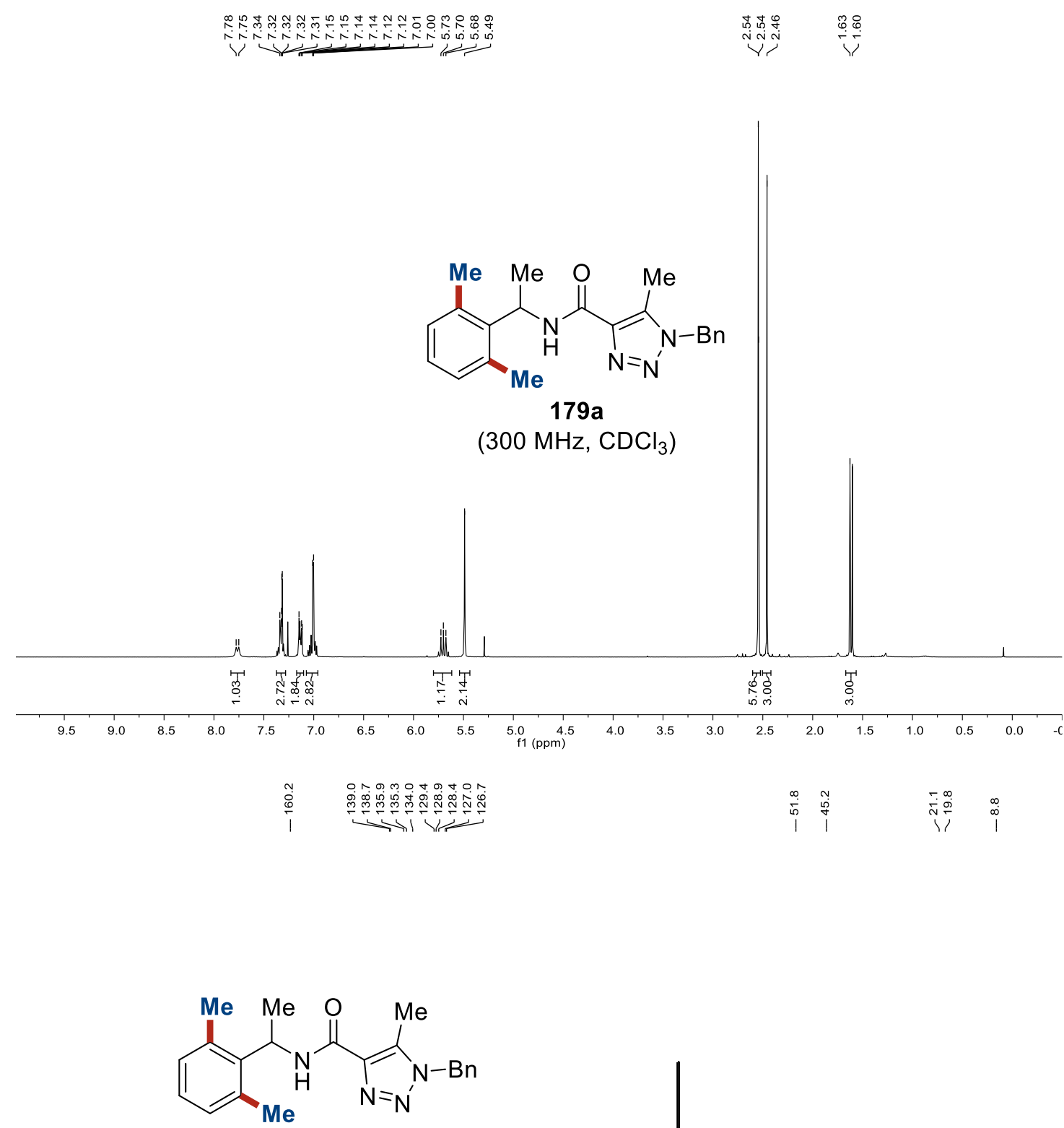

$179 a$

(125 MHz, $\mathrm{CDCl}_{3}$ )

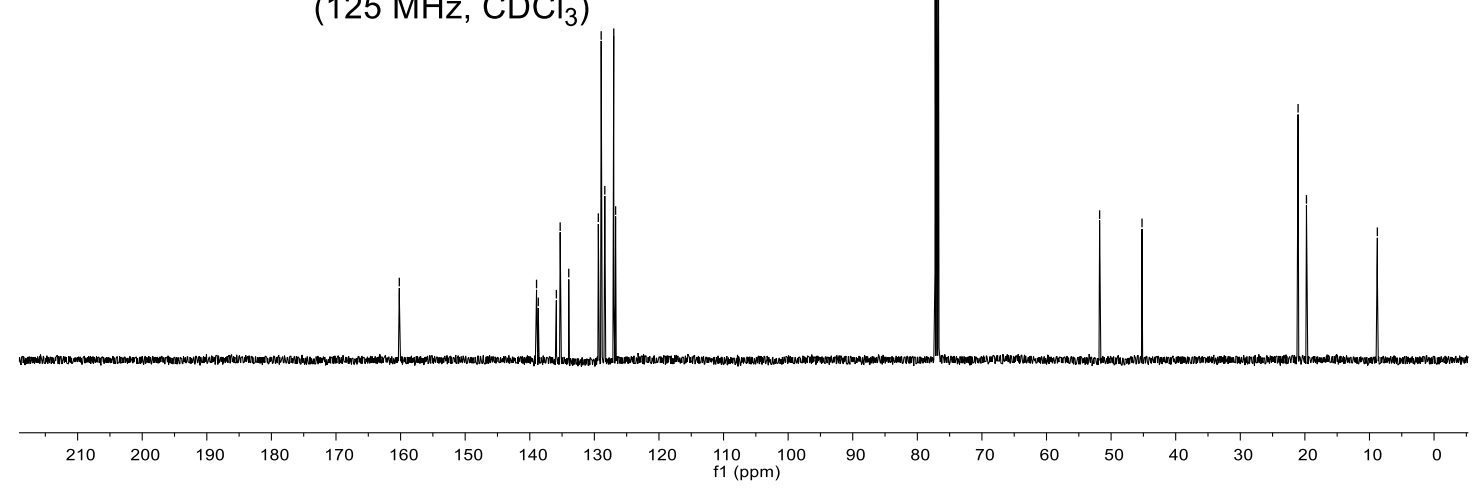


$\underbrace{\underbrace{2}}$

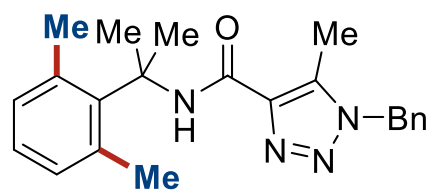

$179 b$

(300 MHz, $\mathrm{CDCl}_{3}$ )
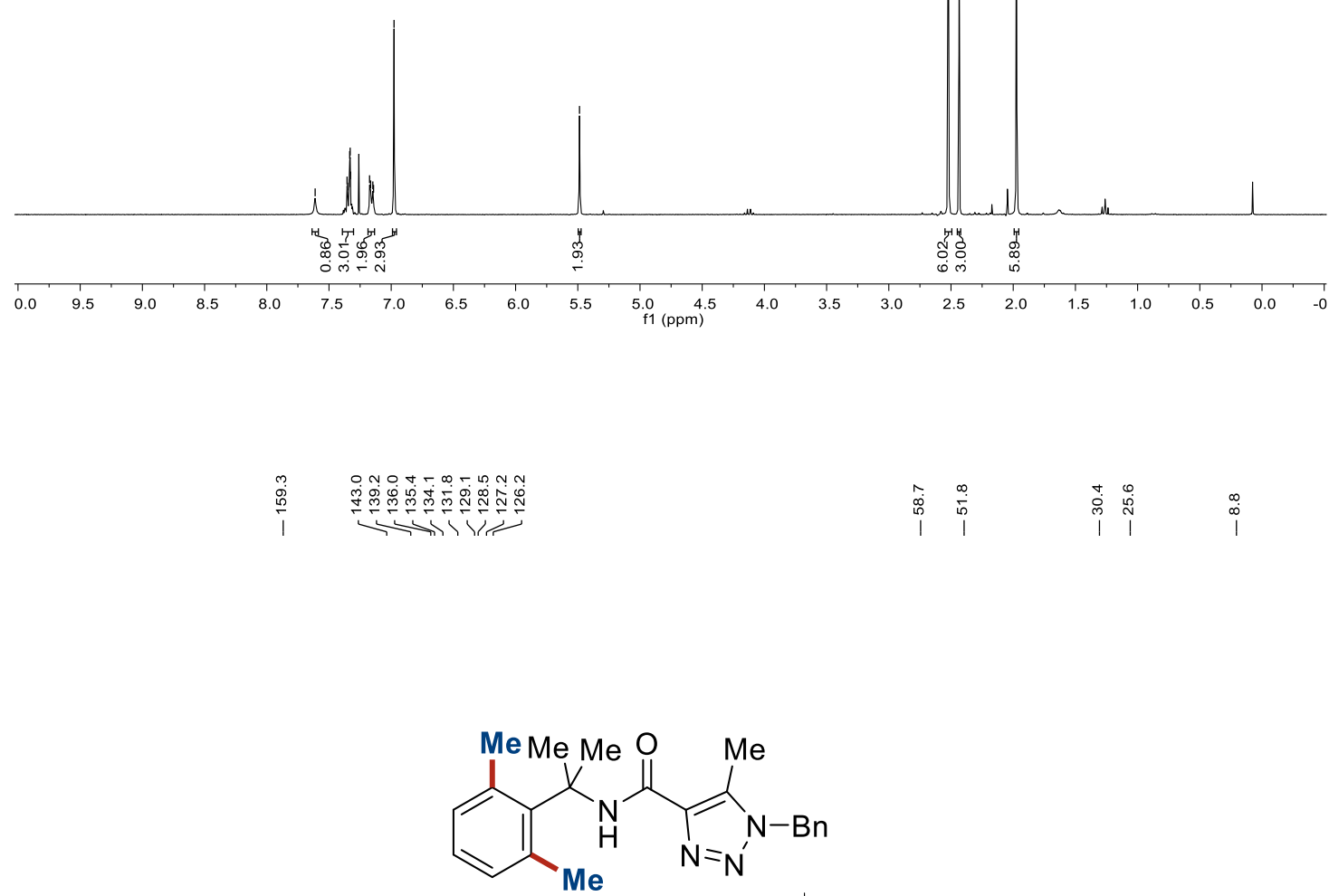

179b

(75 MHz, $\mathrm{CDCl}_{3}$ )
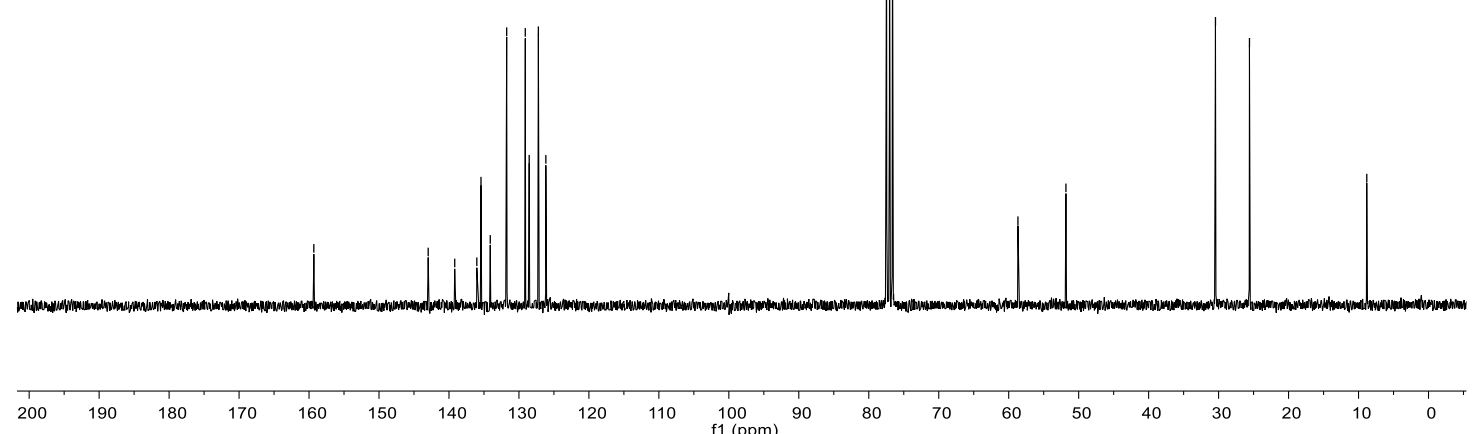


\begin{tabular}{|c|c|}
\hline 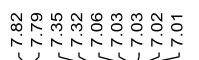 & 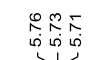 \\
\hline
\end{tabular}
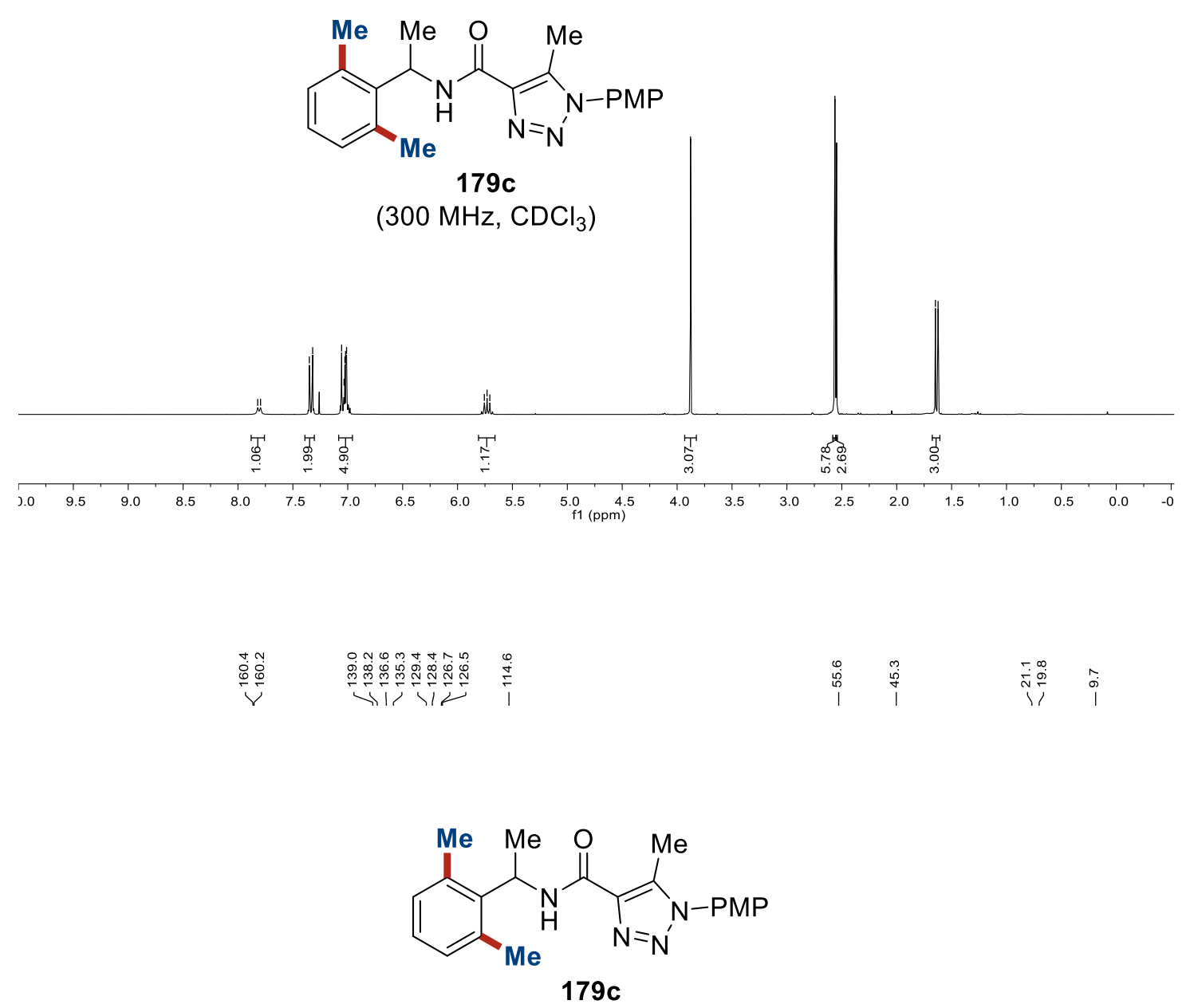

(126 MHz, $\mathrm{CDCl}_{3}$ )

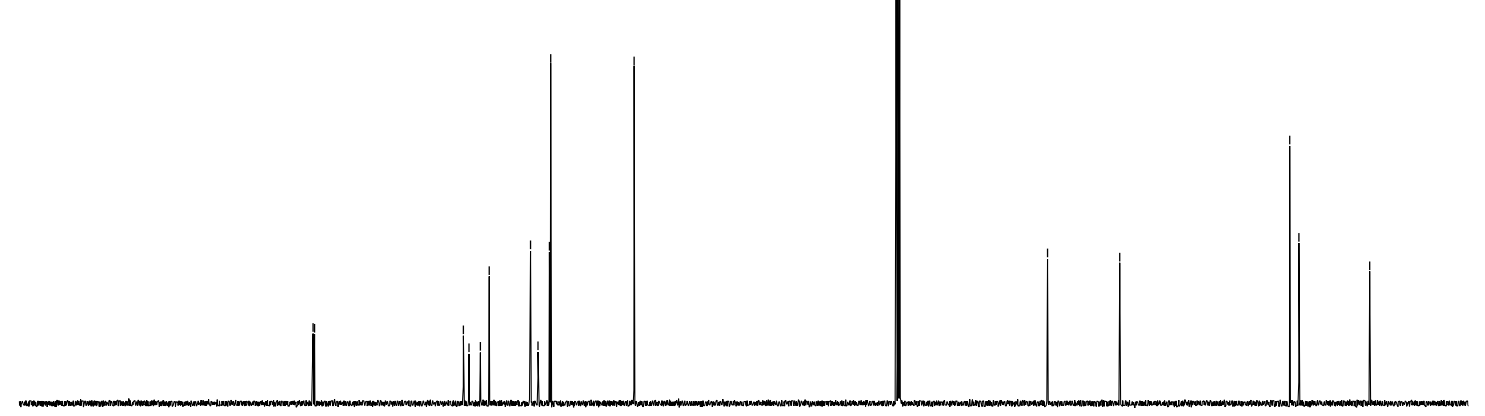

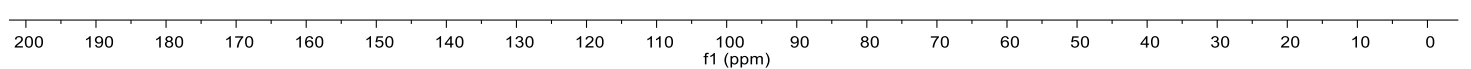




\section{ل望}

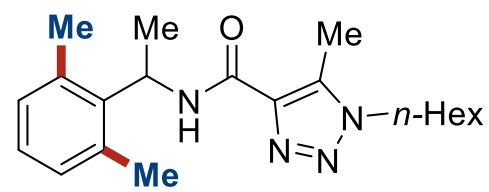

179d

$\left(300 \mathrm{MHz}, \mathrm{CDCl}_{3}\right)$

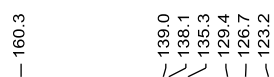

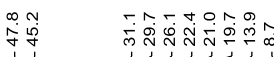

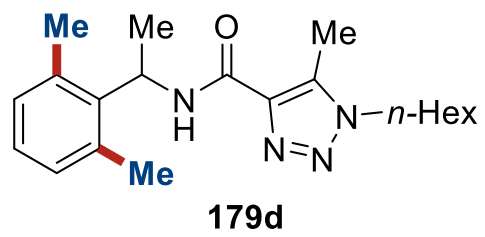

$\left(126 \mathrm{MHz}, \mathrm{CDCl}_{3}\right)$

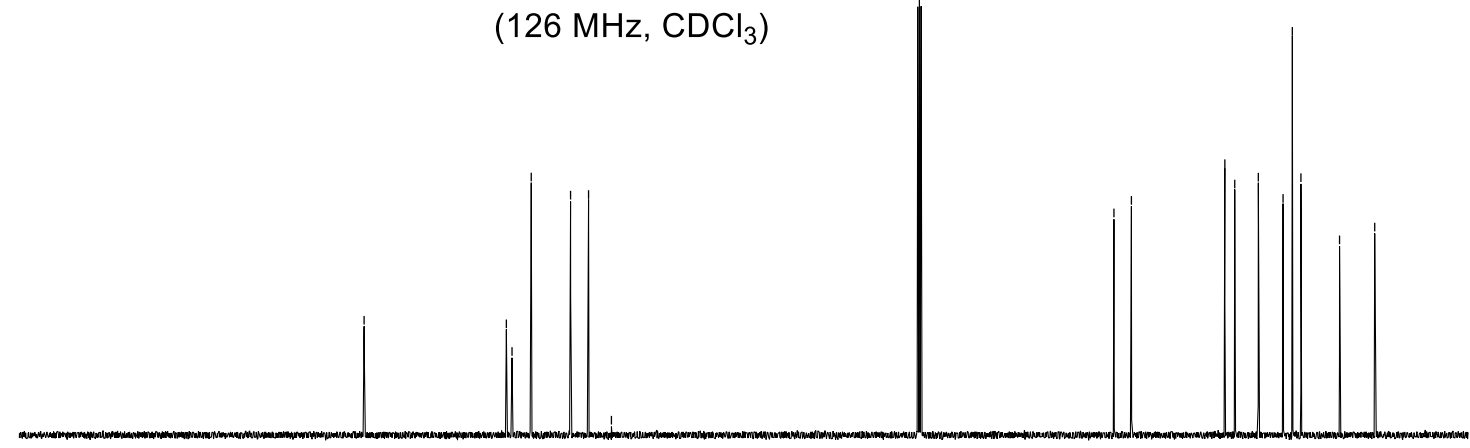

\begin{tabular}{lllllllllll}
\hline 210 & 200 & 190 & 180 & 170 & 160 & 150 & 140 & 130 & 120 & $110 \begin{array}{l}100 \\
\mathrm{f} 1(\mathrm{ppm})\end{array}$
\end{tabular} 


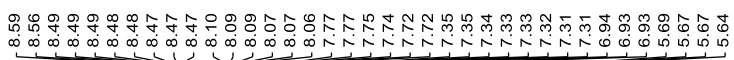

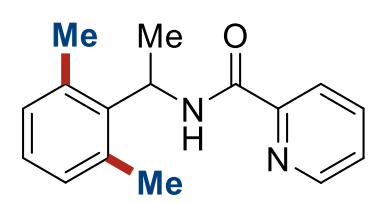

$179 f$

$\left(300 \mathrm{MHz}, \mathrm{CDCl}_{3}\right)$
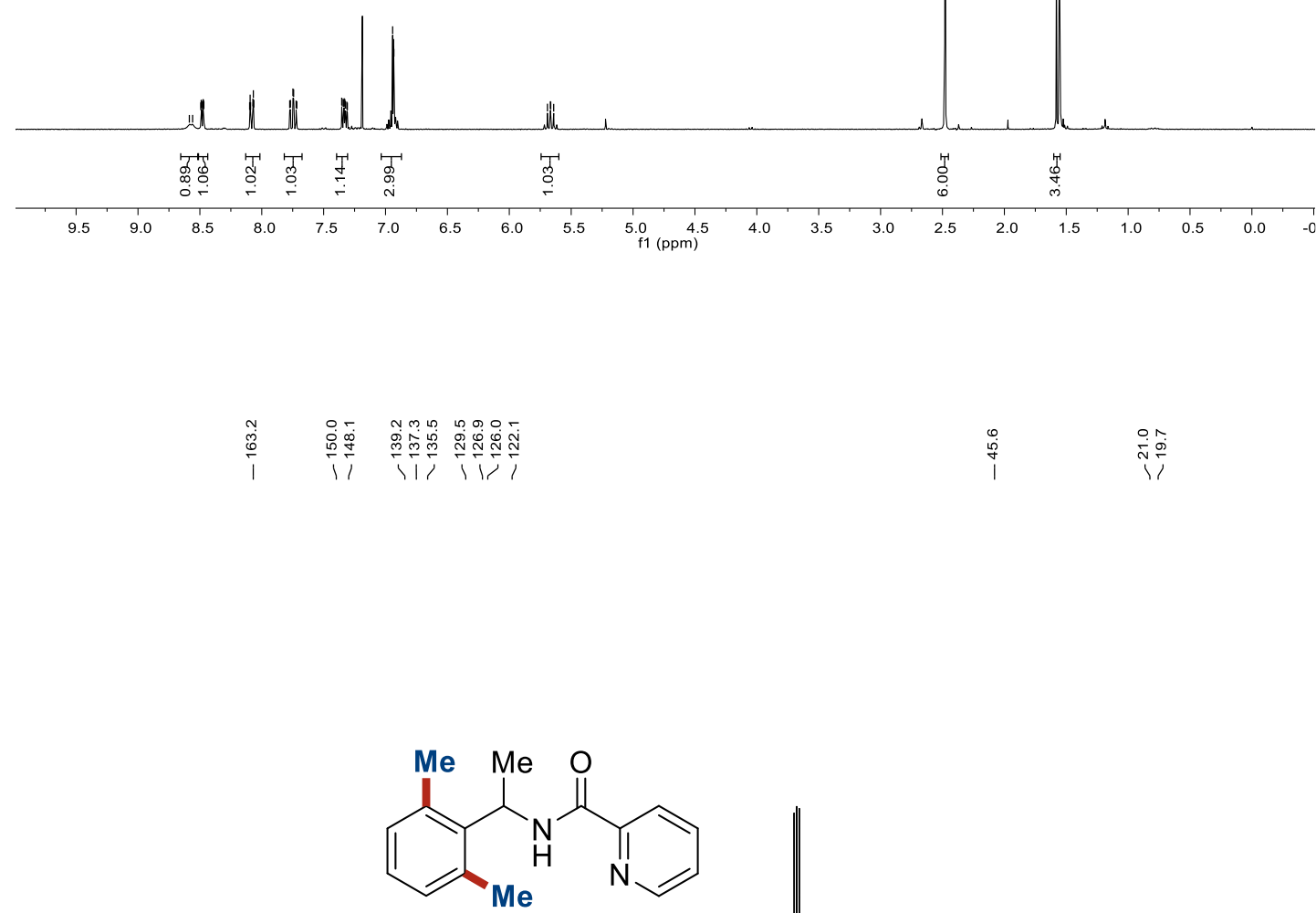

$179 f$

(75 MHz, $\mathrm{CDCl}_{3}$ )
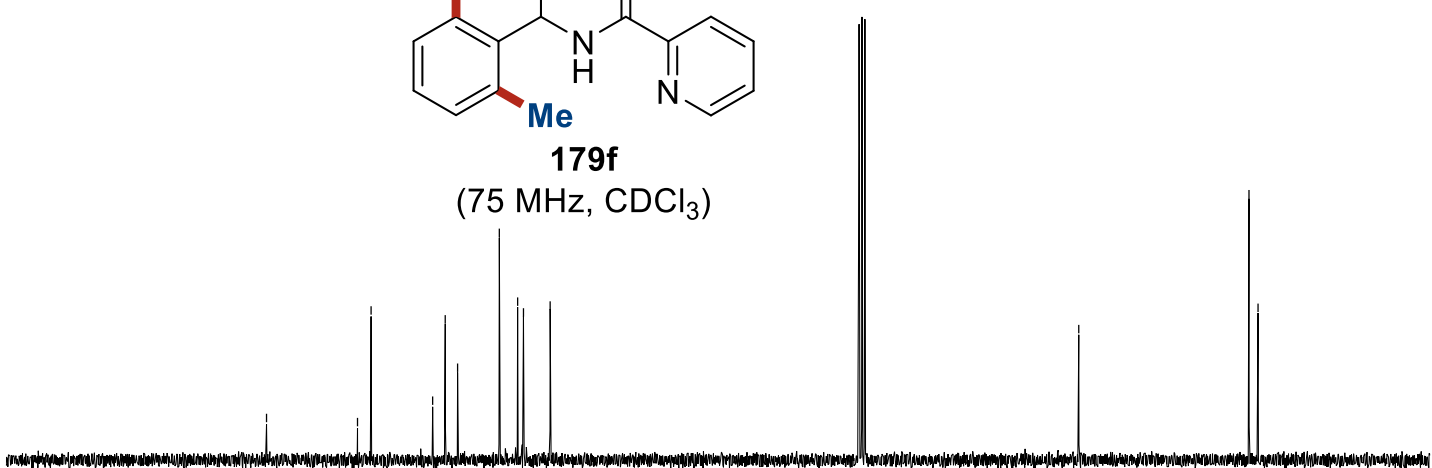

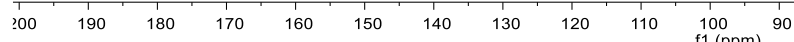

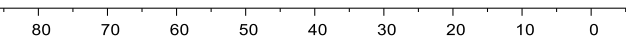




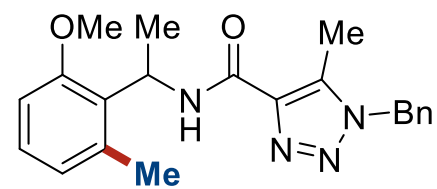

$179 \mathrm{~g}$

(400 MHz, $\mathrm{CDCl}_{3}$ )

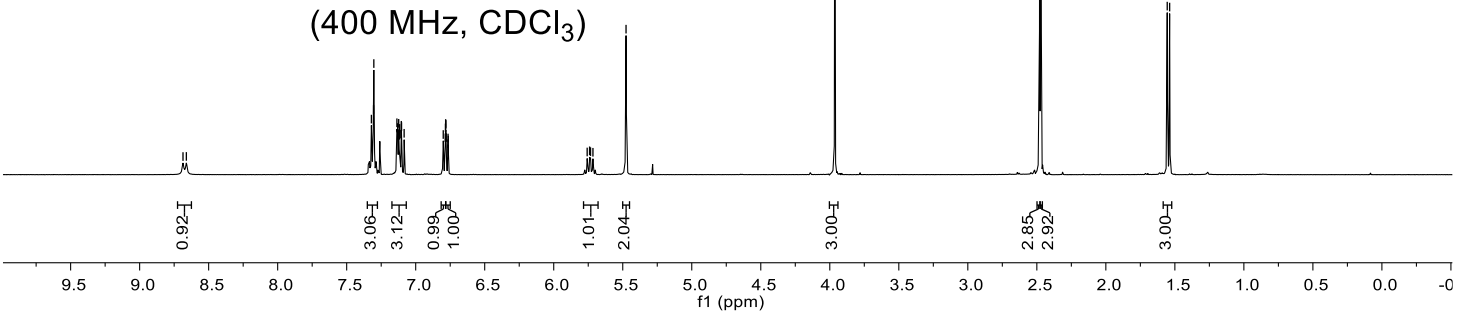

11

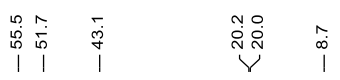<smiles>COc1cccc(C)c1C(C)NC(=O)c1nnn(Br)c1C</smiles>

$179 \mathrm{~g}$

(100 MHz, $\mathrm{CDCl}_{3}$ )

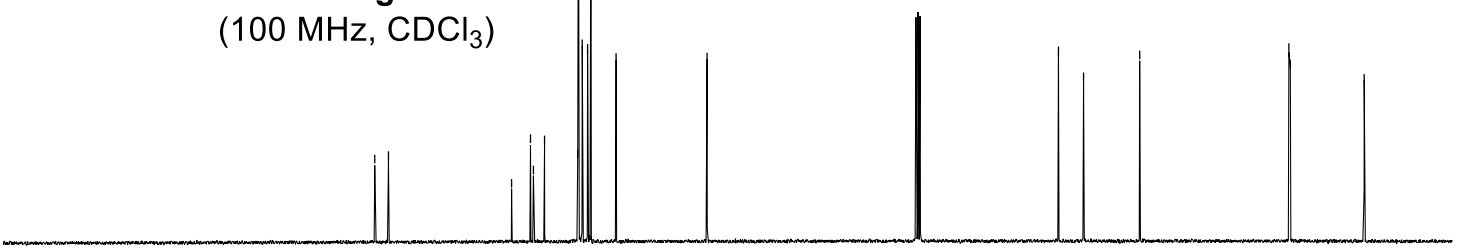

$\begin{array}{llllllllllllllllllllll}210 & 200 & 190 & 180 & 170 & 160 & 150 & 140 & 130 & 120 & 110 & 100 & 90 & 80 & 70 & 60 & 50 & 40 & 30 & 20 & 10 & 0\end{array}$ 

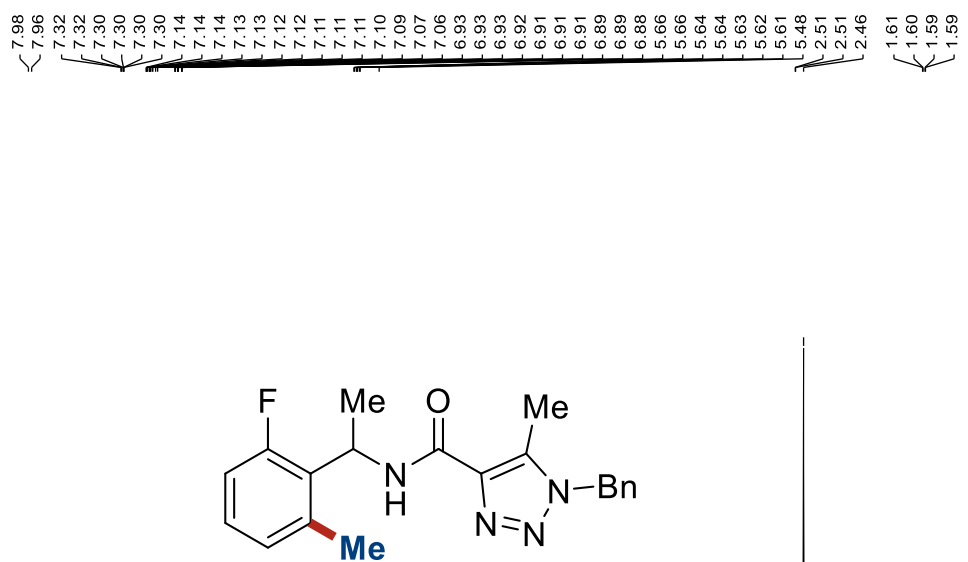

$179 \mathrm{~h}$

$\left(400 \mathrm{MHz}, \mathrm{CDCl}_{3}\right)$

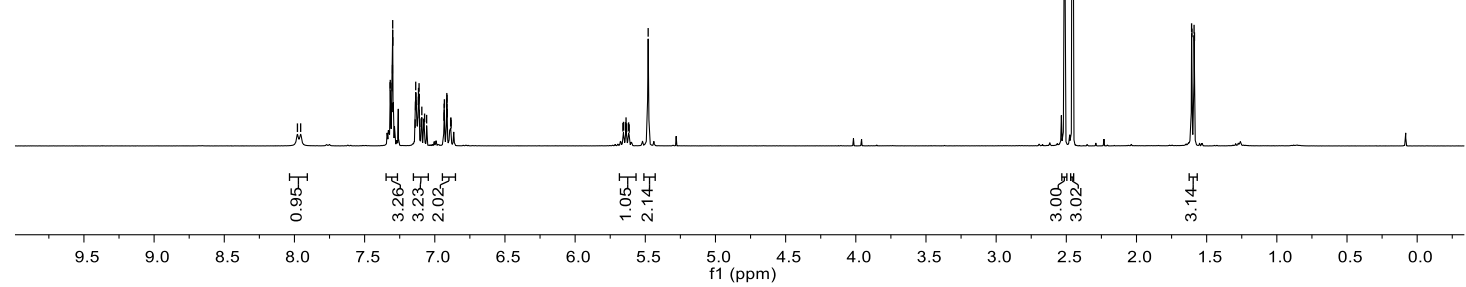

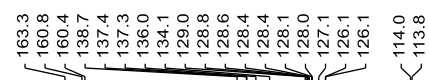

în

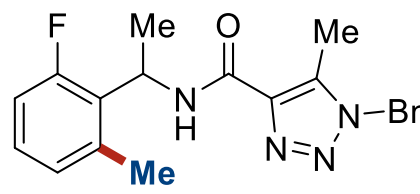

179h

(100 $\left.\mathrm{MHz}, \mathrm{CDCl}_{3}\right)$

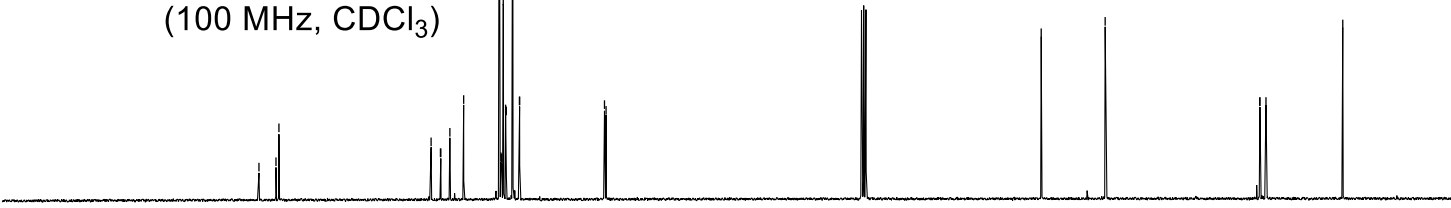

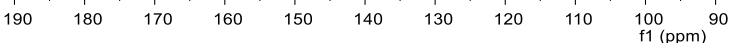


<smiles>Cc1cccc(F)c1C(C)NC(=O)c1nnn(Cc2ccccc2)c1C</smiles>

$179 \mathrm{~h}$

(376 MHz, $\mathrm{CDCl}_{3}$ )

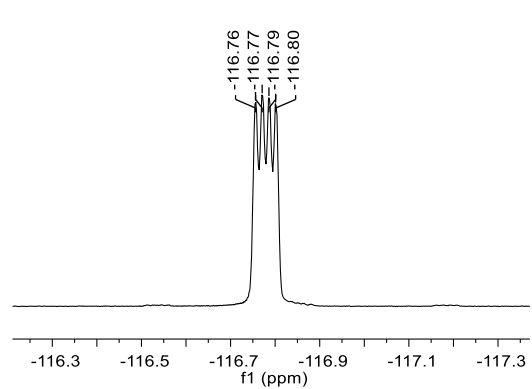

$\begin{array}{lllllllllllllllllllllllllllllll}65 & -70 & -75 & -80 & -85 & -90 & -95 & -100 & -105 & -110 & -115 & -120 & -125 & -130 & -135 & -140 & -145 & -150 & -155 & -160 & -165 & -170 & -175 & -180 & -185 & -1\end{array}$

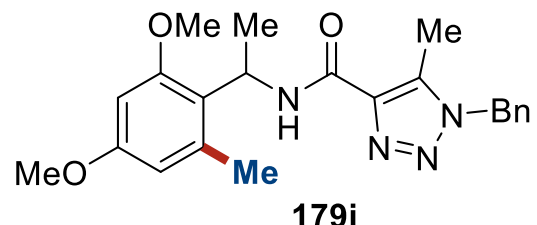

(300 MHz, $\mathrm{CDCl}_{3}$ )

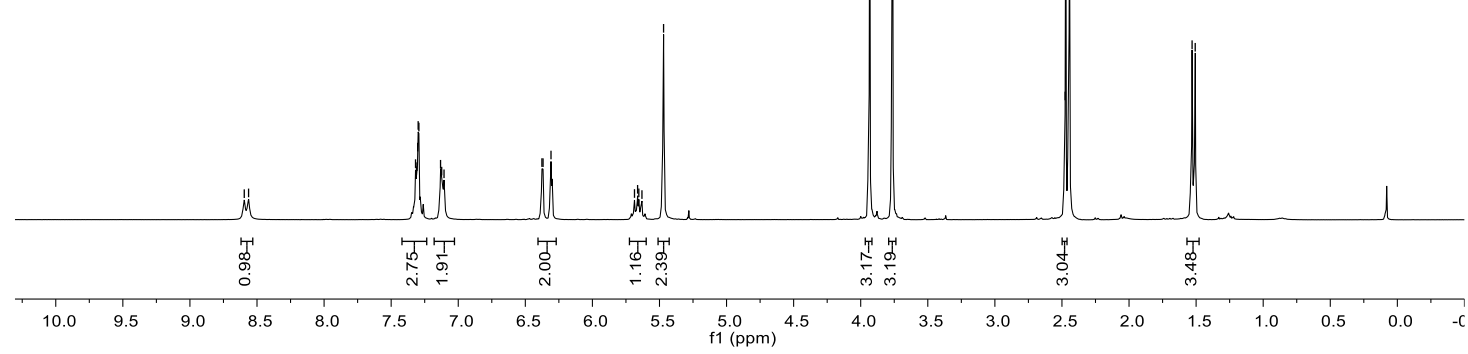




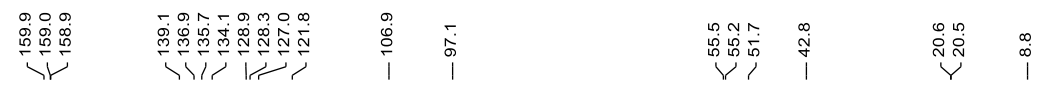<smiles>COc1cc(OC)c(C(C)NC(=O)c2nnn(Cc3ccccc3)c2C)c([N+](=O)[O-])c1</smiles>

$179 i$

(125 MHz, $\mathrm{CDCl}_{3}$ )

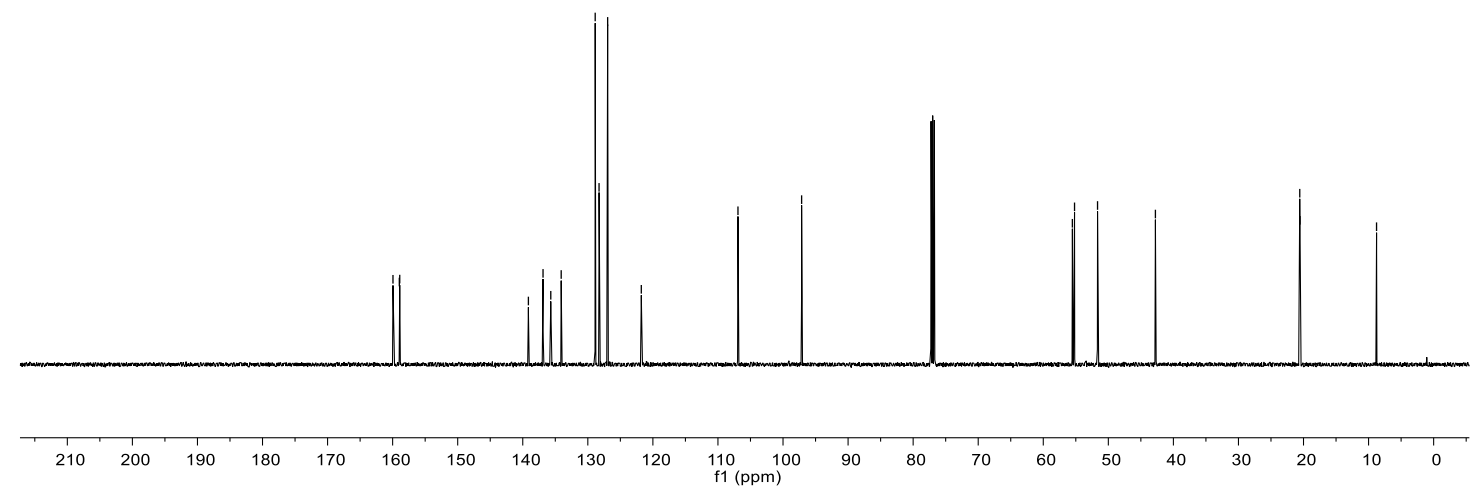

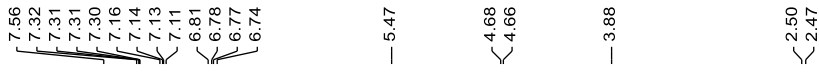<smiles>COc1cccc(OC)c1CNC(=O)c1nnn(Cc2ccccc2)c1C</smiles>

179j

(300 MHz, $\mathrm{CDCl}_{3}$ )

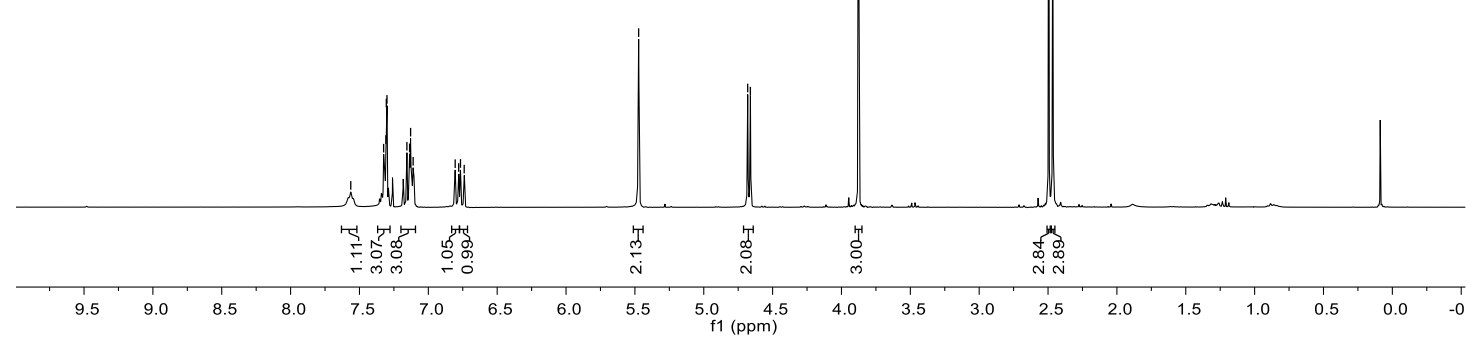




\section{$i_{1}^{1}$}<smiles>COc1cccc(OC)c1CNC(=O)c1nnn(Cc2ccccc2)c1C</smiles>

179 j

(125 MHz, $\mathrm{CDCl}_{3}$ )

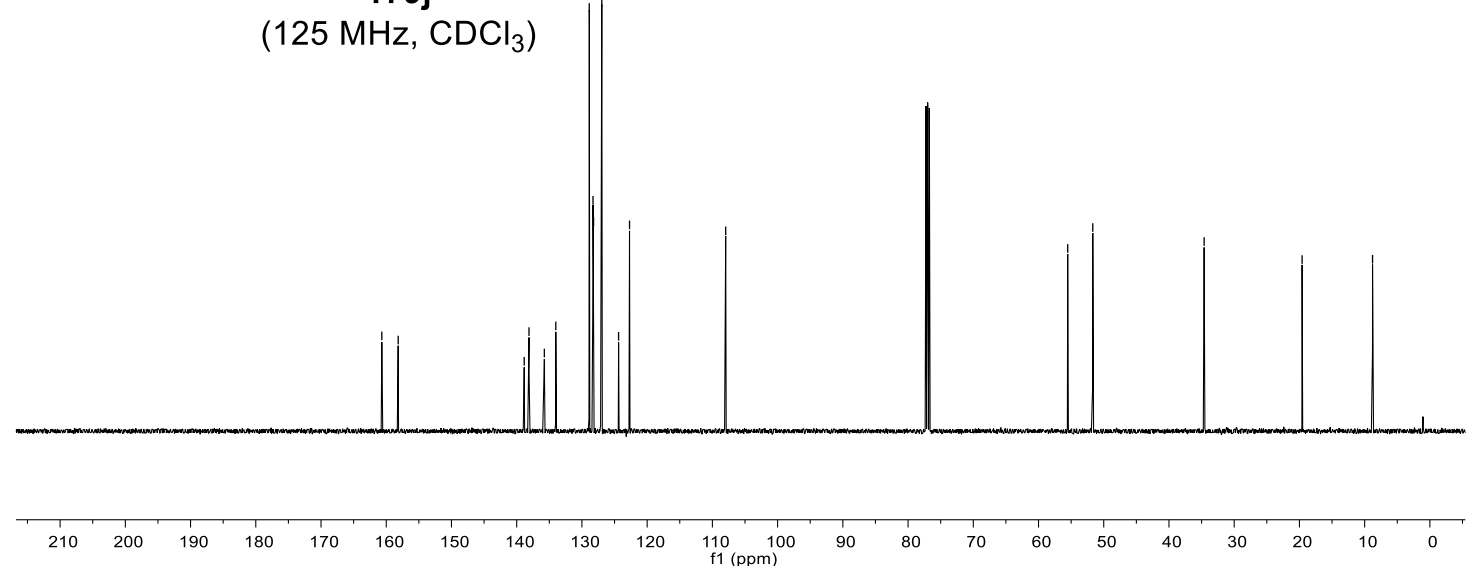

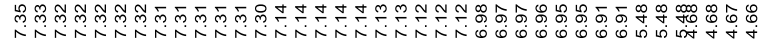

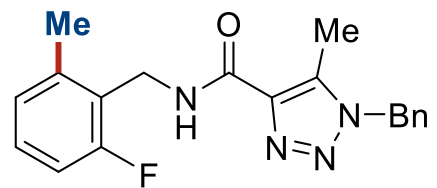

179k

(400 MHz, $\mathrm{CDCl}_{3}$ )

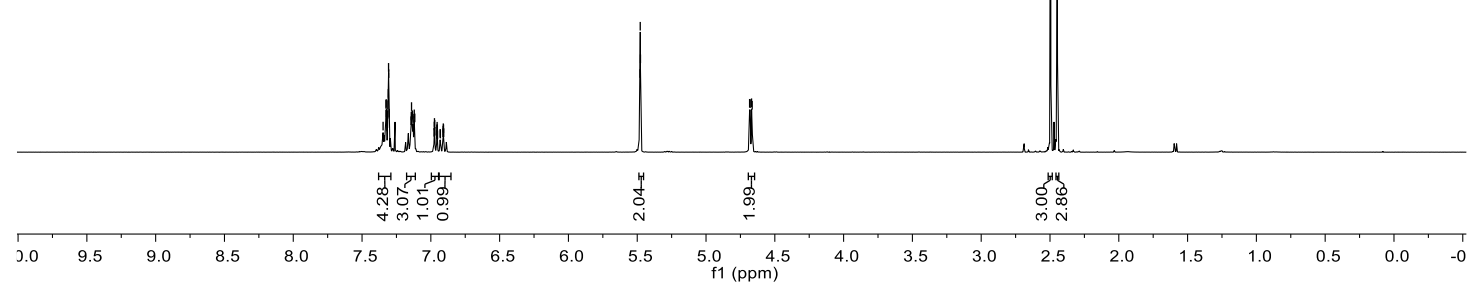




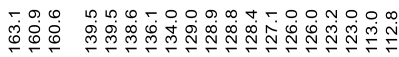

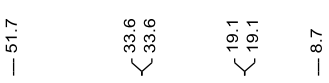

$\overbrace{F}^{M e}$

179k

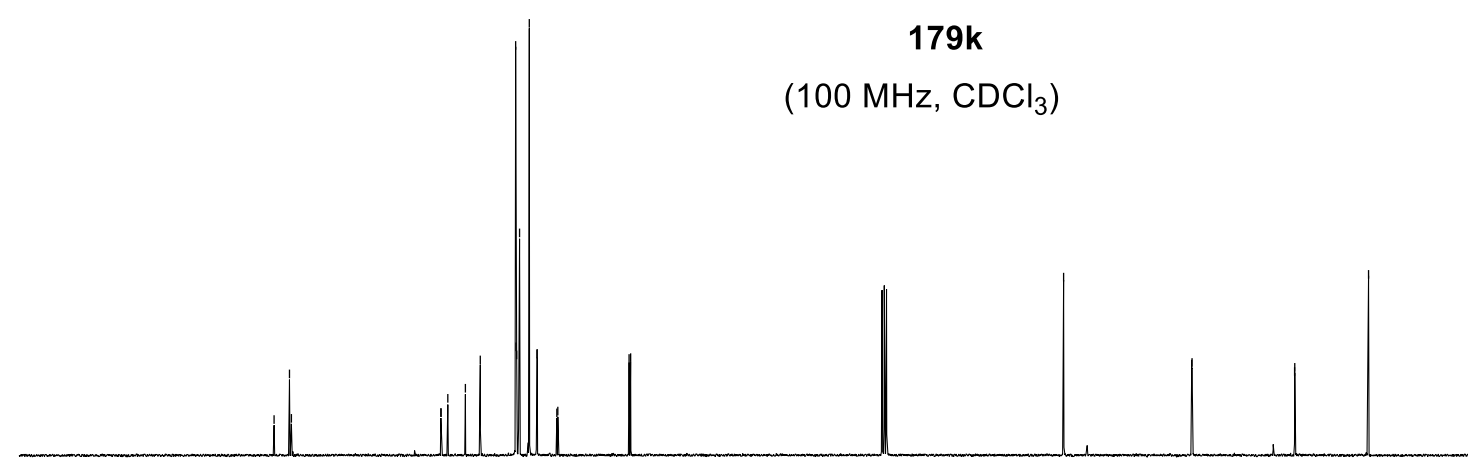

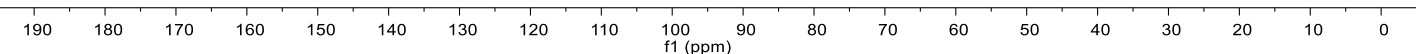

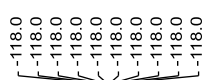

$\overbrace{N=N_{N}^{N-B n}}^{M e}$

179k

$\left(376 \mathrm{MHz}, \mathrm{CDCl}_{3}\right)$

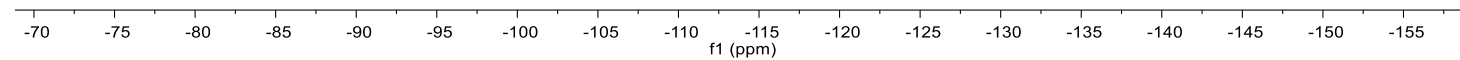




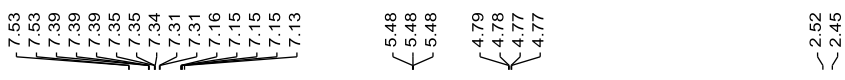<smiles>Cc1cccc(C(F)(F)F)c1CNC(=O)c1nnn(Cc2ccccc2)c1C</smiles>

179I

(300 MHz, $\mathrm{CDCl}_{3}$ )

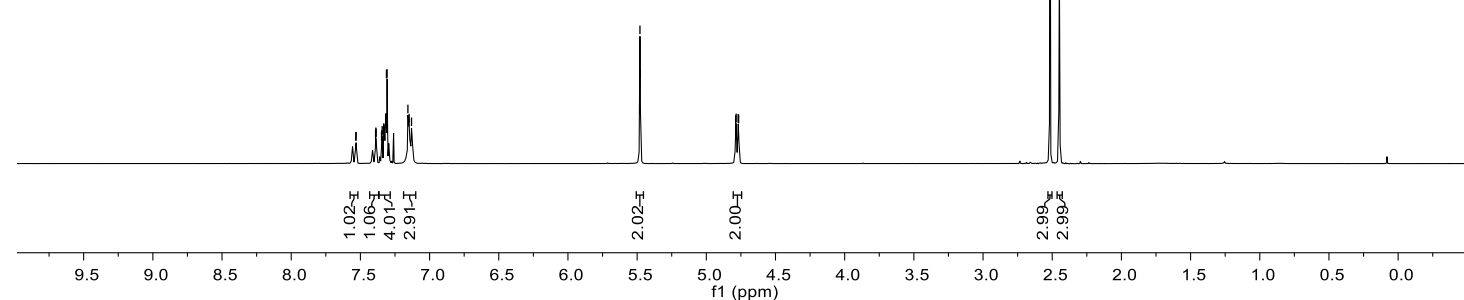

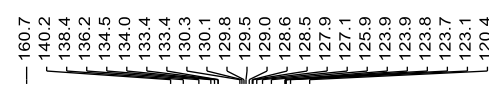

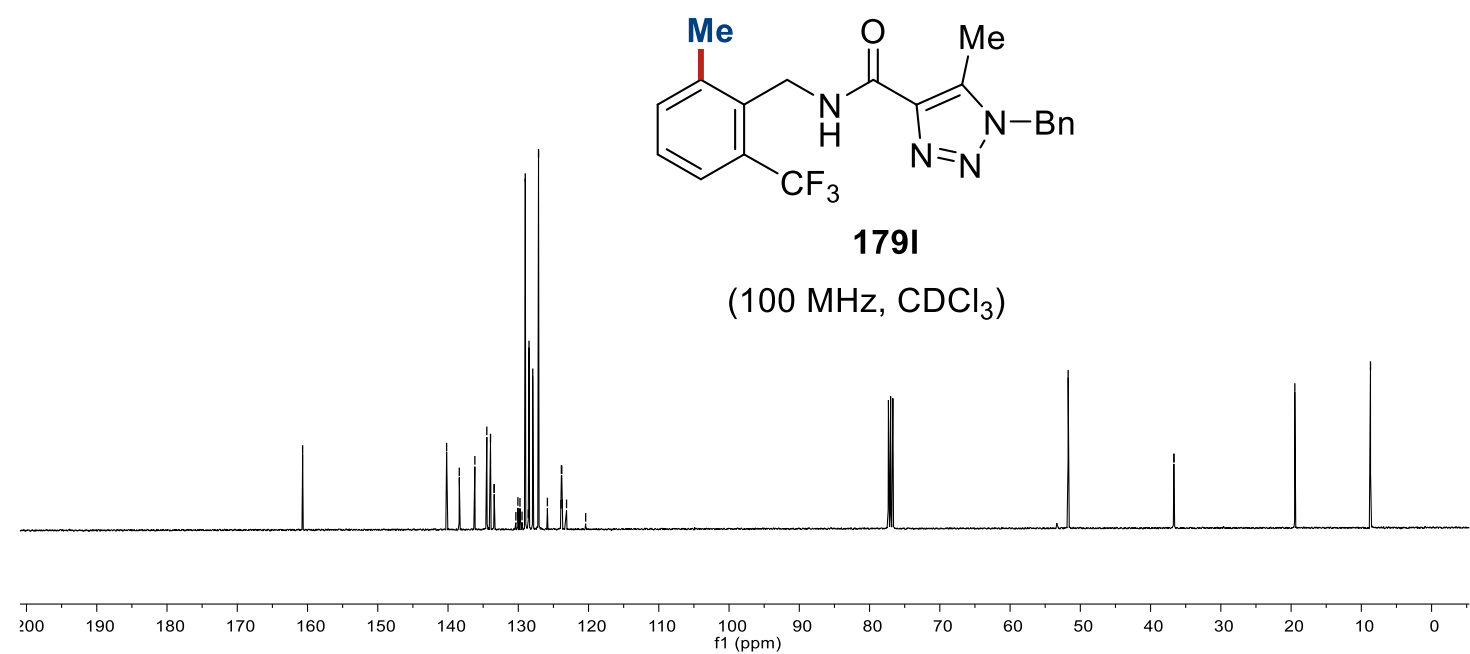




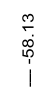

$\underbrace{M e}_{C_{3}}$

179I

(376 MHz, $\mathrm{CDCl}_{3}$ )

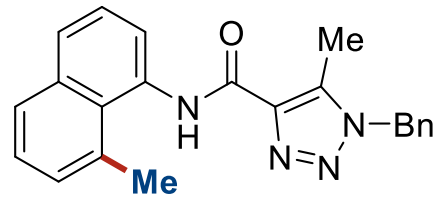

$$
179 \mathrm{~m}
$$

(300 MHz, $\mathrm{CDCl}_{3}$ )

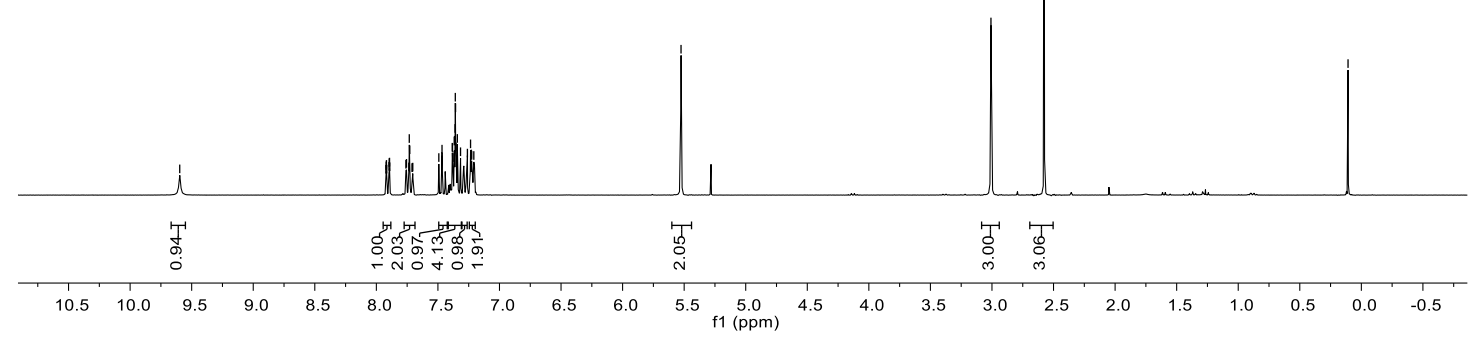




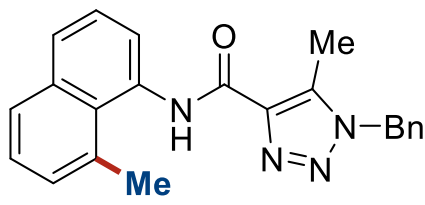

$179 \mathrm{~m}$

(75 MHz, $\mathrm{CDCl}_{3}$ )

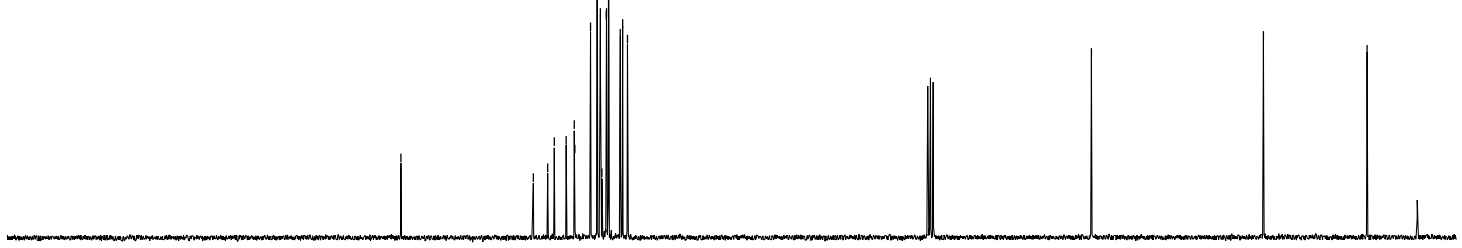

\begin{tabular}{llllllllllllllllllllllll}
\hline 20 & 210 & 200 & 190 & 180 & 170 & 160 & 150 & 140 & 130 & 120 & 110 & 100 & 90 & 80 & 70 & 60 & 50 & 40 & 30 & 20 & 10 & 0
\end{tabular}

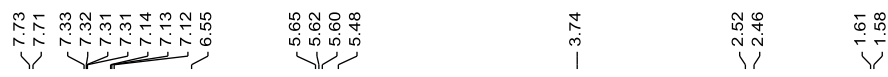<smiles>COc1cc(C)c(C(C)NC(=O)c2nnn(Cc3ccccc3)c2C)c(C)c1</smiles>

$179 n$

(300 MHz, $\mathrm{CDCl}_{3}$ )

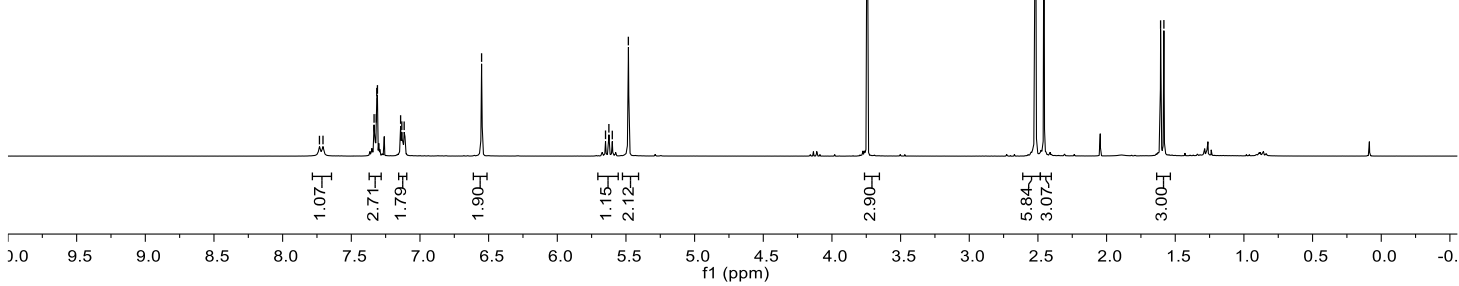



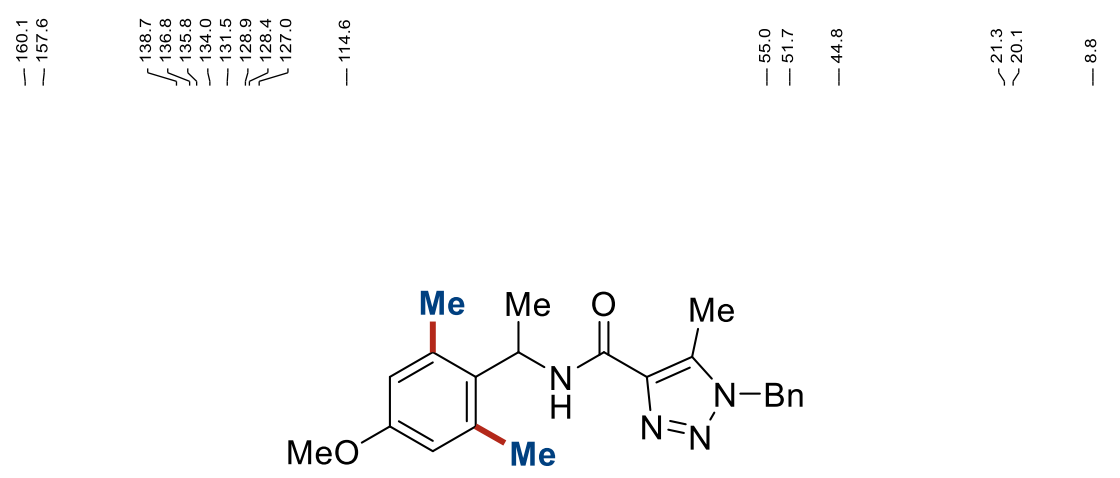

179n

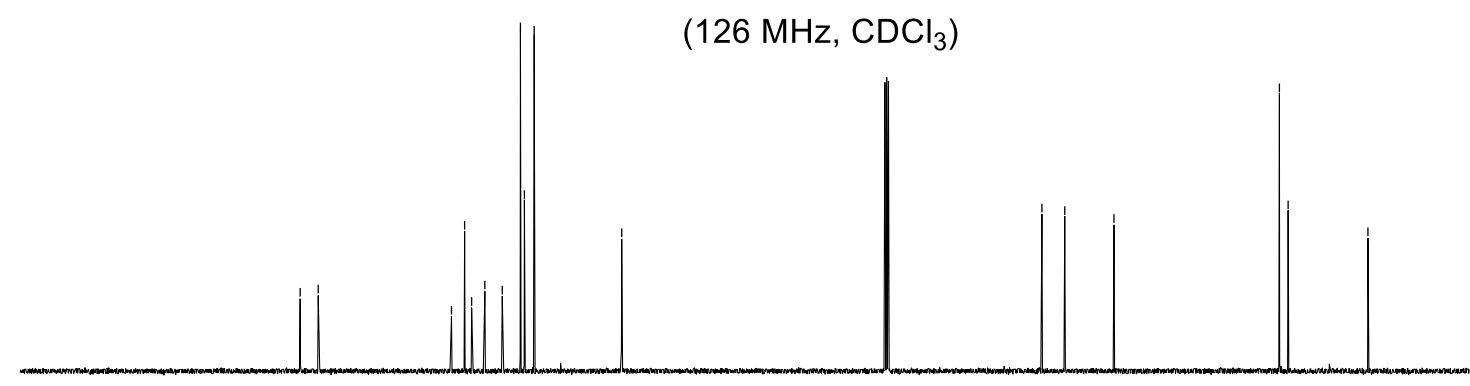

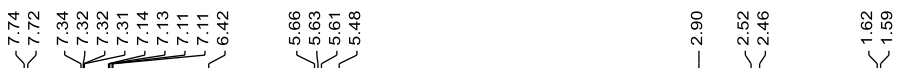

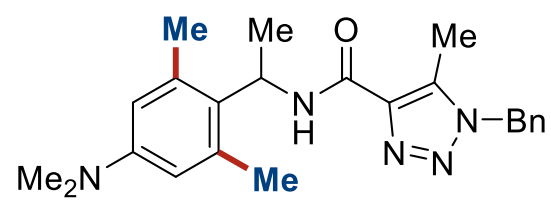

1790

(300 $\mathrm{MHz}, \mathrm{CDCl}_{3}$ )

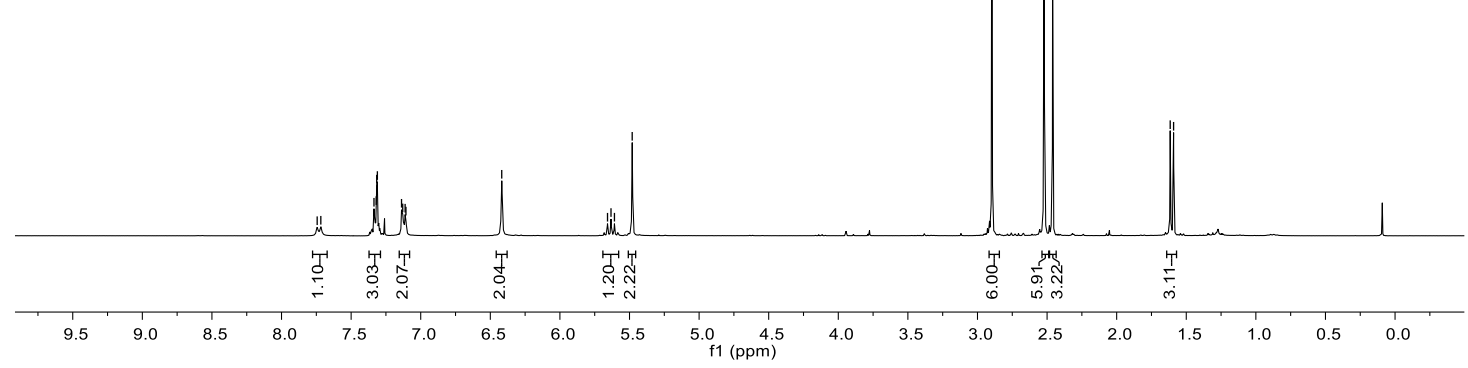




\section{审}

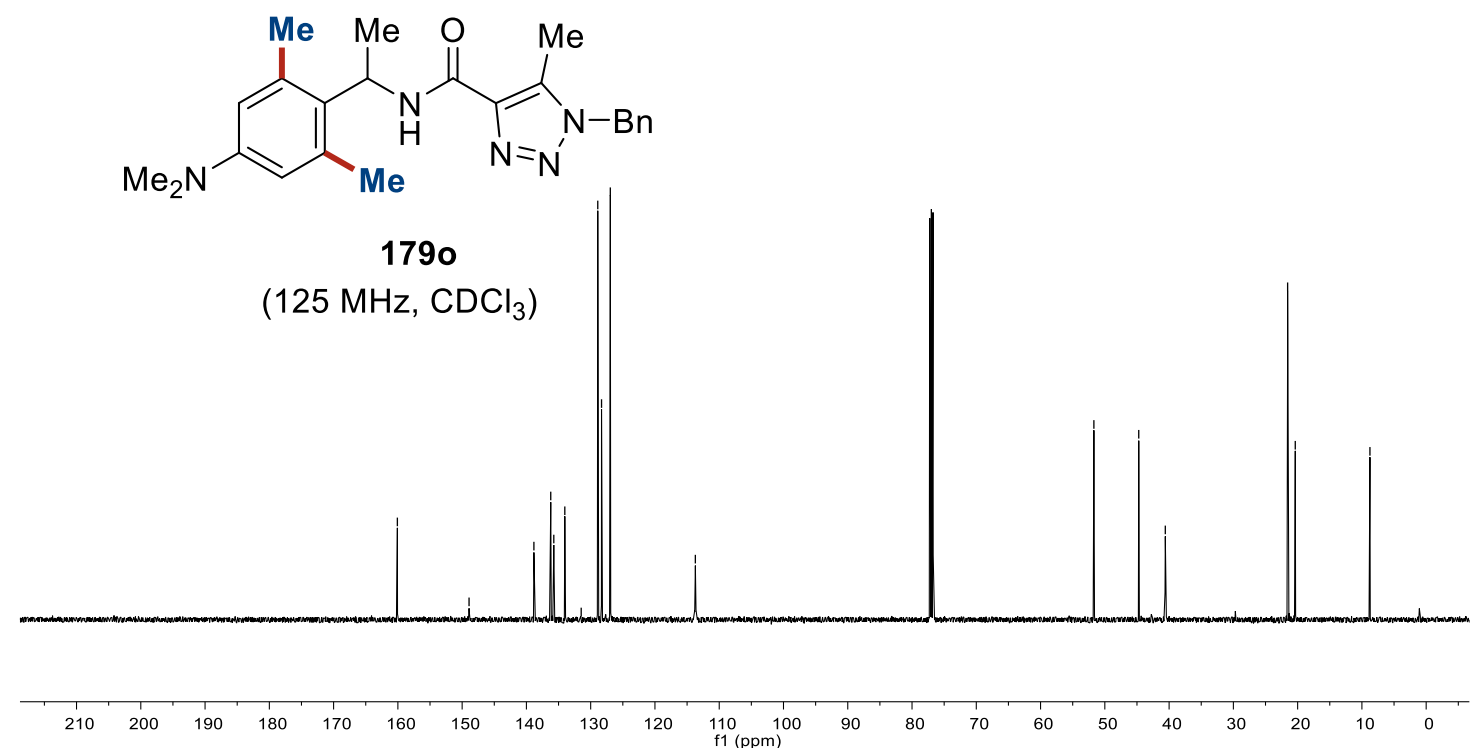

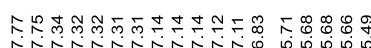

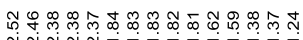

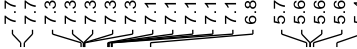

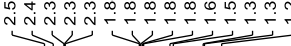<smiles>Cc1cc(C2CCCCC2)cc([N+](=O)[O-])c1C(C)NC(=O)c1nnn(Cc2ccccc2)c1C</smiles>

(300 MHz, $\mathrm{CDCl}_{3}$ )

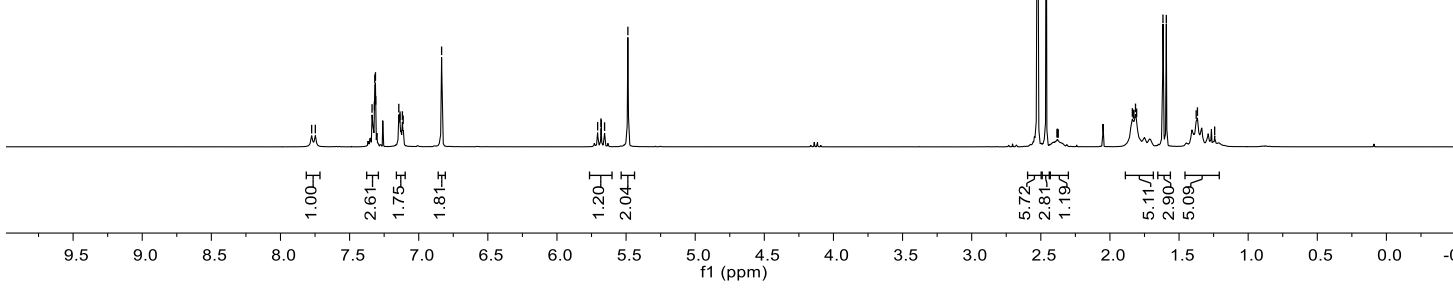



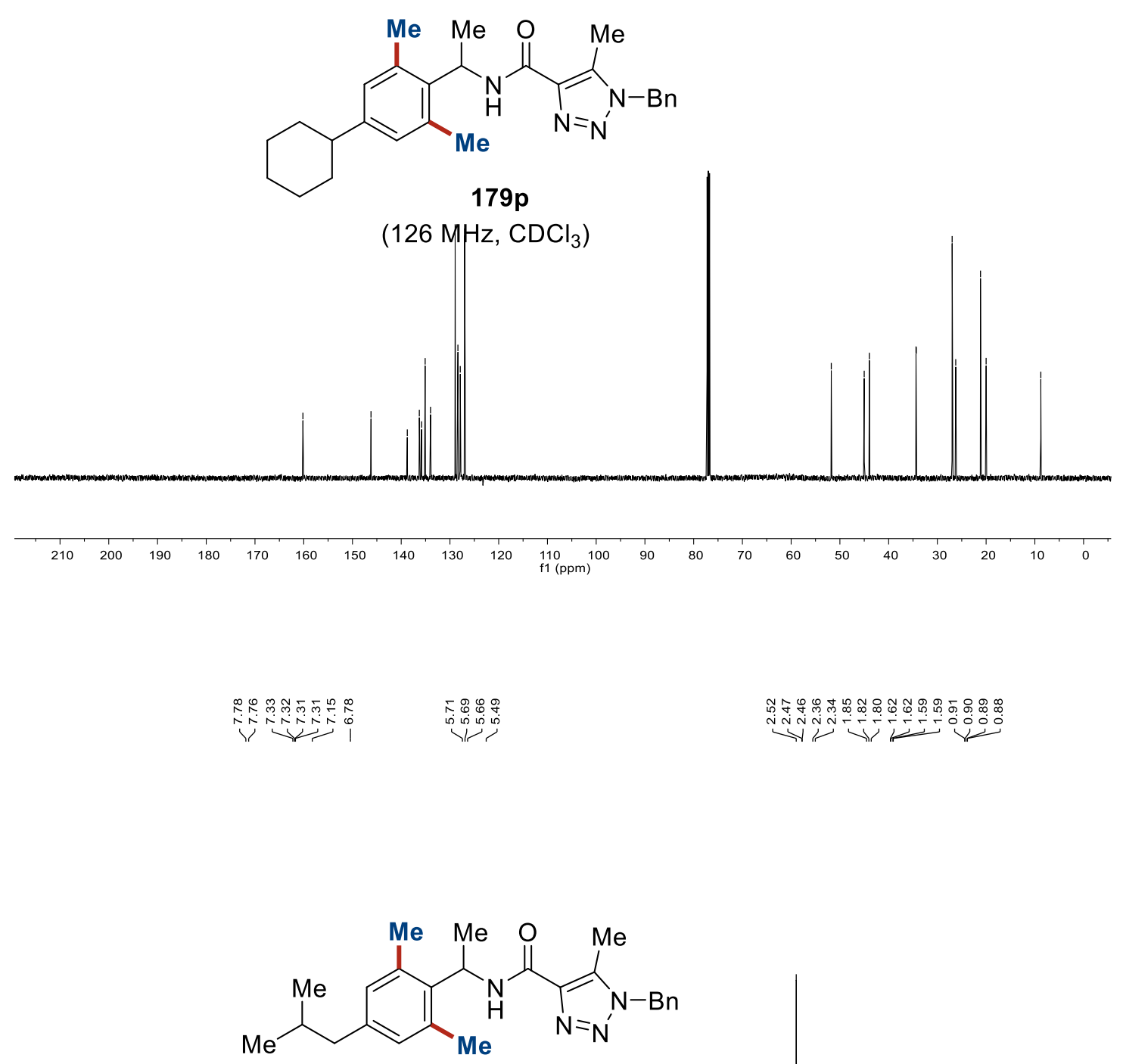

$179 q$

(300 $\mathrm{MHz}, \mathrm{CDCl}_{3}$ )

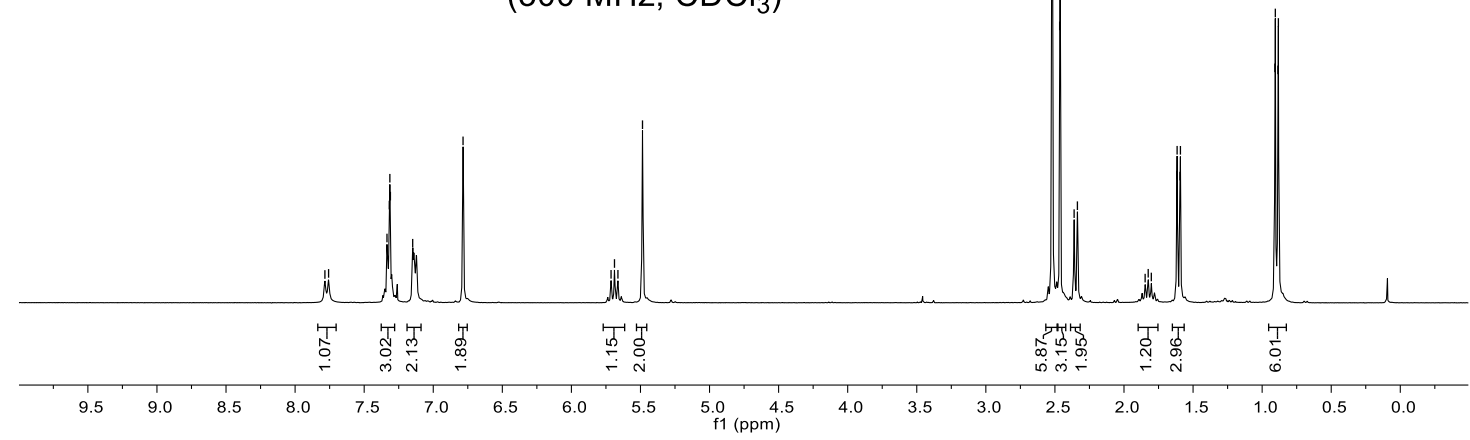




\section{|}

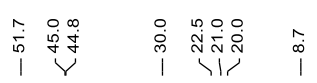<smiles>Cc1cc(CC(C)C)cc(C)c1C(C)NC(=O)c1nnn(Cc2ccccc2)c1C</smiles>

$179 q$

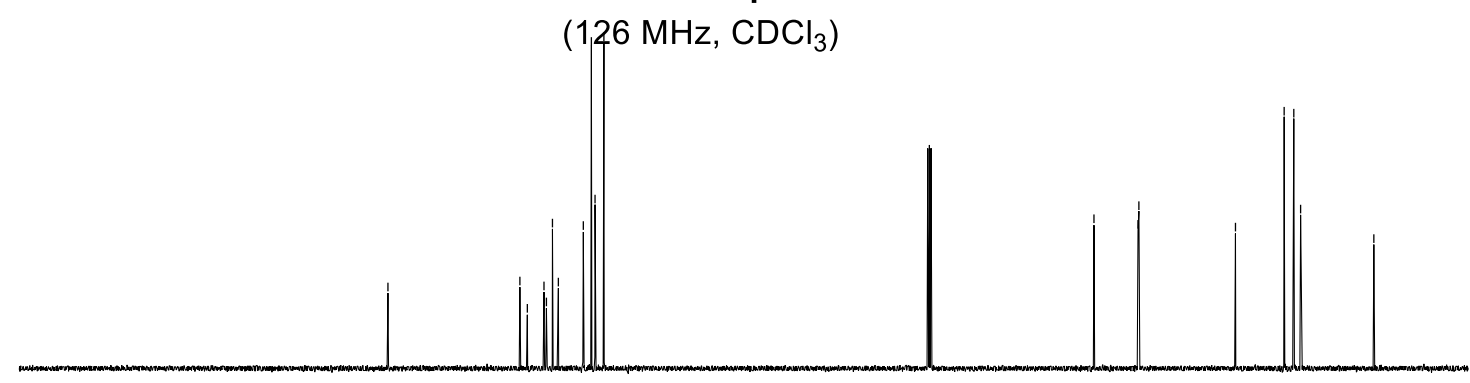

$\begin{array}{llllllllllllllllllllllllllll} & 210 & 200 & 190 & 180 & 170 & 160 & 150 & 140 & 130 & 120 & 110 & 100 & 90 & 80 & 70 & 60 & 50 & 40 & 30 & 20 & 10 & 0\end{array}$

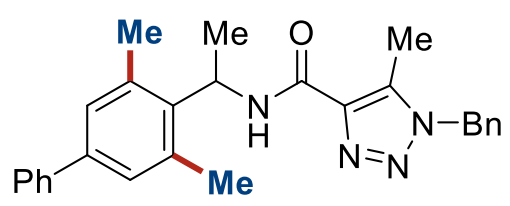

179r

(300 MHz, $\mathrm{CDCl}_{3}$ )

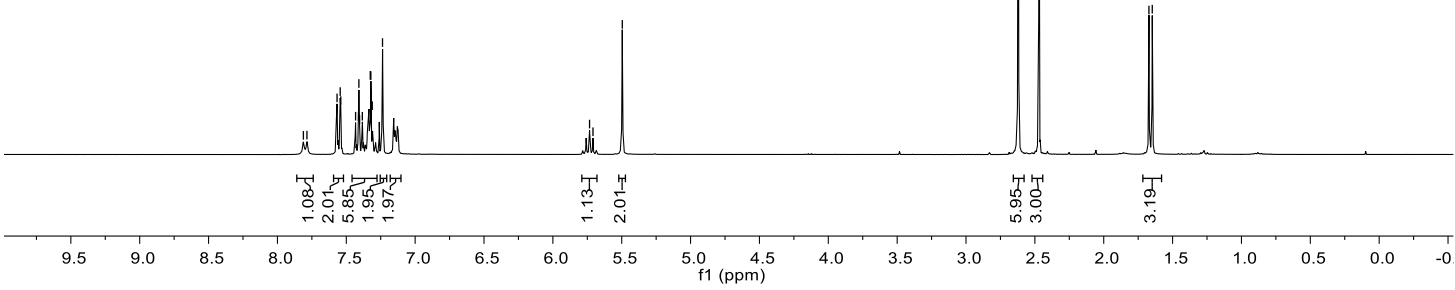




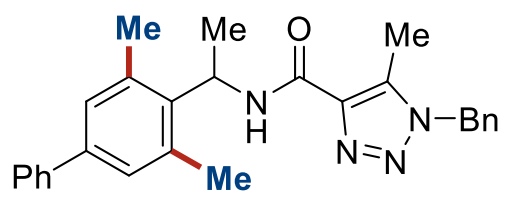

$179 r$

(126 MHz, $\mathrm{CDCl}_{3}$ )

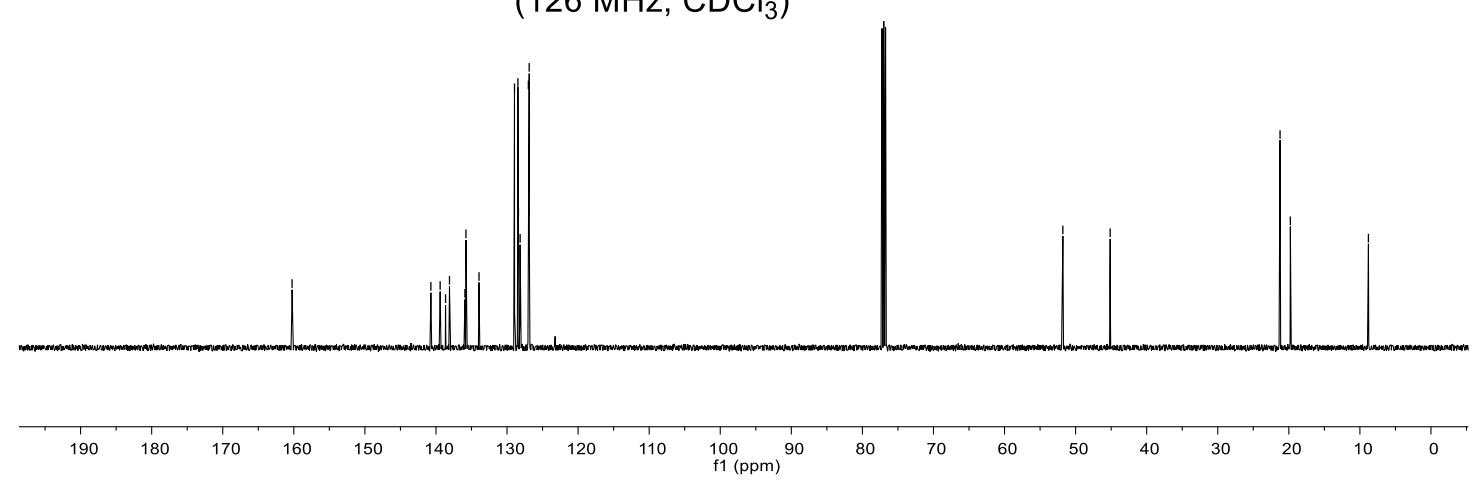

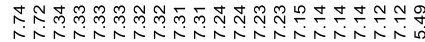

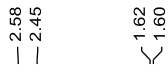<smiles>Cc1cc(C(F)(F)F)cc(C)c1C(C)NC(=O)c1nnn(Cc2ccccc2)c1C</smiles>

179s

(300 MHz, $\mathrm{CDCl}_{3}$ )

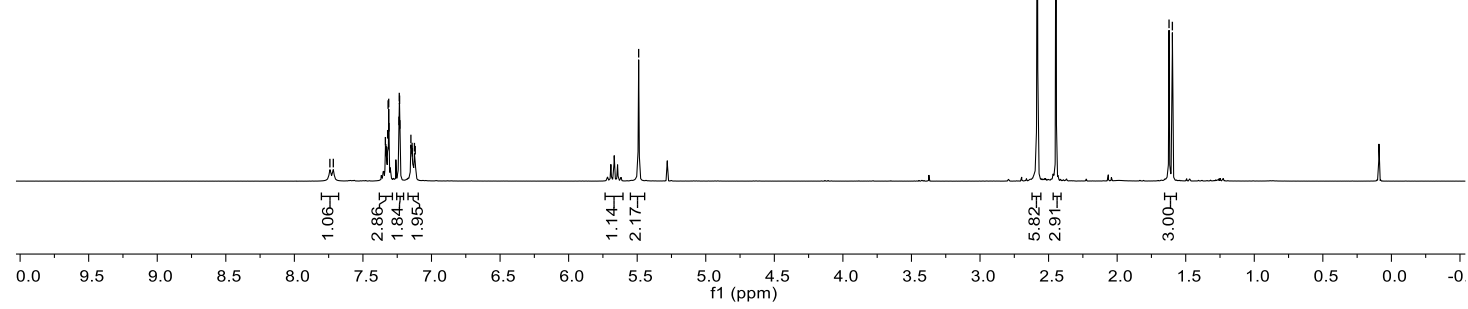



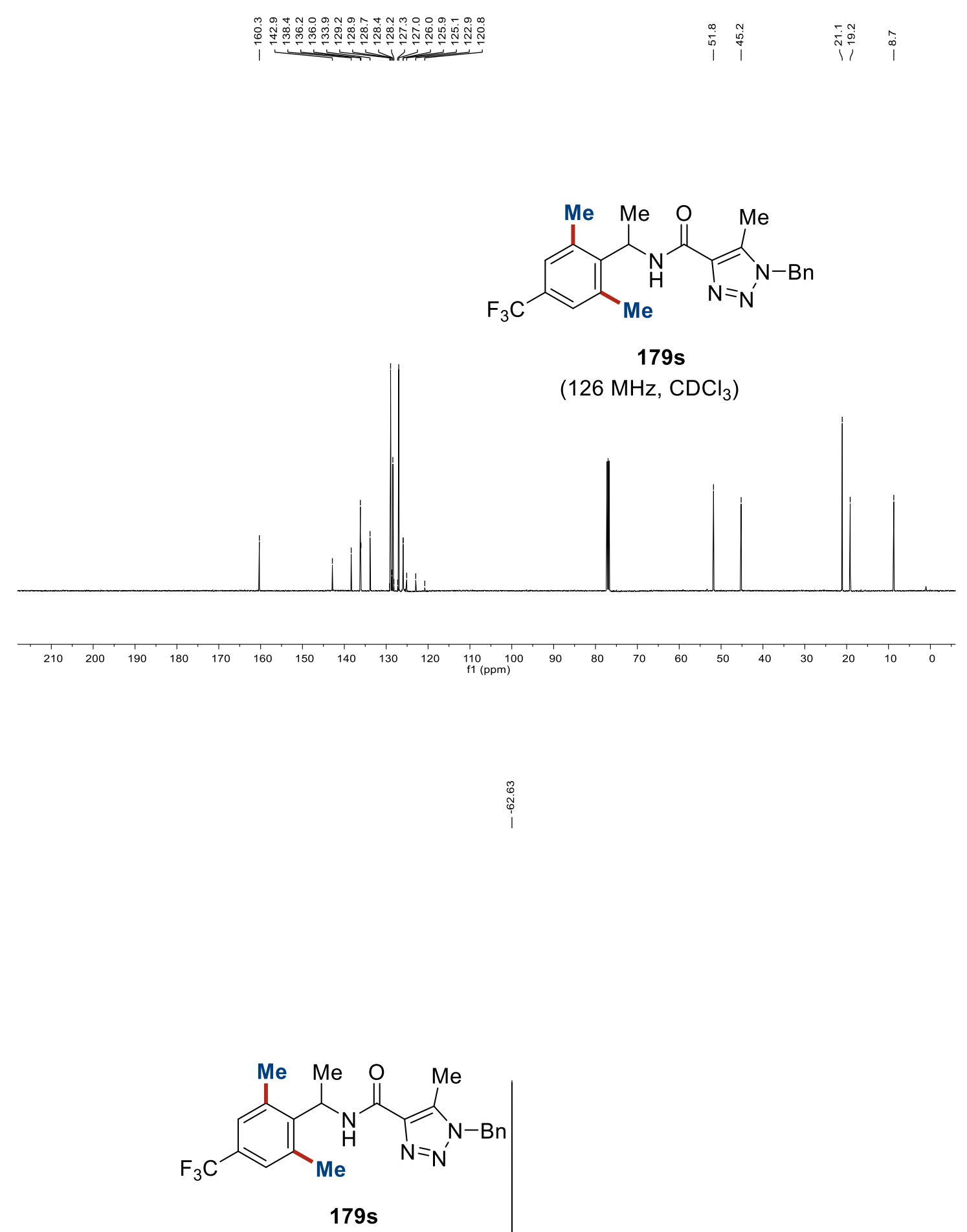

(282 MHz, $\mathrm{CDCl}_{3}$ )

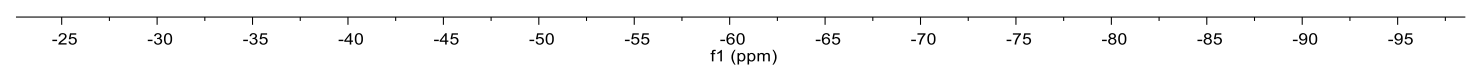




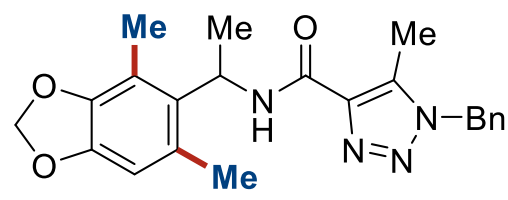

$179 t$

$\left(400 \mathrm{MHz}, \mathrm{CDCl}_{3}\right)$

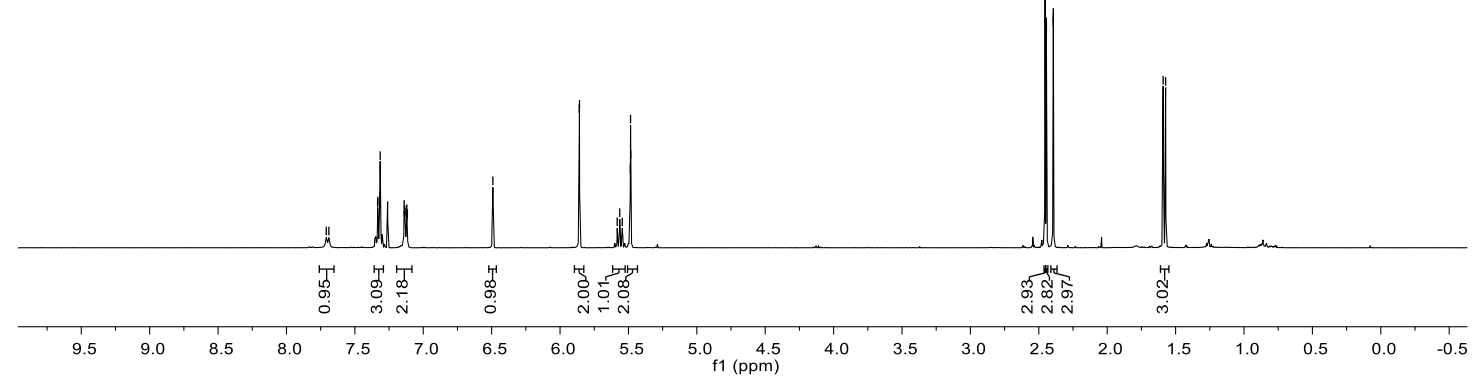

i

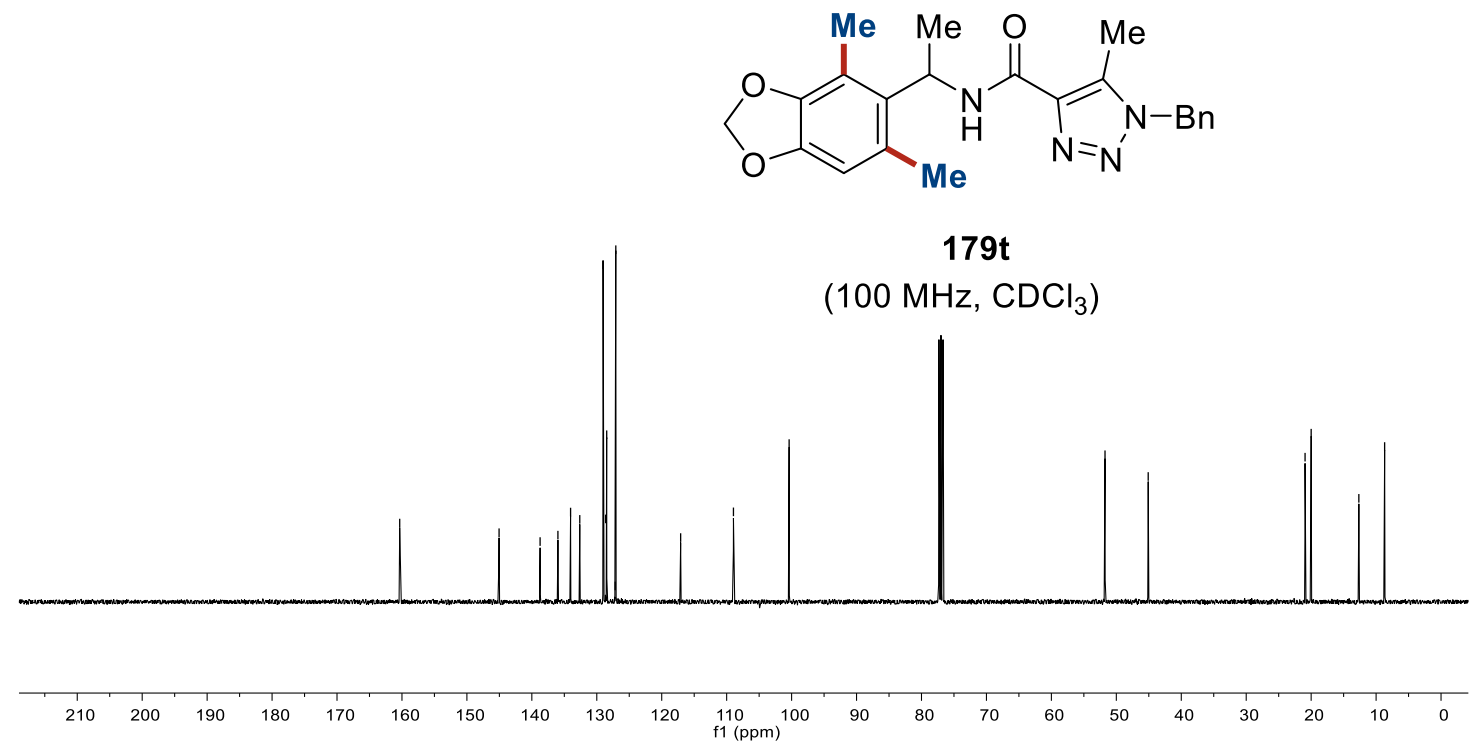




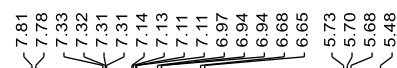

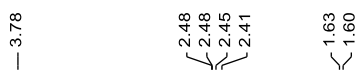<smiles>[M]C(NC(=O)c1nnn(Cc2ccccc2)c1C)c1c(C)ccc(OC)c1C</smiles>

$179 u$

(300 MHz, $\mathrm{CDCl}_{3}$ )

|

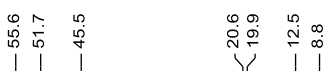<smiles>COc1ccc(C)c(C(C)NC(=O)c2nnn(Cc3ccccc3)c2C)c1C</smiles>

179u

(125 MHz, $\mathrm{CDCl}_{3}$ )

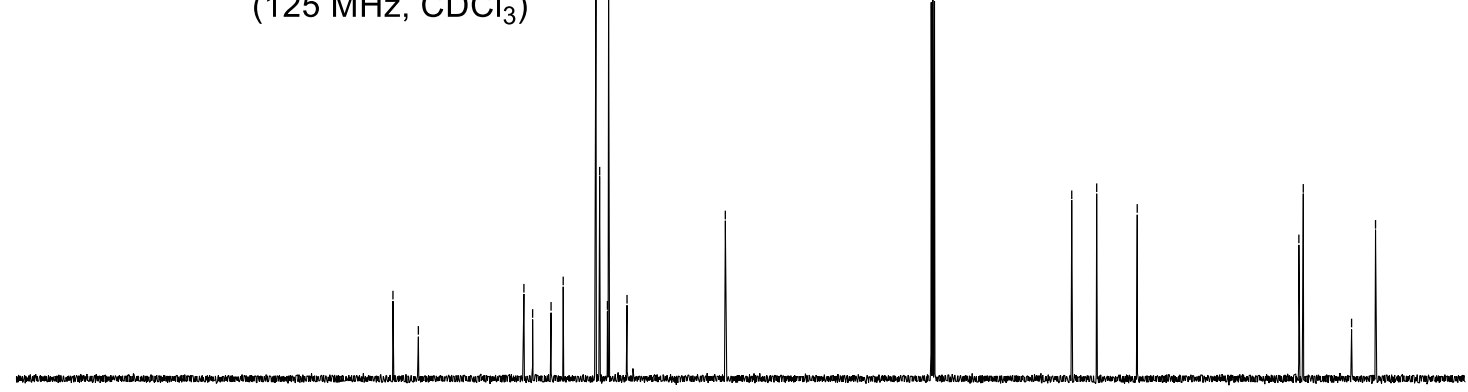

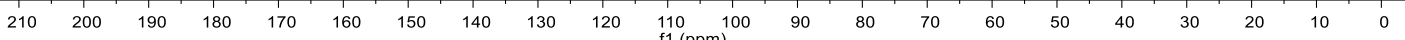




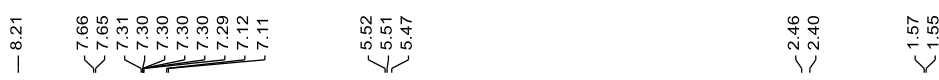<smiles>Cc1cncc(C)c1C(C)NC(=O)c1nnn(Cc2ccccc2)c1C</smiles>

179v

(500 MHz, $\mathrm{CDCl}_{3}$ )

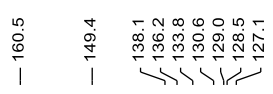

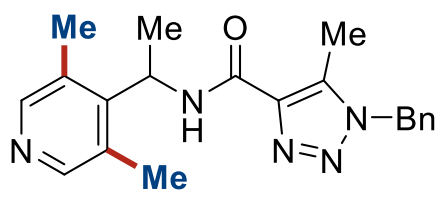

$179 v$

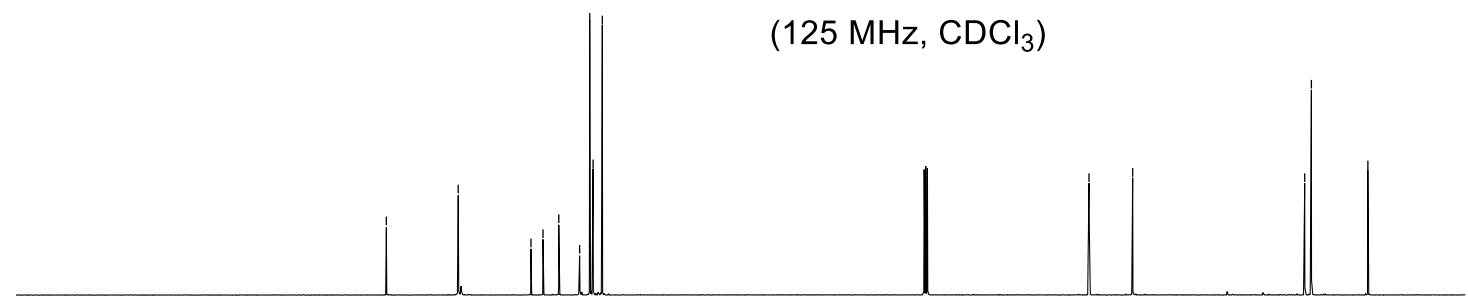

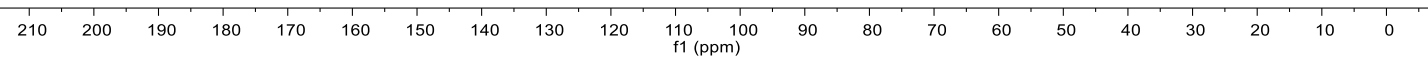




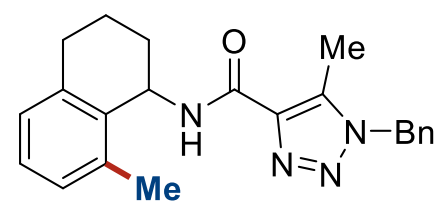

179w

(300 $\mathrm{MHz}, \mathrm{CDCl}_{3}$ )

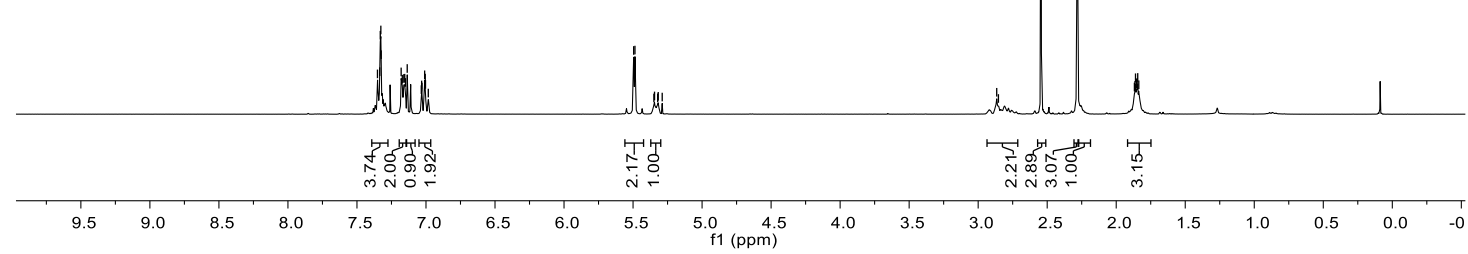

$\underbrace{\infty}$

<smiles>Cc1cccc2c1C(NC(=O)c1nnn(Cc3ccccc3)c1C)CCC2</smiles>

179w

(125 $\mathrm{MHz} \mathrm{CDCl}_{3}$ )

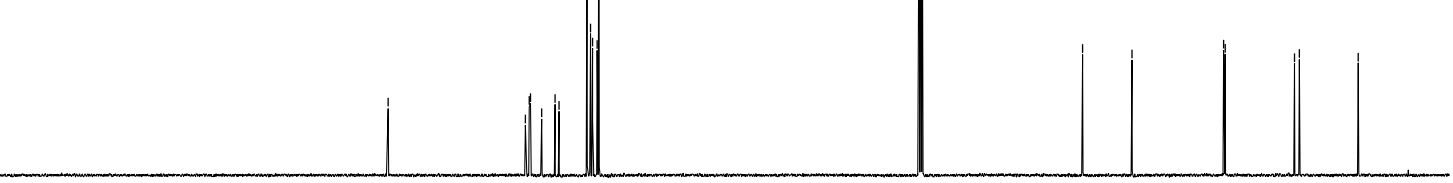

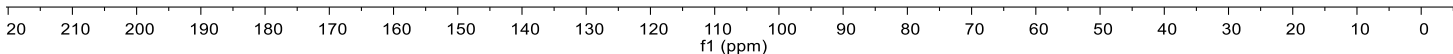




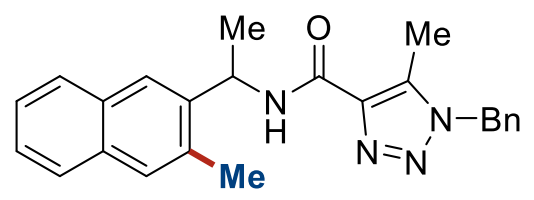

179x

(300 MHz, $\mathrm{CDCl}_{3}$ )

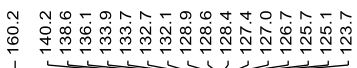

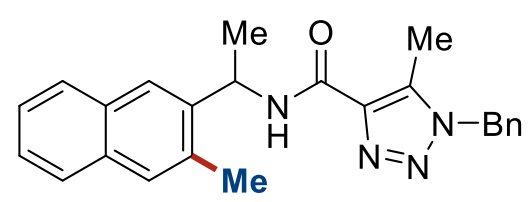

179x

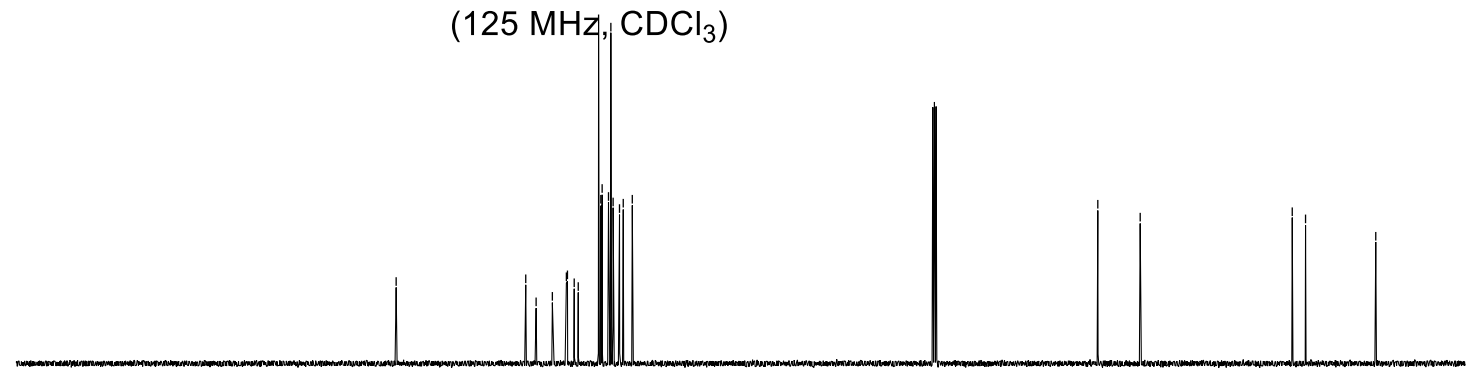

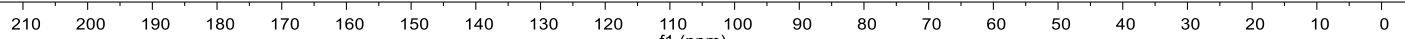




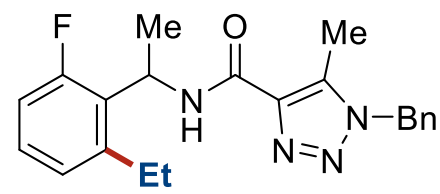

196h

(400 MHz, $\mathrm{CDCl}_{3}$ )

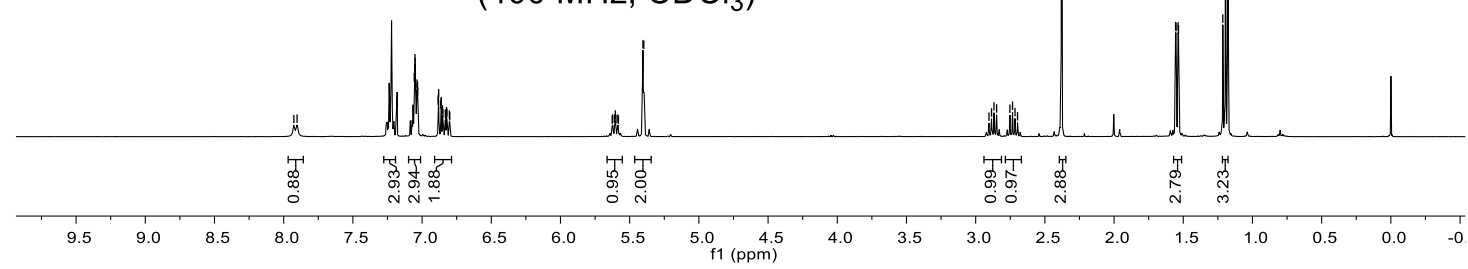

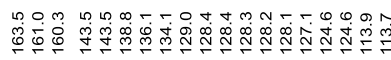

i

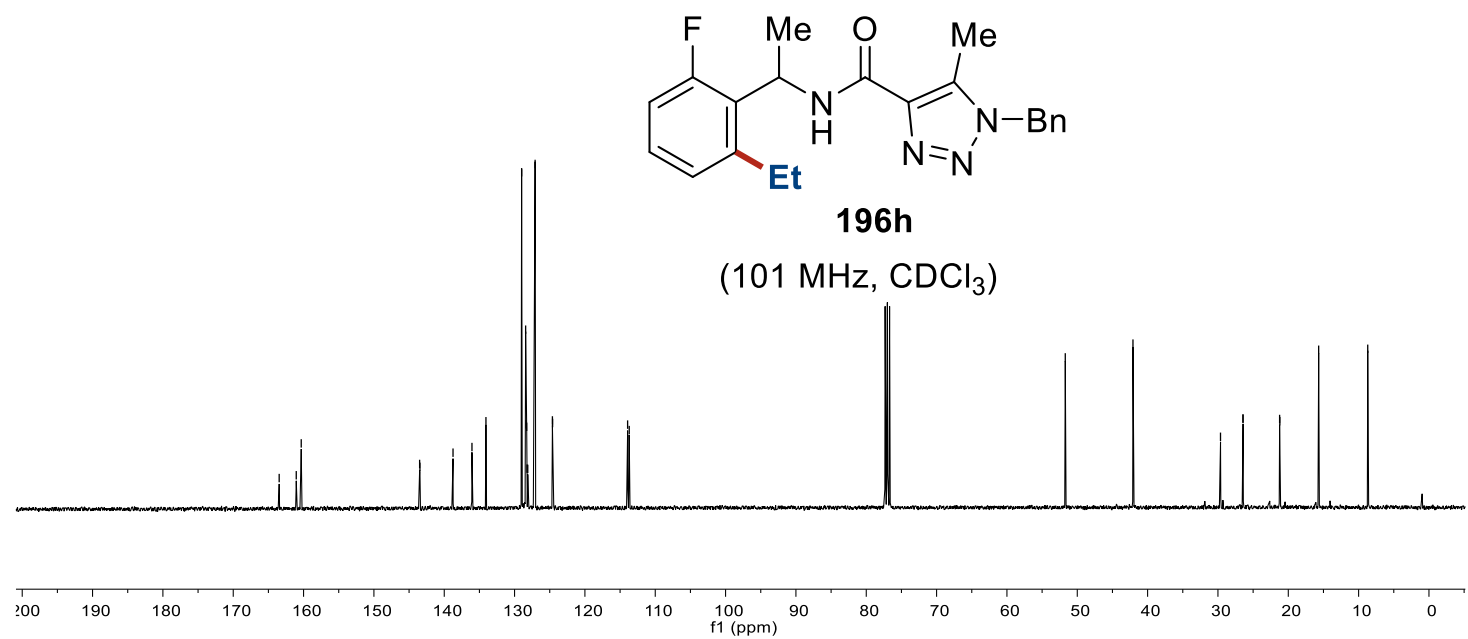




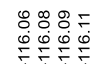

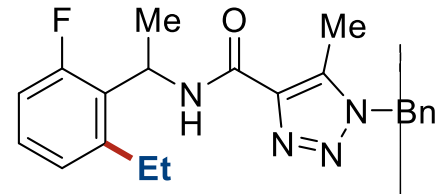

$196 \mathrm{~h}$

(376 MHz, $\mathrm{CDCl}_{3}$ )
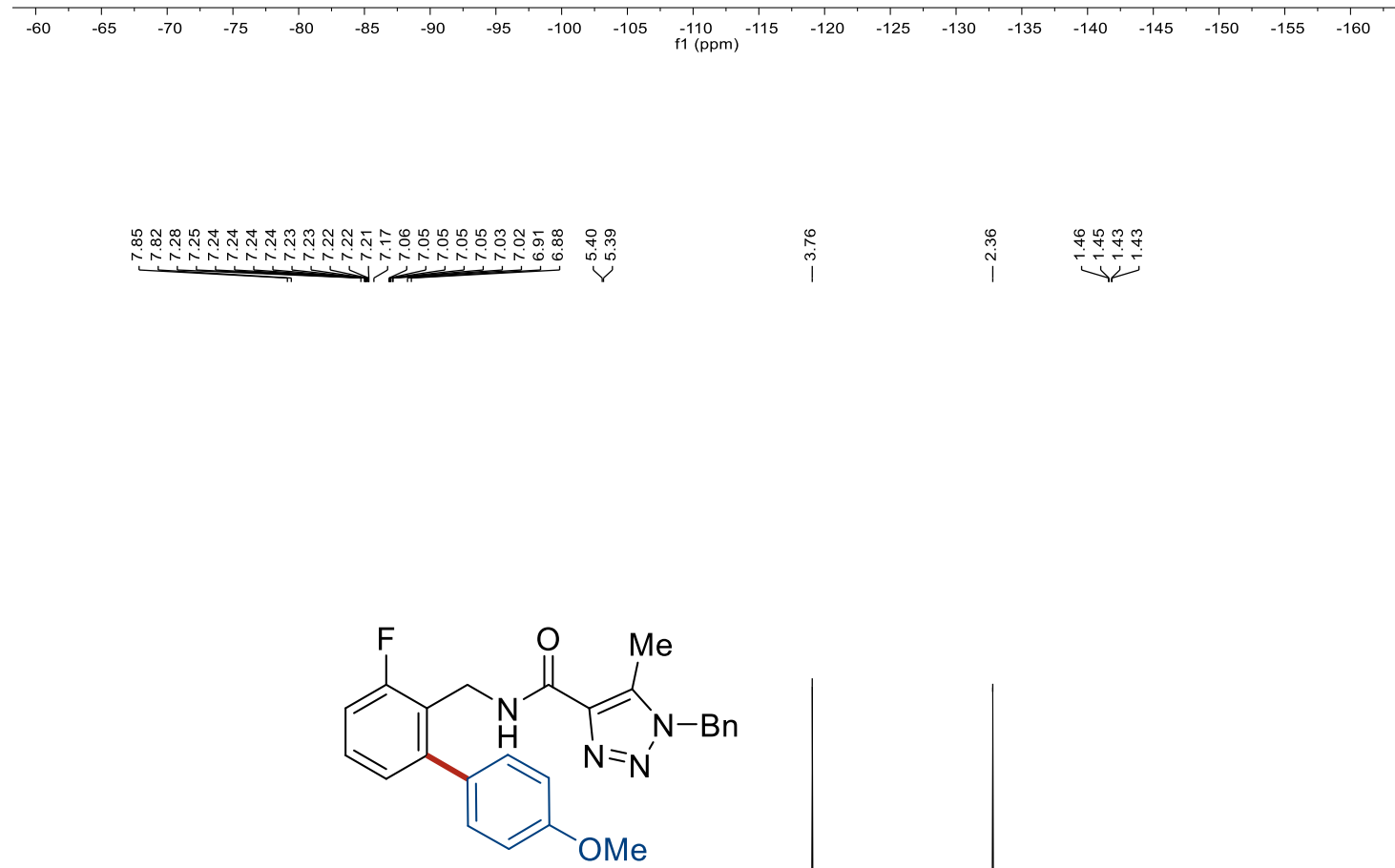

197a

(300 MHz, $\mathrm{CDCl}_{3}$ )

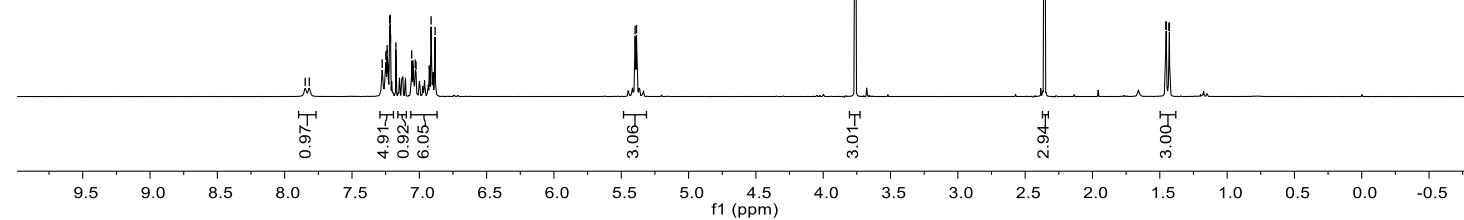




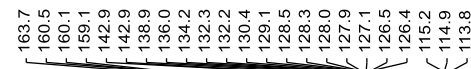

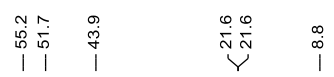<smiles>COc1ccc(-c2cccc(F)c2CNC(=O)c2nnn(Cc3ccccc3)c2C)cc1</smiles>

$197 a$

$\left(75 \mathrm{MHz}, \mathrm{CDCl}_{3}\right.$ )

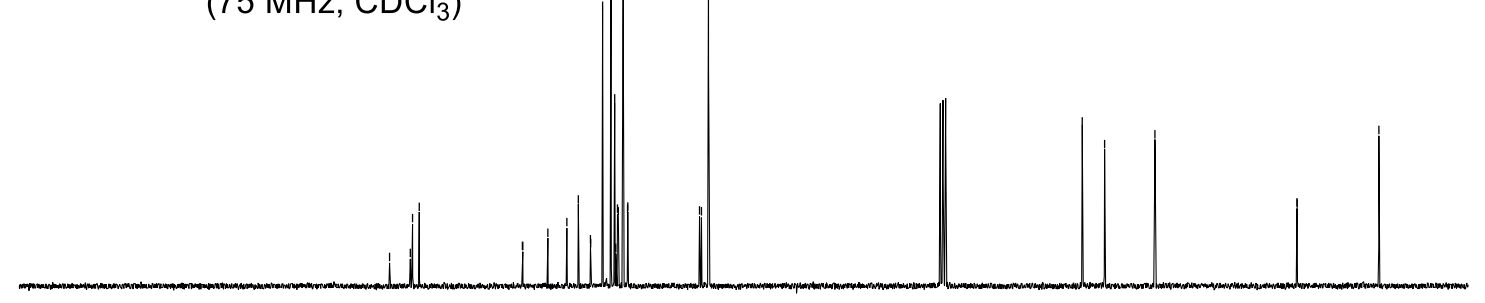

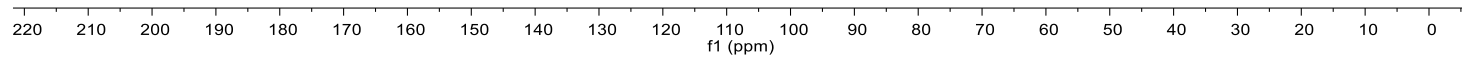

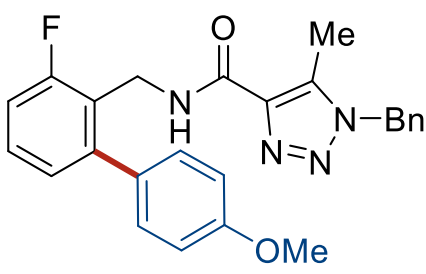

197a

(282 MHz, $\mathrm{CDCl}_{3}$ ) 


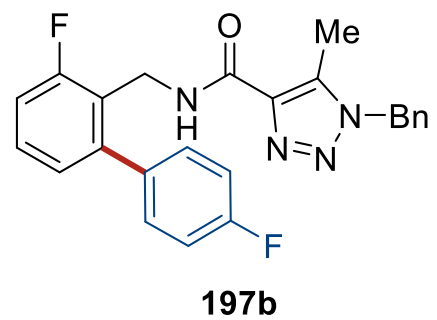

(300 MHz, $\mathrm{CDCl}_{3}$ )
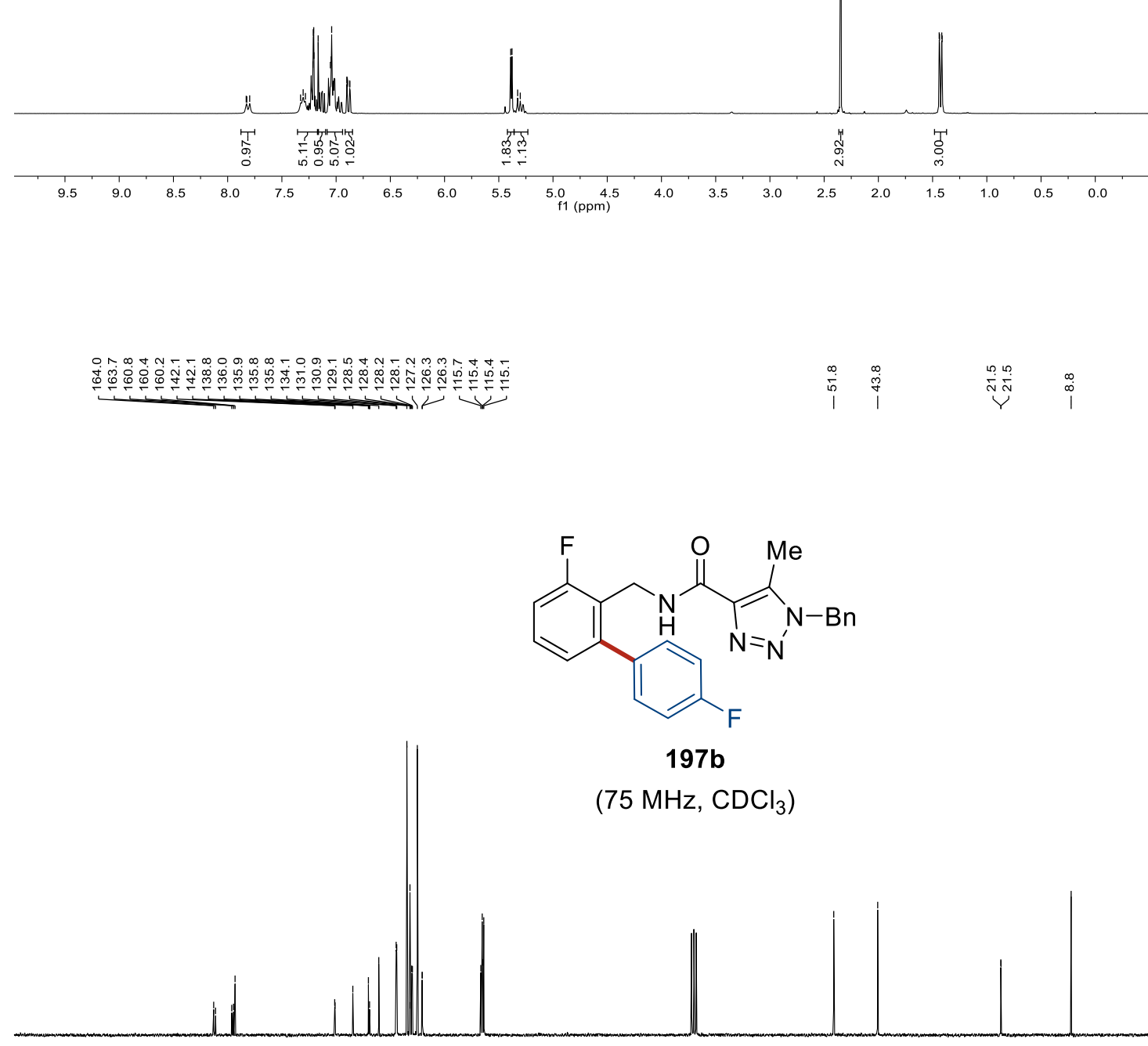

\begin{tabular}{lllllllllllllllllllllll}
\hline 00 & 190 & 180 & 170 & 160 & 150 & 140 & 130 & 120 & 110 & 100 & 90 & 80 & 70 & 60 & 50 & 40 & 30 & 20 & 10 & 0
\end{tabular} 
$\stackrel{2}{*}$<smiles>Cc1c(C(=O)NCc2c(F)cccc2-c2ccc(F)cc2)nnn1Br</smiles>

197b

$\left(282 \mathrm{MHz} \mathrm{CDCl}_{3}\right)$<smiles>COc1ccc(-c2cccc(C(F)(F)F)c2CNC(=O)c2nnn(Cc3ccccc3)c2C)cc1</smiles>

$197 c$

$\left(300 \mathrm{MHz}, \mathrm{CDCl}_{3}\right.$ )

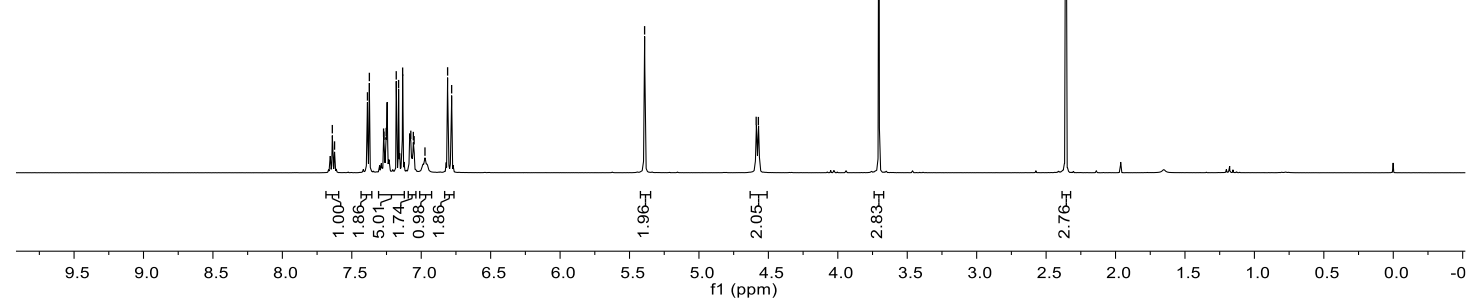




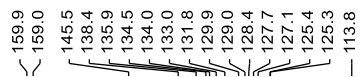

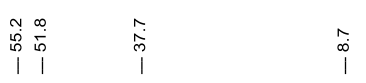<smiles>COc1ccc(-c2cccc(C(F)(F)F)c2CNC(=O)c2nnn(Cc3ccccc3)c2C)cc1</smiles>

197c

(125 MHz, $\mathrm{CDCl}_{3}$ )

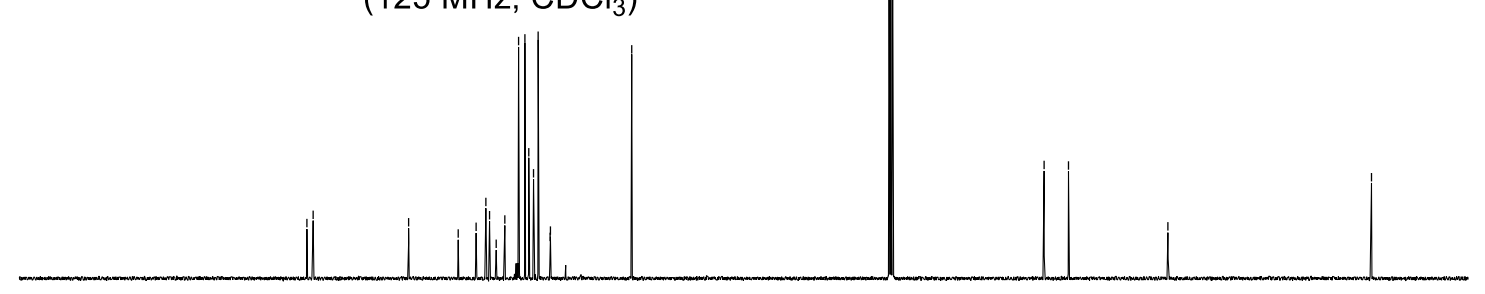

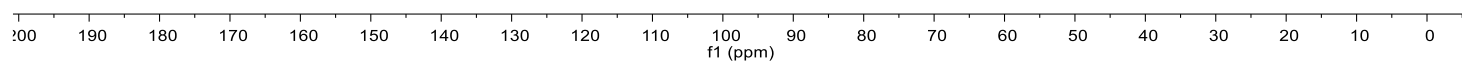<smiles>COc1ccc(-c2cccc(C(F)(F)F)c2CNC(=O)c2nnn(Cc3ccccc3)c2C)cc1</smiles>

$197 \mathrm{c}$

(282 MHz, $\mathrm{CDCl}_{3}$ ) 


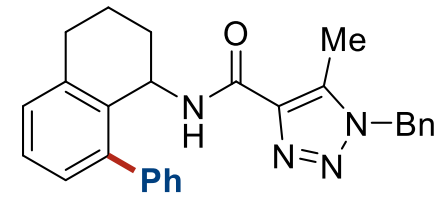

197d

(300 MHz, $\mathrm{CDCl}_{3}$ )

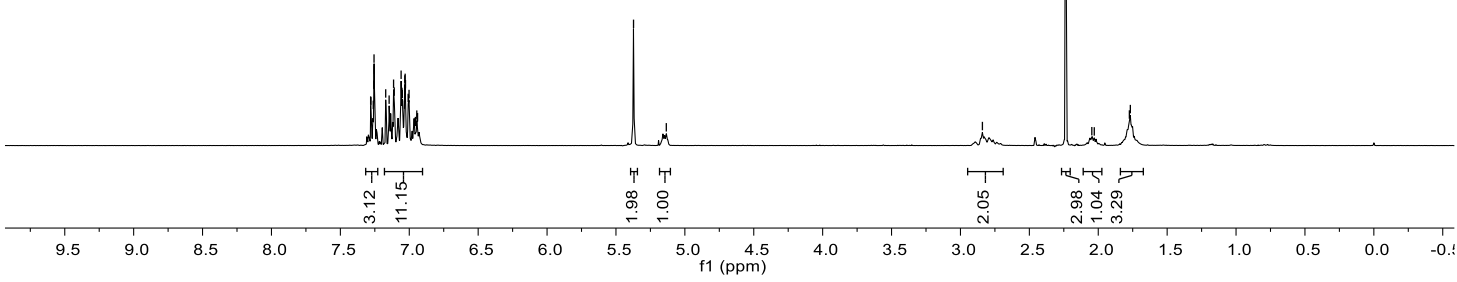

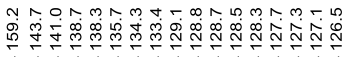

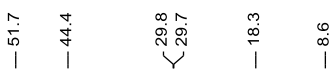

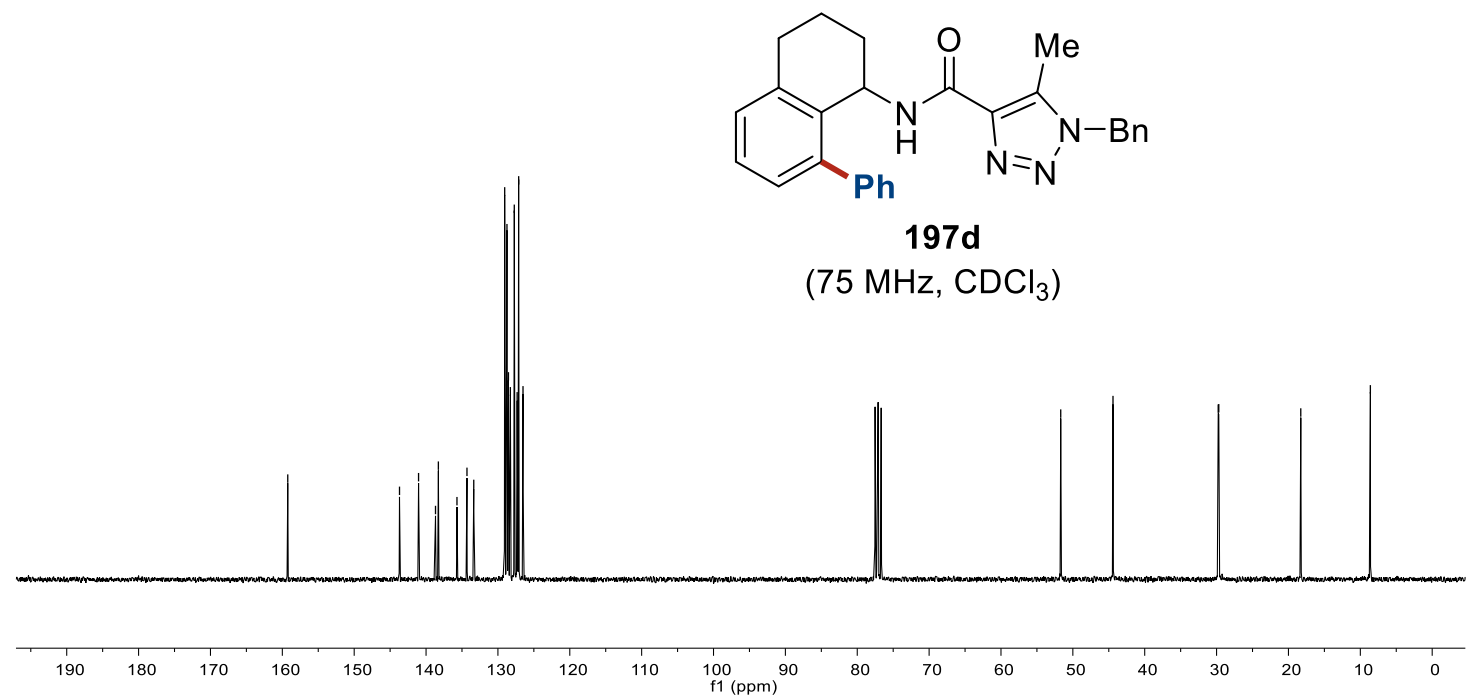




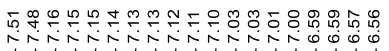

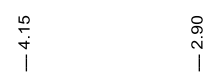
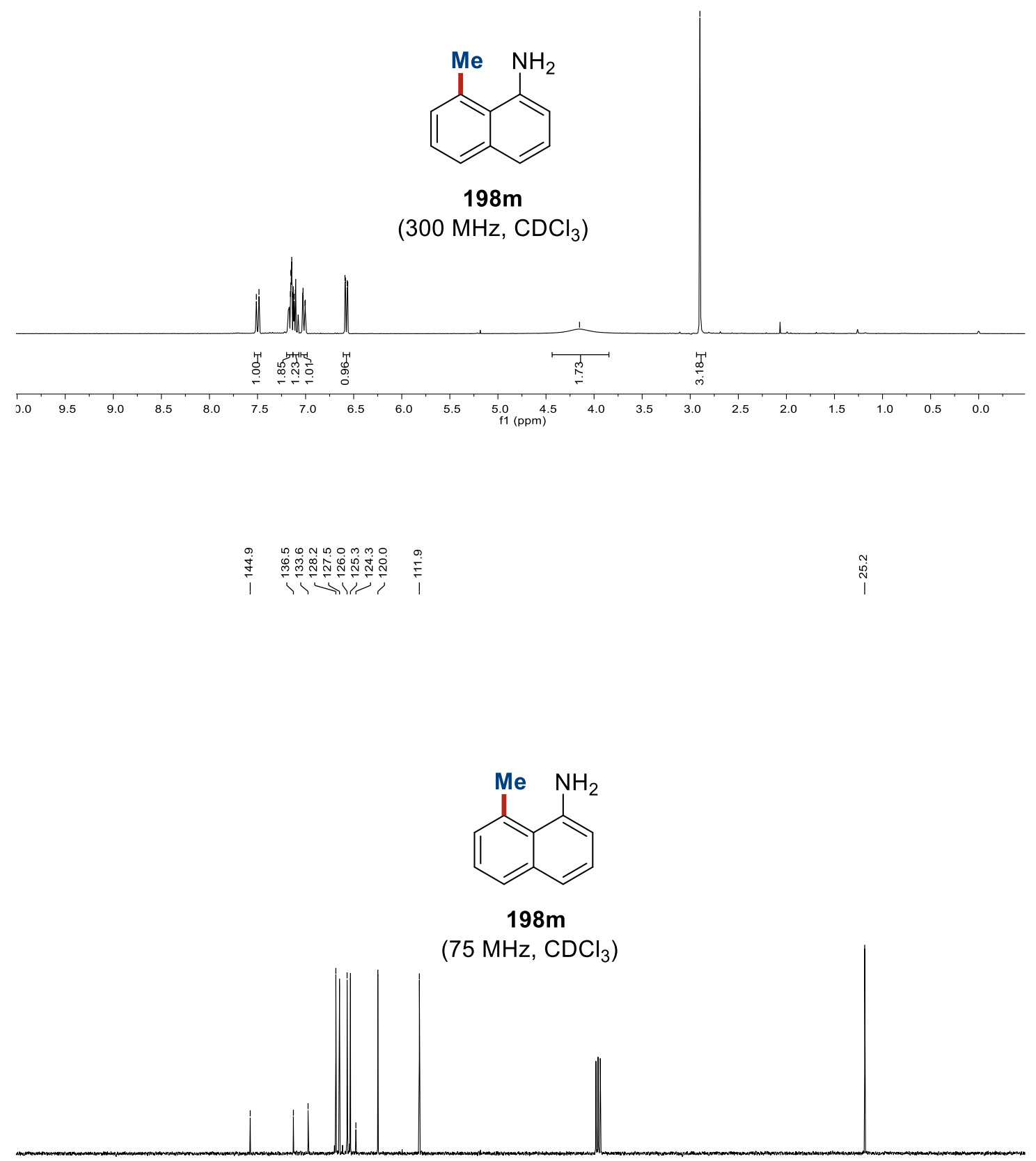

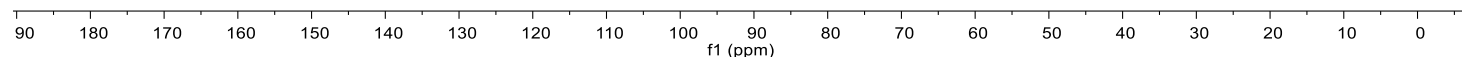



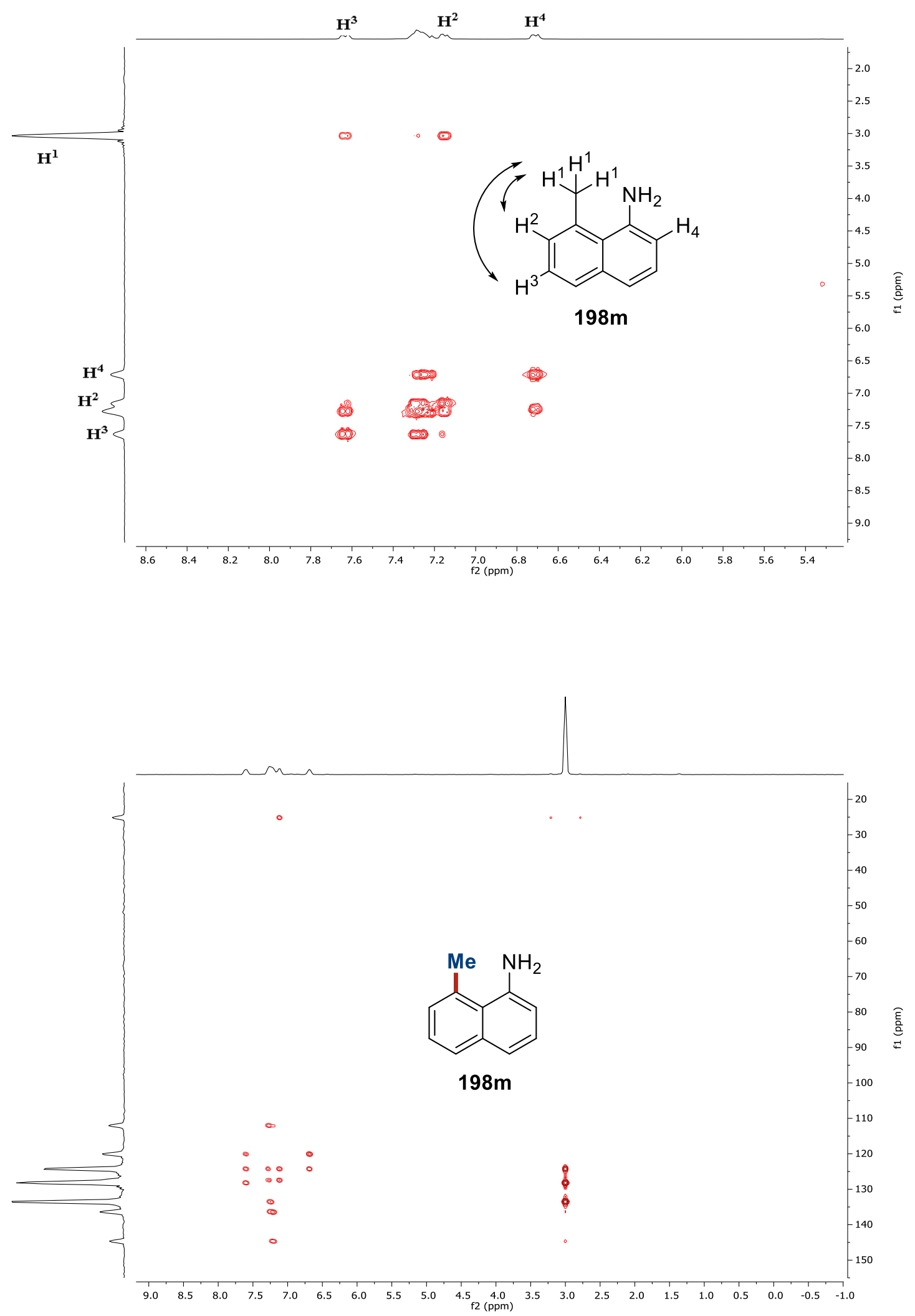


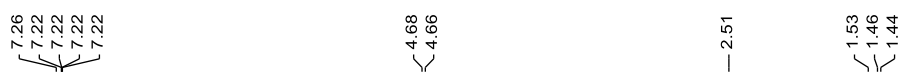

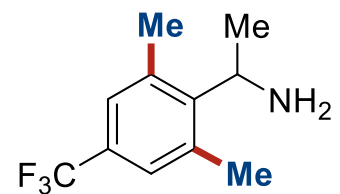

198s

(300 $\mathrm{MHz}, \mathrm{CDCl}_{3}$ )

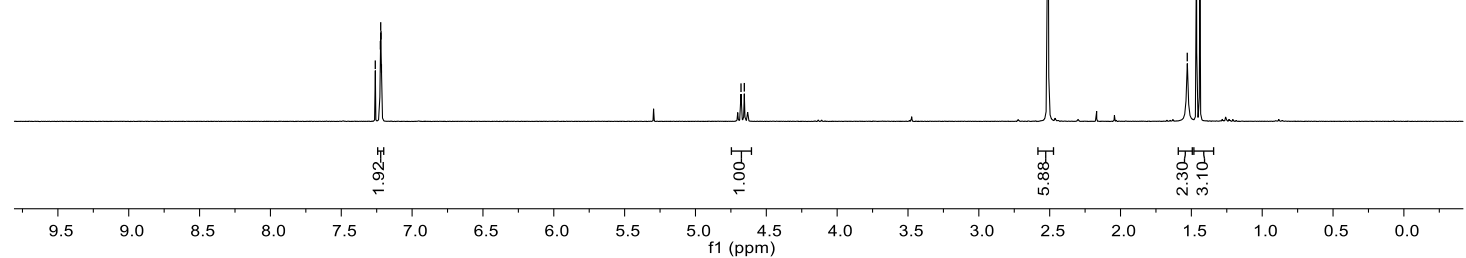

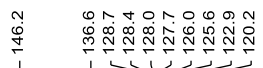

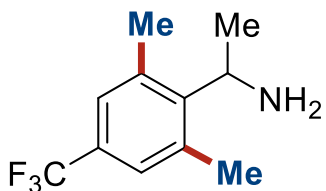

198s

(100 $\mathrm{MHz}, \mathrm{CDCl}_{3}$ )

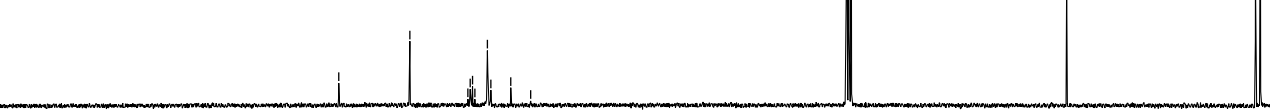

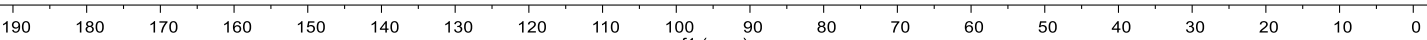




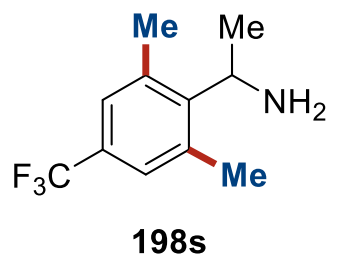

(376 MHz, $\mathrm{CDCl}_{3}$ )
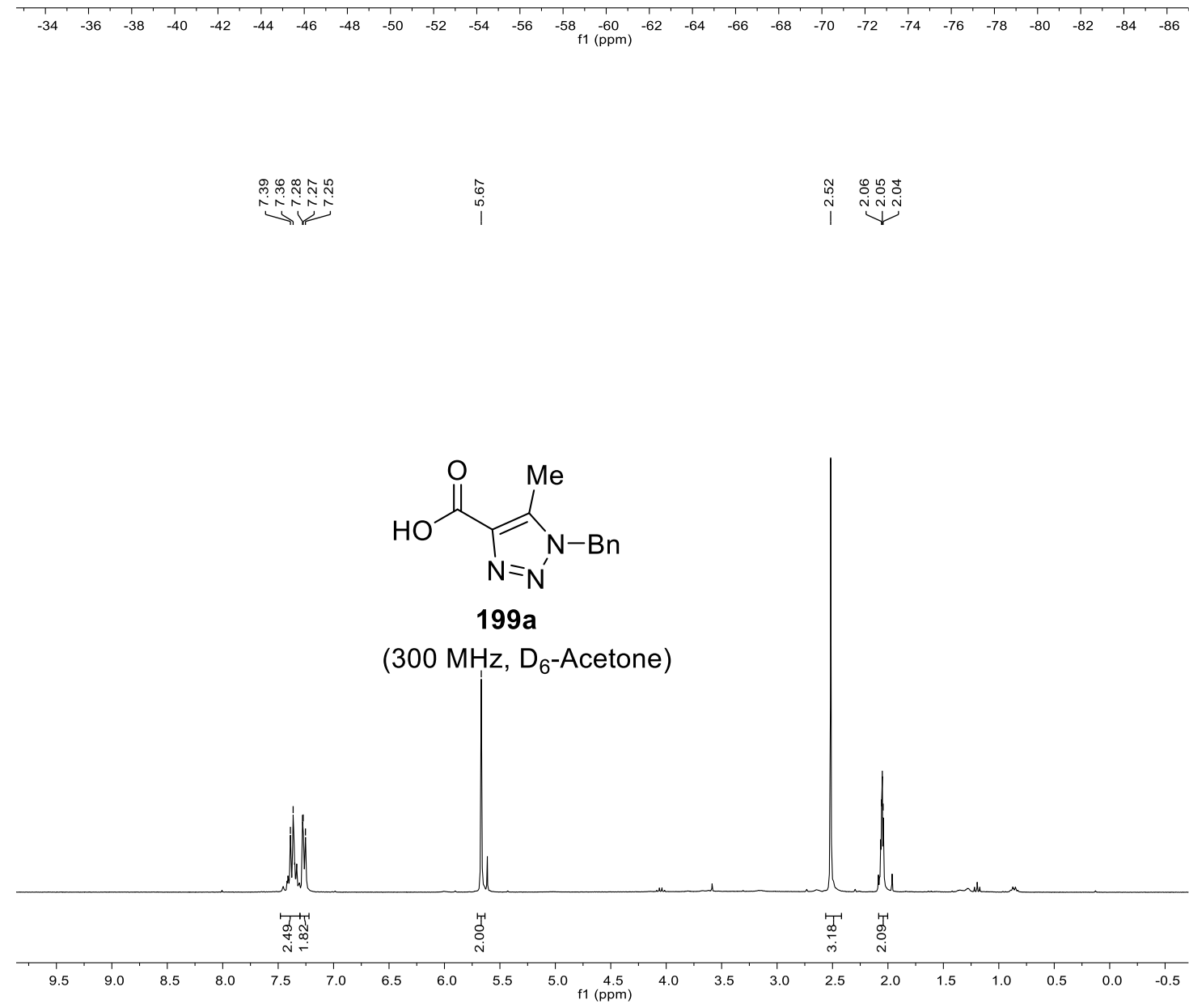


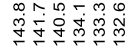

रiा रा

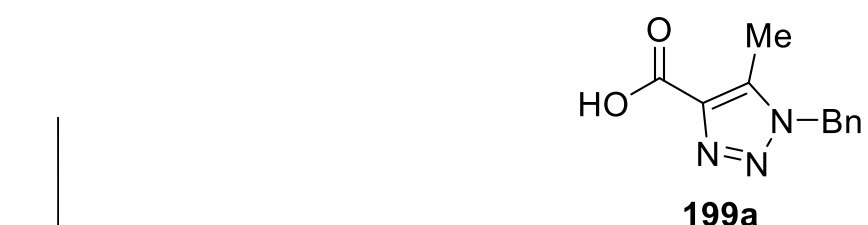

(75 MHz, $\mathrm{D}_{6}$-Acetone)

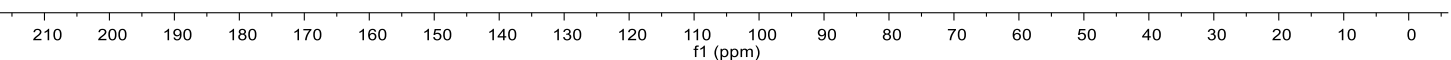

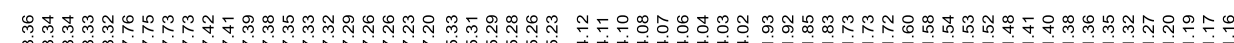

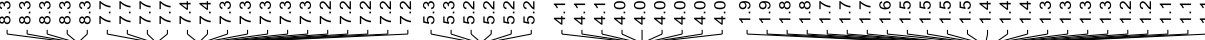

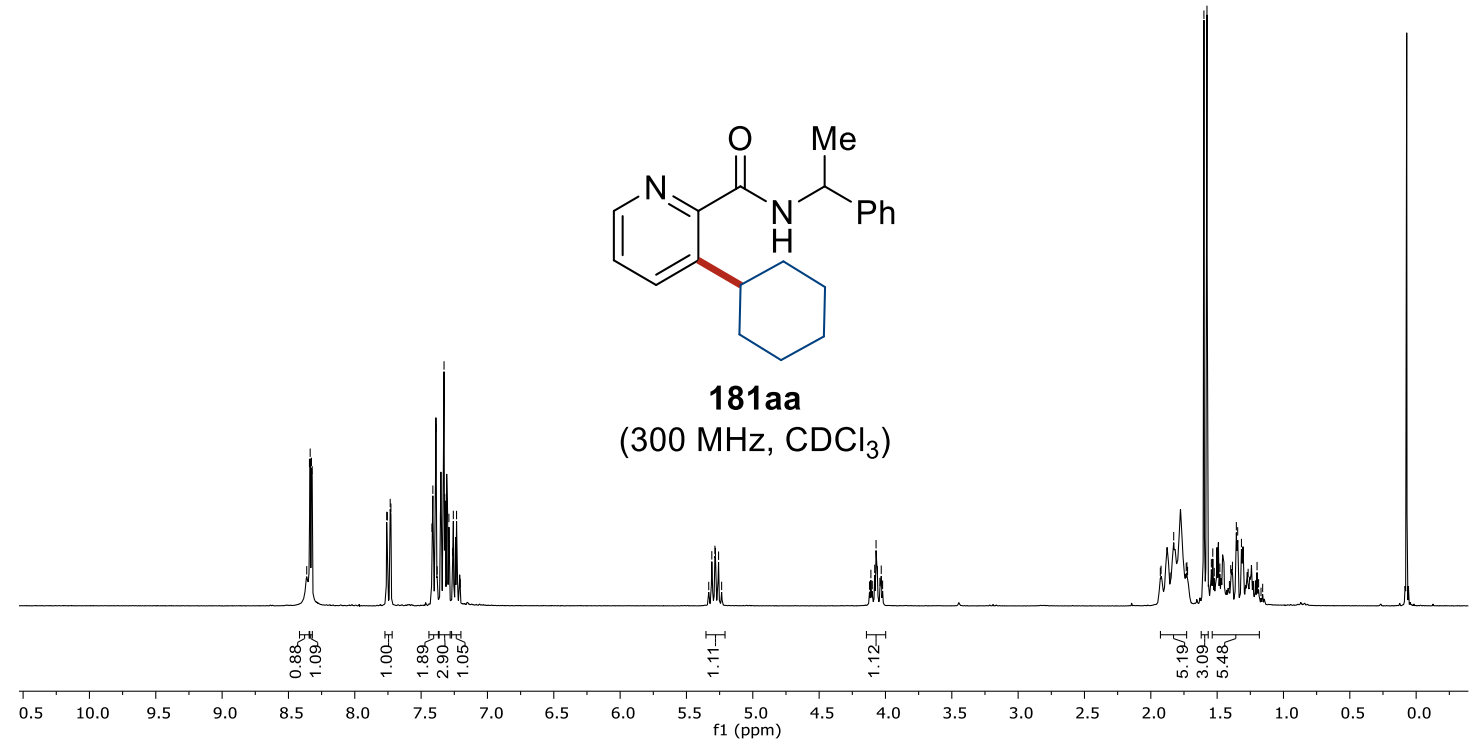



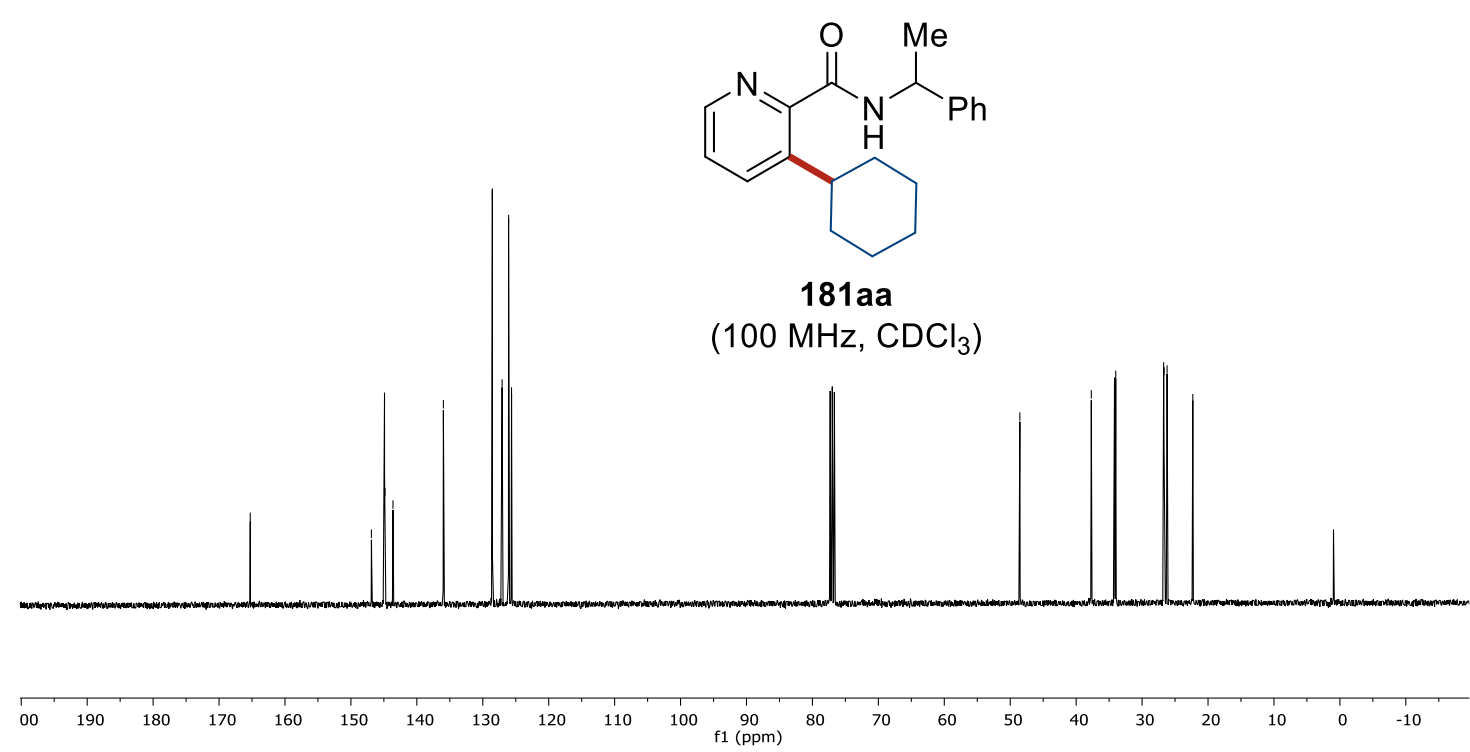

$\underbrace{\underbrace{\infty}_{000}}$

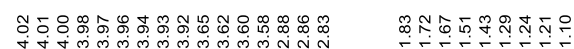<smiles>O=C(NCCc1ccccc1)c1ncccc1C1CCCCC1</smiles>

181 ba

(300 MHz, $\mathrm{CDCl}_{3}$ )

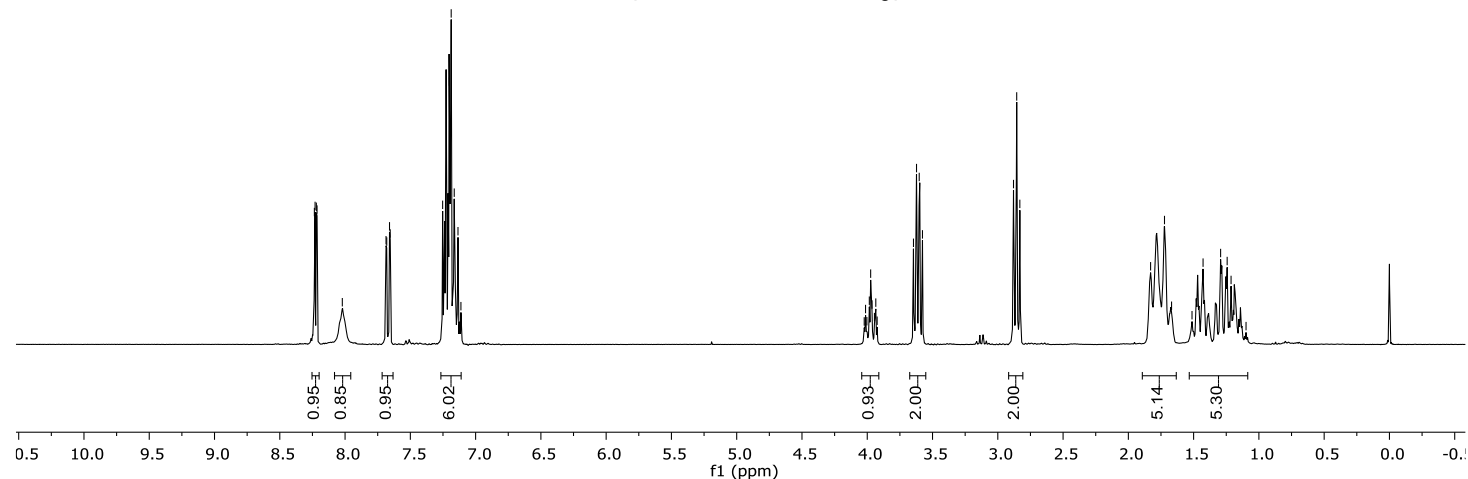




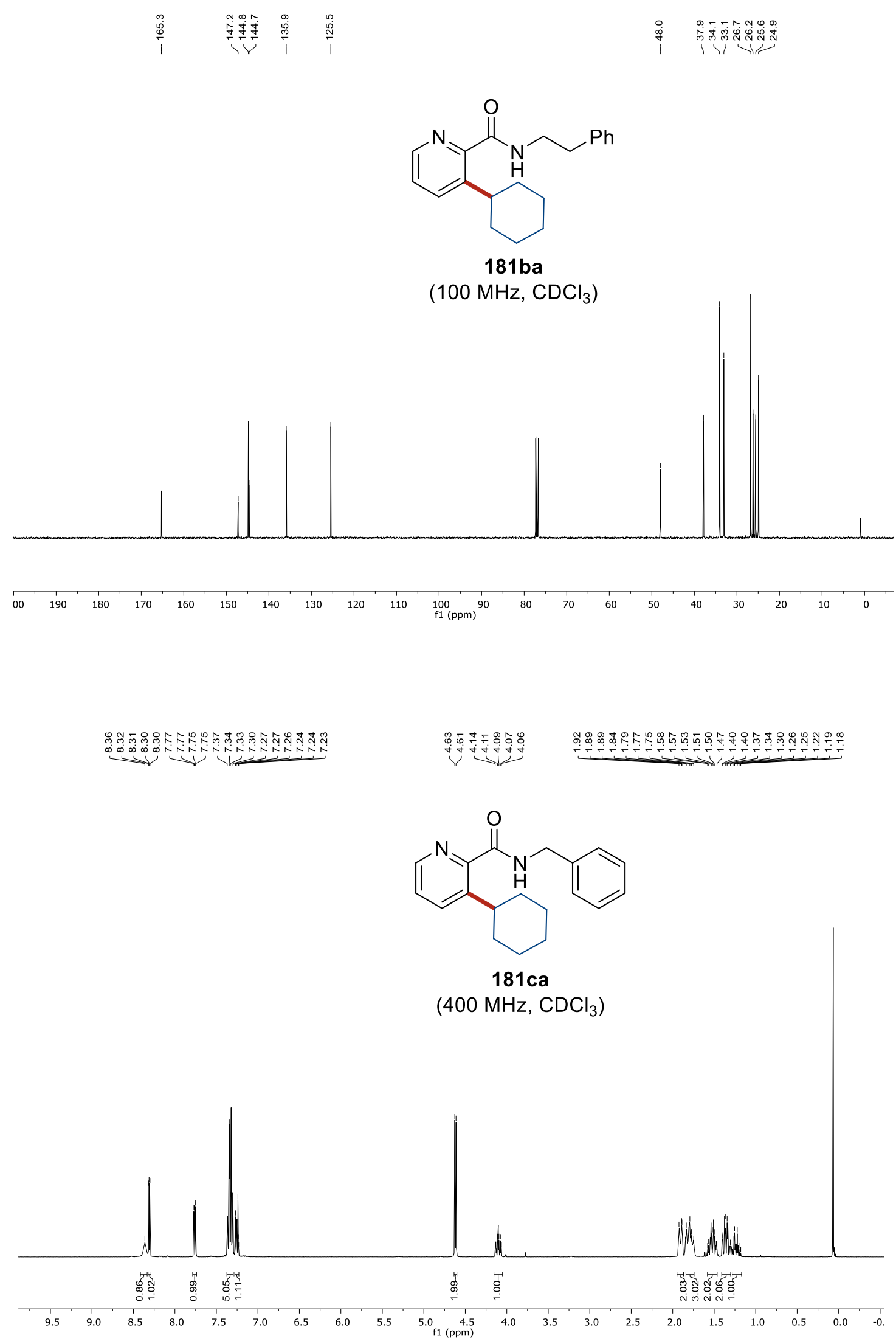




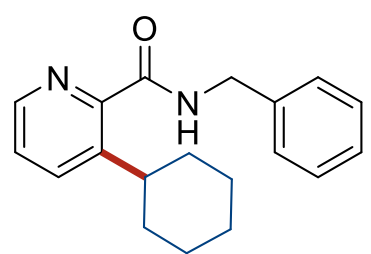

181ca

$\left(100 \mathrm{MHz}, \mathrm{CDCl}_{3}\right.$ )
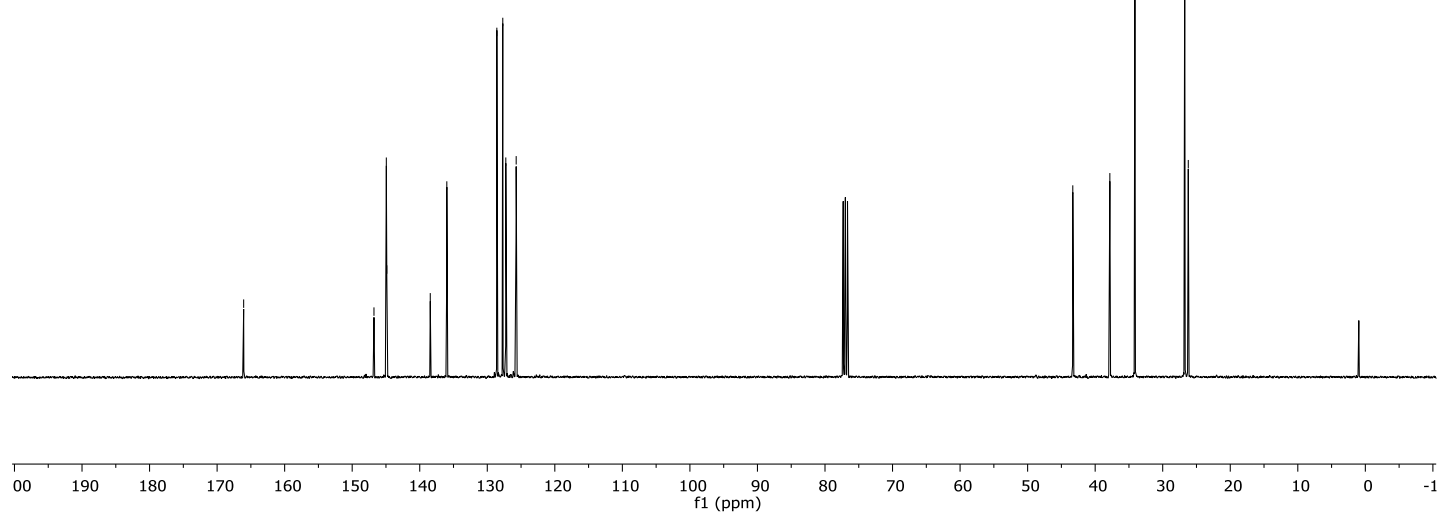

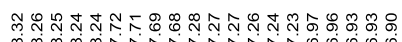

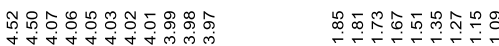

on

نU ن<smiles>O=C(NCc1ccc(F)cc1)c1ncccc1C1CCCCC1</smiles>

$181 \mathrm{da}$

$\left(300 \mathrm{MHz}, \mathrm{CDCl}_{3}\right)$

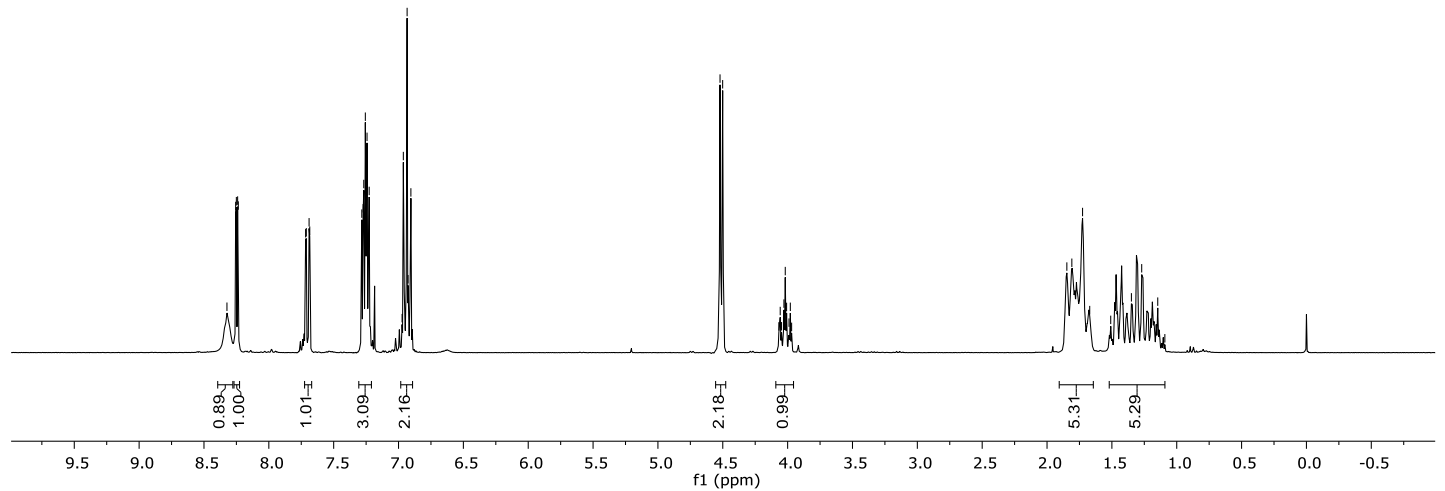




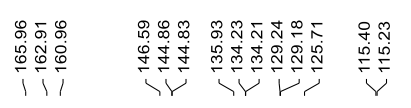

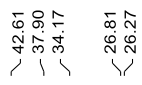<smiles>O=C(NCc1ccc(F)cc1)c1ncccc1C1CCCCC1</smiles>

$181 \mathrm{da}$

$\left(125 \mathrm{MHz}, \mathrm{CDCl}_{3}\right)$

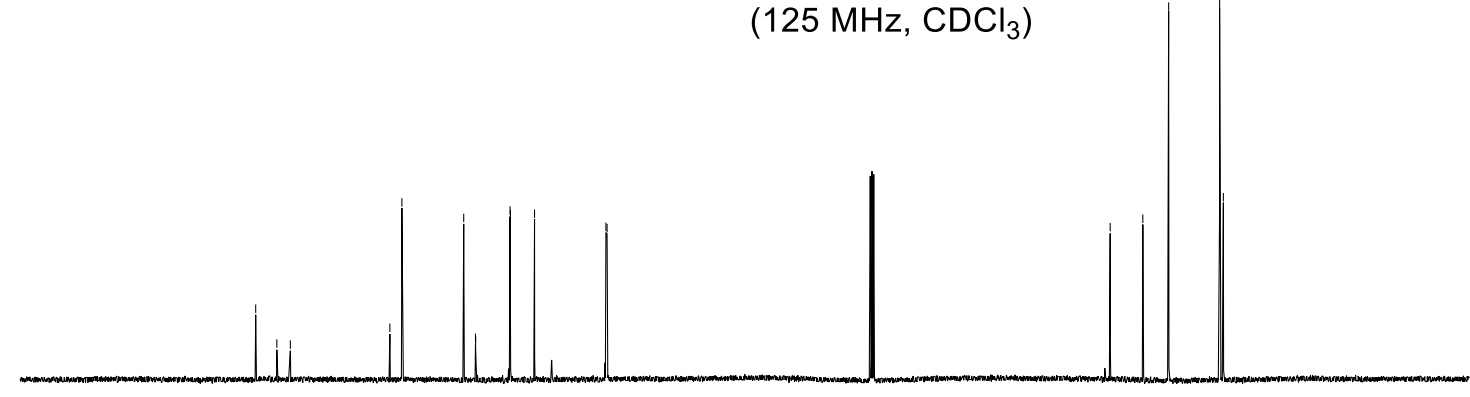

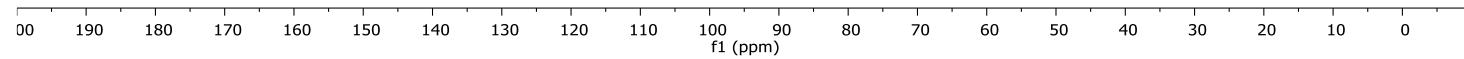

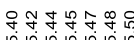

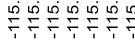

定

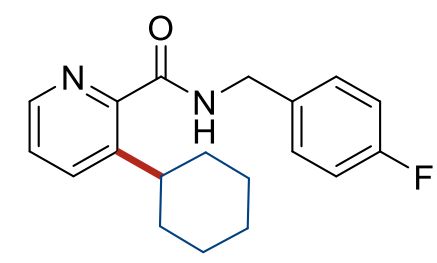

$181 \mathrm{da}$

(275 MHz, $\mathrm{CDCl}_{3}$ )

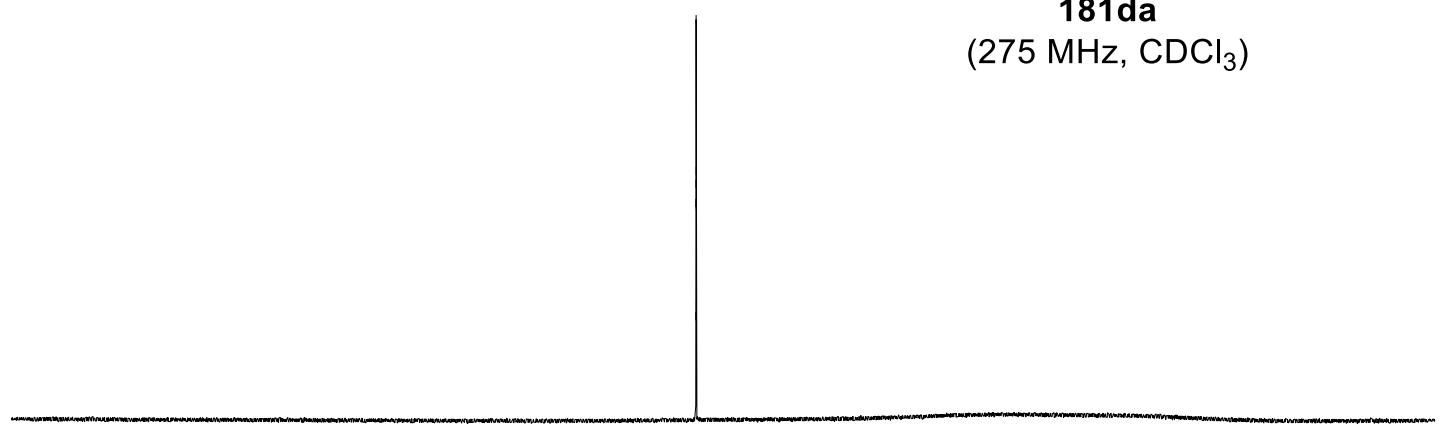

$\begin{array}{llllllllllllllllllllllll}-10 & -20 & -30 & -40 & -50 & -60 & -70 & -80 & -90 & -100 & -110 & -120 & -130 & -140 & -150 & -160 & -170 & -180 & -190 & -200 & -210 & -220 & -230\end{array}$ 


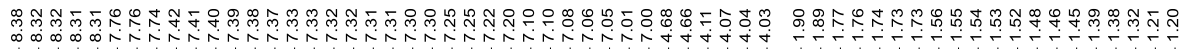<smiles>O=C(NCc1ccccc1F)c1ncccc1C1CCCCC1</smiles>

181 ea

$\left(400 \mathrm{MHz}, \mathrm{CDCl}_{3}\right)$

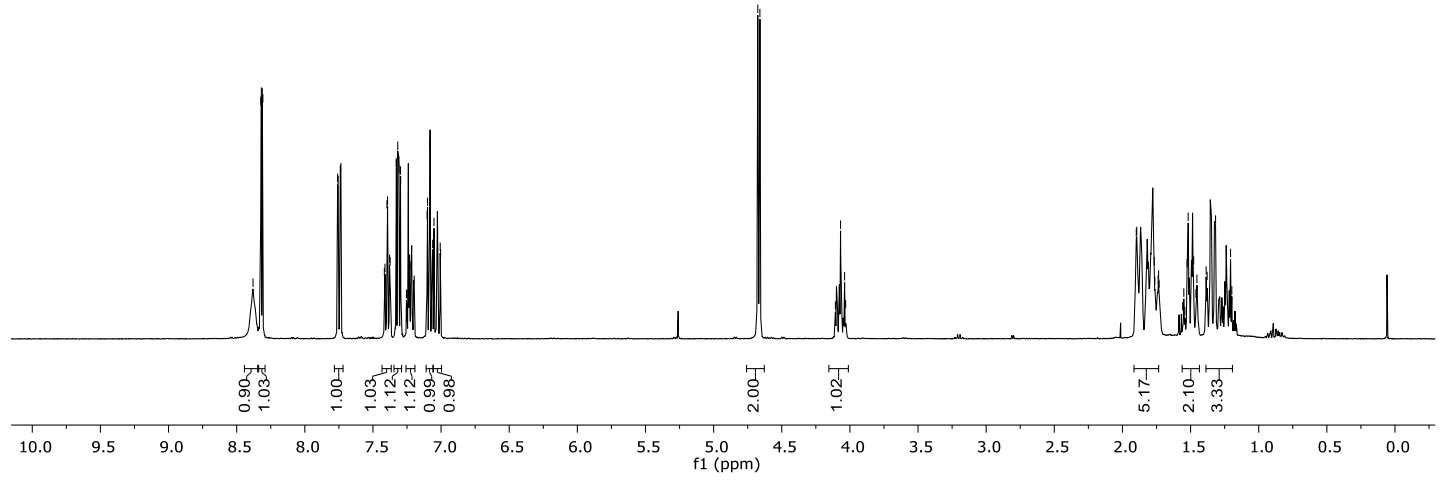

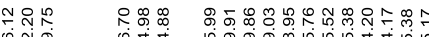

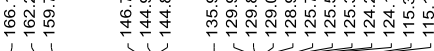

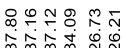

inj<smiles>O=C(NCc1ccccc1F)c1ncccc1C1CCCCC1</smiles>

181ea

$\left(101 \mathrm{MHz}, \mathrm{CDCl}_{3}\right)$

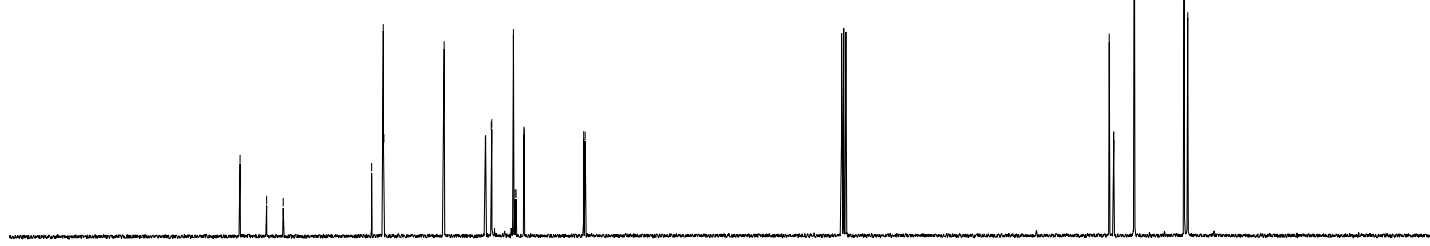

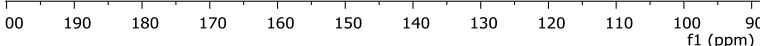




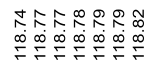<smiles>O=C(NCc1ccccc1F)c1ncccc1C1CCCCC1</smiles>

181ea

(275 $\mathrm{MHz}, \mathrm{CDCl}_{3}$ )

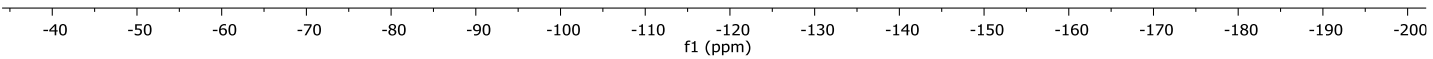

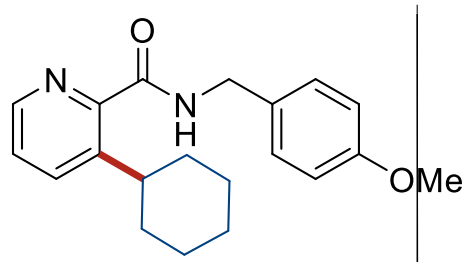

$181 \mathrm{fa}$

$\left(400 \mathrm{MHz}, \mathrm{CDCl}_{3}\right)$

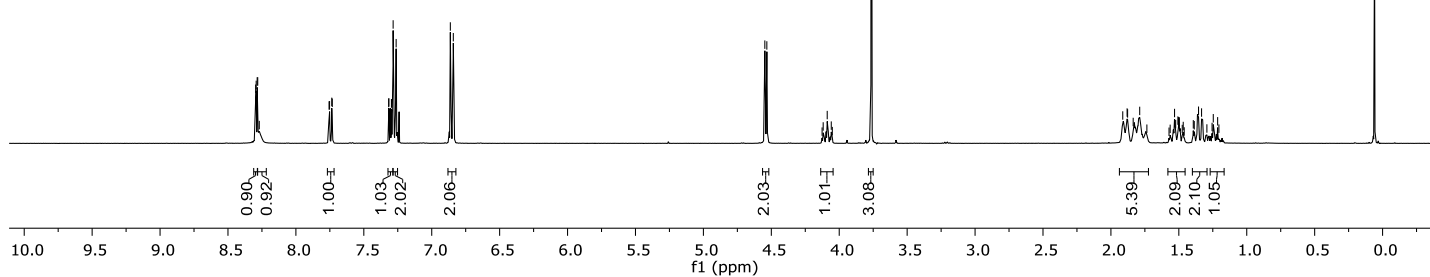




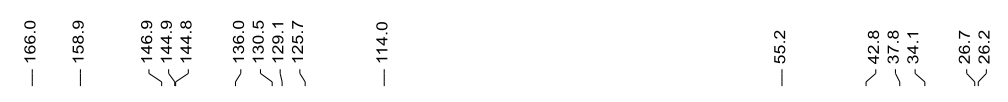<smiles>COc1ccc(CNC(=O)c2ncccc2C2CCCCC2)cc1</smiles>

$181 \mathrm{fa}$

$\left(100 \mathrm{MHz}, \mathrm{CDCl}_{3}\right)$

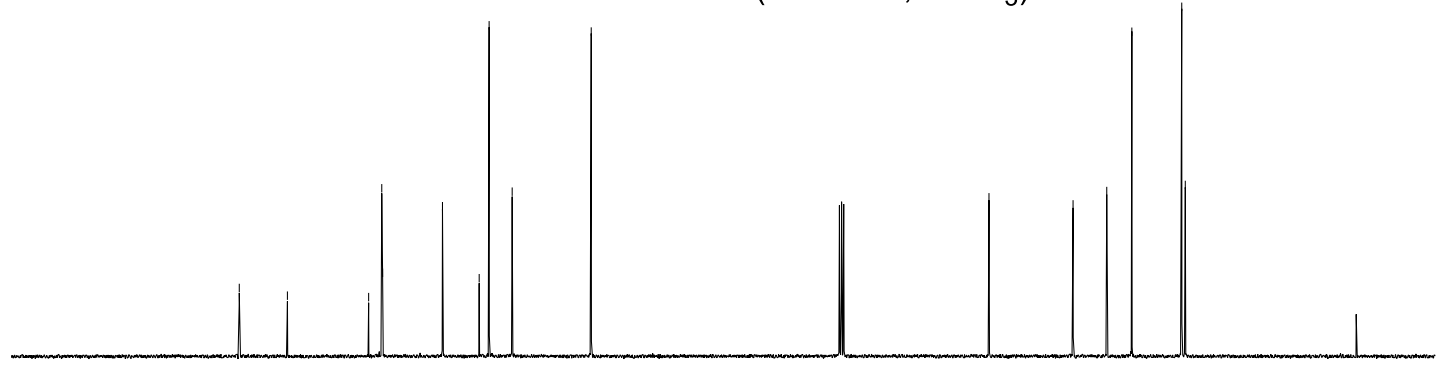

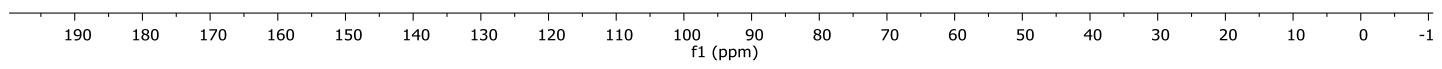

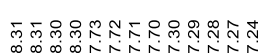

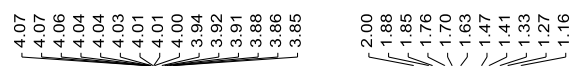

$\underbrace{\infty}_{\infty} \underbrace{\infty}$

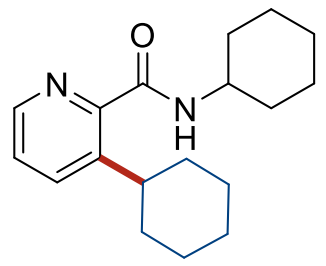

181ga

(400 MHz, $\mathrm{CDCl}_{3}$ )

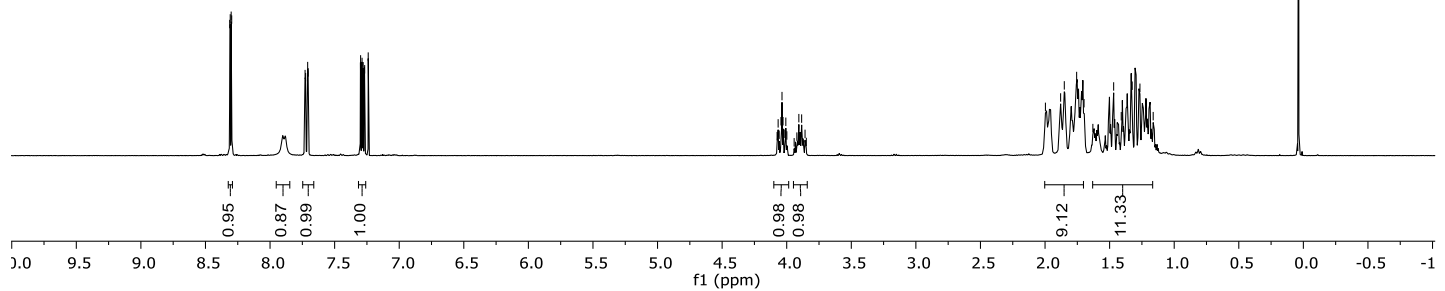




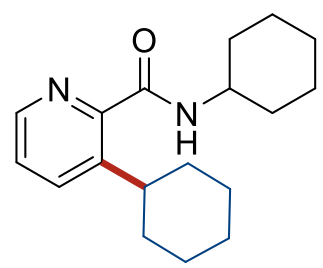

181ga

(100 MHz, $\mathrm{CDCl}_{3}$ )

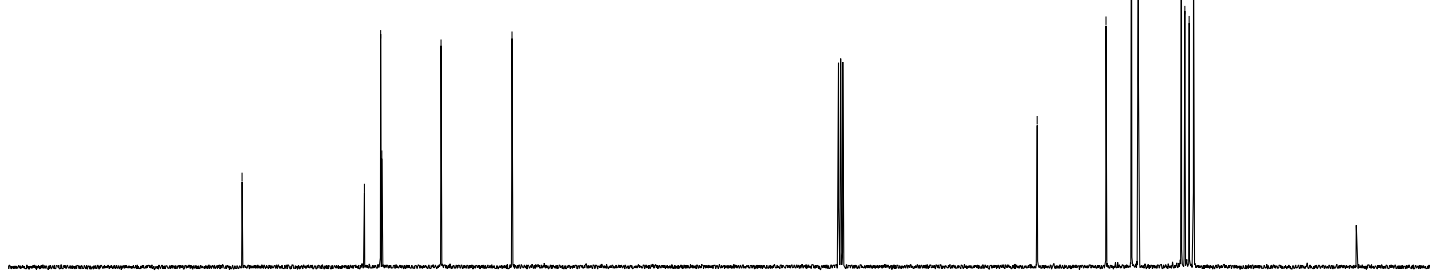

$\begin{array}{llllllllll}190 & 180 & 170 & 160 & 150 & 140 & 130 & 120 & 110 & \begin{array}{c}100 \\ \mathrm{f} 1(\mathrm{ppm})\end{array}\end{array}$

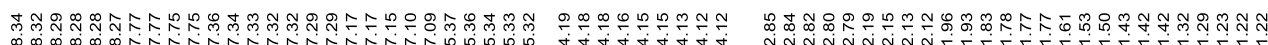

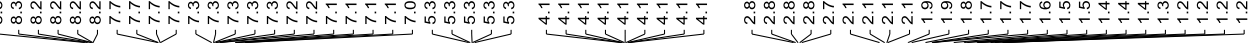

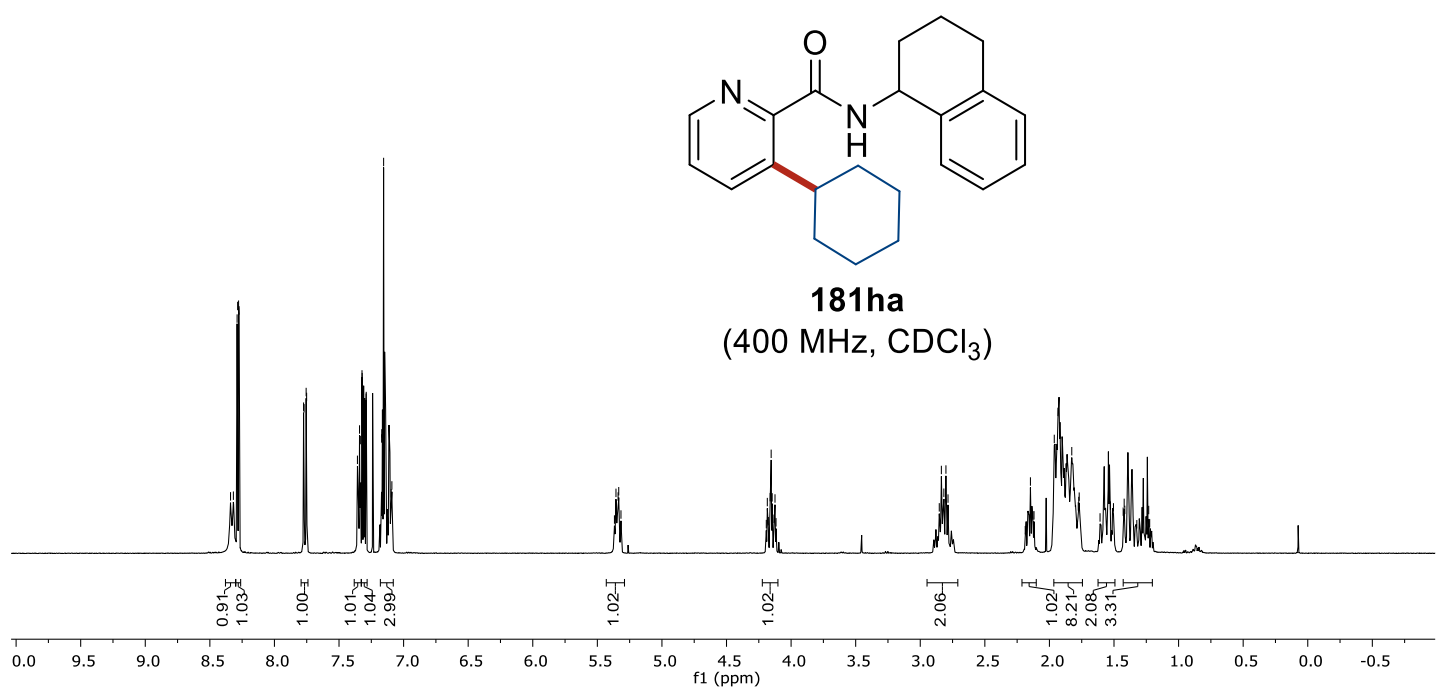


<smiles>O=C(NC1CCCc2ccccc21)c1ncccc1C1CCCCC1</smiles>

181ha

$\left(100 \mathrm{MHz}, \mathrm{CDCl}_{3}\right)$
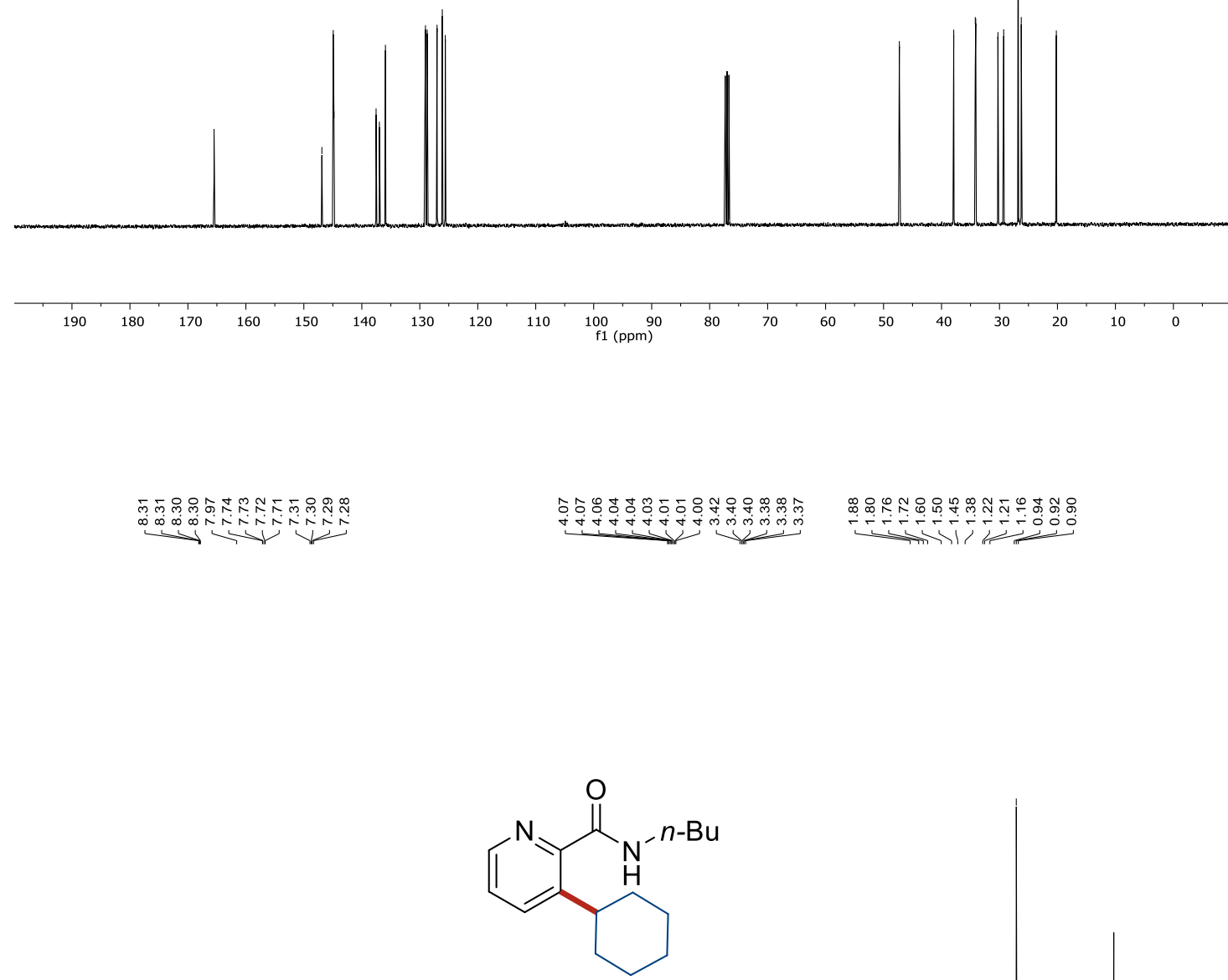

$181 \mathrm{ia}$

(400 $\mathrm{MHz}, \mathrm{CDCl}_{3}$ )

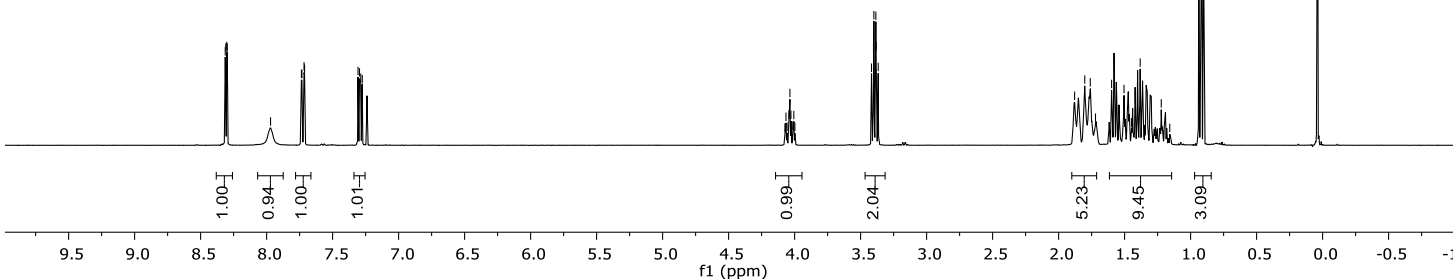




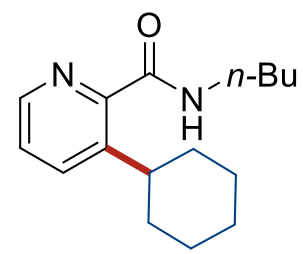

$181 \mathrm{ia}$

(100 MHz, $\mathrm{CDCl}_{3}$ )

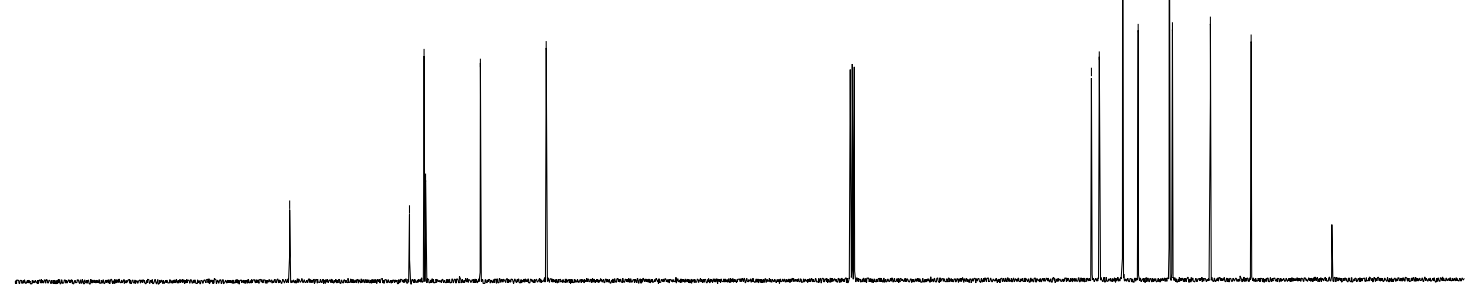

$\begin{array}{lllllllllll}200 & 190 & 180 & 170 & 160 & 150 & 140 & 130 & 120 & 110 & \begin{array}{c}100 \\ \mathrm{f} 1(\mathrm{ppm})\end{array}\end{array}$

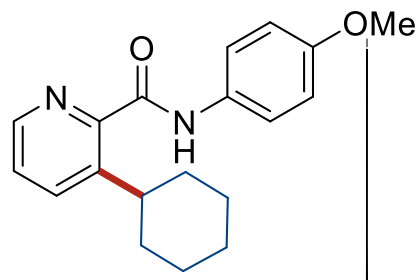

181ja

$\left(300 \mathrm{MHz}, \mathrm{CDCl}_{3}\right)$

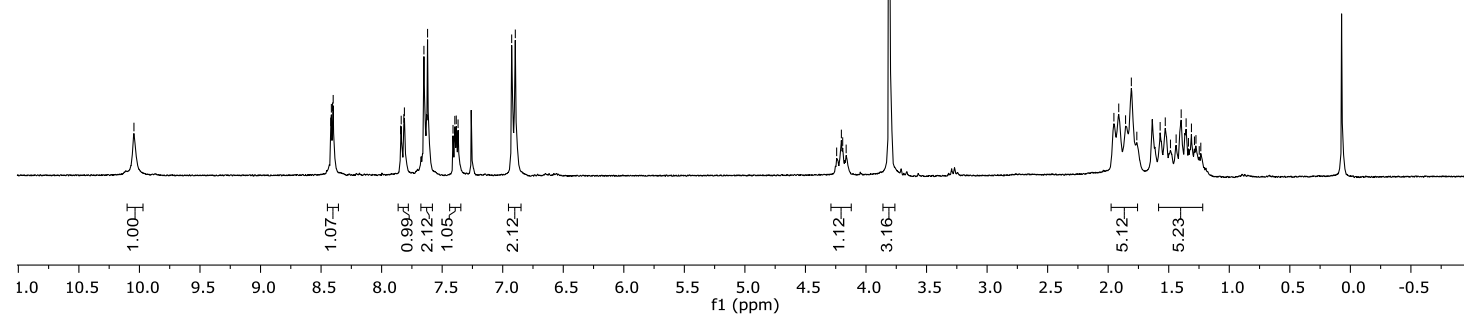


i

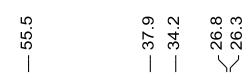<smiles>COc1ccc(NC(=O)c2ncccc2C2CCCCC2)cc1</smiles>

$181 \mathrm{ja}$

$(125 \mathrm{MHz}, \mathrm{CDCl}\})$

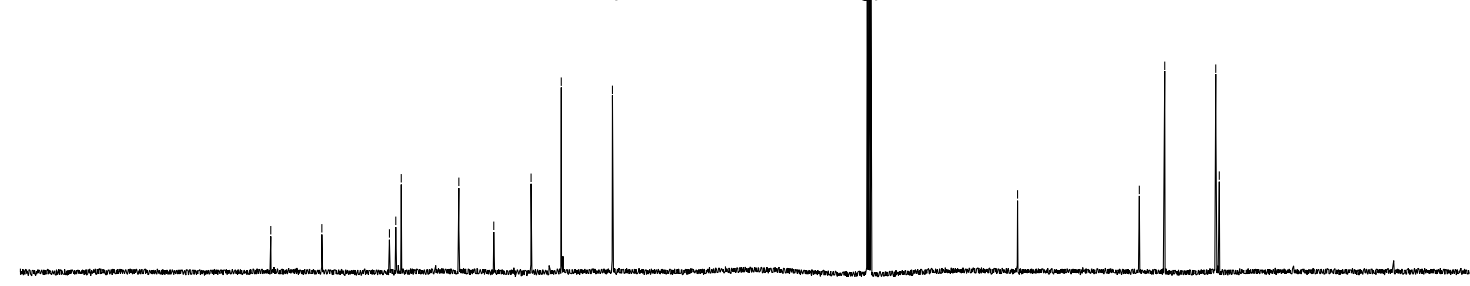

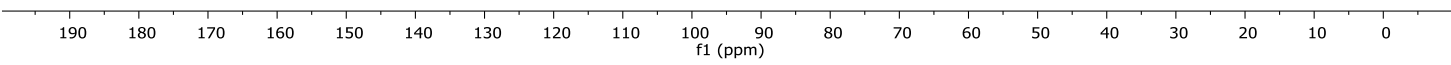

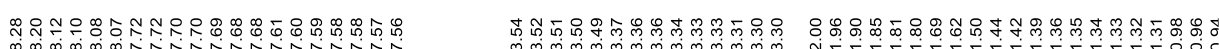

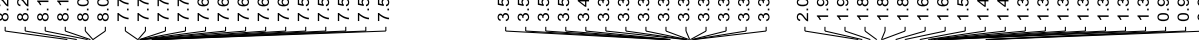

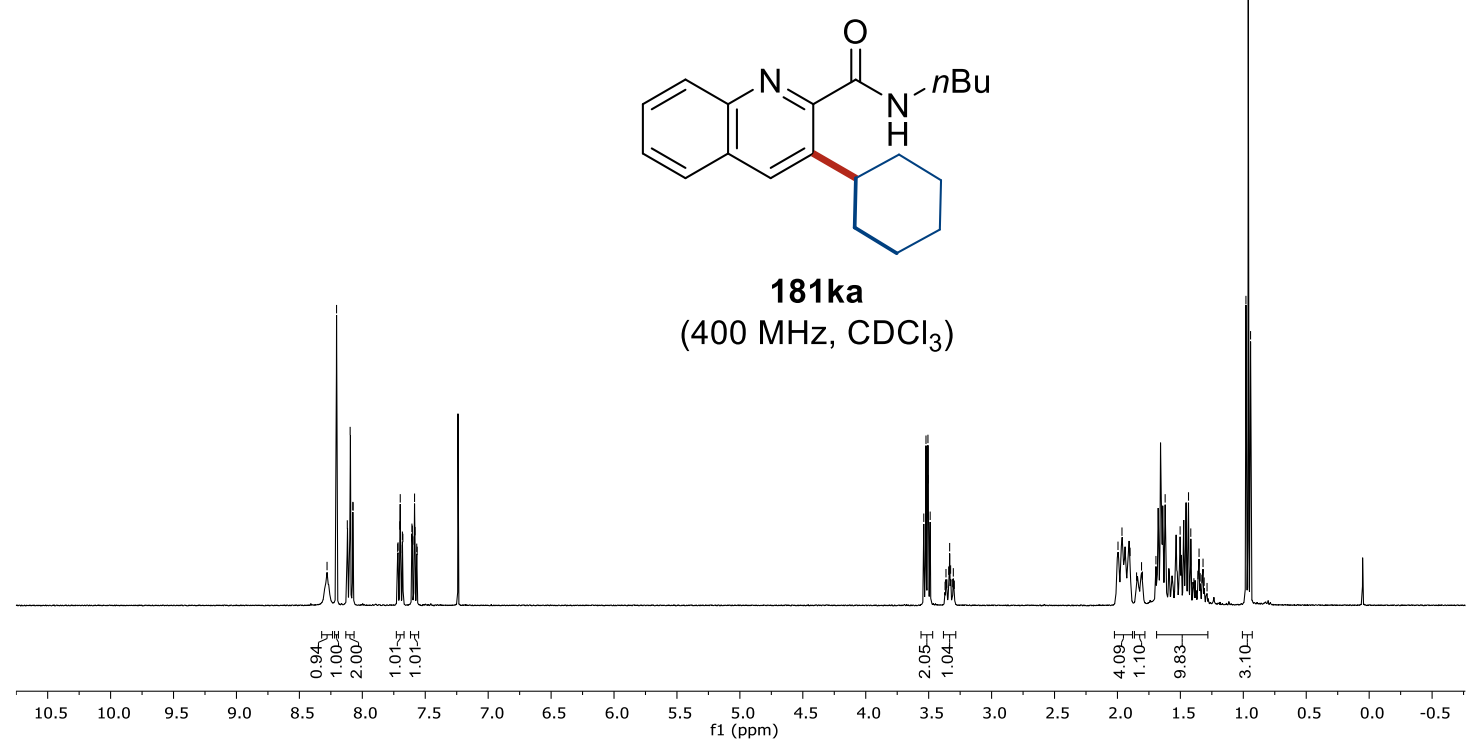




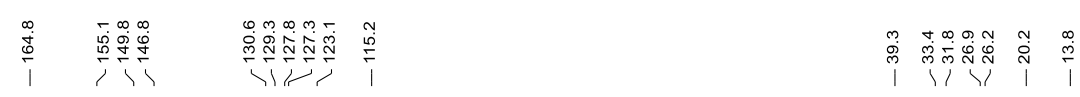

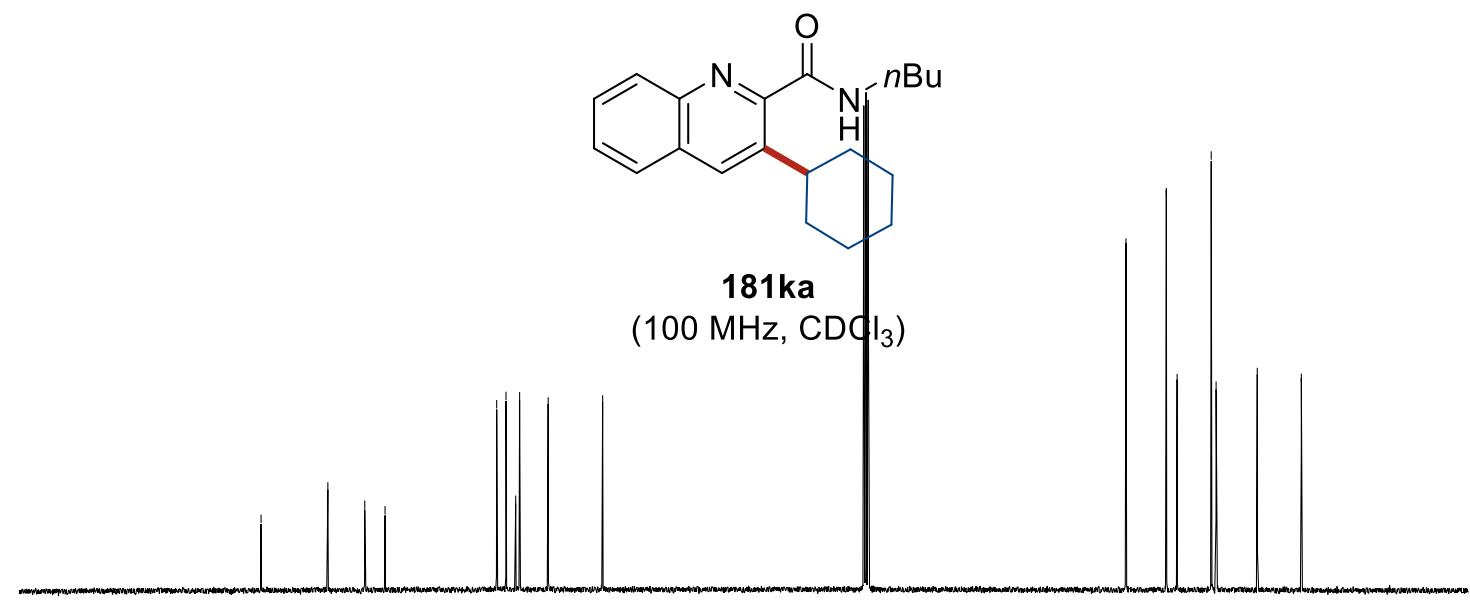

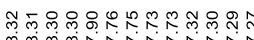

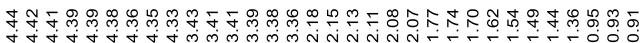

$\underbrace{\infty}_{\infty}$

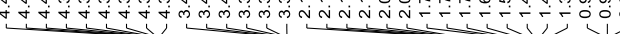

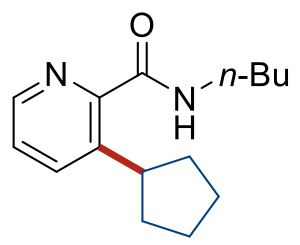

$181 \mathrm{ib}$

$\left(300 \mathrm{MHz} \mathrm{CDCl}_{3}\right)$

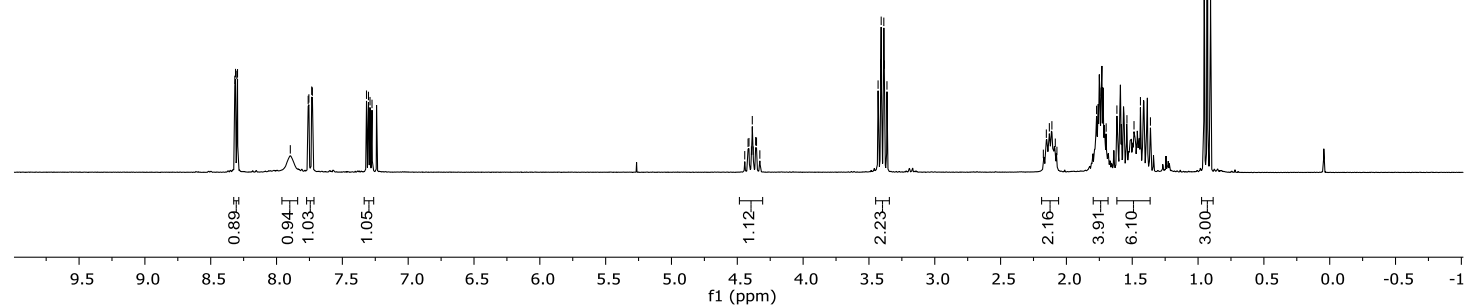




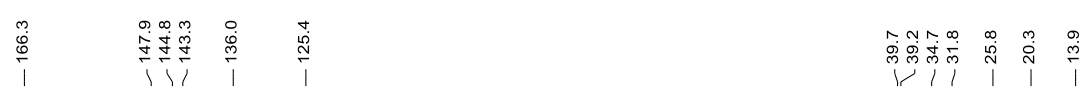

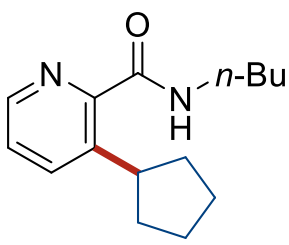

181ib

(125 MHz, $\mathrm{CDCl}_{3}$ )

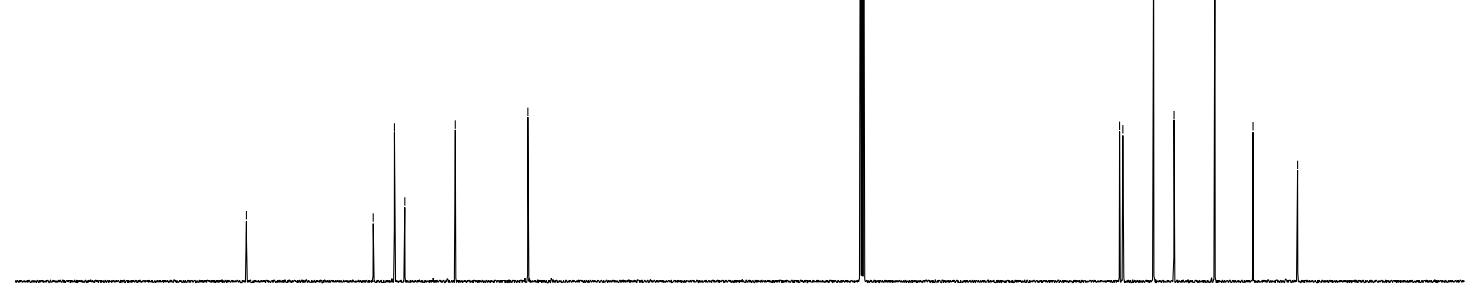

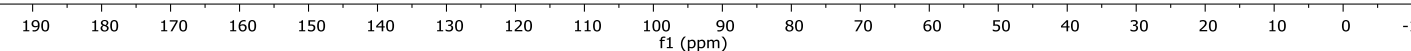

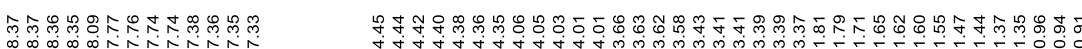
$\underbrace{\infty} \underbrace{\infty}_{\infty} \underbrace{\infty \pi i n i n}$<smiles>CCCCNC(=O)c1ncccc1C1CCOCC1</smiles>

181 ic

(300 MHz, $\mathrm{CDCl}_{3}$ )

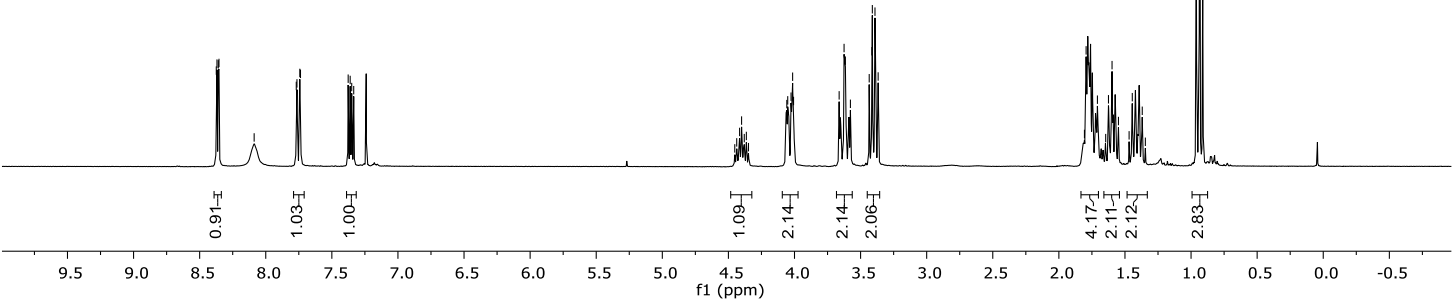



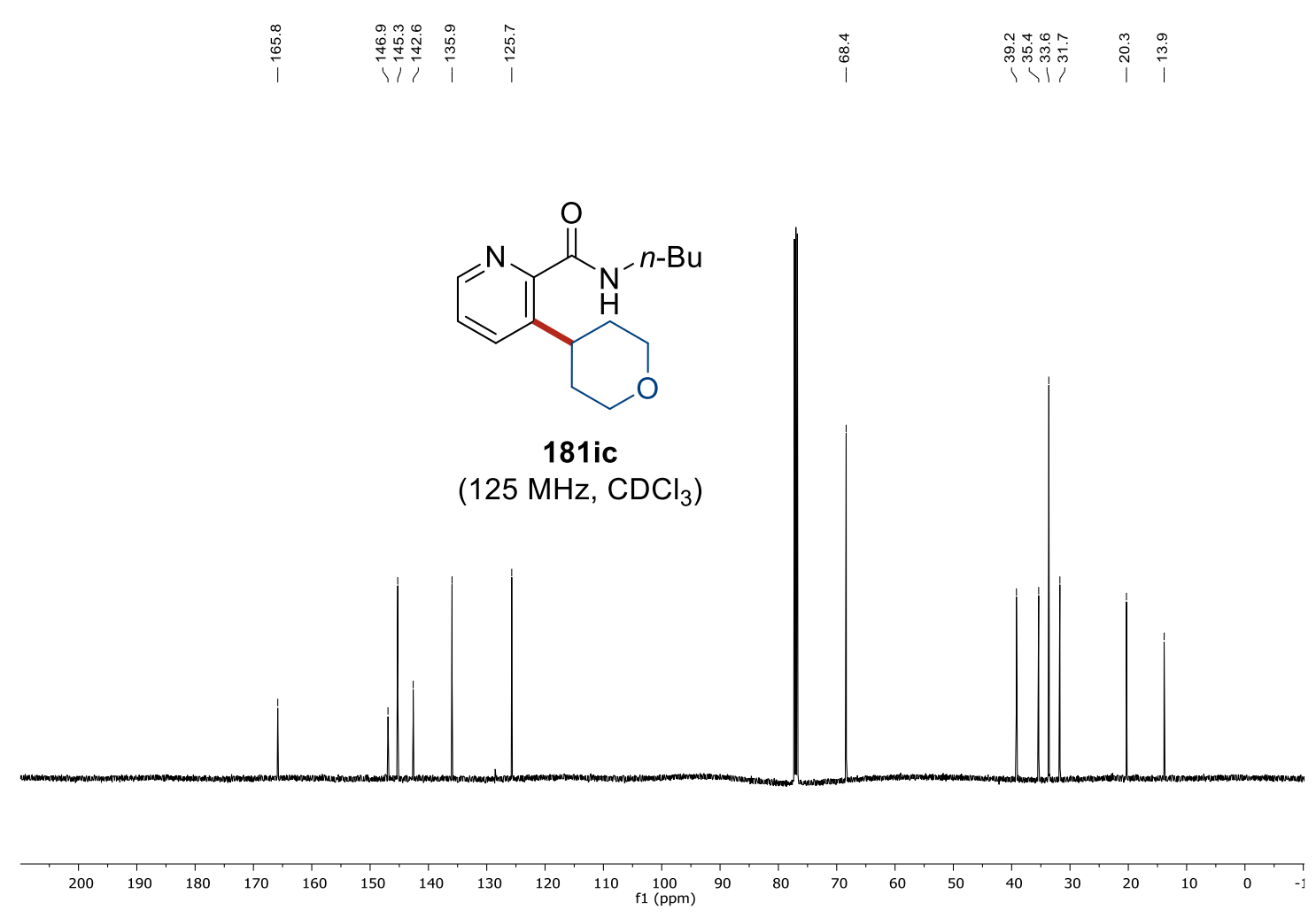

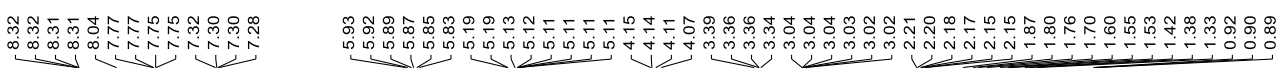

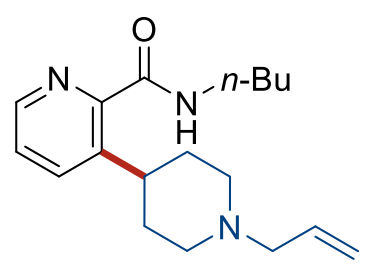

181 id

(400 MHz, $\mathrm{CDCl}_{3}$ )

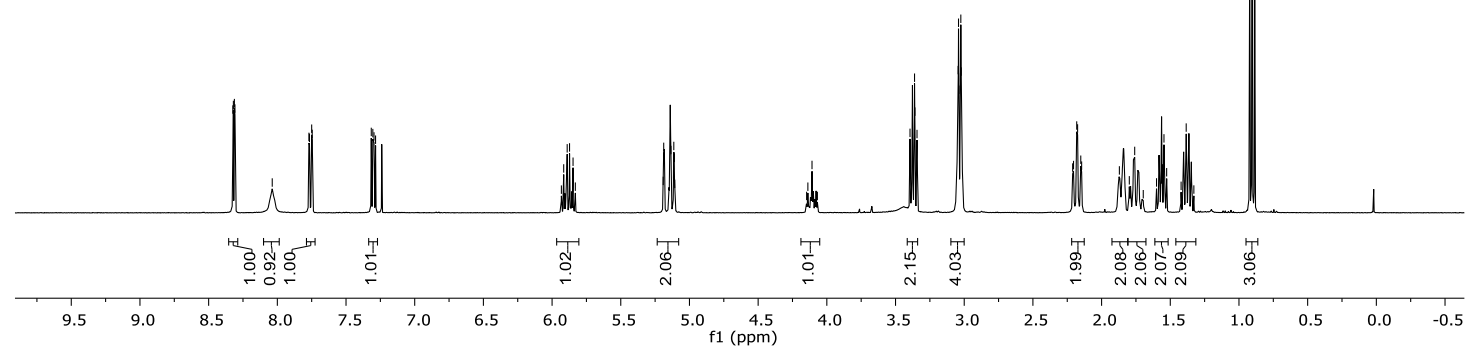



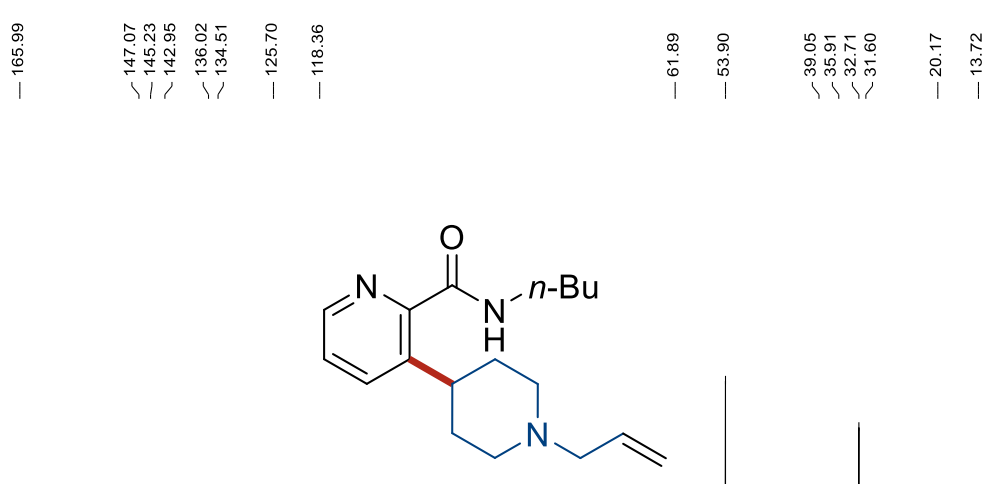

181 id

$\left(100 \mathrm{MHz}, \mathrm{CDCl}_{3}\right.$ )
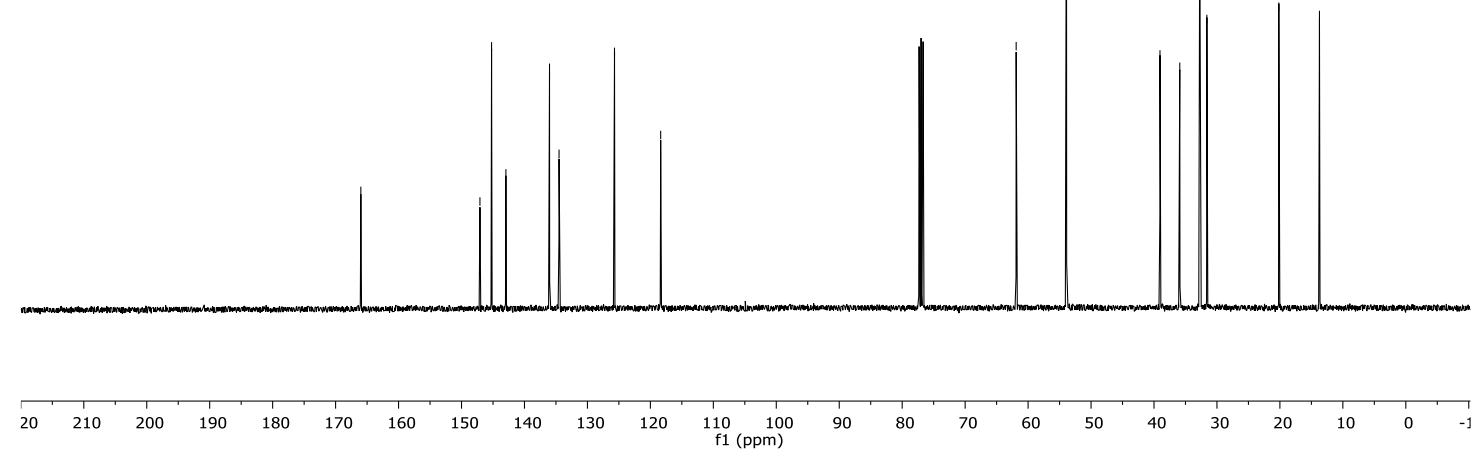

ס유요

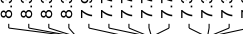

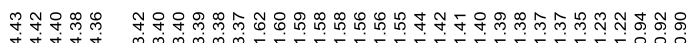

更过

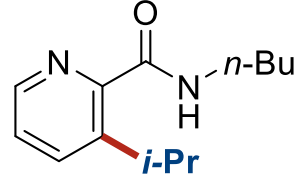

181 ie

$\left(400 \mathrm{MHz}, \mathrm{CDCl}_{3}\right)$

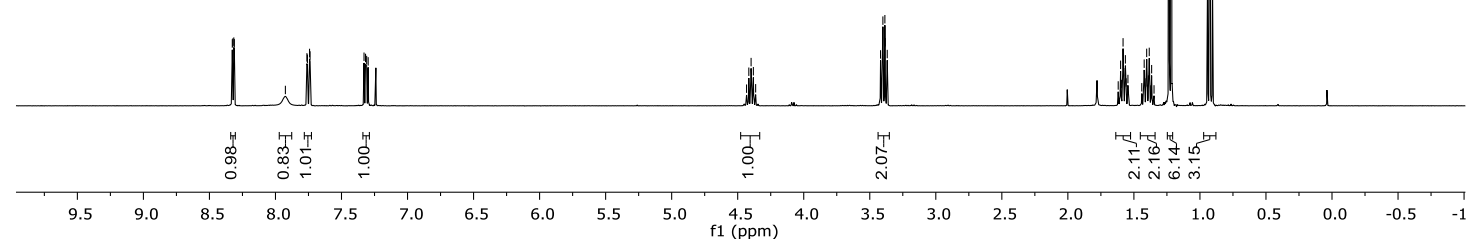




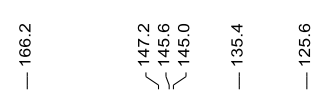

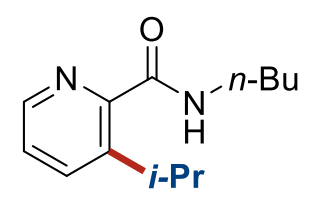

181 ie

(100 $\mathrm{MHz}, \mathrm{CDCl}_{3}$ )
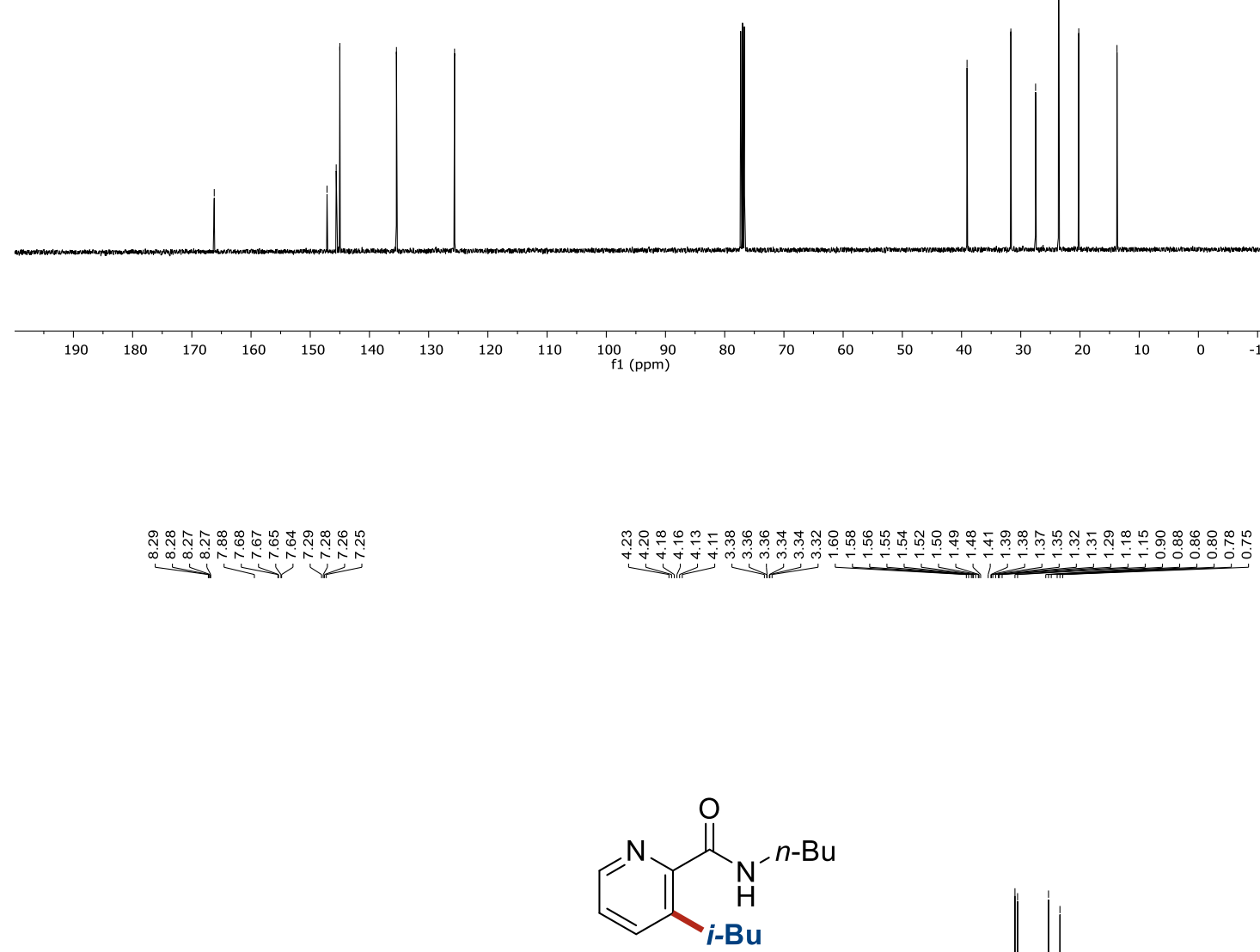

181 if

(300 $\mathrm{MHz}, \mathrm{CDCl}_{3}$ )

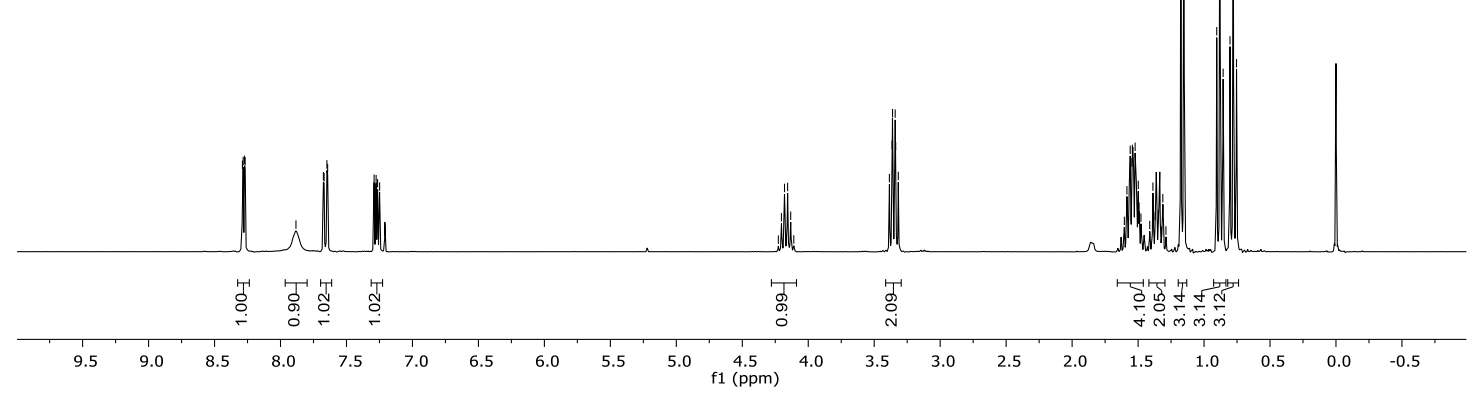



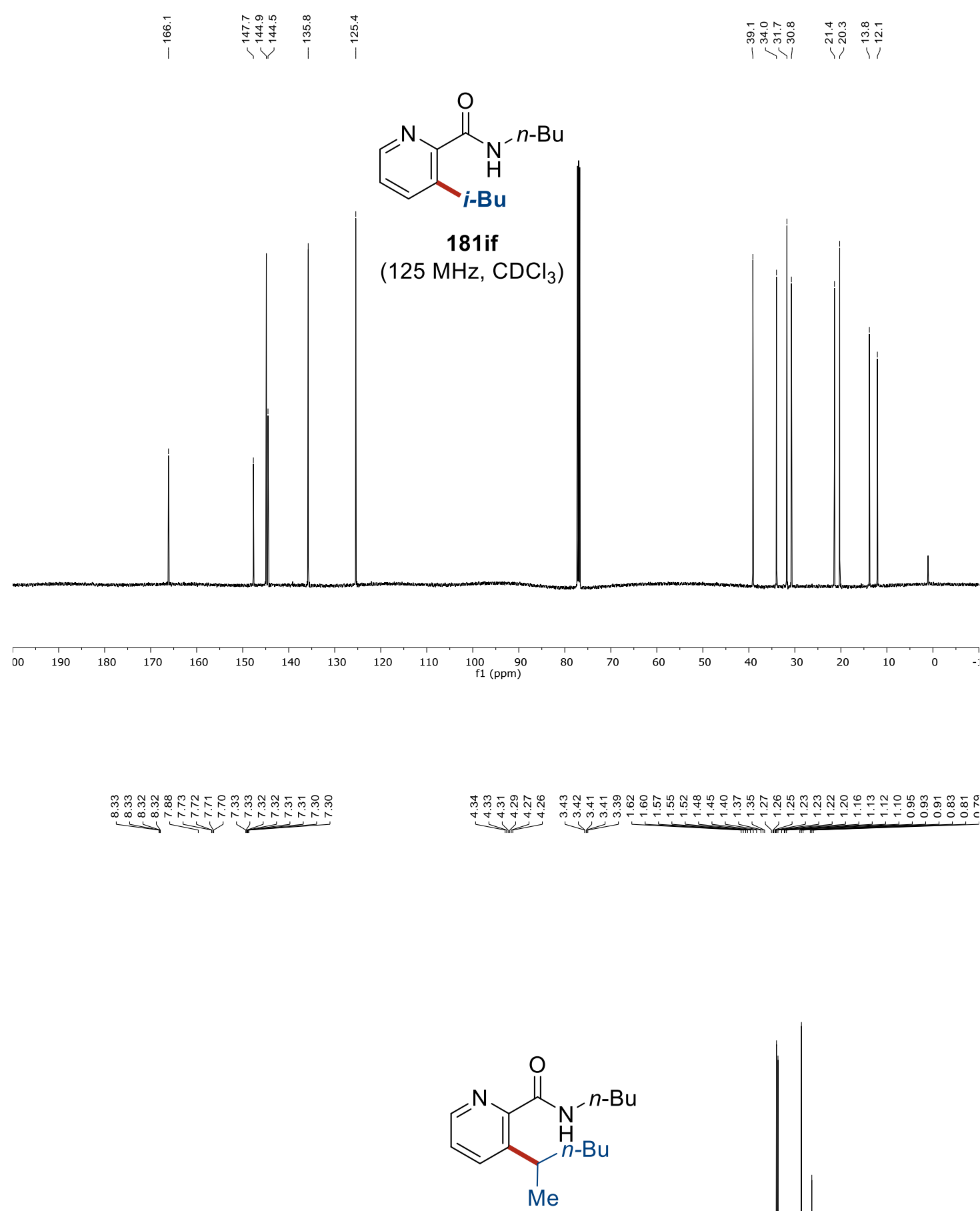

$181 \mathrm{ig}$

(300 MHz, CDCl3)

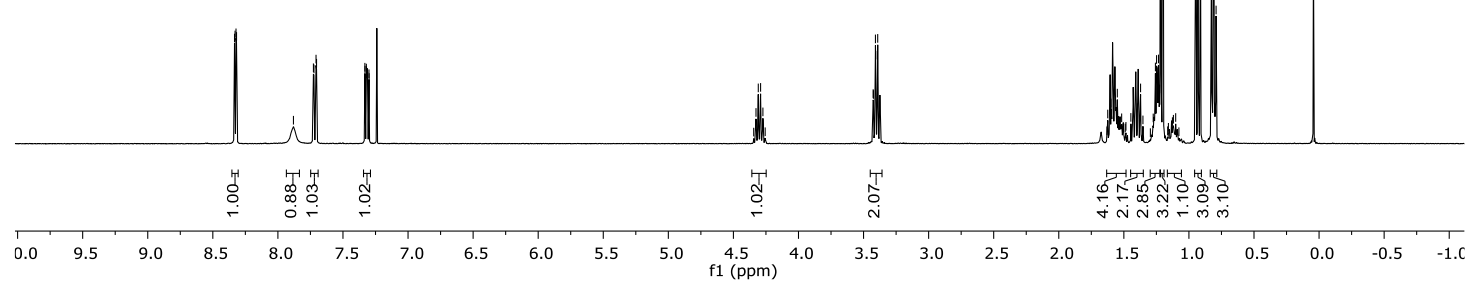




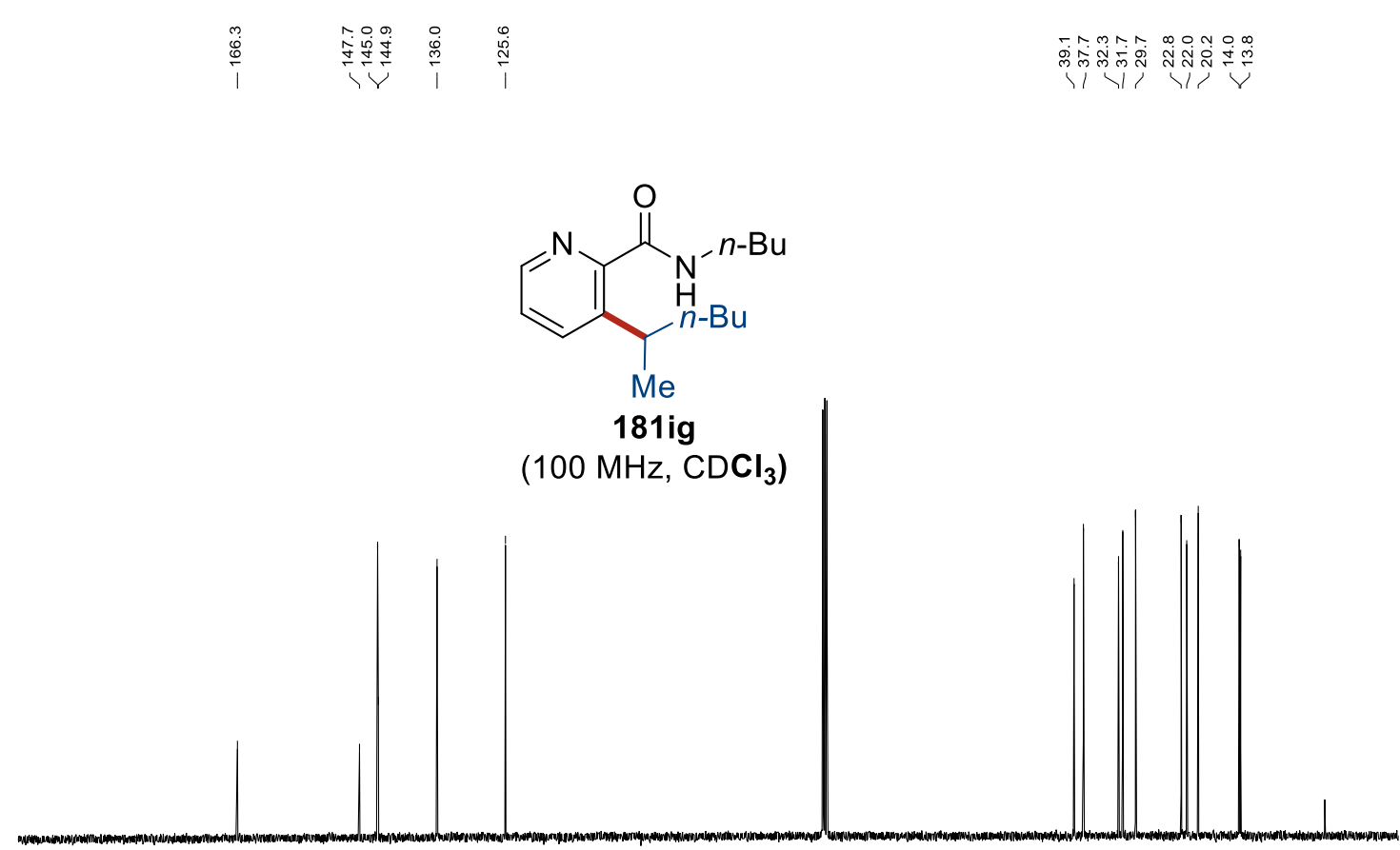

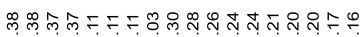

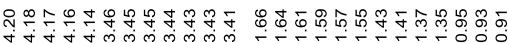

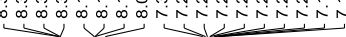

作

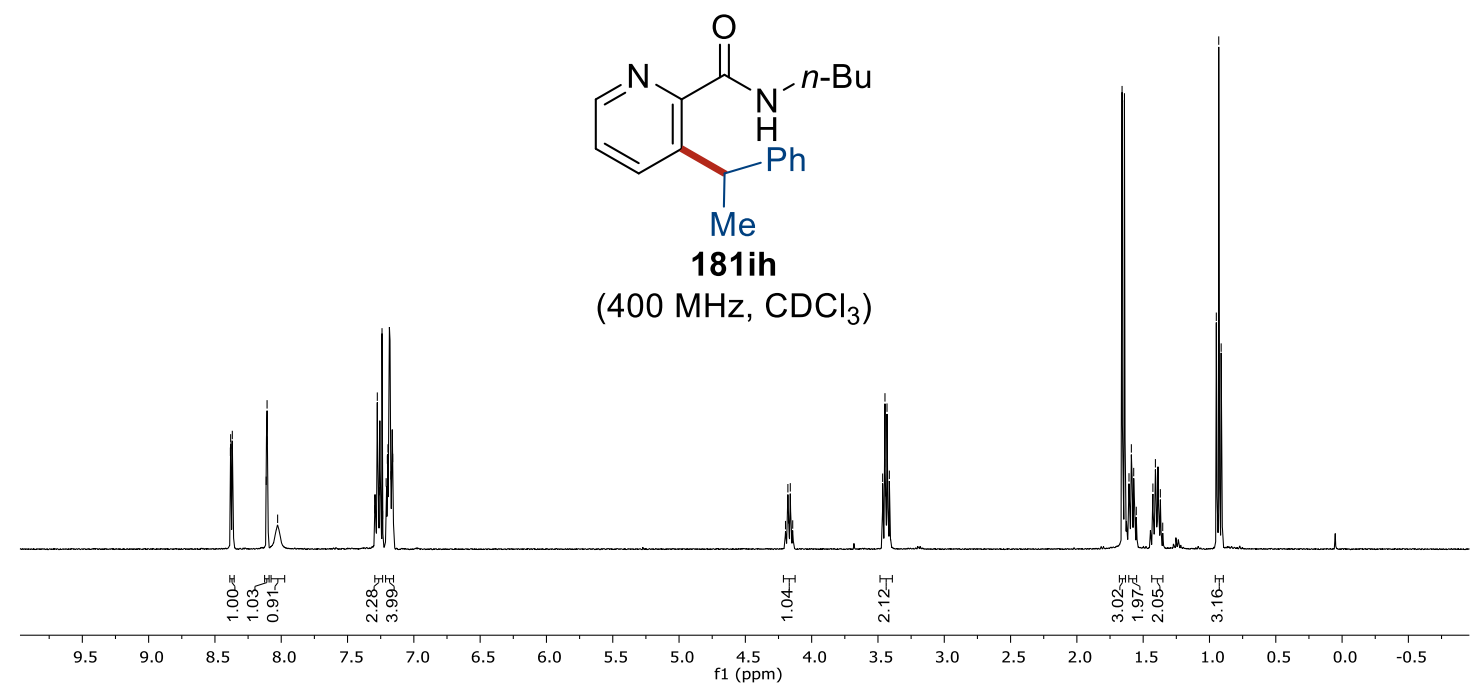




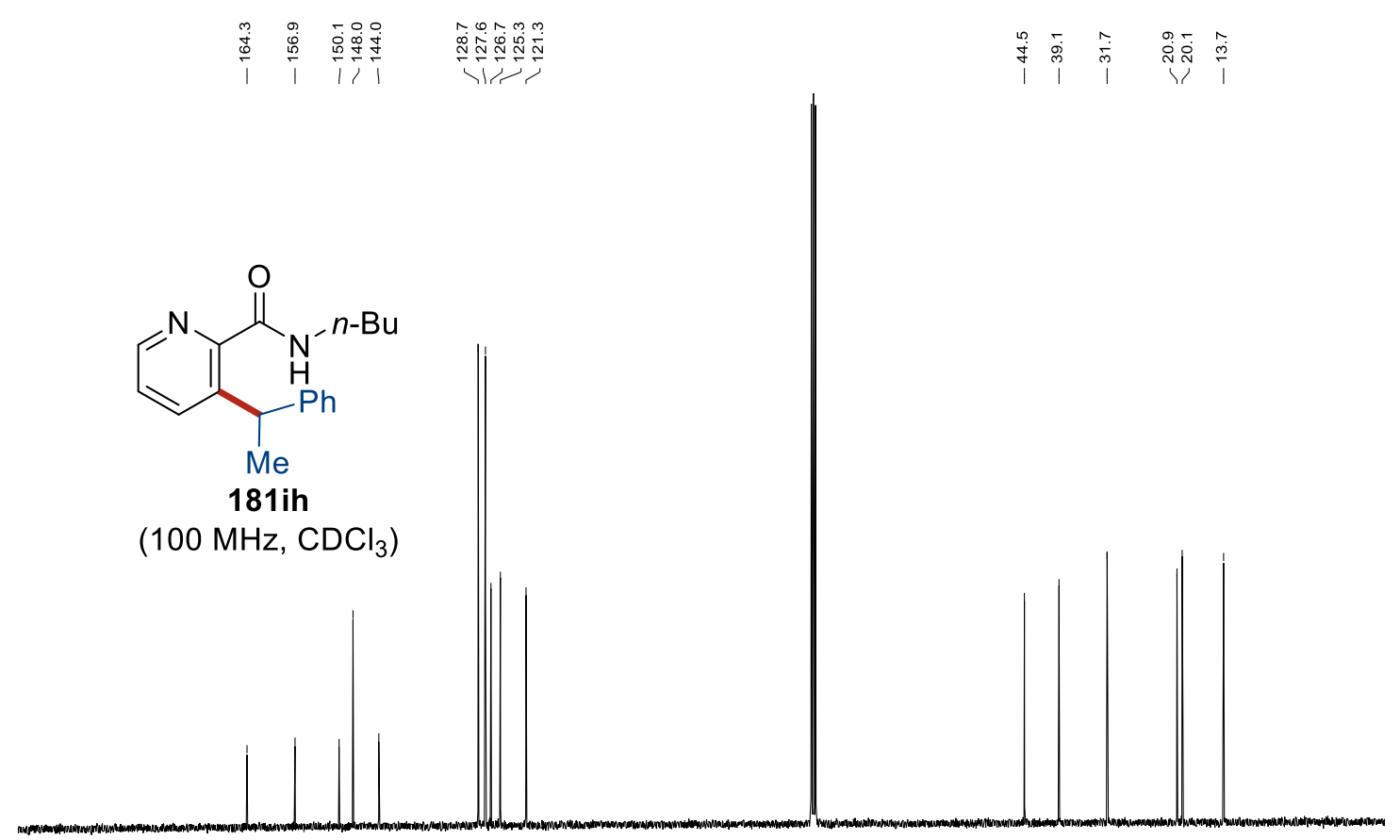

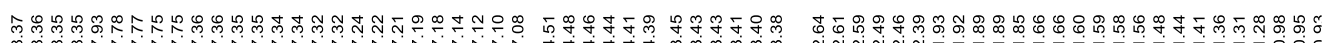

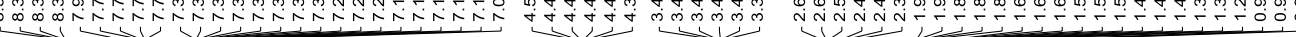

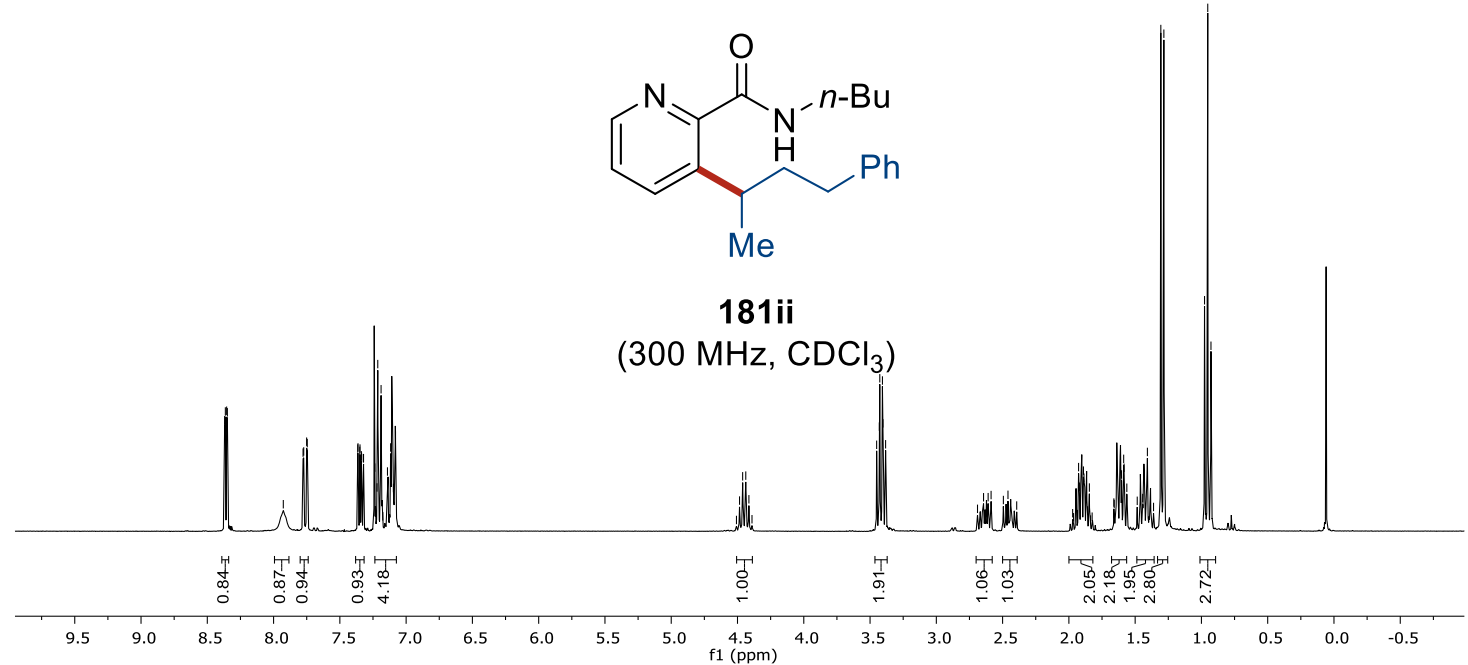




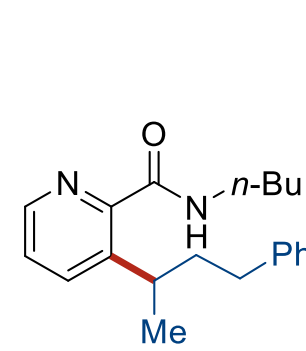

$181 \mathrm{ii}$

(125 MHz, $\mathrm{CDCl}_{3}$ )
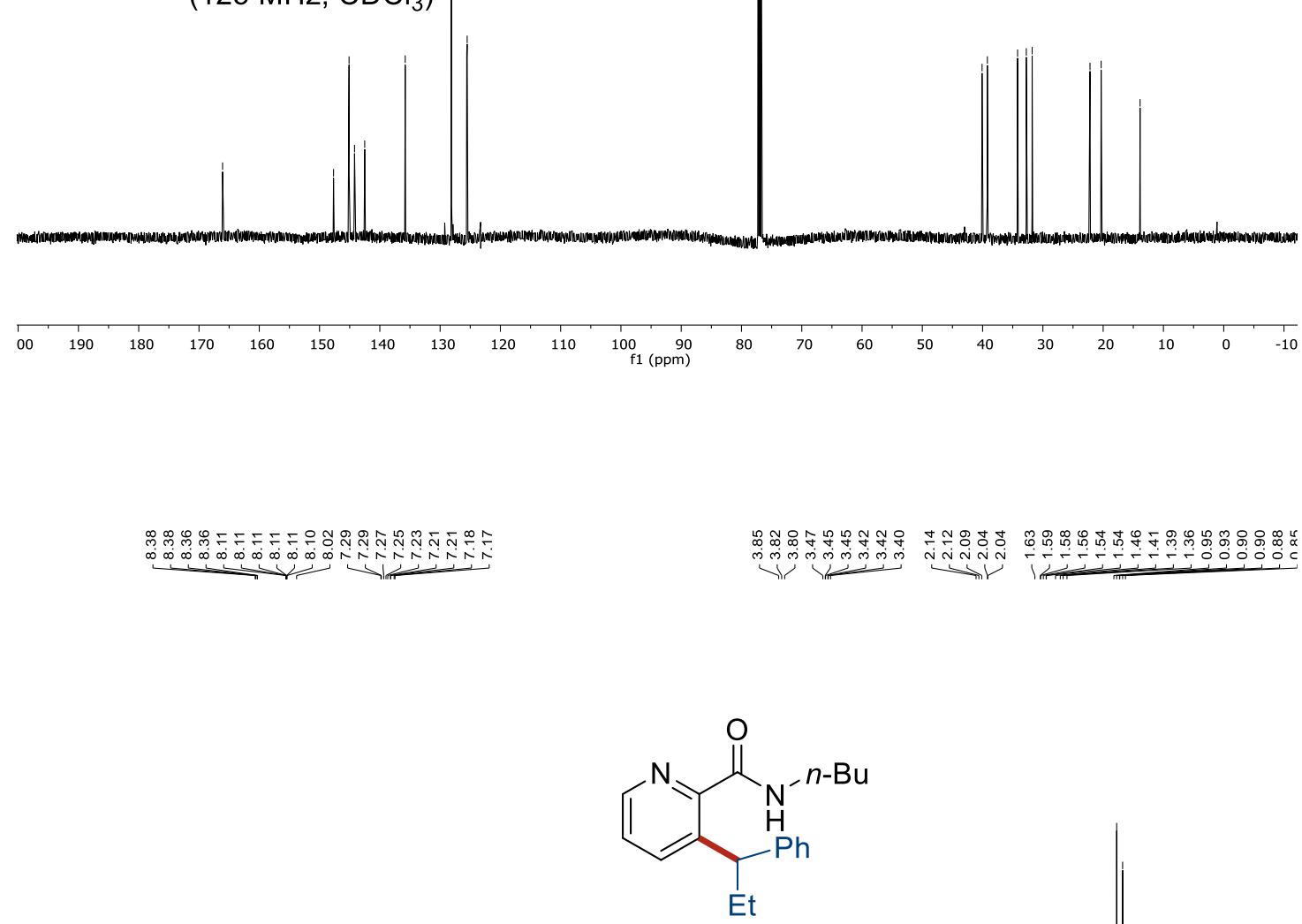

181ij

$\left(300 \mathrm{MHz}, \mathrm{CDCl}_{3}\right)$

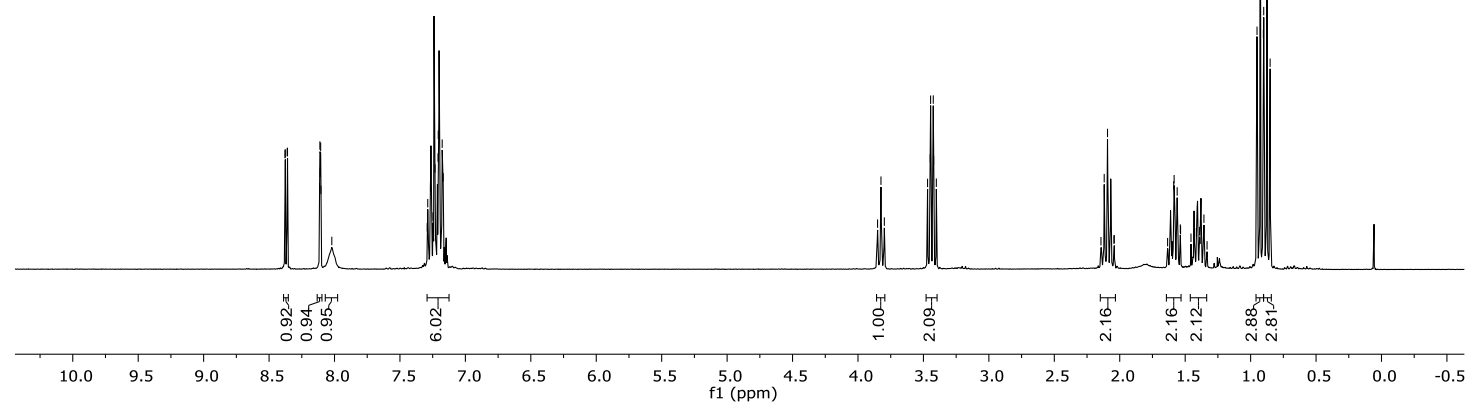



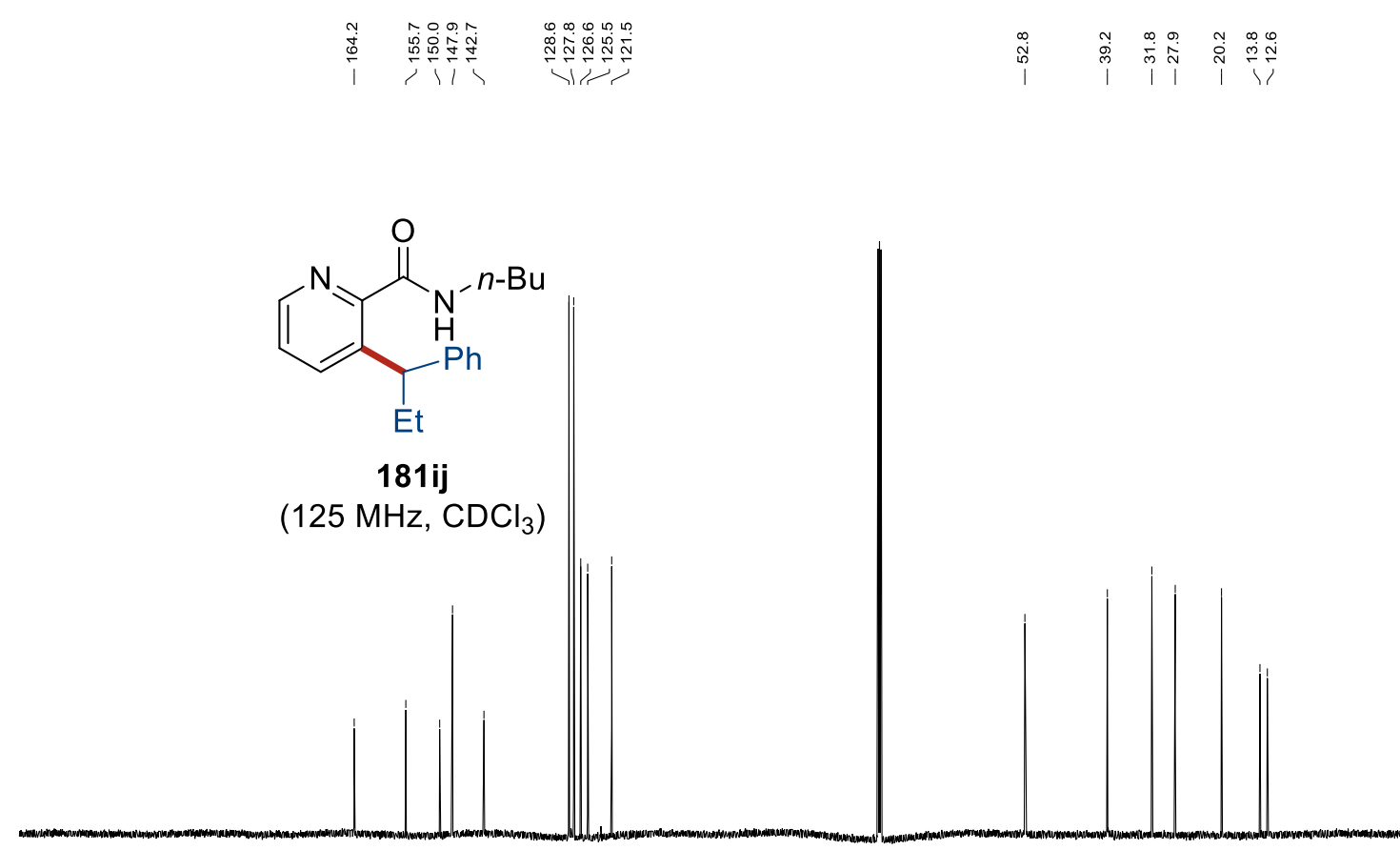

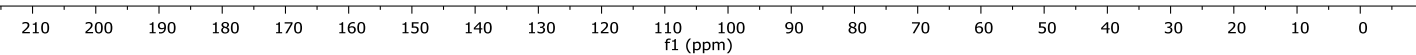

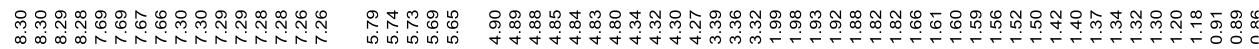

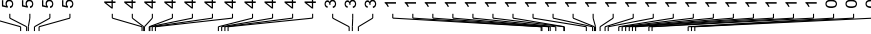

$\underbrace{C_{M}^{N}}_{M e}$

181ik

$\left(300 \mathrm{MHz} \mathrm{CDCl}_{3}\right)$

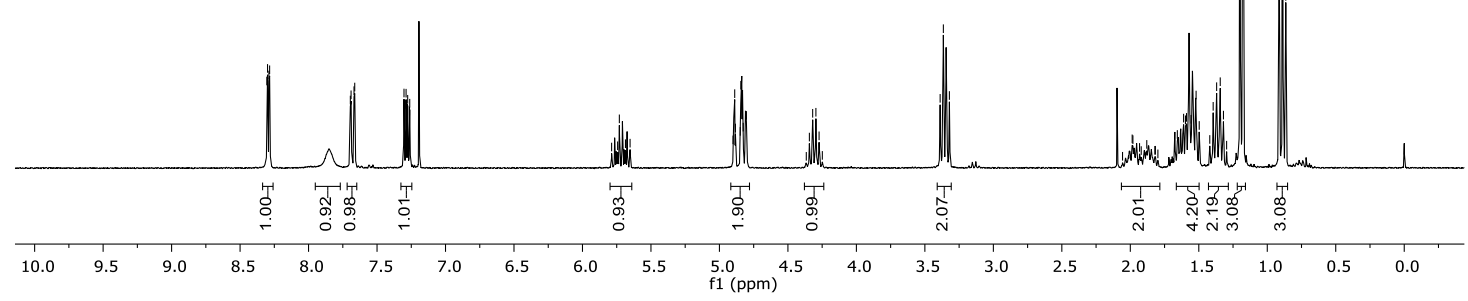




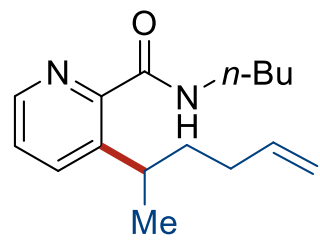

181ik

$\left(125 \mathrm{MHz}, \mathrm{CDCl}_{3}\right)$

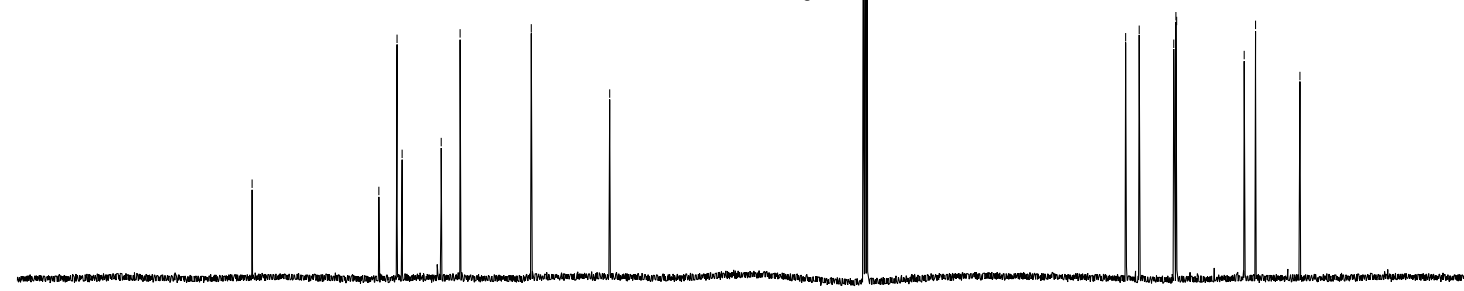

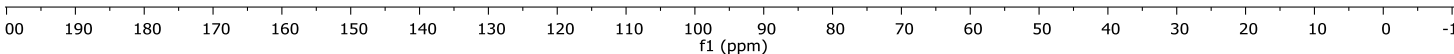

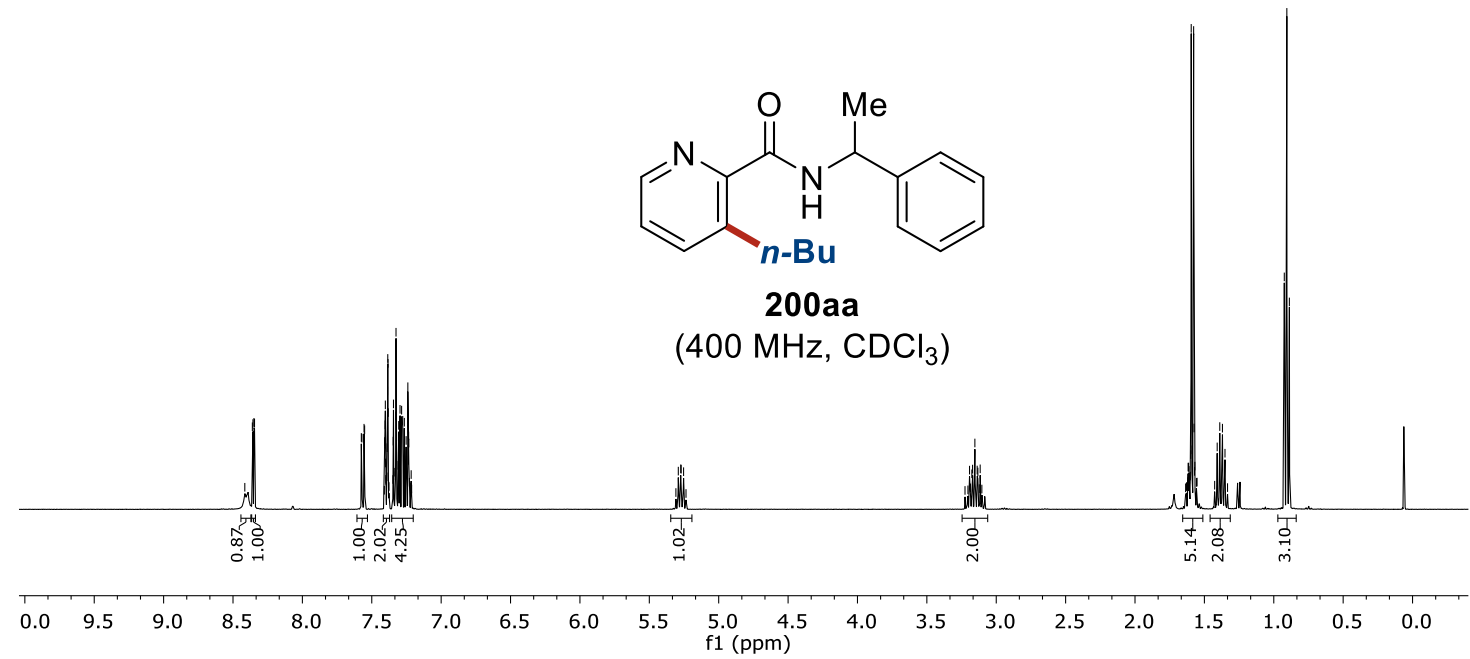




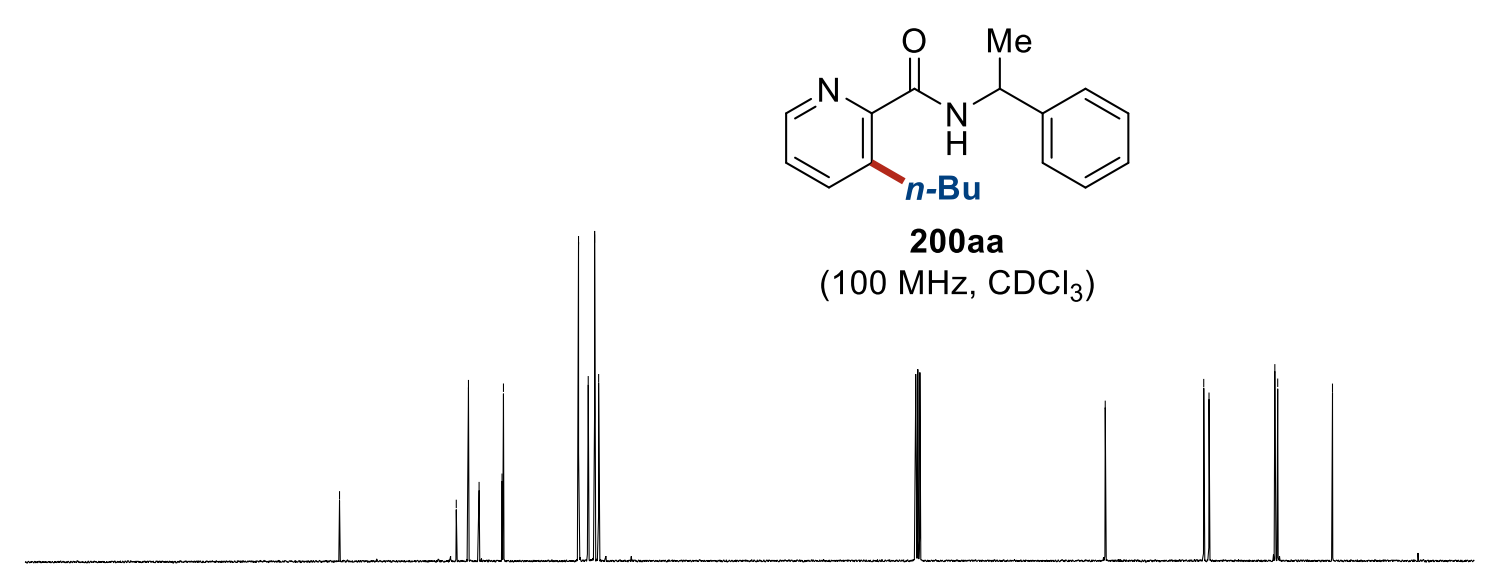

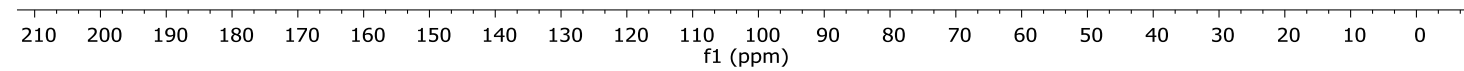

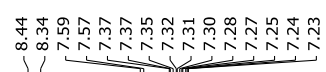

草

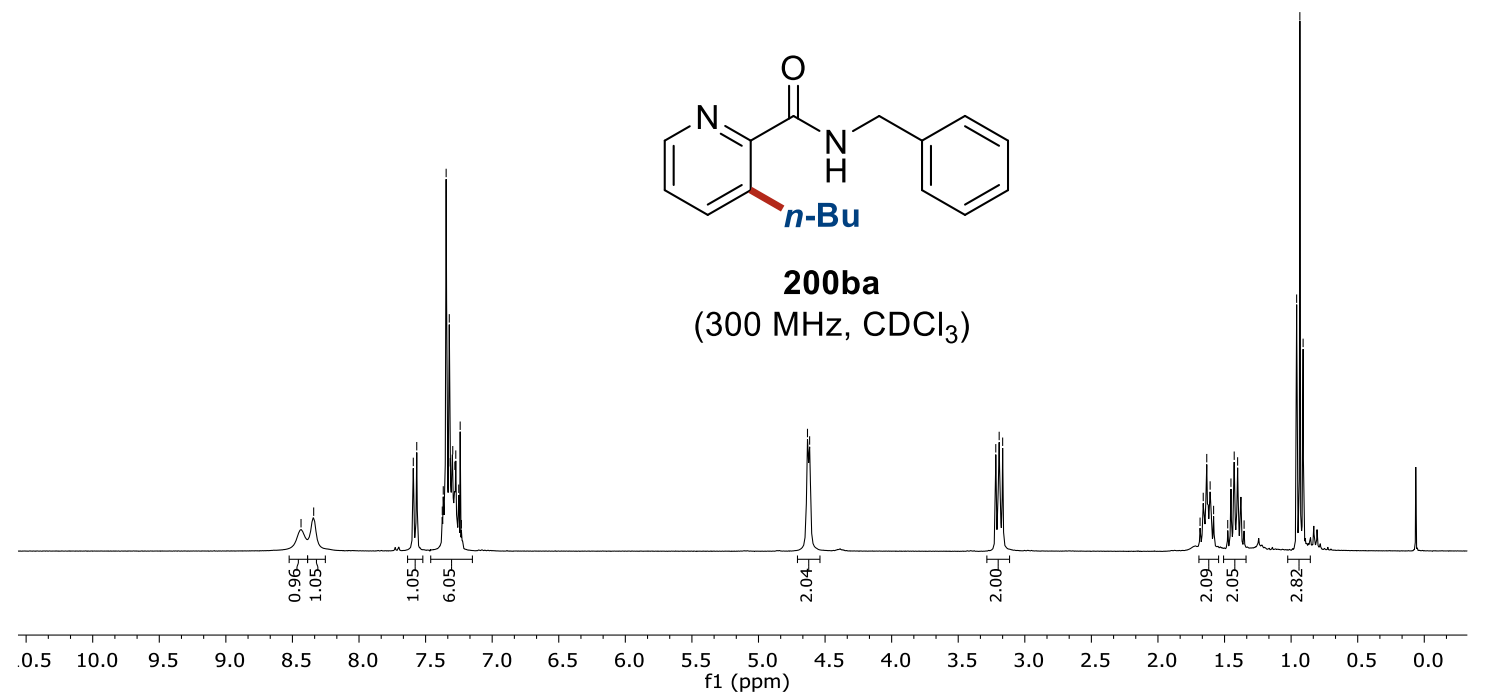




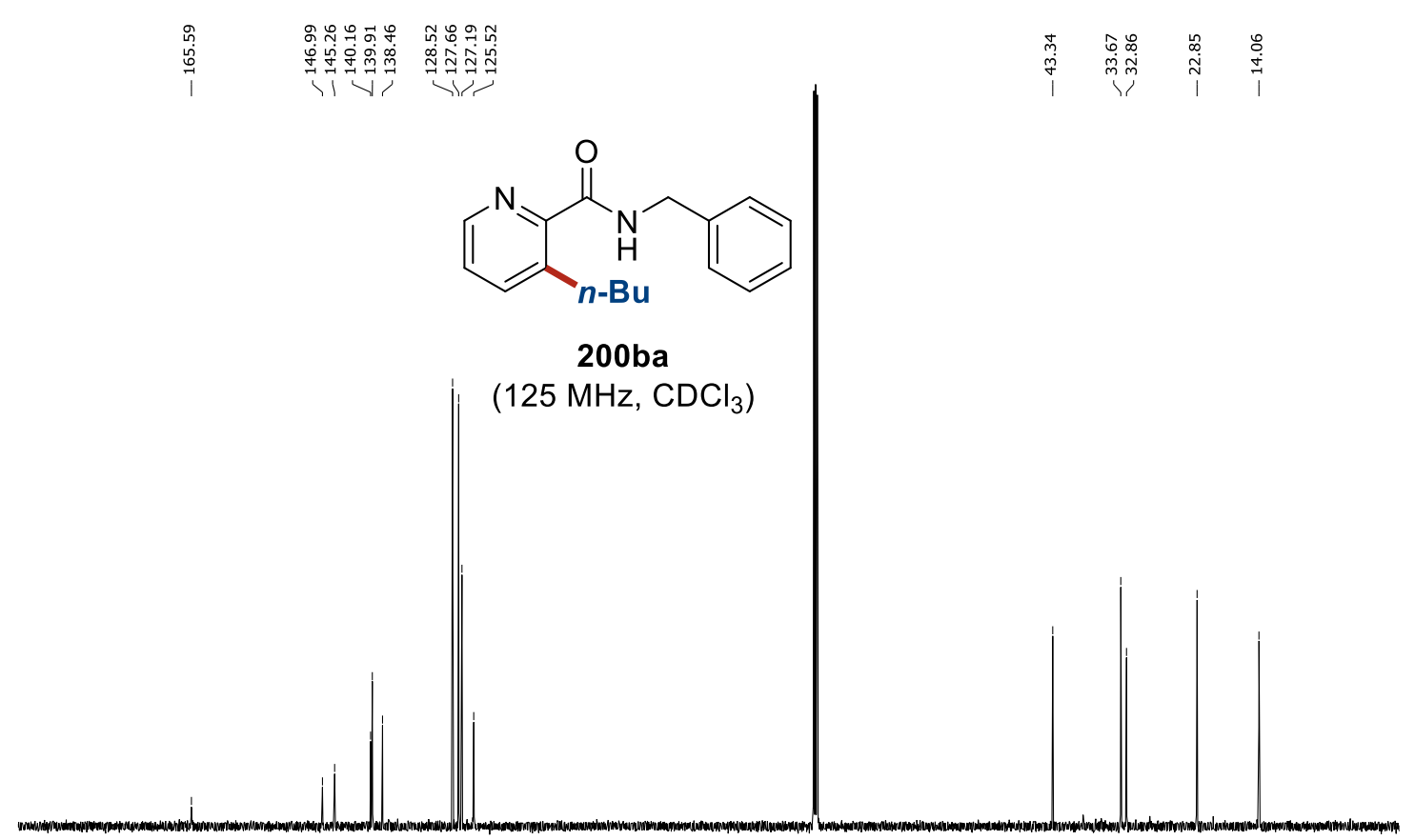

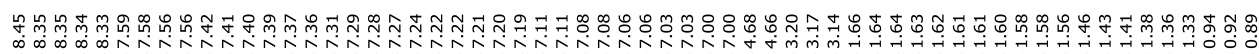

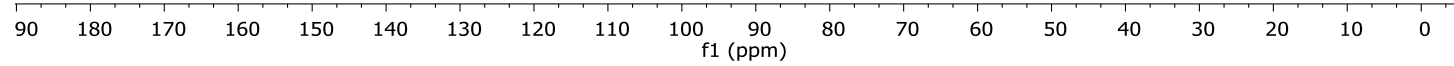

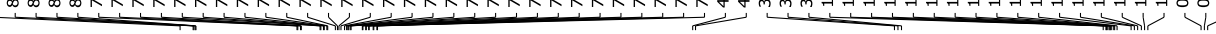

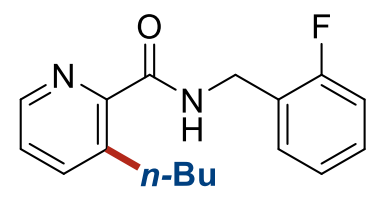

200ca

$\left.300 \mathrm{MHz}, \mathrm{CDCl}_{3}\right)$

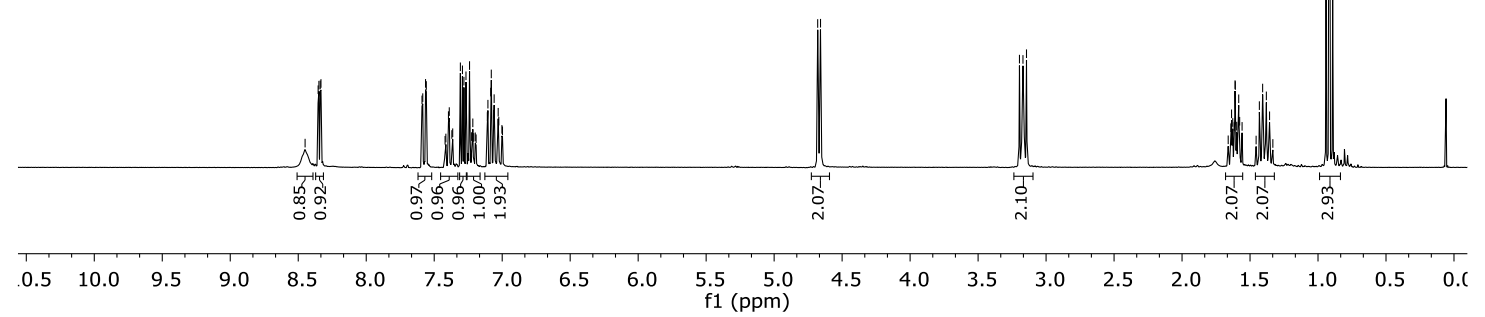


○िم

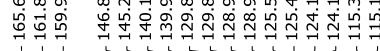

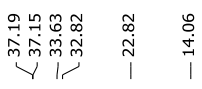

$\overbrace{n-B u}^{C}$

$200 \mathrm{ca}$

(125 MHz, $\mathrm{CDCl}_{3}$ )

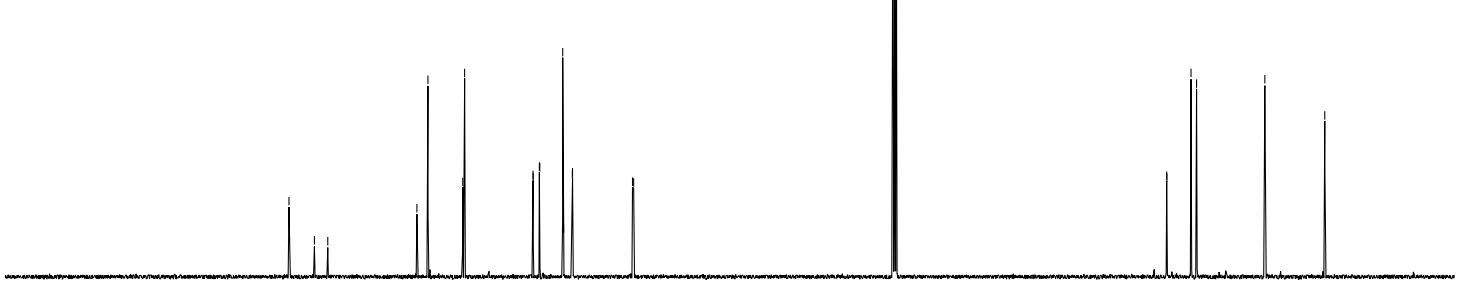

$\begin{array}{llllllllllllllllllll}200 & 190 & 180 & 170 & 160 & 150 & 140 & 130 & 120 & 110 \underset{\mathrm{f} 1(\mathrm{ppm})}{100} & 90 & 80 & 70 & 60 & 50 & 40 & 30 & 20 & 10 & 0\end{array}$

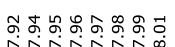

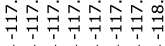

$\underbrace{\mathrm{N}}_{n-\mathrm{Bu}}$

200ca

(275 MHz, $\mathrm{CDCl}_{3}$ )

$\begin{array}{llllllllllllllllllllll}-10 & -20 & -30 & -40 & -50 & -60 & -70 & -80 & -90 & -100 & -110 & -120 & -130 & -140 & -150 & -160 & -170 & -180 & -190 & -20\end{array}$ 


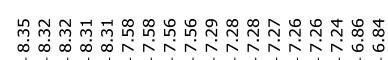

㤏量
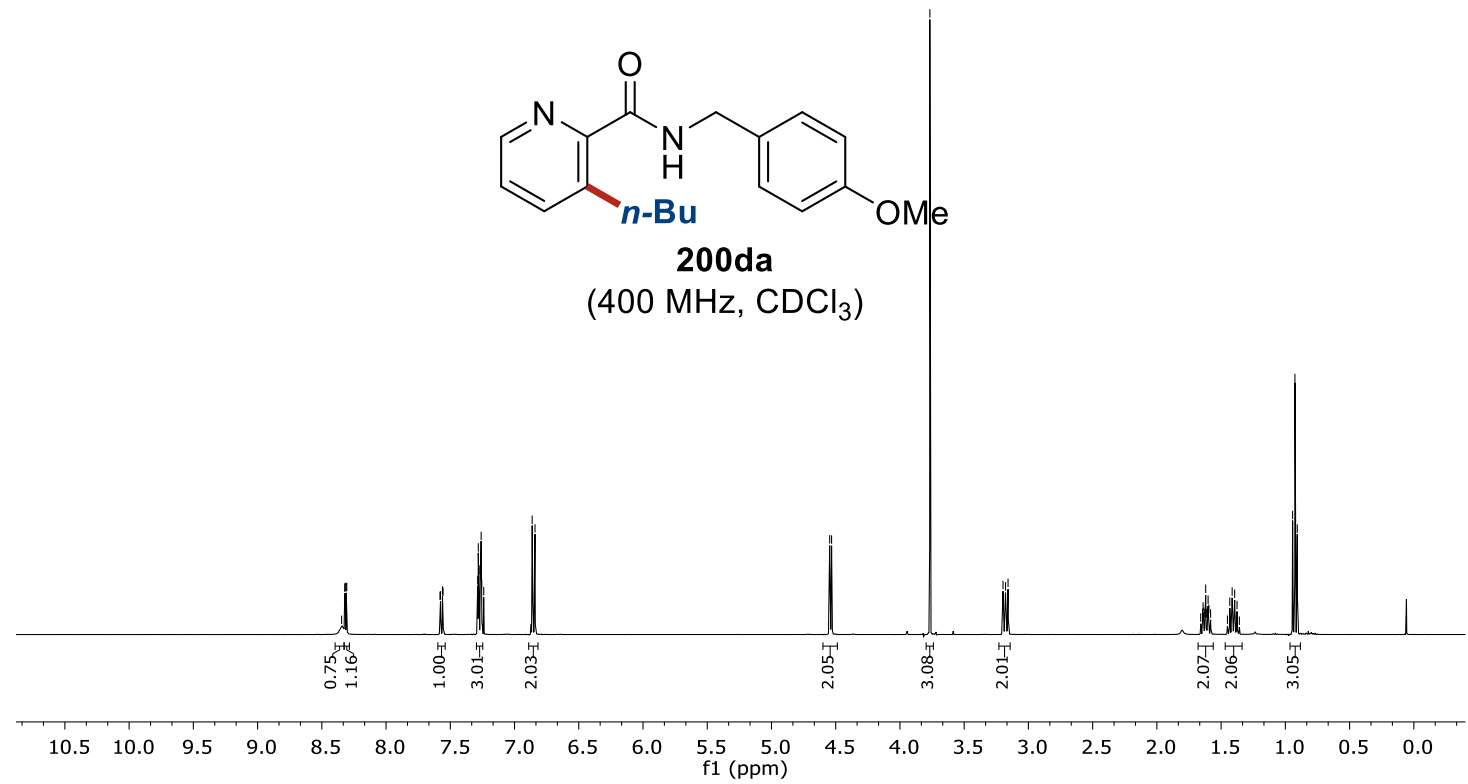

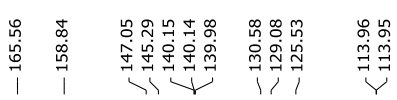

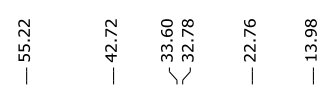

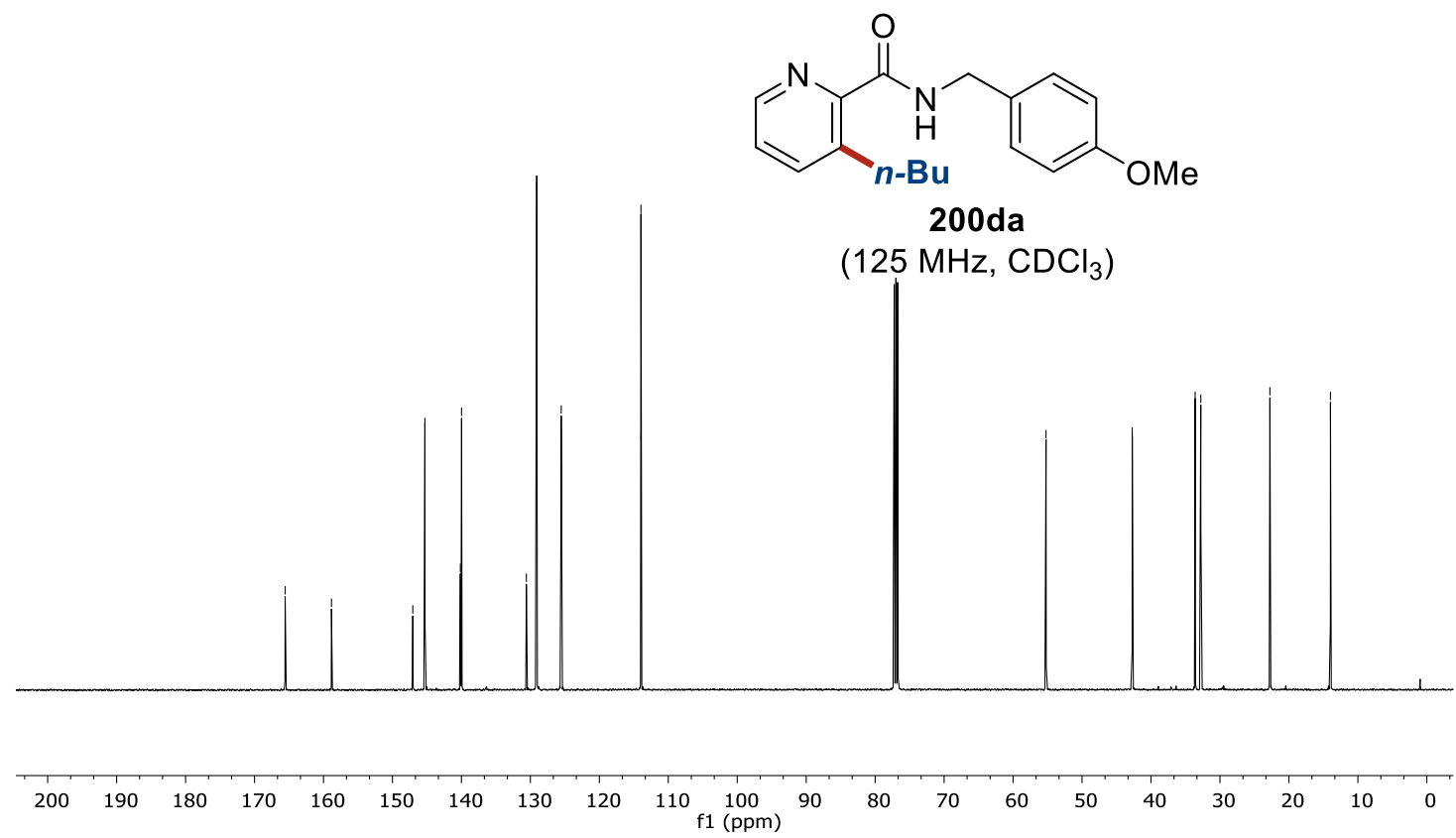




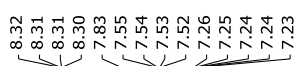

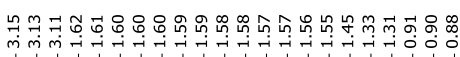

$\overbrace{n-\mathrm{Bu}}^{\mathrm{N}}$

200ea

(400 MHz, $\mathrm{CDCl}_{3}$ )

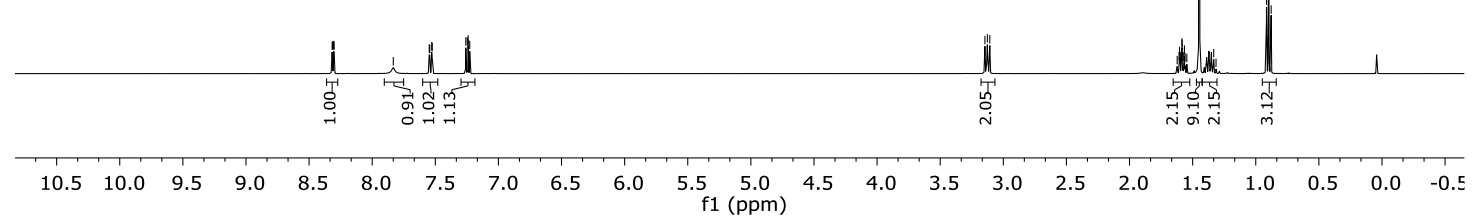

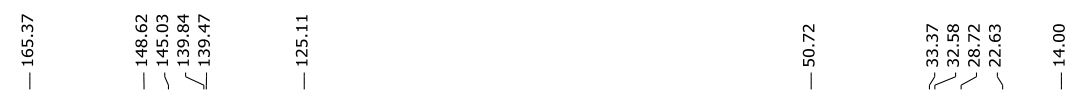<smiles>CCCCOc1cccnc1C(=O)NC(C)(C)C</smiles>

$\left(100 \mathrm{MHz}, \mathrm{CDCl}_{3}\right.$ )

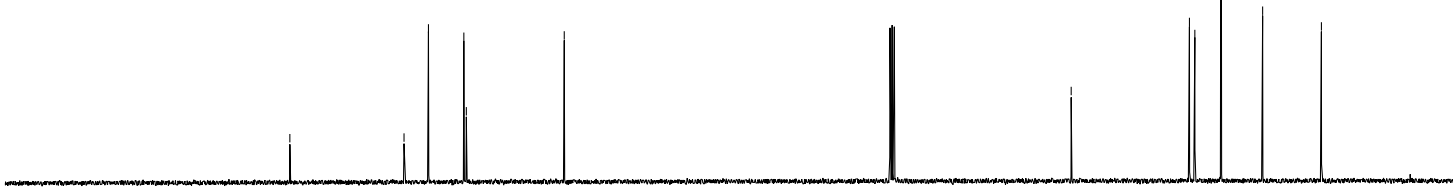

$\begin{array}{lllllllllllllllllllll}200 & 190 & 180 & 170 & 160 & 150 & 140 & 130 & 120 & 110 \underset{\mathrm{f} 1(\mathrm{ppm})}{100} & 90 & 80 & 70 & 60 & 50 & 40 & 30 & 20 & 10 & 0\end{array}$ 

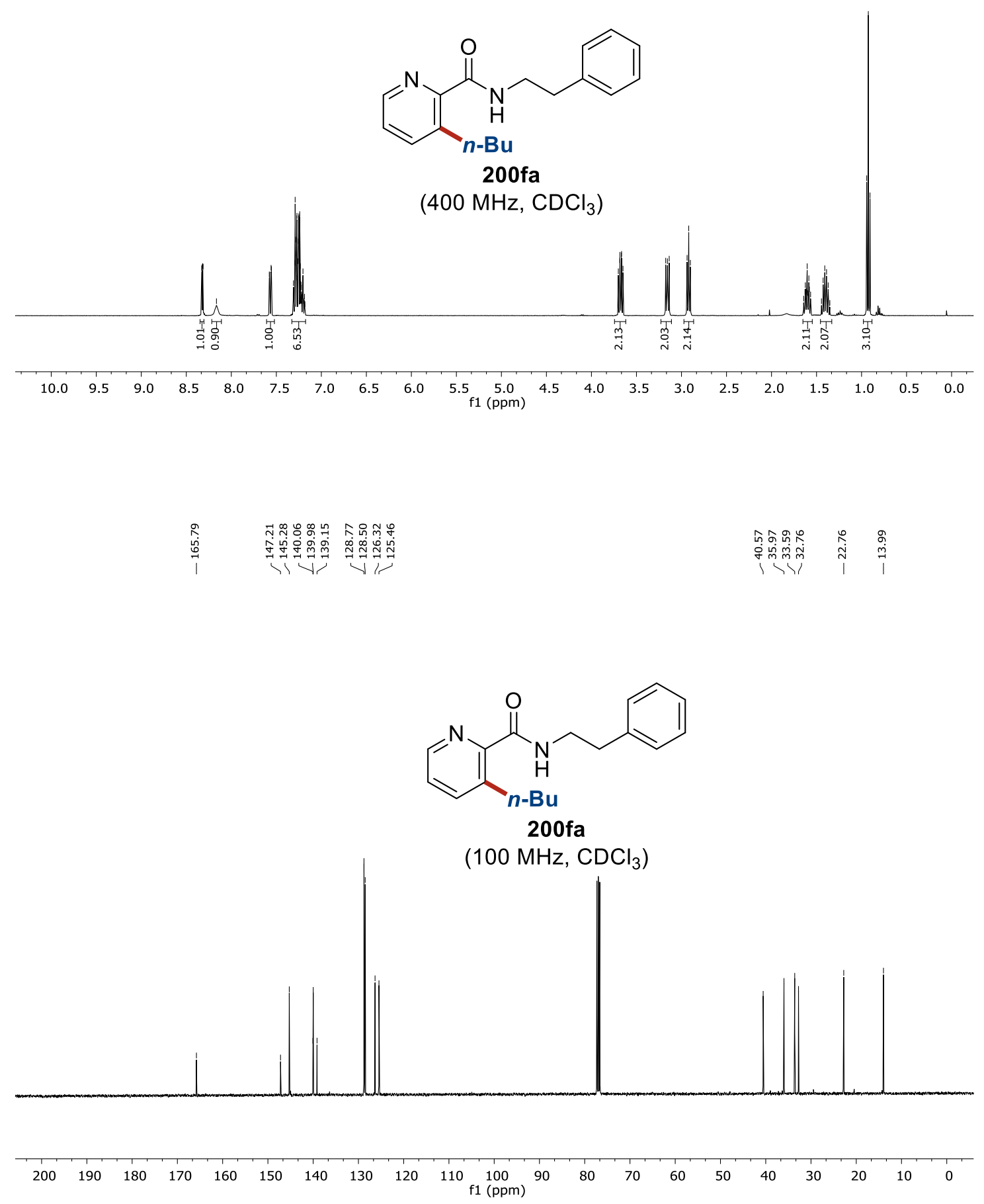
<smiles>CCCCc1cccnc1C(=O)NC1CCCCC1</smiles>

200ga

$\left(500 \mathrm{MHz}, \mathrm{CDCl}_{3}\right)$
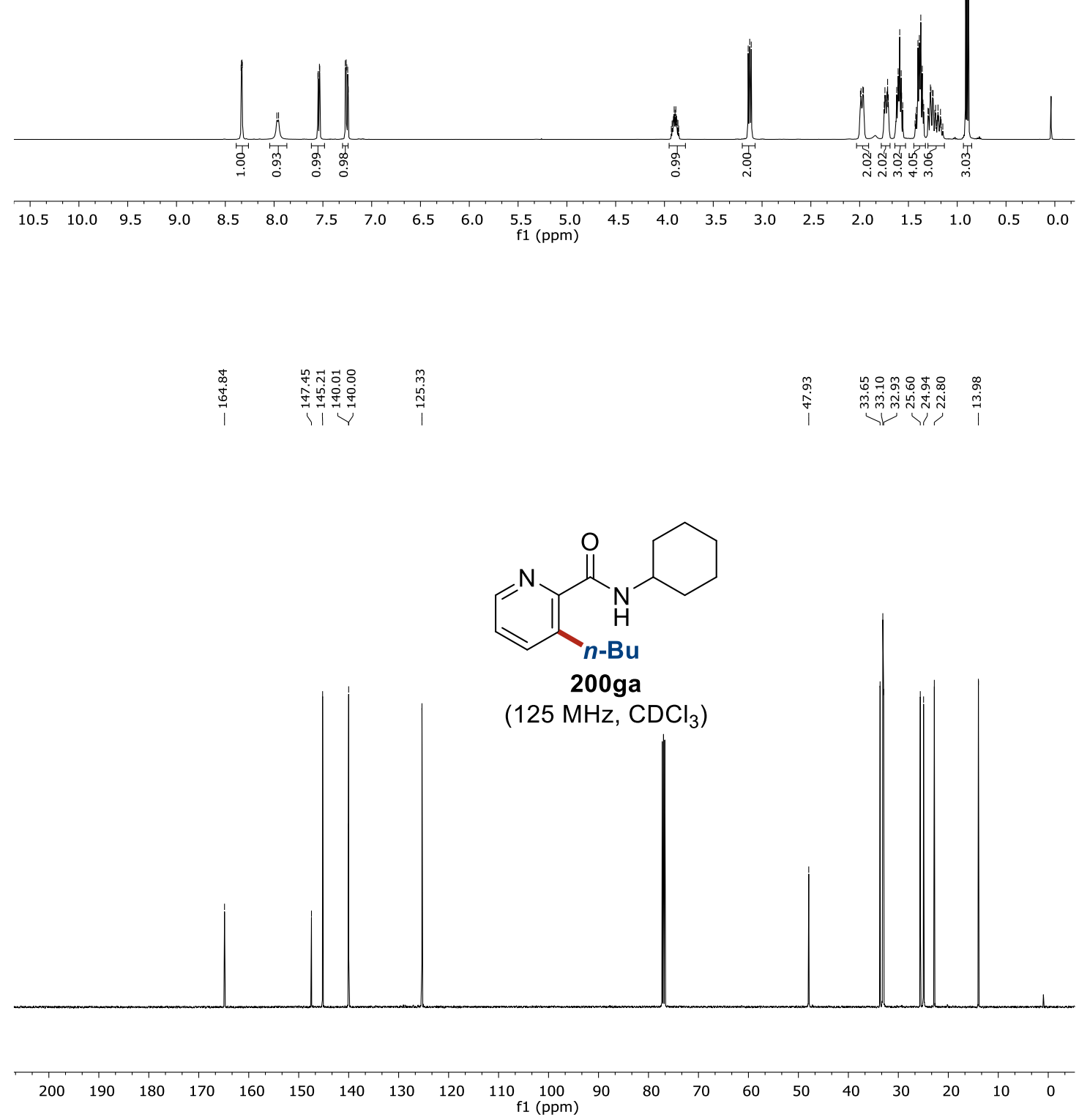


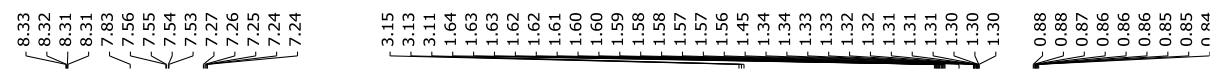<smiles>CC(C)(C)CSc1cccnc1C(=O)NC(C)(C)C</smiles>

200eb

(400 $\left.\mathrm{MHz} \mathrm{CDCl}_{3}\right)$
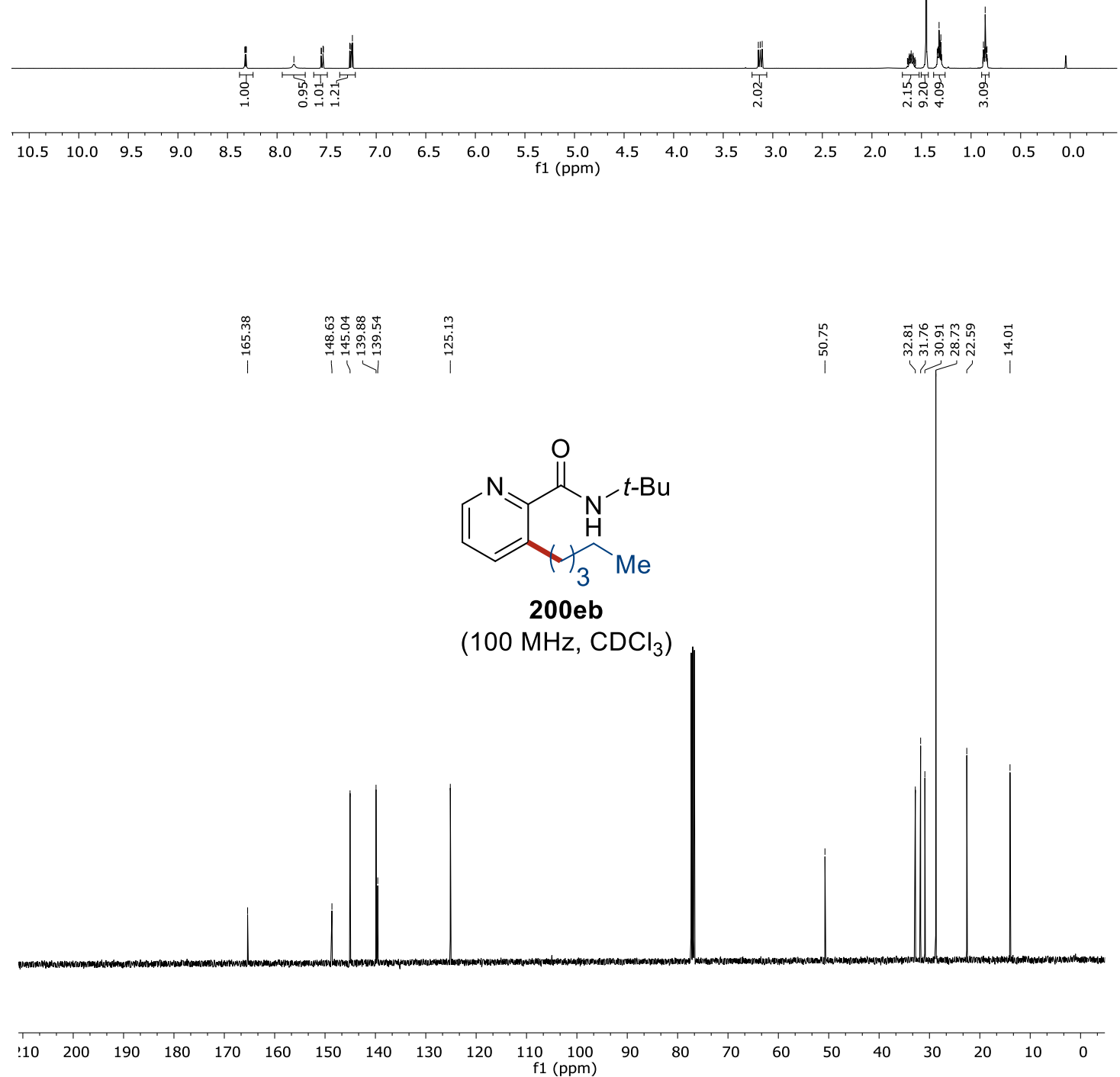


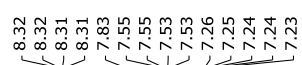

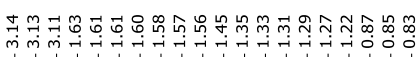

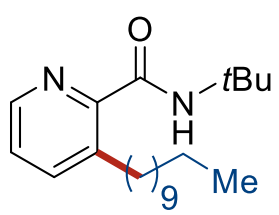

$200 \mathrm{ec}$

(400 MHz, $\mathrm{CDCl}_{3}$ )

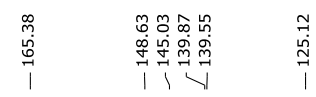

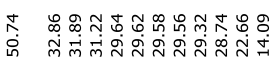

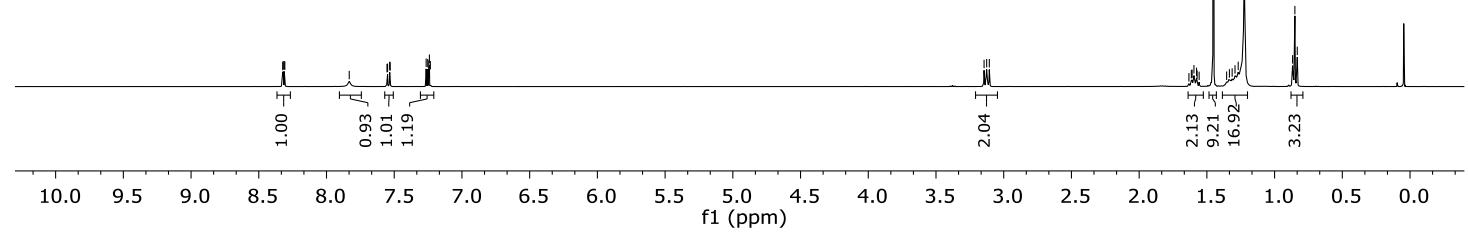<smiles>CCCCc1cccnc1C(=O)NC(C)(C)C</smiles>

200ec

$\left(100 \mathrm{MHz}, \mathrm{CDCl}_{3}\right.$ )

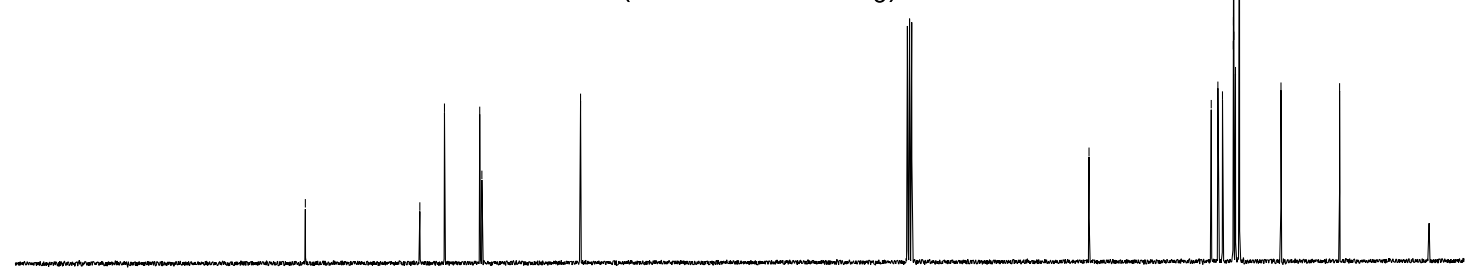

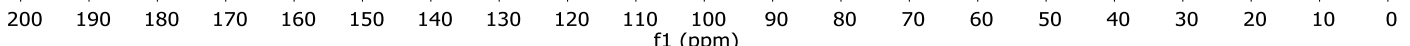




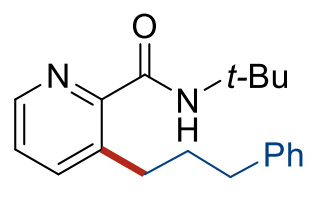

200ed

(400 MHz, $\mathrm{CDCl}_{3}$ )

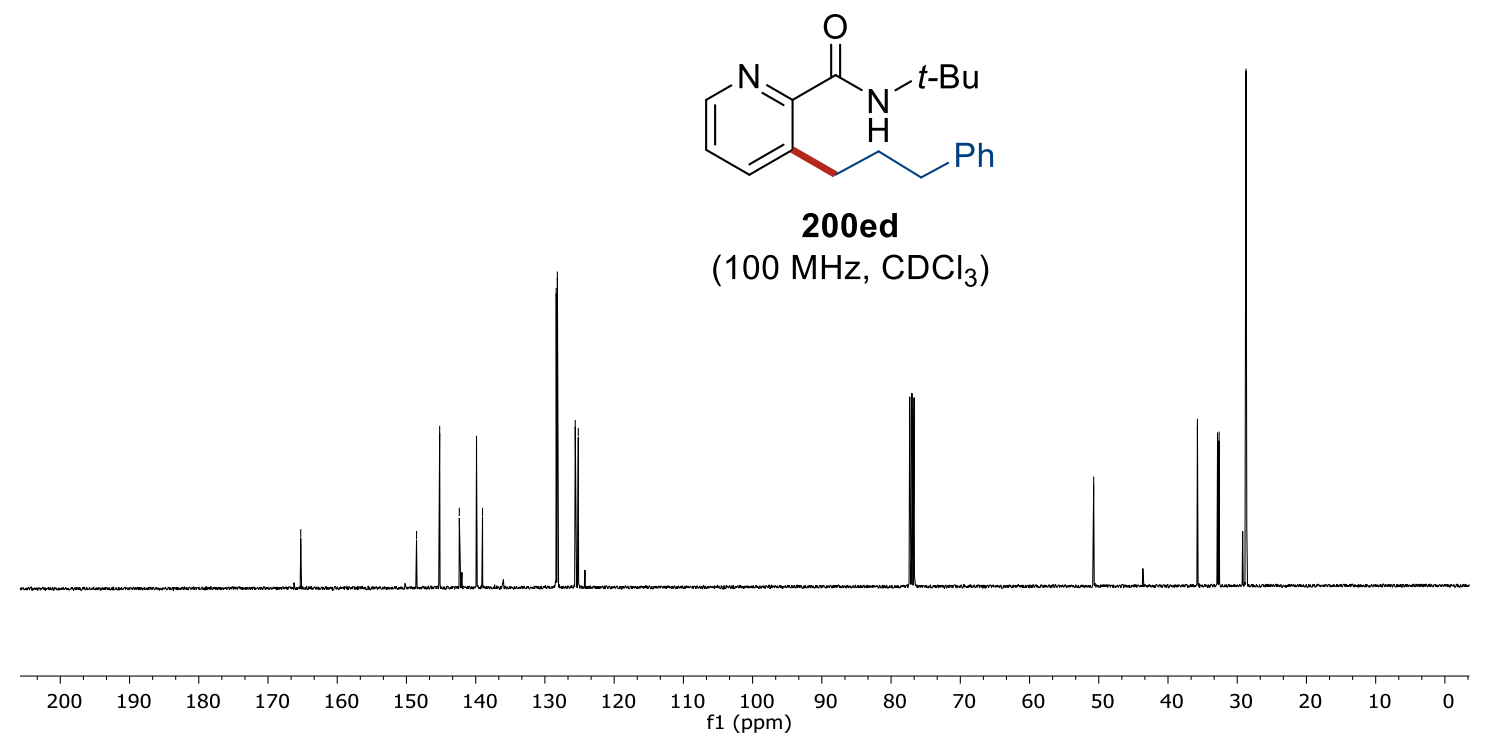


$\underbrace{m}$

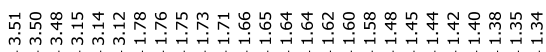<smiles>CC(CCl)c1cccnc1C(=O)NC(C)(C)C</smiles>

200ee

(400 $\mathrm{MHz}, \mathrm{CDCl}_{3}$ )

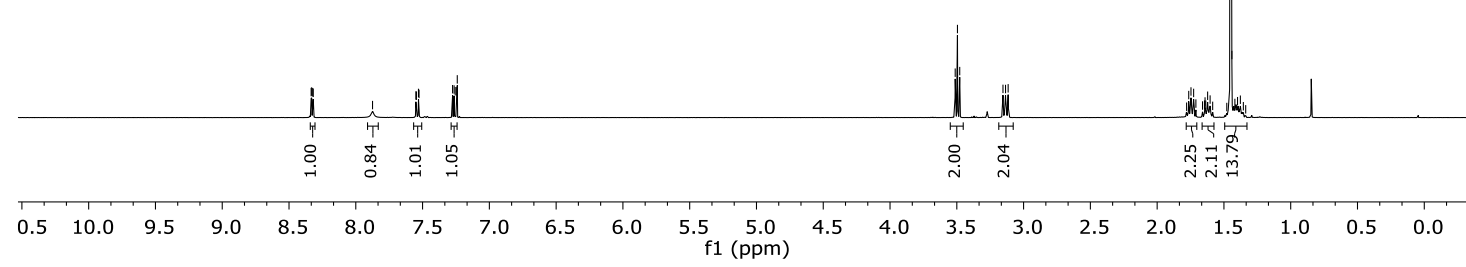

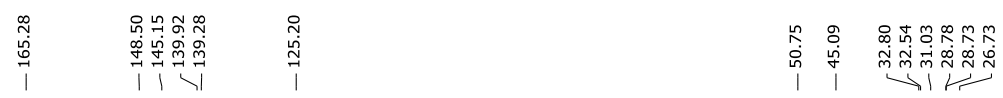<smiles>CC(CCl)c1cccnc1C(=O)NC(C)(C)C</smiles>

200ee

$\left(100 \mathrm{MHz} \mathrm{CDCl}_{3}\right)$

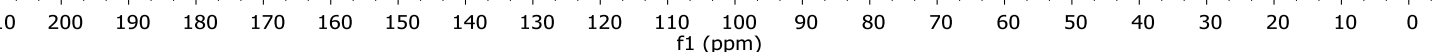




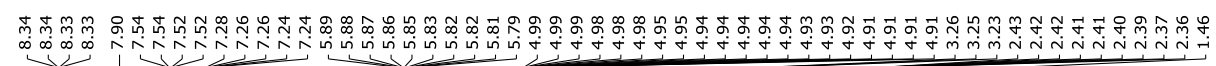<smiles>C=CCCc1cccnc1C(=O)NC(C)(C)C</smiles>

200ef

$\left(400 \mathrm{MHz}, \mathrm{CDCl}_{3}\right)$
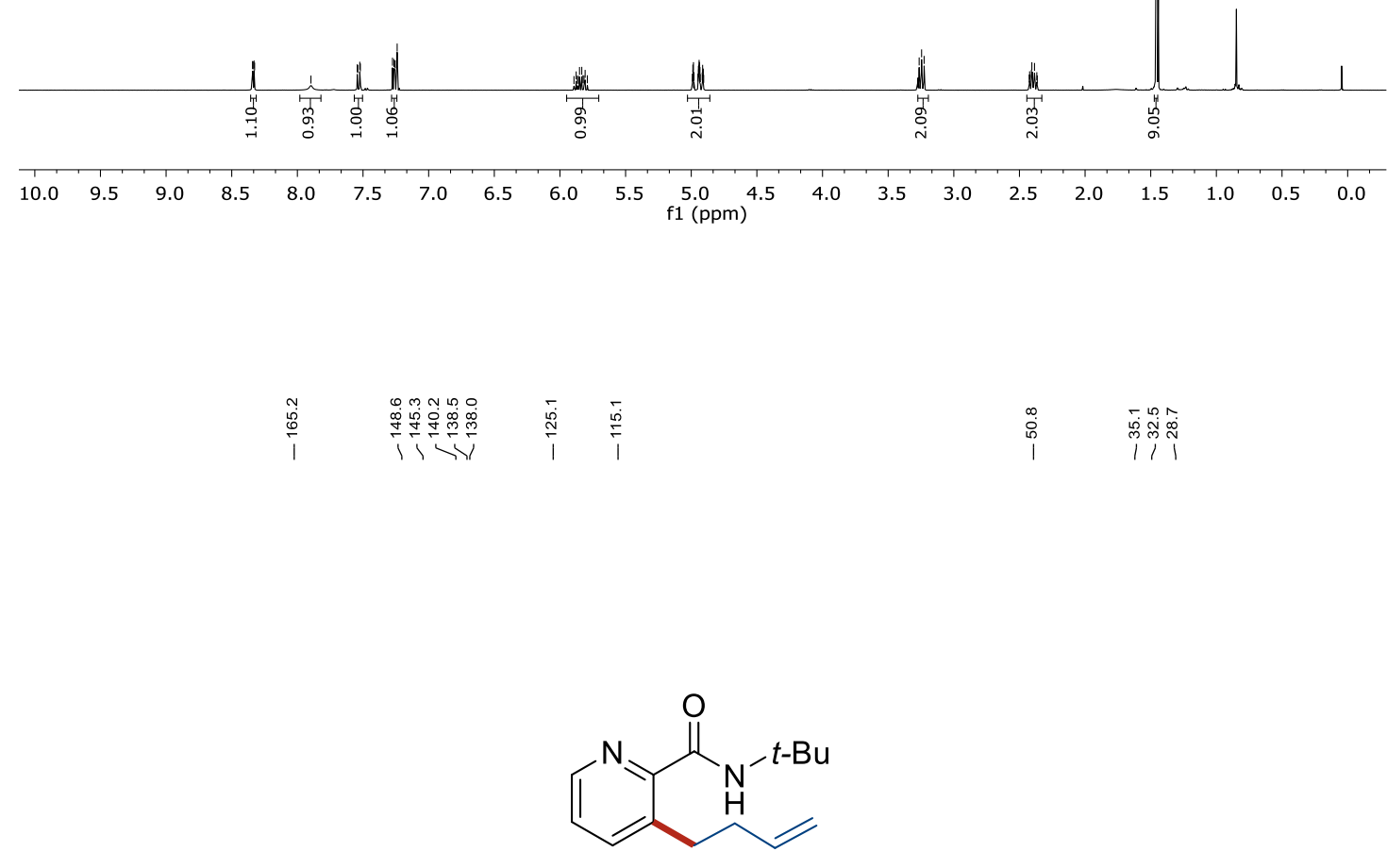

200ef

$\left(100 \mathrm{MHz} \mathrm{CDCl}_{3}\right)$

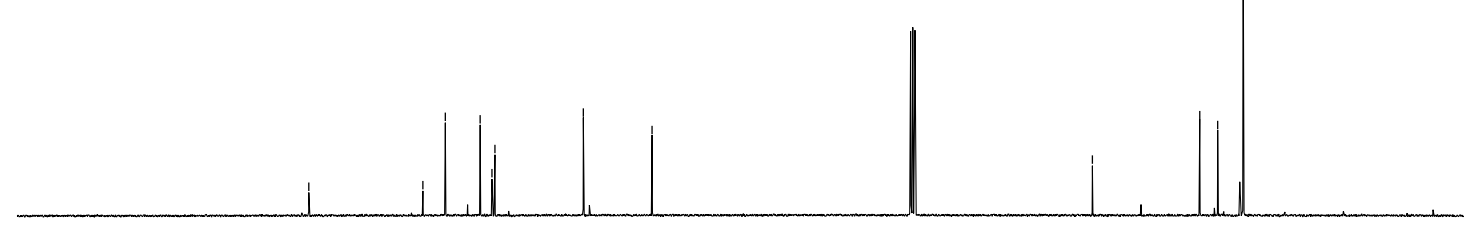

$\begin{array}{lllllllllll}200 & 190 & 180 & 170 & 160 & 150 & 140 & 130 & 120 & 110 & \begin{array}{l}100 \\ \mathrm{f} 1(\mathrm{ppm})\end{array}\end{array}$ 
<smiles>C=CCc1cccnc1C(=O)NC(C)(C)C</smiles>

200eh

$\left(400 \mathrm{MHz}, \mathrm{CDCl}_{3}\right)$

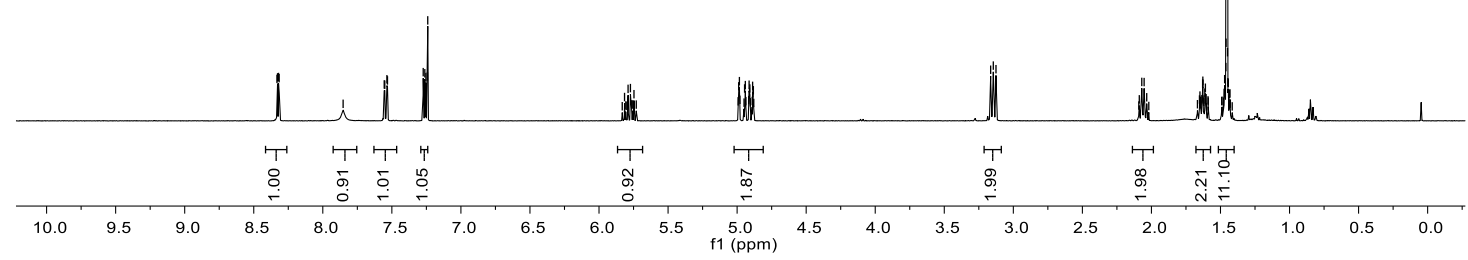

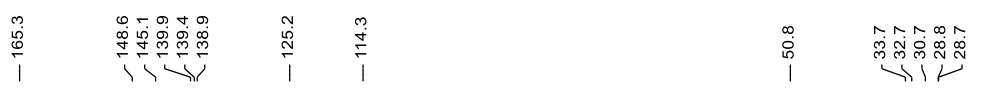<smiles>C=CCc1cccnc1C(=O)NC(C)(C)C</smiles>

200eh

$\left(100 \mathrm{MHz}, \mathrm{CDCl}_{3}\right.$ )

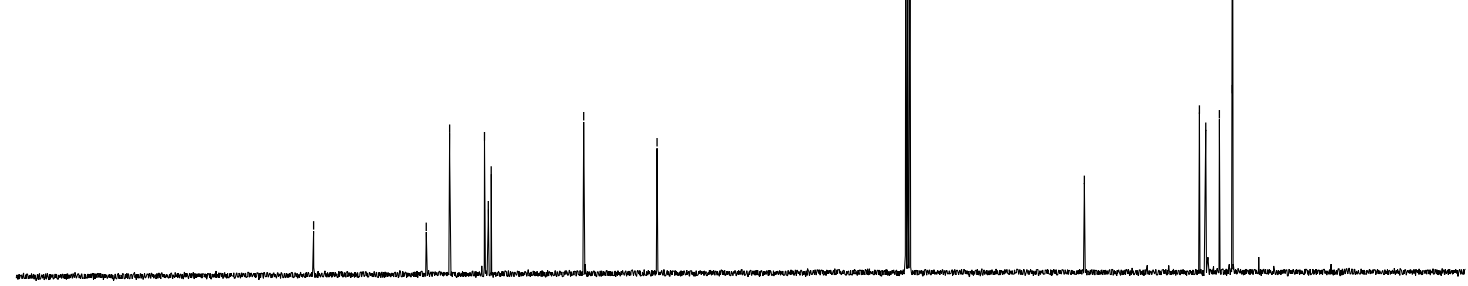

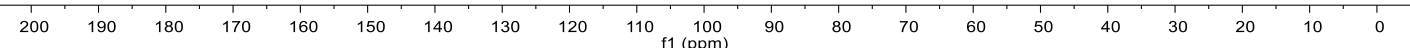




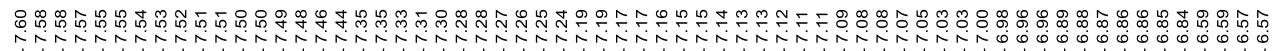

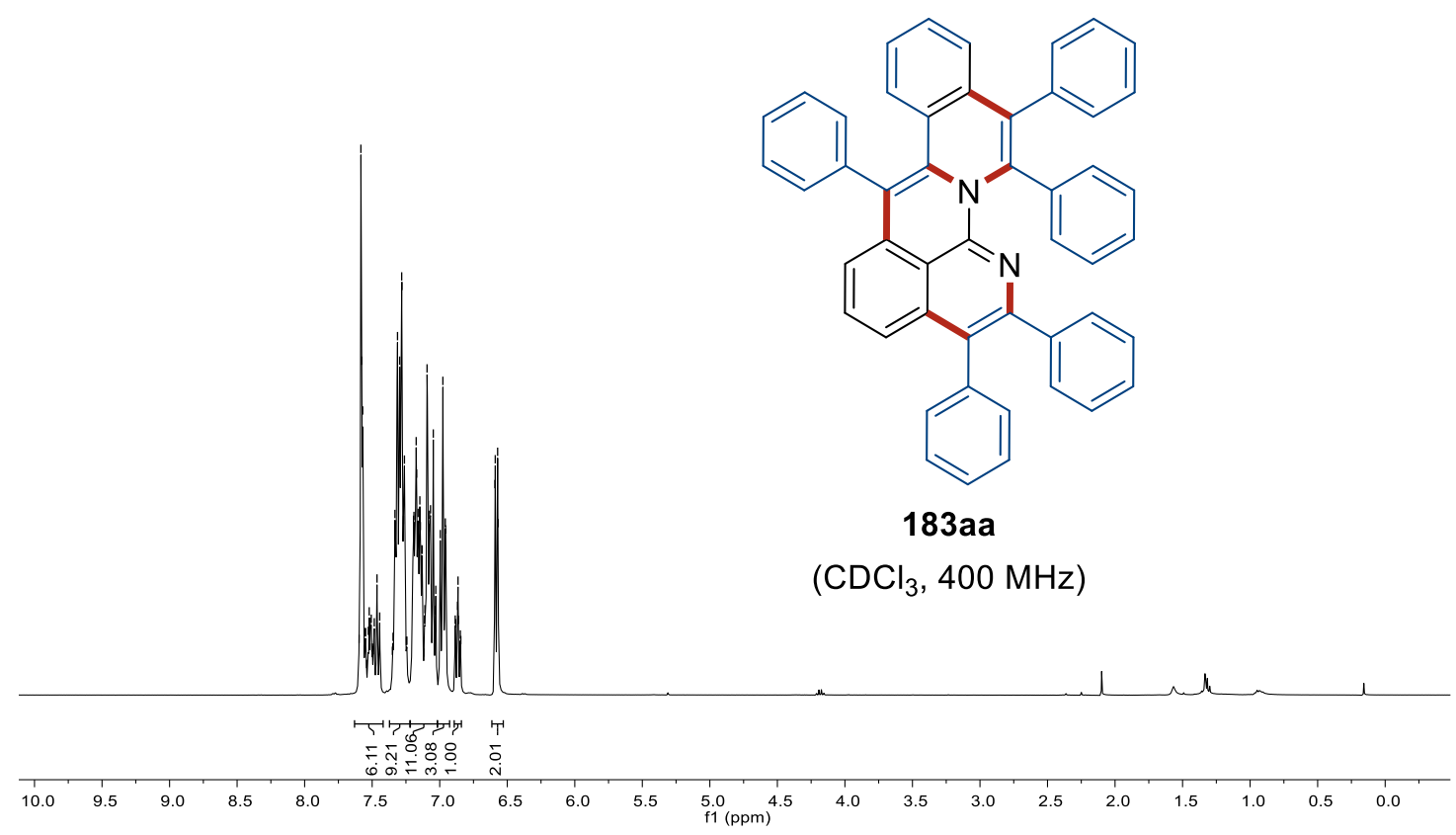

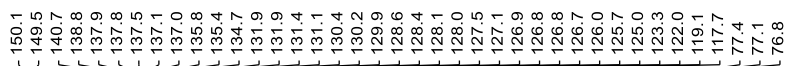

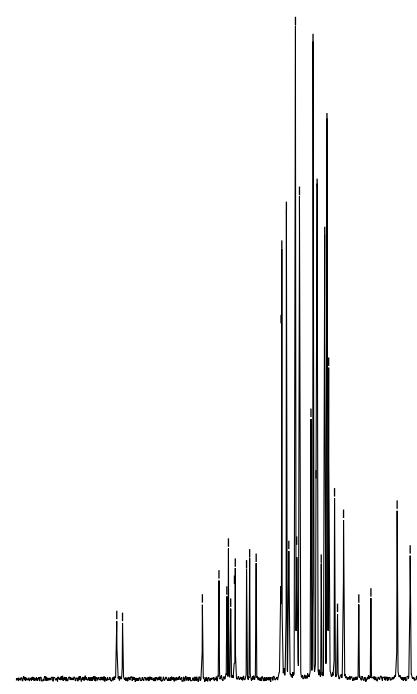<smiles></smiles>

183aa

$\left(100 \mathrm{MHz}, \mathrm{CDCl}_{3}\right)$
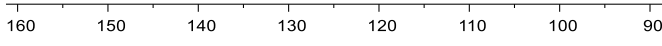

f1 $(\mathrm{ppm})$ 


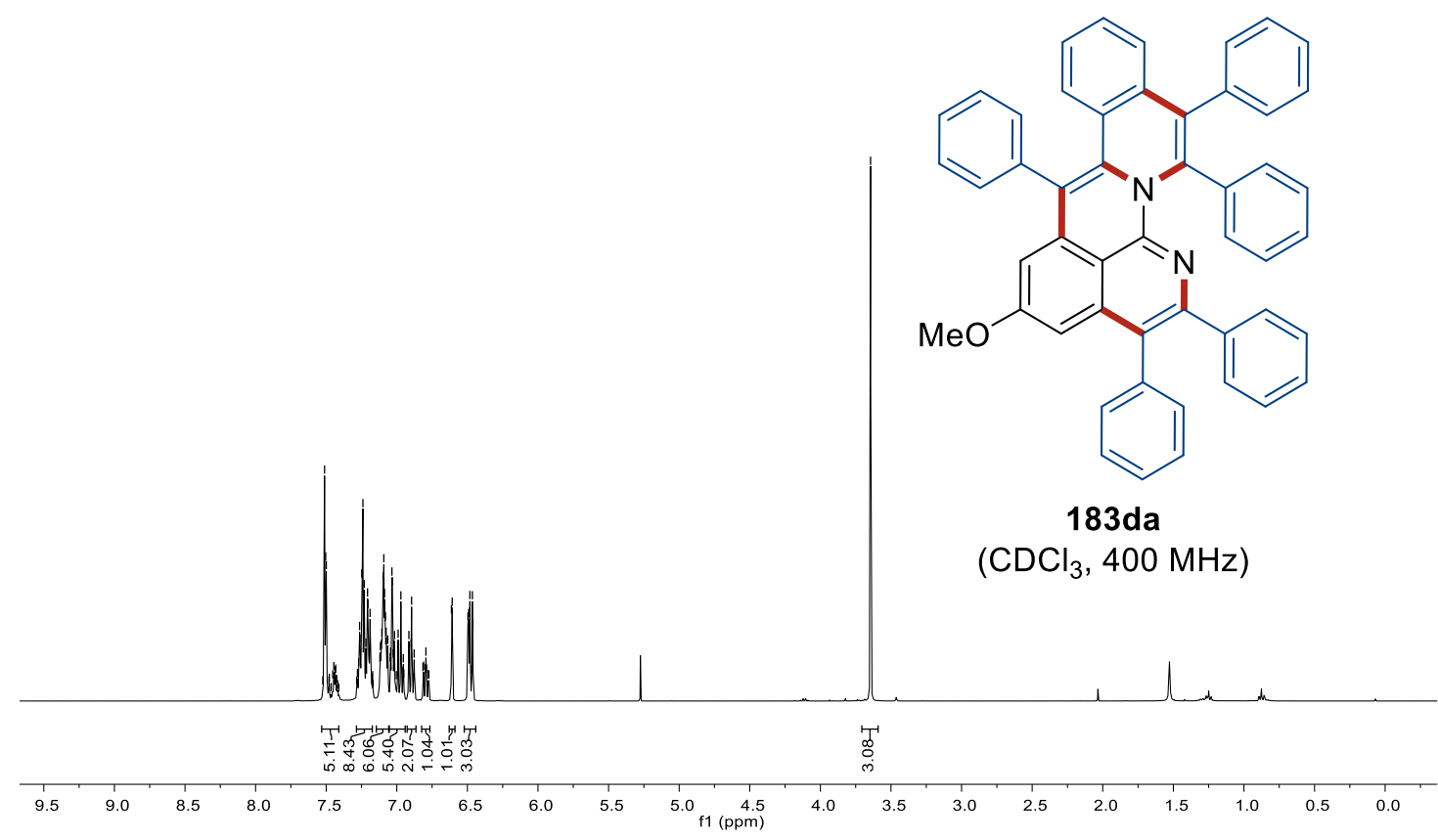

$\frac{1}{1}$

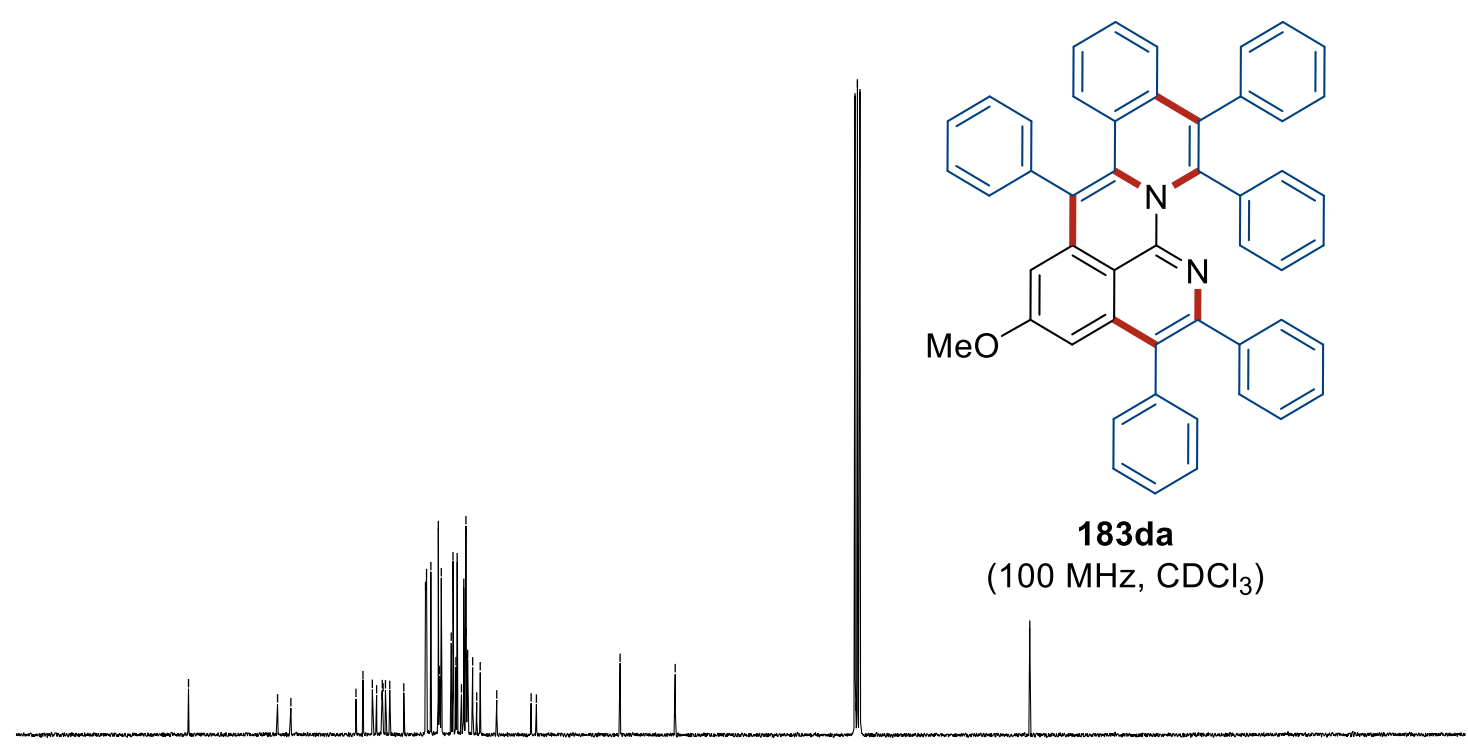

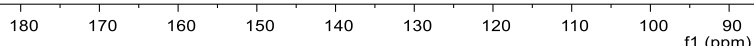




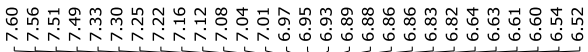

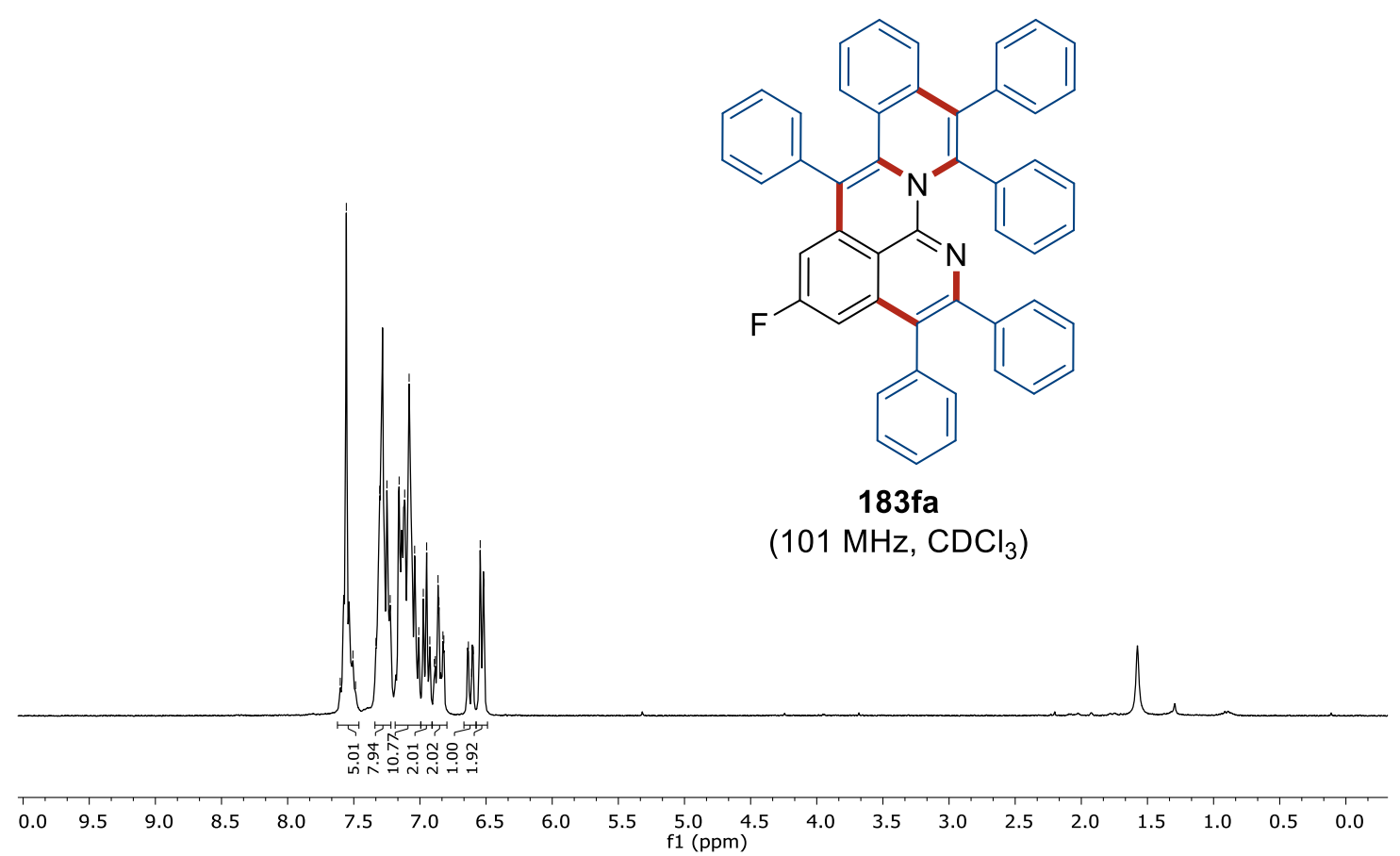

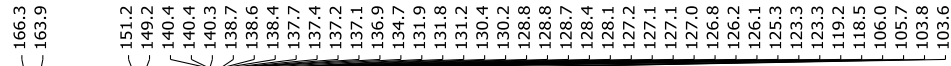

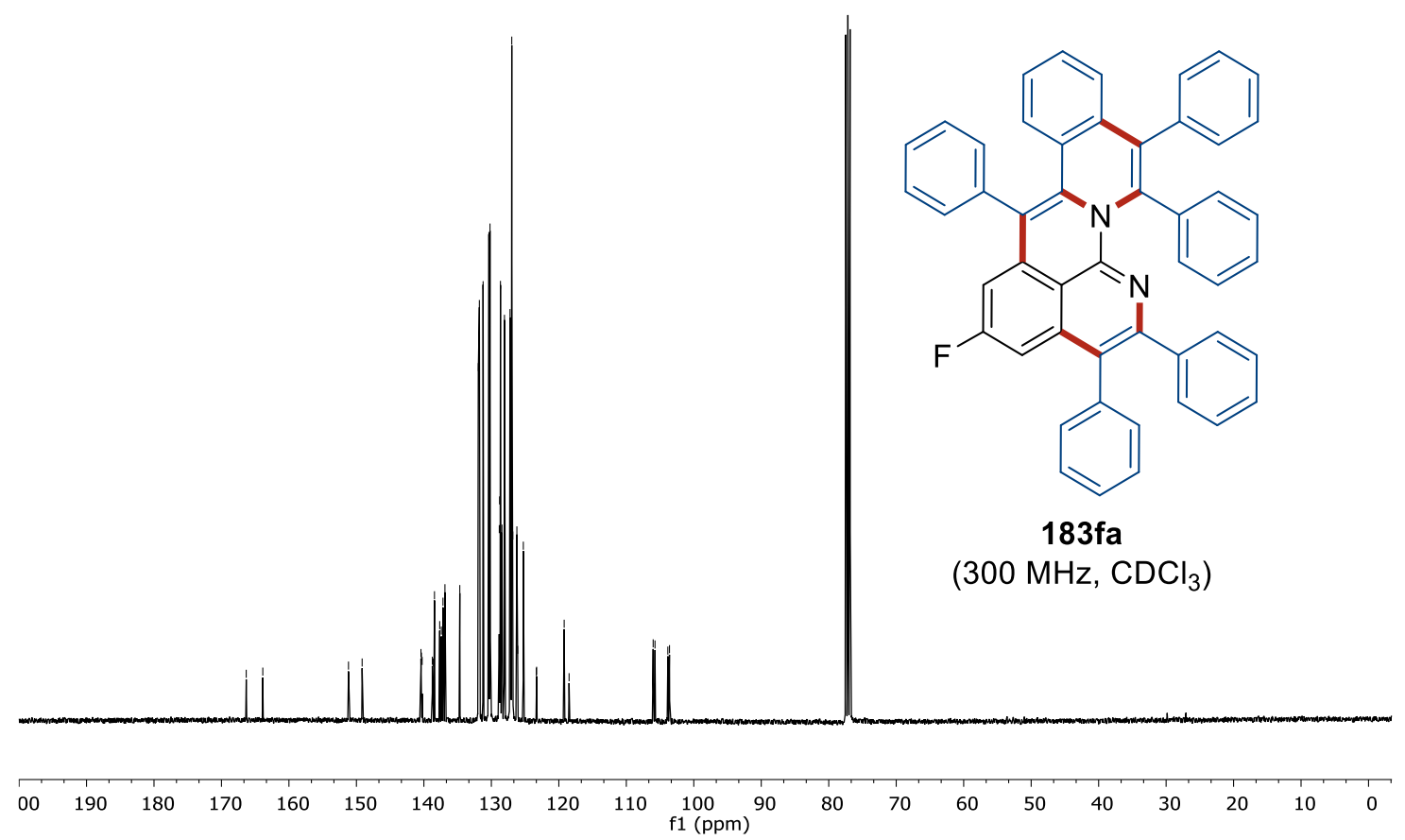




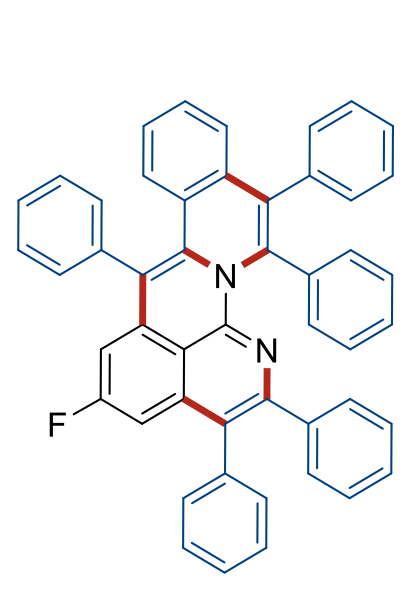

$183 f a$

$\left(471 \mathrm{MHz}^{\mathrm{CDCl}}\right)_{3}$

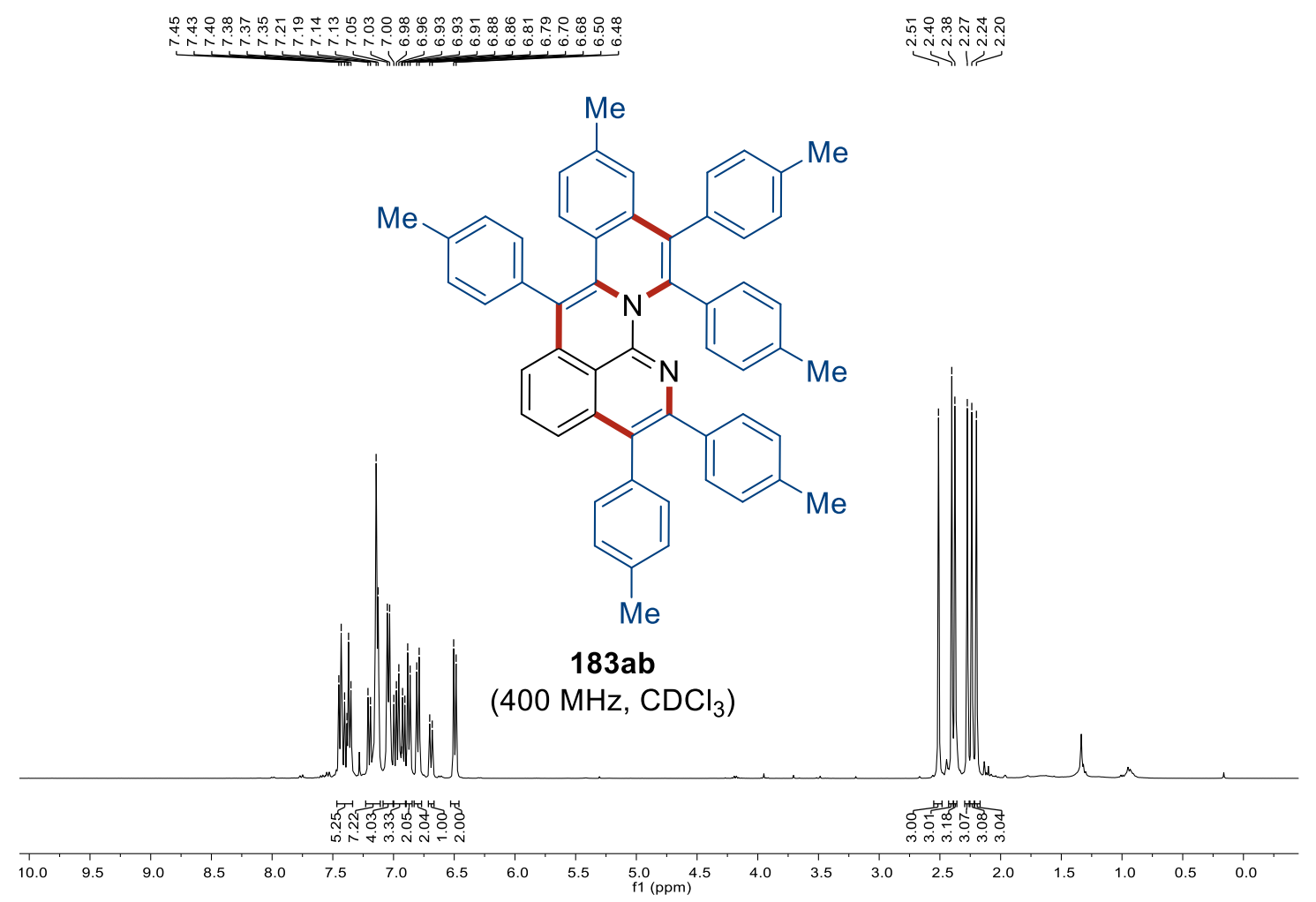




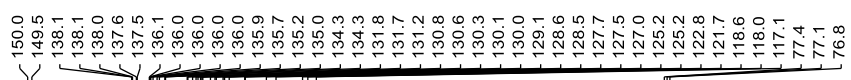
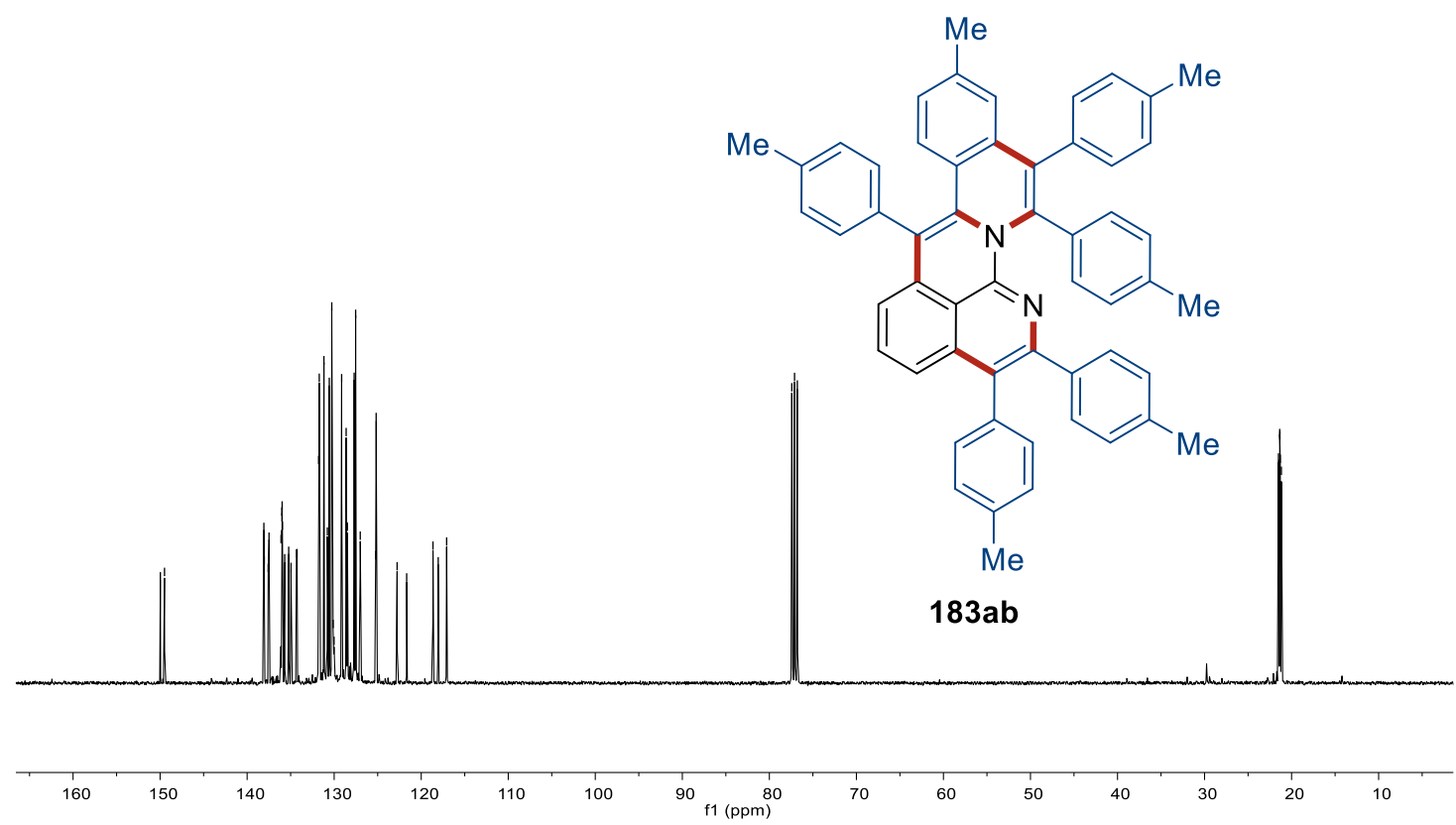

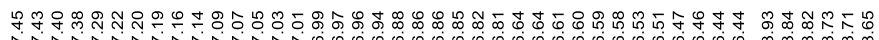

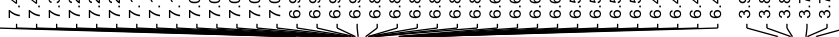

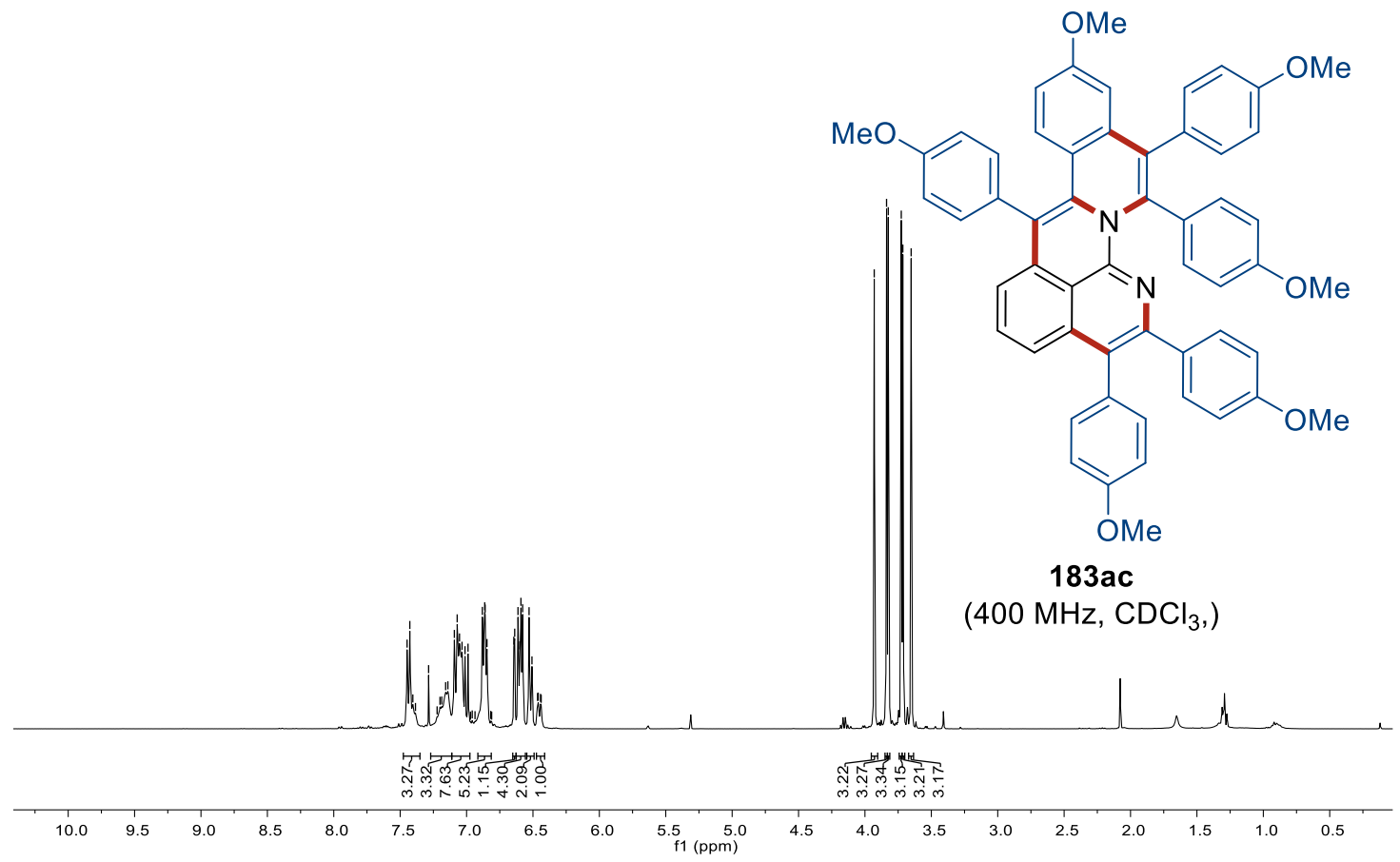




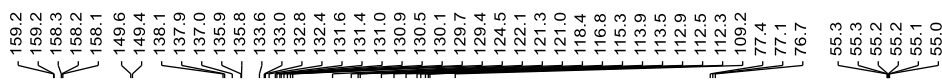

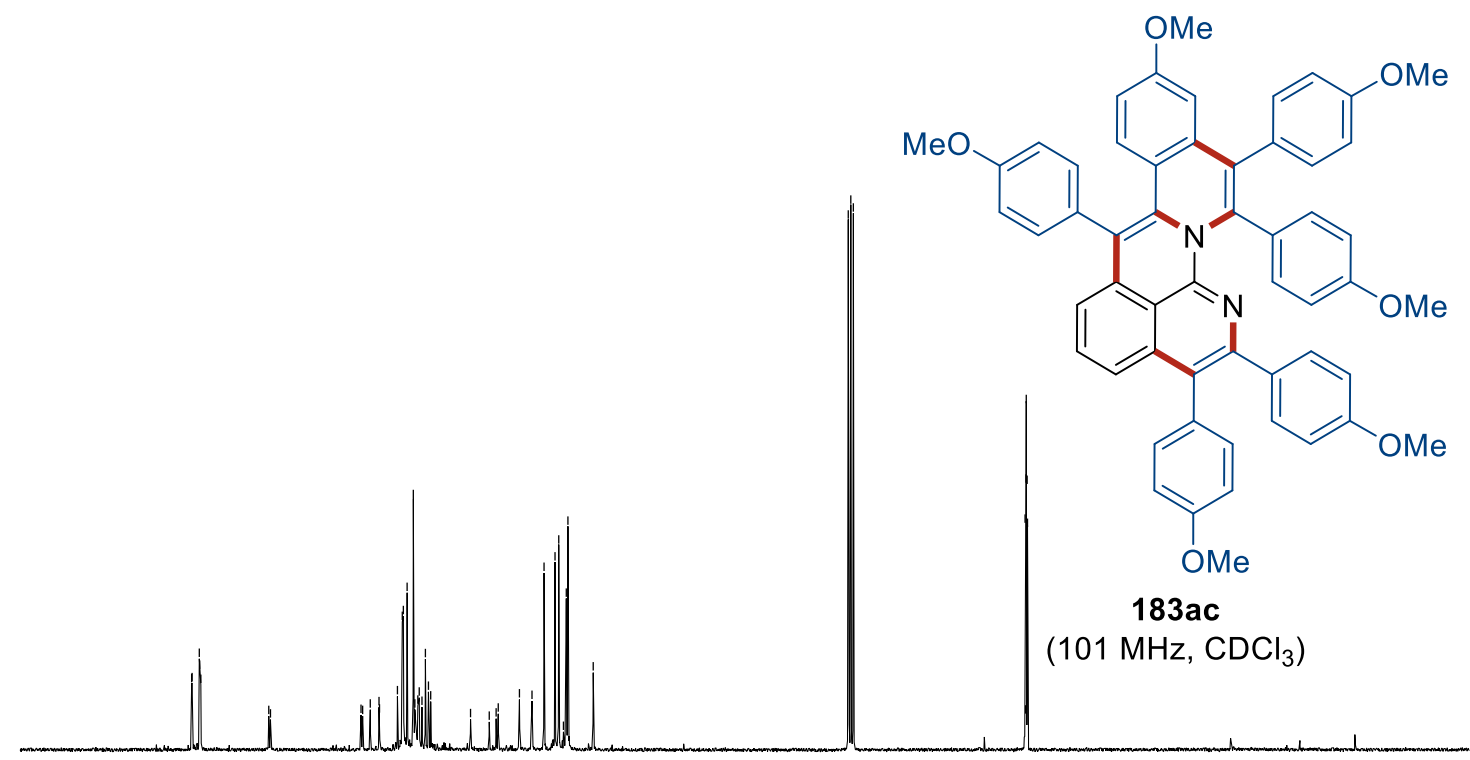

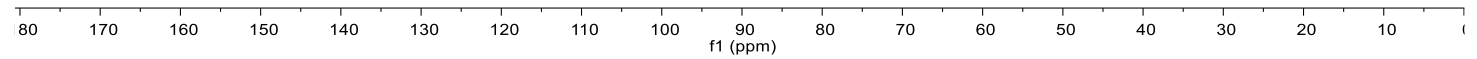

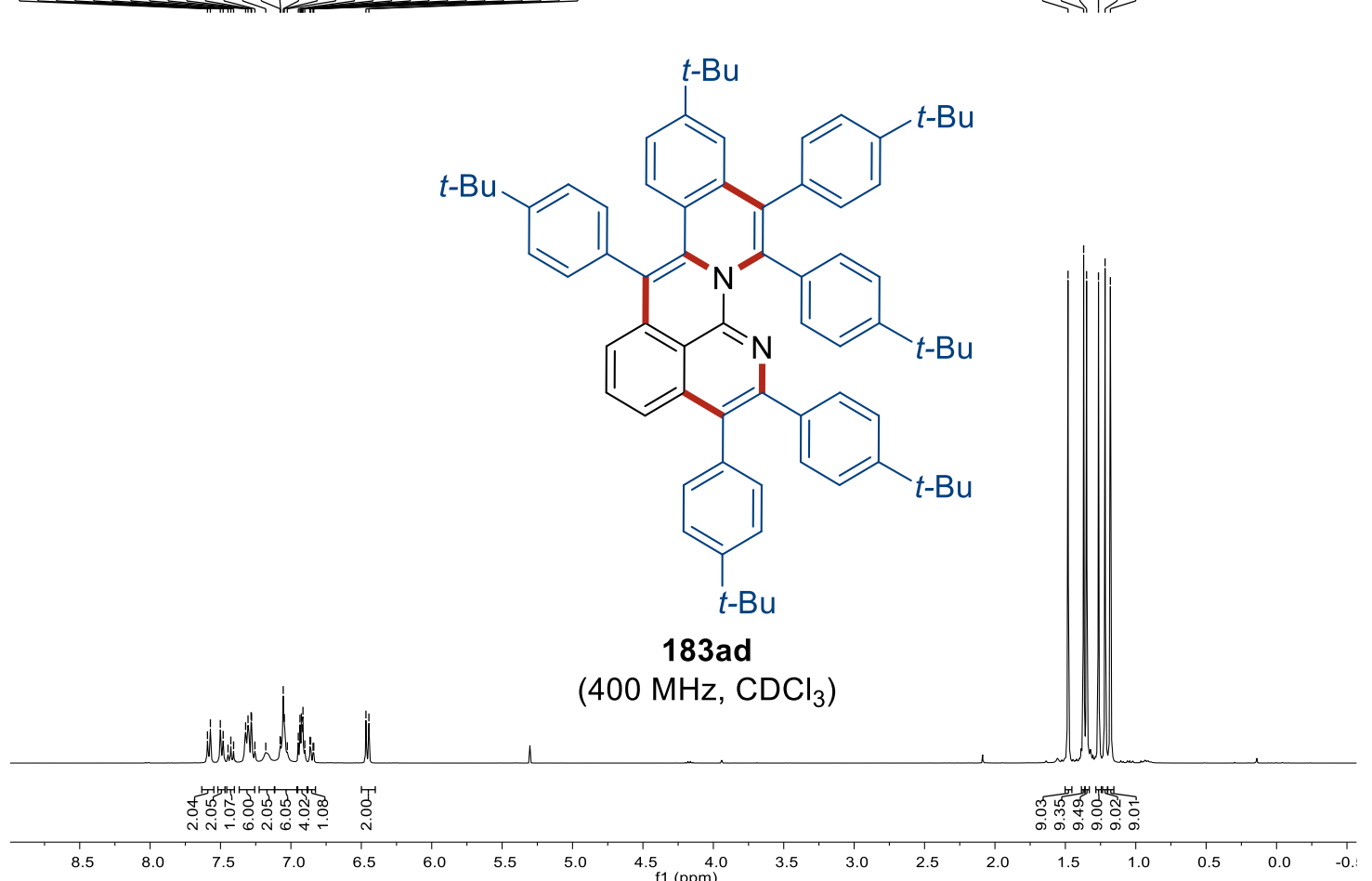




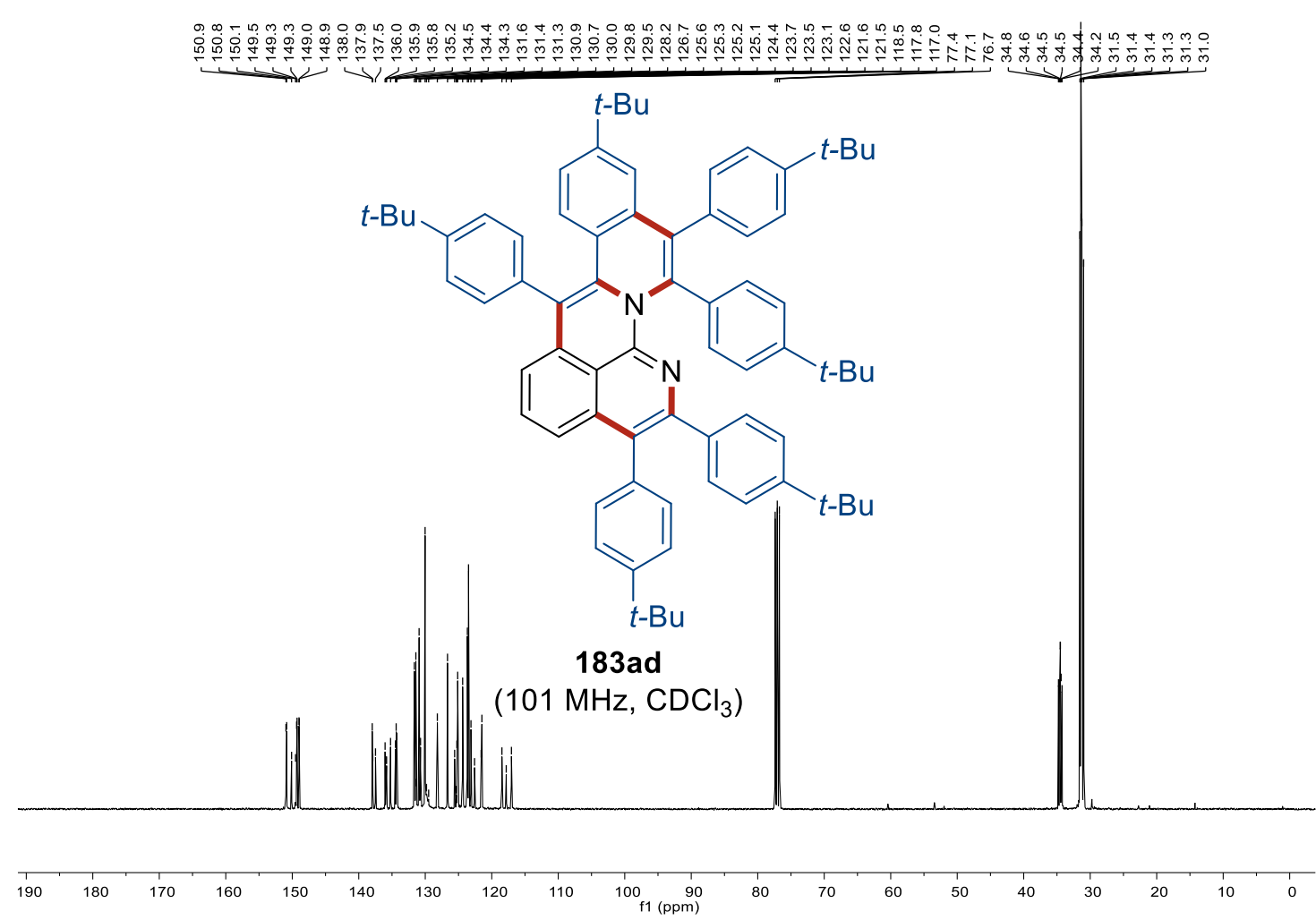

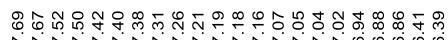

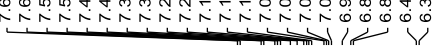

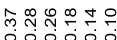

인,

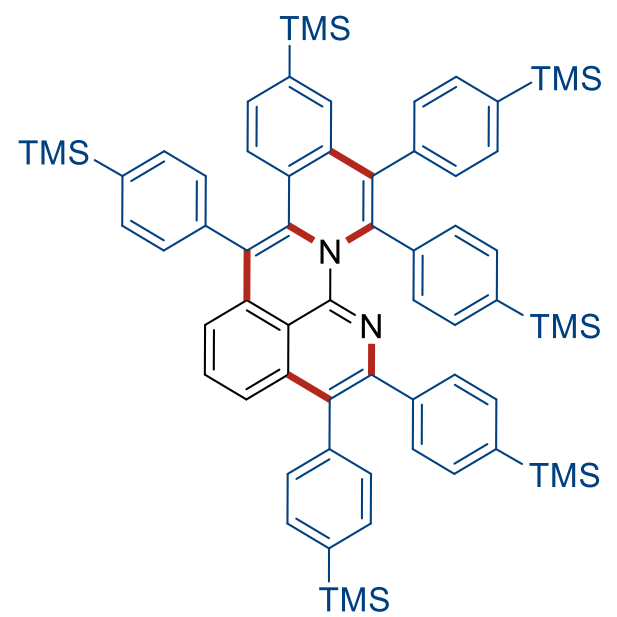

183ae

$\left(400 \mathrm{MHz}, \mathrm{CDCl}_{3}\right.$ )

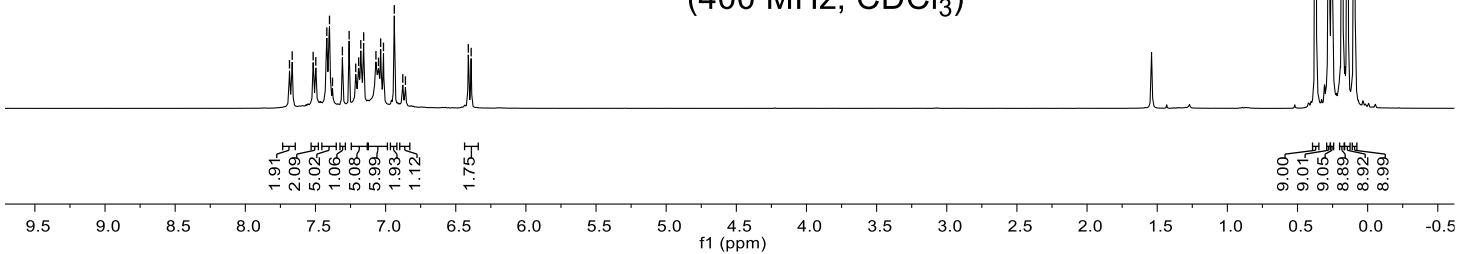




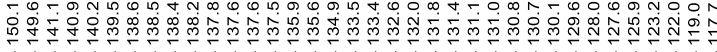

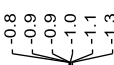

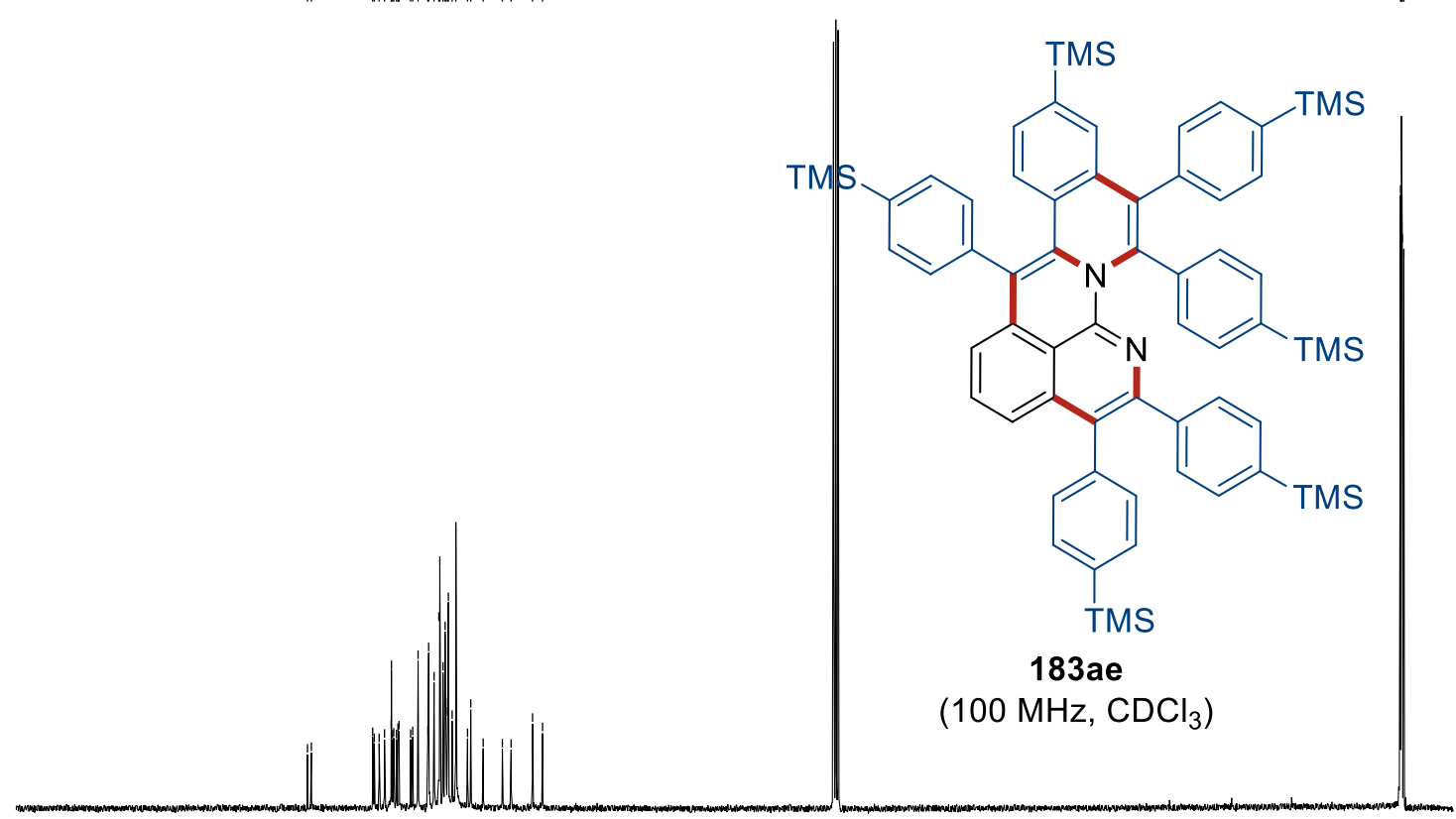

$\begin{array}{llllllllllllllllllll} & 180 & 170 & 160 & 150 & 140 & 130 & 120 & 110 & 100 & 90 & 80 & 70 & 60 & 50 & 40 & 30 & 20 & 10 & 0\end{array}$

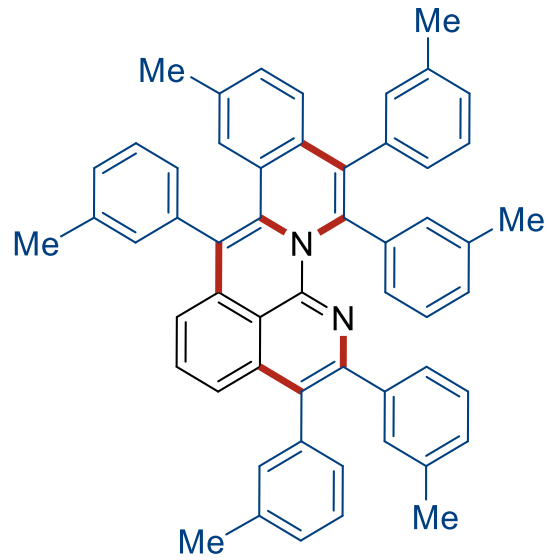

183af

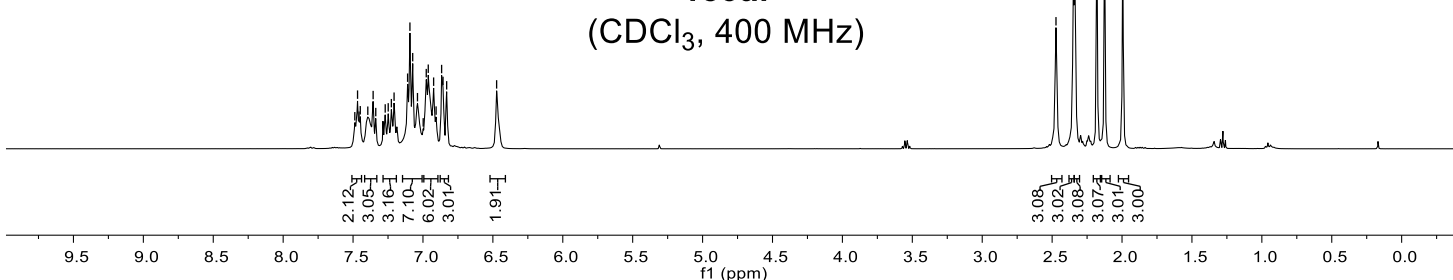



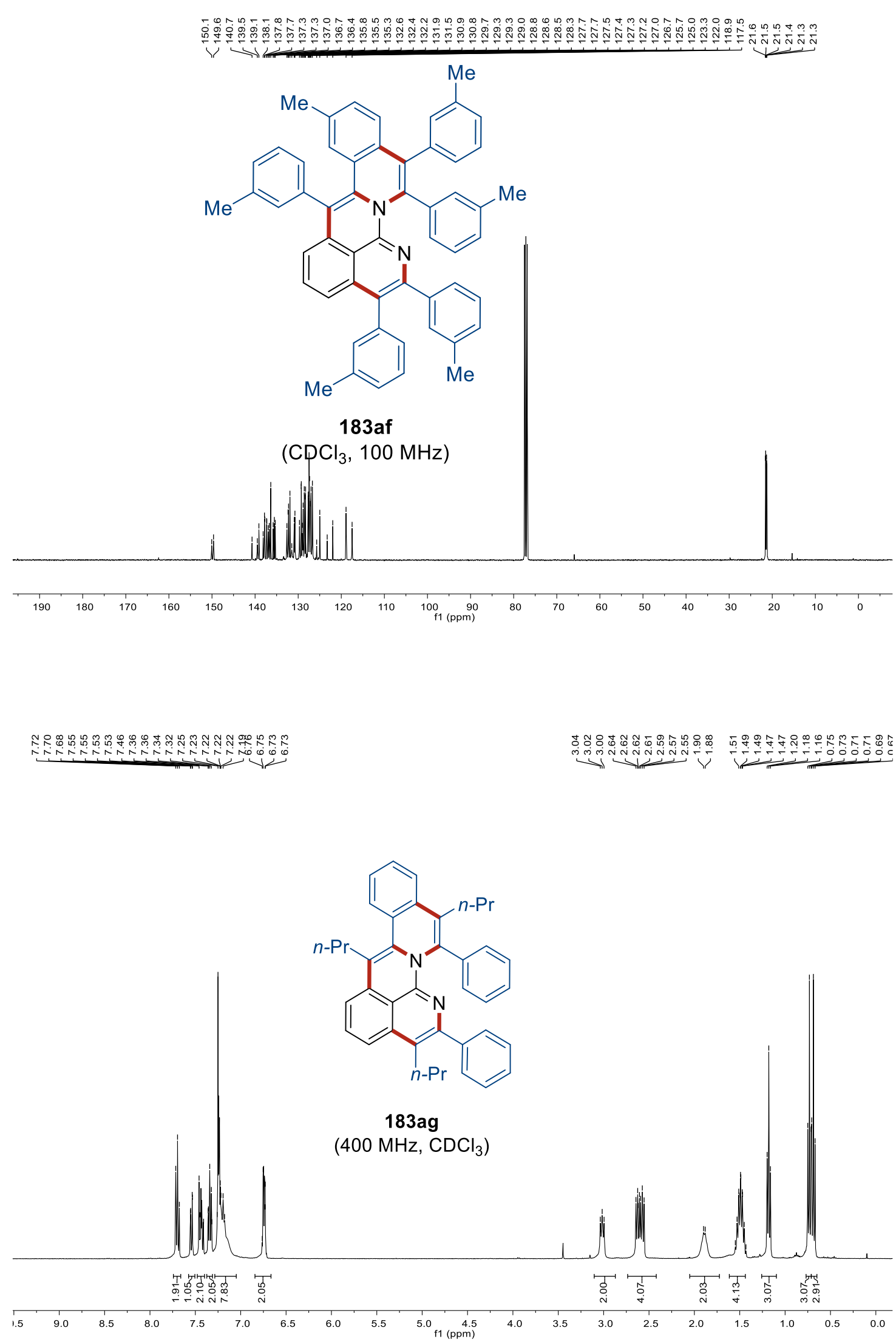

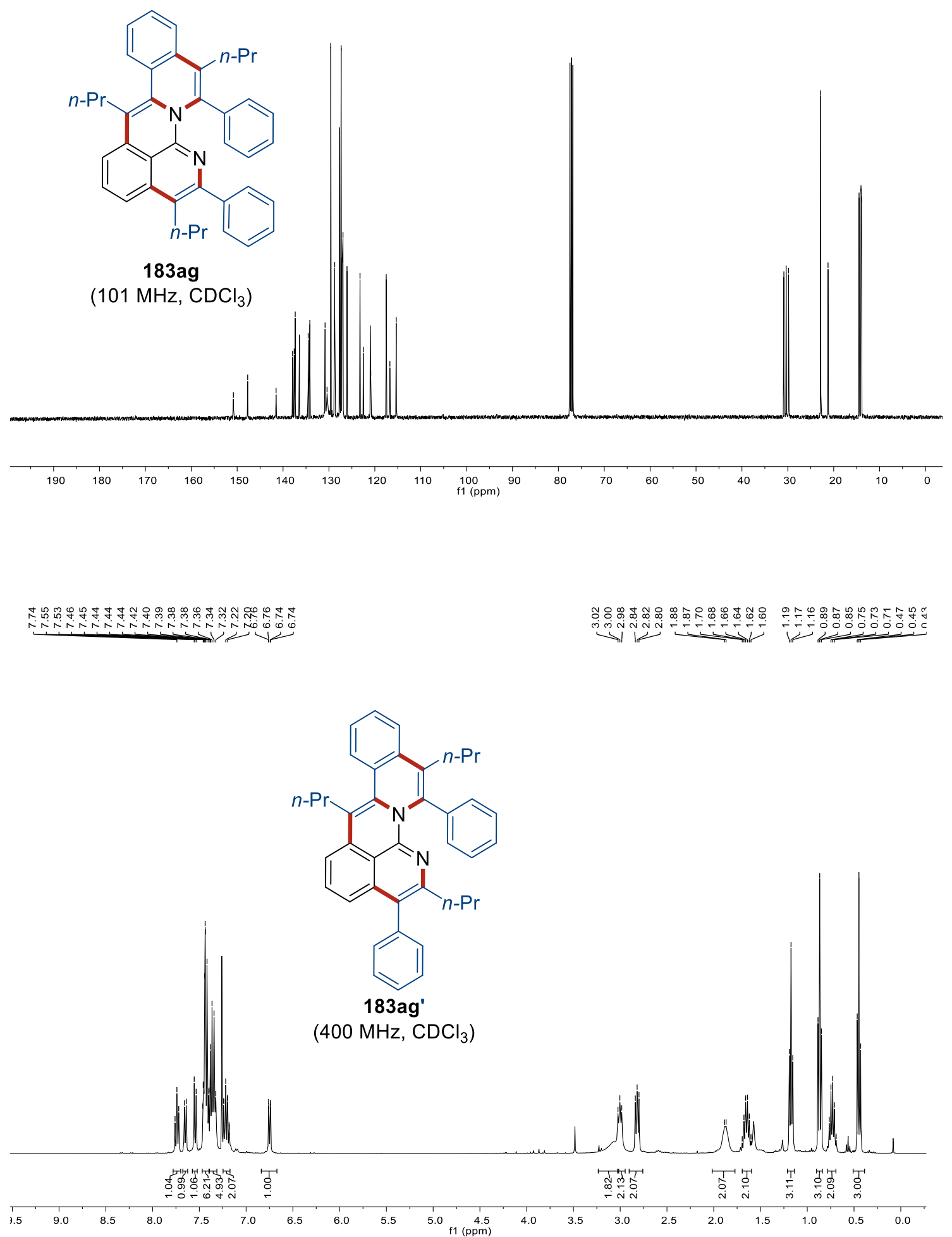


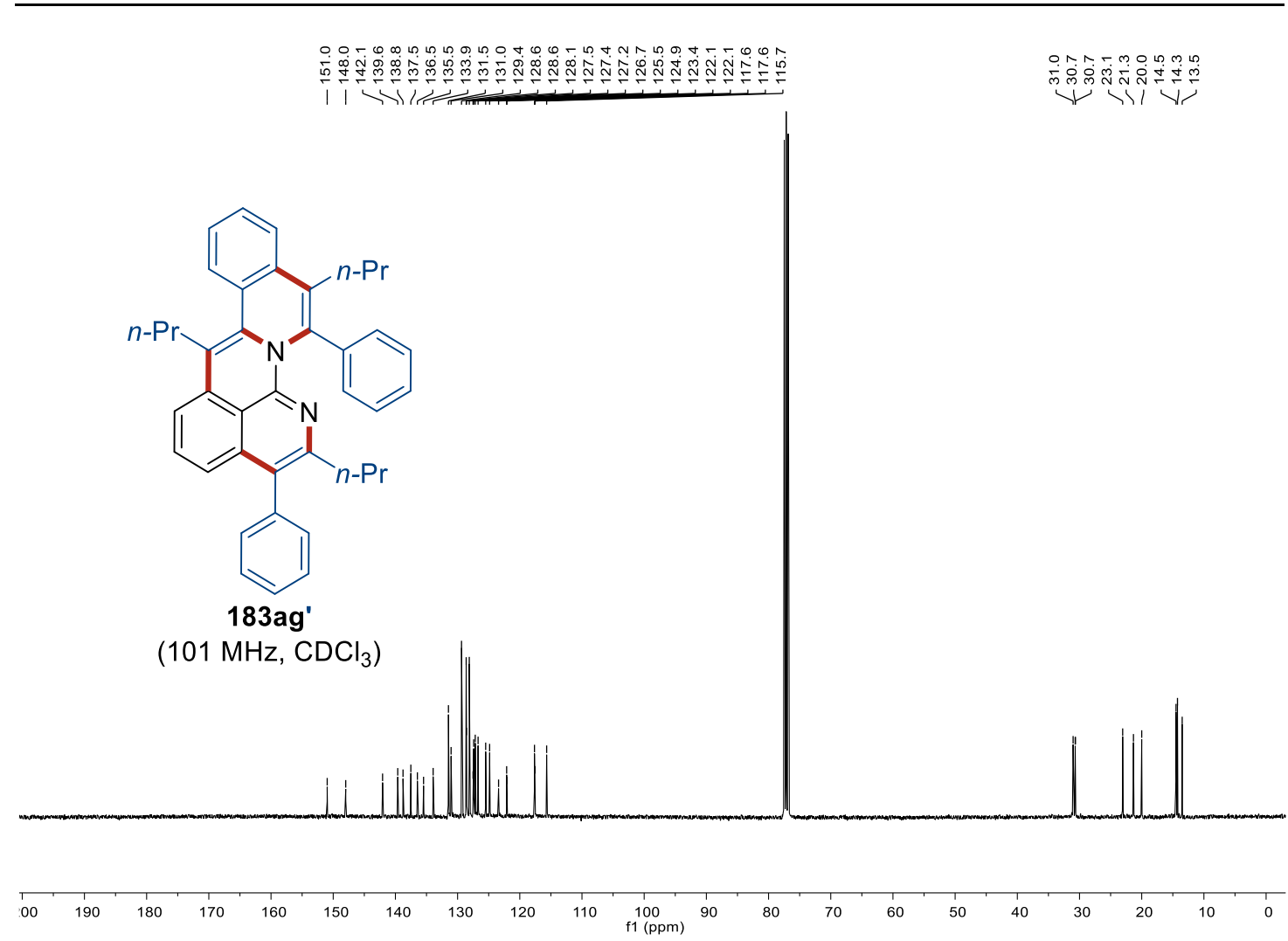

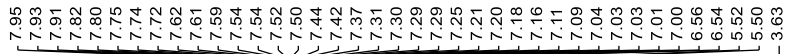

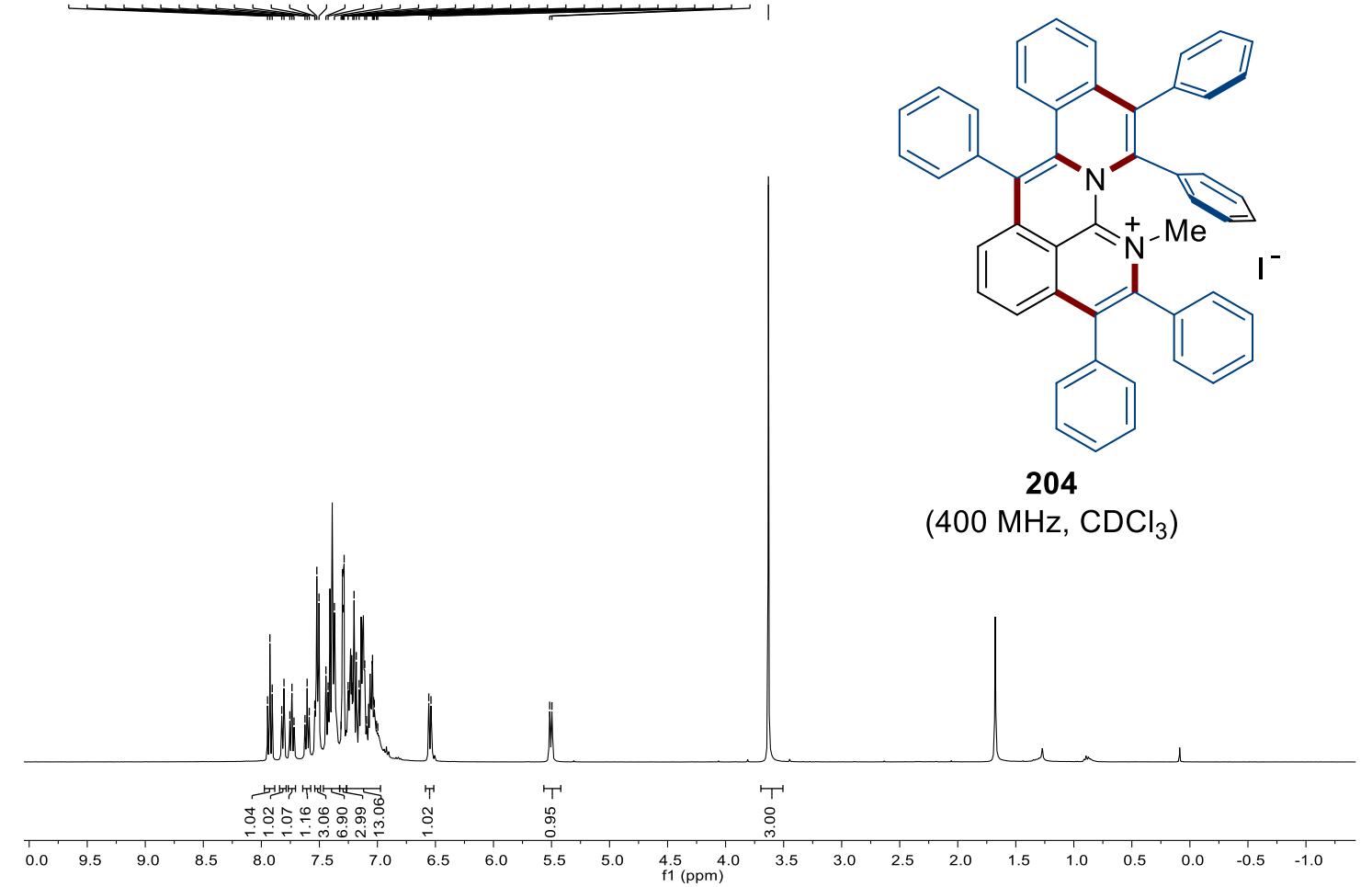


l.

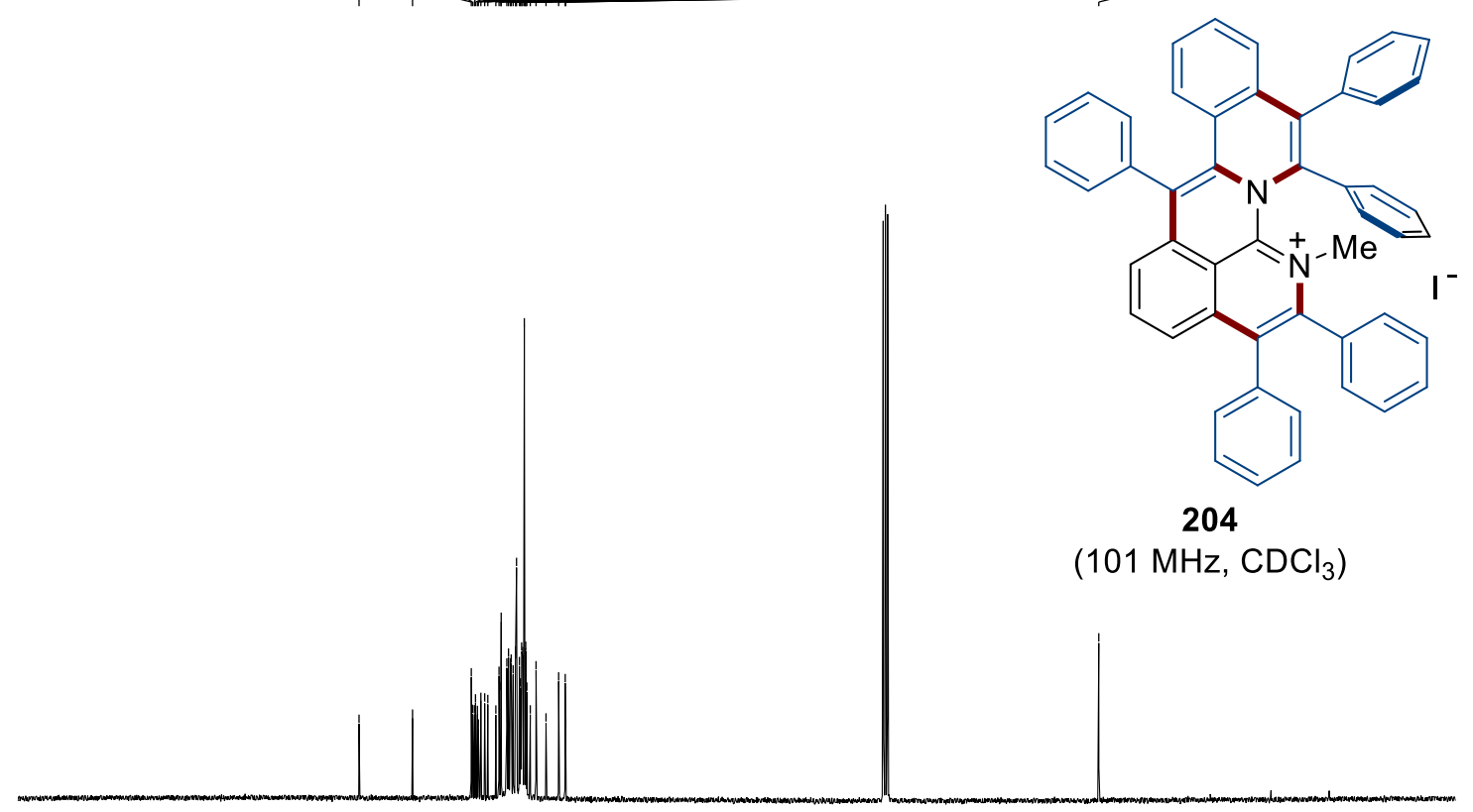

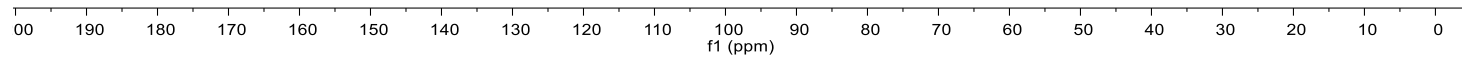

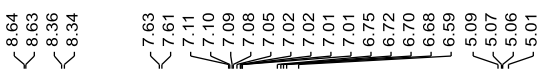

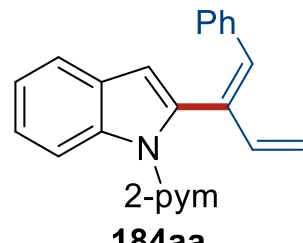

$400 \mathrm{MHz}, \mathrm{CDCl}_{3}$

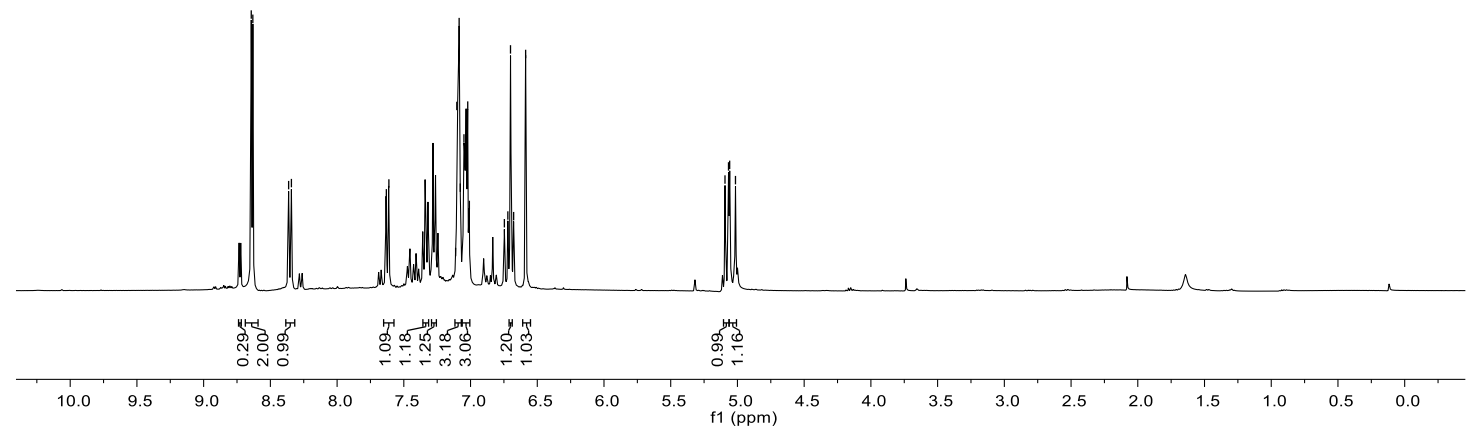




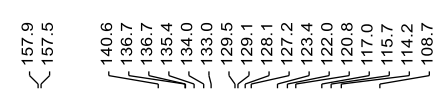

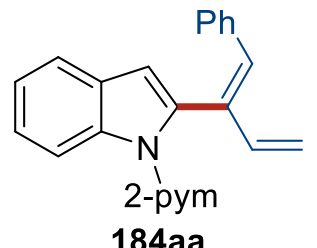

(101 MHz, CDCl3)
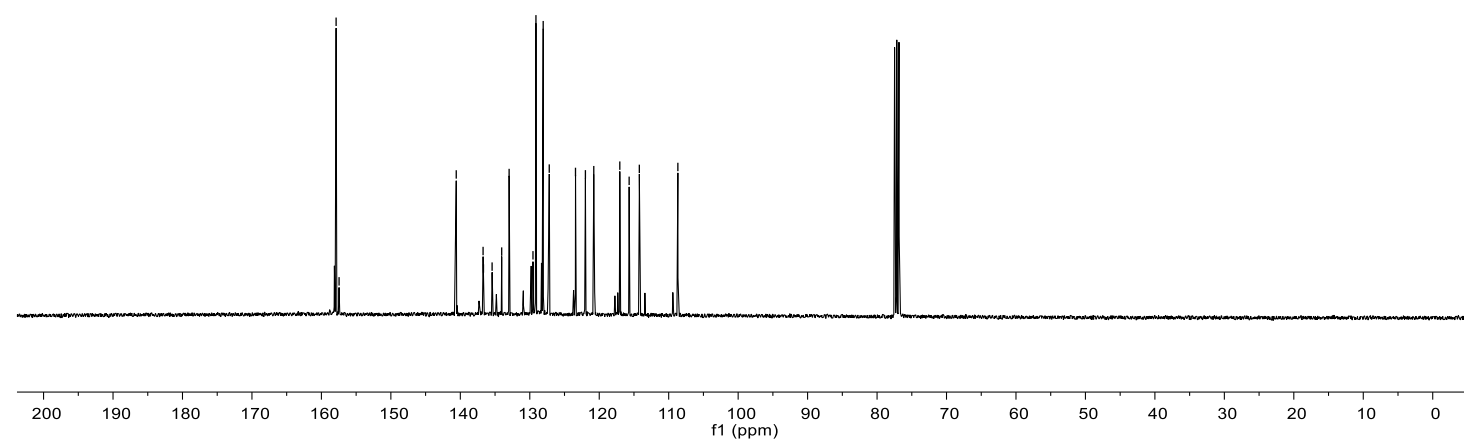

$\underbrace{\underbrace{2}}$

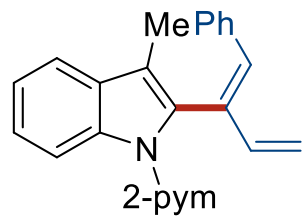

184ba

$\left(400 \mathrm{MHz}, \mathrm{CDCl}_{3}\right)$

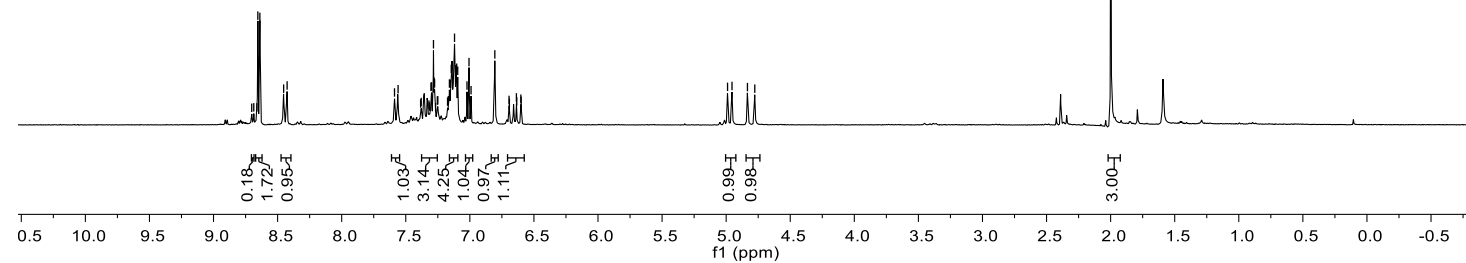




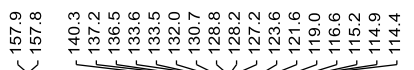

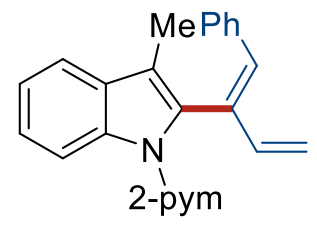

184ba

$\left.\left(101 \mathrm{MHz}^{\mathrm{CDCl}}\right)_{3}\right)$

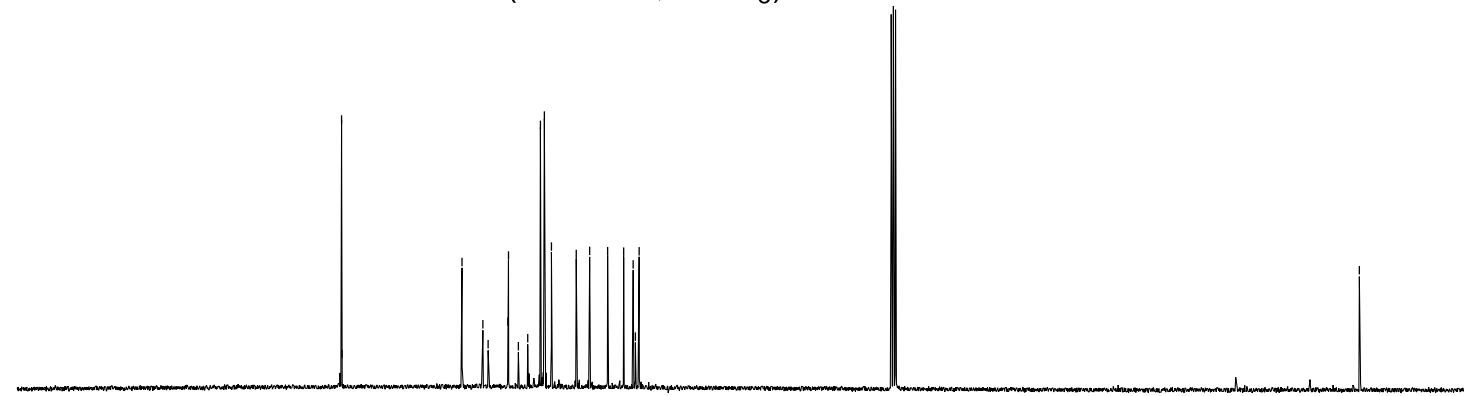

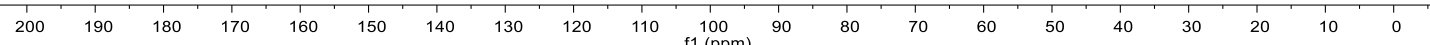

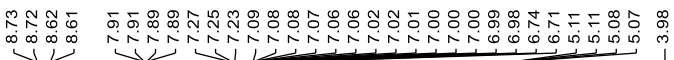

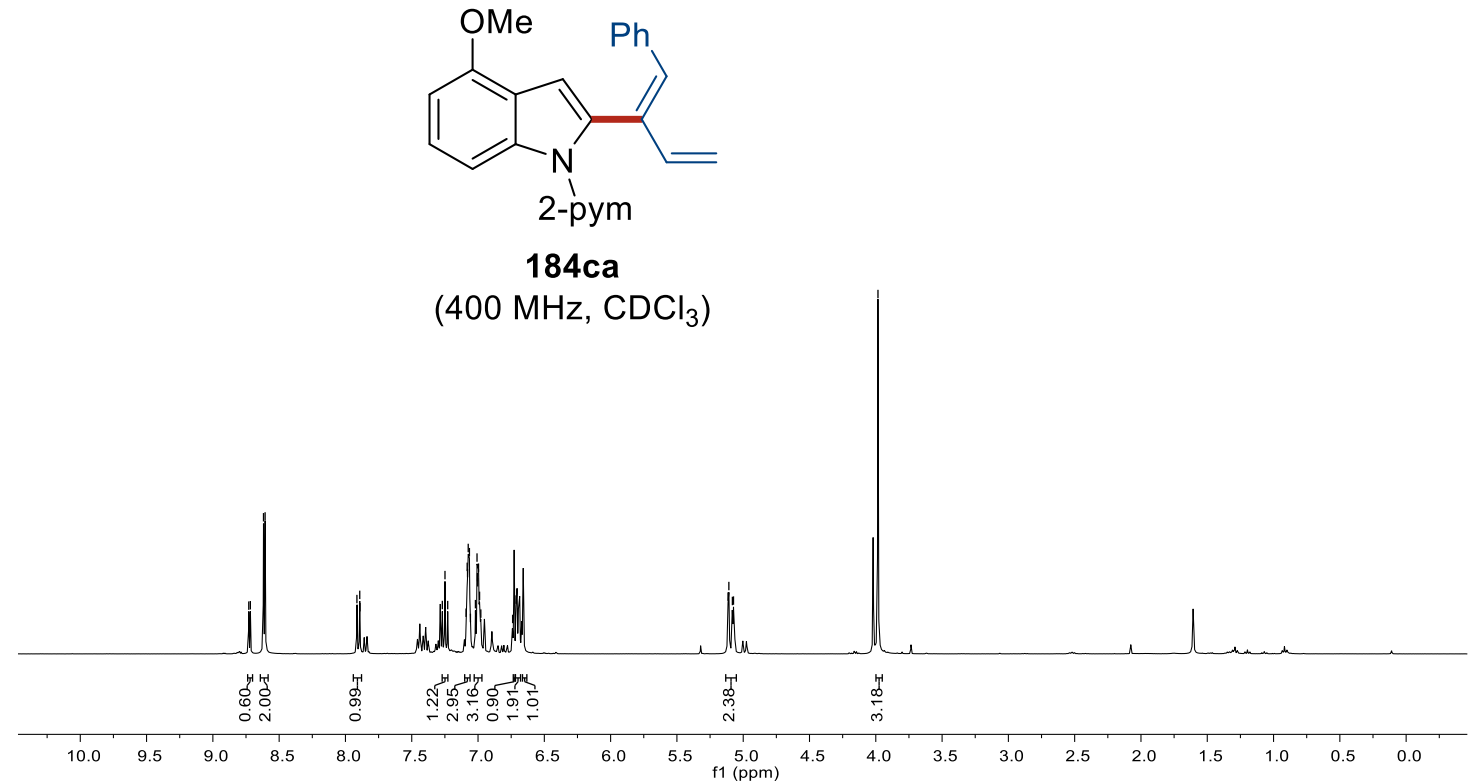




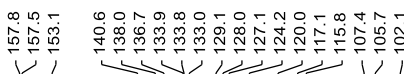

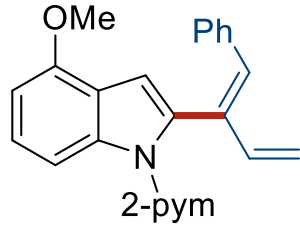

184ca

(101 $\mathrm{MHz}, \mathrm{CDCl}_{3}$ )
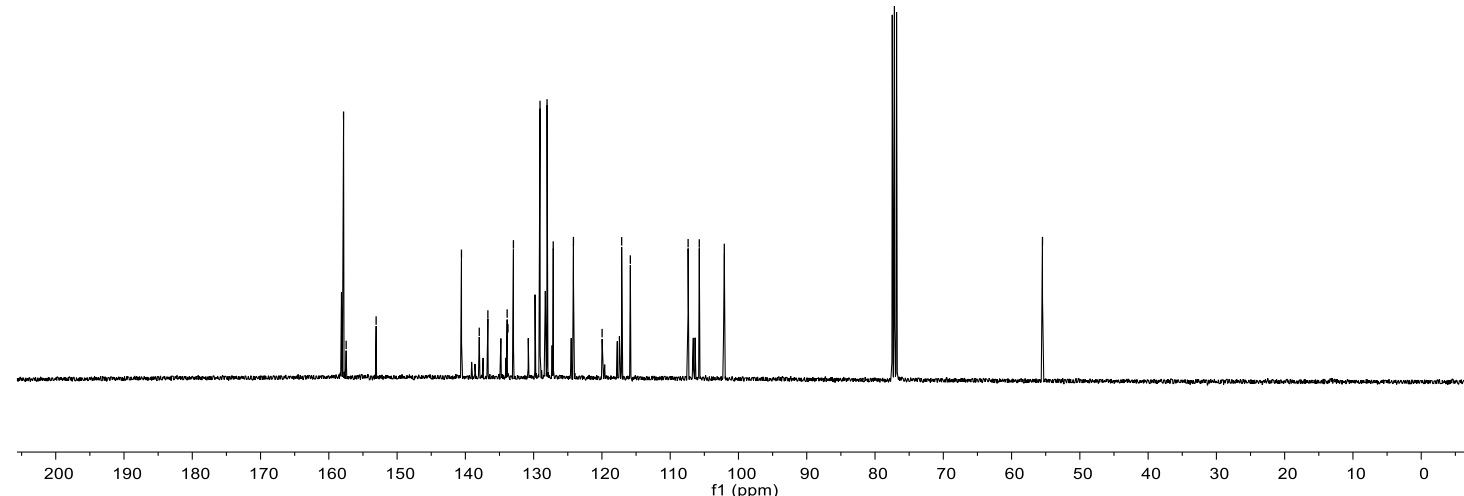

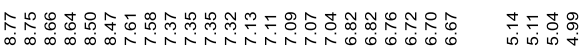

$\longrightarrow$

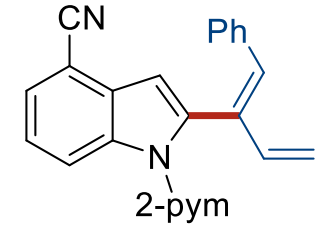

184da

(300 $\mathrm{MHz}, \mathrm{CDCl}_{3}$ )
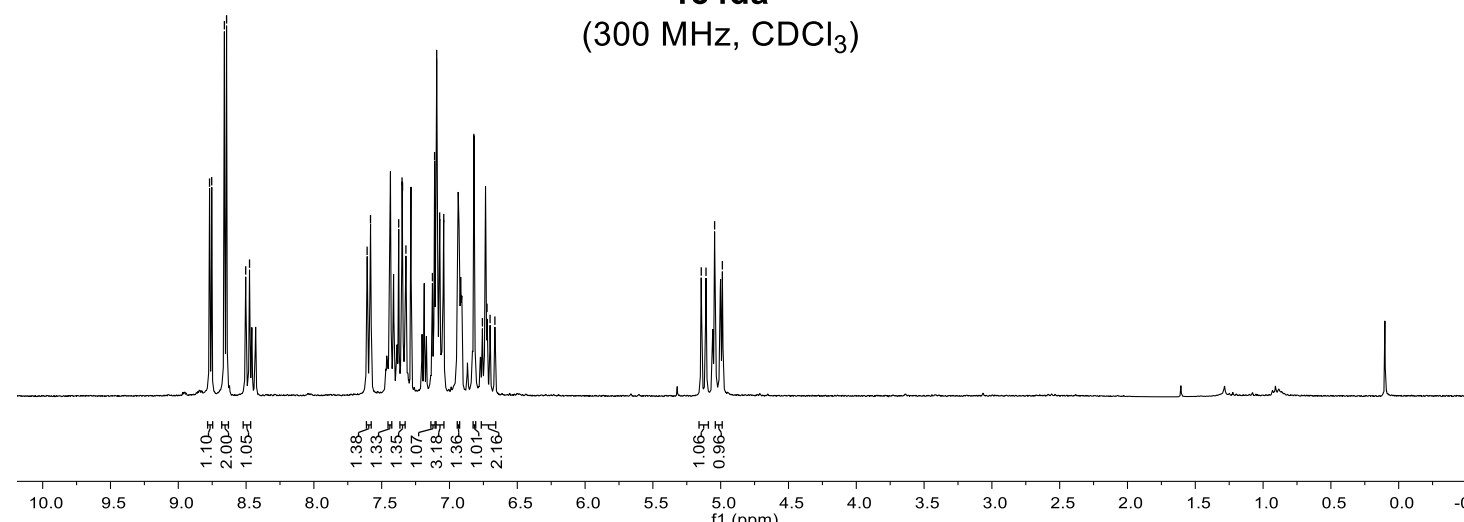


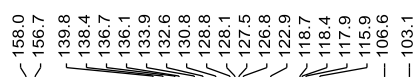

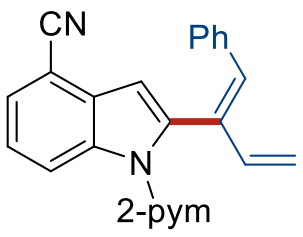

184da

(101 MHz, $\mathrm{CDCl}_{3}$ )

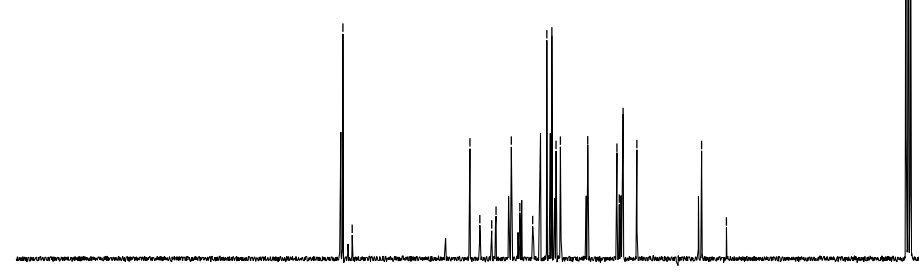

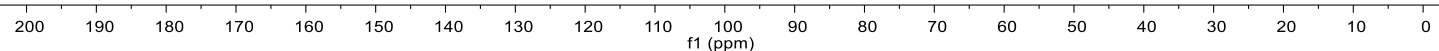

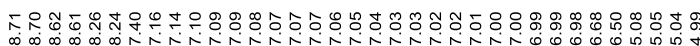

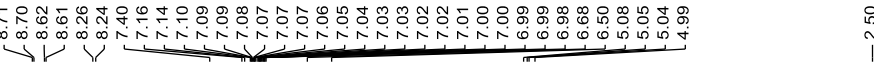

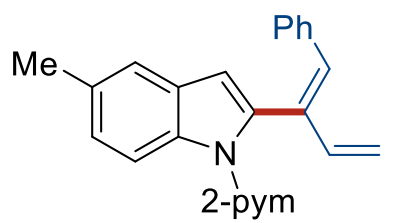

184ea

(400 $\mathrm{MHz}, \mathrm{CDCl}_{3}$ )

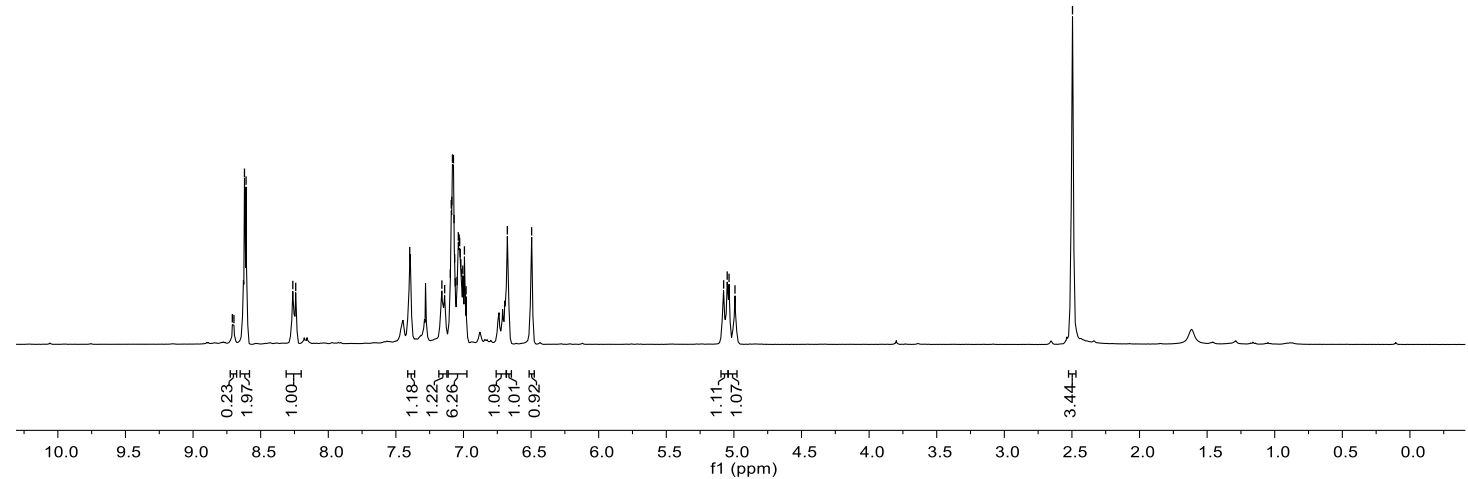


$V^{\infty}$

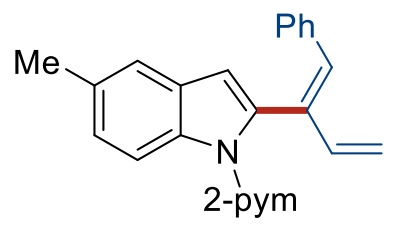

184ea

$\left(101 \mathrm{MHz}, \mathrm{CDCl}_{3}\right)$
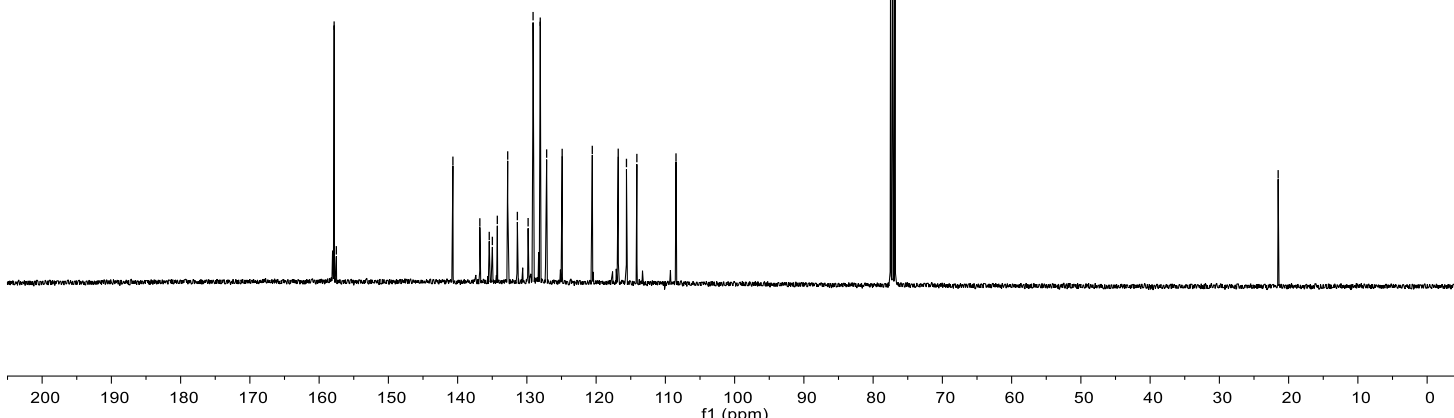

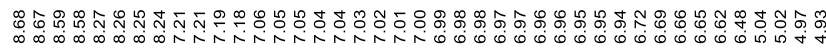

W

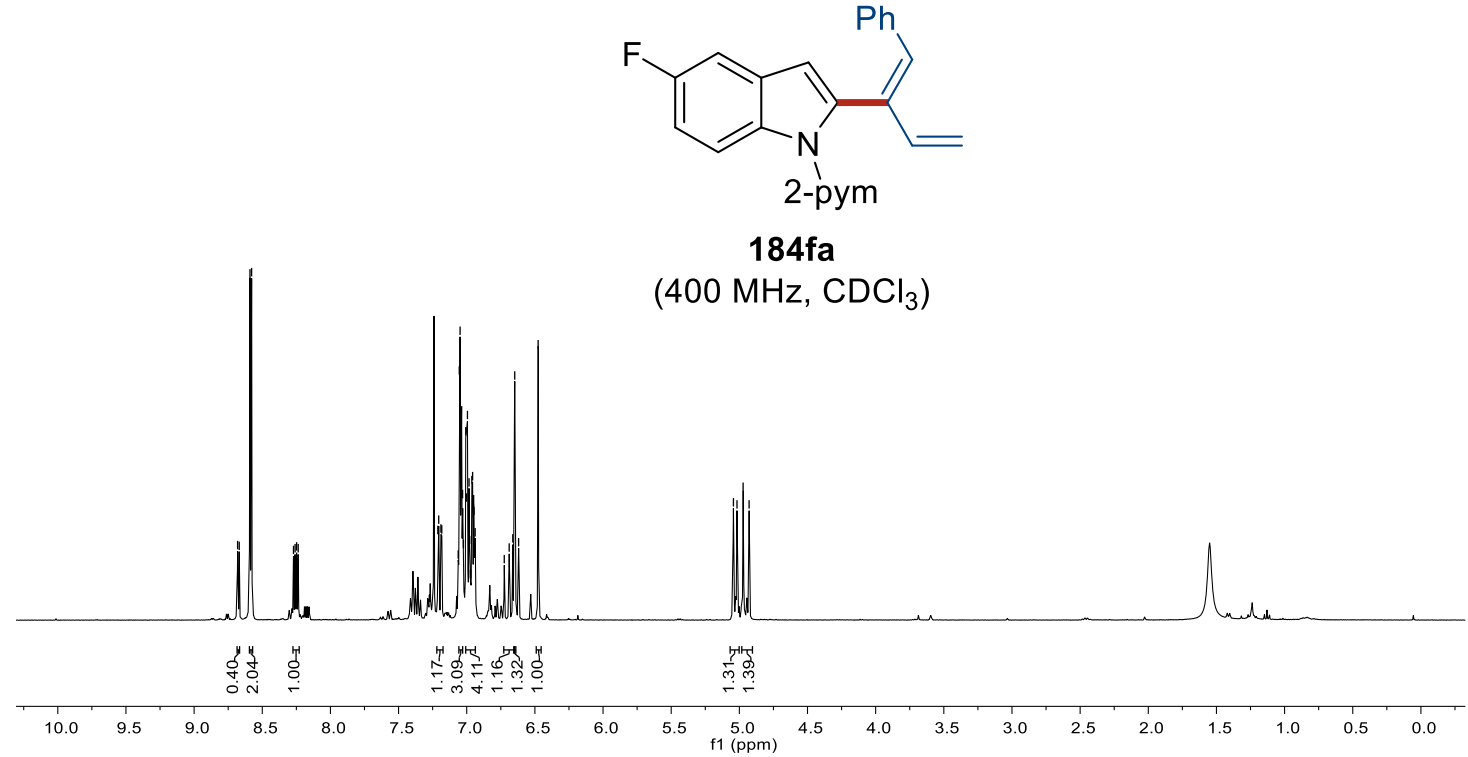



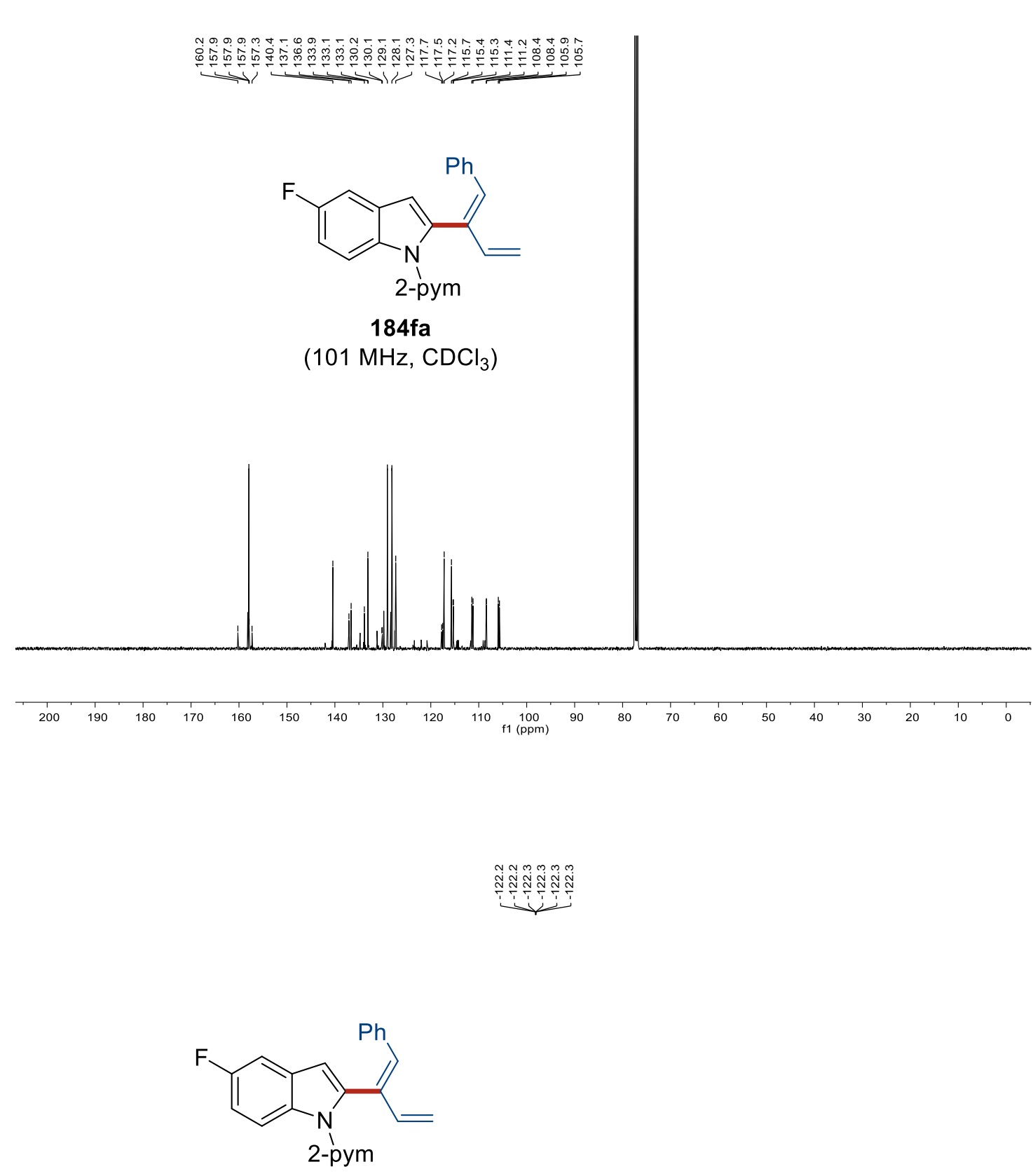

$184 \mathrm{fa}$

(377 MHz, $\mathrm{CDCl}_{3}$ )

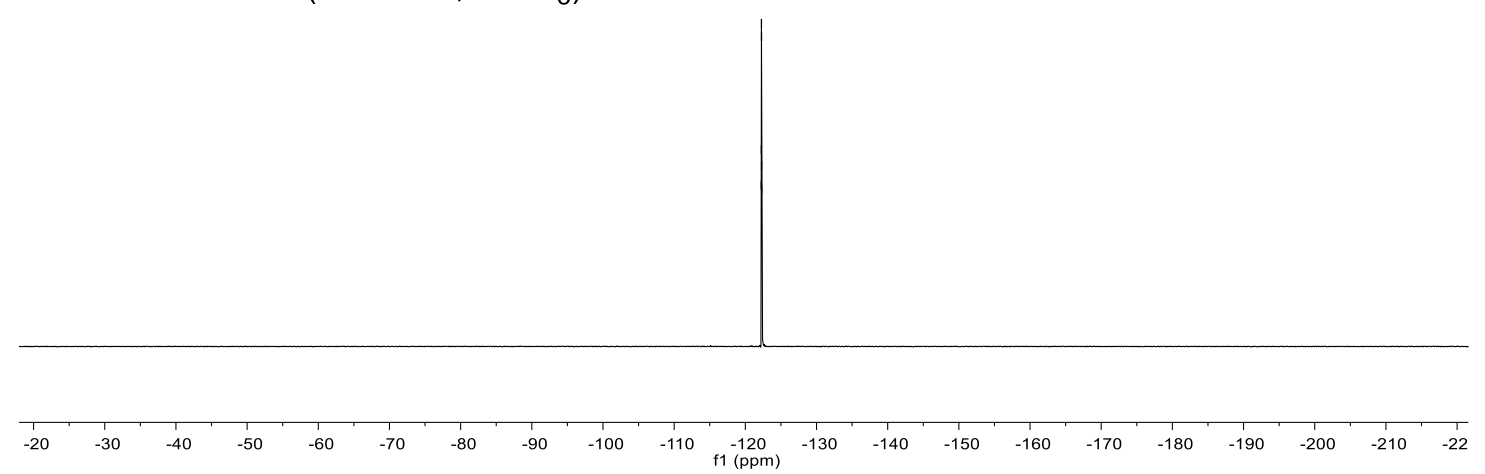




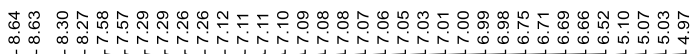

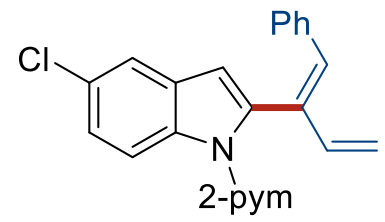

184ga

(300 MHz, CDCl 3 )

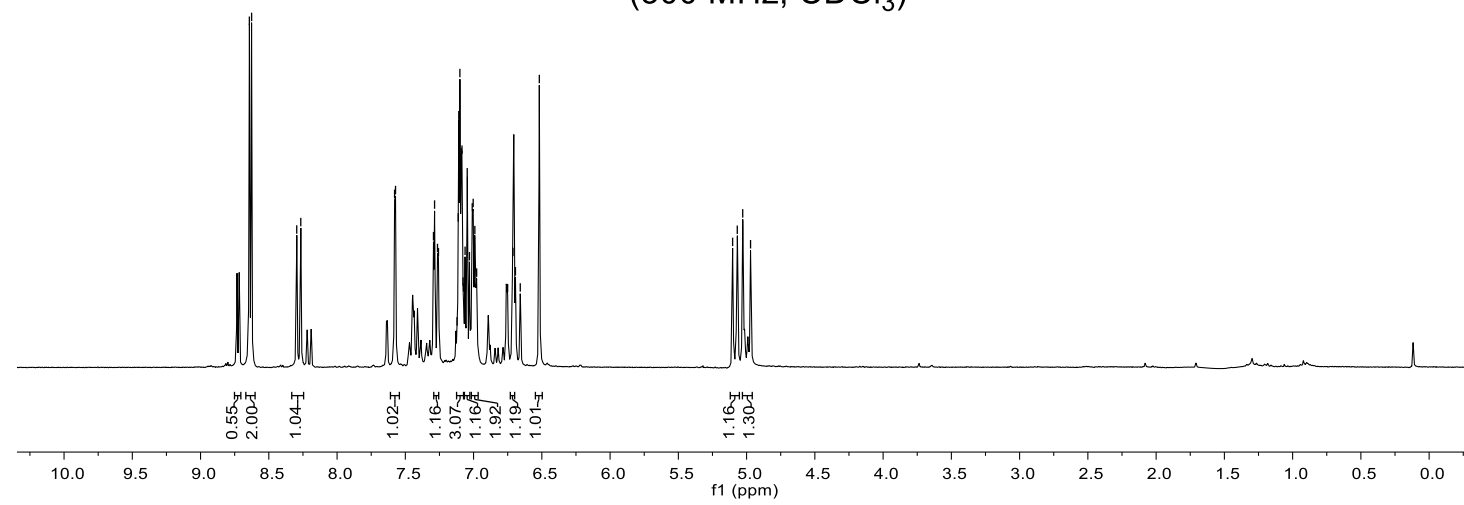

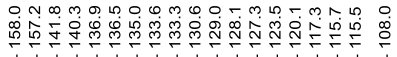

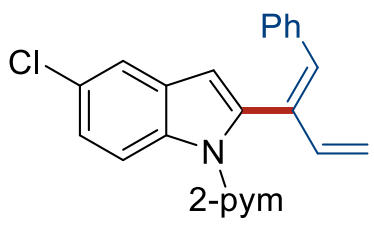

184ga

$\left(101 \mathrm{MHz}, \mathrm{CDCl}_{3}\right)$

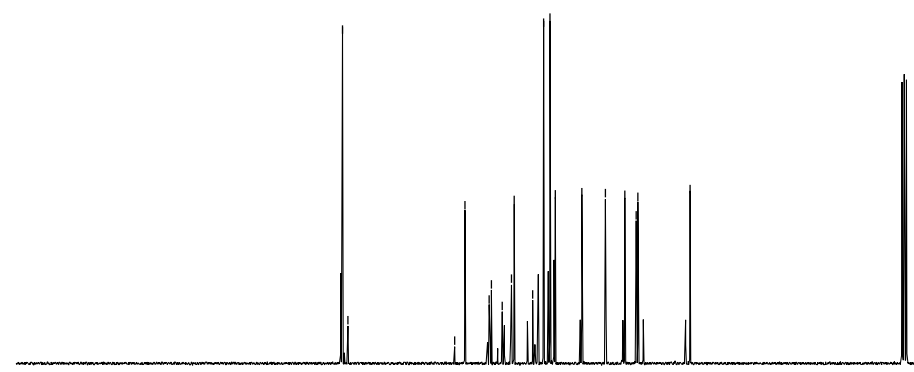

$\begin{array}{lllllllllllllllllllllllllll}1 & 190 & 180 & 170 & 160 & 150 & 140 & 130 & 120 & 110 & 100 & 90 & 80 & 70 & 60 & 50 & 40 & 30 & 20 & 10 & 0\end{array}$ 
$\underbrace{0}_{0}$

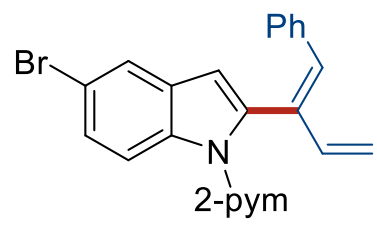

184ha

$\left(400 \mathrm{MHz} \mathrm{CDCl}_{3}\right)$

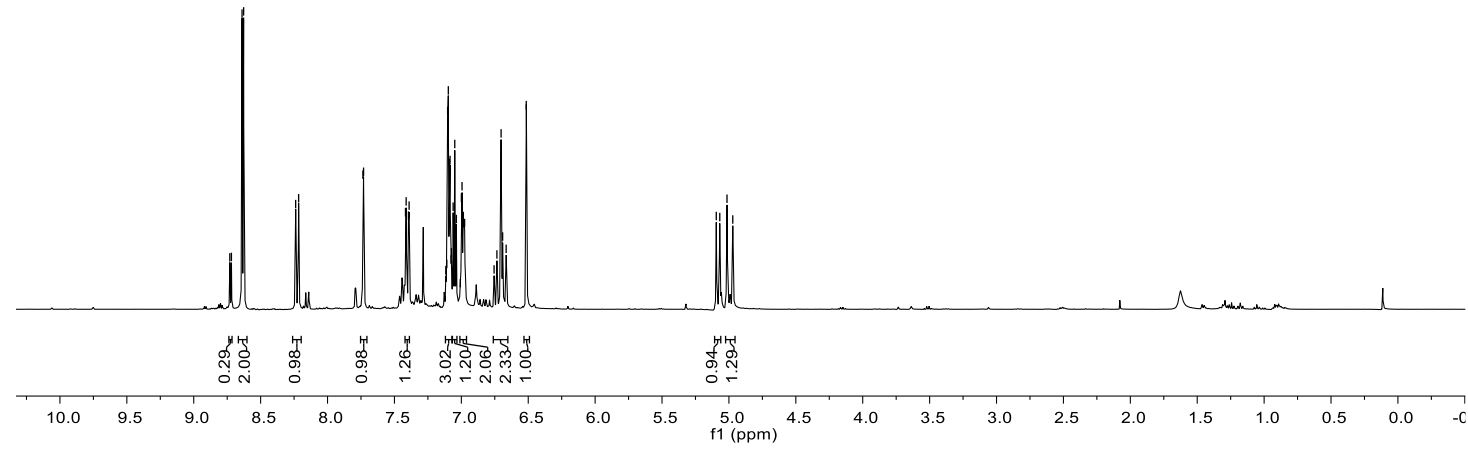

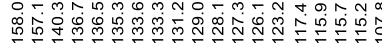

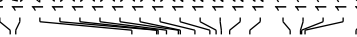

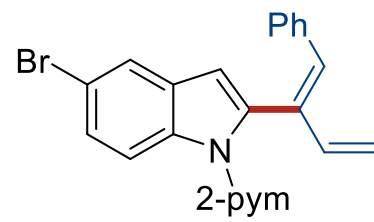

184ha

(101 $\mathrm{MHz}, \mathrm{CDCl}_{3}$ )

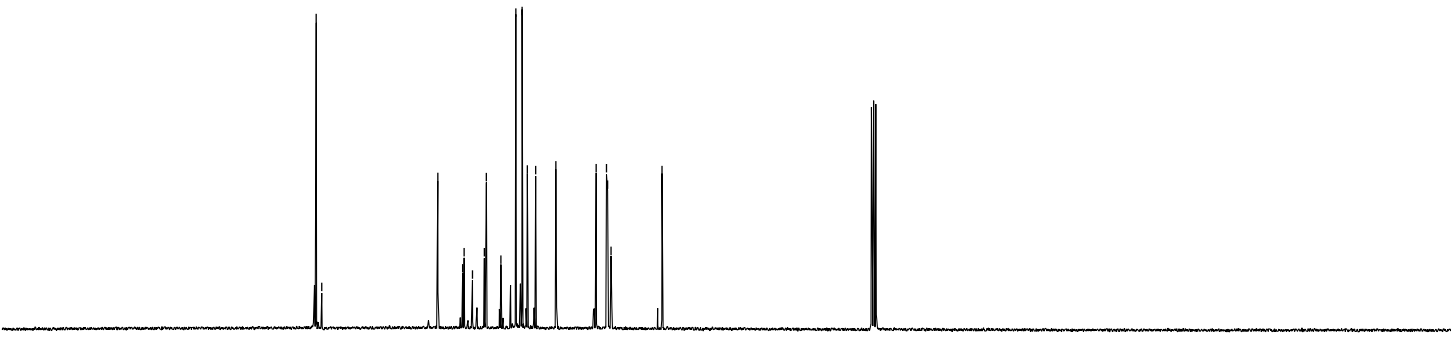

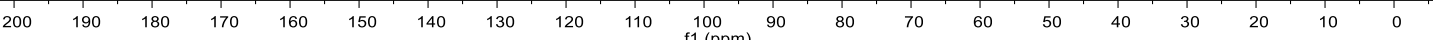




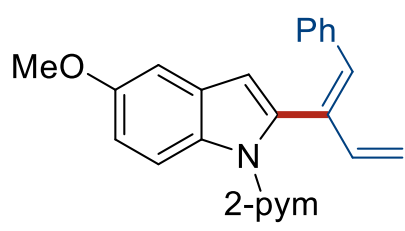

184ia

$\left(400 \mathrm{MHz} \mathrm{CDCl}_{3}\right)$

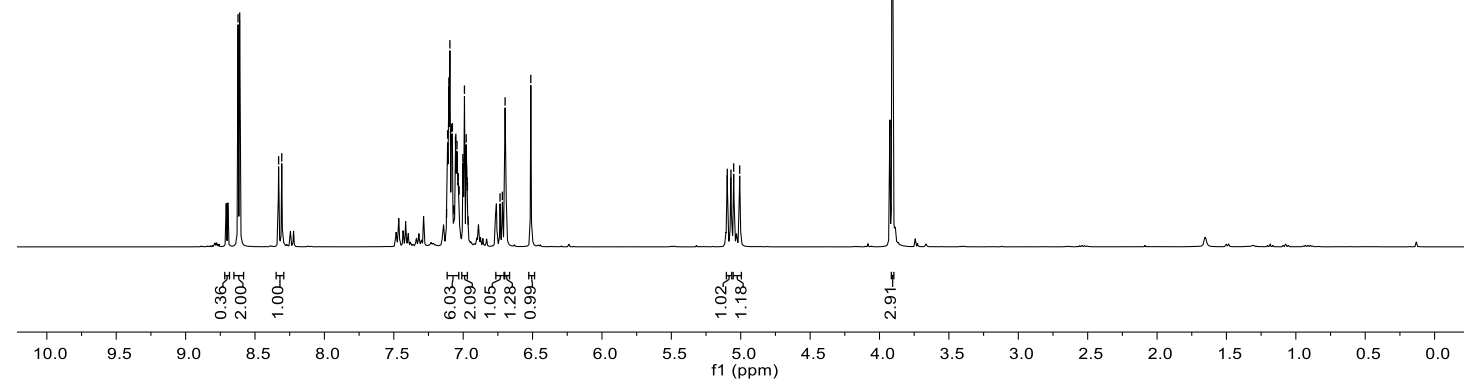

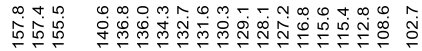

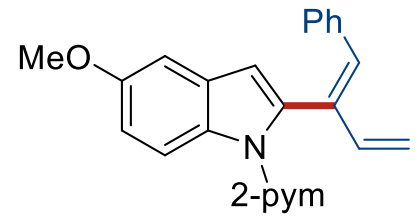

184ia

$\left(101 \mathrm{MHz}, \mathrm{CDCl}_{3}\right)$

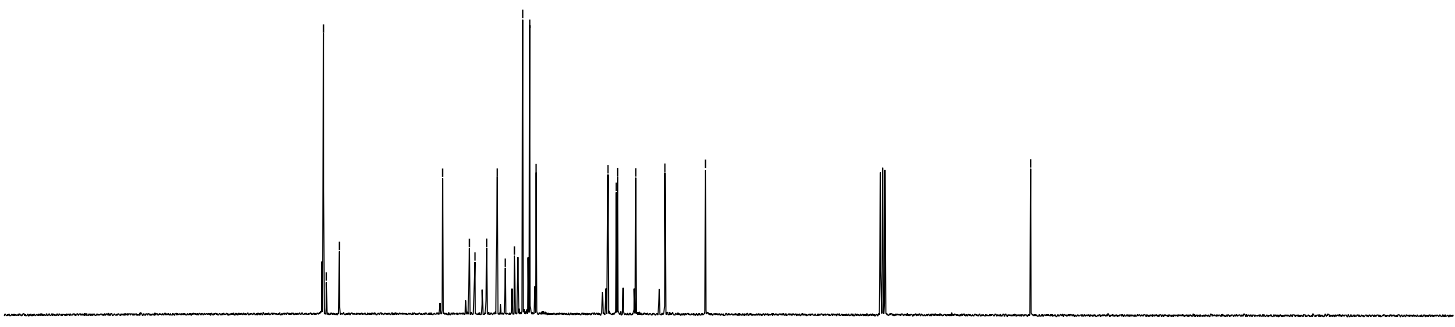

$\begin{array}{lllllllllllllllllllllllllllll}200 & 190 & 180 & 170 & 160 & 150 & 140 & 130 & 120 & 110 & 100 & 90 & 80 & 70 & 60 & 50 & 40 & 30 & 20 & 10 & 0\end{array}$ 


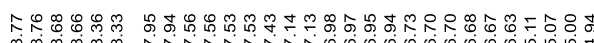

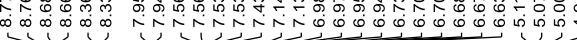

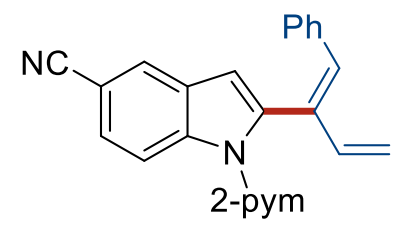

$184 \mathrm{ja}$

(300 MHz, $\mathrm{CDCl}_{3}$ )

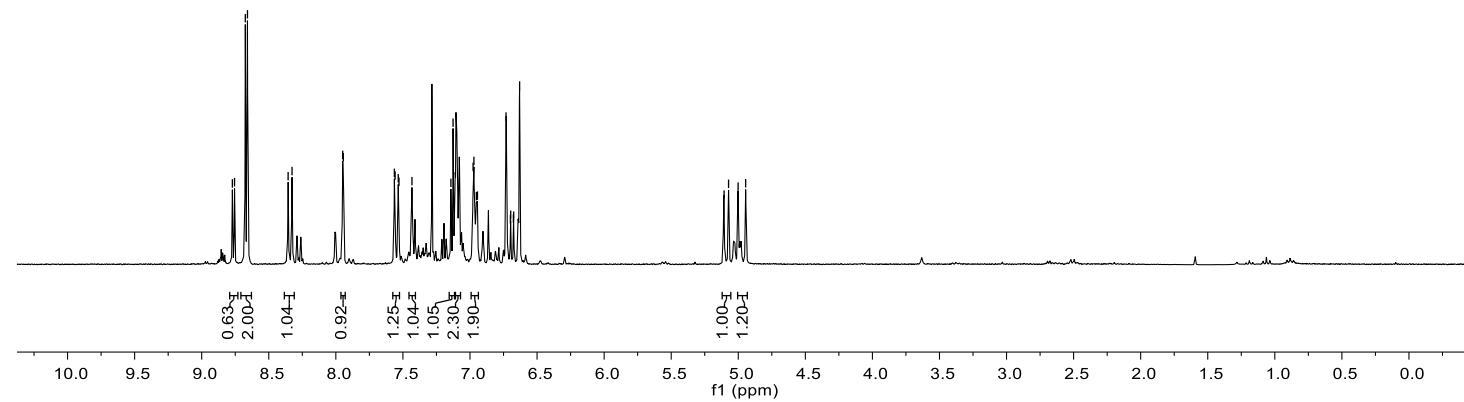

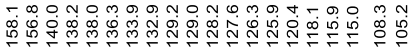

11

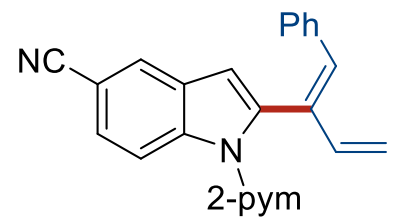

$184 \mathrm{ja}$

(101 $\mathrm{MHz} \mathrm{CDCl}_{3}$ )

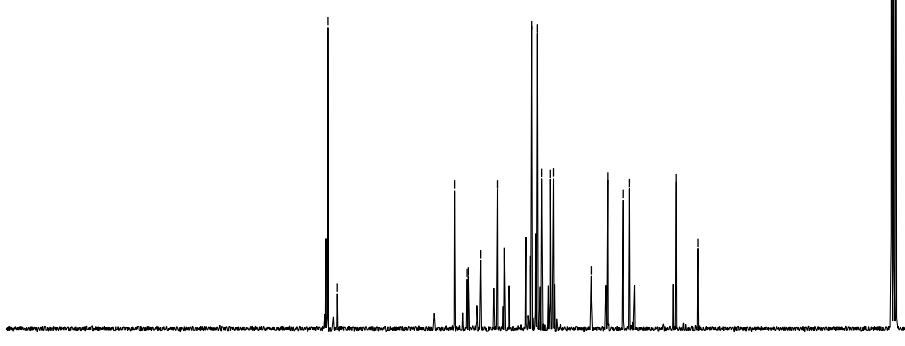

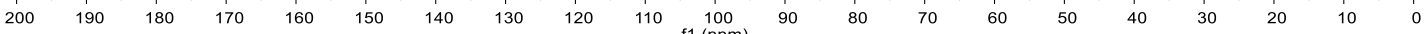




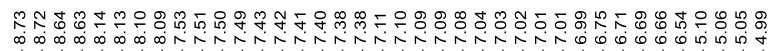

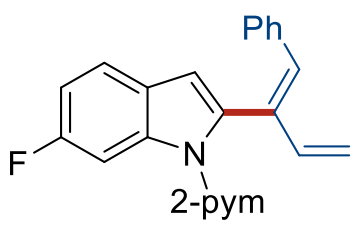

184ka

(300 MHz, $\mathrm{CDCl}_{3}$ )

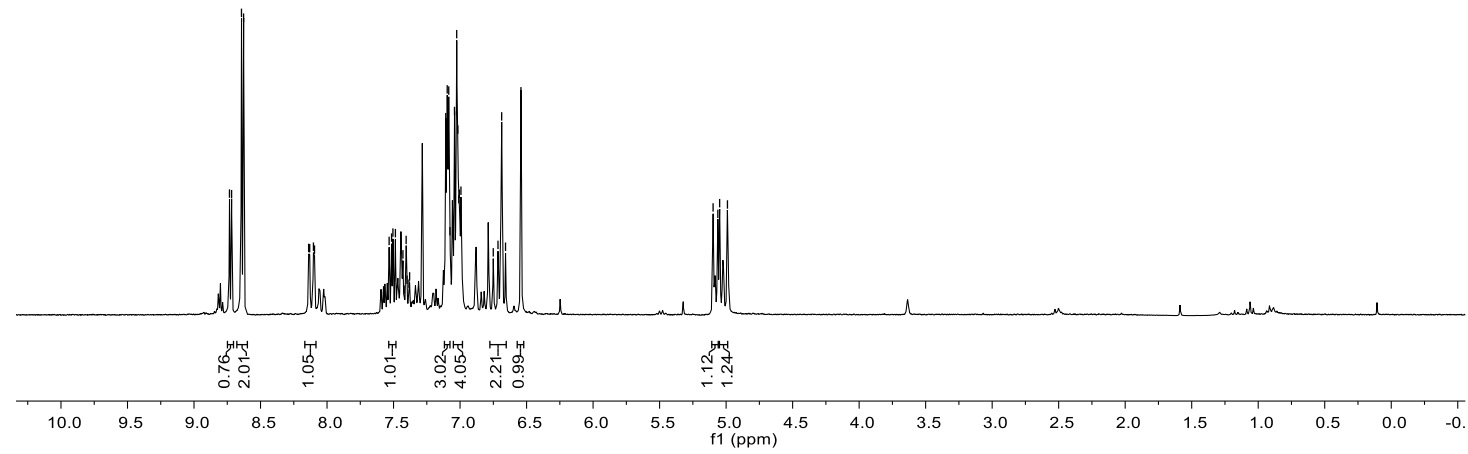

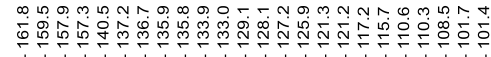

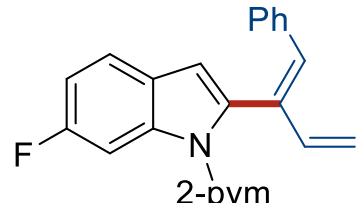

$184 \mathrm{ka}$

(101 MHz, $\mathrm{CDCl}_{3}$ )

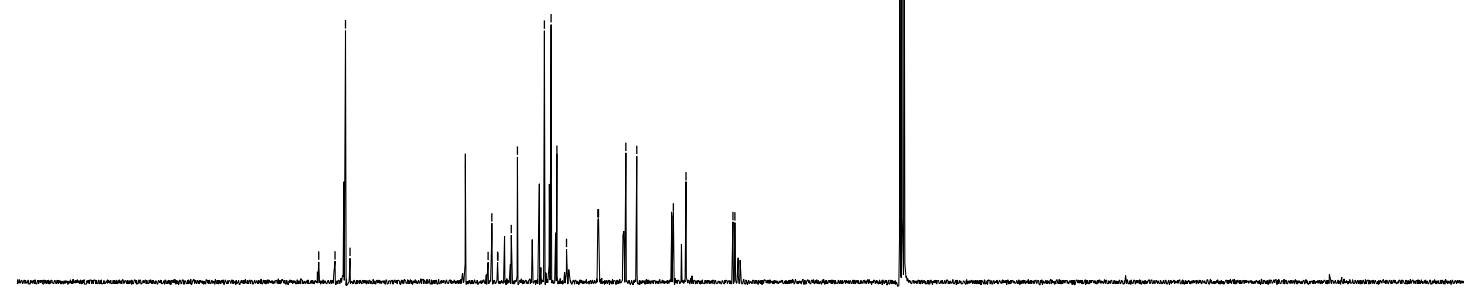

$\begin{array}{llllllllllllllllllllllllllll} & 1 & 190 & 180 & 170 & 160 & 150 & 140 & 130 & 120 & 110 & 100 & 90 & 80 & 70 & 60 & 50 & 40 & 30 & 20 & 10 & 0\end{array}$ 


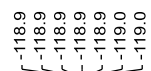

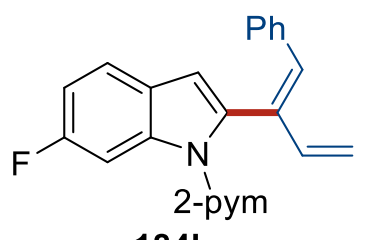

$184 \mathrm{ka}$

(377 MHz, $\left.\mathrm{CDCl}_{3}\right)$

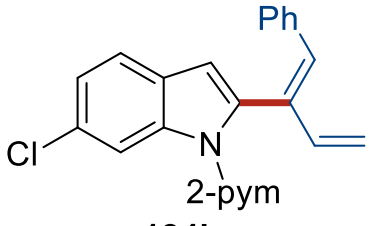

184la

$\left(400 \mathrm{MHz} \mathrm{CDCl}_{3}\right)$

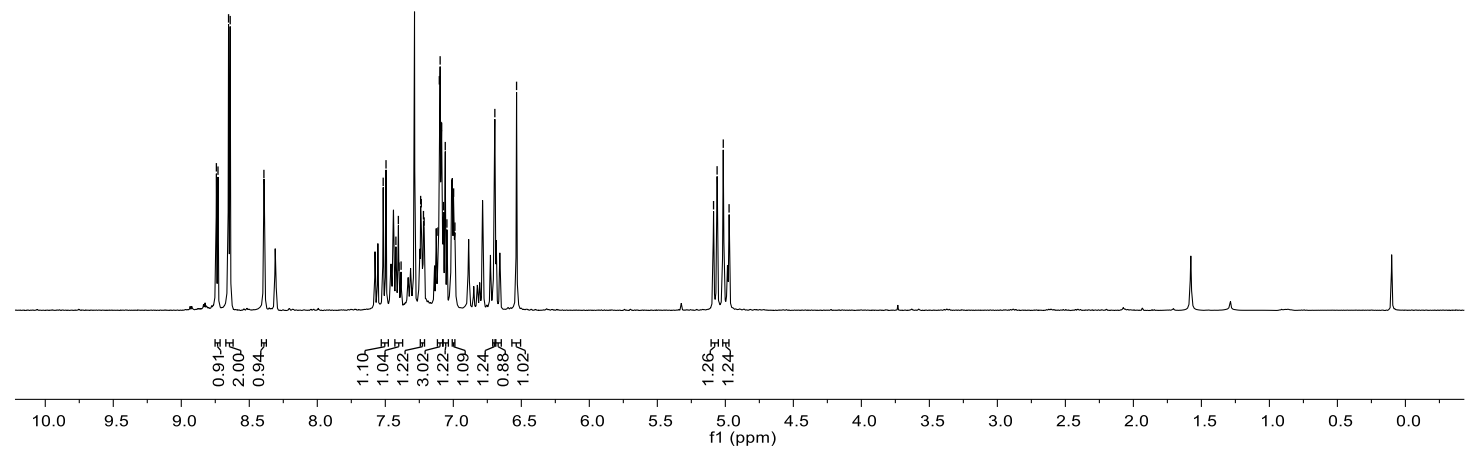




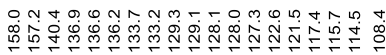

人)<smiles>C=C/C(=C/c1ccccc1)c1cc2ccc(Cl)cc2n1[Po+]</smiles>

184la

(101 MHz, $\mathrm{CDCl}_{3}$ )

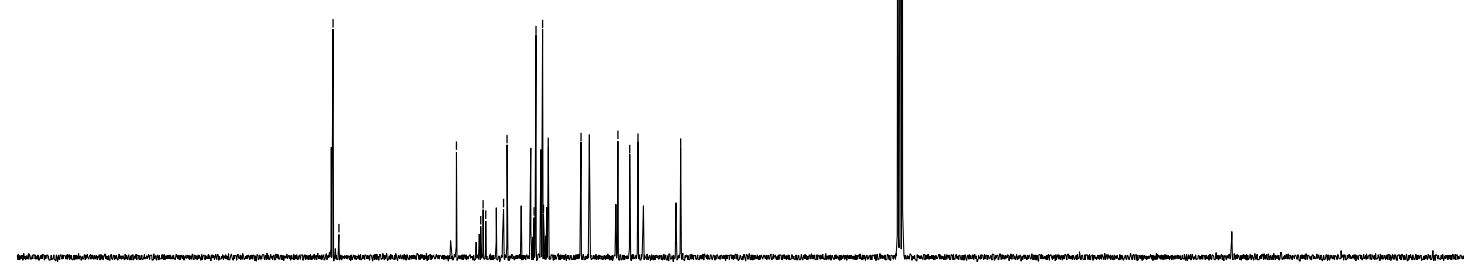

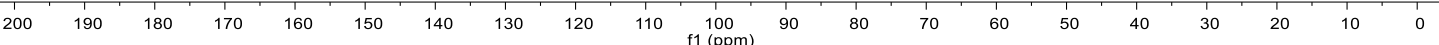

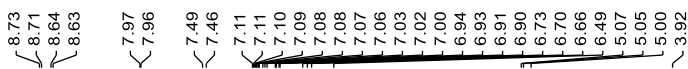

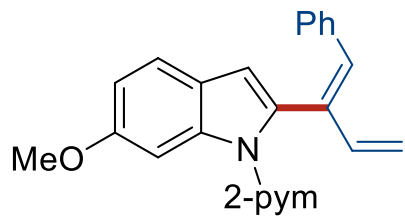

$184 \mathrm{ma}$

$\left(300 \mathrm{MHz}, \mathrm{CDCl}_{3}\right)$

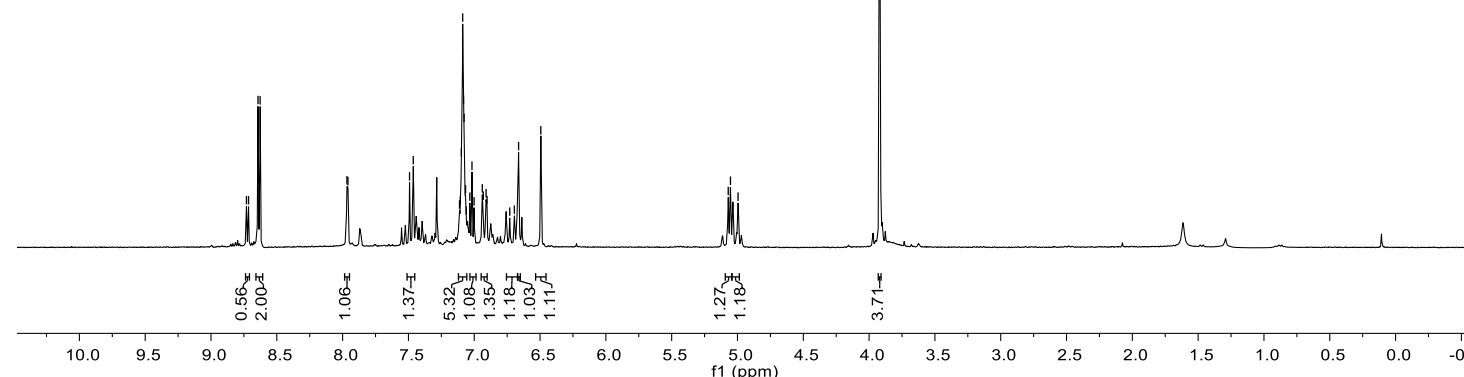



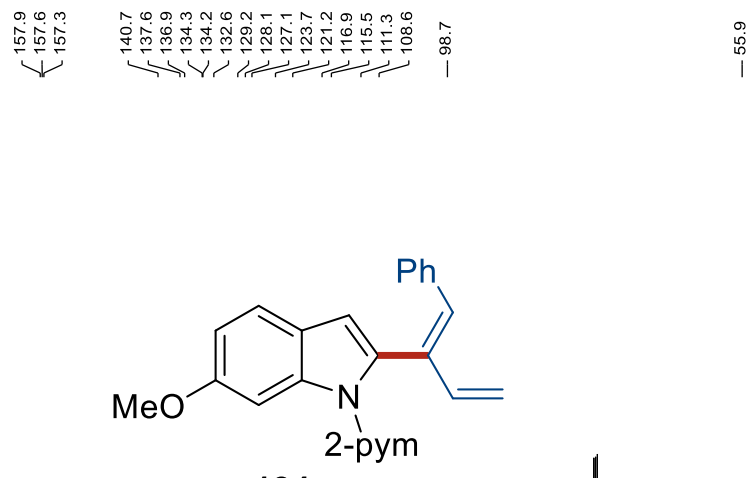

184ma

$\left(126 \mathrm{MHz}, \mathrm{CDCl}_{3}\right)$

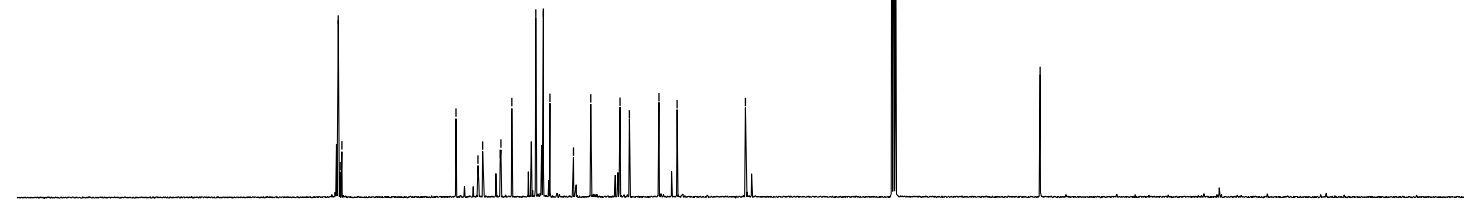

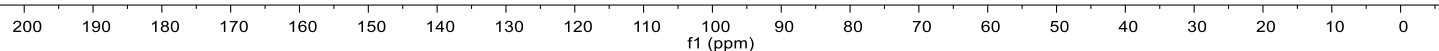

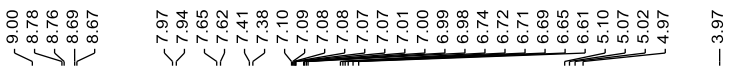

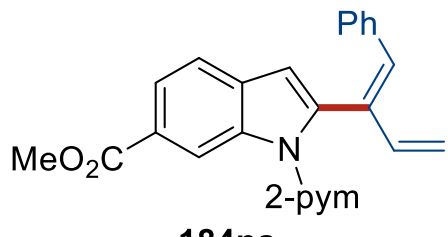

(300 MHz, $\mathrm{CDCl}_{3}$ )

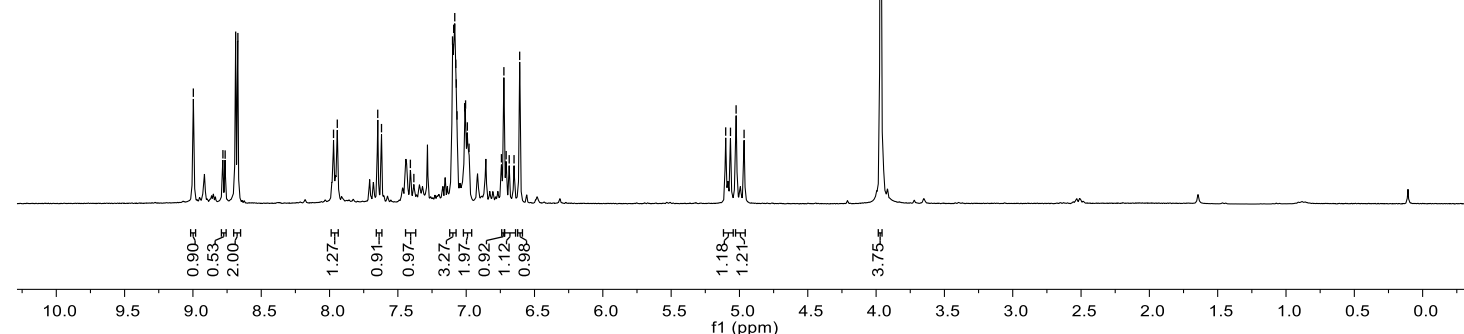


$\underbrace{1}$

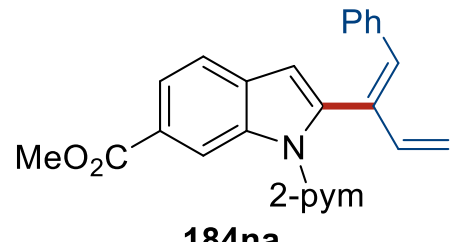

$\left(101 \mathrm{MHz}, \mathrm{CDCl}_{3}\right)$

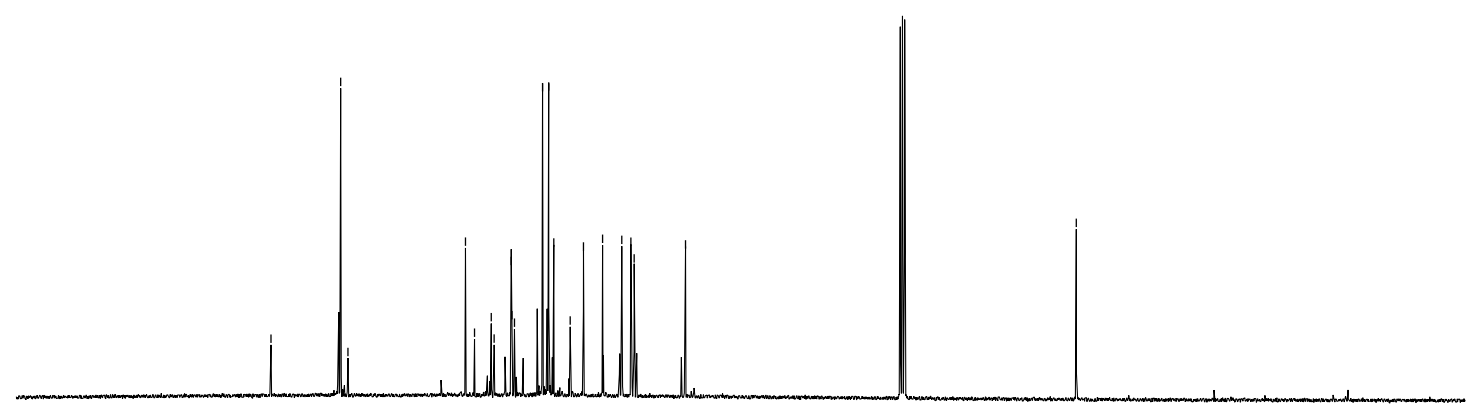

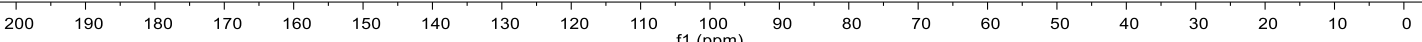

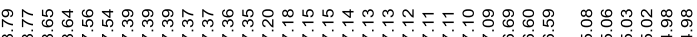

$\underbrace{\circ}$

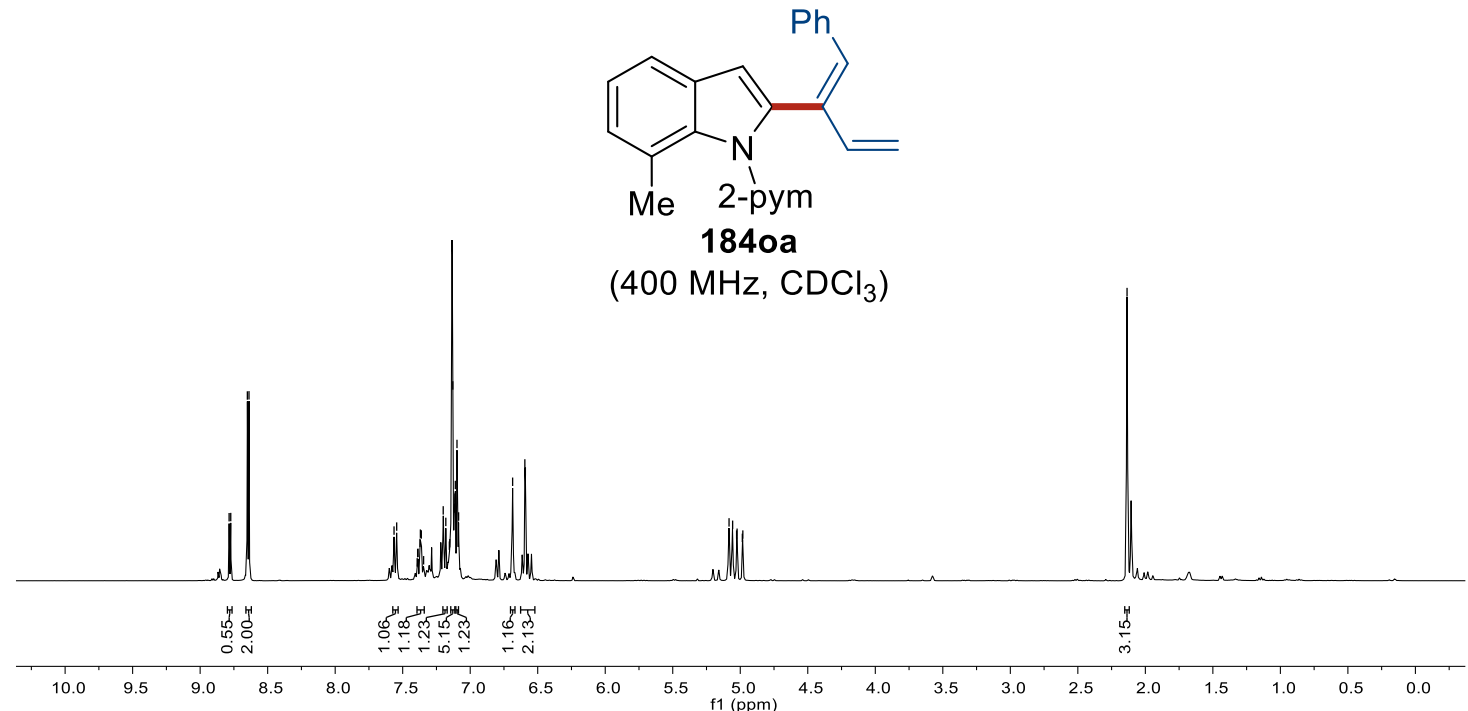




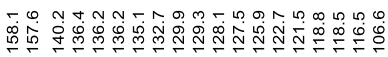

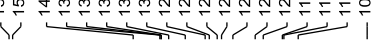
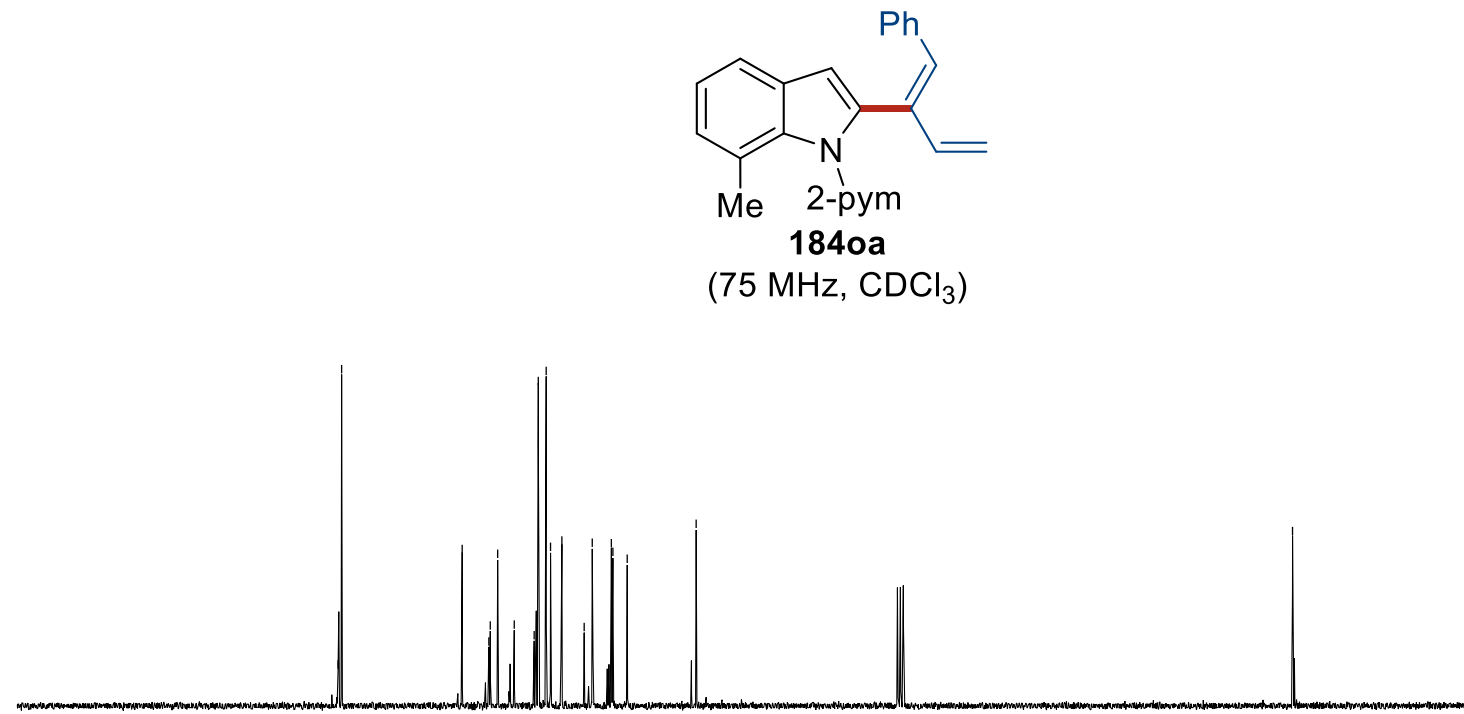

$\begin{array}{lllllllllllllllllllllll}1 & 190 & 190 & 180 & 170 & 160 & 150 & 140 & 130 & 120 & 110 & 100 & 10 & 80 & 70 & 60 & 50 & 40 & 30 & 20 & 10 & 0\end{array}$

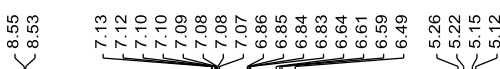

$\underbrace{\infty}$

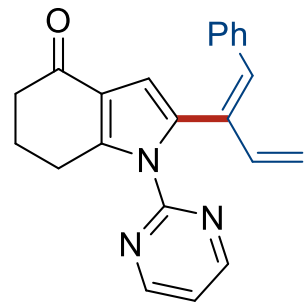

(Z)-3pa

(400 MHz, $\mathrm{CDCl}_{3}$ )

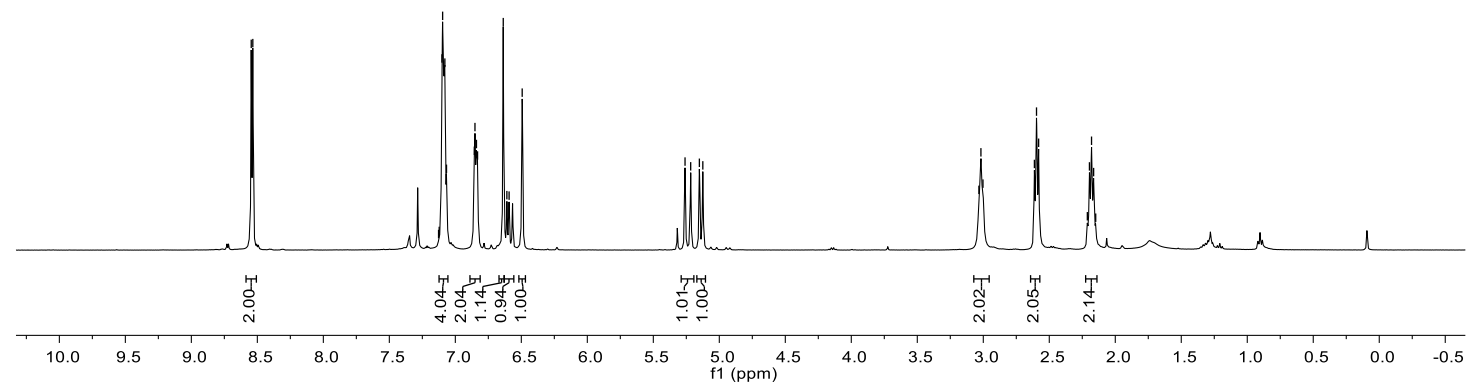




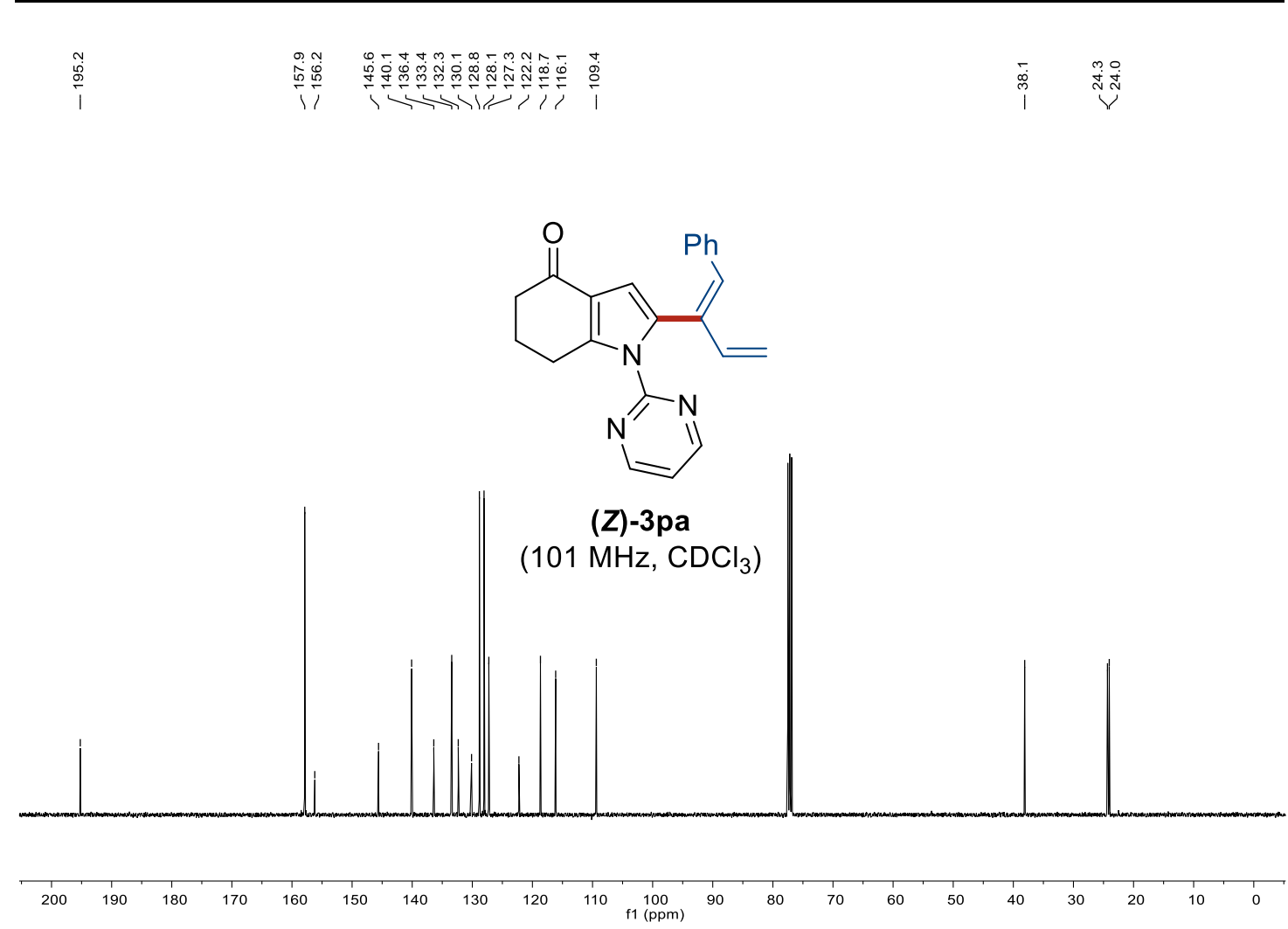

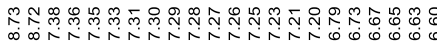

约

ํำ

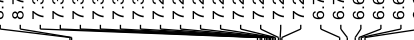

पi

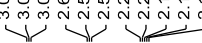

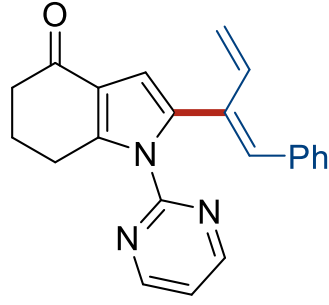

(E)-184pa

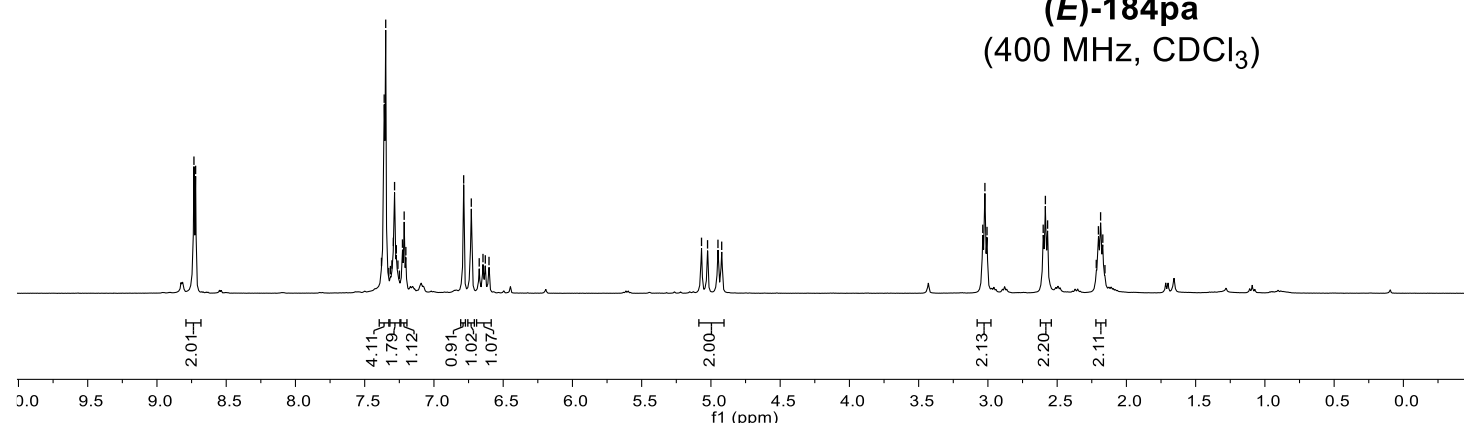




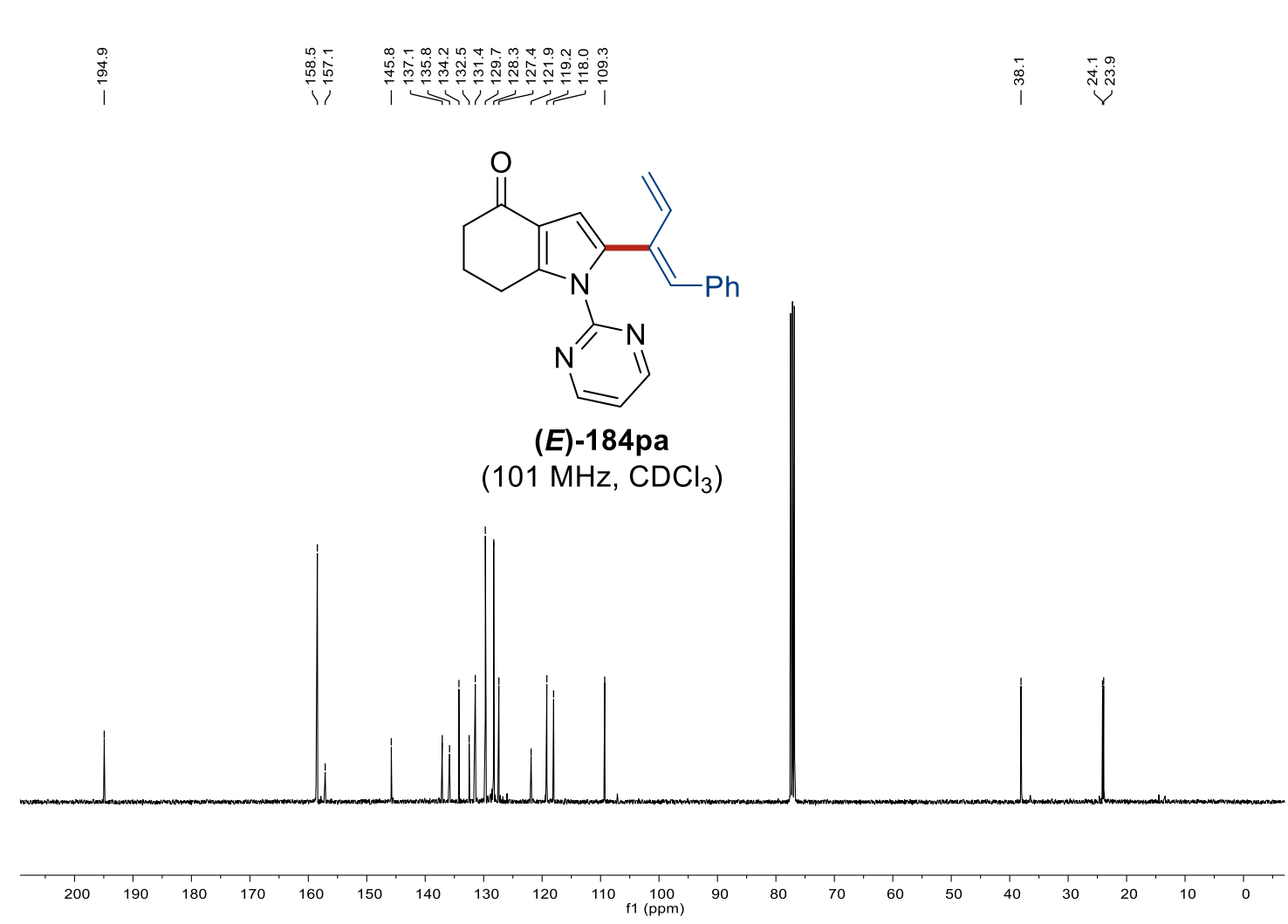

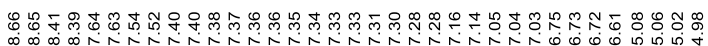

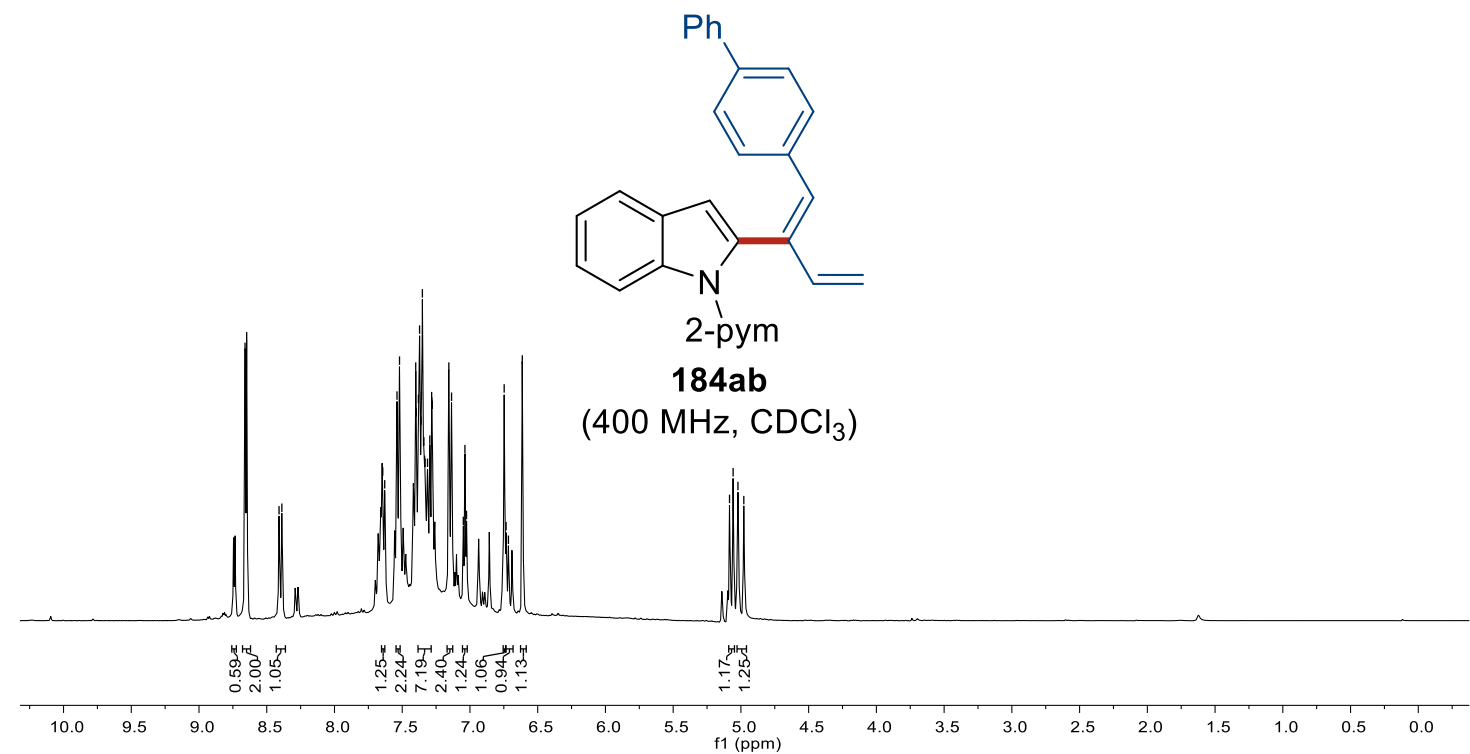




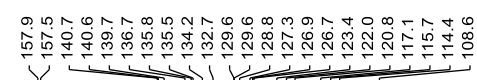

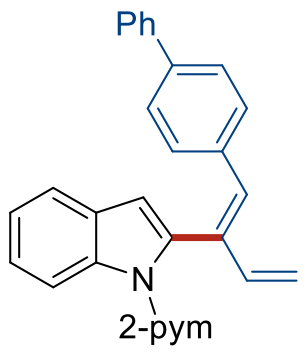

184ab

$\left(126 \mathrm{MHz}, \mathrm{CDCl}_{3}\right)$

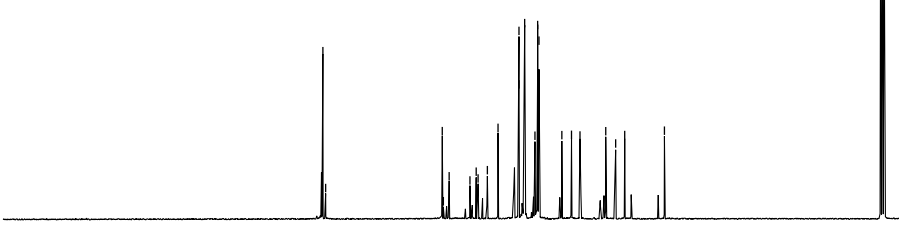

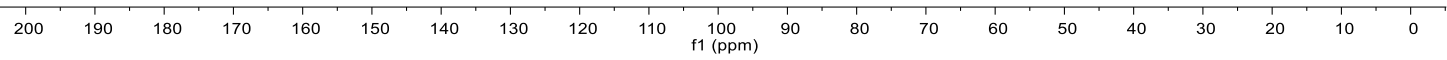

$\underbrace{1}$

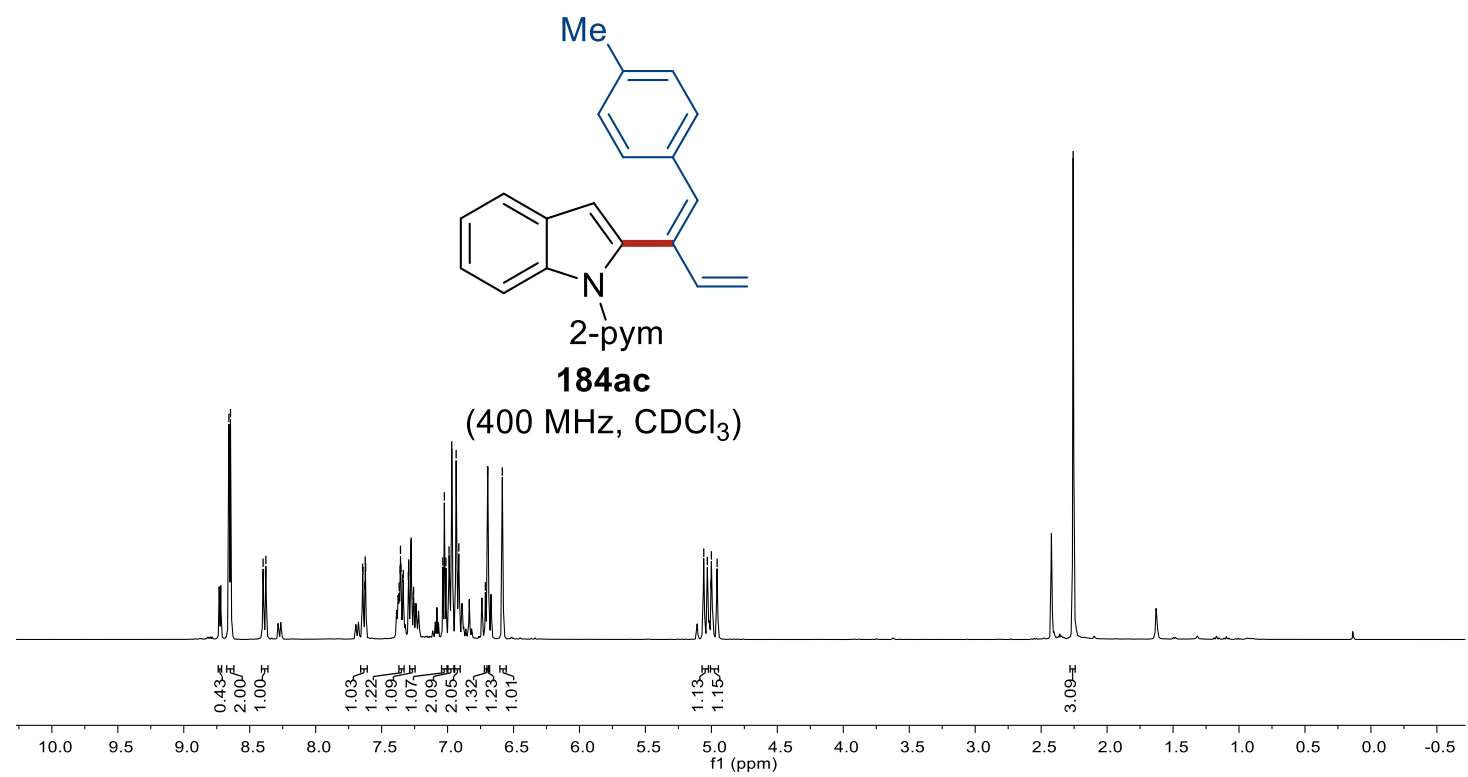




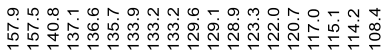

I.

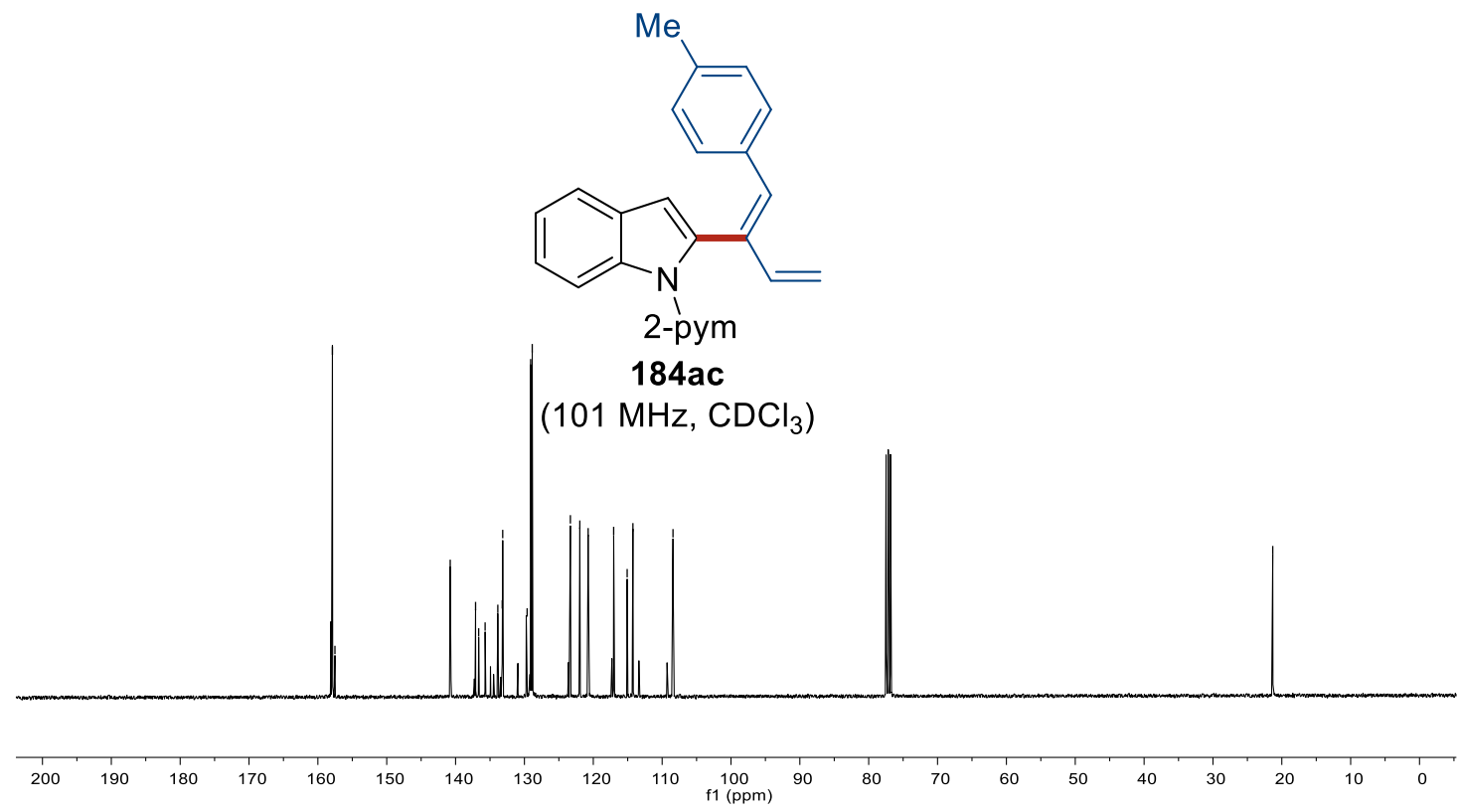

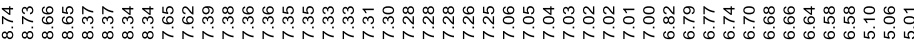

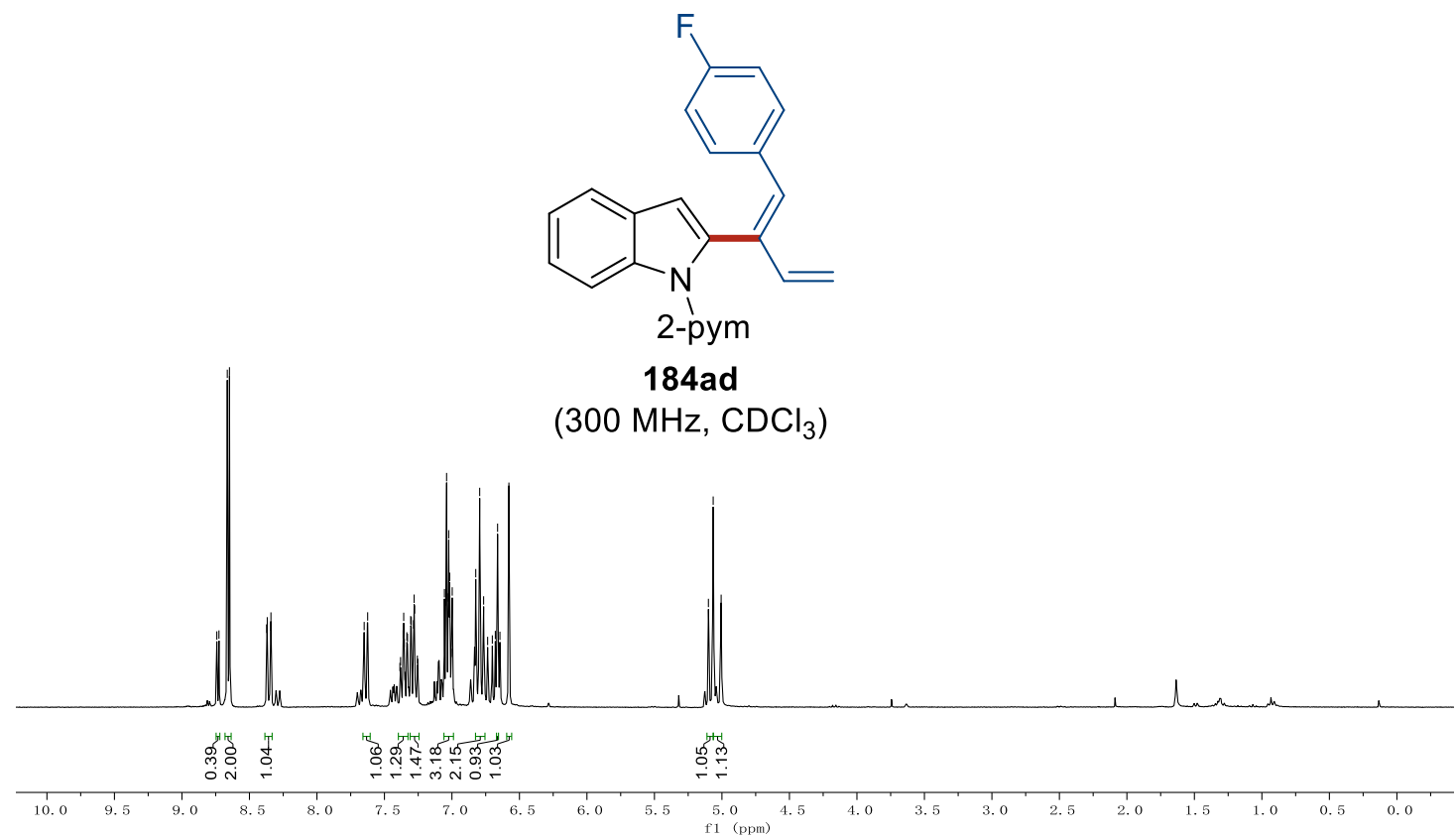




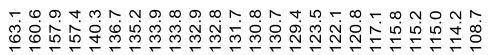

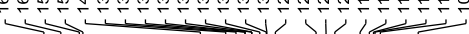

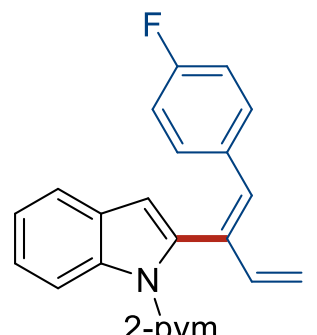

$184 a d$

$\left(101 \mathrm{MHz}^{\mathrm{CDCl}}{ }_{3}\right)$

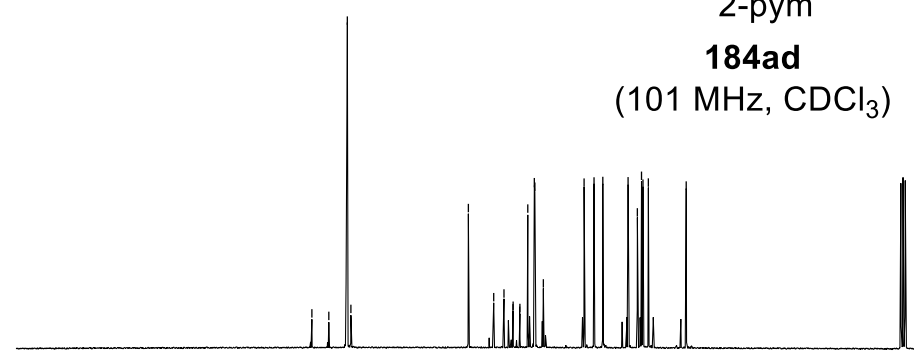

$\begin{array}{llllllllllllllllllllll} & 1 & 190 & 180 & 170 & 160 & 150 & 140 & 130 & 120 & 110 & \begin{array}{c}100 \\ \mathrm{f} 1(\mathrm{ppm})\end{array} & 90 & 80 & 70 & 60 & 50 & 40 & 30 & 20 & 10 & 0\end{array}$

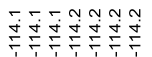

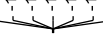

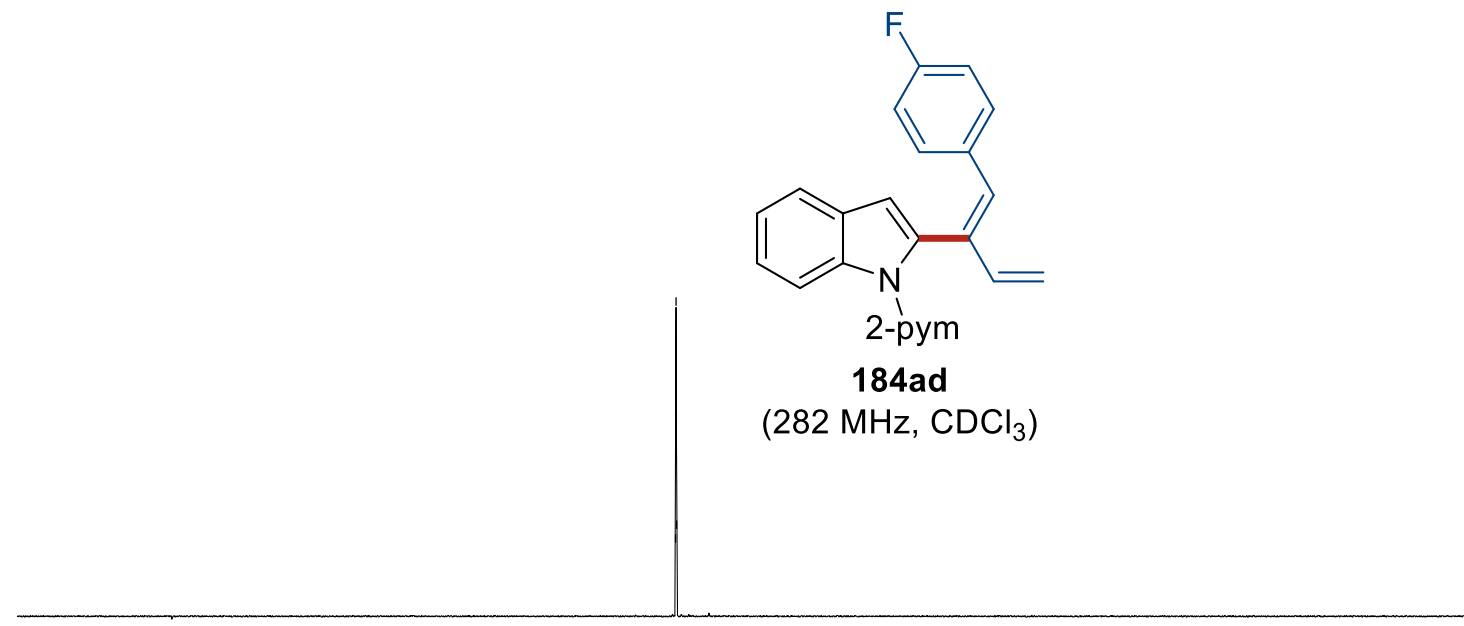

$\begin{array}{lllllllllllllllllllllllllllllllllllll}-50 & -55 & -60 & -65 & -70 & -75 & -80 & -85 & -90 & -95 & -100 & -105 & -110 & -115 & -120 & -125 & -130 & -135 & -140 & -145 & -150 & -155 & -160 & -165 & -170 & -175 & -180 & -185 & -190\end{array}$ 


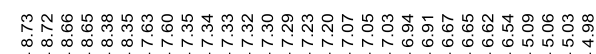

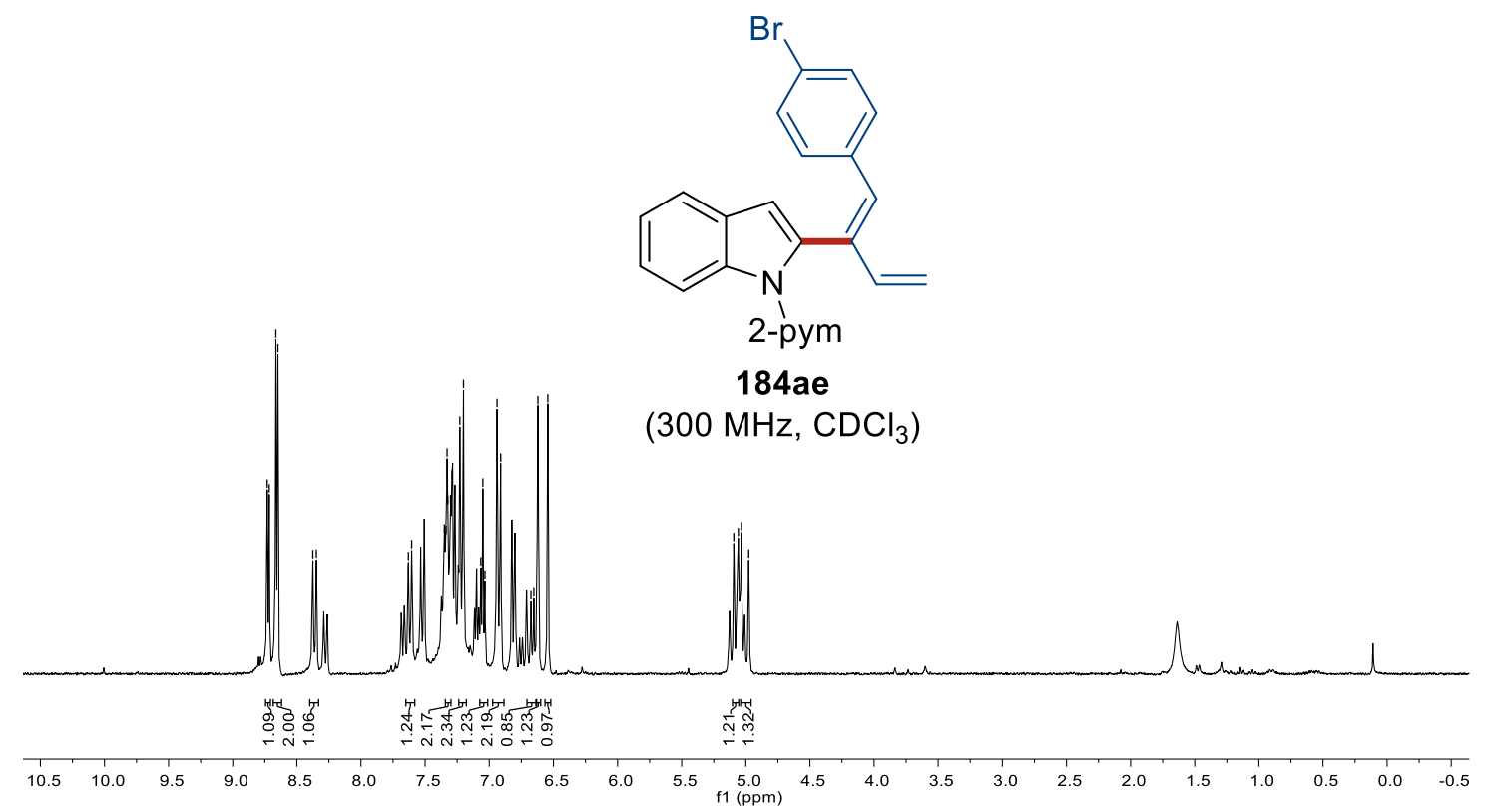

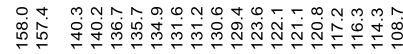

$\checkmark$ या

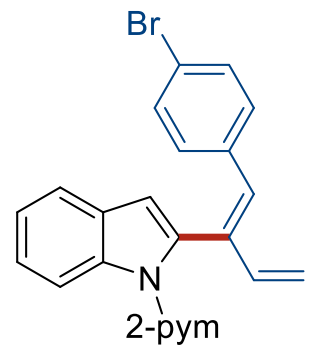

184ae

(126 MHz, $\mathrm{CDCl}_{3}$ )

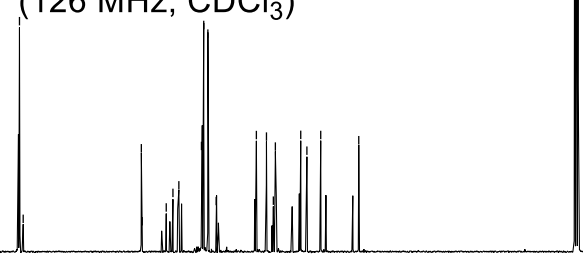

$\begin{array}{lllllllllllllllllllllllll} & 200 & 190 & 180 & 170 & 160 & 150 & 140 & 130 & 120 & 110 & 100 & 90 & 80 & 70 & 60 & 50 & 40 & 30 & 20 & 10 & 0\end{array}$ 
$\underbrace{-10}$

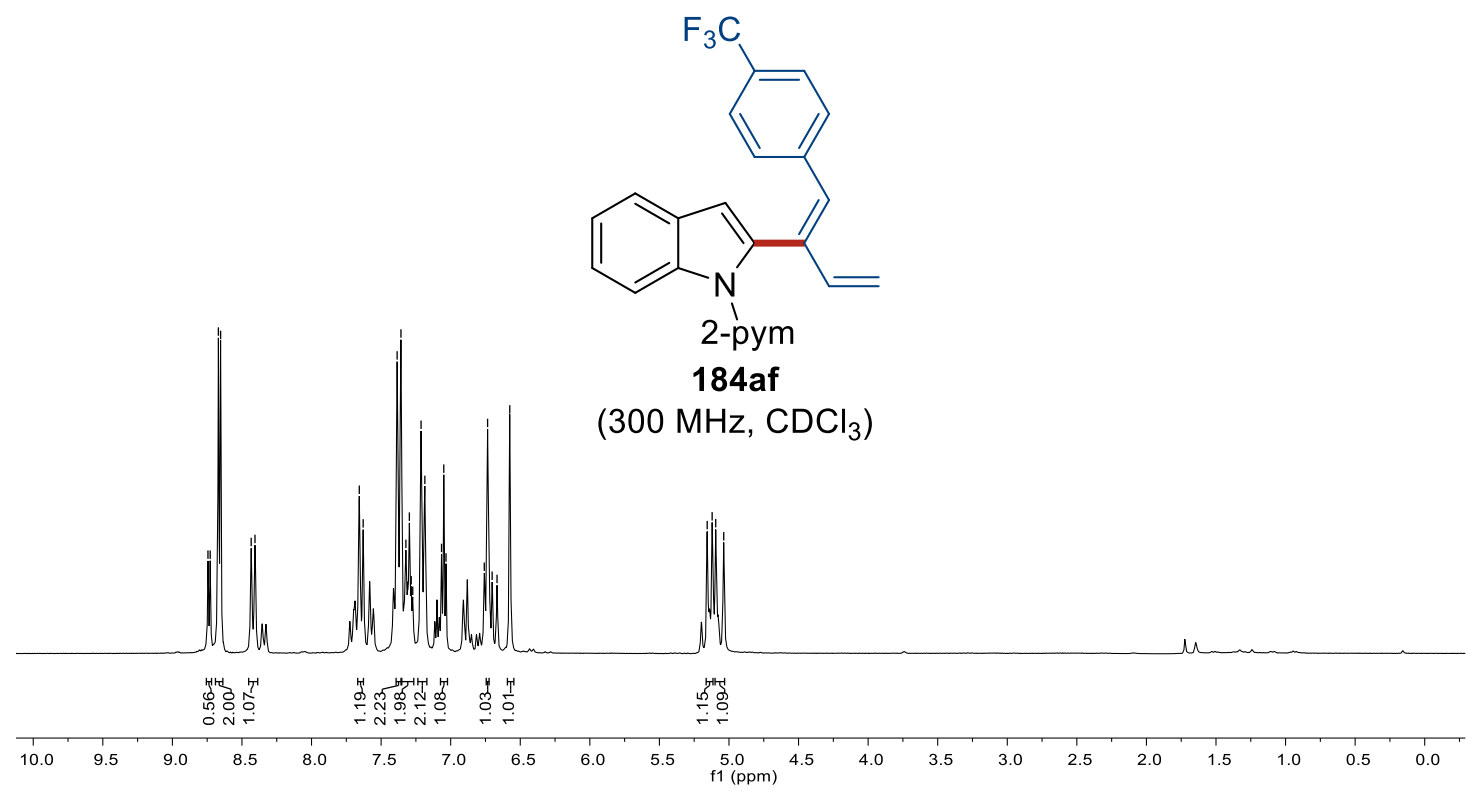

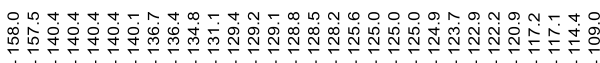

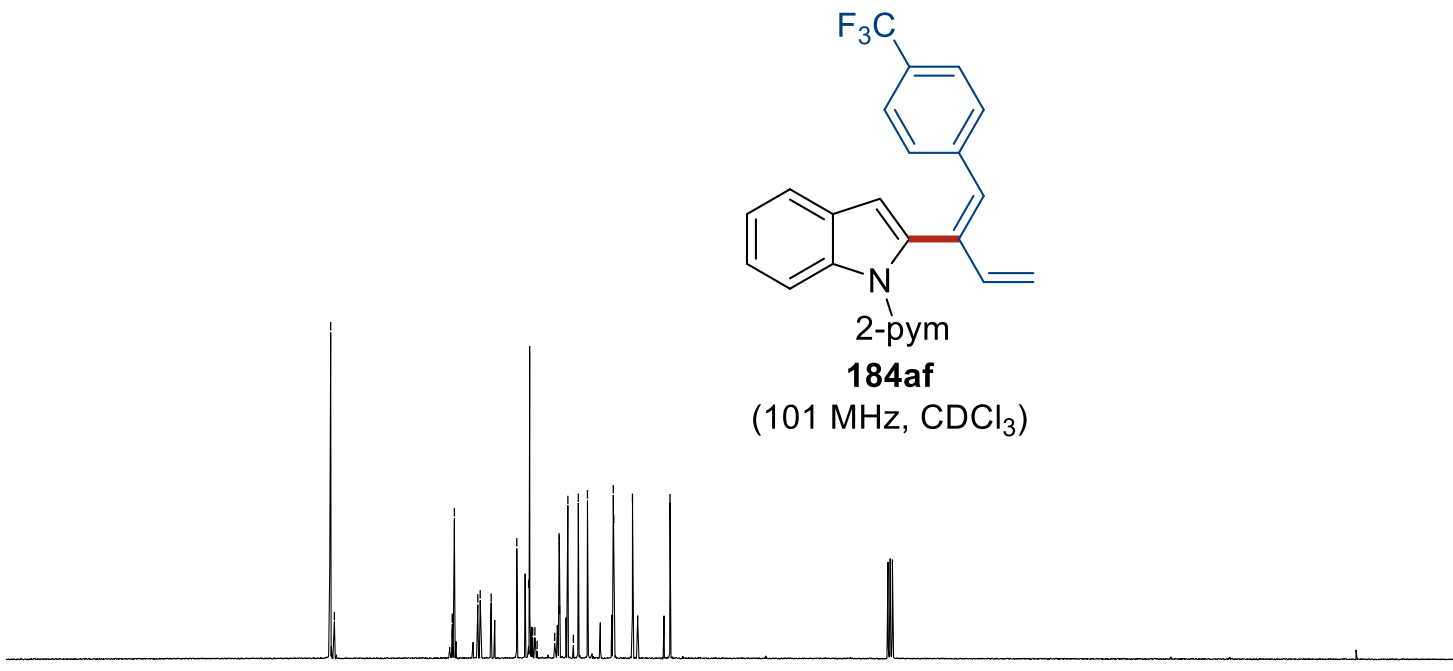

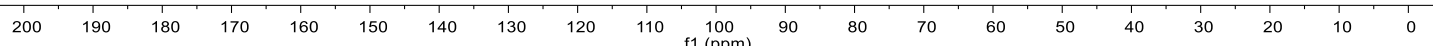




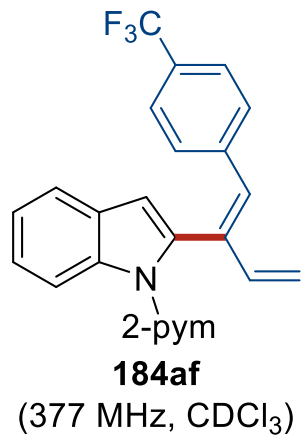

(377 MHz, $\mathrm{CDCl}_{3}$ )

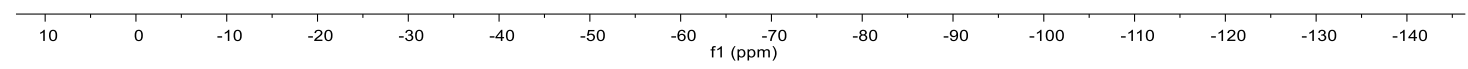

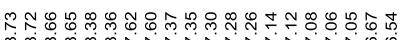

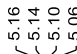

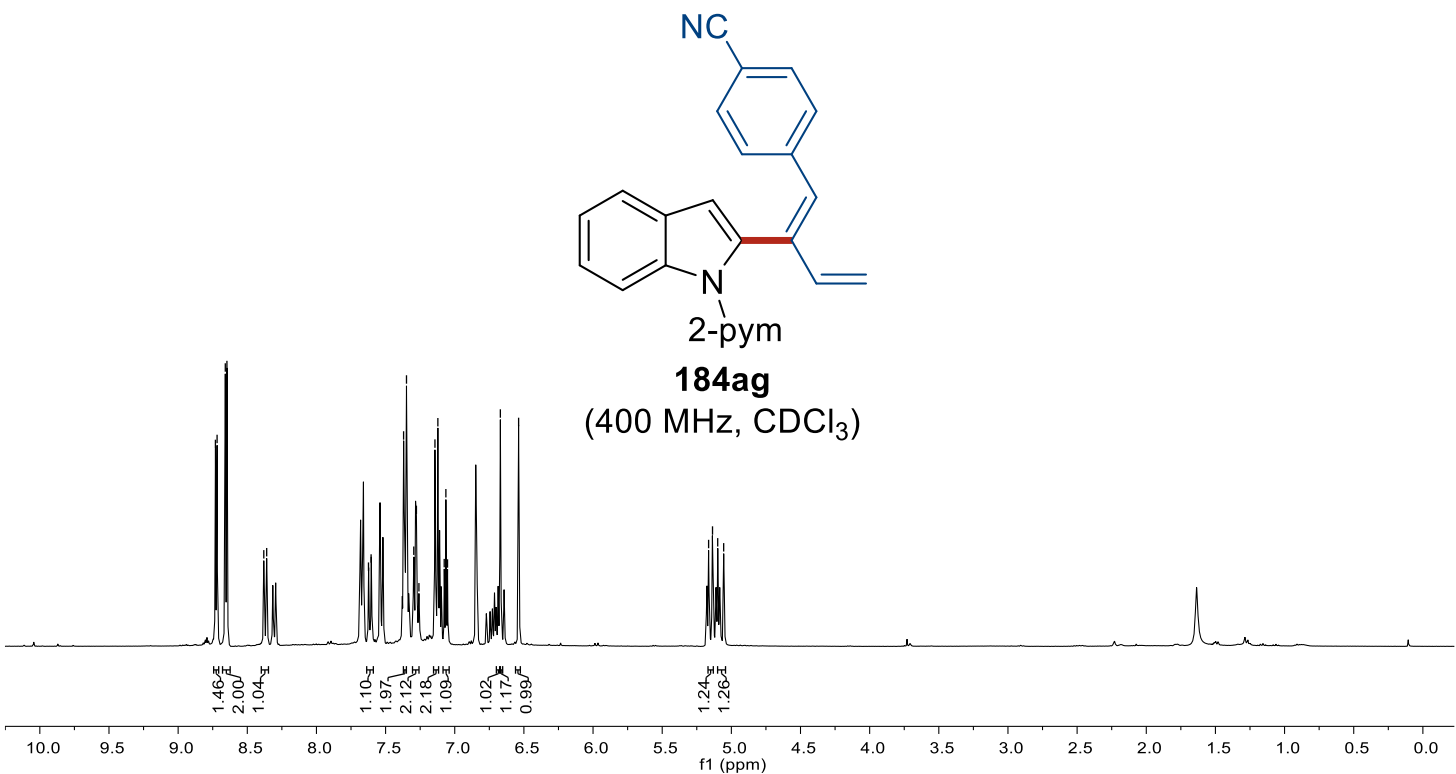

2-pym

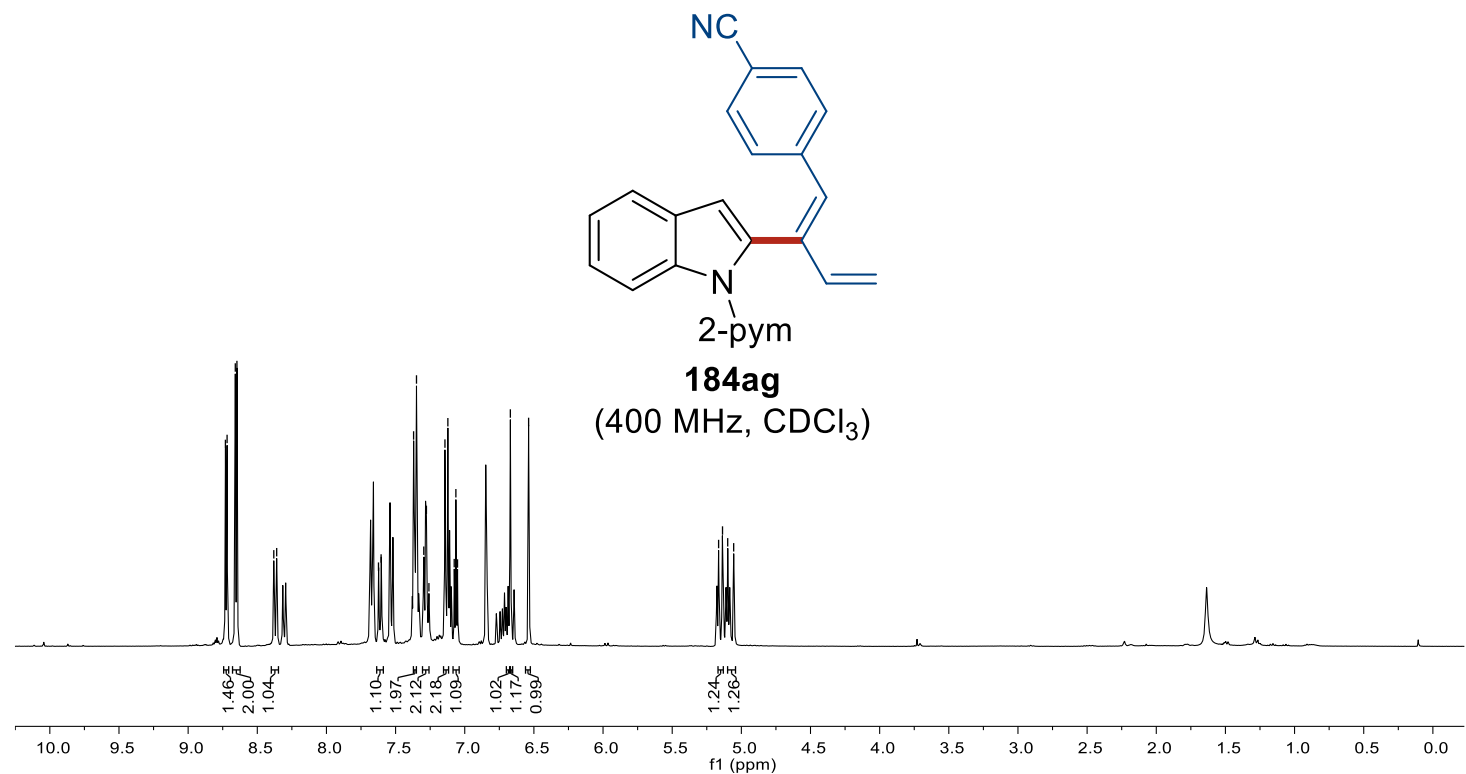




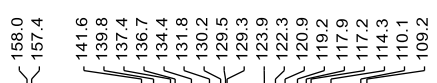

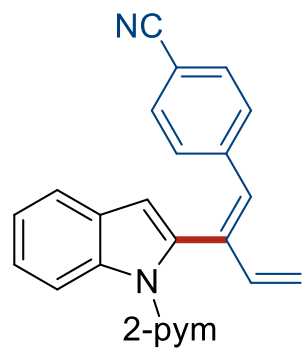

184ag

$\left(101 \mathrm{MHz}, \mathrm{CDCl}_{3}\right)$

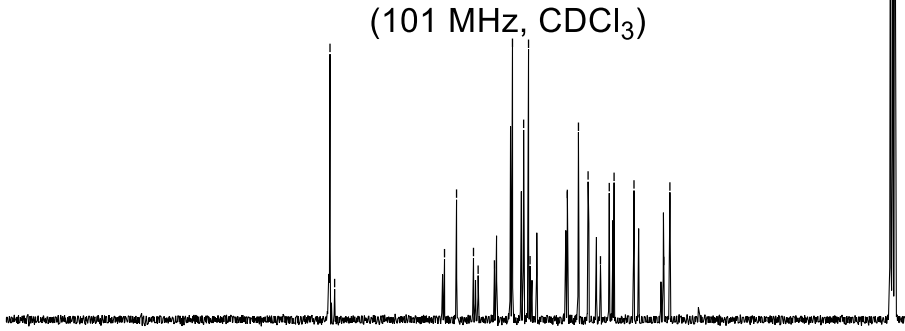

$\begin{array}{lllllllllllllllllllll}200 & 190 & 180 & 170 & 160 & 150 & 140 & 130 & 120 & 110 & \begin{array}{c}100 \\ \mathrm{f} 1(\mathrm{ppm})\end{array} & 90 & 80 & 70 & 60 & 50 & 40 & 30 & 20 & 10 & 0\end{array}$

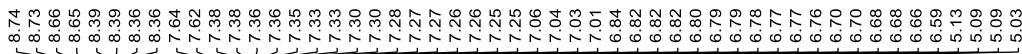

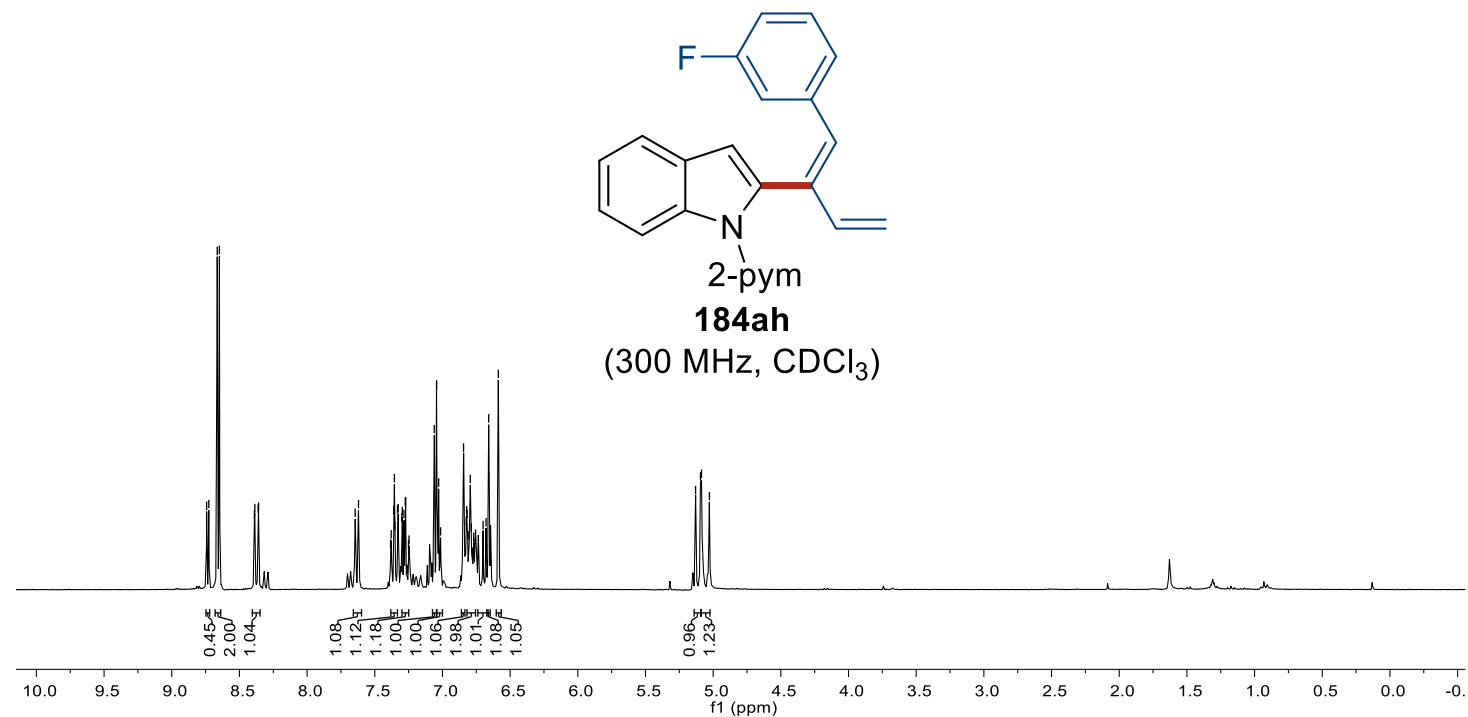




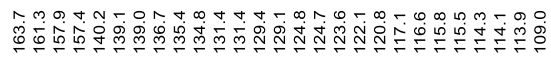

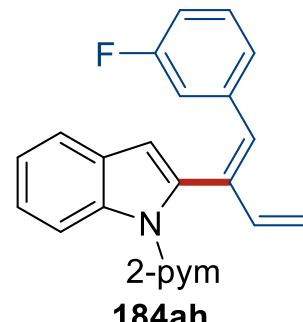

(101 MHz, $\mathrm{CDCl}_{3}$ )

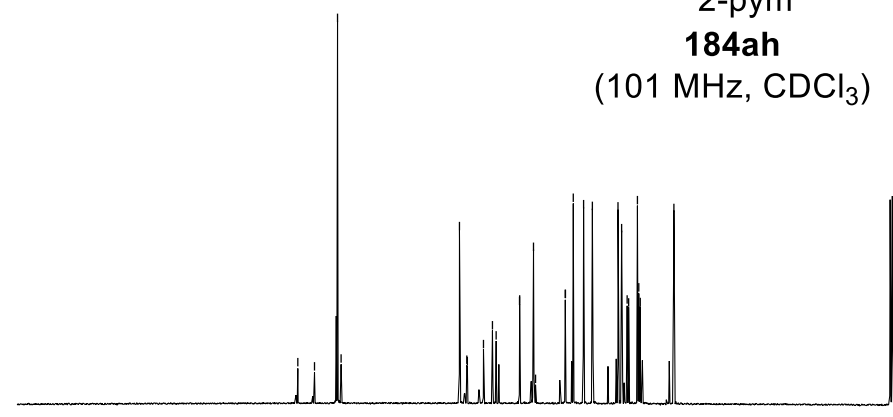

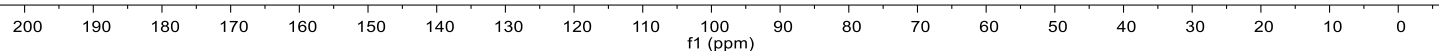
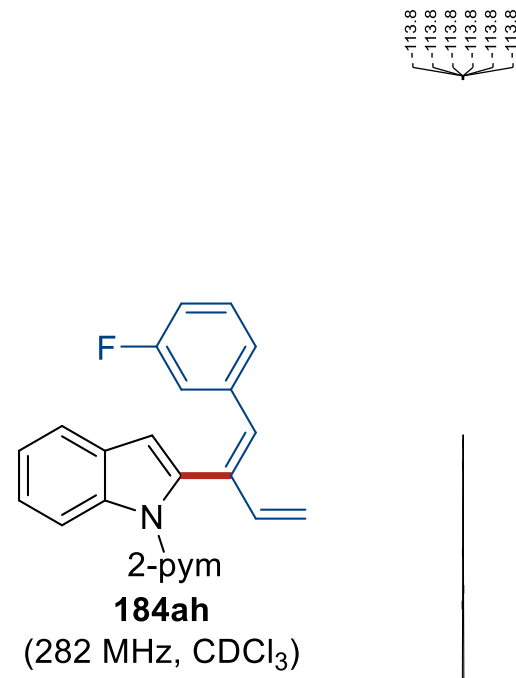

\begin{tabular}{llllllllllllllllllll}
\hline-65 & -70 & -75 & -80 & -85 & -90 & -95 & -100 & -105 & -110 & -115 & -120 & -125 & -130 & -135 & -140 & -145 & -150 & -155 & -160
\end{tabular} 


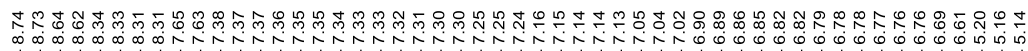
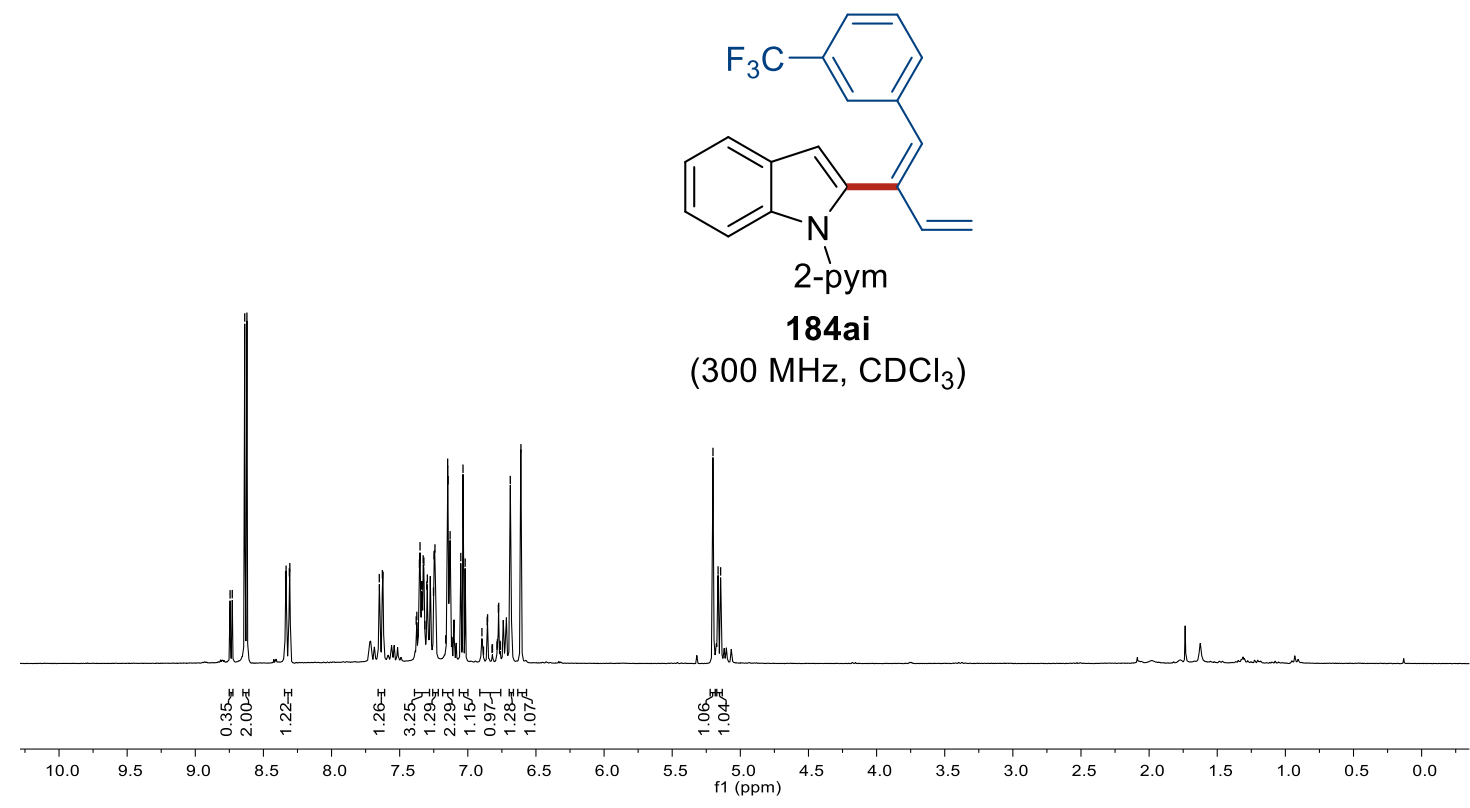

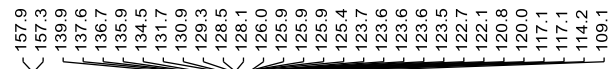

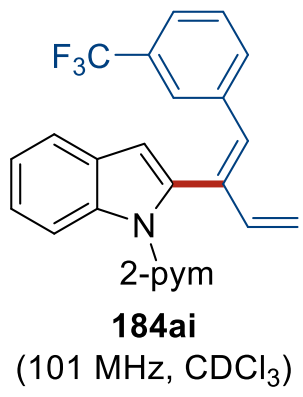

$\left(101 \mathrm{MHz}, \mathrm{CDCl}_{3}\right)$

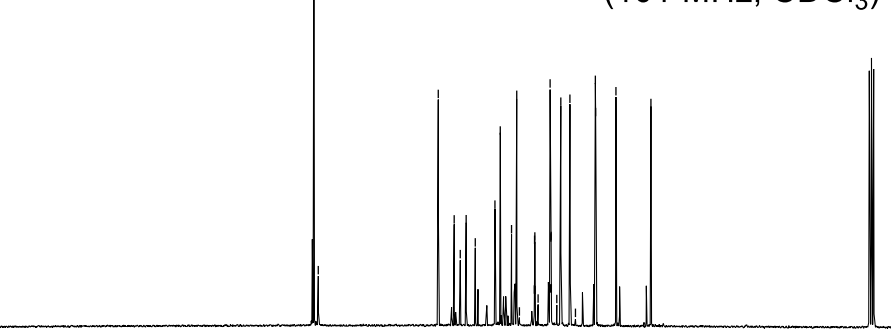

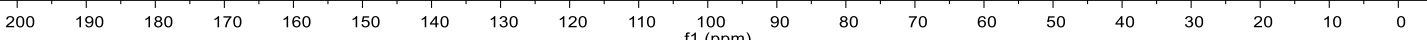




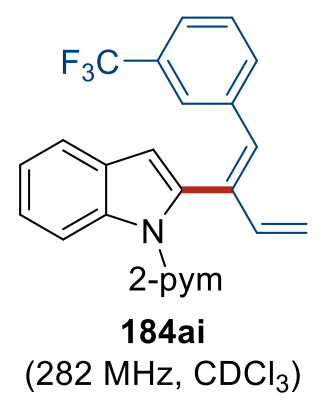

$\begin{array}{rlllllllllllllllllllllllllllllllllllllllll}5 & 0 & -5 & -10 & -15 & -20 & -25 & -30 & -35 & -40 & -45 & -50 & -55 & -60 & -65 & -70 & -75 & -80 & -85 & -90 & -95 & -100 & -105 & -110 & -115 & -120 & -125 & -130 & -135 & -140 & 1\end{array}$

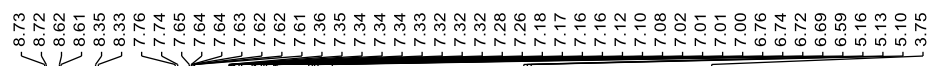

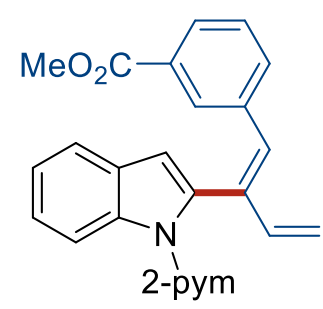

184aj

(400 MHz, $\mathrm{CDCl}_{3}$ )

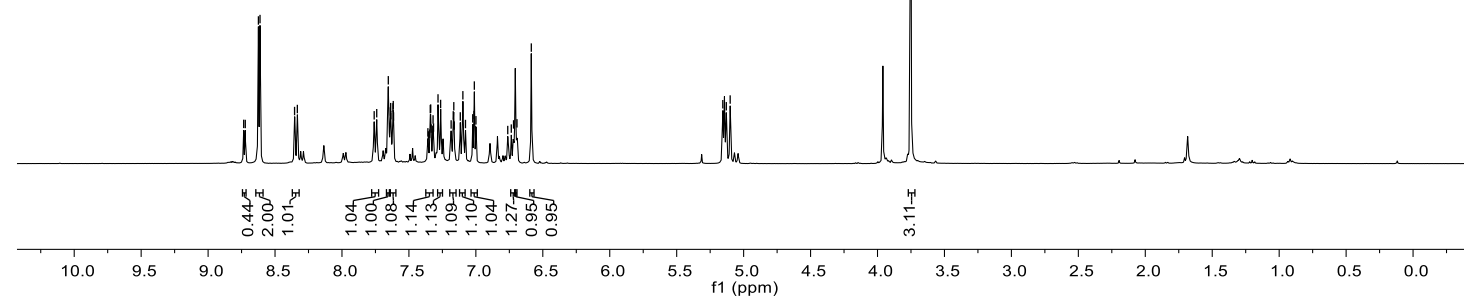




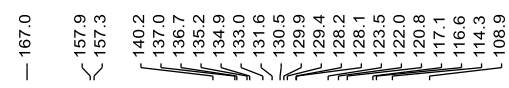

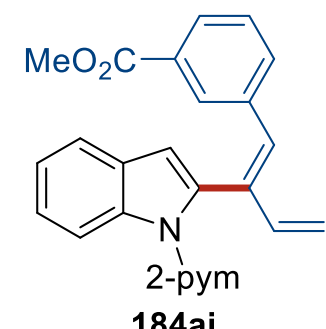

$\left.\left(126 \mathrm{MHz}^{\mathrm{CDCl}}\right)_{3}\right)$

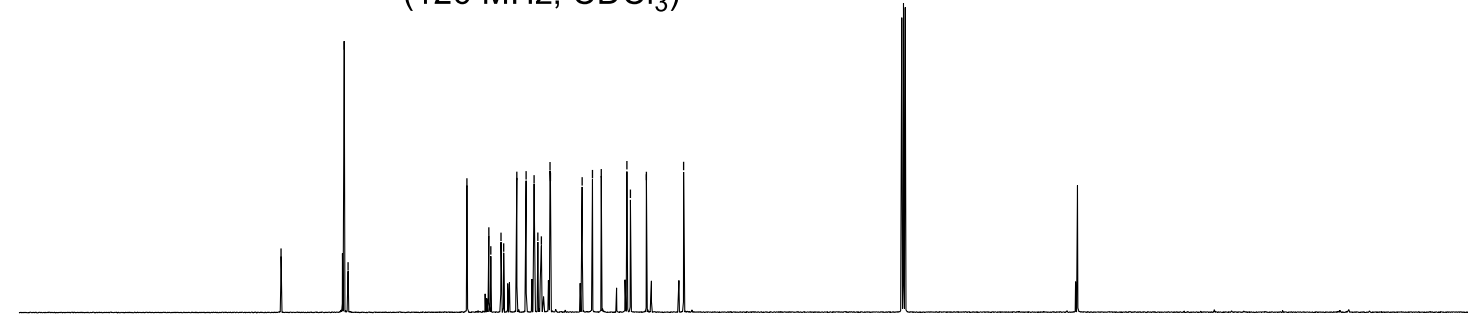

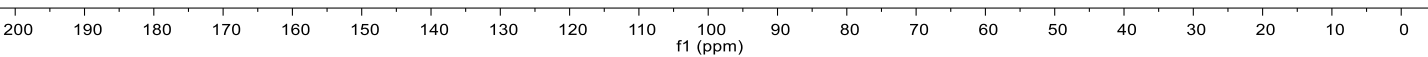

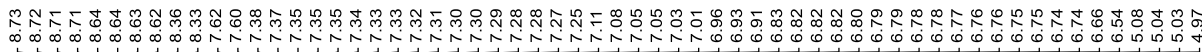

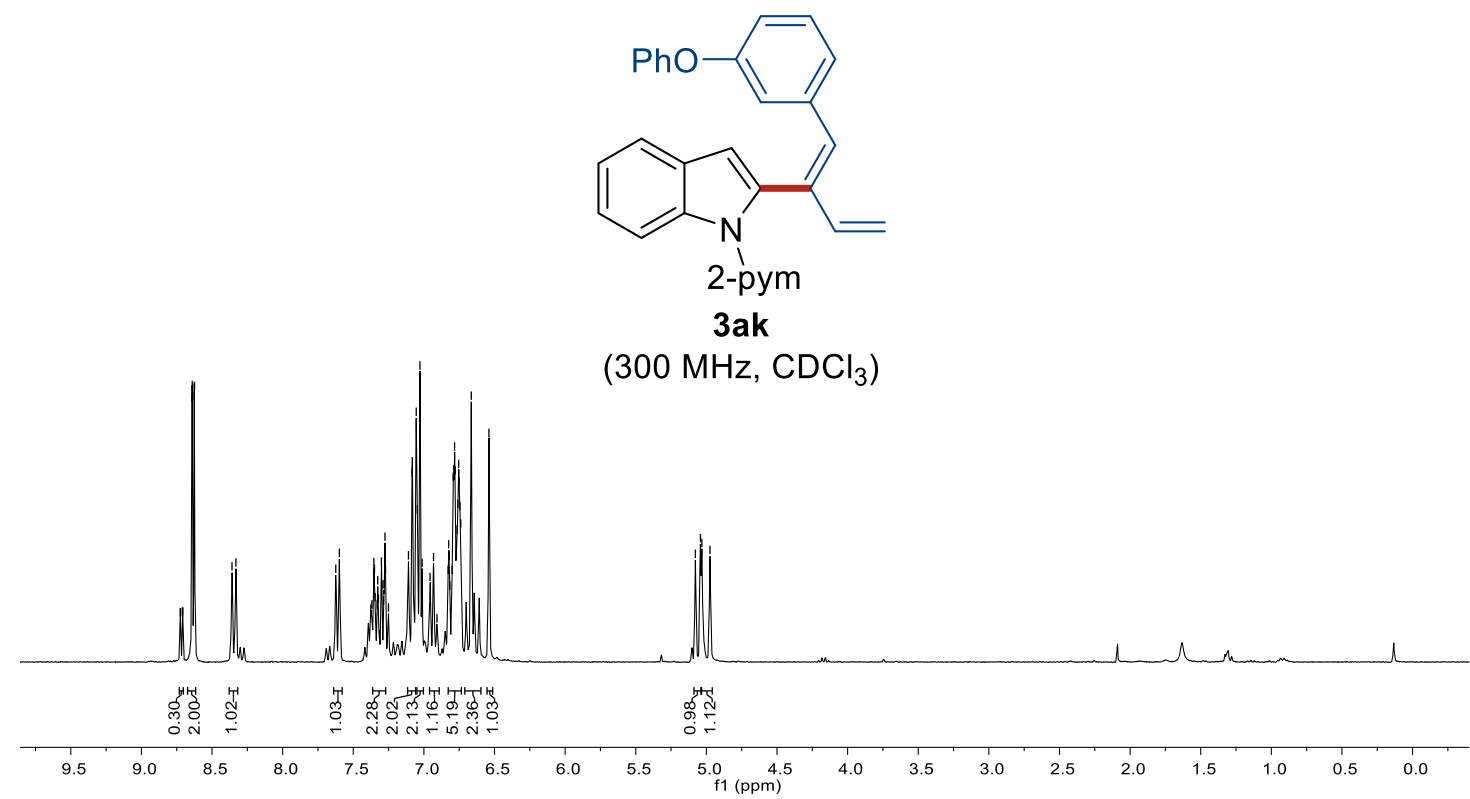




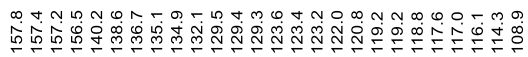

v

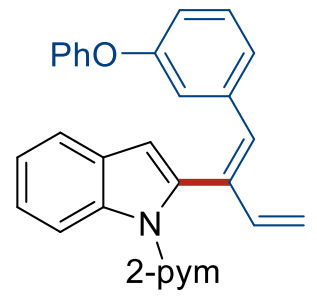

184ak

$\left(101 \mathrm{MHz}^{\mathrm{CDCl}} \mathrm{CDC}_{3}\right)$

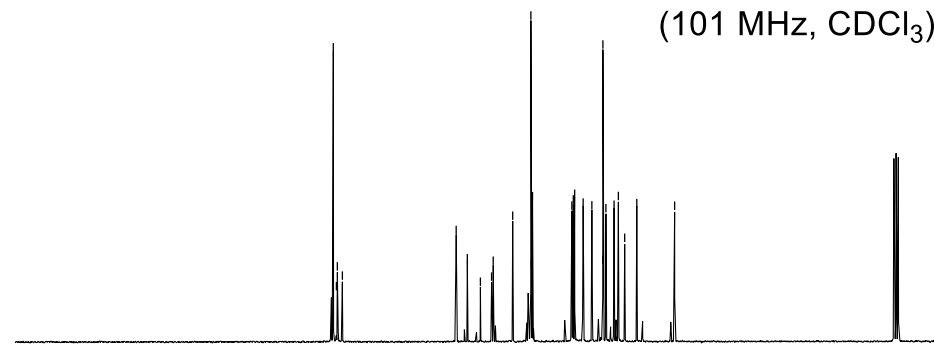

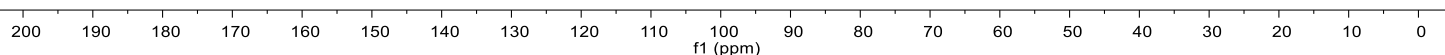

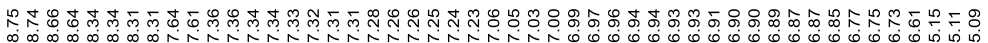

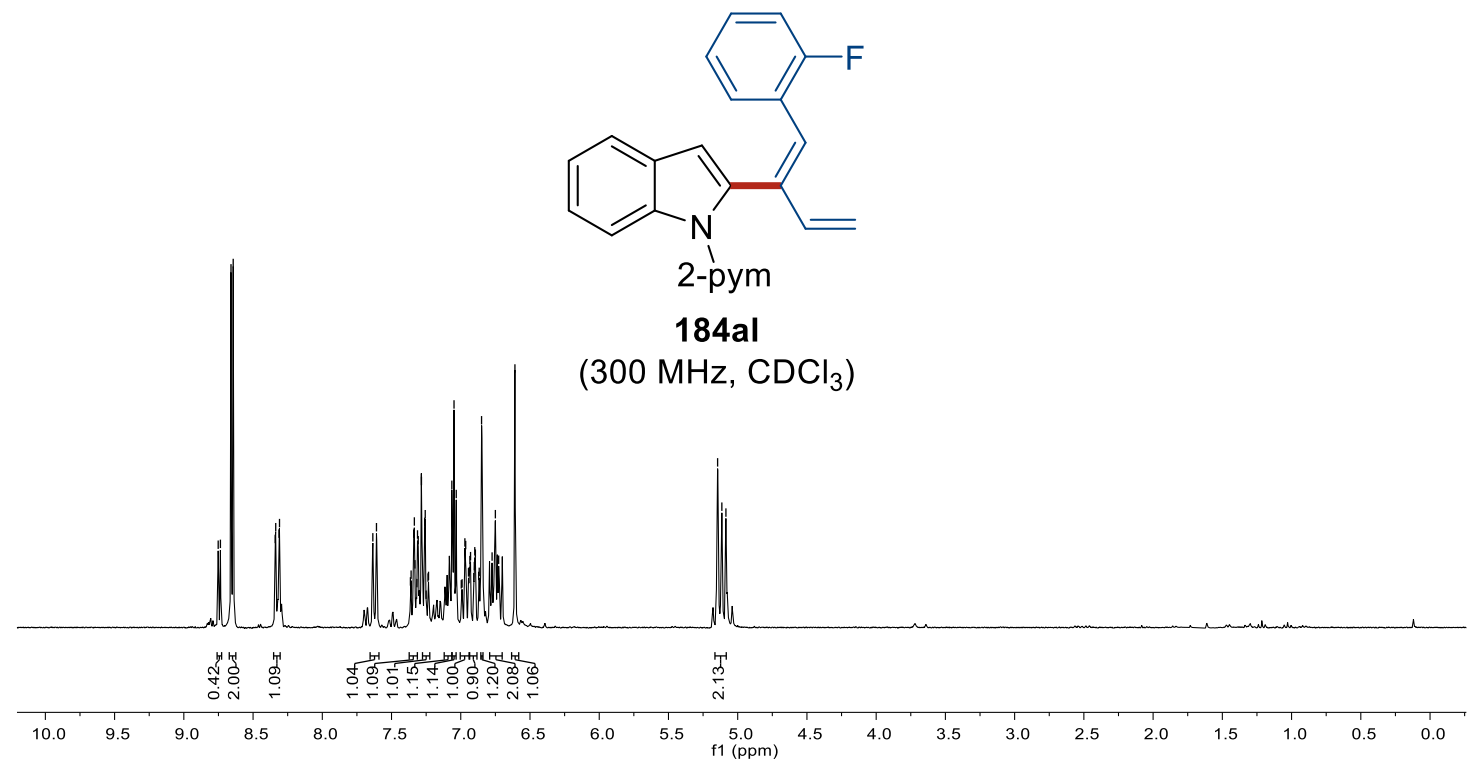




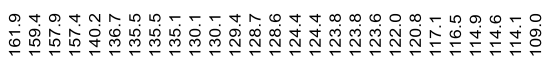
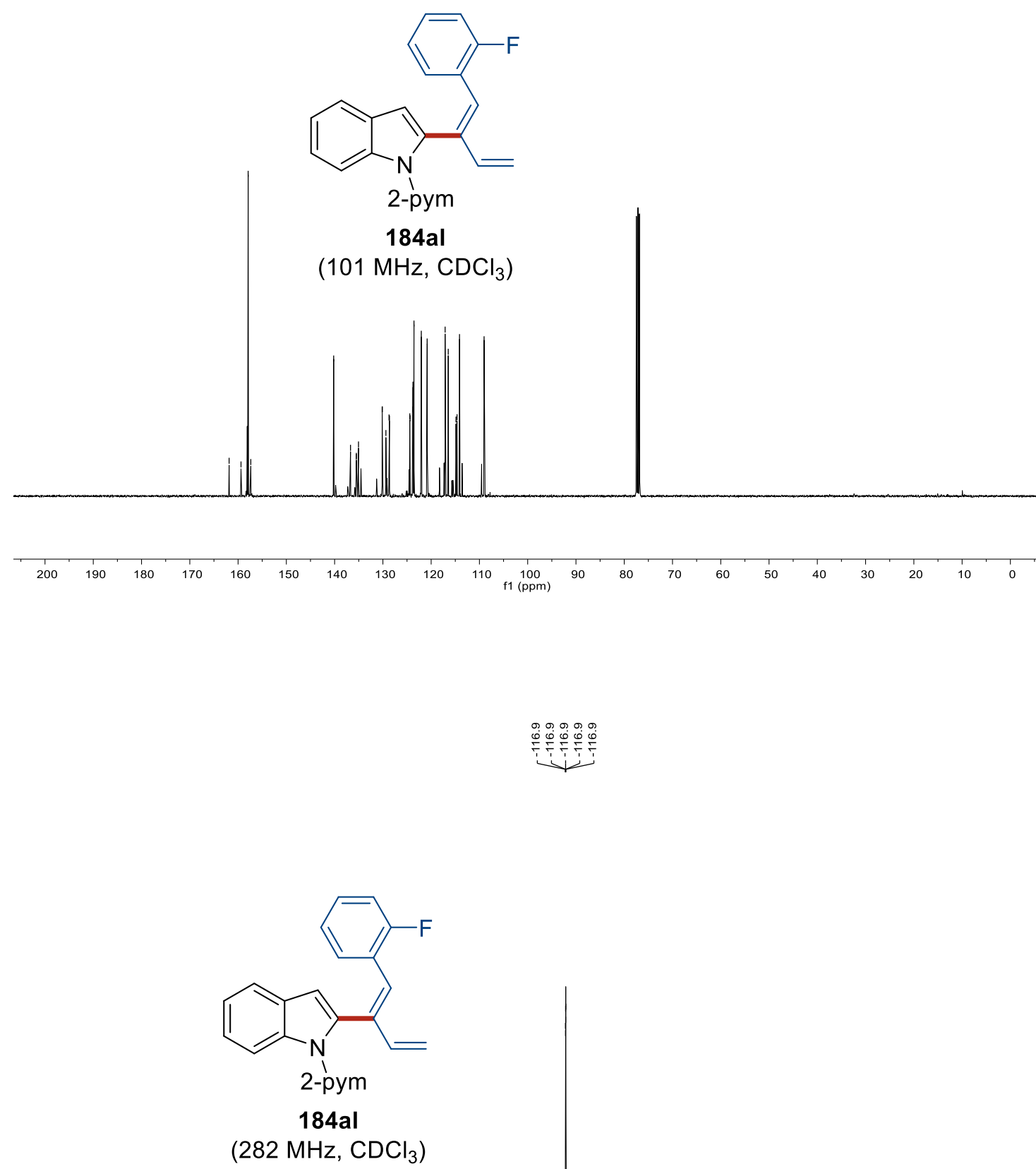

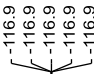

$\left.\mathrm{z}, \mathrm{CDCl}_{3}\right)$

\begin{tabular}{lllllllllllllllllllllllllllllllllll}
\hline & -55 & -60 & -65 & -70 & -75 & -80 & -85 & -90 & -95 & -100 & -105 & -110 & -115 & -120 & -125 & -130 & -135 & -140 & -145 & -150 & -155 & -160 & -165 & -17
\end{tabular} 
$\underbrace{0}_{\infty}$

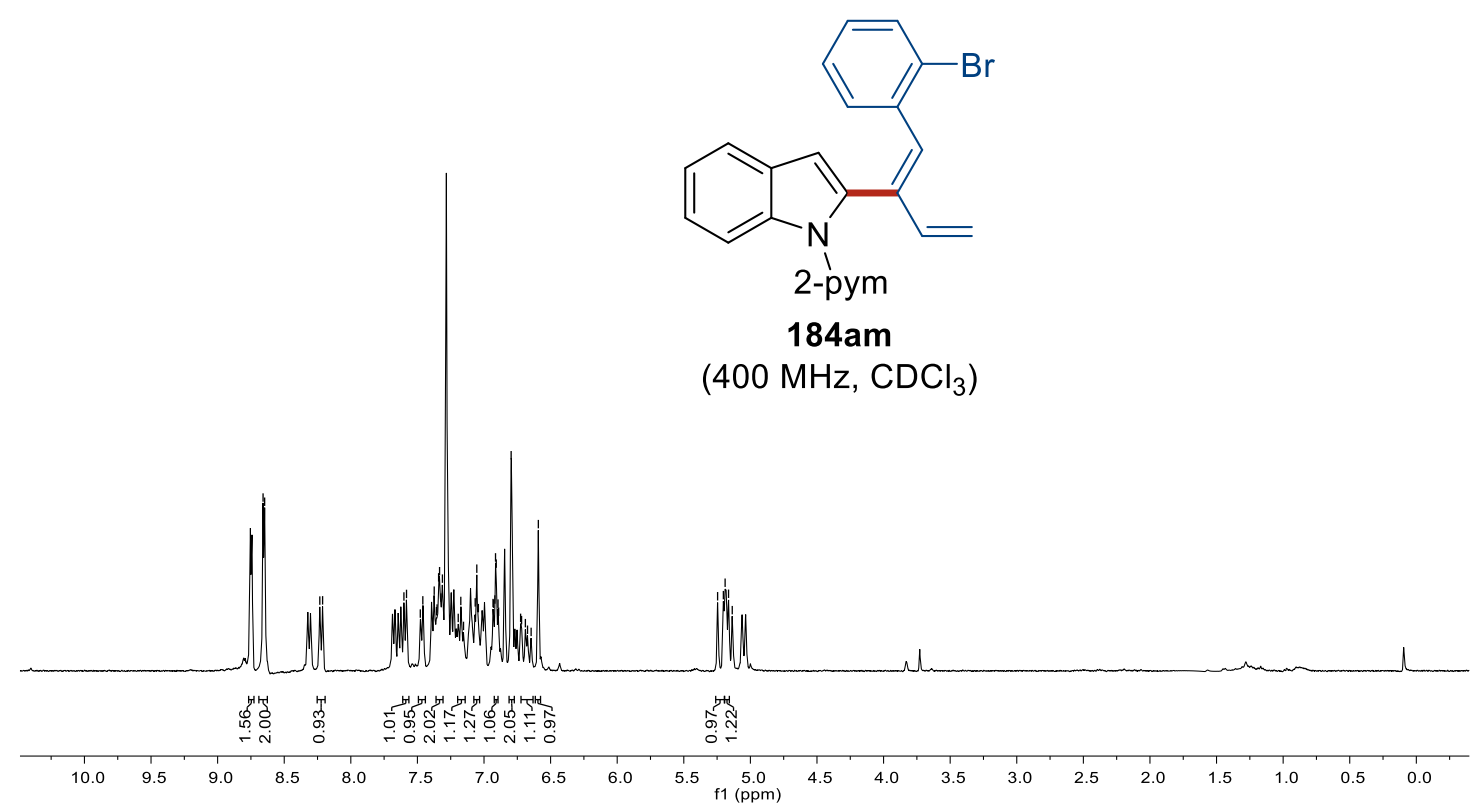

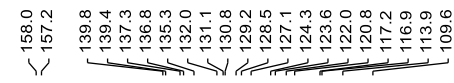

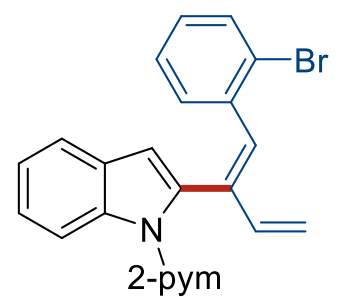

184am

$\left(101 \mathrm{MHz}^{\mathrm{CDCl}} \mathrm{CDC}_{3}\right)$

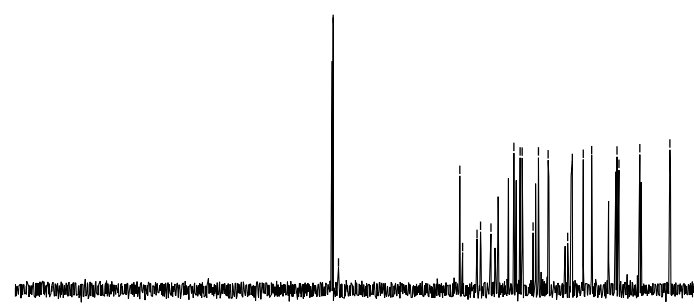

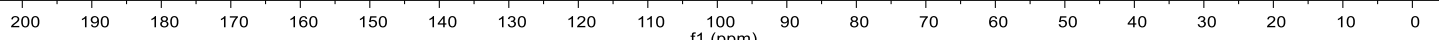




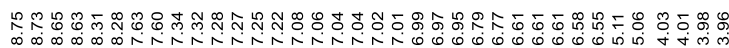

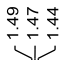

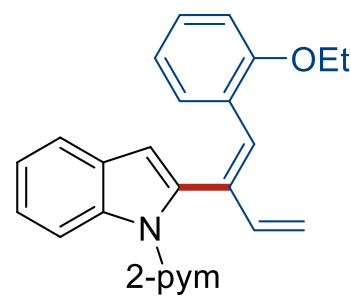

184an

(300 $\left.\mathrm{MHz} \mathrm{CDCl}_{3}\right)$

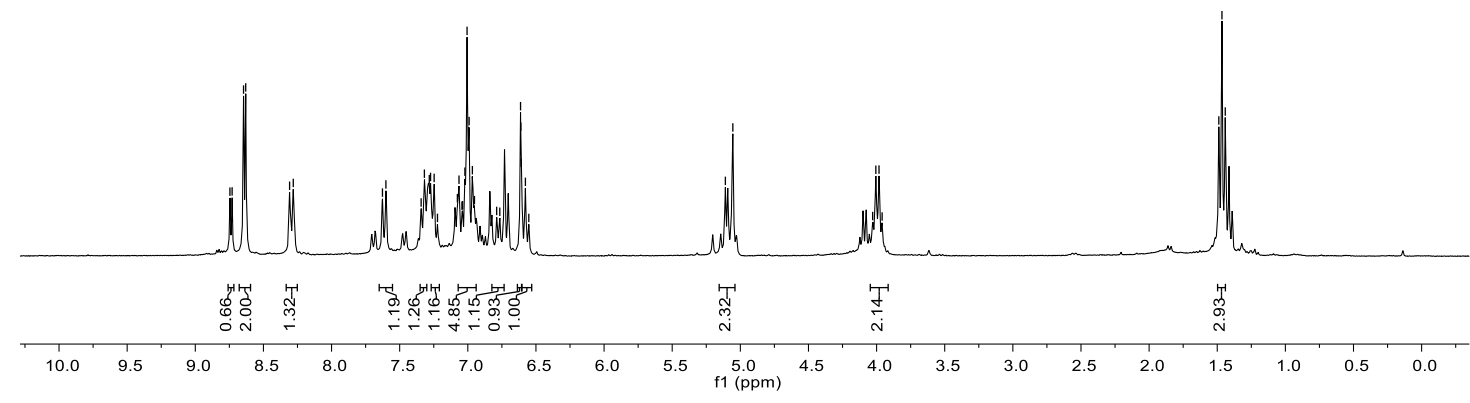

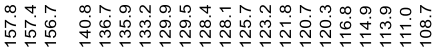
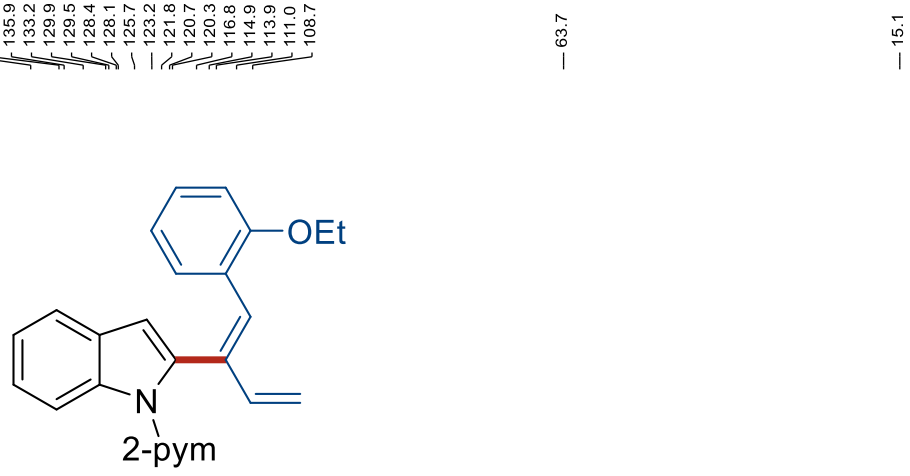

184an

$\left.\left(75 \mathrm{MHz}^{\mathrm{CDCl}}\right)_{3}\right)$

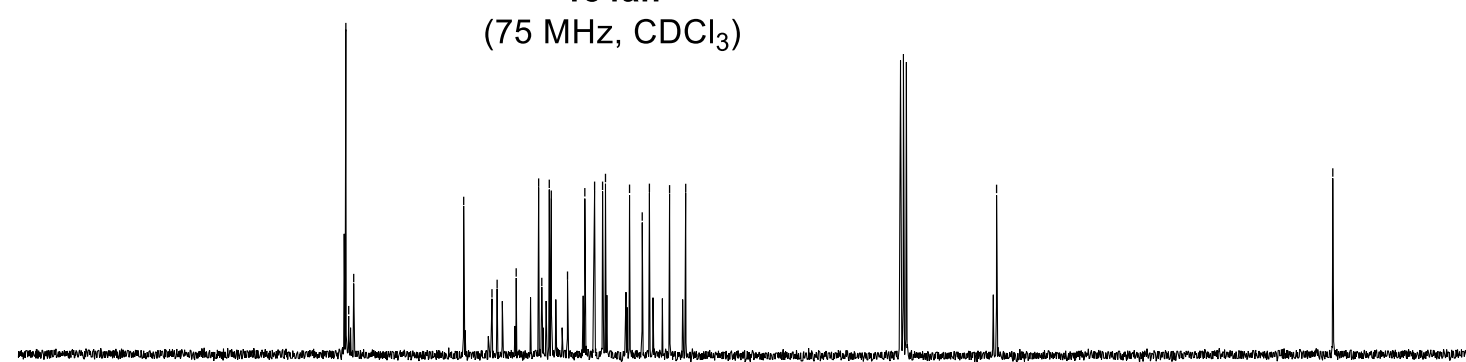

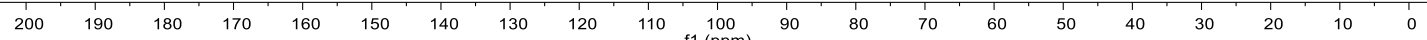




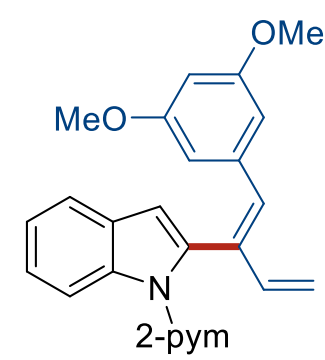

184ao

(300 MHz, $\mathrm{CDCl}_{3}$ )

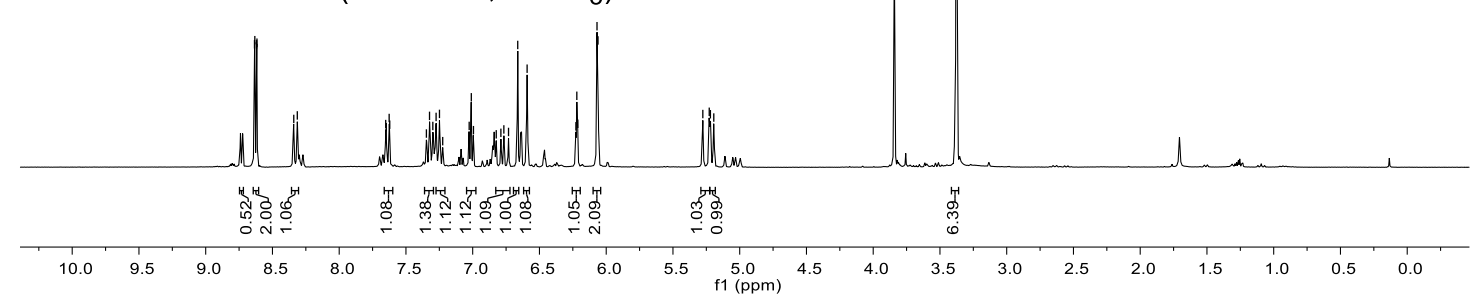

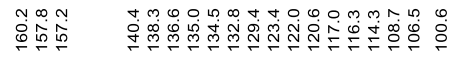

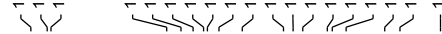

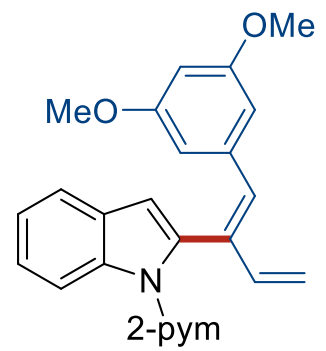

184ao

(75 MHz, $\mathrm{CDCl}_{3}$ )

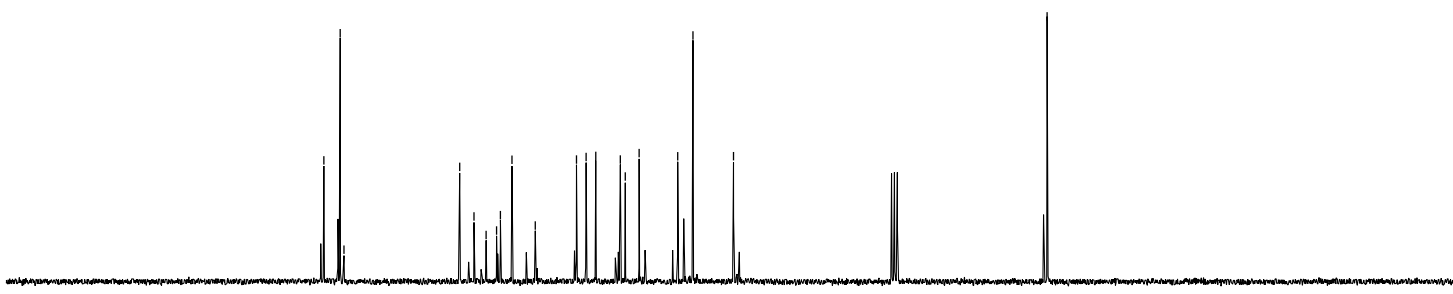

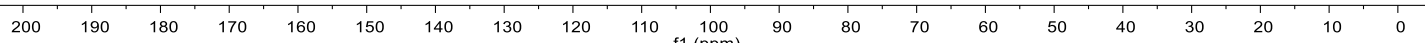




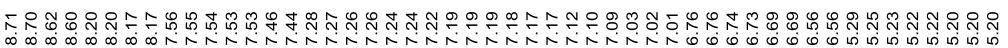

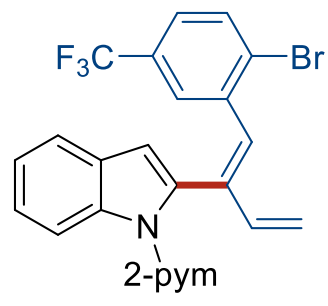

184ap

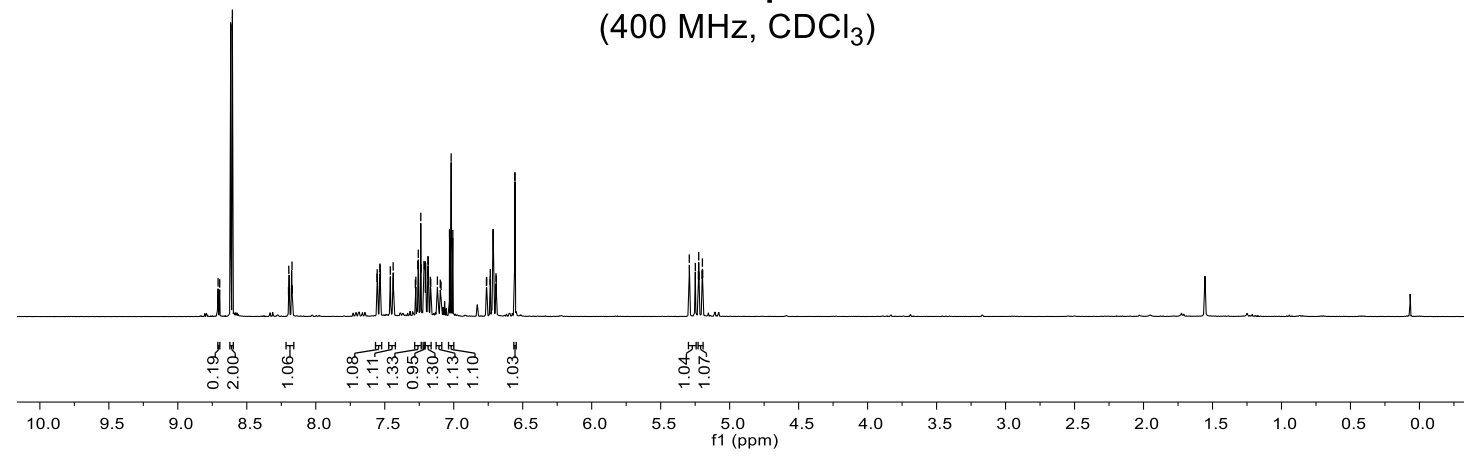

$\underbrace{1} \underbrace{1}$

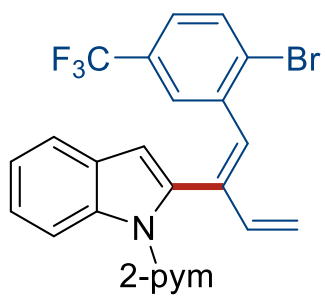

184ap

(101 MHz, $\mathrm{CDCl}_{3}$ )

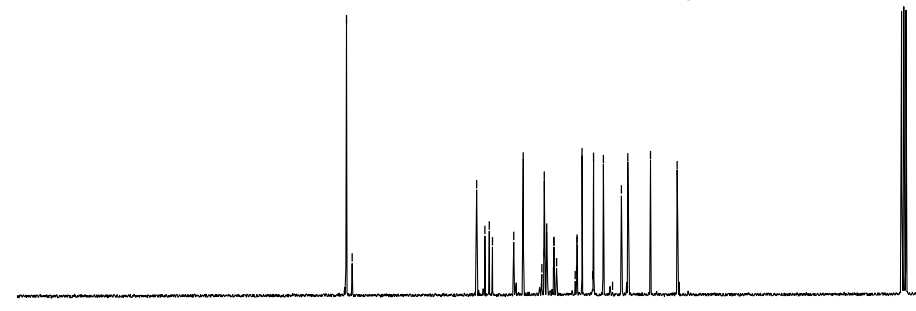

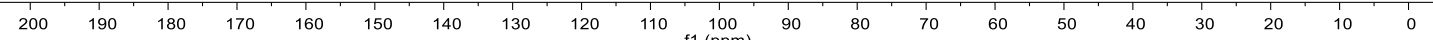




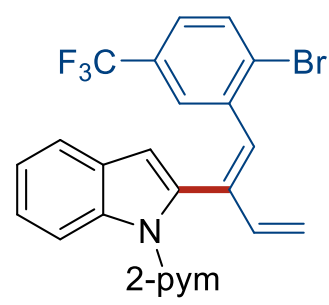

184ap

(376 MHz, $\mathrm{CDCl}_{3}$ )

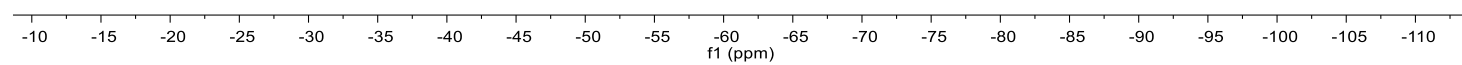

Y)

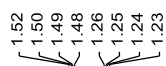

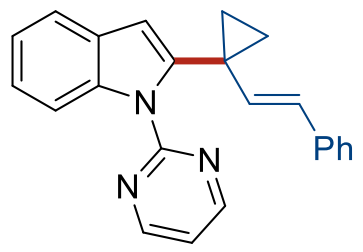

186aa

(300 $\mathrm{MHz}, \mathrm{CDCl}_{3}$ )

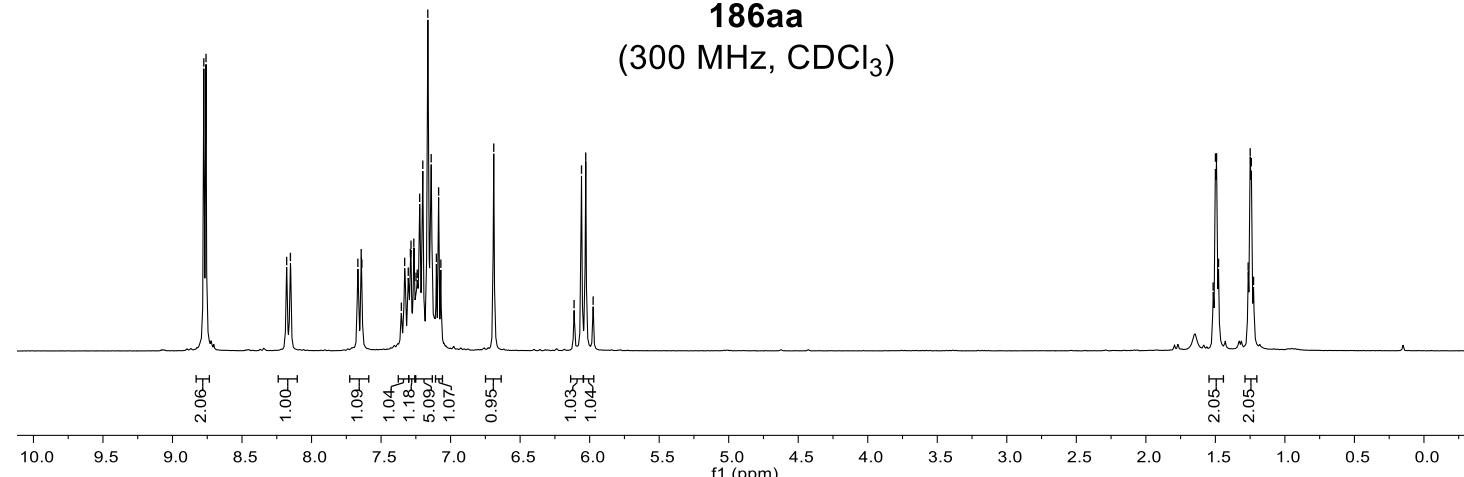




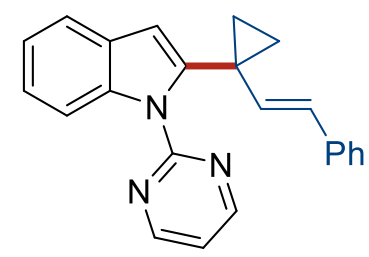

$186 a a$

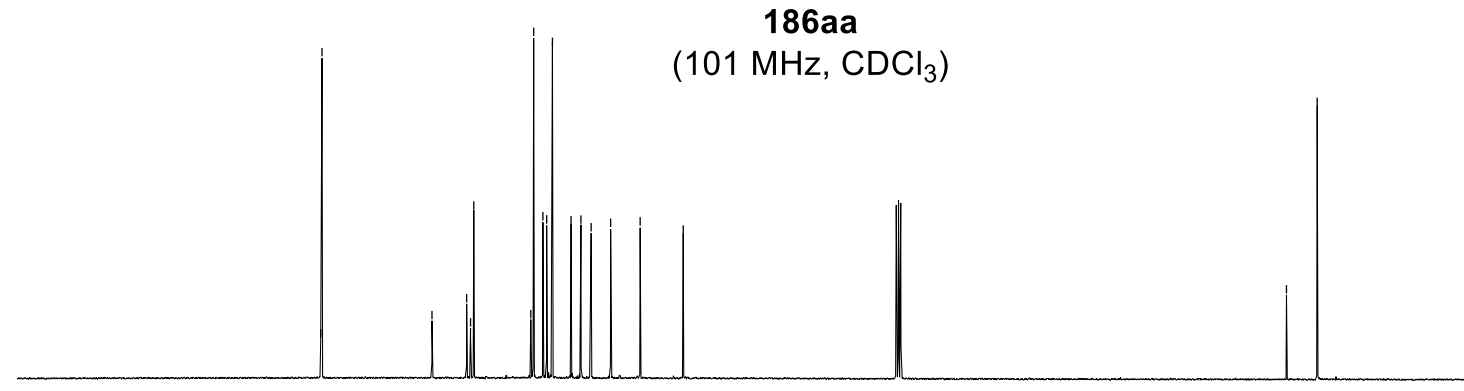

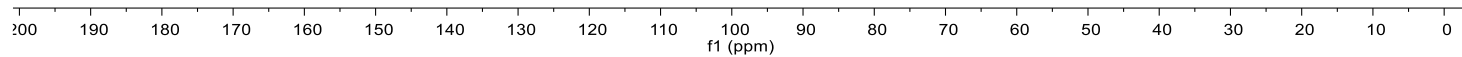

Yு

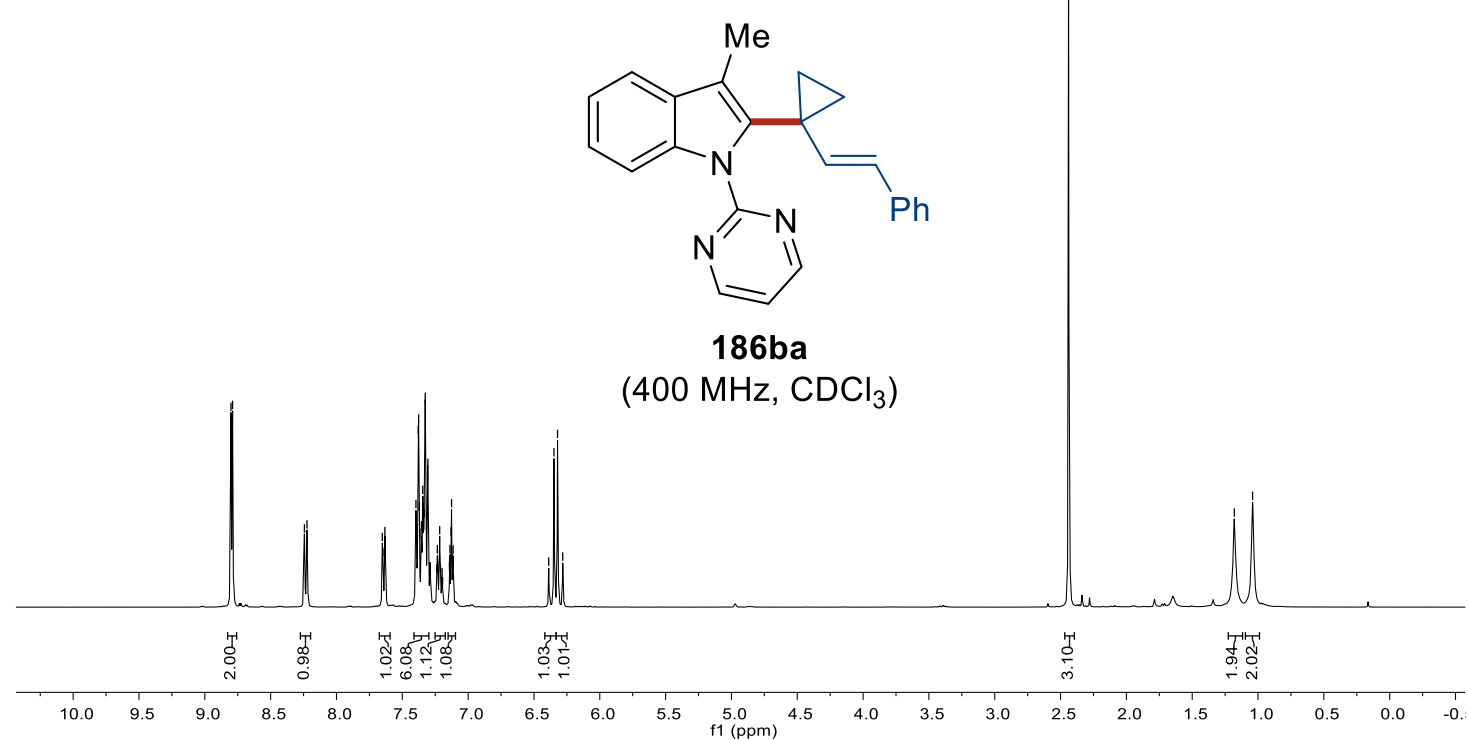



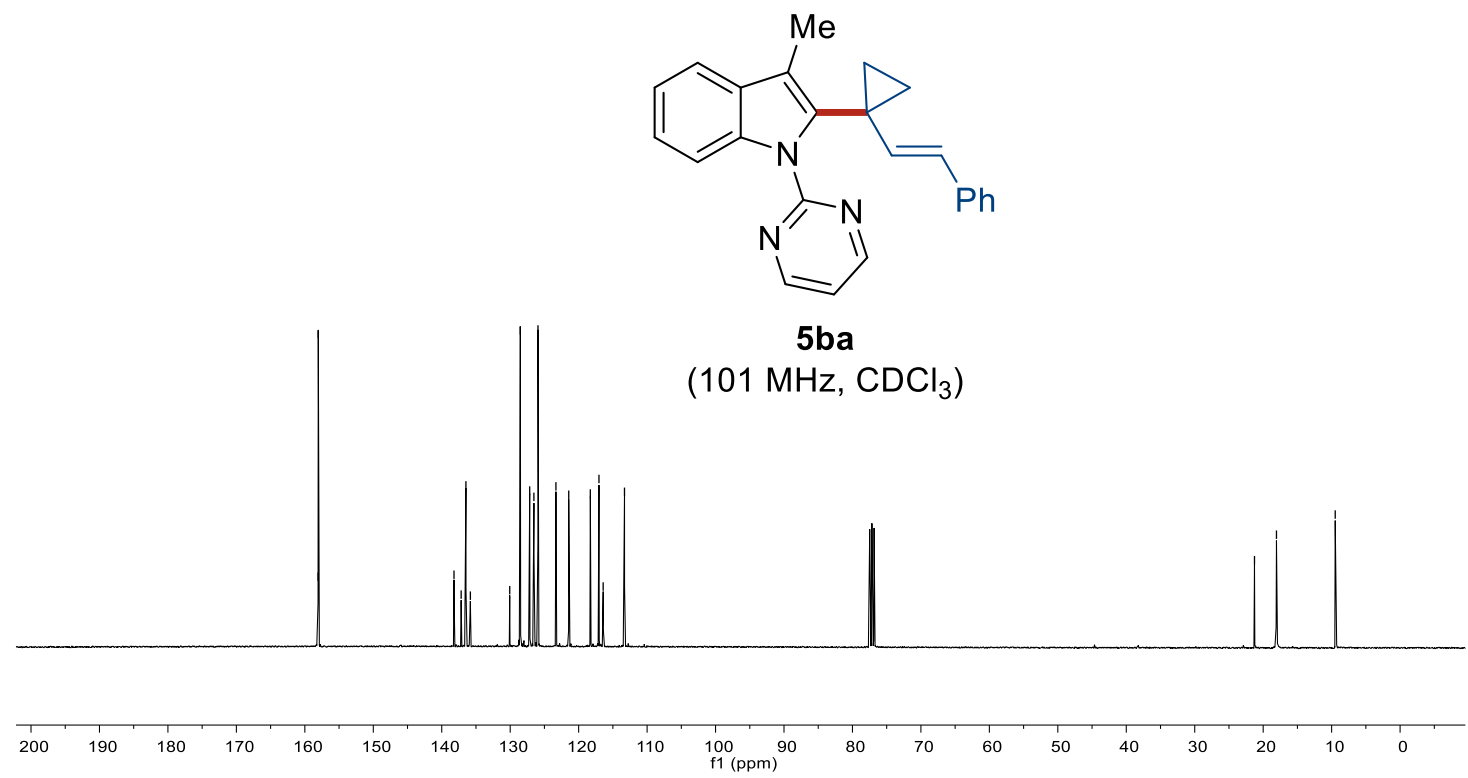

ソำ

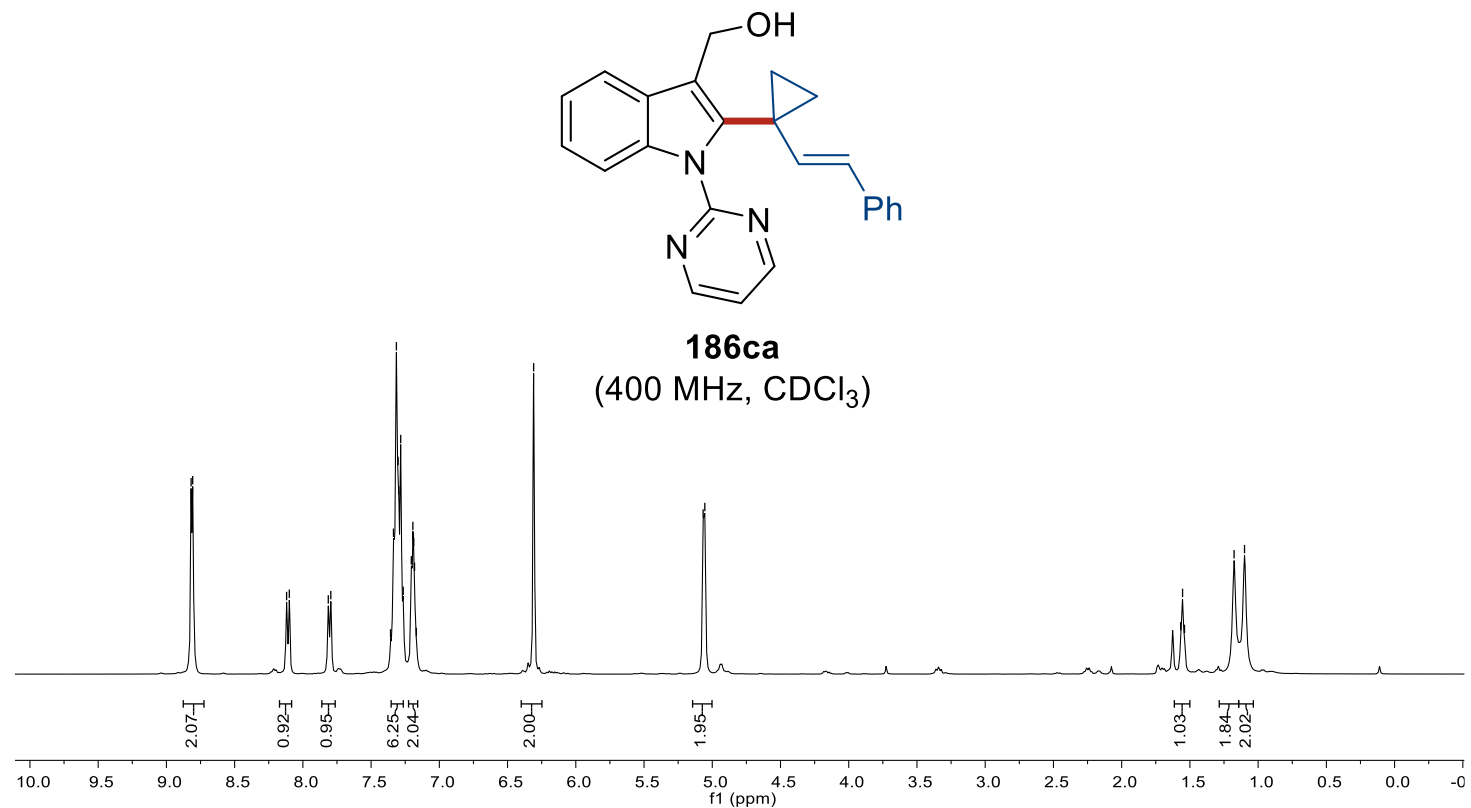


<smiles>OCc1c(C2(/C=C/c3ccccc3)CC2)n(-c2ncccn2)c2ccccc12</smiles>

186qa

(101 $\mathrm{MHz}, \mathrm{CDCl}_{3}$ )
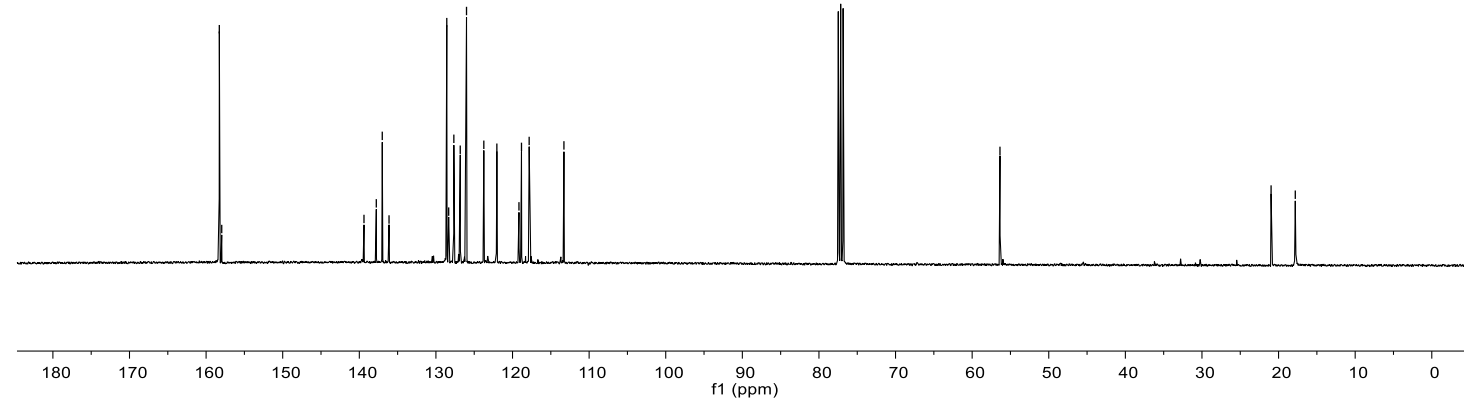

l)

经

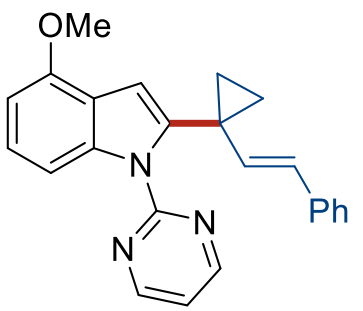

186ca

(300 $\mathrm{MHz}, \mathrm{CDCl}_{3}$ )
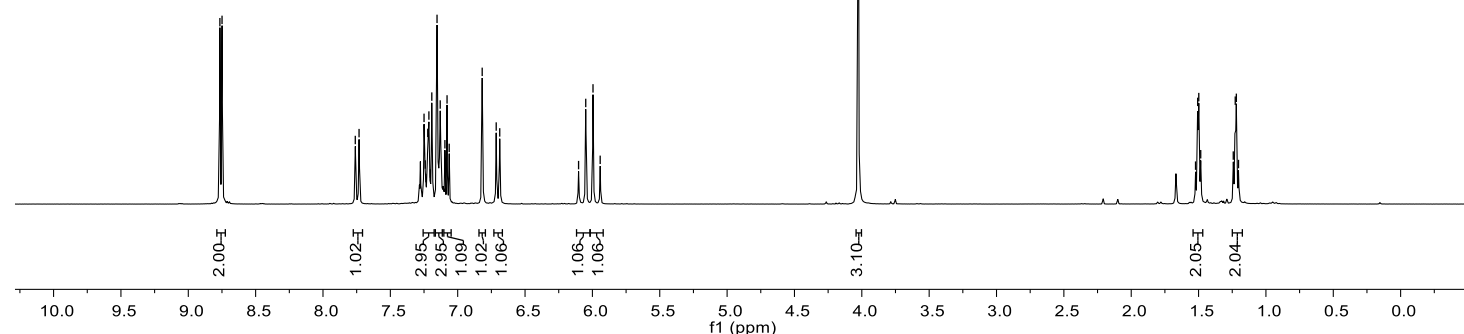


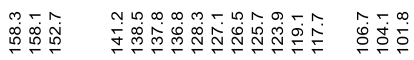

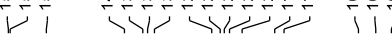

$\stackrel{\substack{0 \\ \stackrel{\leftrightarrow}{0}}}{1} \quad \stackrel{\substack{\infty \\ \infty}}{1}$<smiles>COc1cccc2c1cc(C1(/C=C/c3ccccc3)CC1)n2-c1ncccn1</smiles>

$186 \mathrm{ca}$

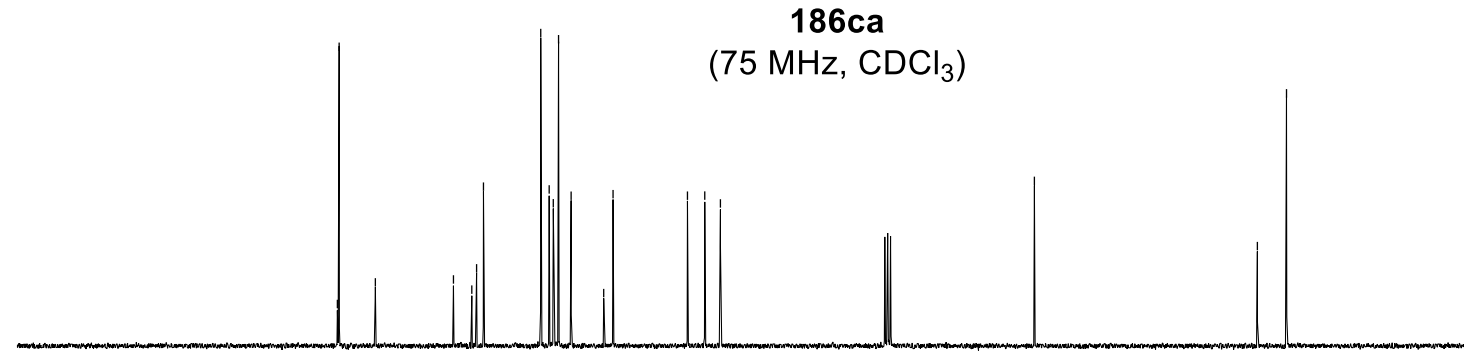

$\begin{array}{lllllllllllllllllllllll} & 190 & 180 & 170 & 160 & 150 & 140 & 130 & 120 & 110 & \begin{array}{c}100 \\ \mathrm{f}(1 \mathrm{ppm})\end{array} & 90 & 80 & 70 & 60 & 50 & 40 & 30 & 20 & 10 & 0\end{array}$

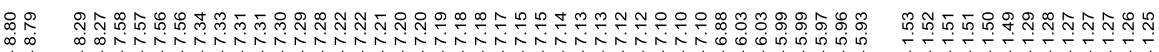

$\mathrm{V}^{\infty} \mathrm{Y}^{\infty}$

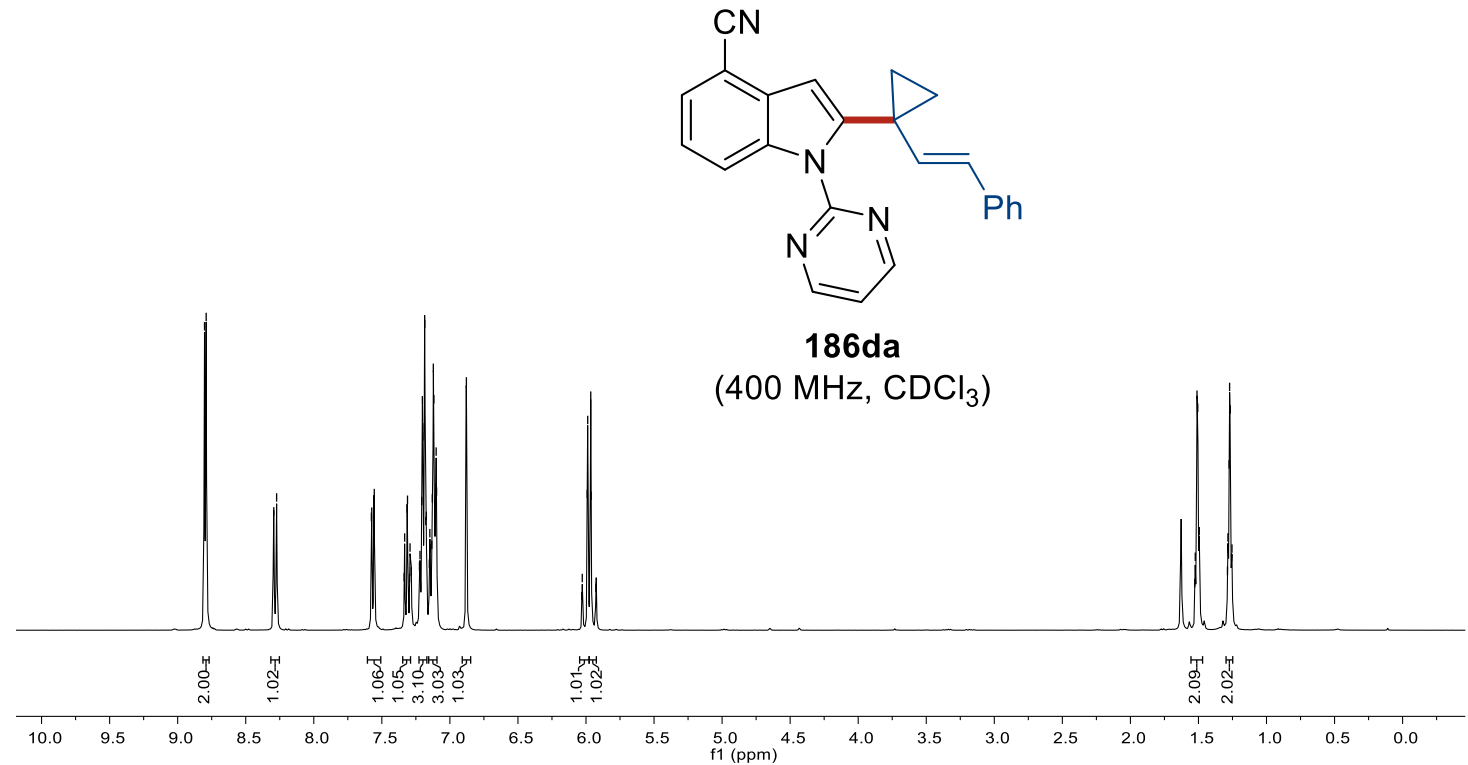


$\underbrace{j}$

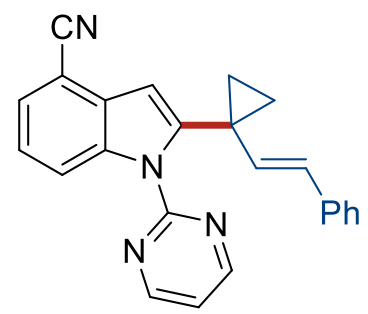

186da

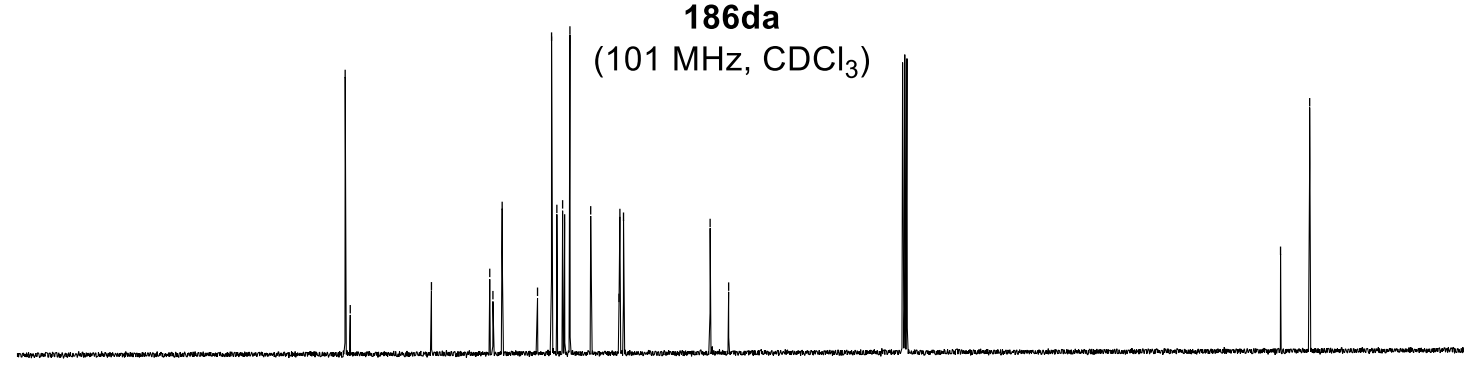

$\begin{array}{llllllllllllllllllll}1 & 1 & 1 & 1 & 1 & 1 & 1 & 1 & 1 & 1 & 1 & 1 & 1 & 1 & 1 & 1 & 1 & 1 & 1 & 1 \\ \mathrm{f} 1(\mathrm{ppm}) & 90 & 80 & 70 & 60 & 50 & 40 & 30 & 20 & 10 & 0\end{array}$

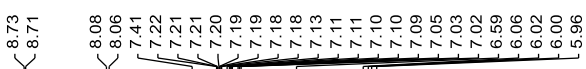

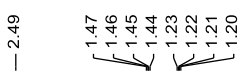

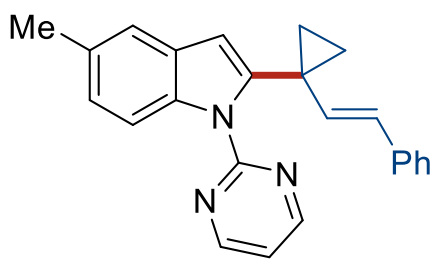

186ea

(400 MHz, $\mathrm{CDCl}_{3}$ )

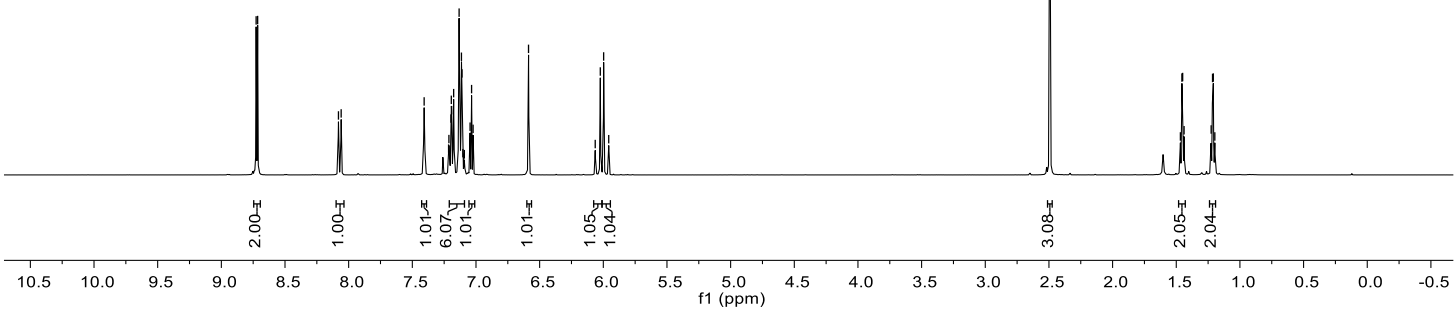




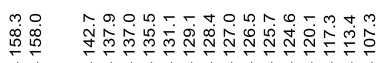

$i \sqrt{2}$

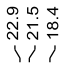<smiles>Cc1ccc2c(c1)cc(C1(/C=C/c3ccccc3)CC1)n2-c1ncccn1</smiles>

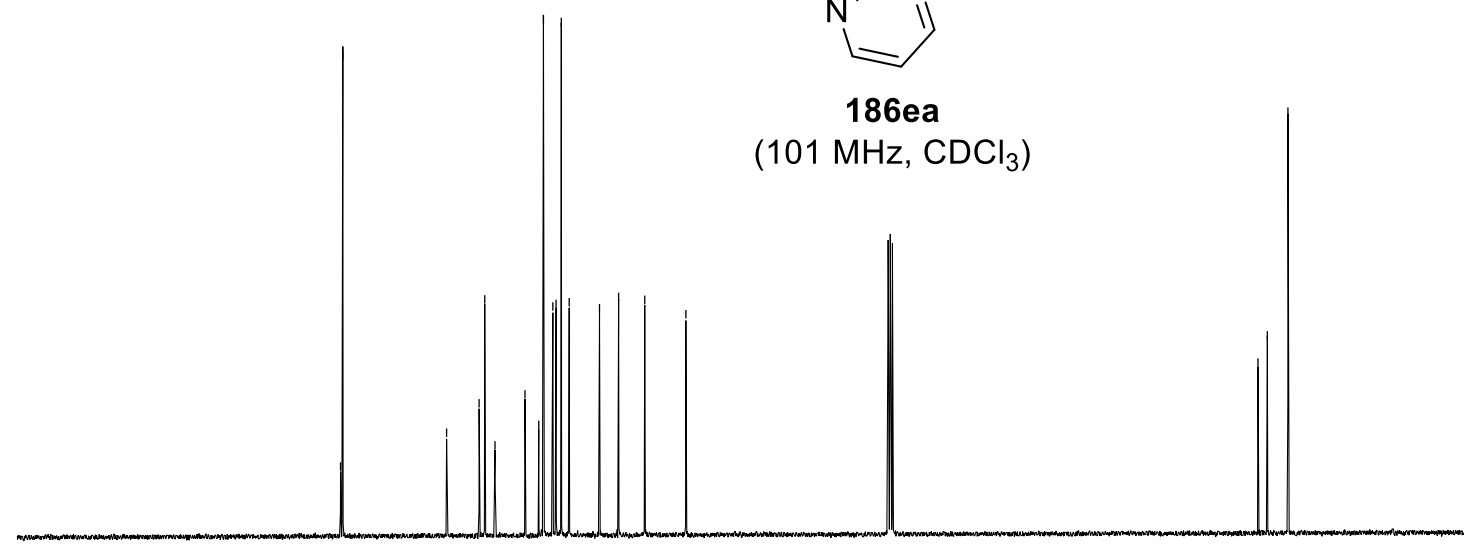

$\underbrace{\infty}$

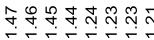

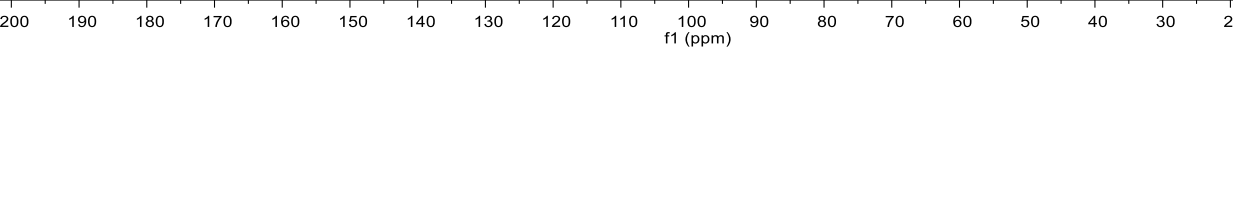<smiles>Clc1ccc2c(c1)cc(C1(/C=C/c3ccccc3)CC1)n2-c1ncccn1</smiles>

186ga

$\left(400 \mathrm{MHz}, \mathrm{CDCl}_{3}\right)$

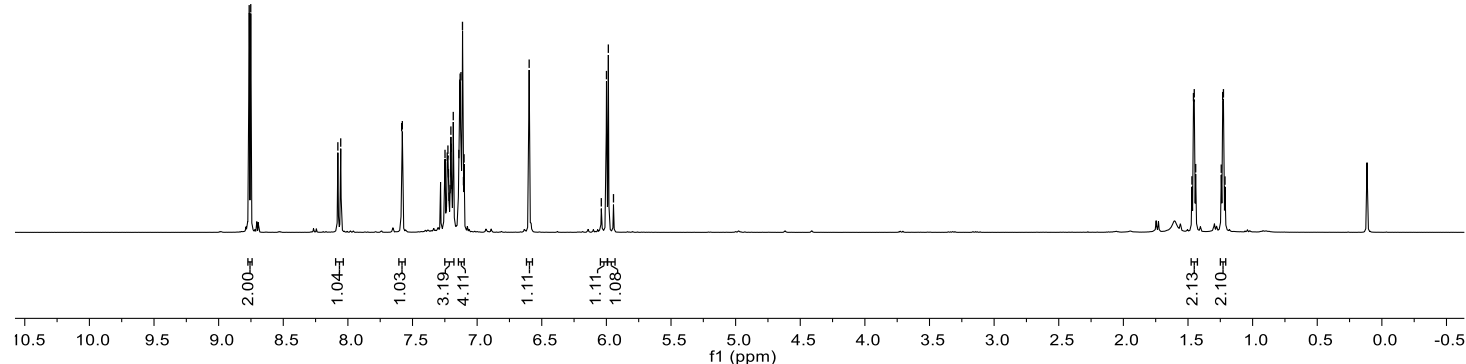




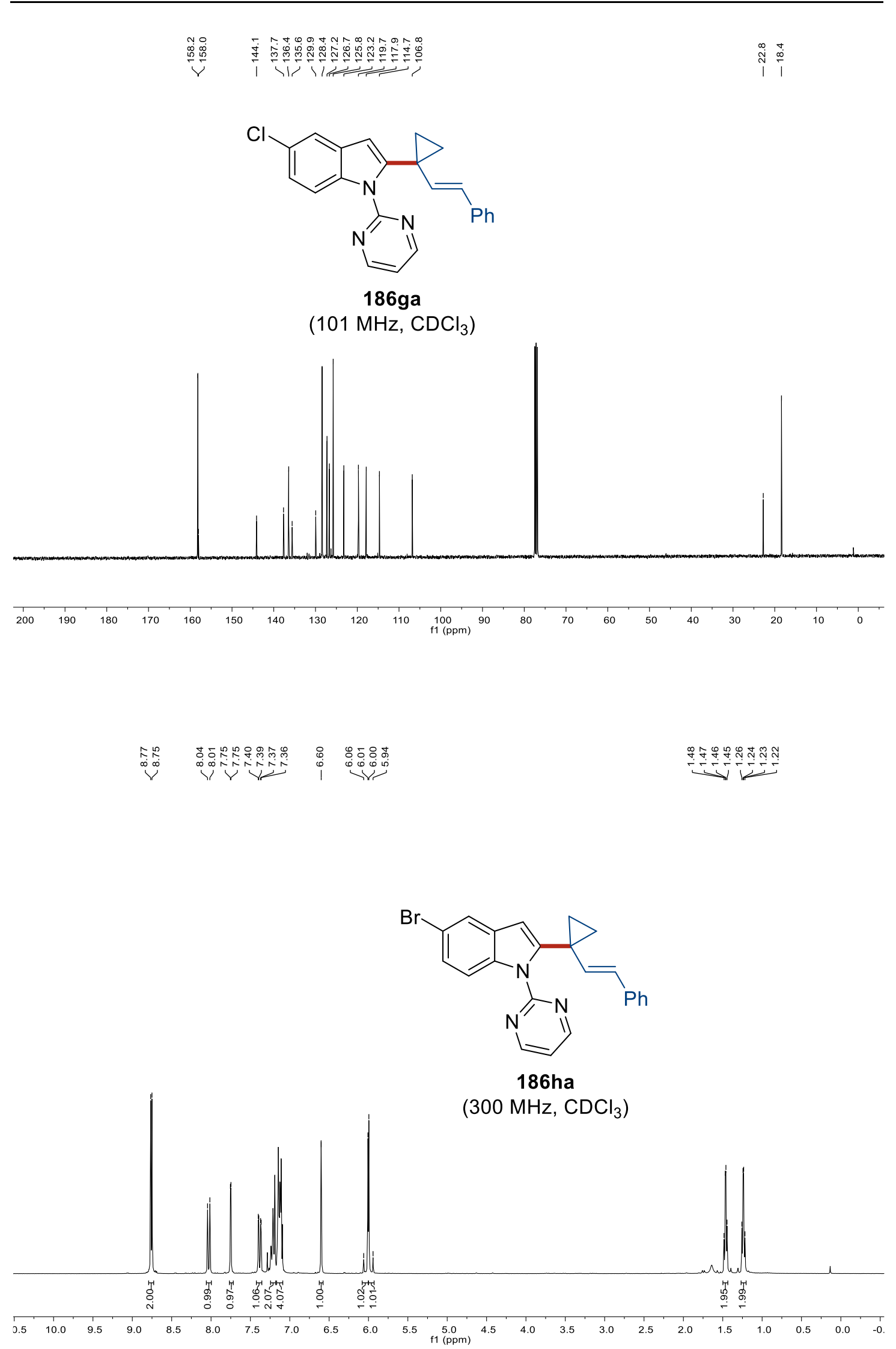




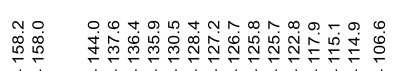

$\checkmark \underbrace{2}$

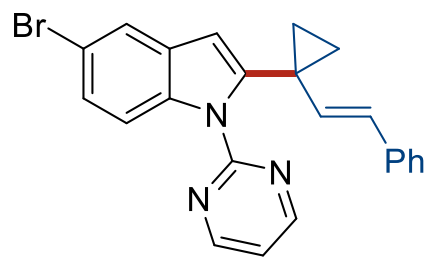

186ha

(101 $\mathrm{MHz} \mathrm{CDCl}_{3}$ )
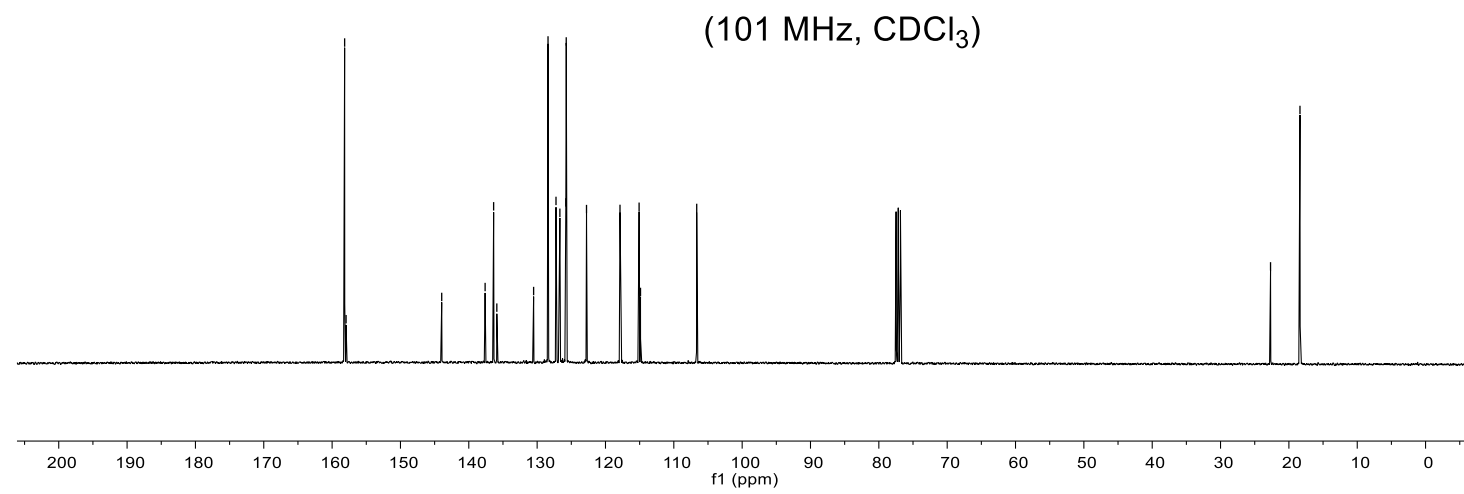

$\underbrace{\infty}$

인

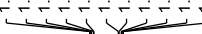

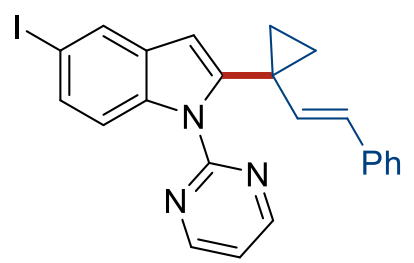

186ra

(400 $\mathrm{MHz} \mathrm{CDCl}_{3}$ )

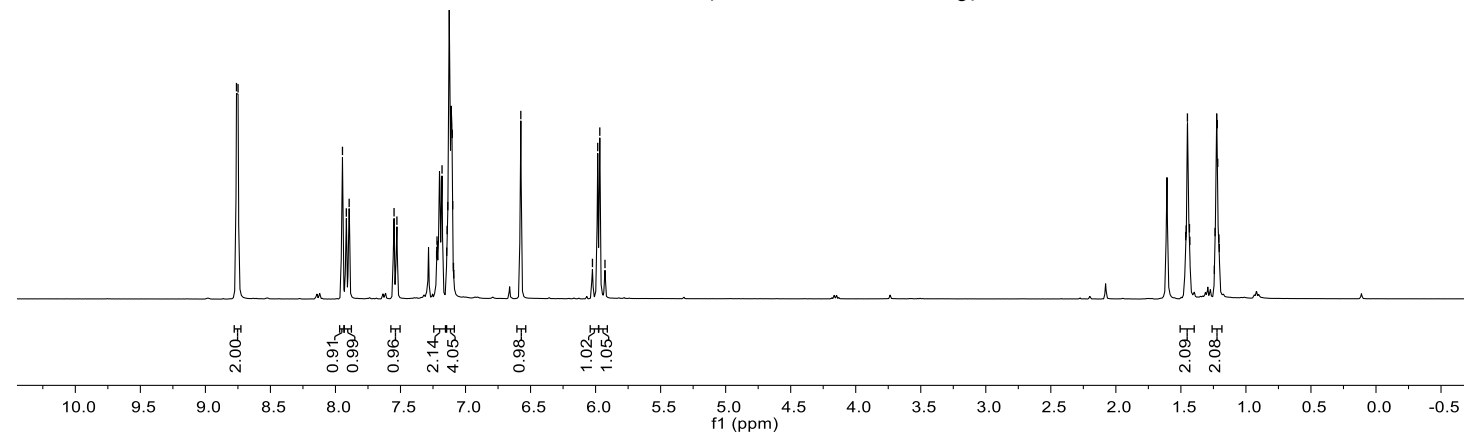




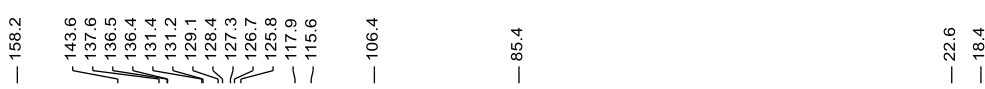<smiles>Ic1ccc2c(c1)cc(C1(/C=C/c3ccccc3)CC1)n2-c1ncccn1</smiles>

186ra

(101 MHz, $\mathrm{CDCl}_{3}$ )

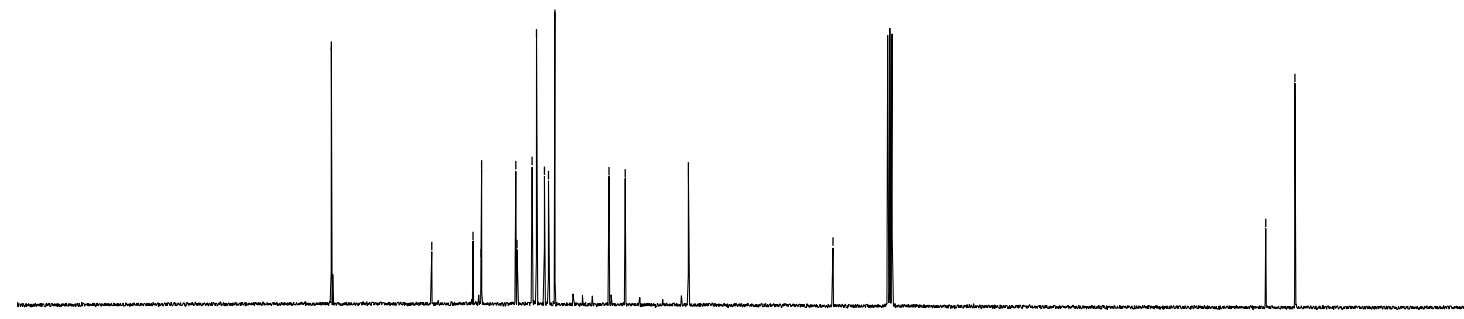

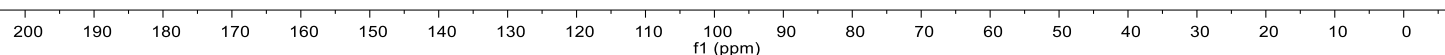

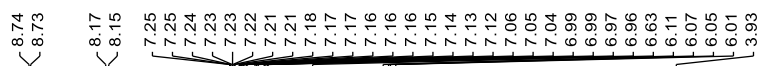

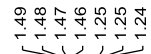



186ia

(400 MHz, $\mathrm{CDCl}_{3}$ )

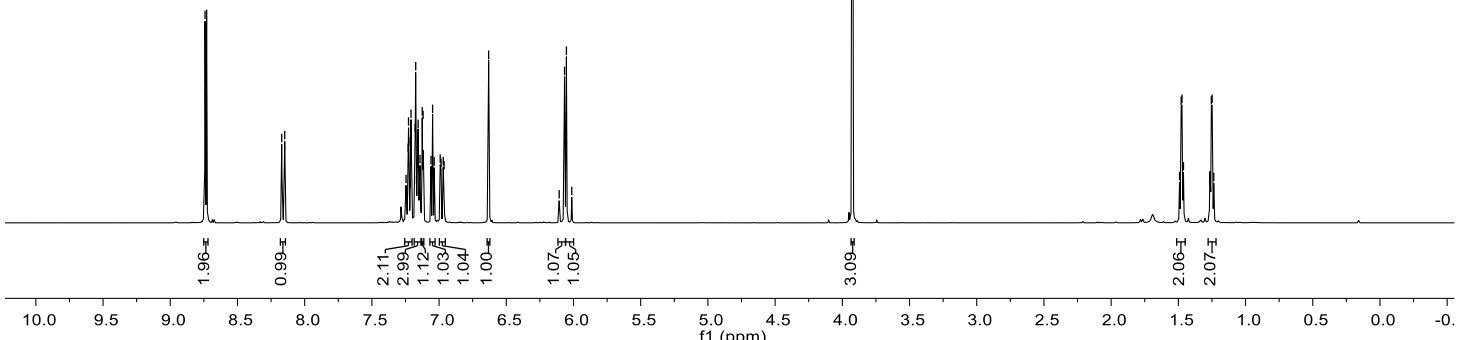



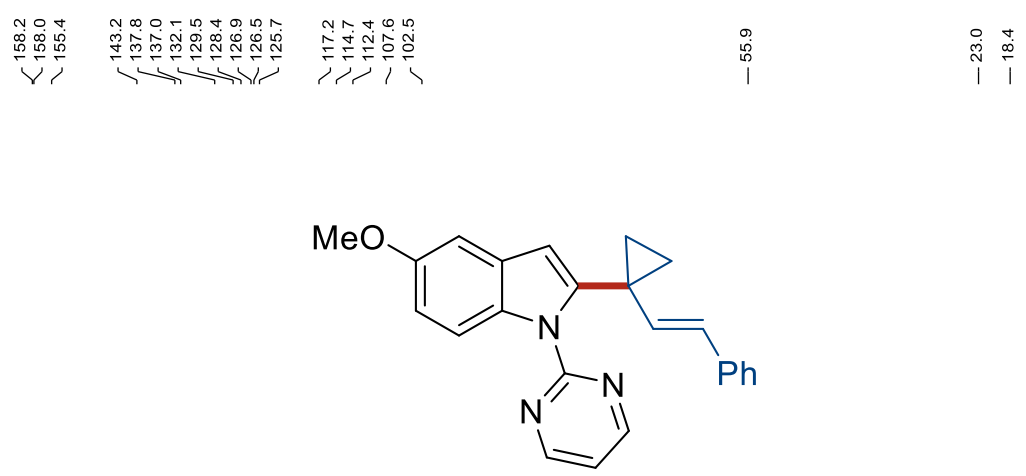

186ia

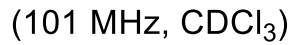
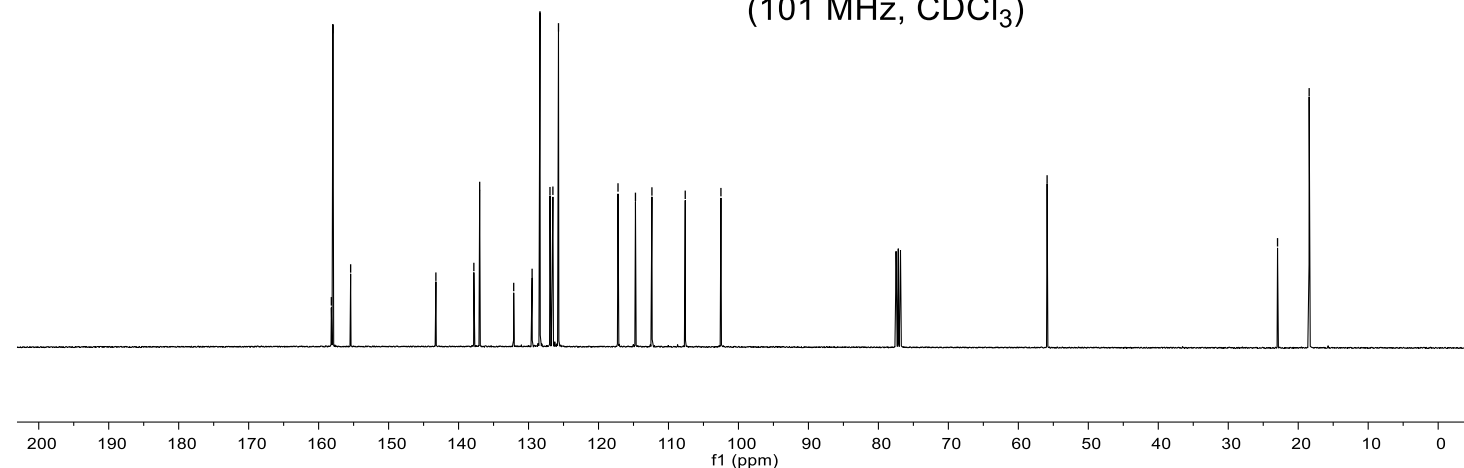

$\underbrace{\infty}_{\infty}$
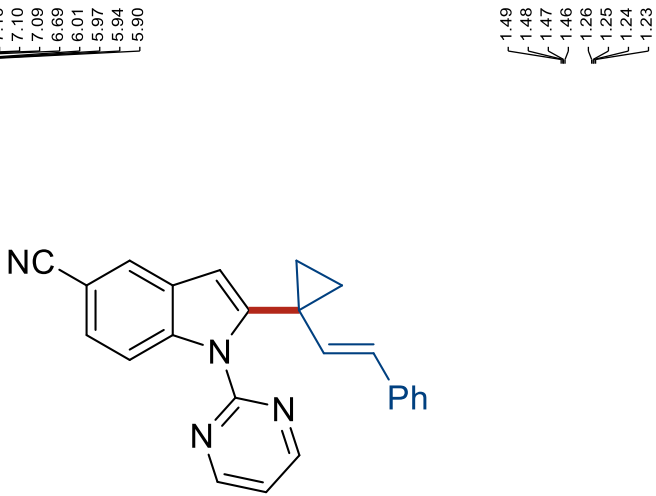

186ja

$\left(400 \mathrm{MHz}, \mathrm{CDCl}_{3}\right.$ )

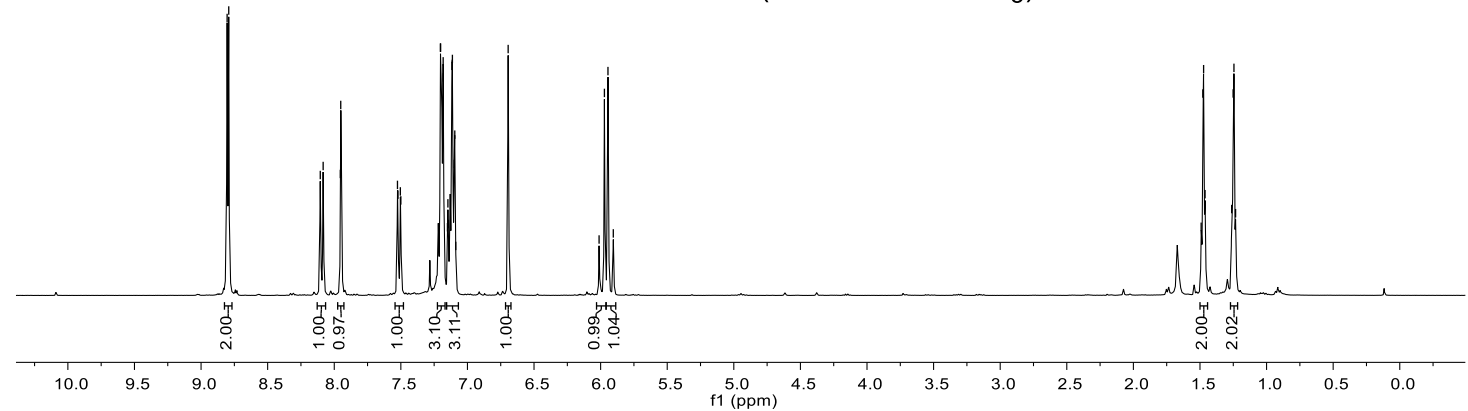




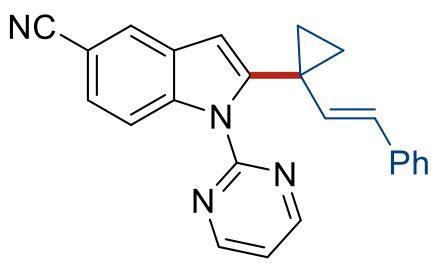

$186 \mathrm{ja}$

$\left(101 \mathrm{MHz}^{\mathrm{CDCl}}{ }_{3}\right)$
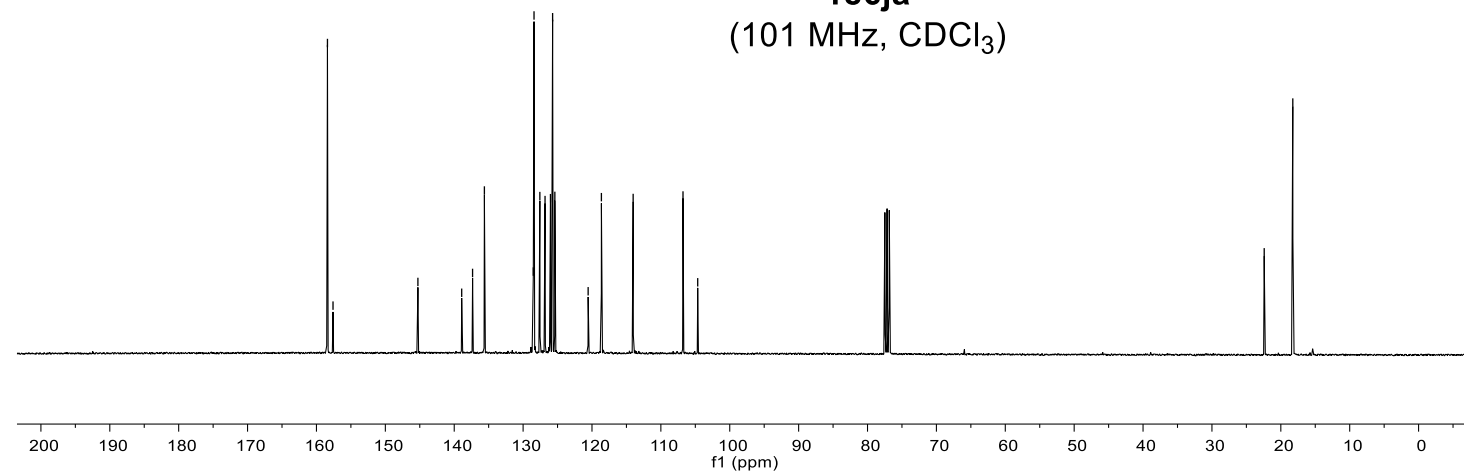

$\underbrace{\infty}$

赵

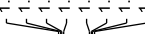

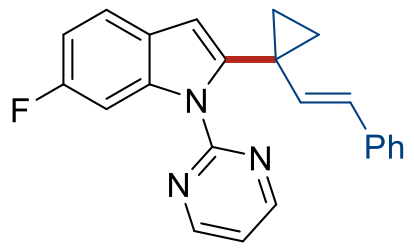

186ka

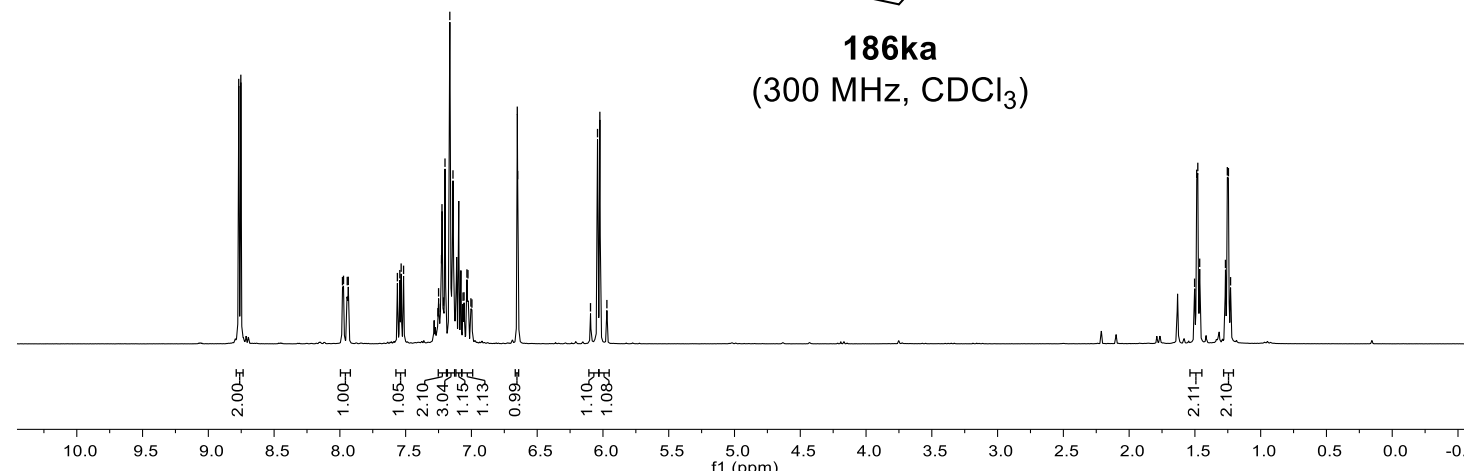



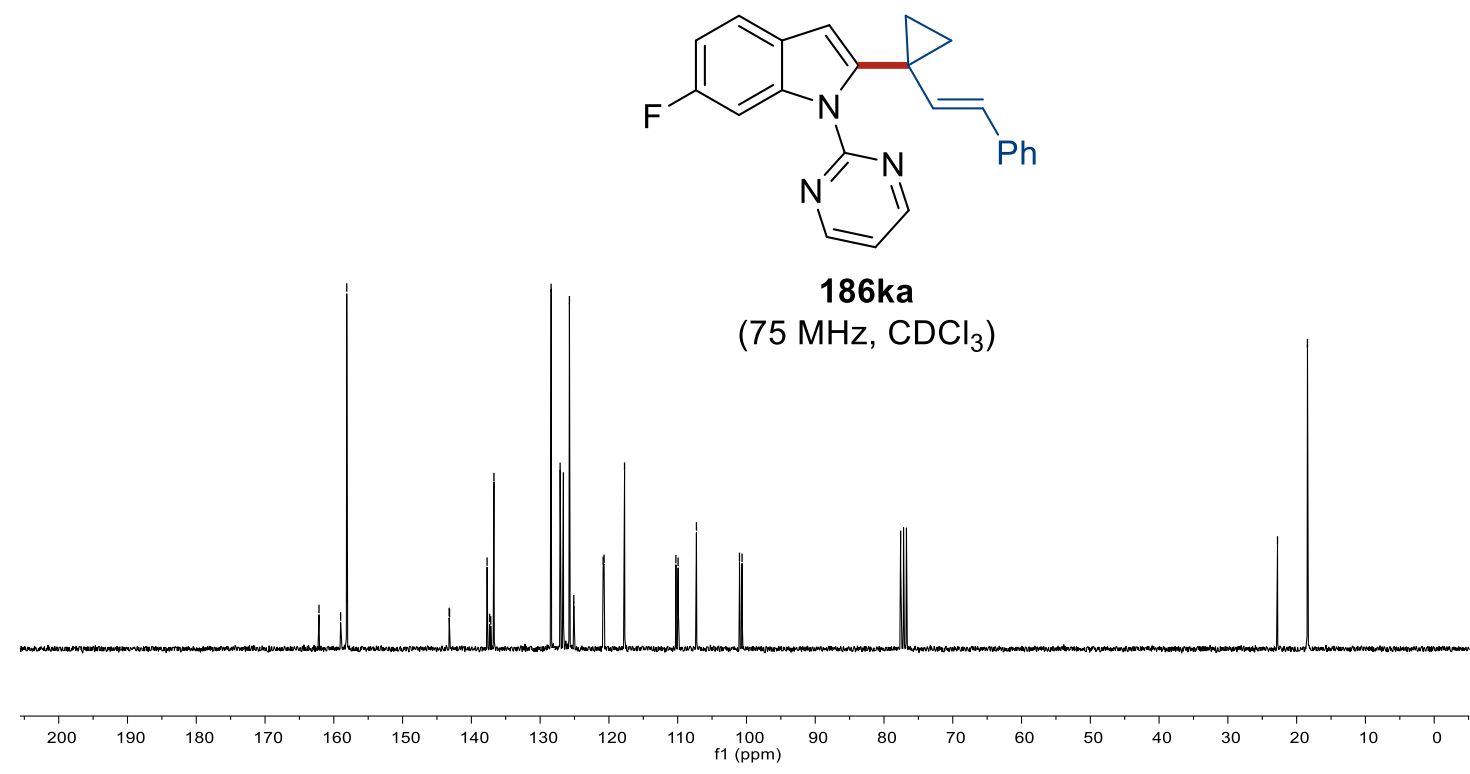

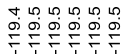

ن<smiles>Fc1ccc2cc(C3(/C=C/c4ccccc4)CC3)n(-c3ncccn3)c2c1</smiles>

$186 \mathrm{ka}$

$\left(282 \mathrm{MHz}, \mathrm{CDCl}_{3}\right)$

$\begin{array}{llllllllllllllllllllllllllll}-70 & -75 & -80 & -85 & -90 & -95 & -100 & -105 & -110 & -115 & -120 & -125 & -130 & -135 & -140 & -145 & -150 & -155 & -160 & -165 & -17\end{array}$ 
$\underbrace{\infty}$

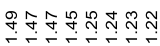<smiles>Clc1ccc2cc(C3(/C=C/c4ccccc4)CC3)n(-c3ncccn3)c2c1</smiles>

186la

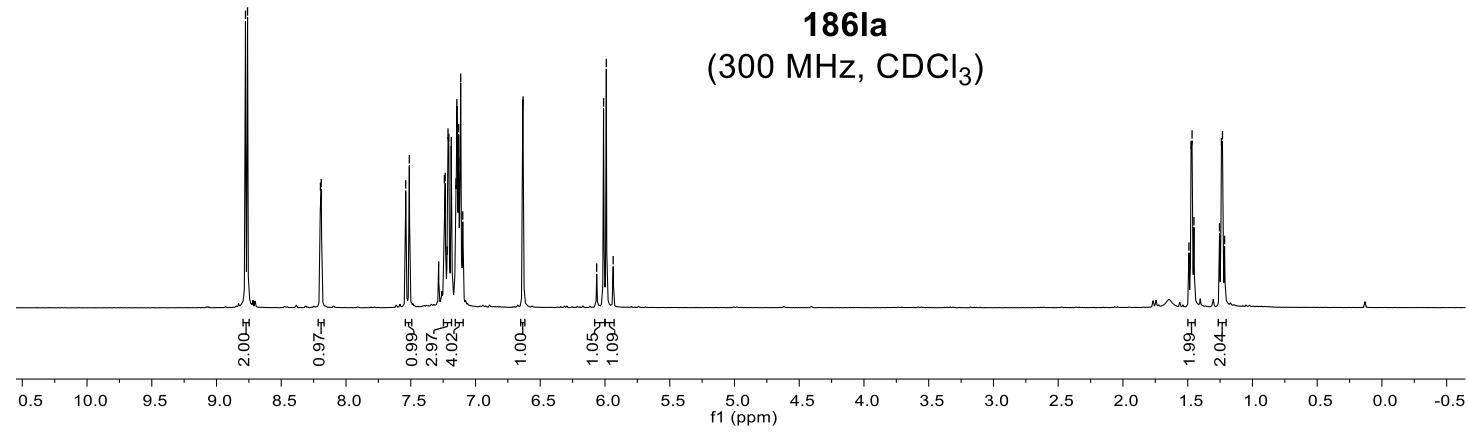

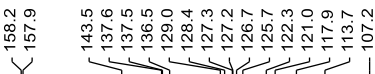

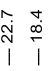

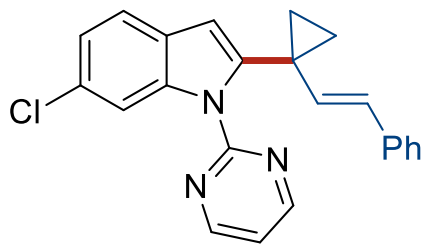

186la

(101 $\mathrm{MHz}, \mathrm{CDCl}_{3}$ )

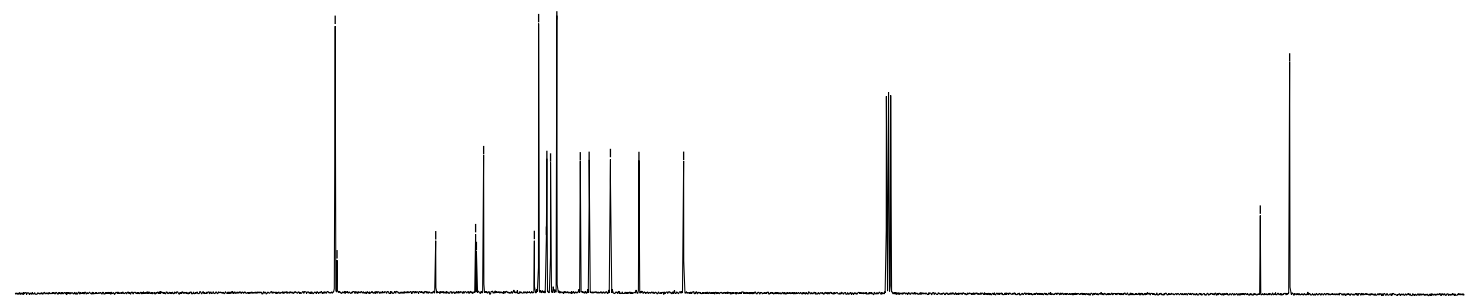

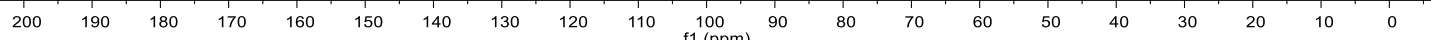




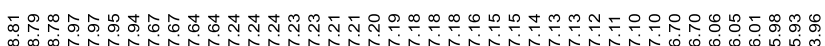

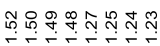

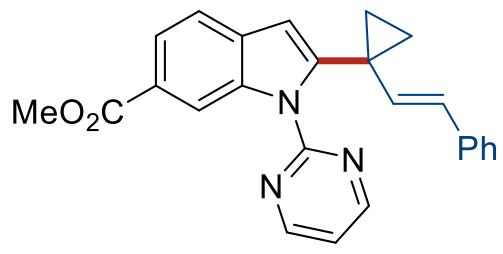

186na

(300 MHz, $\mathrm{CDCl}_{3}$ )

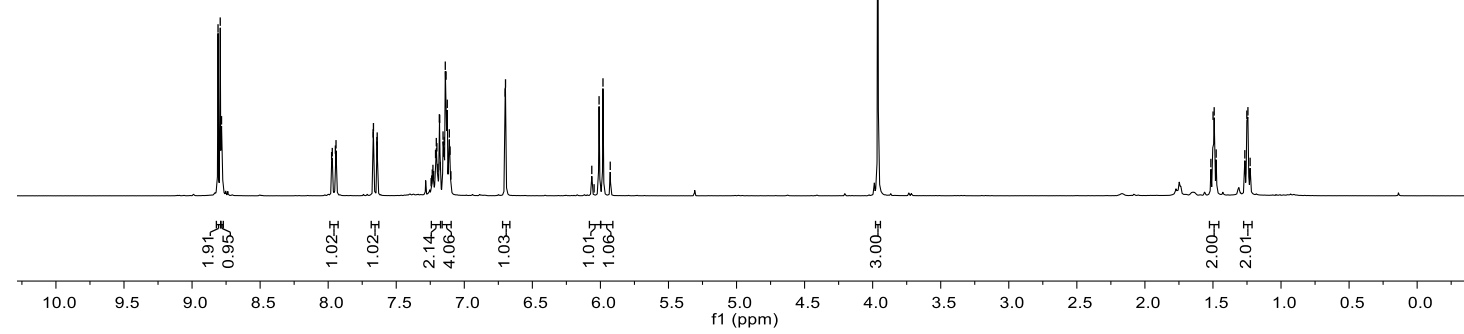

$\underbrace{1} \underbrace{\infty}_{0}$

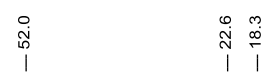

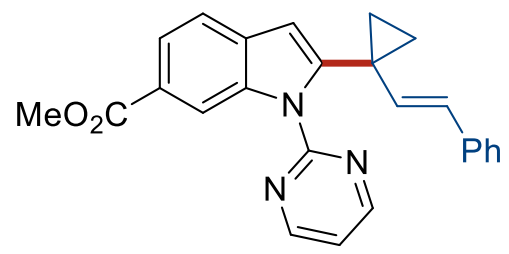

186na

$\left(101 \mathrm{MHz}, \mathrm{CDCl}_{3}\right)$

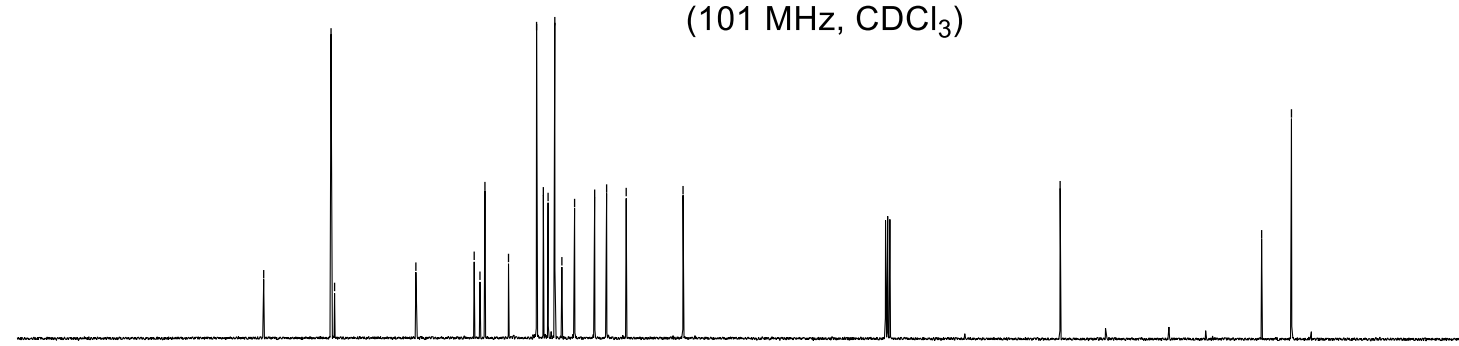

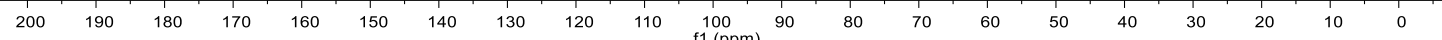




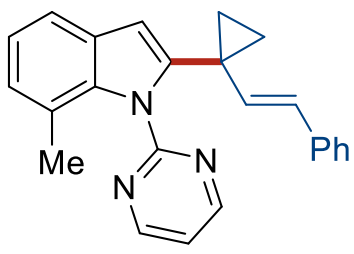

$1860 a$

(300 $\mathrm{MHz}, \mathrm{CDCl}_{3}$ )
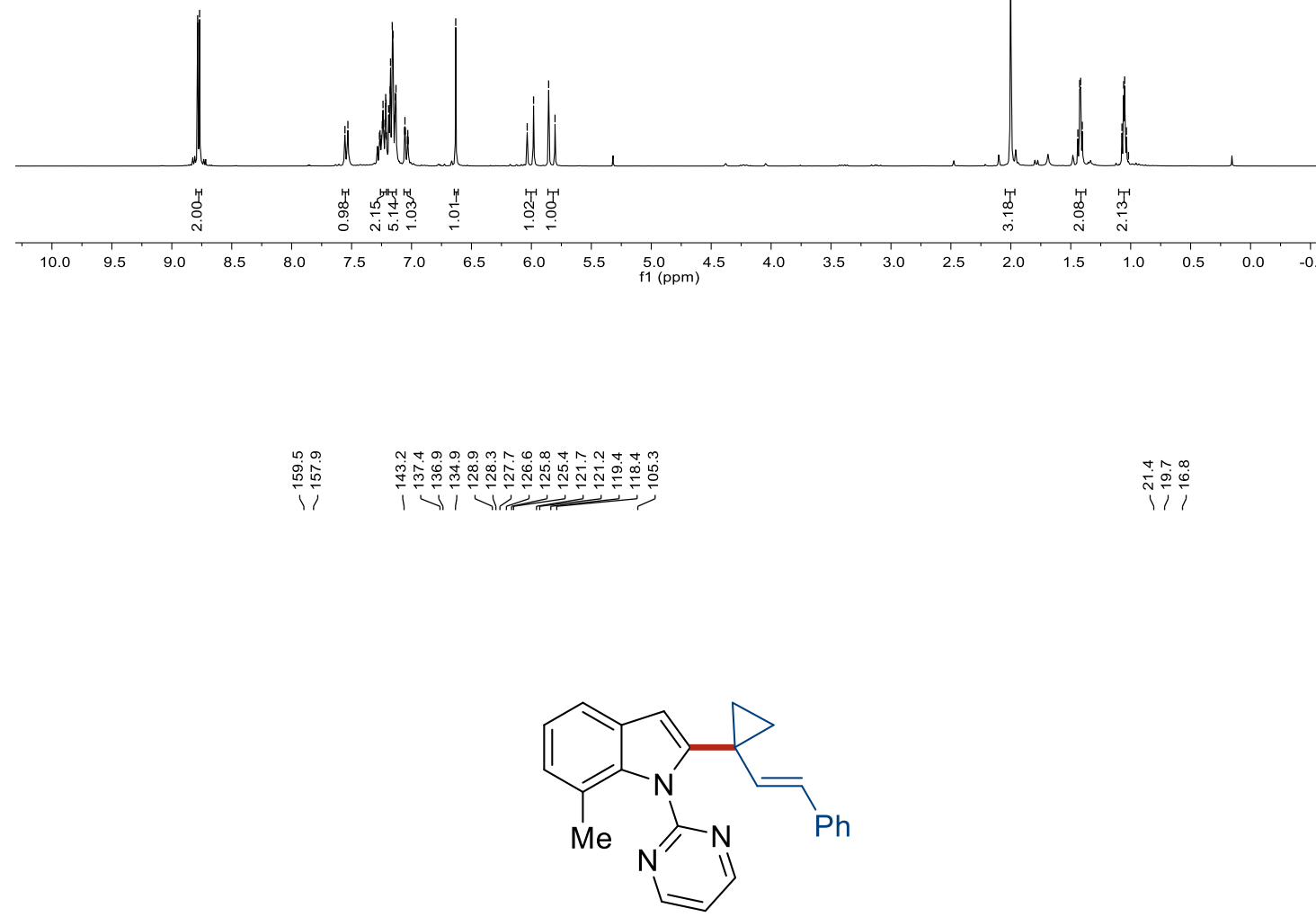

186oa

(101 $\mathrm{MHz} \mathrm{CDCl}_{3}$ )

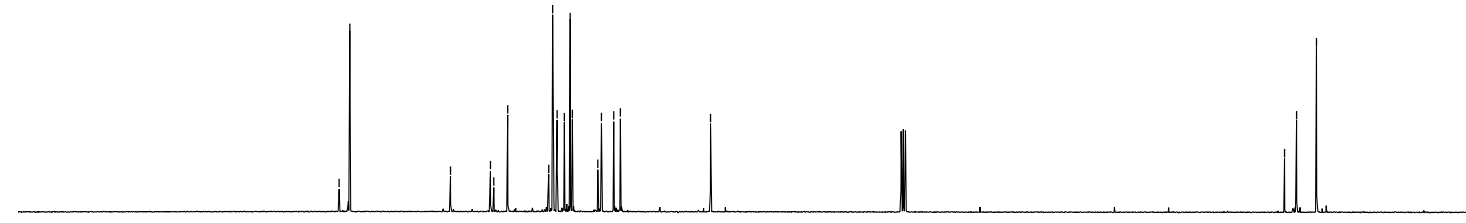

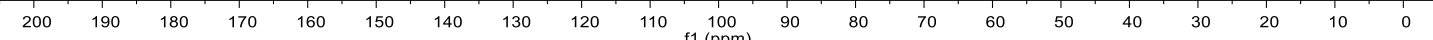




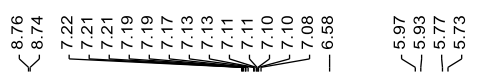

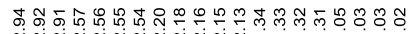

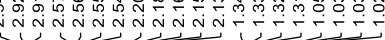<smiles>O=C1CCCc2c1cc(C1(/C=C/c3ccccc3)CC1)n2-c1ncccn1</smiles>

186pa

$\left.\left(400 \mathrm{MHz}^{\mathrm{CDCl}}\right)_{3}\right)$

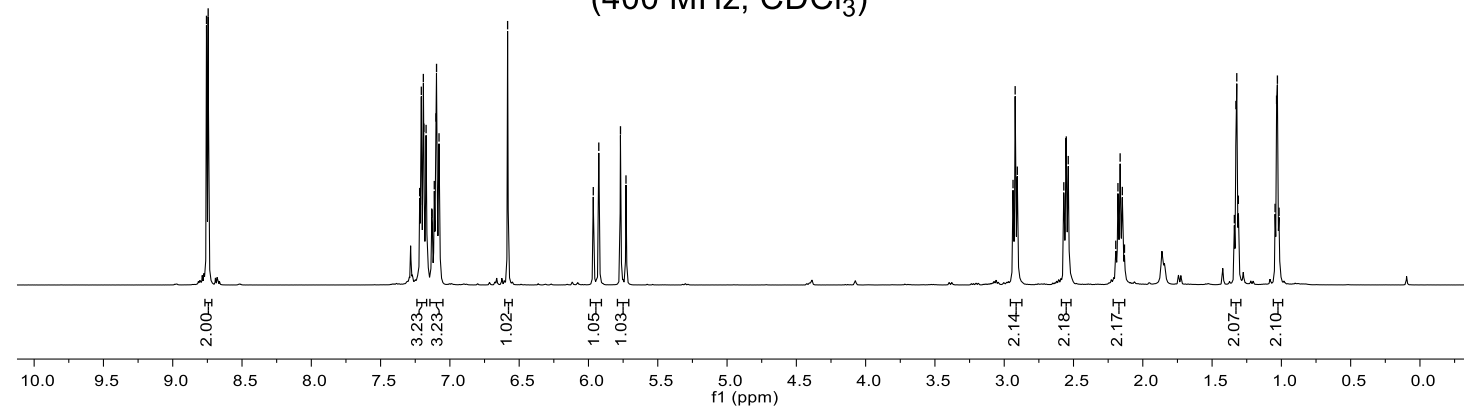

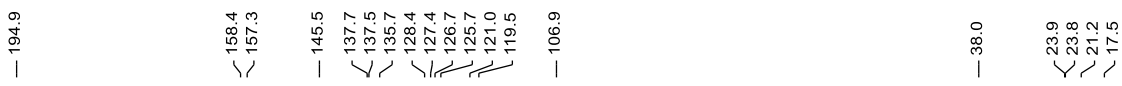<smiles>O=C1CCCc2c1cc(C1(/C=C/c3ccccc3)CC1)n2-c1ncccn1</smiles>

$186 \mathrm{pa}$

$\left(101 \mathrm{MHz}, \mathrm{CDCl}_{3}\right)$

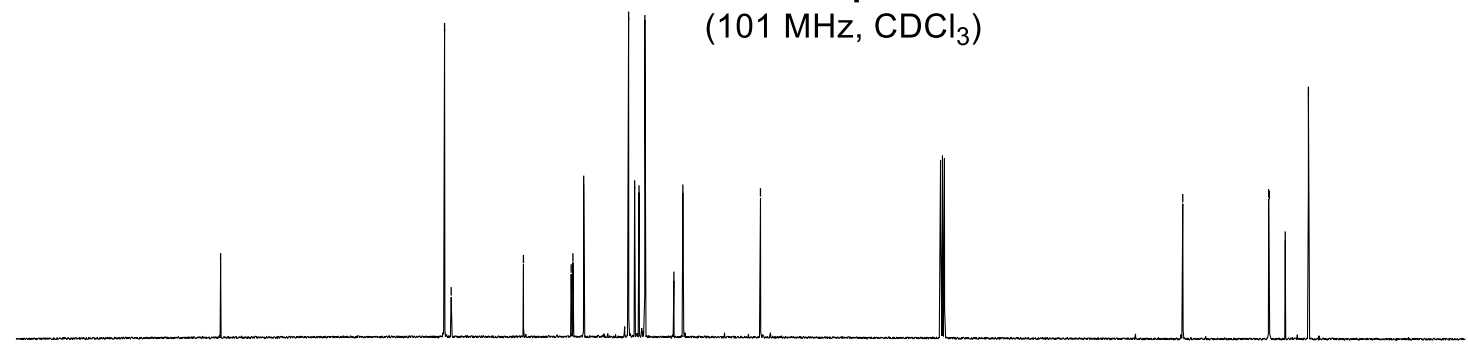

$\begin{array}{llllllllllllllllllllllllllllllll} & 1 & 210 & 200 & 190 & 180 & 170 & 160 & 150 & 140 & 130 & 120 & 110 & 100 & 90 & 80 & 70 & 60 & 50 & 40 & 30 & 20 & 10 & 0\end{array}$ 


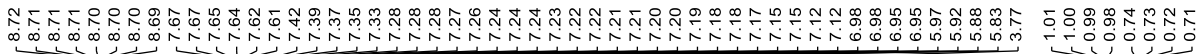

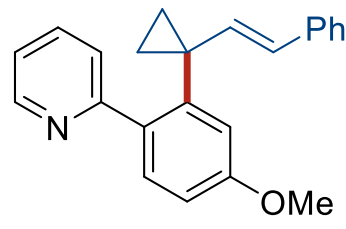

186sa

(300 $\left.\mathrm{MHz} \mathrm{CDCl}_{3}\right)$

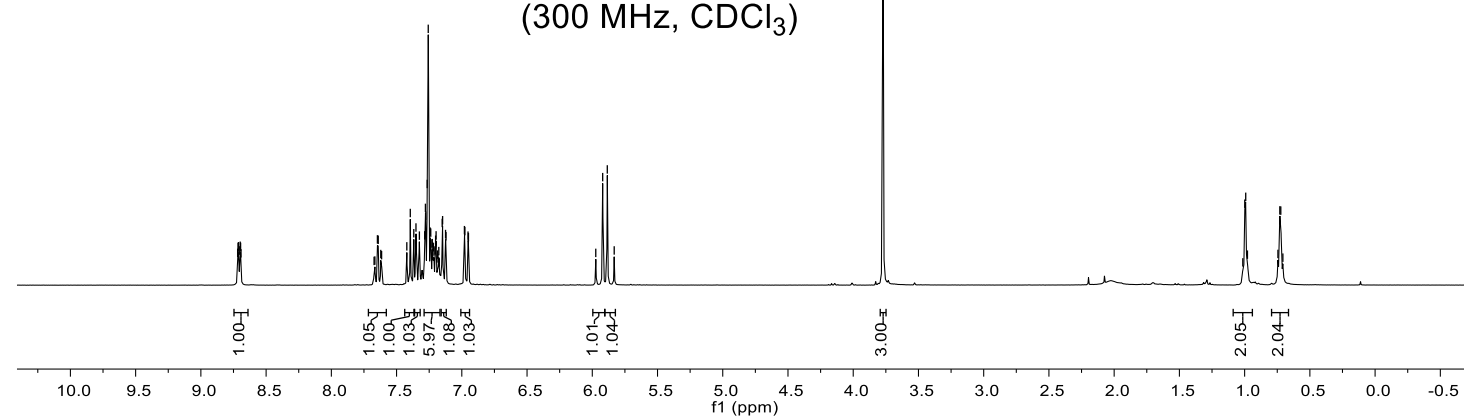

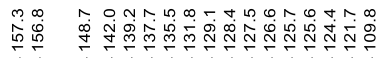

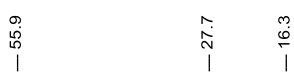

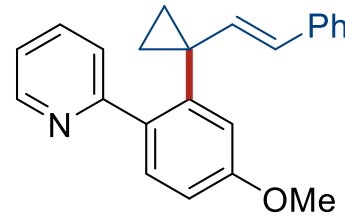

186sa

(75 MHz, $\mathrm{CDCl}_{3}$ )

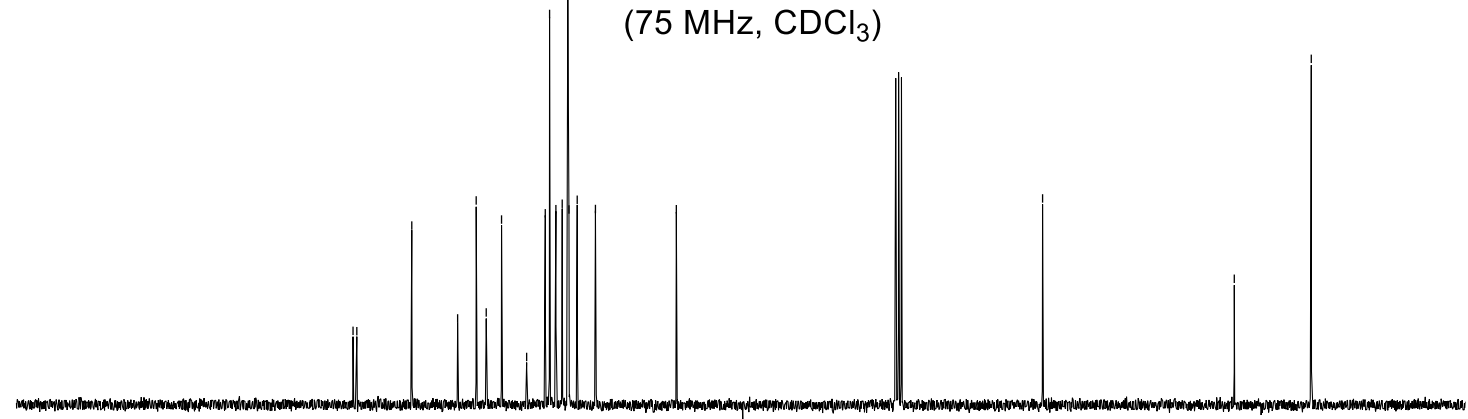

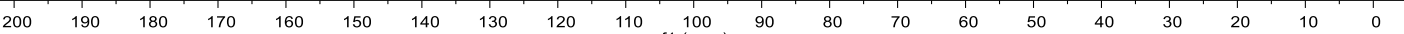


$\bigcup_{\infty}^{\infty}$

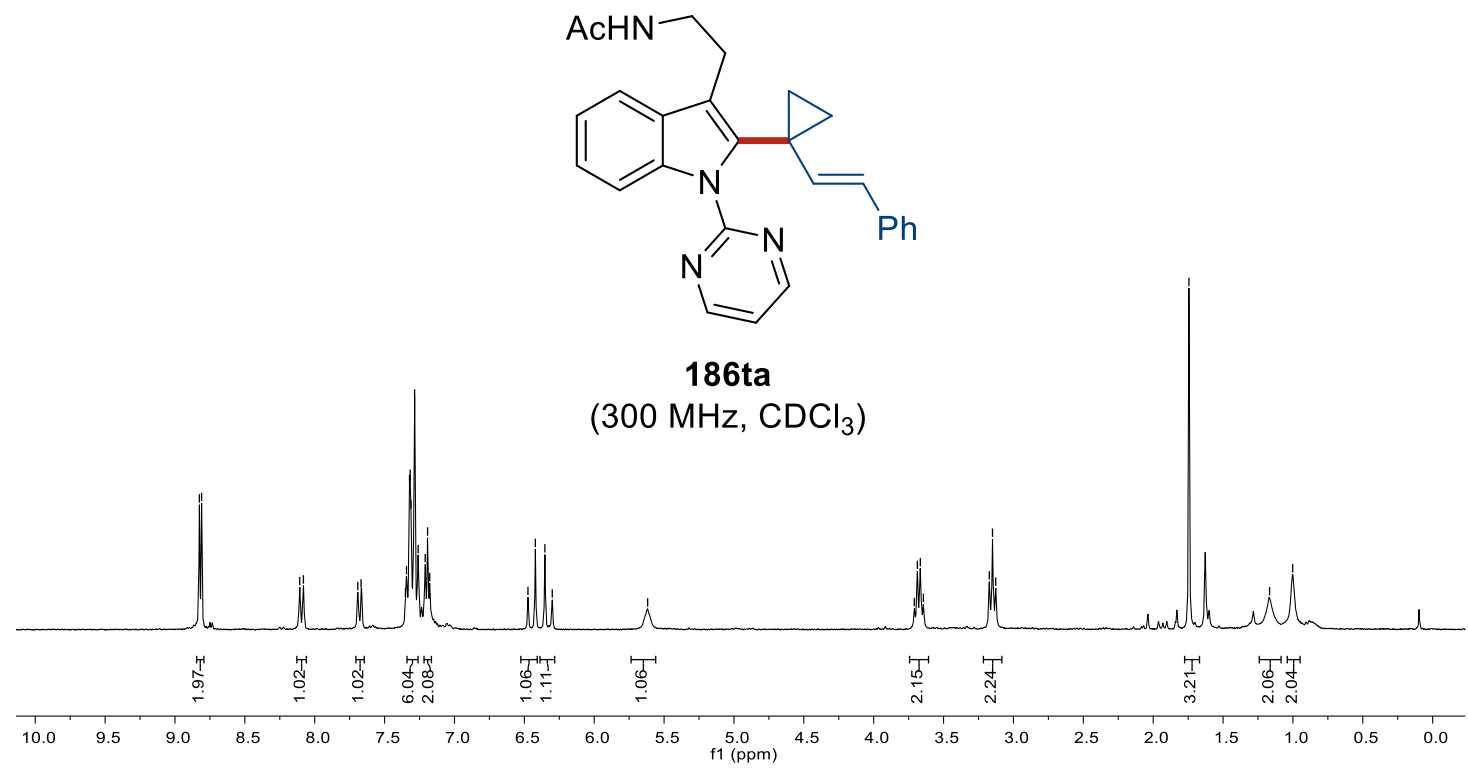

i V V

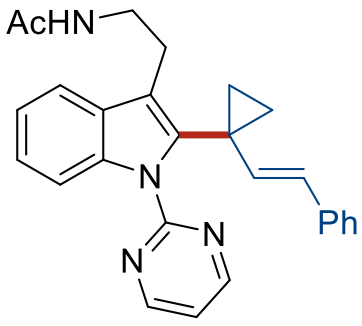

186ta

$\left(101 \mathrm{MHz}^{\mathrm{CDCl}} 3\right.$ )

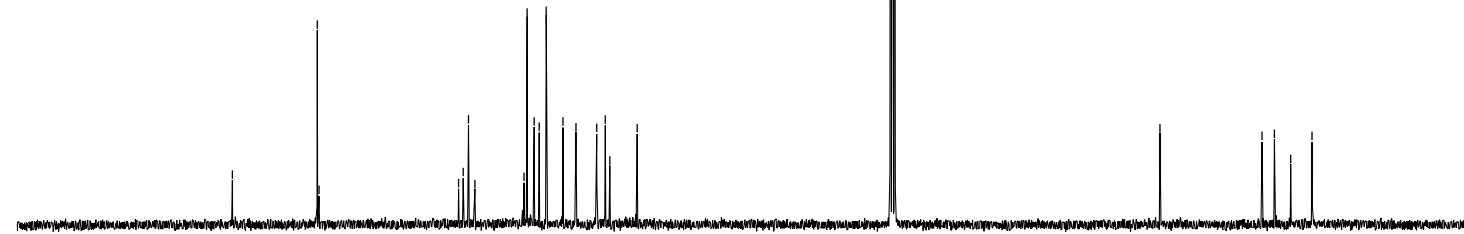

$\begin{array}{llllllllllllllllllllllllll}0 & 190 & 180 & 170 & 160 & 150 & 140 & 130 & 120 & 110 & 100 & 90 & 80 & 70 & 60 & 50 & 40 & 30 & 20 & 10 & 0\end{array}$ 
$\underbrace{\substack{n \\ 0.0}}$

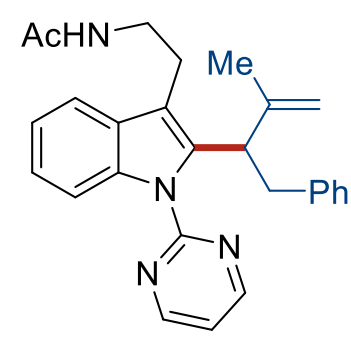

186ta'

$\left.\left(300 \mathrm{MHz}^{\mathrm{CDCl}}\right)_{3}\right)$

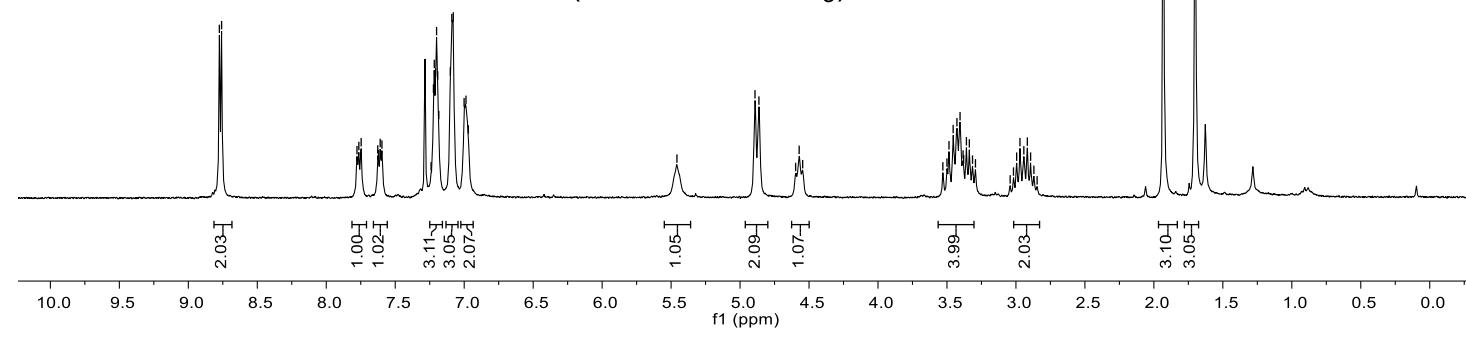

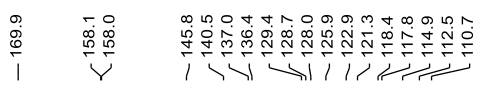

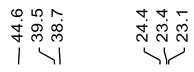

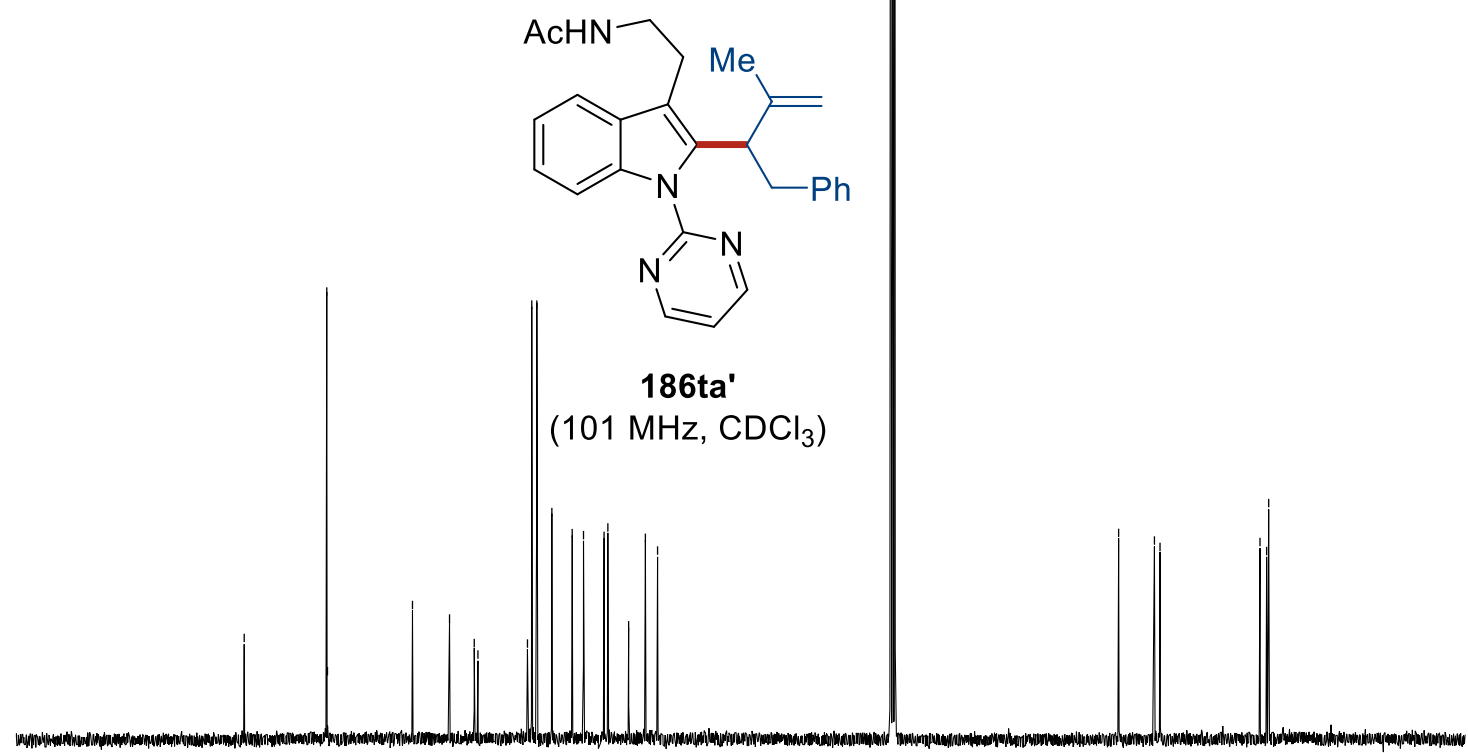

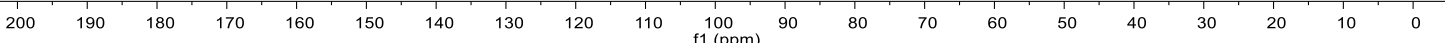


铍

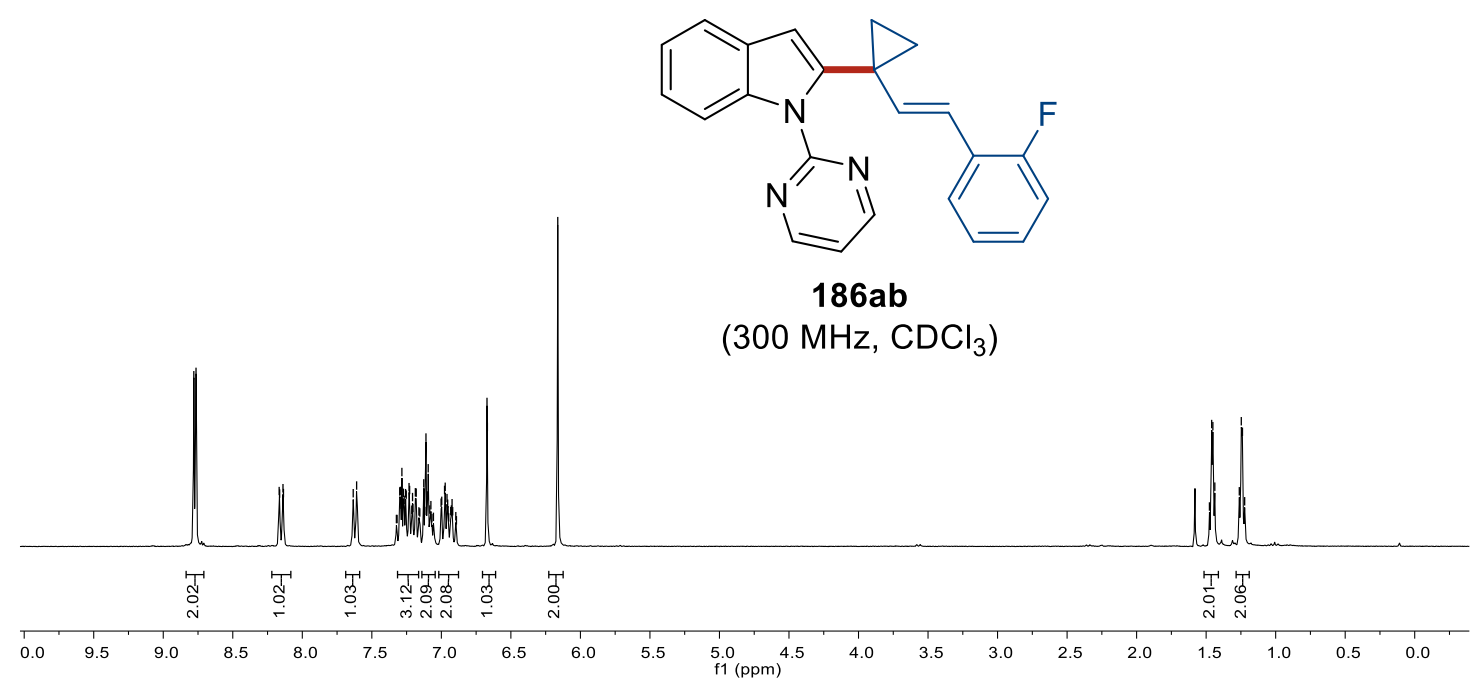

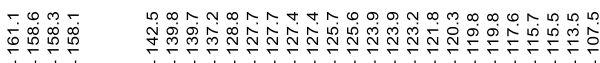

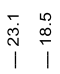

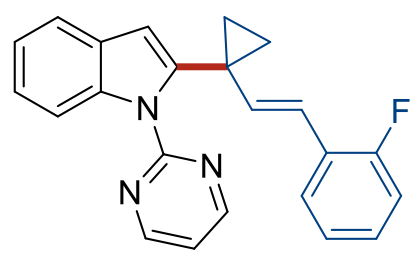

$186 a b$

(101 MHz, $\mathrm{CDCl}_{3}$ )

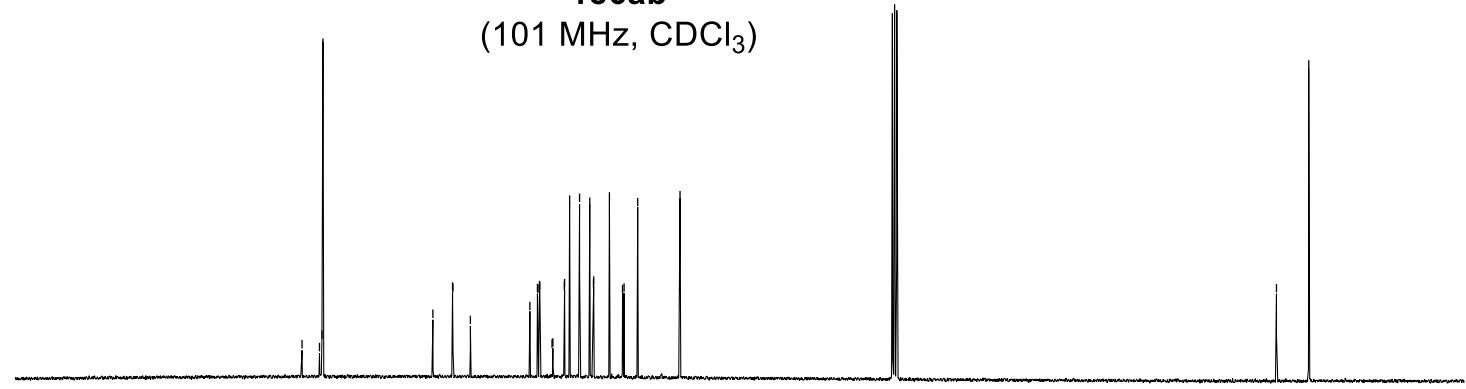

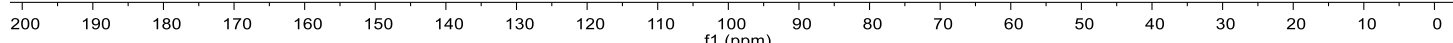



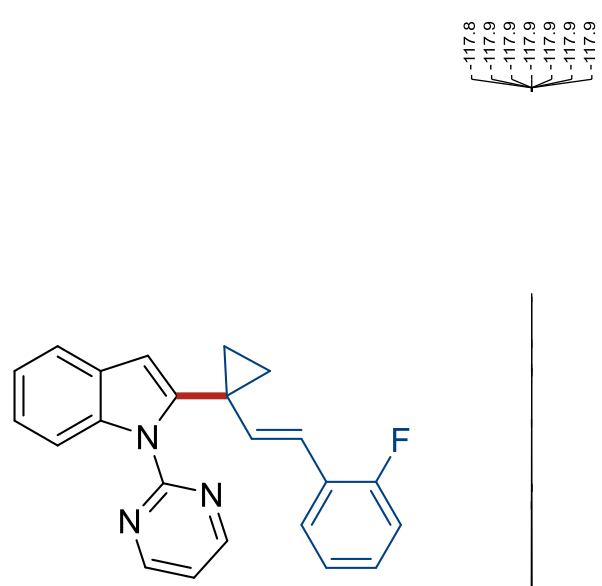

$186 a b$

(377 MHz, $\mathrm{CDCl}_{3}$ )

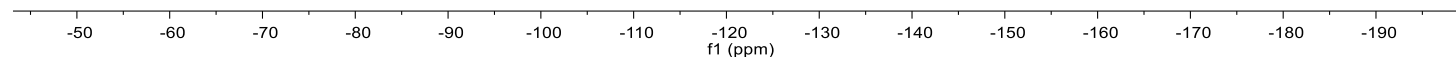

$\underbrace{\infty}$

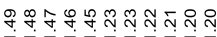

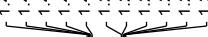

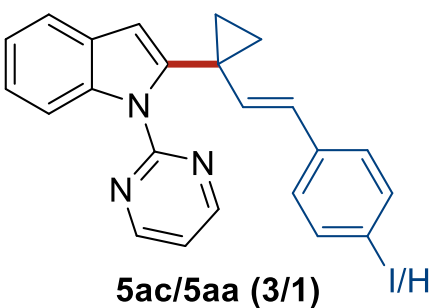

(400 $\mathrm{MHz} \mathrm{CDCl}_{3}$ )

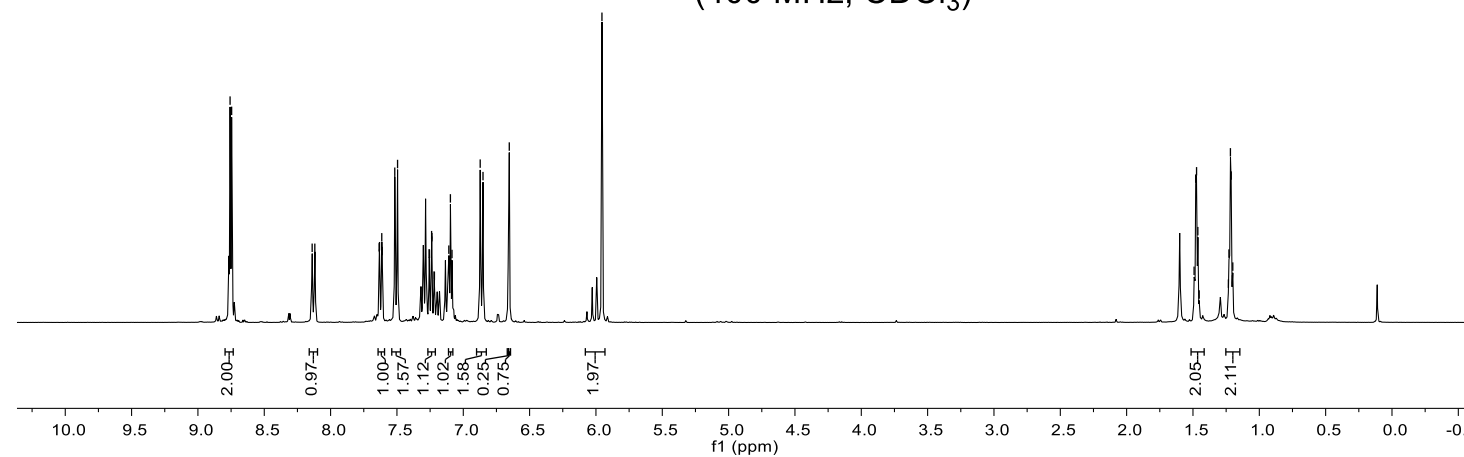




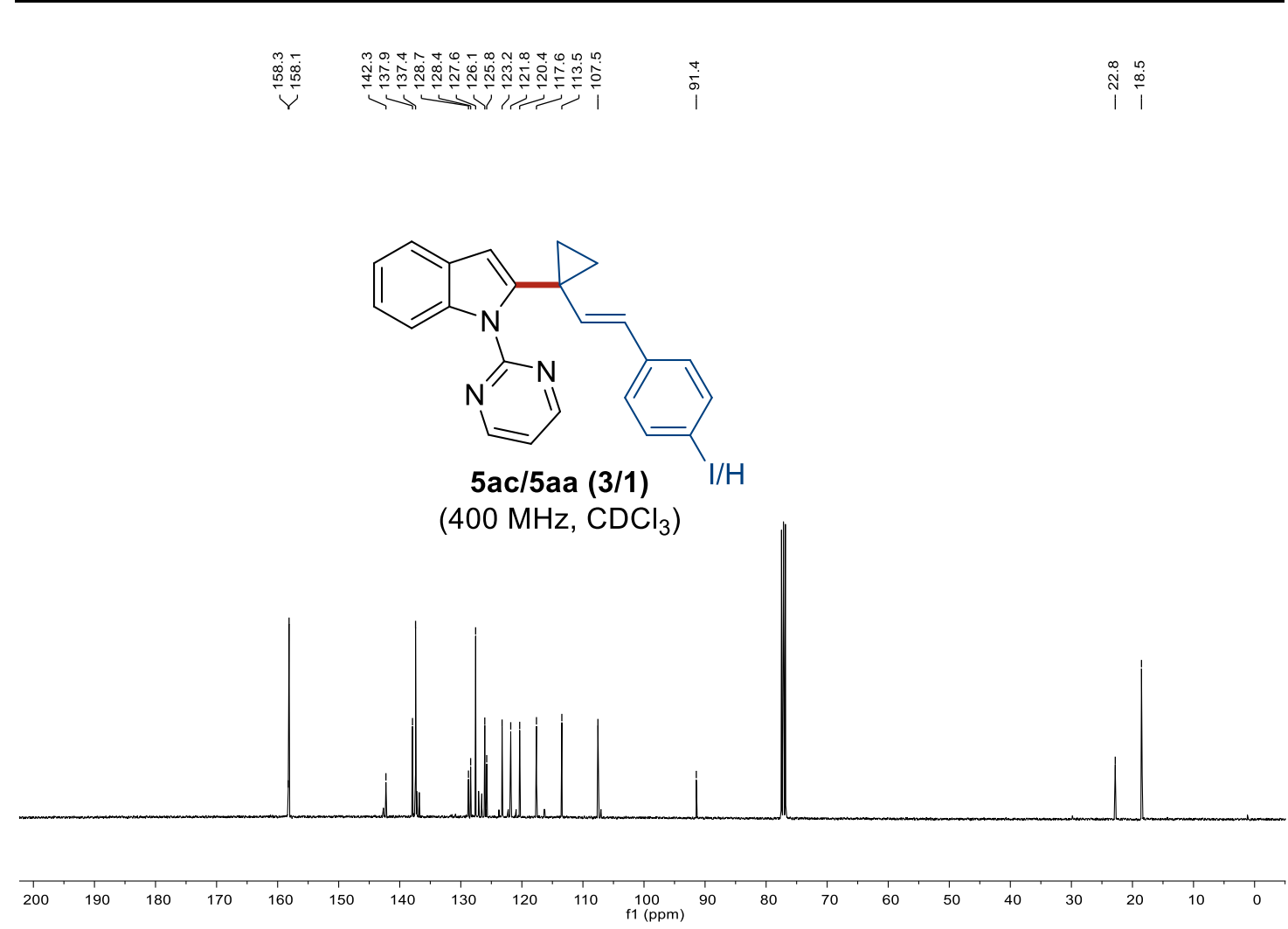

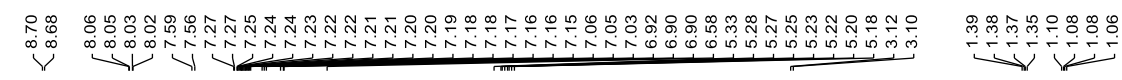

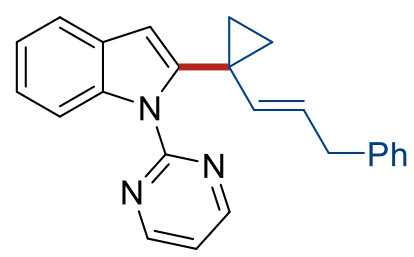

186ad

(300 MHz, $\mathrm{CDCl}_{3}$ )

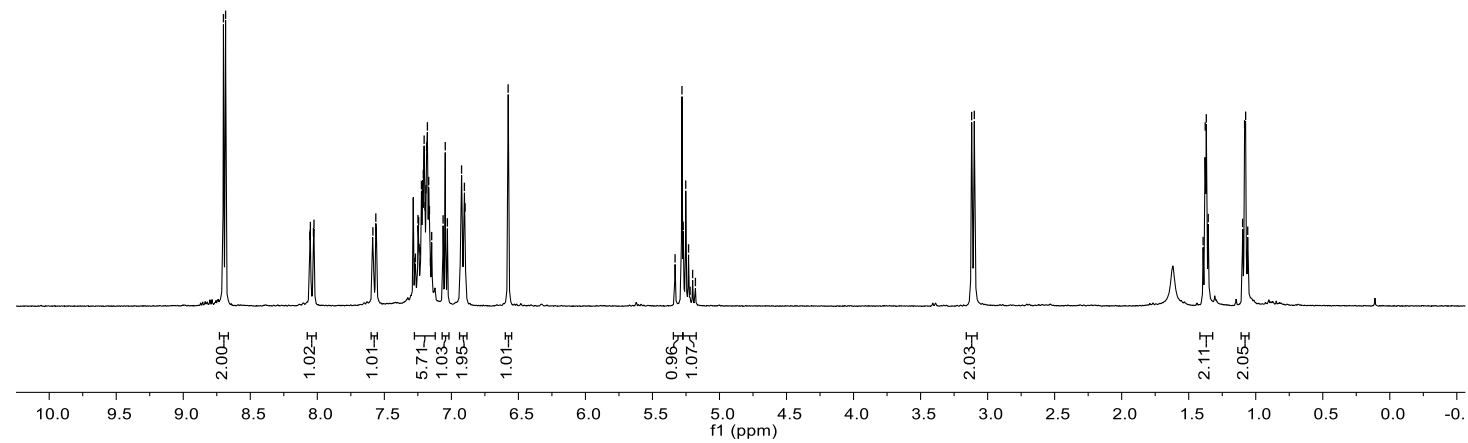




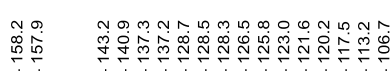

$\underbrace{1 / 1}$

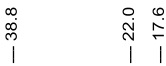

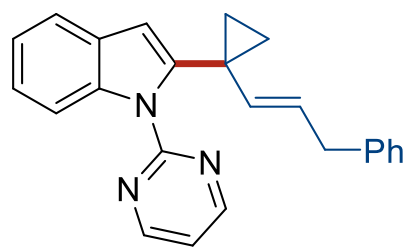

$186 \mathrm{ad}$

(101 MHz, $\mathrm{CDCl}_{3}$ )

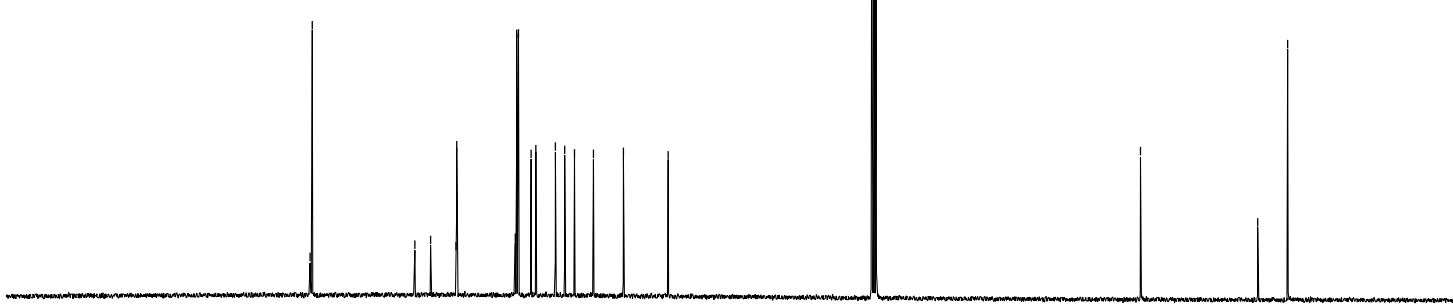

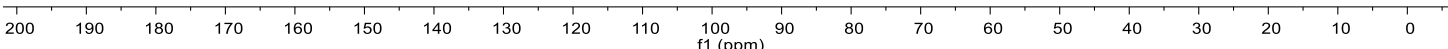

V)

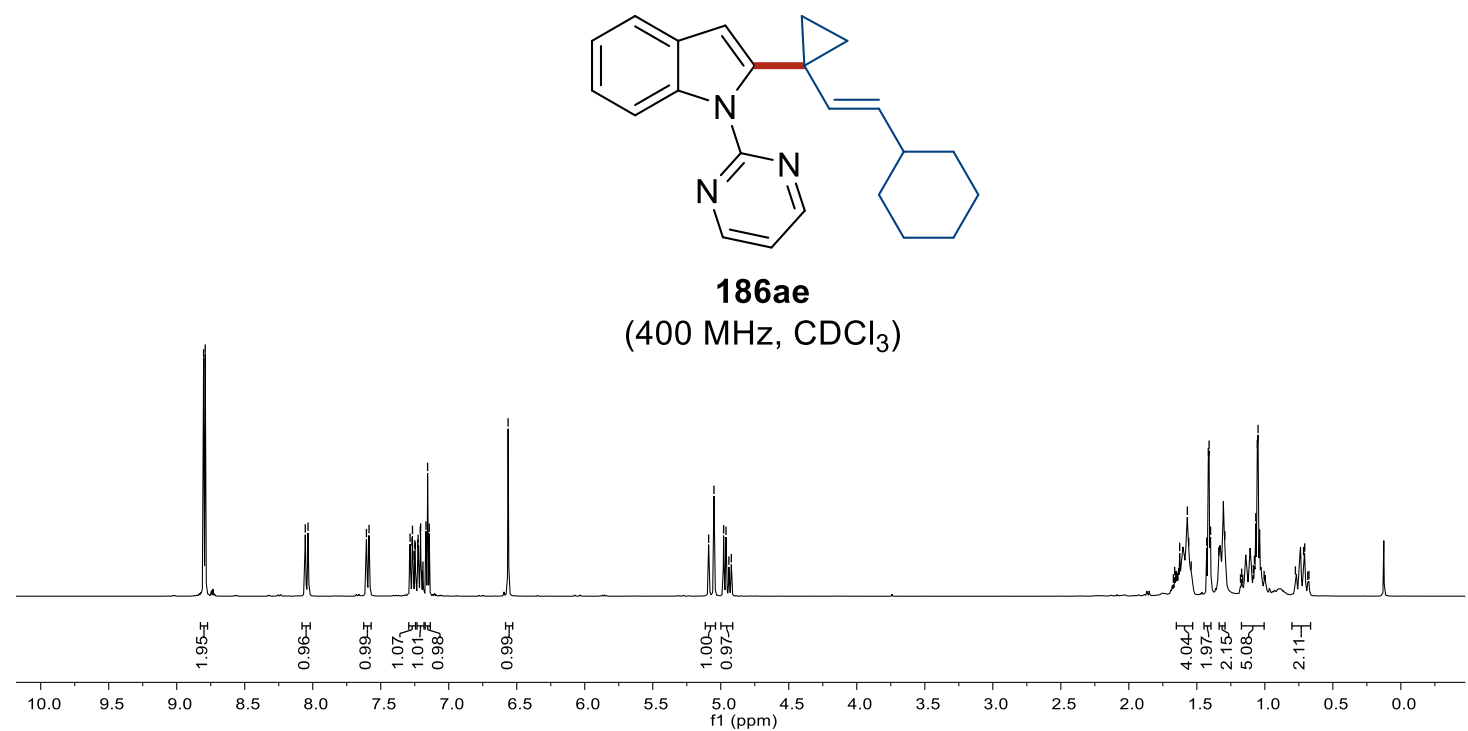



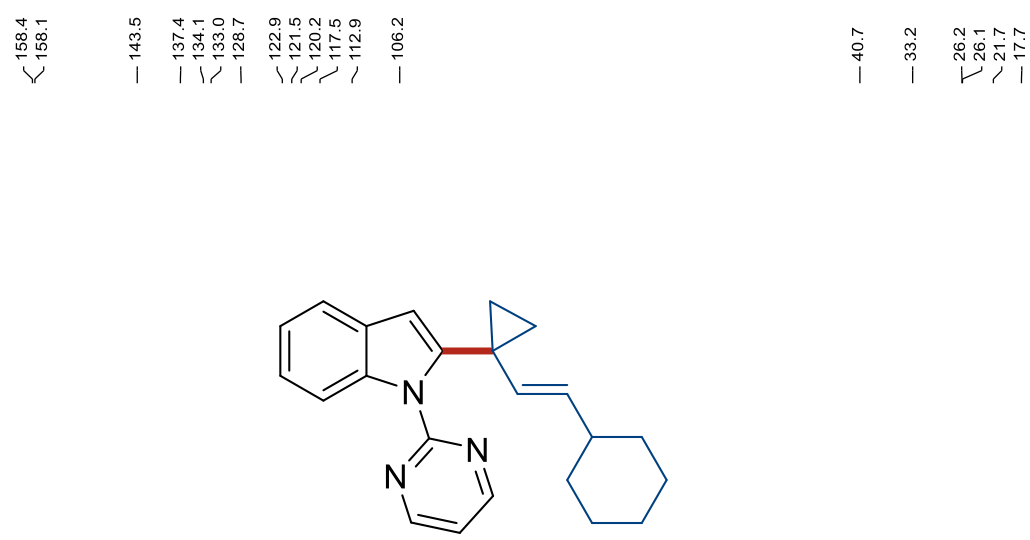

186ae

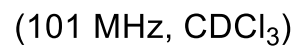
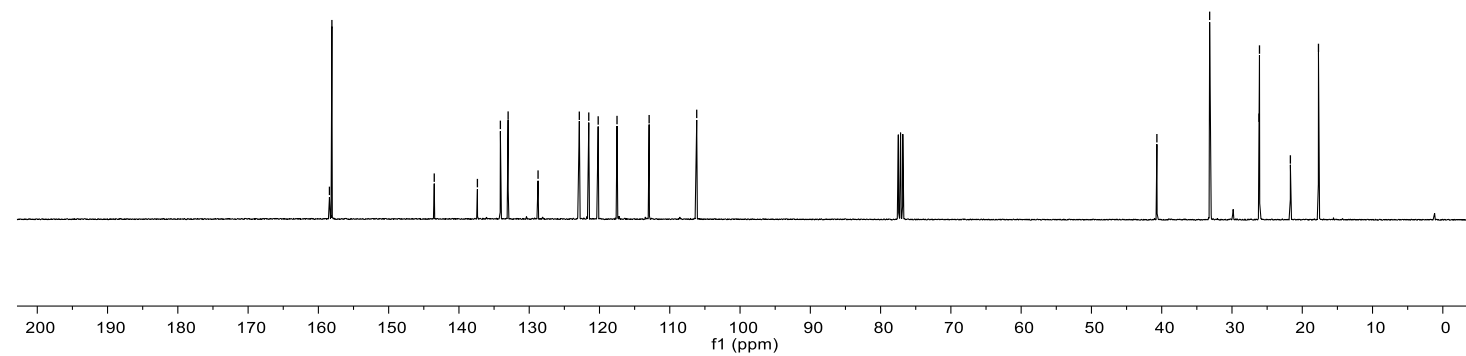

象象

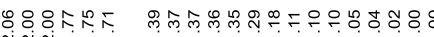

$V^{\infty} \underbrace{\infty}$

4

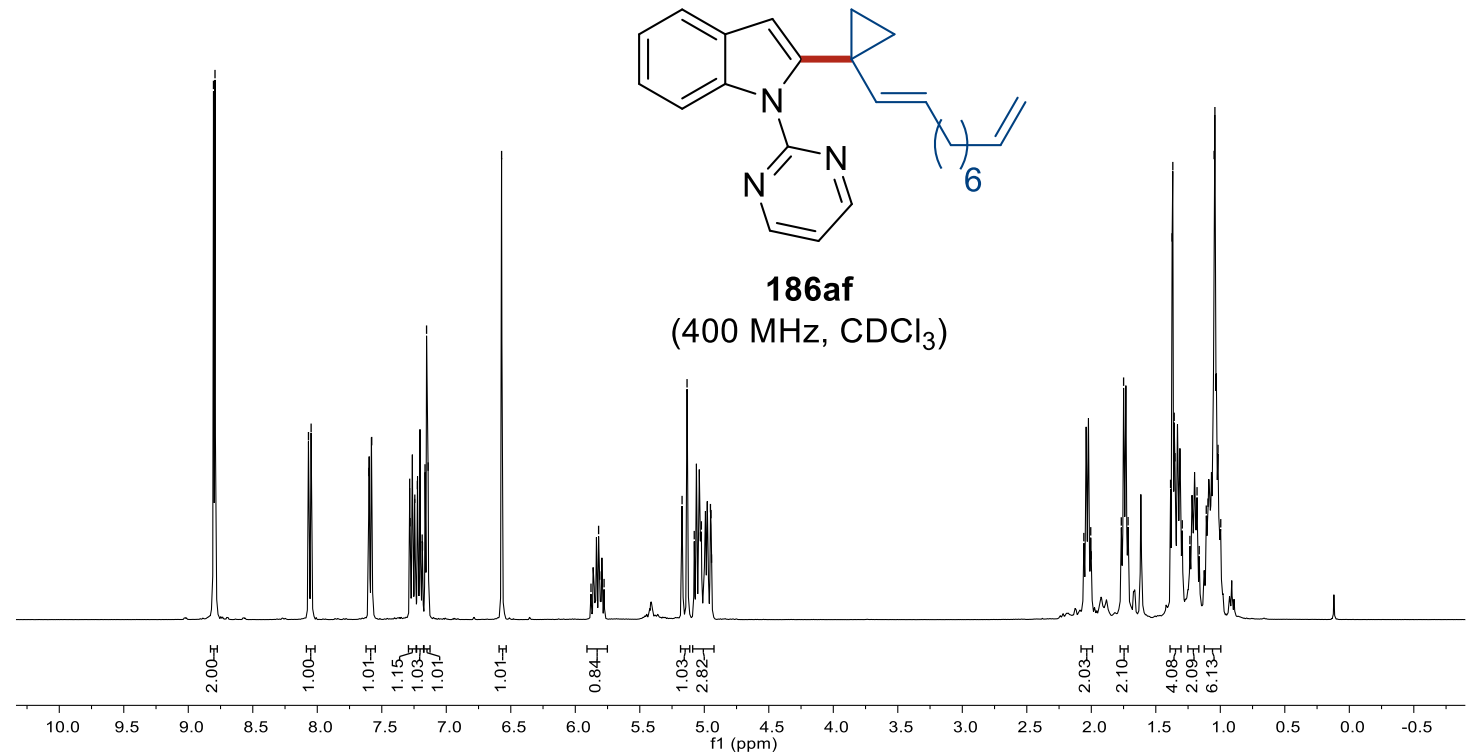




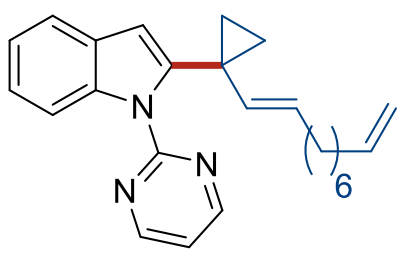

186af

$\left.\left(101 \mathrm{MHz}^{\mathrm{CDCl}}\right)_{3}\right)$

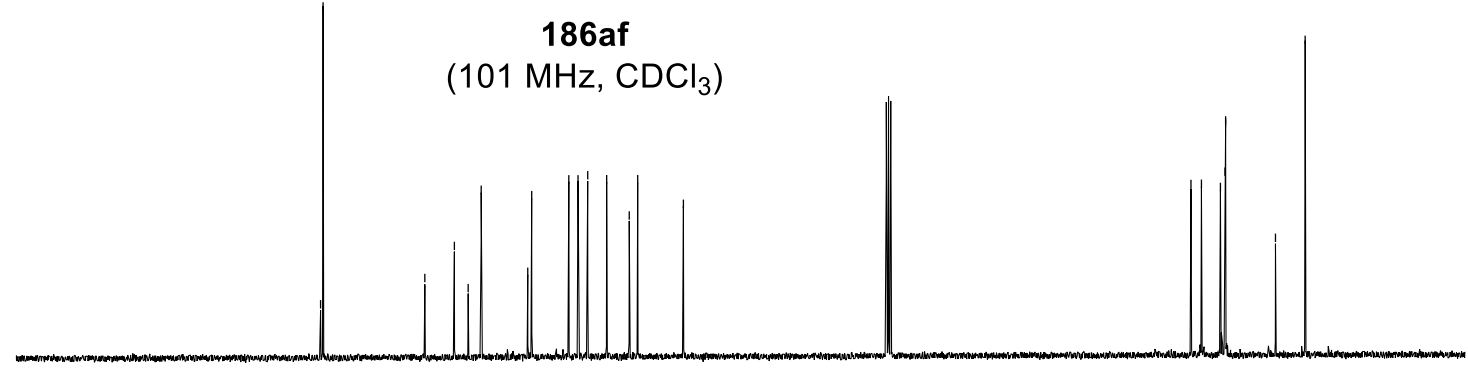

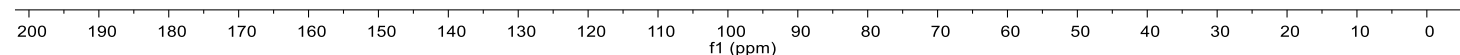

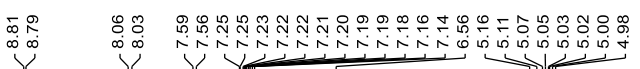

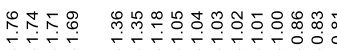

$\Rightarrow$ ए

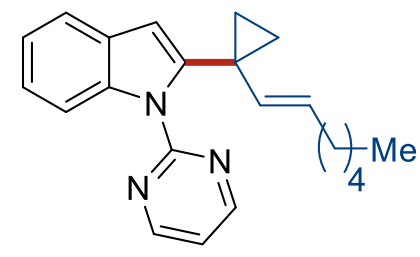

186ag

(300 MHz, $\mathrm{CDCl}_{3}$ )

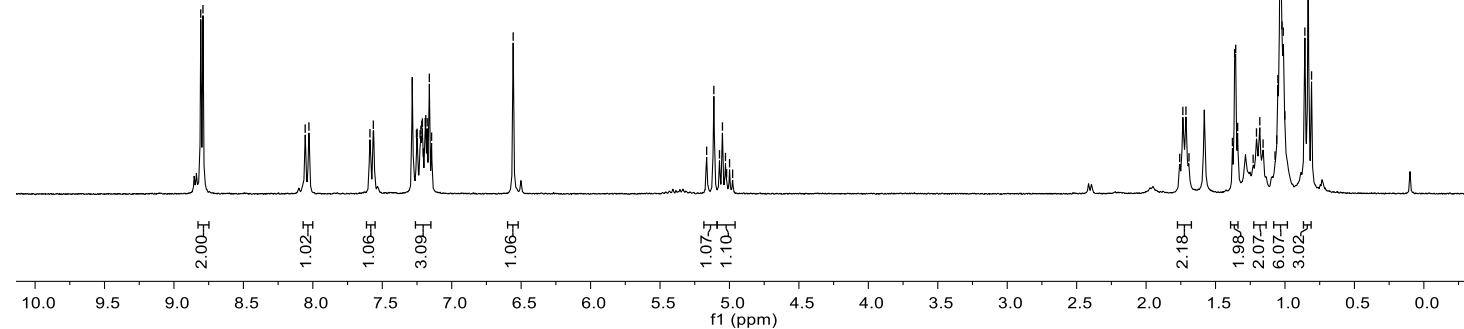




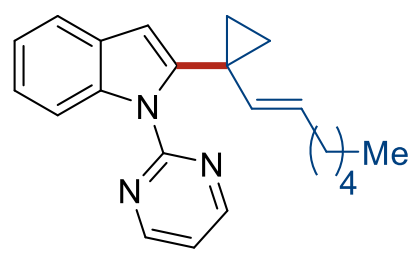

186ag

$\left(101 \mathrm{MHz}^{\mathrm{CDCl}}{ }_{3}\right)$

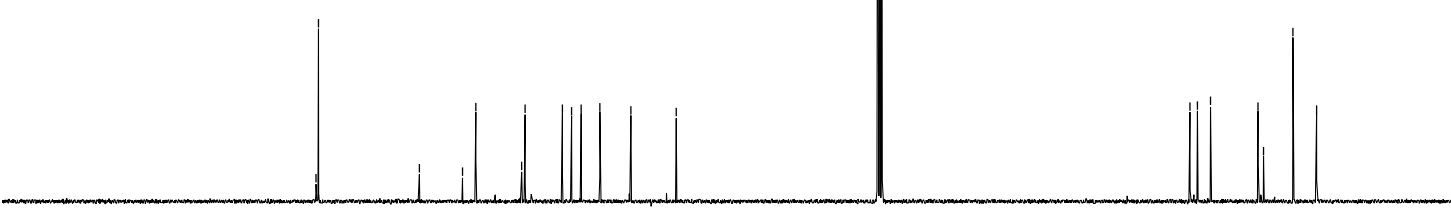

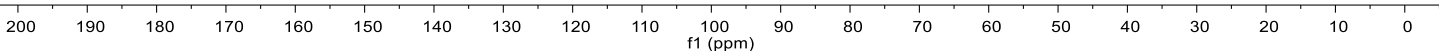
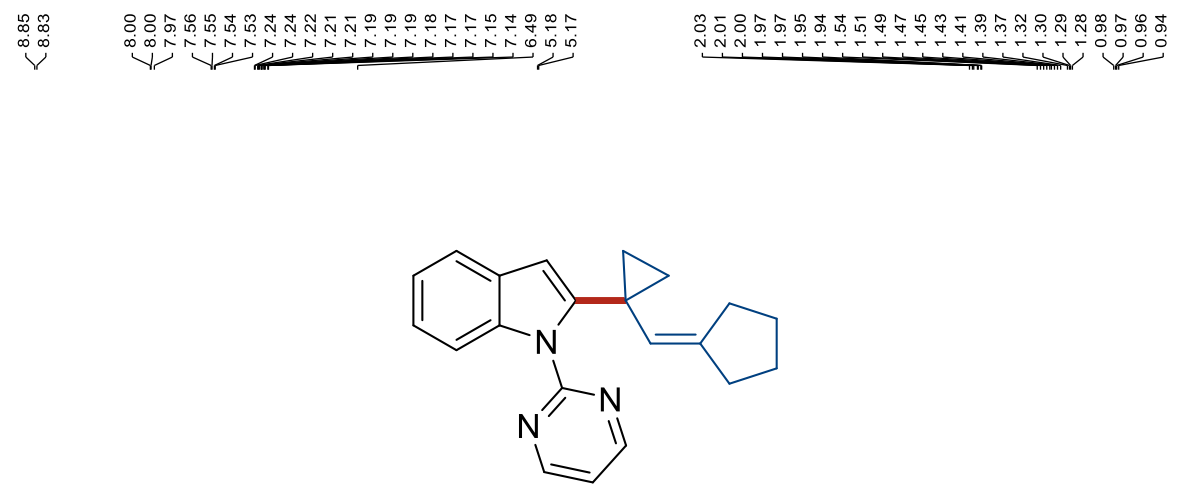

186ah

(300 MHz, $\mathrm{CDCl}_{3}$ )

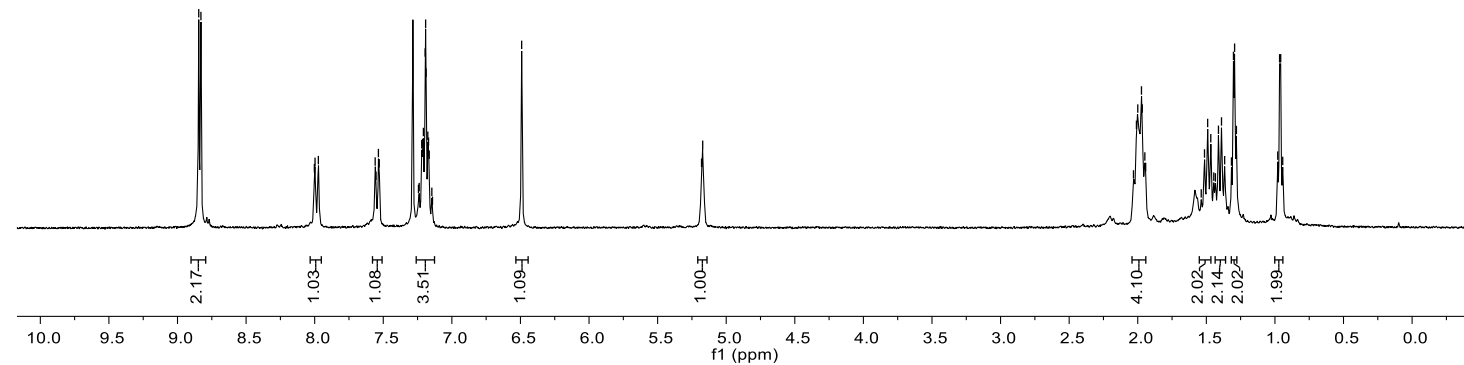




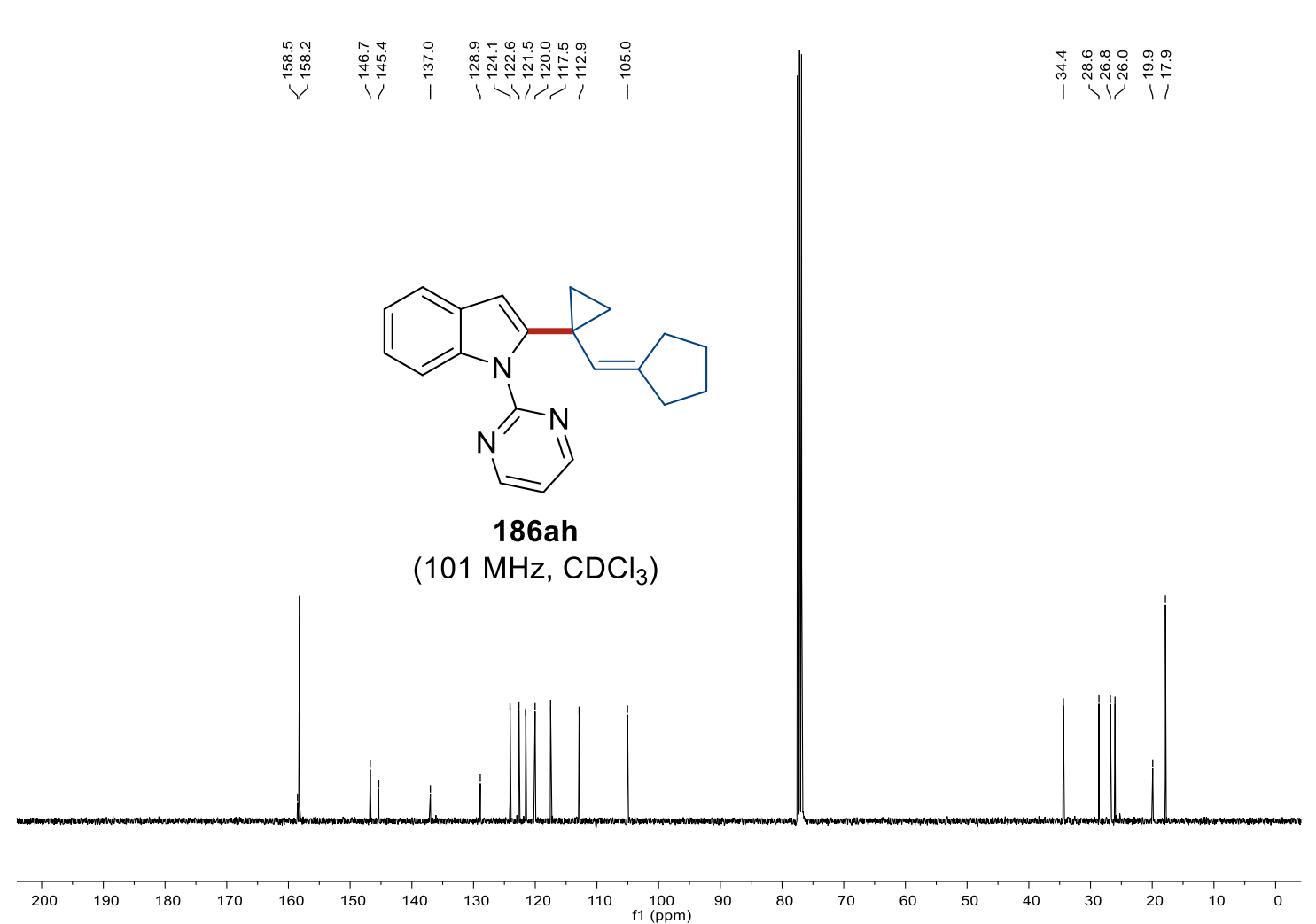

Y

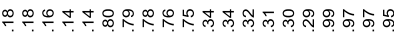

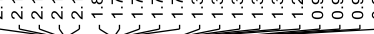

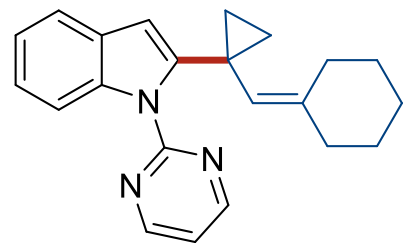

186aj

(300 MHz, $\mathrm{CDCl}_{3}$ )

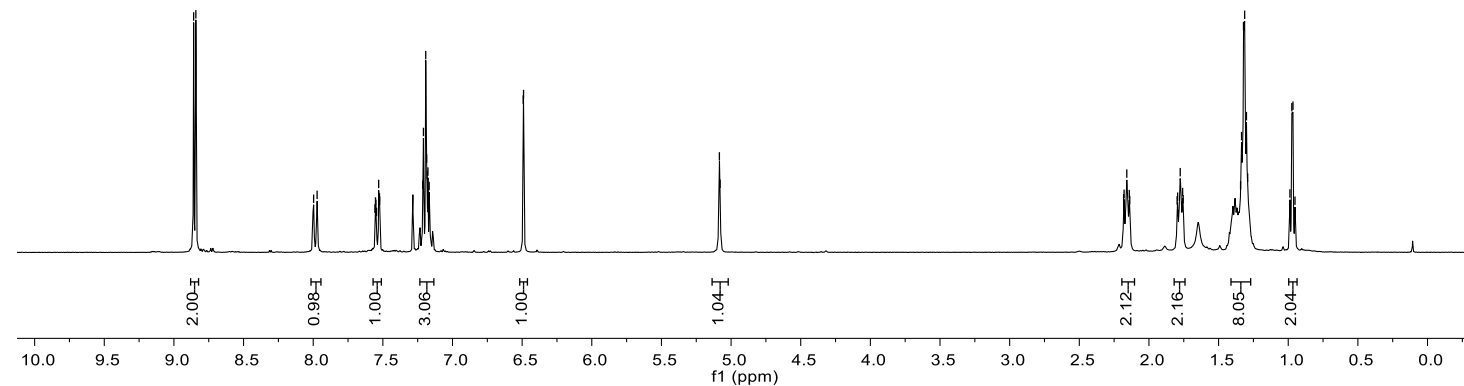



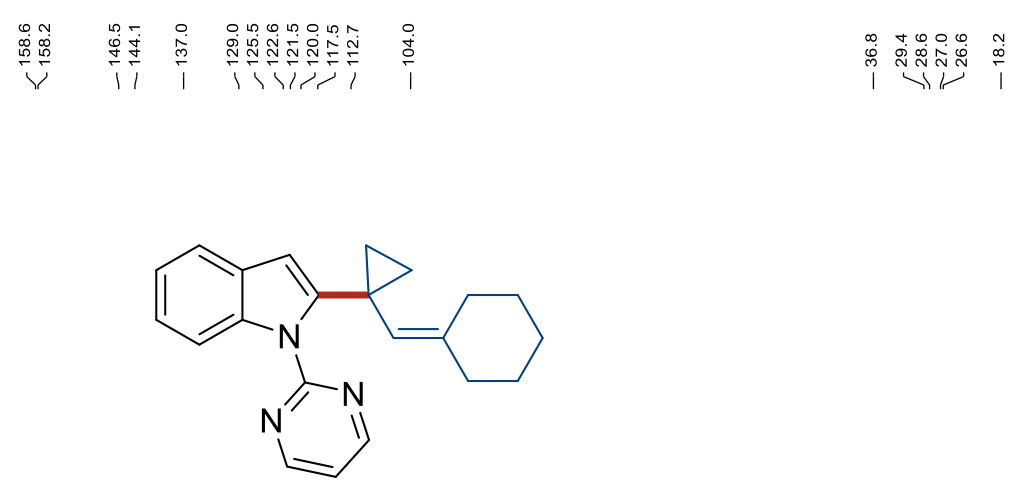

186aj

$\left(101 \mathrm{MHz}^{\mathrm{CDCl}}\right)_{3}$

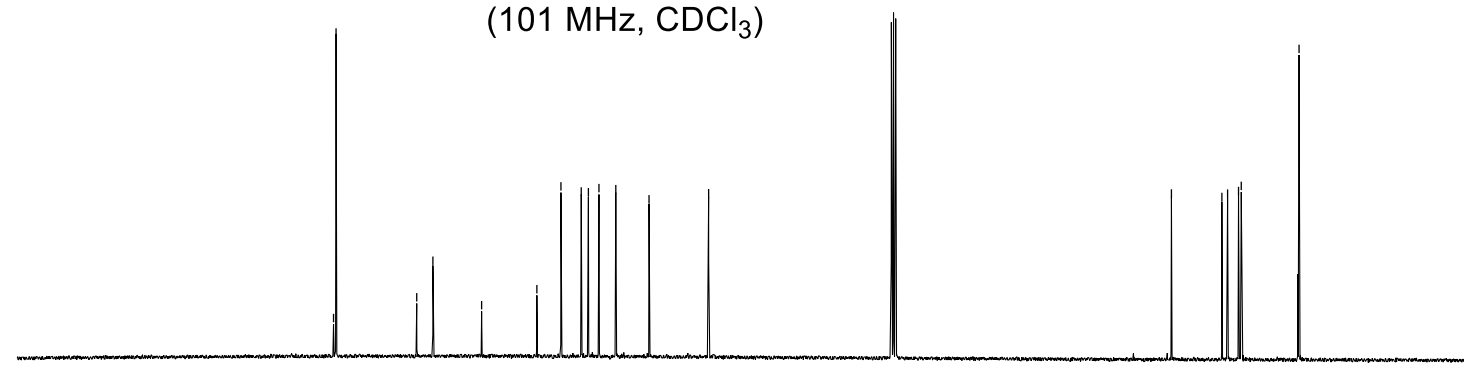

$\begin{array}{llllllllllllllllllllllllllll}1 & 190 & 180 & 170 & 160 & 150 & 140 & 130 & 120 & 110 & 100 & 90 & 80 & 70 & 60 & 50 & 40 & 30 & 20 & 10 & 0\end{array}$ $\underbrace{\substack{\infty \\ \infty}}$

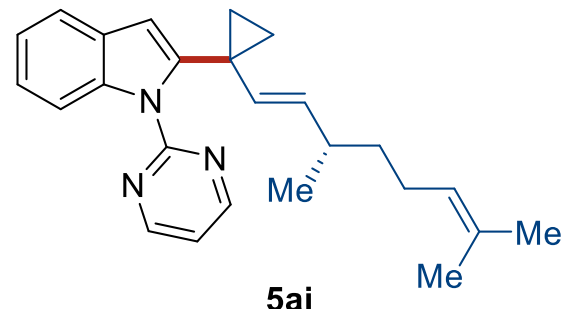

$\left(400 \mathrm{MHz}, \mathrm{CDCl}_{3}\right)$

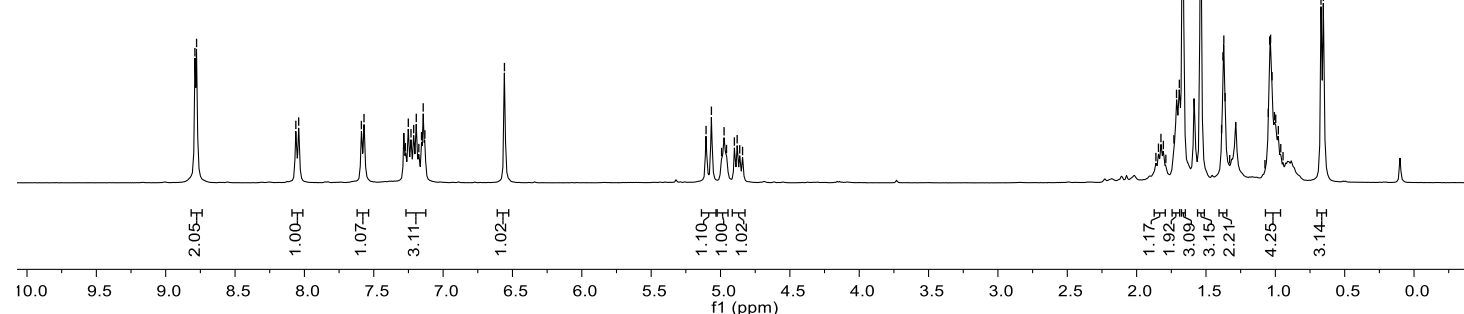




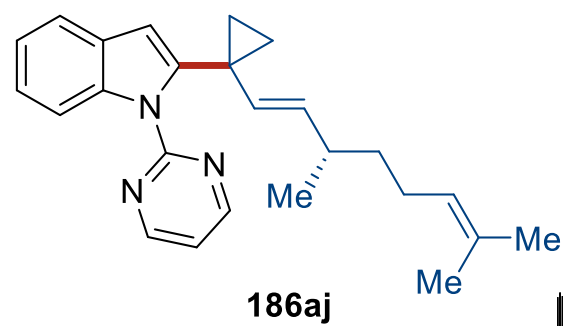

(400 MHz, $\mathrm{CDCl}_{3}$ )
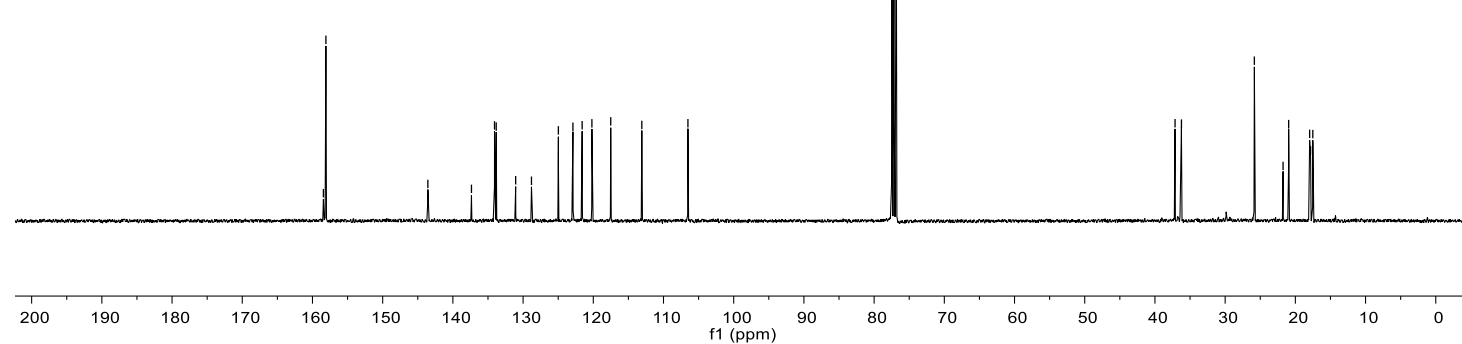

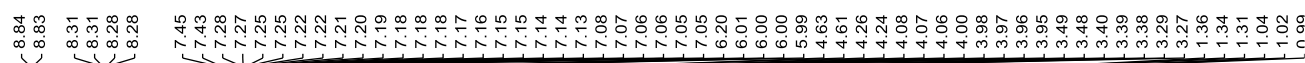<smiles>CCOC(=O)C1=C(C(=O)OCC)C(c2ccccc2)C(c2cc3ccccc3n2-c2ncccn2)=CC1</smiles>

208

(300 MHz, $\mathrm{CDCl}_{3}$ )

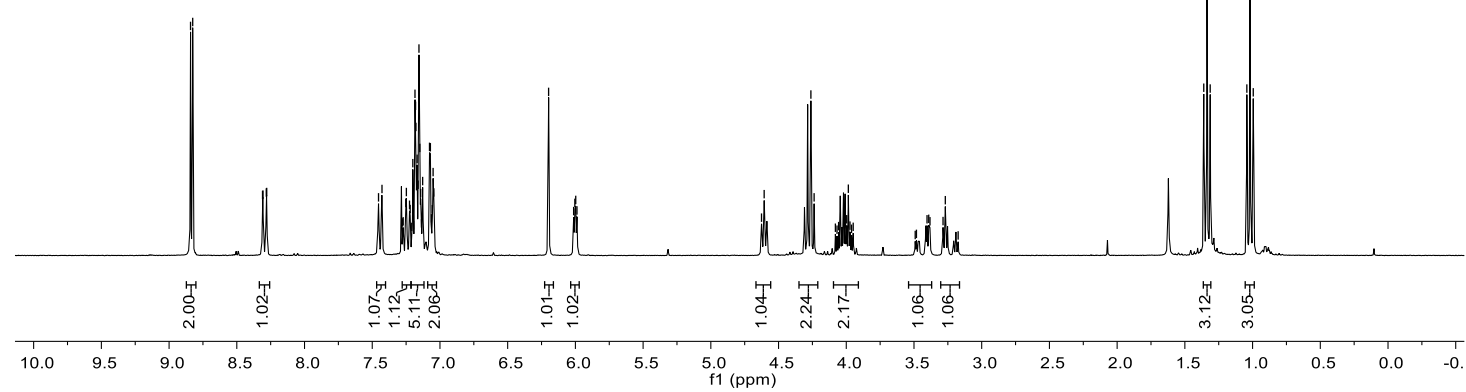




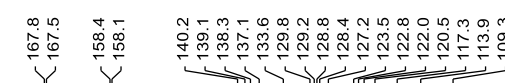

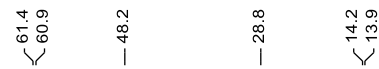

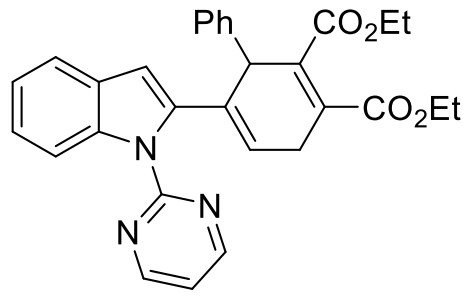

208

$\left(75 \mathrm{MHz}, \mathrm{CDCl}_{3}\right.$ )

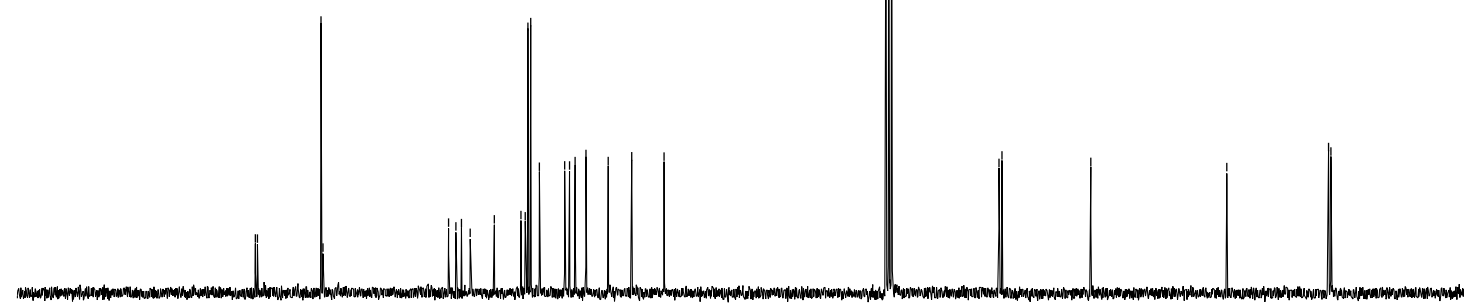

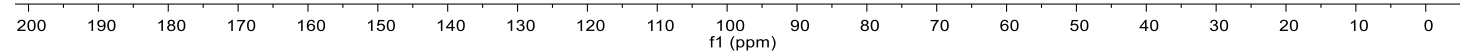

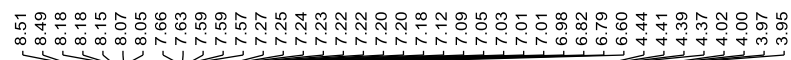

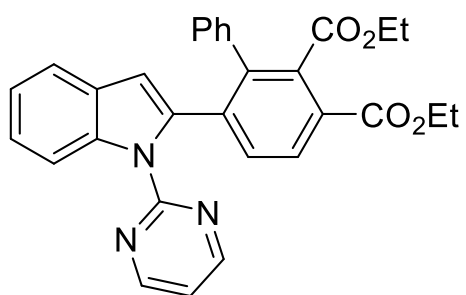

209

$\left(300 \mathrm{MHz}, \mathrm{CDCl}_{3}\right)$

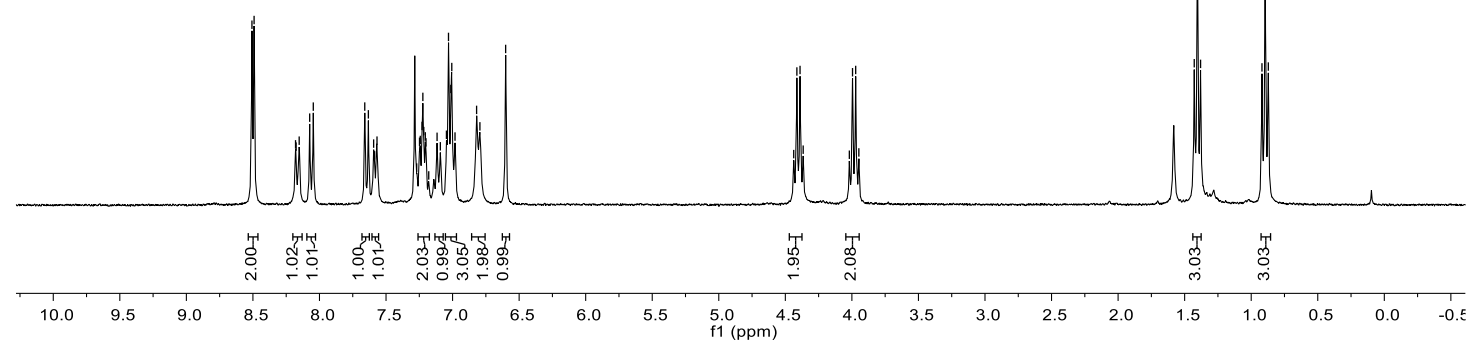




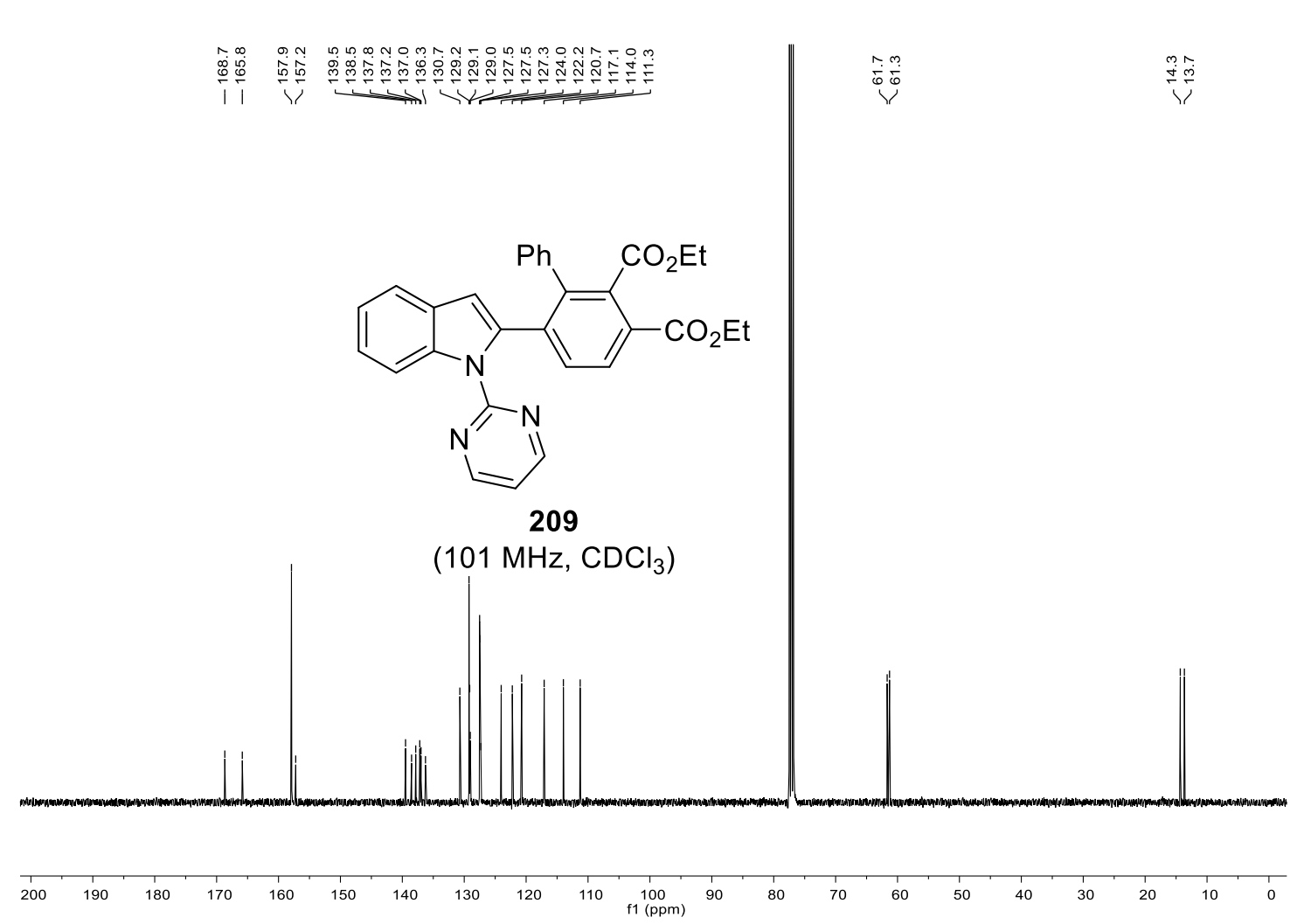




\section{Acknowledgements}

First and foremost, I would like to express my deepest gratitude to my supervisor Prof. Dr. Lutz Ackermann for giving me the opportunity to conduct my $\mathrm{PhD}$ in his researchgroup. Thank you for your support, constant encouragement, your valuable guidance and your interest in my projects. His conscientious academic spirit and modest, openminded personality will inspire me in my future academic study.

I gratefully acknowledge China Scholarship Council (CSC) for the financial support during my research stay in Germany.

I am grateful to Prof. Dr. Konrad Koszinowski for accepting to be my second supervisor. I also would like to thank, Prof Dr. Dr. h.c. Lutz-F. Tietze, Prof. Dr. Manuel Alcarazo, Dr. Michael John and Prof. Dr. Johannes C. L. Walker for agreeing to take part in my defense.

I would like to thank the people from our research group with whom I had the opportunity to collaborate: Dr. Weijun Kong, Dr Gianpiero Cera, Dr Huawen Huang, Dr Santhivardhana Reddy Yetra, Dr. Lars Finger, Dr. Qingqing Bu, Dr. Cuiju Zhu, Dr. Joachim Loup, Dr. Torben Rogge, Dr. Weiping Liu, Dr. Rositha Kuniyil, Dr. Wei Wang, Nikolaos Kaplaneris, Isaac Choi, Isaac Maksso, Sachiyo Nakanowatari. I would also like to extend my gratitude to Dr. Christopher Golz for their assistance with X-ray diffraction analysis, as well as to all the members of the analytical departments (NMR and mass spectrometry) at the IOBC for their continuous support to our research work.

I would like to thank Stefan Beußhausen for taking care of the instruments of our research group, especially the GC-MS, the glovebox and the SPS, which I used almost on a daily basis in the last four years. Thank you for your invaluable help! My gratitude also goes to Karsten Rauch for his continuous support to our lab work, and especially for the preparation of dry solvents and catalysts. I would like to thank Mrs. Gabriele Keil-Knepel and Mrs. Bianca Spitalieri for their continuous assistance with administrative tasks.

I also would like to express my gratitude to all past and present members of the Ackermann research group, especially to members of Lab 308, Karsten Rauch, Dr. Joachim Loup, Dr. 
Torben Rogge, Dr. Thomas Müller, Dr. Gianpiero Cera, Dr. Wei-Jun Kong, Dr. Yulei Wang, Dr. Antonio del Vecchio, Dr. Krzystof Kuciński, Dr. Antonis Messinis, Dr. Cong Tian, Jiayu Mo, Isaac Maksso. I would like to sincerely thank all the people who proofread this thesis: Nikolaos Kaplaneris, Dr. Xuefeng Tan, Dr. Yulei Wang, Dr.Ramesh C. Samanta, Dr. Antonio del Vecchio, Dr. Wei Wang, Shoukun Zhang, Long Yang, Maximilian Stangier, Adelina Kopp, Jun Wu. I also would like to thank all the people who previously corrected manuscripts, supporting information, posters, abstracts and proposals for me: Dr. Torben Rogge, Dr. Joachim Loup, Dr. Weijun Kong, Dr. Xuefeng Tan, Dr. Lars Finger, Dr. Wei Wang, Dr. Krzystof Kuciński, Dr. Rositha Kuniyil. 


\section{Curriculum Vitae}

\section{Personal Information}

\begin{tabular}{ll}
\hline Name: & Zhigao Shen \\
Date of Birth: & 05.02.1989 \\
Place of Birth: & Shaodong, Hunan (P. R. China.) \\
Gender & Male \\
Nationality & Chinese
\end{tabular}

Academic Education

10/2016-11/2020 PhD Candidate in Organic Chemistry

Institut für Organische und Biomolekulare Chemie,

Georg-August-Universität Göttingen

Supervisor: Prof. Dr. Lutz Ackermann

Thesis: C-H Activation by Iron(III), Manganese(II) And Rhodium(III)

Catalysis

09/2012-07/2015 M. Sc. in Pharmaceutical Chemistry

China Pharmaceutical University

Supervisor: Prof. Dr. Yisheng Lai

Thesis: Chiral lon-pair Organocatalyst Promotes Highly Enantiosele-

ctive 3-exo lodo-cycloetherification of Allyl Alcohols

09/2008-07/2012 B.Sc. in Pharmacy

Department of Pharmacy

Yanbian University 


\section{Conferences}

24-29/09, 2017 Poster of the 1st Summer School on Organic Catalysis for Energy

Conversion, Göttingen, Germany.

22-26/06, 2019 The Young Scholar Forum of Xiangtan University, Xiangtan,

China

31/08-02/09, 2019 Poster of the $12^{\text {th }}$ International School of Organometallic

Chemistry (ISOC12) Collegi Universitari, Camerino, Italy.

27-31/07, 2020 ISCHA Virtual Symposium, Göttingen, Germany.

10-14/11, $201516^{\text {th }}$ Tetrahedron Symposium Asia Edition, Shanghai, China

08-13/09, 2013 The $24^{\text {th }}$ International Society of Heterocyclic Chemistry

Congress, Shanghai, China.

\section{Publications}

1. Weijun Kong,' Zhigao Shen,' L. H. Finger, L. Ackermann, Electrochemical Access to Aza-Polycyclic Aromatic Hydrocarbons: Rhodaelectro-Catalyzed Domino Annulations, Angew. Chem. Int. Ed. 2020, 59, 5551-5556. ('Both authors contributed equally)

2. Zhigao Shen,' H. Huang,' C. Zhu, S. Warratz, L. Ackermann, $\mathrm{MnCl}_{2}-$ Catalyzed C-H Alkylation on Azine Heterocycles Org. Lett. 2019, 21, 571-574. ('Both authors contributed equally)

3. S. R. Yetra,' Zhigao Shen, 'H. Wang, L. Ackermann, Thiocarbonyl-Enabled Ferrocene C-H Nitrogenation by Cobalt(III) Catalysis: Thermal and Mechanochemical, Beilstein J. Org. Chem. 2018, 14, 1546-1553. ('Both authors contributed equally)

4. Zhigao Shen,' G. Cera,' T. Haven, L. Ackermann, Tri-Substituted Triazole-Enabled CH Activation of Benzyl and Aryl Amines by Iron-Catalysis, Org. Lett. 2017, 19, 37953798. ('Both authors contributed equally) 
5. C. Zhu, J. C. A. Oliveira, Zhigao Shen, H. Huang, L. Ackermann, Manganese(II/III/I)Catalyzed C-H Arylations in Continuous Flow, ACS Catal. 2018, 8, 4402-4407.

6. W. Liu, G. Cera, J. C. A. Oliveira, Zhigao Shen, L. Ackermann, $\mathrm{MnCl}_{2}$-Catalyzed CH Alkylations with Alkyl Halides, Chem. Eur. J. 2017, 23, 11524-11528.

7. Zhigao Shen,' X. Pan,' Y. Lai,' J. Hu, X. Wan, X. Li, H. Zhang, W. Xie, Chiral Ion-Pair Organocatalyst Promotes Highly Enantioselective 3-exo lodo-Cycloetherification of Allyl Alcohols, Chem. Sci. 2015, 6, 6986-6990. ('These authors contributed equally)

8. Zhigao Shen,' Z. Xia,' H. Zhao, J. Hu, X. Wan, Y. Lai, C. Zhu, W. Xie, Synthesis of Naked Amino-pyrroloindoline via Direct Aminocyclization of Tryptamine, Org. Biomol. Chem. 2015, 13, 5381-5384. ('Both authors contributed equally)

9. X. Yu, J. Hu, Zhigao Shen, H. Zhang, J.-M. Gao, W. Xie, Stereospecific Construction of Contiguous Quaternary All-Carbon Centers by Oxidative Ring Contraction, Angew. Chem. Int. Ed. 2017, 56, 350-353.

10. Z. Xia, J. Hu, Zhigao Shen, X. Wan, Q. Yao, Y. Lai, J.-M. Gao, W. Xie, Enantioselective Bromo-oxycyclization of Silanol, Org. Lett. 2016, 18, 80-83.

11. Z. Xia, J. Hu, Zhigao Shen, Q. Yao, W. Xie, $\mathrm{Re}_{2} \mathrm{O}_{7}$ Catalyzed Dienone-phenol Rearrangement, RSC Adv. 2015, 5, 38499-38502. 


\title{
Erklärung
}

Ich versichere, dass ich die vorliegende Dissertation in dem Zeitraum von Oktober 2016 bis November 2020 am Institut für Organische und Biomolekulare Chemie der

\author{
Georg-August-Universität Göttingen \\ auf Anregung und unter Anleitung von
}

\section{Herrn Prof. Dr. Lutz Ackermann}

selbstständig verfasst und keine anderen als die angegebenen Hilfsmittel und

Quellen verwendet habe.

Göttingen, den 11.2020 